\title{
Biological and Medical Research with Accelerated Heavy Ions at the Bevalac, 1977-1980
}

\author{
M. C. Pirruccello and C. A. Tobias, Editors
}

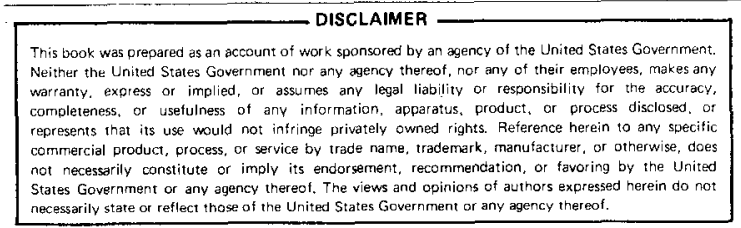

Biology and Medicine Division

Lawrence Berkeley Laboratory

University of California

Berkeley, California 94720

This work was supported by the Office of Health and Environmental Research of the U.S. Department of Energy under Contract W-7405ENG-48. 


\section{DISCLAIMER}

This report was prepared as an account of work sponsored by an agency of the United States Government. Neither the United States Government nor any agency Thereof, nor any of their employees, makes any warranty, express or implied, or assumes any legal liability or responsibility for the accuracy, completeness, or usefulness of any information, apparatus, product, or process disclosed, or represents that its use would not infringe privately owned rights. Reference herein to any specific commercial product, process, or service by trade name, trademark, manufacturer, or otherwise does not necessarily constitute or imply its endorsement, recommendation, or favoring by the United States Government or any agency thereof. The views and opinions of authors expressed herein do not necessarily state or reflect those of the United States Government or any agency thereof. 


\section{DISCLAIMER}

Portions of this document may be illegible in electronic image products. Images are produced from the best available original document. 
(c) Copyright by the Regents of the University of California, 1980. All rights reserved. 


\section{PREFACE}

Over a time span of fourteen years, four major reviews of studies with accelerated heavy ions have been published by the Biology and Medicine Division of Lawrence Berkeley Laboratory. This report is the fifth in that series. The others are: "Biomedical Studies with Heavy-Ion Beams," March 1967 (UCRL-17357); "Initial Radiobiological Experiments with Accelerated Nitrogen Ions at the Bevatron," December 1971 (LBL-529); "Radiobiological Experiments Using Accelerated Heavy Ions at the Bevatron," September 1973 (LBL-2016); and "Biological and Medical Research with Accelerated Heavy Ions at the Bevalac, 1974-1977," Apri1 1977 (LBL-5610).

The first of these reports was written in conjunction with a proposal submitted in 1967 for building a heavy-ion accelerator (the Omnitron). The current report, which comes only five years after heavy beams became available at the Bevalac, is an account of a massive interdisciplinary and multilaboratory research effort, and spells out some pathways for the future when the uses of heavy-ion beams are likely to be more widespread than now. Much of the first report was an attempt to extrapolate knowledge about heavy ions as it existed fourteen years ago, and that report contains several speculations and untested ideas. We have now verified in a quantitative manner many of the early ideas. For example, we have demonstrated that certain heavy ions have a low oxygen effect and that clinical applications of heavy-ion beams to cancer therapy are definitely feasible. Due to advances in accelerator art, therapy and diagnosis can be done on a large scale in the future with biomedical accelerators. Some fields of research that seemed only marginally important in 1967, such as heavy-ion radiography and microscopy, are developing into elaborate disciplines. Other research areas developed very differently from the way they were originally visualized. For example, in 1967 we proposed the injection of radioactive nuclides into the ion source of an accelerator in order to obtain radioactive beams.
Instead, we have shown that portions of inert beams become radioactive whenever they are passed through an absorber. Heavy ions have also proven to be excellent tools for basic research on physical interactions and molecular and cellular effects.

The need for petter diagnosis and therapy of cancer has not diminished. Interdisciplinary studies with heavy-ion beams produced at the Bevalac have much to offer and need to be continued. We believe that the importance of this field to medicine also warrants the construction of medical research accelerators in the near future.

The Bevalac and fundamental research with heavy-ion beams are supported by the Biological and Environmental Research Divison of the U.S. Department of Energy. Much of the cancer research effort is sponsored by the National Cancer Institute; NASA supports investigations related to the safety of space flight. These additional sources of support are cited within individual chapters. The editors wish to express their appreciation for the excellent technical assistance of those people involved in the production of this volume: Loretta Lizama, editorial assistance; Diana Morris, word processing operations; Shirley Ashley, manuscript production; Michael Baublitz, proofreading; Robert Stevens, many of the technical illustrations; Alice Ramirez, composition; John Flambard, cover design; and the entire staff of the Photography Department.
M. C. Pirruccello
C. A. Tobias

Lawrence Berkeley Laboratory November, 1980 



\section{CONTENTS}

PART I. OVERVIEW

Introduction and Overview

E. L. Alpen .................................... 3

International Cooperation in Heavy-Ion Research

C. A. Tobias............................... 7

The Bevalac Biomedical Facility

J. R. Alonso, J. Howard, T. Criswe11, W. Chu, F. Lothrop, and C. Weber.......... 11

PART II. RADIOLOGICAL PHYSICS AND CHEMISTRY RESEARCH

Instrumentation for Measuring Heavy-Ion Fields

J. R. Alonso, E. V. Benton, W. Chu, J. Llacer, J. Richier, and C. A. Tobias ....... 21

Experimental Heavy Particle Physics

A. Nuclear Interactions and Radiation Dosimetry

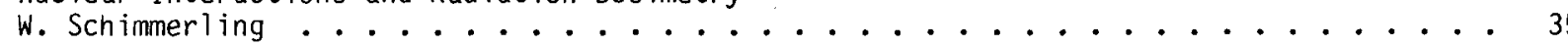

B. Fragmentation Studies Using Nuclear Track Detectors

E. V. Benton, D. J. Hildebrand, R. P. Henke, W. Heinrich, and C. A. Tobias . . . . . . 42

Microdosimetry Research

J. Howard, S. B. Curtis, H. H. Rossi, and P. J. Kliauga . . . . . . . . . . . . 47

Energy Transfer from Heavy Particles

A. Chatterjee and J. L. Magee ......................... 53

Radiation Chemistry of Heavy Particles

J. L. Magee and A. Chatterjee ........................... 63

PART III. CELLULAR AND MOLECULAR RADIOBIOLOGY OF HEAVY IONS

Physical and Cellular Radiobiological Properties of Heavy Ions in Relation to Cancer Therapy Applications

E. A. Blakely, C. A. Tobias, F.Q.H. Ngo, and S. B. Curtis . . . . . . . . . . . 73

Hypoxic Cell Radiosensitizers and Heavy Charged Particle Radiations

W. M. Saunders and J. D. Chapman . . . . . . . . . . . . . . . . . . . 87

Cellular Damage and Repair Following Heavy-Ion Irradiation

F.Q.H. Ngo, E. A. Blakely, T.C.H. Yang, M. J. Yezzi, and C. A. Tobias ......... 89

Effects of Combined Low- and High-LET Radiations

F.Q.H. Ngo, E. A. Blakely, and C. A. Tobias . . . . . . . . . . . . . . 103

Macromolecular Lesions and Cellular Radiation Chèmistry

R. Roots, E. A. Blakely, A. Chatterjee, and C. A. Tobias ............... 113

Silicon: Radiobiological Cellular Survival and the 0xygen Effect

E. A. Blakely, F.Q.H. Ngo, P. Y. Chang, L. Lommel, and C. A. Tobias . . . . . . . . 119

Heavy-Ion Cell-Cycle Response and Progression Effects

E. A. Blakely, F.Q.H. Ngo, P. Y. Chang, L. Lommel, W. Kraft-Weyrather, G. Kraft,

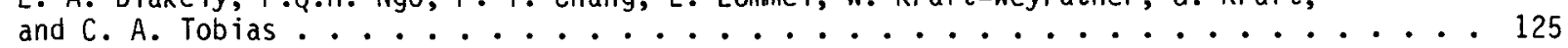

The Repair-Misrepair Model of Cell Survival

C. A. Tobias, E. A. Blakely, and F.Q.H. Ngo . . . . . . . . . . . . . . . 137 
Cell Transformation and Mutagenes is

T.C.H. Yang, F.Q.H. Ngo, J. Howard, and C. A. Tobias . . . . . . . . . . . . . 149

Specific Gene Mutations Induced by Heavy Ions

M. Freeling, C. W. Karoly, and D.S.K. Cheng . . . . . . . . . . . . . . 159

PART IV. HEAVY-ION RADIOBIOLOGY OF TISSUES AND TUMORS

In Vivo Tumor Radiobiology of Heavy Charged Particles

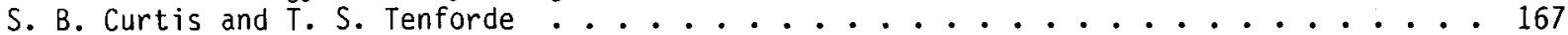

Heavy-Ion Radiobiology of Multicellular Tumor Spheroids

A. Rodriquez and E. L. Alpen . . . . . . . . . . . . . . . . . . . 177

The Relative Biological Effect of High Z, High LET Charged Particles on

Spermatogonial Killing

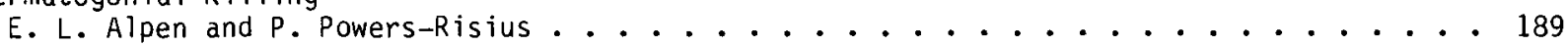

Survival of Intestinal Crypt Cells After Exposure to High Z, High Energy Charged

Particles

E. L. Alpen, P. Powers-Risius, and M. McDonald . . . . . . . . . . . . 199

Estimate of the Therapeutic Ratio for Charged Particle Beams

T. S. Phillips and L. S. Goldstein . . . . . . . . . . . . . . . . 207

Response of Mouse Skin and Bone Marrow to Heavy Charged Particles

E. J. Ainsworth . . . . . . . . . . . . . . . . . . . 225

Dose Response for Rat Skin Tumors Induced by Single and Split Doses of Argon Ions

F. J. Burns and R. E. Albert . . . . . . . . . . . . . . . . . . . . 233

Response of Rat Spinal Cord to Single and Fractionated Doses of Accelerated Heavy Ions

J. L. Leith, M. McDonald, P. Powers-Risius, S. F. Bliven, R. E. Walton,

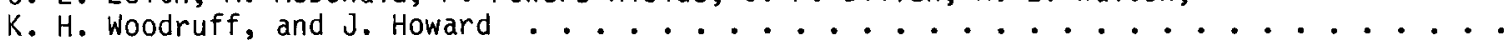

Genetic Effects of Heavy-Ion Beams on Mice

L. S. Goldstein .................................

Carcinogenesis with Heavy-Ion Radiation in the Harderian Gland of Mice

E. L. Alpen, R.J.M. Fry, P. Powers-Risius, and E. J. Ainsworth ............

Cataractogenic Effects of Heavy Charged Particles in Mice

E. J. Ainsworth, J. G. Jose, V. V. Yang, and M. E. Barker . . . . . . . . . . . 261

PART V. SHORT- AND LONG-TERM EFFECTS OF LOW FLUENCE HZE PARTICLES

Abundances, Energy and LET Spectra of HZE Particles in Space

S. B. Curtis and E. V. Benton ...........................

Thermophysical Lesions Caused by HZE Particles

C. A. Tobias, M. J. Malachowski, A. L. Nelson, and D. E. Philpott . . . . . . . . 277

HZE Particle Effects in the Mammalian Brain: Relevance to Manned Space Flight

L. M. Kraft, F. E. D'Amelio, and E. V. Benton . . . . . . . . . . . . . 287

Life Span Studies on Mice Exposed to Heavy Charged Particles or Photons: Preliminary Results

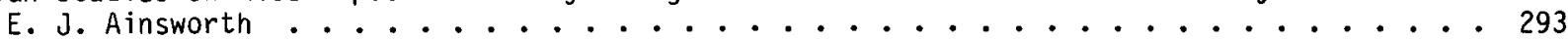

PART VI. MEDICAL APPLICATIONS OF HEAVY IONS

SECTION A. RADIATION THERAPY

Clinical Results in Heavy Particle Radiotherapy

J. R. Castro, J. M. Quivey, W. M. Saunders, K. H. Woodruff, G.T.Y. Chen,

J. T. Lyman, S. Pitluck, C. A. Tobias, R. E. Walton, and T. C. Peters ........ 
Radiological Physics of Heavy Charged-Particle Beams Used for Therapy

J. T. Lyman, J. Howard, L. Kanstein, and J. R. Alonso . . . . . . . . . . . . . 319

Heavy Charged-Particle Treatment Planning

G.T.Y. Chen, S. Pitluck, and J. T. Lyman ...................... 331

\section{SECTION B. HEAVY-ION RADIOGRAPHY AND MICROSCOPY}

Projection Radiography and Tomography

C. A. Tobias, J. I. Fabrikant, E. V. Benton, and W. R. Holley . . . . . . . . . 335

Heavy-Ion Mammography and Breast Cancer

J. I. Fabrikant, C. A. Tobias, M. P. Capp, W. R. Holley, K. H. Woodruff,

and E. A. Sickles . . . . . . . . . . . . . . . . . . . 347

Heavy-Ion Radiography Applied to Charged Particle Radiotherapy

G.T.Y. Chen, J. I. Fabrikant, W. R. Holley, C. A. Tobias, and J. R. Castro........ 359

Active Heavy-Ion Radiography and Computerized Tomography

J. Llacer, W. Chu, C. A. Tobias, J. I. Fabrikant, and J. R. Alonso . . . . . . . 367

Heavy-Ion Microscopy

G. Kraft, T.C.H. Yang, T. Richards, and C. A. Tobias . . . . . . . . . . . 375

High Energy Beams of Radioactive Nuclei and Their Biomedical Applications

A. Chatterjee, E. L. Alpen, J. Llacer, J. R. Alonso, and C. A. Tobias . . . . . . 383

SECTION C. FOCAL LESIONS

Recent Advances in Pituitary Research

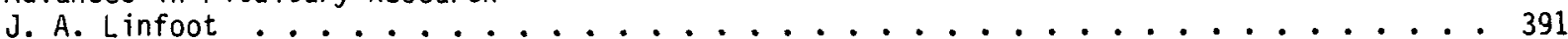

Focal Lesions in the Central Nervous System

J. I. Fabrikant, T. F. Budinger, C. A. Tobias, and J. L. Born . . . . . . . . . 399

Restriction of the Spread of Epileptogenic Discharges in Cats by Intracranial Irradiations

C. T. Gaffey, V. J. Montoya, J. T. Lyman, and J. Howard . . . . . . . . . . . . . . 407

PART VII. THE FUTURE

A Future Dedicated Medical Ion Accelerator

R. A. Gough, J. R. Alonso, T. Elioff, and H. Grunder . . . . . . . . . . . . . 415

STAFF LIST. . . . . . . . . . . . . . . . . . . . . . . . . . . 421

PROGRAM ADVISORY COMMITTEE. . . . . . . . . . . . . . . . . . . . . . 423 
PART I. OVERVIEW

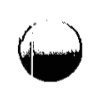


$\bullet$

$\bullet$ 


\title{
INTRODUCTION AND OVERVIEW
}

\author{
E. L. Alpen
}

The Lawrence Berkeley Laboratory is the birthplace both of the accelerator technology that now dominates modern physical science, and of the biomedical applications of these accelerators. Since the first small 4-inch Cyclotron was built by $E$. 0 . Lawrence in 1930, a series of increasingly complex accelerators has been designed and built at Berkeley, culminating with the Bevalac, a high energy heavy-ion accelerator.

Since the late 1930s there has been a parallel ongoing effort to utilize these unique machines for biological and medical research. Almost from the beginning there has been an interest in the use of beams of accelerated particles to treat cancer and other diseases in man. The first treatments of human beings with neutron beams were delivered in 1938 by R. S. Stone and $h$ is colleagues using the Berkeley 37-inch Cyclotron (Stone et a 7., 1940). The scientific rationale for the use of accelerated atomic nuclei was originally proposed by Robert Wilson in 1946, based on the advantageous depthdose distributions achieved with heavy ions stopping in matter (Wilson, 1946). A year later, when the 184-inch Synchrocyclotron was completed by E. O. Lawrence and his associates (Brobeck et al., 1947), the first biological and biophysical investigation of high energy accelerated nuclei were started by Tobias and his colleagues (Tobias et al., 1952). Within a few years, exposures of patients for therapeutic purposes began (Tobias et a1., 1955a,b, 1958).

Beginning in the early 1950s, beams of protons, deuterons, and helium ions were used for irradiation of the pituitary gland in humans with endocrine disorders (Lawrence et al., 1953, 1959; Tobias et al., 1955a,b). This program is.still active; over 700 patients have been treated. In 1975, the program was expanded to include the treatment of human cancers with helium ions at the 184-inch Synchrocyclotron, and with heavier ions at the Bevalac (Castro et al:, 1980). At both of these machines, beam delivery systems have been built that provide large, uniform, precisely calibrated beams for patient treatments. Sophisticated control systems have been developed that provide the high degree of safety and reliability needed for human treatments.

In the early 1970s, with accelenator design expertise increasing (Grunder et al., 1971; white. et al., 1971; Isaila et al., 1972), it became possible to examine the biological effects of high-energy, heavy ions.... Synchrotrons at Princeton and Berkeley accelerated ions with atomic numbers between 6 and 18, at energies that permitted several biological studies (LBL, 1971, 1973; Hall and Lehnert, 1973; Underbrink et al., 1973). The biological effectiveness of these high $Z$ ion beams indicated a likelihood of an enhanced therapeutic potential when comparisons were made with lower $Z$ ion beams (Tobias, 1973).

The construction of the Bevalac accelerator complex (Ghiorso et al., 1973) for physics and nuclear science research al so expanded the opportunity for biological and medical studies with charged particles.

In 1974 the programmatic decision was made to establish the Bevalac as a national accelerator facility to conduct biomedically related heavy-ion research. DOE, then ERDA, established line item funding in the budget of the agency for accelerator operations. From the beginning it was established that the beam would be available to all potential users throughout the nation. procedures were developed and have operated smoothly for time assignment. A review committee, the Program Advisory Committee, which reports to the Director of $L B L$, examines all beam time proposals. The proposals are approved or disapproved on scientific merit, and, if approved, assigned a priority for beam time. Local support is provided to outside users to permit efficient utilization of beam time.

A broad and comprehensive study to investigate the biomedical applications of high-energy heavy ion beams was begun at LBL in the early 1970s. LBL-5610 summarizes research on heavy ions through 1977 (LBL, 1977); the present document covers progress during the intervening years. As research has revealed the basis for a large number of applications of high-energy particle beams, heavy-ion radiation medicine and biology is increasingly receiving the attention of an expanding international community. Tobias describes the interest of the French, Russian, Japanese, and Canadian groups in a following chapter.

A primary advantage of charged particle therapy is that it offers a precise delivery of radiation dose to the tumor with a concomitantly low dose to surrounding tissues. Clinically, potential damage to healthy, surrounding tissue usually limits the dose that can be safely delivered to a tumor. Compared to other radiation modalities, heavy-ion beams can cause more biological damage to the tumor at a lower rate of damage to healthy tissue. : Even a small additional dose to the tumor should increase the probability of local control. The sparing effect of particles on normal tissues and the comparatively high level of damage to tumors is a result of two factors: first, physical dose can be delivered at depth with a lower entrance dose; and second, the physical dose delivered by stopping particles is biologically more effective due to specific energy deposition properties of charged particles. 
Other available modalities (e.g., protons, pions, neutrons) share this feature of enhanced biologically effective dose at depth to varying degrees, and it is therefore of interest to compare the importance of this effect for available radiotherapeutic modalities.

The dual panels of Figure 16 shown by Blakely et al. in the first chapter of Part III demonstrate how the consideration of both physical dose and the corresponding enhancement of biological killing effectiveness influences the comparison of the modalities. The abscissa in these plots is the product of two ratios: the physical dose at the entrance, and the enhanced biological killing effectiveness at depth to the enhanced biological killing effectiveness for the entrance dose. The comparison of the biological effectiveness is based on measurements made with cultured cells in vitro. The top panel was constructed for a $10 \mathrm{~cm} \times 10 \mathrm{~cm}$ field at 10 to $14 \mathrm{~cm}$ tissue depth. The bottom panel was constructed for a $10 \mathrm{~cm} \times 10 \mathrm{~cm}$ field at 14 to $24 \mathrm{~cm}$ tissue depth.

In both plots, the conventional radiation modalities ( $X$ rays and gamma rays) occupy the least desirable upper left portion of the plot (notably short on the abscissa scale, as are. neutrons). For the smaller, more shallow target volume, it appears that the $308 \mathrm{MeV} / \mathrm{amu}$ carbon, $425 \mathrm{MeV} / \mathrm{amu}$ neon beams, and $65 \mathrm{MeV}$ negative pions are superior in their ratio of biologically effective doses.

Argon and silicon ions, and protons and nelium ions, are intermediate on the abscissa, but quite different from each other with respect to their placement on the ordinate axis, a point that will be discussed later.

For a larger, deeper tumor, the relative placement of the therapy modalities changes except for the location of the proton and helium data. At this range, as seen in the lower panel of this figure, the $400 \mathrm{MeV} / \mathrm{amu}$ carbon and $228 \mathrm{MeV} / \mathrm{amu}$ helium beams are quite similar, as are the $557 \mathrm{MeV} / \mathrm{amu}$ neon beam and the $85 \mathrm{MeV}$ pion beam. The neutron beams and low-LET modalities have deteriorated considerably in their effective dose ratio on the abscissa. The effect of argon and silicon has been estimated at energies of $700 \mathrm{MeV} / \mathrm{amu}$ and $670 \mathrm{MeV} / \mathrm{amu}$, respectively. Their locations are intermediate on the abscissa, but are clearly almost a factor of two better in effective dose ratio than the neutron beams.

Physical dose distributions are enormously important but the biological effectiveness factor must also be considered when comparing effectiveness of various treatment modalities. The capacity to kill hypoxic cells is considered the other major advantage of high-LET modalities. This factor constitutes the ordinate in the vector plots of Figure 16.
The radiobiological oxygen effect is usually studied by measuring cell survival curves for different combinations of particle beams and comparing those obtained in the presence of air and in nitrogen. The ratio of doses at which the same biological effect is obtained is termed the oxygen eninancement ratio (OER).

$$
O E R=\frac{D_{0} \text { nitrogen }}{D_{0} \text { air }} \text { (at constant survival) }
$$

Detailed studies have shown that the OER is reduced from a level of 3 to nearly unity when ionizations per unit track length of stopping charged particles are increased. The most advantageous position for a therapeutic modality is therefore located at the lower right portion of the vector plots in Figure 16 .

For both treatment depths in the upper and lower panels of this figure, it is clear that neutrons, silicon, and argon beams are superior to the other modalities in reducing the oxygen effect. The low LET modalities and protons are clearly inferior in this regard. Pions, and helium, carbon and neon beams are intermediate in their measured potential for reducing the oxygen effect.

In considering these various biological and physical aspects, it is essential to weigh the merits of both depth-effect advantages, and reduction of the oxygen effect. The superiority of heavy ions compared to other modalities with respect to both effective dose delivery and reduced oxygen effect will be significant, especially for tumors where hypoxic fractions have resulted in local treatment failure.

These comparisons continue to support our interest in continued development of high energy charged particle beams for radiotherapy.

Furthermore, research on imaging with heavy ions, described in Part VI of this report, encourages us to believe that significant new scientific advances are possible through application of heavy-ion research in this area. Because of the special property of heavy ions that their stopping is determined by electron density rather than photoelectron interactions, as is the case for $x$ rays, it is possible that new and previously unavailable findings $c$ an be achieved through heavy-ion imaging.

Finally, it would be inappropriate, in a laboratory known for its contributions to basic research, not to point out the enormous significance to fundamental radiation biology, biophysics, and chemistry of the findings described in this report and yet to be discovered by investigators using heavy-ion beams. Using the beams available at the Bevalac, we have a special opportunity to apply the special properties of accelerated heavy-ion beams to test fundamental assumptions of our science. 
References

Brobeck, W. M., E. 0. Lawrence, K. R. McKenzie, E. M. McMillian, R. Serber, D. C. Sewell, and R. L. Thornton. 1947. Initial performance of the 184-inch cyclotron of the University of California. Phys. Rev. 7l, 449.

Castro, J. R., J. M. Quivey, J. T. Lyman, G.T.Y. Chen, C. A. Tobias, and T. L. Phillips. 1980. Current status of heavy charged particle radiotherapy at Lawrence Berkeley Laboratory. Cancer 46, 633-641.

Ghiorso, A., H. A. Grunder, W. Hartsough, G. Lambertson, E. Lofgren, K. Lou, R. Main, R. Mobley, R. Morgada, W. Salsig, and F. Selph. 1973. The Bevalac: An economical facility for very high energetic heavy particle research. IEEE Trans. Nucl. Sci. NS-20, 155. (Abstract).

Grunder, H. A., W. D. Hartsough, and E. J. Lofgren. 1971. Acceleration of heavy ions at the Bevatron. Science 174, 1128-1129.

Hal1, E. J. and S. Lehnert. 1973. The biophysical properties of $3.9 \mathrm{GeV}$ nitrogen ions. IV. OER and RBE determinations using cultured mammalian cells. Radiat. Res. 55, 431-436.

Isaila, M. V., W. Schimmerling, K. G. Vosburgh, M. G. White, R. C. Fritz, and P. J. McNulty. 1972. Acceleration of argon ions to $1.17 \mathrm{x}$ $10^{10}$ electron volts. Science 117, 424-425.

LBL (Lawrence Berkeley Laboratory). 1971. Initial Radiobiological Experiments with Accelerated Nitrogen Ions at the Bevatron. Lawrence Berkeley Laboratory Report LBL-529.

LBL (Lawrence Berkeley Laboratory). 1973. Radiobiological Experiments Using Accelerated Heavy Ions at the Bevatron. Lawrence Berkeley Laboratory Report LBL-2016.

LBL (Lawrence Berkeley Laboratory). 1977. Biological and Medical Research with Accelerated Heavy Ions at the Bevalac, 1974-1977. Lawrence Berkeley Laboratory Report LBL-5610.

Lawrence, J. H., C. A. Tobias, J. A. Linfoot, J. L. Born, A. Gottschalle, and R. P. Kling. 1953. Heavy particles: The Bragg curve and suppression of pituitary function in diabetic retinopathy. Diabetes 12, 490-501.
Lawrence, J. H., J. L. Born, C. A. Tobias, R. Carlson, F. Sangalli, and G. Welch. 1959. Clinical and metabolic studies in patients after alpha particle subtotal or total hypophysectomy. Medicine in Japan $5,859-862$.

Stone R. S., J. H. Lawrence, and P. C. Aebersold. 1940. A preliminary report on the use of fast neutrons in the treatment of malignant disease. Radiology 35, 322-327.

Tobias, C. A. 1973. Pretherapeutic investigations with accelerated heavy ions. Radiology 108, 145-158.

Tobias, C. A., Anger, H. 0., and J. H. Lawrence. 1952. Radiological use of high energy deuterons and alpha particles. Am. J. Roentgeno 1. 67, 1-27.

Tobias, C. A., J. E. Roberts, J. H. Lawrence, B.V.A. Low-Beer, H. O. Anger, J. L. Born, R. McCombs, and C. Huggins. 1955a. Radiation Hypophysectomy with High Energy Proton Beams. Lawrence Berkeley Laboratory Report UCRL-3035.

Tobias, C. A., J. E. Roberts, J. H. Lawrence, B.V.A. Low-Beer, H. O. Anger. J. L. Born, R. McCombs, and C. Huggins. 1955b. Irradiation hypophysectomy and related studies using $340-\mathrm{MeV}$ protons and $190 \mathrm{MeV}$ deuterons. Proceedings International Conference on Peaceful Use of Atomic Energy, Geneva To, 95-106

Tobias, C. A., J. H. Lawrence, J. L. Born, R. K. McComb, J. E. Roberts, H. 0 Anger, B.V.A. Low-Beer, and C. B. Huggins. 1958. Pituitary irradiation with high energy proton beams. A preliminary report. Cancer Res. 18, $121-134$.

Underbrink, A. G., L. A. Schairer, and A. H. Sparrow. 1973. The biophysical properties of 3.9 GeV nitrogen ions. V. Determination of relative biological effectiveness for somatic mutations in Trandescantia. Radiat. Res. 55, $437-446$.

White, M. G., M. Isaila, K. Predec, and H. L. Allen. 1971. Acceleration of nitrogen ions to $7.4 \mathrm{GeV}$ in the Princeton particle accelerator. Science 174, 1121-1123.

Wilson, R. R. 1946. Radiological use of fast protons. Radiology 47, 487-491 
6

Blank 


\title{
INTERNATIONAL COOPERATION IN HEAVY-ION RESEARCH
}

\author{
C. A. Tobias
}

The international ties of scientific collaboration of the Lawrence Berkeley Laboratory date back over half a century to the time when Ernest 0. Lawrence conceived and constructed $h$ is first cyclotron. The cyclotron not only became the sole source for the production of artificial radioisotopes in biology and medicine, but also provided a unique base for neutron and charged particle research. Ernest and $h$ is brother John Lawrence set the tone for international scientific collaboration, which has fluorished to the present time. They opened their laboratories to visiting foreign scientists, many of whom later became leaders in their scient if ic fields and most continued their scientific exchange and collaboration with us. For example, the late George Hevesy, who originated biological tracer research, depended on this laboratory to supply most of his $32 \mathrm{p}$ and $49 \mathrm{Fe}$ to Denmark. Years later, Hevesy came to LBL for an extended visit and performed the first "suicide" experiments with radioisotopes.

When, in 1939, the first attempts to produce low energy carbon beams at LBL proved successful, a scientist from India, Nag Chawdhury, was among the postdoctoral fellows involved in this research at Berkeley. One of his goals was to build the first cyclotron in Calcutta, and he began this task with machine parts donated by LBL. He later became Vice Chancellor of Nehru University, New Delhi. Work is underway in 1980 to extend the acceleration energy of several heavy ions at the Calcutta cyclotron to $250 \mathrm{MeV} / \mathrm{amu}$.

Following World War II a group of Swedish scientists, led by The Svedberg, the inventor of the ultracentrifuge, visited $L B L$. At that time, research had begun on the initial biomedical. applications of protons, deuterons, and helium ions at the new 184-inch Synchrocyclotron. Assisted by an exchange of information with our accelerator scientists, Svedberg's group proceeded to design and build the first Swedish proton synchrotron in Uppsala to be used for biomedical and for basic physics research. During 1954 to 1957 two LBL students, Victor Burns (now at U.C. Davis) and Charles Soudhaus (now at U.C. Irvine), spent postdoctoral years working with the Uppsala group and at the Karolinska Institute. Cornelius Tobias spent a sabbatical year there as we11. Good cooperation developed with the Swedish group that has now lasted for many years. The biophysicist Borje Larson and his group developed the first "ridge filter" to modulate proton beams, and that group has pioneered proton therapy of cancer; these developments have aided us in the application of heavy-ion research to oncology.
The introduction, by the late Swedish neurosurgeon Herbert 01 ivercrona, of pituitary surgery for the treatment of advanced metastatic breast cancer encouraged John Lawrence and our group in the early 1950s to develop hel ium-ion hypophysectomy. This procedure has proven successful for the treatment of pituitary tumors, notably acromegaly and Cushings disease, and is now used routinely as the therapy of choice for these endocrine and metabolic diseases. Pituitary irradiation also appears to have promise for the control of metastatic, hormone-dependent carcinomas and of diabetes mellitus with associated vascular disease. Currently, we are studying localized focal lesions in the central nervous system with Bevalac accelerated carbon and neon particles, and the future therapeutic use of focal irradiation of the pituitary and of local regions of the brain and spinal cord also appear promising. Among the conditions to be treated with heavy ions are arteriovenous malformations and vascular fistulae. L. Leksell, professor of neurosurgery at the Karolinska Institute, Stockholm, did pioneer work with J. Ray on the therapy of these conditions. The first patient treated with a helium Bragg peak for arteriovenous malformation in 1980 was referred from Oxford University by R. Berry, and the treatment at $L B L$ was based in part on experience gained by L. Leksell with gamma rays.

The first low-energy heavy-ion accelerators were built in the 1960 s at $L B L$ and at Yale University. The first biological experiments were carried out at LBL by Joseph Sayeg and his colleagues. Norwegian scientists who were at LBL as postdoctoral fellows at that time also performed basic biophysical studies with these beams. Tor Brustad, of the Norsk Hydro Institute in $0 \mathrm{slo}$, demonstrated the enzyme inactivating properties of heavy ions, and Thormod Hendricksen of Oslo University obtained the first free radical yields on the effects of accelerated heavy ions on biological macromolecules. During this period we had extensive association with the French scientists, Maurice Tubiana, of the Institut Gutave-Roussy, and Paul Blanquet, of Bordeaux University Medical School. G. Tilsjar Lentules of Julich University in Germany was the first scientist to demonstrate the effects of accelerated protons on the electroencephalogram.

For the initial heavy-ion radiobiological studies, human cells in culture were used by paul Todd, Pennsylvania State University. Edward Barendson of TNO in the Netherlands initially developed the stable T-1 cell strain for radiological work. He spent a sabbatical period here, and participated in the studies of the oxygen effect. Later one of our staff members, Stanley 
Curtis, spent a sabbatical year at Rijswijk and brought from Holland to our laboratory the WAGI Rij rat rhabdomyosarcoma tumor system. Biomedical research with rhabdomyosarcoma then became an integral part of our heavy-ion research program.

Visiting Japanese scientists working with us during the time the SuperHILAC was developed were Nabuo Oda of the Japanese Institute Technology, Tokyo, and Sahaya Nakai of the National Institute of Radiological Sciences, Chiba, Japan. Oda specialized in the physics of delta-ray production by heavy ions in their passage through matter; Nakai made basic contributions to the genetic crossover frequencies caused by accelerated heavy ions in yeast cells. Collaboration between $H$. H. Smith of the Brookhaven National Laboratory and T. Tazima of the Institute of Genetics, Tokyo University, and John Lyman of LBL demonstrated that low-energy accelerated heavy ions are highly mutagenic and tumorigenic in plants. J. Madvanath of India worked in radiation biophysics at LBL on heavy ion effects on leukocytes; today he is working with the National Radiological Institute in Bombay.

The U.S.S.R. has built a low-energy heavy-ion accelerator, and there nave been exchange visits between the U.S.S.R. and the U.S. relating to space science. The exchange of information produced at these visits resulted in a joint American-Soviet report on the hazards of space radiation. In 1965, Cornelius Tobias participated in a visit to the U.S.S.R. to study progress in neuroradiobiology, and visited the accelerator laboratories at Dubria. Soviet physicists held a colloquium relating to the potential biomedical uses of charged particles. Soon afterwards, the Soviet scientists built a proton biomedical facility at Dubna. Extensive developments have followed at the Moscow F.E.T.P. accelerator, which has a large proton cancer therapy program, and at the Leningrad proton synchrotron, where there is a pituitary irradiation program.

\section{CURRENT INTERNATIONAL COLLABORATIONS}

The Bevalac is the only accelerator in the world capable of producing heavy ions with substantial beam penetration qualities that are suitable for medical applications in humans. The current international collaborations which have centered on medical applications presently involve nine countries and international organizations.

\section{France}

There is a formal agreement between the Gustave-Roussy Institut of Cancer Research, Villejuif, France and LBL for cooperative heavyion-related biomedical research that extends to the exchange of scientists, and scientific information. The synchrotron at Saclay is being extended to produce heavy ions, especially carbon beams, and Maurice Tubiana and Jean Dutreix plan to develop heavy-ion cancer therapy. Edmund
Malaise and his associates at the Institut are studying oncological radiobiology. Roland Gilet spent several months at LBL with Stanley Curt is studying heavy-ion-induced cell division delay. Marcelle Guichard recently spent several months at LBL investigating heavy charged particle radiation effects on human melanoma cells growing in immunodeficient mice. Current $7 y$, Jacques Richier from Lyon University is on a French government fellowship to study radiological pnysics problems related to the fragmentation of heavy ions and the production of radioactive beams with Aloke Chatterjee at LBL. Cooperative research with 0 . Isabelle of Université Clermont Ferrand is $p l a n n e d$ to study the visual effects of heavy-ion and radiobiological damage in the retina.

\section{$\underline{\text { Japan }}$}

There is interest in Japan in heavy-ions research, including the use of low-energy ion guns for micro-etching and implantation, and high-energy heavy-ion physics, radiography, and therapy. Since 1977 we have participated in meetings of the United States-Japan Cooperative Radiation Oncology Program. The first meeting, held in Berkeley, had 14 Japanese physicians and scientists participating. Two meetings which were held in Japan had several LBL staff members attending, and the next meeting is scheduled for November 1980.

The Japanese scientists have a heavy-ion accelerator at the Institute of Physical and Chemical Research near Tokyo. We visited this HILAC that produces beams $7.5 \mathrm{MeV} / \mathrm{amu}$; there are plans to increase the energy to greater than $100 \mathrm{MeV} / \mathrm{amu}$. Sadayoshi Fukumoto and Yasuo Hirao will present plans for a Japanese medical heavyion accelerator at the next American-Japanese meeting.

At the initiative of Nabuo $0 d a$ and $K$. Sakamoto, formal agreement proposals have been sent to the U.S. National Science Foundation and the Japanese Research Council to support the exchange and collaboration of scientific research with heavy ions. The fields covered by the agreement are radiological physics and chemistry, molecular and cellular radiobiology, and tissue radiobiology. Shigefumi Okada of Tokyo University is currently spending a sabbatical at $L B L$, investigating the role of DNA strand breaks in cellular injury due to heavy ions. Other Japanese scientists are presently visiting the nuclear science department of LBL.

\section{West Germany}

There is an intense collaboration between scientists at LBL and several laboratories in Germany. A radiological physicist, Eckhart Schneider (Strahlencentrum Giessen) visited and participated at the Bevalac for about one year. A radiobiologist, Christine Lücke-Huhle (GFK Karlsruhe), spent six months with Eleanor Blakely and our cell group. Her work on cell kinetics and spheroids was particularly interesting 
because the results with heavy ions could be directly compared to similar experiments she has performed with pi mesons at SIN.

Edward Alpen was an invited participant at the symposium on relativistic heavy-ion research held at the heavy-ion accelerator laboratory (GSI) at Darmstadt in 1978. Because there are plans to extend the energy of the existing accelerator Unilac at GSI from $10 \mathrm{MeV} / \mathrm{um}$ to several $\mathrm{GeV} / \mu \mathrm{m}$, there is a great interest in our experience with the Bevalac.

Currently Gerhard Kraft and Wilma KraftWeyrather are spending a year with our radiation biophysics group. Gerhard is collaborating with us in developing heavy-ion microscopy and a novel electrooptial technique for the study of radiation effects in DNA. Wilma and Eleanor Blakely are carrying out special experiments on the role of radiosensitizers. With Director Putlitz we are making plans for continued collaboration with GSI in the field of basic physical and biological research.

\section{Switzerland}

There are scientific relationships with the radiobiology laboratory of the University of Zurich. Hans Blattmann spent a year with our radiation biophysics group at LBL studying dosimetry and beam control techniques; he is applying this to pion dosimetry at Zurich. Scientists working with Heidi Fritz-Niggli are studying vascular effects due to pi meson stars in the newborn rat brain; they propose to study these vascular effects and also genetic effects induced by our heavy-ion beams at LBL.

\section{Sweden}

A Swedish postdoctoral fellow, Bengt Langstrom, spent a year in our laboratory in 1976 studying the properties of radioactive beams for radiochemical synthesis of labeled compounds. With improvement in our laboratory techniques of generating and measuring radioactive beams, there are plans for Langstrom's second visit toward further research with the radioactive beams.

\section{England}

There are continuous collaborations with British radiation scientists concerning research in heavy-ion radiobiology and radiation therapy. Some of the basic rationale for using heavy-ion beams in cancer therapy relates to the radiobiological oxygen effect which was studied so carefully by L. H. Gray, who often visited LBL. Among scientific visitors to our laboratory were Roger Berry of London who worked on the radiobiological oxygen effect and Roger Tym. Jack Fowler, the current Director of the Gray Laboratory, and Elizabeth Travis of NCI, are currently collaborating in research with heavy-ion beams on long term effects on lung tissue. W. G. Burns of Harwell has initiated plans for a one year visit with our group to study chemical physics.

\section{Canada}

There are many collaborative research programs with Canadian radiation scientists. R. L. Skarsgard's group at the pi meson facility in Vancouver participates in the cooperative particle dosimetry group. Gordon Whitmore of the Canadian NRC and University of Toronto is serving as a member of the laboratory's heavy-ion program advisory committee. We have collaborated with scientists at the University of Western Ontario on $X-r a y$ and heavy-ion microscopy.

Don Chapman of the University of Alberta in Edmonton spent a year with our group doing research on the mechanisms of action of hypoxic cel1s. A radiotherapist from the Cross Cancer Hospital of Edmonton, Raoul Urtasun also spent several months here. Beginning with these two scientists, interest developed on the Edmonton campus to build a heavy-ion accelerator for research in the biomedical and physical sciences. Initial plans were prepared and submitted with cooperation from LBL, and the investigators, who are headed by Brian Lentle, received a grant from the government of Alberta in 1980 to plan the accelerator. The design specifications for the Alberta heavy-ion accelerator are similar to the specifications of a future biomedical research accelerator that is designed to replace the Bevalac. If the two machines could be designed and perhaps built as a joint enterprise, much effort and cost could be saved. William Saunders, who was trained at Stanford and at Edmonton, recently joined LBL in experimental radiotherapy, and he will be involved in the design studies of both accelerators.

\section{China}

There are preliminary but rapidly developing scientific relationships with China. Several delegations of scientists have visited LBL, and the President has announced that the Chinese will build a high energy particle accelerator with American collaboration. Recently a visiting Chinese delegation included a detailed study of heavy-ion biomedical facilities and research programs. The delegation, headed by Liu Da of qinghua University, expressed a wish that biomedical studies be included among the topics of joint research.

\section{International Atomic Energy Agency}

We have had a number of scientific interactions with the staff of IAEA, which includes participation in symposia. Several nations that now do not have a heavy-ion particle facility wish to participate in the studies in some manner. In India, Pakistan, and the Middle East there are large numbers of cancer patients and a great desire to use modern investigative and therapeutic techniques. The staff of IAEA also suggested applications for accelerated heavy ions in applied fields where little heavy-ion research has been carried out so far: the development of agriculturally useful plant mutants induced by highly mutagenic heavy ions, and the use of heavy 
ions in immunological research, particularly in relation to preparing vaccines against certain diseases. Heavy-ions may be able to kill

pathogenic miocroorganisms while preserving their antigenic properties.

\section{SUMMARY}

The rapidly growing research applications of heavy ions in basic biology and medicine have stimulated interest in this field in many countries. LBL, with its unique facilities and its scientific programs, is the focal point of interest. Plans are underway in several countries, including France, Japan, West Germany, and Canada, to build heavy-ion facilities, and to collaborate with our staff at LBL in heavy-ion research in physics, biology, and medicine. 


\title{
THE BEVALAC BIOMEDICAL FACILITY
}

\author{
J. R. Alonso, J. Howard, T. Criswell, W. Chu, F. Lothrop, and C. Weber
}

\section{ACCELERATOR DESCRIPTION}

The Bevalac, shown in Figure 1 , is a highenergy heavy-ion accelerator complex that was created in 1974 by Tinking the SuperHILAC and Bevatron accelerators. The complex is operated by the LBL Accelerator and Fusion Research Division, H. Grunder, division head, and R. Force, operations chief.

The SuperHILAC, an Alvarez-type linear accelerator, produces beams of ions as heavy as lead at energies up to $8.5 \mathrm{MeV} / \mathrm{amu}$. SuperHILAC beams are transported to the Bevatron through an $800 \mathrm{ft}$ beam transport line which has twelve bending and thirty focusing magnets that can be rapidly and efficiently tuned by a sophisticated computer monitoring and control system.

The Bevatron is a weak-focusing synchrotron that accepts injected particles for a period of several hundred microseconds. During acceleration, the beam is bunched while the magnetic guide field is increased so that the particles remain in basically the same circular orbits. When the desired energy has been obtained, the guide field is held steady ("flat-topped") while beam is drawn out of the ring by slow resonant extraction. The beam is then directed to the desired experiment through the magnet switchyard and beam delivery network known as the External Particle Beam (EPB) system.

The whole acceleration process from injection to extraction requires less than two seconds. After time is allowed for magnet ramp-down and resetting, pulses can be delivered at a rate of one every four or six seconds, depending on the final energy. The extracted beam pulse (spili) width can be varied from a few milliseconds to about a full second, and extraction energies are continuously variable from about $50 \mathrm{MeV} / \mathrm{amu}$ to about 2 GeV/amu.

\section{COMPUTER CONTROL AND TIME SHARING}

Both the SuperHILAC and the Bevalac are operated by virtually identical data processing and control equipment. Each accelerator has a system tailored to its particular needs for control and monitoring.

The SuperHILAC operates at 36 pulses/sec, accepting ions from two injectors and delivering ions to as many as three different experimental areas. Injection and delivery are time shared at the rate of 36 pulses/sec. Usually, light ions from one injector are delivered to the Bevalac at a 2 pulse/sec rate, and heavy ions from the second injector are delivered to SuperHILAC research teams at $34 \mathrm{pu} / \mathrm{ses} / \mathrm{sec}$. The control system is tailored to regulate and switch the accelerating, focusing, and steering components from injector to injector and ion to ion at each pulse. Thus, the SuperHILAC nuclear science program, which uses heavy ions (krypton and heavier), is not noticeably affected by the SuperHILAC's role as an injector for the Bevalac.

At the Bevatron, a pulse rate of 10 to 15 pulses/min is standard. Only one injector is used (either the SuperHILAC or the local $20 \mathrm{MeV}$ unit), and beam switching is performed in the EPB system. The computer control system at the Bevatron has been designed to adjust and regulate the synchrotron magnetic current, the pulse rate, the acceleration parameters, the magnetic extraction parameters, and the magnet currents in the EPB beam lines to a high degree of accuracy and reproducibility.

\section{RESEARCH PROGRAMS}

Beam time on the Bevalac is divided between two disciplines: one-third for biomedical research and two-thirds for nuclear science studies. Nuclear science experiments cover a wide range of fields including elementaryparticle production with heavy ions, nuclear hydrodynamic studies, nuclear fragmentation, cosmic-ray simulation, and atomic physics (electron capture and loss of relativistic ions). The biomedical program consists of radiotherapy, radiobiology, and radiography, as described in the rest of this report. Phase I and Phase II therapy trials began in 1979 and will continue through the spring of 1981. Integration of the varied and active nuclear science and biomedical programs has placed great demands on available accelerator time. The traditional mode of Bevalac operation has been revised so that therapy is conducted during the day shift four days per week, usually Tuesday through Friday. Radiobiology is performed on some weekends and overnights, and the nuclear science research consumes the remainder of the time such that the one-third to two-thirds proportioning is maintained.

Machine reliability and beam-on-target times are constantly monitored and improved wherever possible. Our present availability figures are about $82 \%$ for the biomedical program and about $75 \%$ for nuclear science. The most one can expect from this combination of complex machines is about $85 \%$, which is about $92 \%$ for each machine individualiy.

The ion species and beam currents that are routinely provided to the biomedical facility are summarized in Table 1 (LBL, 1977). Improvements to the Bevatron's local injector have made it 


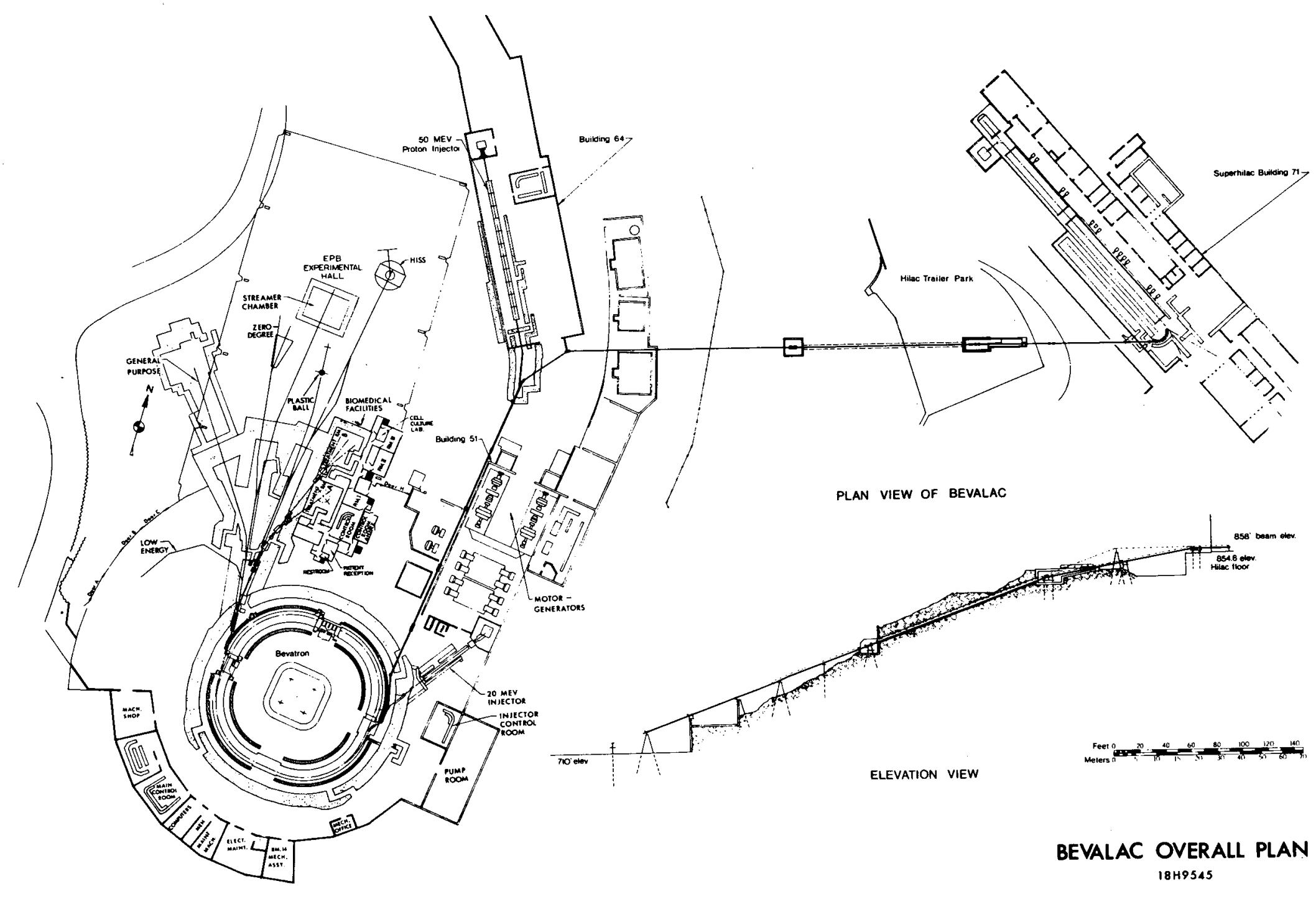

Figure 1. Overall plan of the Bevalac. (XBL 7910-12578) 
Table 1. Ion Species Provided to the Biomedical Facility

\begin{tabular}{|c|c|c|c|}
\hline Ion and Source & $\begin{array}{c}\text { Energy } \\
\text { (MeV/amu) }\end{array}$ & $\begin{array}{c}\text { Rate } \\
\text { (particles/pulse) } \\
(\mathrm{rad} / \mathrm{min})\end{array}$ & $\begin{array}{l}\text { Range to } \\
\text { Bragg Peak }(\mathrm{cm})\end{array}$ \\
\hline 4Helium (local) & 225 & $\begin{array}{l}5 \times 10^{9} \\
10 \text { to } 50\end{array}$ & 30.4 \\
\hline 12Carbon (local) & 400 & $\begin{array}{l}5 \times 10^{9} \\
4,000\end{array}$ & 25.9 \\
\hline 12Carbon (Bevalac) & 400 & $\begin{array}{l}1.5 \times 10^{10} \\
12,000\end{array}$ & 25.9 \\
\hline 20 Neon (Bevalac) & 670 & $\begin{array}{l}1 \times 10^{9} \\
1,200\end{array}$ & 32 \\
\hline 28 silicon & 670 & $\begin{array}{l}1 \times 10^{9} \\
1,000-2,000\end{array}$ & 23.2 \\
\hline 40 Argon (Bevalac) & 500 & $\begin{array}{l}2 \times 10^{8} \\
4,000\end{array}$ & 12.3 \\
\hline
\end{tabular}

Dose rates are given for a beam diameter at the $80 \%$ level (assuming a Gaussian distribution). Nitrogen and oxygen have been accelerated at low intensities from the Bevatron, but Bevalac beams of these ions have not been requested by biomedical users.

possible for carbon-ion therapy to be accommodated either by the Bevatron or the SuperHILAC, which provides a significant increase in scheduling flexibility.

\section{THE BEVALAC BIOMEDICAL FACILITY}

The extracted beam can be directed to any of ten nuclear science areas or two biomedical areas. The layout of the beam delivery system is shown in Figure 2. The beam lines that access the biomedical research area take off from Channel II and are capable of delivering beams of energies as high as $1 \mathrm{GeV} /$ nucleon. Higher energy beams are generally of little interest because of their extremely long range: Both biomedical beam lines are designed to be achromatic so that small energy variations in the beam will not affect the longitudinal location of the beam spot. These energy variations are typically less than $0.1 \%$, although they can be detected by: measurable range changes (a few tenths of $a$ : millimeter) in certain experiments.

\section{Irradiation Areas}

Figure 3 shows the layout of the beam lines and facilities for the two irradiation caves. One is designed for patient therapy, and the other is designed for biological studies and physical (microdosimetry) studies. Instrumentation is complete for each irradiation area.

\section{Treatment Facility}

A patient treatment facility (Alonso et al., 1979) has been created out of Biomed Cave 1, as shown in Figure 4 . The treatment room has been equipped with the Philips/MEL Ram-type therapy couch, laser patient alignment units, X-radiography units, and the necessary heavy-ion dosimetry equipment. Auxiliary equipment for the couch has been built so that patients may be treated in the recumbent, sitting, or standing positions. A movable optical bench has been installed to allow absorbers and collimators to be placed close to the patient. The floors are carpeted, decorative walls have been fastened to the concrete block structure, and the equipment is as unobtrusive as possible. The patient area just outside the treatment area includes a waiting room, a changing area, an examination and preparation room, and a treatment planning room.

Patients are received in Building 55, the Research Medicine building; the examinations and treatment preparations are done there. Patients are brought to the therapy area (about 150 yards distant) at the time their therapy is scheduled. All therapy is done on an outpatient basis.

EMI Medical Inc. has just delivered one of two CT scanners that have been designed to the specifications of LBL and Massachusetts General Hospital. These units, which are modifications of the companv's 7070 scanner, will scan either a sitting or a recumbent patient. The sitting 


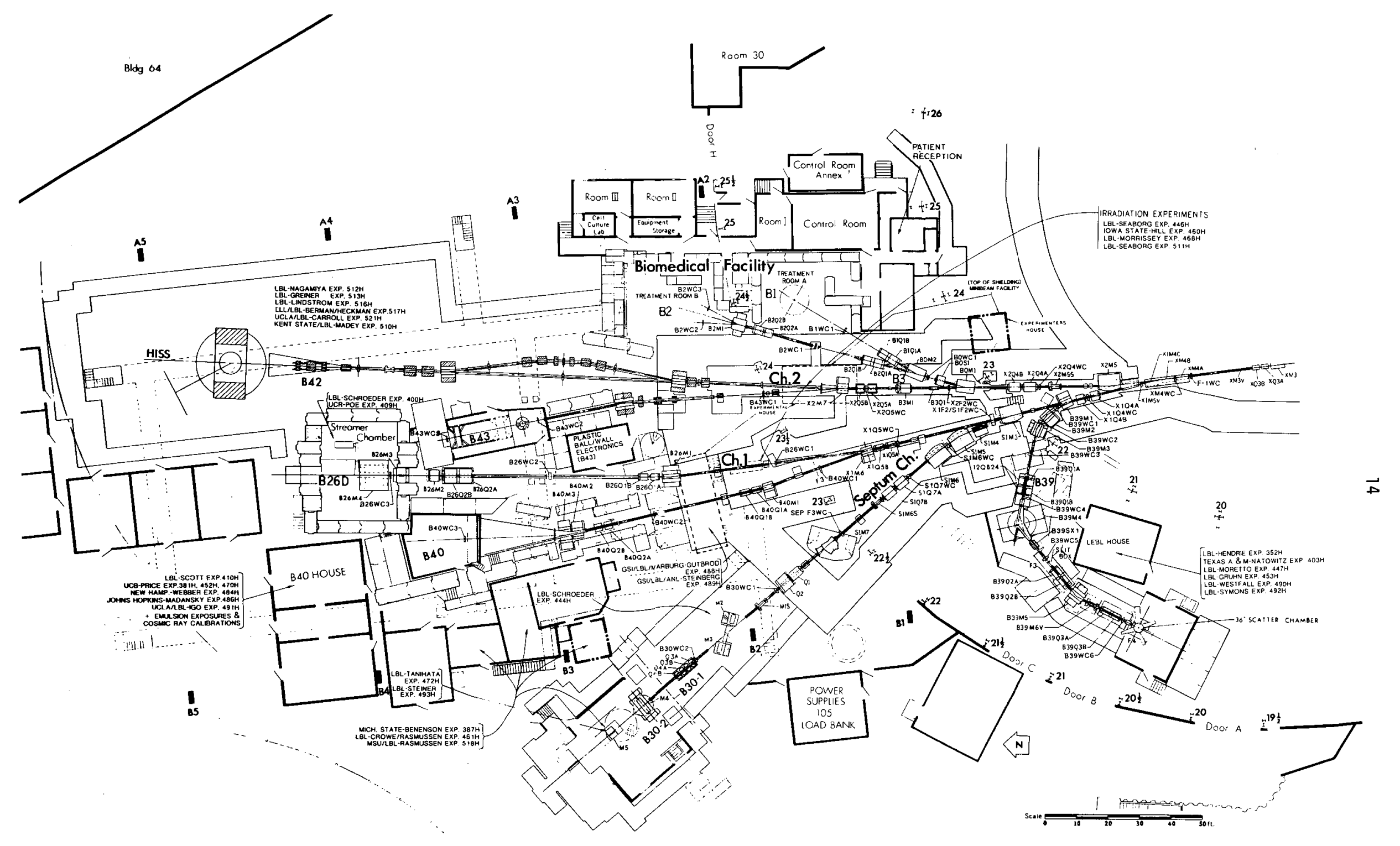

Figure 2. Plan view of the Bevatron/Bevalac beam delivery areas. (XBL 7912-13729) 


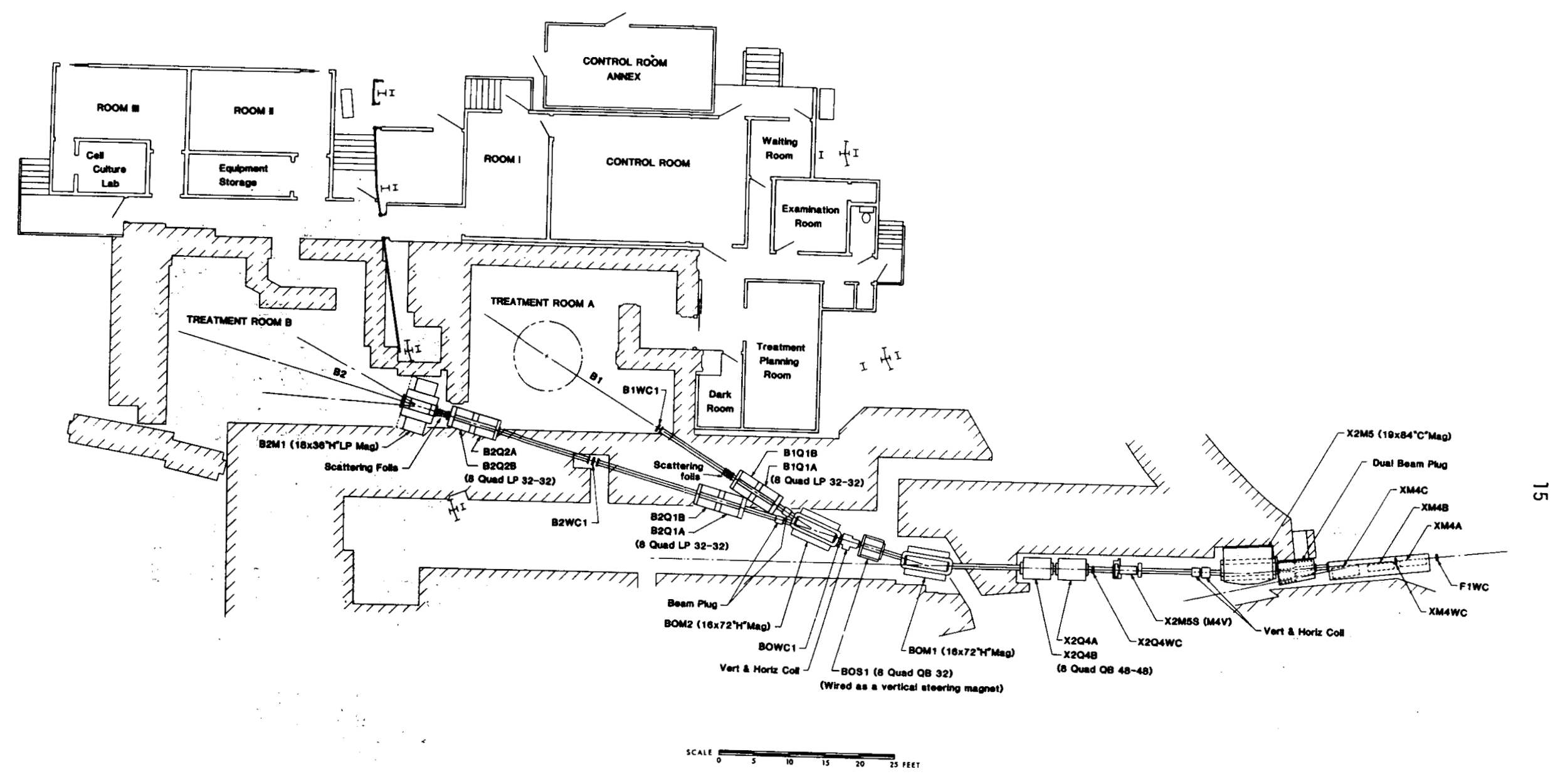

BIOMEDICAL BEAM BI \& B2 19F5235

Figure 3. Biomedical beam B1 and B2. (XBL 7910-12579) 


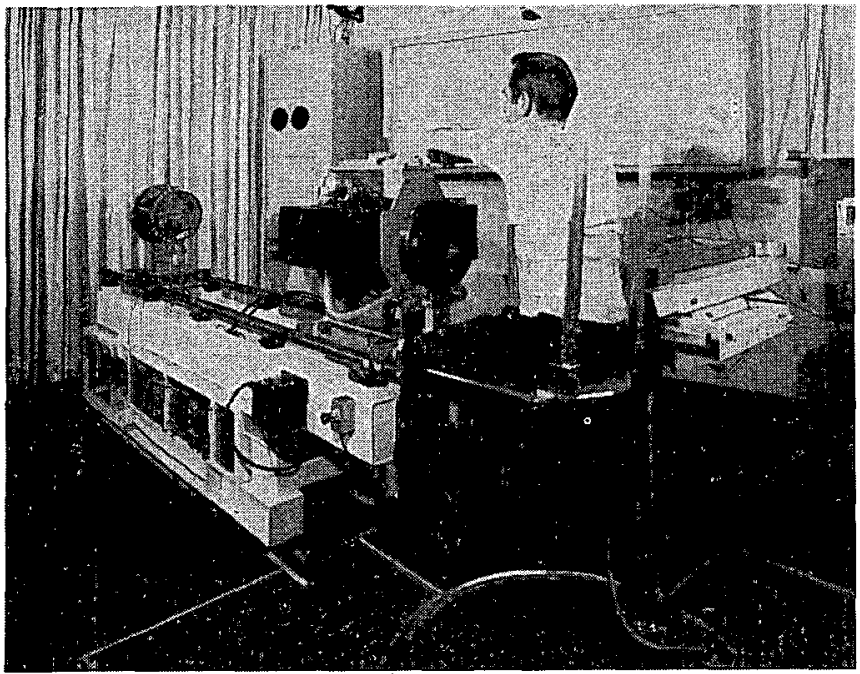

Figure 4. Treatment room showing chair attachment to the Philips positioner. Beam comes through the curtain and passes through dosimetry apparatus before reaching the patient. Shown on the optical rails are an ion chamber, two collimators and the range-modulating water column. (CBB 780-13486)

(or standing) position is frequently encountered at the Bevalac because beam delivery is in the horizontal direction and efficacious dose delivery to a tumor site requires that the patient be upright. CT scans done at local hospitals cannot show the organ positions as they are positioned during treatment; this is a serious limitation that will be overcome with LBL's on-site CT scanner.

\section{Biology Facility}

Adjacent to and just downstream from the treatment room is the biology research room, Cave II. Precisely aligned rails are positioned along two possible beam paths and about $32 \mathrm{~cm}$ below the center of the beam line. Dosimetry, collimation and absorption devices, and the biology samples are secured to the rails by means of clamp-on jigs, which assure precise positioning each time a unit is placed in the beam line. An X-ray facility is also included to facilitate positioning or comparative exposures of samples to $X$ rays under conditions almost identical to those for heavy ions. The effects of combined $x-r a y$ and heavy-ion irradiation are also being explored. Control and monitoring of beam delivery is directed from the central biomedical control room. Dose delivery into the biology research area is as fully accurate and reproducible as it is into the patient area.

Two preparation rooms are located next to the biology irradiation area. One is equipped primarily for animal experiments and has a nolding area for animal cages and sufficient table space for the necessary preirradiation procedures. The second room, which is designed for cell and tissue culture work, contains incubators, hoods, an autoclave, microscope, Coulter counter, and other appropriate apparatus.

\section{BEAM DELIVERY}

The beam in both biomedical areas can be made about as small as a $1 \mathrm{~cm}$ circle. For large fields, a system of scattering foils and occluding rings, adapted from the 184-inch Synchrocyclotron, has been developed to produce beam sizes of $20 \times 20 \mathrm{~cm}$ or larger with uniform dose profile to $2 \%$ over the entire field. Using this occluding ring system, the beam loss of approximate 1 y $50 \%$ compares favorably with losses incurred by taking only the central portion of an overly enlarged Gaussian-shaped beam. Dose rates for large spot sizes are considerably reduced, but intensities are still adequate for therapy.

To deliver dose to tumors with thicknesses ranging from 4 to $10 \mathrm{~cm}$, a series of modulators of the stopping depth, referred to as spiral ridge filters (Lyman and Howard, 1977), have been devised for treatment. A family of these filters has been fabricated to cover the full range of treatment situations, i.e., different amounts of Bragg peak spread for a number of ion species and energies. The absolute depth of penetration, or range, of an ion beam is adjusted by setting the energy of the incident ion to be equivalent to a range of about 25 to $30 \mathrm{~cm}$ in water. The thickness of an energy absorber can then be varied until the exact desired range is reached. The energy absorber used here is a computercontrolled variable water column (Lyman and Howard, 1977).

Current beam intensities from the Bevalac permit a single therapeutic dose of approximately 200 rad to be delivered to the patient in about 2 min. During the 20 to 30 minutes normaliy required for patient positioning, rapid beam switching (in less than one minute) now permits biology experimenters to use the beam in Cave II, providing that they can utilize the same ion, energy, and focus. This technique has increased tremendously the output of biological work, and makes the combined therapy-biology program an efficient user of accelerator time.

\section{Dosimetry and Control}

Dosimetry is performed with one secondary emission chamber (SEM) and three multisegment ion chambers (Lyman and Howard, 1977), one upstream of the occluding ring and the other two downstream and close to the patient. They are calibrated daily. One of the downstream chambers is monitored independently of the main computer system. The separate monitor will stop all beam delivery independent of the computer if the beam dose delivery exceeds a specified maximum.

Under continuing development is MEDUSA (Medical Dose Uniformity Sampler), which is designed to provide rapid, on-line monitoring of beam position and profile (Alonso et al., 1979). 
This 16-plane multiwire proportional chamber produces detailed and accurate images by Fourier convolution reconstruction techniques. (See Part II for a detailed report on this project.)

Normal operation of the accelerator for therapy requires about 30 beam pulses to achieve the desired 200 rad fraction. Intensity variations from pulse to pulse may be smoothed out by monitoring the integrated dose and stopping beam delivery in midpulse at the desired total dose. A rapid beam cutoff system with accuracy of better than $2 \%$ has been installed and is used routinely for both biology and therapy. The cutoff system uses a signal derived from the biomedical dosimetry computer and sent to the Bevalac's beam extraction computer to interrupt delivery within about a millisecond.

Control and recording of all biomedical activities is handled through a PDP $11 / 45$ computer and peripherals. The control program, AVISHAI, has been designed to monitor and store all treatment and irradiation parameters, including ion chamber readings, beam intensity readings (from the Bevalac), water column settings, and patient couch position. The control program also asserts control functions, starting and stopping the irradiations according to the instructions given at the computer console.

To facilitate tuning the biomedical beam lines, beam monitoring information developed by the biomedical dosimetry and control system is transmitted to the Bevalac main control room, which allows the accelerator operators to steer and focus the beam precisely to the target(s).

\section{Radioactive Beams}

Peripheral nuclear fragmentation reactions of primary Bevalac heavy-ion beams in $10 \mathrm{w}-2$ targets are used to produce secondary beams of radioactive nuclei (Chatterjee and Tobias, 1977; Alonso et al., 1979). The large cross section and small deflection of the projectile fragments lead to high production and delivery efficiency for these beams. Dispersive beam transport al lows good separation and purification of the desired secondary beams. $11 \mathrm{C}$ and $19_{\mathrm{Ne}}$ beams of high purity and good intensity (almost $0.2 \%$ of the primary beam current) are presently being used for biomedical experiments. These radioactive beams are a part of the regular Bevalac beam inventory and are available in the biomedical area within a few minutes of the researcher's request.

Positron emission of the decaying ${ }^{11_{C}}$ or $19 \mathrm{Ne}$ is from the stopping point of the particle, and directional detection of the escaping gamma rays permits a precise determination of the location of the stopping particle. A special imaging device called PEBA (Positron Emitter Beam Analyzer) that has 72 sodium iodide crystal detectors is being developed, and so far has been able to measure beam stopping points to an accuracy of $\pm 1 \mathrm{~mm}$ (Llacer et al., 1978).
The uses of these positron emitting beams are primarily in nuclear medicine and verification of radiotherapy treatment planning. Present and envisioned applications in nuclear medicine diagnostic procedures are numerous and include implantation of radioactive atoms to measure the microcirculation in vital organs. Another potential value of these beams is in the localization of the Bragg peak in relation to the tumor volume to be treated. This verification is important to effective heavy-ion cancer radiotherapy, because the beam itself will confirm the accurate compensation of tissue inhomogeneities in a particular patient. A detailed discussion on radioactive beams $c$ an be found in Part VI Section B of this report.

IMPROVEMENTS IN PROGRESS

\section{Beam Sharing}

Several methods of beam sharing among various users are being implemented to improve the utilization of beam time. Current standard methods include septum splitting (same ion, same energy) and quick switching between target areas (same ion, same energy). The septum split physically separates the extracted beam, sending some of it to Channel I users and the rest to Channel II users on the same Bevalac pulse. Quick switching is accomplished between Channel II users, normally therapy and biomed, in about a minute, and diverts all of the beam from one target area to another. Under test currently and soon to become operational is the mezzanine mode (same ion, different energies). In this mode an ion beam is first accelerated to a desired energy, and a portion of the beam is extracted and delivered to one target area. The remainder is then accelerated to a second, final energy, extracted, and delivered to a second target area of the EPB. Mezzanine operation will permit a new degree of freedom in operating for diverse research requirements.

The ultimate flexibility in beam sharing would be for the Bevatron to accept different ions on different pulses and accelerate each ion type to a different energy. This requires rapid changes for the full set of transferline magnets and for all of the injection, acceleration, and extraction parameters for the Bevatron. Although this goal requires considerable effort, the success of the time-sharing system at the SuperHILAC establishes confidence that this capability $c$ an be implemented at the Bevatron.

\section{Local Injector}

Construction of a more powerful radiofrequency acceleration system for the local injector is underway with the aim of providing low intensity neon beams for Bevatron injection. Further improvements to the source will permit increasing the intensity of local neon beams to a level sufficient for therapeutic doses. This will add the same flexibility of injector choice we now have with carbon. 


\section{Beam Scanning}

The capability to scan a pencil beam over the desired field is being developed as an improved technique for beam delivery, especially when correlated CT scan information may be used to model the beam profile into the irregular shapes normally needed for therapy. The first step, currently in progress, is to accomplish twodimensional scanning. Development and testing of a prototype scanning system is being pursued as part of a comprehensive research and development effort on the optimized design for a dedicated medical ion accelerator.

\section{Uranium Capability for the Bevalac}

A long planned upgrading program for the Bevalac to accelerate high-intensity uranium beams to relativistic energies is in progress (LBL, 1979). The project includes the design and construction of a third injector for the SuperHILAC, incorporating a pre-accelerator linac section that will produce high currents of low charge state, very heavy ions. These ions will be accelerated through the existing SuperHILAC, transported through an upgraded transfer line and injected into the Bevatron through a new injection system.

To accelerate these partially stripped ions, the vacuum in the Bevatron must be improved from its present $10^{-7}$ Torr to 10-10 Torr. This will be acomplished by the insertion of a cryopumped vacuum liner that will create a high vacuum area within the present enclosure. Acceleration, extraction, and beam delivery equipment and techniques will conform to the existing ones with only minor modifications.

This project will open up an entirely new area of physics for exploration by nuclear scientists. Although the very heavy ions perhaps will not find many immediate uses in the biomedical program, the general upgrading of the accelerators will provide significant increases in beam intensities for all ion species. The impact of the project on Bevalac operation will be a shutdown of about six months duration starting in the summer of 1981 for the installation of the new vacuum liner and initial tests.

\section{REFERENCES}

Alonso, J.R., J. Howard, and T. Criswell. 1979. The Bevalac Radiotherapy Facility. Lawrence Berkeley Laboratory Report LBL-8961.

Alonso, J.R., C.A. Tobias, and W.T. Chu. 1979. Computed Tomographic Reconstruction of Beam Profiles with a Multi-wire Chamber. Lawrence Berkeley Laboratory Report LBL-8947.

Alonso, J.R., A. Chatterjee, and C. A. Tobias. 1979. High Purity Radioactive Beams at the Bevalac. Lawrence Berkeley Laboratory Report LBL-8951.

Chatterjee, A. and C. A. Tobias. 1977. Radioactive beams. Biological and Medical Research with Accelerated Heavy Ions at the Bevalac, 1974-1977, pp. 59-75. Lawrence Berkeley Laboratory Report LBL-5610.

LBL (Lawrence Berkeley Laboratory). 1977. Bevatron/Bevalac Users' Handbook. Lawrence Berkeley Laboratory, Publication 101.

LBL (Lawrence Berkeley Laboratory). 1979. High Intensity Uranium Beams from the SuperHILAC and the Bevatron, Preliminary Design Report and Technical Description. Lawrence Berkeley Laboratory, publication 5014.

Llacer, J., A. Chatterjee, H. C. Jackson, and M. V. Zunzunegui. 1978. An Imaging Instrument for Positron Emitting Heavy Ion Beam Injection. Lawrence Berkeley Laboratory Report LBL -8138 .

Lyman, J. T., and J. Howard. 1977. Biomedical research facilities and dosimetry. Biological and Medical Research with Accelerated Heavy Ions at the Bevalac, 1974-1977, pp. 26-35. Lawrence Berkeley Laboratory Report LBL-5610. 


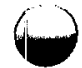

PART II. RADIOLOGICAL

PHYSICS AND CHEMISTRY RESEARCH 


\title{
INSTRUMENTATION FOR MEASURING HEAVY-ION FIELDS
}

\author{
J. R. Alonso, E. V. Benton, ${ }^{*}$ W. Chu, J. Llacer, J. Richier, and C. A. Tobias
}

There are a number of physical beam characteristics that should be measured for radiobiological experiments with heavy ions and for meeting the requirements of cancer therapy with these particles. We are developing three different techniques to measure some of those characteristics: MEDUSA, a two-dimensional beam intensity profile measuring device; a beam quality analyzer that gives detailed information about the individual components of a complex fragmented beam; and nuclear track tecnniques for beam characterization by measurements of damage regions in plastics.

\section{MEDUSA (Medical Dose Uniformity Sampler}

(W. T. Cnu, J. R. Alonso, and C. A. Tobias)

For radiotnerapy program and radiobiology experiments at the Bevalac it is important to have a flat dose distribution across the heavyion beams. For example, the radiotherapists often prescribe a uniformity of doses within a few percent fluctuation over a $20 \mathrm{~cm}$ diameter field size. Currently such wide, flat fields are generated by transporting the heavy-ion beams through a scatterer-occluding $r$ ing assembly (Crowe et al., 1975; Koehler et al., 1977). Measurements of the beam flatness and its daily verification are important as small variations in beam alignment on the occluding rings can cause substantial skewing of the intensity distribution at the irradiation sites. The measurements by the usual techniques of scanning ion chambers across the radiation field or by densitometry of pnotographic plates exposed to the beam are laborious and time consuming.

A 16-plane, multiwire, proportional chamber has been constructed and used at the Biomedical beam area (Alonso et al., 1979). This instrument, called MEDUSA (Fig. 1), comprises 16 planes of multiwire chamber stacked together, with their wire directions staggered in such a way that they uniformly cover the entire $180^{\circ}$ space. Each plane is made of 64 wires, each $4 \mathrm{~mm}$ apart. The sensitive area is a circle with a diameter of $25.6 \mathrm{~cm}$. The chamber is made by alternately stacking the 16 signal planes and hign voltage planes. The chamber is flushed with dry nitrogen gas with a low flow rate $(0.1 \mathrm{cc} / \mathrm{sec})$ and holds a minute positive pressure generated by a gas buboler connected to the exit gas port of the chamber.

When the ionizing radiation passes through the chamber, the electrons resulting from the ionization are collected in each wire and stored in integrating capacitor connected to each signal wire. The voltage on the capacitor is therefore proportional to the beam intensity over the area

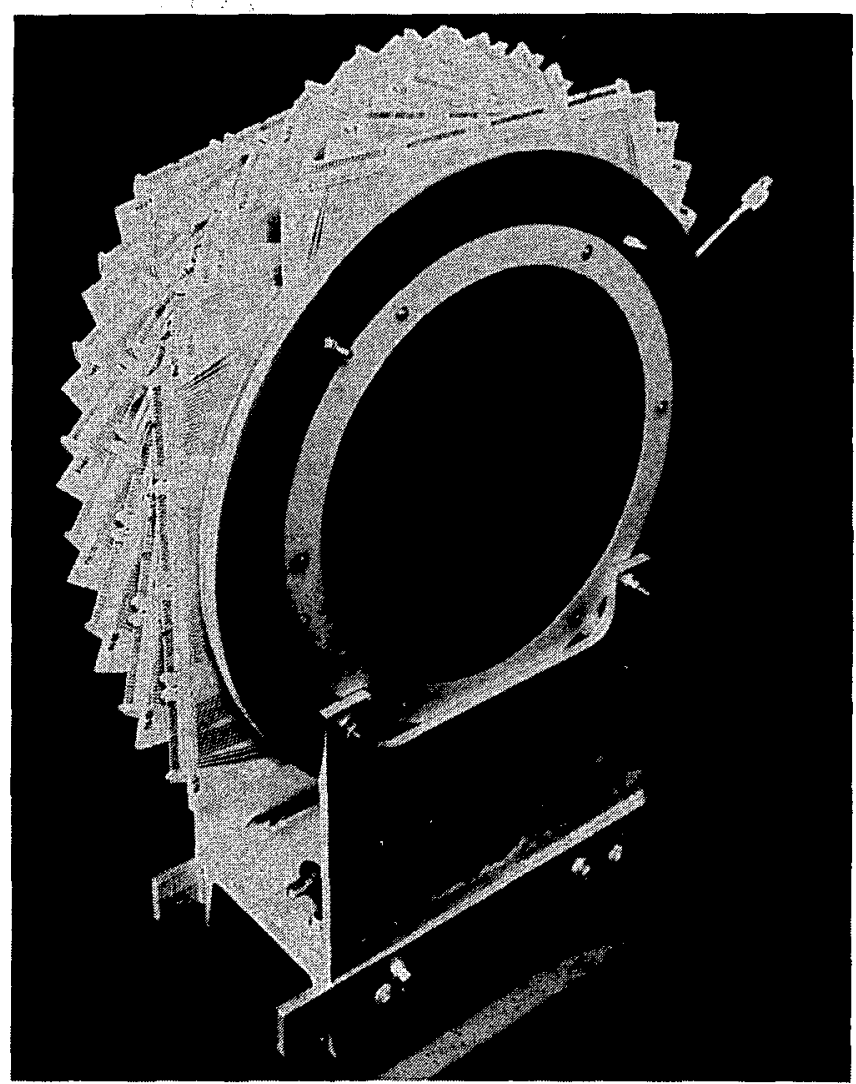

Figure 1. MEDUSA. (CBB 783-3335)

of a strip, which is centered on the particular wire and extends midway toward the adjacent two wires. The data collected on the 64 wires of a plane are the line integrals of the twodimensional profile of the beam intensity along the wires. In other words, the data represent the one-dimensional projection of the twodimensional beam intensity profile along the direction of the wires of the particular plane. Based on the 16 projections, the beam intensity profile is readily reconstructed.

At the end of each data collecting cycle, capacitor voltages are sequentially sampled, digitized, and stored in buffer memory under the control of the LSI-11 microcomputer. Upon command, the data in the buffer are serially transmitted to the host computer, a PDP 11/34 computer located at the Biomedical facility. The data are corrected for different sensitivities of the individual wires which mainly stem from the mismatching in capacitor values and other components. The 1024 data points $(16 \mathrm{planes}$ of 64 wires) are used to reconstruct the beam profile, usually of $64 \times 64$ array with $4 \mathrm{~mm}$ pixel size. 
The reconstruction takes less than $20 \mathrm{sec}$, which will be reduced to a few sec when the array processor is used. The software developed by us for the reconstruction is based on the back-projection with Fourier convolution technique (Shepp and Logan, 1974). The reconstructed image is color coded according to the intensities and displayed on a RAMTEK system which has $512 \times 512$ pixel matrix with each pixel of 12-bit refreshable memory.

Some examples of the heavy-ion beam profiles produced by the MEDUSA are shown in Figure 2 . A11 examples are taken with $20 \mathrm{Ne}$ ion with the energy of $670 \mathrm{MeV} /$ nucleon. Figure $2 \mathrm{~A}$ shows a beam spread by $28 / 64$ in $\mathrm{Pb}$ as the first scatter, $3 / 8$ in $\mathrm{Cu}$ as the second scatter, and an occluding ring-and-post system. The beam intensity within a circle of the diameter of $18.8 \mathrm{~cm}$ is $93.0 \pm 2.4 \%$ (std dev), with the hot point normalized at $100 \%$. Simple algebraic means of the intensities in. pixels in either vertical or horizontal lines within the circle are also plotted. When the $\mathrm{Cu}$ second scatter in the beam in the above example is replaced with a $12 \mathrm{~cm}$ spiral ridge filter, the range of the beam is spread (i.e., the modified or extended Bragg peak), but a similar beam profile is obtained as shown in Figure 2B. This picture shows the beam intensity profile at the range of $1 \mathrm{~cm}$ beyond the proximal peak, obtained by placing $13 \mathrm{~cm}$ of plastic in the beam upstream of the MEDUSA. The beam profile is not as uniform as in the unmodified beam as shown in Figure $2 A$.

In Figure 2C, a similar beam with 20/64 in Po as the first scatter and a $12 \mathrm{~cm}$ spiral ridge filter with the occluding ring-and-spot is shown at the midpeak of the modified Bragg peak. In this example the intensity profile of the beam across the horizontal line through the center of the MEDUSA is also plotted. The top two lines in the plot represent 100 and $90 \%$, where the $100 \%$ is the normalized hot spot. The size of the beam that lies between 90 and $100 \%$ is $11.5 \mathrm{~cm}$, and the mean intensity is $95.9 \pm 2.8$.

An example of an actual therapy beam shaped by an irregularly shaped collimator to conform to the shape of the treatment volume is shown in Figure 2D. The beam is modified by using $28 / 64$ in $\mathrm{PD}$ as the first scatter and a $10 \mathrm{~cm}$ spiral ridge filter plus an occluding ring. The range of the beam is adjusted to align the distal peak with the distal edge of the treatment volume. Finally, the beam goes through the collimator, which is placed in front of the MEDUSA located at the isocenter.

The MEDUSA nas proved to be a versatile equipment when used in the Biomedical facility for routine beam profile measurements and for new beam developments. It produces satisfactory beam profiles using one Bevalac beam pulse. In actual practice, however, three or more beam pulses with reduced intensity are used to average out the movements of the beam from pulse to pulse. Now we are exploring the possibility of using the system for imaging purposes, which is discussed in Part VI of this report.
BEAM QUALITY ANALYZER

(J. Llacer, J. Richier, and C. A. Tobias)

Heavy ions are being used in a variety of basic and applied biomedical studies, including cell, tissue and organ radiobiology, and cancer diagnosis (radiography) and therapy. In the majority of cases, the pure heavy-ion beams delivered by the Bevalac accelerator do not have the characteristics of penetration depth, crosssectional width, or Bragg peak width that are desired for a specific application. Metal foils, rotating or fixed ridge filters, and/or variable depth water absorbers are then interposed between the beam delivery port and the subject of irradiation, generating a substantial number of fragment nuclei in the beam. Although it is feasible to obtain good information routinely about the dose delivered to the subject under all the irradiation conditions encountered, we do not have detailed knowledge of what particles generate the delivered dose. Cnatterjee et al. (1976) have described the general characteristics of the complex beams that can be used for biomedical applications based on information obtained from cosmic-ray data. Studies by Blakely et al. (1979) make use of calculated data for complex beams in order to understand the inactivation of human kidney cells by heavy-ion beams. These studies show that dose alone is not a sufficient description of a beam with a substantial number of fragments, but it is necessary to be able to separate its components because of their different biological effects.

The process of nuclear fragmentation becomes considerably more complex as the atomic number of the ion increases, and it is not possible to calculate theoretically all the involved parameters. There is, at this time, a substantial research program within the Biology and Medicine Division to measure the physical characteristics of heavy-ion beams used in biomedical research (see Schimmerling in Part II). Although that program wil provide detailed and accurate information on the fundamental nuclear processes occurring in the ion beams of biomedical interest, there is still a need for some simple instrumentation that can provide an experimenter or physician with reasonably quick information of particle composition and dose contributed by each different nucleus in any of the large number of configurations that can be assembled. The information does not need to separate different isotopes of a same element, for example, but it should be reliable and reproducible, and data processing should take place within a short time after the completion of a measurement.

During the investigations of the possible use of solid-state detectors for heavy-ion radiography and tomography, it became evident that detector configurations similar to the ones that are proving useful for that task are also excellent for beam quality analysis. This chapter discusses the first detector configuration we have used, showing the results of a series of experiments carried out with a beam of $530 \mathrm{MeV} / \mathrm{n}$ silicon ions. Planned modifications and improvements in the system will be described. 


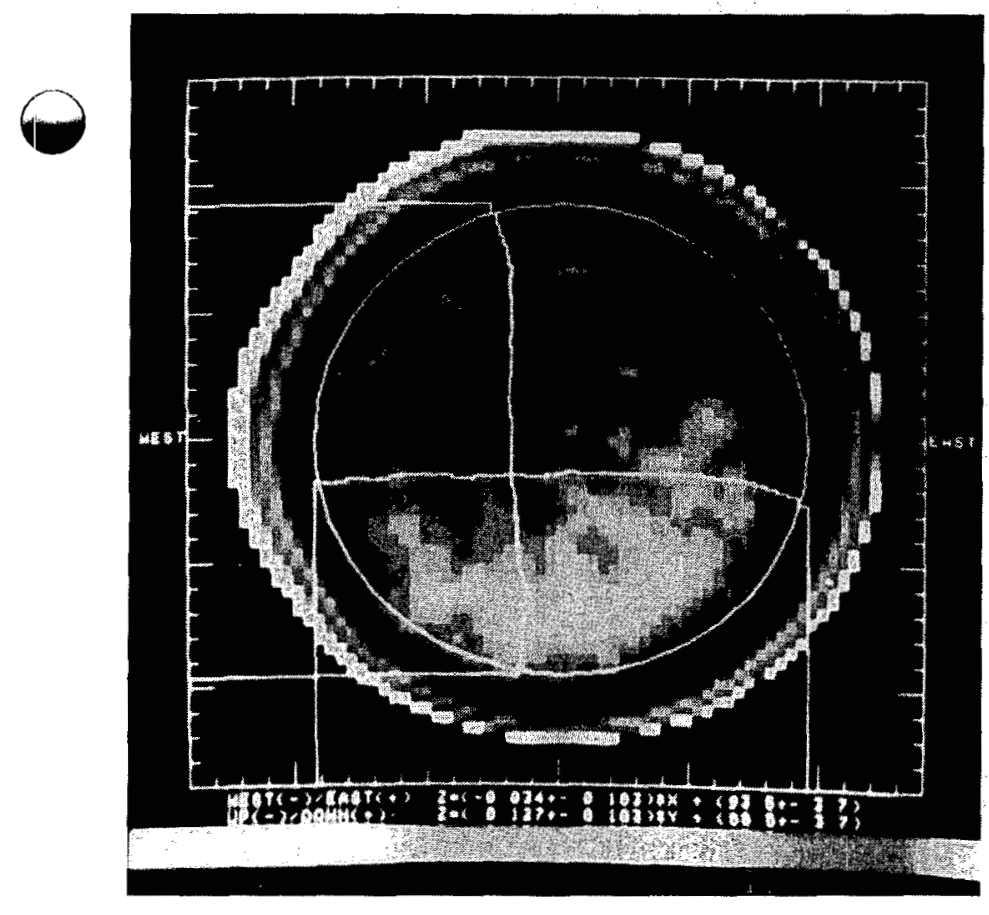

Figure 2A. The intensity profile of $670 \mathrm{MeV} /$ nucleon $20_{\text {Ne beam. (XBB 808-10088) }}$

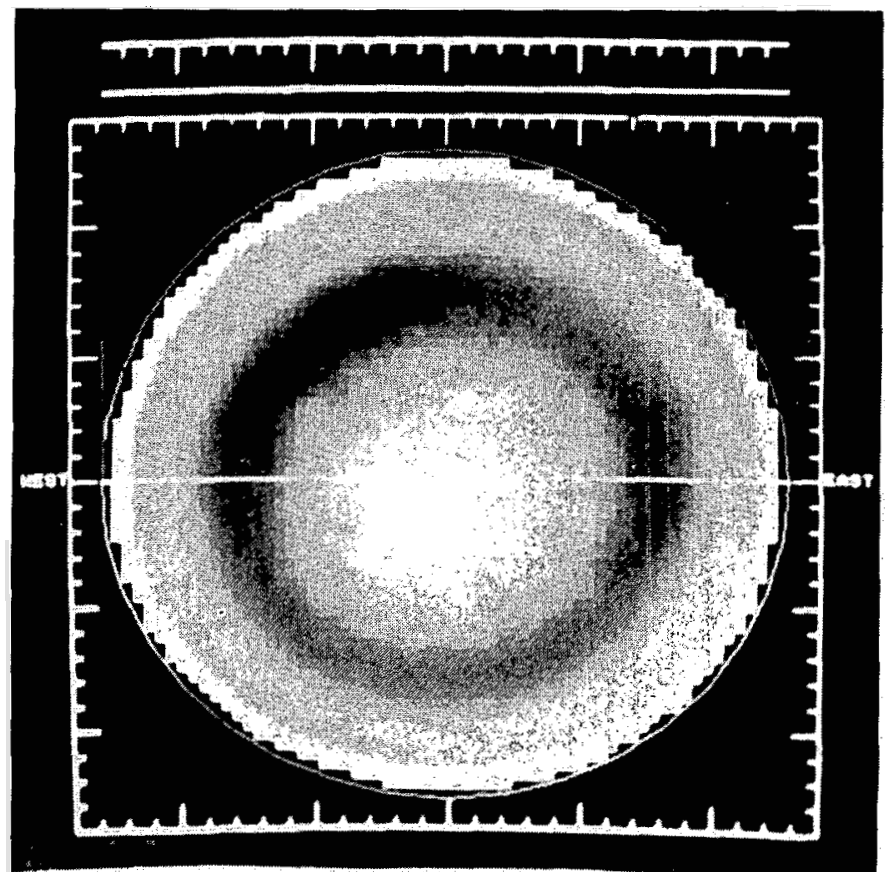

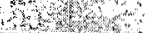

Figure 2C. The beam intensity proifle at the middle of the modified Bragg peak. (XBB 808-10090)

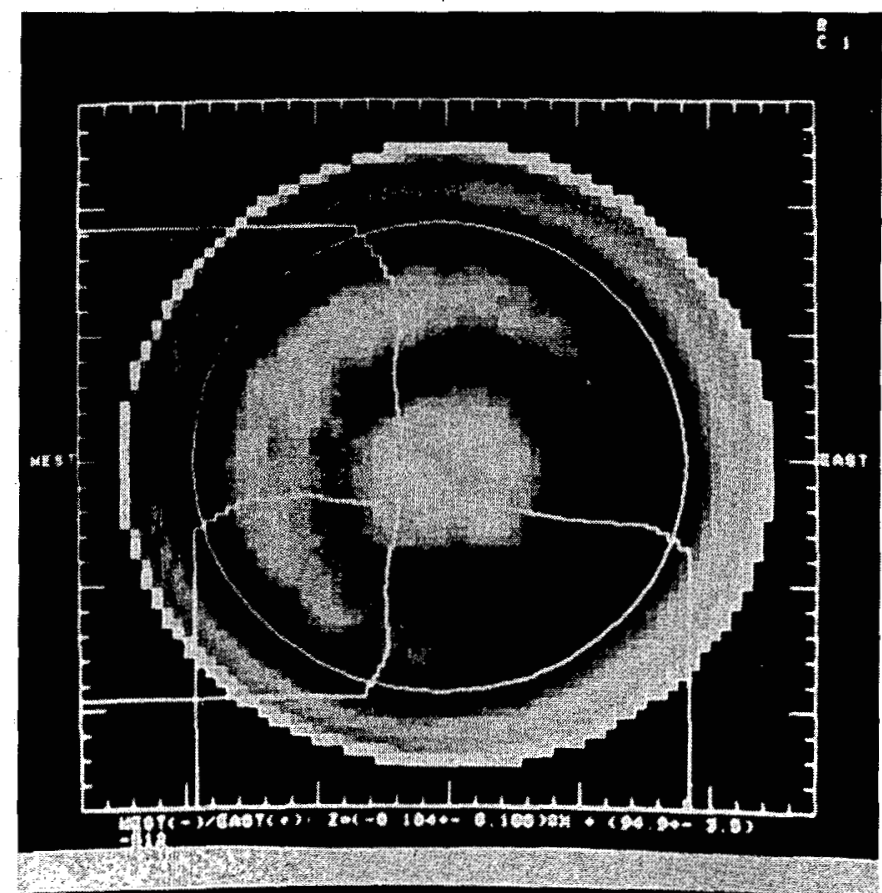

Figure 2B. The beam intensity distribution at the proximal peak of the modified Bragg peak. (XBB 808-10089)

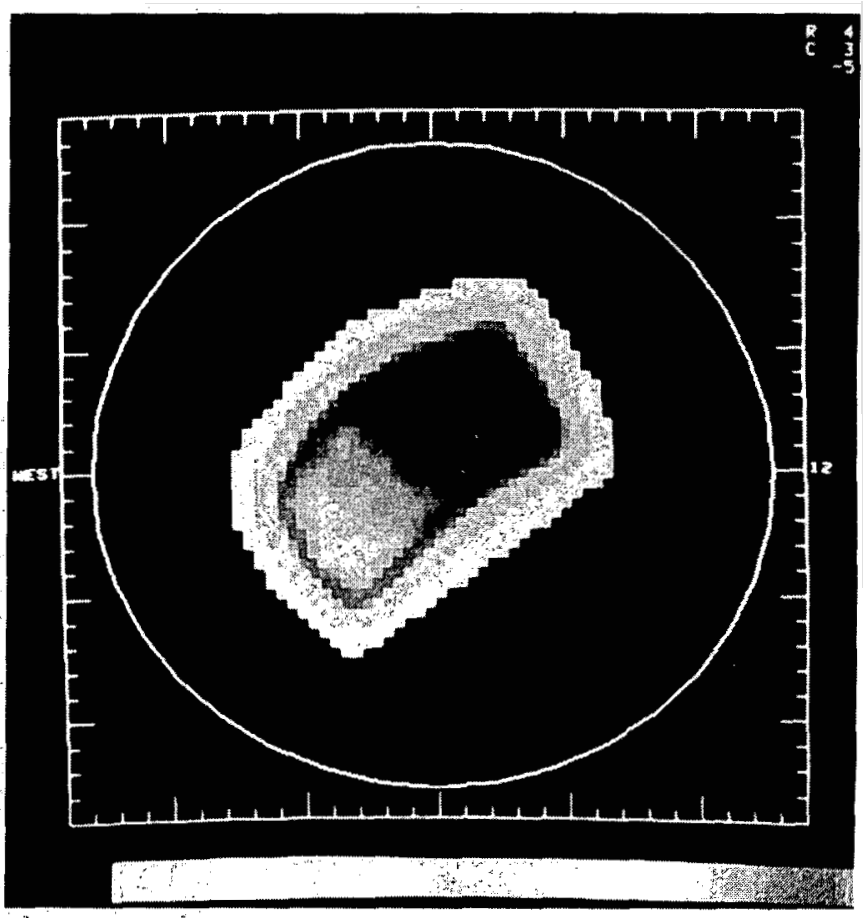

Figure 20. An example of the actual therapy beam, shaped by an irregular collimator, at the isocenter. (XBB 808-10092) 


\section{Initial Project Aims}

Figure 3 shows the Bragg ionization curve for the silicon ion beams used in the experiments. The initial beam energy was $530 \mathrm{MeV} / \mathrm{n}$ and a lead foil scatterer of $0.4 \mathrm{~mm}$ thickness was used. Eight locations $(A-M)$ have been indicated in the curve corresponding to the length of the water column absorber of interest for cell survival experiments with that beam. At each of one of the points, a measurement of beam composition was to be made. In order to facilitate the analysis, the beam was broken into a number of components:

1. parent $28 \mathrm{Si}$ or fragment $27 \mathrm{Si}$ nuclei, 2. fragment $\mathrm{Al}$ and $\mathrm{Mg}$ nuclei, considered individually,

3. fragment $\mathrm{Ne}$ and $\mathrm{Na}$ nuclei, considered together,

4. fragment $F, 0, N$, and $C$ nuclei, considered together,

5. fragment $B, B e, L i, H e$, and $H$ nuclei, considered together.
For each component, at points $A$ through $M$ of the Bragg curve, we wanted to obtain: (1) mean LET (stopping power) for each individual particle or group of particles, $\mathrm{dE}_{j}$; (2) relative number of particles of each type, $f_{n i}$; (3) relative ionization generated by a 11 the particles of each type, $f_{E i}$.

Further, by using the Bragg curve of Figure 3 as normalization, we wanted to obtain estimates of: (4) dose contributed by particles of each type, $d_{j}$ and (5) number of particles of each type, $n_{i}$.

\section{Detector and Electronics Configuration}

Figure 4 shows schematically the configuration of absorbers and collimators used in the silicon beam line and the position of the two solid-state detectors. The inset shows the cross section of the two detectors in detail. The

first detector consists of a $190 \mu \mathrm{m}$ thick silicon

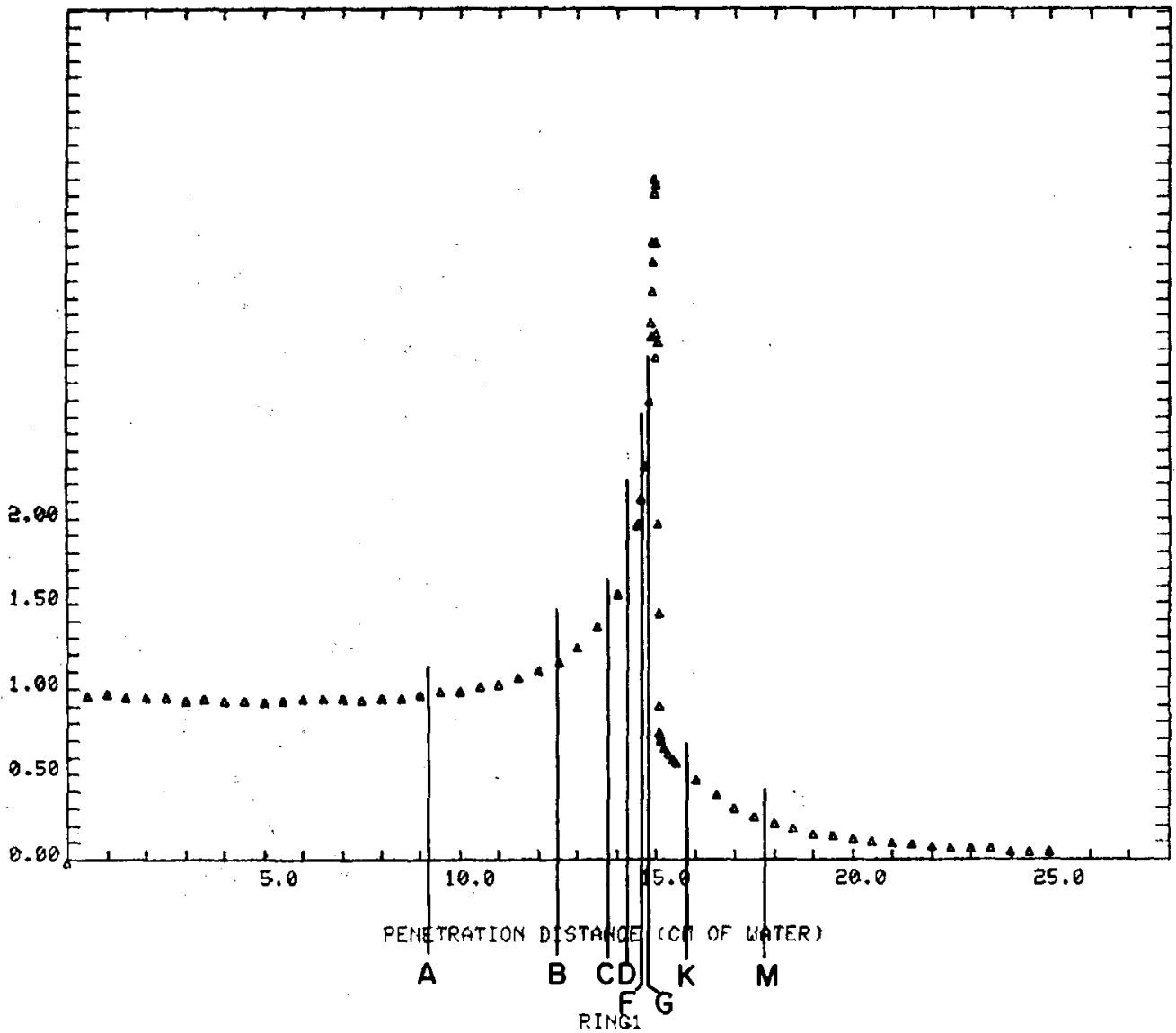

Figure 3. Bragg ionization curve for a $530 \mathrm{MeV} / \mathrm{n}$ (nominal) silicon ion beam after a lead foil scatterer of $0.4 \mathrm{~mm}$ thickness. Locations A through $M$ correspond to the eight points where measurements of beam composition were made: $A=8.15 \mathrm{~cm}$ of water, $B=12.55, C=13.75, D=14.34, F=14.75$, $G=14.85, K=15.95$, and $M=17.95$. (XBL 808-11442) 


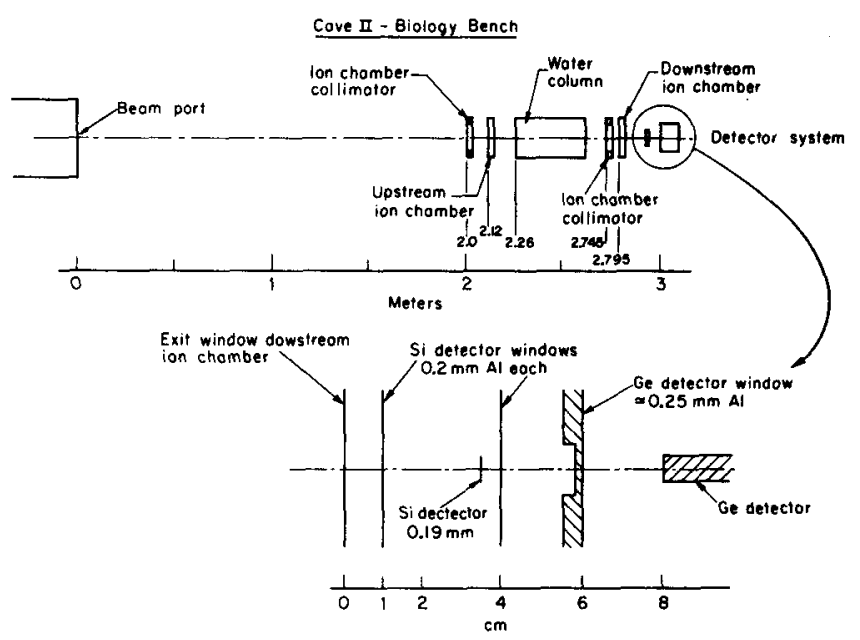

Figure 4. Description of the experimental setup used for the experiments. Inset shows detector configuration. (XBL 808-3609)

\section{Data Analys is}

The tecnnique of particle identification from the time-coincident "dE" and "E" signals was used with heavy-ion beams of biomedical interest at LBL Dy Maccabee (1973) and Maccabee and Ritter (1974). Goulding and Harvey (1975) reviewed the field of particle identification, and contributed important algoritnms and circuits to the art. Schimmerling and Curtis (1977) made time-offlight measurements with $3.5 \mathrm{GeV}$ nitrogen ions. There is, in addition, a large number of examples in the literature of the use of "dE," "E," and time-of-flight information for particle identification. We have encountered substantial problems, nowever, in attempting to analyze our data by methods that are usually intended for particle identification after thin absorbers. For our simple detector system, without time-offlight information, the most valuable option that we nave found for relating $d E$ to $E$ signals for a given particle is to use an empirical relationsnip of the form:

$$
E=\frac{k}{(d E)^{\alpha}}
$$

where $k$ and $\alpha$ are constants which depend on the particle and position within the Bragg curve. This relationship holds for fragments which are heavy enough to stop fully within the germanium detector.

The range of parameters $k$ and a defining the boundaries of a particle of a given type have been determined empirically. This was done by starting at point $A$ of Figure 3 , where the simple law (Sachs et al., 1966):

$$
(E+d E) \cdot d E \propto A Z^{2}
$$

could be applied without difficulty to unambiguously identify the fragments. Proceeding then in a logical sequence we were able to identify particles from points $B$ to $M$ of Figure 3 . Equation 1 was then used to define boundaries between particles for computer data analysis.

For lighter fragments, which do not stop fully in the second detector, a similar procedure can be followed starting at point $A$, identifying the individual fragments by a calculation of the energy loss expected in the detector by the different particles, assuming an initial velocity equal to the initial, parent beam velocity at the water column entrance. The calculation carries out the integration for each particle over the water column length, plus the detector length. The extension from points $B$ to $M$ follows again at logical sequence.

\section{Experimental Results}

Figure 5 shows an energy spectrum of $\mathrm{dE}$ for position $A$ in the Bragg curve. Peaks corresponding to all the elements from $B$ to $S i$ have been 


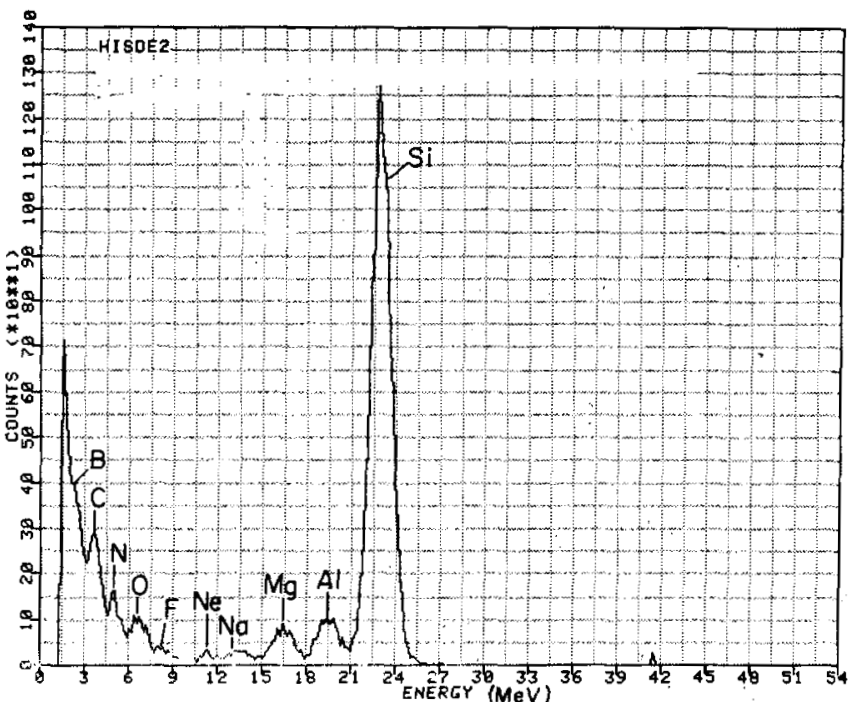

Figure 5. Histogram of $\mathrm{dE}$, energy deposited in the thin silicon detector, for the silicon ion beam at position A of the Bragg cruve. Peaks for all nuclei from boron to silicon are clearly defined. (XBL 808-11443)

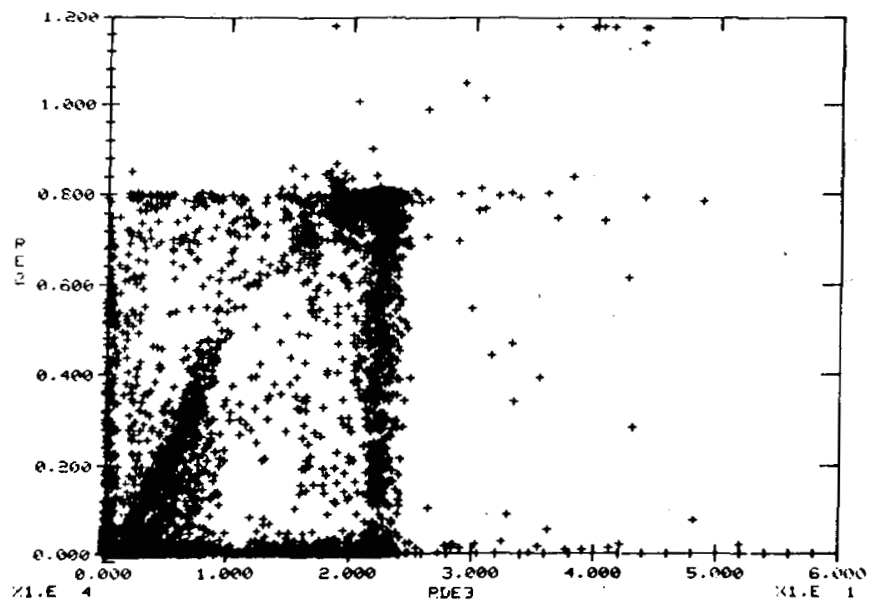

A

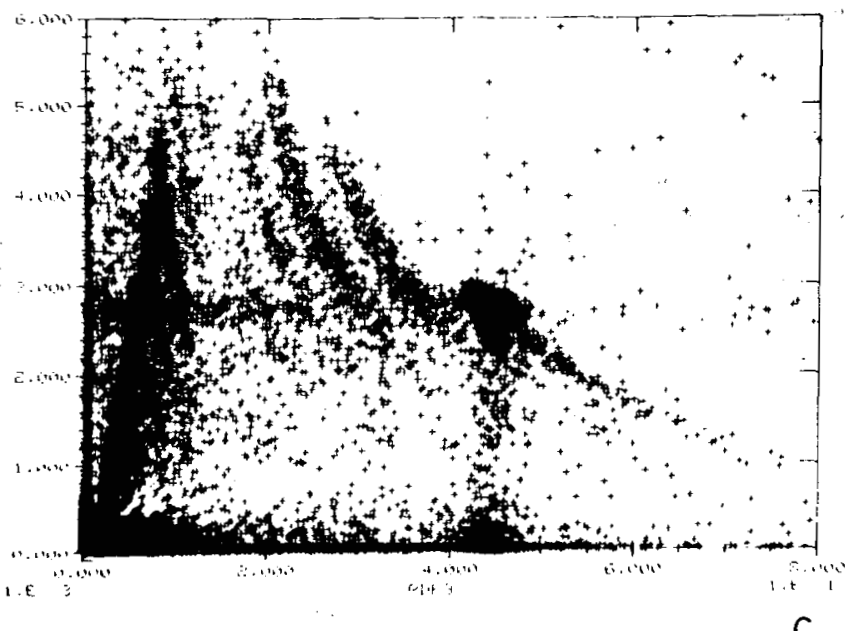

identified unequivocally by the relationship of equation 2 and by energy loss calculations. Lower energy events have been removed from the display.

Figure $6 A$ snows scatter plots of $E$ vs. $d E$ for positions $A, B, C$, and $D$ in the Bragg curve. Figure $6 B$ shows the results for positions $F, G$, $K$, and $M$. Each plotted point $(+)$ corresponds to one analyzed event. The vertical axis (RE2) corresponds to the energy $E$ deposited in the residual energy detector, while the horizontal axis (RDE3) shows the energy dE deposited in the thin silicon detector and is a measure of LET for single particles. The scales are calibrated in absolute energy ( $\mathrm{MeV}$ ), and are expected to be correct to within 2 to $5 \%$.

Observing the scatter plot for position A (Fig. 6A), we notice the main peak for the parent silicon ions as well as the clusters corresponding to the nuclei that appear in the dE spectrum of Figure 5. The vertical column of events under the main silicon peak corresponds to primaries that have not deposited their full energy in the long residual range detector due to escaping from
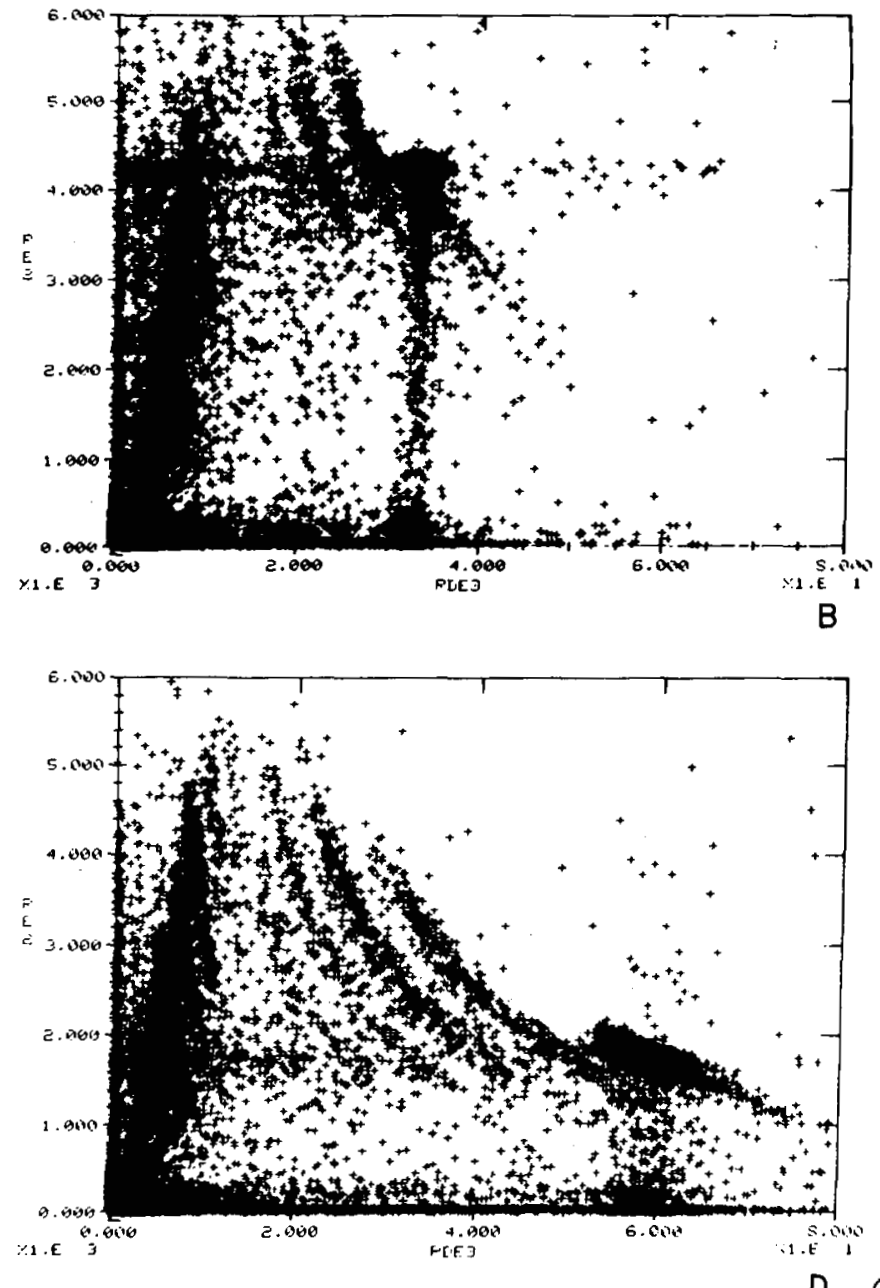

Figure 6A. Scatter plots of signals dE vS. E, (RE2 vs. RDE3 in the plots) for positions A, B, C, and D in the Bragg curve. Each event recorded is shown by a cross (+). Energies are in MeV. Note different scales for position A. (XBL 808-10927) 

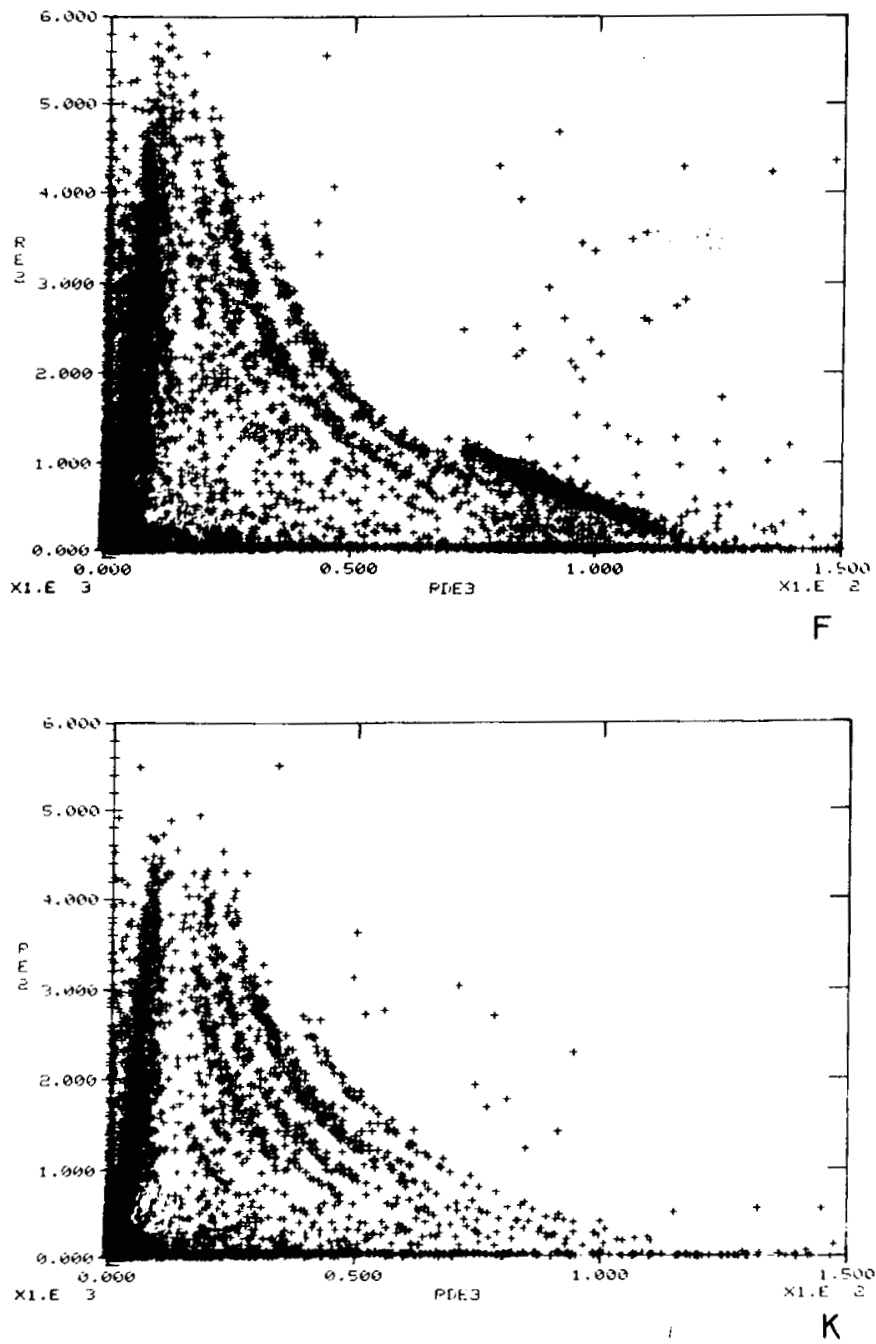
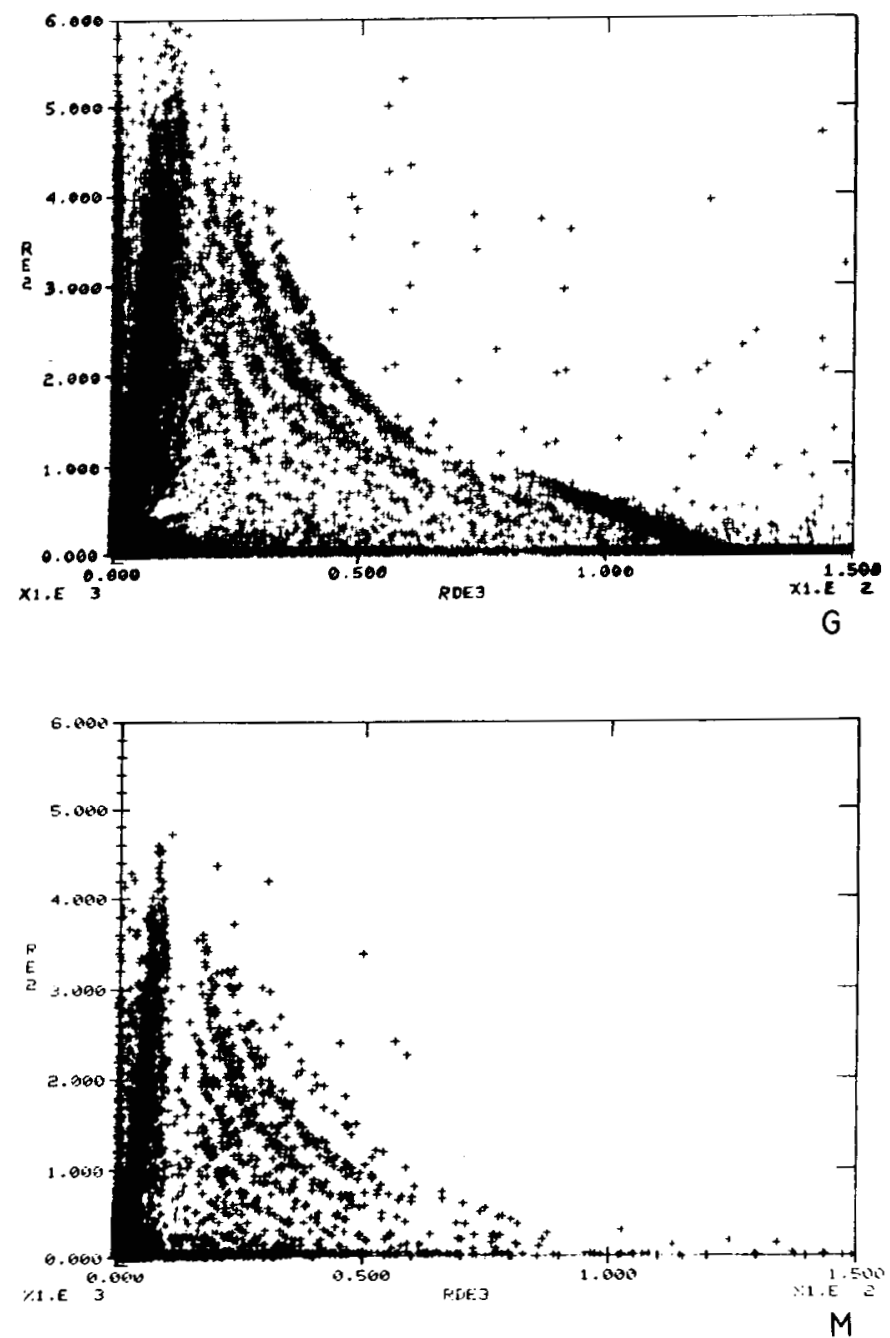

Figure 6B. Scatter plots of signals dE vs. E (RE2 vs. RDE3 in the plots) for positions $F, G, K$, and $M$. (XBL 808-10926)

the walls, or from fragmentation in the detector itself.

As we progress from point $A$ towards $F$ and $G$, near the Bragg peak, the dE signal for the parent silicon ions moves towards higher energy, while the residual energy $E$ decreases. Curves described by the relation of equation 1 are becoming more apparent for the heavier fragments, while some approximately straight lines appear clustered to the left of the scatter plots corresponding to the lighter fragments that do not stop fully in the residual energy detector.

As we approach the Bragg peak, the silicon peak becomes quite broad in both $E$ and $d E$. Beyond the Bragg peak, points $K$ and $M$, there is no more silicon, and the heavier fraginents also start disappearing. The scatter plots show a considerable number of events along both the dE and $E$ axes. Those in the RDE $3=0$ axis have been caused by a setting of the event trigger electronics which was slightly out of adjustment. Those events along the RE2 $=0$ axis are due to the fact that the silicon dE detector has a larger transverse dimension than the $E$ detector. Both types of events have been removed during data analysis.

Table 1 shows the data obtained directly by the analysis of the data of Figures $6 \mathrm{~A}$ and $6 \mathrm{~B}$ following the methods outlined above. Figure 7 shows a plot of the average energy loss (stopping power) measured for each $k$ ind of particle, or group of particles in silicon. Theoretical values for silicon are also shown. The number of silicon particles entering the water column for each of the individual experiments at points A to $M$ were not monitored in this first experiment: For that reason, the fraction of detected particles, $f_{n i}$, and the fraction of total energy loss, $f_{E} i$, are only consistent within each individual column of Table 1 . Each column adds up to $100 \%$.

In order to obtain estimates of dose $d_{j}$ contributed by each type of particle, or group of particles, and of the number $n_{j}$ of particles 
Table 1. Data Obtained Directly from Experimental Results

\begin{tabular}{|c|c|c|c|c|c|c|c|c|}
\hline \multirow{2}{*}{$\begin{array}{l}\text { Particle } \\
\text { or Group }\end{array}$} & \multicolumn{8}{|c|}{ Position in Bragg Curve } \\
\hline & A & B & c & D & $F$ & G & k & M \\
\hline \multirow{2}{*}{ 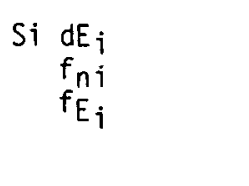 } & $\begin{array}{l}0.12 \mathrm{MeV} / \mu \mathrm{m} \\
26.0 \%\end{array}$ & $\begin{array}{l}0.19 \\
18.3\end{array}$ & $\begin{array}{l}0.25 \\
15.0\end{array}$ & $\begin{array}{l}0.32 \\
15.2\end{array}$ & $\begin{array}{l}0.50 \\
9.6\end{array}$ & $\begin{array}{l}0.60 \\
4.6\end{array}$ & - & - \\
\hline & $70.4 \%$ & 60.8 & 55.0 & 63.0 & 57.6 & 45.0 & - & - \\
\hline Al & $\begin{array}{l}0.10 \mathrm{MeV} / \mu \mathrm{m} \\
3.8 \% \\
8.6 \%\end{array}$ & $\begin{array}{l}0.14 \\
2.6 \\
6.6\end{array}$ & $\begin{array}{l}0.18 \\
2.1 \\
6.2\end{array}$ & $\begin{array}{l}0.21 \\
2.2 \\
6.1\end{array}$ & $\begin{array}{l}0.26 \\
1.5 \\
4.6\end{array}$ & $\begin{array}{l}0.27 \\
1.3 \\
5.8\end{array}$ & $\begin{array}{l}0.28 \\
1.0 \\
6.7\end{array}$ & $\begin{array}{l}0.24 \\
0.1 \\
1.0\end{array}$ \\
\hline $\mathrm{Mg}$ & $\begin{array}{l}0.09 \mathrm{MeV} / \mu \mathrm{m} \\
1.97 \% \\
4.0 \%\end{array}$ & $\begin{array}{l}0.12 \\
2.4 \\
9.0\end{array}$ & $\begin{array}{l}0.14 \\
2.6 \\
5.7\end{array}$ & $\begin{array}{l}0.16 \\
2.7 \\
9.7\end{array}$ & $\begin{array}{l}0.21 \\
2.3 \\
5.0\end{array}$ & $\begin{array}{l}0.21 \\
2.0 \\
3.2\end{array}$ & $\begin{array}{l}0.22 \\
3.2 \\
1.75\end{array}$ & $\begin{array}{l}0.24 \\
0.6 \\
4.4\end{array}$ \\
\hline$(\mathrm{Na}, \mathrm{Ne})$ & $\begin{array}{l}0.06 \mathrm{MeV} / \mu \mathrm{m} \\
1.5 \% \\
2.03 \%\end{array}$ & $\begin{array}{l}0.075 \\
3.4 \\
4.47\end{array}$ & $\begin{array}{l}0.11 \\
2.5 \\
4.6\end{array}$ & $\begin{array}{l}0.126 \\
2.6 \\
4.26\end{array}$ & $\begin{array}{l}0.15 \\
3.3 \\
5.8\end{array}$ & $\begin{array}{l}0.12 \\
3.5 \\
6.8\end{array}$ & $\begin{array}{l}0.16 \\
5.4 \\
21.4\end{array}$ & $\begin{array}{l}0.16 \\
4.0 \\
21.7\end{array}$ \\
\hline$(C, N, O, F)$ & $\begin{array}{l}0.03 \mathrm{MeV} / \mathrm{mm} \\
12.3 \% \\
8.14 \%\end{array}$ & $\begin{array}{l}0.043 \\
14.5 \\
10.9\end{array}$ & $\begin{array}{l}0.05 \\
18.4 \\
15.2\end{array}$ & $\begin{array}{l}0.053 \\
15.6 \\
10.7\end{array}$ & $\begin{array}{l}0.07 \\
20.4 \\
17.1\end{array}$ & $\begin{array}{l}0.07 \\
23.5 \\
26.5\end{array}$ & $\begin{array}{l}0.07 \\
17.2 \\
29.8\end{array}$ & $\begin{array}{l}0.07 \\
16.0 \\
37.7\end{array}$ \\
\hline$(\mathrm{H}, \mathrm{He}, \mathrm{Li}, \mathrm{Be}, \mathrm{B})$ & $\begin{array}{l}0.0056 \mathrm{MeV} / \mu \mathrm{r} \\
54.6 \% \\
6.9 \%\end{array}$ & $\begin{array}{l}0.012 \\
58.9 \\
12.2\end{array}$ & $\begin{array}{l}0.013 \\
59.7 \\
13.0\end{array}$ & $\begin{array}{l}0.012 \\
61.7 \\
9.4\end{array}$ & $\begin{array}{c}0.012 \\
62.9 \\
9.8\end{array}$ & $\begin{array}{l}0.012 \\
65.0 \\
12.7\end{array}$ & $\begin{array}{l}0.010 \\
73.0 \\
17.9\end{array}$ & $\begin{array}{l}0.010 \\
79.2 \\
25.0\end{array}$ \\
\hline
\end{tabular}

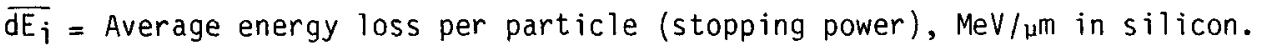

$f_{n i}=$ Fraction of detected particles at a particular position in Bragg curve. Each column is normalized to 100.

$f_{E j}=$ Fraction of total energy loss at a particular position in Bragg curves. Each column is normalized to 100.

Notice that entries $f_{n}$ and $f_{E} i$ are only consistent within each column. Results are not normalized to a fixed number of silicon particles in the beam before entering the water column.

at each position, the numerical values of the Bragg curve of Figure 3 were used to normalize the variables $f_{E} i$ and $f_{n i}$ of Table 1 to a value of 1.0 for silicon particles at zero water column. Table 2 shows the resulting estimates.

The estimates for $d_{j}$ were obtained, for position $A$ for example, from the expression:

$$
d_{j}=f_{E i} B_{A}
$$

where $B_{A}$ is the value of the Bragg curve at position $A$ in Figure 3 . The results of the estimates for $d_{i}$ are shown in Figure 8 .

The estimates for $n_{i}$ were obtained from the expression

$$
n_{i}=K\left(d_{j} / d E_{j}\right)
$$

where $k$ was chosen so that for $d j=1$, at zero water column for silicon particles, and the 


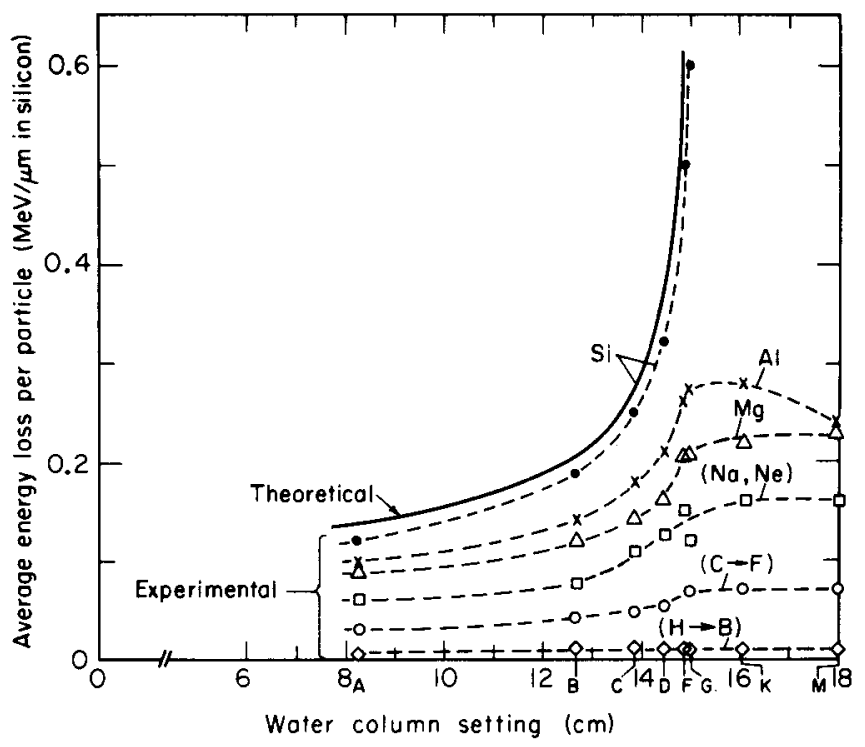

Figure 7. Average energy loss $\mathrm{dE}_{i}$, in silicon for the measured types of particles at points $A$ through $K$ of the Bragg curve. Theoretical values for silicon are also shown. (XBL 808-3608) corresponding theoretical value for $d E_{j}, n_{j}$ became equal to 1 . The results are plotted in Figure 9 .

\section{Discussion}

The data presented in the scatter plots of Figures $6 \mathrm{~A}$ and $6 \mathrm{~B}$ contain a large amount of information about the complex process of nuclear fragmentation in thick targets. Extracting that information is only a question of generating the appropriate computer software to analyze the data in a meaningful manner. The grouping of particles to facilitate analys is and the choice of the three parameters presented in Table 1 has been dictated by the need to use an available software system for data analys is that is flexible but slow. The results presented in Table 1 and Fiqure 7 are, however, quite reliable. An interpretation that at point $G$ of the Bragg curve $45 \%$ of the dose received by a thin sample is due to silicon ions and $26.5 \%$ is due to fragments including $C, N, 0$, and $F$ can be expected to be correct to within a few percent.

An appropriate detector to identify and count the primary silicon ions before entering the

Table 2. Data Obtained by Normalizing the Data of Table 1 to a Standard Bragg Curve Obtained in Conditions Similar to Those of the Present Experiment

\begin{tabular}{|c|c|c|c|c|c|c|c|c|}
\hline \multirow{2}{*}{$\begin{array}{l}\text { Particle } \\
\text { or Group }\end{array}$} & \multicolumn{8}{|c|}{ Position in Bragg Curve } \\
\hline & A & $B$ & C & D & $F$ & $\mathrm{G}$ & K & $M$ \\
\hline $\begin{array}{l}\text { Si } d_{i} \\
n_{j}\end{array}$ & $\begin{array}{l}0.67 \\
0.558\end{array}$ & $\begin{array}{l}0.73 \\
0.38\end{array}$ & $\begin{array}{l}0.80 \\
0.32\end{array}$ & $\begin{array}{l}1.19 \\
0.37\end{array}$ & $\begin{array}{l}1.70 \\
0.34\end{array}$ & $\begin{array}{l}1.74 \\
0.29\end{array}$ & - & $\begin{array}{l}- \\
-\end{array}$ \\
\hline A1 & $\begin{array}{l}0.083 \\
0.083\end{array}$ & $\begin{array}{l}0.079 \\
0.056\end{array}$ & $\begin{array}{l}0.09 \\
0.05\end{array}$ & $\begin{array}{l}0.116 \\
0.055\end{array}$ & $\begin{array}{l}0.136 \\
0.052\end{array}$ & $\begin{array}{l}0.22 \\
0.13\end{array}$ & $\begin{array}{l}0.032 \\
0.011\end{array}$ & $\begin{array}{l}0.002 \\
0.001\end{array}$ \\
\hline $\mathrm{Mg}$ & $\begin{array}{l}0.038 \\
0.042\end{array}$ & $\begin{array}{l}0.06 \\
0.05\end{array}$ & $\begin{array}{l}0.08 \\
0.057\end{array}$ & $\begin{array}{l}0.108 \\
0.067\end{array}$ & $\begin{array}{l}0.148 \\
0.07\end{array}$ & $\begin{array}{l}0.124 \\
0.06\end{array}$ & $\begin{array}{l}0.008 \\
0.004\end{array}$ & $\begin{array}{l}0.01 \\
0.004\end{array}$ \\
\hline$(\mathrm{Na}, \mathrm{Ne})$ & $\begin{array}{l}0.019 \\
0.032 \\
\end{array}$ & $\begin{array}{l}0.054 \\
0.07: 2\end{array}$ & $\begin{array}{l}0.067 \\
0.061\end{array}$ & $\begin{array}{l}0.081 \\
0.064\end{array}$ & $\begin{array}{l}0.17 \\
0.113\end{array}$ & $\begin{array}{l}0.264 \\
0.22\end{array}$ & $\begin{array}{l}0.104 \\
0.065\end{array}$ & $\begin{array}{l}0.05 \\
0.031\end{array}$ \\
\hline$(C, N, O, F)$ & $\begin{array}{l}0.078 \\
0.26\end{array}$ & $\begin{array}{l}0.13 \\
0.302\end{array}$ & $\begin{array}{l}0.222 \\
0.444\end{array}$ & $\begin{array}{l}0.20 \\
0.377\end{array}$ & $\begin{array}{l}0.51 \\
0.71\end{array}$ & $\begin{array}{l}1.03 \\
1.47\end{array}$ & $\begin{array}{l}0.144 \\
0.206\end{array}$ & $\begin{array}{l}0.086 \\
0.123\end{array}$ \\
\hline$(\mathrm{H}, \mathrm{He}, \mathrm{Li}, \mathrm{Be}, \mathrm{B})$ & $\begin{array}{l}0.066 \\
1.17\end{array}$ & $\begin{array}{l}0.146 \\
1.21\end{array}$ & $\begin{array}{l}0.189 \\
1.454\end{array}$ & $\begin{array}{l}0.178 \\
1.483\end{array}$ & $\begin{array}{l}0.29 \\
2.41\end{array}$ & $\begin{array}{l}0.49 \\
4.08\end{array}$ & $\begin{array}{l}0.086 \\
0.86\end{array}$ & $\begin{array}{l}0.057 \\
0.57\end{array}$ \\
\hline & & & & & & & & \\
\hline
\end{tabular}

$d_{i}=$ Dose, normalized to 1.0 for silicon particles at zero water column.

$n_{i}=$ Number of particles, normalized approximately to 1.0 for silicon at zero water column.

These data are presented only as tentative. 


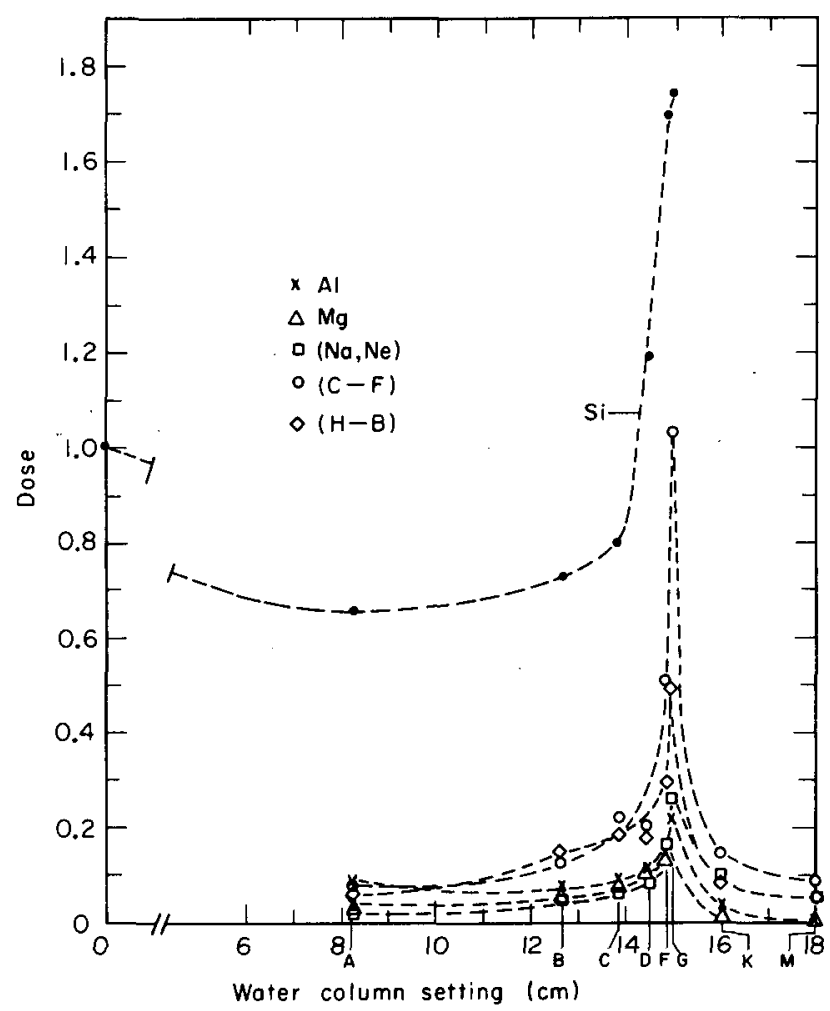

Figure 8. Estimate of the dose contributed to the Bragg curve of Figure 3 by each $k$ ind of particle. Results may be only qualitatively correct. (XBL 808-3611)

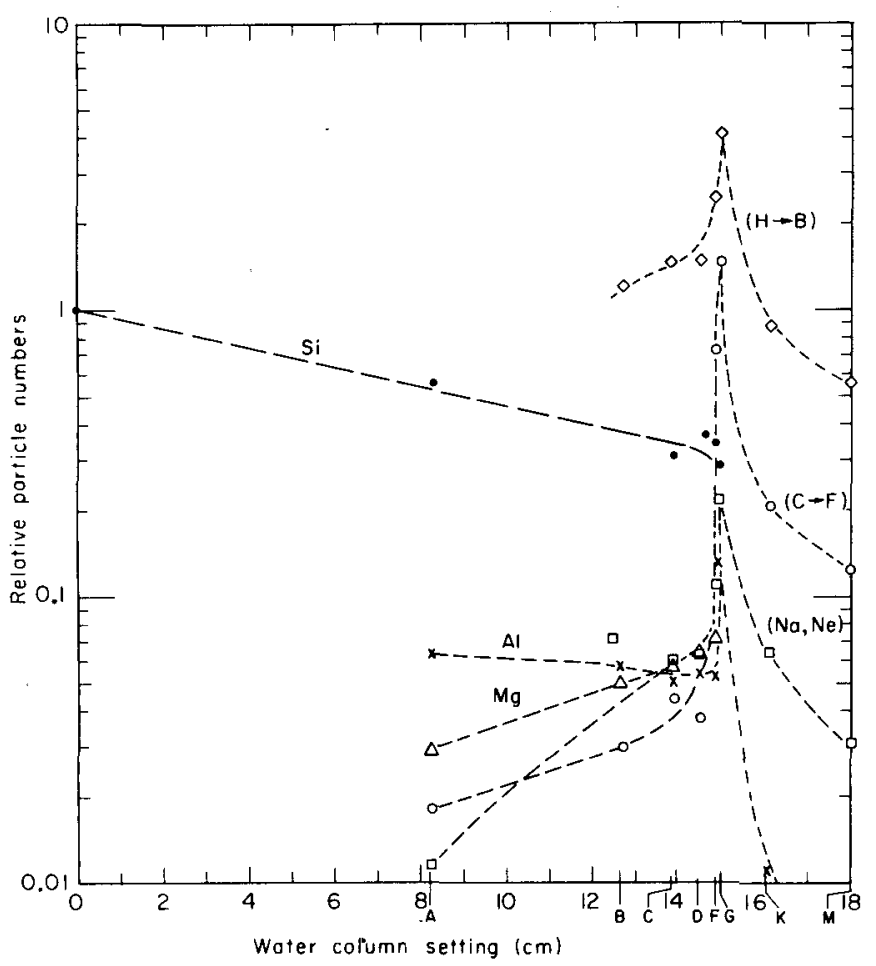

Figure 9. Estimates of particle number vs. distance. Results may be on ly qualitatively correct. (XBL 808-3610) water column was not available, consequently the estimates of Table 2 are less reliable. Although the thickness of the detector windows has been taken into consideration in calculating the normalization of the data to the Bragg curve of Figure 3 , small errors in registration can lead to significant errors in the estimates near the Bragg peak. The data for the dose contributed by each type of particle, shown in Figure 8, are qualitatively correct. The sharp peak in the number of particle, $n_{i}$, shown in Figure 9 , that appears to exist at the lightest elements must be interpreted with caution. As the data stand, we find that for each parent silicon particle entering the water column, there are four particles of the lightest group ( $H$ through $B$ ) detected at position $G$. This result seems quite likely, but the reason for the drop to 0.86 light particles at point $K$ is not too clear. A possible explanation for the result is that the majority of light particles near the Bragg peak have low velocity and they fall below the level of detection at points $K$ and $M$. This point will be investigated further. The numbers vs. distance curve of Figure 9 for silicon shows an approximately exponential nature, which agrees with the theoretical work of Cnatterjee et al. (1976).

\section{Future Developments}

The results of this first experiment are quite encouraging: they point out the capability of fairly simple detector systems to yield significant information to an experimenter or physician about the detailed characteristics of complex beams. It is now possible to experimentally confirm beam fragmentation theories, test the correlation between biological observations and beam characteristics, and perform routine beam characterization measurements to ensure reproducibility in biological experiments or in radiation treatment. We are planning or implementing a number of steps to make more accurate measurements and to make data reduction simpler and faster.

1. A new de detector will be placed before the water column absorber. This detector will be used as a particle identifier and counter. In the new configuration, every particle will be accounted for and we expect to be able to reproduce a Bragg curve directly from the measurements alone. 2. Improvements in the electronics system will remove spurious signals from both the $\mathrm{dE}$ and the $\mathrm{E}$ channels.

3. Utilization of new software for data acquisition will allow increasing the data acquisition rate by a factor of nearly ten. 4. Utilization of an interactive data analys is software system will allow the experimenter to have. an almost instantaneous return of desired parameters after an experiment is finished. The system uses a fast array processor and the data will be presented in a color video display with large storage capacity.

5. Interaction with the different experimenters of the Radiation Biophysics Group 
interested in beam characterization and analys is will increase gradually as the system's capabilities to supply information are improved.

This project is being developed concurrently with a demonstration of the capability for tomographic image reconstruction with heavy ions. The development of larger detector arrays with corresponding electronics for image reconistruction will automatically benefit the beam analysis project. Fundamentally, the two.projects differ only in the way the data are analyzed, i.e., different software systems are needed to carry out the two different jobs. The development of the two systems will be carried on concurrently, using as many parts in common as possible.

\section{NUCLEAR TRACK TECHNIQUES}

$$
\text { (E. V. Benton) }
$$

Solid-state track detectors or dielectric track detectors have been in use for about fifteen years and have found numerous applications in different fields of science and technology. In many dielectrics, paths of ionizing charged particles are revealed as the result of damage created by the energy left behind in the detector by the incident charged particles. These damage trails can be delineated and made visible through a selective chemical etching of the radiation-damaged material along the charged-particle trajectory. This process results in an etch pit, or, in some cases, a narrow channel referred to as a track. Further etching can enlarge tracks to a convenient size for direct viewing with an ordinary optical microscope. This phenomenon has been found to be general and includes many minerals, glasses, and polymers. Although the number and variety of applications of solid-state detectors is very broad and still rapidiy growing, one of the most useful areas lies in nuclear science and technology (Fleischer et al., 1975; Francois et al., 1980).

The sensitivity of these detectors (the ability to record visible tracks) varies widely from that of minerals such as mica which record tracks of particles only as heavy as argon or iron at the end of their range, to that of plastics, such as Lexan or cellulose nitrate, and lately to a new and very sensitive track. detector called CR-39 which records protons ip to at least $20 \mathrm{MeV}$ (Ruddy et al., 1980).

On the submicroscopic scale, a particle damage trail and the subsequent formation of an etch track can be viewed in terms of the chemical attack of a very narrow linear region, as shown is Figure 10. On the right is the tip of a track resulting from a chemical attack of a damaged region. The degree of radiation damage is greatest in the immediate vicinity of the particle trajectory, and the etch rate (normal to the leading end of the etch pit) has its greatest value $V_{T}$, the track etch rate. The degree of damage and the etch rate both decrease as the distance from the trajectory increases.

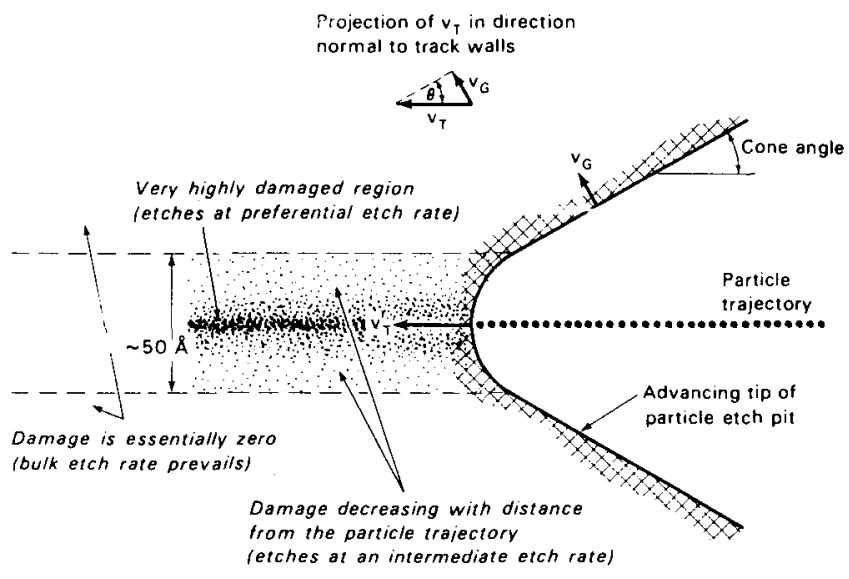

Figure 10. Damage trail and track etching on a submicroscopic scale. (XBL 807-10680)

At a certain distance (estimated to be somewhere between 25 and $50 \AA$ ) the damage falls off to some small value, and the etch rate normal to the interior of the track is the etch rate of the undamaged bulk material, $V_{G}$. Since the advancing tip of the etch pit maintains its shape while moving forward at the rate $V_{T}$ in the undamaged region, the angle made by the walls of the etch pit with the particle trajectory must be such that the projection of $V_{T}$ in the direction of. the normal to these walls will be $V_{G}$. This is also shown in Figure 10 . Consequently, the track cone angle, $\theta$, is given by sin $\theta=V_{G} / V_{T}$. If one observes track formation on the scale of an optical microscope, the rounded pits shown in Figure 10 appear perfectly sharp. $V_{T}$ can be expressed as a monotonically increasing function of the particle's restricted energy loss rate RE $L_{W}$, the rate of energy loss to electrons with energy less than $w$ (Henke and Benton, 1972). The function REL is identical to

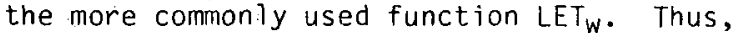
as LET varies along the particle damage trail, $V_{R}$ and $\theta$ also vary.

The stages of evolution of an etch track are shown in Figure 11, which is a side view of a track with a dip angle $\delta=45^{\circ}$ and a cone angle $\theta=15^{\circ} \%$ The positions of the detector surface are labeled in a time sequence from 0 to 6 . Thus; one can see that the appearance of a track can vary considerably from a small etch cone to a larger etch cone; and then eventually to a shallow etch pit. From a detailed knowledge of the etch-track geometry, it is possible to ident ify, the particle as to its charge, energy and, in some cases, mass (Henke and Benton, 1972; Beriton and Henke, 1968).

\section{Accelerator Applications}

There are numerous useful applications of solid-state track detectors around accelerators. At LBL, the following procedures have been used: 


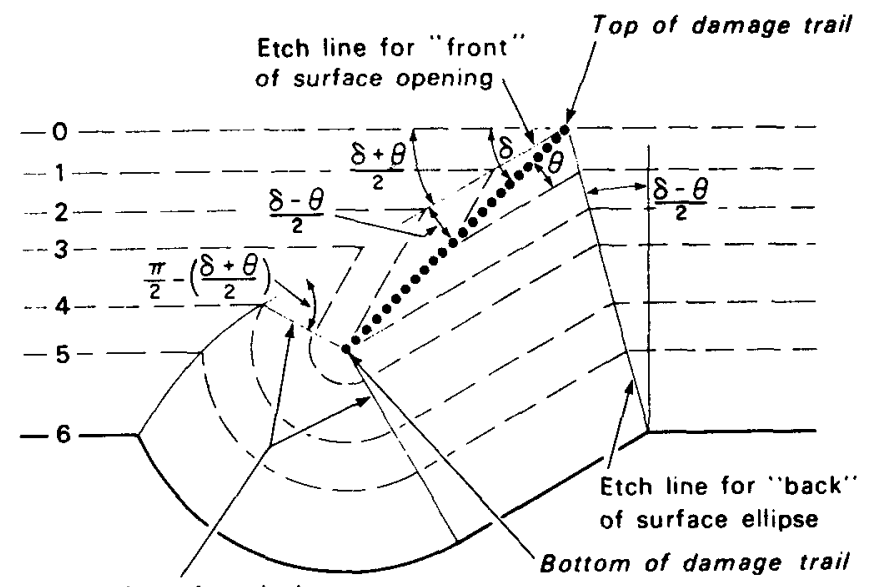

Intersection of conical

and spherical track portions

Figure 11. Sequential stages of track etching for a particle damage trail of finite length; $\delta$ is the angle between particle trajectory and detector surface; $\theta$ is the track cone angle. (XBL 807-10697)

\section{Fluence Mapping}

Sheets of cellulose nitrate detector exposed normally to the beam and quickly processed in $\mathrm{NaOH}$ have routinely been used during the tune-up and experiment preparation phases in order to obtain quickly a picture of the extent of radial distribution and homogeneity of the beam at the experimenter's station. The advantages of track detectors over ordinary $\mathrm{film}$ in this application is that one obtains a picture of the heavy ions of interest without accompanying lighter ions or electron contaminations. Since individual tracks can be observed, it is possible to get a true picture of the heavy-ion beam distribution.

\section{Energy Measurement of Particle Beams}

Although the conventional beam dosimetry technique provides reliable information on the energy distribution and fluence of the particles as well as integrated quantities such as the Bragg curve, they cannot provide this information for individual beam pulses or even for a few beam pulses because they must define the curve through repeated measurements. In contrast, the plastic detector--wedge energy--degrader configuration can analyze individual pulses (or average over a larger number of pulses, if desired) and can be quickly processed and read with a conventional light microscope in about 10 min which is quite competitive with the time required by conventional dosimetry. The basic principles behind this work are straightforward and applicable to low-energy proton machines as well, if CR-39 is used as the detector (Benton et al., 1977).

\section{LET Spectra Determination}

Since, for normally incident particles onto a detector surface, particle-track diameters can be related to particle LET, it is possible to obtain first visually and then through measurement the purity of the beam and to measure the distribution of LET present in the beam of particular configuration. Figure 12 is a photomicrograph of a $510 \mathrm{MeV} /$ nuc neon-ion incident normally on a sheet of CR-39 detector. The high-energy neon particles are clearly seen as the small-diameter circular tracks. The larger dark tracks are those due to higher LET particles present in the beam.

An example of particle beam distribution obtained at the Bevalac is shown in Figure 13. The distribution on the left is that due to $670 \mathrm{MeV} / \mathrm{amu} 28 \mathrm{Si}$ ions while the one on the right is the same beam as measured after passing through $10 \mathrm{~cm}$ of water. In both cases, the detector used was CR-39 with track diameters shown in $\mu \mathrm{m}$. The distribution on the right shows a significant tail of lower diameter tracks due to lighter beam fragments, while the mean of the distribution has been shifted to larger track diameters due to slowing down in water.

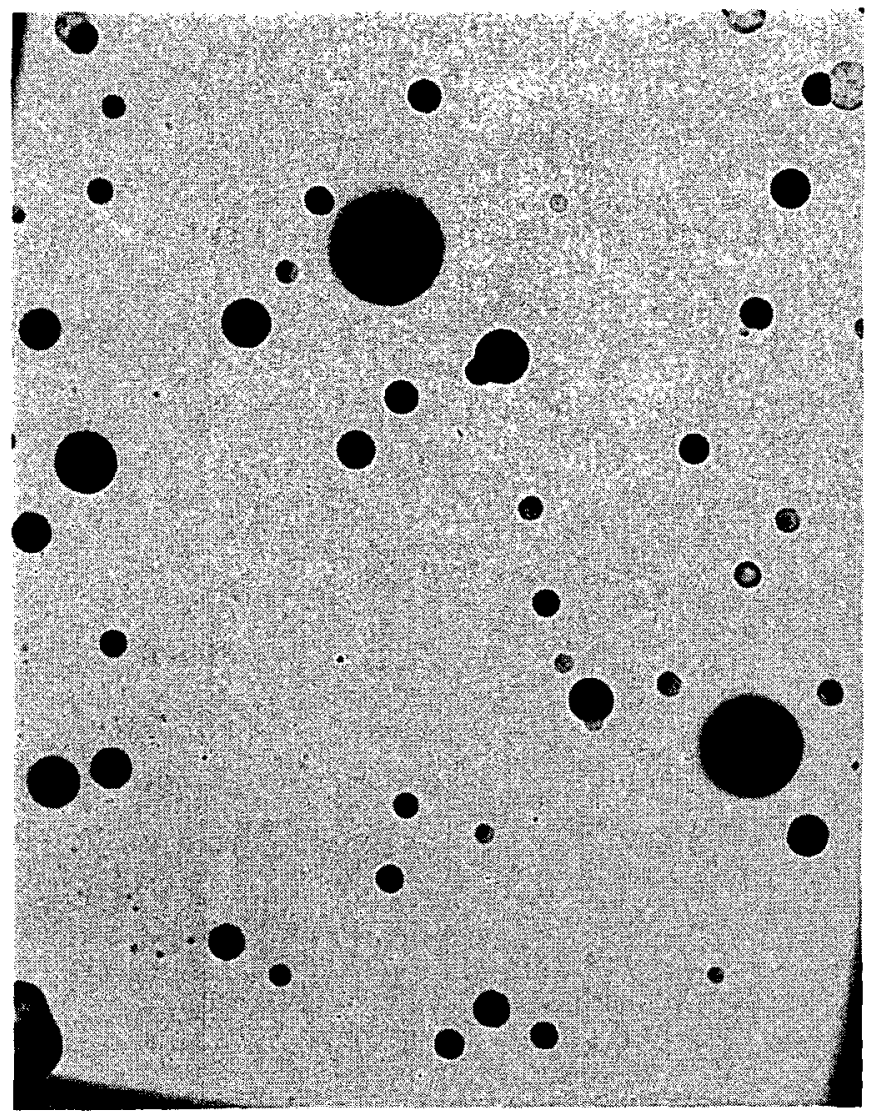

Figure 12. Photomicrograph of $570 \mathrm{MeV} / \mathrm{amu}$ neon ions normally incident on a sheet of CR-39 detector. The largest tracks are those due to higher-LET particles. (XBB 807-8513) 


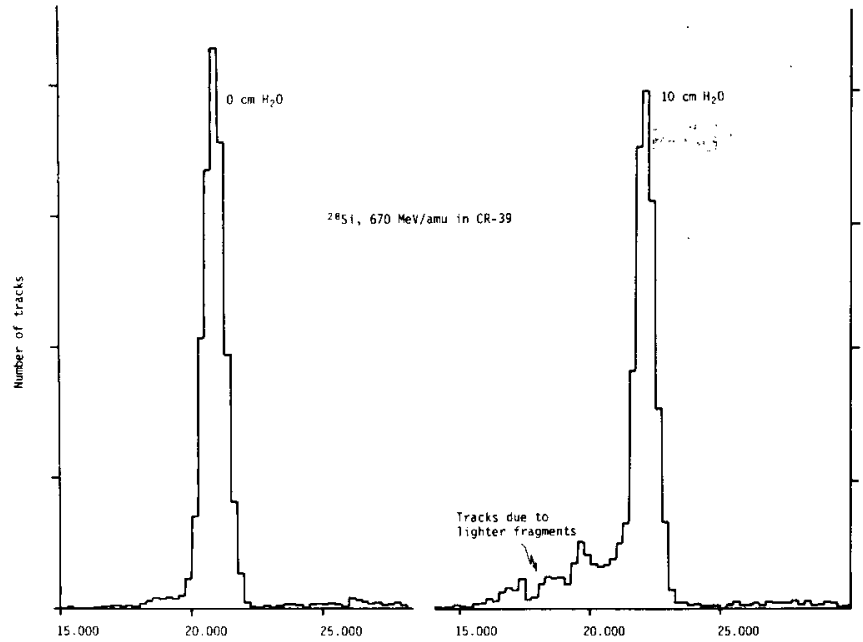

Figure 13. Distribution of track diameters of $670 \mathrm{MeV} / \mathrm{amu} 28 \mathrm{Si}$ ions in CR-39. Distribution on the left is that due to pure beams; distribution on the right shows lighter fragments due to passage through $10 \mathrm{~cm}$ of water. (XBL 807-10714)

Three-Dimensional Stopping-Point Distribution Determination

For biomedical applications that require radiation using the Bragg peak, it may be necessary to determine the three-dimensional LET distribution within a certain defined volume or the three-dimensional stopping-point distribution of particles. Since plastic track detectors are close to tissue equivalent, they can be assembled into stacks simulating the experimental volume. After exposure and processing, the individual layers within such stacks can be analyzed to yield a true three-dimensional picture of the stopping heavy-ion beam. Complex geometry involving widely different types of stopping materials such as bone, tissue, and air can be simulated with phantoms and stacks of plastic track detectors in order to define the LET distributions at different depths.

\section{Fragmentation Studies}

Since heavy charged-particle beams fragment during the passage through matter and since this fragmentation can significantly change the ionization characteristics of the chargedparticle beam, in some radiobiological experiments it is necessary to know the extent of the fragmentation and the smearing of the Bragg peak that occurs as the result of incident particles losing neutrons, protons, or larger fragments of incident nuclei. These types of studies, described earlier, can be effectively performed by the use of multilayered stacks of solid-state track detectors.
Heavy-Particle Radiography

We have shown some time ago that heavy particles can be used to produce radiographs with high depth resolution and contrast. Through the use of stacks of solid-state track detectors, small differences in the stopping power of objects can be detected and permanently recorded (Benton et al., 1973). This method is now under active development and is described in Part VI, Section $B$ of this report.

\section{FOOTNOTE AND REFERENCES}

*Department of Physics, University of San Francisco, San Francisco, CA.

Alonso, J. R., C. A. Tobias, and W. T. Chu. 1979. Computed tomographic reconstruction of beam profiles with a multiwire chamber. IEEE Trans. Nuc1. Sci. NS-26, 3077-3079.

Benton, E. V. and R. P. Henke. 1968. A new method for charge determination of heavy, cosmic ray particles. Nucl. Instrum. Methods 58, 241-244.

Benton, E. V., R. P. Henke, and C. A. Tobias. 1973. Heavy-particle radiography. Science $182,474-476$.

Benton, E. V., R. P. Henke, and C. A. Tobias. 1977. Calibration of high-energy heavyparticle beams with plastic nuclear track detectors. Nucl. Track Detection 1, 139-143.

Blakely, E. A., C. A. Tobias, T.C.H. Yang, K. C. Smith, and J. T. Lyman. 1979. Inactivation of human kidney cells by high-energy monoenergetic heavy-ion beams. Radiat. Res. 80, $122-160$

Chatterjee, A., C. A. Tobias, and J. T. Lyman. 1976. Nuclear fragmentation in therapeutic and diagnostic studies with heavy ions. Spallation Nuclear Reactions and Their Applications, (Shen and Merker, eds.), pp.169-191. Reidel, Dordrecht, Holland.

Crowe, K., L. Kanstein, J. Lyman, and F. Yeater. 1975. Lawrence Berkeley Laboratory Report LBL -4235 .

Fleischer, R. L., P. B. Price, and R. M. Walker. 1975. Nuclear Tracks in Solids. University of California Press, Berkeley.

Francois, H. et al., ed. 1980. Solid State Nuclear Track Detectors. Pergamon Press, Oxford and New York.

Goulding, F. S. and B. G. Harvey. 1975. Identification of nuclear particles. Annu. Rev. Nucl. Sci. 25, 167-240.

Henke, R. P. and E. V. Benton. 1972. On geometry of tracks in dielectric nuclear track detectors. Nucl. Instrum. Methods 97, 483-489. 
Koehler, A. M., R J. Schneider, and J. M. Sisterson. 1977. Medical Physics 4, 297.

Maccabee, H. D. 1973. Composition of highenergy heavy-ion beams: Preliminary measurements. Radiat. Res. 54, 495-509.

Maccabee, H. D. and M. A. Ritter. 1974. Fragmentation of high-energy oxygen-ion beams in water. Radiat. Res. 60, 409-421.

Ruddy, F. H., C. C. Preston, R. Gold, E. V. Benton, and J. H. Roberts. 1980. CR-39 polymer, a promising new solid state track recorder for high energy neutron applications. HEDL-SA 2149.
Sachs, M. W., C. Chasman, and D. A. Bromley. 1966. Heavy ion reaction product identification by measurement of $\mathrm{dE} / \mathrm{dx}$ and $\mathrm{E}$. Nucl. Instrum. Methods 41, 213-225.

Schimerling, W. and S. B. Curt is. 1977. Velocity spectrometry of $3.5 \mathrm{GeV}$ nitrogen ions. Radiat. Res. 72, 1-17.

Shepp, L. A. and B. F. Logan. 1974. The Fourier reconstruction of a head section. IEEE Trans. Nucl. Sci. NS-21, 21-43. 


\title{
EXPERIMENTAL HEAVY PARTICLE PHYSICS
}

\author{
W. Schimmerling, E. V. Benton, ${ }^{*}$ D. J. Hildebrand, ${ }^{\dagger}$ R. P. Henke, \\ W. Heinrich, $¥$ and C. A. Tobias
}

\section{A. NUCLEAR INTERACTIONS AND RADIATION DOSIMETRY}

\section{(W. Schimmerling)}

The maximum energy deposition of heavy-ion beams can be made to occur at a precisely defined range in any material by selection of the particle species and of its incident energy. This choice is determined by the amount of material in the target volume (e.g., tissue in radiation therapy) and the amount of material required for beam control and modification ( $r$ idge filters, collimators, beam-flattening foils). The range of heavy ions used for biomedical research is $20-30 \mathrm{~g} / \mathrm{cm}^{2}$. The mean free path for nuclear interactions is $-20 \mathrm{~g} / \mathrm{cm}^{2}$ for beams with the required incident energies (Wilson and Costner, 1975; Jaros et al., 1978). Thus, a significant fraction of the incident heavy-ion beam will undergo a nuclear reaction. The products of these nuclear interactions will, in general, have different biological effectiveness, or quality, in the target volume. In the case of radiation therapy and the irradiation of animals, the nuclear interaction products will constitute the distal dose as well as the dose to nontarget tissues.

In order to characterize the radiation field of heavy-ion beams, it is necessary to know the nuclear interaction cross section, the probability of producing one or more secondary particles of a given kind, the energy spectrum and the angular distribution of these secondary particles, and their multiplicity, i.e., the number of fragments or reaction products that are produced in any single interaction. Since these reactions can take place at any depth in the material traversed by the beams, it is also necessary to know these quantities as a function of particle energy : for all the particles that may be present. Such information is not at present available; its acquisition is partly the objective of the developing field of heavy-ion physics. "An : introductory review of this field (Curtis and Schimmerling, 1977), and several excellent reviews covering the field of heavy-ion physics in depth are also available (Scott, 1978; Goldhaber and Heckman, 1978).

The knowledge of the nuclear physics of heavy ions is not, by itself, sufficient to evaluate the radiation field of a heavy-ion beam. "The beams have finite size and are also modified by beam-transport elements, collimators, and multiple coulomb scattering. Even with a complete knowledge of the physical quantities related to all these phenomena, the calculation and prediction of the radiation field is a cumbersome and complex task that requires sophisticated calculation techniques. Further- more, the requirements of biomedical research differ from the usual physics experiment in two important ways. First, we are interested in the transport of heavy ions through thick targets. Second, it is necessary to know all the particles that have gone through a given target. Every particle traversing a biological sample contributes to the observed effect, regardless of whether it has triggered an electronic logic signal or not.

This chapter describes the Radiation Biophysics Group research programs in the nuclear physics of heavy-ion beams and their transport through thick targets. This work is intended to provide the information required for heavy-ion dosimetry, for therapeutic applications, and for fundamental studies in radiation chemistry, radiobiology, and biochemistry. The target materials of interest are tissue constituents, as well as the components of scattering foils, ridge filters, collimators, and ionization chamber gases. The most important beams of interest are the helium, carbon, and neon beams at energies of 200-700 MeV/A which are used for therapy or considered for future therapeutic use. Higher energies and heavier particles may be of interest in the study of single particle effects, the production of heavier radioisotope beams, and the production of microlesions.

This chapter describes measurements of neutron background obtained at the Medical Facility of the 184-inch Synchrocyclotron that are relevant to heavy-ion treatment at the Bevalac, experiments to study the fundamental physics of neutron production and of fragmentation into charged particles, and the development of transport calculations for beam modelling. A need exists for instrumentation to measure beam quality at several levels of sophistication. A particle identification spectrometer, developed for fragmentation studies, is described in this chapter, along with the use of plastic track detectors pioneered for this use by $E$. Benton and his collaborators. J. Llacer et al. describe a prototype position-sensitive germanium detector that can be used as an on-line heavy-ion imaging instrument: and as a beam quality monitor (chapter II-1). The reader is further referred to the articles by Chen et al, and Lyman et al. in PartivI of this report, where the interaction of heavy ions with matter is discussed in the context of treatment planning and verification.

\section{NEUTRON PHYSICS AND DOSIMETRY}

Altnough the dose contributions of secondary protons and neutrons are small (in most beams, less than $3 \%)$, these particles are an important source of background radiation in and around the 
medical caves. Such information becomes especially important when sensitive tissues are close to the treatment area. For example, in the treatment of certain eye tumors with a narrow nelium-ion beam, parts of the patient's eye will be outside the target volume but close to the edge of the charged-particle radiation field. In this case it is important to ensure that the neutron dose to the lens of the eye is below the cataractogenic threshold. This threshold has been estimated at 75-100 rad for fission neutrons (Ham, 1960; Upton, 1968).

The distribution of neutrons produced in the various elements of the helium medical beam was measured (Smith et al., 1980) using activation detectors along the beam line and perpendicular to it, in a plane containing the patient isocenter. The radioactive yield of different activation detectors was unfolded from the known energy dependence of the activation cross sections to obtain a neutron energy spectrum, and the unfolded spectra were corrected for radioactivity induced by charged particles.

The results of this investigation allowed us to identify the most significant sources of neutrons in the beam line. These were the water column and the collimators used for beam shaping close to the patient. The relative contribution of these sources was obtained, as was the neutron dose perpendicular to the beam axis in a plane containing the patient isocenter. The dose in soft tissue due to neutrons and protons decreases from about $5 \times 10^{-3} \mathrm{rad}$ on the beam axis to. about $1 \times 10^{-3} \mathrm{rad}$ at $20 \mathrm{~cm}$ from the beam axis, per plateau rad of incident beam. The dose increases somewhat when a phantom is inserted in the beam to simulate the patient's head, as shown in Figure 1 , where the perpendicular dose distribution has been plotted for both cases. A Gaussian distribution has been fitted to the rising portion of the curves for ease in the use of these data. The full width at half maximum (FWHM) is $22 \mathrm{~cm}$ with a phantom of the beam and $27 \mathrm{~cm}$ without a phantom. These measurements give an upper limit of the absorbed dose in soft tissue that will typically result in a dose of less than 10 rad to the eye, which is well below the threshold for cataract incidence determined for fission neutrons.

In a separate series of experiments performed at the Bevalac in collaboration with physicists from Kent State University, we have measured the probability (double differential cross section) of producing neutrons at a given energy and a given angle with respect to an incident heavy-ion beam, when nuclei in the beam interact with the nuclei in targets of carbon, aluminum, copper, lead, and uranium. The energy of the neutrons is obtained from the time of flight (TOF) between the target and each of: the neutron detectors, which were placed at different angles around the target. The neutron detectors are $1 \mathrm{~m}$ wide, 12.5 or $25 \mathrm{~cm} \mathrm{high,} \mathrm{and} 10 \mathrm{~cm}$ thick. In addition to the TOF, we recorded the light output and the position at which each neutron generated the recoil charged particles that gave rise to the detected signal. This resulted in a more accurate assignment of the detector efficiency

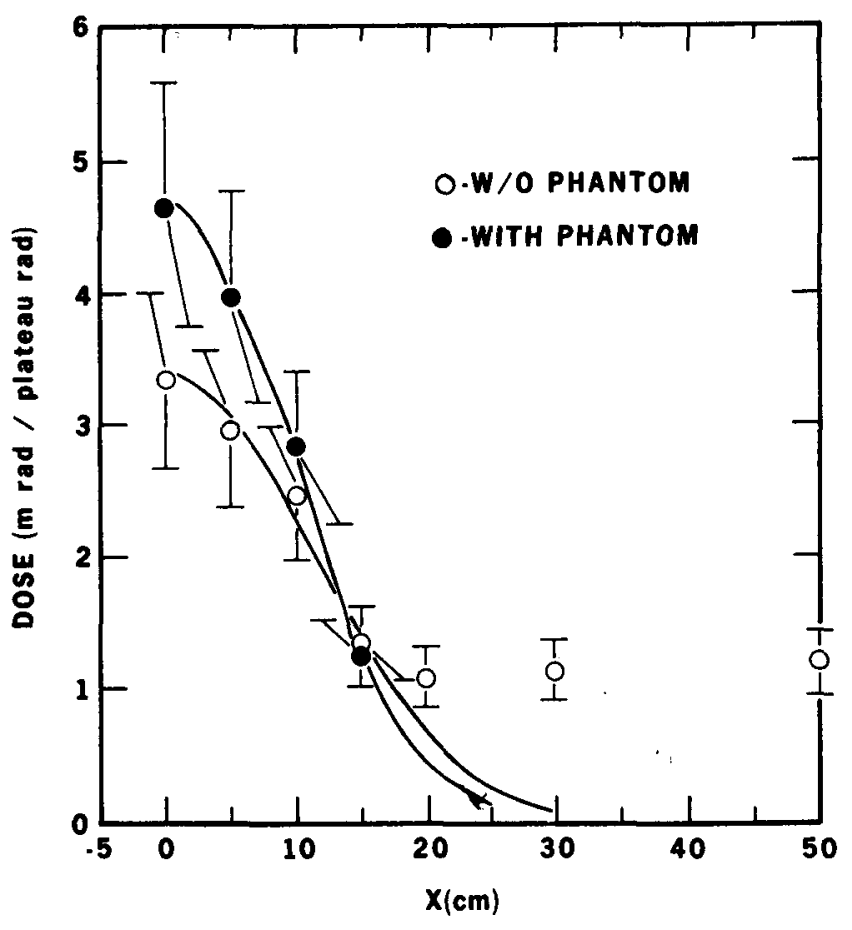

Figure 1. Absorbed dose (mrad per plateau rad of the primary helium beam) due to neutrons, as a function of distance from the beam axis, in a plane containing the patient isocenter. The data have been obtained by unfolding the radioactive yield from the known energy dependence of the activation cross sections to obtain a neutron spectrum, and using fluence-to-dose conversion factors derived by Rindi (1977) from a review of published data. The open circles correspond to a measurement without a phantom, and the full circles correspond to a measurement with a phantom. The curves are Gaussian functions fitted to the rising portion of the data. The error bars are mainiy due to the uncertainty in the fluence-to-dose conversion factors (estimated at $\pm 25 \%)$. (XBL 791-7886)

and angular distribution.

The experiments performed to date are listed in Table 1. The possibility of performing measurements at forward angles is a particular advantage of neutron experiments. On the other hand, it is more difficult to detect neutrons than charged particles. Several detectors can be used to verify the detection of one charged particle; however, neutrons are detected through a generaliy destructive nuclear interaction. Thus, a neutron can only be detected once, which makes neutron measurements especially vulnerable to background effects such as room-scattered neutrons. The later experimental runs were designed to study these effects on our detectors. Shadow bars were used to block neutrons originating in the targets, and to estimate the effect of neutrons originating in extraneous structures. The maximum shadow bar correction was $\sim 35 \%$ at neutron energies of $\sim 50 \mathrm{MeV}$ in the least favorable experimental geometry. 
Table 1. Summary of Neutron Runs

\author{
December 1977. : (No Shadow Bars) \\ Detector Angles: $15^{\circ}, 30^{\circ}, 45^{\circ}, 60^{\circ}, 90^{\circ}, 120^{\circ}, 150^{\circ}$ \\ $250 \mathrm{MeV} / \mathrm{A}$ Neon on $\mathrm{C}, \mathrm{Al}, \mathrm{U}$ \\ $400 \mathrm{MeV} / \mathrm{A}$ Neon on $\mathrm{C}, \mathrm{Al}, \mathrm{Cu}, \mathrm{Pb}, \mathrm{U}$
}

October 1978 (No Snadow Bars)

Detector Angles: $0^{\circ}, 10^{\circ}, 20^{\circ}, 30^{\circ}, 45^{\circ}, 135^{\circ}$

$400 \mathrm{MeV} / \mathrm{A}$ Alphas on $\mathrm{C}$, U

$400 \mathrm{MeV} / \mathrm{A}$ Carbon on $\mathrm{U}$

March 1979

(With Shadow Bar Runs)

Detector Angles: $30^{\circ}, 45^{\circ}, 60^{\circ}, 90^{\circ}$

$400 \mathrm{MeV} / \mathrm{A}$ Neon on $\mathrm{C}, \mathrm{Al}, \mathrm{CU}, \mathrm{U}$

The neutron detector efficiencies were calculated with a Monte Carlo code developed by our Kent State collaborators and estimated to be accurate to $\sim 5 \%$ (excluding threshold uncertainties) on the basis of comparisons with available experimental data (Cecil et a1., 1979). An unambiguous determination of TOF required that only one beam particle be incident on the target during the 200 nsec sampling time; therefore, pileup circuitry was designed and built to reject beam pulses in beam-monitoring detector, which were accompanied by a second pulse within $\pm 200 \mathrm{nsec}$. This circuit has been made available as part of routine biomedical dosimetry instrumentation. Use of high current, high counting rate photomultiplier bases (Kerns, 1977) allowed a beam-counting rate of $\sim 5 \times 10^{5}$ /pulse with a pile-up rejection rato of 30 to $40 \%$.

Tnree different energy regions can be distinguished in the neutron spectra (Schimmerling et al., 1979, 1980): (1) a low energy "evaporation" region, (2) a high-energy exponential tail reflecting the internal momentum distribution of the nucleons, and (3) an intermediate energy region reflecting quas ifree and preequilibrium processes. The intermediate energy region becomes less pronounced with increasing angle, and is almost nonexistent at $90^{\circ}$. The $30^{\circ}$ spectrum shows a broad shoulder in the carbon target, around $150 \mathrm{MeV}$, "which we interpret as a signature of quasifree nucleon collisions.

The cross sections for different angles approach similar values below - $15 \mathrm{MeV}$, as shown in Figure 2. The almost isotropic distribution at the lowest energies is expected for neutron evaporation from the target. The cross section integrated in energy falis off exponentially with angle. The total integrated cross section is proportional to the fifth power of the sum of the radii of the target and the projectile. This

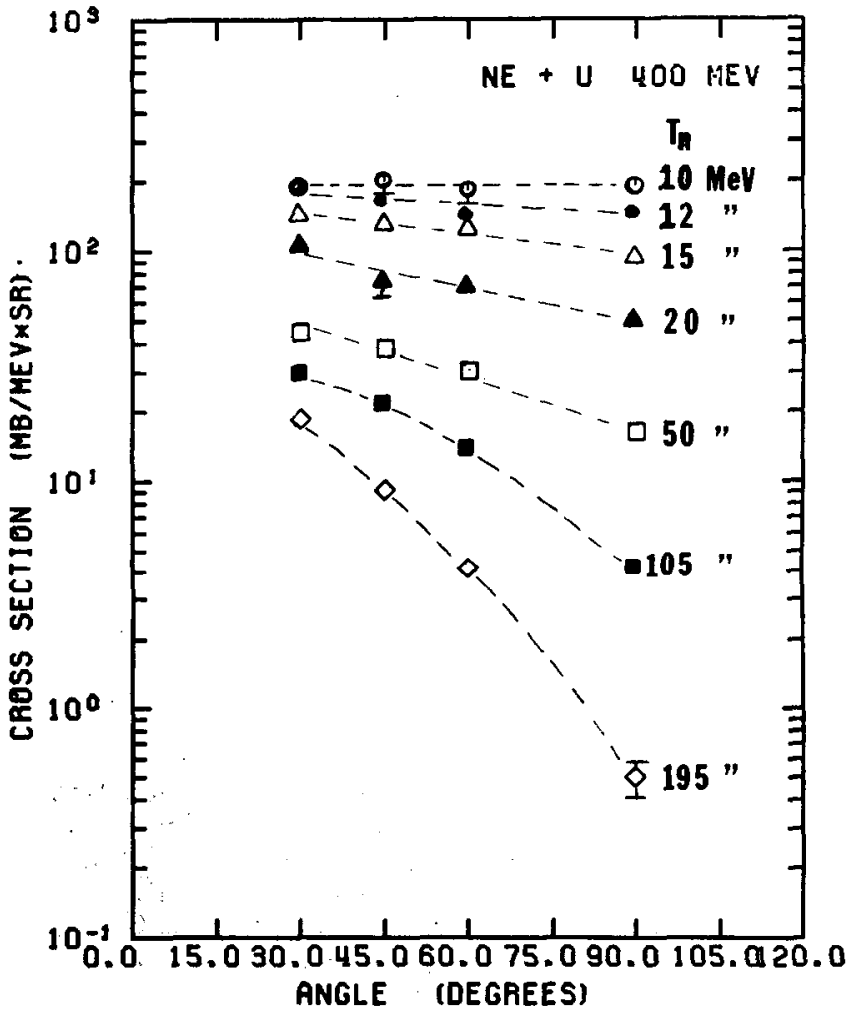

Figure 2. Double differential cross section for neutron production in the collision on neon ions with a uranium target for an incident beam energy of $400 \mathrm{MeV} / \mathrm{A}$, as a function of neutron detection angle, for several neutron energies. (XBL 806-10520) 
representation implies that the average multiplicity of neutrons, taken as the ratio of the measured cross section to the geometric cross section, is proportional to a geometric interaction volume. The average multiplicity for neutrons abut $25 \mathrm{MeV}$ was 2, 2.5, 4, and 9 for carbon, aluminum, copper, and uranium targets, respectively (Schimmerling et al., 1979; Madey et al., 1980).

Neutron production experiments provide an insight into the coulomb effects that modify the spectrum of charged particles, especially protons. The ratio of measured neutron-production cross sections (Schimmerling et a1., 1979) to that for the production of protons in similar reactions (Sandoval et al., 1980; Nagamiya et al., 1980) as a function of energy is greater by a factor of $\sim 2$ tnan what is expected from firestreak calculations (without Coulomb effects) performed by $G$. Westfall of LBL. Different numbers of neutrons and protons contribute to the projectiletarget interaction at different impact parameters. The cross-section ratios given oy the firestreak calculation provide a scaling factor for comparison with the experimental data. Preliminary results of some recent calculations (Gyulassy and Kauffman, 1980) indicate that Coulomb effects may be sufficiently large to explain most of the observed results. Preliminary analys is of the data for other targets indicates tnat, in the case of aluminum where coulomb effects would not be expected to be significant, the $n / p$ ratios are close to unity at $30^{\circ}$. At $90^{\circ}$, however, there still remains a significant discrepancy.

The Kent State group is planning further studies of neutron-producing heavy-ion reactions at higher energies, and for heavier incident beams beyond the range of interest of biomedical research. Within the Radiation Biophysics Group, further work on neutron production is also planned, with the aim of understanding the corresponding physics for particles of interest in biomedical research and space applications, and of improving the computer codes used to calculate neutron doses in the Biomedical Facility.

\section{MEASUREMENTS OF HEAVY-ION FRAGMENTATION}

The objective of this research is the complete characterization of simulated biomedical heavy-ion beams at the Bevalac by the direct measurement of particle fluxes emerging from a thick absorber, and of their energy spectra. The apparatus is presently set up in the Beam 40 beam line. The goals of this research will be approached in several steps:

1. Measurements along the "central" or beam axis, using unmodified beams of carbon, neon, and argon (with the same energies as are used in biomedical research) as a function of the thickness of a polymethylmethacrylate (PMMA) absorber, similar in purpose to the standard biomedical water column.

2. Central axis measurements, using ridge filters or beam scatterers, to simulate biomedical beams at the experimental location.

3. Measurements, as above, away from the beam axis, to obtain the distributions across the beam spot. Since, to a first approximation, in-scattering equals outscattering in the absorber, the distributions should not vary significantly until a critical region near the edge of the beam spot is reached.

4. Radiobiological and radiation chemistry experiments in the completely characterized beam, to ascertain the significance of the different beam components.

5. Development of calculations to predict the physical characteristics of heavy-ion beams in arbitrary configurations.

Preliminary approaches to the direct measurement of the physical characteristics of the heavy-ion beam radiation field, in configurations of interest to biomedical applications, have been discussed previously (Curt is and Schimmerling, 1977; Schimmerling et al., 1977). The main limitation on all of this preliminary work, as well as on other attempts to characterize the heavy-ion beam radiation field (Maccabee, 1973; Maccabee and Ritter, 1974), lies in the fact that particles are identified by three properties: mass, charge, and energy (or velocity). Thus, at least three independent measurements of every particle are required to identify it completely.

In order to completely identify every particle in the radiation field, and measure its energy, we have designed and built a multidetector, particle-identification spectrometer consisting of a channel plate TOF telescope, pulse ionization cnambers, and a ten-element silicon detector telescope, as well as scintillation counters and multiwire proportional chambers for beam definition. Photographs of the apparatus, as currently installed in the Beam 40 irradiation cave, are shown in Figure 3 .

The incident beam intensity is defined by two plastic scintillators, $S_{0}$ (not shown) and $S_{1}$. $S_{0}$ is located $\sim 50 \mathrm{ft}$ upstream of the apparatus and, in conjunction with $S_{1}$, can be used to measure the beam energy by ToF. Two scintillators, $S_{2}$ and $A_{1}$, define the spot at the exit side of the absorber that serves as the source of the observed central axis fragments and primary beam particles. Scintillator $S_{2}$ is a $1-\mathrm{cm}$ diameter disk embedded in a $4 \times 4$ in PMMA light guide, and $A_{2}$ is a $4 \times 4$ in scintillator with a $1-\mathrm{cm}$ diameter central hole tilled with an opaque plastic. The two scintillators are used in anticoincidence to define the particle source, and contribute a uniform thickness across the beam spot.

The multiwire proportional counter MWC provides a computer-accessible readout of the Deam profile that can be used for beam focusing and to unfold the beam intensity distribution across the beam spot for normalization. The multiwire proportional chambers VPM3 and VPM5 are of the delay-line readout type (Grove et al., 1972). They provide the position of every 

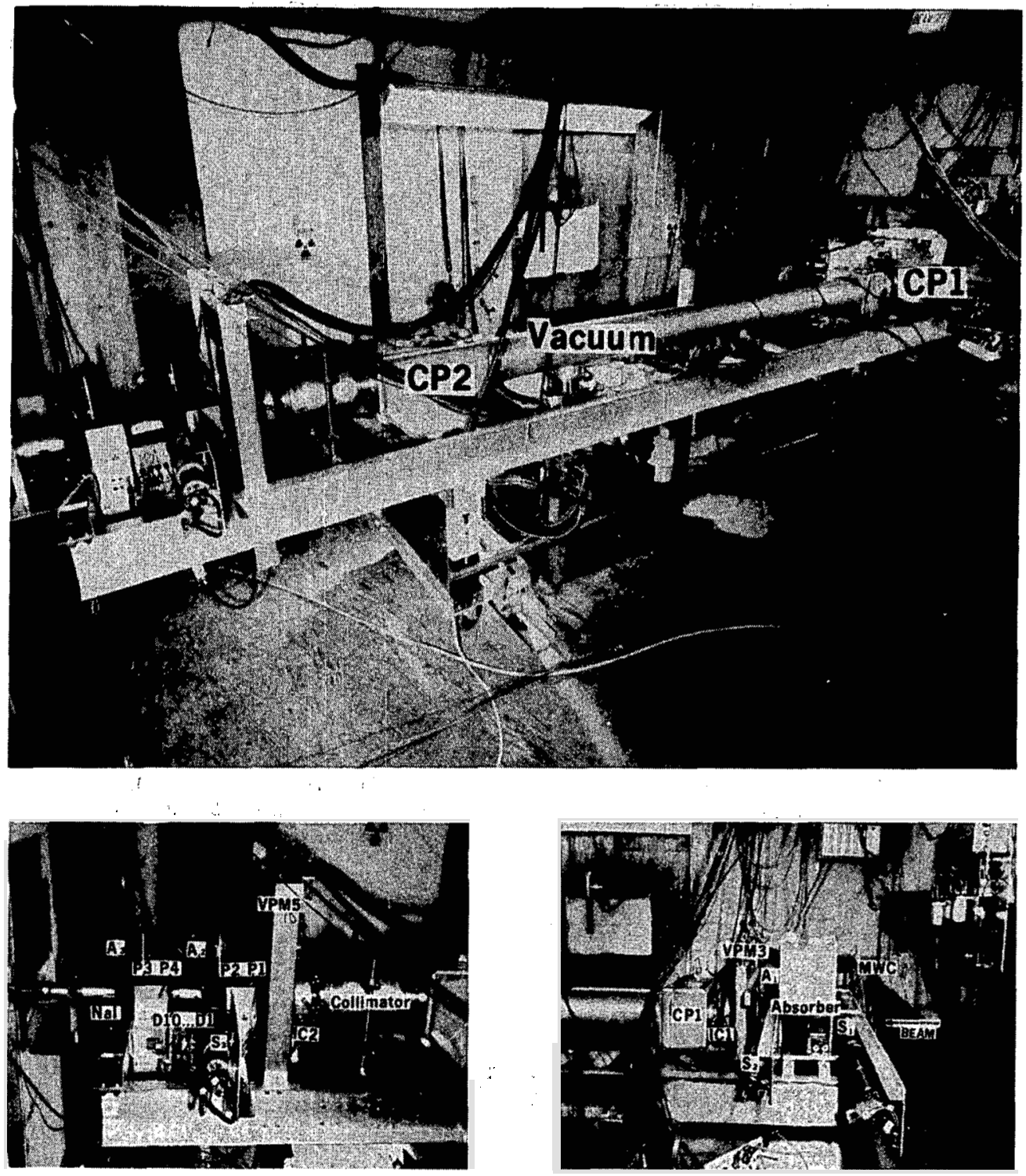

Figure 3. Photographs of the multidetector particle identification spectrometer used for the fragmentation experiments described in the text. Top: View of the complete apparatus as installed. The beam is incident from the right. The vacuum enclosure containing the TOF telescope is labelled showing the locations of the channel plate detectors. Bottom left: close-up view of the downstream end of the apparatus. Bottom right: close-up view of the upstream end of the apparatus. The detectors are described in the text. (XBB 803-3439A)

particle traversing the spectrometer, and are used for electronic collimation and partial rejection of multifragment events. There are four $100 \mu \mathrm{m}$ thick, position-sensitive solid-state detectors, $P_{1}-P_{4}$ (Lamport et al., 1976) that are also being tested for similar use in these experiments.

The TOF telescope consists of two $0.005 \mathrm{~g} / \mathrm{cm}^{2}$ thick foils, each viewed by a channel-plate electron muitiplier that amplifies the secondary electrons produced in the foil (Gabor et al., 1975). These are labelled CP1 and CP2 in.the vacuum enclosure shown in Figure 3 . The measured TOF resolution is $120 \mathrm{psec}$ (FWHM).
The solid state detector (Greiner, 1972) consists of ten 3-mm thick, 1-in diameter silicon detectors, labelled $\mathrm{Dl}$ and D1O. The central, 3/4-in diameter sensitive portion of the telescope is defined by two scintillators with a central,3/4-in diameter hole, $A 2$ and $A 3$, in anticoincidence with a thin scintillator $S_{3}$. A 3 -in diameter, $3-i n$ thick NaI crystal at the downstream end of the apparatus serves as a total absorption detector for some of the fragments that do not stop in the solid-state detectors.

Two ionization chambers, operated in a pulse mode, provide a charge measurement for each event 
(Epstein et al., 1971). Ionization chamber $I_{1}$ measures the total charge emerging from the absorber, while $\mathrm{IC}_{2}$ identifies only the charge of the fragments traversing the TOF telescope. The absorber is a remotely controllable, double PMMA wedge with fixed additional inserts and with computer readable settings.

The response of every detector in the spectrometer to a single particle satisfying the electronic logic requirements is recorded on magnetic tape by a dedicated PDP-11/34 computer. Data acquisition proceeds using the $L B L$ version of a recently available software package, QDA/ MULTI, adapted for this detector configuration.

The complexity of the spectrometer is dictated by the broad energy and mass range of the particles to be detected.: Some of these, the lightest and most energetic, will traverse the entire spectrometer without stopping. The heaviest fragments, e.g. stopping argon, will deposit $\sim 8,000$ times more energy per unit length than an energetic proton, but will have a residuai range too short to penetrate the first few solid-state detectors. Therefore, particle identification will have to be based on different criteria depending on whether the particle stops in the spectrometer, traverses it without stopping, or stops before reaching the first few solid-state detectors. In the last case, isotopic identification is no longer possible, and charge resolution in the pulse ionization chamber will be used to identify the elements in the radiation field.

Multiple fragments will be rejected by examination of the particle identitication variance distribution in the solid-state detectors (Greiner, 1972), by comparisons between the two ionization chambers, and by detection in the wire chambers. The angle accepted by the detector is determined by $C P 2$, and is of the order of a few mrad. The entire apparatus can be rotated about $\mathrm{S}_{2}$ which makes extension to off-axis measurements possible.

In order to normalize the measurements to the exit point of the absorber (the point of interest), the solid-state detector telescope can be brought up close against $\mathrm{S}_{2}$. In conjunction with one of the veto counters (to detect particles escaping from it), this telescope can be used to measure the total flux into a substantial fraction of the exit solid angle for each fragment. This measurement, together with a Monte Carlo transport code, should enable a fairly accurate normalization to be made.

The combination of TOF and the solid-state detector telescope is particularly powerful for particles that do not stop in the detector. Figure 4 shows a two-dimensional scatter plot of

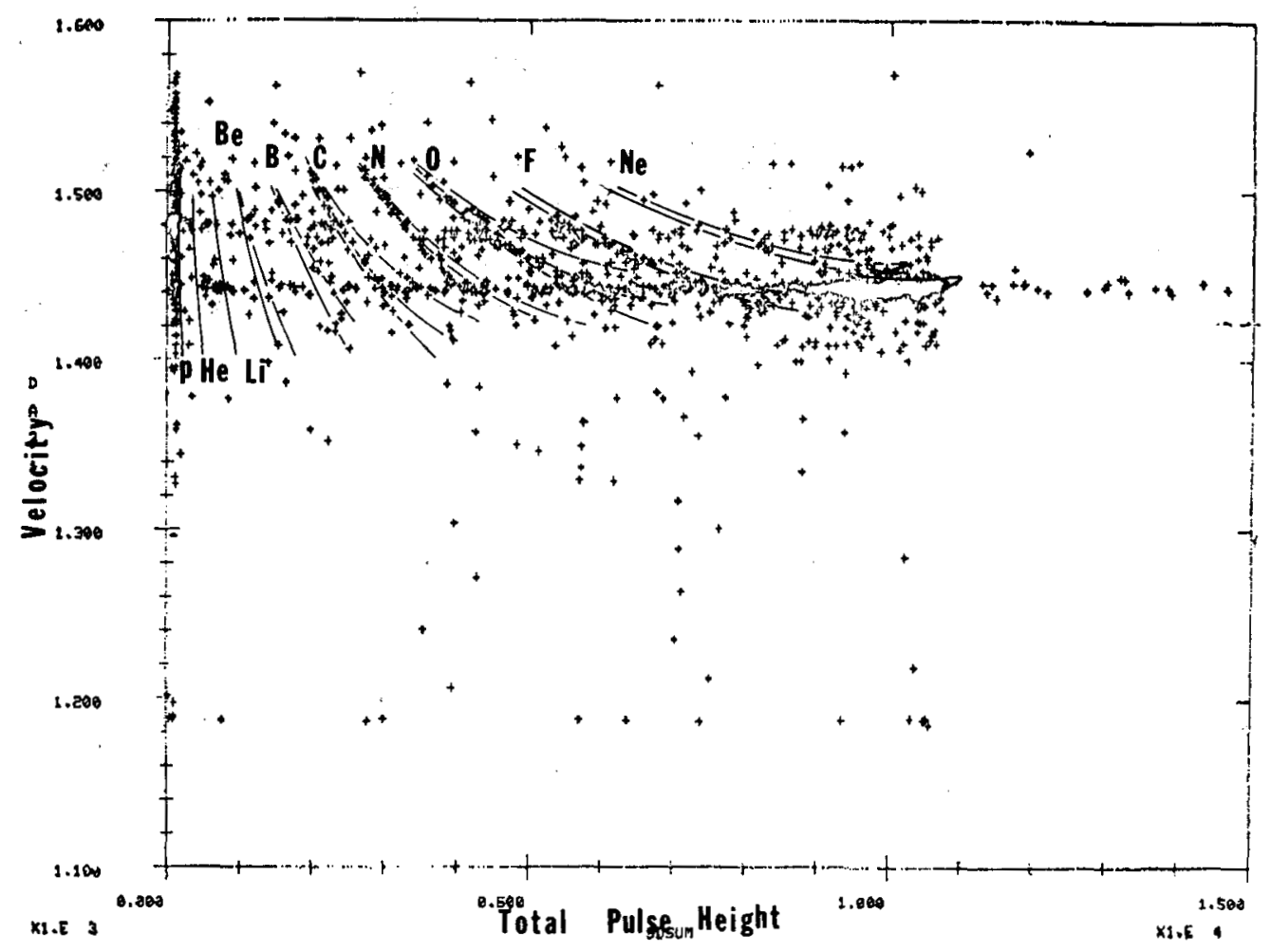

Figure 4. Two-dimensional scatter plot of velocity in the fragmentation spectrometer TOF telescope vs. total energy deposited in the solid-state detectors. The labelled curves are calculated from range-energy relations. For each element, the rightmost curve corresponds to a light isotope and the leftmost curve to a heavier isotope. The units on the coordinate axes are arbitrary. (XBL 806-10521) 
velocity vs. the total energy deposited in the solid-state telescope, for a $19 \mathrm{Ne}$ test beam with an incident energy of $680 \mathrm{MeV} / \mathrm{A}$, after traversing $27.9 \mathrm{~g} / \mathrm{cm}^{2}$ of PMMA equivalent. The residual energy of the primary beam at this depth was $226 \mathrm{MeV} / \mathrm{A}$. Curves calculated from rangeenergy relations have been superimposed on the data of Figure 4. The elements are well separated into distinct groups that can also be resolved by simple inspection. This would not be possible using only the information obtained from energy deposition in the solid-state detector, since the low-velocity component of one element overlaps with the nigner velocity component of its neighbors. The TOF information is seen to be indispensable for the identification of these fragments, most of which do not stop in the detector.

The dynamic range of the detector had not been optimized for this measurement, and the statistics are also inadequate to evaluate the isotopic resolution of the apparatus. The elemental composition can, nevertheless, be obtained. The distribution of different elements is shown in Figure 5. A calculation of the dose contributed by each element, taken as the product of relative $L E T_{\infty}$ and fractional fluence, is presented in Table 2, which shows that approximately $23 \%$ of the dose at this depth is due to fragments.

Distribution such as those shown in Figure 5 reflect the beam composition at the exit of the 3.39-m long TOF detector. In order to evaluate the beam composition at the exit of the absorber, it is necessary to calculate or measure the geometrical acceptance of the detector, i.e., the fraction of fragments of each type and energy that are detected, relative to the number that are emitted from the exit face of the absorber. A particle transport computer code was developed to calculate this acceptance for our spectrometer. The program uses a Monte Carlo method to evaluate the transmission of the primary beam (and of secondary and subsequent generations of nuclear interaction fragments) through an absorber of arbitrary thickness. It also includes the effects of multiple coulomb scattering and the angular dependence of nuclear fragmentation (Greiner et al., 1975; Wefel et al., 1979):

Table 2. Relative Dose Components in Degraded $19 \mathrm{Ne}$ Beam

\begin{tabular}{ccc}
\hline Particle & Relative LET & Relative Dose \\
\hline Ne & 1 & 1 \\
F & 0.71 & 0.11 \\
N & 0.56 & 0.07 \\
C & 0.43 & 0.03 \\
B & 0.32 & 0.01 \\
Be & 0.22 & 0.007 \\
\end{tabular}

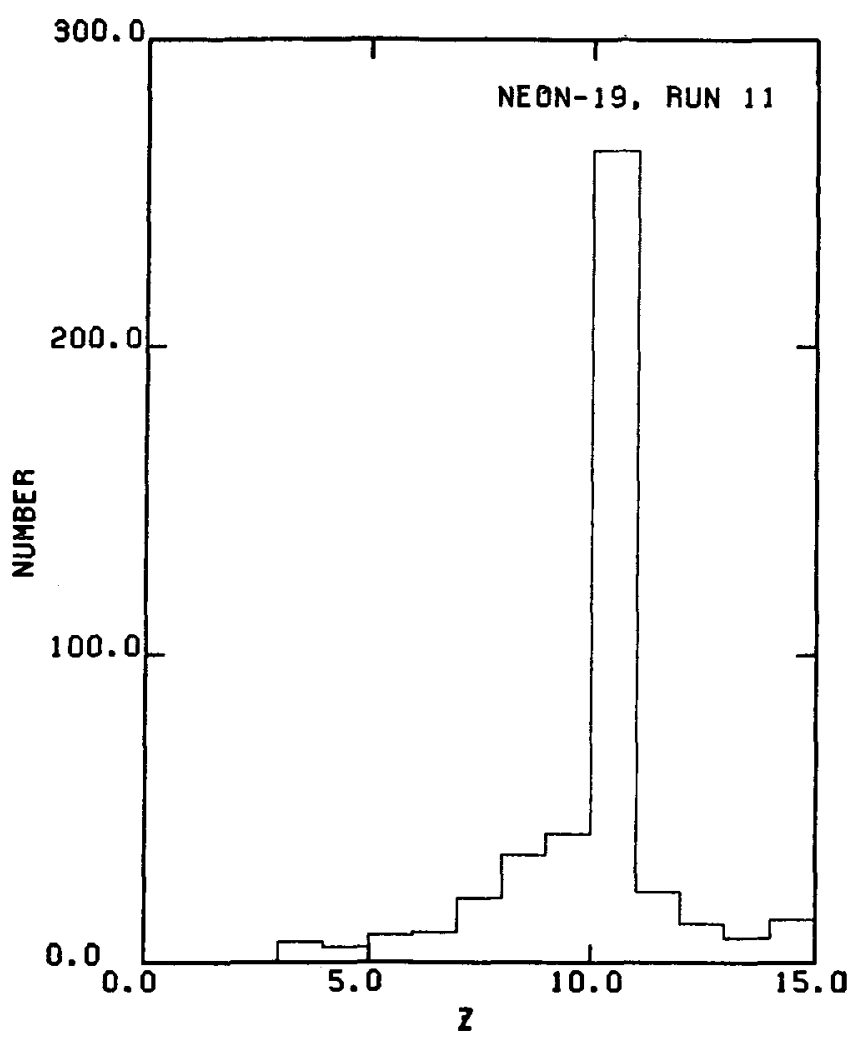

Figure 5. Relative composition of the ${ }^{19} \mathrm{Ne}$ test beam described in the text, after traversing $27.9 \mathrm{~g} / \mathrm{cm}^{2}$, of PMMA equivalent. The elements with atomic number $Z$ greater than that of the incident beam are artifacts due to detection of multiple fragments. The units on the ordinate are arbitrary. (XBL 806-10519)

Table 3 illustrates the geometrical acceptance for protons, deuterons and carbon, in the case of a carbon beam with an incident energy of $450 \mathrm{MeV} / \mathrm{A}$, after traversing $30 \mathrm{~g} / \mathrm{cm}^{2}$ of PMMA, as a function of the energy per nucleon of the detected particle. Carbon was chosen for these initial; calculations because experimental values of the projectile: fragmentation cross sections are available (Linastrom et a1., 19/5a). Estimates of these cross sections must be used for other beams (Silberberg and Tsao, 1973) until experimental values are obtained as a result of this work and related heavy-ion research.

The significance of such measurements may be understiood by reference to several recent results in radiobiology. OER values are different for particles with similar LET values (Curtis, 1970). Explanations for this discrepancy are based on the different-energy densities found along the tracks of different heavy ions, which results from differential interactions of the chemical intermedaries of biological effects. Recent investigations in cellular radiobiology ( $B$ lakely et al., 1979) confirm this effect. Tissue radiobiology studies with neavy-ion beams have also shown that a discontinuous dependence of 
Table 3. Geometrical Acceptance for a $450 \mathrm{MeV} / \mathrm{A}$ Carbon Beam and Penetrating Particles, After Traversing $30 \mathrm{~g} / \mathrm{cm}^{2}$ of PMMA

\begin{tabular}{cccc}
\hline $\begin{array}{c}\text { Particle Energy } \\
(\text { MeV/A) }\end{array}$ & $Z=1$ & $\begin{array}{c}\text { Geometrical } \\
Z=2\end{array}$ & $\begin{array}{r}\text { Acceptance }(\%) \\
Z=6\end{array}$ \\
\hline & 0 & 0 & $8.1 \pm 1.0$ \\
75 & 0 & 0 & $9.5 \pm 0.6$ \\
125 & $2.8 \pm 0.5$ & $5.9 \pm 0.4$ & $11.0 \pm 0.2$ \\
175 & $5.1 \pm 0.5$ & $7.3 \pm 0.4$ & 0 \\
225 & $5.6 \pm 0.4$ & $7.3 \pm 0.3$ & 0 \\
275 & $6.0 \pm 0.4$ & $8.2 \pm 0.3$ & 0 \\
325 & $6.2 \pm 0.3$ & $9.5 \pm 0.2$ & 0 \\
375 & $8.4 \pm 0.4$ & $10.0 \pm 0.6$ & 0 \\
425 & $9.8 \pm 0.8$ & 0 & 0 \\
475 & 0 & 0 & 0 \\
\hline
\end{tabular}

*Errors are quoted only for the Monte Carlo statistics.

effect on LET exists for carbon, neon, and argon beams (Alpen et al., 1980). The nuclear interaction effects studied by this work cannot be excluded as a possible explanation of these results. Recent work on potentially lethal damage and the nonadditive effects of mixed LET radiations (Ngo et al., 1980) may make the contribution of particles of different LET to the total ionization, measured as absorbed dose, even more relevant for an understanding of fractionated irradiations.

The results to be obtained in this work are also important in other ways. For example, present calculations of range-energy curves are accurate only at the $2 \%$ to $3 \%$ level. These experiments will directly measure the energy of different particles as a function of absorber thickness, and can be expected to yield accurate range-energy data. The ionization produced by every particle is recorded in the ionization chambers as a precisely measured charge. From a knowledge of stopping power, it is thus possible to obtain $W$ values in various ionization chamber gases, as well as the ionization distribution.

\section{B. FRAGMENTATION STUDIES USING} NUCLEAR TRACK DETECTORS

(E. V. Benton, D. J. Hildebrand, R. P. Henke, W. Heinrich, and C. A. Tobias)

Plastic nuclear track detectors have an atomic composition close to biological matter. Thick stacks of these detectors are therefore good phantoms to monitor the distributions of projectile particles and fragments in tissue. In experiments using a beam of high-energy heavy ions, the fragmentations in different target materials can oe investigated by measuring the fragments behind the target with plastic track detectors. The stopping densities of fragments as a function of depth measured this way is the basis to test and inprove model calculations for the propagation of heavy-ion beams through matter.

An initial experiment was performed at the Bevalac with a beam of $570 \mathrm{MeV} / \mathrm{A} 40 \mathrm{Ar}$. A variable water column was used as target material, and adjusted to $9.28 \mathrm{~cm}$ water. A stack of 251 sheets of 250-um thick Lexan was exposed to $3.01 \times 10^{4}$ beam particles $/ \mathrm{cm}^{2}$. After standard processing of the Lexan sheets, readout and evaluation were performed with a Quantimet scanner (Vidicon camera). The stack was scanned foil by foil; the scanned area per foil was $3.79 \mathrm{~cm}^{2}$.

In the next step, the features were traced from foil to foil and particle trajectories were established. The atomic number of particles was obtained from a least squares analys is that compared feature data along the trajectories with calculations based on the track etch rate of argon tracks (Henke and Benton, 1979).

The differential energy spectra of beam particles and fragments in different depths of absorber were calculated numerically for steps of absorber of $\Delta X=0.5 \mathrm{~g} / \mathrm{m}^{2}$ and for energy intervals of $\Delta E=1 \mathrm{MeV} / \mathrm{A}$, considering the energy loss and fragmentation (Al lkofer and Heinrich, 1974). The spectrum of the beam particles was assumed to have a Gaussian form with $\langle E\rangle=570 \mathrm{MeV} / \mathrm{A}$ and $\sigma=0.01\langle E\rangle$.

The calculation of the fragmentation of the A beam was performed for water, since Lexan has a composition that is very similar. The fragmentation probabilities in water are dominated by the cross sections for hydrogen because twothirds of all nuclei are hydrogen. Energydependent fragmentation cross sections for a hydrogen target were calculated from an empirical formula of Silberberg and Tsao (1973). For the collisions with oxygen nuclei, the fragmentation cross sections were determined from the hydrogen target data using a scaling factor that is based 
on a factorization of these cross sections as measured for heavy ions at Berkeley (Lindstrom et al., 1975a; Westfall et a1., 1978). The fragmentation cross sections for collisions with oxygen were assumed to be energy-independent. Total cross sections were calculated from empirical formulas (Lindstrom et al., 1975b).

The points in Figure 6 are the measured fluence of particles with LET350>1.5 $\mathrm{GeV} \mathrm{cm}^{2} / \mathrm{g}$. The calculated number of tracks depends on the cross sections. The empirical formula of Silberberg and Tsao (1973) used in this calculation has an error of $\pm 30 \%$. The two curves shown in Figure 6 were calculated for the upper limit and the lower limit of these cross sections. The calculated depth in the stack cannot be directly compared with the experimental data because we did not consider the other materials in the beam. We adjusted the curve of the calculated track density to the experimental data by a shift in the abscissa.

The width of the measured Bragg peak corresponds to the registration range of the primary particles (between 0 and $\sim 70 \mathrm{MeV} / \mathrm{A}$ ). The distribution of primary fluence is a rectangle smoothed only a little by the range straggling of the particles $( \pm 0.3 \mathrm{~mm})$. Overlapping features near the stopping point are registered as one event and account partiy for the deviation from the expected shape.

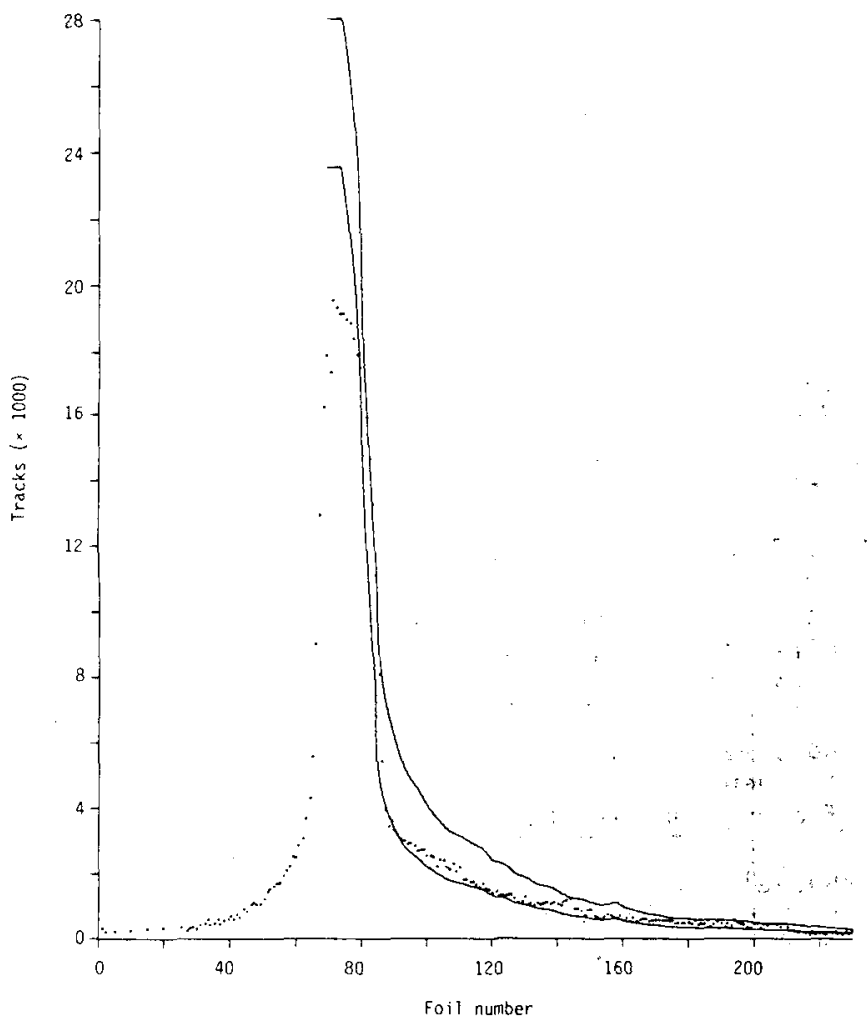

Figure 6. Tracks counted in the Lexan foils. Dots: experimental results. Upper and lower curve: calculations for the upper and lower limits of the cross sectons. (XBL 807-10683)
The preliminary results of Figure 6 show that the measured track densities behind the Bragg peak are within the limits of the calculated values. The statistical accuracy of the experimental data is much better than the uncertainties of the cross sections. Therefore, it must be possible to determine fragmentation cross sections from this experiment. To do this, Z-specific stopping-point distributions of all traced tracks will have to be compared with the results of a calculation with higher numerical accuracy.

Considering that the procedures of data acquisition and analys is are both innovations in the application of plastic nuclear-track detectors (and these data represent the first attempt at application), the result is encouraging. The results of a numerical calculation of the fragmentation in water are comparable with the experiment. The method of plastic nuclear track detector analys is presented here can be used to study particle-specific cross section over large energy intervals in a single experiment.

\section{ACKNOWLEDGEMENTS}

The neutron dosimetry studies were performed in collaboration with A. R. Smith, J. B. McCaslin, R. H. Thomas, A. Henson, L. Kanstein, L. D. Stephens, J. Ozawa, and F. W. Yeater, and were partially funded through a grant from the National Cancer Institute No. 5P01CA19138. The neutron production experiments were performed in collaboration with J. Kast and D. Ortendanl of LBL, and R. Madey, B. D. Anderson, R. A. Cecil, and $A$. R. Baldwin of Kent State University. The LBL researchers were partially funded oy the National Aeronautics and Space Administration through P.0. No. L-22395-A. The nuclear fragmentation studies are being performed in collaboration with T. S. Subramanian, S. B. Curtis, C. A. Tobias, V. Perez-Mendez, and H. Crawford of $L B L$, and $A$. Sadoff of Ithaca College, New York; they are partially funded by a grant from the National Cancer Institute, No. Ro1-CA23247. E. Benton's work is funded by NASA, Grant NAS9-15152.

\section{FOOTNOTES AND REFERENCES}

* Department of Pnysics, University of San Francisco, San Francisco, CA.

† Digital Equipment GMBH, Frankfurt, Federal Republic of Germany.

* Gesamthochschule Siegen, Federal Republic of Germany.

Allkofer; 0 . C. and W. Heinrich. 1974. Attenuation of cosmic ray heavy nuclei fluxes in the upper atmosphere by fragmentation. Nucl. Phys. B71, 429-438.

Alpen, E. L., P. Powers-Risius, and M. A. McDonald. 1980. Survival of intestinal crypt cells after exposure to high-z, high-energy charged particles. Radiat. Res., in press. 
Blakely, E. A., C. A. Tobias, T.C.H. Yang, K. C. Smith, and J. T. Lyman. 1979. Inactivation of human kidney cells by high-energy monoenergetic heavy-ion beams. Radiat. Res. 80 , $122-160$.

Cecil, R. A., B. D. Anderson, and R. Madey. 1979. Improved predictions of neutron detection efficiency for hydrocarbon scintillators from $1 \mathrm{MeV}$ to about $300 \mathrm{MeV}$. Nucl. Instrum. Methods 161, 439-447.

Curtis, S. B. 1970. The effect of track structure on OER at high LET. Charged Particle Tracks in Solids and Liquids, pp. 140-142. Institute of Physics and The Physical Society, London.

Curtis, S. B. and W. Schimmerling. 1977. Nuclear physics of accelerated heavy ions. Biological and Medical Research with Accelerated Heavy Ions at the Bevalac, 1974-1977, pp. 36-48. Lawrence Berkeley Laboratory Report LBL-5610.

Epstein, J. W., J. I. Fernandez, M. H. Israel, J. Klarmann, and R. A. Mewaldt. 1971. Largearea pulse ionization chamber of measurement of extremely heavy cosmic rays. Nucl. Instrum. Methods 95, 77-85.

Gabor, G., W. Schimmerling, D. Greiner, F. Bieser, and F. Lindstrom. 1975. High resolution spectrometry for relativistic heavy ions. Nuc1. Instrum. Methods 130, 65-71.

Goldnaber, A. S. and H. H. Heckman. 1978. High energy interactions of nuclei. Ann. Rev. Nucl. Sci. 28, 161-205.

Greiner, D. E. 1972. A versatile, highresolution particle identifier-theory. Nucl. Instrum. Methods 103, 291-308.

Greiner, D. E., P. J. Lindstrom, H. H. Heckman, Bruce Cork, and F. S. Bieser. 1975. Momentum distribution of isotopes produced by fragmentation of relavistic $12 \mathrm{C}$ and 160 projectiles. Phys. Rev. Lett. 35, 152-155.

Grove, R., I. Ko, B. Leskovar, and V. PerezMendez. 1972. Phase compensated electromagnetic delay lines for wire chamber readout. Nuc 1. Instrum. Methods 99, 381-385.

Gyulassy, M., and S. K. Kauffman. 1980. Coulomb Effects in Relavistic Nuclear Collisions. Lawrence Berkeley Laboratory Report LBL-10279.

Ham, W. T. 1960. Fast neutron radiation hazards. Fast Neutron Physics, (J. Marion and J. L. Fowlers, eds.), Vol. I, pp. 841, Interscience, New York.

Henke, R. P. and E. V. Benton. 1980. A method of automated HZE-particle Z-spectra measurement in plastic nuclear track detectors. Solid State Track Detectors. Pergamon Pres $\overline{s, \text { Oxford }}$ and New York.
Jaros, J., A. Wagner, L. Anderson, 0. Cnamberlain, R. Z. Fuzesy, J. Gallup, W. Gorn, L. Schroeder, S. Shannon, G. Shapiro, and H. Steiner. 1978. Nucleus-nucleus total cross section for light nuclei at 1.55 and $2.89 \mathrm{GeV} / \mathrm{c} /$ nucleon. Phys. Rev. C18, 2273.

Kerns, C. R. 1977. A high-rate phototube base. IEEE Trans. Nucl. Sci. NS-24, 353-355.

Lamport, J. E., G. M. Mason, M. A. Perkins, and A. J. Tuzzolino. 1976. A large area circular position sensitive Si detector. Nucl. Instrum. Methods 134, 71-76.

Lindstrom, P. J., D. E. Greiner, H. H. Heckman, B. Cork, and F. S. Bieser. 1975a. Isotope Production Cross Sections from the Fragmentation of the 160 and $12 \mathrm{C}$ at Relativistic Energies. Lawrence Berkeley Laboratory Report LBL-3650.

Lindstrom P. J., D. E. Greiner, H. H. Heckman, B. Cork, and F. S. Bieser. 1975b. Fragmentation of $40 \mathrm{Ar}$ at $100 \mathrm{Gev} / \mathrm{C}$. Proceedings of the 14th International Cosmic Ray Conference, vol. 7, pp. 2317-2318.

Maccabee, H. D. 1973. Composition of heavy ion beams: Preliminary measurements. Radiat. Res. 54, 495-509.

Maccabee, H. D. and M. A. Ritter. 1974. Fragmentation of high-energy oxygen-ion beam in water. Radiat. Res. 60, 409-421.

Madey, R., R. A. Ceci1, B. D. Anderson, A. R. Baldwin, W. Schimmerling, J. W. Kast, and D. Ortendahl. 1980. Neutron production by relativistic neon ions on carbon, aluminum, copper, and uranium. International Conference on Nuclear Physics, Berkeley, August 24-30. (Abstract).

Nagamiya, L., L. Anderson, W. Bruckuer, 0. Chamberlain, M.-C. Lemaire, S. Schnetzer, G. Shapiro, H. Steiner, and I. Tanihata. 1980. Proton and pion spectra at large angles in relativistic heavy-ion collisions. Phys. Lett., in press.

Ngo, F. Q., E. A. Blakely, and C. A. Tobias. 1980. Sequential exposures of mammalian cells to low- and high-LET radiations. I. Lethal effects following $x$-ray and neon ion irradiation. Radiat. Res., in press.

Rindi, A. 1977. An analytical expression for the neutron flux-to-absorbed dose conversion factor. Health Phys. 33, 264.

Sandoval, S., H. H. Gutbrod, W. G. Meyer, A. M. Poskanzer, R. Stock, J. Gosset, J. C. Jourdain, C. H. King, Ch. Lukner, Nguyen Van Len, G. D. Westfa11, and K. L. Wolf. 1980. Spectra of $p, d$, and $t$ from relativistic nuclear colTisions. Phys. Rev. C, in press.

Schimmerling, W., S. B. Curtis, and K. G. Vosburgh. 1977. Velocity spectrometry of $3.5 \mathrm{GeV}$ nitrogen ions. Radiat. Res. 72, 1-17. 
Schimmerling, W., J. W. Kast, D. Ortendahl, R. Madey, R. A. Cecil, B. D. Anderson, and A. R. Baldwin. 1979. Measurement of the inclusive neutron production by relativistic neon ions on uranium. Pnys. Rev. Lett. 43, 1985-1987.

Schimmerling, W., J. W. Kast, D. Ortendah1, G. Westfa11, R. Madey, R. A. Ceci1, B. D. Anderson, and A. R. Baldwin. 1980. Inclusive neutron production by relativistic heavy ions on uranium. International Conference on Nuclear Physics, Berkeley, August 24-30. (Abstract).

Scott, D. K. 1978. The current experimental situation in heavy ion reactions. Theoretical Methods in Medium-Energy and Heavy Ion Physics (K. W. MCEvoy and W. A. Friedman, eds.) pp. 3-188. Plenum Press, New York.

Silberberg, R. and C. H. Tsao. 1973. Partial cross sections in high energy nuclear reactions and astrophysical application. Astrophys. J. Suppl. 25, 315-368.

Smith, A. R., W. Schimmerling, L. L. Kanstein, J. B. MacCaslin, and R. H. Thomas. 1980.
Neutron flux-density and secondary-particle energy spectra at the 184-inch synchrocyclotron medical facility. Med. Phys., in press.

Upton, A. C. 1968. Effects of radiation on man. Annu. Rev. Nucl. Sci. 18, 495.

Wefel, J. P., J. M. Kidd, W. Schimmerling, and K. G. Vosburgh. 1979. Differential fragmentation cross section for $7.3 \mathrm{GeV}$ nitrogen ions incident on protons. Phys. Rev. C19, 1380-1392.

Westfal1, G. D., L. W. Wilson, P. J. Lindstrom, H. J. Crawford, D. E. Greiner, and H. H. Heckman. 1978. Fragmentation of Relativistic $56 \mathrm{Fe}$. Lawrence Berkeley Laboratory Report LBL-7162.

Wilson, J. W. and C. M. Costner. 1975. Nucleon and heavy-ion total and absorption cross section for selected nuclei. National Aeronautics and Space Administration Technical Note NASA TN D-8107. 


\title{
MICRODOSIMETRY RESEARCH
}

\author{
J. Howard, S. B. Curtis, H. H. Rossi, " and P. J. Kliauga*
}

The energy deposition patterns deep within tissue caused by the various heavy-ion beams produced at the Bevalac are created by the passage of many different primary and secondary particles, varying in LET from minimum ionizing electrons through high energy protons and helium ions up to lower energy heavy particles of the type making up the primary beam. Thus, a broad spectrum of energy depositions can be expected at any point deep within a body or water phantom.

Biological effects produced by these beams at any point are a function of the relative contributions of the various particles to the overall energy deposition pattern at that point. Many biological effects depend on the rate at which energy is deposited per unit track length by a charged particle. Thus, for a given amount of energy deposited by the beam in a given volume, the biological effects will depend upon how that energy is deposited, i.e., whether in a few large amounts or in many smaller amounts. Microdosimetry deals with the direct measurement of energy losses in small gas volumes that mimick small spheres of tissue. By obtaining spectra of a microdosimetric quantity such as the lineal energy, $y$, information can be gained on the "quality" of the radiation in question. (The lineal energy, $y$, is defined as the quotient of the energy imparted to matter in the volume of interest by a single energy deposition event and the mean chord length in that volume.) Such beam quality measurements may be useful in understanding and interpreting biological results obtained at various depths behind a water column. They may also be used in comparing the quality of one beam geometry with another and in checking the reproducibility of beam quality from one week or month to the next. Finally, there is one theory of biological action (Kellerer and Rossi, 1972) that uses average quantities calculated from measured spectra of 1 ineal energy for predicting certain biological effects.

Although, at present, there may not be agreement as to the value for understanding, biological effects of energy deposition spectra in tissue volumes with characteristicidimensions. of 1 or $2 \mu \mathrm{m}$, it is generally agreed that energy deposition spectra obtained in such tissue volumes will give a measure of the quality of the radiation field at the position of the measurement that can be used to characterize the. beam and may provide a bas is for some insight into the biological effects observed. To this end, a capability for measuring energy deposition spectra in the various Bevalac beams is being developed. In addition, an experiment has been designed to measure y spectra as a function of impact parameter or distance from a particle trajectory.

\section{BACKGROUND}

Considerable help has been obtained from both the Columbia group headed by H.H. Rossi and the Los Alamos group headed by J. Dicello. Indeed, the experiment to measure the energy deposition spectra as a function of impact parameter has been designed totally by the Columbia group. As described below, both groups have made independent measurements on the various beams.

In January 1977, Ross $i$ and his group from Columbia University made an initial study using argon and carbon beams. Measurements were made using 4-in wall-less counters with $1 / 4$ in and 1 in spherical fields. After a limited amount of data was collected, a collaboration between LBL and Columbia was established to overcome the difficulties of executing such a complex experimental program from such a distance. In addition, it was felt that even 4 -in wall-less counters might be too small to measure adequately the energy deposition spectrum, and that a large tank $2 \mathrm{ft}$ in diameter and $8 \mathrm{ft}$ long should be fabricated to determine wall effects and to measure spectra as a function of impact parameter. The construction of a special 8-in heavy-ion wall-less counter specifically for use at the Bevalac was begun at Columbia.

During this time, J. Dicello of LoS Alamos Scientific Laboratory (LASL) requested time to make microdosimetric measurements on the specific conditions used during the biological experiments of M.R. Raju (LASL) at LBL. Therefore, in the latter part of July 1977, Dicello and M. Zaider brought their complete system to LBL and made measurements at various positions in a neon beam using a 1-in walled counter (Zaider et al., 1980). At that time rt was relt that LBL should have its own detèctor system, and a collaboration was formed with the LASL group for the design of a gas flow system, pile-up rejection system, and a data acquisition and analys is program.

The next series of measurements were made in the summer of 1978 when four different beams of approximately the same energy (500-600 Mev/amu) were made available to the Los Alamos group with help from the LBL group. The four ions measured were: iron, neon, argon, and carbon. In addition, two different types of proportional counters were used in making the measurements: a 1 -in walled counter and a $1 / 2$ in wall-less counter with a $1 / 16$ in diameter helical electrode defining the field. The intent was to determine whether the wall-less counter would measure an appreciably different spectrum than the walled counter using beams with approximately the same initial energy. Small differences were noted at the low energy deposition (low $y$ ) portion of the spectrum. 
While the wall-less chamber can be used in some situations where the range of the delta rays is small, the Columbia group felt its dimensions were inadequate for use with the Bevalac beams. In May 1979 these measurements were reported at the Sixth International Congress of Radiation Research in Tokyo (Dicello, private communication) but they have not yet been published.

During the summer of 1978, the fabrication of the large tank at LBL was completed, and the counter and associated equipment were installed. The 8-in heavy-ion counter was transported from Columbia to LBL and P. Kliauga and associates from Columbia came to $L B L$ to make measurements with the tank. The electrode structure for both the tank and the 8-in counter are quite similar (Fig. 1): a $1 \mathrm{ml}$ stainless steel wire for the collector with a $1 / 4$ in diameter helix surrounding it. The sensitive volume of the counter is defined by field tubes to be $1 / 4$ in long.

Measurements were made with a $570 \mathrm{MeV} / \mathrm{amu}$ argon beam and a $400 \mathrm{MeV} / \mathrm{amu}$ carbon beam. Also mounted inside the tank (see Fig. 1) is a small scintillator (1/16 by $1 / 4$ by 2 in) light pipe photomultiplier combination on a moveable carriage used to locate and provide coincidence with the primary particle for the measurement of spectra as a function of impact parameter, and an 18-in diameter by $1 / 4$ in tissue equivalent plastic disc used to establish equilibrium conditions. The disc system also would give an indication of the effect of wall spacing on the energy deposition spectrum. Some data with intermediate-sized beams were obtained, as well as preliminary data on the effect of wall distance from the counter (Kliauga et al., 1978a, 1978b).

In March of 1978, a program was established to build a gas flow system. designed at LASL and a pile-up rejection system designed at $L B L$. The final piece of apparatus for the flow system was received in May 1980 (although we were able to operate the system earlier using a borrowed piece of equipment). Both the flow system and the pile-up rejection system were in operation by the summer of 1979 and were used to take measurements with the 8 -in counter. However, because of a defective calibration source, the complete system was not ready for optimal operation until June 1980 .

P. Kliauga returned to LBL in March 1980 to make some measurements with the 8 -in counter and the large tank counter. Preliminary results from these measurements indicated that the tank should be even larger to completely describe the full spectrum. Since it is practically impossible to fill uniformly a space $2 \mathrm{ft}$ or larger in diameter with beam, the plans for taking broad beam spectra with the 8 in counter or the large tank counter were abandoned. We decided instead to concentrate on the impact parameter measurements, from which broad beam effects could be calculated, and the Columbia-LBL group made these measurements in the plateau of a neon beam in June 1980. The impact parameter was varied out to a distance of $24 \mu \mathrm{m}$ (tissue equivalent) from the edge of the counter.

\section{EXPERIMENTAL RESULTS}

The beams produced by the Bevalac for biomedical research have energies typically of the order of 500-700 MeV/amu. The maximum delta-ray energies produced are a few MeV, which leads to about $1 \mathrm{~cm}$ ranges in tissue. Fortunately, such energetic events are improbable, and a realistic estimate of the container size required to introduce negligible distortion in the measured spectra must take into account the strongly energy-dependent cross section for delta-ray production. A simple computer simulation comparing total ionization in a finite field of heavy ions to total ionization in an infinite field of heavy ions showed that an error of about

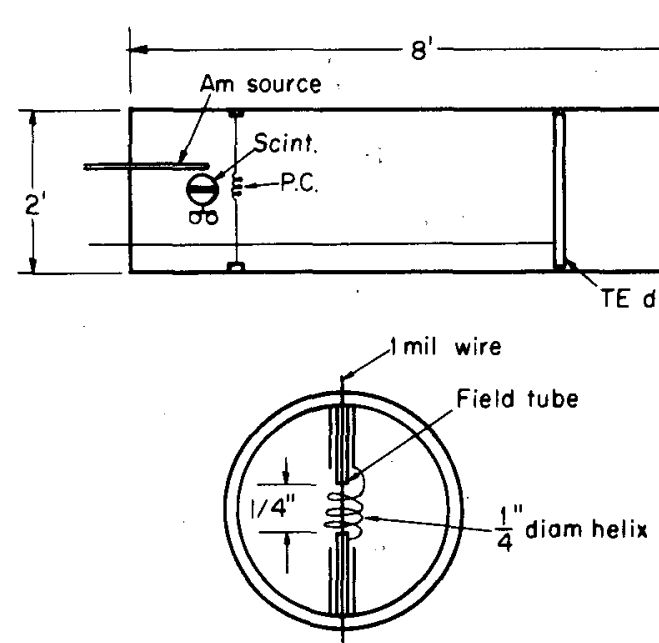

PROPORTIONAL COUNTER

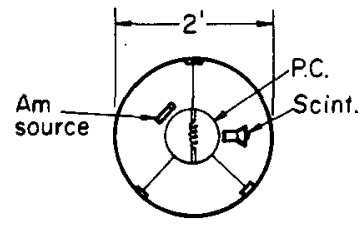

mylar window
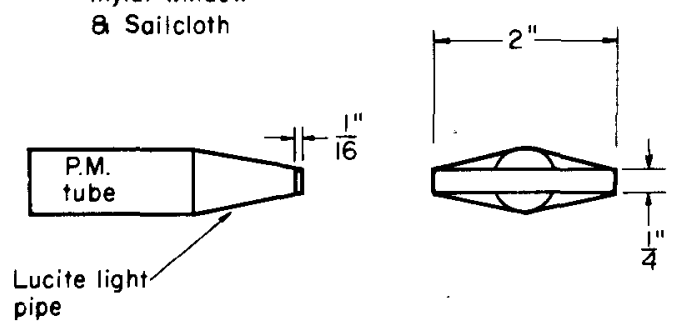

SCINTILLATOR ROTATABLE

Figure 1. Detail of the large tank counter with its associated equipment. (XBL 807-3529) 
$10 \%$ could be expected for a beam of $30 \mathrm{~cm}$ radius at 68 Torr (simulating $1 \mu \mathrm{m}$ tissue in a 1/4 in counter) TE gas.

Since beams that completely fill the tank uniformly cannot be produced, broad-beam spectra with complete delta-ray equilibrium cannot be taken at 68 Torr simulating a 1 m site size. On the other hand, because of the broad range of energies involved it is difficult to obtain broad-beam spectra at large site sizes (high gas pressures) because of the large amount of energy deposited in a proportional counter by a heavy ion.

As a concrete example, at $100 \mathrm{keV} / \mu \mathrm{m}$ (450 $\mathrm{MeV} / \mathrm{amu} 40_{\mathrm{Ar}}$ at the plateau) and $1 \mu \mathrm{m}$ path length, the ion produces about 30,000 ion pairs, which correspond to about $10 \mathrm{mV}$ out of the preamplfier with no gas gain. At $3,000 \mathrm{keV} / \mathrm{um}$ (40 Ar near the Bragg peak), the ion produces about $300 \mathrm{mV}$ out of the preamplifier. With an $8 \mu \mathrm{m}$ site diameter, these figures become $80 \mathrm{mV}$ and $2.4 \mathrm{~V}$, respectively. However, in order to see the delta-ray part of the spectrum, at least a moderate gas gain and sensitivity are needed. Thus, it becomes impossible to observe both primary and secondary contributions to the spectra at the same gas gain or with the same preamplifier sensitivity. Furthermore, at these high gas pressures and low gas gain (small field strength) ion recombination is almost certainly a serious problem.

For these reasons, it appears that the most practical method of obtaining accurate microdosimetric spectra of heavy ions is by taking "differential" spectra, i.e., spectra of a narrowly collimated beam at various distances (impact parameters) from the sensitive site (collecting volume).

An additional experimental problem occurs with spectra taken near the peak regions. After traversing most of their range through a water absorber, the heavier ions such as neon and argon undergo considerable fragmentation and produce a large number of low- $Z$ fragmentation products. These fragmentation products have approximately the same velocity as the beam particles, and are directed strongly forward. Therefore, they are highly susceptible to the "v" type of wall effect (Kliauga et al., 1978b), and they are responsible for much of the low-LET (small event sizes) observed.

The following is a discussion of our results from the work in March, 1980. The data from the experiments performed in June 1980 have not yet been analyzed. All of the experiments performed in March were with ${ }^{12} \mathrm{C}$ ions of approximately $470 \mathrm{MeV} / \mathrm{amu}$. Only one gas pressure was used, corresponding to a unit-density site size of $1 \mu \mathrm{m}$ diameter simulated by the proportional counter.

The pile-up rejection system was fully operational as was the analog system. An interim data acquisition system was operational but there was no on-line analysis system and the raw data were taken back to Columbia University and entered by hand into a PDP $11 / 45$ computer. The spectra were taken at two different electronic gains in order to obtain a better resolution at the low energy end, and they were combined into a single normalized spectrum for analysis.

Figure 2 is a Bragg curve of the ${ }^{12} \mathrm{C}$ beam used in the experiments. Sufficient time was available for only two points: the plateau and peak regions. Figures $3 A$ and $3 B$ are spectra taken on the plateau region with the heavy ion counter.

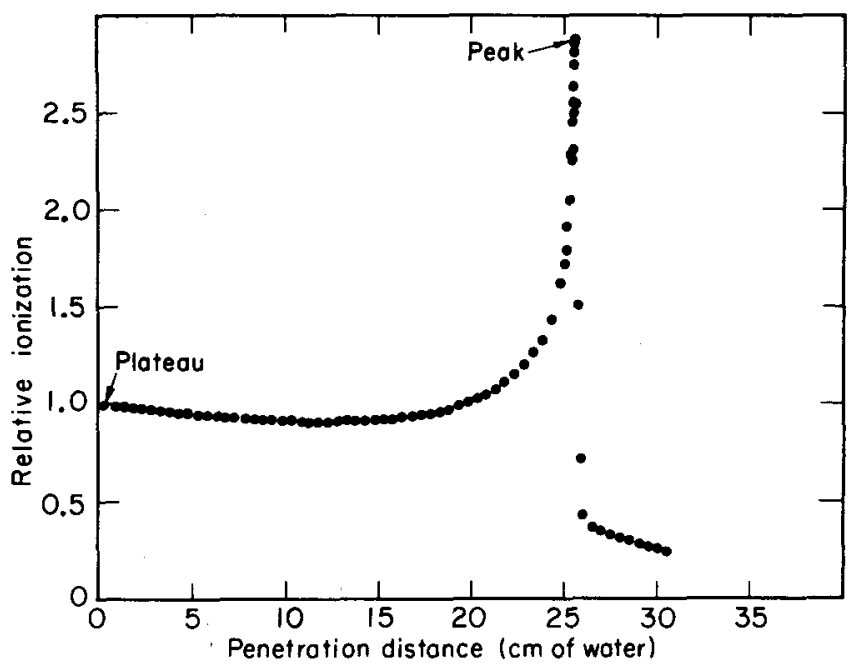

Figure 2. Dose vs. depth curve of a $474 \mathrm{MeV} / \mathrm{amu}$ T2C beam, taken with Bevalac instruments. (XBL 807-3526)

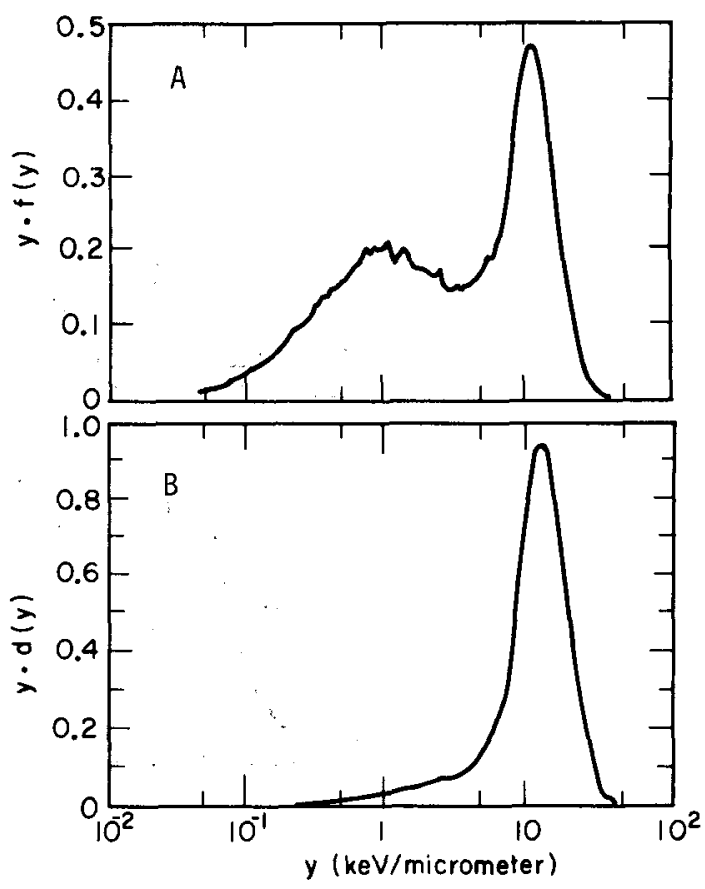

Figure 3. Microdosimetric spectra: plateau position, taken with the 8-in heavy-ion counter. (XBL 807-3527) 
Figure $3 \mathrm{~A}$ is the conventional frequency distribution in which $y f(y)$ is plotted as a function of $y$ on a $\log$ scale. : The area under such a curve represents the relative probability of an event within the interval of: $y$ considered. Figure $3 B$, the dose distribution, shows $y d(y)$ as a function of $y$ on a log scale, and the area is proportional to dose. In Figure $3 A$, note the broad peak centered at about $1 \mathrm{keV} / \mathrm{\mu m}$, which is often called the delta-ray peak.

Figures $4 A$ and $4 B$ are spectra taken at the same location, but with an identical type of cylindrical wal1-less counter mounted in the large aluminum tank. The only difference between Figures 3 and 4 is the environment of the counters. The delta-ray peak in Figure $4 \mathrm{~A}$, still centered at about $1 \mathrm{keV} / \mathrm{\mu m}$, is much more prominent, and it shows the influence of wall on the smaller counter in suppressing these events. Figures $5 \mathrm{~A}$ and $5 \mathrm{~B}$ are spectra taken near the peak of the Bragg curve, about $1 \mathrm{~mm}$ upstream, with the large tank. The large number of events in the broad peak at small y valves are probably all fragmentation products, which are numerous at this depth.

\section{Wal1-Effect Measurements}

The tissue equivalent plastic-disc, shown in Figure 2, was used to test for the amount of wall-effect and presence of charged particle equilibrium. 'Since time was not available to accumulate full spectra, high electronic ga in was used to focus on the low-energy part to the spectrum that we call the delta-ray peak. The

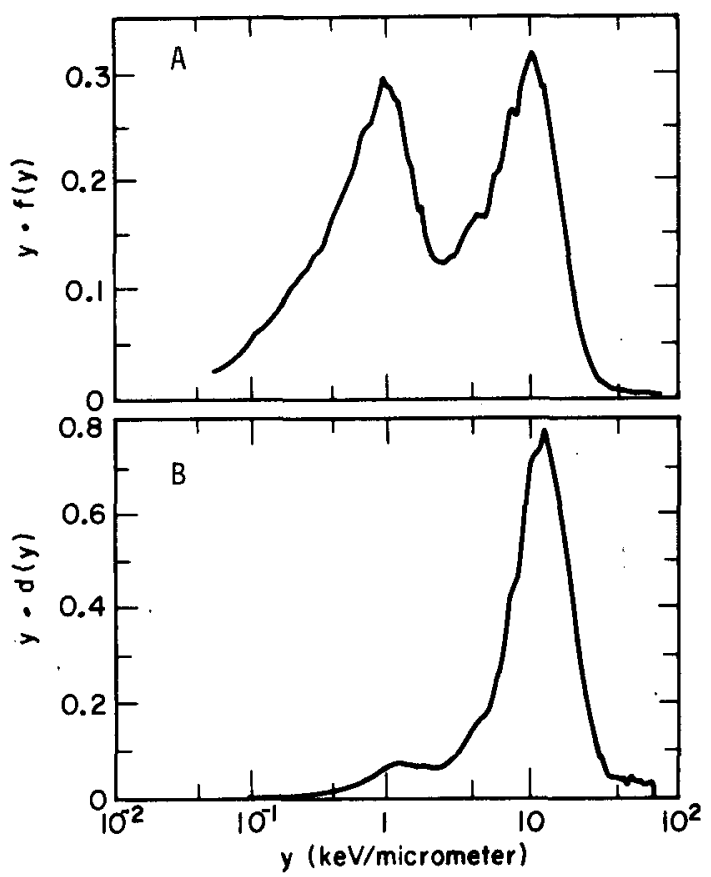

Figure 4. Microdosimetric spectra: plateau position, as Figure 3, but taken with the large tank. (XBL 807-3525) variable actually measured is the integral of the spectrum from $480 \mathrm{eV}$ to $4.8 \mathrm{keV}$ divided by the number of monitor counts (beam particles), and the variable is thus a measure of the number of low-energy events in this interval per beam particle. Figure 6 shows the results. As expected, as the disc is moved further from the counter, the number of low-energy events

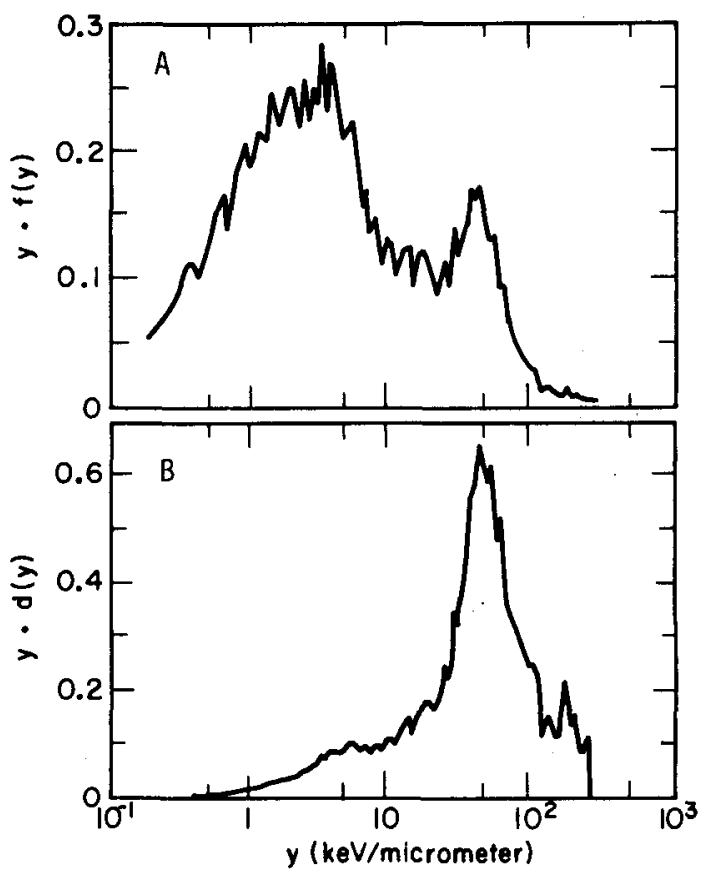

Figure 5. Microdosimetric spectra: peak position, taken with the 8 in counter. (XBL 807-3523)

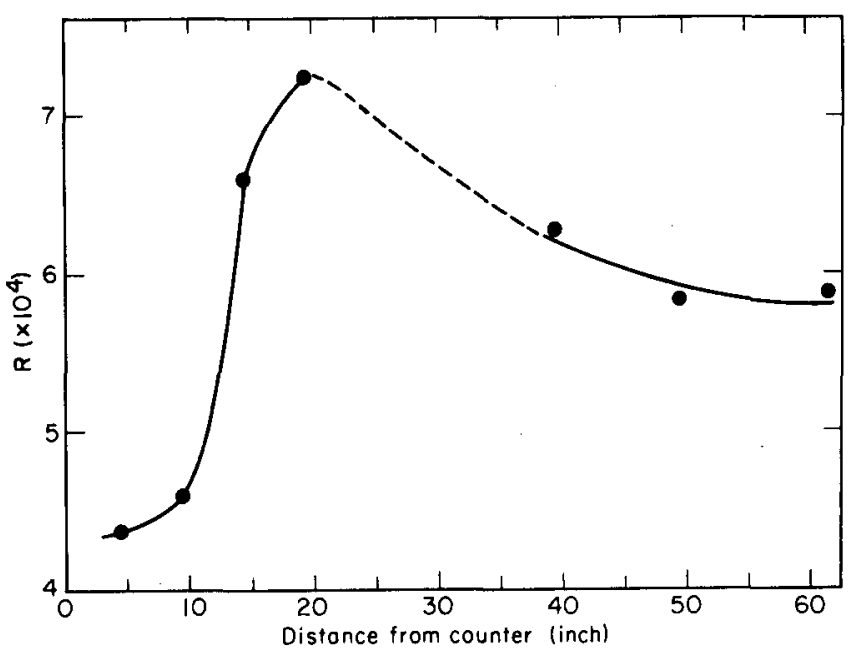

Figure 6. Ratio of the number of events in the energy interval from $480 \mathrm{eV}$ to $4.8 \mathrm{keV}$ divided by the number of beam particles as a function of distance of the tissue-equivalent disc from the counter; taken at the plateau position. (XBL $807-3528$ ) 
increases (the "wall-effect" decreases). However, instead of leveling off at some point, which would indicate the minimum size of counter required to be truly wall-less, the variable reaches a peak and decreases again as the disc is moved even further. Delta rays are evidently scattered out of the beam, and there is no compensation for this loss if the beam diameter is too small. In other words, charged particle equilibrium does not exist because the beam is not broad enough; consequently larger beam diameters are required. (This discussion of experimental results was excerpted from Columbia University Annual Report C00-4733-3 1980 which will soon be published.)

\section{CONCLUSIONS AND FUTURE PLANS}

A reliable, practical microdosimetry system is now in place at the Bevalac, and systematic extensive studies will begin soon. Future plans include (1) reducing the size of the scintillator and/or replacing it with another device (such as a solid-state detector) that will give us better localization of the beam, and (2) providing additional scalers and scintillators for better normalization of the data.

It is hoped that the detailed impact parameter experiments will lead to a design for a small, easily set up instrument for routine microdosimetry of the beams used for both radiotherapy and radiobiology. The controversy between walled and wall-less detectors (as well as the degree of wall-lessness necessary) will have to be resolved.

\section{FOOTNOTES AND REFERENCES}

* Department of Radiology, College of Physicians and Surgeons, Columbia University, NY.

Kellerer, A. M. and H. H. Rossi. 1972. The theory of dual radiation action. Curr. Top. Radiat. Res. 8, 85-158.

Kliauga, P. J., R. D. Colvett, L. J. Goodman, and Y.M.P. Lam. 1978a. Columbia University Annual Report C00-4733-1, 61. Columbia University, New York.

Kliauga, P. J., R. D. Colvett, L. J. Goodman, and Y.M.P. Lam. 19780. Microdosimetry of $400 \mathrm{MeV} / \mathrm{amu} 12 \mathrm{C}$ and $450 \mathrm{MeV} / \mathrm{amu} 40 \mathrm{Ar}$ beams. Proceedings, Sixth Symposium on Microdosimetry. Harwood Academic Publishers, Ltd., London.

Zaider, M., J. F. Dicello, M. R. Raju, M. Takai, and J. Howard. 1980. Microdosimetry of pretherapeutic neon beams at LBL. Radiat. Res., in press. 
•

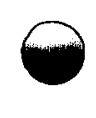
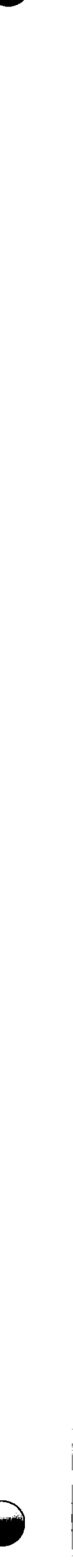


\title{
ENERGY TRANSFER FROM HEAVY PARTICLES
}

\author{
A. Chatterjee and J. L. Magee
}

Several mechanisms are responsible for degrading the kinetic energy of a charged particle in matter, but for charged particles produced in the Bevalac and with energies of biomedical interest (about $500 \mathrm{MeV} / \mathrm{n}$ or less), the energy loss through electronic interaction (excitation or ionization) is the single most important mechanism. No other mechanism is considered here. The energy loss is at first purely a physical phenomenon, but in media composed of molecules, chemical effects inevitably occur, and in living systems, biological effects occur. The three classes of effects (physical, chemical, and biological) occur on different time scales a) though there may be significant overlapping.

We often say that the purely physical phase of the energy deposit is over in $10^{-15} \mathrm{sec}$. It is convenient to think of time in a local sense, i.e., the origin of time is taken at every position as the time of the energy deposit that affects the region. From the uncertainty principle, $\Delta t \Delta E \approx \hbar$, we can identify a $\Delta t$ for an energy loss, and we get $\Delta t \approx 10^{-17} \mathrm{sec}$ for a $100 \mathrm{eV}$ loss and $\Delta t \approx 10-16 \mathrm{sec}$ for $\Delta E=10 \mathrm{eV}$. The reference time $10^{-15} \mathrm{sec}$ is the estimated local time for secondary electrons to fall into the subexcitation range (i.e., below $7 \mathrm{eV}$ ).

The diffusion-controlled track reactions of the chemical phase start at about $10^{-11} \mathrm{sec}$. Between $10^{-15} \mathrm{sec}$ and $10^{-11} \mathrm{sec}$ many significant events occur, and at the present time the only way to investigate them is theoretically. In this chapter we are primarily concerned with features of the initial energy deposit at 10-15 $\mathrm{sec}$, but some remarks are made below on the presumed processes by which the energy transforms through the $10^{-11} \mathrm{sec}$ reference time and beyond. Although the very earliest events can create biological damage, most biological processes occur on a much longer time scale and will not be considered here.

The physical energy deposit forms the bas is of all observed effects, and in this chapter we attempt to bring out the important features of such energy deposits (or track'structure). Controversies exist among workers in this field: regarding the information on track structure or the physical parameters that are significant for the analysis of track structure effects. For: example, Kellerer and Rossi (1978) assume that if one knows the energy absorbed in a critical volume of $1 \mu \mathrm{m}$ size, one may be able to predict quantitatively the eventual biological effects. Paretzke (1980) claims that one has to know the detailed cross section of physical processes and then evaluate the energy loss events in various channels before any correlation with chemistry or biology can be made. Paretzke has completed extensive and detailed calculations using Monte
Carlo methods and cross-section data in the gas phase. It is still unknown in what way energy loss processes in the gas phase are relevant to phenomena in condensed phases. Ritchie et al. (1980) are examining condensed phase effects on initial physical events.

In this chapter we present some recent views regarding energy transfer from energetic heavy particles with emphasis on the effects in the matter that absorbs the energy. For concreteness we use water as the absorbing medium. Our approach has been to estimate the radial energy density appropriate for $10^{-15} \mathrm{sec}$ around heavy particle trajectories. The final goal is to use this energy distribution in correlated studies of other effects. The distribution itself is composed of two qualitatively different regions which we call "core" and "penumbra." So far we have investigated the radiolytic decomposition of water (chapter II-5), and we expect that this type of track model will be useful in biological applications.

\section{THE PATTERNS OF ENERGY DEPOSIT}

\section{Stopping Power of Heavy Particles}

The best known physical quantity relating to a particle track is the stopping power. It is defined as the energy loss suffered by the incident particle in traversing unit length in the medium along its path and it is sometimes referred to as the linear energy transfer (LET $)_{\infty}$ ). Any description of track structure usually nas stopping-power theory as the starting point.

The stopping power of heavy particles in matter has been investigated (Northcliffe and Schilling, 1970; Ziegler, 1977). Figure 1 shows the $L T_{\infty}$ for six different particles in water as a function of energy per unit mass (E). These curves were obtained using a formula proposed by Pierce and Blann (1968). Table 1 contains a summary of the effective charges ( $Z_{\text {eff }}$ ) on particles in water as a function of $E$. The effective charge is given by:

$$
Z_{\text {eff }}:=Z\left[1-\exp \left(-0.95 v_{r}\right)\right] \text {, }
$$

where $Z$ is the atomic number of the particle, $v_{r}$ is the relative velocity given by $v_{r}=v / v_{0} z^{2} / 3^{r}$, $v$ is the particle velocity, and $v_{0}$ is the average velocity of the electron in the $H$ atom $\left(v_{0}=e^{2} / \hbar\right)$. The stopping power $\left(L E T_{\infty}\right)$ of a particle with an energy per nucleon $E^{\infty}$ is given in terms of the stopping power of the proton at the same energy: 


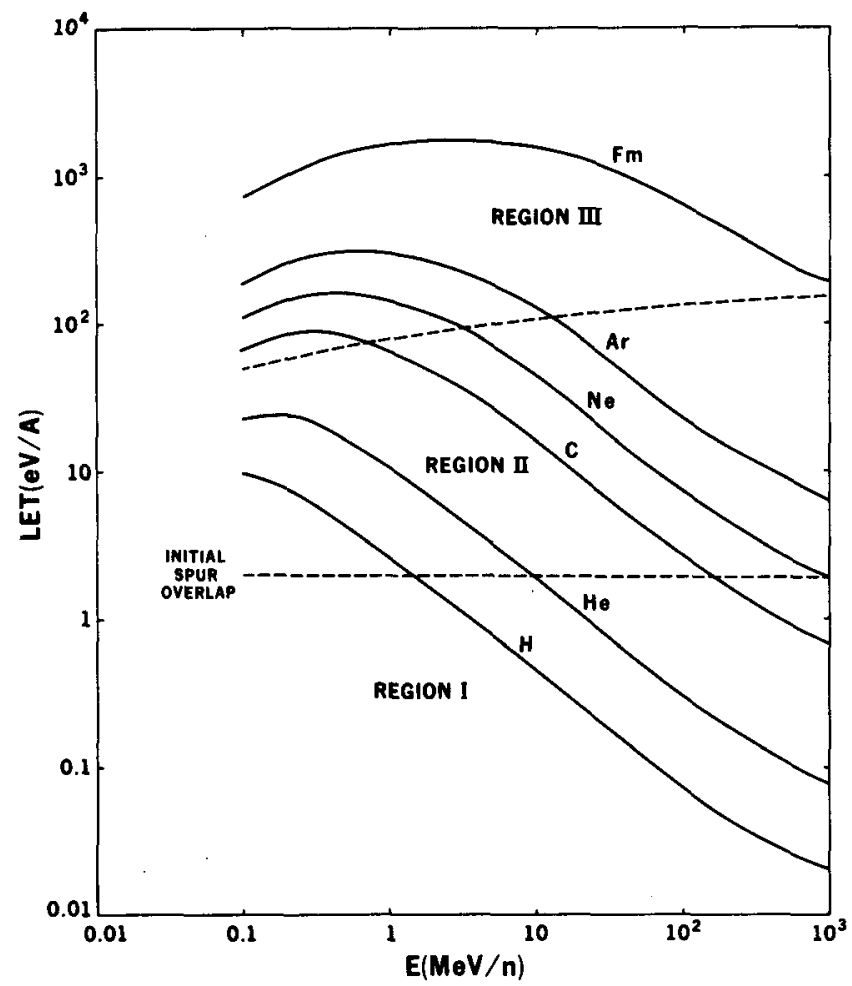

Figure 1. The stopping power (in eV/A) of six selected particles in $\mathrm{H}_{2} \mathrm{O}$ vs. specific energy (in MeV per nucleon). Data are constructed from Ziegler (1977). Region I is considered as lowLET, region II as medium-LET, and region III as high-LET. Such separation has proven to be useful for radiation chemistry studies with heavy particles. (XBL 7911-12802)

Table 1. Root Mean Square Charge States

\begin{tabular}{ccccccc}
\hline E (MeV $/ N)$ & Fermium & Argon & Neon & Carbon & Helium & Hydrogen \\
& & & & & & \\
\hline 1000 & 99.50 & 18.00 & 10.00 & 6.00 & 2.00 & 1.00 \\
800 & 99.39 & 18.00 & 10.00 & 6.00 & 2.00 & 1.00 \\
600 & 99.17 & 18.00 & 10.00 & 6.00 & 2.00 & 1.00 \\
400 & 98.67 & 18.00 & 10.00 & 6.00 & 2.00 & 1.00 \\
200 & 96.76 & 18.00 & 10.00 & 6.00 & 2.00 & 1.00 \\
100 & 92.54 & 17.99 & 10.00 & 6.00 & 2.00 & 1.00 \\
80 & 90.51 & 17.99 & 10.00 & 6.00 & 2.00 & 1.00 \\
60 & 87.38 & 17.97 & 10.00 & 6.00 & 2.00 & 1.00 \\
40 & 82.02 & 17.92 & 10.00 & 6.00 & 2.00 & 1.00 \\
20 & 70.84 & 17.62 & 9.97 & 6.0 & 2.00 & 1.00 \\
10 & 58.45 & 16.86 & 9.83 & 5.98 & 2.00 & 1.00 \\
8 & 54.47 & 16.47 & 9.74 & 5.96 & 2.00 & 1.00 \\
6 & 49.47 & 15.88 & 9.58 & 5.93 & 2.00 & 1.00 \\
4 & 42.78 & 14.87 & 9.25 & 5.84 & 2.00 & 1.00 \\
2 & 32.65 & 12.79 & 8.40 & 5.55 & 1.99 & 1.00 \\
1 & 24.40 & 10.52 & 7.27 & 5.03 & 1.96 & 1.00 \\
0.8 & 22.14 & 9.79 & 6.87 & 4.83 & 1.93 & 1.00 \\
0.6 & 19.49 & 8.88 & 6.34 & 4.54 & 1.89 & 0.99 \\
0.4 & 16.22 & 7.67 & 5.60 & 4.11 & 1.82 & 0.98 \\
0.2 & 11.77 & 5.85 & 4.41 & 3.35 & 1.63 & 0.93 \\
0.1 & 8.47 & 4.36 & 3.37 & 2.63 & 1.40 & 0.85 \\
& & & & & & \\
\hline
\end{tabular}




$$
\left(\frac{d T}{d x}\right)_{\text {ion }\left(z_{e f f}\right)}=\left(\frac{d E}{d x}\right)_{\text {proton }} z_{e f f}^{2} .
$$

The proton stopping power was calculated using the Bragg rule with data for the atomic targets $H$ and 0 obtained from Ziegler (1977). The actual data scatter widely, and a considerable amount of smoothing was made; the low-energy stopping power of heavy particles cannot be considered as well known.

The concept of $L T_{\infty}$ was first introduced by Zirkle et a). (1952) in order to distinguish it from stopping power. While stopping power deals with energy lost by a charged particle in a medium, $L E T_{\infty}$ refers to the energy absorbed "locally" by the medium. The weakness in the concept of linear energy transfer lies in an ambiguity as to just how much of the total energy lost by the particle should be considered as locally deposited. Energy lost to bremsstrahlung is clearly not locally deposited, but in the stopping of heavy particles in the energy range of biomedical interest this effect is very small. However, even in loss phenomena involving electronic interactions only, energy can be deposited over large regions (compared to cellular dimensions) by high energy delta-rays. In such cases, partial LET values have sometimes been used, limiting the LET with cutoff procedures that depend on recoil electron energy, $\varepsilon$. or recoil electron penetration distance $r$. In the former case, the restricted LET is designated $\mathrm{LET}_{\varepsilon}$, and in the latter case, LETr.

Use of $\mathrm{LET}_{\varepsilon}$ has been made in the dosimetry of high-energy heavy particles with plastic foils. Experimental evidence indicates that energy-transfers up to about $350 \mathrm{eV}$ contribute to local damage which responds to an etching technique (Benton, 1969). Thus, it seems that LET 350 seems to be relevant to the analys is of foil etching methods. For living tissues such parametrization has not been accomplished yet. At present, it is our belief that the data of neither radiation biology nor radiation chemistry will be found to be simple functions of any one physical parameter related to the pattern of energy deposit (Magee and Chatterjee, 1980).

\section{Radial Energy Density}

In the region of energy loss through electronic interaction, there are two gross categories of collisions: glancing and knock-on. For heavy charged particles-(but not for electrons), there is an approximate equipartition of energy loss between these two categories (Chatterjee et al., 1973).

On the most general grounds, it can be argued that the tracks of $n$ igh energy particles are composed of two parts; we have designated them "core" and "penumbra." The core is created by the glancing collisions of the particle with excitations in the range from 6.5 to $100 \mathrm{eV}$ (the stopping medium is water). Bohr's adiabatic criterion sets a limit of the radial distance from the trajectory of the incident particle within which molecules can be excited by this mechanism.

Similar limits have also been obtained by Brandt and Ritchie (1974), based on plasma oscillations, and can be given by:

$$
r_{c}=\frac{\beta_{c}}{\Omega_{p}} .
$$

We call $r_{c}$ the core radius. In equation $3, B$ is the velocity of the particle in terms of the velocity of light, $c$; and $\Omega_{\mathrm{p}}$ is the plasma oscillation frequency.

The penumbra is composed of the tracks of the knock-on electrons. Of course, some of the energy of these electrons is lost in the core, but most of it is used to create the region which is generally much larger than the core, called the penumbra. It has a radius which we designated as $r_{p}$. Based on our previous detailed calculations on radial-energy density (Chatterjee et al., 1973), $r_{p}$ can be approximately fitted to the following empirical formula:

$$
r_{p}=396(v)^{2.7}
$$

where $v$ is in units of $10^{9} \mathrm{~cm} / \mathrm{sec}$.

Based on detailed studies (Chatterjee and Tobias, 1980), the distribution of energy in the core and in the penumbra can be empirically given as follows:

$$
\begin{aligned}
& { }_{\text {core }}=\frac{\mathrm{LET}_{\infty} / 2}{\pi r^{2}}+ \\
& \frac{\mathrm{LET}_{\infty} / 2}{2 \pi r_{c}^{2} \ln \left(\sqrt{\mathrm{e}} r_{p} / r_{c}\right)} r \leqslant r_{c}, \\
& \rho_{\text {pen }}(r)=\frac{\operatorname{LET} / 2}{2 \pi r^{2} \ln \left(\sqrt{\mathrm{e}} r_{p} / r_{c}\right.} r_{c}<r \leqslant r_{p} .
\end{aligned}
$$

Here, LET is the total energy loss per unit path-length and $r$ is the radial distance from the particle trajectory. The first term in equation 5 is the energy density due to the glancing collisions; the second term gives a contribution from the energy lost by the knock-on electrons in their penetration of the core. The energy density of equation 6 arises from knock-on electrons which are stopped between $r_{C}$ and $r_{p}$.

The track dimensions $r_{c}$ and $r_{p}$ depend only on the particle velocity $B$ and not on charge or mass. The energy density, however, depends on $L E T_{\infty}$ and thus on the effective charge of the particle. The general particle track, therefore, depends on two parameters that can be taken as energy per nucleon (E) and atomic number of particle $(Z)$. The actual effective charge $Z_{\text {eff }}$ 
Table 2. Track Parameters Independent of Particle Charge

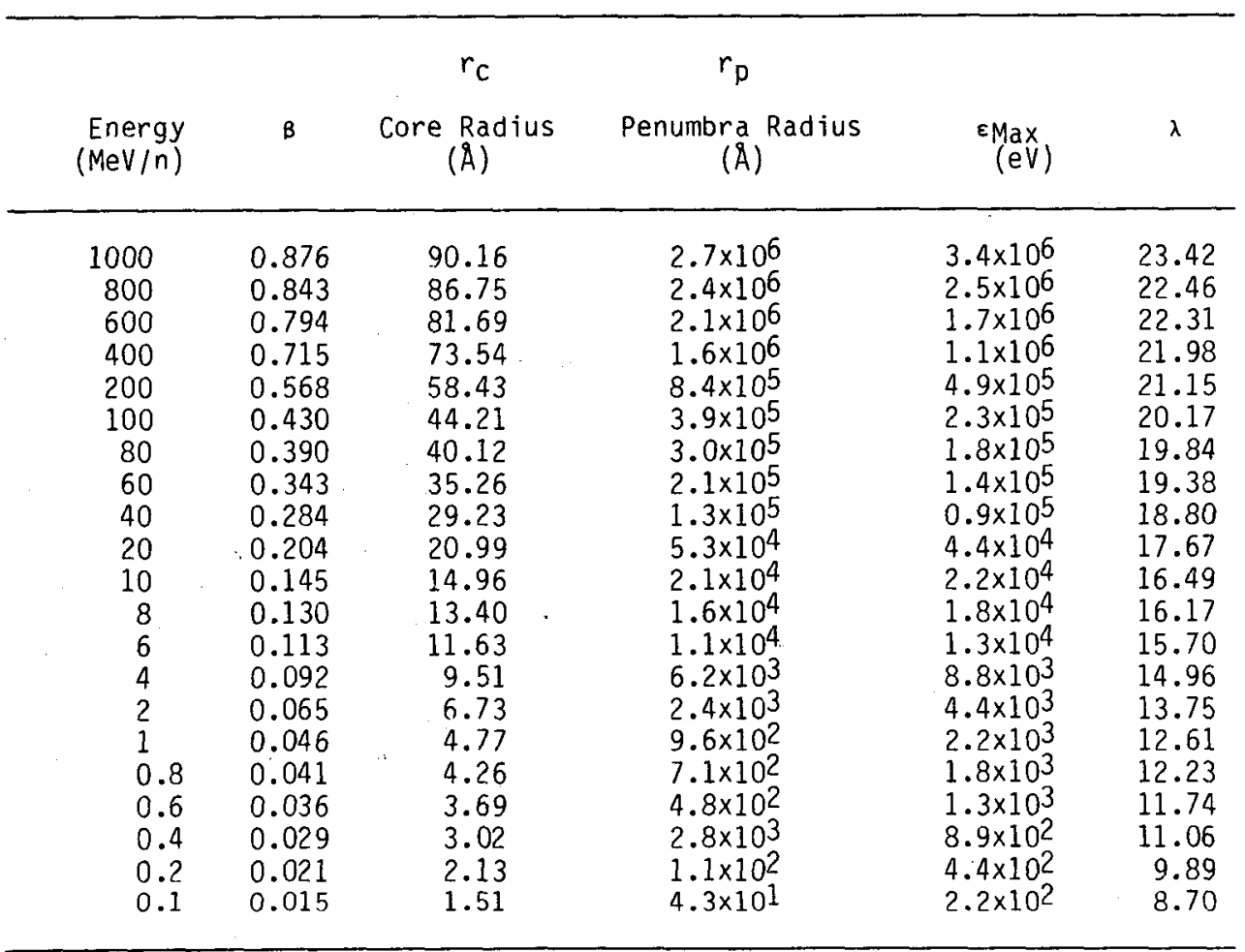

is determined by the interaction of the particle with the medium; it depends on the velocity and cannot be set arbitrarily. Table 2 contains values of some of the track quantities that depend on the velocity only. The last column of Table 2 gives $\lambda$, the ratio of the "initial" energy density in the core to that in the near $r_{C}$.

The densities given by equations 5 and 6 are average values, and the actual initial values may fluctuate greatly around them. For example, the core is comprised of spurs which contain, on the average, $40 \mathrm{eV}$; if the LET is small enough, the core is made up of a string of spurs rather than a continuous distribution of intermediates. The penumbra is made up of tracks of knock-on electrons, and it is always more inhomogeneous than the core.

A schematic model for the transverse section of a heavy particle track is presented in Figure 2. The circular region in the center is the core, bounded by the Bohr adiabatic radius, $r_{c}$. The tracks of the knock-on electrons tenuousiy occupy the region of the penumbra, bounded by $r_{C}$. and $r_{p}$. The radius $r_{p}$ cannot be calculated analyticaliy from simple physical arguments; the maximum extent of the region is dependent in a complicated manner on the energy of the secondary electrons and also on the diffusion distance of these electrons before they become subelectronic.

Previously, our theoretical evaluation of radial energy density could be compared with experiments only at low energies (Chatterjee, et al., 1973). But recently Varma and Baum of Brookhaven National Laboratory (1980) have made

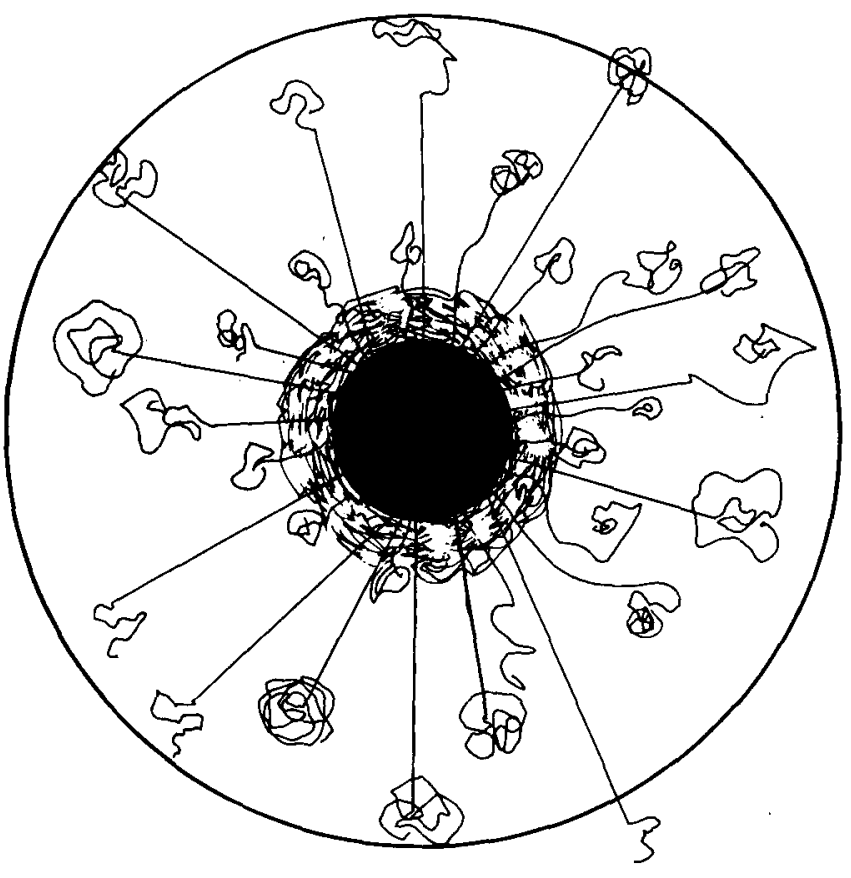

Figure 2. Schematic model of track (crosssectional view). The radi $i r_{c}$ and $r_{p}$ are shown: the lines in the penumbra indicate individual electron tracks. The energy deposition at the boundary separating the core and the penumbra is not as dense as it is in the core, but in this region the tracks of electrons do overlap. (XBL 778-3712) 
some measurements with $377 \mathrm{MeV} / \mathrm{n} 20 \mathrm{Ne}$ ions. They measured dose as a function of radial distance from the trajectory. A variable pressure ionization chamber and a double-mesh wall ionization chamber (approximating a wall-less ionization chamber) were used. Linear energy transfer restricted in radial distance (LETr) was also determined, and the data were extrapolated to obtain LET $T_{\infty}$. They simulated distances from $58 \AA$ to $1010 \mu \mathrm{m}$ in a tissue equivalent gas (by varying pressure). Their measured values, when expressed at percent LET were within $\pm 6 \%$ of the values computed from our semiempirical formula given by equations 5 and 6 . In Table 3, $\mathrm{LET}_{r}$ values are presented showing the comparison between the theoretical estimates and experimentally obtained data of Varma and Baum for a few typical radial distances varying between $2.9 \mu \mathrm{m}$ and $174 \mu \mathrm{m}$ for $377 \mathrm{MeV} / \mathrm{n} 20 \mathrm{Ne}$ ions. It is clear from the table that the value of $r_{p}$ (the maximum radial distance with in which the total LET is contained) is somewhat smaller than experimentally measured values tend to indicate.

More experiments with Bevalac ions are in progress. When the data are available, more extensive comparisons will be made with the theoretical calculations. If needed, improvements will be sought in the theoretical basis for the calculations.

In the present state of development of the theoretical model, the production of secondary electrons is based on the Rutherford scattering formula, which is a very rough approximation. Such an approximation was adopted for ease in calculation. We expect to refine the calculations by introducing a spectrum of low energy (less than 1,000 ev) electrons, which is more realistic. Efforts to measure the distribution of the low energy electrons are in progress and are described below.

\section{Low Energy Knock-On Electron Spectrum}

A sizeable fraction of the recoil electrons produced when a heavy ion passes through matter has low energy (less than a few keV). Such electrons have a short range in matter (a few $\mu \mathrm{g} / \mathrm{cm}^{2}$ ). In order to measure the true primary energy distribution, a target $<10^{-2} \mu \mathrm{g} / \mathrm{cm}^{2}$ equivalent must be used. This low target density is achieved by a differentially pumped gas jet. Measurements of the electron energies are made using a time-of-flight technique which takes advantage of the characteristics of the chevron channel multiplier.

A schematic view of the spectrometer than has been built in our laboratory is shown in Figure 3. The gas jet fore-chamber is a high vacuum vessel pumped by two $1,000 \mathrm{l} / \mathrm{sec}$ diffusion pumps. Each pump has a water-cooled baffle system, but no valve, in order that the maximum pumping speed can be achieved. The chamber is equipped with all the necessary plumbing needed to support the diffusion pumps, and a large capacity back-pump is used. The chamber is also equipped with an ion gauge and associated electronics for measurement of the interior vacuum.

The "start" sample detector utilizing a chevron channel multiplier provides a signal when a heavy ion passes through a carbon foil (not shown in the figure) located at known distance from the gas jet. Time resolution of this detector is <100 psec. After passing through the "start" detector, heavy ions interact (impinging perpendicular to the plane of the diagram) with atoms in the gas jet, producing primary electrons. The physical dimensions of the gas jet must be reasonably small and well defined. A collimator and transmission grid $10 \mathrm{~cm}$ away provides a window into an electron accelerating field. Electrons produced by the heavy ions fly through the field-free region for a time determined by their energy; if their tracks are oriented properly, they are detected by the chevron channel multiplier. A silicon detector is used to verify that an ion has traversed the gas jet.

Signals are processed as a time-sampled spectrum to avoid statistical biasing of the spectrum by the faster electrons. The start detector provides a trigger signal related to the time when heavy ion passes through the gas jet. By inspecting the output of the electron detector for a fixed-time increment at regulartime delays from the start pulse, a histogram of counting, rate vs. flight time can be made. Absolute energy. calibration can be made by taking advantage of the sensitivity of the channel plates: to soft $X$ rays. When a heavy ion inter-

Table 3. LETr Values for a 377 MeV./n Neon Beam from the Bevalac

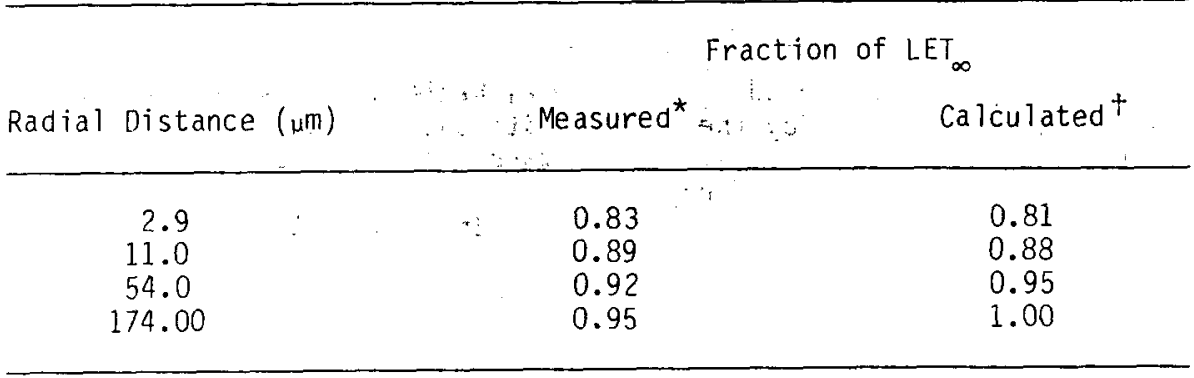

* Measured in tissue equivalent gas. tCalculated in liquid water. 


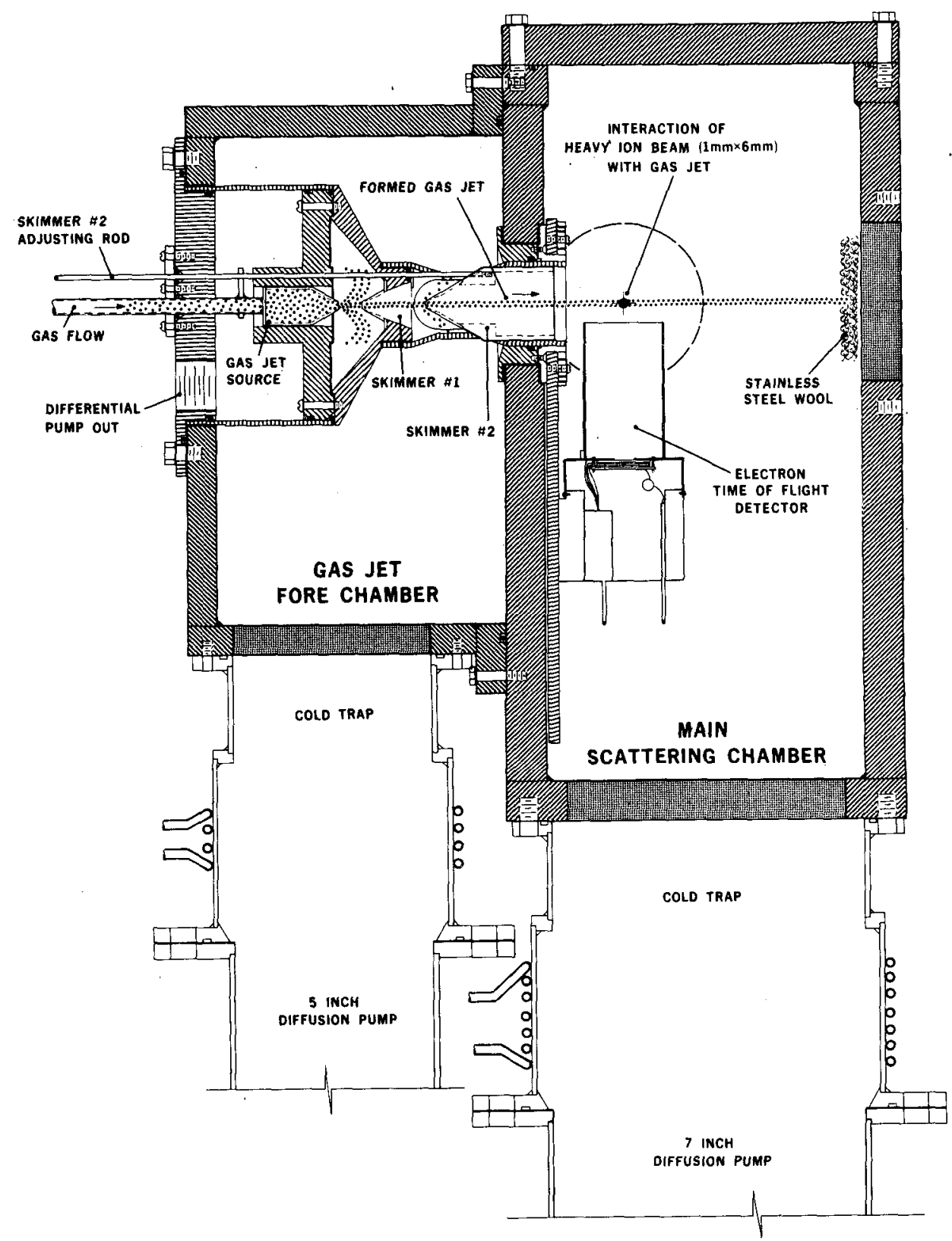

Figure 3. A schematic view of the secondary electron spectrometer spectrum of low-energy electrons (less than $1 \mathrm{keV}$ ) will be measured by the time-of-flight technique and using a chevron-channel plate analyzer. A special gas jet will be used so that secondary electrons, once produced, will have low probability $\left(<10^{-2}\right)$ of undergoing further collision before they are detected. Heavy particle trajectories will be perpendicular to the plane of the figure. (XBL 756-1588)

acts with the gas atoms, $x$ rays are produced. In some cases, these $X$ rays are detected by the channel plate; so in the time spectrum, a small peak appears at a time corresponding to the $x$-ray travel time to the detector.

Initially, instead of the gas jet, we will use a thin carbon foil to duplicate the measurements made by Toburen (1972) and Toburen and Wilson (1972). We will use hydrogen and helium beams of a few MeV from the van de Graaf facility. Such measurements will enable us to calibrate our spectrometer before measurements with gas jets are made, and subsequently data are taken with Bevalac ions.

In the first experiments with gas jets, we will use simple atomic gases such as neon and argon. Simple diatomic gases will also be used. The electron spectrum measurements will also be made with a tissue equivalent gas. We hope that the results of these measurements will enable us to make a substantial improvement in calculation of the various physical quantities associated 


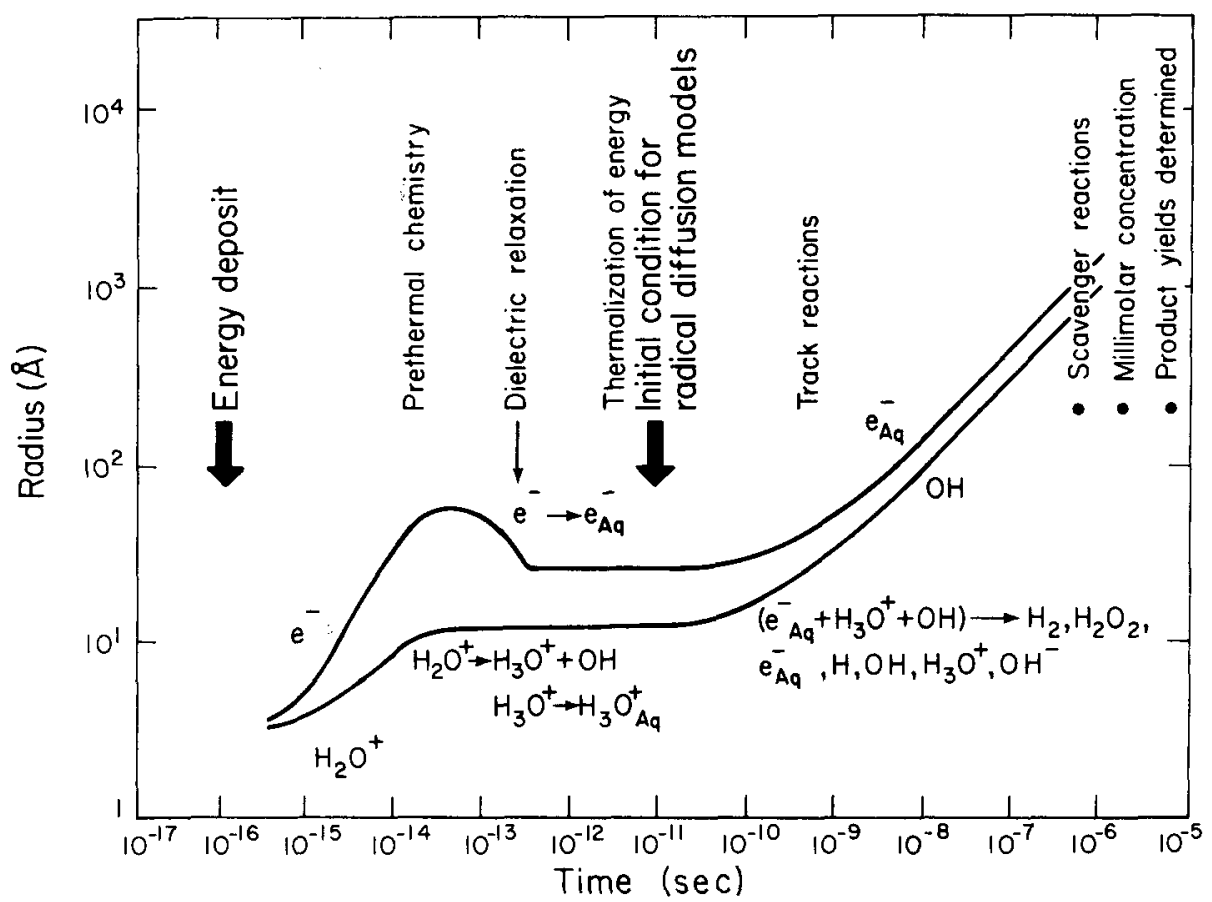

Figure 4. Space-time development of small isolated energy deposit (spur) in a dilute aqueous system. In the prethermal time period $\left(t<3 \times 10^{-12} \mathrm{sec}\right)$, phenomena involve electronic motion for the most part; the ion-molecule reaction creating $\mathrm{H}_{3} \mathrm{O}^{+}$and the longitudinal relaxation of $\mathrm{H}_{2} \mathrm{O}$ require only proton motion. In the postthermal period, the species that have already been created undergo diffusioncontrolled reactions with each other (indicated by the parenthes is on the left of the equation). (XBL 794-1326)

with track structure of heavy particles.

\section{Space-Time Development of Deposited Energy}

So far, most of the discussion has centered around the energy transfer phenomena that occur in less than $10^{-15} \mathrm{sec}$. In chapter I I-5 we discuss the chemical effects of particle tracks without considering the many events that occur between the initial energy deposition and the thermalization time of $10^{-11} \mathrm{sec}$. Several assumptions had to be made to initiate the radiation chemical calculation, and the validity of the assumptions are judged on the bas is of the agreement with experiment of the chemical results obtained, essentially on a microsecond time scale. The time period between $10^{-15} \mathrm{sec}$ and 10-11 sec needs to be investigated, and "it seems that theory is the only available approach. Recently, such an effort has begun at the oak Ridge National Laboratory by a research team led by J. E. Turner. The procedure is based on a Monte Carlo code developed earlier. We fully expect that knowledge of track structure at $10^{-11}$ sec will be increased greatly as this investigation proceeds.

We have worked on a concept of the local space-time evolution following a small isolated energy loss in water (Fig. 4). From a chemical point of view, the distribution in space of $\mathrm{e} \overline{\mathrm{a}} \mathrm{q}, \mathrm{H}_{3} \mathrm{O}^{+}$, and $\mathrm{OH}$ at $10^{-11} \mathrm{sec}$ is what is meant by "initial track structure." The radical diffusion model uses such distribution to calculate the reactions during track expansion and the radical reactions with scavengers; all of these processes are complete in a few microseconds, and the product yields are determined. The events that occur before thermalization (a few psec) have not, of course, been observed directly. However, the ion-molecule reaction that forms $\mathrm{H}_{3} \mathrm{O}^{+}$and $\mathrm{OH}$ at $\mathrm{t}=2 \times 10^{-14} \mathrm{sec}$ (it is the fastest chemical process of radiation chemistry), and the hydration of the electron at the longitudinal relaxation time for water $\left(\approx 4 \cdot x 10^{-13} \mathrm{sec}\right)$ are widely accepted. In any case, the reactive intermediates are formed in the prethermal period. At thermalization, all of the deposited energy except that tied up in dissociation of water to form the intermediates is distributed as a local temperature rise in the track.

Biological systems are, for the most part, concentrated aqueous solutions, and many of the same processes (as those shown in Fig. 4) occur on their irradiation. However, reactions of the biological molecules can occur at any time, including the prethermal period.

Clearly, the tracks used by the radiation chemist are related to the patterns of initial 
energy deposit obtained by purely physical considerations. The chemical "initial" patterns have lost a certain amount of the original detail--both in the distribution of energy in species and in space. The radiation chemist also has a cutoff in the size of pattern of interest, e.g., scavengers destroy radicals before they can move some maximum distance such as $10^{3} \AA$ or so in dilute aqueous systems.

It has long been customary in considerations of radiation chemistry to ignore mechanical effects produced by the heating in particle tracks. The neglect of such effects is certainly permissible for low-LET radiations and tracks of particles with small Z, but mechanical efforts in the tracks of high-Z particles, particularly in the low energy region, may be important. Unfortunately, the theory of these effects has not been developed. Plesset and Prosperetti (1977) have presented an elementary treatment of bubble dynamics which is a fragment of the required theory.

Along with the energy degradation processes discussed here, there is an increase in local pressure as the local temperature builds up. In a picosecond or so (local time), the high pressures in a track produce shock waves that run away into the undisturbed medium. Shock waves in 1 iquids are we 11 understood: shocks produced by particle tracks are weak (Zel'dovich and Raizer, 1967), there is no great volume change, and the molecules are not expected to be damaged. This situation results from the fact that the repulsive potentials of molecules are large for decreased separations, so the high pressures are relieved by a slight volume increase. On the other hand, the high temperature initially created cannot dissipate on the same time scale as the pressure is relieved, so the elevated temperatures remain. If there is enough excess energy, a bubble will form and expand until the heat conductivity cools the region and allows the bubble to collapse. These phenomena are so poorly understood that we do not know how to estimate the condition for bubble formation; we do not expect it to occur except for the heaviest particles and in a low energy region. Perhaps of more importance is the fact that we do not know what effects to expect from bubble formation, either in dilute aqueous solutions or in biological systems. These matters are scheduled for investigation. Uncertainties abound throughout, and there are many mechanisms that must be understood before a complete theoretical foundation exists for the early-time phenomena.

\section{DISCUSSION}

The energy deposition by an energetic heavy particle in the electronic phase $(<10-15 \mathrm{sec})$ in water is quite well understood. The resulting energy density is summarized in equations 5 and 6. It is possible that the knock-on electron spectrum investigation described here will allow us to make some refinements in this energy density, but no major changes are expected. We show in chapter II -5 how this result can be used in a model for the radiation chemistry of dilute aqueous solutions, and we believe that it will be of value in models for radiation effects in biological systems such as cells. The fact that the latter are concentrated solutions in their simplest description introduces difficulties that have not been fully analyzed. The initial energy deposit and flow are expected to be different in such systems.

From the time that energy is transferred to the molecules of a medium, a large number of complicated processes occur before the medium undergoes permanent changes. This complexity exists even in relatively simple systems such as water and is greatly accentuated if the water contains organic materials. A mammalian cell contains (besides $80 \%$ water) DNA and RNA (which are strictly polymers), and proteins (which are macromolecules). In such a complicated system almost no knowledge exists about the amount of energy transferred to DNA or RNA. Certain assumptions can be made to calculate the energy transferred to these molecules by the primary particle (in about $10^{-16} \mathrm{sec}$ ), but the fate of this transferred energy is a problem of even higher order of complexities.

We have much information on energy transfer to large molecules by low-LET radiations. Thermoluminescence experiments suggest the possibility that excitation energy is transferred via special structures. Configurations which may be involved in such energy transfer processes are the alpha-helical structure of polypeptides in proteins and planarly stacked purine and pyrimidine bases in double helical structures of DNA. Energy transfer processes can also be affected by migration of electrons through a "conduction band" and subsequent trapping of electrons in positive holes. From ESR studies we also know that free radicals are formed in irradiated large biological molecules. Migration of these dissociated free radicals may play an important role in the energy transfer process.

Most of the experiments done to elucidate the above modes of energy transfer have been done in environments not at all similar to those encountered in normal cellular systems. For example, measurements have frequently been made in the dry or frozen state. If water molecules are around the macromolecules, the energytransfer process and its effects may be quite different, so the results are not directiy applicable to biology. Furthermore, the experiments have generally been performed with low-LET radiations, and thus are probably not applicable to heavy particle radiations.

There is no way to go directly from the energy deposition patterns presented here to biological applications. We plan to increase our knowledge of the chemical effects by theoretical and experimental studies of concentrated solutions. The next phase of the program will involve biological systems more explicitly.

\section{REFERENCES}

Benton, E. V. 1969. The restricted energy loss 
criterion for registration of charged particles in plastics. Nucl. Inst. Methods 67, 343-352.

Brandt, W. and R. H. Ritchie. 1974. Primary processes in the physical stage. Physical Mechanism in Radiation Biology (R. D. Cooper and R. W. Wood, eds.) pp. 20-50. U. S. Atomic Energy Commission. CONF-721001, Washington, D. C.

Chatterjee, A., H. D. Maccabee, and C. A. Tobias. 1973. Radial cutoff LET and radial cutoff dose calculations for heavy charged particles in water. Radiat. Res. 54, 479-494.

Chatterjee, A. and C. A. Tobias. 1980. Analytical expression for radial distribution of energy deposited by a penetrating heavy particle. Radiat. Environ. Biophys., in press.

Kellerer, A. M. and H. H. Rossi. 1978. A generalized formulation of dual radiation action. Radiat. Res. 75, 471-488.

Magee, J. L. and A. Chatterjee. 1980. Parameters of heavy particle irradiations. Radiat. Res., in press. (Abstract).

Northcliffe, L. C. and R. F. Schilling. 1970. Range and stopping-power tables for heavy ions. Nucl. Data A7, 233-463.

Paretzke, H. G. 1980. Track structure calculations and their accuracy. Sixth International. Congress of Radiation Research (S. Okada, M. Imamura, T. Terashima, and H. Yamaguchi, eds.) pp. 157-164. Japanese Association of Radiation Research, Tokyo, Japan.

Pierce, T. E. and M. Blann. 1968. Stopping powers and ranges of $5-90 \mathrm{MeV} 32 \mathrm{~s}, 35 \mathrm{Cl}$, $79 \mathrm{Br}$, and $127_{\mathrm{I}}$ ions in $\mathrm{H}_{2}, \mathrm{He}, \mathrm{N}_{2}, \mathrm{Ar}$, and $\mathrm{Kr}$ : A semi-empirical stopping power theory for heavy ions in gases and solids. Phys. Rev. 173, 390-405.
Plesset, M. S. and A. Prosperetti. 1977. Bubble dynamics and cavitation. Ann. Rev. Fluid Mech. 9, 145-185.

Ritchie, R. H., R. N. Hamm, H. A. Wright, and J. E. Turner. 1980. Physical phase effects in charged particle track structure. Sixth International Congress of Radiation Research (S. Okada, M. Imamura, T. Terashima, and $H$. Yamaguchi, eds.) p. 165. Japanese Association for Radiation Research, Tokyo, Japan.

Toburen, L. H. 1972. K-shell ionization of carbon by fast protons. Phys. Rev. A5, $2482-87$.

Toburen, L. H. and W. E. Wilson. 1972. Distributions in energy and angle of electrons ejected from molecular hydrogen by $0.3-1.5 \mathrm{MeV}$ protons. Phys. Rev. A5, 247-256.

Varma, M. N. and J. W. Baum. 1980. Energy deposition in nanometer regions by $377 \mathrm{MeV} /$ nucleon $20 \mathrm{Ne}$ ions. Radiat. Res. 81, 355-363.

Zel'dovich, Y. B. and Y. P. Raizer. 1967. Physics of Shock Waves and High Temperature Phenomena vol. 2, pp. 685-784. Academic Press, New York.

Ziegler, J. F. L. 1977. Hydrogen stopping powers and ranges in all elements. The Stopping Power and Ranges of Ions in Matter (J. F. Ziegler, ed.) vols. 3 and 4. Pergamon Press, oxford.

Zirkle, R. E., D. F. Marchbank, and K. D. Kuck. 1952. Exponential and sigmoidal survival curves resulting from alpha- and $x$-irradiation of Aspergillus spores. J. Cell. Comp. Physiol. 39 (Supp1. 1), 75-85. 
62

Blank 


\title{
RADIATION CHEMISTRY OF HEAVY PARTICLES
}

\author{
J. L. Magee and A. Chatterjee
}

The physical aspects of the energy deposit of fast particles in matter are considered in chapter II-4. These energy deposits also initiate chemical effects which are merged with the early physical processes $\left(10^{-15} \mathrm{sec}<\mathrm{t}<10^{-11}\right.$ $\mathrm{sec}$, and become dominant on a longer time scale ( $t>10^{-11} \mathrm{sec}$ ). In a biological system, the early chemical processes tend to be the same as would occur in a nonliving system; but on an appropriate time scale ( $t>10^{-3} \mathrm{sec}$ ), biological processes take over. Here we are only concerned with radiation chemistry and its relationship to the initial energy deposit. The primary objective of our track studies is, however, application to biology.

The simplest system to use in theoretical considerations of radiation chemistry is the dilute aqueous solution. In such a system the energy is absorbed almost exclusively in water, and the principal chemistry is that of radiation decomposition of water. The various observed radiation products depend upon the additives that are present, but the basic radiation chemistry is essentially the same for all dilute solutions.

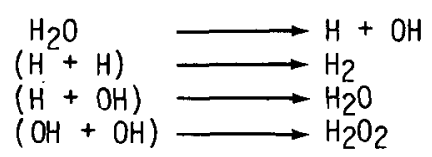

This set of equations is a simplified set which applies to the radiolysis of acid solutions. Equation 1 indicates the initial decomposition of $\mathrm{H}_{2} \mathrm{O}$ by the radiation; hydrated electrons primarily formed are converted into $H$ atoms by the acid, a process which requires about $6 \times 10^{-11}$ sec. None of the early phenomeria that occur at shorter times are considered explicitly in this treatment, and we start with an assumed spatial distribution of $\mathrm{H}$ and $\mathrm{OH}$ radicals at $6 \times 10^{-11}$ sec.

The parentheses around the left sides of equations 2 through 4 indicate that the recombinations are track processes. Under conditions normally considered in radiation chemistry (low dose rates), tracks are independent. This means that radicals that escape their siblings in a track react with a scavenger before they can react with a radical or product from another track. A typical scavenger reaction is:

$$
\mathrm{OH}+\mathrm{Fe}^{+2} \longrightarrow \mathrm{OH}^{-}+\mathrm{Fe}^{+3}
$$

which occurs in the Fricke dosimeter. The yield of $\mathrm{Fe}^{+3}$ per $100 \mathrm{eV}$ absorbed in the Fricke system, $\mathrm{G}\left(\mathrm{Fe}^{+3}\right)$, is given by:

$$
\mathrm{G}\left(\mathrm{Fe}^{+3}\right)=\mathrm{G}_{\mathrm{OH}}+3 \mathrm{G}_{\mathrm{H}}+2 \mathrm{G}_{\mathrm{H}_{2} \mathrm{O}_{2}},
$$

where the terms on the right indicate the amounts (in terms of $G$ values) of the respective products which are produced in the tracks.

The radiolytic decomposition of water in a dilute solution is a fundamental problem. Yields of all products are given by equations like equation 6 . The fundamental quantities $\mathrm{G}_{0 H}$, $G_{H}$, and $G_{H_{2} O_{2}}$, of course, depend upon the track structure. $\mathrm{O}_{2}$ ' The track structure in water is expected to be virtually the same as that in a biological system (such as a cell in suspension). The study of radiation chemistry of dilute solutions, therefore, provides another method to investigate the structure of tracks as they are likely to be present in an irradiated biological system but at times much longer than they are accessible to purely physical measurements.

\section{OVERVIEW OF TRACK PROBLEMS FOR RADIATION CHEMISTRY}

Heavy particle tracks are all similar in that they consist of cores and penumbras (see chapter. II-4). We consider tracks in a differential, or track segment sense, and two parameters determine the track structure. An accelerated particle has an atomic number $Z$ and $a$ velocity $v$. The energy per unit mass $E$ is determined as $E=M C^{2}\left[\left(1-\beta^{2}\right)-1 / 2-1\right]$, where $M$ is the proton mass and $B$ is the particle velocity, $v$, divided by the velocity of light, $c$. The effective charge $Z^{\star}$ is a unique function of $B$; it depends on the balance between ionization and electron capture, and $Z^{*}$ determines the LET. The spectrum of knock-on electrons determines the dimensions of the penumbra, and it depends on $B$.

The radiation chemistry of the heavy particles should form an understandable pattern as one considers particles over the accessible ranges of $Z$ and $v$, or $B$. No result of particle irradiation can be expected to be a function of one parameter on $1 y$, such as the LET.

All low-LET radiations are composed of electrons, and thus the penumbras of heavy particle tracks are low-LET radiations. Any type of radiation is mixed in the sense that it contains tracks with various energy densities. One of our objectives in this study is to understand the relationship between the effects of electron and heavy particle irradiations.

The high-energy electron track is composed of a core with widely spaced spurs and a penumbra made up of electron tracks that expand and react 
without overlapping. A high-energy proton track is expected to have a similar structure. The similarity of these two tracks can relate the heavy particle and low-LET radiations, and furnish a means to define the concept of "quality" of radiation more precisely.

Consider the differential yields of a proton and an electron of the same velocity in the high energy region. We have shown (Magee and Chatterjee 1978a,b, 1980b) that the following integro-differential equation applies:

$$
\begin{aligned}
G_{i}^{\prime}\left(E_{i}\right)=\left(1-f_{i}\right) G_{s p}+ \\
f_{i} \frac{\int_{\varepsilon_{0}}^{\varepsilon_{\max }^{i}} G_{e}(\varepsilon) w_{i}\left(E_{i}, \varepsilon\right) d \varepsilon}{\int_{\varepsilon_{0}}^{\varepsilon_{\max }^{i}} w_{j}(E, \varepsilon) d \varepsilon},
\end{aligned}
$$

where $G^{\prime}\left(E_{j}\right)$ is the differential yield of the ith particle (proton or electrons); $G_{s p}$ is the average yield of a spur (notice that this quantity is independent of the particle); $f_{i}$ is the fraction of energy loss in knock-on electrons for the ith particle); $\varepsilon_{0}$ and $\varepsilon_{\text {max }}$ are the lower and upper limits of the knock-on spectrum for the $i$ th particle; $w_{j}\left(E_{j}, E\right)$ is the probability per unit energy that a knock-on electron will be produced with energy $\varepsilon$ by the $i$ th particle of energy $E_{i}$; and $G_{e}(E)$ is the yield per $100 \mathrm{eV}$ for an electron which starts with energy $\varepsilon$, and is stopped in the medium.

The validity of equation 7 depends upon the fact that all of the entities of the track, i.e., the spurs along the core and the knock-on electrons of the penumbra, develop their chemical yields independently.

Equation 7 furnishes a precise relationship between the electron and proton differential yields; and at high velocities, they are almost the same. To a first approximation,

$$
w_{i}\left(\dot{E}_{i}, \varepsilon\right) \simeq \frac{1}{\varepsilon} \text {. }
$$

The lower limit of the integral $\varepsilon_{0}^{i}$ is taken as $100 \mathrm{eV}$ for both particles. The upper l'imits are different,

$$
E_{\max }^{\mathrm{e}}=\frac{1}{2} \mathrm{E}_{\mathrm{e}}
$$

because an electron can only lose nalf its energy, : and

$$
\varepsilon_{\max }^{p}=\frac{m c^{2}}{1-B^{2}},
$$

where $m$ is the electron mass. The fractions of energy in knock-on electrons vary slightly between the two particles, although they are both $\sim 0.5$.

\section{Yields of Electron Tracks}

When electron tracks are considered, the integro-differential equation 7 involves only one dependent variable, $G_{e}$, and the equation can be considered as a self-consistent equation in $\mathrm{G}_{e}(\varepsilon)$. We require that $\mathrm{G}_{\mathcal{e}}(\varepsilon)$ is known from $\varepsilon=\varepsilon_{0}$, or $100 \mathrm{eV}$, to $\varepsilon=10^{t} \mathrm{eV}$. In the high energy region, equation 7 can be transformed into a differential equation. Notice in this connection that the left side of this equation is:

$$
G_{e}^{\prime}(E)=\frac{d}{d E} E G_{e}(E)
$$

The differential equation derived from equation 7 for the electron has been investigated and an approximate solution valid at high velocity has been obtained (Magee and Chatterjee, 1978a,b). This solution is:

$$
\begin{aligned}
G_{e}(n) & =G_{s p}+ \\
& \frac{a_{0}}{n}\left\{1+\frac{0.1936}{n}+\frac{0.1587}{n^{2}}+\frac{0.2752}{n^{3}}\right\}
\end{aligned}
$$

where $a_{0}$ is a constant to be obtained from calculation or experiment, and a new independent variable $n=\ln E / 2 E_{0}$ is introduced. The analytical solution given in equation 10 is valid for electron energies above $20 \mathrm{keV}$. In the very low energy region, an electron track model was used; in the intermediate energy region, equation 7 was used in the integro-differential form along with the track model (Magee and Chatterjee 1978a).

The unknown function of these equations, $G_{e}(E)$, or $G_{e}(n)$, is the yield of any product in dilute solution. We investigated the yields of the Fricke dosimeter for all electron energies from $100 \mathrm{eV}$ to $10^{7} \mathrm{eV}$. The yields for any electron irradiation for which the electron spectrum is known can be obtained, using these results.

The specific effects of electron track ends are given in this treatment. We see from equation 10 that as $n$ gets large (the bracket approaches unity), the term in 0.6 continues to make a contribution to $G_{e}(n)$, so that the asymptotic value $G_{S p}$ is not actually reached in the range of energies that are of interest to experimentalists. The term in $n^{0.6}$ can be considered as giving the track end contribution. 
The nature of $G_{S p}$ has been considered in a special study (Magee and Chatterjee, 1980a). The spur yield is made up of contributions from all energy losses between 0 and $100 \mathrm{ev}$, with an average value which we have estimated as $40 \mathrm{eV}$. We have shown how our approximate concepts of the phenomena that occur early (in less time than $10^{-12} \mathrm{sec}$ ) are compatible with observed radiation chemical yields.

\section{Yields of Heavy Particle Tracks}

Equation 7 can be used to calculate yields for all heavy particles that have spurs which do not interact much as they expand. In practice, this means the tracks of $\mathrm{H}$ and $\mathrm{He}$ at energies above about $100-200 \mathrm{MeV} / \mathrm{n}$. The radiation chemistry of these particles at high energies are thus well known from results with fast electrons. For particles of higher $Z$ and for all particles at lower energy, two problems must be solved: (1) the effects of the overlapping of spurs along the core, and (2) the effects of the overlapping of the core and penumbra. These problems are considered in a paper by Magee and Chatterjee (1980b). The physical energy deposit can be separated into parts assigned to the core and penumbra, respectively. In chapter II-4, the average energy density in a heavy particle track was shown to vary as $r^{-2}$ where $r$ is the radial distance from the trajectory. The separation technique (which has been investigated and which appears promising) is to assign all of the energy that is initially deposited at an energy density greater than a critical value ol to the core. One can see the reason for such a suggestion in a consideration of the competititon of the radicals in the track between recombination and reaction with a scavenger which is in a spatially constant concentration. The rate of the recombination varies as $\mathrm{kc}^{2}$ where $k$ is the rate constant and $c$ the concentration that is initially proportional to the energy density in the deposit; the initial rate of scavenger reaction varies as $k_{s} c_{s} c$, where $k_{s}$ is the rate constant and $c_{S}$ is the scavenger concentration. If the scavenger reaction rate is larger, it is reasonable to assume that the energy deposit is like that of an isolated electron track; if the recombination is larger, the energy deposit is like that of a track core. If the two rates are equal:

$$
k c_{1}=k_{S} c_{S} \text {, }
$$

where $c_{1}$ is the radical concentration for this condition, we see that one would expect the initial energy deposit to be proportional to $\mathrm{k}_{\mathrm{s}} \mathrm{C}_{\mathrm{s}} / \mathrm{k}$ or the same value for all particles and energies.

The use of a critical energy density, $\rho 1$, for the separation of a track into core and penumbra was investigated in a consideration of the Fricke dosimeter system irradiated with heavy particles (Chatterjee and Magee, 1980; Magee and Chatterjee 1980b). The concept appears to be promising and can perhaps be refined. Calculated values of $r_{1}$ (the radial position at which the energy density is initially at the critical value) are shown in Figure 1 as a function of specific energy for six representative particles. The particles selected for study cover the entire heavy particle spectrum: $H, D$, and He have been widely used for many years; $\mathrm{C}$, Ne, and $\mathrm{Ar}$ are the principal particles accelerated in the Bevalac; $\mathrm{Fm}$ has atomic number 100 , and is representative of the very heavy particles. We see in Figure 1 that values of $r_{1}$ merge on the low energy side with the penumbra radius, $r p$, and on the high energy side with the "physical core" radius, $r_{c}$. For energies below the one at which $r_{1}=r_{p}$ for a given particle, the heavy particle track has no penumbra.

We call the core defined by $r_{1}$ the "chemical core" because its size depends upon chemical reaction rate criteria. The concept of core more often used, for example in chapter II-4, depends upon the initial energy deposit only, and such a core can be called a "physical core."

Treatment of the Chemical core

Several considerations of the core expansion have been made, and they all lead to a rather simple picture of a core made up of a string of

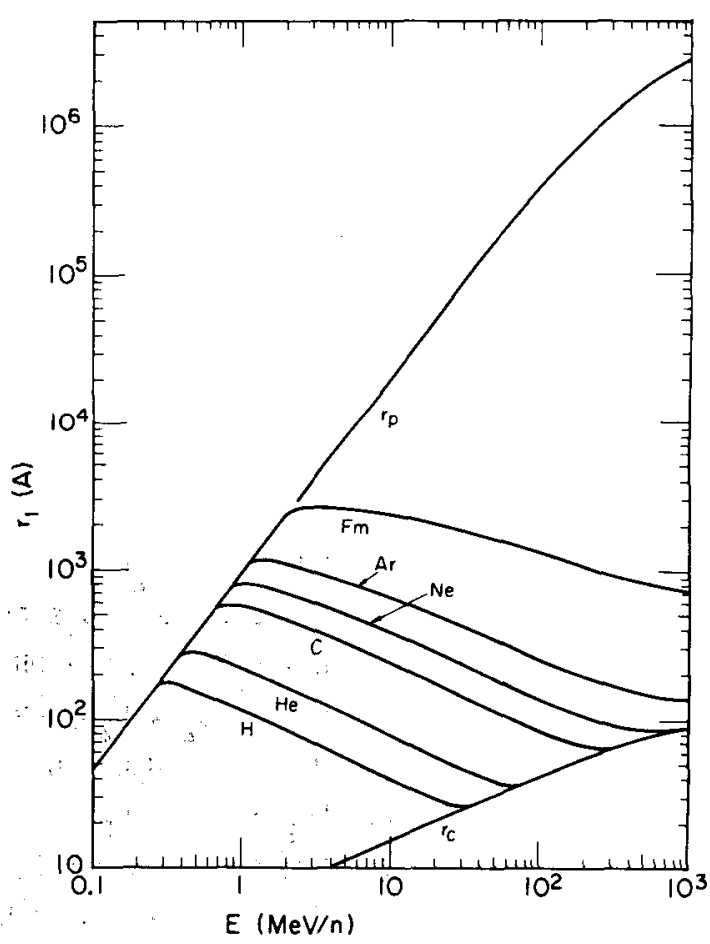

Figure 1. Chemical core radii $r_{1}(E)$ (in $A$ ) for the six selected particles vs. specific energy $E$ (in $\mathrm{MeV} / \mathrm{n}$ ). At low energies, all values of $r 1(E)$ are equal to the penumbra radius, $r_{p}$; at high energies, all values of $r_{1}(E)$ become equal to the physical core radius, $r_{c}$. Of course, $r_{c}$ and $r_{p}$ are common for all particles. (XBL 7910-3807) 
spurs that expand, and react with each other and a scavenger. : The basic treatment was introduced by Ganguly and Magee (1956). The type of differential equation that allows us to calculate the rate of radical reaction in the expanding track (in a one-radical model) is:

$$
\begin{aligned}
\frac{d v}{d t}=- & \frac{2 k v^{2}}{\left[2 \pi\left(r^{2}+4 D t\right)\right]^{3 / 2}} \cdot \\
& {\left[1+\frac{\left[2 \pi\left(r^{2}+4 D t\right)\right]^{1 / 2}}{Z_{1}}\right], }
\end{aligned}
$$

where $v$ is the number of radicals in a spur, $r$ is the initial radius of the spur, $D$ is the diffusion coefficient, $t$ is the time, and $z_{1}$ is the average separation of spurs in the track. Notice that as the expansion occurs, and the second term in the bracket becomes larger than unity:

$$
\frac{d v}{d t}=-\frac{2 n v^{2}}{\left[2\left(r^{2}+4 D t\right)\right] Z_{1}}
$$

Chatterjee and Magee (1980) have shown how to obtain the water decomposition yields using the Fricke dosimeter system and a numerica? technique. of course the $\mathrm{Fe}^{+3}$ yields are obtained through equation 6 . The reactions that occur in this system, along with their reaction rate constants, are shown in Table 1 . A set of coupled equations for the reactions $A$ and $B$ of Table 1 , as they occur in the core, is obtained. The initial conditions are compatible with the initial energy deposit. As time increases, the radicals initially in the physical core expand and engulf the radicals surrounding them, the reactions IA and IB of Table 1 occur. The process continues until the entire core is enguifed (i.e., to the radius $r_{1}$ ), and then the reactions continue (without further engulfing) until the scavenger reaction time $t=\left(k_{s} c_{s}\right)^{-1}$ is reached. The radicals $\mathrm{H}$ and $\mathrm{OH}$, and the molecules $\mathrm{H}_{2}$ and $\mathrm{H}_{2} \mathrm{O}_{2}$, existing at that time, are designated as the primary water decomposition products.

Results of some calculations of the water decomposition in the core are shown in Figure 2. In this approximation the yield depends only on the energy per unit distance assigned to the core (LET core). Calculations for all particles fall on the same curve.

\section{Treatment of the Penumbra}

The yield of the penumbra must be given by an integration over the electron spectrum. Consider the expression:

Table 1. Reactions in the Fricke System

A. Recombination of Primary Radicals
1. $\mathrm{H}+\mathrm{H} \rightarrow \mathrm{H}_{2}$
$1 \times 10^{10}$
2. $\mathrm{H}+\mathrm{OH} \rightarrow \mathrm{H}_{2} \mathrm{O}$
$2.4 \times 10^{10}$
3. $\mathrm{OH}+\mathrm{OH} \rightarrow \mathrm{H}_{2} \mathrm{O}_{2}$
$4 \times 10^{9}$

B. Reactions of Radicals and Product Molecules
4. $\mathrm{H}+\mathrm{H}_{2} \mathrm{O}_{2} \rightarrow \mathrm{H}_{2} \mathrm{O}+\mathrm{OH}$
$1 \times 10^{8}$
5. $\mathrm{OH}+\mathrm{H}_{2} \mathrm{O}_{2} \rightarrow \mathrm{H}_{2} \mathrm{O}+\mathrm{HO}_{2}$
$5 \times 10^{7}$
6. $\mathrm{OH}+\mathrm{H}_{2} \rightarrow \mathrm{H}_{2} \mathrm{O}+\mathrm{H}$
$6 \times 10^{7}$
7. $\mathrm{HO}_{2}+\mathrm{H} \rightarrow \mathrm{H}_{2} \mathrm{O}_{2}$
$1 \times 10^{10}$
8. $\mathrm{HO}_{2}+\mathrm{OH} \rightarrow \mathrm{H}_{2} \mathrm{O}+\mathrm{O}_{2}$
$1 \times 10^{10}$
9. $\mathrm{HO}_{2}+\mathrm{HO}_{2} \rightarrow \mathrm{H}_{2} \mathrm{O}_{2}+\mathrm{O}_{2}$
$2 \times 10^{6}$

C. Scavenger Reactions
10. $\mathrm{H}+\mathrm{O}_{2} \rightarrow \mathrm{HO}_{2}$
11. $\mathrm{HO}_{2}+\mathrm{Fe}^{+2} \rightarrow \mathrm{HO}_{2}^{-}+\mathrm{Fe}^{+3}$
$1 \times 10^{10}$
12. $\mathrm{OH}^{2}+\mathrm{Fe}^{+2} \rightarrow \mathrm{OH}^{-}+\mathrm{Fe}^{+3}$
$2 \times 10^{6}$
13. $\mathrm{H}_{2} \mathrm{O}_{2}+\mathrm{Fe}^{+2} \rightarrow \mathrm{OH}^{-}+\mathrm{OH}+\mathrm{Fe}^{+3}$
$3 \times 10^{8}$
56 


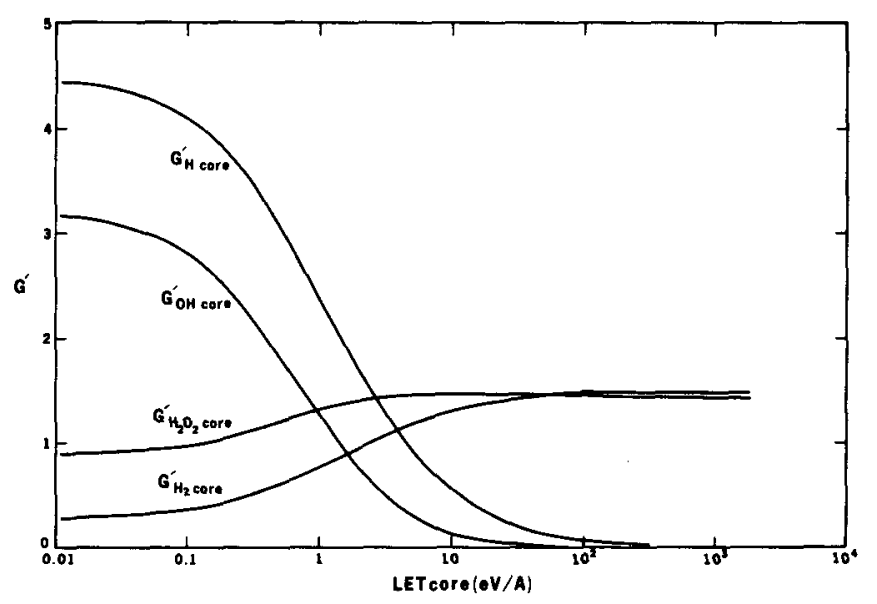

Figure 2. Differential yields of water decomposition products in the chemical cores of heavy particles vs. LET core

(XBL 805-9561)

$$
G_{p e n}^{\prime}(E)=\frac{\int_{\varepsilon_{1}}^{\varepsilon_{\max }} G_{\mathrm{e}}\left(\varepsilon^{\prime}\right) e(E, \varepsilon) d \varepsilon}{\int_{\varepsilon_{1}}^{\varepsilon_{\max }} w(\varepsilon, \varepsilon) d \varepsilon},
$$

where $\varepsilon_{1}$ and $\varepsilon_{\max }$ are appropriate 1 imits, and $w$ is the function for the ordinary knock-on electron spectrum. All of the knock-on electrons originate on the track axis and go into the chemical core region which is bounded by $r_{1}$ (see Fig. 1), the position of the critical energy density $\rho_{1} ; \varepsilon_{1}$ is the minimum energy that allows knock-on electrons to reach $r_{1}$, and must be taken as the lower limit. The penetration of electrons in the penumbra is a statistical problem which has been considered by Chatterjee and Tobias (1980), who found that the penumbra radius is essentially proportional to $\varepsilon_{\max }$. This result means that the average:penetration is proportional to the energy. Thus, we:take:

$$
\varepsilon^{\prime}=\varepsilon-\varepsilon_{1} \text {, }
$$

as the appropriate argument in the electron yield expression, i.e., $G_{e}\left(\varepsilon^{\prime}\right)$ because electrons which go beyond $r_{1}$ leave this amount of energy $\ldots$ in the penumbra.

This calculation has been carried out for water decomposition, and $G_{\text {pen }}^{\prime}(E)$ values are. shown in Figure 3 for $\mathrm{OH}$ and $\mathrm{H}_{2} \mathrm{O}_{2}$ production. The penumbra contributions tend to be determined by $E$, the energy per nucleon of the particle. There are various lower limits of energy for the existence of a penumbra contribution, and these are indicated by the vertical lines. We see from Figure $1, r_{1}=r_{p}$ at small energies for all particles.

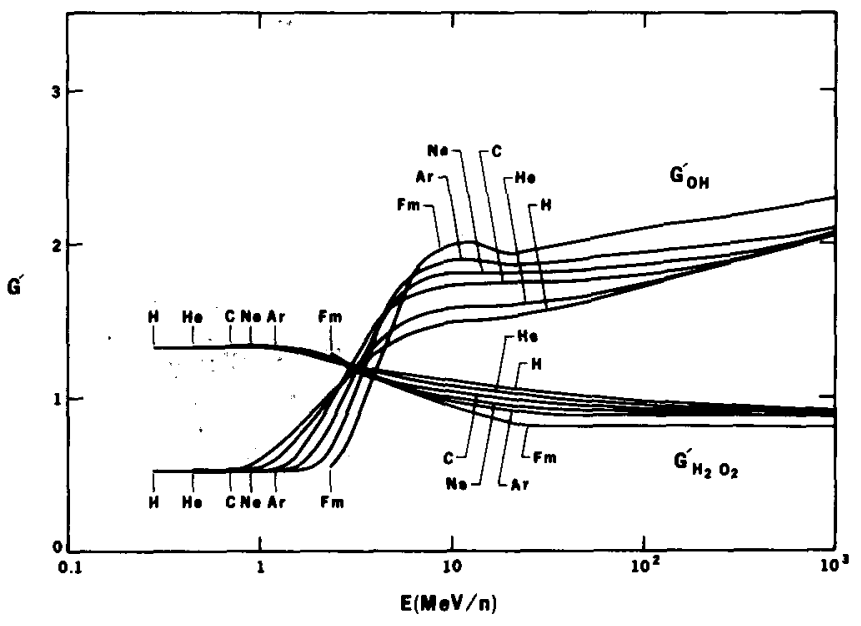

Figure 3. Differential yields of the water decomposition products $\mathrm{OH}$ and $\mathrm{H}_{2} \mathrm{O}_{2}$ in the penumbras of the six representative heavy particles vs. specific energy $E$.

(XBL 805-9673)

\section{Heavy-Particle Track Model}

The differential yield of a heavy particle is given by the equation:

$$
\begin{aligned}
G^{\prime}(E)= & \left.F_{\text {core }} G^{\prime} \text { core }^{(L E T} \text { core }\right)+ \\
& \left(1-F_{\text {core }}\right) G_{\text {pen }}^{\prime}(E)
\end{aligned}
$$

Any particle, of course, has a value of $Z$; at energy $E$, the particle has an LET and an $F_{\text {core; }}$ $\mathrm{LET}_{\text {core }}=\mathrm{F}_{\text {core }} \mathrm{LET}$.

Differential yields obtained in this way have the least possible sensitivity to calculational procedures. The contributions of the core and penumbra are determined individually in the most reliable manner possible. If the total yield $[G(E)]$ is desired, it is obtained by an integration, using equation 9 .

The track differential yields of the water decomposition products $\mathrm{OH}$ and $\mathrm{H}_{2} \mathrm{O}_{2}$ are shown in Figure 4 . We use equation 6 to obtain yields of the Fricke dosimeter, and these are shown in Figure 5 Several sets of experimental points are plotted for comparison. In the low energy region for the lightest particles $H$ and $H e$, for which most data are available, there is so much scatter in the experimental points that it is difficult to assess the agreement. Another difficulty prevents a satisfactory comparison at the high energies for $C$, Ne and Ar. Fragmentation of these ions occurs under the experimental conditions (see chapter II-1 and Chatterjee et al., 1976), and we have not actually made a calculation for the particles of the experiments. Analysis of the fragmentation will be made in the future, and then a meaningful comparison will be possible. 


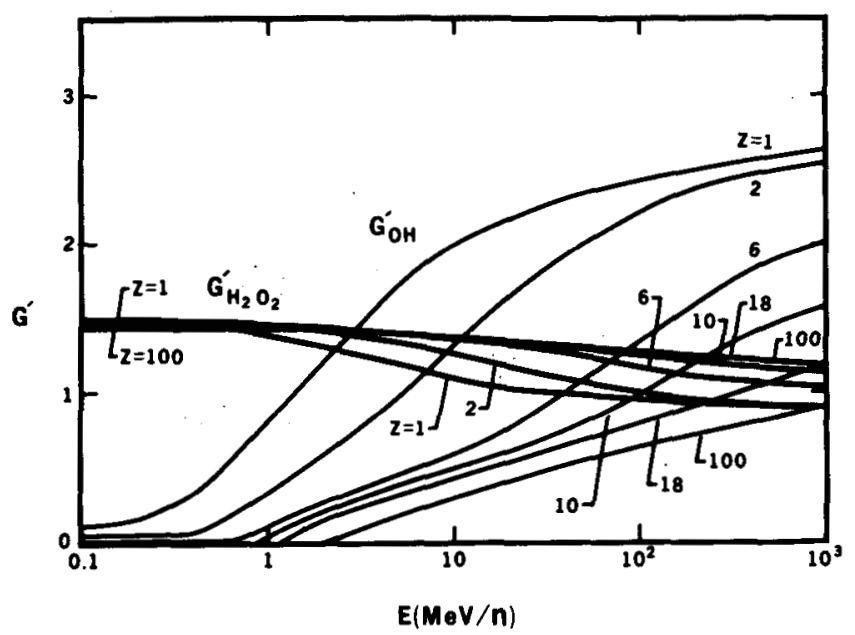

Figure 4. Differential yields of the water decomposition products $\mathrm{OH}$ and $\mathrm{H}_{2} \mathrm{O}_{2}$ in the tracks of the six representative heavy particles vs. specific energy $E$.

(CBB 807-8444)

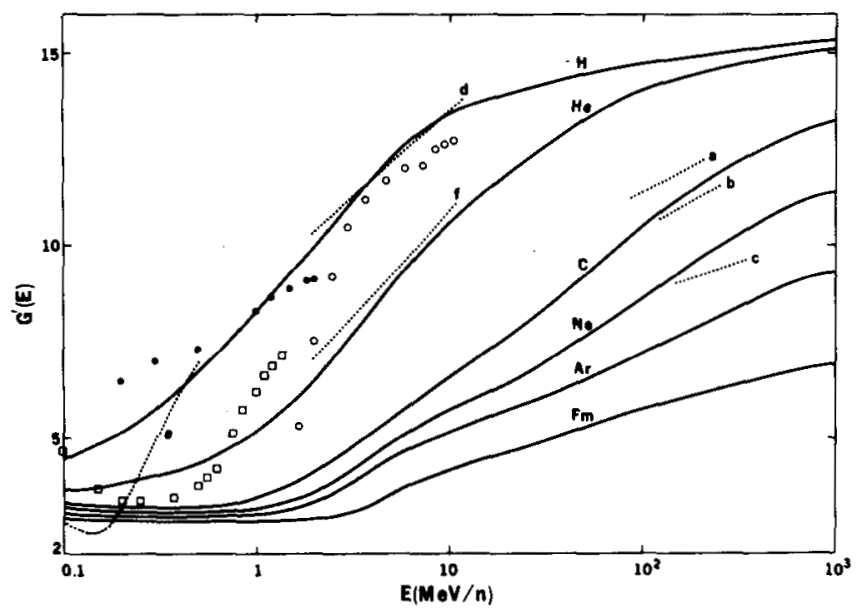

Figure 5. Calculated differential yields $G^{\prime}(E)$, for the six representative particles vs. specific energy $E$, are given by the solid curves.

Estimates of values for $\mathrm{C}$, Ne, and Ar from direct experimental measurements by Jayko et al. (1978) are indicated by the dashed lines $a, b$, and $c$, respectively. In the low energy region (below $10 \mathrm{MeV} / \mathrm{n})$, estimates have been made from analys is of integral G-values by various authors. for $H$ : - Hart et al. (1956); dashed curve e, Pucheault and Julien (1972). For D: o, Hart et al., (1956); dashed curve d, Schuler and Allen (1957); For He: $\square$, Gordon and Hart (1961); dashed curve $f$, Schuler and Allen (1957).

(XBL 803-8508)

\section{DISCUSSION}

The radiation chemistry of dilute aqueous solutions has been considered for heavy particles with representative $Z$ values from 1 to 100 over the range of energies from 0.1 to $100 \mathrm{MeV} / \mathrm{n}$. The possibility exists that other effects will be discovered, but it is likely that most of the significant phenomena are already taken into account, and that Figures 4 and 5 give a good overall view of the yields of heavy particles in aqueous solutions. Several parameters are involved in the calculations, and thus there is room for adjustment of the model. Also, the yields depend on scavenger concentration. We plan to improve the model by theoretical studies of its dependences on the various parameters and also by experimental studies of additional chemical systems. A particularly interesting system for experimental investigation is a modified Fricke dosimeter (Gupta et a1., 1978) which has large $G$ values for low-LET radiations (up to 85 or so). This system will allow us to investigate the criterion for separation of core and penumbra and, in fact, will help us find the limits in which such a separation is valid.

All of these considerations apply to tracks that react completely with scavengers before they react with neighboring tracks. This is always the situation in any system at low rates of irradiation. Thus, the track model set forth nere should be directly applicable to any aqueous system under a low-dose rate.

The principal objective of this track study is the development of a model track which will be useful in interpreting the results of heavyparticle radiation biology. Efforts to use the ideas generated in the study have been made over the past three years and will continue. We visualize the problem as that of merging the purely chemical effects of the radiation with the biological effects that occur on a longer time scale. The initial chemical effects in a biological system are, of course, the same as would be expected in a nonliving system, and then the strictly biological reactions (enzyme catalyzed) take over. We have considered the problem of cell survival and tried to determine how far one can go in explaining observed survival curves if one assumes that the process which causes cell death is a radical-radical reaction. As usual, the results are mixed with some indications that the mechanism is reasonable, and with others that it is significantly incomplete. A preliminary report entitled "A radical diffusion model of cell survival" was presented at the Sixth International Congress of Radiation Research (Magee, 1979), and the work is continuing.

\section{REFERENCES}

Chatterjee, A. and J. L. Magee. 1978. Relationship of the track structure of heavy particles to the physical distribution and chemical effects of radicals. Sixth Symposium on Microdosimetry ( $\mathrm{J}$. Booz and H. G. Ebert, eds.) vol. I, pp. 283-294. 
Chatterjee, A. and J. L. Magee. 1980. Radiation chemistry of heavy particle tracks. II. The Fricke dosimeter system. J. Phys. Chem., in press.

Chatterjee, A. and C. A. Tobias. 1980. Analytical expression for radial distribution of energy deposited by a penetrating heavy particle. Radiat. Evironm. Biophys., in press.

Chatterjee, A., C. A. Tobias, and J. T. Lyman. 1976. Nuclear fragmentation in therapeutic and diagnostic studies with heavy ions. Spallation Nuclear Reactions and Their Applications (Shen and Merke, eds.) pp. 169-191. D. Reidel Publishing Co., Dordrecht, Holl and.

Ganguly, A. K. and J. L. Magee. 1956. Theory of radiation chemistry. III. Radical reaction mechanism in the tracks of ionizing radiations. J. Chem. Phys. 25, 129-134.

Gordon, S. and E. J. Hart. 1961. Chemical yields of ionizing radiations in aqueous solutions: Effect of energy of alpha particles. Radiat. Res. 15, 440-451.

Gupta, B. L., R. M. Bhat, K. R. Gomathy, and B. Susheela. 1978. Radiation chemistry of the ferrous sulfate-benzoic acid-xylenol orange system. Radiat. Res. 75, 269-277.

Hart, E. J., W. J. Ramler, and S. R. Rocklin. 1956. Chemical yields of ionizing particles in aqueous solutions; effect of energy of protons and deuterons. Radiat. Res. 4 , 378-393.

Jayko, M., A. Appleby, E. Christman, A. Chatterjee, and J. Magee. 1978. Radiation Chemistry of Particles Accelerated by the Bevalac. Lawrence Berkeley Laborabory Report LBL-7432, pp. 1-22.
Magee, J. L. 1979. A radical diffusion model of cell survival. Sixth International Congress of Radiation Research. Japanese Association for Radiation Research, Tokyo, Japan.

(Abstract).

Magee, J. L. and A. Chatterjee. 1978a. Theory of the chemical effects of high-energy electrons. J. Phys. Chem. 82, 2219-2226.

Magee, J. L. and A. Chatterjee. 1978b. Theory of electron tracks with explicit consideration of track-end effects. Sixth Symposium on Microdosimetry (J. Booz and H. G. Ebert, eds.) vol. I, pp. 157-168.

Magee, J. L. and A. Chatterjee. 1979. Chemical track effects in condensed systems and implications for biological damage. Sixth International Congress of Radiation Research TS. Okada, M. Imamura, T. Terashima, and $H$. Yamaguchi, eds.), pp. 166-173. Japanese Association for Radiation Research, Tokyo, Japan.

Magee, J. L. and A. Chatterjee. 1980a. A spur unfolding model for the radiolys is of water. Radiat. Phys. Chem. 15, 125-132.

Magee, J. L. and A. Chatterjee. 1980b. Radiation chemistry of heavy particle tracks. I. General considerations. J. Phys. Chem., in press.

Pucheault, J. and R. Julien, 1972. Radiolys is by protons: Dose-rate and LET effects. Proceedings of the Third Tihany Symposium on Radiation Chemistry (J. Dobos and P. Hedwig, eds) vol. II, pp. 1191-1199. Akadémiai Kiadó, Budapest.

Schuler, R. H. and A. 0. Allen. 1957. Radiation chemistry studies with cyclotron beams of variable energy: yields in aerated ferrous sulfate solution. J. Am. Chem. Soc. 79, 1565-1572. 
$\odot$

$\ominus$

\section{.

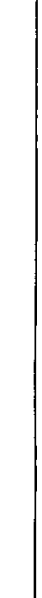

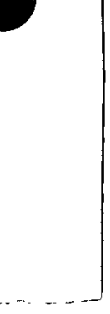

\section{.}

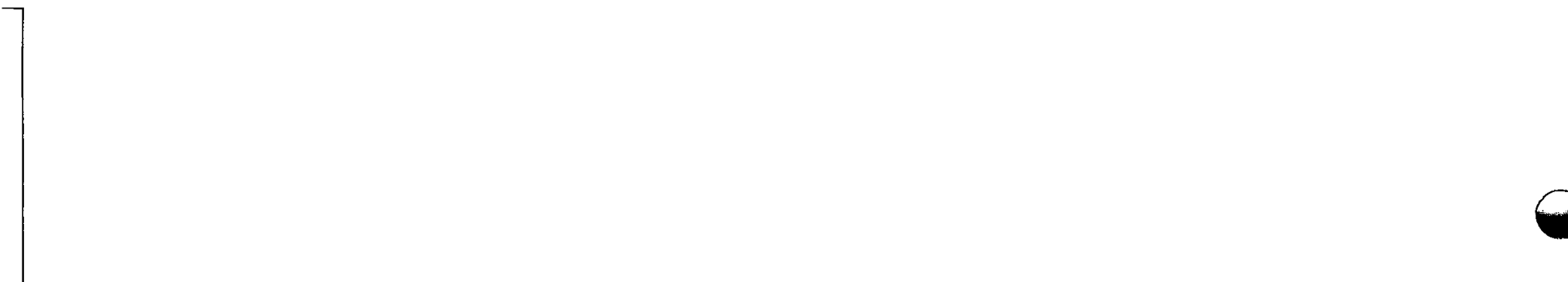


PART III. CELlular

AND MOLECULAR RADIOBIOLOGY OF HEAVY IONS 


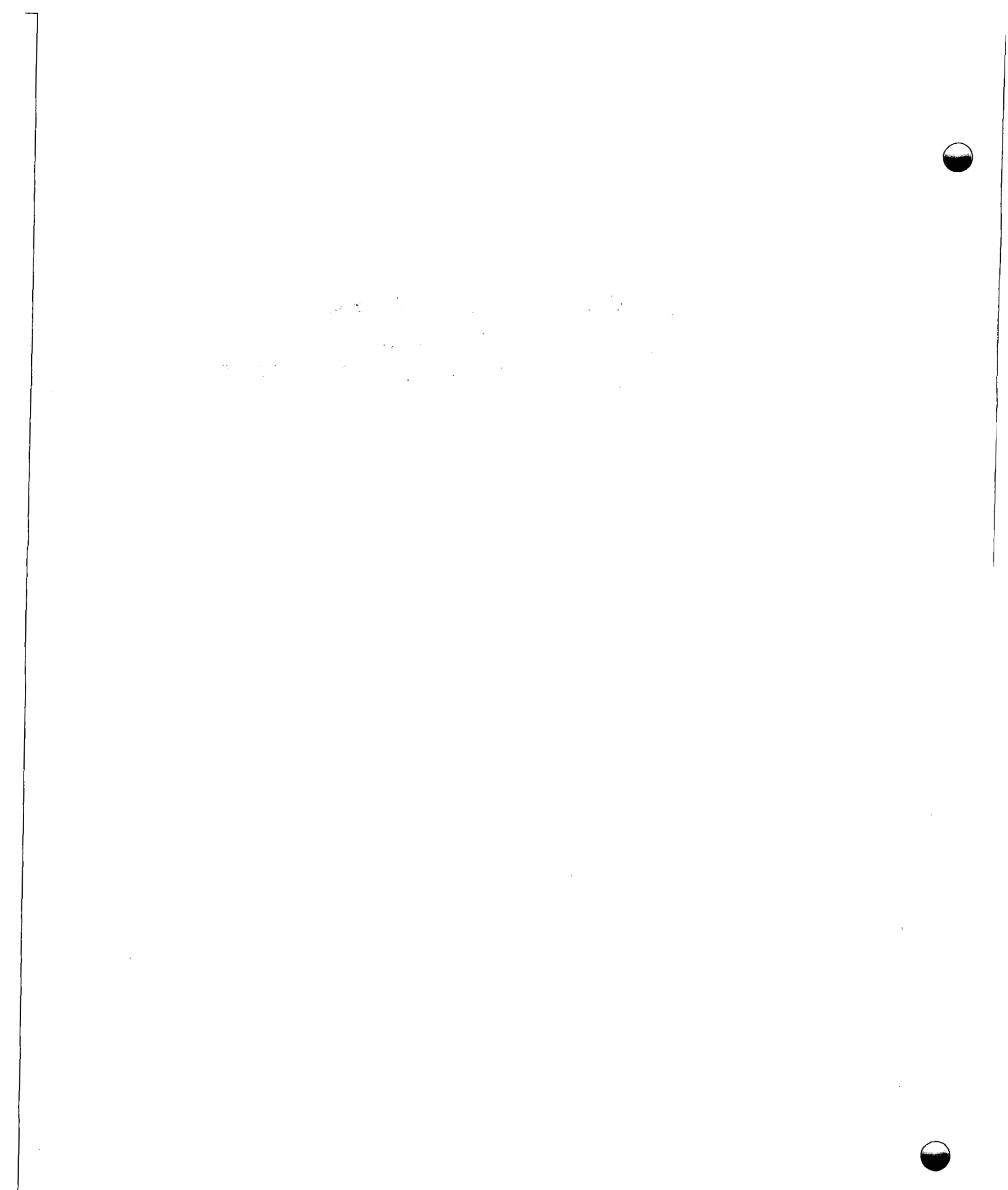




\title{
PHYSICAL AND CELLULAR RADIOBIOLOGICAL PROPERTIES OF HEAVY IONS IN RELATION TO CANCER THERAPY APPLICATIONS
}

\author{
E. A. Blakely, C. A. Tobias, F. Q. H. Ngo, and S. B. Curtis
}

Precise delivery of tumor dose, with concomitant low dose to surrounding tissue, is a primary feature of charged particle therapy. Although normal tissue damage usually limits the treatment dose that can be delivered, comparisons with conventional modalities show that heavy ions can provide effective dose to tumors at depth without exceeding normal tissue tolerance. Any enhanced incremental dose to the tumor should increase the probability of local control. The sparing effect of particles on normal tissues and the comparatively high level of damage to tumors is the result of two factors: first, physical dose can be delivered at depth with a lower entrance dose; and second, the physical dose delivered by stopping particles is biologically more effective because of the specific energy deposition properties of charged particles.

Biologically effective dose distributions are not the only criterion considered in the selection of a therapeutic modality. Reduction of the biological radioresistance of hypoxic cells is considered the other major feature of high-LET radiations. Heavy ions markedly decrease the difference in radiosensitivity between hypoxic and oxic cells. Other radiobiological characteristics of heavy ions that are undergoing study for their possible clinical significance are: the reduction in repair of heavy-ion-induced lesions, the reduction in differences of cell sensitivity in various cell ages during the division cycle, the increased synchronizing effect of heavy ions, and the increased radiosensitivity of hyperploid cells.

The evaluation of relative advantages of various radiation modalities requires quantitative physical and biological data. Major physical aspects of heavy-ion beams are discussed in Parts II and VI of this report). Spec if ic biological aspects of heavy ions are discussed in each chapter of Part III. This chapter deals with a quantitative comparison of biological properties of monoenergetic and extended Bragg peak heavy-ion beams, based on in vitro cellular survival experiments.

\section{RBE AND OER}

Cellular in vitro measurements of RBE and OER values have been made at numerous residual ranges of each of the Bevalac beams as they have become available since 1974 (Blakely et al., 1978, 1979; Chapman et al., 1977, 1978; Curt is et al., 1980; Fu and Phillips, 1976; Gerner and Leith, 1977; Goldstein et al., 1980; Hall et al., 1977; Leith et al., 1975, 1977; Lücke-Hühle et al., 1979; Ngo et al., 1980; Phillips et al., 1977, 1979; Raju et al., 1978; Raju, 1980a,b; Roots et al., 1980; Schilling et al., 1977; Yang et al., 1979, 1980).
The beams studied include: carbon (308-400$474 \mathrm{MeV} / \mathrm{amu}$ ), neon (425-557-670 MeV/amu), silicon (530 and $670 \mathrm{MeV} / \mathrm{amu}$ ) and argon ( $570 \mathrm{MeV} / \mathrm{amu}$ ).

Using a variety of experimental systems, eight or more mammalian cell lines have been used to study beams with monoenergetic narrow $(4 \mathrm{~cm})$, and broad $(10 \mathrm{~cm})$ Bragg peaks. Most of this large body of RBE and OER information from cultured cells has recently been summarized (Raju, 1980a) and shows good agreement despite the technical differences between the various systems. Figures 1-3 describe the principal observations made with respect to the aerobic and hypoxic biological response of a single cell line (human kidney $\mathrm{T}-1$ ) to irradiations at various ranges (indicated in Figures $4 A$ and $4 B$ ) of monoenergetic and Bragg peak extended beams. Representative survival curves from which the OER and RBE values were extracted are shown in Figure 5 .

The left panel of Figure 1 depicts carbon $400 \mathrm{MeV} / \mathrm{amu}$ monoenergetic data. In this beam the OER remains high and the RBE low over the greatest part of the beam's range. However, within the last two centimeters of range the values quickly change, with the hypoxic RBE at $10 \%$ survival reaching a maximum of $3.9 \pm 1.0$ and the OER dropping to a low of $1.6 \pm 0.3$ at the closest range studied upstream of the Bragg peak.

The middle panel demonstrates what happens when the Bragg peak of the $400 \mathrm{MeV} / \mathrm{amu}$ carbon beam is spread to $10 \mathrm{~cm}$ with a spiral ridge filter (SRF). The stopping of additional particles over the last $10 \mathrm{~cm}$ upstream of the peak results in a reduction of the oxygen effect and an increasse of the RBE over a broader region of the range, especially the last several centimeters. Notice that the physical dose plotted at the top of this panel is deliberately sloped to give less dose in the distal peak, where the particles are most effective. An evaluation of the success of two filter designs for isoeffectiveness at the $50 \%$ survival level for this cell line will be made later in the chapter. The RBE and OER data from the $10 \%$ survival level are presented here for illustrative purposes.

The results from the $308 \mathrm{MeV} / \mathrm{amu}$ carbon beam with a $4 \mathrm{~cm}$ peak are presented in the right panel of Figure 1. The RBE is somewhat higher and the OER lower over this shorter extended peak than that measured for the $400 \mathrm{MeV} / \mathrm{amu}$ beam with the longer peak. This trend is common to most of the beams. The lower the initial beam energy, the higher the RBE and lower the OER over the full width of the extended peak. However, in most cases the RBE was lower and OER higher in the proximal peak than in the distal peak of each beam. 
CARBON

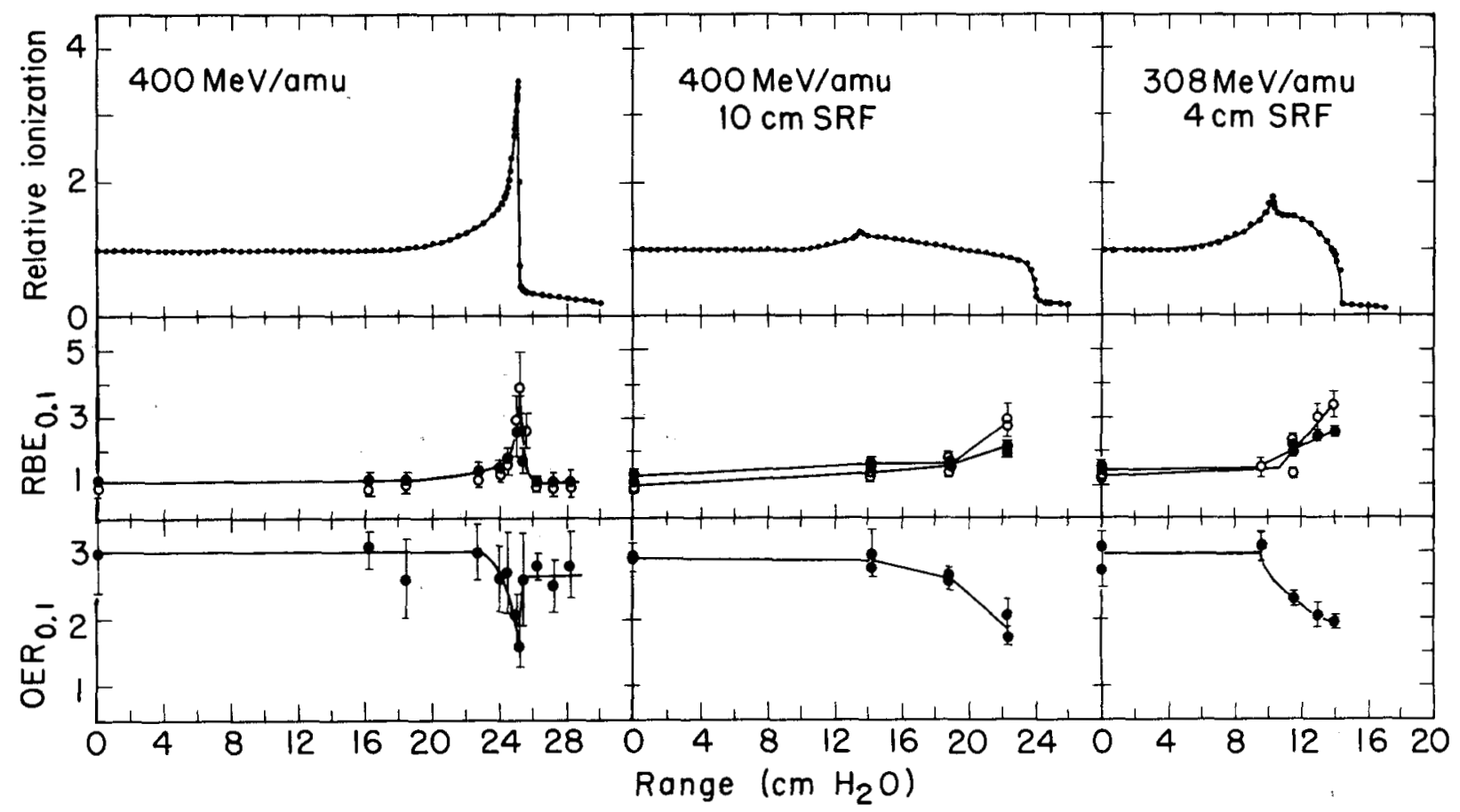

Figure 1. Physical Bragg ionization curves and aerobic (solid symbols) and hypoxic (open symboTs) RBE-10 and OER-10 measurements as a function of range for monoenergetic and 10-cm extended Bragg peak $400 \mathrm{MeV} / \mathrm{amu}$ carbon beams, and a 4-cm extended Bragg peak $308 \mathrm{MeV} / \mathrm{amu} \mathrm{Carbon}$ beam. Biological data are based on human kidney T-1 survival in vitro. (XBL 808-3632)

\section{NEON}

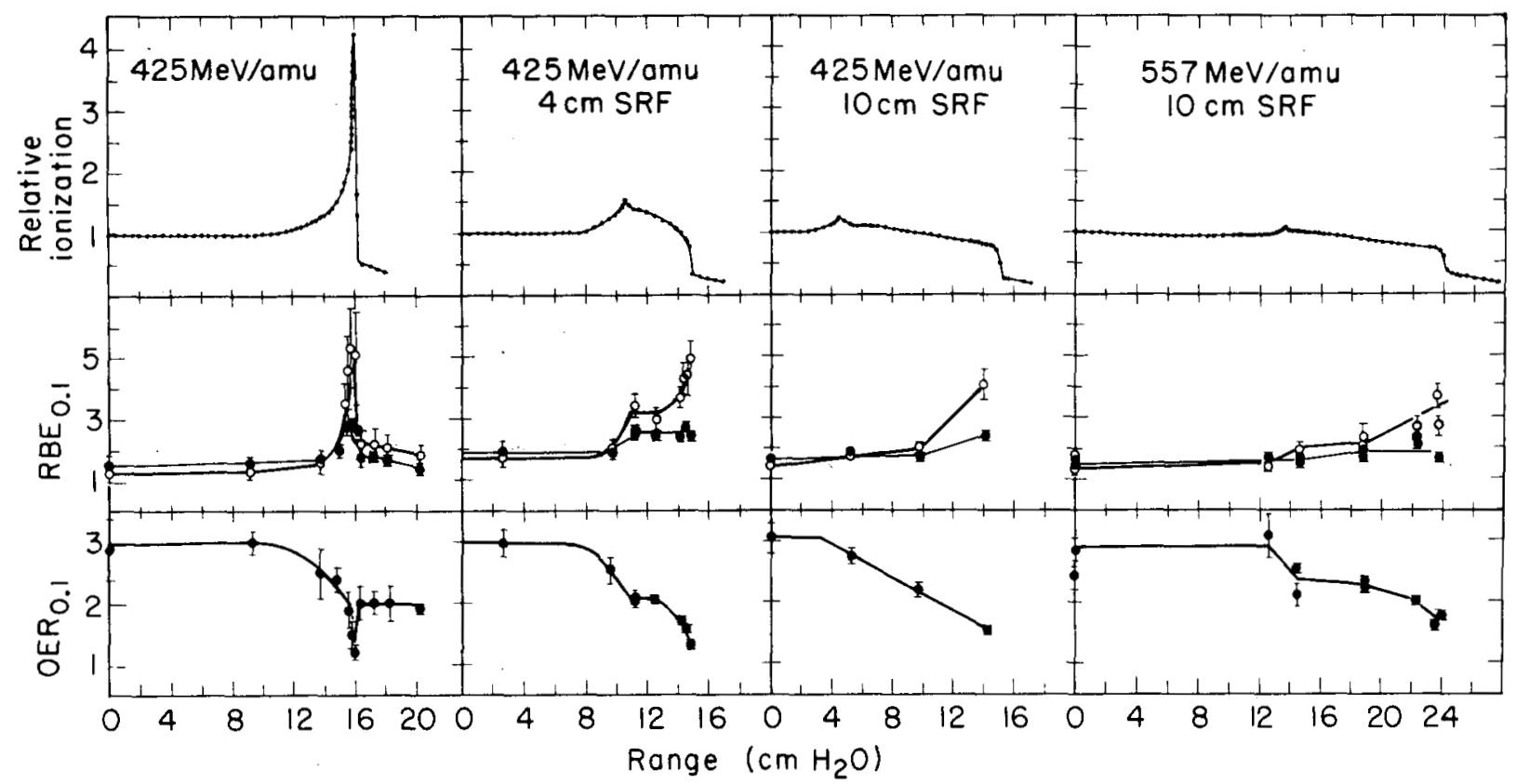

Figure 2. Physical Bragg ionization curves and aerobic (solid symbols) and hypoxic (open symbols) RBE-10 and OER-10 measurements as a function of range for mononenerget ic and 4-cm and 10-cm extended Bragg peak $425 \mathrm{MeV} / \mathrm{amu}$ neon beams, and a 10-cm extended Bragg peak $557 \mathrm{MeV} / \mathrm{amu}$ neon beam. Biological data are based on human kidney T-1 survival in vitro. (XBL 808-3631) 


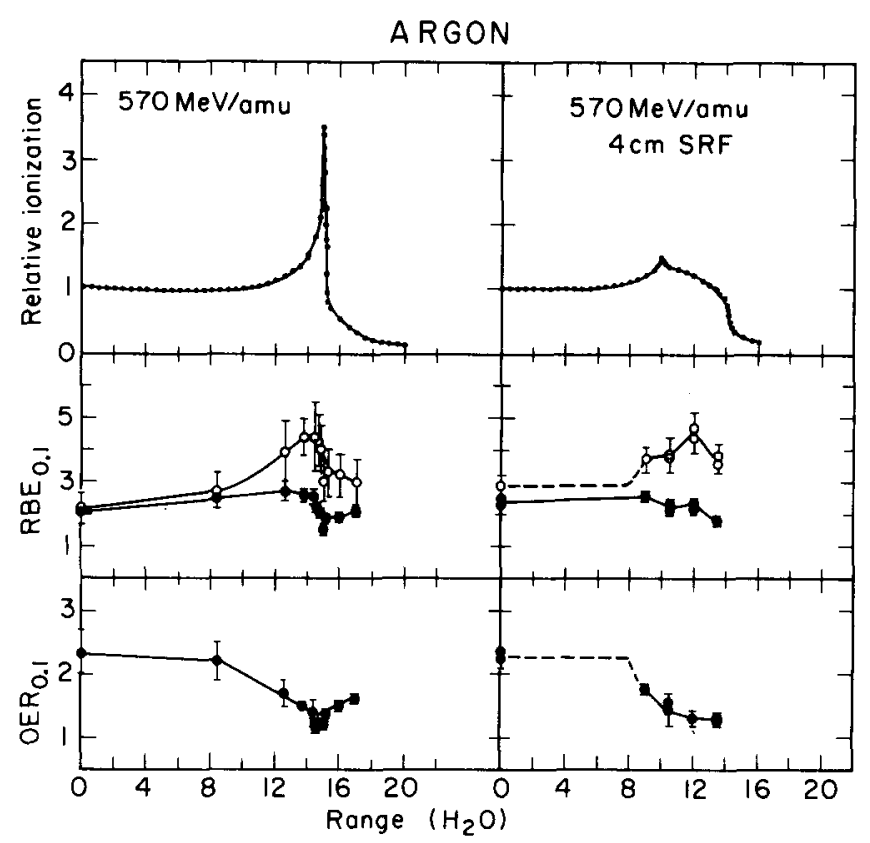

Figure 3. Physical Bragg ionization curves and aerobic (solid symbols) and hypoxic (open symbols) RBE-10 and OER-10 measurements as a function of range for a monoenergetic and a $4-\mathrm{cm}$ extended Bragg peak $570 \mathrm{MeV} / \mathrm{amu}$ argon beam. Biological data are based on human kidney $T-1$ survival in vitro. (XBL 808-3630)

The neon data are summarized in Figure 2. The $425 \mathrm{MeV} / \mathrm{amu}$ monoenergetic data in the extreme left panel are similar to the carbon data, except that the RBE and OER values change earlier upstream of the neon peak and attain a maximum hypoxic RBE of $5.3 \pm 1.3$ and a minimum OER of $1.2 \bullet 0.2$.

The data for the $425 \mathrm{MeV} / \mathrm{amu}$ beam with the 4-cm filter show that the OER is less than 2.0, and that the hypoxic RBE is greater than 3.0.over most of the peak region. At a $10 \%$ survival level these effects are less pronounced for the . $425 \mathrm{MeV} / \mathrm{amu}$ and $557 \mathrm{MeV} / \mathrm{amu}$ beams with the $10-\mathrm{cm}$ peak, as shown in the two panels on the right side of Figure 2. The RBE vialues are somewhat flattened, but the OER values are still. suppressed.

Figure 3 illustrates the monoenergetic and 4-cm extended peak RBE and OER data as a function: of range for argon beams with an initial energy. of $570 \mathrm{MeV} / \mathrm{amu}$. The monoenergetic argon data is: unique in that the hypoxic and aerobic RBE values. are maximized in a broad peak of several cent $i-y_{1}$ meters that lies upstream of the physical Bragg peak. The hypoxic RBE value of $10 \%$ survival drops significantly from $4.4 \pm 0.6$ at $1.2 \mathrm{~cm}$ upstream of the Bragg peak, to $3.0 \pm 0.6$ at the closest peak position. The aerobic RBE value drops from $2.6 \pm 0.2$ to $1.5 \pm 0.2$ over the same range.

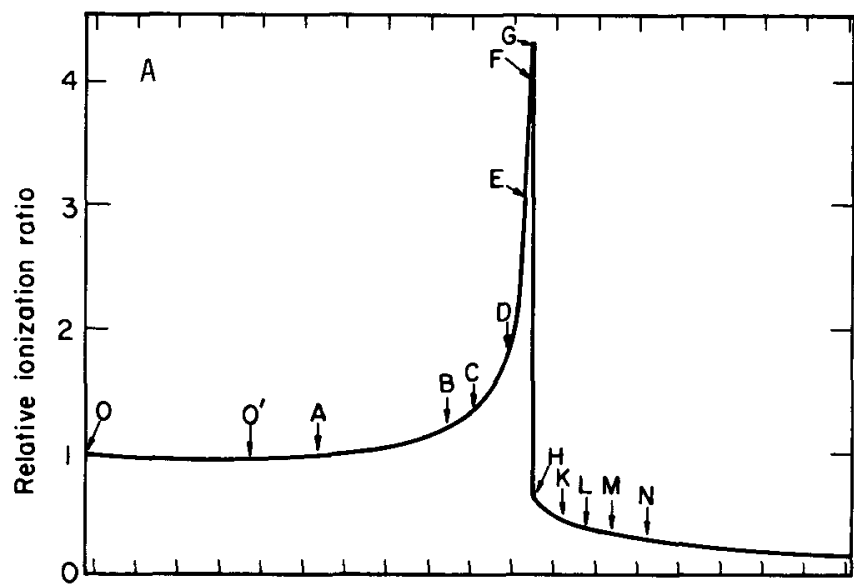

$\mathrm{cm}$ of water

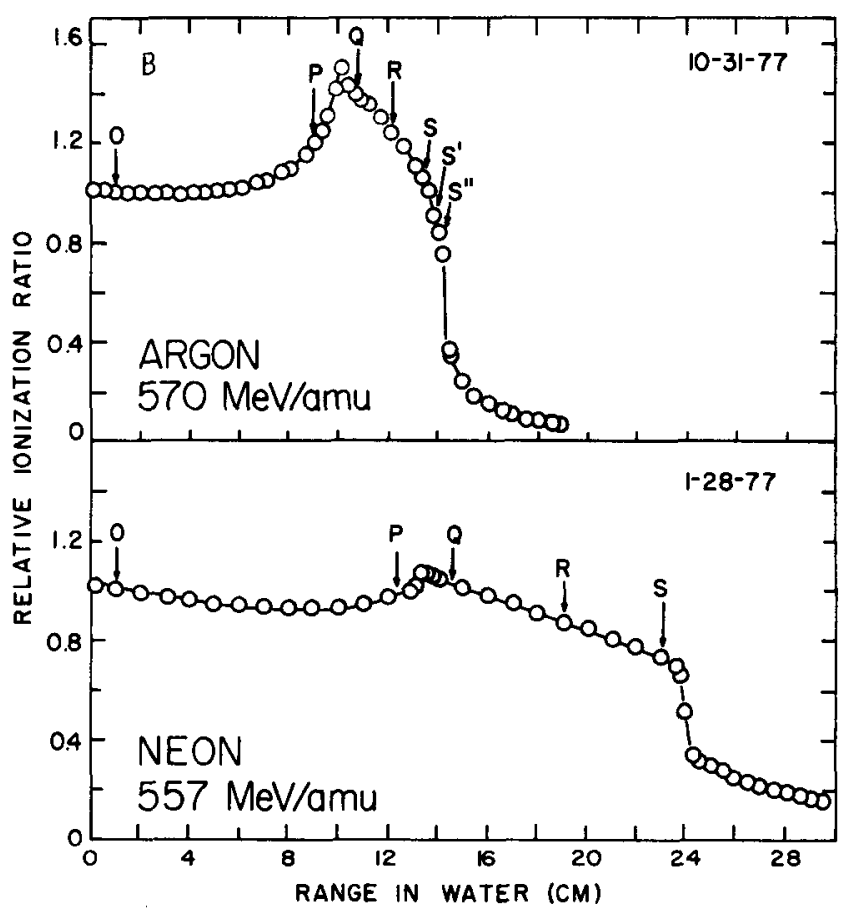

Figure 4.: Beam ranges studied with respect to the monoenerget ic Bragg peak (upper pane1) and the 4-cm: and $10-\mathrm{cm}$ extended Bragg peaks (lower panels). [(A) XBL 777-3591A; (B) XBL 785-8601]

The 4-cm extended-peak RBE and OER values plotted in the right hand panel of Figure 3 show a rather flat aerobic RBE response, with the greatest effect being the high flat hypoxic peak RBE. values which are a result of the very low OER $(<1.4)$ across the entire filter. RBE characteristics as a function of range are the major consideration in the design of ridged filters. The unique LET spectra and primary beam fragmentation properties of argon are quite different from carbon and neon beams of simitar range which have been studied. 


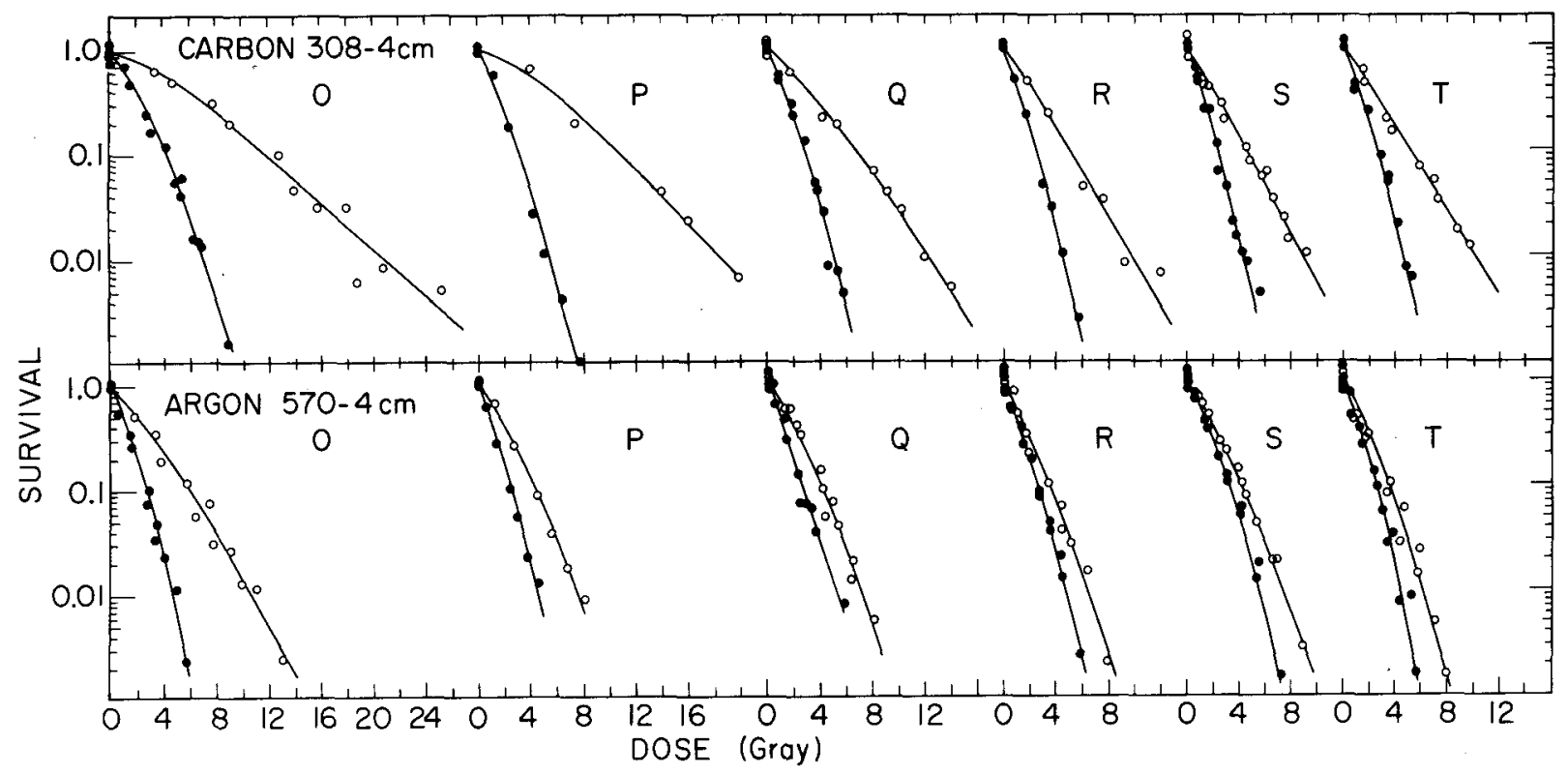

Figure 5. Aerobic (0) and hypoxic (0) survival curves measured at ranges designated in Figure 4 of a $308 \mathrm{MeV} / \mathrm{amu}$ carbon and a $570 \mathrm{MeV} / \mathrm{amu}$ argon beam. The Bragg peaks had been extended to $4 \mathrm{~cm}$. Survival was measured in vitro with human kidney T-1 cells. (XBL 809-3682)

The results described above led to the proposal to accelerate an ion on the periodic table between neon and argon. It appeared that there might be an advantage in using an intermediate ion which would still have an OER advantage like argon, but would generally produce less fragmentation and less overkill effect than the argon beam, and, therefore, give an effective dose distribution at depth that would be more like neon. The preliminary results of our silicon studies are presented in Part III of this report and indicate substantial support for this hypothesis.

\section{RANGE FILTERS AND EXTENOED REGIONS OF ISOEFFECT}

The objective of range filter design is to extend the effective Bragg peak region, by accumulating stopping particles over the broader dimensions required in radiotherapy, to give a region of isoeffective cell killing. There are several parameters to consider in this task, including the beam characteristics of energy deposition and fragmentation, the model for cell inactivation which is used to predict the low dose response in the mixed LET radiation fields, the specific available cell line sensitivities selected for the modeling and their RBE-LET dependence, and the dose level desired for the isoeffect.

Most of the range filters presently in use are ridge filters that were designed by John Lyman based on physical beam parameters and available biological data. As cellular information accumulated with the initial filter designs, the information was used to design better filters. A representative biological doseresponse profile was developed, and several filters of a newer spiral design were tooled to extend Bragg peaks to a width of 4 or $10 \mathrm{~cm}$. In some cases different particles require different filters in order to achieve isoeffective killing across the extended peak. In other cases, the physical and biological properties of beams appear to be similar enough to allow use of the same filter for isoeffectiveness (see below).

In order to demonstrate the isoeffectiveness of the available filters using a single cell line (T-1), the repair-misrepair (RMR) model for cellular inactivation (Tobias et al., 1980) was used to computer fit heavy-ion survival data by least-squares regression, and to calculate aerobic RBE values at the $50 \%$ survival level. The RMR model was selected because it yields a fit to cell survival data that is representative of fits made with other available models. This model also has other characteristics useful for analytical interpretation.

The RBE-50 values for the various ranges studied were multiplied by the measured physical dose at each range studied. The resultant normalized biologically effective dose (BED) has been plotted over each of five Bragg curves of physical dose in Figure 6 . The same $4-\mathrm{cm}$ and 10-cm SRF was used for each beam studied. The data for the $4-\mathrm{cm}$ carbon and neon beams show fairly good success in attaining uniformity of aerobic cell killing across the peak. However, the corresponding OER values plotted below each Bragg curve, and the corresponding hypoxic biologically effective dose values (not snown), revealed that it is not possible to design 


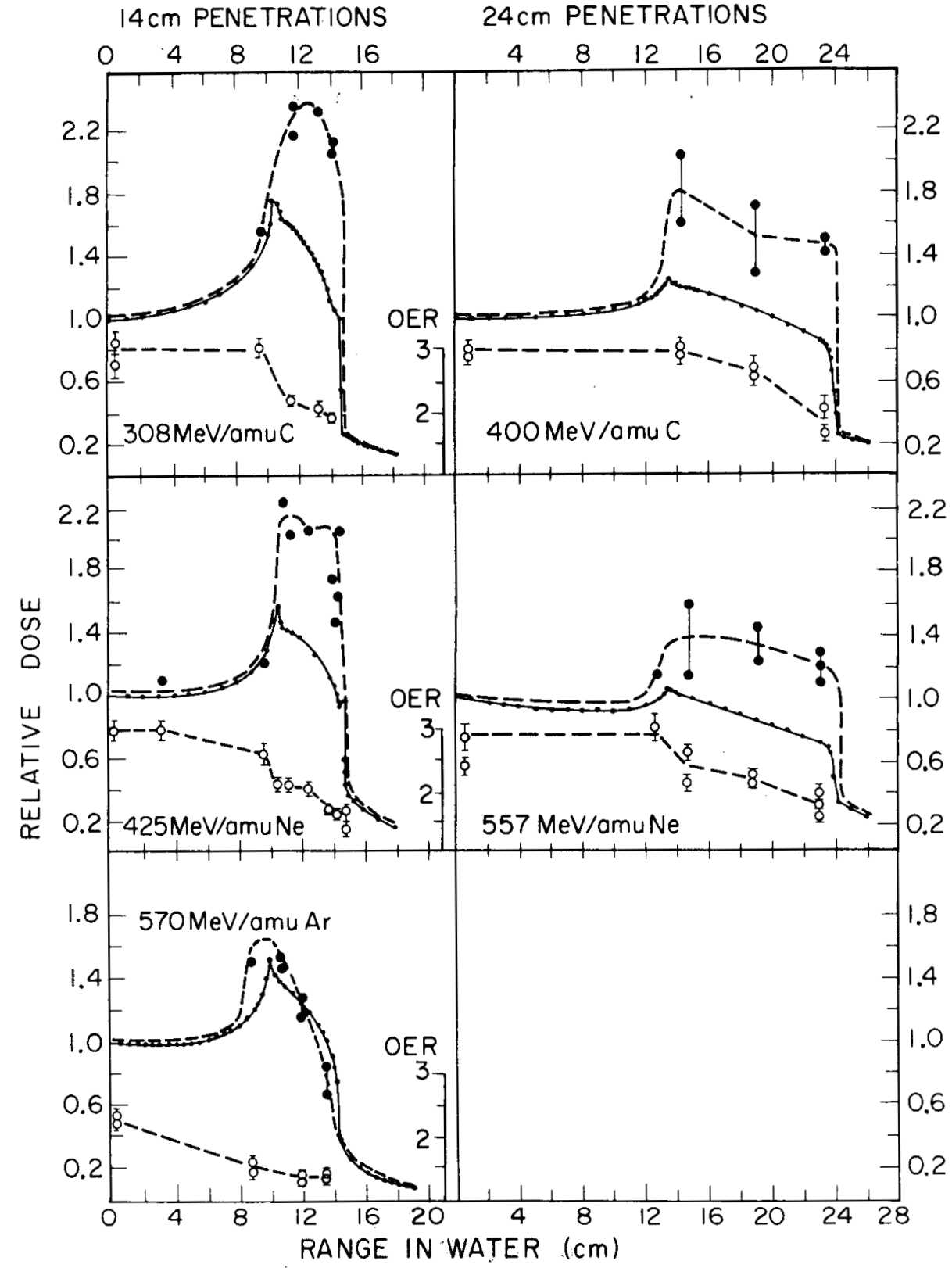

Figure 6. Physical Bragg ionization curves $(\bullet)$, and biologically effective dose $(0)$ and 0 ER $(0)$ at $50 \%$ survival as a function of range for heavy-ion beams with $4-\mathrm{cm}$ extended Bragg peaks at $14-\mathrm{cm}$ range penetration, and with $10-\mathrm{cm}$ extended Bragg peaks at $24-\mathrm{cm}$ range penetration.

(XBL 809-3681)

filters to simultaneously achieve isoeffectiveness for both aerobic and hypoxic cells. The carbon peak OER values are around 2.0 and the neon midpeak OER values are similar, but the OER decreases to about 1.6 in the distal neon peak. The $308 \mathrm{MeV} / \mathrm{amu}$ carbon and $425 \mathrm{MeV} / \mathrm{amu}$ neon beams with 4-cm extended peaks are, in general, very much alike in terms of the measured parameters at this range for this cell line.
The biologically effective and physical dose plots for the longer-ranged $400 \mathrm{MeV} / \mathrm{amu}$ carbon beam with the 10-cm SRF are depicted in the upper right hand panel of Figure 6 . There is quite a bit of scatter in the replicate estimates of RBE-50 in the proximal and midpeak regions, and less scatter in the distal position. However, the filter design of physical dose appears to slightly overcompensate for effective dose in the 
distal peak. More physical dose in the distal end of the peak is needed for isoeffectiveness across the full range of the peak. The OER value for this long range carbon beam is rather high, averaging about 2.5 to 2.6 over the $10 \mathrm{~cm}$ width, but ranging from $2.8 \pm 0.2$ in the proximal peak to $1.9 \pm 0.2$ in the distal peak.

The biologically effective and physical doses for the $557 \mathrm{MeV} /$ amu neon beam with the $10 \mathrm{~cm}$ SRF are plotted in the lower right hand panel of Figure 6 . Notice that data from two replicate monolayer experiments show proximal and midpeak scatter for neon too; however, the isoeffect is somewhat flatter across the $10 \mathrm{~cm}$ of the extended peak. The OER values across the peak of this beam average about 2.1 to 2.3 , and range from $2.3 \pm 0.2$ in the proximal peak to $1.6 \pm 0.1$ in the distal peak.

The final panel in the lower left of Figure 6 presents the $570 \mathrm{MeV} / \mathrm{amu}$ argon OER values and physical and biologically effective doses as a function of range. This beam is different from the others because it shows that for the 4-cm filter design the biologically effective dose is quite similar to the physical dose, except that it is slightly less effective in the distal end of the peak. However, the normalized peak to plateau dose ratio is still quite advantageous $(\sim 1.5)$ in a narrower region straddling the physical proximal peak. This beam is also quite unique because of its extremely low OER, which averages about 1.4 across the entire width of the peak, including the preproximal and distal regions.

The 4-cm filter design appears to be adequate for the carbon and neon beams, but it is not optimal for the argon beam. The BED distribution can be optimized for the argon beam by using a SRF design with a much less sloped physical dose. The 10-cm filter design appears to slightly overcompensate for biological killing in the distal peak of the $400 \mathrm{MeV} / \mathrm{amu}$ carbon and $557 \mathrm{MeV} / \mathrm{amu}$ neon beams.

\section{COMPARISON OF CELL LINE SENSITIVITIES}

The observations described in the above discussion are made from data on a single cultured cell line. In order to address the question of specific variations in heavy-ion radiosensitivity between different cell lines, the aerobic RBE results for eight cell lines at $10 \%$ survival have been summarized in Figure 7 as a function of mean $\mathrm{LET}_{\infty}$. Results from both high energy monoenergetic and ridge-filtered heavy-ion beams are given. Data from several groups of experimenters are included in the figure.

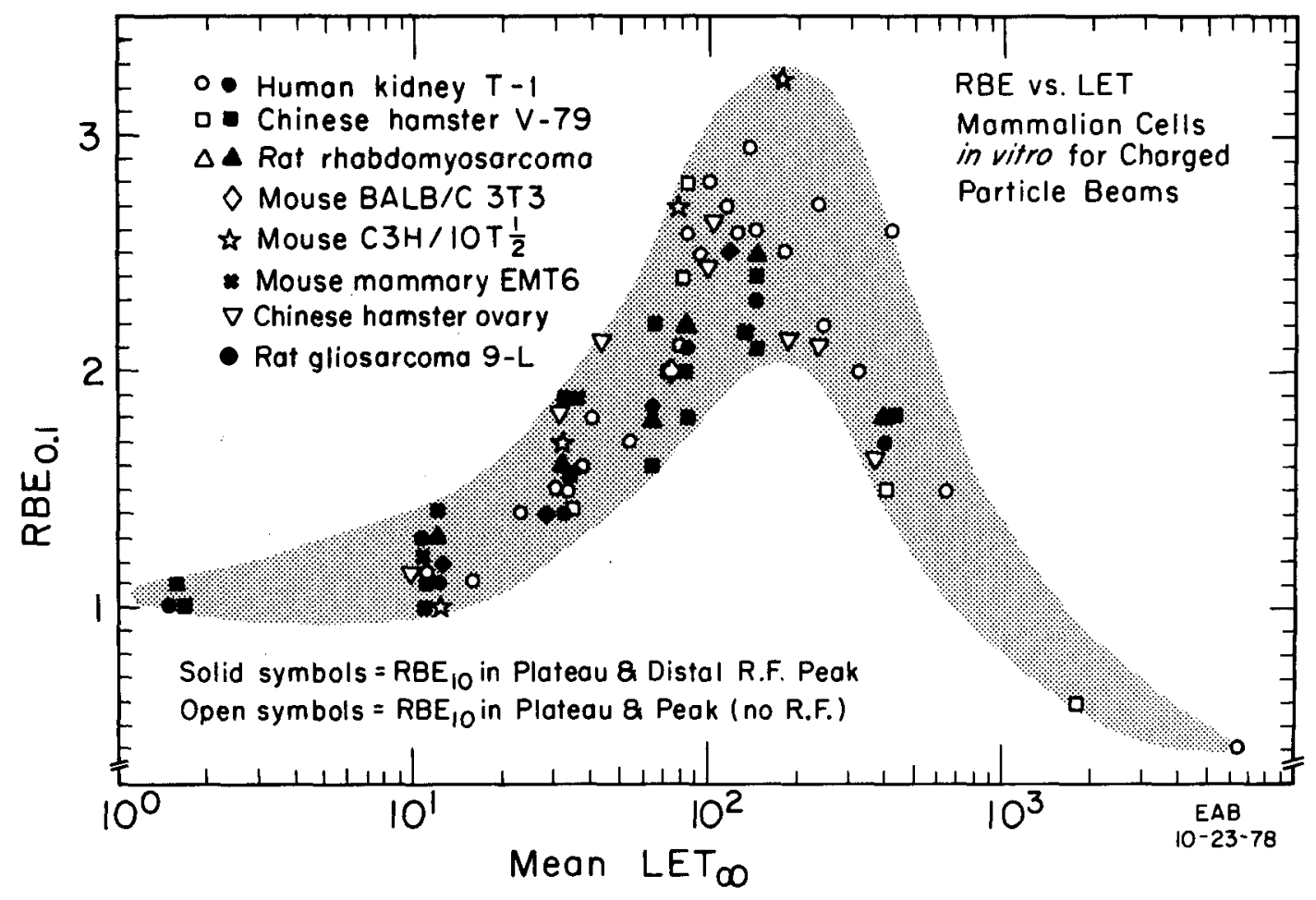

Figure 7. Aerobic RBE-10 vs: mean LET for monoenergetic and extended Bragg peak data using mammalian cell survival data measured in vitro. The results are compiled from nine different cell systems (Blakely et al., 1978, 1979; Lücke-Hühle et al., 1979; Ngo et al., 1980; Roots et al., 1980; Chapman et al., 1977; Curtis et al., 1980; Leith et al., 1975; Yang et a1., 1979, 1980; Goldstein et al., 1980). (XBL 7810-3663A). 
The cell survival data include a variety of tissue culture techniques (e:g., monolayers, stirred suspensions, sealed viais) used by various investigators, and with concomitant variations in the geometry of radiation exposures. These differences, as well as inherent cell-line characteristics, account for the considerable scatter of the data. Nevertheless, there is a region that shows aerobic RBE increasing from 1.0 at low-LET levels (of less than $10 \mathrm{keV} / \mu \mathrm{m}$ ), to a maximum RBE of 3.0 at about 140 to $160 \mathrm{KeV} / \mu \mathrm{m}$. The RBE then decreases rapidly at higher LET. The mean LET values for most of the Bevalac data lie in the ascending region of the RBE plot. We believe that some of the scatter of data represent real differences in the radiation sensitivity of the different cell strains, which will be discussed below. Although not demonstrated here, the RBE is a rapidly changing function of the survival level. At lower doses, the maximum RBE is greater for the LET values presented.

\section{STUDIES WITH THE R-1 RHABDOMYOSARCOMA CELL LINE}

An extensive amount of survival information on the range filtered Bevalac beams has been obtained using the R2D2 subline of the rat tumor R-1 sarcoma (Curtis et al., 1980). Two ranges of carbon and neon ions (13-14 and 22-24 cm) and one range of argon ions $(12 \mathrm{~cm})$ were studied using both the 4 and 10-cm SRF to extend the peak regions. The cells in suspension were continually stirred during the irradiation. An entire survival curve was obtained using the cell suspension in one vessel by withdrawing measured samples sequentially after small dose increments had been delivered. This technique was adapted from one used by Chapman et al. (1977). As an example of the data obtained, Figure 8 presents the dose vs. depth curves for the three short range beams spread to $10-\mathrm{cm}$ peak width (middle pane 1), as well as the RBE values (top panel) and $O E R$ values (bottom panel) at $10 \%$ survival, in the plateau and at three positions in the extended peak region. Results of RBE and OER studies with the rhabdomyosarcoma cell line will be discussed below.

\section{CELL LINE COMPARISONS}

In order to examine in more detail the differences in cell line radiosensitivities to heavy ions, comparisons of two pairs of cell lines irradiated in monoenergetic and extended Bragg peaks have been made. The aerobic RBE values at $10 \%$ survival from the entire R2D2 data set, and the comparable set- of kidney T-1 monolayer data, nave been plotted in Figure 9 against

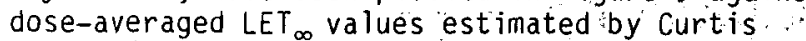
et al. (1980). The RBE values for both sets of data were referenced to $225-k V p X$ rays. It is obvious from the figure that the scatter of data in this more complete comparison of two cell lines does not clearly resolve any specific cell line differences. In the mixed LET fields of extended Bragg peaks it appears that both these cell lines show a RBE maximum at an LET near $200 \mathrm{keV} / \mathrm{\mu m}$. If there are differences in the LET at which the maximum RBE occurs for these cells,

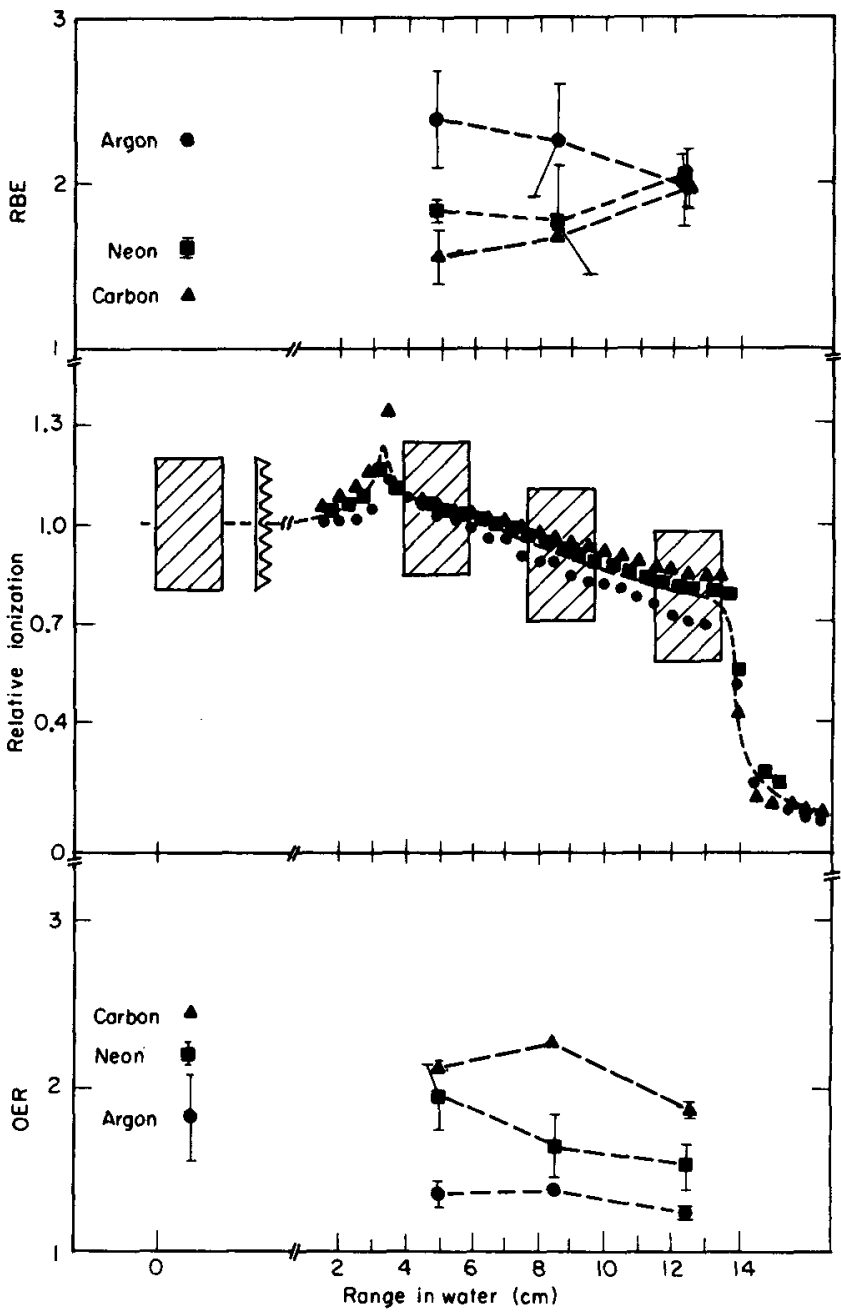

Figure 8. Physical depth-dose distributions, RBE values for oxic cell suspensions at the $10 \%$ survival level, and OER values of the $10 \%$ survival level are shown for carbon, neon, and argon beams with $10-\mathrm{cm}$ extended peak regions and a range of approximately $14 \mathrm{~cm}$ in water. The error bars represent one standard deviation for RBE and OER values measured in two or four separate expriments. (XBL 808-3617)

they are small and not resolvable in highly mixed LET fields.

Figure 10 illustrates some clarification of this point by comparing data on another pair of cultured cell lines... In this study, human T-1 kidney and Chinese hamster $V-79$ : cells were irradiated in monolayer in monoenergetic beams, where the LET spectrum is considerably narrower. The aerobic RBE values are plotted against track averaged $L E T_{\infty}$ values of " Tobias (published in Blakely et al., 1980) for various positions in the unmoderated $570 \mathrm{MeV} / \mathrm{amu}$ argon beam. The figure shows a slight difference in the LET at which the RBE is maximized. This is most clearly seen at the $50 \%$ survival level. It is believed that differences in the cross-sectional inactivation characteristic for each cell line are 


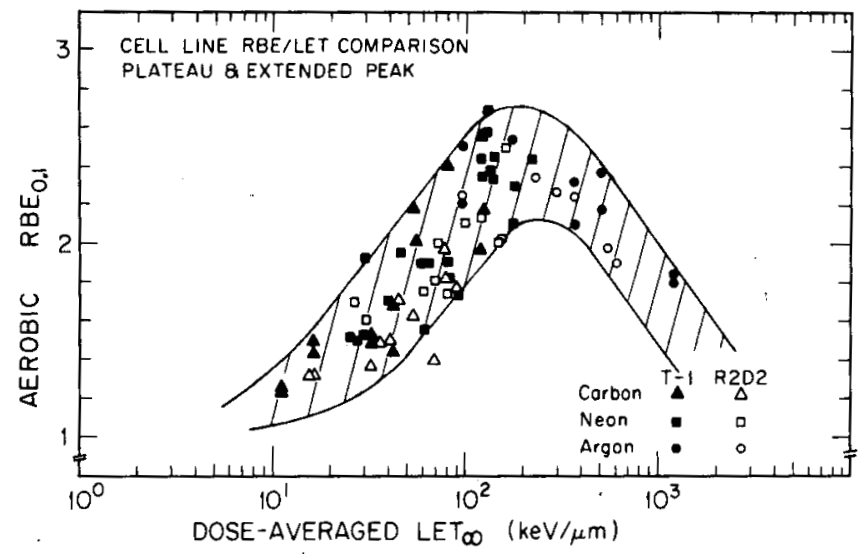

Figure 9. Aerobic RBE-10 values vs. dose-averaged $\overline{\mathrm{LET}}_{\infty}(\mathrm{keV} / \mu \mathrm{m})$ from plateau and extended heavy-ion Bragg-peak data using human kidney $T-1$ cells monolayers (Blakely et al., 1978) and rat rhabdomyosarcoma cell suspensions (Curtis et al., 1980). (XBL 809-3679)

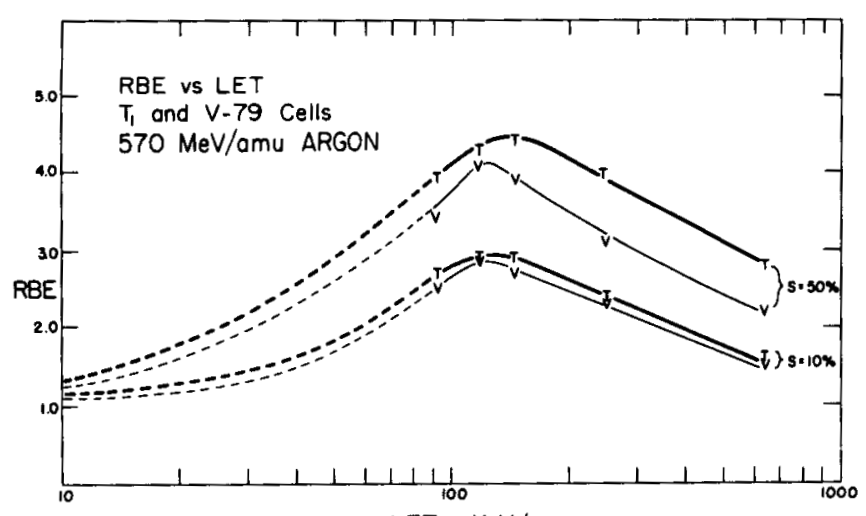

LET $T_{\infty} \mathrm{KeV} / \mu \mathrm{m}$

Figure 10. LET dependence of the RBE values for human kidney $T-1$ cells and Chinese hamster $V-79$ cells measured with cell monolayers in vitro for a monoenergetic $570 \mathrm{MeV} / \mathrm{amu}$ argon beam. The RBE for $V-79$ cells has a peak at a lower LET than for human kidney cells. At $50 \%$ survival the effect is marked; at $10 \%$ survival the effect is not significant. (XBL 795-9634)

responsible for RBE differences like these. Figure 11 demonstrates the consequences of this effect on the design of SRF, where the mixture of LET values is greater.

Figure 11 presents the physical depth dose distribution for a carbon beam of $400 \mathrm{MeV} / \mathrm{amu}$ initial energy with a $10-\mathrm{cm}$ extended. Bragg peak. The biologically effective doses (physical dose times aerobic RBE at $50 \%$ survival normalized to entrance dose times derobic RBE) for three different established cell lines from in vitro experiments are also plotted. The Chinese

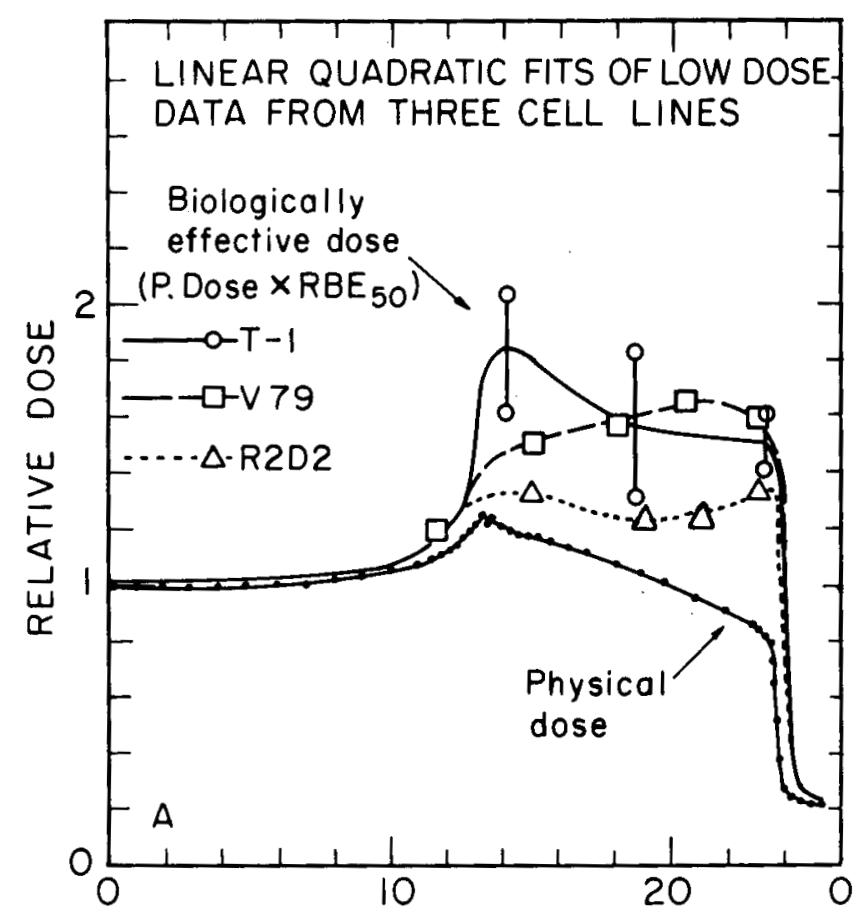

RANGE $\left(\mathrm{cm} \mathrm{H} \mathrm{H}_{2} \mathrm{O}\right)$

Figure 11. Physical Bragg ionization (0) curve for a $400 \mathrm{MeV} / \mathrm{amu}$ carbon ion beam with a $10 \mathrm{~cm}$ extended Bragg peak. The biologically effective dose (BED = physical dose $\times$ RBE-50) is plotted at range positions studied for the human $k i d n e y$ T-1 cell monolayers (0) (Blakely et al., 1978); Chinese hamster $V-79$ cell suspensions ( $\square$ ) (Chapman et al., 1977); and rat rhabdomyosarcoma cell suspensions $(\Delta)$ (Curtis et al., 1980). (XBL 809-3677)

hamster V-79 data from Chapman et al. (1977) and the rat rhabdomyosarcoma data from Curtis et al. (1980), were both taken from experiments in which the cells were irradiated in suspension chambers $1.5 \mathrm{~cm}$ wide. The human kidney $\mathrm{T}-1$ data from Blakely et al. (1978) were irradiated in monolayer.

For this comparison, each cell line has been fitted to the linear quadratic model. Experimental variations (both biological and physical) may account for many of the differences; however, the survival results do not appear to indicate perfect isokilling across the $10 \mathrm{~cm}$ extended peak for each line. Rather, for this spiral filter, there appears to be a slight undercompensation for biological killing in the distal peak for the $v-79$ cel1s, while there is an overcompensation for killing in the distal peak for the T-1 cells. John Lyman has used this type of information to revise filter designs. 
COMPARISONS OF OER AND OGF

In general, comparisons of measured OER values have shown that for charged particle mean LET values greater than $100-200 \mathrm{keV} / \mu \mathrm{m}$, the OER is very low (see Fig. 12). However, such comparisons also show rather large differences in LET dependence of OER be low $100-200 \mathrm{keV} / \mu \mathrm{m}$, especially where there are differences in the fragmentation dose component (Blakely et al., 1979).

It therefore becomes of interest to compare OER values from several systems in the rangefiltered beams. Figure 13 is a plot of oxygen gain factors (OGF) versus residual range for Bevalac beams with the 4-cm and 10-cm SRF. The data are from Blakely et al. (1978) and Curtis et al (1980). The OGF is the ratio of the OER obtained with the reference low-LET radiation source to the OER obtained with the high-LET test beam. Comparisons of OGF values eliminate differences in the efficiency of oxygen removal between experimental techniques. The results of Figure 13 show that for both cell lines, the OGF for both filters is greatest for argon, with neon

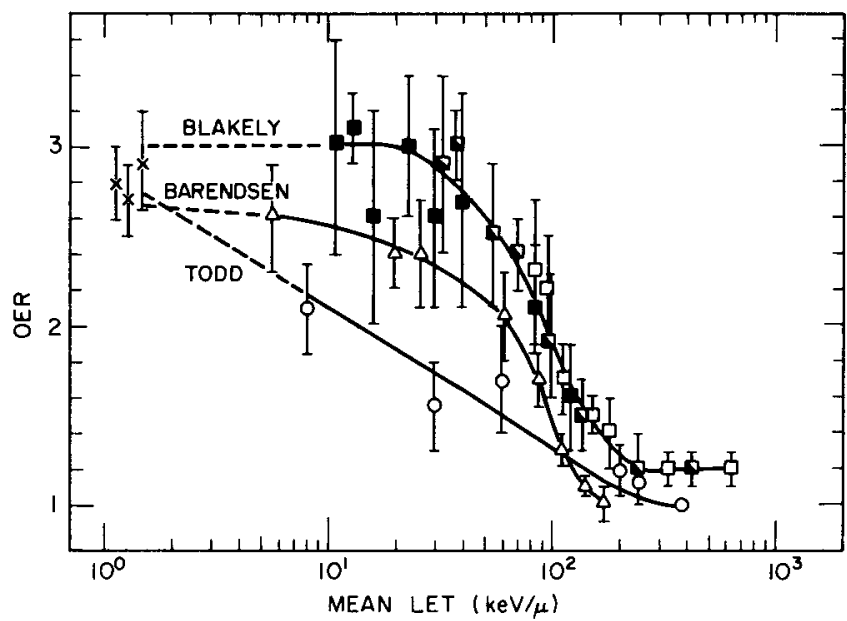

Figure 12. OER ( $95 \%$ confidence limits) vs. track-average mean LET: carbon ( $\mathbf{D})$, neon ( $\mathbf{\nabla})$, and argon ( $\square$ ) beams from the Bevalac (Blakely et al., 1979); low-energy helium-ion data (Barendsen et al., 1966), high-energy (up to $10 \mathrm{MeV} / \mathrm{amu}$ ) heavy-ion data (Todd, 1967); and $X-r a y$ data $(X)$. (XBL 787-3362)

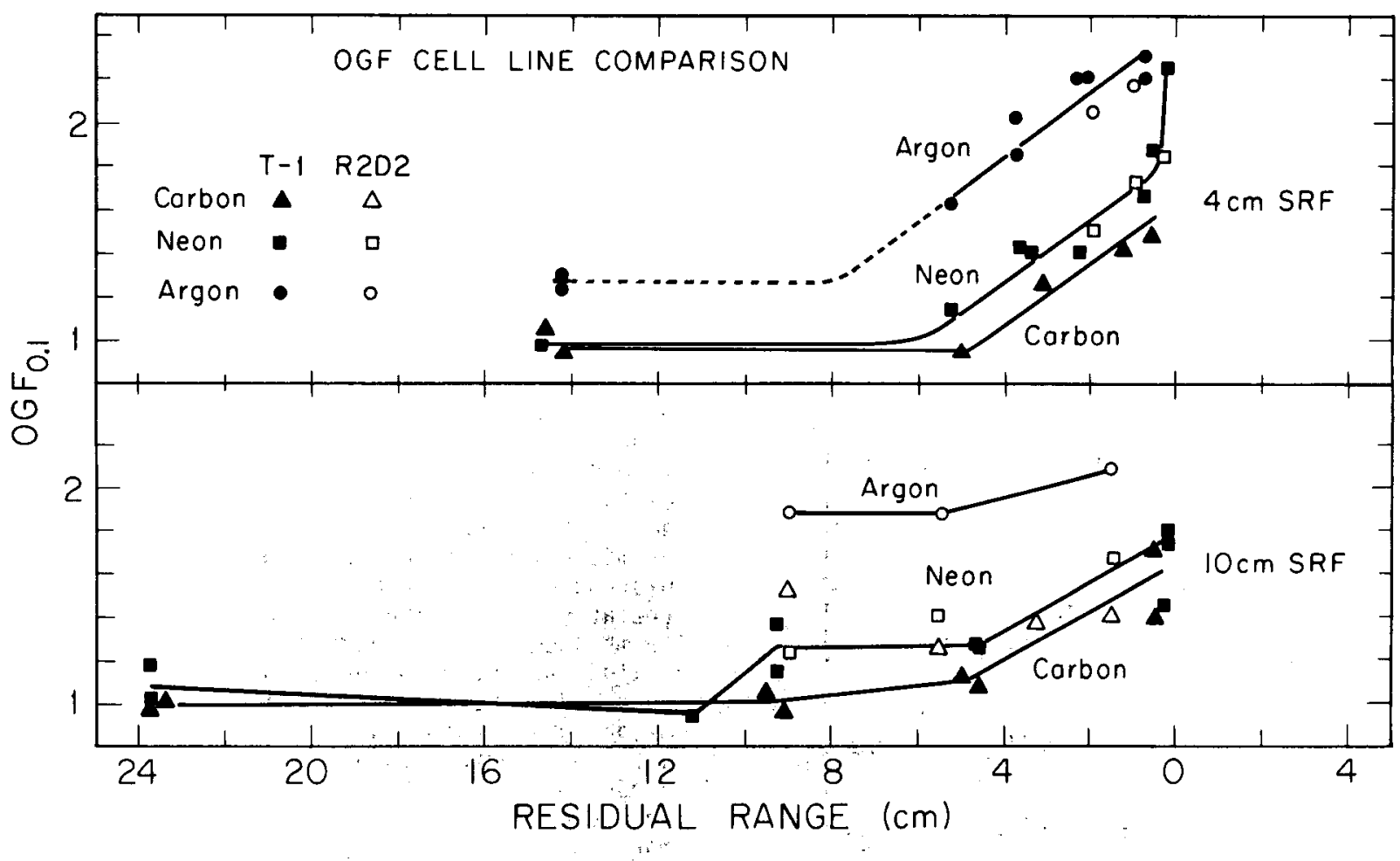

Figure 13. OGF as a function of residual range for: $308 \mathrm{MeV} / \mathrm{amu}$ carbon, $425 \mathrm{MeV} / \mathrm{amu}$ neon, and $570 \mathrm{MeV} / \mathrm{amu}$ argon beams with a 4-cm extended Bragg peak (upper panel); $400 \mathrm{MeV} / \mathrm{amu} \mathrm{Carbon}$, $557 \mathrm{MeV} / \mathrm{amu}$ neon, and $570 \mathrm{MeV} / \mathrm{amu}$ argon beams with a 10-cm extended Bragg peak (lower panel). Data are from human kidney T-1 cell monolayer survival measurements (Blakely et al., 1978) and rat rhabdomyosarcoma cell suspensions survival measurements (Curt is et al., 1980).

(XBL 809-3678) 
and carbon showing successively less gain at the two ranges studied.

\section{SINGLE PORT AND CROSS-FIRED EXPOSURES}

In order to evaluate more realistically the therapeutic potential of Bevalac beams, especially with the limited range-filter-designs available, simulated dual opposed-port exposures were made. The experimental description of our method is detailed in "Figure 14 for a specific beam condition. The data obtained from this technique using carbon, neon, silicon, and argon beams of $\sim 14 \mathrm{~cm}$ range are presented in Figure 15 in a stylized version for simplicity.

In general, the results of single-port and dual opposed-port exposures indicate that (1) all charged particle beams studied have superior

\section{SIMULATED CROSS-FIRED BEAMS}

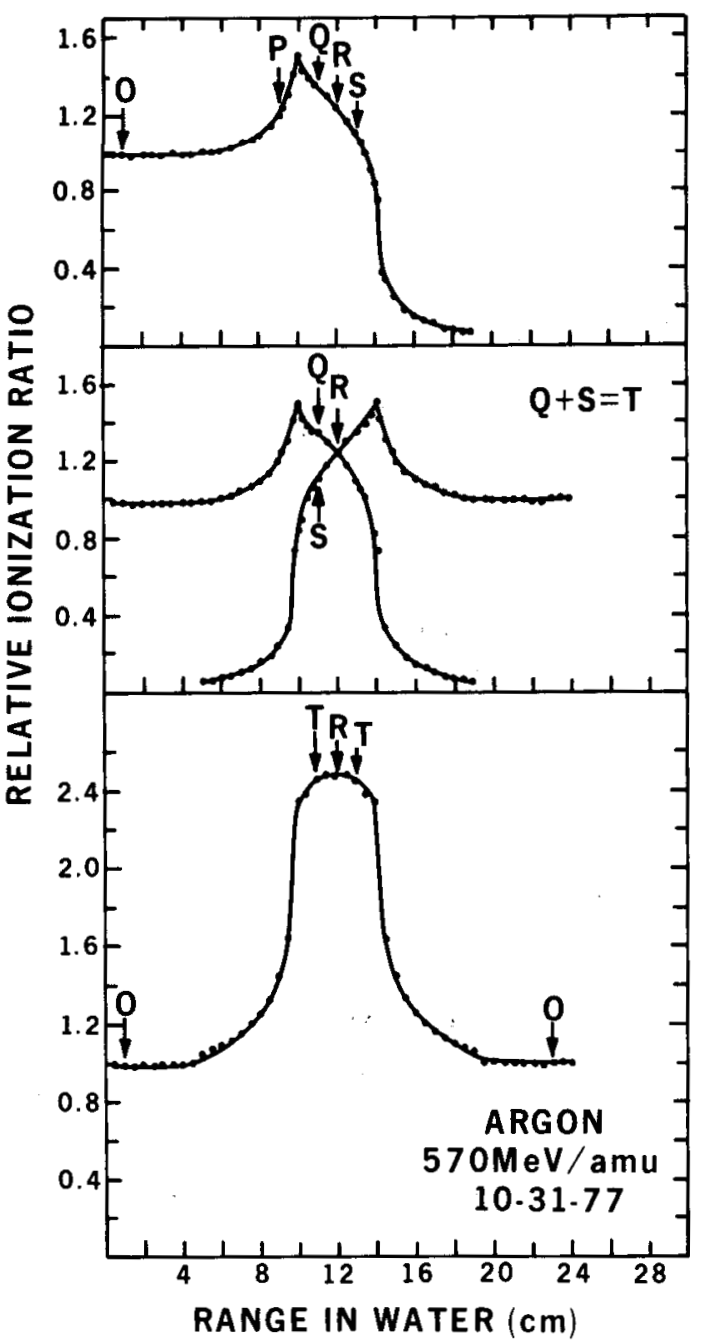

Figure 14. Physical Bragg ionization curves for single-port and simulated cross-fired dual parallel-opposed port $570 \mathrm{MeV} / \mathrm{amu}$ argon beams. (XBL 785-8658)

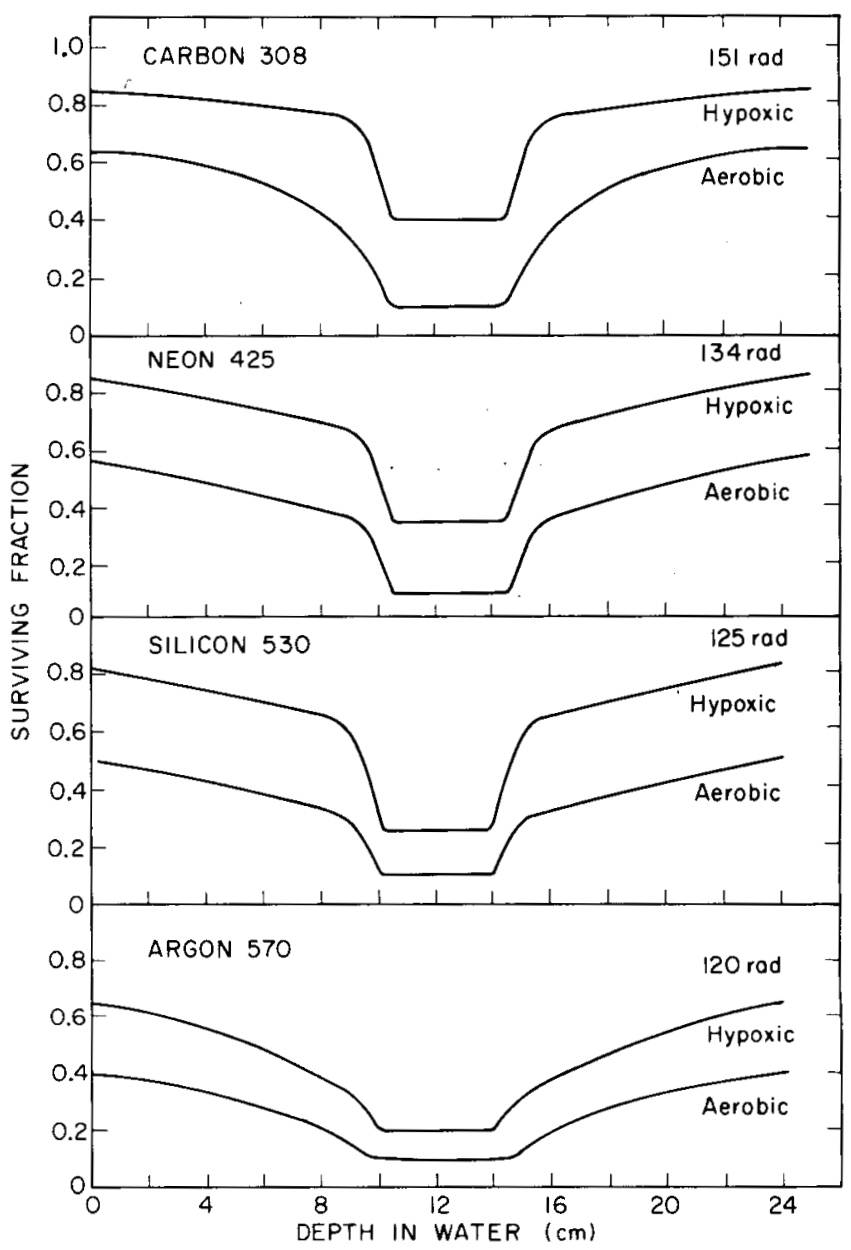

Figure 15. Human kidney cell depth survival curves following cross fire of carbon, neon, silicon, and argon beams for hypoxic and aerated cells with a 4-cm extended Bragg peak. The oxygen effect in the "tumor" is smallest for silicon and argon. (XBL 809-3680)

physical depth-dose advantage over conventional treatment modalities at the same tissue penetration depths; (2) for both the maximum penetration depths studied, the midpeak-to-plateau physical dose ratio decreased slightly with increasing atomic number of the particle; (3) midpeak-toplateau ratios of biological effectiveness (relative to $225-k V P X$ rays) for all particle beams studied were greater than one, with a maximum advantage observed for the $14-\mathrm{cm}$ range neon and carbon beams; (4) midpeak-to-plateau oxygen gain factors were all greater than one with the greatest advantage observed for the argon and silicon beams; and (5) dual opposedport exposure of particle beams demonstrated that more uniformly low OER values, and reduced survival can be achieved simultaneously over the cross-fired extended Bragg peak region, compared to single-port exposures. The cross-fired exposures eliminate some of the imperfections in SRF design, and permit a reduced entrance and exit dose for an equivalent tumor dose from a single port exposure. 


\section{COMPARISON OF THERAPY MODALITIES}

The evaluation of therapeutic advantages of heavy ions described above led us to a comparison of these beams with other available therapy modalities. The two key features of treatment needs are first, to deliver high killing dose to a localized tumor target while sparing normal tissue lying in the treatment volume, and second, to reduce the radioresistance of hypoxic cells. Figure 16 is a vector representation that has been constructed for conventional low-LET sources (protons, neutrons, negative pions; helium, carbon, neon, silicon and argon ion beams). The vector plots were made to describe the treatment of two targets: a $10 \mathrm{~cm} \times 10 \mathrm{~cm}$ field at 10-14 cm tissue depth (upper panel), and a $10 \mathrm{~cm} \times 10 \mathrm{~cm}$ field at $14-24 \mathrm{~cm}$ t issue depth (lower panel). In vitro cell data from the following papers were used to construct this plot: Barendsen, 1968; Berry, 1971; Berry and
Andrews, 1964; Bewley et al., 1976; Blakely et a 1., 1978, 1979, 1980; Broerse et al., 1968; Chapman et al., 1977, 1978; Curt is et al., 1980; Dertinger et al., 1976; Fu and Phillips, 1976; Gerner and Leith, 1976, 1977; Goldstein et al., 1980; Gragg et al., 1976; Hall et al., 1974, 1977, 1978; Heyder and Pohlit, 1979; Leith et al., 1975, 1977; Li et al., 1979; Lucke-Hühle et al., 1979; Mill et a1., 1976; Ngo et al., 1977, 1980; Phillips et al., 1977, 1979; Raju et al., 1971, 1972a,b, 1975, 1978; Raju, 1980a,b; Rini et al., 1979; Robertson et al., 1975; Roots et al., 1980; Schilling et al., 1977; Skarsgard et al., 1979a,b; Tobias et al., 1979, 1980; Todd et al., 1974, 1975; Tremp et al., 1979; Ueno and Grigoriev, 1969; Weibezahn et al., 1979.

The most advantageous position on the figure is located at the lower right quadrant. For the smaller, more shallow target volume (upper panel of Fig. 16), it appears that $308 \mathrm{MeV} / \mathrm{amu}$ carbon,

VECTOR REPRESENTATION Of THERAPY MODALITIES

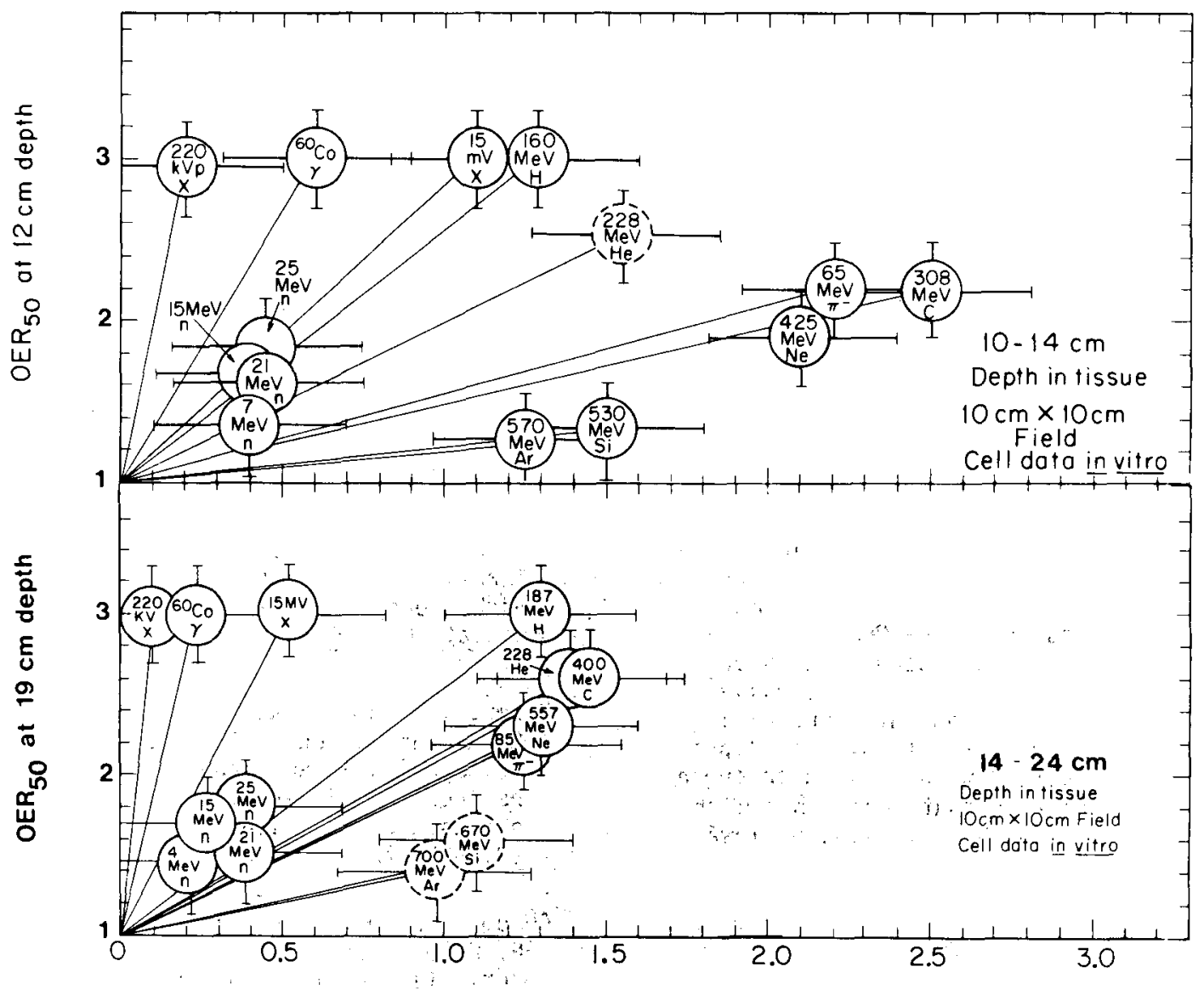

Ratio of biologically effective doses =

$\left[\frac{\text { Dose (mid target volume) }}{\text { Dose (entronce) }}\right] \times\left[\frac{R B E_{50} \text { (mid target volume) }}{R B E_{50} \text { (entrance) }}\right]_{\text {aerobic }}$

Figure 16. Vector representation of therapy modalities for treatment of: a small, shallow field (upper panel) and a large, deep field (lower panel). (XBL 808-3623B) 
$425 \mathrm{MeV} / \mathrm{amu}$ neon, and $65 \mathrm{MeV}$ negative pion beams are superior in their ratio of biologically effective doses, with the neon beam having a slightly less effective dose ratio, but a superior OER advantage. Argon ( $570 \mathrm{MeV} / \mathrm{amu}$ ), silicon ( $530 \mathrm{MeV} / \mathrm{amu}$ ) and several neutron energies (5 to $25 \mathrm{MeV}$ ) have the best OER advantage, and argon and silicon beams are better than neutrons with respect to effective dose ratio.

Helium ions and protons show an enhanced biologically effective dose ratio, but are most similar to the low-LET radiations with respect to OER, which results in their intermediate placement in the vector plot. For a larger, deeper tumor (lower panel of Fig. 16), the relative placement of each of the therapy modalities is altered, except for the location of the $187 \mathrm{MeV} / \mathrm{amu}$ proton and $225 \mathrm{MeV} / \mathrm{amu}$ helium data. At this range, the $400 \mathrm{MeV} / \mathrm{amu}$ carbon and $228 \mathrm{MeV} / \mathrm{amu}$ helium beams are quite similar, as are the $557 \mathrm{MeV} / \mathrm{amu}$ neon beam and the $85 \mathrm{MeV} / \mathrm{amu}$ pion beam. The neutron beams and low-LET modalities have deteriorated considerably in their effective dose ratio, however, the neutron OER remains very low. Argon and sijicon data (estimated for a $700 \mathrm{MeV} / \mathrm{amu}$ and $670 \mathrm{MeV} / \mathrm{amu}$ beam, respectively), are expected to be as low or lower on the OER scale than the neutron beams, with the additional advantage of being almost a factor of two better in effective dose localization.

\section{SUMMARY}

A variety of experiments have been carried out in vitro on several mammalian cell lines with carbon, neon, silicon and argon beams at 14 and $24 \mathrm{~cm}$ depth penetration. The results of these experiments substantiate the conceptual basis for physical and radiobiological advantages of accelerated heavy-ion beams in cancer therapy. The best biologically effective depth dose ratio for situations corresponding to therapy needs can be obtained with accelerated carbon beams. A1l other heavy beams tested, as well as pions, are markedly better than the effective depth dose ratios achievable with neutrons, $X$ or gamma rays. A significant depression of the OER at the various depths required for therapy has been achieved with silicon and argon beams, while these beams still retained advantageous biologically effective depth dose ratios. The depression of the oxygen effect with silicon or argon ion beams is greater than that achievable with neutrons or pions, or with heavy ions of lower atomic number.

\section{ACKNOWLEDGEMENT}

The authors wish to acknowledge the outstanding cooperation of the LBL Accelerator Division and the Bevalac operations staff. The excellent technical assistance of T. Yang, T. Tenforde, J. Howard, P. Chang, L. Lomme l, K. Smith, 0. Oleszko, L. Craise, K. Crabtree, W. Schilling, D. Tse, S. Tenforde, I. Madfes, M. Yezzi, F. Abrams, C. Perez, K. Knight, and $R$. Zamora was an essential component of this work. We thank $R$. Stevens for assistance in drafting the figures, and L. Hawkins and Diana Morris for typing the manuscript. This investigation was supported by Grant CA 15184 awarded by the National Cancer Institute DHEW.

\section{REFERENCES}

Barendsen, G. W. 1968. Response of cultured cells, tumors and normal tissues to radiations of different linear energy transfer. Curr. Top. Radiat. Res. 4, 295-356

Barendsen, G. W., C. J. Koot, G. R. van Kersen, D. K. Bewley, S. B. Field, and C. J. Parnell. 1966. The effect of oxygen on impairment of the proliferative capacity of human cells in culture by ionizing radiations of different LET. Int. J. Radiat. Biol. 10, 317-327.

Berry, R. J. 1971. Hypoxic protection against fast neutrons of different energies. A review. Eur. J. Cancer 7, 145-152.

Berry, R. J. and J. R. Andrews. 1964. The response of mammalian tumor cells in vivo to radiations of differing ionization densities (LET). Ann. N.Y. Acad. Sci. 114, 48-59.

Bewley, D. K., B. Cullen, S. B. Field, S. Hornsey, B. C. Page, and R. J. Berry. 1976. A comparison for use in radiotherapy of neutron beams generated with 16 and $42 \mathrm{MeV}$ deuterons on beryllium. Br. J. Radiol. 49, 360-366

Blakely, E. A., C. A. Tobias, F.Q.H. Ngo, T.C.H. Yang, K. C. Smith, P. Y. Chang, and M. J. Yezzi. 1978. Comparison of helium and heavy ion beams for therapy based on cellular radio- biological data. Int. J. Radiat. Oncol. Biol. Phys. 4 (Supp7. 2), 93-94. (Abstract).

Blakely, E. A., C. A. Tobias, T.C.H Yang, K. C. Smith, and J. T. Lyman. 1979. Inactivation of human kidney cells by high-energy mono- energetic heavy-ion beams. Radiat. Res. 80, 122-160.

Broerse, J. J., G. W. Barendsen, and G. R. van Kersen. 1968. Survival of cultured human cells after irradiation with fast neutrons of different energies in hypoxic and oxygenated conditions. Int. J. Radiat. Biol. $13,559-572$.

Chapman, J. D., E. A. Blakely, K. C. Smith, and R. C. Urtasun. 1977. Radiobiological characterization of the inactivating events produced in mammalian cells by helium and heavy ions. Int. J. Radiat. Oncol. Biol. Phys. 3, 97-102.

Chapman, J. D., E. A. Bläkely, K. C. Smith, R. C. Urtasun, J. T. Lyman, and C. A. Tobias. 1978. Radiation biophysical studies with mammalian cells and a modulated carbon ion beam. Radiat. Res. 74, 101-111. 
Cohen, M., D.E.A. Jones, and D. Green. 1972. Central axis depth-dose data for use in radiotherapy. Br. J. Radiol. (Supplement 11).

Curtis, S. B., W. A. Schilling, T. S. Tenforde, K. A. Crabtree, S. D. Tenforde, J. Howard, and J. T. Lyman. 1980. Survival of oxygenated and hypoxic tumor cells in the extended-peak regions of heavy charged-particle beams. (Manuscript in preparation).

Dertinger, H., C. Lücke-Hühle, H. Schlag, and K. F. Weibezahn. 1976. Negative pion irradiation of mammalian cells. I. Survival characteristics of monolayers and spheroids of Chinese hamster lung cells. Int. J. Radiat. Biol. 29, 271-277.

Fu, K. K. and T. L. Phillips. 1976. The relative biological effectiveness and oxygen enhancement ratio of neon ions for the EMT6 tumor system. Radiology 120, 439-441.

Gerner, E. W. and J. T. Leith. 1977. Interaction of hyperthermia with radiations of different linear energy transfer. Int. J. Radiat. Biol. 31, 283-288.

Gerner, E., J. Leith, and M. Boone. 1976. Mammalian cell survival response following irradiation with $4 \mathrm{MeV} x$ rays or accelerated helium ions combined with hyperthermia. Radiology 119, 715-720.

Goldstein, L. S., T. L. Phillips, K. K. Fu, G. Y. Ross, and L. J. Kane. 1980. Biological effects of accelerated heavy ions: I. Single doses in normal tissues, tumors and cells in vitro. (Submitted to Radiat. Res.).

Gragg, R. L., R. M. Humphrey, and R. E. Meyn. 1976. The response of Chinese hamster ovary cells to fast neutron radiotherapy beams. I. Relative biological effectiveness and oxygen enhancement ratio. Radiat. Res. $65,71-82$.

Hall, E. J. 1974. RBE and OER values as a function of neutron energy. Eur. J. Cancer 10, 297-299.

Hall, E. J. and M. Astor. 1979.' The oxygen enhancement ratio for negative pi mesons. Int. J. Radiat. Oncol. Biol. Phys. 5, 55-60.

Hall, E. J., R. P. Bird, H. H. Rossi, R. Coffey, J. Varga, and Y. M. Lam. 1977. Biophysical studies with high energy argon ions. II. Determinations of the relative biological effectiveness, the oxygen enhancement ratio, and the cell cycle response. Radiat. Res. 70, $469-479$.

Hall, E. J., A. M. Kellerer, H. H. Rossi, and Y. M. Lam. 1978. The relative biological effectiveness of $160 \mathrm{MeV}$ protons. II. Biological data and their interpretation in terms of microdosimetry. Int. J. Radiat. Oncol. Biol. Phys. 4, 1009-1013.

Heyder, I. R. and W. Pohlit. 1979. Radiobiological data for clinical dosimetry in pion tumor therapy. Radiat. Environ. Biophys. 16, 251-260.

Leith, J. T., V. Arcellara, J. T. Lyman, and K. T. Wheeler. 1975. Response of a rat brain tumour to irradiation with accelerated neon ions. Int. J. Radiat. Biol. 28, 91-97.

Leith, J. T., P. Smith, P. Ross-Riveros, and K. T. Wheeler. 1977. Cellular response of a rat brain tumour to a therapeutic neon ion beam. Int. J. Radiat. Biol. 32, 401-407.

Li, G. M., G. A. Hahn, P. Fisher, P. Fessenden, and M. Bagshaw. 1979. Interaction of negative pi-meson with $x$-irradiation. Presented at the VI International Congress of Radiation Research, May 13-19, Tokyo, Japan. (Abstract).

Lücke-Hühle, C., E. A. Blakely, P. Y. Chang, and C. A. Tobias. 1979. Drastic G2 arrest in mammalian cells after irradiation with heavy ion beams. Radiat. Res. 79, 97-112.

Mill, A. J., J. D. Lewis, and W. S. Hall. 1976. Response of Hela cells to irradiation with negative pi mesons. Br. J. Radiol. 49, $166-171$.

Ngo, F.Q.H., A. Han, H. Utsumi, M. M. Elkind. 1977. Comparative radiobiology of fast neutrons: Relevance to radiotherapy and basic studies. Int. J. Radiat. Oncl. Biol. Phys. $\underline{3}$, 187-193.

Ngo, F.Q.H., E. A. Blakely, and C. A. Tobias. 1980. Sequential exposures of mammalian cells to low- and high-LET radiations. I. Lethal effects following $x-r a y$ and neon-ion irradiation. Radiat. Res, in press.

Phillips, T. L. and K. K. Fu. 1976. Biological effects of $15 \mathrm{MeV}$ neutrons. Int. J. Radiat. Oncol. Biol. Phys. 1, 1139-1147.

Phillips, T. L., K. K. Fu, and S. B. Curtis. 1977. Tumor biology of helium and heavy ions. Int. J. Radiat. Oncol. Biol. Phys. 3, 109-113.

Raju, M. R. 1980a. Heavy Particle Radiotherapy: Academic Press, New York.

Raju, M. R. 1980b. Radiobiologic properties of pions and heavy ions - a comparison. J. de L'Association Canadienne des Radiologistes 31 , 26-29.

Raju, M. R., M. Gnanapurani, U. Madhavanath, J. Howard, and J. T. Lyman. 1971. Relative biological effectiveness and oxygen enhancement ratio at various depths of a $910 \mathrm{MeV}$ helium ion beam. Acta Radiol. 10, 353-357.

Raju, M. R., M. Gnanapurani, B. I. Martins, J. Howard, and J. T. Lyman. 1972a. Measurement of OER and RBE of a $910 \mathrm{MeV}$ helium beam using cuitured cells (T-1). Radiology 102, $425-428$.

Raju, M. R., M. Gnanapurani, C. Richman, B. I. Martins, and G. W. Barendsen. $1972 \mathrm{~b}$. 
RBE and OER of negative pi mesons for damage to cultured T-1 cells of human kidney origin. Br. J. Radiol. 45, 178-181.

Raju, M. R., J. F. Dicello, T. T. Trujillo, and M. Kligerman. 1975. Biological effects of the Los Alamos meson beam on cells in cuiture. Radiobiology 116, 191-193.

Raju, M. R., H. I. Amols, E. Bain, S. G. Carpenter, R. A. Cox, and J. B. Robertson. 1978. A heavy particle comparative study. Part III'. OER and RBE. $\mathrm{Br}$. J. Radiol. 51, 712-729.

Raju, M. R.; H. I. Amols, E. Bain, S. G. Carpenter, R. A. Cox, and J. B. Robertson. 1979. OER and RBE for negative pion beams of different peak widths. Br. J. Radiol. 52, 494-498.

Rini, F. J., E. J: Hall, and S. A. Marino. '1979. The oxygen enhancement ratio as a function of neutron energy with mammalian cells in culture. Radiat. Res. 78, 25-37.

Robertson, J. B., J. R. Williams, R. A. Schmidt, J. B. Little, D. F. Flynn, and H. D. Suit. 1975. Radiobiological studies of a high-energy modulated proton beam utilizing cultured mammalian cells. Cancer 35, 1664-1677.

Roots, R., T. C.'Yang, L. Craise, E. A. Blakely, and C. A. Tobias. 1980. Rate of rejoining of DNA breaks induced by accelerated carbon and neon ions in the spread Bragg peak. Int. J. Radiat. Res. 38, 203-210.

Skarsgard, L. D. 1979. The biological properties of pions. Proceedings, Sixth International Congress of Radiation Research (S. Okada, M. Imamura, T. Terashima, and H. Yamaguchi, eds.) Japanese Association for Radiation Research, Tokyo.

Skarsgard, L. D., R. M. Henke lman, G.K.Y. Lam, B. Paicic, C. J. Eaves, and I. Ito. 1979. Pre-clinical studies of negative $p i-m e s o n s$ at Triumf. Treatment of Radioresistant Cancers (M. Abe, K. Sakamoto, and T. L. Phillips, eds.). Elsevier/North Holland Biomedical Press.

Schilling, W. A., S. B. Curtis, T. S. Tenforde, K. E. Crabtree, J. T. Lyman, and J. Howard.
1977. Comparison of the radiation response of rat tumor cells exposed at various positions in the extended Bragg peak of heavy ion beams. Radiat. Res. 70, 642-643. (Abstract).

Tobias, C. A., E. L. Alpen, E. A. Blakely, J.R. Castro, A. Chatterjee, G.T.Y. Chen, S. B. Curt is, J. Howard, J. T. Lyman, and F.Q.H. Ngo. 1979. Radiobiological basis for heavy-ion therapy. Treatment of Radioresistant Cancers (M. Abe, K. Sakamoto, and T. L. PhilTips, eds.) Elsevier/ North-Holland Biomedical Press.

Tobias, C. A., E. A. Blakely, F.Q.H. Ngo, and T.C.H. Yang. 1980. The repair-misrepair model of cell survival. Radiation Biology and Cancer Research (A. Meyn and R. Withers, eds.) pp. 195-230. New York, Raven Press.

Todd, P. W. 1967. Heavy ion irradiation of cultured human cells. Radiat. Res. Suppl. 7, 196-207.

Todd, P., B. I. Martins, J. T. Lyman, J. H. Kim, and C. B. Schroy. 1974. Spatial distributions of human cell survival and oxygen effect in a therapeutic helium ion beam. Cancer $34,1-5$.

Todd, P., C. R. Shonk, G. West, M. M. Kligerman, and J. Dicel10. 1975. Spatial distribution of effects of negative pions on cultured human cells. Radiology 116, 179-180.

Tremp, J., H. Blattmann, and H. Fritz-Niggli. 1979. Cell survival over the depth profile after irradiation with a negative pion beam. Radiat. Environ. Biophys. 16, 267-272.

Ueno, Y. and Y. G. Grigoriev. 1969. The RBE of protons with energy greater than $126 \mathrm{MeV}$. Br. J. Radiol. 42,475 .

Weibezahn, K. F., H. Dertinger, H. Schlag, and c. Lïcke-Hïhle 1979. Biological effects of negative pions in monolayers and spheroids of Chinese hamster cells. Radiat. Environ. Biophys. 16, 273-277.

Yang, T.C.H., C. A. Tobias, E. A. Blakely, L. M. Craise, I. S. Madfes, C. Perez, and J. Howard. 1980. Enhancement effects of high energy neon particles on the viral transformation of mouse C3H1OT1/2 cells in vitro. Radiat. Res. $81,208-223$. 


\title{
HYPOXIC CELL RADIOSENSITIZERS AND HEAVY CHARGED PARTICLE RADIATIONS
}

\author{
W. M. Saunders ${ }^{*}$ and J. D. Chapman ${ }^{\dagger}$
}

Beams of heavy ions are expected to be superior to the beams of photons presently used for cancer therapy. Heavy-ion beams have a better distribution of absorbed dose, and a greater biological effectiveness than photon beams. Furthermore, the chemical effect of oxygen in sensitizing radiobiological damage is diminished for these beams. This means that the increase in RBE observed with increasing LET is greater for hypoxic cells than for oxygenated cells, i.e., the OER is decreased. Radioresistant hypoxic cells are thought to be an important cause of local tumor recurrence after photon irradiation, so the favorable increase in RBE for hypoxic cells irradiated with heavy ions could prove beneficial in radiotherapy.

Although the oxygen effect is lessened in a spread Bragg peak, it is not eliminated. Typical results are given in the first column of Table 1 , which shows the OER decreasing as the mass of the ion increases, but not reaching 1.0. These data are from the work of Chapman et al. (1978), who also studied the effect of hypoxic cell sensitizers on the OER in spread Bragg peaks. These sensitizers are from a class of electron-affinic drugs that decrease the radioresistance of hypoxic cells without affecting the radiation response of well-oxygenated cells. The effect of such drugs on the OER of spread Bragg peaks is summarized in the right-hand columns of Table 1: There is a significant decrease in OER for each of the three hypoxic cell sensitizers studied, and for every beam of heavy ions studied. The rows in Table 1 are in order of increasing LET. In the "no drug" column, there is a substantial drop in OER from top to bottom. However, the other columns show much less variation; that is, there is little, if any, drop of OER with increasing LET.

This lessening of the dependence of OER or LET may be of practical importance in the heavyion cancer treatment program. For a given spread Bragg peak, the OER decreases and the RBE increases from proximal to distal peak. This is a reflection of the increasing mean LET from proximal to distal peak. The ridge filters used for cancer treatments are shaped to compensate for the increasing RBE across the peak by decreasing the physical dose such that there is constant killing of oxygenated cells across the peak. However, the OER does vary across the peak, so the killing of hypoxic cells will not be constant. The data in Table 1 suggest that this variation could be minimized by the addition of the hypoxic cell sensitizer.

The data in Table 1 are not applicable to the treatment of cancer in humans because the high

Table 1. Oxygen Enhancement Ratios* for Chinese Hamster Cells Irradiated with $250-k V X$ rays or Heavy Charged Particle Beams in the Presence of Hypoxic Cell Sensitizers

\begin{tabular}{|c|c|c|c|c|}
\hline & $\begin{array}{l}\text { No } \\
\text { Orug }\end{array}$ & $\begin{array}{l}5 \mathrm{mM} \\
\text { Metronidazole }\end{array}$ & $\begin{array}{c}5 \mathrm{mM} \\
\text { Misonidazole }\end{array}$ & $\begin{array}{c}5 \mathrm{mM} \\
\mathrm{R} 0-07-0741\end{array}$ \\
\hline $250-k V \times$ rays & 2.8 & 1.60 & 1.27 & 1.17 \\
\hline $\begin{array}{l}\text { Helium ions }(8 \mathrm{~cm} \text {, } \\
\text { spread peak) }\end{array}$ & 2.40 & 1.62 & 1.30 & 1.13 \\
\hline $\begin{array}{l}\text { Carbon ions } \\
(\text { plateau })\end{array}$ & 2.55 & 1.78 & 1.46 & 1.23 \\
\hline $\begin{array}{c}\text { Carbon ions }(4 \mathrm{~cm} \text {, } \\
\text { spread peak) }\end{array}$ & 1.65 & 1.40 & 1.23 & 1.15 \\
\hline $\begin{array}{l}\text { Neon ions }(4 \mathrm{~cm} \text {, } \\
\text { spread peak) }\end{array}$ & 1.57 & -- & 1.10 & -- \\
\hline $\begin{array}{l}\text { Argon ions }(4 \mathrm{~cm} \text {, } \\
\text { spread peak) }\end{array}$ & 1.43 & - & 1.28 & -- \\
\hline
\end{tabular}

*At $10 \%$ survival level. 
dose of drugs used (5 mM) would be toxic in humans. The next step in this project will be, therefore, to measure the OER across the peak, with and without hypoxic cell sensitizers in concentrations that could safely be administered to humans $(0.5 \mathrm{mM}$ and $1.0 \mathrm{mM})$. A pre liminary experiment has already been performed by Chapman, Blakely, and Saunders, using a hypoxic cell sensitizer that is about to be released for use in humans in the United States (RO-05-9963). The data from that experiment are presently being analyzed.

\section{FOOTNOTES AND REFERENCE}

* Department of Radiation Oncology, University of California, San Francisco, CA.

t Department of Radiation Oncology, W. W. Cross Cancer Institute, University of Alberta, Edmonton, Alberta, Canada

Chapman, J. D., R. C. Urtasun, E. A. Blakely, K. C. Smith, and C. A. Tobias. 1978. Hypoxic cell sensitizers and heavy charged particle radiation. Br. J. Cancer 37 (Suppl. III), 184-188. 


\title{
CELLULAR DAMAGE AND REPAIR FOLLOWING HEAVY-ION IRRADIATION
}

\author{
F. Q. H. Ngo, E. A. Blakely, T. C. H. Yang, M. J. Yezzi, and C. A. Tobias
}

Whi le gamma or $x$ rays produce ionization in matter essentially by secondary electrons, heavy charged particles cause ionization events in a more complex manner. The major components involved in the energy transfer processes by the passage of a heavy-ion track include the primary particles, secondary electrons or delta-rays, and nuclear fragments. The relative contribution from each of these components is a function of the incident energy and the atomic number and it varies with the depth of penetration in tissue. The physics of heavy-ion beams are described in this report by Chatterjee et al. and Schimmerling et al. (see Part II). Understanding the biophysical and biological effects of heavy ions has been a continuous effort at LBL. In this chapter, we review the work accomplished in the past few years on repair and expression of heavyion caused damage in mammalian cells in culture.

Much of our knowledge in radiation biology stems from the work with low-LET irradiation. At the cellular level, two operationally distinct repair processes have been established: repair of sublethal damage (SLD) (Elkind and Sutton, 1960), and repair of potentially letnal damage (PLD) (Phillips and Tolmach, 1966). Repair of SLD describes the survival increase of fractionated or split-dose exposures relative to that of single-dose exposures on the bas is of same total dose. Repair of PLD often refers to a net increase in survival as irradiated cells are left in a suboptimal culture condition (e.g., medium with deficient nutrients, cells grown to a plateau phase, low temperatures) for sometime before trypsinization takes place. These repair processes are generally expected to be associated with low-LET radiation.

As ide from repair, it has also been shown that radiation damage can be made expressed or fixed, which results in cell killing. Expression of damage nas been demonstrated by brief treatments of heat (Belli and Bonte, 1963; Westra and Dewey, 1971) or anisotonic solutions to the irradiated cells (Munro, 1969; Dettor et al., 1972; Raaphorst and Kruuv, 1976; Utsumi and Elkind, 1979).

The information derived from the studies of repair and expression of damage with heavy ions is fundamental to heavy-ion radiobiology, and it is essential to the ultimate development of biophysical models such as the repair-misrepair model (Tobias et al., 1980; Tobias, et al., Part III). Experiments of this nature are parallel to the studies on heavy-ion induced macromolecular lesions (Roots et al., Part III), mutation, and transformation (Yang et al., Part III), and complementary to the effects on cell proliferation kinetics (Blakely et al., Part III) caused by the high-LET radiation.

\section{DOSE-FRACTIONATION EFFECTS WITH HEAVY IONS}

Repair and Expression of Damage Following Fractionated Exposures

Dose-fractionation effects in chinese namster V79 cells nave been studied with unmodified carbon, neon, and argon beams of incident energies 400,425 , and $570 \mathrm{MeV} / \mathrm{MeV} / \mathrm{amu}$, respectively. As shown in Figure 1, two reference

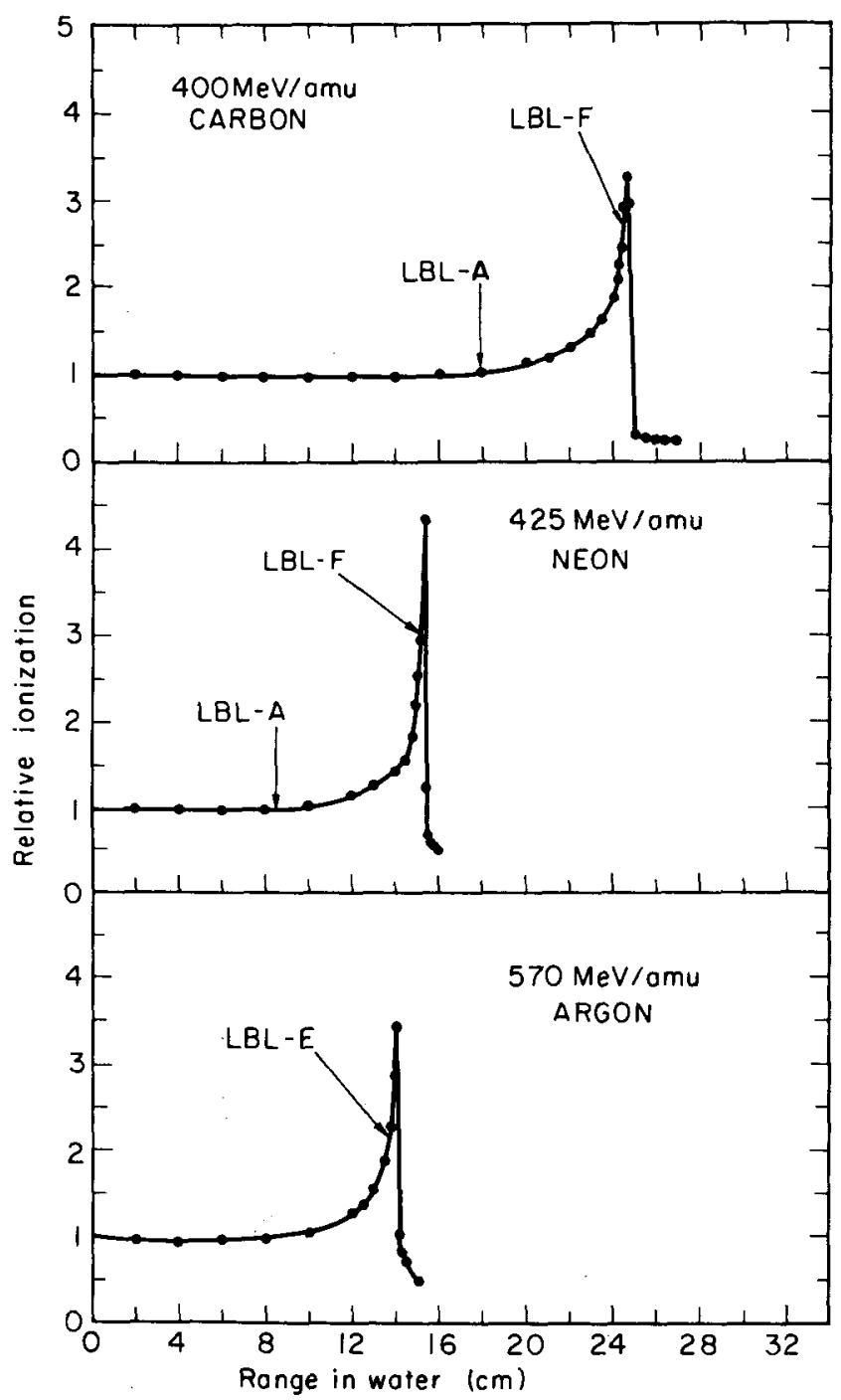

Figure 1. The Bragg ionization curves for $400 \mathrm{MeV} / \mathrm{amu} / 2 \mathrm{C}, 425 \mathrm{MeV} / \mathrm{amu} 20 \mathrm{Ne}$, and $4 \mathrm{Ar}_{\mathrm{Ar}}$ produced at Bevalac. The positions at which cell monolayers were irradiated are indicated by the arrows. The radiological properties at each irradiation positions are given in Table 1.

(XBL 7810-3671) 
positions along the Bragg curve have been chosen for these measurements: a position at the plateau region and a position near the Bragg peak. The results are shown in Figure 2. For carbon and neon beams, repair of sublethal damage is evident at the plateau position where the single-dose survival curves exhibit substantial shoulders. In contrast to this, at the peak positions of the same beams where the survival curves show little or no shoulder, there appears to be a potentiation effect due to the split-dose exposures.

This study suggests that for some biological systems there may be a fractionation gain factor for neavy-ion therapy, at least for the carbon and neon beams. Studies are needed with beam configurations (ridge-fitered) concurrently used in therapy in order to quantify the gain factor.

\section{Further Studies on Split-Dose Potentiation}

The potentiation effect following split-dose irradiation with Bragg-peak carbon, neon, and argon ions was somewhat surprising since it was unexpected on the basis of survival curve shapes.

A physical explanation for this observation can be ruled out since the probability for a finite number of particles to hit a critical target molecule is the same for a given dose whetner this dose is delivered all at once or in two fractions. It appears that a biological explanation is more likely. Since asynchronous cell populations were used in these experiments, an immediate consideration was whether the apparent potentiation only reflected an accumulation of cells in a more serisitive stage in the cell cycle at the time the second doses were delivered. We attempted to clarify this pnenomenon using a number of methods; our investigation concentrated on the $425 \mathrm{MeV} / \mathrm{amu}$ neon-ion beam near the Bragg peak (position designated $\mathrm{LBL}-\mathrm{F}$ as in $\mathrm{Fig} .1$ ).

\section{Cell-Stage Distribution}

In order to know the change of cell-stage distribution following heavy-ion irradiation, we applied the method of flow microfluorometry (FMF) which measures the DNA content of the cell population in question (Ngo et al., 1979). These experiments were conducted using the FMF spectroscopy at the Chemical Biodynamics Laboratory of $L B L$, and the mathematical analys is of the DNA nistogram was made by a computer program developed in the laboratory of J. Bartholomew. Figure 3 shows the changes in the distribution of cells in three phases of the division cycle, $G_{1}, S$, and $G_{2}+M$, as a function of incubation time after a single neon exposure given to an asynchronous population of $V 79$ cells.

The dose chosen for the FMF study corresponded to about $10 \%$ surviving level. Under the assumption that these FMF data are applicable to the survivors, one expects that within the initial $5 \mathrm{~h}$ after a first dose there is a small synchrony effect which leaves a population slightly more sensitive to the second dose; this effect should be more profound $8 \mathrm{n}$ after the first dose. This expectation was based on the observation that cells in $G_{2}+M$ phase are more sensitive to neon particles than those in $G_{1}$ or $S$ (Ngo et al., Part III). An attempt to correlate the cell-stage distribution derived from FMF to survival measurements of split-dose experiment is hampered by the inability of the FMF to

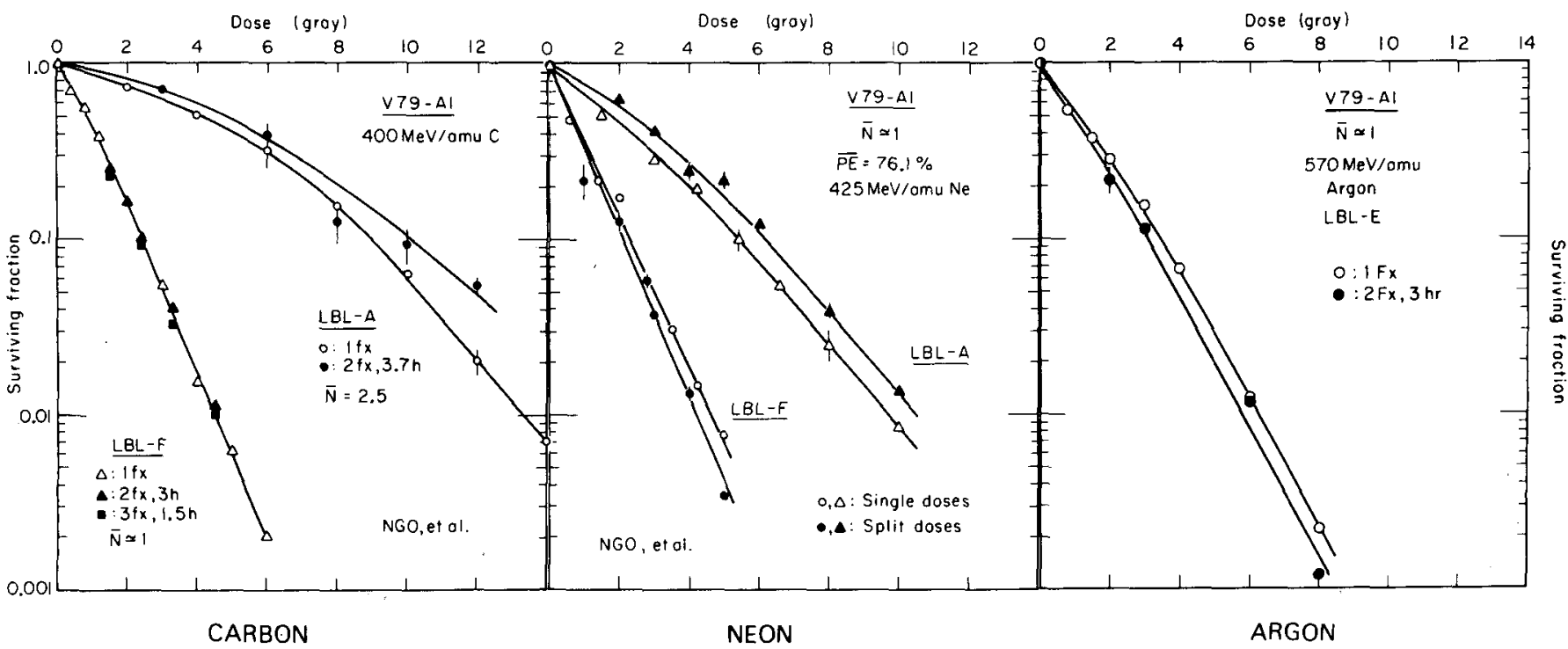

Figure 2. Survival data for asynchronous $V 79$ cells irradiated with single doses (open symbols) or fractionated doses (closed symbols) of carbon, neon, and argon ions at the Bragg curve positions:as designated. (XBL 7810-3668A) 


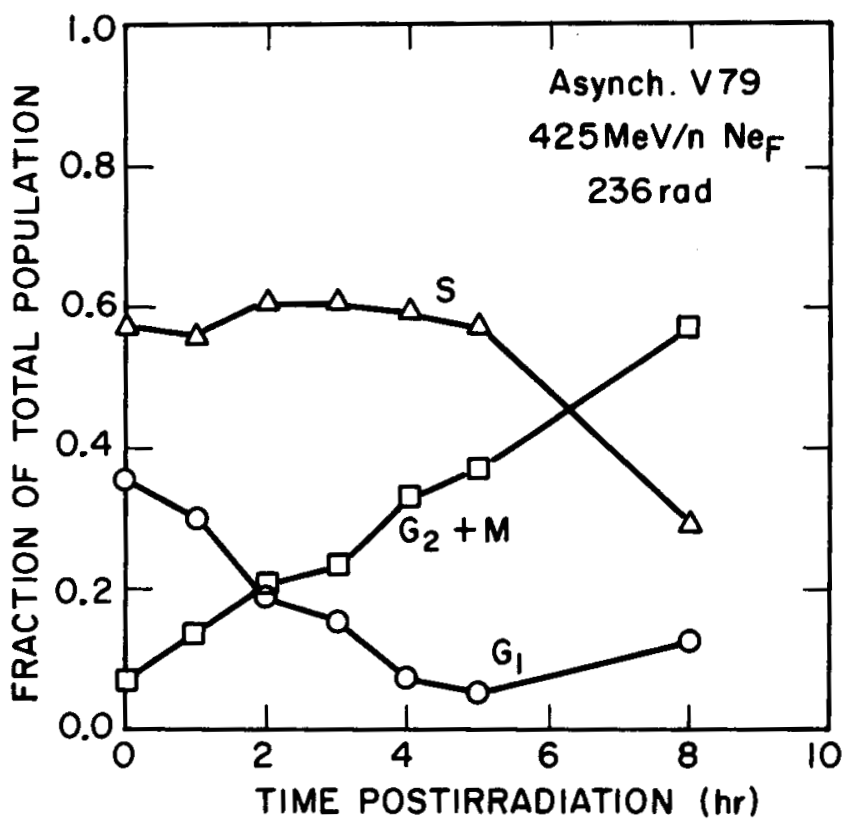

Figure 3. The redistributions of the relative fractions of $V 79$ cells in their $G_{1}, S$, and G2M stages of the cell cycle postirradiation of a neon-ion exposure at position $F$. The data were oDtained from FMF measurements of DNA content. (XBL 808-3651) distinguish between nonsurviving and surviving cells. Other types of experiments as discussed below were subsequently planned in order to better understand the significance of synchrony effect on the observed split-dose potentiation.

\section{Split-Dose Experiments with Room-Temperature Incubation}

At room temperatures, 179 cells are halted from progression, which is now one feature that can be used to help understand the split-dose effect. In the left panel of Figure 4 , cells were synchronized by mitotic selection method and about 5.5 nours later at $37^{\circ} \mathrm{C}$ they were irradiated with single- or split-dose schemes. The temperature during the course of split doses was about $22^{\circ} \mathrm{C}$, and the cultures were maintained in a $5 \% \mathrm{CO}_{2}$ incubator. The data clearly show that for the fractionated irradiation there is a small potentiation that can be observed without the presence of cell progression.

Another experiment, shown in the right panel of Figure 4, compares the split-dose effects with and without cell progression for dosefractionation intervals up to 7 hours. The data indicate that under either room temperature or $37^{\circ} \mathrm{C}$ incubation temperature tnere was a net potentiation which was slightly greater when
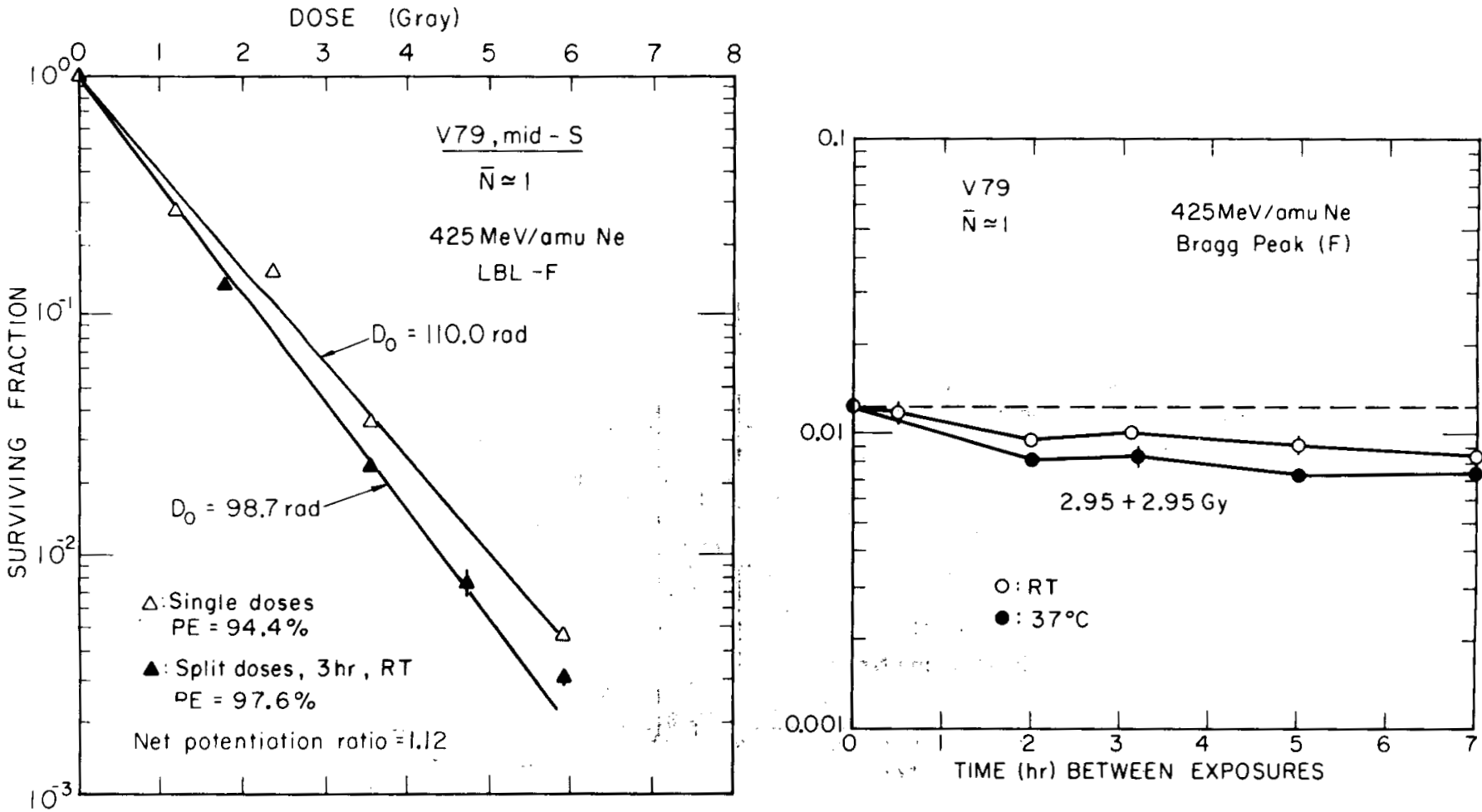

Figure 4. Further studies on the dose-fractionation effects on V79 cells with 20 Ne at position F. Left panel: survival data for single- and split-doses synchronized V79 cells in mid-S stage following splitdose irradiations. Right panel: survival data of asynchronous 179 cells irradiated with two doses. separated by various incubation times. (XBL 806-3389A) 
$37^{\circ} \mathrm{C}$ was used. These results imply that cell synchrony does play. a role in the expression of two-dose potentiation, consistent with the FMF measurements (Fig. 3 ), but clearly syncnrony is not the only factor.

\section{Split-Dose Experiments with Partially Synchronized Cells}

Still another method by which the split-dose effects were investigated was demonstrated by the experiments shown in Figure 5 using partially synchronized cells. In the left panel, synchronized cells were irradiated in the neon plateau and Bragg peak position at various times after mitosis. As expected, the amplitude of the cell-stage variation in response to the charged particles was larger for the lower-LET position (plateau $\mathrm{Ne}_{A}$ ) than for the nigh-LET position (Bragg peak $\mathrm{Ne}_{\mathrm{F}}$ ). In this experiment, the resistant phase lasted longer than we expected from our experience with cell-age response curves of $V 79$ cells. We suspect that this may be a result of inadequate temperature control for cell progression at the later phase of the experiment. However, this deficiency does not affect our interpretation of the study on split-dose effect. The curve with the closed circles traces the survival response of two-dose irradiation where the first dose was given at 2.5 hours and the second dose was given at various times later during which the samples were incubated under conditions similar to the control single-dose samples. At 2.5 nours, the cells were probably in late $G_{1}$ or on the $G_{1} / S$ border. Since $G_{1}$ cells are probably not retarded in their progress by ionizing radiation, as suggested by Sinclair (1972) and also by our. FMF measurements shown in Figure 3 , we expect that the irradiated cells after the first dose continue to progress toward $S$ stage. Thus, the more sensitive response associated with the two-dose scheme substantiates the potentiation effect.

This conclusion is also supported by the data shown on the right panel of Figure 5. In this experiment, the first dose of the two-dose scheme was delivered 6.5 nours after mitosis. For the plateau neon, the split-dose curve lies above the
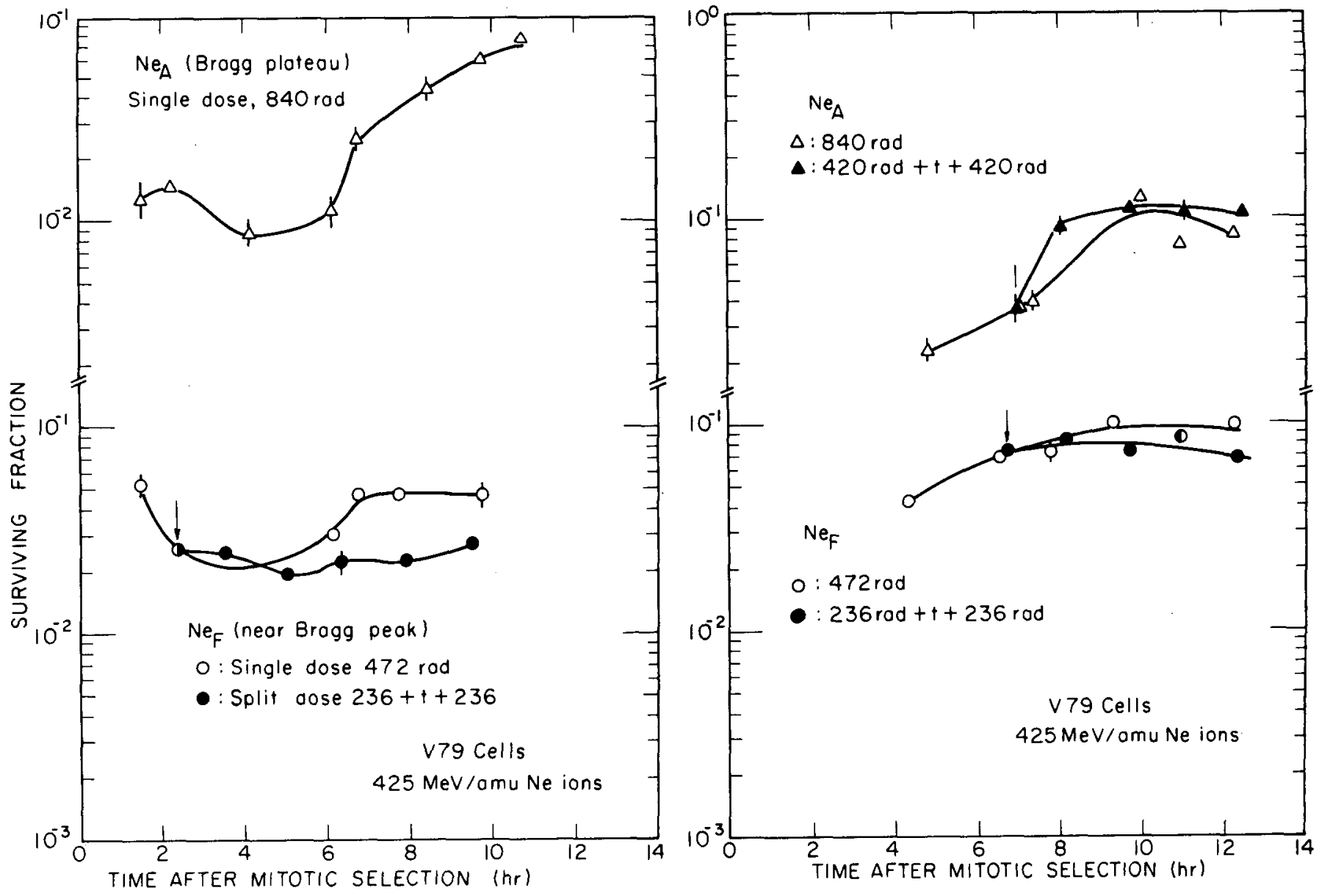

Figure 5. Survival responses of $V 79$ cells synchronized at mitosis and irradiated at various cell-cycle stages with single doses (open) or split doses (closed) of neon ions at either the Bragg plateau (upper curves) or the peak region (lower curves). The split-dose treatments started at $2.5 \mathrm{~h}$ (left panel) or $\sim 7 \mathrm{hrs}$ (right panel) after mitotic selection, and the samples were kept at $37^{\circ} \mathrm{C}$ between exposures. (XBL 808-3566A) 
single-dose curve, which indicates repair of sublethal damage. For the Bragg peak neon, however, there appears to be a small potentiation due to split doses.

Evidence for a Net Potentiation Following Split-Dose Irradiation with a Lower Energy Heavy-Ion Beam

The radiation quality for low-energy neavy ions is considerably different from the energetic charged particles available at the Bevalac. For example, the $8.5 \mathrm{MeV} / \mathrm{amu}$ argon ions produced at the SuperHILAC are characterized by almost negligible nuclear fragments compared to the few nundred MeV/amu neon or argon ions. In addition, the radius of the delta-ray penumbra spreading out laterally from the track of the primary particle is many times smaller for the lower energetic particles (Cnatterjee et al., 1973; Cnatterjee and Schaefer, 1976). "Furthermore, the mean LET of this ion beam is in the overkill domain, which is far beyond the maximum relative biological effect region.

It is of fundamental interest to find out if this extremely high LET radiation also produces a potentiation effect at fractionated irradiation schedule. To address this question, we have conducted an experiment similar to those in Figure 5 . We used the same mitotic-cell population for both $X$ rays and the high-LET argon particles. ( $X$ rays were chosen as a reference radiation.) Figure 6 shows the cell-age response curves of $1,200 \mathrm{rad}$ $X$ rays and 2,000 rad argon ions. The overkill characteristic and the almost negligible doseresponse variation with respect to cell stage are apparent for the argon ions. Nevertheless, a two-dose potentiation was still evident with the overkill particle bean which contrasts the two-dose repair demonstrated with $X$ rays.

Repair of Sublethal Damage vs. Protein Synthesis

In order to better understand repair of sublethal damage, the molecular mechanisms involved in this repair process need to be studied. Using a temperature-sensitive mutant of the chinese namster ovary cells and $x$ rays, Yezzi conducted experiments to determine if protein synthes is plays a role in this process (Yezzi et al., 1980). Upon treatment with $40^{\circ} \mathrm{C}$ the mutant exhibits an innibition of protein synthesis, whereas the wild type cell was essentially unaffected. Some results of this study are shown in Figure 7. The data show that when the cultures were incubated at $40^{\circ} \mathrm{C}$ for: $2 \mathrm{n}$ before the first dose and maintained at $40^{\circ} \mathrm{C}$ during a $2 n$ dose-fractionation interval, repair of radiation damage was reduced in the mutant compared to the wild type. These observations imply that protein synthesis is involved in the repair of sublethal X-ray damage.

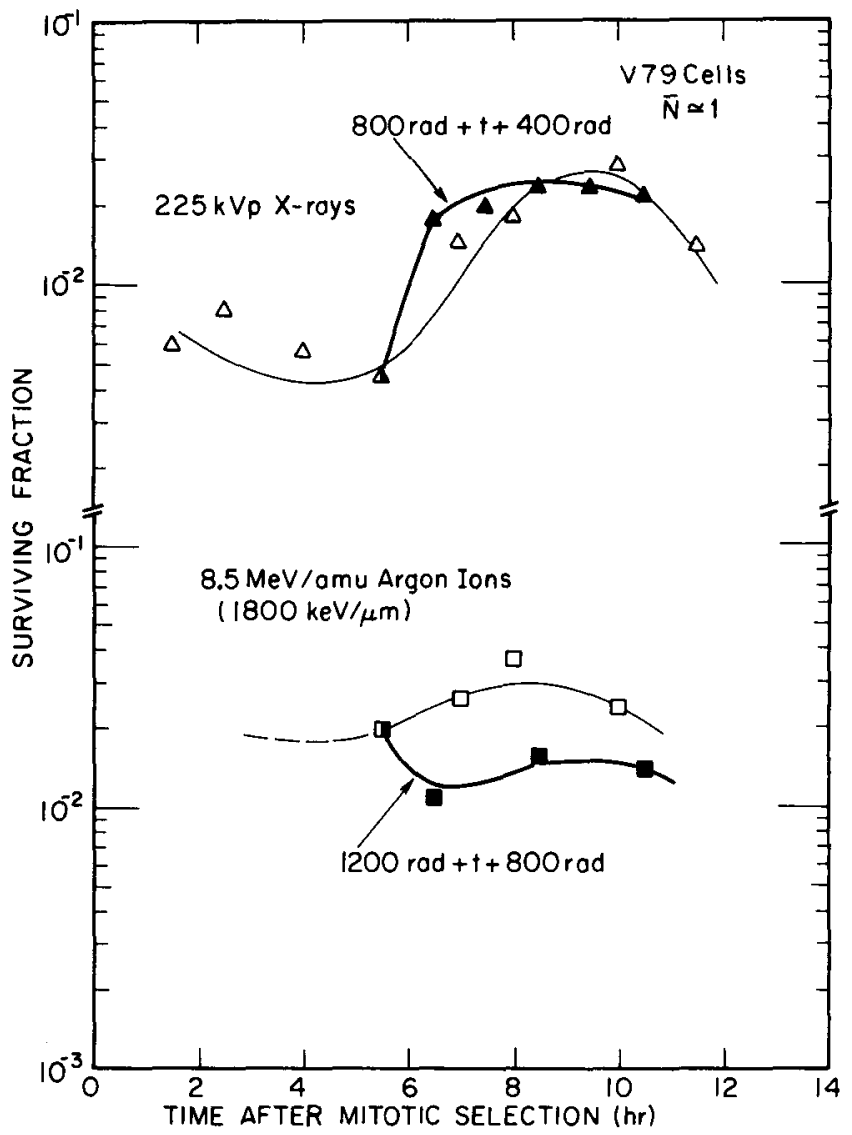

Figure 6. Survival responses of $V 79$ cells synchronized at mitosis and irradiated at various cell-cycle stages with single (open) or split (closed) doses of $x$ rays (upper) or argon ions (lower). The same starting cell population was used for botn radiation types. The split-dose treatments started at $5.5 \mathrm{~h}(\sim \operatorname{mid} \mathrm{S})$ after mitotic selection, and between exposures the samples were kept at $37^{\circ} \mathrm{C}$. (XBL 7910-3823)

\section{REPAIR AND EXPRESSION OF POTENTIALLY LETHAL DAMAGE}

\section{Expression of Radiation-Induced Potentially Letha 7 Damage in Actively Growing Cells by Anisotonic Treatments}

The effects of treatment with anisotonic solutions on cellular radiosensitivity have been studied by a number of investigators (Raaphorst and Kruuv, 1976; Dettor et a 1., 1972; Utsumi and Elkind, 1979). An exposure to a nonlethal dose of nypo- or nypertonic solutions can ennance the cellular response to $X$ rays or fast neutrons (Ngo et al., 1977). Utsumi and Elkind (1979) found that the effect on cells exposed to $x$ rays followed by $0.5 \mathrm{M} \mathrm{NaCl}$ in phosphate buffer saline (PBS) was mainly a reduction in the repair of 

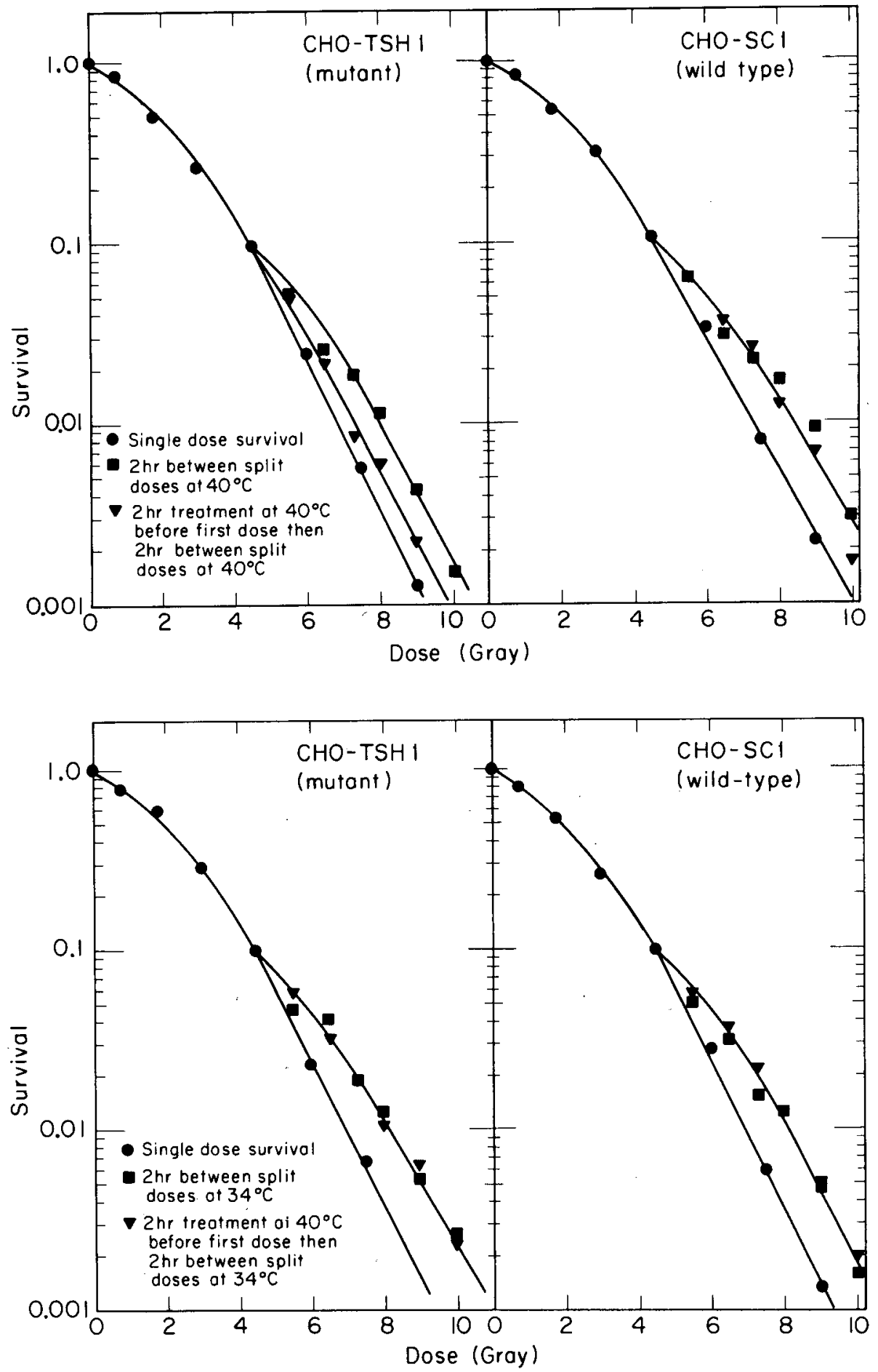

Figure 7. Survival data of a temperature-sensitive mutant, CHO-TSHI, and its wild type, CHO-SCI. $225 \mathrm{kVp} X$ rays were given in graded single doses or in fractionated doses according to the protocols described in the figures. (XBL 805-3338A) 
PLD, and has little or no effect on repair of suolethal damage.

We have designed experiments by incorporating anisotonic salt treatments with exposure to heavy ions of various quality to investigate the following questions:

1. Is there a sequence dependence between salt treatment and radiation on the radiosensitization effect of the anisotonic treatments?

2. How does the sensitization effect of anisotonic treatments vary with radiation quality?

3. Can the action of a single-nit mechanism with radiation be modified by treatment with salt solutions?

4. Does the repair rate of the PLD in question differ depending on radiation quality?

For this study, we chose carbon and neon particles at the Bevalac with mean $L E T_{\infty}$ values of $16,38,85$, and $234 \mathrm{keV} / \mathrm{\mu m}$. The anisotonic and isotonic solutions were prepared in PBS with concentrations of $\mathrm{NaCl}$ at $0.04 \mathrm{M}$ (nypotonic), $0.5 \mathrm{M}$ (nypertonic) and $0.14 \mathrm{M}$ (isotonic). The $\mathrm{pH}$ of all the salt solutions was adjusted to 7.3-7.4, a normal pH for the cultured cells. The exposure time of cultures to each salt solution was 20 minutes at $37^{\circ} \mathrm{C}$. The $\mathrm{NaCl}$ concentrations for the hypo- and hypertonicity were selected because eacn treatment alone is nonlethal, and their radiosensitization effects for cells post $X$-irradiation are approximately the same.

The survival responses of $V 79$ cells exposed to each radiation and then treated with the $0.14 \mathrm{M} \mathrm{NaCl} / \mathrm{PBS}$ solution are shown in Figure $8 \mathrm{~A}$. Treatment of the cells with the isotonic solution did not significantly alter the radiation responses, and was used as our controls. Experiments have Deen completed with both $0.04 \mathrm{M}$ and $0.05 \mathrm{M} \mathrm{NaCl} / \mathrm{PBS}$ (Ngo et al., 1980a). However, due to space limitation, only the survival data pertaining to the control and the hypertonicity are presented here. Figure 8B-E demonstrates the effects of $0.5 \mathrm{M} \mathrm{NaCl} / \mathrm{PBS}$, given either prior to and during irradiation or immediately subsequent to radiation. The data clearly show that the postirradiation treatment is more effective than treatment given before and during irradiation, and that the radiosensitization effect of the hypotonic treatment diminishes with increasing LET. These results imply that anisotonicity can effectively inhibit the repair process of some radiation-induced lesions after they are formed. Furthermore, fast radiation cnemistry is not required in this inhibition mechariism.

To examine the salt effects of the initial and final slopes of the radiation survival curves as a function of LET, we have computer-fitted the survival data for each salt treatment including $0.04 \mathrm{M} \mathrm{NaCl} / \mathrm{PBS}$ (survival data not shown). Two classical models were used for the fitting; the linear quadratic model (Sinclair, 1966; Kellerer and Rossi, 1972; Chadwick and Leenhouts, 1973):

$$
S=z \operatorname{Exp}\left[-\alpha D-B D^{2}\right]
$$

and the modified single-nit multitarget model (Zimmer, 1961; Bender and Gooch, 1962):

$$
S=z e^{-a D}\left[1-\left(1-e^{-b D}\right)^{n}\right]
$$

The survival parameters $D_{0}$ (the mean lethal dose), and $\mathrm{D}_{\mathrm{q}}$ (the quasitnresnold dose) were determined from equation (2) as described elsewhere (Ngo et al., 1980b). Table 1 summarizes the computer fitted values of the parameters $\alpha$, $B, D_{0}$, and $D_{q}$ for postirradiation treatments of $0.04 \mathrm{M}, 0.14 \mathrm{M}$, and $0.5 \mathrm{M} \mathrm{NaCl} / \mathrm{PBS}$ for each of the radiation qualities.

Hence, the changes in the initial and the final slopes of the survival curve with respect to the controls can be defined by the enhancement ratios:

$$
(\alpha)_{\text {anisotonicity }} /(\alpha) \text { isotonicity }
$$

and

$$
\left(D_{0}\right)_{\text {isotonicity }} /\left(D_{0}\right) \text { anisotonicity, }
$$

respectively. Figure 9 demonstrates such changes as a function of LET. As snown on the right panel of Figure 9, the effect of salt treatments on the final slope (nigh-dose region) decreases as LET increases, and there is virtually no difference between hypo- and hypertonic postirradiation treatments. On the other hand, the left panel shows that there may be some differences between the hypo- and hypertonicity results with respect to effects on the initial slope (the low dose region), particularly for the lower LET carbon beam. In the hypertonic situation, the ennancement ratio for the inibiat slopes appears to decrease with increasing LET, but does not reach 1 at the nighest LET radiation used in this study. In the hypotonic situation; this ratio does not differ from 1 significantly, which suggests that hypotonicity is less effective compared to nypertonicity in modifying the single-nit mechanism.

Preliminary results have been obtained regarding, the repair rate of the PLD which can be made lethal by the salt treatments. Figure 10 shows the diminution of the sensitization effect as the time between treatments of radiation and $0.5 \mathrm{M} \mathrm{NaCl/PBS}$ was prolonged. The kinetics of the loss of sensitization represents the repair kinetics associated with the PLO. The half-time of the initial repair was about 15 minutes and was essentially independent of the quality of radiation that induced the lesions. This relatively fast repair kinetics is quite different from that for sublethal damage, supporting the conclusion reached earlier by Utsumi and Elkind (1979) that this type of potentially lethal damage differs from sublethal damage. 

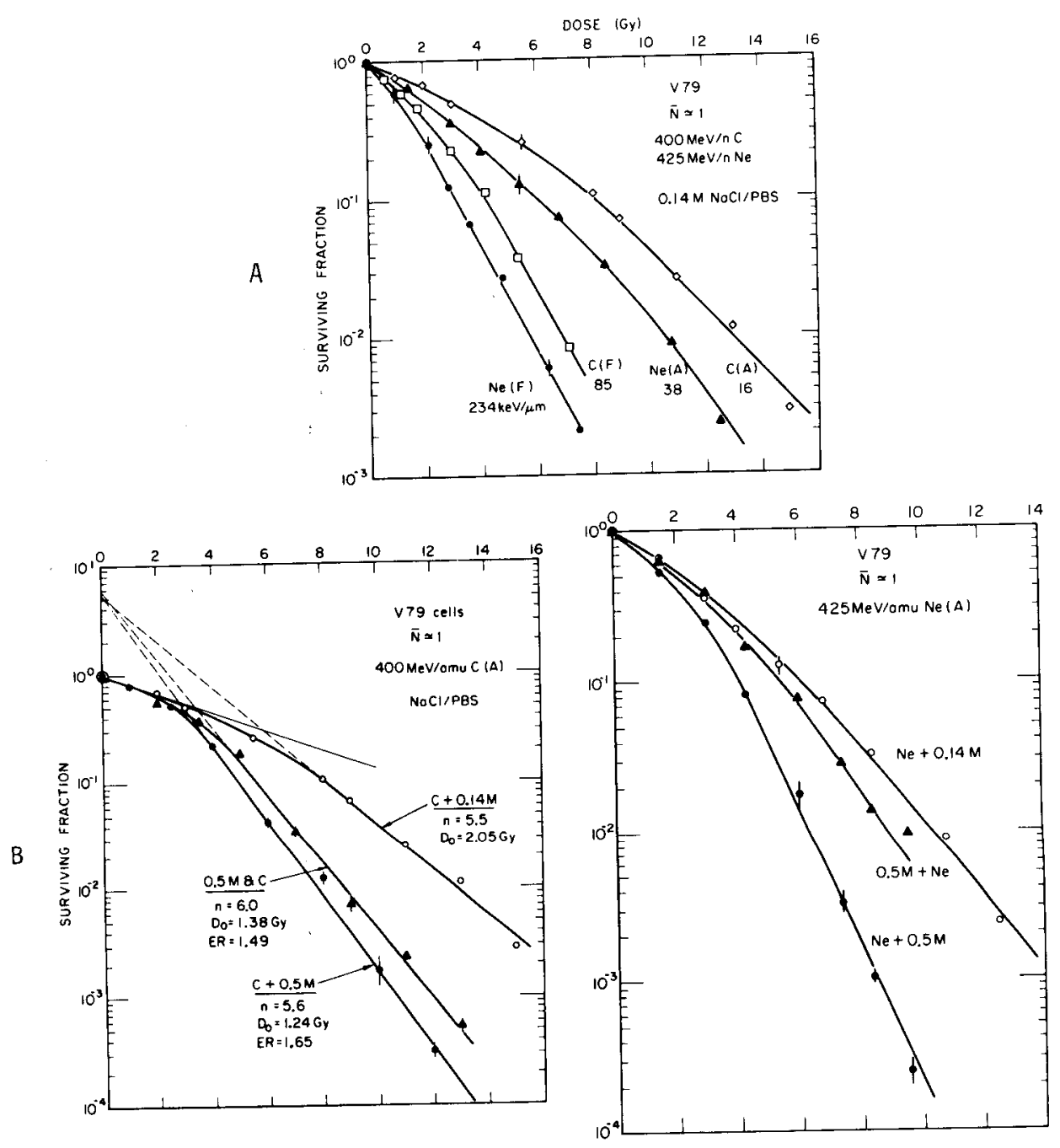

C
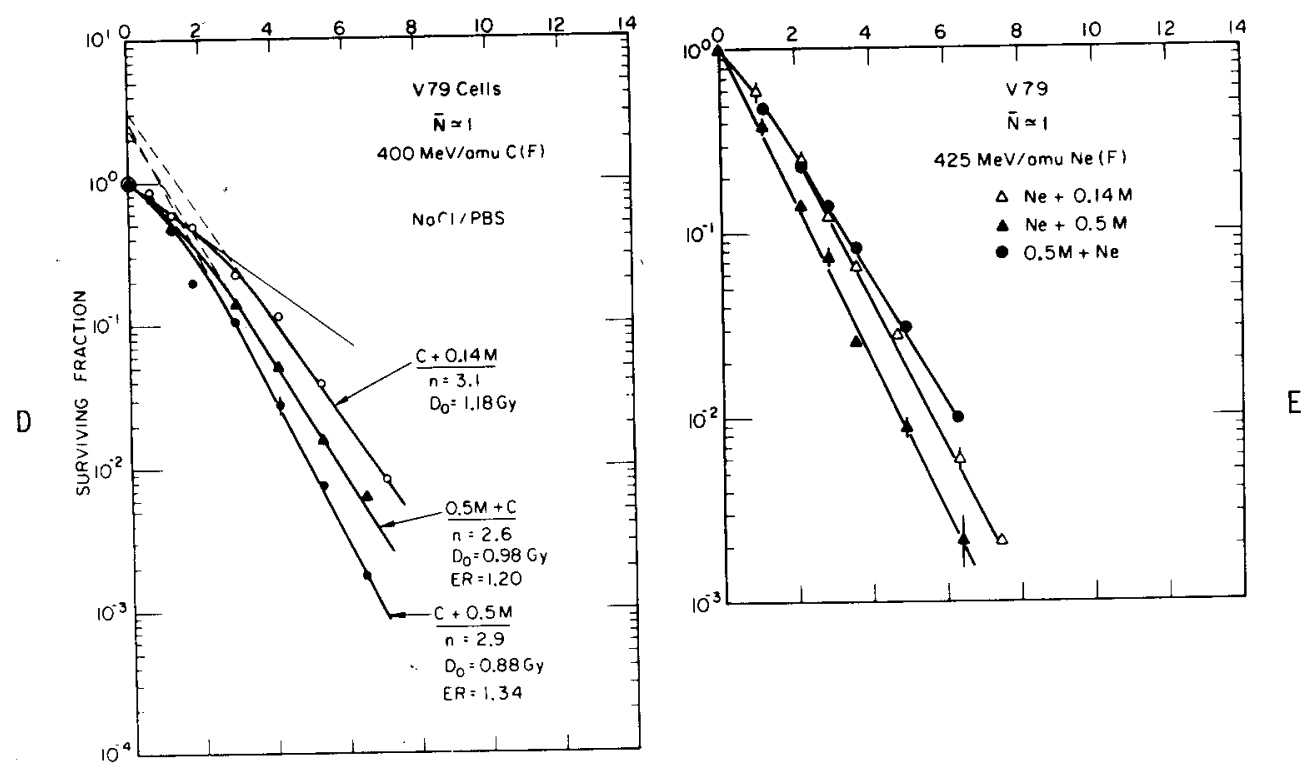

Figure 8. (A) Survival data of V79 cells irradiated various heavy-ion beams of four different LET values. The cells were treated with isotonic salt/PBS solution for 20 minutes at $37^{\circ} \mathrm{C}$ postirradiation. (B) througn (E): The effects of hypertonic solution $(0.5 \mathrm{M} \mathrm{NaCl} / \mathrm{PBS})$ given before and during irradiation or immediately after irradiation. For each radiation, data obtained with postirradiation treatment of isotonicity served as controls. (XBL 806-3387A) 
Table 1. Computer-Fitted Values for the Parameters $\alpha, \beta, D_{0}$, and $D_{q}$ for Equations 1 and 2

Survival

Curve

$\mathrm{NaCl} / \mathrm{PBS}$ Parameters $\mathrm{C}$ (Position A) Ne (Position A) C (Position F) Ne (Position F)

\begin{tabular}{|c|c|c|c|c|c|}
\hline $0.05 \mathrm{M}$ & $\begin{array}{l}\alpha\left(x \quad 10^{-3}\right) \\
B\left(x \quad 10^{-6}\right) \\
D_{0} \\
D_{q}\end{array}$ & $\begin{array}{l}3.330(0.633) * \\
2.708(0.550) \\
156.3(0.2) \\
269.3(52.5)\end{array}$ & $\begin{array}{l}3.440(0.330) \\
5.545(0.412) \\
129.0(0.2) \\
161.6(33.7)\end{array}$ & $\begin{array}{ll}6.608 & (1.059) \\
4.875 & (1.959) \\
110.0 & (0.2) \\
105.1 & (15.7)\end{array}$ & $\begin{array}{l}9.248(0.656) \\
0.755(1.264) \\
103.8(0.0) \\
18.9(0.9)\end{array}$ \\
\hline $0.14 \mathrm{M}$ & $\begin{array}{l}\alpha\left(x 10^{-3}\right) \\
\beta\left(x 10^{-6}\right) \\
D_{0} \\
D_{q}\end{array}$ & $\begin{array}{ll}1.692 & (0.171) \\
1.444 & (0.140) \\
292.0 & (0.2) \\
497.8 & (96.7)\end{array}$ & $\begin{array}{ll}2.896 & (0.270) \\
1.484 & (0.265) \\
230.1 & (0.2) \\
288.3 & (45.2)\end{array}$ & $\begin{array}{l}3.640(0.310) \\
4.428(0.533) \\
163.0(0.2) \\
184.4(34.4)\end{array}$ & $\begin{array}{l}6.535(0.418) \\
2.346(0.680) \\
126.1(0.1) \\
51.1(4.4)\end{array}$ \\
\hline $0.04 M$ & 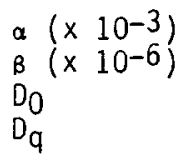 & $\begin{array}{l}1.125(0.696) \\
6.688(0.704) \\
134.0(0.4) \\
230.8(86.7)\end{array}$ & $\begin{array}{ll}3.090 & (0.351) \\
6.453 & (0.438) \\
123.0 & (0.2) \\
154.1 & (35.0)\end{array}$ & $\begin{array}{l}7.963(0.709) \\
3.505(1.313) \\
101.6(0.1) \\
47.8(4.2)\end{array}$ & $\begin{array}{l}9.061(0.399) \\
1.036(0.769) \\
103.9(0.0) \\
18.9(0.7)\end{array}$ \\
\hline
\end{tabular}

*Figures in parentheses $=95 \%$ confidence interval.

The values of the survival parameters derived from least-squared fitting of the $V 79$ cell survival data to equations (1), and (2) given in the text. Comparisons are made for hypertonic $(0.5 \mathrm{M} \mathrm{NaCl})$, isotonic $(0.14 \mathrm{M} \mathrm{NaCl})$, and hypotonic $(0.04 \mathrm{M} \mathrm{NaCl})$ treatments postirradiation of four radiation qualities. The units for these parameters are: $\alpha\left(\operatorname{rad}^{-1}\right), B\left(\operatorname{rad}^{-2}\right), D_{0}(\operatorname{rad})$, and $D_{q}(\operatorname{rad})$.

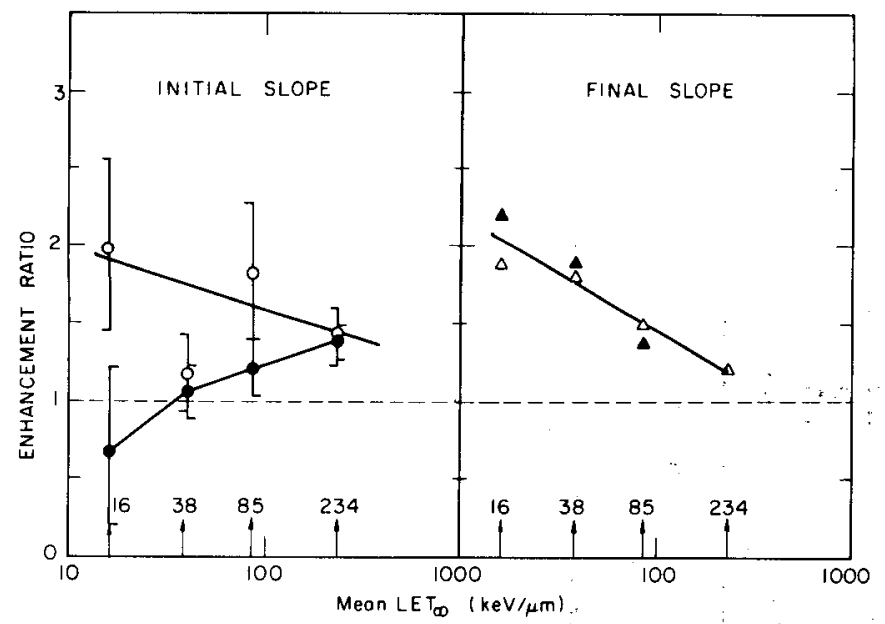

Figure 9. The effects of postirradiation treatments with nypertonicity $(0.5 \mathrm{M} \mathrm{NaCl}$, closed symbols) on the initial slope (left panel) and the final slope (right panel) of survival curves as a function of LET values. The enhancement ratios are as defined in the text, and the calculations were made based on the best-fit $\alpha$ and $D_{0}$ values given in Table 1 . The error bars represent $95 \%$ confidence limits. (XBL 805-3363A)

\section{Repair of Potentially Lethal Damage in Confluent Cells}

Repair of PLD inflicted by heavy ions or $X$ rays has been investigated by $T$. Yang using the delayed plating technique. Mouse embryo cells, C3H10T1/2, which are widely used in chemicaland radiation-induced transformation studies, were grown to a conflict phase at which cell proliferation stopped because of contactinhibition characteristics. Survivals are compared between confluent cells that were trypsinized immediately after irradiation and those that were kept in confluent phase for various lengths of time before plating. Prolonged incubation of these cells, up to two days, showed no appreciable effect on plating efficiency. Some typical experiments are snown in Figure 11. Figures $11 \mathrm{~A}$ and $11 \mathrm{~B}$ show a rather interesting finding: repair of PLD was almost completed within $6 \mathrm{~h}$ after X-irradiation while it could take as long as $24 \mathrm{~h}$ to a completion after an exposure of argon particles.

A comparison of the survival curves in Figure $11 \mathrm{C}$ and 110 indicates that repair of PLD in the $10 T 1 / 2$ cells occurs in three types of radiation with widely different LET. In fact, this phenomenon was found with all heavy ion 
Table 2. Repair Factors for Potentially Lethal Damage Caused by Ionizing Radiations of Various Qualities in Confluent Mouse 10T1/2 Cells in Culture

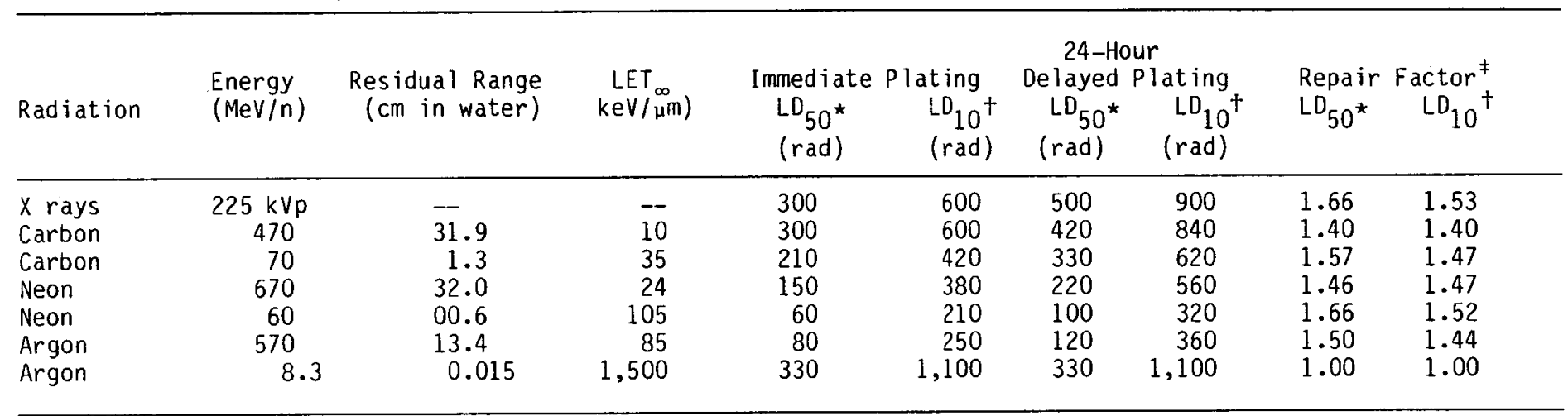

$\star_{L} D_{50}=$ dose required to yield 50 survival.

$T_{L D} 10=$ dose required to yield 10 survival.

${ }_{\text {Repair }}$ Factor $=\frac{\text { Dose that yields a given surviving fraction by a delayed plating }}{\text { Dose that yields the same surviving fraction by immediate plating }}$ 


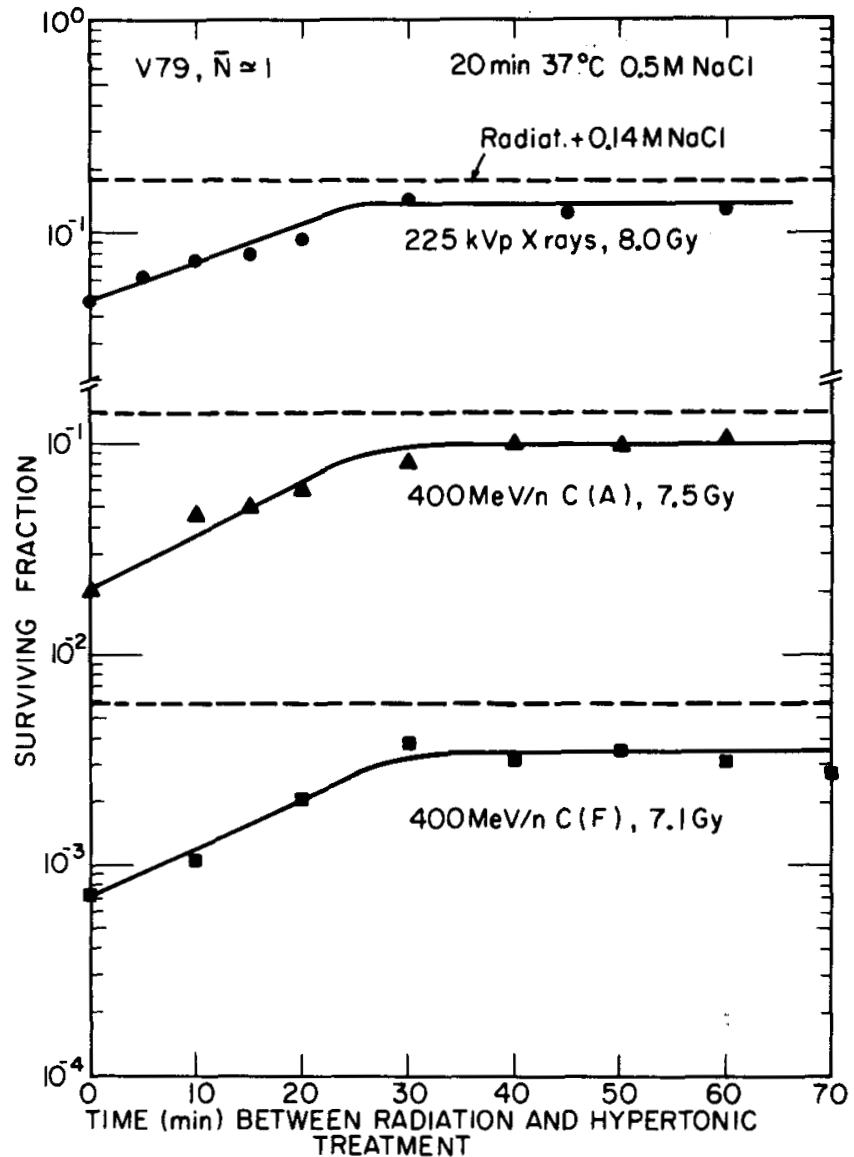

Figure 10. PLD repair kinetics for actively growing V79 cells. The PLD can be made lethal by hypertonic salt treatments. Comparisons are made between $X$-rays, carbon plateau $(A)$, and carbon peak region (F) at doses as indicated. (XBL 805-3346).

beams of appreciable incident energy (Table 2): The generality of PLD repair observed nere after nigh-LET irradiation may not be entirely inconsistent with the lack of PLD repair with fast neutrons or alpha particles reported in the literature, since most of the published work measured repair for only comparatively short postirradiation times (e.g., Snipley et al., 1975; Raju et al., 1977): Tabile 2 shows an exception with a low-energy argon particle beam with an LET value corresponding to the overkill region. There, repair of PLD appears to be absent (repair factor $=1.00$ ).

\section{SUMMARY}

Considerable information has been gained with respect to the effects of dose fractionation, and the repair of sublethal and potentially lethal damage for neavy ions with a wide range of LET values.
Two distinct cellular processes are apparent with dose fractionation schemes: cells can repair sublethal damage, and can also be made more vulnerable by a preexposure of radiation, an effect we have called "potentiation." We have shown that the potentiation is a consequence of at least two mechanisms. With asynchronously growing cells, radiation-induced synchrony is one mechanism; a second mechanism, as evident from synchronized-cell experiments, is yet unknown. In this regard, we nypothesize that an injured cell may become more sensitive to the second set of injurious events, presumably during its course of repair where conformational or tertiary alterations of critical molecules are required. This nypothesis needs quantitative cellular biochemistry experiments to be proven.

Note that an enhancement of radiobiological effects by nigh-LET dose fractionation has been observed by other investigators: e.g., crypt cell survival studies (Goldstein and Phillips in Part IV), and life span studies (Ainsworth in Part V). The correlation between those observations and the potentiation described in this paper remains to be elucidated.

The fact that there is a sparing effect in the plateau region of the Bragg curve with dose fractionation schedules, and that a potentiation effect exists near the peak region implies that there may be a depth-RBE advantage for fractionated heavy-ion therapy. This possible advantage would be more apparent if the two-dose potentiation effect observed in $V 79$ cells is relevant to the rapidly proliferating human tumors. This potential usefulness remains to be evaluated by our clinical team.

Two different processes of PLD repair have been observed. In actively growing hamster $V 79$ cells, the repair of the PLD that can be expressed by hypertonicity treatment is rapid, with a half time of 15 minutes, and the kinetics appears to be independent of radiation quality. In confluent mouse cells, the repair time of PLD inflicted by hign-LET radiation may be as long as 24 hours, much slower than that observed with $X$ rays. The differences found in these cellular studies indicate that one is dealing with a complexity of repairable damage or processes, some of which are LET dependent. Furthermore, this investigation further substantiates a current concept that an apparent single-nit inactivation process, as implied by an exponen$t i a l$ dose-effect curve, cannot exclude the existence of repair processes (Ngo et al., 1980b).

\section{ACKNOWLEDGEMENT}

The authors would like to acknowledge the excellent technical assistance of P. Chang, $L$. Lommel, L. Craise, K. Smith, O. Olezsko, and D. Tse. This work was supported by the National Cancer Institute Grant CA15184 and the NASA Radiation Effects Research Program, Contract No. T3516G. M. Yezzi is a recipient of an NIH Training Grant No. 403845-32951. 


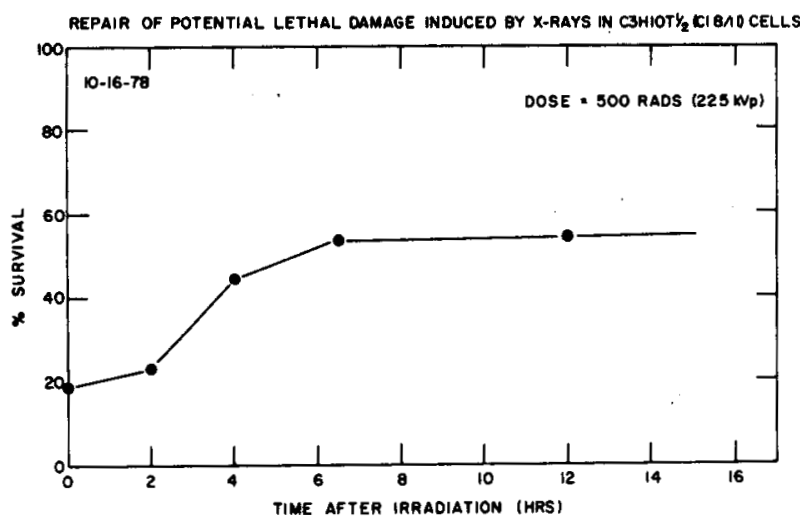

A

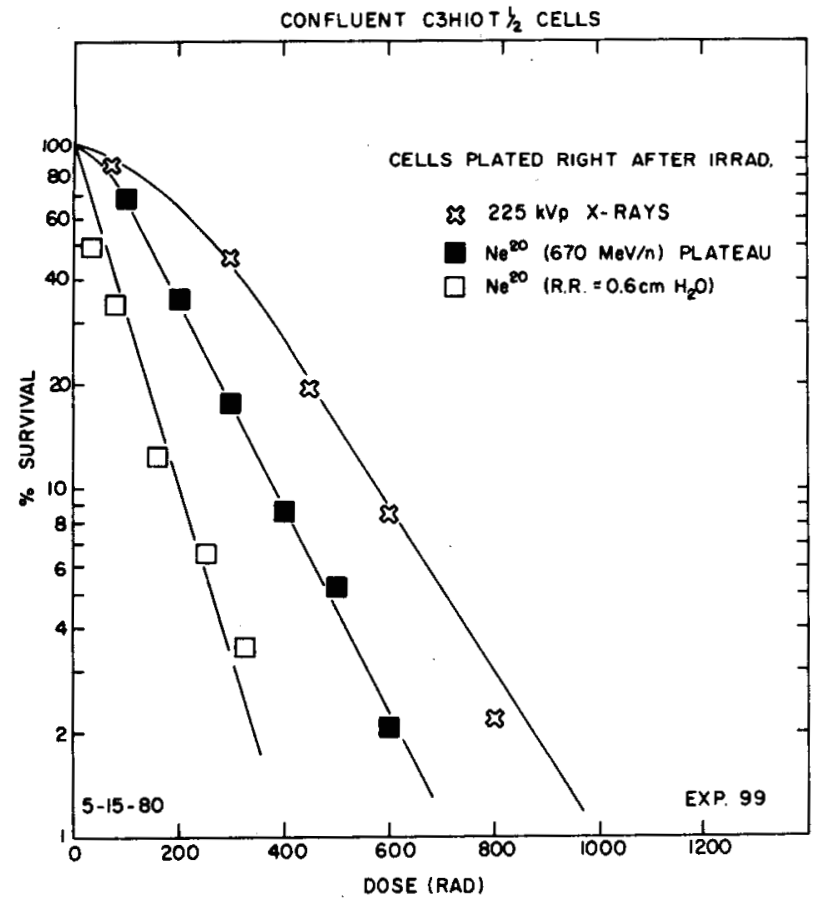

C
REPAIR OF POTENTIAL LETHAL DAMAGE INDUCED gY ARGON PARTICLES $(570 \mathrm{MeV} / \mathrm{n}$ ) IN C3H $10 \mathrm{~T} / \mathrm{k} / \mathrm{C}$ (C18/9) CELLS

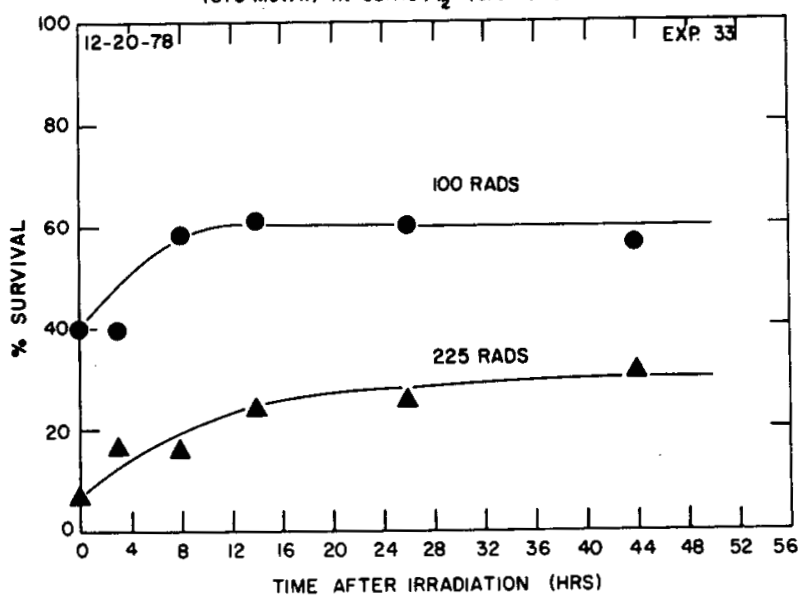

B

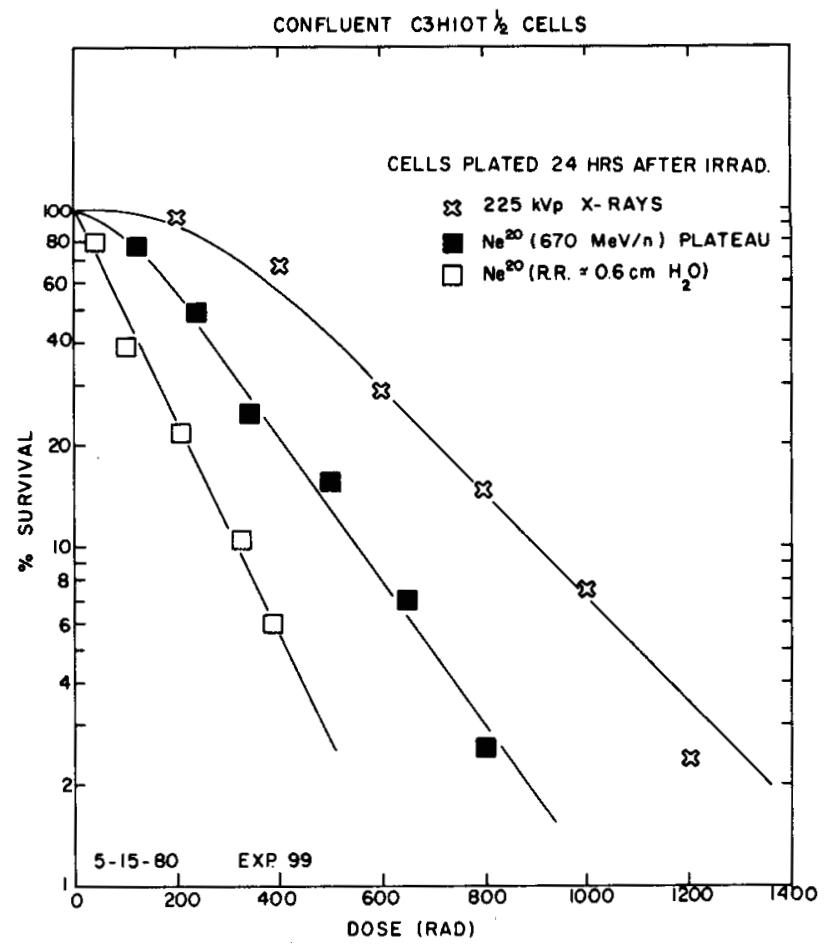

D

Figure 11. Repair kinetics of PLD in confluent C3H10Tl/2 cells irradiated with $X$ rays $(A)$ or argon ions $(B)$, as assayed by delayed plating. Survival data shown in $(C)$ and $(D)$ demonstrate repair of PLD $24 \mathrm{~h}$ postirradiation for three different radiation qualities. [(A) 7811-13162; (B) XBL 791-7712;

(C) XBL 807-10657; (D) XBL 807-10656] 


\section{REFERENCES}

Belli, J. A. and F. J. Bonte. 1963. Influence of temperature on radiation response of mammalian cells in tissue culture. Radiat. Res. 13, 556-593.

Bender, M. A. and Gooch P. C. 1962. The kinetics of $X$-ray survival of mammalian cells in vitro. Int. J. Radiat. Biol. 5, 133-145.

Chadwick, K. H. and H. P. Leennouts. 1973. A molecular theory of cell survival. Phys. Med. Biol. 18, 78-87.

Chatterjee, A., H. D. Maccabee and C. A. Tobias. 1973. Radial cutoff LET and radial cutoff dose calculations for heavy charged particles in water. Radiat. Res. 54, 470-494.

Cnatterjee, A. and H. J. Schaefer. 1976. Microdosimetric structure of heavy ion track in tissue. Radiat. Environ. Biophys. 13, 215-277.

Dettor, C. M., W. C. Dewey, L. F. Winans, and J. S. Noel. 1972. Ennancement of X-ray damage in synchronous chinese hamster cells by hypertonic treatments. Radiat. Res. 52 , $352-372$.

Elkind, M. M. and H. Sutton. 1960. Radiation response of mammalian cells grown in culture. I. Repair of X-ray damage in surviving Chinese namster cells. Radiat. Res. 13, 556-593.

Kellerer, A. M. and H. H. Rossi. 1972. The theory of dual radiation action. Curr. Top. Radiat. Res. Q. 8, 85-158.

Munro, T. R. 1969. Effects of nypertonic sucrose on the radiosensitivity of Chinese namster cells. Radiat. Res. 13, 556-593.

Ngo, F.Q.H., A. Han, H. Utsumi, and M. M. Elkind. 1977. Comparative radiobiology of fast neutrons. Relevance to radiotherapy and basic studies. Int. J. Radiat. Oncol. Biol. Phys. 3 , 187-193.

Ngo, F.Q.H., E. A. Blakely, C. A. Tobias, P. Y. Chang, and K. C. Smith. 1978. Fractionation gain factor from therapeutic heavy-ion beams. Int. J. Radiat. Oncol. Biol. Pnys. 4 (Supp 1.2), 92. (Abstract).

Ngo, F. Q. H., E. A. Blakely, and C. A. Tobias. 1979. Potentiation of cell killing due to dose fractionation: An effect that influences Elkind repair. Int. J. Radiat. Onc. Biol. Phys. 5 (Supp 1. 2), 131. (Abstract).

Ngo, F.Q.H., C. A. Tobias, E. A. Blakely, P. Y. Chang, 0. Oleszko, and L. Lomel. 1980a. Radiation quality, damage repair, and anisotonicity. Radiat. Res. 83,402 (Abstract).

Ngo, F.Q.H., E. A. Blakely, and C. A. Tobias. 19800. Sequential exposures of mammaliancells to low- and nigh-LET radiations. I. Lethal effect following $X$-ray and neon-ion irradiation. Radiat. Res., in press.

Phillips, R. A. and L. J. Tolmach. 1966. Repair of potentially lethal damage in X-irradiated HeLa Cells. Radiat. Res. 29, $413-432$

Raaphorst, G. P. and J. Kruuv. 1976. Effect of tonicity on radiosensitivity of mammalian cells. Int. J. Radiat. Biol. 29, 493-500.

Raju, M. R., J. B. Frank, E. Bain, T. T. Trujillo, and R. A. Tobey. 1977. Repair of potentially lethal damage in $\mathrm{CHO}$ cells by $X$ and $\alpha$ irradiation. Radiat. Res. 71, 614-621.

Snipley, W. U., J. A. Stanley, V. D. Courtenay, and S. B. Field. 1975. Repair of radiation damage in Lewis lung carcinoma cells following in situ treatment with fast neutrons and gamma rays. Cancer Res. 35, 934-938.

Sinclair, W. K. 1966. The shape of radiation survival curve of mammalian cells cultured in vitro. Biopnysical Aspects of Radiation Quality, pp. 21-43. Technical Report Series 58. International Atomic Energy Commission, Vienna.

Sinclair, W. K. 1972. Sensitivity to mitotic delay and stage in the cycle. Curr. Top. Radiat. Res. Q. 7, 323-327.

Tobias, C. A., E. A. Blakely, F.Q.H. Ngo, and T.C.H. Yang. 1980. The repair-misrepair model of cell survival. Radiation Biology in Cancer Research (R. E. Meyn and R. H. Withers, eds.) pp. 195-230. Raven Press, New York.

Utsumi, H. and M. M. Elkind. 1979. Potentially lethal damage versus sublethal damage: Independent repair processes in actively growing Chinese namster cells. Radiat. Res. 77, $346-360$

Westra, A. and W. C. Dewey. 1971. Variation in sensitivity to neat shock during the cell-cycle of Chinese namster cells in vitro. Int. J. Radiat. Biol. 19, 467-477.

Yezzi, M., C. A. Todias, and E. A. Blakely. 1980. Repair of sublethal X-ray damage in a temperature sensitive mammalian cell. Radiat. Res. 83, 406. (Abstract).

Zimmer, K. G. 1961. Quantitative Radiation Biology. 01 iver and Boyd, Edinburgh, Scotland. 
102

Blank 


\title{
EFFECTS OF COMBINED LOW- AND HIGH-LET RADIATIONS
}

\author{
F. Q. H. Ngo, E. A. Blakely, and C. A. Tobias
}

\section{BACKGROUND}

Damage interactions between high- and low-LET radiation has been a fundamental research subject in radiation biology for sometime. The possibility of independence between the actions of the two radiation modalities was investigated during the fifties and sixties with regard to chromosome aberrations (Wolff et al., 1958; Natarajan and Narayana, 1963), cell inactivation (Barendsen, 1960; Todd, 1964), and lethality in dogs and rodents (Blair, 1964). Most "of these workers have concluded that high-and low-LET radiation act independently. This topic was reopened in the late seventies as fast neutrons were being re-evaluated for radiotherapeutic purposes. However, the majority of the experiments were not entirely consistent with the previous conclusions. These later experiments demonstrated that fast neutrons and $X$ rays or gamma rays show some sort of an interactive effect (Railton et al., 1975; Durand and Olive, 1976; Hornsey et al., 1977). Nevertheless, since fast neutrons produce a considerable low-LET component, the interactions observed may be explained by interactions of sublethal lesions produced by this component of neutron irradiation and by the external $X$ or gamma rays (Katz and Sharma, 1974).

Using fission-spectrum neutrons at the Janus reactor (Argonne National Laboratory), Ngo et al. (1977b) found that the nigh-LET radiation produces damage that interacts with $x$-ray-induced damage in living cells. Since the gamma-ray contamination in the Janus neutrons is reduced to only $3-4 \%$ of the neutron dose, this observation of damage interaction raised the question of true independence between nigh- and low-LET radiations.

Beginning in late 1977, we have carried out systematic studies on the radiobiological effects of combined radiation modalities using cultured mammalian cells. In this chapter, we will: discuss briefly some of these findings.

\section{RATIONALE :}

In the urgent development of energy supply for our society, be it from the solar source via space satellite stations or nuclear reactions including nuclear fission and nuclear fusion, the radiation risk for the workers potentially. exposed to a mixture of radiation qualities is a major consideration. Whether nigh- and low-LET radiations exnibit an independent or a synergistic effect in biological systems is an important question that will nave a significant impact on the estimates of human risk.

On the other hand, biological effects of mixed-radiation beams are also of particular interest in the advancement of radiotherapy. Due to insufficient beam time and other practical reasons, a large number of patients have been treated with combined fast neutrons and photons in the few clinical neutrons facilities in this country. In fact, in the current national protocols for the clinical neutron trial, the combined mode has become a major arm for comparison studies. Although recent clinical results suggested that in some situations combined neutron and photon treatments appear to be better than each of the two modalities used alone, there is a lack of biological basis upon which a scientifically sound explanation can be offered. More basic radiobiological studies are required before potential advantages or disadvantages can be realized and predicted for the use of mixed modalities in cancer therapy. This has been emphasized by the Committee for Radiation Oncology Studies (CROS, 1978). While fundamental studies on mixed radiations have immediate relevance to the national neutron therapy program, such information should also provide direct implications for radiotherapy with pi mesons or heavy charged particles, as each of these particle beams consists of a wide spectrum of LET distributions. For practical reasons similar to those found with fast neutrons, combinations of heavy ions and photons may prove to be a worthwhile consideration in the future.

\section{CLASSIFICATION OF INTERACTIVE EFFECTS}

A variety of terminology has been used in the literature for the combined effects of two different deleterious agents on biological systems (Dewey et al., 1972; Steel and Peckham, 1979; ICRU, 1979; Tobias et al., 1980b). We distinguish the interactive processes as follows. Assume that the surviving fractions for a dose of agent $A$ and a dose of agent $B$ are $S_{A}\left(D_{A}\right)$ and $S_{B}\left(D_{B}\right)$, respectively, and the surviving fraction when the two radiations are given together is $S\left(D_{A}+D_{B}\right)$. We define the interactions as:

1. Independent, if

$$
S\left(D_{A}+D_{B}\right)=S_{A}\left(D_{A}\right) \cdot S_{B}\left(D_{B}\right)
$$

2. Protective, if

$$
S\left(D_{A}+D_{B}\right)>S_{A}\left(D_{A}\right) \cdot S_{B}\left(D_{B}\right)
$$

3. Synergistic, if

$$
S\left(O_{A}+D_{B}\right)<S_{A}\left(D_{A}\right) \cdot S_{B}\left(D_{B}\right)
$$

These interactive effects are discussed further by Tobias et al. in Part III. 
RESULTS AND DISCUSSION

Does an Apparent Exponential Survival Curve Imply No Repair?

High-LET radiations cause the so-called single-nit inactivation on living cells, as usually indicated by an exponential doseeffective response. One question is whether those cells that survive a dose of radiation are not nit by any particle.

We have investigated this problem using mixed radiation techniques. The details of the methods and materials of this study have been reported (Ngo et al., 1980b). Figure 1 shows the data from experiments performed by exposing $V 79$ namster cells to a $425 \mathrm{MeV} / \mathrm{amu}$ neon-ion beam and 225-kVp X.rays. The data in the left panel, pooled from two to three experiments, show that the survival curve for the nigh-LET particles was essentially exponential, whereas for $X$ rays the curve had a substantial shoulder. In the right panel, cells were either irradiated with neon ions alone, or with neon ions preceded by a priming $X$-ray dose. The time required between the two radiation treatments was less than $0.5 \mathrm{n}$ during which the cells were kept at ice temperature.
If we assume that the survivors from the neon-ion irradiation were not damaged at all, one would expect that $X$ rays and neon ions act independently on cell inactivation. For the combined treatments described above, survival results expected from independent action between $X$ rays and neon ions are illustrated by the dashed curves. However, the observed survival data for the X-plus-neon irradiation lie below the predicted curves for independence, which suggests there is synergism between the high- and low-LET radiations. In this case, the synergistic effect can be estimated by the change in Do of the neon survival curves with and without a priming $X$-ray dose. These results imply that at least some of the survivors of neon irradiation are sublethally injured.

The question arises as to whether all the subletnal damage due to the high-LET neon-ion beam is caused by the low-LET component associated with the primary particles. We have estimated that for a dose of neon-iog radiation, $75 \%$ of the dose was due to high-LET $20 \mathrm{Ne}$ particles (mean $\mathrm{LET}_{\infty}=284 \mathrm{keV} / \mu \mathrm{m}$ ), approximately $22 \%$ of the dose was due to high- and medium-LET neavy nuclear fragments (mean $L E T_{\infty}=58 \mathrm{keV} / \mu \mathrm{m}$ ), and only about $3 \%$ of the dose was due to relatively low-LET fast protons, nelium ions, and

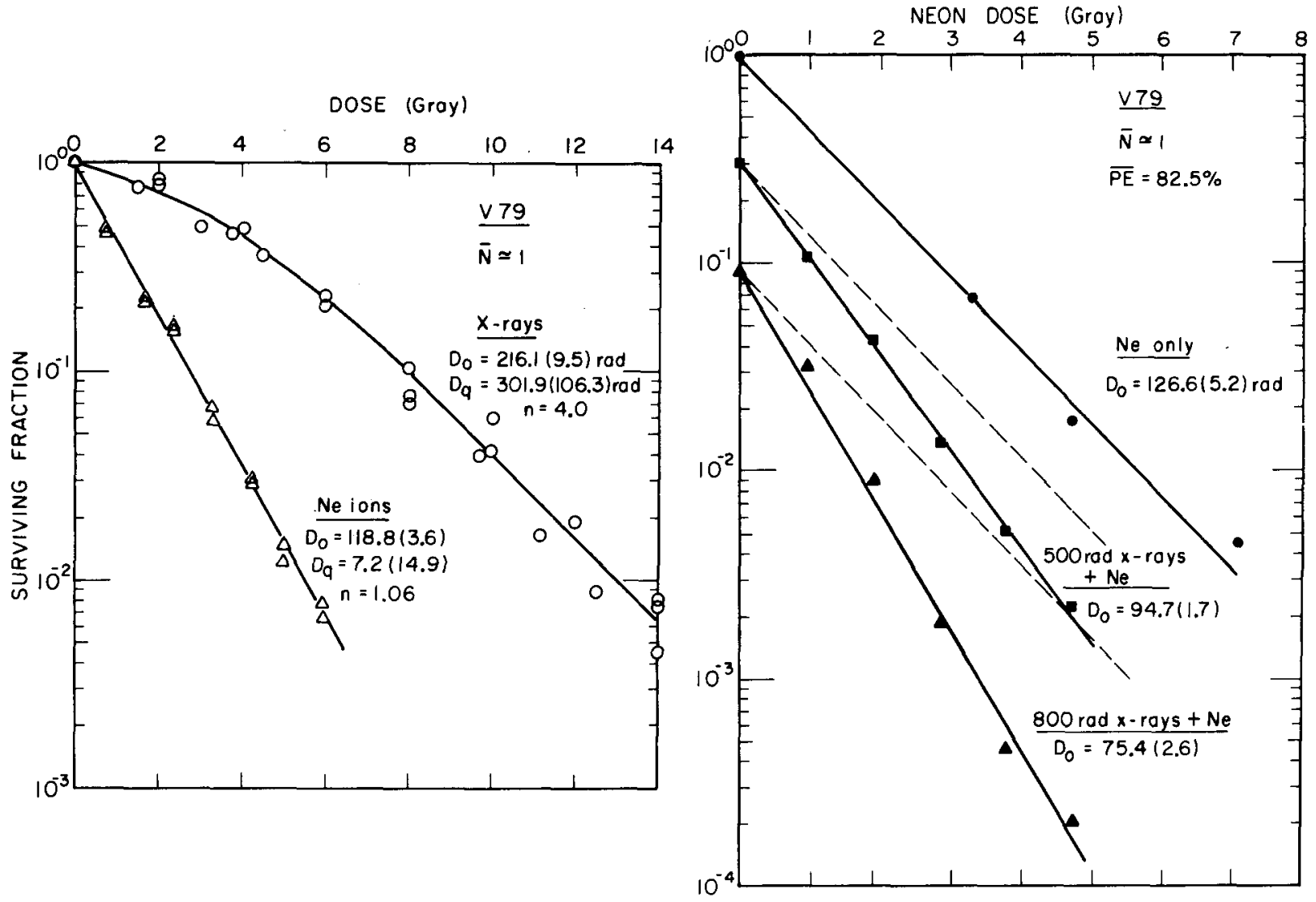

Figure 1. Left panel: Survival data of asynchronized Cninese namster V79 cells exposed to Bragg peak neon ions (mean $L_{E}=234 \mathrm{keV} / \mu \mathrm{m}$ ), or to $225-\mathrm{kVp} X$ rays. Right panel: The effects of two different primary $X-r a y$ doses on the neon-ion survival curve of the same cells. The dashed lines assume an independent action between $X$-rays and neon ions. $D_{0}$ is the mean. letnal dose, $D_{q}$ is the quasi-threshold dose and $n$ is the extrapolation number. $P E$ and $N$ denote average plating efficiency and average cell multiplicity, respectively. (XBL 791-3023B) 
fast neutrons (Ngo et al., 1980b). Since, per unit of absorbed dose, the neon ions are almost equivalent to $x$ rays in causing damage interaction, the lesions induced by the 3\% low-LET component of the high-LET beam cannot account for the entire damage interaction observed.

Another example that supports this view is illustrated in Figure 2. In these experiments, an $8.5 \mathrm{MeV} / \mathrm{amu}$ argon-ion beam was chosen because of its low incident energy which makes it ineffective to cause nuclear fragment reactions along the particle paths. The data shown in the left panel compare the survival curves between $X$ rays and argon ions. The single-nit inactivation kinetics associated with the hign-LET particles are apparent, and their ineffectiveness in cell killing relative to $X$ rays is presumably due to the excess energy density deposited per particle track. We have also shown that there was little or no variation in the survival response with respect to cell cycle stages for this particle beam (Fig. 6, in chapter III-3). Nevertheless, the data shown in the right panel of Figure 2 indicate that there is an interaction between the heavy ions and $X$ rays. Quantitative analyses suggest that a dose of argon ions acts like half of the dose of $x$ rays in causing sublethal lesions in the irradiated cell population.
These results are consistent with the view that the high-LET primary particles, not necessarily the low-LET components, are capable of producing nonlethal events.

\section{Damage Interaction Vs. Cell-Cycle Stages}

A number of radiobiological properties have been shown to vary throughout the cell-division cycle. These include nonrejoining DNA double strand breaks, certain types of cnromosomal damage, repair of sublethal damage, survival, growth delay, mutations, and transformation. In order to better understand the synergistic interaction between low- and high-LET irradiations, we have attempted quantitative measurements on the degree of damage interaction as a function of cell-cycle stages. Preliminary results have been reported elsewhere (Ngo et al., 1979).

Exponentially growing $V 79$ cells were synchronized by the mitotic selection method (Sinclair, 1969). Mitotic cells were collected and allowed to attach in 35-mm diameter plastic dishes. The cells were irradiated with $425 \mathrm{MeV} /$ amu neon ions and $x$ rays, either alone or in combination, at various cell-cycle stages as they grew at $37^{\circ} \mathrm{C}$ under optimal culture conditons.
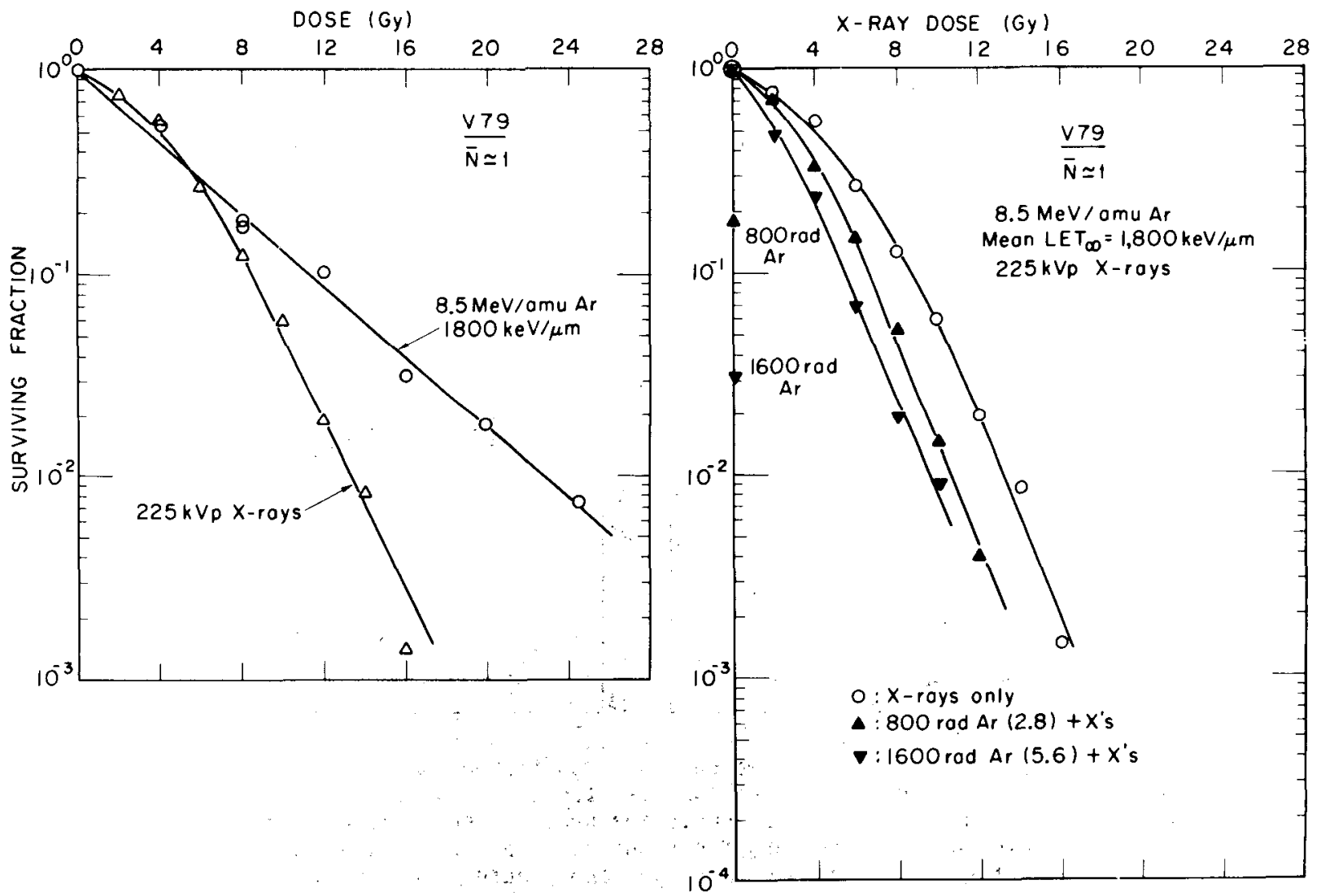

Figure 2. Left panel: Survival data of asynchronized Chinese hamster V79 cells exposed to $X$ rays or to argon ions produced at the SuperHILAC. Right panel: the effects of primary doses of argon ions on the $X$ ray survival curve. The combined-radiation survival curves were normalized with their origins coinciding with the origin for the $X$ ray only survival curve. $800 \mathrm{rad}$ and $1,600 \mathrm{rad}$ of argon particles correspond to approximately 2.8 and 5.6 particles per cell nucleus, respectively. $N$ denotes average cell multiplicity. (XBL 797-3658A) 
Upon completion of irradiation, cells were trypsinized and plated for single-cell survival. In order to minimize the potential biological and physical variations, it is desirable to perform this type of experiment in a single day using the same population of mitotic cells. However, because of the size of the experiment, some previous attempts have failed due to either undesirable quality of the synchrony, or unexpected interuption of the heavy-ion beam.

In the experiments discussed below, these technical difficulties were largely overcome. The data shown in Figure 3 are from two separate experiments (pane?s A,B,D from one experiment, and $C$ from another). In Figure $3 A$, the surviving
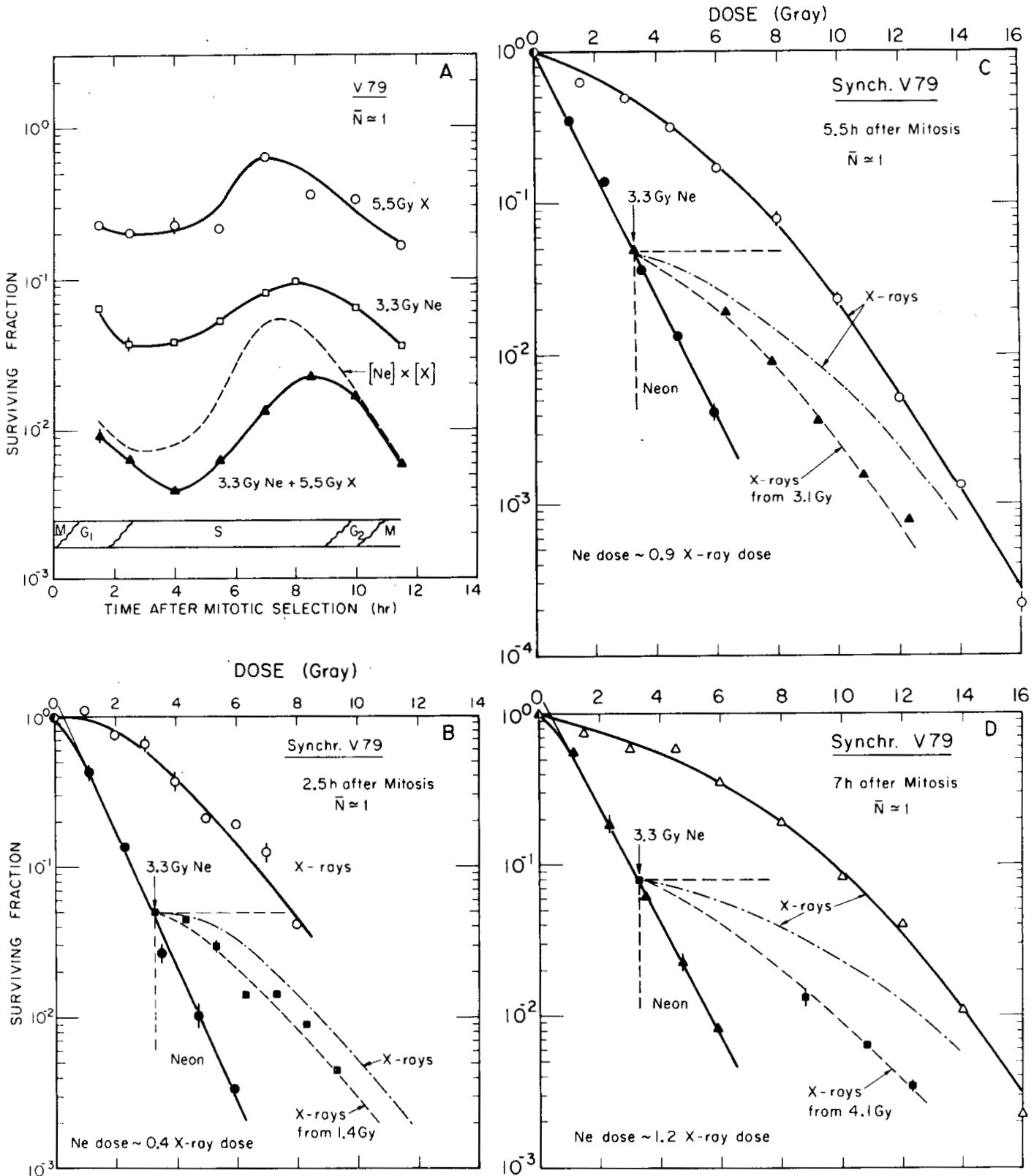

Figure 3. Panel A: The upper two curves demonstrated the measured variation in radioresponse of $V 79$ cells with cell-cycle stages following a single X-ray or a single neon-ion dose. The lowest curve measured the survival from the same respective doses of neon ions and X-rays given in a rapid sequential manner at various cell stages. The dashed curve was constructed from the upper two survival curves on the basis of independence between $X$ rays and neon ions. The cell-cycle stages at various times after mitosis were estimated in the lower portion. Panel B, C, D: The interactive effects of a primary dose of neon ions on the $X$ ray survival for cells in (B) $G_{1} / S$, (C) mid $S$, and (D) late $S$ stages. The survival curves of each synchronized population for $X$-ray or neon-ion irradiation alone are shown as references. The combined radiation treatments were 330 rad neon ions plus graded X-ray doses. (XBL-794-3421A) 
fractions are plotted as a function of cell-cycle stages for a single dose of $x$ rays, or neon ions, or the same doses of $X$ rays and neon ions given with little delay. The dashed curve was constructed assuming the two radiations act independently. There is a variation in the degree of the synergistic effects throughout the cell cycle. The degree of interaction was small for cells at $G_{1}, G_{1} / S, G_{2}+M$ phases, and was relatively large during the time of DNA synthesis. This property is more quantitatively demonstrated in Figures $3 \mathrm{~B}, 3 \mathrm{C}$, and 30 for cells at the $G_{1} / S$ border $(2.5 \mathrm{n}$ after mitosis), early $S$ ( $5 n$ after mitosis), and late $S$ ( $7 \mathrm{n}$ after mitosis), respectively. Each of these synchronized populations was irradiated with 3.3 Gy of neon and several graded doses of $X$ rays. By comparing and fitting the survival data of the combined irradiations to some portion of the $X$-ray survival curve, we have estimated that in terms of damage interaction, $3.3 \mathrm{~Gy}$ of neon irradiation is equivalent to $1.4 \mathrm{~Gy}, 3.1 \mathrm{~Gy}$, and 4.1 Gy of $X$ rays for cells at these respective shapes (2.5 $\mathrm{n}, 5.5 \mathrm{~h}$, and $7 \mathrm{~h}$ after mitosis). The ratio of 3.3 Gy to the equivalent $X-r$ ay doses yields the effectiveness of neon particles relative to $X$ rays in causing interaction with $X$ rays for each of these cell stages. These ratios are noted at the lower left corner in each panel. Hence, among the cell stages studied in this experiment, late-S shows the greatest interaction between neon and $X$-irradiation, and $G_{1} / S$ shows the least interaction.
Figure 4 shows an experiment performed with $570 \mathrm{MeV} / \mathrm{amu}$ argon ions (position 8 , mean $\mathrm{LET}_{\infty}=$ $117 \mathrm{keV} / \mu \mathrm{m})$ and $X$ rays. A similar change in the degree of damage interaction with respect to cell stages was observed. In addition, the data demonstrate that at each cell stage the extent of interaction increases proportionally to the size of the priming dose.

\section{Decay of Damage Interaction}

The kinetics of the loss of interaction has been studied by changing the dose-fractionation time between two radiation treatments. Tnis topic nas been discussed in a previous publication (Ngo et al., 1980); here, we present some follow-up work. In Figure 5 , the dashed-dotted curve represents an hypothetical prediction based on independence between 3.3 Gy neon ions and 5.5 Gy $X$ rays, and the actual data are indicated by the dashed curve. The data points were obtained when cells were first irradiated with both radiations at a late $S$ stage $(7$ nours after mitosis), or they were irradiated with the neonion dose at the same stage and incubated before the $X$-ray dose was given. The survival increase above the dashed curve at about 9 hours, which is consistent with repair of radiation damage inflicted by the nigh-LET particles. The survival decrease afterwards is partly due to cell progression into $G_{2}$ and $M$ stages.
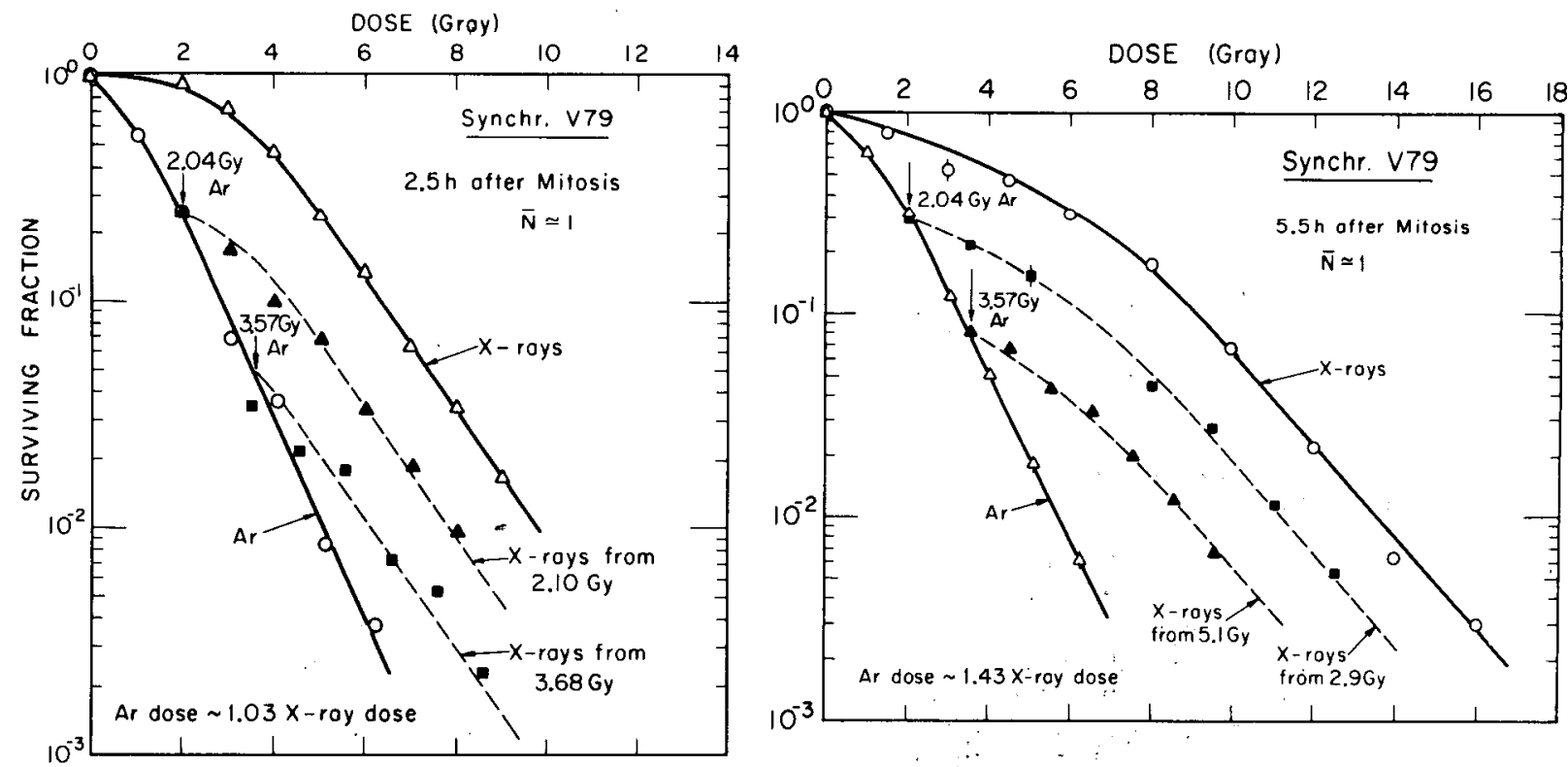

Figure 4. The effects of argon-ion irradiations on the X-ray survival responses for cells in $\overline{G_{1} / S(2.5} \mathrm{n}$, left panel), and mid $S(5.5 \mathrm{~h}$, right panel) stages. The data indicate that the extent of damage interaction increases from $G_{1} / S$ to mid $S$, and at each stage the degree of interaction increases in proportion to the size of the priming dose. The data yield an estimate of the equivalent X-ray dose per unit of argon-ion dose at each cell stage, as indicated in the lower portion on each panel. (XBL 794-3419A) 


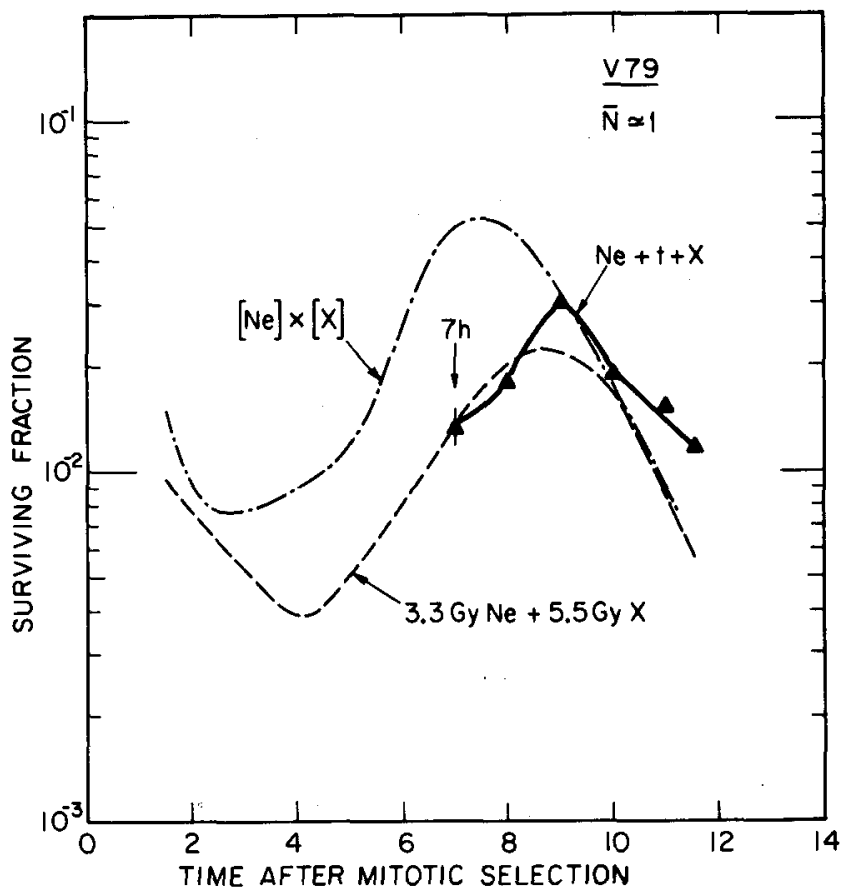

Figure 5. Survival data of synchronized cells irradiated at late $S$ phase witn neon ions and $X$-rays given together or with various time intervals between exposures. See text for details. (XBL 794-3418)

Another experiment, as illustrated in Figure 6 , was designed to investigate how long the synergistic effect lasts. Unsynchronized cells were irradiated with high energy plateau argon ions and $X$ rays at the doses and sequences indicated. The incubation time between the two types of radiations varied from 0 up to $24 n$. For the argon followed by $X$-ray sequence, the survival first increased promptly, and then decreased at a slower rate reaching a minimum at about 7 to $9 \mathrm{~h}$, after which the survival increased again arriving at a plateau at about $12 \mathrm{n}$. The first increase can be attributed to repair of heavy-ion induced damage. The decreased kinetics are probably due to a combined effect of a continuous decay in the degree of damage interaction and argon-induced cell synchrony effects.

The overall kinetics apparently depend upon the sequence of treatment as evident from the survival data for the $X$ rays plus argon ions sequence. The survival due to the combined radiation exposures did not reach the dashed line for independent action until approximately $12 \mathrm{n}$ after the first dose. Based on these results we conclude that with respect to cell killing of V79 cells by sequential treatments of low- and nigh-LET radiation:

1. there is a synergistic effect that decays with time, which separates the two radiations;

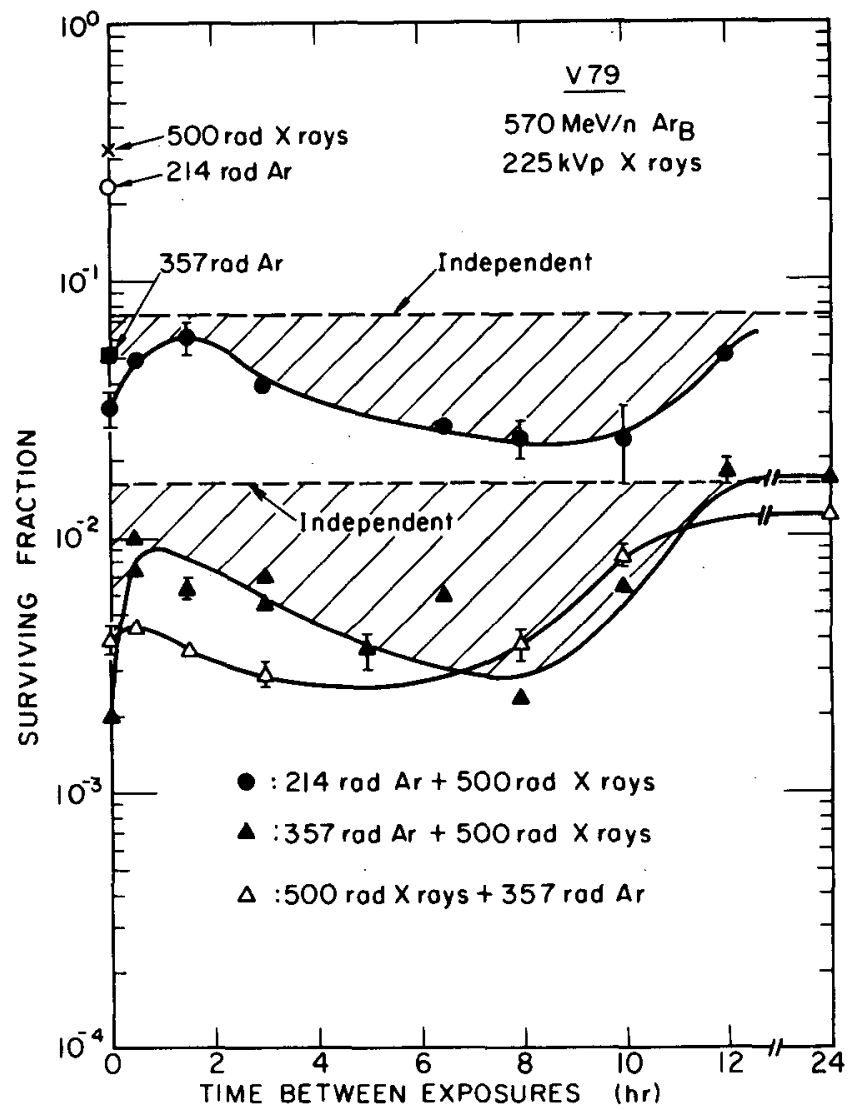

Figure 6. Survival data of asynchronous cell poputations showing the change of response as a function of dose-fractionation time and the sequence of radiations between argon ions and $x$-rays. Samples were incubated at $37^{\circ} \mathrm{C}$ oetween treatments with the two modalities.

(XBL 808-3564)

2. there is a cell synchrony effect that has an initial phase that overlaps with the synergistic effect;

3. the survival kinetics resulting from the synergistic and synchrony effects differ depending on the radiation sequence.

\section{A Comparison of Various Heavy-Ion Beams on Their Ability to Produce Interaction with $X$ rays}

Relationships between RBE and LET have been established for a number of biological end points. Those most thoroughly studied are the cell inactivation and oxygen ennancement effect (Barendsen et al., 1966; Todd, 1967; Blakely et al., 1979). RBE VS. LET relationships have also been reported for chromosome aberrations (Barendsen, 1979), G2 block (Luicke-Hïhle et al., 1979), direct against indirect radiation effects (Chapman et a1., 1979; Roots et a)., 1980), variation of cell-stage response (Skardsgard, 1966; Bird and Burke, 1975), and mutation (Cox et al., 1977). 
It is of basic interest to compare the degree of synergistic interaction of various nigh-LET beams with $X$ rays. Such information $c$ an be utilized, for example, to determine the relative effectiveness of sublethal lesion production as a function of LET (Ngo et al., 1980). To gain information in this regard, mixed-radiation experiments have been conducted using asynchronized $V 79$ cells for charged particles with mean LET values ranging from 16 to $1,800 \mathrm{keV} / \mu \mathrm{m}$. Some experiments were performed with synchronized populations (e.g., Figs. 3 and 4 ). Figure 7 snows the data obtained with five radiation qualities from carbon, neon, and argon particles produced at the Bevalac. The physical and radiological characteristics of these particle beams are described in Table 1.

By our definitions described earlier, two doses of $X$ rays administered together, with little or no time delay, act in a synergistic manner. Experiments were designed to determine how effective per unit dose a high-LET radiation beam is, compared to $X$ rays, in causing synergistic interaction with $x$ rays. Survival was measured for cells that were irradiated with a priming dose of heavy ions, which was immediately followed by graded X-ray doses. These data were compared to the survival data from experiments where the priming neavy-ion dose was replaced by the same absorbed dose of $x$ rays. To make such comparisons, the survival data for the combined modes of irradiation have been plotted according to the transformations described by equations (1) and (2):

the equivalent dose

$$
D_{\text {eq }}=D_{H I}+D_{X}
$$

and the equivalent survival

$$
S_{e q}=\frac{S_{x}\left(D_{x=n i}\right)}{S_{n i}\left(D_{n i}\right)} \cdot S_{m i x}\left(D_{e q}\right)
$$

where the subscripts $h i$ and $x$ represent heavy ions and $x$ rays, respectively. In equation (2), the first term is a normalization factor which is the survival ratio of $x_{-}$and heavy-ion radiations both given at the same dose size as the priming dose of heavy ions. The second term is the surviving fraction measured from a comoined irradiation with heavy ions and $X$ rays.

Using these transformations, three alternative outcomes are possible. First, if per unit of absorbed dose a heavy ions beam is as effective as $X$ rays in causing an interaction effect, the combined-radiation survival data should be superimposed on the $x$-ray-only survival curve. Second, if the neavy-ion beam is less effective than $x$ rays the combined-radiation data should $l$ ie above the $X$-ray-only survival curve. Third, if the heavy-ion beam is more effective than $X$ rays, the combined radiation data should lie below the $x$-ray-only survival curve.
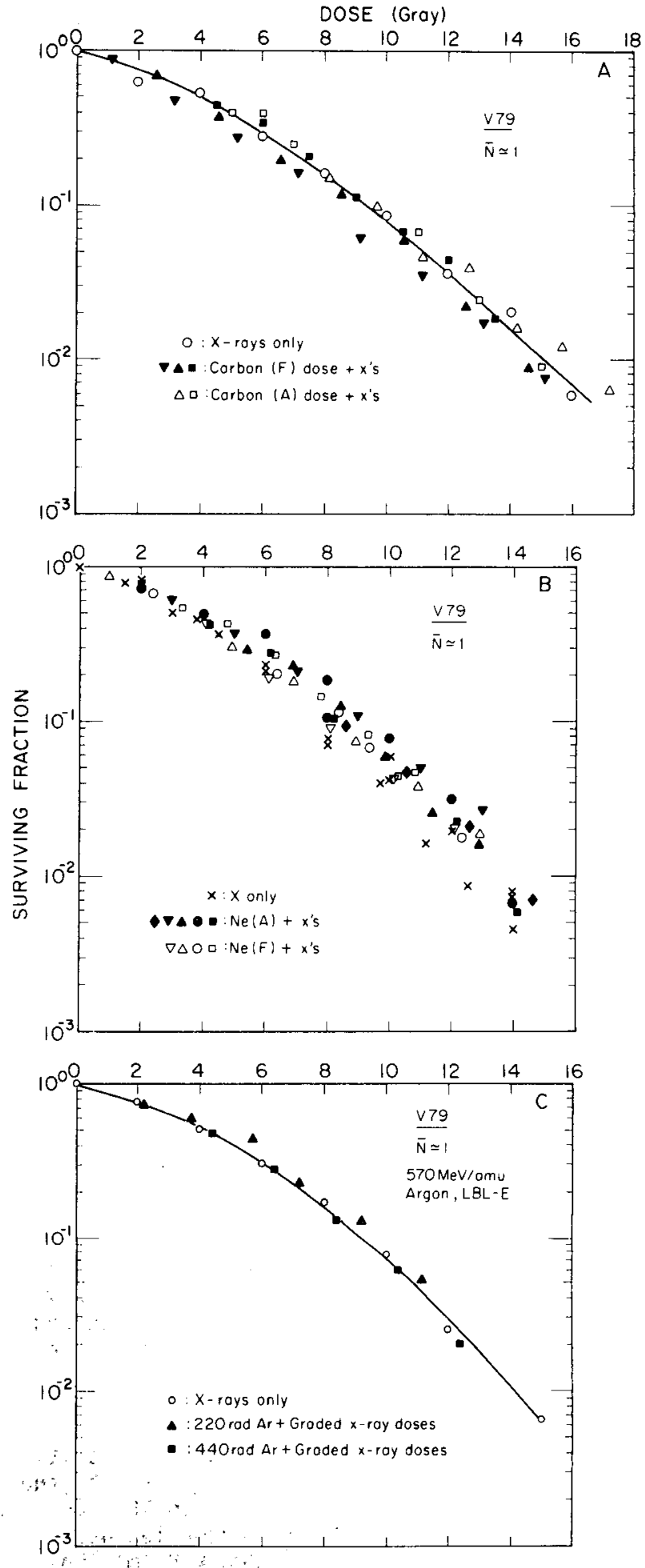

Figure 7. Evaluation of the effects of five different nigh-LET radiations on the $X$-ray response curve of asynchronous V79 cells. Cells were irradiated with various single doses of a heavy-ion beam and immediately followed by graded doses of $X$-rays. Survival data for $X$-irradiation only were obtained with the same stock of cells as those used for the combined radiation treatments in eacn experiment. The survival data from the combined treatments were plotted according to the transformations described in the text. (XBL 791-3025A) 
Table 1. The RBE values for V79 Cell Inactivation and the Relative Effectiveness for Causing Damage Interaction Between $X$ Rays and Charged Particle Beams

\begin{tabular}{|c|c|c|c|c|c|c|c|c|}
\hline Particle & Position & $\begin{array}{l}\text { Residual } \\
\text { Range } \\
\mathrm{cm} \text { in water) }\end{array}$ & $\begin{array}{l}\text { Energy } \\
(\mathrm{MeV} / \mathrm{n})\end{array}$ & $\begin{array}{l}\text { Energy in } \\
\text { Ce11 Layer } \\
(\mathrm{MeV} / \mathrm{n})\end{array}$ & $\begin{array}{c}\text { Mean } \\
\mathrm{LET}_{\infty}^{\dagger} \\
(\mathrm{keV} / \mu \mathrm{m})\end{array}$ & $(10 \%$ & $\begin{array}{l}\mathrm{RBE}^{*} \\
\text { Survival) }\end{array}$ & $\begin{array}{l}\text { Relative } \\
\text { Interaction } \\
\text { Effectiveness }\end{array}$ \\
\hline$\frac{12}{6} \mathrm{C}$ & A & 6.8 & 400 & 175 & 16 & & 1.20 & 0.9 \\
\hline${ }_{10}^{20} \mathrm{Ne}$ & A & 6.8 & 425 & 235 & 38 & & 1.47 & $0.8-0.9$ \\
\hline${ }_{6}^{12} \mathrm{C}$ & $F$ & 0.14 & 400 & 24 & 85 & & 2.92 & $1.1-1.2$ \\
\hline${ }_{10}^{20} \mathrm{Ne}$ & $F$ & 0.14 & 425 & 31 & 234 & & $2: 91$ & 0.8 \\
\hline $\begin{array}{l}40 \\
18^{A r}\end{array}$ & $E$ & 0.29 & 570 & 54 & 245 & & 2.19 & 1.0 \\
\hline $\begin{array}{l}40 \\
18^{A r}\end{array}$ & plateau & & 8.5 & $\sim 6$ & 1,800 & & 0.70 & 0.5 \\
\hline
\end{tabular}

* Distance between position of cell monolayer and Bragg peaks.

+ Values are based on calculations by Blakely et al., 1979.

F With respect to $225-k V p X$ rays.

The solid curves in Figures $7 A$ and $C$ are fitted to the survival data for $X$ rays only; such a curve is not shown in Figure 7B. Nevertheless, within experimental fluctuations for each set of data, all the heavy-ion beams in this study are comparable to $x$ rays in their effectiveness in interacting with $X-r a y$-induced damage. There is a possibility that carbon ( $F$ ) may be slightly more effective than $X$ rays, whereas neon $(A)$ and neon (F) are slightly less effective. Note that the interaction effectiveness for a low-energy argon beam with $\mathrm{LET}_{\infty}=1,800 \mathrm{keV} / \mu \mathrm{m}$ was reduced to approximately nalf of the effectiveness for $x$ rays (Fig. 2 ).

The effectiveness of sublethal lesion production is almost independent of the quality or LET of radiation. This is in contrast to the relationship snown in Table 1 between RBE for cell inactivation and LET. An exception was observed for the LET value $(1,800 \mathrm{keV} / \mu \mathrm{m})$ that corresponds to the overkill region. For this radiation, both cell inactivation and sublethal lesion production are less effective compared to $x$ rays.

\section{Mechanisms for the Interaction Effect}

At least three mechanisms could be attributed to the synergistic interaction observed. First, the sublethal lesions produced by the neon-ion beam and $X$ rays may undergo physical interactions that may result in lethal lesions. This mechanism is similar to the cooperative interaction of lesions for hign-LET radiation-induced lethal effects suggested by Tobias (1971), and the interaction of sublesions which lie within some small distance of each other proposed by Kellerer and Rossi (1978).

A second mechanism may involve the inhibition of repair. Presumably, the damage caused by one radiation may affect the repair of damage produced by another radiation. The ideas of misrepair (Tobias et al., 1980a; Elkind, 1980) and of saturation repair (Laurie et al., 1972; Alper, 1977) have been discussed by a number of inves-

tigators and are pertinent to this consideration. A third mechanism is that lethality occurs because at a critical point for the cell to survive, e.g., postirradiation mitosis, too many lesions remain to be repaired correctly. Hence, this mechanism may be called saturation of damage.

At their late $S$ phase, V79 cells have the largest capacity for repair (Elkind and Sinclair, 1965). This radiobiological property is probably true to some extent for the nigh-LET particles, as their cell-age response curves are qualitatively similar to $x$ rays (see $F i g .3 A$ ). Since we found a greater synergistic interaction in the $S$ phase (Probably the largest effect in late $S$ ), it appears that the second mechanism, i.e., innibition of repair, is the most probable mechanism to explain the interaction effect. 


\section{MIXED-BEAM RADIOTHERAPY}

Radiotherapy using a low-LET radiation source combined with fast neutrons is now in progress in the United States. Two-port exposures that would irradiate tumors with a mixed radiation field have potential uses for future particle radiotherapy. Some potential radiobiological advantages may be inferred from the work described in this chapter:

1. A therapeutic gain may be: anticipated due to the synergistic effect if the overlapping fields of low- and nigh-LET radiations can be localized to the tumor target region. However, since this effect may diminish in a few hours, it requires a rapid delivery of the two modalities.

2. A synchronization effect exists that may favor sterilizing rapidly proliferating tumors by a hign-LET plus low-LET radiation sequence.

3. The greater synergistic effect for cells in $S$ phase than those in $G_{1}$ phase may provide a favorable differentiation between tumor and normal tissue for some types of cancer using mixed-beam therapy.

\section{FUTURE WORK}

The work reported briefly in this chapter represents our initial efforts in the area of mixed-radiation biology. This field of research should be expanded to other dimensions such as tumor and normal tissue studies, and carcinogenic effects. It may bring about new and unexpected findings in radiation biology and cancer research, results that one would not be able to predict on the basis of the effects observed with one radiation modality alone.

In the future, we plan to investigate the mixed-radiation effect and its dependency upon: (1) dose size, particularly in low dose region; (2) oxygen effect; and (3) cells with a Gl stage that is longer than that of the $V 79$ cells presently used.

In order to better understand the mechanism underlying the radiobiological effects of combined radiation treatments, biological studies at the molecular level are necessary. In this regard, damage in the genetic materials due to mixed radiation of low- and nigh-LET natures will be examined. Furtnermore, we will continue to develop a biophysical model to assist our understanding of the mechanism of interaction effects, and to predict results from the action of combined modalities.

\section{ACKNOWLEDGEMENT}

We thank P. Cnang, 0. Olezsko, and L. Lommel for their support and assistance in maintaining an excellent cell culture laboratory, and in successfully carrying out the often tedious experiments. Technical assistance was also offered by our graduate and undergraduate students, including M. Yezzi, I. Madfes, R. Zamora, and $K$. Knight. This work was supported by the National Cancer Institute, Grant CA-15184.

\section{REFERENCES}

Alper, T. 1977. Elk ind recovery and sublethal damage: A misleading association Br. J. Radiol. 50, 459-467.

Barendsen, G. W. 1979. Influence of radiation quality on the effectiveness of small doses for induction of reproductive death and chromosomal aberrations in mammalian cells. Int. J. Radiat. Biol. 36, 49-63.

Barendsen, G.W., T.L.J. Bensker, A. J. Vergroesen, and L. Budke. 1960. Effect of different ionizing radiations on numan cells in tissue culture. II. Biological experiments. Radiat. Res. 13, 841-849.

Barendsen, G. W., C. J. Koot, G. R. Van Kersen, D. K. Bewley, S. B. Field, and C. J. Parnell. 1966. The effect of oxygen on impairment of the proliferative capacity of human cells in culture by ionizing radiations of different LET. Int. J. Radiat. Biol. 10, 317-327.

Bird, R. and J. Burki. 1975. Survival of synchronized Chinese hamster cells exposed to radiation of different linear energy transfer. Int. J. Radiat. Biol. 27, 105-120.

Blakely, E. A., C. A. Tobias, T.C.H. Yang, K. C. Smith, and J. T. Lyman. 1979. Inactivation of human kidney cells by high-energy monoenergetic neavy-ion beams. Radiat. Res. $80,122-160$.

Blair, H. A. 1964. On Addition of Modes of Radiation Injury in Producing Lethality in Dogs and Rodents. University of Rochester Atomic Energy Project Report, UR-654.

Chapman, J. D., S. D. Doern, A. P. Reuvers, C. J. Gillespie, A. Chatterjee, E. A. Blakely, K. C. Smitn, and C. A. Tobias. 1979. Radioprotection by DMSO of mammalian cells exposed to $X$ rays and heavy charged-particle beams. Radiat. Environ. Biophys. 16, 29-41.

Cox, R., J. Thacker, D. T.. Goodhead, and R. J. Munson. 1977. Mutation and inactivation of inammalian cells by various ionizing radiations. Nature (London) 267, 425-427.

CROS (Committee on Radiation Oncology Studies). 1978. Proposal for a program in particle beam radiation therapy in the United States. Cancer Clinical Trials 1, 153-208.

Dewey, W. C., L. E. Stone, H. H. Miller, and R. E. Giolak. 1971. Radiosensitization with 5-bromodeoxyuridine of Chinese hamster cells $x$-irradiated during different phases of the cell cycle. Radiat. Res. 47, 672-688.

Durand, R. E. and P. L. 01 ive. 1976. Irradiation of multi-cell spheroids with fast neutrons 
vs. X-rays: A qualitative difference in subletnal damage repair capacity or kinetics. Int. J. Radiat. Biol. 30, 589-592.

Elkind, M. M. 1980. Cells, targets, and molecules in radiation biology. Radiation Biology in Cancer Research ( $R$. E. Meyn and $H$. R. Withers, eds.) pp. 71-93. Raven Press, New York.

Elkind, M. M. and W. K. Sinclair. 1965. Recovery in $x$-irradiated mammalian cells. Curr. Top. Radiat. Res. Q. 1 , 167-220.

Hornsey; S., U. Andreozzi, and P. R. Warren. 1977. Sublethal damage in cells of the mouse gut after mixed treatment with $x$ rays and fast neutrons. Br. J. Radiol. 50, 513-517.

ICRU (International Commission on Radiation Units and Measurements). 1979. Report 30. Washington, D.C.

Katz, R. and S. C. Sharma. 1974. Cellular survival in a mixed radiation environment. Int. J. Radiat. Biol. 26, 143-146.

Kellerer, A. M. and H. H. Rossi. 1978. A generalized formulation of dual radiation action. Radiat. Res. 75, 471-488.

Laurie, J., J. S. Orr, and C. J. Foster. 1972. Repair processes and cell survival. Br. J. Radiol. 45, 362-368.

Lücke-Hiihle, C., E. A. Blakely, P. Y. Chang, and C. A. Tobias. 1979. Drastic G2 arrest in mammalian cells after irradiation with heavyion beams. Radiat. Res. 79, 97-112.

Natarajan, A. T. and K. R. Narayanan. 1963. Repair from Genetic Radiation Damage ( $F$. H. Sobels, ed.) p. 413. Pergamon Press, 0xford.

Ngo, F.Q.H. and M. M. Elkind. 1977a. Damage interaction in $V 79$ Cninese hamster cells resulting from the combined exposure to $x$-rays and fast neutrons. Radiat. Res. 70, 706 . (Abstract).

Ngo, F.Q.H., A. Han, and M. M. Elkind. $1977 b$. on the repair of sublethal damage in $V 79$ cninese namster cells resulting from irradiation with neutrons combined with $x$-rays. Int. J. Radiat. Biol. $32,507-511$.

Ngo, F.Q.H., E. A. Blakely, and C. A. Tobias. 1978. Does an exponential survival curve of irradiated mammalian cells imply no repair? Radiat. Res. 74, 588. (Abstract).

Ngo, F.Q.H., E. A. Blakely, C. A. Tobias, and P. Y. Chang. 1979. High-LET radiations produce sublethal lesions. Paper presented at the Sixth International Congress of Radiation Research, May 13-19, Tokyo, Japan.

Ngo, F.Q.H., C. A. Tobias, and E. A. Blakely. 1980a. Evaluation of interactive effects on combined modalities. Paper presented at the Third International Symposium on Cancer
Therapy by Hyperthermia, Drugs and Radiation. June 22-26, Fort Collins, Colorado.

Ngo, F.Q.H., E. A. Blakely and C. A. Tobias. 19800. Sequential exposures of mammalian cells to low-and nign-LET radiations. I. Lethal effect following $x$-ray and neon-ion irradiation. Radiat. Res., in press.

Railton, R., R. C. Lawson, and D. Porter. 1975. Interaction of gamma-rays and neutron effects on the proliferative capacity of Chinese hamster cells. Int. J. Radiat. Biol. 27, $75-82$.

Roots, R., A. Chatterjee, E. A. Blakely, and C. A. Tobias. 1980. Cellular radiation chemistry as a function of radiation quality. Radiat. Res. 83, 495. (Abstract).

Sinclair, W. K. 1969. Methods and criteria of mammalian cell synchrony. Recent Results in Cancer Research, pp. 90-105. SpringerVerlag, New York.

Skarsgard, L. D., C. M. Pujara, and S. Richardson. 1966. The effects of nigh LET radiation on recovery and radiation sensitivity throughout the cell cycle in mammalian cells. Paper presented the Third International Congress Radiation Research, Cortina, di Ampezzo, June 26, pp. 204.

Steel, G. G. and M. J. Pecknam. 1979. Exploitable mechanisms in combined radiotherapychemotherapy: The concept of additivity. Int. J. Radiat. Oncol. Biol. Phys. 5, 85-91.

Tobias, C. A. 1971. Physical energy transfer and biological effect. Advances in Medical Physics (J. S. Laughlin, ed.) pp. 28-50. Boston.

Tobias, C. A., E. A. Blakely, F.Q.H Ngo, and T.C.H. Yang. 1980a. The repair-misrepair model of cell survival. Radiation Biology in Cancer Research (R. E. Meyn and H.R. Withers, eds.) pp. 195-230. Raven Press, New York.

Tobias, C. A., E. A. Blakely, and F.Q.H. Ngo. 1980b. Interactions between deleterious agents on living cells. Radiat. Res. 83, 402. (Abstract).

Todd, P. W. 1964. Reversible and irreversible effects of ionizing radiations on the reproductive integrity of mammalian cells cultured in vitro. PhD. Thesis, University of California, Berkeley.

Todd, P. W. 1967. Heavy-ion irradiation of cultured human cells. Radiat. Res. (Suppl.) 7, 196-207.

Wolff, S., K. C. Atwood, M. L. Randolph, and H. E. Luippold. 1958. Factors limiting the number of radiation induced chromosome exchanges. I. Distance. Evidence from noninteractions of $X$-ray and neutron-induced breaks. J. Biophys. Biochem. Cytol. 4 , $365-372$. 


\title{
MACROMOLECULAR LESIONS AND CELLULAR RADIATION CHEMISTRY
}

\author{
R. Roots, E. A. Blakely, A. Chatterjee, and C. A. Tobias
}

Our studies of the interaction of densely ionizing particles with macromolecules in the living cell may be divided into four parts: characterization of lesions to cellular DNA in the unmodified Bragg ionization curve; characterization of lesions to cellular DNA in the spread Bragg curve as used in radiation therapy; elucidation of the cellular radiation chemistry characteristic of nigh vs. low LET radiation qualities; and the introduction of novel techniques designed to give a better understanding of the fundamental properties of induction of lesions and their repair potentials in high LET radiation.

INDUCTION AND REPAIR OF DNA LESIONS IN THE UNMODIFIED BRAGG IONIZATION CURVE

Our early track segment studies of DNA damage in mammalian cells induced by densely ionizing particle radiation have been reported (Ritter, 1976; Ritter et a1., 1977, Tobias et a1., 1977). In these investigations, the induction and repair of DNA breaks were examined after exposures to several tens of kilorad.

Our later, more detailed, investigations of the characteristics of DNA lesions with respect to radiation quality in the unmodified Bragg peak were done with a maximum absorbed dose of 2,000 rad. For these experiments, monolayers of human kidney $T-1$ cells were irradiated at ice temperature and either processed for DNA strand break assays or reincubated for various time periods after irradiation. The number of total breaks was assayed by the DNA uncoiling technique pioneered by Rydoerg (1975). The repair capacity of human kidney $T-1$ cells to rejoin DNA breaks induced by accelerated heavy-ion beams of $C^{6+}$, $\mathrm{Ne}{ }^{10^{+}}$, and $\mathrm{Ar}^{18^{+}}$(308 to $\left.500 \mathrm{MeV} / \mathrm{n}\right)$ is highly' dependent upon the quality of the radiation. The ionization densities at the various positions in the unmodified Bragg ionization curve were expressed both as $\mathrm{LET}_{\infty}(\mathrm{keV} / \mu \mathrm{m})$ and in terms of the factor $\left(Z^{*}\right)^{2} / 8^{2}$ where $Z^{*}$ is the charge of the stripped nucleus and $B$ is the ratio of its velocity to the velocity of light. The latter expression gives a better common fit and was therefore used.

As the ionization density increased, the number of DNA breaks per particle also increased. up to a certain LET-value, after which it levels off as illustrated in Figure 1. The number of DNA breaks per cell per rad decreases noticeably as the ionization density increases, so that the total number of DNA breaks per cell per rad is at least fourfold higher at a few $\mathrm{keV} / \mathrm{\mu m}$ than at $100 \mathrm{keV} / \mathrm{\mu m}$. Figure 1 also shows that the number of DNA breaks per particle that cannot rejoin (repair) increases as a function of LET. This

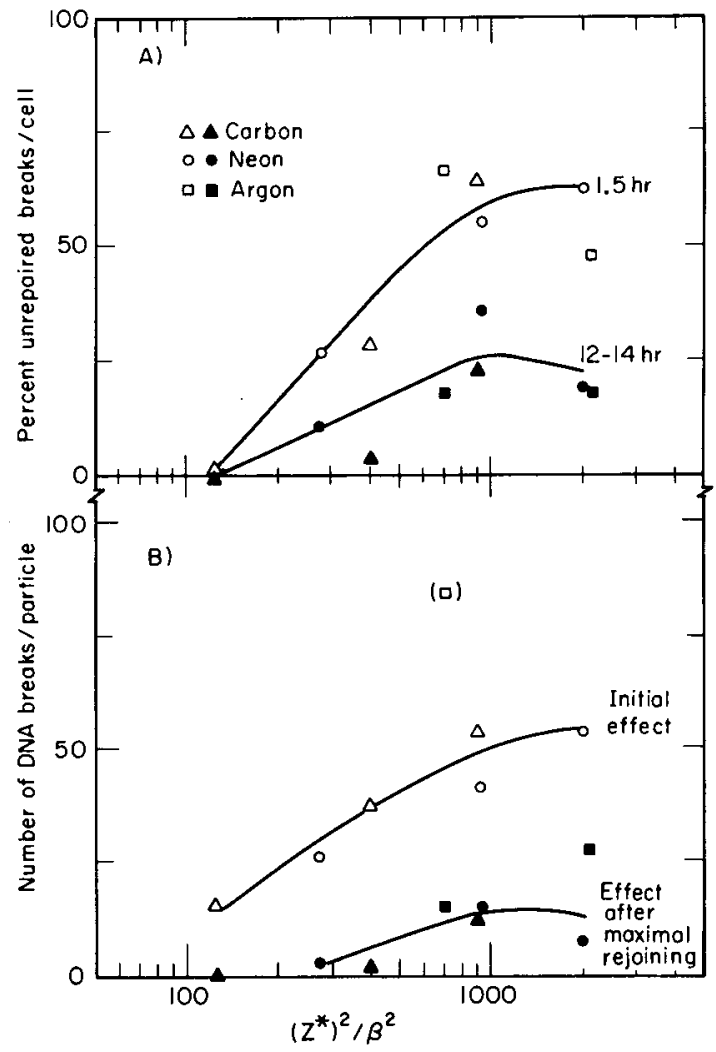

Figure 1. (A) The percentage of the initial number of DNA breaks induced by accelerated heavy ions $(2,000$ rad) left unrejoined after 1.5 or 12 to $14 \mathrm{~h}$ of postirradiation incubation plotted as a function of the energy deposition, $\left(Z^{*}\right)^{2} / B^{2}$, for $\mathrm{C}^{6+}(\Delta, \Delta), \mathrm{Ne}^{10^{+}}(0,0)$, or $\mathrm{Ar}^{18^{+}}(\square, \boldsymbol{\square})$ is shown. (B) The number of ONA strand breaks per particle plotted against energy deposition, $\left(Z^{*}\right)^{2} / B^{2}$, after $2,000 \mathrm{rad}$ of $\mathrm{C}^{+}(\Delta, \Delta), \mathrm{Ne}^{10^{+}}$ $(0,0)$, or $\mathrm{Ar}^{18^{+}}(\square, \square)$ particle irradiation is shown. Initial number of breaks $(\Delta, 0, \square)$; number of nonrejoined breaks after 12 to $14 \mathrm{~h}$ post-

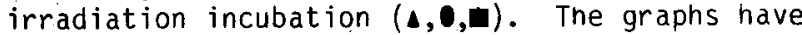
been fitted by eye to the carbon and neon data since the argon data represent only one experiment. (XBL 7.85-3111)

impaired rejoining capacity becomes maximal in the 100 to $200 \mathrm{keV} / \mathrm{um}$ range $\left.\left(Z^{*}\right)^{2} / \mathrm{B}^{2}=1,000\right)$. Therefore, although several times more DNA breaks are made per cell per rad at $1-2 \mathrm{keV} / \mu \mathrm{m}$, than at $100-200 \cdot \mathrm{keV} / \mu \mathrm{m}$, the proportion of breaks per particle that are difficult or impossible to repair becomes much higher at $100-200 \mathrm{keV} / \mathrm{um}$ than at the low LET values.

The rate of DNA repair is dependent upon radiation quality, as illustrated in Figure 1. 
At low LET values more than $90 \%$ of the breaks of the initial break yield are rejoined after 1-2 hours of postirradiation incubation, as opposed to only $40 \%$ in the 100 to $200 \mathrm{keV} / \mu \mathrm{m}$ range. Evidently, many of the breaks in the $100-200 \mathrm{keV} / \mathrm{\mu m}$ range (approximately $25 \%$ ) fail to rejoin altogether as shown in the 12-14 hours postirradiation incubation curve in Figure 1 . These irrepairable DNA lesions are thought to consist of ONA double strand breaks, and probably involve a fairly large number of DNA nucleotides.

The peak RBE value for cell killing occurs in the 100-200 keV/um range (Blakely et a 1., 1979; Roots et al., 1979); similarly, the peak RBE value for irrepairable DNA strand breaks also occurs in this same range.

Induction and repair of DNA breaks in leporine photoreceptor cells in vivo after heavy-ion irradiation in the plateau regions of high energy $\mathrm{Ne}^{10^{+}}$and $\mathrm{Ar}^{18^{+}}$have been investigated by Keng and Lett (1978) and Lett (1979). Following doses of 100 to 2,000 rad, DNA strand-break rejoining proceeded rapidly in situ; however, a few hours after irradiation, a transient degradation was seen at all doses. The rejoining of DNA strand breaks then resumed giving apparently "fully restored" DNA tnat did not deteriorate within a period of a few months postirradiation.

CHARACTERIZATION OF LESIONS TO CELLULAR DNA IN THE SPREAD BRAGG PEAK

There appears to be a marked difference in the rate of rejoining of DNA breaks irradiated at numerically equivalent LET $_{\infty}$ values in the spread as opposed to the unmodified Bragg peak. This reflects a large component of relatively low-LET radiation when the Bragg peak is spread by the use of a ridge filter. DNA lesions induced in the $10 \mathrm{~cm}$ spread Bragg ionization curves of $400 \mathrm{MeV} / \mathrm{n}$ carbon and $557 \mathrm{MeV} / \mathrm{n}$ neon particle beams were studied using suspension cultures of Chinese hamster 679 cells (Roots et al., 1980a). Cells suspended in $\mathrm{Ca}^{++}$-free medium were added to specially designed $3 \mathrm{~cm}$ wide, $1.5 \mathrm{~cm}$ deep glass irradiation vessels which were placed in the proximal, middle, and distal positions of the $10 \mathrm{~cm}$ Bragg peak spread by brass ridge filters and were irradiated with 2,000 rad at ice temperature. A small stirring magnet kept the cells in suspension during irradiation.

Aliquots of $0.5 \mathrm{ml}$ of the cell suspension were removed before the samples were irradiated and just after irradiation, and were placed in $2 \mathrm{ml} \mathrm{Ca}^{++}$-free medium for postirradiation incubation at $37^{\circ} \mathrm{C}$ for $0.5,1.0,2.0$, and 4-5 hours. After incubation, the cell suspension was centrifuged, the MEM decanted, and the cell pellet assayed for DNA breaks. To determine the initial number of breaks, $0.5 \mathrm{ml}$ aliquots of the irradiated cell suspension were added to $2 \mathrm{ml}$ cold $\mathrm{Ca}^{++}$-free MEM and centrifuged for pelleting at ice temprature. The DNA strand break yield was estimated by the DNA uncoiling technique which was used previously for determination of DNA lesions in the unmodified Bragg peak (Roots et al., 1979).

Table 1 illustrates the dramatic difference in the rejoining rate of DNA strand breaks between cells irradiated in the unmodified Bragg curve at $100-150 \mathrm{keV} / \mathrm{\mu m}$ LET values and in the

Table 1. DNA Strand Break Rejoining Capacity with Respect to Radiation Quality

Fraction of Initial Strand-Break Yield

Type of Radiation

after 1-2 nours postirradiation

incubation after prolonged postirradiation incubation

* Koch and Painter (1975) and Roots and Smith (1975).

t Roots et al. (1979).

\# Roots et al. (1980a). 
$10 \mathrm{~cm}$ spread Bragg curve $(30-135 \mathrm{keV} / \mu \mathrm{m})$ of high energy carbon and neon particle beams. In the former case, track segment. $\mathrm{LET}_{\infty}$ values were calculated while in the latter case dose-averaged $\mathrm{LET}_{\infty}$ values for the midpoints of the $1.5 \mathrm{~cm}$ proximal, mid, and distal irradiation positions were calculated by $S$. Curtis. These values are 30,38 , and $65 \mathrm{keV} / \mu \mathrm{m}$, respectively, for the carbon beam, and 58, 75, and $135 \mathrm{keV} / \mathrm{\mu m}$, respectively, for the neon beam. We recognize that LET $_{\infty}$ values cannot be used as a common parameter to account for the microdistribution of the energy deposition effected by different kinds of high energy particles, in part due to the contribution by fragmentation caused both in the absence and in the presence of a ridge filter.

The DNA strand break rejoining rate was approximately the same in the three different positions of the Bragg curve for both the carbon and neon irradiations in the $10 \mathrm{~cm}$ spread Bragg peak; the initial strand break rejoining half time is approximately $20 \mathrm{~min}$. For X-ray irradiated cells, the initial rate of rejoining of DNA breaks is approximately $10 \mathrm{~min}$. Table 1 summarizes the contrast between the rejoining efficiency of DNA strand breaks in cells irradiated in the spread Bragg peak, where approximately $90 \%$ of the initial number of DNA breaks are rejoined in 1-2 hours, as opposed to the results for cells irradiated in the unmodified Bragg peak where only $40 \%$ of the initial number of breaks are rejoined within 1-2 hours postirradiation in the 100 to $200 \mathrm{keV} / \mathrm{um}$ range (Fig. 1). The final extent of irrepairable DNA breaks is $<10 \%$ for cells irradiated in the spread Bragg curve while it is about $25 \%( \pm 6.8 \%)$ for cells irradiated in the unmodified. Bragg curve in the $100-200 \mathrm{keV} / \mu \mathrm{m}$ range.

\section{CELLULAR RADIATION CHEMISTRY OF DENSELY IONIZING PARTICLE BEAMS}

Various aspects of cellular radiation chemistry have been studied in bacterial and mammalian cells for. low linear energy transfer: (LET $T_{\infty}$ ) radiation, including hydroxyl-radical mediated cell inactivation and. DNA damage, the effects of the superoxide, radical ion, and the mechanism(s) of the oxygen effect. Only a few. high LET studies have been done with various biological systems to assess the importance of the radiolytic products of water as a function of radiation quality (Brustad, 1962; Takeshita; and Sawada, 1974; Chapman et al., 1979; Antoku; 1975; Singh et al., 1976a,b). However, most of this work was done with fast neutrons or charged particle beams having highly composite LET values.

$\therefore 1$ in $: 1$

We have investigated the extent of the nydroxyl-radical mediated lethality of mammalian cells in track segment studies for various high-LET radiations, including $\mathrm{He}^{2+}, \mathrm{Li}^{3+}, \mathrm{C}^{6+}$, $\mathrm{Ne}^{+} \mathrm{O}^{+}$, and $\mathrm{Ar} 18^{+}$of initial energies from 11.4 'to $570 \mathrm{MeV} / \mathrm{n}$ (Roots et al., 1980b). We have obtained some results from experiments with $225 \mathrm{kVp} \times$ rays done for comparative purposes (Roots, unipublished data).
Cell monolayers, grown in glass vessels, were irradiated in Dulbecco's phosphate buffered saline (PBS) or PBS containing a hydroxyl radical scavenger under aerobic conditions. The decreased cell sensitivity achieved after maximal scavenging of the nydroxyl radical is a measure of cell lethality mediated by this free radical species. Cellular radiosensitivity was defined as the reciprocal of the radiation dose to give ten percent survival, $1 / 0_{10} \%$. To be able to compare radiosensitivities obtained for different ion species, the radiosensitivity in the absence of the hydroxyl radical scavenger (ethylene glycol) was converted to unity for each separate LET value. The radiosensitivity obtained after maximal removal of $\mathrm{OH}-$ radicals by ethylene glycol was then normalized with respect to the nonprotected case.

In Figure 2, the radiosensitivites obtained for various radiation qualities have been plotted against track segment $\mathrm{LET}_{\infty}$ values. The extent of hydroxyl radical dependent lesions is governed by particle velocity and $Z$ number, but $L E T_{\infty}$ is not suitable for correlation. In the case of the low energy particle beams $\left(11.4 \mathrm{MeV} / \mathrm{n} \mathrm{Li}^{2+}\right.$ and $15.6 \mathrm{MeV} / \mathrm{n} \mathrm{C}^{6+}$ ) or the low $Z$ value $(228 \mathrm{MeV} / \mathrm{n}$ $\mathrm{He}^{2+}$ ) where fragmentation, is minimal, the decrease in the extent of OH-radical induced cell lethality with respect to LET corresponds to the

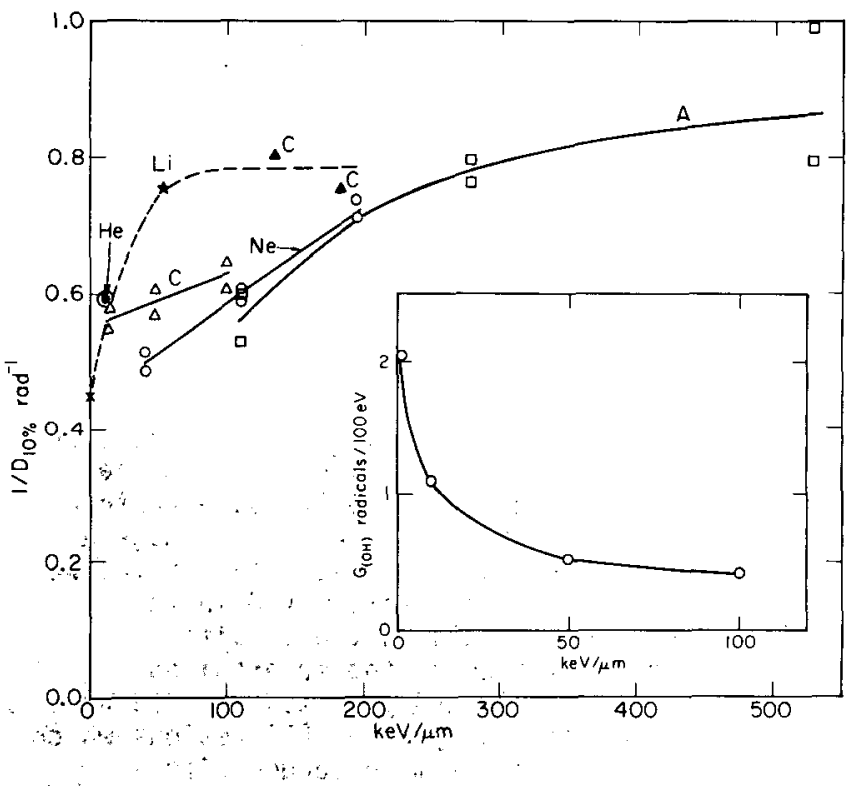

Figure 2. Radiosensitivity ( $\left.1 / 0_{10} \% \mathrm{rad}^{-1}\right)$ of Chinese hamster ovary cells protected maximally against $\mathrm{OH}$-induced lethality as a function of track segment mean $\mathrm{LET}_{\infty}(\mathrm{keV} / \mu \mathrm{m})$. Accelerated particle beams were used: $11.4 \mathrm{MeV} / \mathrm{n} \mathrm{Li}{ }^{+}$(*), 15.6 MeV/n $\mathrm{C}^{6+}(\mathbf{4}), 228 \mathrm{MeV} / \mathrm{n} \mathrm{He}{ }^{2+}(0), 308 \mathrm{MeV} / \mathrm{n}$ $\mathrm{C}^{+}(\Delta), 425 \mathrm{MeV} / \mathrm{n} \cdot \mathrm{Ne}^{+} \mathrm{O}^{+}(0)$, and $570 \mathrm{MeV} / \mathrm{n} \mathrm{A18^{+ }}$ (a). The insert shows the dependence of $\mathrm{G}(\mathrm{OH})$ on the value of LET (neutral pH). Data are taken from a figure published by $I$. $V$. Vereschinskii and A. K. Pikaev in Introduction to Radiation Chemistry, Israel program for Scientific Transiations (1964). (XBL 805-3313) 
decrease in $\mathrm{G}(\mathrm{OH})$ as a function of LET as depicted in the insert in the figure. Our data indicate that $\mathrm{OH}$-radical mediated damage becomes insignificant at high-LET values where radicalradical recombination is high; however, these studies are not yet completed.

In summary, from the work with $225 \mathrm{kVp} X$ rays used as a reference point for the high LET studies, the OH-radical mediated cell inactivation is approximately $60 \%$ of the total radiosensitivity for aerobic cells. In hypoxic cells, the $\mathrm{OH}$-radical mediated cell inactivation is only about $20 \%$ (Roots, unpublished data). Our interpretation is that in hypoxic cells, the majority of $\mathrm{OH}$-radical induced DNA lesions is quickly restored by endogenous nydrogen donating species and that hydroxyl radical mediated lesions can result in oxygen dependent as well as oxygen independent damage because $\mathrm{OH}-$ radical action contributes to about one-fifth of the radiosensitivity in anoxic cells. Further, the contribution to cell killing by $\mathrm{OH}$-radicals is highly dependent on radiation quality as shown in Figure 2 for irradiated aerobic cells. An assessment of $\mathrm{OH}-\mathrm{radical}$ induced damage in anaerobic mammallian cells has not yet been done for high LET studies; however, it is anticipated that as soon as the oxygen effect disappears with increasing LET, the $\mathrm{OH}-\mathrm{radical}$ induced effects will be the same for aerobic as for anaerobic cells. Since the OH-radical mediated lethality is greatly reduced for irradiated anaerobic cells, the $\mathrm{OH}$-radical effect probably will be fairly independent of LET in anaerobic cells as already suggested from the work of Brustad (1962) and Manney et al. (1963) for high LET studies with haploid yeast cells.

\section{CHARACTERIZATION OF THE TYPES \\ OF DNA BREAKS INDUCED WITH HIGH LET RADIATION USING A MODEL SYSTEM}

We have begun studies of the detrimental effects of various agents (e.g., heavy metals or radiation) to cellular ONA using a system that separates the DNA lesions into DNA single-strand breaks, DNA double-strand breaks, and damage at the nucleosomal level. For this assay system we are using DNA from Simian virus SV 40 in the African green monkey cell as the sensitive target. The small size of the supercoiled covalently closed SV40 ONA permits quantification of the above mentioned types of DNA lesions which is not possible with the much larger size mammalian DNA.

\section{REFERENCES}

Antoku, S. 1975. Chemical protection of cultured mammalian cells against fast neutrons. Int. J. Radiat: Biol. 27, 287-292.

Blakely, E. A., C. A. Tobias, T. C. Yang, K. C. Smith, and J. T. Lyman. 1979. Inactivation of human kidney cells by high-energy monoenergetic heavy-ion beams. Radiat. Res. 80 , 122-160.
Brustad, T. 1962. Heavy ions and some aspects of their use in molecular and cellular radiobiology. Adv. Biot. Med. Phys. 8, 161-224.

Chapman, J. D., S. D. Doern, A. P. Reuvers, C. J. Gillespie, A. Chatterjee, E. A. Blakely, K. C. Smith, and C. A. Tobias. 1979. Radio- protection by DMSO of mammalian cells exposed to $x$-rays and to heavy charged particle beams.

Radiat. Environ. Biophys. 16, 29-41.

Keng, P. C. and J. T. Lett. 1978. Rejoining of DNA strand breaks in the photoreceptor cells of the rabbit retina in situ after exposure to $400 \mathrm{MeV} /$ nucleon neon ions. Radiat. Res. 83 , 387 .

Koch, C. J., and R. B. Painter. 1975. The effect of extreme nypoxia on the repair of DNA single-strand breaks in mammalian cells. Radiat. Res. 64, 256-269.

Lett, J. T. 1979. Late effects in leporine tissues after heavy-ion irradiation. Sixth International Radiation Research Society Meeting (S. Okada et al., eds.) p. 132. Japanese Association for Radiation Research, Tokyo, Japan.

Manney, T. R., T. Brustad, and C. A. Tobias. 1963. Effects of glycerol and of anoxia on the radiosensitivity of haploid yeasts to densely ionizing particles. Radiat. Res. 18 , $374-388$.

Ritter, M. A. 1976. DNA strand break rejoining in Chinese hamster cells as a junction of radiation quality. Ph.D. Thesis, University of California, Berkeley.

Ritter, M. A., J. E. Cleaver, and C. A. Tobias. 1977. High LET radiation induce a large proportion of non-rejoining breaks in mammalian cell DNA. Nature (London) 266, 653-655.

Roots, R., and K. C. Smith. 1975. Rejoining of DNA single-strand breaks in mammalian cells incubated in buffer or in medium after aerobic or anaerobic $x$-irradiation. Int. J. Radiat. Biol. 27, 595-602.

Roots, R., T. C. Yang, L. Craise, E. A. Blakely, and C. A. Tobias. 1979. Impaired repair capacity of DNA breaks induced in mammalian cellular DNA by accelerated heavy ions. Radiat. Res. 78, 38-49.

Roots, R., T. C. Yang, L. Craise, E. A. Blakely, and C. A. Tobias. 1980a. Rejoining capacity of DNA breaks induced by accelerated carbon and neon ions in the spread Bragg peak. Int. J. Radiat. Biol., in press.

Roots, R., A. Chatterjee, E. A. Blakely, and C. A. Tobias. 1980b. Cellular radiation chemistry as a function of radiation quality. Proceedings of the International conference on oxygen and Oxy-radicals in Chemistry and Biology. May 25-29, Austin, Texas, in press. 
Rydberg, B. 1975. The rate of strand separation in alkali of DNA of irradiated mammalian cells. Radiat. Res. 61, 274-287.

Singh, D. R., J. M. Mahajan, and D. Krishnan. 1976a. Effect of chemical protectors on fastneutron reversions in yeast. Int. J. Radiat. Biol. 30, 585-588.

Singh, D. R., J. M. Mahajan, and D. Krishnan. 1976b. Effect of dimethyl sulfoxide (DMSO) on radiation-induced heteroallelic reversion in diploid yeast. Mutat. Res. 37, 193-200.
Takeshita, K. and S. Sawada. 1974. Lethal effects of 14-MeV fast neutrons on frog eggs and cultured mammalian celis. Biological Effects of Neutron Irradiation, pp. 245-255. International Atomic Energy Commission, Vienna.

Tobias, C. A., E. A. Blakely, and R. J. Roots. 1977. Cellular and molecular radiobiology of heavy ions. Biological and Medical Research with Accelerated Heavy Ions at the Bevalac, 1974-1977, pp. 76-110. Lawrence Berkeley Laboratory Report LBL-5610. 
118

Blank 


\title{
SILICON: RADIOBIOLOGICAL CELLULAR SURVIVAL AND THE OXYGEN EFFECT
}

\author{
E.A. Blakely, F.Q.H. Ngo, P.Y. Chang, L. Lommel, and C.A. Tobias
}

A comparison of the RBE values and OER properties of monoenergetic carbon, neon, and argon beams with initial energies of several hundred MeV/amu has been made as a function of residual range (Blakely et al., 1979). The biologically effective peak-to-plateau dose ratio was maximized for carbon and neon, and then diminished somewhat for argon. However, argon ions nad a superior LET distribution for optimal reduction of the radiobiological oxygen effect. The analysis suggested the possible therapeutic usefulness of accelerating and biological testing of an ion beam with an atomic number intermediate between neon and argon. Tobias et al. (1979) proposed, therefore, that perhaps a silicon or phospnorus beam would retain an effective depth dose profile while maintaining a lower OER. An analys is of the $1 / \beta^{2}$ dependence of the cellular inactivation cross section of the $400 \mathrm{MeV} / \mathrm{amu}$ carbon, $425 \mathrm{MeV} / \mathrm{amu}$ neon, and $570 \mathrm{MeV} / \mathrm{amu}$ argon beams (see Fig. 1) also supported this hypothesis.

Two preliminary silicon experiments have been completed. This chapter reports measured and calculated Bragg curve physical dose information, and cell survival studies under aerobic and hypoxic conditions using monoenergetic silicon beams of two initial energies (530 MeV/amu and $670 \mathrm{MeV} / \mathrm{amu}$ ). In addition, some limited cell

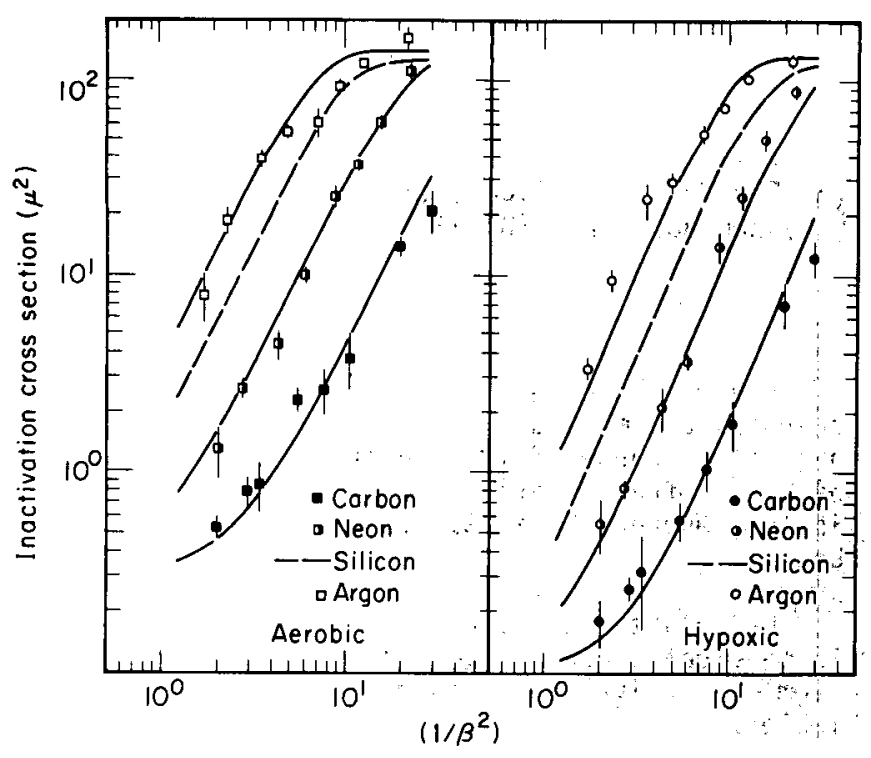

Figure 1. Human kidney $T-1$ cell inactivation cross section versus $1 / \beta^{2}$ for $400 \mathrm{MeV} / \mathrm{amu}$ carbon, $425 \mathrm{MeV} / \mathrm{amu}$ neon, and $570 \mathrm{MeV} / \mathrm{amu}$ argon beams and estimated for a $530 \mathrm{MeV} / \mathrm{amu}$ silicon beam. (XBL 7811-12333A) survival information is presented from an experiment with a $530 \mathrm{MeV} / \mathrm{amu}$ silicon beam which has been moderated with a spiral ridge filter to extend the $B r a g g$ peak to $4 \mathrm{~cm}$.

\section{METHOOS}

The cell culture and oxygen depletion techniques used in the series of experiments to be presented have been described in detail elsewhere (Blakely et al., 1979). Briefly, approximately two days prior to irradiation $\sim 7.0 \times 10^{4}$ exponentially growing human kidney $T-1$ cells are plated on to $35 \mathrm{~mm}$ diameter glass petri dishes. Twenty minutes prior to irradiation, the dishes are each removed from the incubator, the medium is removed by aspiration, and $0.3 \mathrm{ml}$ of fresh medium is replaced before loading each dish individually into specially designed aluminum and glass chambers.

Each sample is flushed with gas at approximately 160 to $240 \mathrm{~cm}^{3} / \mathrm{m}$ in for $20-30$ minutes. A gas mixture of $95 \%$ air $+5 \% \mathrm{CO}_{2}$ is used for the aerobic samples, and $95 \%$ nitrogen $+5 \% \mathrm{CO}_{2}$ for the hypoxic samples. Just prior to irradiation, the chambers are sealed off with a slight positive pressure of 25 to $38 \mathrm{~cm}$ of water.

Dosimetry for the heavy charged particle irradiations was performed using the transmission parallel-plate ionization chambers described by Lyman and Howard (1977). The alignment of equipment on the optical bench is similar to that described earlier, except that the variable thickness water absorber, downstream ionization chamber and sample nolder were about $60 \mathrm{~cm}$ further downstream than they were previously, in order to allow for greater natural radial expansion of the beam. When a ridge filter was used, it rotated just upstream of the variable water column: : Dose rates were about 300-500 rad/ min for both sets of experiments.

In order to ensure a uniform dose distribution across the radial diameter. of the beam, lead scattering foils were inserted upstream of the biomedical cave. The thickness of lead used for the $530 \mathrm{MeV} / \mathrm{amu}$ beam was $0.45 \mathrm{g-cm}-2$ in one experiment, and $0.9 \mathrm{~g}-\mathrm{cm}^{-2}$ in the other. More lead $\left(2: 7 \cdot \mathrm{g}-\mathrm{cm}^{-2}\right)$ was used with the $670 \mathrm{MeV} / \mathrm{amu}$ beam. The beam diameter was usually 3 to $5 \mathrm{~cm}$, and there was about a $6 \%$ dose variation across the cell monolayer.

\section{Physical Characterization of the Beams}

Figure 2 presents the Bragg curves measured for the $530 \mathrm{MeV} / \mathrm{amu} \mathrm{silicon}$ beams (monoenergetic and $4 \mathrm{~cm}$ extended peak), and the $670 \mathrm{MeV} / \mathrm{amu}$ 


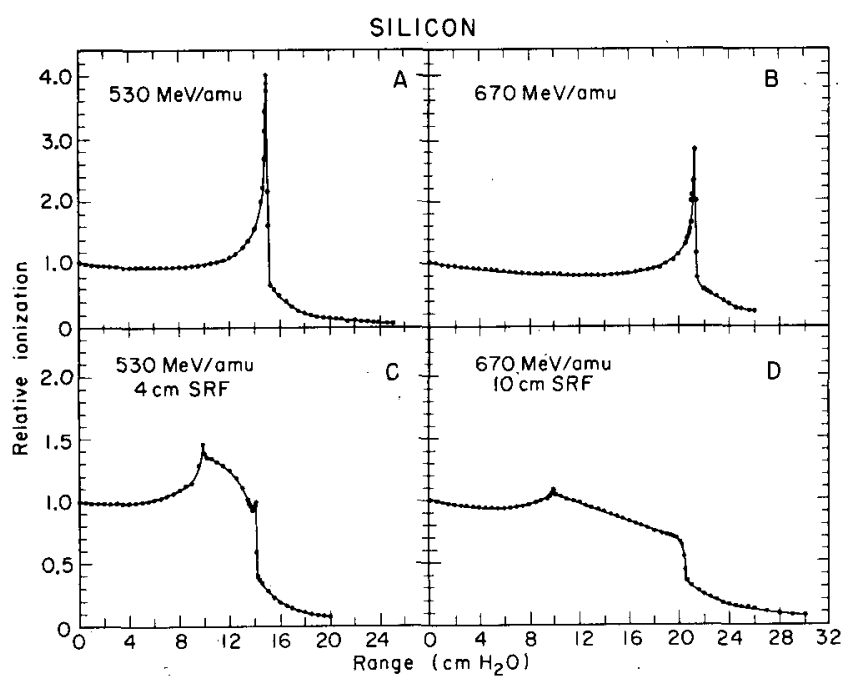

Figure 2. Silicon Bragg ionization relative dose curves vs. range, measured for: (A) monoenergetic beam with an initial energy of $530 \mathrm{MeV} / \mathrm{amu}$; (B) monoenergetic beam with an initial energy, of $670 \mathrm{MeV} / \mathrm{amu}$; (C) modulated $530 \mathrm{MeV} / \mathrm{amu}$ beam with a $4 \mathrm{~cm}$ extended Bragg peak; (D) modulated $670 \mathrm{MeV} / \mathrm{amu}$ beam with a $10 \mathrm{~cm}$ extended Bragg peak. (XBL 808-3628)

silicon beams (monoenergetic and $10 \mathrm{~cm}$ extended peak). The peak to plateau physical dose ratio is 4 for the monoenergetic $530 \mathrm{MeV} / \mathrm{amu}$ beam, and decreases to 3 for the longer ranged $670 \mathrm{MeV} / \mathrm{amu}$ beam. The spiral ridge filters designed to extend the Bragg peak 4 or $10 \mathrm{~cm}$, which have been used in comparative beam studies, were also used for the silicon beams. It should be noted, however, that filters designed to optimize silicon physical dose distributions for isoeffective killing may be more appropriate.

Panels $A$ and $B$ of Figure 3 demonstrate ionization measurements in dose/particle for the $530 \mathrm{MeV} / \mathrm{amu}$ and $670 \mathrm{MeV} / \mathrm{amu}$ silicon beams, respectively. The solid circles represent normalized Bragg ionization ratios. The experimental points were compared to a calculated Bragg curve (solid line). The difference between the measured Bragg curve (solid circles) and the computed Bragg curve is due to fragmentation; this difference is shown by open symbols in Figure 3 .

Beyond the particle range, the fragmentation effects (circles with solid points) are measured directly. The contribution from fragments in the monoenergetic beam increases from a small value at the plateau to a maximum a few millimeters upstream from the Bragg peak. Fragmentation effects for the $530 \mathrm{MeV} / \mathrm{amu}$ silicon beams appear to be intermediate between the fragmentation levels of neon and argon, with a maximum of $38 \%$ fragmentation in the Bragg peak. The fragmentation is slightly higher $(41 \%)$ at the same position in the $670 \mathrm{MeV} / \mathrm{amu}$ silicon beam.
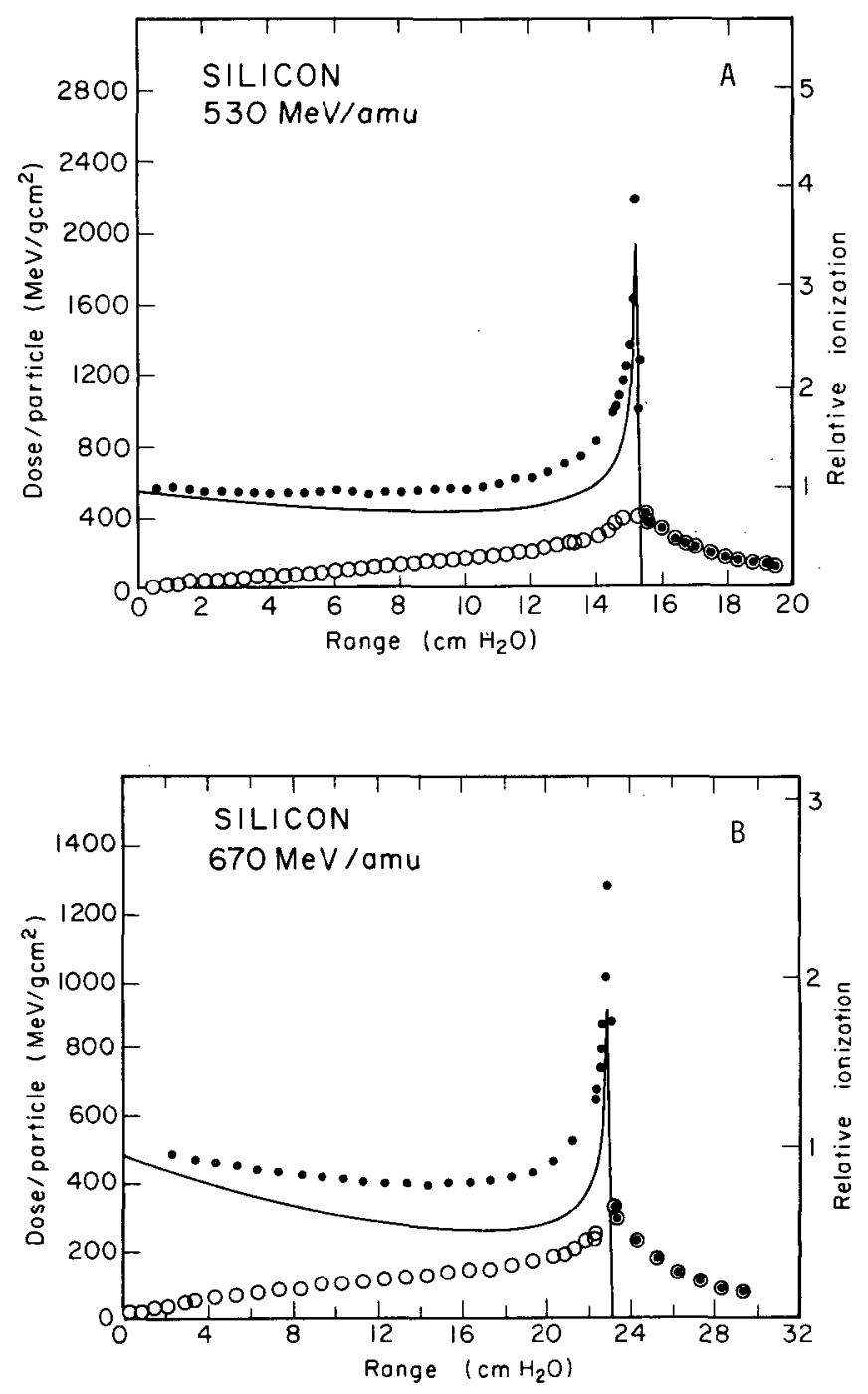

Figure 3. Silicon dose depth curves in water. Actual normalized ionization ratios measured (0). Calculated ionization contributions from secondary fragments $(0)$. Calculated Bragg curve (-). (A) $530 \mathrm{MeV} / \mathrm{amu}$ silicon; (B) $670 \mathrm{MeV} / \mathrm{amu}$ silicon. [(A) XBL 808-3626; (B) XBL 808-3627]

Fragmentation effects for the two beam energies are more different in the plateau. At a residual range of $10 \mathrm{~cm}$ upstream of the Bragg peak, the fragmentation in the $530 \mathrm{MeV} / \mathrm{amu}$ beam is $15 \%$, whereas it is $31 \%$ for the $670 \mathrm{MeV} / \mathrm{amu}$ beam.

Calculated mean track LET values for the monoenerget ic beam range positions (where track segment survival was measured) are presented in Table 1.

\section{X-Ray Dosimetry}

$X$ irradiation of the cells was performed with a Philips X-ray unit (Type 11645) operated at $220 \mathrm{kVp}$ and $15 \mathrm{~mA}$ with filtration of $0.25 \mathrm{~mm} \mathrm{Cu}$ 
Table 1. Silicon LET Values

\begin{tabular}{|c|c|c|c|c|c|c|c|c|c|c|}
\hline $\begin{array}{l}\text { Beam } \\
\text { Position }\end{array}$ & 0 & $A$ & B & C & $D$ & E & $F$ & G & $K$ & $M$ \\
\hline $\begin{array}{l}\text { Residual } \\
\text { Range* } \\
\text { (cm water) }\end{array}$ & 13.3 & 6.8 & 2.4 & 1.2 & 0.54 & 0.29 & 0.14 & 0.04 & $-1 \cdot 0$ & -3.0 \\
\hline $\begin{array}{l}\text { Mean track } \\
\text { average LET } \\
\text { of primary } \\
\text { beam }(\mathrm{keV} / \mathrm{\mu m})\end{array}$ & 58 & 71 & 107 & 140 & 174 & 220 & 340 & 580 & -- & -- \\
\hline $\begin{array}{l}\text { Dose average } \\
\text { LET of } \\
\text { fragments } \\
(\mathrm{keV} / \mu \mathrm{m})\end{array}$ & 32 & 40 & 56 & 80 & 138 & 165 & 197 & 191 & 161 & 104 \\
\hline
\end{tabular}

and $1 \mathrm{~mm} \mathrm{Al}$, and a half-value layer of $0.75 \mathrm{~mm}$ $\mathrm{Cu}$. The dose rate was usually $270 \mathrm{rad} / \mathrm{min}$, as measured with a calibrated Victoreen condenser 250 R-meter at a target distance of $24 \mathrm{~cm}$. Further details of Fricke chemical dosimetry and thermoluminescent dosimetry (TLD) are provided elsewhere (Blakely et al., 1979). The exact gas delivery method and geometry used for the heavyion exposures were duplicated for the $X$-ray experiments. X-ray OER values of $2.9 \pm 0.3$ are obtained at the $10 \%$ survival with these methods.

\section{RESULTS}

Aerobic and hypoxic cell survival curves for the silicon beams studied are shown in composite Figure 4. In most cases, results are from a single experiment, although in a few cases, the results are pooled from two experiments. The continuous lines in the figures represent best fits made to the data by eye.

The data from the monoenergetic $530 \mathrm{MeV} / \mathrm{amu}$ beam (positions 0 to $M$ ) is qualitatively. consistent with the changes observed in the shapes of the survival curves for identical positions in the $425 \mathrm{MeV} / \mathrm{amu}$ neon and $570 \mathrm{MeV} /$ amu argon beams (Blakely et al, 1979). In the "plateau" of the beam (positions 0 and A), survival curves have a definite shoulder, and there is a large difference in cell killing between aerobic and hypoxic curves. These differences are dramatically reduced as the range to the Bragg peak is diminished. The survival curves change abruptly beyond the stopping points of primary beam particles, that is beyond the Bragg peak at positions $K$ and $M$. Survival curves are nearly exponential at the Bragg peak, but shoulders reappear for curves in the region downstream from the peak. Cell killing here is due to the dose from fragments only.

Survival curves measured in the simulated, dual parallel-opposed positions (where the $4 \mathrm{~cm}$ extended peaks of the $530 \mathrm{MeV} / \mathrm{amu}$ silicon beam overlap) are designated "XF-4" in Figure 4. A more detailed description of cross-fired beam experiments is presented by Blakely et al. in Part III. The aerobic and hypoxic cross-fired peak survival curves are similar to the survival curves which would lie between positions C and D on the monoenergetic silicon beam.

The pair of survival curves which are labelled $D^{\star}$ in Figure 4 represent a $0.54 \mathrm{~cm}$ residual range position on the $670 \mathrm{MeV} / \mathrm{amu}$ beam. A comparison of the $D^{*}$ survival curves with the 0 curves of the $530:$ MeV/amu beam shows only slight differences, with the higher energy beam being less effective. A statistical analys is is underway to determine how significant the differences are.

\section{RBE and OER values}

Figure 5 demonstrates how the OER and aerobic hypoxic RBE values at $10 \%$ survival vary as a function of range for the $530 \mathrm{MeV} / \mathrm{amu}$ silicon beam. The aerobic and hypoxic RBE values are both a little less than 2.0 over the first $12 \mathrm{~cm}$ of range. At greater depth, the RBE values quickly increase, reaching a maximum within the last two centimeters upstream of the Bragg peak. The maximum hypoxic RBE is 4.6 ; the maximum 


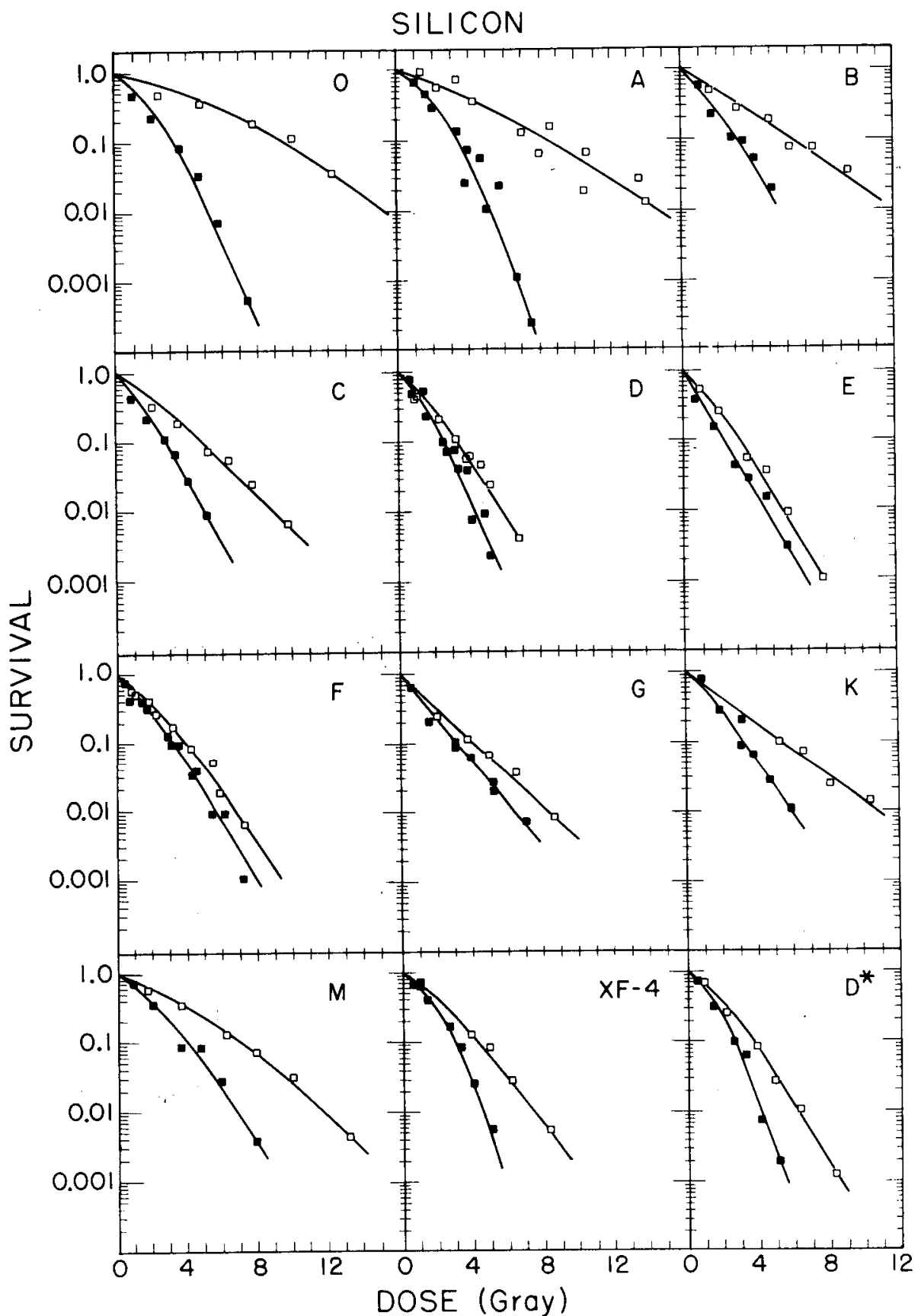

Figure 4. Silicon cell survival data: aerobic ( $\mathbf{a})$, hypoxic ( $\square$ ). The letter in the upper right of each set of curves designates the range studied in Table 1. Positions 0 througn $M$ are from a monoenergetic $530 \mathrm{MeV} / \mathrm{amu}$ beam. Position XF-4 is the cross-fired peak position using the $530 \mathrm{MeV} / \mathrm{amu}$ beam with the $4 \mathrm{~cm}$ extended Bragg peak as described in the text. Position $D^{*}$ represents the $D$ position in a $670 \mathrm{MeV} / \mathrm{amu}$ beam. Data are from one or two experiments for each set of curves. (XBL 808-3647) 


\section{SILICON}

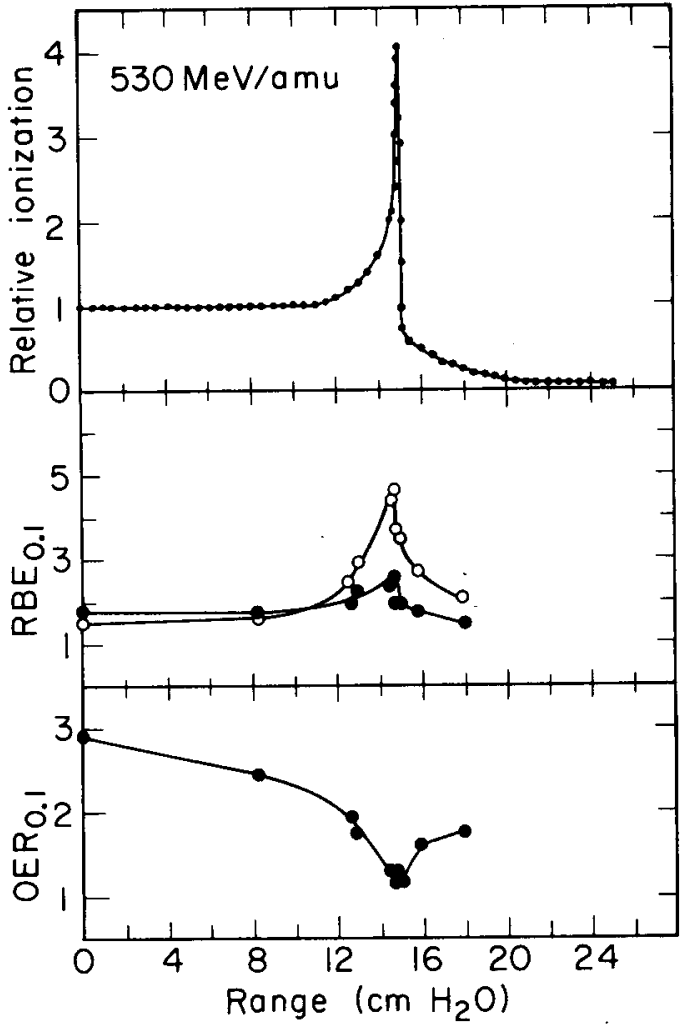

Figure 5. $530 \mathrm{MeV} / \mathrm{amu}$ siliccon Bragg ionization curve, and aerobic and nypoxic RBE and OER at 10\% survival as a function of beam range in water. (XBL 808-3624) aerobic RBE is 2.5. Both peaks occurred at position $E$ with a residual range of $0.29 \mathrm{~cm}$.

The OER values are initially very high in the plateau of the silicon beam (2.9 at position 0$)$. The values decrease slowly with depth at first, and then after $12 \mathrm{~cm}$ of range quickly drop to a low of 1.2 in the Bragg peak. The OER is low over a $3 \mathrm{~cm}$ region straddling the Bragg peak.

\section{DISCUSSION}

Figure 6 is a composite plot of OER and hypoxic RBE values at $10 \%$ survival over the last $7 \mathrm{~cm}$ of residual range of $400 \mathrm{MeV} / \mathrm{amu}$ carbon, $425 \mathrm{MeV} / \mathrm{amu}$ neon, and $530 \mathrm{MeV} / \mathrm{amu}$ silicon, and $570 \mathrm{MeV} / \mathrm{amu}$ argon beams. The comparison generally indicates increasing effectiveness and decreasing oxygen effect with increasing atomic number of the particle, especially in the last few centimeters of range near the Bragg peak. The order of the relationship of the effects for the beams continues even at residual ranges beyond the peak, where beam fragments are primarily responsible for cell killing.

The carbon and neon beams both show sharp and rather narrow RBE peaks and OER valleys; whereas, the silicon and argon RBE peaks and OER valleys are somewhat broader. The neon, silicon, and argon RBE values peak upstream of the Bragg peak; whereas carbon RBE values peak at the closest residual range measured near the Bragg peak. The width of the RBE peak increases dramatically as a function of residual range with increasing atomic number from neon to argon.

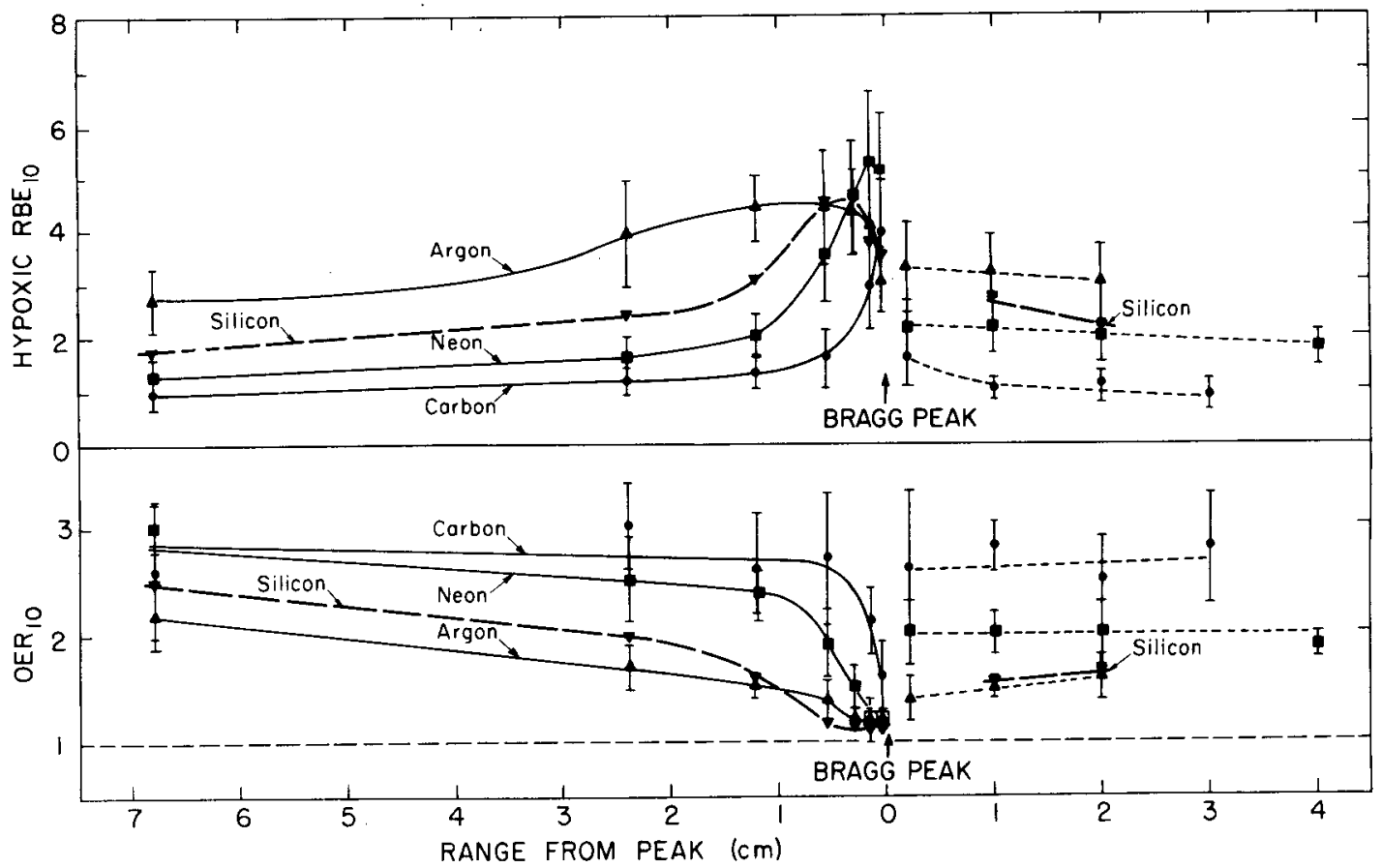

Figure 6. OER and nypoxic RBE at $10 \%$ survival as a function of beam range: carbon $(0)$, neon (D), silicon ( $\boldsymbol{\nabla}$ ) and argon ( 1 ). Error bars represent 95\% confidence interval. (XBL 808-3603) 
The effects of beam range modulation by spiral ridge filters on cell killing is highly dependent on the mixture of stopping particles at each location across the desired region of isoeffectiveness. Range modulation of the RBE and OER properties of the beam therefore occurs concomitantly.

The single cross-fired silicon experiment was completed to examine plateau and cross-fired peak RBE and OER values. The hypoxic RBE at 10\% survival was 3.4 in the cross-fired peak, and 1.5 in the plateau. These RBE values are more like the comparable neon values than like the comparable argon values. The OER at $10 \%$ survival is 1.4 for the cross-fired peak, which is much closer to the arqon cross-fired DER of 1.3 than to the neon cross-fired OER of 1.8. On the bas is of this comparison the silicon beam has an LET distribution that is more favorable than neon or argon for therapeutic purposes which require an argon-like low OER, but with a peak-to-plateau RBE ratio more like neon.

The comparison of RBE and OER values from the same residual range position for silicon beams of two energies ( 530 and $670 \mathrm{MeV} / \mathrm{amu}$ ) demonstrates the expected results. The hypoxic RBE value at $10 \%$ survival was 4.4 for the $D$ position of the $530 \mathrm{MeV} / \mathrm{amu}$ silicon beam, and the OER was 1.3. The hypoxic RBE value at $10 \%$ survival was 3.9 for the comparable $D^{*}$ position of the $670 \mathrm{MeV} / \mathrm{amu}$ beam, and the OER was 1.4. Consistent with previous results with other beams, the silicon beam with the higher initial energy had a slightly lower RBE and a slightly higher OER than the silicon beam with the lower initial energy.

In summary, the silicon data from two experiments are consistent with expectations based on measurements from neon and argon beams of similar range. The radiobiological properties of the silicon beam appear to be quantitatively intermediate between the neon and argon results. Limited extended peak and high energy survival measurements indicate that silicon may be of significant therapeutic usefulness at depths of both 14 and $24 \mathrm{~cm}$.

\section{ACKNOWLEDGEMENTS}

The authors wish to acknowledge the outstanding cooperation of R. Force, F. Lothrop, H. Grunder, M. Tegawa, J. Alonso, R. Miller, J. Howard, K. Crebbin, R. Gisser, and other members of the LBL Accelerator Division, whose skilled efforts made these silicon beam studies possible. The excellent technical assistance of T. Yang, L. Craise, I. Madfes, M. Yezzi, F. Abrams, K. Knight, R. Zamora, and G. Kraft was an essential component of this work. This work was supported, in part, by the National Cancer Institute (Grant .CA15184).

\section{REFERENCES}

Blakely, E. A., C. A. Tobias, T.C.H. Yang, K. C. Smith, and J. T. Lyman. 1979. Inactivation of human kidney cells by high energy monoenergetic heavy ion beams. Radiat. Res. 80 , $122-160$.

Lyman, J. T. and J. Howard. 1977. Dosimetry and instrumentation for helium and heavy ions. Int. J. Radiat. Oncol. Biol. Physics 3 , 81-86.

Tobias, C. A., E. L. Alpen, E. A. Blakely, J. R. Castro, A. Chatterjee, G.T.Y Chen, S. B. Curtis, J. Howard, J. T. Lyman, and F.Q.H. Ngo. 1979. Radiobiological basis for heavy-ion therapy in Treatment of Radioresistant Cancers. (M. Abe, K. Sakamoto, and T. L. Phillips, eds.) pp. 159-183. Elsevier/North-Holland Biomedical Press, Amsterdam. 


\title{
HEAVY-ION CELL-CYCLE RESPONSE AND PROGRESSION EFFECTS
}

\author{
E. A. Blakely, F. Q. H. Ngo, P. Y. Chang, L. Lommel, W. Kraft-Weyrather,* \\ G. Kraft," and C. A. Tobias
}

Variations in the radiosensitivity of cultured mammalian cells at different phases of the division cycle have been known since the early 1960s (Terasima and Tolmach, 1961, 1963; Sinclair and Morton, 1963, 1966). It was not, however, until the late 1960s and early 1970s that highLET radiations were found to diminish the amplitude of the variation between the most sensitive and the most resistant phases. This has been observed with fast neutrons (Sinclair, 1970; Hal1, 1968; Masuda, 1971; Hall et al., 1972, 1975; Sapozink and Djordjevic, 1974; Gragg et a1., 1978), with alpha particles (Hall et al., 1972; Raju et al., 1975), with pions (Raju et al., $1978 a, b)$, and with heavy-ion particle beams (Skarsgard 1966; Elkind, 1970; Bird, 1972; Bird and Burki, 1971, 1975; Hall et al., 1977; Raju et al., 1980).

Although it is generally agreed that the variation in radiosensitivity to high-LET radiation is reduced compared to that observed for $X$ rays, there is not sufficient data in the literature to make quantitative comparisons as to the magnitude, quality, and LET dependence of the reduction. More systematic studies are needed to establish the LET dependence of this reduction phenomenon.

The cellular age response to low-LET radiations can be generalized into two forms: one for cells with a short $G_{1}$ phase, and one for cells with a long $G_{1}$ phase (Sinclair, 1972). Both forms show a similar response at cell cycle ages beyond the sensitive $G_{1} / S$ interface, through a maximally resistant late $S$ phase, and finally a sensitive $G_{2}$ and $M$ population. However, in those cells with a long $G_{1}$ phase, there is an additional radioresistance early in $G_{1}$ that declines at the $G_{1} / S$ transition to a value about equal to that for mitotic cells.

Most reports that demonstrate a reduced age variation with high-LET radiation indicate that the variation seen is qualitatively similar to $X$ rays. However, there is also conflicting evidence for this point, since Raju et al. (1975) have found that cells are most sensitive to alpha particles in the late $S$ phase, whereas the peak of radioresistance appears to be in $G_{2}$ or early $G_{1}$. This apparent reversal in the most sensitive and most resistant stages of the cell cycle for cells exposed to alpha particles compared to those exposed to $x$ rays has not been explained.

In addition to studies of cell age radiosensitivity using the clonogenic capacity of the irradiated cell as an end point, division delay and/or $G_{2}$ block induced by $n$ igh-LET radiations have also been studied with neutrons (Schneider and Whitmore, 1963; Ngo et a1., 1977), with pions
(Schlag et al., 1978), with alpha particles (Raju et al., 1980), and with heavy-ion beams (Skarsgard, 1964; Elkind, 1967; Lücke-Hühle et al., 1979; M. Collyn et al., private communication).

Lücke-Hühle et a1. (1979) quantitatively measured cell cycle progression effects induced by high-energy heavy ions at the Bevalac using Chinese hamster $V-79$ monolayers in track segment experiments with monoenergetic heavy-ion beams. They observed a profound $\mathrm{G}_{2}$-b lock that had an LET dependence similar to the cell killing end point, but with a greater biological effectiveness. An example of the drastic $G_{2}$ block measured after 400 rad of carbon peak ions is given in Figure 1. In contrast to gamma and $X$ rays, they found heavy ions did not affect cell traversal through the $G_{1}$ and $S$ phase.

This chapter will present a summary of age response and cell progression studies completed or currently in progress at the Bevalac and SuperHILAC.

METHODS

\section{Radiation Sources}

The Bragg curves for the $403 \mathrm{MeV} / \mathrm{amu}$ carbon and $429 \mathrm{MeV} /$ amu neon Bevalac beams studies are plotted in Figure 2. Dosimetry and irradiation geometry are discussed in detail elsewhere (Blakely et al., 1979). The positions marked "F" represent residual ranges in the Bragg peak of each beam. The mean track average LET values, calculated by Tobias (and published in Blakely et al., 1979), are $85 \mathrm{keV} / \mu \mathrm{m}$ for the carbon peak position, and $234 \mathrm{keV} / \mathrm{um}$ for the neon peak. A single experiment was also done in the plateau of the carbon beam, indicated as position $0^{\prime}$ in Figure 2. Carbon particles at this residual range have a mean $\mathrm{LET}_{\infty}$ of approximately $11 \mathrm{keV} / \mu \mathrm{m}$. Lead scattering foils were inserted upstream from the last set of quadrupole and steering magnets to ensure adequate radial beam un iformity.

For: age response studies, $8.5 \mathrm{MeV} / \mathrm{amu}$ and $570 \mathrm{MeV} / \mathrm{amu}$ argon ion beams were also used (Bragg curves not shown)... Positions designated " $B$ " and "E," with residual ranges of $2.4 \mathrm{~cm}$ and $0.29 \mathrm{~cm}$, respectively, were used for the high energy exposures. The mean LET $T_{\infty}$ of particles at these ranges were $117 \mathrm{keV} / \mathrm{\mu m}$ and $245 \mathrm{keV} / \mathrm{\mu m}$, respectively. A plateau position was used with the low energy beam, and the mean LET of these particles was $1,800 \mathrm{keV} / \mu \mathrm{m}$.

$x$-irradiation of the cells was performed with a Philips X-ray unit operated at $225 \mathrm{kVp}$ and 


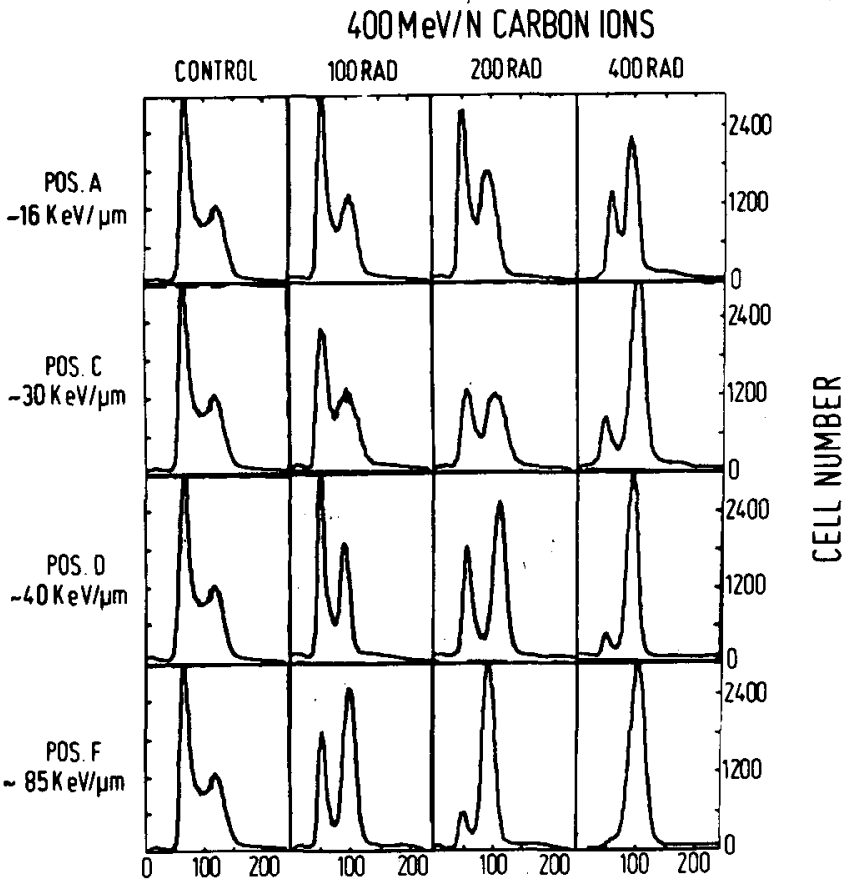

CHANNEL NUMBER [PROPORTIONAL TO DNA CONTENT]

Figure 1. DNA histograms from CHV-79 cells after 100, 200, and $400 \mathrm{rad}$ or $400 \mathrm{MeV} / \mathrm{amu}$ carbon ions at different positions of beam range. (From Lücke-Hühle et a1., 1979). (XBL 8010-12583)
$15 \mathrm{~mA}$ with a total filtration of $0.35 \mathrm{~mm} \mathrm{Cu}$ and a half-value layer of $1.09 \mathrm{~mm} \mathrm{Cu}$. The dose rate was usually $140 \mathrm{rad} / \mathrm{min}$, as measured with a calibrated Victoreen: condenser 250 R-meter at a target distance of $40 \mathrm{~cm}$. Samples were irradiated through the supporting plastic growing surface of a pair of flasks each placed vertical to the horizontal axis of both the $X-r a y$ and the heavy-ion beams.

\section{Cell cycle Parameters}

Chinese hamster lung $V-79$ cells (obtained from Dr. R. B. Painter, San Francisco) were routinely grown as monolayers. in Eagle's minimum essential media with Earle's salts supplemented with $12.8 \%$ fetal bovine serum and antibiotics. The mean population doubling time at $37^{\circ} \mathrm{C}$ in humidified air with $5 \% \mathrm{CO}_{2}$ was 10 to $11 \mathrm{~h}$. The $v-79$ cell-cycle generation time was approximately 10.5 hours as shown in the tritium pulse-labelled experiment ( $15 \mathrm{~min}, 0.05 \mu \mathrm{Ci} / \mathrm{ml}, 17 \mathrm{Ci} / \mathrm{m}$ mole) plotted in Figure 3. Cells were synchronized by mitotic selection. Cell synchronization methods used were similar to those of Sinclair and.Morton (1965). For T-1 cells, we found it necessary to preshake the cell population five times prior to the final selection. The mean generation time of the $V-79$ cells (estimated from Figure 3 ) has been subdivided into an average $G_{1}+M / 2$ of $1.75 \mathrm{~h}$, an average $S$ of $7.5 \mathrm{~h}$, and an average $\mathrm{G}_{2}+\mathrm{M} / 2$ of $1.20 \mathrm{~h}$.

Human kidney T-1 cells (provided by Dr. G. W. Barendsen, Rijswijk, The Netherlands) were routinely maintained under identical conditions as the $V-79$ cells. The mean population doubling

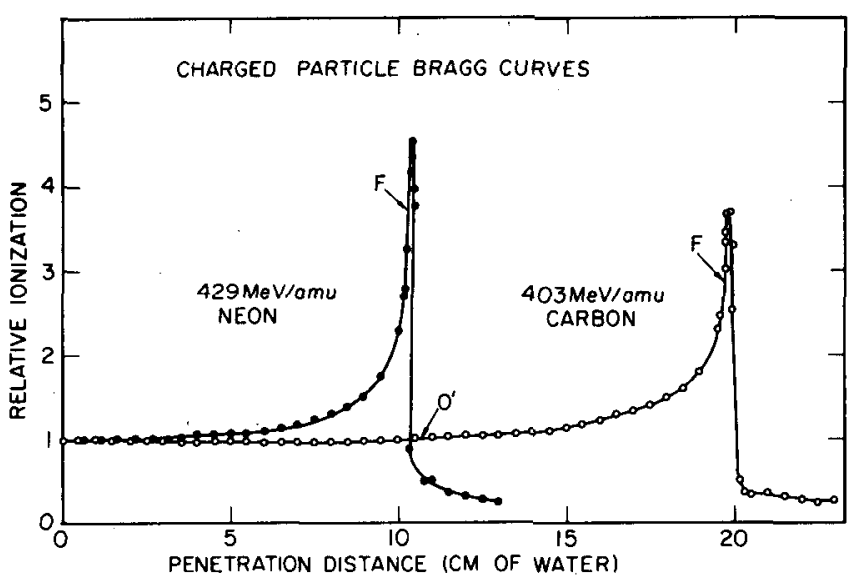

Figure 2. Bragg ionization curves for $429 \mathrm{MeV} / \mathrm{amu}$ neon and $403 \mathrm{MeV} /$ amu carbon beams showing beam range positions used for studies of cell. cycle response and progression effects. (XBL 805-3355)

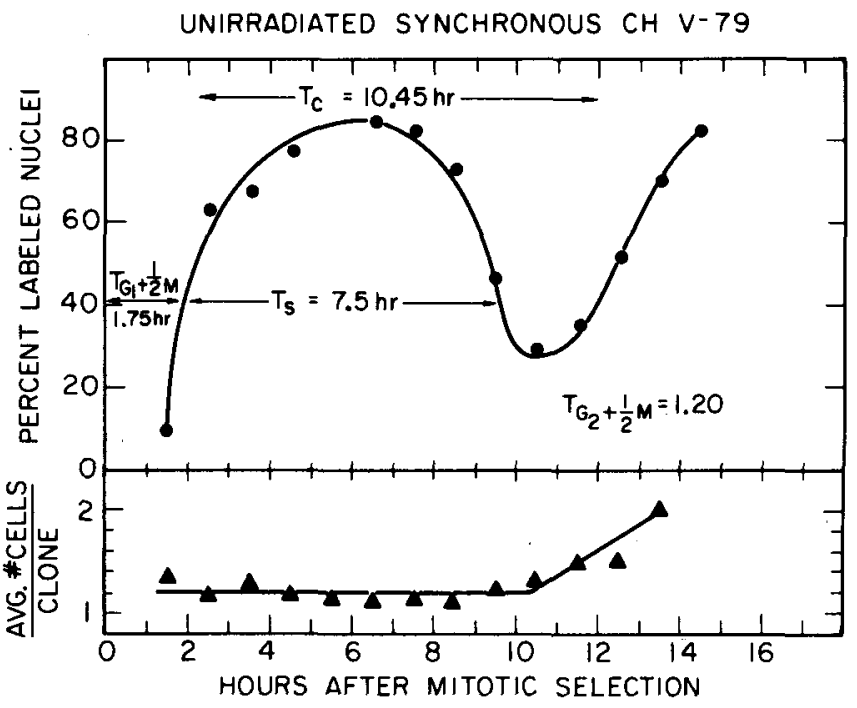

Figure 3. Percent of synchronized Chinese hamster cells labelled with a 15 min pulse of. ${ }^{3} \mathrm{H}-\mathrm{TdR}$ at various times after mitotic selection. Cell cycle parameters have been estimated based on a generation time of 10.45 hours.

(XBL 805-3353) 
time was about $22 \mathrm{~h}$. The concentration of glutamine used for stock cultures was doubled for experimental T-1 cultures. Published T-1 cell cycle generation times have estimated a $G_{1}$ phase of $14 \mathrm{~h}$, an $S$ phase of $8 \mathrm{~h}, \mathrm{G}_{2}$ phase of $5 \mathrm{~h}$, and a mitotic phase of $0.8 \mathrm{~h}$ for a $27 \mathrm{~h}$ population doubling time (Galavazi and Bootsma, 1966). Our T-1 subclone has a $22 \mathrm{~h}$ doubling time, and is believed to have a shortened $G_{1}$ phase of approximately $9 \mathrm{~h}$.

\section{PLM Technique}

Twenty-four to forty-eight hours prior to irradiation, $6.5 \times 10^{5}$ exponentially growing $\checkmark-79$ cells were plated into $25 \mathrm{~cm}^{2}$ plastic, $T-f$ lasks in order to achieve a final cell number of $2-3 \times 10^{6}$ at the time of the experiment. Immediately prior to exposure, cultures were pulse labelled with $3 \mathrm{H}-\mathrm{TdR}$ (New England Nuclear) for $15-20$ minutes $(0.1 \mu \mathrm{Ci} / \mathrm{ml}, 20 \mathrm{Ci} / \mathrm{mM})$. Cultures were rinsed well and held at room temperature briefly after the ${ }^{3} \mathrm{H}-\mathrm{TdR}$ pulse, and during the radiation exposure for a total time that did not exceed 30 minutes. This process labels the $S$ phase cohort. The cultures were then immediately returned to $37^{\circ} \mathrm{C}$ unt il they were fixed, without colcemid, at the appropriate time interval. Control sham-irradiated samples were treated in an identical manner to control for the temperature excursion at $25^{\circ} \mathrm{C}$. After fixation, Kodak NTB-2 photographic liquid emulsion diluted 1:1 with distilled water was thinly layered onto the growing surface. After one week of exposure in the dark the autoradiographs were developed and prepared for scoring. Approximately 200-300 mitoses were scored per point for the determination of percent labelled mitoses (PLM).

Scoring labelled S-phase nuclei from synchronized populations (as was done in Figure 3 ) is a much less tedious microscopic task compared to scoring labelled metaphase figures from asynchronous PLM experiments. However, PLM experiments have the advantage of requiring less timedependent cell preparation, which is still a major consideration for experiments requiring. cyclotron beam availability. Within beam time limitations it was possible to prepare asynchronous cultures that could, at short not ice, be labelled with $3_{H-T d R}$. In addition, PLM experiments enable one to examine the duration of immediate delays in $G_{2}$ phase and mitosis for cells that continue to cycle. However, it should be noted that the progression of cells that will survive and those doomed to die cannot be distinguished with this technique. Division delay studies by Elkind et al. (1963) with $X$ rays, and more recently by Ngo et al. (1977) with neutrons, however, have indicated that division delay is the same for bothikilled and surviving cells.

The technique described above yields plots of percent labelled mitoses at specific times postirradiation. Control samples for each experiment provide an estimate of the duration of the $\mathrm{G}_{2}+\mathrm{M} / 2$ phase. Preliminary estimates of the increased duration of this interval, after irradiation with $X$ rays or heavy ions, were obtained from fits of the data made by eye. The PLM information represents perturbations in cycle $k$ inetics of a population of $S$ phase cells that moves from $S$, through $G 2$ phase, into $M$ phase. Further work is planned with synchronized populations when more beam time becomes available. Such experiments will help clarify some of the complicating factors inherent in PLM experiments of the design just described.

\section{Mitotic Index}

The percentage of mitotic cells was determined from 500 to 4,000 cell samples from each flask. When there were insufficient mitoses in the sample, especially at short time points after irradiation, more cells were scored.

\section{Cell Survival}

Cell cultures prepared in an identical fashion as described above for the PLM technique, except for the pulse treatment, were irradiated at various doses with each radiation tested. After the exposure, celis (both irradiated and unirradiated) were trypsinized, counted, and plated into four to eight flasks containing complete growth medium at appropriate dilutions. Colonies that formed after incubation at $37^{\circ} \mathrm{C}$ for 6 days ( $\mathrm{V}-79$ cells) or 11 days (T-1 cells) were stained and counted.

\section{RESULTS}

\section{Cell Age Response}

Cell age responses of synchronized Chinese hamster $V-79$ cells to $225-k V p X$ rays or $425 \mathrm{MeV} / \mathrm{amu}$ Bragg peak neon ions (234 keV/um) are presented in Figure 4A. The data were taken from a single experiment in which the cell growth after mitotic selection was controlled indentically for both $X$ and neon irradiations. The results show that at doses of $X$ rays and neon ions that yield approximately identical killing of $G_{1}$ cells, the amplitude of the survival variation throughout the cell cycle for $X$ rays is approximately 1.5 times greater than for neon.

It should be noted, however, that this cellcycle variation amplitude increases with increasing dose of neon ions to a lesser degree than for $X$ rays. This point is clearly demonstrated in $F$ igure $4 B$ where cells from. the same mitotic population were used to obtain whole survival curves at $G_{1} / S$ and late $S$ phase with either neon ions or $X$ rays. Per unit of absorbed dose, the difference in survival response between the two ages is much reduced for neon compared to $X$ rays. This is consistent with the concept that cell-age response variation decreases with increasing LET.

Survival curves of $\mathrm{CHV}-79$ cells at $\mathrm{G}_{1} / \mathrm{S}$ and mid-S stages from a $570 \mathrm{MeV} / \mathrm{amu}$ argon experiment are shown in Figure 5 . The cellis were exposed at the " $B$ " position of the Bragg curves where the 

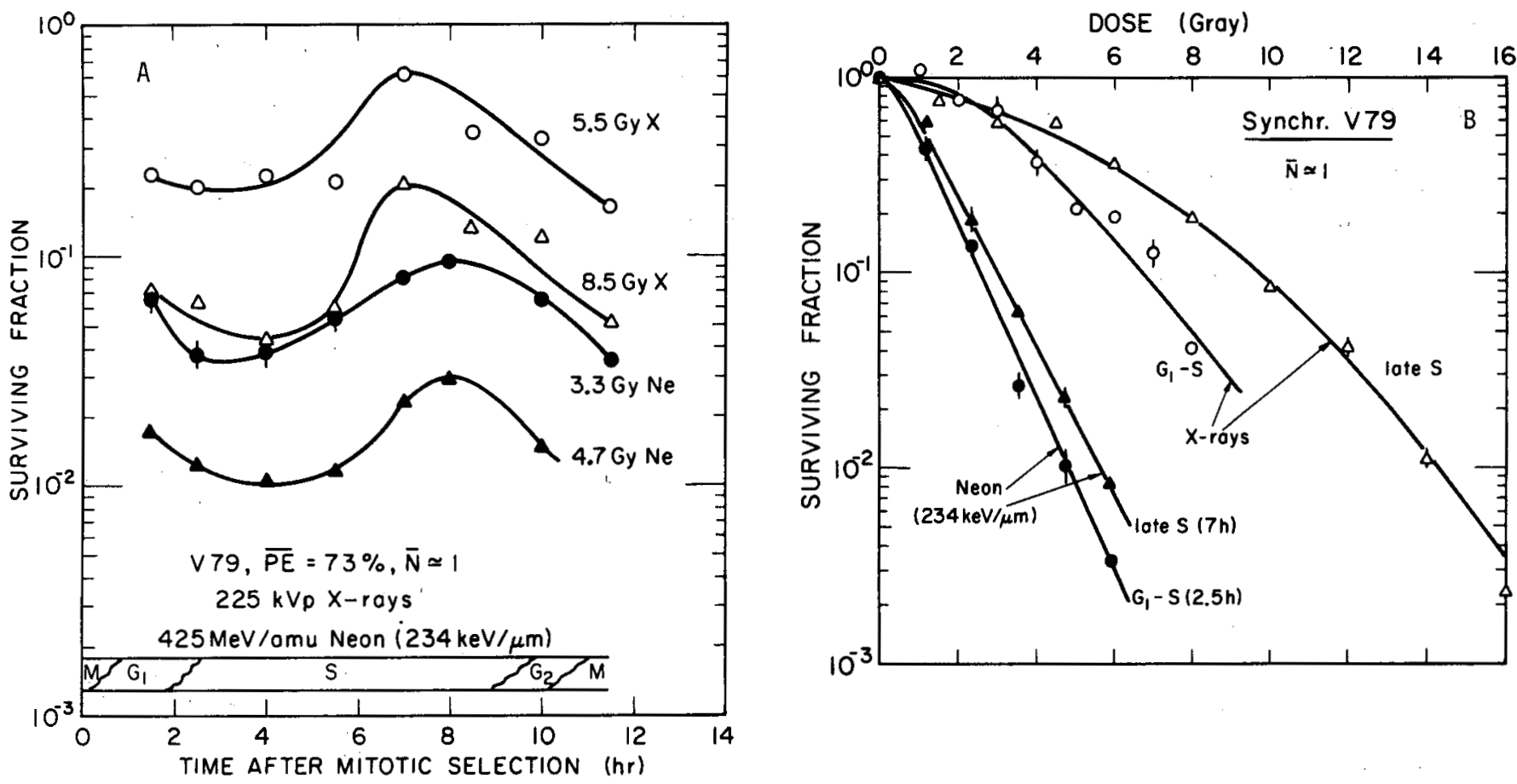

Figure 4. Radiation response of synchronized Chinese hamster $V-79$ cells to 225-kVp X rays and Bragg peak $425 \mathrm{MeV} / \mathrm{amu}$ neon ions, determined in the same population. (A) Age response for 5.5 and 8.5 Gy of $X$ rays, and 3.3 and 4.7 Gy of Bragg peak neon ions. (B) Survival dose-response of synchronized $G_{1} / S(2.5 \mathrm{~h})$ and late $S(7 \mathrm{~h})$ phase Chinese hamster $V-79$ cells to $X$ rays and Bragg peak neon ions. [(A) XBL 794-3416A; (B) XBL 794-3420]

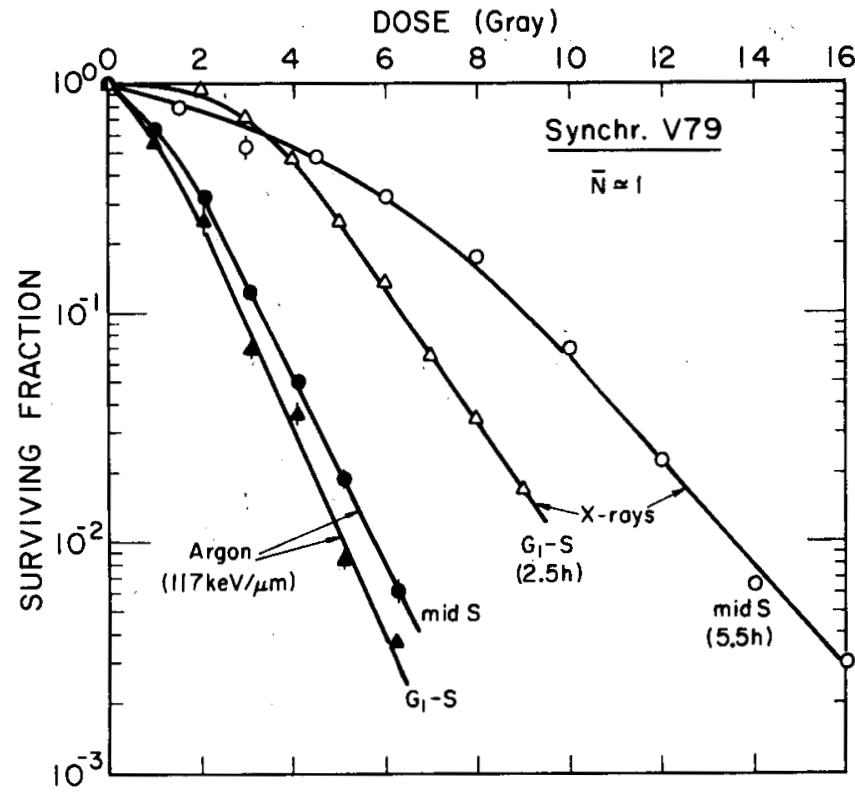

Figure 5. Survival dose-response of synchronized $\mathrm{G}_{1} / \mathrm{S}(2.5 \mathrm{~h})$ and mid $S(5.5 \mathrm{~h})$ phase Chinese hamster $V-79$ cells to $X$ rays and Bragg plateau $570 \mathrm{MeV} / \mathrm{amu}$ argon ions. (XBL 794-3423)
LET is $117 \mathrm{keV} / \mathrm{\mu m}$. The reduction of the age response is also obvious in this figure.

The cell age response of $\mathrm{CHV}-79$ cells exposed to $X$ rays or to $8.5 \mathrm{MeV} / \mathrm{amu}$ argon ions is illustrated in Figure 6 . The low-energy argon

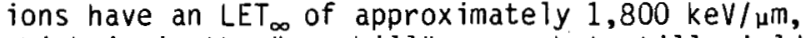
which is in the "overkill" range but still yields an exponential survival curve (not shown). The amplitude of the age response is quite diminished with this charged particle beam.

The relationship between the RBE for cell killing and the cell survival level is depicted in Figure 7. The Bragg peak $425 \mathrm{MeV} / \mathrm{amu}$ neon ion data are in the upper panel, and the Bragg plateau $570 \mathrm{MeV} / \mathrm{amu}$ argon data are in the lower panel. In the upper panel, the relationship for asynchronous cells is also shown. For neon ions, the RBE curves for the synchronized populations were derived from survival curves given in Figure 4B. The RBE curve for the asynchronous population was derived from survival curves of three different experiments. For argon ions, the RBE curves were derived from survival curves shown in Figure 5 . The results indicate that as a consequence of the heavy-ion reduction in the age response, $S$ phase cells generally have a greater RBE than do $G_{1} / S$ phase cells. Aerobic RBE values at low dose (high survival) for the neon and argon exposures are between 2.0 and 4.0 . 


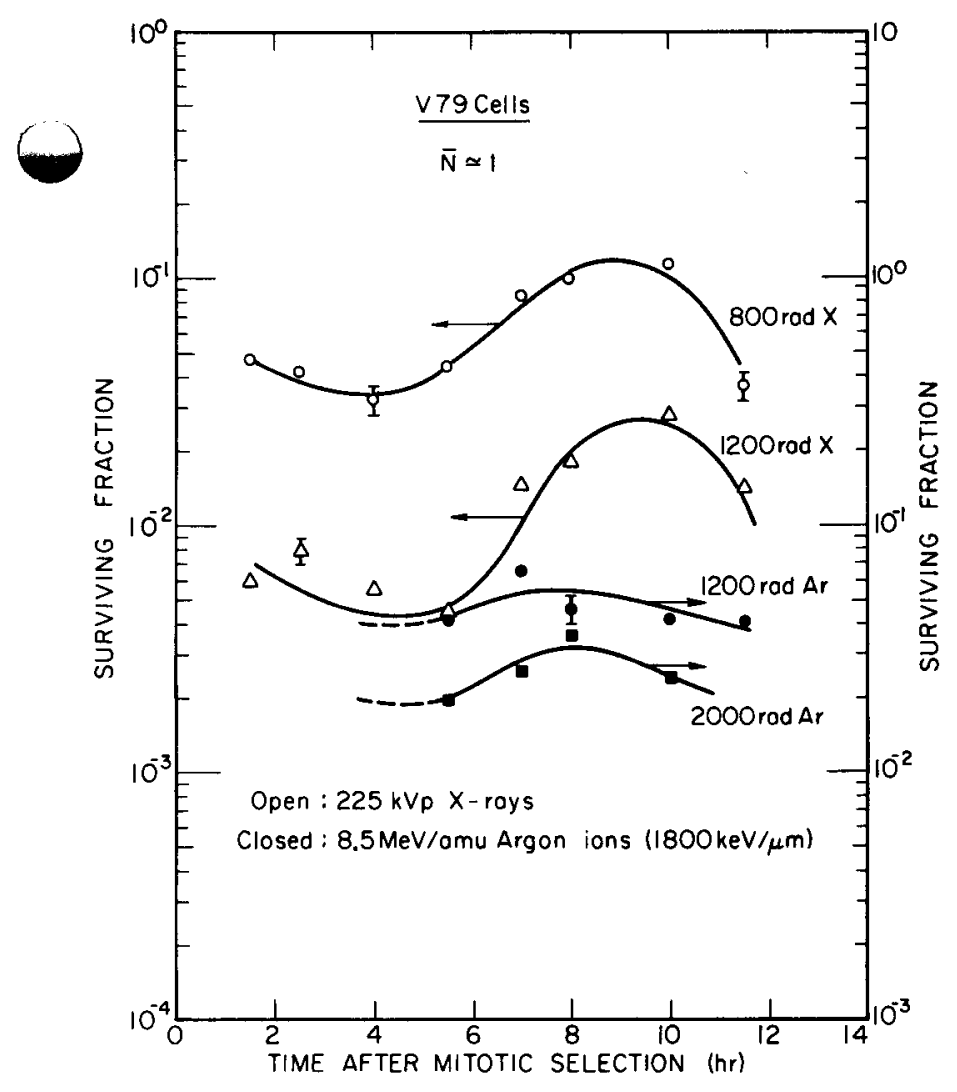

Figure 6. Age response for 8.0 and 12.0 Gy of $\bar{X}$ rays, and 12.0 and 20.0 Gy of Bragg plateau $8.5 \mathrm{MeV} / \mathrm{amu}$ argon ions determined in the same population of synchronized Chinese hamster cells. (XBL 7910-3825)

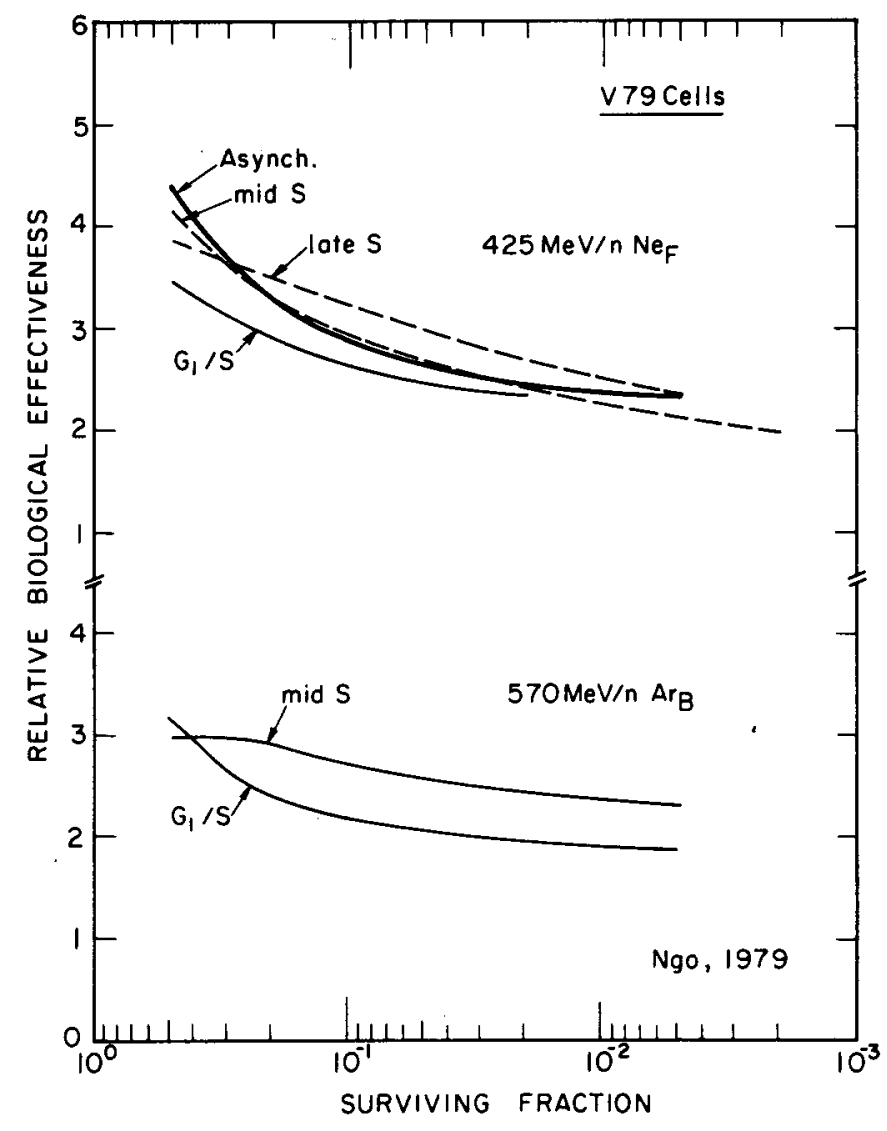

Figure 7. The relationship between the RBE for cell kiTling and the surviving fraction of Bragg peak $425 \mathrm{MeV} / \mathrm{amu}$ neon ions, and Bragg plateau $570 \mathrm{MeV} / \mathrm{amu}$ argon ions for synchronized CHV-79 cells at various stages of the cell cycle. In the upper panel, the relationship for asynchronous cells is also shown. The asynchronous data were obtained from three different experiments. (XBL 808-3567)

Synchronized human kidney cell survival response to Bragg peak argon ions is presented in $F$ igures $8 \mathrm{~A}$ and $8 \mathrm{~B}$. The argon survival curves are exponential at this LET and show a major reduction in differences between cells of different ages compared to those seen. at low-LET (data not shown). The amplitude of the age response shows some structure, but is quite flat in contrast to published X-ray age response. studies with T-1 cells (Vos et al., 1966).. $S$-phase cells are the most radioresistant, and. the $G_{1} / S$ boundary appears to be the most radiosensitive phase.

\section{PLM Studies}

Figure 9 presents the cell survival measured for the asynchronous Chinese hamster cells, with 225-kVp X rays, and peak carbon and neon ions. Despite almost a threefold difference in LET, both sets of particle data show an approximately identical survival curve with an RBE relative to $x$ rays of 2.9 at $10 \%$ survival. The LET-dependence of cell survival and cycle delay will be discussed in some detail later.
Figure 10 shows three unirradiated control experiments done on different days with slightly varying times for transporting the samples from the radiation source buildings to the cell Taboratory. This figure is intended to qualitatively illustrate the level of our resolution. The curve through the points was best-fitted by eye. Notice that the maximum level of incorporation is very high (over $95 \%$ ). The cell-cycle parameters estimated from these curves are in reasonable agreement with the synchronized pulselabelled experiment shown earlier ( $\mathrm{Fig} .3$ ). For the irradiated experiments to follow, the curve of each treatment was analyzed with respect to its own control.

The $X$-ray data from a single experiment with doses of 250,500 , or 750 rad are shown in Figure 11A. The eye-fitted curves show a marked shift to the right with increasing dose, which indicates a prolongation of $G_{2}$ and/or $M$ phases for those $S$ phase cells that made it to mitosis. The duration of $S$ phase only shows a slight 

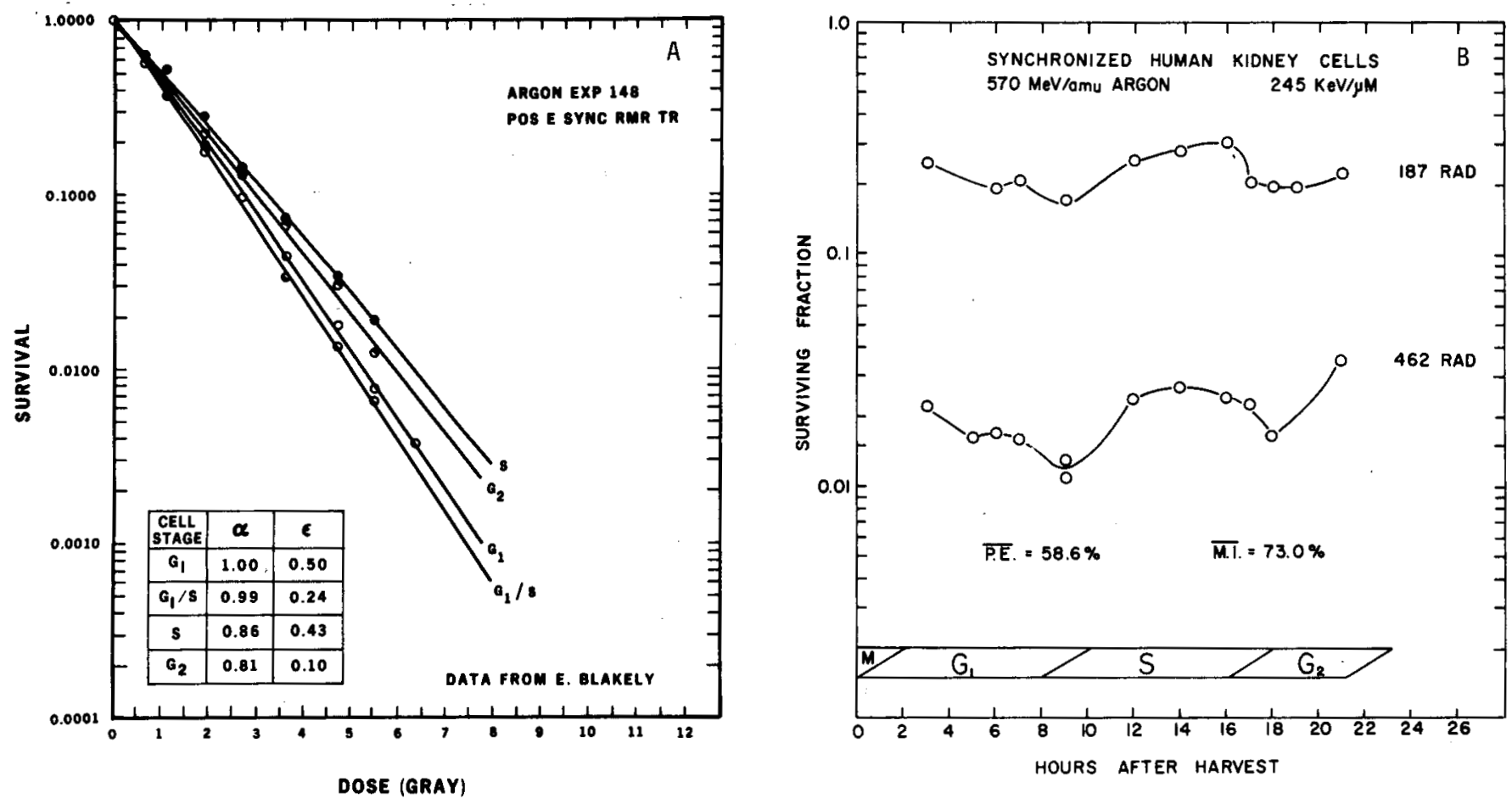

Figure 8. Radiation response of synchronized human kidney T-1 cells to Bragg peak $570 \mathrm{MeV} / \mathrm{amu}$ argon ions, determined in the same population. (A) Survival dose response of synchronized $\left(G_{1}, G_{1} / S, S, G_{2}\right)$ human kidney cells to Bragg peak argon ions. (B) Age response for 1.87 and 4.62 Gy of Bragg peak argon ions. [(A) XBL 794-9460; (B) XBL 795-9607]

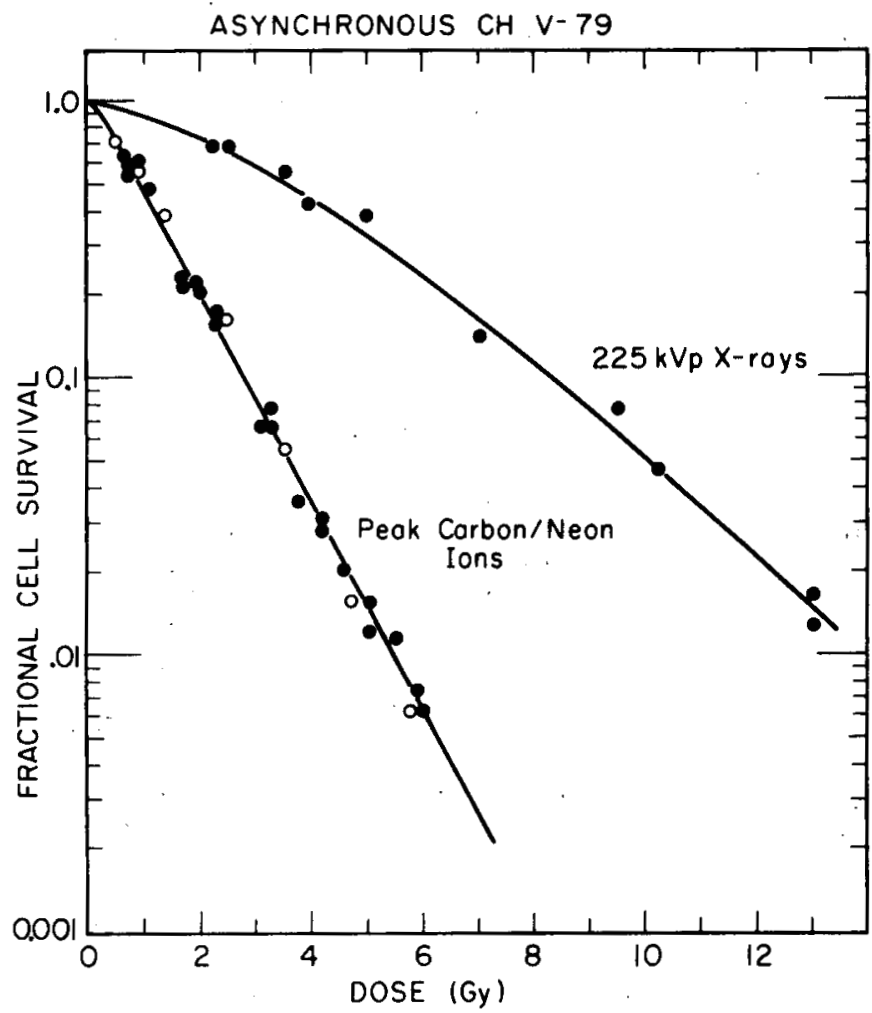

Figure 9. Survival dose resporise of asynchronized $\mathrm{CHV}-79$ cells to $225-\mathrm{kVp} X$ rays and Bragg peak $403 \mathrm{MeV} / \mathrm{amu}$ carbon and $429 \mathrm{MeV} / \mathrm{amu}$ neon ions. (XBL 805-3354)

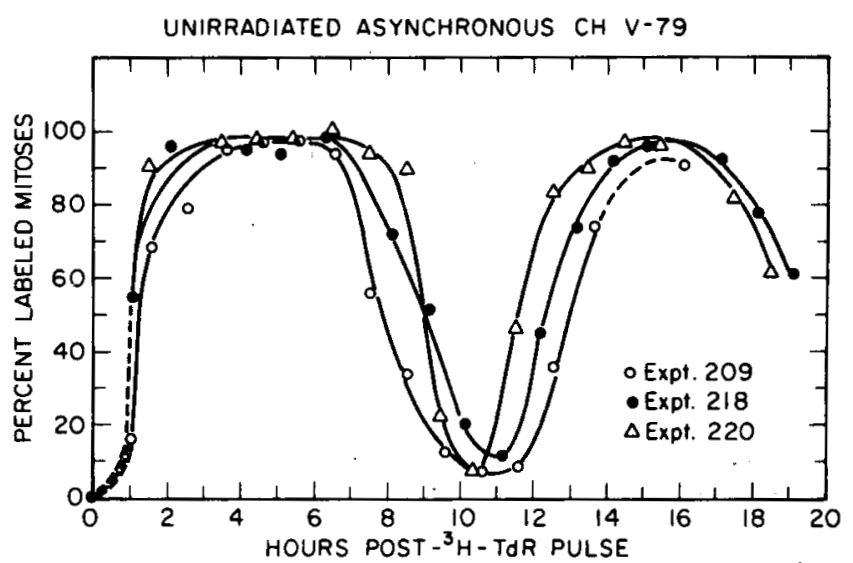

Figure 10. Pulse labelled mitoses plot for unirradiated asynchronous $\mathrm{CHV}-79$ cells pulsed at

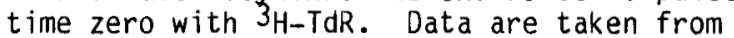
three different experiments. (XBL 808-3357) 

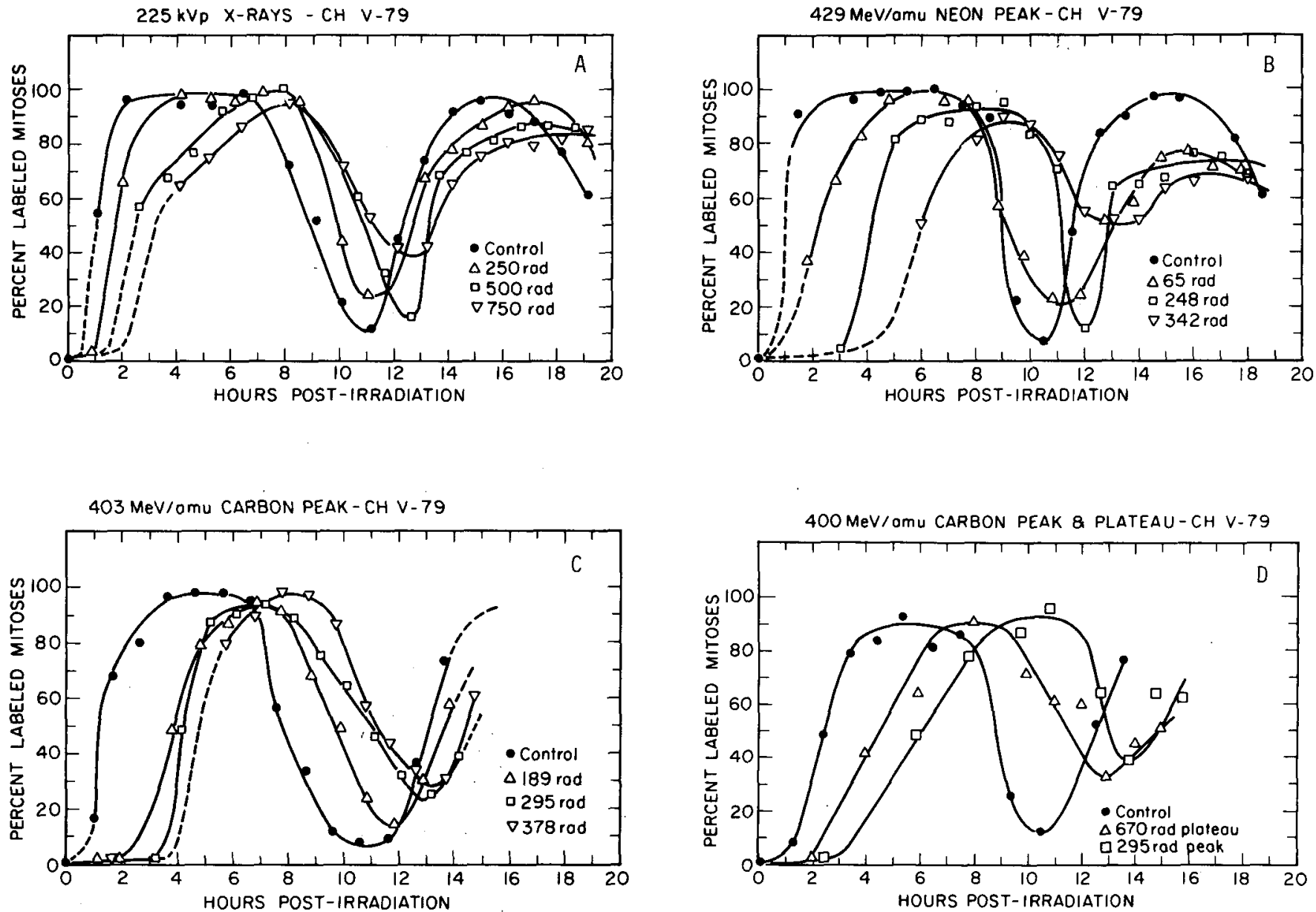

Figure 11. Pulse labelled mitoses plots for asynchronous CHV-79 cells pulse labelled at time zero with 3 H-TdR and irradiated with: (A) $2.5,5.0$, or 7.5 Gy of $225-k$ Vp $X$ rays (B) 0.65 , 2.4, or 3.42 Gy of Bragg peak $42.9 \mathrm{MeV} / \mathrm{amu}$ neon ions; (C) $1.89,2.95$, or 3.78 Gy of Bragg peak $403 \mathrm{MeV} / \mathrm{amu}$ carbon ions; (D) 6.7 Gy of Bragg plateau $400 \mathrm{MeV} / \mathrm{amu}$ Carbon ions or $2.95 \mathrm{~Gy}$ of Bragg peak $400 \mathrm{MeV} / \mathrm{amu}$ carbon ions. [(A) XBL 805-3358; (B) XBL 805-3356; (C) XBL 805-3359; (D) XBL $809-3676]$

indication of prolongation at the highest $X$-ray $: s$ dose studied, and cannot be demoristrated as significant based on the present inadequate level of incorporation of the label is as high as the controls in Figure 10, but the maximum incorporation appears to be slightly decreased. with higher dose. ment are illustrated in Figure 1.1 B. Neon doses that cause an even more dramatic shift of the curves to the right are lower than those of the: $x-r a y$ experiment. For the high dose, the second: wave of labelled mitoses is probably perturbed by the cells that are blocked in $M$, or that were: at $G_{1}$ at the time of irradiation but have moved to mitosis. Both of these.. factors complicate the interpretation of these curves at this time postirradiation.

Figure $11 \mathrm{C}$ shows the results of each of three doses of Bragg peak carbon ions. Notice that it $\therefore \rightarrow$

was more difficult to estimate the delays for this experiment. As will be seen later, the delayiplot as a function of dose shows more scatter for the carbon than for the neon or X-ray data. : ? :

Figure 110 shows data from an early PLM experiment in which cells were irradiated in the Bragg peak or in the plateau of a: $400 \mathrm{MeV} / \mathrm{amu}$ carbon beam, at doses that resülted in equivalent cell killing. Despite the dose correction for survival RBE, a G2 + M delay of the cycling cells (jirradiated with Bragg peak ions while they were in "S phase) was" induced, which was greater than the delay induced by the low-LET carbon plateau ions.

\section{DISCUSSION}

Figure 12 summarizes the specific $G_{2}+M$ delay examined as a function of dose for both $x-r a y$ and heavy-ion experiments. The preliminary 


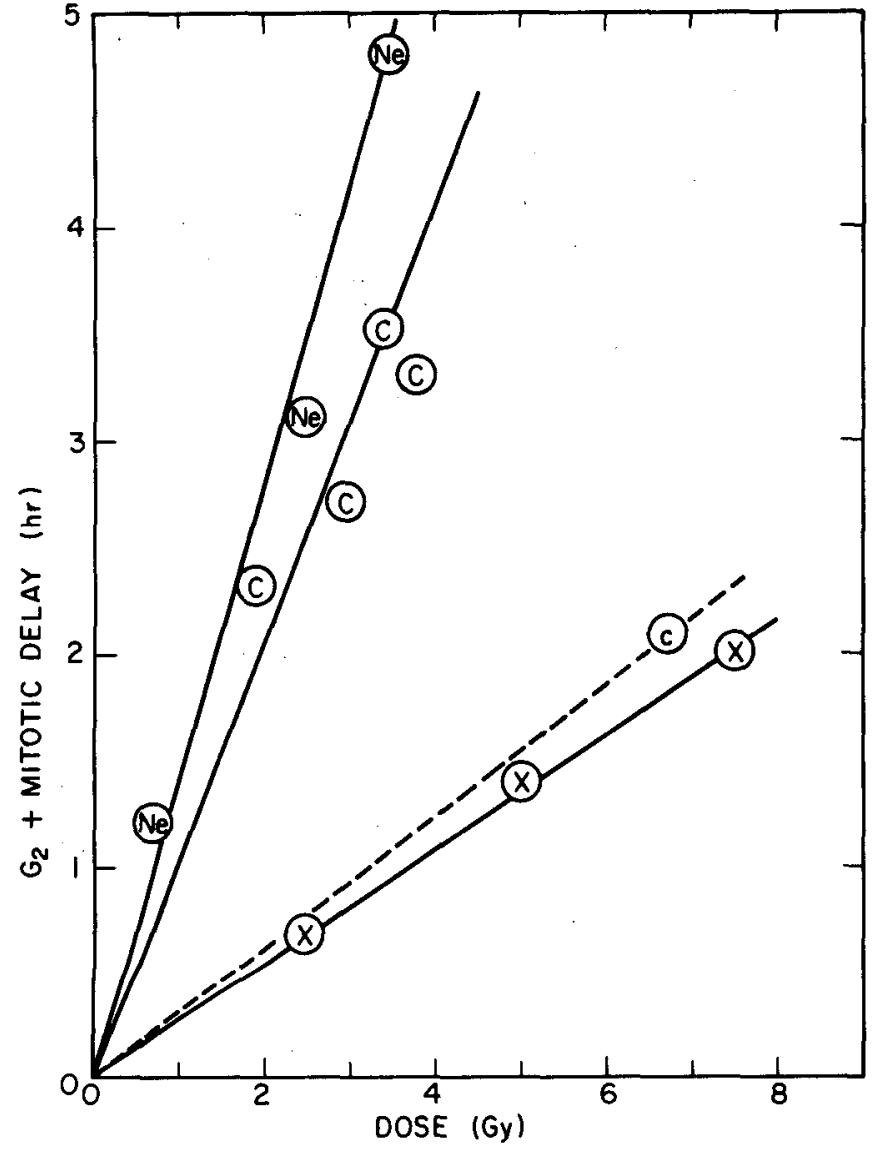

Figure 12. Duration of $\mathrm{G}_{2}+$ mitotic delay as a function of dose of 225-kVp $x$ rays (X); Bragg plateau $400 \mathrm{MeV} / \mathrm{amu}$ carbon ions (c); Bragg peak $403 \mathrm{MeV} / \mathrm{amu}$ carbon ions (C); or Bragg peak $429 \mathrm{MeV} / \mathrm{amu}$ neon ions (Ne). (XBL 805-3369)

data are admittedly only approximations but they do indicate a linear dose response. The slope of the $X$-ray curve is about $0.2 \mathrm{~min} / \mathrm{rad}$. The slope of the dotted line drawn through the single carbon plateau point is very similar (close to 0.2 ) which would be expected since carbon plateau at position $0^{\prime}$ has a low LET of $11 \mathrm{keV} / \mu \mathrm{m}$. Despite the almost identical survival curves obtained with the neon and carbon peak ions, the slopes of the lines for the mitotic delay end point are slightly different for the two ions $(0.8 \mathrm{~min} / \mathrm{rad}$ for neon and $0.6 \mathrm{~min} / \mathrm{rad}$ for carbon). With this type of plot it is possible to calculate an $R B E$ value for the $G_{2}+$ mitotic delay induced by the particle beams by taking the ratio of doses of $x$ ray and a particle beam that yields the same level of effect. This has been done at several levels of $G_{2}+M$ delay and the results are plotted in Figure 13.

Figure 13 shows RBE as a function of heavyion dose for the two end points $\left(G_{2}+\right.$ mitotic delay and cell killing). The top two lines on the figure indicate that the RBE for the delay end point is independent of heavy-ion dose in the dose region studied. This reflects the linear

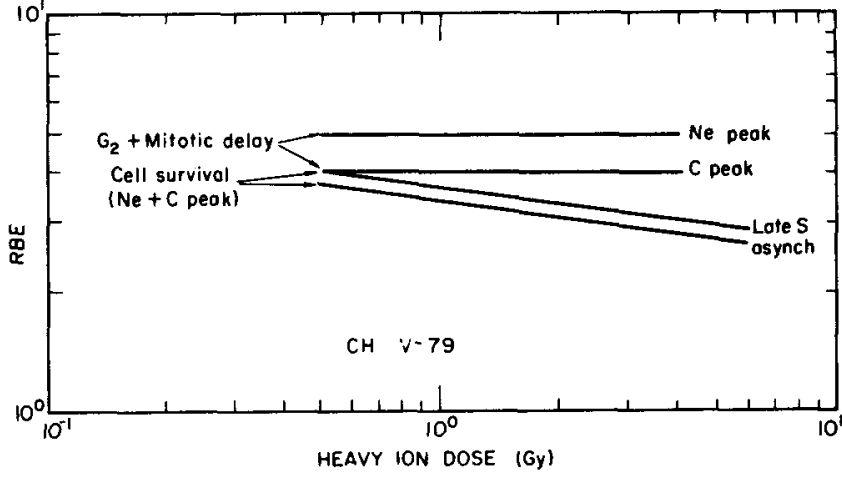

Figure 13. RBE for two end points (cell survival and $G_{2}+M$ delay) vs. heavy-ion dose. Survival data show response of asynchronous and late $S$ phase CHV-79 cells to Bragg peak $403 \mathrm{MeV} / \mathrm{amu}$ carbon ions and $429 \mathrm{MeV} / \mathrm{amu}$ neon ions. (XBL 805-3370)

relationship between the X-ray and heavy-ion response which was seen in Figure 12 . The effect is the same at all delay levels studied, with the RBE for the neon peak observed as greater than that for the carbon peak. In contrast, the lowest lines on the figure demonstrate that the RBE for cell killing has a dose dependence, with a higher RBE at the low-dose end for asynchronous cells irradiated with the neon or carbon peak ions. Cell killing for synchronized late $S$ phase V-79 cells was measured in separate experiments (Ngo et al., 1980). These data are more representative of the population we are studying. The $S$ phase cells are more $X$-ray radioresistant, and the RBE for this synchronized population is greater than that seen for the asynchronous population. Note that the RBE for $\mathrm{G}_{2}+$ mitotic delay is greater than the RBE for cell killing, especially at high doses and even at doses that give equivalent cell killing.

Figure 14 is a plot of RBE as a function of mean average LET. The open circles represent the $10 \%$ cell survival end point, and the closed circles show the result for the $50 \%$ survival level. The data are based on carbon, neon, and argon ion experiments. A single curve has been drawn through all the points, although there is evidence (Blakely et al., 1979) to indicate it is more appropriate to draw a line through the data from each ion at LET values above $100 \mathrm{keV} / \mathrm{\mu m}$. To simplify the discussion, this has not been done. Carbon and neon at 85 and $234 \mathrm{keV} / \mu \mathrm{m}$, respectively, yield the same RBE for cell killing because these data appear to lie on equal sides of a peak in the RBE curve. The open triangles show the LET dependence of RBE for the $\mathrm{G}_{2}+M$ delay.

A straight line has been drawn through the points, but in fact we need to examine the results at more LET values to understand the relationship fully. It also appears that the maximum $R B E$ of the $G_{2}+M$ delay end point may have been missed by not examining an LET of 


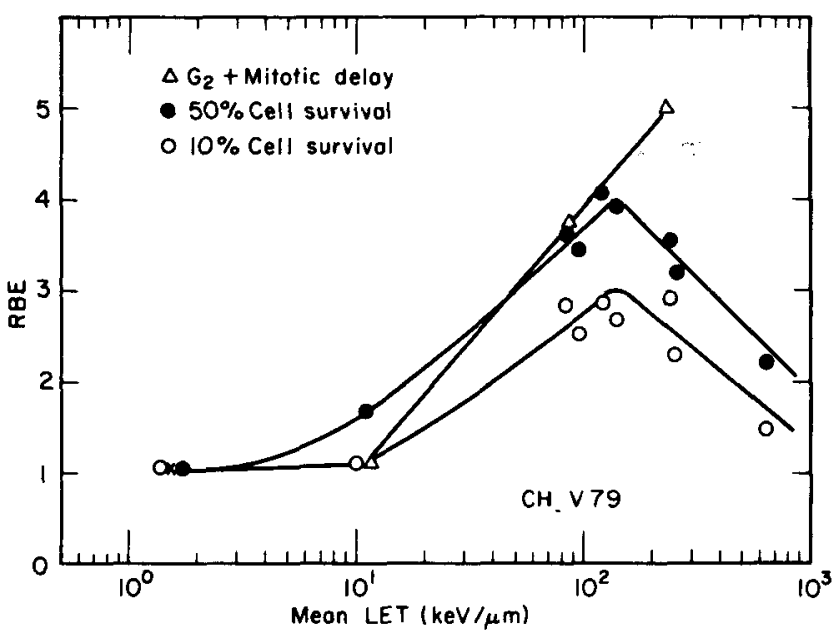

Figure 14. RBE for two end points (cell survival track average $\mathrm{LET}_{\infty}(\mathrm{keV} / \mathrm{\mu m})$. Data are taken from CHV-79 cell experiments with 400-403 MeV/amu carbon, 425-429 MeV/amu neon, and $570 \mathrm{MeV} / \mathrm{amu}$ argon-ion beams. (XBL 805-3368) at $50 \%$ and $10 \%$, and $G_{2}+M$ delay) vs. mean

energies and found that radiation-induced delays of cellular progression through the cell cycle are optimally affected by particles depositing about $100 \mathrm{keV} / \mu \mathrm{m}$.

The PLM curves for X-ray or heavy-ion irradiated $V-79$ cells in the present report indicate that within the resolution of the curve fits made by eye, there is no obvious increase in the duration of the $S$ phase subsequent to irradiation. This observation appears to support the heavy-ion results of Lücke-Hühle et al. (1979), and the pion results of Schlag et al. (1979), but indicates PLM curves may not be the most sensitive way to estimate $S$ phase delay.

The PLM studies of Geard (1980) show only a slight, and probably not significant, increase in the duration of $S$ phase after irradiation with helium ions, even at LET values between 63 and $138 \mathrm{keV} / \mu \mathrm{m}$. In contrast, Raju et al. (1980) using FMF techniques, concluded that there was a significant prolongation of the $S$ phase of $V-79$ cells irradiated with alpha particles compared to comparable cell killing doses of $X$ rays. Work is in progress to resolve these differences, and to elucidate the mechanism of the $G_{2}$ block and division delay for low and high LET radiations.

\section{ACKNOWLEDGEMENT}

tha $\mathrm{keV} / \mu \mathrm{m}$, or that it may peak at a greater LET than the cell killing end point. Skarsgard (in Elkind and Whitmore, 1967) using Chinese hamster cells $\left(\mathrm{CH}_{2} \mathrm{~B}_{2}\right)$ and low-energy heavy-ion beams of helium, lithium, oxygen, boron, and carbon has shown that the RBE values for mitotic delay, chromatid exchanges, and survival all reach maxima for LET values of approximately 150 to $200 \mathrm{keV} / \mathrm{\mu m}$, and then fall off for higher values of LET. Skarsgard used higher doses for his RBE determination for mitotic delay than we have used here; however, our $R B E$ values for $G_{2}+M$ delay are similar to his, with both sets of data reaching a maximum of about 4.0 to 5.0 .

In contrast to Skarsgard's results, however, we do not see an exponential dose dependence for mitotic delay. Our dose dependence appears to.. be linear, similar to that seen by Lücke-Hühle et al. (1979). The latter authors used the flow: microfluorometry (FMF) method to analyze the number of cells arrested in $G_{2}+M_{1}$ and: observed similar (i.e., 4.4 to 4.7). RBE values for carbon $400 \mathrm{MeV} / \mathrm{amu}$ and neon $425 \mathrm{MeV} / \mathrm{amu}$ : Bragg peak Bevalac beams, when the RBE was calculated as a ratio of the slopes of the plots of maximum number of accumulated $\mathrm{G}_{2}+M$ cells. vs. dose. They obtained even higher values (i.e., 8.3 for caroon peak) when they calculated the RBE by comparing doses yielding $50 \%$ of the maximum: number of cells in $\mathrm{G}_{2}+M$ at 8 hours after irradiation. These differences may be a result of the fact that the FMF technique scores all cells in the $G_{2}+M$ block no matter at what stage the irradiation or block occurred, whereas the PLM technique only scores the $\mathrm{G}_{2}+M$ block incurred by cells in $S$ phase at the time of irradiation. Using PLM techniques, Geard (1980) studied cell cycle progression of Chinese hamster $\checkmark-79$ cells irradiated with helium ions of various
The authors wish to acknowledge the assistance of the LBL Accelerator Division and the Bevalac operations staff for their skills and patience in providing Bevalac beams under the stringent time constraints required for these experiments. The outstanding technical assistance of R. Roots, K. Smith, 0. Oleszko, and M. Yezzi is gratefully acknowledged. This investigation was supported by the National Cancer Institute DHEW, Grant No. CA-15184.

\section{FOOTNOTES AND REFERENCES}

* Presently on leave from GSI, Darmstadt, West Germany.

Bird, R. P. 1972. Sensitivity of Synchronized Mammalian Cells to High LET Radiation. Ph.D. Thesis, University of California, Berkeley.

Bird, R. P. and J. Burki. 1971. Inactivation of mammalian cells at different stages of the cell. cycle as a function of radiation linear energy transfer. Biophysical Aspects of Radiation Quality, pp. 241-250. IAEA, Austria.

Bird, R. P. and H. J. Burki. 1975. Survival of synchronized Chinese hamster cells exposed to radiation. of different linear energy transfer. Int. J. Radiat. Biol. 17, 105-120.

Blakely, E. A., C. A. Tobias, T.C.H. Yang, K. C. Smith, and J. T. Lyman. 1979. Inactivation of human kidney cells by high-energy monoenergetic heavy-ion beams. Radiat. Res. 80, 122-160. 
Elkind, M. M., A. Han, and K. W. Volz. 1963. Radiation response of mammalian cells grown in culture. IV. 'Dose dependence of division delay and postirradiation growth of surviving and non-survivig Chinese hamster cells. J. Nat). Cancer Inst. 30, 705-721.

Galavazi, G. and D. Bootsma. 1966. Synchronization of mammalian cells in vitro by inhibition of the DNA synthesis. II. Population dynamics. Expt. Cell Res. 41, 438-451.

Geard, C. R. 1980. Changes in cell cycle progression of. Chinese hamster $V-7.9$ cells induced by high LET charged particle. Radiat. Res. 83, 696-709.

Gragg, R. L., R. M. Humphrey; H. D. Thames, Jr., and R. E. Meyn. 1978. The response of Chinese hamster ovary cells to fast neutron radiotherapy beams: III. Variation in relative biological effectiveness with position in the cell cycle. Radiat. Res. 76, 283-291.

Hall, E. J: 1969. Radiobiological measurements with 13 MeV neutrons: Br. J. Radiol. 42, 805-813.

Hall, E. J., W. Gross, R. F. Dvorak, A. M. Kellerer, and H. H Rossi. 1972. Survival curves and age response functions for Chinese hamster cells exposed to $X$ rays or high LET alpha-particles. Radiat. Res. 52, 88-98.

Hal1, E. J., L. Roizin-Towle, R. B. Theus, and L. S. August. 1975. Radiobiological properties of high energy. cyclotron-produced neutrons used in radiotherapy. Radiology 117, 173-178.

Hal1, E. J., R. P. Bird, H. H. Rossi, R. Coffey, J. Varga, and Y. M. Lam. 1977. Biophysical studies with high energy argon ions. II. Determinations of the relative biological effectiveness, the oxygen enhancement ratio, and the cell cycle response. Radiat. Res. 70, 469-479.

Lücke-Hühle, C., E. A. Blakely, P. Y. Chang, and C. A. Todias. 1979. Orastic G? arrest in mammalian cells after irradiation with heavy ion beams. Radiat. Res. 79, 97-112.

Masuda, K. 1971 Survival of synchronized L cells irradiated with $14 \mathrm{MeV}$ neutrons. Int. J. Radiat. Biol. 20, 85-86.

Ngo, F.Q.H., A. Han, H. Utsumi, and M. M. Elkind. 1977. Comparative radiobiology of fast neutrons: Relevance to radiotherapy and basic studies. Int. J. Radiat. Oncol. Biol. Phys. 2., 187-193.

Ngo, F.Q.H., E. A. Blakely, and C. A. Tobias. 1980. Sequential exposures of mammalian cells to low- and high-LET radiation. I. Letha? effects following $x$ ray and neon-ion irradiation. Radiat. Res, in press.

Raju, M. R., R. A. Tobey, J. H. Jett, and J. A. Walters. 1975. Age response for line CHO
Chinese hamster cells exposed to $x$-irradiation and alpha particles from plutonium. Radiat. Res. 63, 422-433.

Raju, M. R., H. I. Amols, S. C. Carpenter, R. A. Tobey, and R. Walters. 1978a. Age response for CHO cells exposed to negative pions. Radiat. Res. 76, 219-223.

Raju, M. R., H. I. Amols, E. Bain, S. G. Carpenter, J. F. Dicello, J. P. Frank, R. A. Tobey, and R. A. Walters. 1978b. Biological effects of negative pions. Int. J. Radiat. Oncol. Biol. Phys. $3,327-33 \overline{4}$.

Raju, M. R., E. Bain, S. G. Carpenter, J. H. Jett, R. A. Walters, J. Howard, and P: PowersRisius 1980a. Effects of argon ions on synchronized Chinese hamster cells. Radiat. Res. (Submitted for publication).

Raju, M. R., T. S. Johnson, N. Tokita, S. Carpenter, and J. H. Jett. $1980 \mathrm{~b}$. Differences in cell-cycle progression delays after exposure to $238 \mathrm{Pu}-\mathrm{a}$ pha particles compared to $X$ rays. Radiat. Res. (Submitted for publication).

Sapozink, M. D and B. Djordjevic. 1974. Effect of hypoxia on synchronous HeLa cells irradiated with fast neutrons. Radiology 110, 691-698.

Schlag, H., K. F. Weibezahn, and C. Lücke-Hühle. 1978. Negative pion irradiation of mammalian cells. II. A comparative analysis of cell cycle progression after exposure to negative pi mesons and cobalt $\gamma$-rays. Int. J. Radiat. Biol. 33, 1-10.

Schneider, D. 0. and G. F. Whitmore. 1963. Comparative effects of neutrons and $X$ rays on mammalian cells. Radiat. Res. 18, 286-306.

Sinclair, W. K. 1970. Dependence of radiosensitivity upon cell age. Time and Dose Relationships in Radiation Biology as Applied to Radiotherapy (V. P. Bond, H. D. Suit, and V. Marcial, eds.) p. 97-116. Report BNL-50203 C-58.

Sinclair, W. K. 1972. Sensitivity to mitotic delay and stage in the cycle. Curr. Topics in Radiat. Res. Q. 7, 264-285.

Sinclair, W. K. and R. A. Morton. 1963. Variations in $x$ ray response during the division cycle of partially synchronized Chinese hamster cells in culture. Nature 199, 1158-1160.

Sinclair, W. K. and R. A. Morton 1965. X ray and ultraviolet sensitivity of synchronized Chinese hamster cells at various stages of the cell cycle. Biophys. J. 5, 1-25.

Sinclair, W. K. and R. A. Morton. 1966. X ray sensitivity during the cell generation cycle of cultured Chinese hamster cells. Radiat. Res. 29, 450-474.

Skarsgard, L. D. 1964. Survival and mitotic delay in Chinese hamster cells after heavy ion 
and $x$-irradiated. Radiat. Res. 22, 235. (Abstract).

Skarsgard, L. D. 1967. Quoted in M. M. Elkind and G. F. Whitmore. The Radiobiology of Cultured Mammalian CeT1s, p. 420-421. Gordon and Breach, New York.

Skarsgard, L. D. 1970. Referred to in M. M. Elkind. Damage and repair processes relative to neutron and charged particle irradiation. Curr. Top. Radiat. Res. Q.7, 1-103.

Skarsgard, L. D., C. M. Pujara, and S. Richardson. 1966. The effect of high LET radiation on recovery and radiation sensitivity throughout the cell cycle in mammalian cells. Third International Congress of Radiation Research, Cortina, p. 24. (Abstract).
Skarsgard, L. D., B. A. Kinlman, L. Parker, C. M. Pujara, and S. Richardson. 1967. Survival chromosome abnormalities, and recovery in heavy-ion and $X$-irradiated mammalian cells. Radiat. Res. Suppl. 7, 208-221.

Terasima, T. and L. J. Tolmach. 1961. Changes in $x$ ray sensitivity of HeLa cells during the division cycle. Nature 190, 1210-1211.

Terasima, T. and L. J. Tolmach. 1963. Variations in several responses of HeLa cells to $x$-irradiation during the division cycle. Biophys. J. 3, 11-32.

Vos, O., H.A.E.M. Schenk, and D. Bootsma. 1966. Survival of excess thymidine synchronized cell populations in vitro after x-irradiation in various phases of the cell cycle. Int. J. Rad. Biol. 11, 495-503. 
136

Blane 


\title{
THE REPAIR-MISREPAIR MODEL OF CELL SURVIVAL
}

\author{
C. A. Tobias, E. A. Blakely, and F. Q. H. Ngo
}

In studying heavy-ion radiobiology, we attempted to use several well-known models, such as the single hit multitarget (Sinclair, 1966), the linear quadratic (Chadwick and Leenhouts, 1973, 1978; Kellerer and Rossi, 1972, 1978), and a form of the repair saturation model (Green and Burki, 1972). None of these models fitted the experimental results particularly well; the need for further development became particularly evident when we began to study the effects of split doses and of mixed modalities (see Ngo et al., Part III).

During the last three years we proposed a new model, the repair-misrepair model (RMR), to interpret radiobiological experiments with heavy ions (Tobias et. al., 1980a). In building this model, attempts were made to have a framework that actually corresponds to certain biological processes that are known to occur in irradiated cells.

In using the RMR model it became apparent that some of its features are suitable for handling the effects produced by a variety of environmental agents in addition to ionizing radiation. These include UV radiation, temperature, and perhaps the effects of some environmental poisons. In the discussion to follow, some general statements will be given that relate to this model, as well as some examples of applications. The rigorous mathematical derivations will not be presented here; they are being prepared for publication (see $\mathrm{Ngo}$ et al., in Part III; Albright, 1980).

\section{GENERAL REQUIREMENTS FOR THE RMR MODEL}

A quantitative description of the interaction of deleterious agents with cells requires a consideration of several aspects in addition to dose-effect relationships. For example, a model must include time-dependent phenomena in order to cover possible repair and enhancement effects, a statistical treatment that can deal both with molecular and much liarger dimensions, and an adequate mechanism for handiing biological complexity.

\section{DESCRIPTION OF THE REPAIR-MISREPAIR MODE}

We assume that two separate sequences of events take place in an irradiated cell. The first sequence begins with an initial energy transfer consisting of ionizations and excitations, culminating via fast secondary physical and chemical processes in established macromolecular lesions in essential cell structures. Most available experimental data and theoretical calculations indicate that this physicochemical phase is extremely fast. The second sequence contains the responses of the cell to the lesions and consists of the processes of recognition and molecular repair.

The time occurrence of the two processes outlined above can be calculated in a number of ways. Our treatment depends on the following basic assumptions:

1. For a given agent, the essential macromolecular lesions fall into one, or at most a few specific classes.

2. These lesions are established in a time span of about $10^{-3}$ seconds or less.

3. In a living cell external observers do not possess the ability to determine the exact location and nature of each lesion without destroying the cell. However, information on the overall yield of various types of lesions is available from assay procedures on groups of cells that are chemically analyzed after they are killed. 4. Living cells can recognize essential lesions in their own structures. They probably fail to identify the nature of the radiation that caused the lesions because the energy transfer occurs much too fast for macromolecular recognition.

5. In normal cells there exists one repair process or at most a few enzymatic repair processes for each essential macromolecular lesion. The enzymatic repair processes may last for hours and minutes, and can be separated in time from the initial physicochemical and later genetic phases.

These assumptions have been discussed in some detail (Tobias et al., 1980a).

A schematic drawing showing the effects of ionizing radiations is given in Figure 1 . This figure is also a blueprint for the modes of theoretical calculations one may wish to carry out. It is divided into three phases.

Phase I: Initial Energy Transfer and Chemical Modification

During the initial, physicochemical phase of interactions one or more types of "relevant" lesions are established, in the macromolecular structures of cells. These lesions are defined as U.lesions. U stands for "uncommitted," which is what the lesions are before they are subjected to enzymatic repair, modification, or amplification.

A large array of experimental and theoretical knowledge has accumulated to indicate that the $U$ lesions are formed in less than $10^{-3}$ seconds 


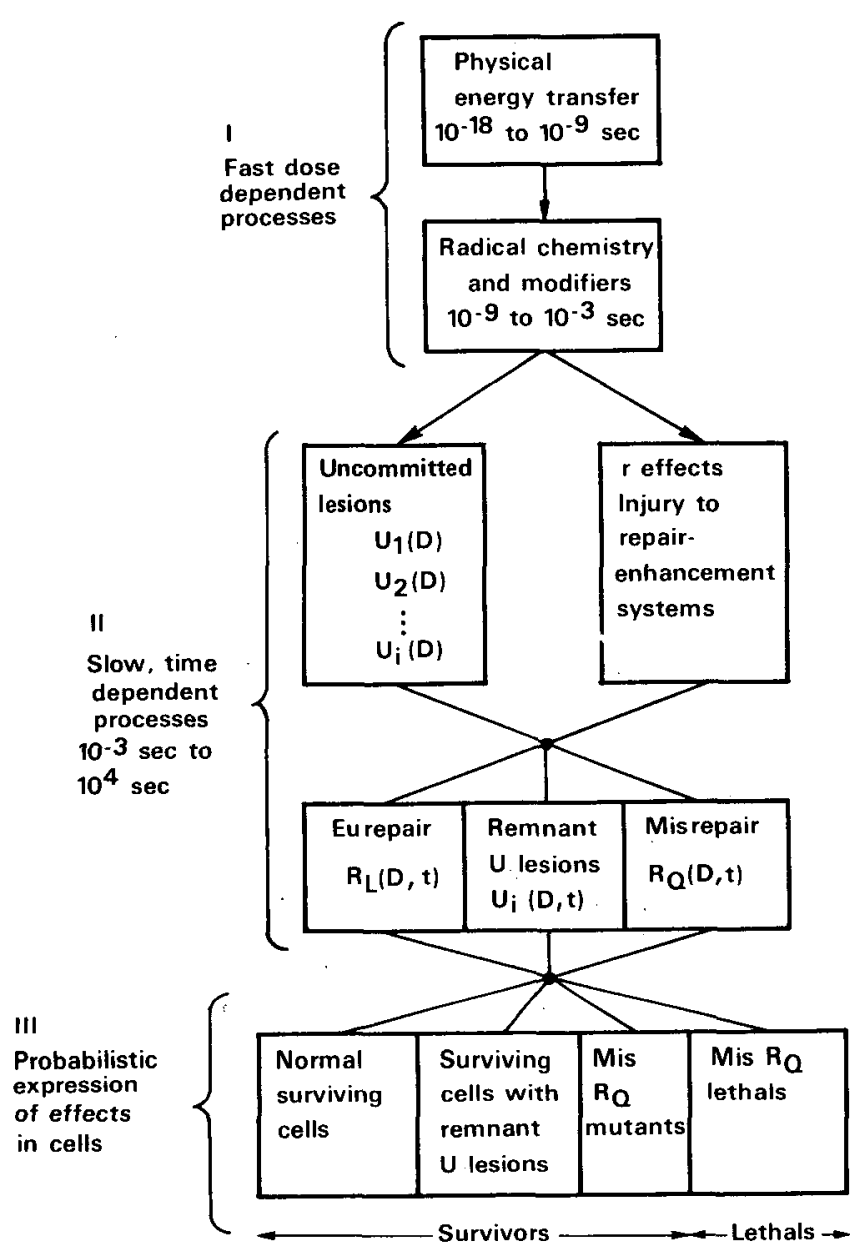

Figure 1. Schematic drawing for the repairmisrepair model as described in the text. At the end of Phase I the cells have various types of uncommitted lesions that are either repaired, misrepaired, or remain as remnant $U$ lesions. Radiation may also cause effects on the repair system; these effects are represented as $r$ effects, which then become expressed during Phase III. (XBL 808-3588)

at ambient temperatures and that the effect of most, if not all, important chemical protectors and sensitizers is over by that time. There is strong evidence that various types of molecular injury to the genetic apparatus, specifically to DNA, represent $U$ lesions. U lesions can be chemically defined and the'ir quantities measured only if we use assay procedures that usually kill living cells. In this manner the dose dependent yield of specific $U$ lesions $c$ an be established. In individual living cells, however, a "biological uncertainty principle" prevents us from precisely identifying and locating specific $U$ lesions during Phase I. In order to do this we would have to apply physical methods that would themselves potentially produce more $U$ lesions (Tobias et al., 1980a).
Typical $U$ lesions in the genetic apparatus, including those made by toxic chemical compounds in DNA, are: base alterations and interaction, single-strand scission, covalent interstrand crosslink, double-strand scission, intercalation of foreign molecules in the DNA structure, DNARNA crosslinks, and DNA-protein crosslinks. Elsewhere in the cell we know less about the nature of $U$ lesions; they might be membrane injury, injury to centromeres, centrioles, microtubules of the mitotic spindle, or mitochondrial injury. Although the potential list of $U$ lesions appears formidable, analys is of cell survival data indicate that for a given deleterious agent usually a single specific type of $U$ lesion is more important than all others combined. For example, the prevalent $U$ lesions from UV radiation are pyrimidine dimers in DNA; for heavy accelerated nuclei there is some evidence that $U$ lesions are double-strand scissions of DNA.

\section{Phase II: Transformations of Lesions}

Even though external observers cannot detect the location and nature of individual $U$ lesions with certainty, the macromolecular apparatus can do this in times of $10^{-4}$ sec or longer because of the proximity of the lesions to the detecting apparatus, and the possibilities for using low lying quantum states as well as electrostatic and magnetic forces. Vibration and rotation of nucleic acid chains and their characteristic relaxation times may have an important part in recognizing lesions.

Phase II, then, is recognition of $U$ lesions and biochemical responses to these lesions which may be characterized in molecular "repair," or "enhancement." Whereas several classes of repair processes have been well documented (Cleaver, 1978), it is also known that in dying cells there is increased DNAase activity. Recent mammalian cell studies by Ngo et al. (Part III), have identified a split dose effect that may be regarded as enhancement.

\section{$r$ Effects}

We recognize $r$ effects as injuries on repair systems that usually do not kill cells by them-. selves; these lesions, however, do modify the responses of living cells to the presence of $u$ lesions. The $r$ effects usually encompass. changes in the rate of $U$ lesion repair or enhancement and slow down the rate of progression of normal cellular processes. $r$ effects could be due to molecular lesions in repair enzymes, however, at this early state of our knowledge, it appears more likely that $r$ effects either modify the rate of action of existing enzymes or change the rate of formation of new enzymes. At least some of the $r$ effects are probably caused in Phase I; their consequences are detected by measuring rate processes in Phase II. In our earlier publication (Tobias et al., 1980a) we have not specified $r$ effects as a special class. We wish to enlarge the importance of this aspect of the problem, by recognizing that $r$ effects can seriously modify cellular responses to deleterious agents. 
With the passage of time $U$ lesions are enzymatically altered by repair mechanisms. The consequences are repaired states, which are assumed to have greater time stability than $U$ lesions. Split-dose experiments with mammalian cells have convinced us that $X$-ray and heavy-ion induced $U$ lesions have typical half lives at $37^{\circ} \mathrm{C}$ of about 10 to 100 minutes, whereas $R$ states may last at least for one and possibly for several cell generations.

Repair has obvious connotations for $U$ lesions in DNA: mending single-strand breaks, replacing damaged nucleotide bases, severing interstrand links, or rejoining pieces of DNA with two strands. Since molecular repair is known to consist of error prone processes (Bernardi and Nino, 1978) we made the following definitions.

Eurepair is perfect repair, where the reestablished DNA molecule has in every respect the same structure (including coding) as the undamaged DNA at the same location. Misrepair is either incomplete repair or repair where the structural integrity of DNA is complete, but the coding sequence is changed in some manner. It is obvious that eurepair, if completed rapidly, will not cause lethality or permanent alteration to cell behavior. In contrast, misrepair can be the cause of both mutation and of lethal effects.

We know from a large body of literature that the result of one type of misrepair is abnormal chromosome rejoinings. It is widely believed that these are the result of at least two lesions in different points of the genome. We suggest that for ionizing radiations: (1) a single type of $U$ lesion is more important than others: (2) there are many of these $U$ lesions produced in the mammalian cells at an LO50 dose;

(3) there are two types of repair processes: one proceeding proportionally with the mean concentration of $U$ lesions present, and the other proportionally to the square of the density of $U$ lesions.

\section{Mathematical Approach}

The processes described above and shown in Figure 1 have been analyzed by two different mathematical techniques. The first method followed by Tobias et a1. (1980b, and this paper) employs differential equations to calculate the time dependence of the mean number of lesions in the cell. This was followed by the use of Poisson statistics in order to calculate survival and mutation probabilities. This method has the advantage of relative simplicity and ease of the process of calculation and of the final formalism. However, a weakness stems from the fact that the second order process described here does not exactly follow Poisson statistics and that the procedure is not correct when the number of lesions per cell is very small.

The second method regards the RMR processes s a branching Markov chain. If $U_{n}$ and $L_{n}$ denote the uncommitted and lethal states with $n$ lesions, and $S_{0}$ is the survival state, then the steps of evolution of the cell through three, two, one, and no lesions can be represented in a diagram as:

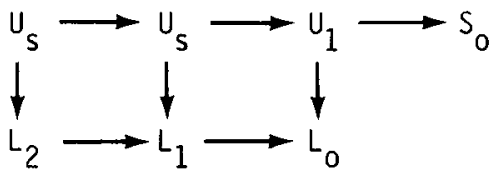

The goal of the Markov formulation is the calculation of the probability $P_{s}(t)$ that the cell has evolved to survival at time $t$. $N$. Albright has recently completed such a calculation (Albright, 1980).

The results of the two mathematical approaches are qualitatively and quantitatively similar. Differences, amounting to a few percent occur at low doses and short time intervals.

\section{The Fate of $U$ Lesions}

For the mean number of lesions per cell, $U$, we obtain a first order quadratic differential equation:

$$
\frac{d U}{d t}=-\lambda U(t)-k U^{2}(t)
$$

In this equation $\lambda$ is the coefficient of linear repair and $k$ the coefficient of quadratic repair. The assumption that $\lambda$ and $k$ are constants independent of time and dose is equivalent to the assumption that the $r$ effects of a dose (D) of ionizing radiation are negligibly small. With the further assumption that $U(t=0)=U_{0}(D)$ and $U(t \rightarrow \infty)=0$ and $\varepsilon=\lambda / k$, the solution of equation 1 is:

$$
U(t)=\frac{U_{0} e^{-\lambda t}}{1+\frac{U_{0}}{\varepsilon}\left(1-e^{-\lambda t}\right)}
$$

$U(t)$ is sometimes also called remnant $U$ lesion, $U_{r}$. We may further postulate that all $U$ lesions turn either into linearly repaired or quadratically repaired moieties $R_{L}$ and $R_{0}$, or remain as remnant lesions. This leads us to a conservation equation:

$$
U(t)+R_{L}(t)+R_{Q}(t)=U(0)=U_{0}
$$

and equation 3 agrees with the definition:

$$
R_{L}=\int_{0}^{t} \lambda U(t) d t
$$

and

$$
R_{Q}=\int_{0}^{t} k U^{2}(t) d t
$$


In order to use equation 1, we must know the dose-dependent yield of various types of $U$ lesions per cell as a function of time. Equation 1 can accept any analytical function for yield. In DNA for example, strand breaks induced by $x$ rays are usually proportional to dose (Ritter et al., 1977):

$$
U_{0}=\alpha D
$$

From equations 4 and 2 we can evaluate the quadratic repairs that have occurred as a function of time and dose. For example, when the repair time is large, $t \rightarrow \infty$, then the total number of quadratically repaired lesions, $R_{Q}$ is:

$$
R_{Q}=\alpha D-\varepsilon \ln \left[1+\frac{\alpha D}{\varepsilon}\right]
$$

The second term in the right hand side of equation 6 corresponds to the number of lesions that have been repaired by the linear process, assuming there is no linear misrepair.

In Figure 2 we plot the manner in which the relationships described by equations 1-6 appear in survival plots. The shaded area indicates the mean number of $U$ lesions as functions of time after exposure to radiation. The treatment here describes a "memory" effect: as long as there are remnant $U_{r}$ lesions in a cell, we might conjecture that by the presence of $U$ lesions the cell "remembers" a previous dose of a deleterious agent. If a second deleterious agent affects the cell at time $t$, the new $U_{r}$ lesions produced by this agent could be added to the $U_{r}$ lesions already present. We assume, on the other hand, that the forms $R_{L}$ and $R_{0}$ represent "final" states in the sense that they do not specifically interact with a second dose of the deleterious agent. From the equations and the graph, it is clear that we used simple kinetics; if it turns out that the kinetics of enzyme repair are more complex than we assumed, the mathematics can be readily modified without changing the overall method of approach.

\section{Phase III: Expression of Biological Effects}

The consequence of the presence of $R_{L}, R_{Q}$, and remnant $U$ lesions to individual cells is governed by the probability of how many of a given type lesion can be found at a given time in a given cell. Let $\phi$ be the probability that a linear repair process results in eurepair; let $\psi$ be the probability that a quadratic repair is lethal misrepair. An analysis of survival curves indicates that for wild type cells and low-LET radiation $0.95 \leq \emptyset \leq 1$ and $\psi \simeq 1$; for repair deficient mutants $\phi<<1$.

If we assume that the Poisson theory is valid for the distribution of $R_{L}$ and $R_{0}$ lesions, then the time dependent RMR survival probability $S$ is:

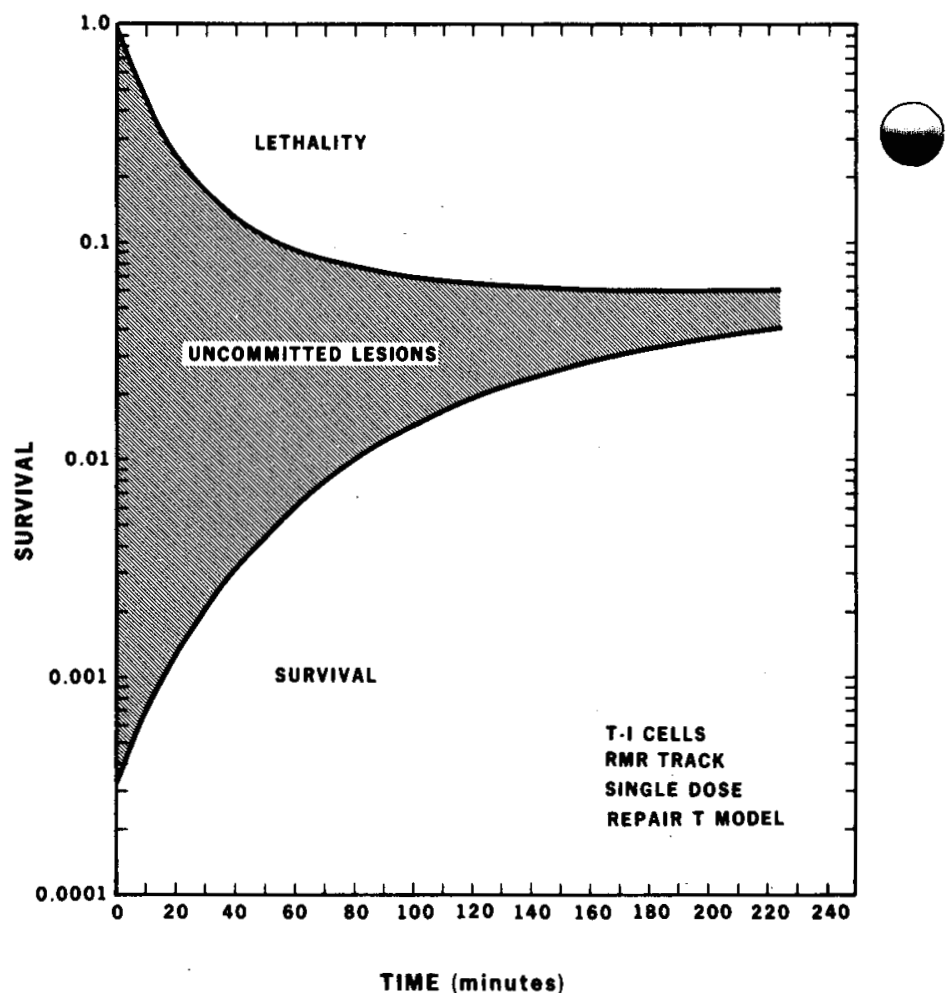

Figure 2. Time dependence of the probabilities for survival (s) and lethality $[1-L(t)]$ as a function of time elapsed after a single dose. The shape of the survival curves depends on the values $\lambda$ and $k$. The number of cells with uncommitted lesions decreases with time.

(XBL 794-9457)

$$
S=e^{-U_{0}}\left(1+\frac{U_{0}}{\varepsilon}\left(1-e^{-\lambda t}\right)\right) \phi_{E}
$$

Substituting for $U_{0}$ from equation 5 and assuming that $t \rightarrow \infty$ :

$$
S=e^{-\alpha D}\left(1+\frac{\alpha D}{\varepsilon}\right)^{\phi \varepsilon}
$$

Equation 8 has three adjustable constants: $\alpha, \phi$ and $\varepsilon$. These constants can be evaluated by least-squares calculations from accurate survival data. However, the experimental data are usual.ly not sufficiently accurate to evaluate all three constants from a single survival curve. For determination of the constant $\alpha$ it appears better to obtain a repairless $\left(r^{-}\right)$mutant. If the rate of linear repair is zero, $\lambda=\varepsilon=0$. Then:

$$
S_{\left(r^{-}\right)}=e^{-\alpha D} \text { and }-\ln S_{\left(r^{-}\right)}=\alpha D=U_{0}
$$


The repair ratio is the ratio of survivors with or without repair. This is:

$$
\frac{S}{S}_{\left(r^{-}\right)}=\left(1+\frac{\alpha D}{\varepsilon}\right)^{\phi_{\varepsilon}}
$$

ه can be directly obtained from the initial ratio of the slopes of the survival curves of the wild type and $\left(r^{-}\right)$mutants:

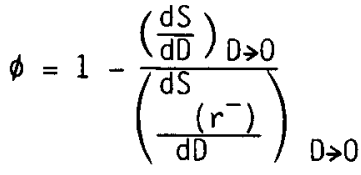

For purposes of practical analys is of repair curves, it is sometimes useful to plot the log repair function:

$$
\ln (r)=\alpha D+\ln S=\phi_{\varepsilon} \ln [1+(\alpha D / \varepsilon)]
$$

The rate of change of this function with dose is also useful

$$
\frac{d}{d D}[\ln (r)]=\alpha \emptyset \frac{\varepsilon}{\varepsilon+\alpha D}
$$

Figure 3 shows the log repair function for some of the survival curves from T-1 cells exposed to silicon beams. Figure 4 shows the survival curves for $T-1$ cells in nitrogen at two experimental points along the silicon Bragg curve, as fitted by the RMR method. The values of $\alpha$ and $\phi$ appear more critical for medical applications than those of $\varepsilon$. More details on experiments exposing T-1 cells to a silicon beam are given by Blakely et al. in Part III.

Analysis of neon and silicon survival curves indicate that a single type of $U$ lesion appears to be sufficient to explain all of the survival data and that the yield of $U$ lesions in aerated cell suspensions is nearly proportional to LET: until saturation occurs. The yield of $U$ lesions in an hypoxic environment increases with higher power of LET, until above $100 \mathrm{keV} / \mu \mathrm{m}$ the hypoxic and oxic a values are nearly equal. "The ratio of the $\alpha$ oxic and $\alpha$ hypoxic constants are approximately the same as the oxygen effect ratio:

$$
[O E R]=\frac{a_{\text {oxic }}}{a_{\text {hypoxic }}}
$$

This is an indication that most of the oxygen effect occurs in the physicochemical phase, i.e., within the first $10^{-3}$ seconds. The ratio of linear to quadratic repair, $\varepsilon$, appears to be of the same order of magnitude at various LET values: We conclude that oxic and hypoxic $U$

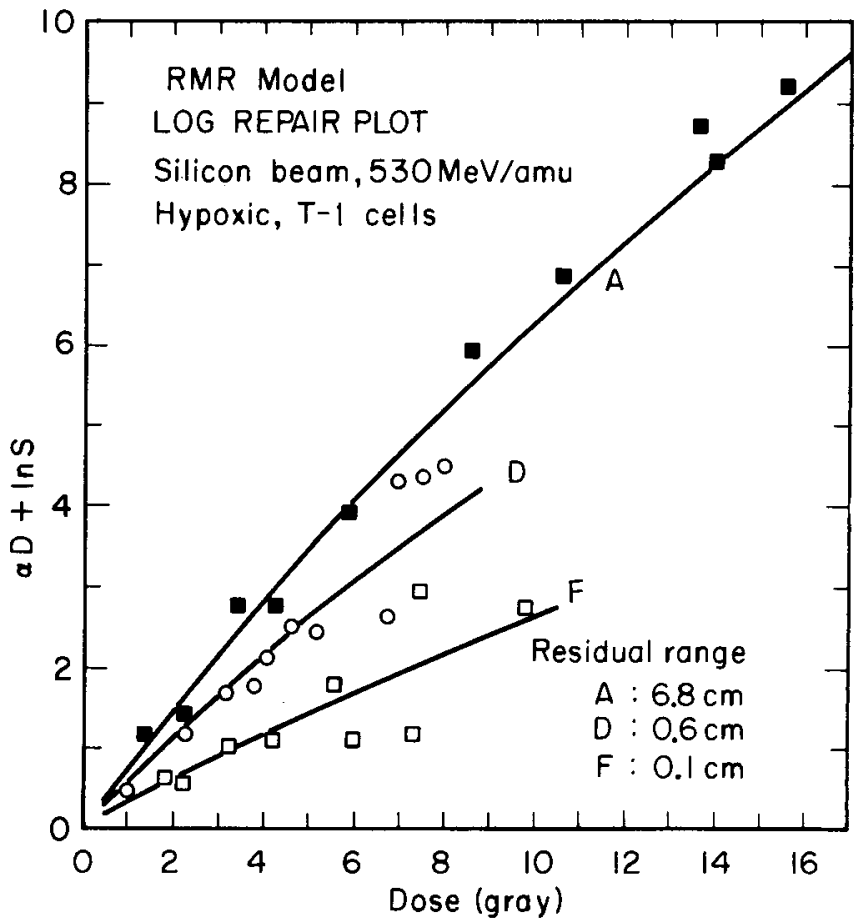

Figure 3. Log-repair plots for survival curves of T-1 cells exposed $530 \mathrm{MeV} / \mathrm{amu}$ silicon beams at various residual range values. At position $F$, where the LET is high, repair is less than at positions $A$ or D. (XBL 8010-3735)

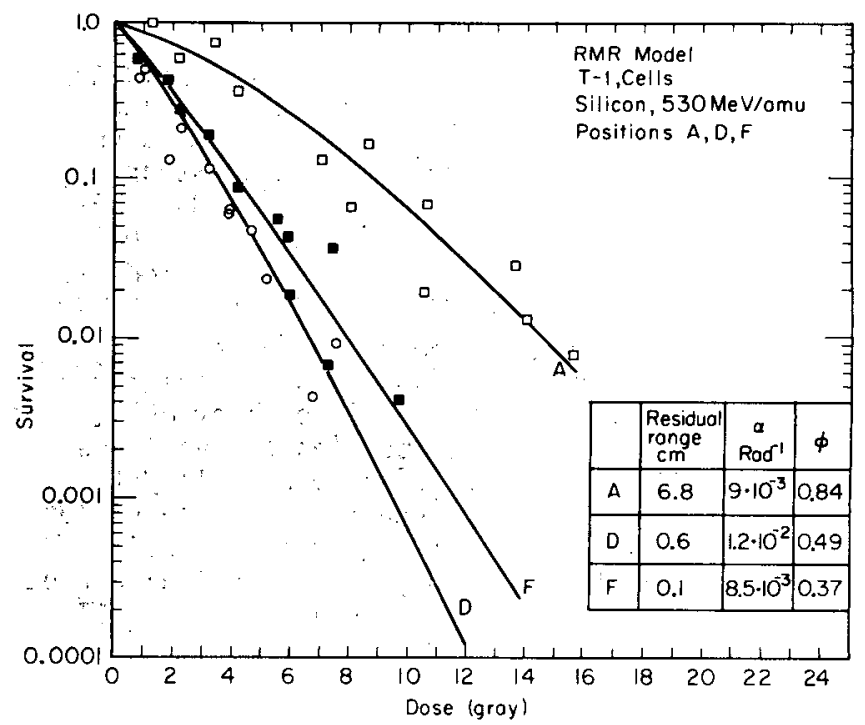

Figure 4. RMR fits to survival data of $T-1$ cells exposed to silicon beams. (XBL 8010-3736) 
lesions in mammalian cells are similar chemically and are repaired at about the same rate.

Speculations with Respect to the Molecular Nature of U Lesions

Highly ionizing radiations are probably capable of producing a wide variety of lesions in DNA. However, the role of double-strand scission has emerged as an important type of lesion with respect to lethality, mutation, and cell transformation. Christensen et al. (1972) showed that the yield of double-strand scissions increases rapidly with increased LET in double stranded forms of bacteriophage $\phi X-174$ both in aerobic and in anaerobic environments. In yeast cells, survival has been linked with the yield of double-strand scissions, and it is suspected that certain repairless mutants are unable to repair double-strand scissions. In mammalian cells in vitro the time rate of repair of double-strand scissions is similar in magnitude to the rate of repair of sublethal damage deduced from split-dose experiments, and proceeds in vivo at $37^{\circ} \mathrm{C}$ with a 80 to 120 min half life. Singlestrand scission repairs much faster (7-10 min half life). We know that the yield of singlestrand breaks decreases with increasing LET, whereas double-strand scission increases as LET increases.

Ionizing radiation can produce double-strand scission at high LET as a one-step process without chemical modification; at lower LET we know that production of double-strand scissions depends on the molecular milieu next to DNA, on the presence of oxygen, or on radiation sensitivity and protection. Thus, double-strand scissions might be the result of a set of complex steps. We also know that enzymatic action in cells can produce double-strand scissions. In genetic recombination, double-strand scissions are created and mended enzymatically.

In order to visualize the action of heavy ions, we postulate that in dense chromatin material a single heavy-ion track might produce a series of double-strand scissions or lesions along the core and penumbra of the track as shown in Figure 5. The production of double-strand lesions might involve the loss of some DNA. As the strands are reconnected, it is likely that the result is misrepair. If misrepair interaction occurs between DNA lesions made by the same heavy-ion track, the amount of misrepair will appear proportional to dose or to particle flux. Thus, intratrack misrepair may appear as linear misrepair even though at the microscopic level it is a higher order process. A more detailed consideration of the relationship is in progress.

We have shown that the linear misrepair coefficient $\phi$ is related to the probability that a single heavy ion will cause misrepair along its track.

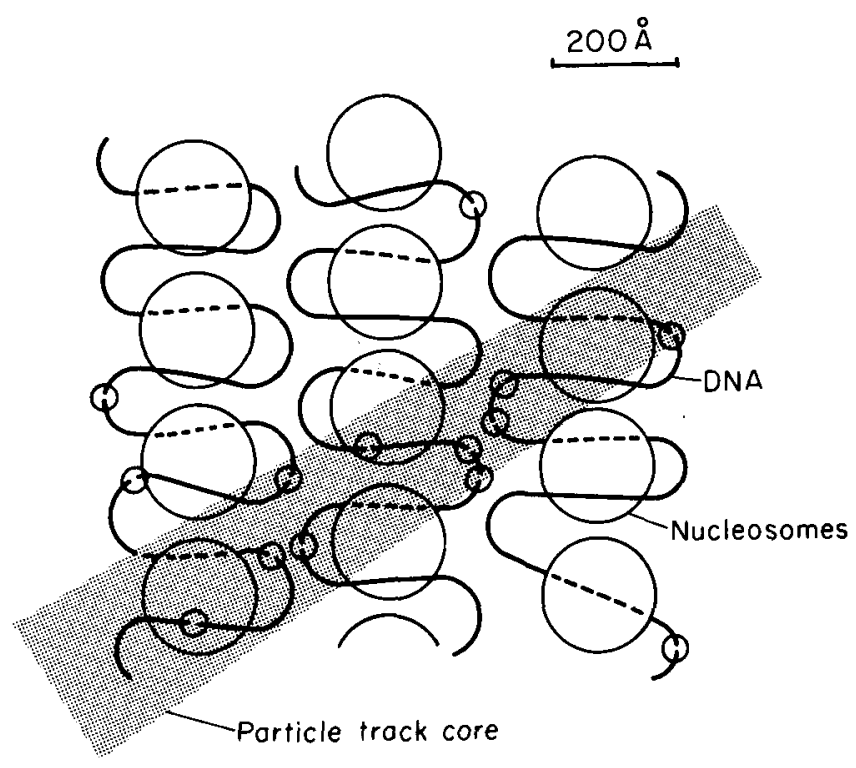

Figure 5. Schematic drawing of the submicroscopic organization of DNA and nucleosomes in the cell nucleus. The shaded area represents the track core. Since the energy density in the track core is very high, it is possible that a single track causes many strand breaks and other deleterious effects in its path. When these lesions are adjacent to one another, the probability for misrepair is high. (XBL 8010-3733)

\section{BIOLOGICAL EFFECTS OF MIXEO BEAMS}

The RMR model allows calculation of survival curves for mixed beams, for example, mixtures of low and high LET radiation delivered simultaneously. This is a complex problem because the spatial distribution of energy deposits, the spatial distribution and state of nucleic acids, and the dependence of repair coefficients on LET must be considered. However, analysis of heavyion data allows some simplifications. Our calculations depend on the validity of the following statements:

1. Low and high LET radiations (L) produce essentially similar $U$ lesions in the same cell strain.

2. The LET dependence of survival appears to be due principally to the yield of $U$ lesions, $\alpha(L)$, and the amount of linear misrepair, $\phi(L)$. The repair rate constant $\varepsilon$ is essentially independent of LET.

Assuming that these considerations hold, two rules can be proposed on the basis of simplified RMR theory:

1. The $U$ lesions present simultaneously in a cell from various LET components are additive. 
2. The coefficient of linear intratrack misrepair for a mixed LET radiation field is a dose-averaged value of the $\phi$ values for each component of the field.

We may express these rules as follows. Let the dose in a mixed field of $k$ components be represented by:

$$
D=\sum_{i=1}^{k} \gamma_{i} L_{i}
$$

where $L_{j}$ represents mean LET of component $i$ and $\gamma i$ is flux density. The concentration of $U$ lesions due to the $i$ component is:

$$
U_{i}=\gamma_{i} \alpha_{i} L_{i}
$$

And the total $U_{0}$ lesions present at $t=0$ is:

$$
U_{0}=\sum_{i=1}^{k} \gamma_{i} \alpha_{i} L_{i}
$$

If $\phi_{j}$ is the coefficient of linear intratrack misrepair for component $i$, then the mixed beam coefficient $\phi$ is:

$$
\phi=\frac{\sum_{i} \phi_{i} U_{i}}{\sum_{i} U_{i}}
$$

The values of equations 14 and 15 can be directly substituted into equation 8 to obtain survival curves for mixed modalities. Ngo et al. (1980) tested the relationships expressed by equation 15 for mixtures of neon particles and $x$ rays.

A typical heavy-ion beam, after it has passed through absorbers, may be described as being a mixture of four general components: (1) the parent beam of monoenergetic parallel particles, with discrete high LET broadened by straggling and multiple scattering; (2) high-LET fragments with atomic numbers smaller than the parent beam, but a spectrum of velocities; (3) a low-LET component representing chiefly fragment protons, helium, and light nuclei of high-velocities; and (4) multiple pronged stars from target nuclei, neutrons, gamma rays, and mesons; these comprise oniy $1-2 \%$ of the local dose.

Work is in progress to mode? the particle distribution in mixed heavy-ion beams, and to calculate survival curves in heavy-ion fields. based on RMR analysis of monoenergetic particle effects.

$\frac{\text { Classification of Mixed Modalities }}{\text { on the Basis of the RMR Model }}$

We believe that the RMR model can be generalized to model the cellular effects of a variety of environmental agents like UV radiation, heat, radiomimetic chemicals, mutagenic agents. These agents have certain features in common. Each may be capable of producing $U$ lesions or uncommitted lesions that have the potential for killing or causing mutations. Generally, the $U$ lesions from each agent are different, however, there may be overlap in the spectrum of $U$ lesions formed by different agents. The repair kinetics. of different $U$ lesions are probably also different. There may be a characteristic differential equation, similar to equation 1 , but perhaps differing in some respects, for each $U$ lesion. Also, it is possible that each agent can have $r$ effects; that is the action of each agent can modify repair of its own lesions or lesions from other modalities. We believe, however, that there are a finite number of classes of $U$ lesions that depend on the molecular structure of the cell.

The following is a classification scheme that was recently proposed by Tobias et al. (1980b). Assume that two modalities $x$ and $y$ produce $U$ lesions $U_{x}$ and $U_{y}$. Modality $x$ has $r$ effects also: $r_{x x}$ on the repair of $x$ and $r_{x y}$ on the repair of $y$. Modality $y$ causes $r_{y x}$ and $r_{y y}$; the surviva? probabilities are $S_{x}$ and $S_{y}$. (Note: for the present discussion, we define the magnitude of $r$ effects as follows: $r_{x y}=\lambda(r)_{y} / \lambda_{y}$; if $r_{x y}=1$, then $\lambda(r)_{y}=\lambda_{y} \cdot$ )

\section{Independence}

If the $U$ lesions from $x$ and $y$ are qualitatively different then $U_{x} \neq \equiv U_{y}$ and the repair pathways are independent. If there are no $r$ effects: $r_{x y}=r_{y x}=1$ and the two environmental agents act independently. The survival probability for the joint application of two agents, S, is:

$$
S=S_{x} \cdot S_{y}
$$

We are not aware of the existence of two toxic environmental agents that are entirely independent of one another. However, if one dose of $x$ rays follows another with a substantial time interval between them, they will have independent effects if the $U$ lesions produced by the first dose are repaired before the second dose was applied: and if there are no $r$ effects.

\section{Simple Synergism}

If two agents produce identical U lesions then if simultaneously $U_{x}$ lesions are produced by $X$ and $U_{y}$ lesions by $Y_{,}$, then the following differential equations hold:

$$
\frac{d U_{x}}{d t}=-\lambda U_{x}-k U_{x}^{2}-k U_{x} U_{y}
$$

$$
\frac{d U_{y}}{d t}=-\lambda U_{y}-k U_{y}^{2}-k U_{y} U_{x}
$$


Adding and rearranging we obtain:

$$
d\left(U_{x}+U_{y}\right)=-\lambda\left(U_{x}+U_{y}\right)-k\left(U_{x}+U_{y}\right)^{2}
$$

Note that if $U=U_{x}+U_{y}$, equation 20 is identical to equation 1. Equations $19 \mathrm{a}$ and $19 \mathrm{~b}$, however, are not identical to 1 . The last term on the right side has been added to each equation. These are interaction terms signifying synergism between $X$ and $Y$. The survival is:

$S\left(U_{x}+U_{y}\right)=\varepsilon^{-\left(U_{x}+U_{y}\right)}\left(1+\frac{U_{x}+U_{y}}{\varepsilon}\right)^{\phi \varepsilon}$

If $X$ and $Y$ are both $X$ rays, equations 19 through 21 signify that $X$ rays are synergistic with themselves. In order to be able to add $19 \mathrm{a}$ and $19 \mathrm{~b}$, simultaneity in time must prevail. If the agent $X$ is administered before $Y$ and a time interval $T$ occurs between the administration of $X$ and $Y$, then the $U_{x}$ lesions must be evaluated at time $T$ and added to the $U_{y}: U=U_{x}(T)+U_{y}$. If a long time interval occurs between the administration of $X$ and $Y$, then $U_{X}(T>\infty)=0$, the synergism terms are zero, and the joint effects of two agents ( $X$ and $Y$ ) are independent, as discussed above.

Two doses of $X$ rays administered together, or with a time interval between, have simple synergism. If a time interval has elapsed, then the remnant $U$ lesions must be added. The magnitude of the remnant lesion $U_{r}$ is expressed in equation 2 .

\section{Potentiation or Amelioration}

If the $U$ lesions caused by agent $X$ and $Y$ are different, $U_{x} \equiv U_{y}$, but $x$ alters the repair kinetics for lesions $U_{y}$ such that $r_{x y} \neq 1$ then $X$ potentiates or ameliorates the effects of $Y$. In the former case the rate of repair of $U_{y}$ lesions is reduced; in the latter case the rate is increased. This should not be confused with chemical radiosensitization or protection, which occur in the physicochemical phase at short time intervais $\left(<10^{-3} \mathrm{sec}\right)$.

We believe that caffeine, which by itself does not produce pyrimidine dimers, potentiates for the action of UV radiation. At the molecular level there are many ways in which potentiation or amelioration might occur. For example, $X$ may induce the repair enzymes for the action of $y$. The enhancement of radiation effects by changing the salt tonicity of the cellular milieu probably belongs in this class.

The effects of temperature on the responses to ionizing radiation is probably a case for potentiation. A temperature of $43^{\circ} \mathrm{C}$ or higher usually inhibits the repair of $U$ lesions caused by ionizing radiation. We believe that the $U$ lesions for temperature effect are different from those due to ionizing radiations, and we expect an asymmetry in the effect. High temperature administered before $X$ rays been shown to have a different result than $X$ rays administered before the temperature is increased.

\section{Asymmetric Synergism}

If the lesion $U_{x} \equiv U_{y}$ and if the $r$ effects $r_{x y}$ and $r_{y x}$ are different from unity, we have an asymmetric combination of synergism combined with potentiation or amelioration. We believe that two doses of high-LET radiation separated by a time interval, or a single dose of high-LET radiation followed by another dose of $X$ rays, behave in this manner. The order in which the two modalities are applied and the magnitude of the time interval between them are both important. F. Ngo of our laboratory has some preliminary evidence that neon is not only synergistic with neon but also potentiates a second dose (see Ngo et al. in Part III).

The addition of a high salt solution to cells that are also being exposed to $X$ radiation, which causes phenomena usually described as PLD repair, probably belongs to the class of asymmetric synergism. High salinity applied before nean radiation has a different degree of effect on repair than high salinity appliied after a dose of neon radiation. The experimental data are shown in Figure 6 .

\section{Cross Reaction of Lesions}

Assume that $X$ and $Y$ produce qualitatively different lesions in the same cell or $U_{x} \not \equiv U_{y}$. It is possible that in the course of repair of $U_{x}$ and $U_{y}$, the repair systems and lesions interact in such a manner that new lesions $U_{z}$ result that are different from both $U_{x}$ and $U_{y}$. This may be the case when UV radiation and $X$ rays are acting jointly on the same cell. The pyrimidine dimers, the most prevalent $U_{x}$ lesions caused by UV radiation; are repaired by excision repair which proceeds via single-strand incisions in ONA. One single-strand scission may interact with another single-strand scission produced by $X$ rays, and the net result might be the appearance of a double-strand scission lesion $U_{Z} ; U_{Z}$ is not efficiently produced by either UV radiation or $X$ rays alone.

USE OF THE RMR MODEL

TO STUDY LOW-DOSE EFFECTS

The traditional method of studying low dose effects was to study the dose-effect relationship at various doses and to extrapolate the results to very low dose levels. The accuracy of such a procedure is poor because of statistical variations. A debate is in progress over whether or not the dose effect relationships for mutation or cell transformation are linear or higher order functions of dose. The importance of this problem is indicated by the extent of public interest, and by the involvement of important national and international study groups. A recent United Nations report (UNSCEAR 1977) and 


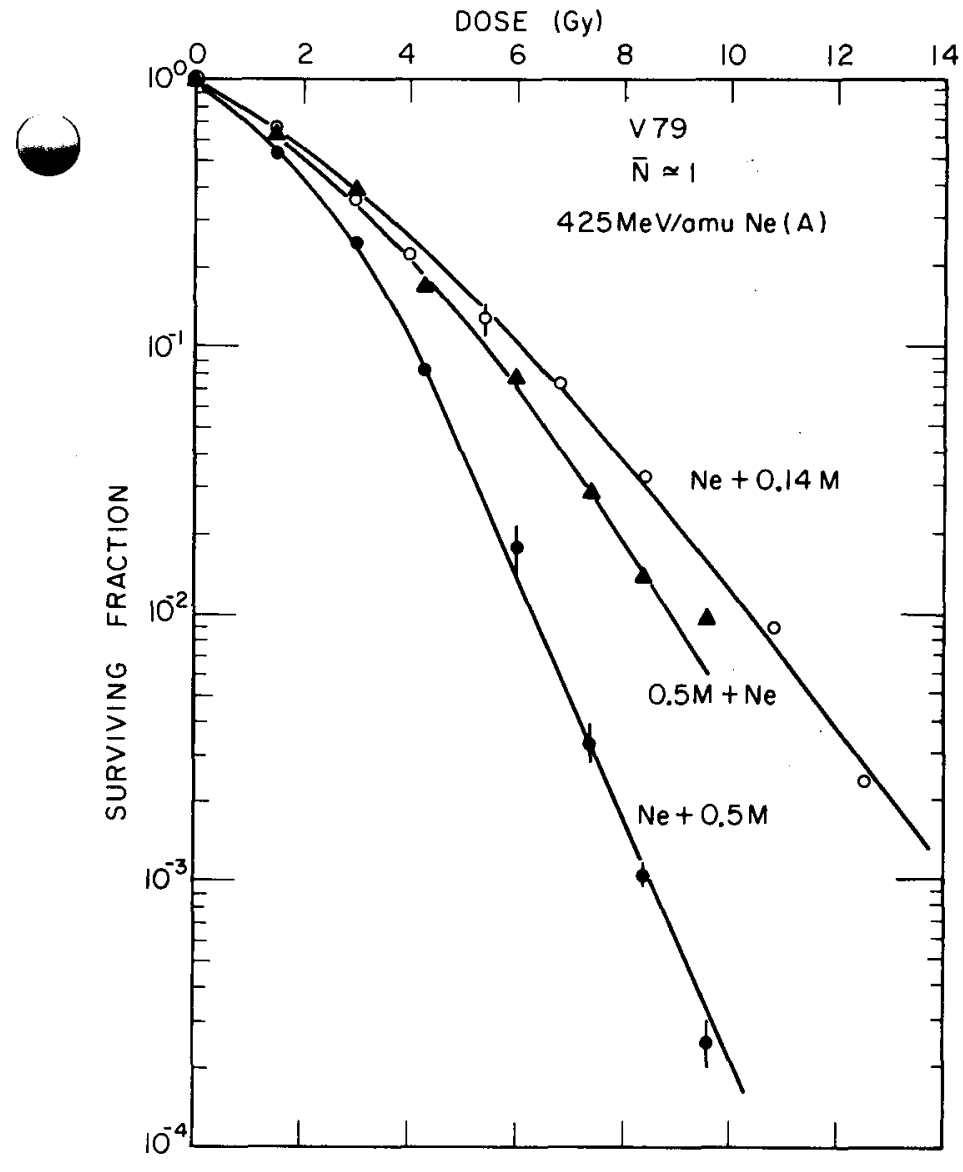

Figure 6. This figure illustrates time asymmetry in the joint effect of neon radiation and hypertonic salt treatment on hamster $V-79$ cells (from the work of F.Q.H. Ngo). High salt concentrations increase the lethal effect of neon particles. However, this PLD effect is smaller when the salt treatment precedes exposure to the neon beam, and larger when the neon radiation is given before the cells are exposed to a hypertonic environment. (XBL 805-3365)

$$
\frac{d U}{d t}=C-\lambda U-k U^{2}
$$

Assuming that at the beginning, when $t=0$, $U_{0}=0$, and as $t \rightarrow \infty U=U_{\infty}$ we obtain:

$$
U=\frac{U_{\infty}\left(1-\frac{U_{\infty}}{\varepsilon^{\star}}\right)\left(1-\varepsilon^{-\lambda^{\star} t}\right)}{1-\frac{U_{\infty}}{\varepsilon^{\star}}\left(1-e^{-\lambda^{\star} t}\right)}
$$

where $\varepsilon^{*}=\lambda^{*} / k$. With the value of $U_{\infty}$ :

$$
\begin{array}{r}
U_{\infty}=-\frac{\varepsilon}{2} \pm \sqrt{\frac{\varepsilon^{2}}{4}+\frac{C}{k}} \\
\lambda^{\star}=\lambda+2 U_{\infty} k
\end{array}
$$

Assume that cells have been in a continuous state of proliferation for a long time $t$, and that the cell division period is $T$. We also assume that cells with lethal misrepair die at the time of cell division or earlier, but that unrepaired $U$ lesions are carried through cell division to the next generation of cells. Then, the $U$ lesions reach a constant level, $U_{\infty}$ when $\mathrm{dU} / \mathrm{dt} \approx 0$ and, in each generation about as many new $U$ lesions are produced as $U$ lesions

disappear due to repair. Equation 22 becomes:

$$
C T=\lambda^{*} U_{\infty} T+k U_{\infty}^{2} T
$$

If all quadratic repair is lethal misrepair and if the coefficient of lethal linear misrepair is $\phi$ as defined above, then the number of lethal lesions produced in the cell per generation is:

$$
k U_{\infty}^{2} T+\lambda^{*}(1-\phi) U_{\infty}^{2} T
$$

The probability of survival per generation is:

$$
S_{\text {gen }}=\exp \left[-k U_{\infty}^{2} T+\lambda^{\star}(1-\phi) U_{\infty} T\right]
$$

a U.S. National Academy of Science (BEIR
Committee No. 3), have not been able to reach an. unequivocal agreement.

We have applied the RMR model to low doses and to continuous background radiation. Even, in the present early form the model may provide: important new insights into the low level irradiation problem.

Assume that there is a continuous and con-. stant level of production of $U$ lesions at the. rate of $C$ per unit time and that the repair. coefficients $\lambda$ and $k$ are constant in time and. independent of dose level. We know that these conditions are not strictly met; for example, $\lambda$ can vary in the course of cell division. However, the assumption may give insight into the nature of the process. Equation 1 must be then rewritten as:
At a given time the remnant lesions per cell $U_{R} \simeq U_{\infty}$... If an acute dose of radiation $x$ is applied producing $U_{x}$ lesions then the new level of $U$ lesion wili be: $U: U_{R}+U_{x}$. If $U_{R}$ is not zero, then the RMR model (equation 8) predicts a finite initial slope for the survival curve even at the lowest $X$ dose levels.

\section{Variants Produced by Misrepair}

In each generation; a fraction of misrepair is not lethal. However, since misrepair involves abnormal DNA sequences, the cells that carry misrepair will be designated as variant mutants. These mutants exist even in the absence of normal recombination. 
Case 1: Assume that the variants (M) have no particular advantage. They would then have the same generation times as the normal cell population. The variants then would not be eliminated, but grow in proportion to "normal" cells. In the course of many generations, multiple mutants could accumulate.

Case 2: It is more likely that the variants produced have an "advantage" or a "disadvantage" in comparison with normal cells that have only uninjured or eurepaired DNA. The advantage might express itself in a variety of ways; for example, $M$ may have a decreased generation time, and greater resistance to physical and chemical agents. A possiblity is that the newly arising $M$ forms have improved repair efficiency when compared to the parent uninjured cells. This occurs if $\varepsilon$ or $\phi$ is greater, or $k$ smaller than norma 1 .

The proportion of cells with advantageous repair may gradually outgrow normal cells; because of better repair, fewer of the $M$ variants will die in each generation.

Increased repair efficiency might come about if the misrepair process alters control genes, causes improved structure of repair enzymes, or produces aneuploidy in which genes that code for repair enzymes reoccur more than in a single copy.

There are various evidences in the literature that some repair systems are under genetic control. In bacteria, the "SOS" repair systems are "inducible" and in certain strains of algae repair can also be induced (Radman, 1975; Howard and Cowie, 1978). In yeast many genetic species are known for repair (Ho and Mortimer, 1976). Loss of a control gene in an inducible system due to radiation injury might result in a "constitutive" repair system.

From the above analysis it is evident that a continuous background level of radiation can potentially cause a shift toward higher repair capacity in the population. Since radiation produces a variety of misrepair, we hypothesize that at a high dose rate, the advantages might be lost. For a given strain of cells then, there might be an optimal radiation background at which, at the expense of producing some lethals, the viable members of the population have an optimal repair capacity.

Since the well-known experiments of Russell on mice $(1963 a, b)$, it has been known that the mutagenic effects of low-LET radiation are largely repaired in germ cells, particularly in oocytes. We must wonder, however, whether or not misrepair occurs along with eurepair in these cases as postulated in our model.

Causes of Generation of New $U$ Lesions in Background

We discussed the possibility that various deleterious agents can produce $U$ lesions similar to lesions produced by ionizing radiation. The rate of generation of such lesions, $C$ in equation 22, might depend not only on cosmic ray background and on natural radioactivity, but also on the combined effects of all environmental agents.

It is also possible that $U$ lesions are generated in cells due to normal biochemical, metabolic and self-reproductive activities. Many cells possess enzymes that can cause incision in DNA and a number of metabolic processes have strong oxidizing agents or free radicals as intermediate products.

Given the rules for additivity of $U$ lesions, and the possibilities that various agents also have $r$ effects, it is quite possible that the quantitative effect of a dose of ionizing radiation is different at various times on the same cellular system. Cells have memories for $U$ lesions generated earlier in time, and cells that have earlier lived in an environment where repair has been advantageous for them, may have developed improved repair capacities.

The problem of the biological effects of low level irradiation cannot be separated from the problems of joint effects of all environmental agents acting on cells together. It is important then to establish the environmental and internal causes of cell death and accumulation of $U$ lesions in normal background environments. The RMR model presents a quantitative framework for testing how the effects of these various agents interact with each other.

In order to understand environmental effects, we need to:

1. analyze the chemical nature of $U$ lesions from each type of environmental agent;

2 . measure the yield of various types of $U$

lesions from specific environmental poisons;

3. quantitatively measure $r$ effects on repair mechanisms.

These procedures would help to establish the degree of toxicity of various mixed environments.

\section{REFERENCES}

Albright, N. L. 1980. A Markov Model of the Repair-Misrepair Process of Cell Survival. Lawrence Berkeley Laboratory Report LBL-11660.

Bernardi, F. and J. Ninio. 1978. The accuracy of DNA replication. Biochimie 60, 1083-1095.

Chadwick, K. H. and H. P. Leenhouts. 1973. A molecular theory of cell survival. Phys. Med. Biol. 13, 78-87.

Chadwick, K. H. and H. P. Leenhouts. 1978. The rejoining of DNA double strand breaks and a model for the formation of chromosomal rearrangements. Int. J. Radiat. Biol. 33 , $517-529$

Christensen, R. C, C. A. Tobias, and W. D. Taylor. 1972. Heavy-ion induced single and double 
strand breaks in $\phi X-174$ replicative form DNA. Int. J. Radiat. Bio1. 22, 457-477.

Cleaver, J.E. 1978 DNA repair and its coupling to DNA replication in eukaryotic cells. Biochim. Biophys. Acta 516, 480-516.

Green, A.E.S. and J. Burki. 1972. A note on survival curves with shoulders. Radiat. Res. $60,536-540$.

Ho, K.Y.S. and R. K. Mortimer. 1973. Induction of dominant lethality by $x$ rays in a radiosensitive strain of yeast. Mutat. Res. 20, 45-51.

Howard, A. and F. G. Cowie. 1978. Induced resistance in Closterium: Indirect evidence for the induction of repair enzyme. Radiat. Res. $25,607-616$.

Kellerer, A. M. and H. H. Rossi. 1972. The theory of dual radiation action. Curr. Top. Radiat. Res. Q. 8, 85-158.

Kellerer, A. M. and H. H. Rossi. 1978. generalized formulation of dual radiation action. Radiat. Res. $75,471-488$.

Ngo, F.Q.H., E. A. Blakely, and C. A. Tobias. 1980. Sequential exposures of mammalian cells to low- and high-LET radiations. I. Lethal effects following $X-r$ ay and neon-ion irradiation. Radiat. Res., in press.
Radman, M. 1975. SOS repair hypothesis:

$\because$ Phenomenology of an inducible DNA repair which is accompanied by mutagenesis. Molecular Mechanisms for Repair of DNA ( $P$. Hanawalt and R. B. Setlow, eds.) pp. 355-367. New York, PTenum Press.

Ritter, M., J. E. Cleaver, and C. A. Tobias. 1977. High-LET radiations induce a large proportion of nonjoining DNA breaks. Nature 266, 653-655.

Russe11, W. L. 1963a. Genetic hazards of radiation. Proc. Am. Phil. Soc. 107, 11-17.

Russell, W. L. 1963b. The effect of radiation dose rate and fractionation on mutation in mice. Repair from Genetic Radiation Damage (F. H. Sobel, ed.) pp. 205-217. Pergammon Press, New York.

Sinclair, W. S. 1966. The shape of radiation survival curves of mammalian cells cultured in vitro. Biophysical Aspects of Radiation Quality, pp. 21-43. Technical Report Series 58. IAEA, Vienna.

Tobias, C. A., E. A. Blakely, F.Q.H Ngo, and T.C.H. Yang. 1980a. The repair-misrepair model of cell survival. Radiation Biology and Cancer Research (A. Meyn and R. Withers, eds.) pp. 195-230. Raven Press, New York.

Tobias, C. A., E. A. Blakely, and F.Q.H. Ngo. 1980b. Interactions between deleterious agents on ${ }^{i}$ living cells. Radiat. Res. 83, 402. (Abstract.) 
148

Blank 


\title{
CELL TRANSFORMATION AND MUTAGENESIS
}

\author{
T. C. H. Yang, F. Q. H. Ngo, J. Howard, and C. A. Tobias
}

Carcinogenesis and mutagenes is are two of the most important biological effects of ionizing radiation. With the progress of cell culture techniques, studies on the dose-response relationship and the mechanism(s) of radiationinduced cell transformation and mutation become possible. The cell systems used by most investigators for studying radiation transformation are established mouse fibroblast lines. When a normal fibroblast is grown in vitro and treated with carcinogens, its cytological and growth characteristics can be altered and show many characteristics of a malignant cell, including the loss of contact inhibition and the ability to form tumors upon reinjection into syngeneic hosts. The oncogenic transformation in vitro is a complex process and in many respects strongly resembles carcinogenesis in vivo. The process of radiation induced cell transformation in vitro requires time and cell proliferation. Several development stages are involved: the initiation and fixation of radiation induced damage, a proliferative phase of expression, and a quiescent phase of expression. Using the present assay technique, an incubation period of 4 to 6 weeks (about the same time as for chemical induced cell transformation and about twice longer than viral (SV40) transformation) is required before the radiation transformed foci of cells can be identified and scored.

As for mutation studies, various mammalian cell systems are available, including Chinese hamster lung (V79), embryonic (Don) or ovary (CHO) cell lines. Most quantitative data on mutation induction by radiation have been obtained on the basis of selection with purine or pyrimidine analogues, i.e., HGPRT- (resistance to 6-thioguanine (6TG) or other purine analogues) and TK- (thymidine kinase deficiency or resistance to thymidine analogues). In addition to the HGPRT genes, mutation at other loci have been found and used for studying: radiation mutagenesis, e.g., resistant to ouabain. Following radiation exposure, a stage of mutation lesion fixation through cell division and à period of expression are necessary before mutants can be identified.

Within the last few years, the transformation and the mutation of cells by ionizing radiation has been studied in depth by many investigators. Most quantitative data, however, have been obtained for low-LET radiation, and a limited amount of information is available for high-LET particles (Cox et a 1., 1977; Yang and Tobias, 1980). In general, high-LET radiation has been found to be more effective in producing cell ransformation and mutation than $X$ or gamma rays. Because of the potential applications of heavyion radiation in cancer therapy, there is a need for information on the carcinogenic and mutagenic effects of heavy ions. We have initiated a research program to systematically study the dose-effect relationships of cell transformation and of mutation for heavy ions with various charges, velocities, and LET values. In the past three years, we have collected data in order to answer some specific questions: (1) How effective are high-LET heavy ions in producing cell transformation? What is the dose-effect relation? (2) Can heavy ions enhance viral transformation and act as a cocarcinogen? Is the cocarcinogenic effect LET dependent? And how? (3) Can cells repair heavy-ion induced enhancement lesions? How much? (4) What is the mutagenic effect of high energy heavy ions? (5) How does the RBE for cell killing compare with that for cell transformation, cocarcinogenic effect, and mutagenesis?

The present goal of this research project is to collect quantitative data that are useful for estimating and understanding the potential risk involved in space flight and in heavy-ion cancer therapy, e.g., the late effects in normal tissues due to mutation and cell transformation. The ultimate goal is to use heavy-ion radiation as a research tool to elucidate the fundamental mechanism(s) of mutagenesis, DNA repair, and carcinogenesis. The mechanistic studies on mutagenesis and cell transformation will help to provide a firm base for developing a sound scientific theory that can be used to evaluate the potential hazards of low dose and low dose rate of both low- and high-LET radiation.

\section{ONCOGENESIS}

Little work on cell transformation with high LET radiation has been reported, and only the carcinogenic effect of neutrons has been studied in reasonable detail (Borek, 1976; Han and Elkind, 1979). Studies on cell transformation with heavy ions were initiated in our laboratory less than a year ago, and some of the preliminary results are reported here. A mouse embryo cell line--C3H10T1/2 (c18)--was obtained from Dr. C. Heidelberger's: laboratory at the University of Southern California. Only well-confluent cells were used for studying radiation cell transformation. After exposure to radiation, cells were trypsinized into singlets and plated into $100-\mathrm{mm}$ dishes at a density that gave about 500 survivors. Medium was changed once a week and cells for transformation were fixed and stained after 5 to 6 weeks of incubation at $37^{\circ} \mathrm{C}$. The plates were checked under a Zeiss dissecting microscope, and the colonies that showed multiple layers of cells, i.e., a loss of density inhibition of growth classified as Type II and III according to Reznikoff et al. $(1973 a, b)$ were scored as transformed. The colony-forming ability was 
determined by plating irradiated "cells into $100-\mathrm{mm}$ dishes at a low density which gave about 100 to 150 survivors per dish at the end of 9 days of incubation. Colonies that contained more than 9 cells were counted as survivors.

Results of two experiments with $470 \mathrm{MeV} / \mathrm{n}$ carbon beam are shown in Figure 1. Note the initial rapid increase of transformation frequency per survival with dose that appears to reach a plateau at about 600 rad for both $X$ rays and $470 \mathrm{MeV} / \mathrm{n} \quad\left(\mathrm{LET}_{\infty} \simeq 10 \mathrm{keV} / \mu \mathrm{m}\right)$ carbon ions. This dose-response relationship is similar to that seen by other investigators. In the range of doses studied, carbon particles with a residual range in water of about $1.4 \mathrm{~cm}(\sim 70 \mathrm{MeV} / \mathrm{n}$; LET $\simeq 34 \mathrm{keV} / \mu \mathrm{m}$ ) consistently gavè a higher frequency of transformation per viable cell than $X$ rays. When the transformation frequency per survivor $\left(-3 \times 10^{-3}\right)$ induced by an $x$-ray dose that gives a $50 \%$ lethality ( 220 rad) is taken as the reference point, the RBE for $470 \mathrm{MeV} / \mathrm{n}$ and $70 \mathrm{MeV} / \mathrm{n}$ carbon ions was found to be about 1.0 and 1.8 , respectively. A comparison between $R B E$ values for cell transformation and cel' killing for various radiation qualities is shown in Table 1. For the same cell line (C3H1OT1/2), the RBE is about the same for both cell killing and oncogenic transformation for a given quality of ionizing radiation. The data obtained with $430 \mathrm{keV}$ neutrons suggest that there may be a significant difference, by a factor about 2 , between the RBE value for cell lethality and cell transformation. This difference, however, becomes less significant when the RBE is taken at lower doses, i.e., at higher survival levels.

It is not known at present whether the RBE for oncogenic transformation is the same as for cell killing for all high-LET radiation. Further investigation on this question is essential, because it will yield insights on the mechanism(s) of radiogenic cell transformation. For example, if the RBE and LET relationship is precisely the same for both end points, cell transformation lesions are likely related to the repairable lethal damages, and the oncogenic transformation of cells may be a result of misrepair of these lesions.

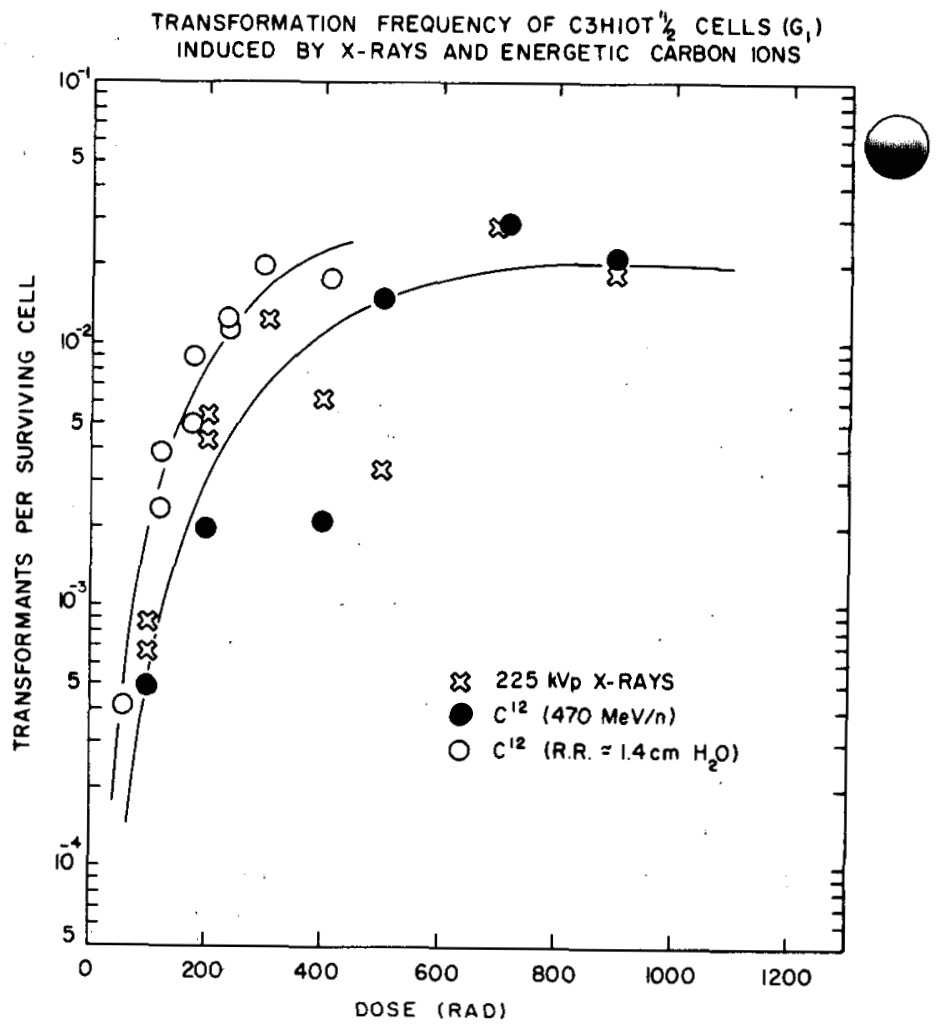

Figure 1. Dose-response curves for $x$ rays and carbon ions in inducing oncogenic transformation of mouse embryo cells (C3H1OT1/2). (XBL 807-10658)

\section{COCARCINOGENIC EFFECT}

There are numerous types of oncogens, e.g., radiation, chemical, and viral oncogens in our environment, and it is possible that some tumors are caused by more than one carcinogen. The interaction between certain types of carcinogens can be more than additive, i.e., synergistic. Animals treated with a combination of chemical carcinogens and oncogenic viruses, for example, develop a higher incidence of tumors than animals inoculated with virus or chemical alone (ChiecoBianchi et al., 1963; Duran-Reynals, 1954).

Table 1. RBE Values for Cell Killing and Transformation for High-LET Radiation:

\begin{tabular}{|c|c|c|c|c|}
\hline \multirow{2}{*}{ Radiation } & \multirow{2}{*}{ Cells } & \multicolumn{3}{|c|}{ RBE (at $50 \%$ surviva 7 ) } \\
\hline & & Survival & Transportation & References \\
\hline $\begin{array}{l}\text { Neütrons } \\
(430 \mathrm{keV})\end{array}$ & $\begin{array}{l}\text { primary hamster } \\
\text { embryo cells }\end{array}$ & 15 & 33 & Borek (1976) \\
\hline $\begin{array}{l}\text { Neutrons } \\
\text { (GENUS) }\end{array}$ & E3H10Tl/2 cells & 2.6 & 2.6 & $\begin{array}{l}\text { Han and Elkind } \\
(1979)\end{array}$ \\
\hline $\begin{array}{l}\text { Carbon } \\
(470 \mathrm{MeV} / \mathrm{n})\end{array}$ & C3H1OTI/2 cells & 1.0 & 1.0 & \\
\hline $\begin{array}{l}\text { Carbon } \\
\left(70^{:} \mathrm{MeV} / \mathrm{n}\right)\end{array}$ & C3H10Tl/2 cells & 1.8 & 1.8 & . \\
\hline
\end{tabular}


Several polycylic hydrocarbons have been shown to act synergistically in vitro with RNA tumor viruses to transform rodents cells (Freeman et al., 1970; Rhim et al., 1971). Radiation has been found to enhance viral transformation of mammalian cells in culture also. The effect of $X$ rays on the sensitivity of hamster kidney (BHK21) cells to transformation by polyoma virus was first examined by Stoker (1963), and an enhancement of viral transformation was observed. Similar effects were found by Pollock and Todaro (1968) when they studied the change in cell transformation frequency of SV40 infected mouse fibroblasts ( $3 T 3$ cells) and human diploid skin fibroblasts irradiated with 250-kVp $X$ rays. The frequency of cell transformation by oncogenic virus can also be enhanced by U.V. radiation (Lytle et al., 1970). Enhancement of viral transformation by other carcinogens is, therefore, a well-established general phenomenon, and radiation can be considered as an effective cocarcinogen.

Heavy ions occur in nature in numerous places, e.g. alpha particles emitted from $210 \mathrm{pb}$ and $210 \mathrm{po}$ in insoluble smoke particles, radionuclides that are by-products of nuclear fuel cycles, contaminants of nuclear research sites, uranium mines, certain nuclear medicine, and cosmic rays in space. Therefore, it is practical as well as important to our fundamental knowledge, to know the potential cocarcinogenic effect of high-LET radiation in cell transformation by oncogenic viruses. We initiated experiments a few years ago to study this interesting problem. Using cultured mouse embryo cells

(C3H10T1/2) as a model system, and the methods developed by Pollock and Todaro (1968), extensive studies have now been carried out on the enhancement of cell transformation by $\$ V 40$ following exposure to carbon, neon, or argon ion beams produced at the Bevalac and SuperHILAC.

Figure $2 \mathrm{~A}$ shows a plot of the average number of tranformants per cell plated as a function of dose. With no radiation, the frequency of transformation by SV 40 virus was about 7 to $8 \times 10^{-4}$. The frequency of transformation was enhanced by radiation two to three times, and reached a peak around 300 rad for $X$ rays and about 200 rad for neon particles (425 MeV/n; $\mathrm{LET}_{\infty} \simeq 34 \mathrm{keV} / \mathrm{um}$ ). At higher doses, as cell:killing becomes dominant, a decrease in the enhancement effect was found. The rate of this decrease was slower for $X$ rays than it was for neon ions. The results of the effect of $X$ rays and neon ions" on "the number of transformants per survivor are shown, in Figure $2 B$. The increase of the enhancement ratio as a function of radiation dose is curvilinear for both neon particles and for $X$ rays. The RBE value for neon ions in the enhancement effect decreased as the dose was increased. For the $50 \%$ lethal dose of neon ions (about 140 rad), which caused a fivefold increase of viral transformation, the RBE was about 2.28 . The $10 \%$ survival dose (about $310 \mathrm{rad})$, gave an enhancement ratio of about 15 and RBE of about 1.84 . Carbon ions (400 MeV/n)

ith LET values of about $11 \mathrm{keV} / \mu \mathrm{m}$ gave the same enhancement ratio for $X$ rays over the dose range studied (up to about 500 rad), as shown in

Figure $3 A$. The kinetics of the enhancement of
EFFECT OF NEON IONS ( $425 \mathrm{MeV} / \mathrm{OMU}$ ) AND X-RAYS (225 kVP) ON SV-4O VIRAL TRANSFORMATION OF C3HIOT $\frac{1}{2}$ CELLS (CI $8 / I 1$ )

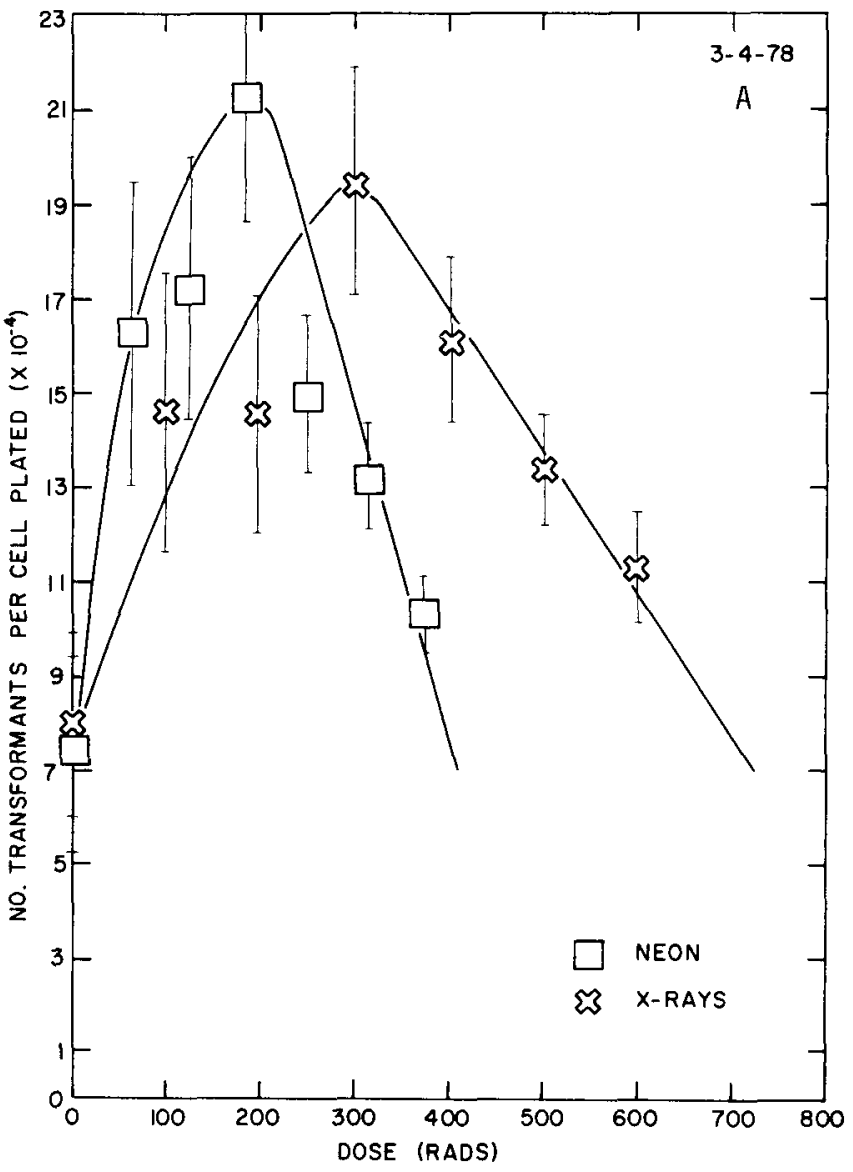

C3HIOT $1 / 2$ CELLS $(\mathrm{CI} 8 / 11)$ INFECTEO WITH SV 40 VIRUS \& IRRADIATED WITH Ne ${ }^{20}$ (425 MeV/amu)

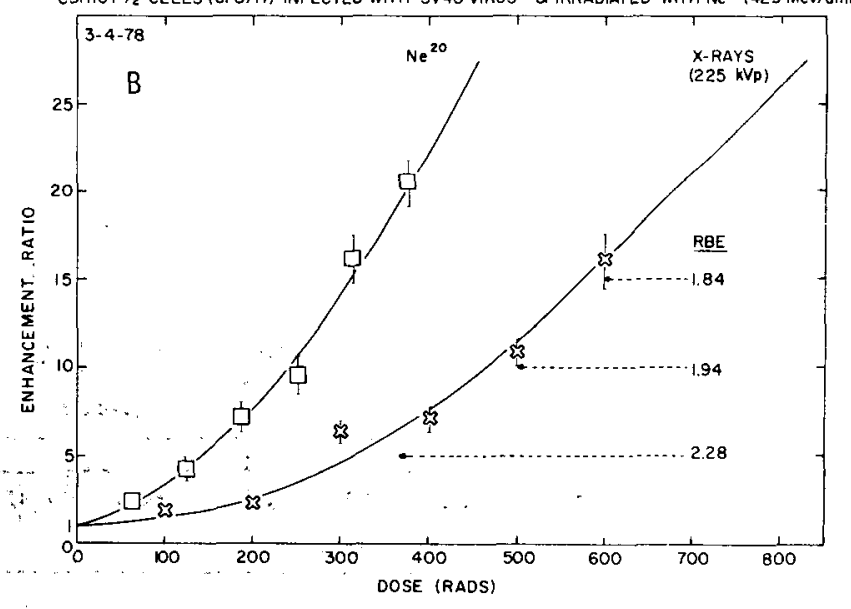

$+\cdots \cdot$

$\because$

Figure 2- Enhancement effect of neon particles $(425 \mathrm{MeV} / \mathrm{n})$ and $\mathrm{X}$ rays in viral transformation. (A) The number of transformants per cell plated in SV4O-infected C 3 HIOT1/2 cells as a function of radiation dose. ( $B)$ The relationship between the enhancement ratio (a ratio of transformant per survivor of irradiated cells to that of nonirradiated cells) and radiation dose. [(A) XBL 785-8403; (B) 784-8251] 
viral transformation, however, approached linearity for $570 \mathrm{MeV} / \mathrm{n}$ argon ions with $\mathrm{LET}_{\infty}$ about 85 to $90 \mathrm{keV} / \mathrm{mm}$ (Fig. 3B). For heavy ions with extremely high LET values, e.g., $5 \mathrm{MeV} / \mathrm{n}$ argon particles ( $L E T_{\infty} \simeq 2,000 \mathrm{keV} / \mu \mathrm{m}$ ), the enhancement effect was much lower than $X$ rays. This may be because the cross-sectional area for cell killing with low energy argon ions is close to the size of nuclear area, i.e., hitting the nucleus with a lower energy argon particle may cause a lethal event (Yang et al., 1977). The cell killing thus becomes a dominant effect for heavy ions with high LET.

Based on our data presently available, the RBE of the enhancement effect increases with LET, reaches a maximum peak around 100 to $200 \mathrm{keV} / \mu \mathrm{m}$, and then decreases to value less than 1 , as shown in Figure $3 \mathrm{C}$. The precise relationship between

ENHANCEMENT OF SV- 40 VIRAL TRANSFORMATION OF C3HIOT $1 / 2$ CELLS (Cl 8//2) WITH X-RAYS (225 kVp) OR CARBON PARTICLES (400 MeV/amu)

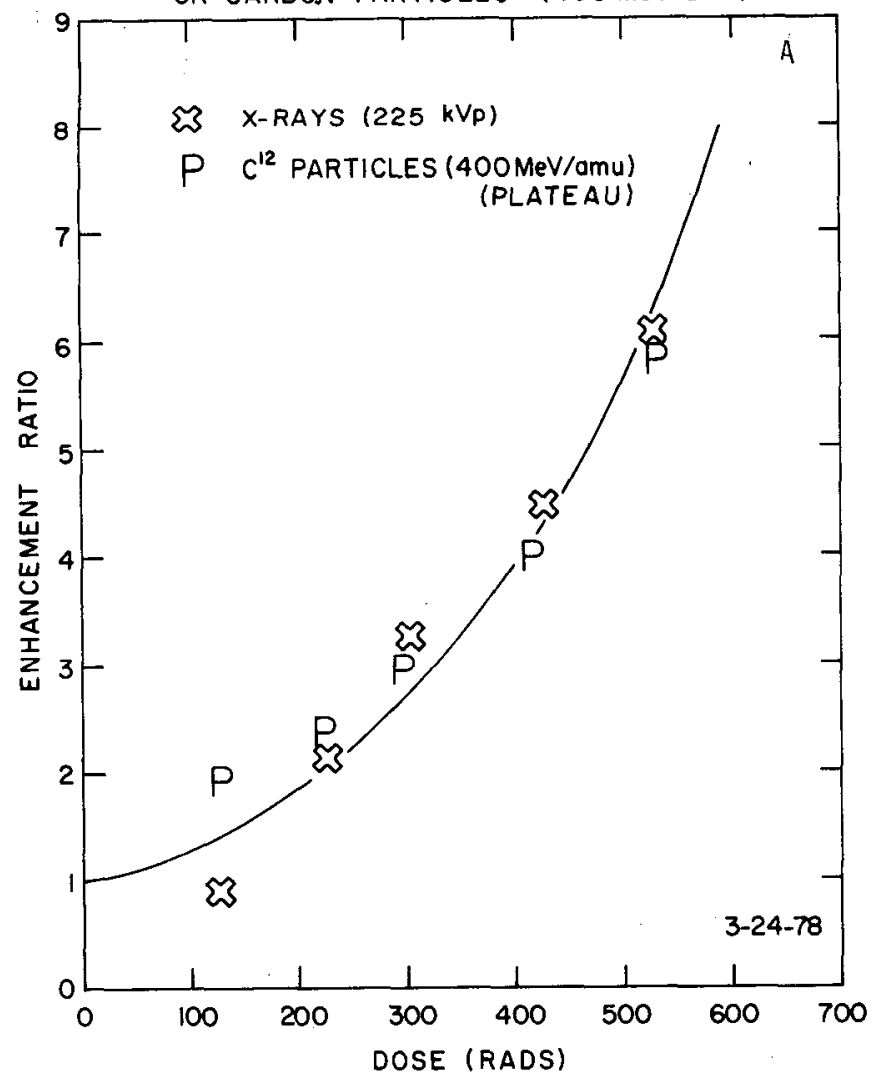

Figure 3. Radiation enhancement effect of heavy ions with various charges and energies.

(A) Enhancement of SV 40 viral transformation of C3HIOT1/2 cells with $X$ rays or $400 \mathrm{MeV} / \mathrm{n}$ carbon particles. (B) Transformation frequency per survival as a function of dose for $570 \mathrm{MeV} / \mathrm{n}$ argon jons and $x$ rays. (C) RBE as a function of LET $T_{\infty}$ for radiation enhancement of viral transformation. [(A) XBL 785-8402; (B) 807-10659; (C) $807-10687]$
RBE and LET, however, will not be known until more data are obtained, especially in the LET region between 100 to $1,000 \mathrm{keV} / \mathrm{\mu m}$.

The question as to whether the radiationinduced lesion that enhances the viral transformation of cells can be repaired is clearly an interesting and important one. For ionizing radiation, there are two well-known repair processes: sublethal damage repair and potential lethal damage repair. The role of these two repair processes in the enhancement effect has been studied, but thus far only a very limited amount of data has been obtained and reported (Coggin, 1969). An extensive study on the effect of potential lethal damage repair on the enhancement of viral transformation by $x$ rays and heavy ions has been done in our laboratory (Yang et al., 1980). When cells were maintained at a confluent
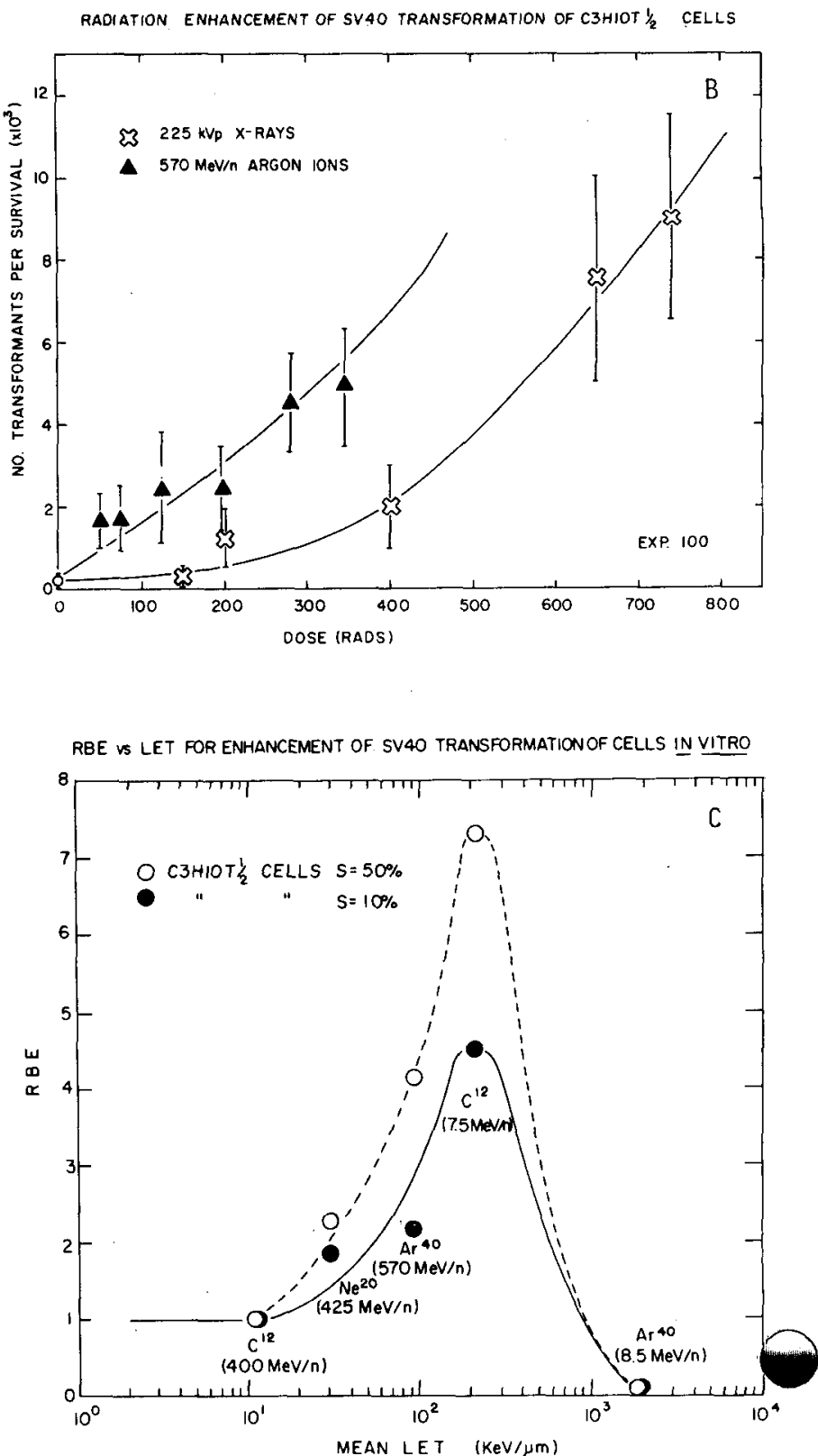
state at $37^{\circ} \mathrm{C}$ for $8 \mathrm{~h}$ after irradiation before they were plated into dishes at low density, there was an increase in cell survival for both

$X$ rays and neon particles (see Ngo et al., Part III). The enhancement effect of viral transformation, however, was decreased for both $X$ rays and neon ions when the viral infection was delayed.

Figure 4 depicts the results of neon ions on cell transformation. The enhancement effect of neon irradiation on the viral transformation of cells decreased the longer the SV4O infection was delayed. The enhancement ratio dropped by a factor of 1.67 , i.e., from about 5 to 3 , when the viral infection was done $8 \mathrm{~h}$ after the cells were irradiated with a dose of 138 rad (LD50). A further decrease of the enhancement ratio was observed when the SV4O infection was performed $18 \mathrm{~h}$ after irradiation. For a dose of $310 \mathrm{rad}$, which is a lethal dose for $10 \%$ survival, the enhancement ratio decreased from about 15 to 6 for an $8 \mathrm{~h}$ delay, and from 15 to 1.8 for an $18 \mathrm{~h}$ delay.

A quantitative study on the effect of sublethal damage repair on enhancement has also been conducted in our laboratory (Yang and Tobias, 1980). Results showed an increase in survival and a decrease in enhancement of viral transformation when cells received a conditioning dose of 250 rad $X$ rays and were then irradiated with various doses after an $8-h$ incubation at $37^{\circ} \mathrm{C}$, as compared to the effects of single exposure. The viral infection was done immediately after the second radiation dose. For cells irradiated

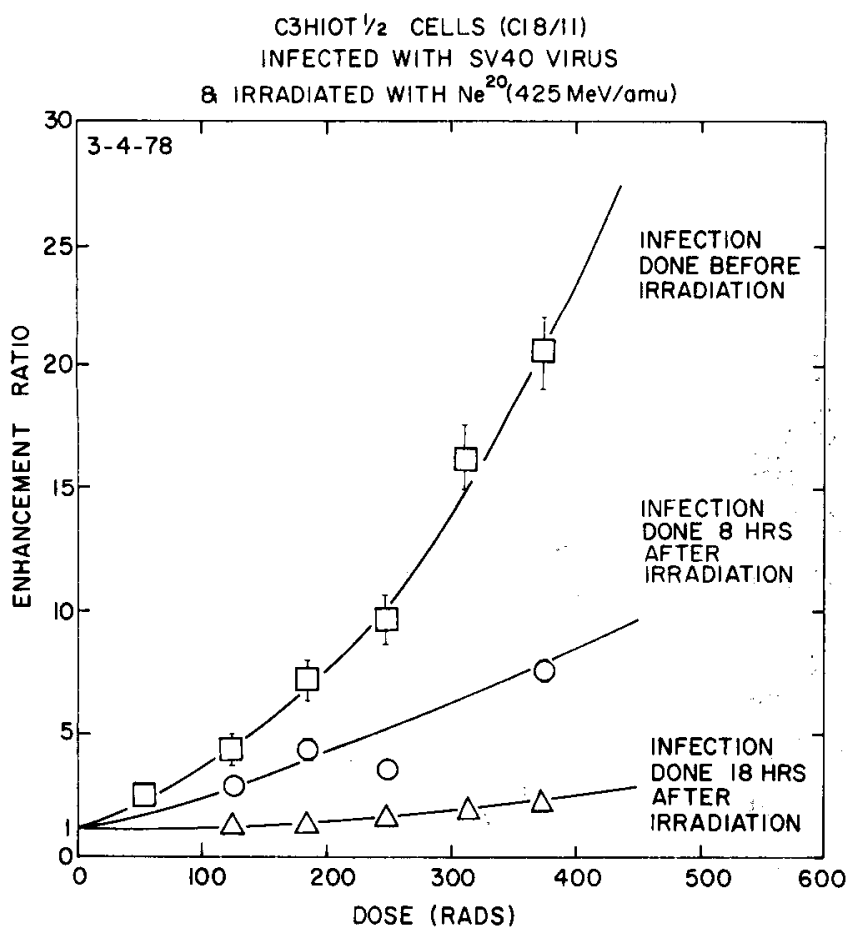

Cigure 4. Effect of SV40 infection time on the enhancement of viral transformation in $\mathrm{C} 3 \mathrm{H} 10 \mathrm{TI} / 2$ cells irradiated with neon particles (425 MeV/n). (XBL 784-8252) with split doses, there was only a shift of the enhancement curve without any change of its slope, which is similar to that of a survival curve.

These experimental results indicate that mammalian cells can effectively repair most of the radiation-induced enhancement lesions. Our more recent studies showed that the kinetics of repair is about the same for both enhancement effect and survival. The biological effect changes exponentially with time and reaches a plateau about $6 \mathrm{~h}$ after $\mathrm{X}$-irradiation. Caffeine, which is a repair inhibitor, applied to cells after irradiation and SV4O infection caused an increase of viral transformation and X-ray enhancement effect; hydroxyurea showed no effect.

To explain the enhanced transformation, it has been suggested that radiation produces strand breaks in cellular DNA that promote the attachment or integration of viral genomes during DNA repair synthesis (Pollock and Todaro, 1968; Coggin, 1969; Lytle et al., 1970; Casto, 1973). At the time we initiated our research work we did not know: (1) what type(s) of DNA strand breaks is important in radiation enhancement effect, or (2) whether the repair of radiation-induced DNA lesions directly aids the integration of viral genomes into the host DNA.

Our data from heavy ion studies have provided new information on these two questions. Since high LET heavy ions are more effective in producing DNA double strand breaks than single strand breaks (Christensen et al., 1972), since the enhancement effect is greater for high-LET heavy particles than for low-LET radiation, and since the enhancement of viral transformation is proportional to the square of the X-ray dose (Yang et al., 1980), DNA double strand breaks appear to be the major lesions involved in the radiation enhancement effect. The repair studies, furthermore, suggest that these double strand breaks may be the repairable ones and that they are related to the slow-repair lesions observed in asynchronous hamster cells by Ritter et al. (1977) and by Roots et al. (1979). Recently we have obtained some experimental results that suggest the repair of radiation-induced enhancement lesions at $G_{1}$ phase does not aid the integration of SV4O viral genomes into cell nuclear DNA. However, the importance of the repair process in $S$ phase in promoting the attachment of viral DNA into host chromosomes of irradiated cells needs further investigation.

\section{MUTAGENESIS}

We initiated studies on the mutagenic potential of energetic heavy ions about six months ago. Two different genetic markers (ouabain resistant and 6-TG resistant) and three cell lines (C3H10T1/2, V79, and CHO cells) were chosen for the mutation work. Using the techniques developed by Landolph and Heidelberger (1979), the induction of ouabain resistant mutation in $\mathrm{C} 3 \mathrm{H} 10 \mathrm{~T} 1 / 2$ cells by U.V. light, $X$ rays, $10.2 \mathrm{meV} / \mathrm{n}$ lithium ions, $8.5 \mathrm{MeV} / \mathrm{n}$ argon particles, and alpha particles of $241_{\text {Am }}$ were examined. 
No mutant was found in cells treated with radiation, except U.V. light. Results showed that ionizing radiation, including high-LET heavy ions, cannot effectively induce ouabain resistant mutation. This finding was not unexpected, since Thacker et al. (1978) reported a negative result in 679 cells treated with gamma rays and accelerated helium ions.

The use of 6-thioquanine to study mutation induction in Chinese hamster ovary cells (CHO) by heavy ions yielded positive results. Asynchronous $\mathrm{CHO}$ cells in exponential growing phase were irradiated with neon ions $(425 \mathrm{MeV} / \mathrm{n}$; $\mathrm{LET} \approx 30 \mathrm{keV} / \mu \mathrm{m})$, subsequently seeded at a low density, and incubated for 8 to 10 days before selecting for mutants with 6-TG medium. The respread method was used to obtain the mutation frequency, and the results are given in Figures 5 and 6 . Both neon ions and $X$ rays increased the number of mutants per viable cell curvilinearly with an increase in dose. Neon particles appear to be highly effective in producing mutation, as compared to $X$ rays. The RBE value, which was determined by taking the mutation frequency per viable cell $\left(-5 \times 10^{-5}\right)$ induced by an $X-r a y$ dose given $10 \%$ survival ( $550 \mathrm{rad})$ as the reference point, was about 3.6. A comparison between the RBE for mutation induction $(-3.6)$ and for cell killing $(\sim 1.4)$ at $10 \%$ survival level indicates that, compared to $X$ rays, neon ions can produce more mutation lesions than lethal injuries.

Using V79 cells, F.Q.H. Ngo has obtained some preliminary data with $15.6 \mathrm{MeV} / \mathrm{n}$ carbon ions. Compared with $225 \mathrm{kVp} X$ rays, low energy carbon particles ( $\mathrm{LT}_{\infty} \sim 126 \mathrm{keV} / \mathrm{um}$ ) appear to be very effective in producing both cell killing and 6-THr mutation, as shown in Figure 6 . These mutation data, although preliminary, do consistently suggest that high-LET radiation can be very mutagenic and are qualitatively in agreement with other investigators' results (Cox et al., 1977).

\section{PLANS FOR FUTURE RESEARCH}

Within a relatively short period of time, our heavy-ion research program on cell transformation and mutagenesis has yielded some interesting information. We now have a better understanding, for example, about the role of the repair mechanism in the radiation ennancement effect, and the nature of molecular lesions that are induced by radiation and may be responsible for the enhancement of viral transformation. Studies on the mutation induction and cell transformation

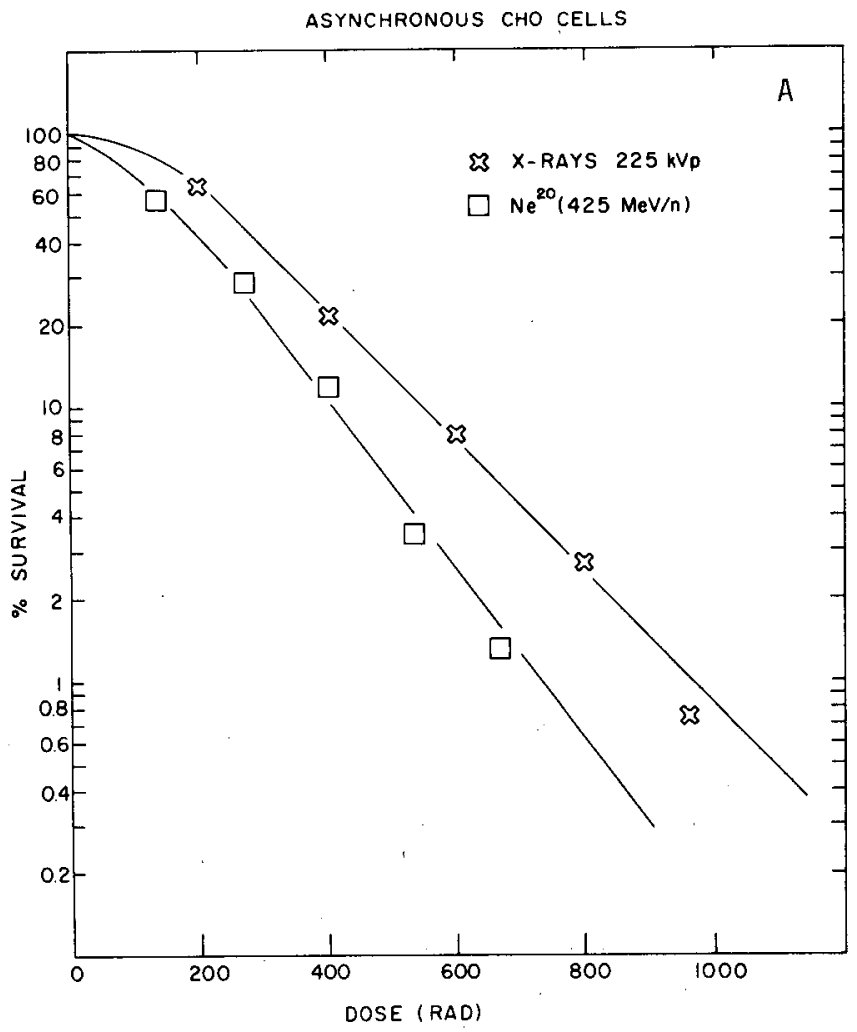

Figure 5. Dose-response curves of $\mathrm{CHO}$ cells irradiated with $X$ rays or neon particles $(425 \mathrm{MeV} / \mathrm{n}):$ : Survival (A) and:6-TGr mutation (B). [(A) XBL 807-10690; (B) 807-10692]

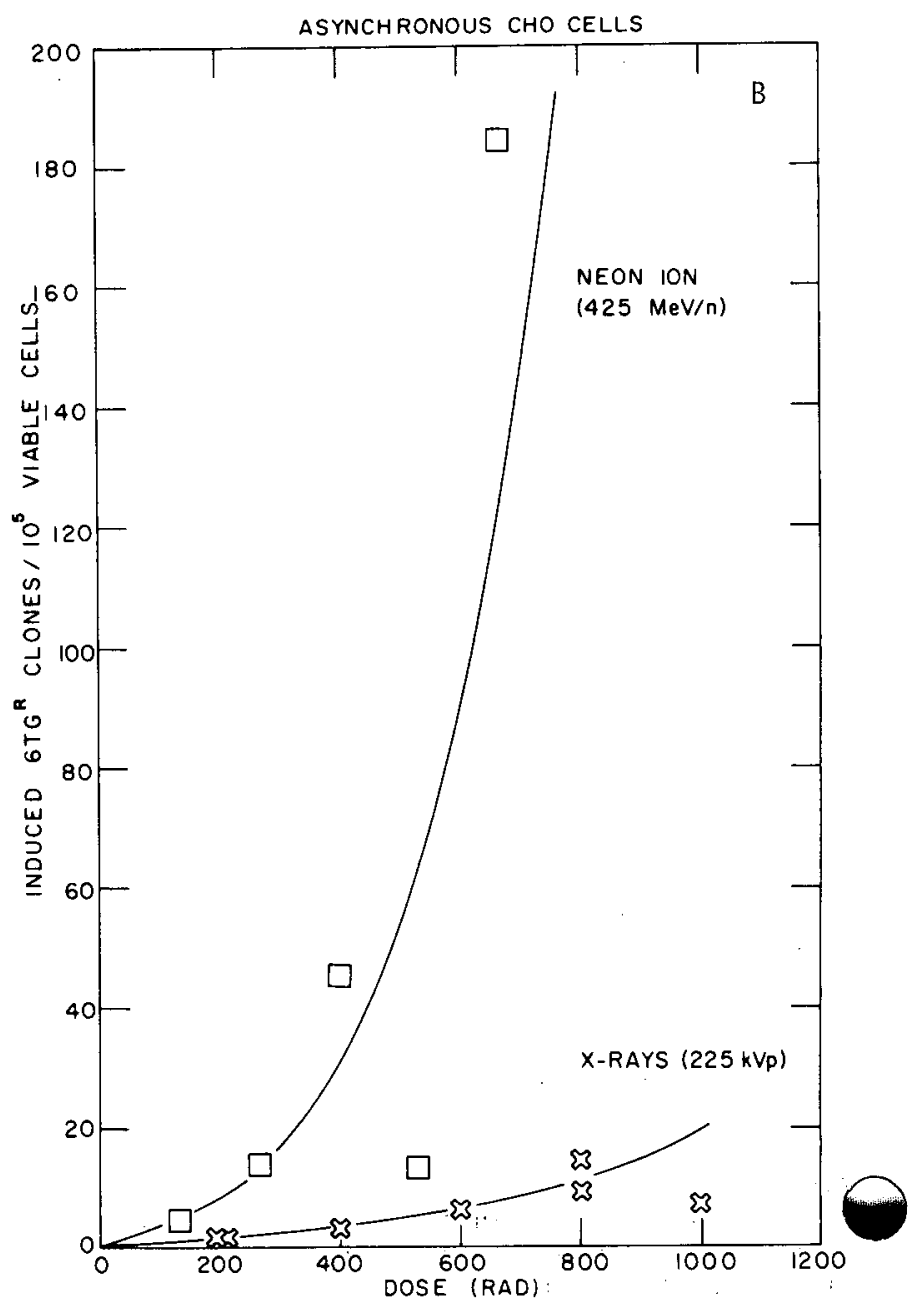



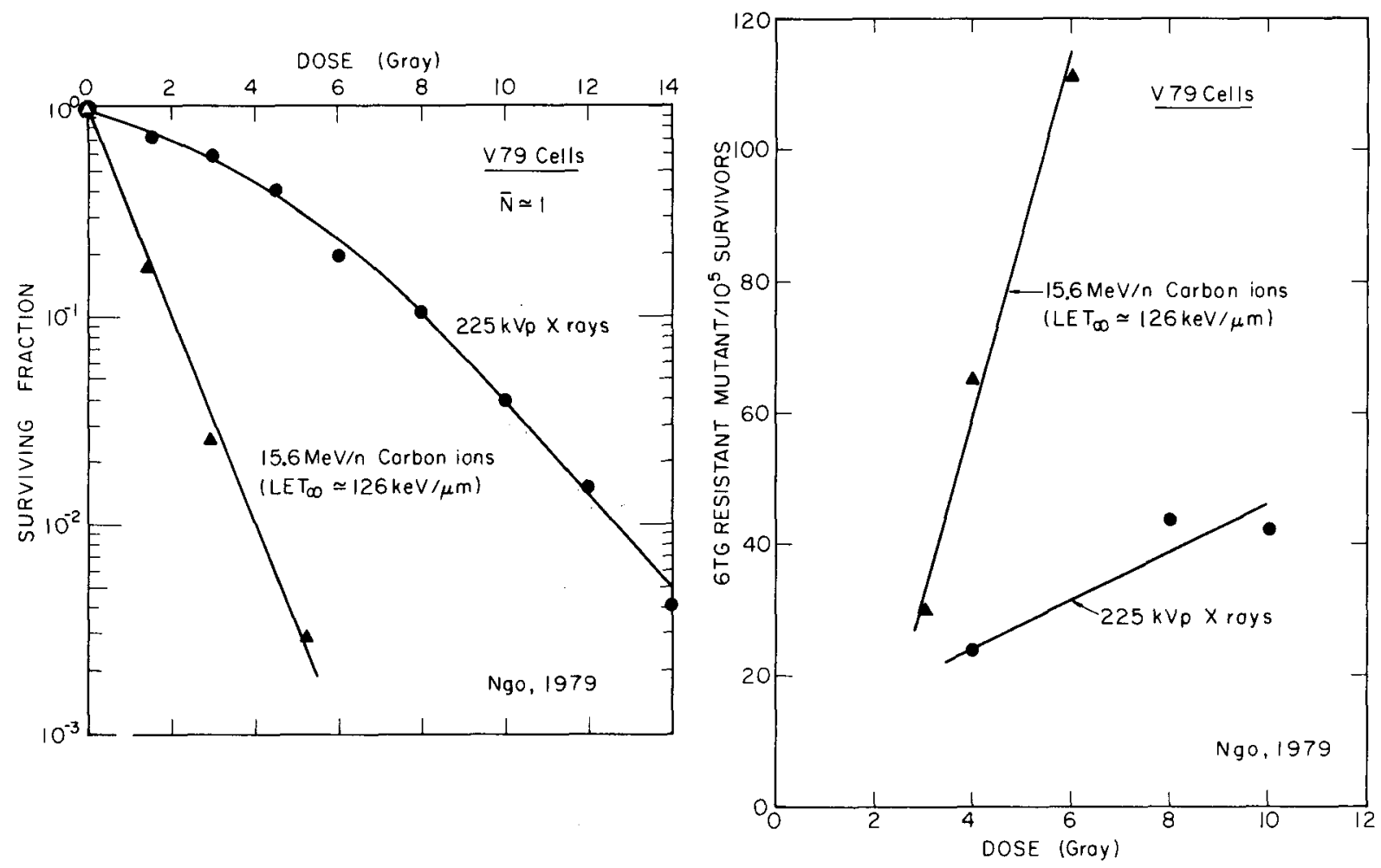

Figure 6 . The effects of carbon ions $(15.6 \mathrm{MeV} / \mathrm{n})$ on cell survival and mutation in 779 cells, as compared to $X$ rays. Mutation data of $V 79$ cells are not corrected with respect to spontaneous mutation ( 3-4 $\times 10^{-4}$ per survivor). (XBL 808-3562A)

with heavy ions give us more insight into the molecular mechanisms of cell transformation. The negative result of obtaining ouabain-resistant mutation with heavy ions and the high yield of cel1 transformation by high-LET radiation suggest that the lesion (possibly point mutation) for ouabain mutation is not the one or the only one involved in the cell transformation.

In the next three years, we $p$ lan to continue our studies on mutation and cell transformation with heavy ions and to determine the cross sec tion of mutation and oncogenic transformation per heavy particle of various LET values and charges: This information will be useful for estimating. the potential hazards of cosmic rays. The effects of fractionated doses of high-LET radiation on mutagenesis and cell transformation and the possible synergistic interaction between high- and low-LET radiation in inducing mutation and oncogenic transformation will be studied. This information will be important for heavy-ion cancer therapy and for assessing radiation risk, since a synergistic effect of cell killing between high- and low-LET radiation has been $\therefore$ well demonstrated (Ngo et al., 1977, 1978).

For our fundamental understanding the mechanism of radiogenic cell transformation and the cocarcinogenic effect of radiation, experiments will be performed to examine the cell cycle dependency of cell transformation and enhancement of viral transformation by radiation. The role of repair mechanisms in oncogenesis, cocarcino- genesis, and mutation will be explored further. We will study the kinetics of repair of transformation lesions, enhancement damages, and mutation injuries induced by heavy-ion radiation. Data from these studies can provide information on the nature of molecular structure alterations in DNA which are related to oncogenes is and mutagenesis.

Because most of human cancers are epithelial in origin, a study on the radiation oncogenesis of epithelial cells is certainly important. We plan to initiate some work on culturing mammalian epithelial cells and conducting some preliminary radiation experiments with these cells.

We canisolate cells transformed by heavy ions by applying the cell transformation technique. The nontransformed and highly oncogenic cells of same cell line will be used as model systems to study the radioresponses of normal and tumor cells. In addition, various chemicals that can modify the expression of tumorigenesis, e.g., retinoic acid, will be tested with the heavy-ion transformed cells. If the transformed cells: are highly sensitive to some modifiers, a prevention of heavy-ion induced oncogenesis through a chemical means may become feasible and useful for cancer therapy and for space travelers.

In sum, we have obtained some interesting results related to cell transformation and mutation, and we believe that a continuation of 
research work with heavy-ion radiation for another three years will result in valuable data that will be important for risk assessment, for radiation cancer therapy, and for a fundamental understanding of carcinogenes is.

\section{ACKNOWLEDGEMENTS}

The authors would like to acknowledge the excellent technical assistance of L. Craise and D. Tse, and thank M. C. Pirruccello for editing. These studies were supported by the NASA Radiation Effects Research Program, NASA (Contract No. T3516G) and the National Cancer Institute (Grant No. CA 15184).

\section{REFERENCES}

Borek, C. 1976. In vitro cell transformation by low doses of $x$-irradiation and neutrons. Biology of Radiation Carcinogenes is (J. M. Yuhas, R. W. Tennant and J. D. Ragen, eds.), pp. 309-326. Raven Press, New York.

Casto, B. C. 1973. Enhancement of adenovirus transformation by treatment of hamster cells with ultraviolet irradiation, DNA base analogs, and dibenz $(a, h)$ anthracene. Cancer Res. 33, $402-407$.

Chieco-Bianehi, L., L. Fiore-Donati, G. DeBenedicts, and G. Tridente. 1963. Influence of urethan on susceptibility to leukemia induction by Graffi virus in adult mice. Nature (London) $199,292-293$.

Christensen, R. C., C. A. Tobias, and W. D. Taylor. 1972. Heavy-ion-induced single- and double-strand breaks in $\phi X-174$ replicative from DNA. Int. J. Radiat. Biol. 22, 457-477.

Coggin, J. H. Jr. 1969. Enhanced virus transformation of hamster embryo cells in vitro. J. Virol. $3,458-462$.

Cox, R., J. Thacker, D. T. Goodhead, and R. J. Munson. 1977. Mutation and inactivation of mammalian cells by various ionising radiation. Nature (London) 267, 425-427.

Duran-Reynals, F. 1954. Studies on the combined effects of fowl pox virus and methylcholanthrene in chicken. Ann. N.Y. Acad. Sci. 54, 977-991.

Freeman, A. E.; P. J. Price, H. J. Igel, J. C. Young, J. M. Maryak, and R. J. Huebner. 1970. Morphological-transformation of rat embryo cells induced by diethylnitrosamine and murine leukemia cells. J. Nat1. Cancer Inst. 44, $65-78$.

Han, A. and M. M. Elkind. 1979. Transformation of mouse C3H10T1/2 cells by single and fractionated doses of $x$-rays and fissionspectrum neutrons. Cancer Res. 39, 123-130.

Landolph, J. R. and C. Heidelberger. 1979. Chemical carcinogens produce mutations to ouabain resistance in transformable $\mathrm{C} 3 \mathrm{H} 10 \mathrm{~T} 1 / 2$ C18 mouse fibroblasts. Proc. Natl. Acad. Sci. USA. 76, 930-934.

Lytle, C. D., K. B.. Hellman, and N. C. Telles. 1970. Enhancement of viral transformation by ultraviolet light. Int. J. Radiat. Biol. 18, 297-300.

Ngo, F.Q.H., A. Han, and M. M. Elkind. 1977. On the repair of sublethal damage in $V 79$ Chinese hamster cells resulting from irradiation with fast neutrons or fast neutrons combined with $X$-rays. Int. J. Radiat. Biol. 32, 507-511.

Ngo, F.Q.H., E. A. Blakely, C. A. Tobias, P. Y. Chang, and K. C. Smith. 1978. Fractionation gain factor from therapeutic heavy-ion beams. Int. J. Radiat. Oncol. Biol. Phys. 4 (Suppl. 2), 92. (Abstract).

Pollock, E. J. and G. J. Todaro. 1968. Radiation enhancement of SV-40 transformation in $3 T 3$ and human cells. Nature (London) 219, 520-521.

Reznikoff, C. A., D. W. Brankov, and C. Heidelberger. 1973a. Establishment and characteristics of a cloned line of $\mathrm{C} 3 \mathrm{H}$ mouse embryo cells sensitive to postconfluence inhibition of division. Cancer Res. 33, 3231-3238.

Reznikoff, C. A., J. S. Betram, D. W. Brankov, and C. Heidelberger. 1973b. Quantitative and qualitative studies of chemical transformation of cloned $\mathrm{C} 3 \mathrm{H}$ mouse embryo cells sensitive to postconfluence inhibition of cell division. Cancer Res. 33, 3239-3249.

Rhim, J. S., B. Creasy, and R. J. Huebner. 1971. Production of altered cell foci by 3 -methylcholanthrene in mouse cells infected with AKR leukemia virus. Proc. Natl. Acad. Sci., U.S.A. $68,2212-2216$.

Ritter, M. A., J. E. Cleaver, and C. A. Tobias. 1977. High-LET radiations induce a large proportion of non-rejoining DNA breaks Nature (London) 266, 653-655.

Roots, R., T.C.H. Yang, L. Craise, E. A. Blakely, and C. A. Tobias. 1979. Impaired repair capacity of DNA breaks induced in mammalian cellular DNA by accelerated heavy ions. Radiat. Res. 78. 38-49.

Stoker, M. 1963. Effect of x-irradiation on susceptibility of cells to transformation by polyoma virus. Nature (London) 200, 756-758.

Thacker, J., M. A. Stephens, and A. Stretch. 1978. Mutation to ouabain-resistance in Chinese hamster cells: Induction by ethyl methanesulphonate and lack of induction by ionizing radiation. Mutat. Res. 51, 255-270.

Yang, T.C.H., E. A. Blakely, A. Chatterjee, G. Welch, and C. A. Tobias. 1977. Response of cultured mammalian cells to accelerated krypton particles. Life Sci. Space Res. 15, 169-174. 
Yang, T.C.H. and C. A. Tobias. 1980. Radiation and cell transformation in vitro. Advances in Biology and Medicine, in press.
Yang, T.C.H., C. A. Tobias, E. A. Blakely, L. M. Craise, I. S. Madfes, C. Perez, and J. Howard. 1980. Enhancement effects of high-energy neon particles on the viral transformation of mouse C 3 H1OT1/2 cells in vitro. Radiat. Res. 81, 208-223. 
158

Blank 


\title{
SPECIFIC GENE MUTATIONS INDUCED BY HEAVY IONS
}

\author{
M. Freeling, " C. W. Karoly, ${ }^{\star}$ and D. S. K. Cheng ${ }^{\star}$
}

Accelerated heavy ions cause chromosome breaks with high efficiency and also induce mutants in specific genes. A general review of heavy-ion radiation genetics can be found in the LBL report covering Bevalac research from 19741977 (Freeling and Yang, 1977). It was concluded that we need to have exact knowledge of the molecular nature of heavy-ion induced mutants in higher plants and animals. There are several practical reasons for studying the nature of DNA lesions induced by heavy ions:

1. In theory, heavy ions should be uniquely capable of inducing mutants that require multiple double-strand break points within a localized piece of DNA. There are several lines of evidence that indicate "regulatory mutations" are induced by break point asSOciated events (Freeling and Woodman, 1979). If so, heavy ions may provide agriculture with a means to increase useful variability which has not been evaluated using sparcely ionizing radiation.

2. Identification of potential sources of environmental mutagens using in situ monitors is just now beginning. The alconol dehydrogenase-1 (Adn1) system expressed in maize pollen has proved one of the more promising in situ monitors (Freeling, 1978, 1980). The heavy ion-induced mutants which we have isolated and studied are now being incorporated into the monitor.

3. Most importantly, heavy ions provide a potential tool with which the geneticist might change the gene in ways not possible using point mutagens or sparcely ionizing radiation. We now know little about the nature of the gene in higher organisms. We should know more when the behaviors of interesting mutants--such as those induced by heavy ions-are reduced to the level of nucleotide sequence.

This report summarizes our heavy-ion research rationale, progress, and plans for the near future. We are engaged in one major and two minor projects. The major project involves selecting a group of maize Adn1 mutants induced by heavy ions and correlating their altered: behavior (e.g., altered organ specificity of expression, stability of expression, etc.) with: a)tered DNA nucleotide sequences and sequence arrangements. This research requires merging the techniques of classical genetics and recombinant DNA technology. Our results to date are encouraging. Our secondary projects involve (1) the use of the Adh gene in the fruit fly, Drosophila melanogaster, as a second system with which to quantify the sort of specific gene mutants induced by heavy ions as compared to $X$ rays, and (2) the development of a maize Adh 1 pollen in situ monitor for environmental mutagens.
HEAVY-ION INDUCED MUTANTS AT Adh1

The Adhl gene in maize presents particular advantages that facilitate comparative mutagenesis studies. The product of the Adhl gene, ADHL, is expressed from the haploid genome of a pollen grain. A typical maize plant sheds several million pollen grains. At specific times during plant development, those cells that give rise to pollen were subjected to 200-500 rad of either $\mathrm{C}^{6+}(500 \mathrm{MeV} / \mathrm{amu})$ or $\mathrm{Ne}^{10+}(400 \mathrm{MeV} / \mathrm{amu})$ at the Bevalac. Some of the cells in the pollen lineage suffered chromosomal damage. If aneuploid gametes result, the pollen aborts because the presence of nearly all chromosomal regions are necessary for pollen tube growth. Thus, severe chromosomal derangements are filtered out. Among the euploid pollen, some will be low or lacking on ADH activity and will, for that reason, be relatively resistant to toxic concentratons of allyl alcohol $(\mathrm{C}=\mathrm{C}-\mathrm{C}-\mathrm{OH}) . \mathrm{ADH}^{+}$ pollen dies in the presence of allyl alcohol because the enzyme catalyzes the formation of highly toxic acrolein ( $\mathrm{C}=\mathrm{C}-\mathrm{CHO})$. Presumptive mutants were recovered as rare kernels in otherwise barren ears. We also used Adh1 alleles which are specifically marked by virtue of unique electrophoretic mobility of their product ADHs, and by other genetic markers. Figure 1 diagrams our selection scheme (reproduced from Freeling and Cheng, 1978).

Our heavy ion-induced mutants fall into three categories: (1) confirmed and characterized new Adhl alleles: Adhl-S1951a ( $\mathrm{Ne}^{10+}$ ) and Adhl-S3020 $\left(\mathrm{C}^{6+}\right),(2)$ complex chromosoma $]$ rearrangements involving the chromosomal arm (1L) on which Adhl maps and generating aneuploids for the Adh1 gene region, and (3) several mutants or aberrants that are still being characterized.

Most interesting are our two heavy-ioninduced Adh1 alleles. S1951a is underexpressed in the scutellum and simultaneously overexpressed in the root. This organ-reciprocal behavior acts in cis to the Adh1 structural gene.

There are several other interesting cases of cis-acting specific gene mutations in higher organisms (reviewed by Paigen, 1979; Dickinson and Carson, 1979), $;$ but the simultaneous regulation of two organs is unique. The second mutant, S3020, was thought to have been a deletion of the Adh1 gene. When pollen from seven S3020/\$3020 homoallelic plants was collected and stained to recognize revertant (blue-opaque) individuals, a phenotypic revertant frequency of about $2 \times 10-6$ was calculated $(15 / 7,352,000)$. This frequency is in line with expectations based on revertant frequencies for 15-ethyl-methanesulfonate induced Adhl structural gene mutants (Freeling, 1978). However, occasional (1/12) \$3020/\$3020 plants 

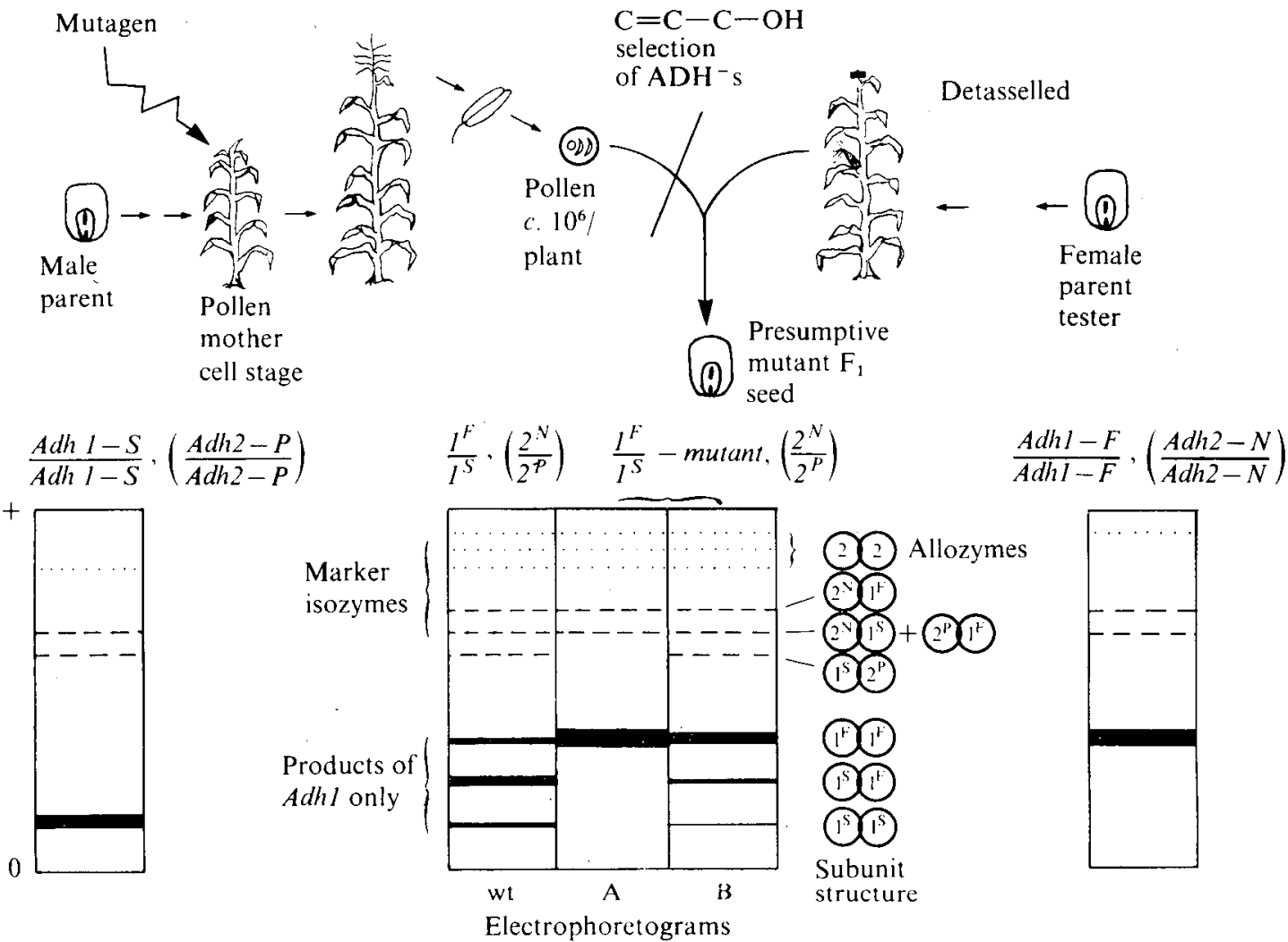

Figure 1. Chemical selection, recovery and classification of Adhl-mutants. The paternal or target Adh1 allele, Adh1-S, is abbreviated 1S, etc. The electrophoretograms depict semiquantitative banding patterns and intensities after native starch gel electrophoresis. O denotes origin; + denotes the anode. The ADH activities reflected in these diagrams are from the wild-type $F_{1}$ (wt), Adh1negative heterozygote $(A)$ and Adn1-underproducer heterozygote $(\bar{B})$ extracts obtained from $F_{1}$ scutellar slivers. The subunit composition(s) of each band is indicated where subunit $1 S$ is specified by Adh1-S, etc. The dashed and dotted bands in the marker isozyme region of gel denote low activity and usually not-present activity, respectively. Reproduced from Freeling and Cheng, 1978, p. 108. (XBL 808-10929)

registered huge $\mathrm{ADH}^{+}$pollen frequencies

$(1 / 30-1 / 500)$, which was totally unexpected of a deletion. Adn1-S3020 is mysterious.

Although we have only two confirmed mutants that affect Adh1 alone, we have several more that are being confirmed, and are trying again with $\mathrm{Ne} 1^{+}$during the summer 1980 . We will need several more mutants before a spectrum of mutant types can be established. However, it is probably significant that our two heavy-ion-induced Adh1 alleles are not within the 21 mutant spectrum established for ethyl methanesulfonate (Freeling, 1978). Preliminary evidence indicates that $\$ 3020$ maps genetically to one end of the Adhl structural gene and prevents recombination in that region. Definitive evidence awaits studies on the DNA sequences that underlie the behavior of our new mutants.
As a second priority in our heavy-ion mutant screening project, chromosomal aberrations which involve $\mathrm{IL}$ are being cataloged. Given the accessibility of the male meiotic stages, pachytene analyses provide the definitive assignment of break points. Figure 2 shows a pollen sample from an $F_{1} p l a n t$ that is independently segregating 1:1 for. ADH-dysfunction and aborted pollen. This situation can be explained in at least two ways. First, that a new Adh1- mutant has been selected with an unlinked reciprocal translocation heterozygote in. its genetic background. Second, the Adhl gene itself could have been bisected and inactivated by a reciprocal translocation break point. In actuality, the first alternative was the case, but we are particularly interested in using heavy ions to disconnect components of Adhl. 


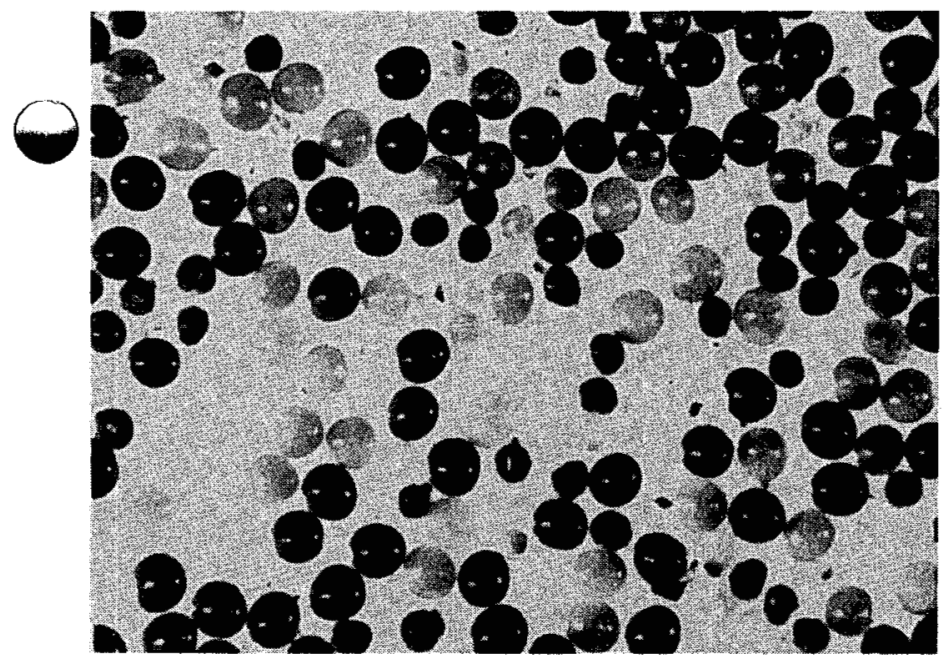

Figure 2. Maize pollen stained specifically for the presence (blue and opaque) or absence (yellow and transluscent of AOHI activity). This population demonstrates the simultaneous 1:1 segregation of $\mathrm{ADH}^{+}$: $\mathrm{ADH}-$ and plump : small

(aborted). In this case, the explanation is an Adh $1^{+} /$Adh 1- heterozygote in a reciprocal

$\overline{\operatorname{trans}}$ iocation heterozygote background. (XBB 808-9054)
MOLECULAR BIOLOGY

OF HEAVY ION INDUCED Adh1 MUTANTS

There is only one way to visualize directly the genetic end point of mutagenesis, and that is to recover and study the mutant lesion as a closed, recombinant DNA molecule. The first step to obtaining primary sequence information on Adh1-A1951a and Adh1-\$3020 is to obtain a recombinant plasmid carrying a sequence complementary to some coding sequence of Adh1; this molecule is called an Adhl "probe." In all likelihood, we now have this probe.

In collaboration with Dr. W. C. Taylor (Department of Genetics, University of California, Berkeley), we have constructed a cDNA library from anaerobic maize root mRNAs. From previous work on radiolabelled anaerobic roots and translation of anaerobic root messages in a rabbit reticulocyte cell-free system (Sachs et al., 1980; Ferl et al., 1980), it was clear that an mRNA population could be prepared where $12 \%$ was expected to be mRNA-Adh1.

Figure 3 is a fluorograph from a twodimensional polyacrylamide separation of

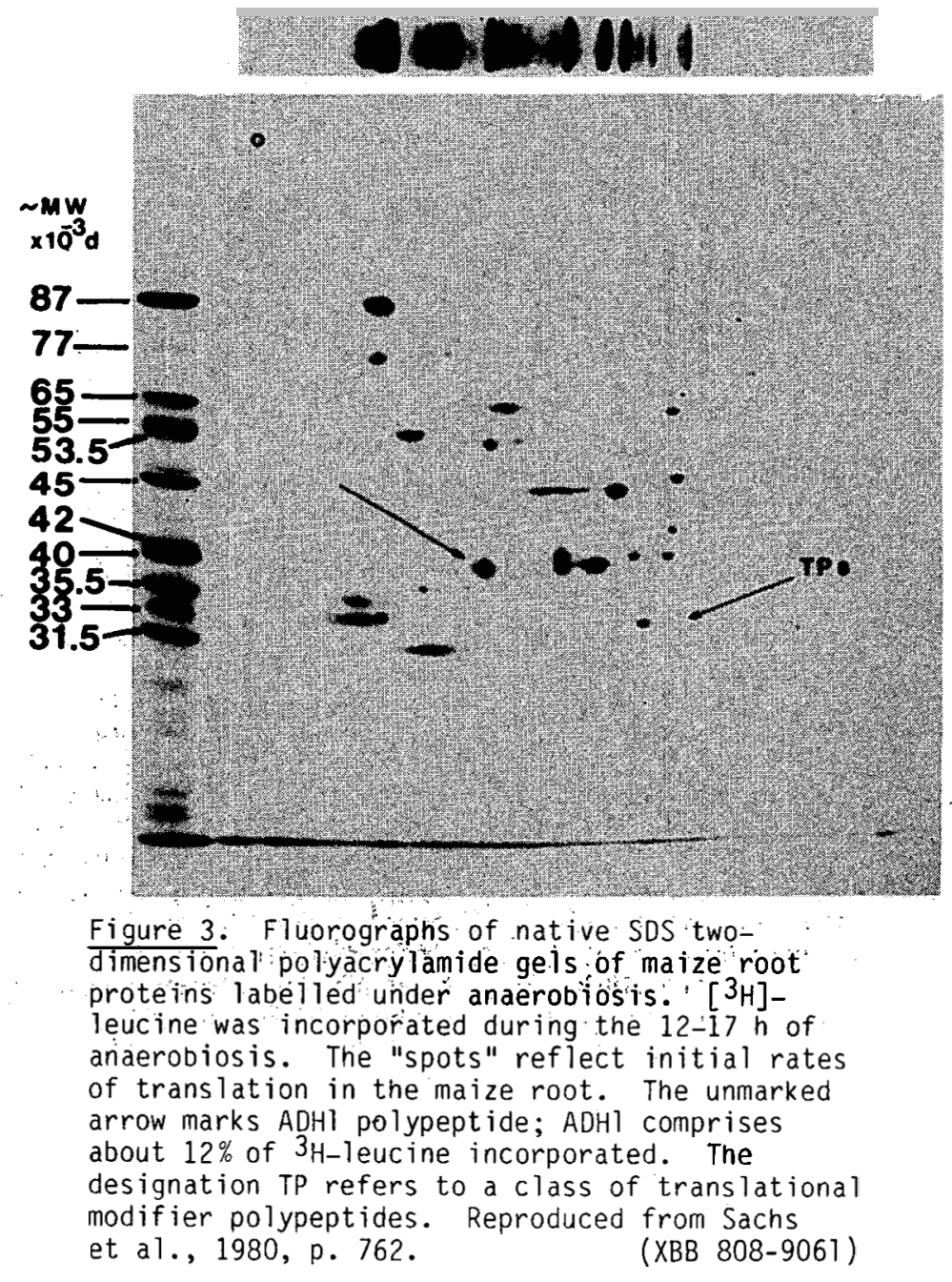


anaerobic peptides labelled with [ $\left.{ }^{3} \mathrm{H}\right]$-leucine for $5 \mathrm{~h}$; Adh1 "spots" are labelled. Polysomal RNA was isolated from maize roots $60 \mathrm{~h}$ after subjecting them to anaerobiosis. Polyadenylated mRNA was purified from the >100s polysomal fractions by oligo (dT) cellulose chromatography. Double-strand cDNA was synthesized from the poly A-RNA templates. The cDNA was tailed with oligo (dC) and inserted into the PstI site of E. coli plasmid pBR322 which had been tailed with oligo (dG). E. coli strain SK2267 was transformed with the recombinant plasmid. Presumptive inserts were identified by pattern of antibiotic resistances inherent to the pBR322 vehicle and confirmed by the filter hybridization technique of Grunstein and Hogness (1975) using polynucleotide kinase-labelled anaerobic mRNA as the probe. The exact methodological details and citations will be reported elsewhere. Our result is an anaerobic cDNA library of over 100 recombinant molecules. We expect that 12 of them will hybridize with Adh1 coding sequence.

We are now using a number of methods to isolate the Adnl probe from the library. In the end, we will use the probe to recover pure InNA-ADH identified by its in vitro translation product.

Given a probe for the Adh1 coding sequence, we can immediately begin a comparison of Adn1S1951a and Adh1-S3020 with their progenitor aTlele: Adh1-S. These comparisons would begin by a casual comparison of the number and sizes of Adhl fragments resulting from genome digestion with various restriction endonucleases; the relevant technique here is Southern hybridization. Eventually, we hope to sequence the mutational lesions cloned in a lambda phage vehicle.

In summary, we almost certainly have an Adh1 probe. In theory, any DNA-level alteration at Adnl can now be known at the level of nucleotide sequence.

\section{DROSOPHILA Adh AS A COMPLEMENTARY SYSTEM}

One of us (C.W.K.) has embarked on a doctoral project seeking to compare the effect of accelerated heavy-ion radiation to that of $x$ rays at a specific gene. We chose Adh in the fruit fly, Drosophila melanogaster, as our target gene for mutagenesis because much is already known about radiation genetics in this organism (review by Sankaranarayanan and Sobels, 1976), and Adh is among its best understood genes (reviewed in Dickinson and Sullivan, 1975).

We have devised a mutagenesis scheme where mature sperm are irradiated with either plateau LET heavy ions or $X$ rays. The irradiated males carry an electrophoretically distinct Adh allele, Adh $\mathrm{F}$, and the close-in markers purple $(\overline{p r})$ and cinnabar (cn). About 200 mutagenized males are mated with a tenfold excess of tester females. These females are heterozygotic at the Adh region of chromosome 2. One chromosome is dominantly marked, with curly (Cy) and has been made null for $A D H$ via a point lesion in the Adh gene (AdhnB); the other chromosome carries a deletion which covers Adh; as well as several flanking genes (Fig. 4A). Therefore, these females are devoid of $\mathrm{ADH}$.

The $\mathrm{F}_{1} \mathrm{~s}$ are $\mathrm{ADH}^{+}$unless an Adh- mutant occurred after mutagenesis of the male parent. ADH- eggs and larvae grow on 1-pentyne-3-ol where ADH's die (Sofer and Hogness, 1972). Therefore, only ADH-low or -negative larvae wil survive (Fig. 4B). If the reason for being Adhis due to a deletion of the Adh region, then an abundance of flies with exposed markers adjacent to the Adh should emerge; a large deletion of this type would probably be viable only in the cy heterozygote. An equal frequancy of $c y$ and unmarked flies emerging with no exposed markers would be the expected result of a mutagen that makes base-substitutions or very small deletions only. All mutants are checked for ADH activity at the level of agarose gel electrophores is and staining. We have tried this system and it seems to work, but we have not yet attempted to generate data comparing the rate or type of mutations induced by heavy ions or $X$ rays. We propose to do this in future studies.

There is a wealth of data to be found in the literature demonstrating that cells of the pollen lineage in maize modify a double-strand break such that no intragenic mutations (point mutations) have been recovered (see Freeling and Cheng, 1978, for review and discussion). L. J. Stadler made this point some time ago (Stadler and Roman, 1948). It seems reasonable to assume that nucleases enlarge the primary lesion such that it almost always causes pollen abortion. On the contrary, irradiation of Drosophila sperm leads to point mutations, as well as chromosomal aberrations. There is excellent evidence that double-strand break points in sperm DNA remain more-or-less intact until after fertilization of the egg (Sankaranarayanan and Sobels, 1976). Therefore, the data obtained in Drosophila should more accurately reflect differences in primary lesions.

\section{POLLEN MUTAGEN MONITORS}

The use of in situ staining of Adh1- mutant maize pollen grains (as in Fig. 2) portends utility as an environmental monitor (Freeling, 1978, 1980). As with the Ames test in Salmonella, the power of the reversion monitor is no greater than the sensitivity of the AdhI- alleles to be reverted. There are over $\overline{100}$ ethyl methanesulfonate-induced point mutants at Adh1. : Adh1S3020, induced by $\mathrm{C}^{6+}$, appears to be a chromosomal aberration that switches Adh1 off. without deleting it (e.g., an insertion or an inversion).

One would not expect point mutagens to revert this mutant allele. Perhaps accelerated heavy ions will. We hope to identify a series of Adn 1alleles, whose reversion to a more normal state can be used to quantify particular lesions in DNA. S3020, among other mutants, may allow us to quantify multiple double-strand breakage agents. Without taking the nature of the test:alleles seriously, certain mutagenic agents could easily go undetected. 
A.

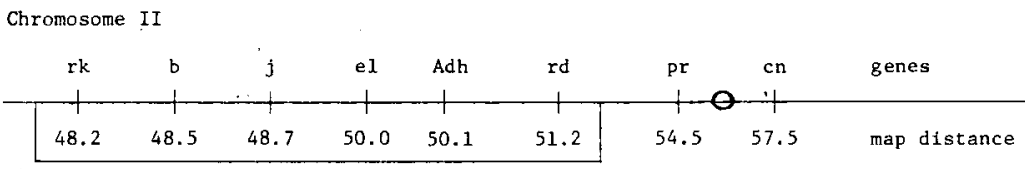

deletion

B.

우

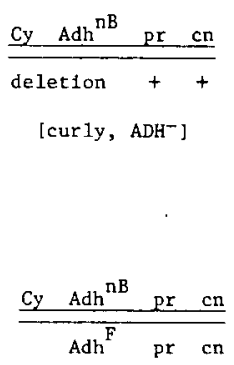

$\left[\mathrm{ADH}^{-}\right.$, curly, purple, cinnabar]

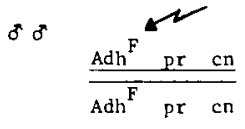

$\left[A D H^{F}\right.$, purple, cinnabar]

1-pentyne-3-o1

$\left[\mathrm{ADH}^{-}\right.$, perhaps markers are exposed]

Figure 4. A scheme for comparative mutagenes is of the Adh region of chromosome II in Drosophila melanogaster. (A) The chromosomal region with gene designations, map position (\% recombination units) and the extent of the deletion on the Scorev 7 chromosomes are indicated. (B) The cross and chemical selection scheme; the cy chromosome is actually CyOnB. Parentheses enclose phenotypes.

(XBL 808-10928)

\section{CONCLUSIONS AND FUTURE DIRECTIONS}

This project seeks exact knowledge on the nature of specific gene mutations induced by heavy ions. Our $\mathrm{C}^{6+}$ - and $\mathrm{Ne}^{10^{+}}$- induced mutants at the Adhl gene in maize are regulatory in nature. They probably do not involve changes in coding sequences. Using CDNA, prepared to isolated mRNA containing mRNA-ADH1, we now have. a library of recombinant plasmids including, in theory, a probe for Adh1. We hope to study the mutants we now have and others now being tested, or to be induced, to obtain a spectrum of heavy-
ion mutations. Data will be at both genetic behavior and nucleotide sequence levels.

A particularly powerful comparative mutagenesis system at Adh of Drosopnila melanogaster is being developed and will be used to compare heavy ions and $X$ rays.

We have begun to apply our basic knowledge $f$ the nature of the gene and heavy-ion mutagenesis to practical problems in agriculture and monitoring the environment for mutagens.

\section{ACKNOWLEDGEMENTS}

We thank the Agricultural Operations crew, California Agricultural Experiment Station, and the crew at the Bevalac. The basic genetics and molecular biology of maize Adh1 are funded by National Institutes of Health Grant GM21734 to M. F.; C.W.K. is a recipient of a Public Health Service predoctoral grant.

\section{FOOTNOTES AND REFERENCES}

* Department of Genetics, University of California,: Berkeley, CA.

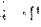

Dickinson; W. J. and H. L. Carson. 1979. Regulation of the tissue specificity of an enzyme by a cis-acting genetic element. Proc. Nat 1. Acad. Sci. U.S.A. 76, 4559-4561.

Dickinson, W. J. and D. T. Sullivan. 1975. Gene-Enzyme Systems in Drosphilia. SpringerVerlag, New York. 
Fer1, R. J., M. D. Brennan, and D. Schwartz. 1980. In vitro translation of maize ADH:

Evidence for the anaerobic induction of MRNA. Biochem. Genet., in press.

Freeling, M. 1978. Allelic variaton at the level of intragenic recombination. Genetics $89,211-224$.

Freeling, M. 1979. Maize Adh1 as a monitor of environmental mutagens. Environ. Health Perspect. 27, 91-98.

Freeling, M. 1980. Toward monitoring specific ONA lesions in the gene using pollen systems. Environ. Health Perspect. In press.

Freeling, M. and D.S.-K. Cheng. 1978. Radiation-induced alcohol dehydrogenase mutants in maize following allyl alcohol selection of pollen. Genet. Res. 31, 107-129.

Freeling, M. and J. C. Woodman. 1979. Regulatory variant and mutant alleles in nigher organisms and their possible origin via chromosomal breaks. The Plant Seed: Development, Preservation and Germination (I. Rubenstein et al., eds.). Academic Press, New York.
Freeling, M. and T.C.H. Yang. 1977. Heavy-ion radiation genetics. Biological and Medical Research with Accelerated Heavy Ions at the Bevalac, 1974-1977, pp. 154-163. Lawrence Berkeley Laboratory Report LBL -5610 .

Grunstein, M. and D. S. Hogness. 1975. Colony hybridization: A method for the isolation of cloned DNAs that contain a specific gene. Proc. Nat 1. Acad. Sci. U.S.A. 72, 3961-3965.

Paigen, K. 1979. Genetic factors in developmental regulation. Physiological Genetics. Academic Press, New York.

Sachs, M. M., M. Freeling, and R. Ok imoto. 1980. The anaerobic proteins of maize. Cell $20,761-767$.

Sankaranarayanan, K. and F. Sobels. 1976. Radiation genetics. The Genetics and Biology of Drosophila, vol. 1C (M. Ashburner and E. Novitski, eds.). Academic Press, New York.

Sofer, W. H. and M. A. Hatk off. 1972. Chemical selection of alcohol dehydrogenase negative mutants in Drosophila. Genetics 72, 545-549.

Stadler, L. J. and H. Roman. 1948. The effects of $X$ rays upon mutation of the gene $A$ in maize. Genetics 33, 273-303. 
PART IV. HEAVY-ION RADIOBIOLOGY OF TISSUES AND TUMORS 


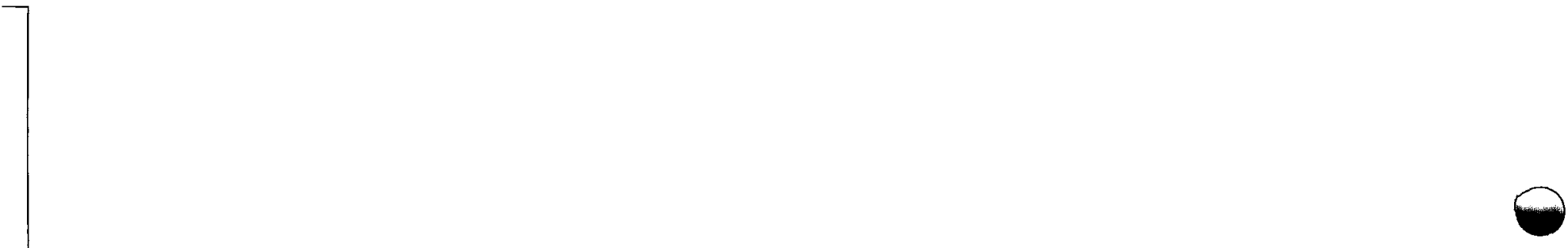

0

- 


\title{
IN VIVO TUMOR RADIOBIOLOGY OF HEAVY CHARGED PARTICLES
}

\author{
S.B. Curtis and T.S. Tenforde
}

The response of tumor cells systems to irradiation with carbon, neon and argon beams at various positions in the plateau and extendedpeak regions of the Bragg ionization curve is being evaluated from experiments conducted both in vivo and in vitro. The radiobiological end points being studied include: tumor volume response, cellular survival after tumor irradiation in situ, cell-kinetic parameters measured by the flow cytofluorometric and time-lapse cinematographic techniques, and survival of oxic and hypoxic cells irradiated in suspension. The results of the first three types of measurements will be presented in this chapter, and the in vitro $O E R$ and $R B E$ determinations for tumor cells irradiated as a suspension will be presented in one chapter by Blakely et al. in Part III.

\section{TUMOR SYSTEMS}

The tumor line used for the majority of our in vivo and in vitro experiments is the R1 rhabdomyosarcoma which was originally developed at the Radiobiological Institute (REPGO/TNO) in the Netherlands by Reinhold (1965, 1966) and by Barendsen and his colleagues (Hermens and Barendsen, 1967, 1969, 1975; Barendsen and Broerse, 1969, 1970). Two sublines of the RI tumor have been used in these experiments: (1) the R1/LBL subline that was used in many of our initial studies of radiation-induced tumor growth delay and cure (Curtis et al., 1978), but which lacks the in vivo to in vitro plating characteristic; (2) the R2D2 subline, which has an acceptable $18 \%$ in vivo to in vitro plating efficiency and has therefore been used for studies of cell survival following tumor irradiation in situ. The R2D2 subline has also been used for limited measurements of radiationinduced growth delay, and has been the tumor subline used for in vitro cell survival studies.

Two other tumor lines have been used in collaborative research efforts involving the tumor radiobiology group at $\mathrm{LBL}$ and the group headed by $E$. Malaise at the Institut GustaveRoussy in Villejuif, France: (1) A humán melanoma cell line has been used for studies of in vivo cell survival, potentially lethal damage repair, and hypoxic cell sensitizer effects in combination with heavy-ion and 60 co gamma irradiation. This tumor line has an $85 \%$ nypoxic fraction of cells when growing in immunodeficient nude mice (Guichard et al., 1977), and has been adapted for cell survival assay using the in vivo to in vitro technique. (2) The EMT6 tumor cell line, which was originally derived from the KHJJ tumor (Rockwell et al., 1972), has been used for in vitro cell-kinetic studies following photon or charged-particle radiation.
In studies performed by J. T. Leith, K. T. Wheeler, and their colleagues, cell survival following in situ tumor irradiation with chargedparticle beams has been measured for rat brain gliosarcoma $9 \mathrm{~L}$ tumors. The radiobiological properties of the $9 \mathrm{~L}$ tumor line, which was originally induced in Fischer rats by intravenous injection of $\mathrm{N}-n$ itrosomethylurea, have previously been described by Leith et a1. (1975a).

\section{RADIATION-INDUCED GROWTH DELAY MEASUREMENTS: RBE VALUES FOR SINGLE AND FRACTIONATED DOSE SCHEDULES AND HYPOXIC CELL SENSITIZER EFFECTS}

\section{Single Radiation Doses}

Measurements were made of the volume response of R1/LBL tumors to graded single doses of orthovoltage $X$ rays, and to beams of accelerated carbon, neon, and argon ions in the plateau region and in the distal position of a 4-cm extended-peak region. Values of radiation-induced growth delay were calculated as the difference in time for the irradiated and nonirradiated tumors to reach twice the volume measured on the day of irradiation. For each radiation modality, the growth delay as a function of absorbed dose is presented in figure 1. The RBE20 and RBE50 values, calculated as the ratio of $X$-ray to charged-particle doses required to produce growth delays of 20 and 50 days, respectively, are given in Table 1. The following conclusions can be drawn from these data: (1) The RBE values for peak neon and argon ions are slightly higher than for peak carbon ions. However, the standard deviations of the RBE values are sufficiently large (see Fig. 1) that the differences observed for the three charged-particle beams in this series of experiments cannot be regarded as statistically significant. (2) The growth delays induced by plateau radiation are significantly different for the three charged-particle beams, as reflected by $R E_{50}$ values of $1.3,1.8$, and 2.9 for the carbon, neon and argon beams, respectively. As a consequence, the peak-toplateau RBE50 ratios for the carbon and neon ions are 1.77 and $1: 44$, respectively, while that for argon ions is 0.86 . The ratio for both the carbon and neon beams is thus significantly greater than unity, which constitutes a favorable condition for tumor therapy in the extended-peak ionization region. In contrast, the peak-toplateau RBE50 ratio for argon ions is less than unity, under which condition the level of tissue damage in the plateau ionization region could exceed that from the peak radiation used for tumor therapy. 


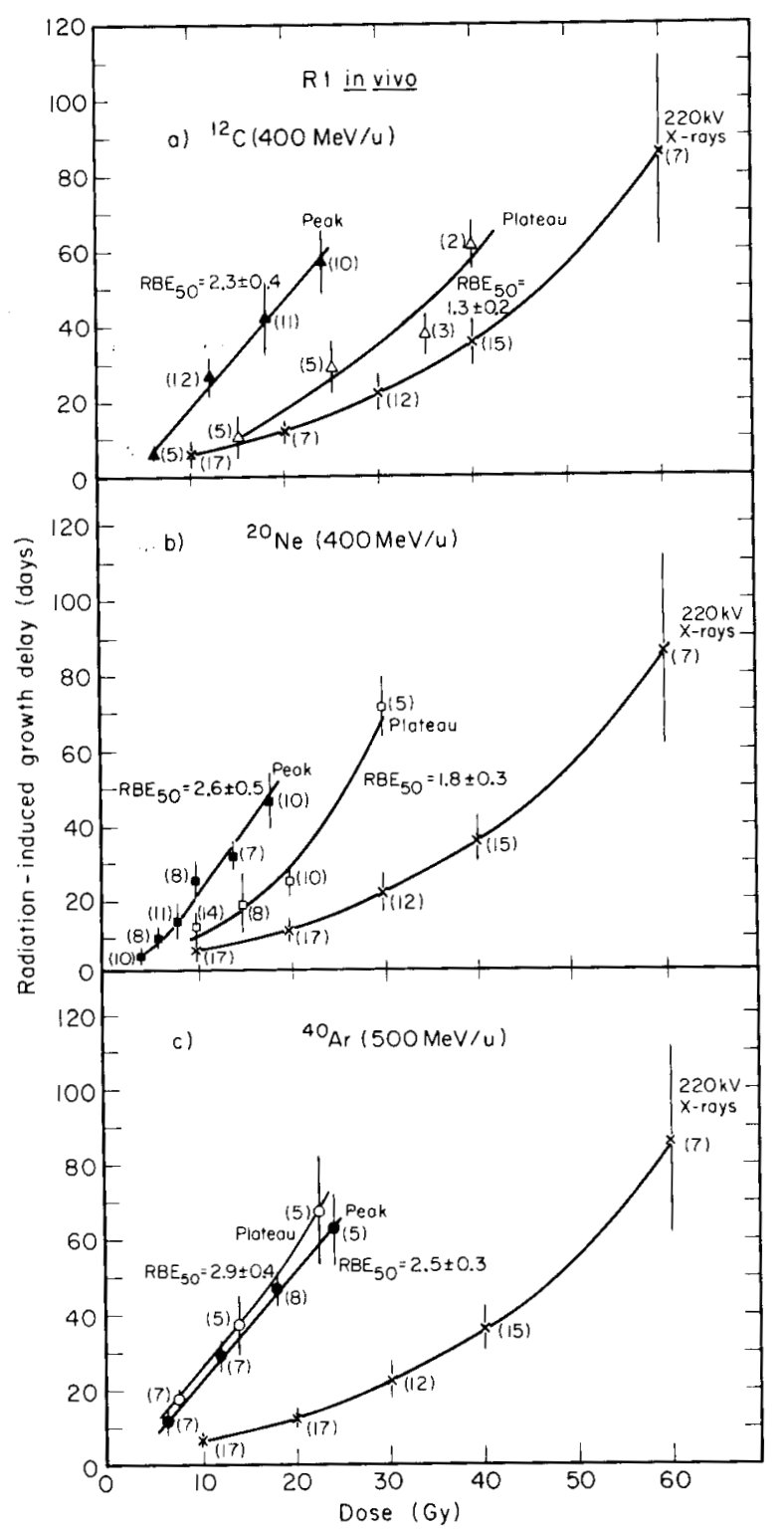

Figure 1. The radiation-induced growth delay to reach twice the volume measured at the time of irradiation is plotted for R1/LBL tumors exposed to $X$ rays and to plateau and peak radiation from beams of carbon, neon and argon ions. Tumors were positioned in the distal $1.5 \mathrm{~cm}$ of a $4-\mathrm{cm}$ extended-peak ionization region for each of the charged-particle radiation modalities. The range in water of the carbon, neon, and argon beams were 24,14 , and $12 \mathrm{~cm}$, respectively. RBE values calculated at the 50-day growth delay level are given for each of the charged-particle beams in both the plateau and peak ionization regions. The number in parentheses next to each data point on the growth delay curves indicates the number of tumors used, and the error bar represents one standard deviation. (XBL 785-3203) 
Table 1. RBE Values for Radiation-Induced Growth Delay of R1 Tumors In Vivo Following Single and Fractionated Dose Schedules

\begin{tabular}{|c|c|c|c|c|c|}
\hline $\begin{array}{l}\text { Fractionation } \\
\text { Schedule }\end{array}$ & Ion & $\begin{array}{l}\text { Initial Energy } \\
(\mathrm{MeV} / \mathrm{u})\end{array}$ & $\begin{array}{l}\text { Tumor } \\
\text { Position }\end{array}$ & $\mathrm{RBE}_{20}{ }^{\dagger}$ & $\mathrm{RBE}_{50}{ }^{\dagger}$ \\
\hline \multirow[t]{6}{*}{$1 \mathrm{Fx}$} & ${ }^{12} \mathrm{C}$ & 400 & Plateau & 1.3 & 1.3 \\
\hline & ${ }^{12} \mathrm{C}$ & 400 & Peak & 2.8 & 2.3 \\
\hline & ${ }^{20} \mathrm{Ne}$ & 400 & Plateau & 1.7 & 1.8 \\
\hline & ${ }^{20} \mathrm{Ne}$ & 400 & Peak & 2.9 & 2.6 \\
\hline & ${ }^{40} \mathrm{Ar}$ & 570 & Plateau & 3.3 & 2.9 \\
\hline & ${ }^{40} \mathrm{Ar}$ & 570 & Peak & 3.0 & 2.5 \\
\hline \multirow[t]{2}{*}{$4 \mathrm{Fx} / 3 \mathrm{~d}$} & ${ }^{12} \mathrm{C}$ & 400 & Peak & 2.3 & $2.0^{\neq}$ \\
\hline & ${ }^{20} \mathrm{Ne}$ & 400 & Peak & 2.8 & 2.6 \\
\hline \multirow[t]{2}{*}{$5 \mathrm{Fx} / 4 \mathrm{~d}$} & ${ }^{12} \mathrm{C}$ & 400 & Peak & 2.6 & 2.8 \\
\hline & ${ }^{20} \mathrm{Ne}$ & 400 & Peak & 3.2 & 3.0 \\
\hline $6 \mathrm{Fx} / 5 \mathrm{~d}$ & ${ }^{12} \mathrm{C}$ & 400 & Peak & 2.7 & 2.3 \\
\hline \multirow[t]{2}{*}{$8 \mathrm{Fx} / 10 \mathrm{~d}$} & ${ }^{12} \mathrm{C}$ & 400 & Peak & 2.4 & 2.3 \\
\hline & ${ }^{20} \mathrm{Ne}$ & 400 & Peak & 2.7 & 2.9 \\
\hline \multirow[t]{2}{*}{$10 \mathrm{Fx} / 11 \mathrm{~d}$} & ${ }^{12} \mathrm{C}$ & 400 & Peak & 2.4 & $2.5^{\neq}$ \\
\hline & ${ }^{20} \mathrm{Ne}$ & 400 & Peak & 3.6 & 3.3 \\
\hline
\end{tabular}

* The peak ionization region was extended to a width of $4 \mathrm{~cm}$ using a variable-thickness absorber (ridge filter), and tumors were positioned in the distal $1.5 \mathrm{~cm}$ region.

+ The $R B E_{20}$ and $R B E_{50}$ values were calculated from the ratio of the $X-r a y$ dose to the charged-particle dose required to produce growth delays of 20 days and 50 days, respectively.

$\neq$

Extrapolated value.

\section{Fractionated Radiation Doses}

Growth delay RBE values have also been measured for peak carbon-ion and neon-ion w.......... radiation administered as 4,5 , and 6 daity $\ldots$ doses, as 8 doses in a 10-day interval, and as : 10 doses in an 11-day interval." The RBE20 and "t RBE 50 values for these fractionation schedules are summarized in Table 1 , and the growth delay. curves for the 5-fraction and 10-fraction schedules are shown in Figure 2. In al1 of the fractionation regimens, recovery was observed with $X$ rays. For the peak carbon ions, substantial recovery was observed only with the 10-fraction schedule. With peak neon ions, there was no evidence of recovery in a fractionated dose schedule.
Hypoxic Cell Sensitizers Combined with Single and Fractionated Doses

Radiation-induced growth delay measurements have been made for RI/LBL tumors exposed to large single doses of $x$ rays in combination with the nypoxic cell sensitizers Ro 07-0582 (misonidazole) and Ro $05-9963$ (the 0-demethylated derivative of misonidazole). The effect of misonidazole on the growth delay induced by single doses of peak carbon and neon jons has also been measured. In addition, the effect of misonidazole combined with $X$ rays, peak carbon ions, or peak neon ions has been determined when the sensitizer and radiation were administered in four daily fractions. In all cases, the sensitizers were administered 30-60 min prior to irradiation in 


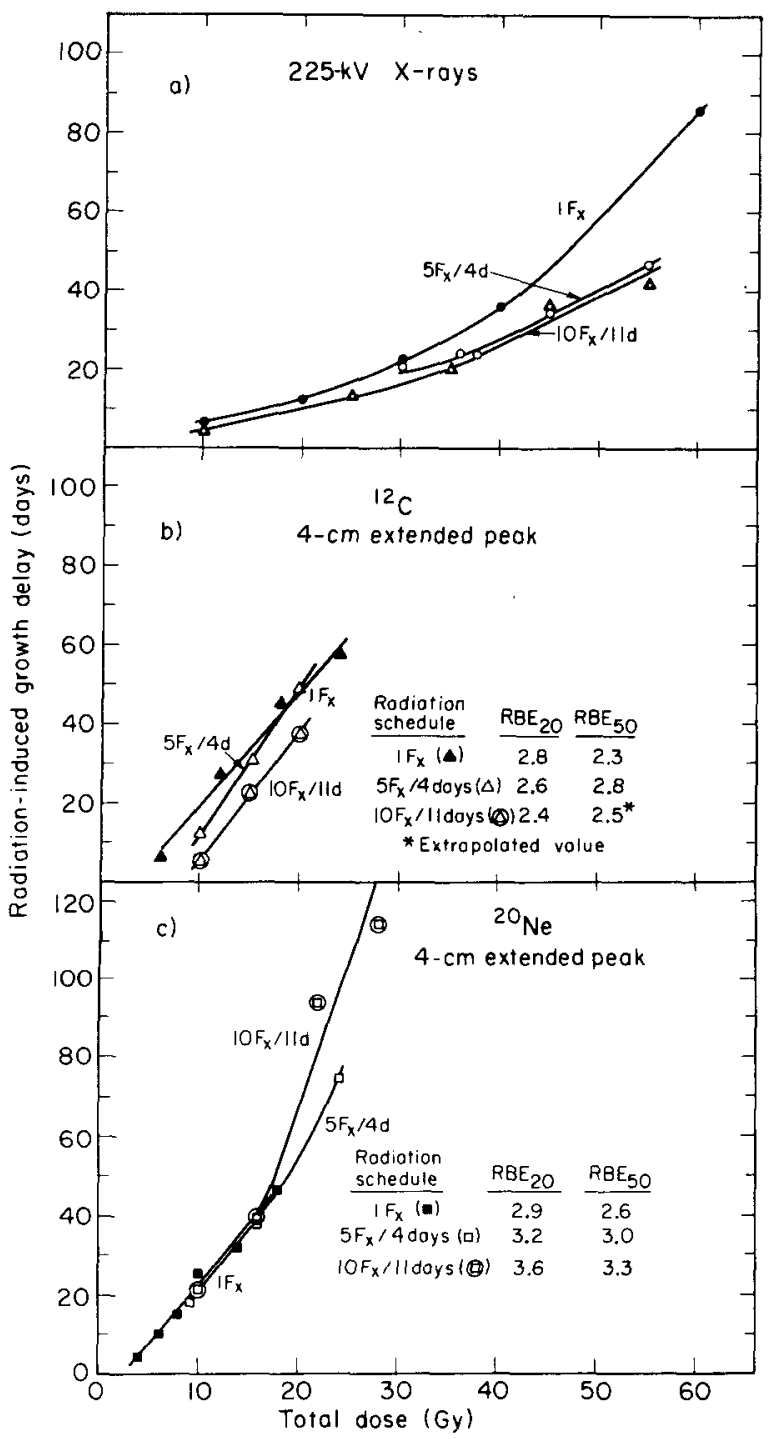

Figure 2. Radiation-induced growth delay curves for R1/LBL tumors are plotted as a function of total dose for single and fractionated radiation schedules with $X$ rays, peak carbon ions, and peak neon ions. For the carbon- and neon-ion exposures, the tumors were positioned in the distal portion of a $4-\mathrm{cm}$ extended peak. RBE values calculated at the 20-day and 50-day growth delay levels are given for peak carbon ions and peak neon ions administered as single doses, as 5 daily fractions, and as 10 fractions in 11 days (i.e., 5 daily fractions for two consecutive weeks). Significant recovery during fractionated charged-particle radiation was observed only for the 10 fraction carbon-ion irradiation. (XBL 805-3287)

order to achieve a maximum drug level in the tumor at the time of radiation. plots of the radiation-induced growth delay as a function of dose are shown for each of these experimental conditions in Figures 3 and 4 , and the RBE values and drug enhancement ratios at the 20-day and 50-day growth delay levels are given in Table 2 .
The results of these studies demonstrate that misonidazole has a significant effect on the response of $R 1 / L B L$ tumors to single doses of $X$ rays, giving enhancement ratios of 1.8 to 2.1 when the drug was administered i.p. at a $500 \mathrm{mg} / \mathrm{kg}$ dose level. For the $4-\mathrm{cm}$ extended-peak regions of carbon and neon beams, misonidazole was found to exert a small potentiating effect on tumor cell killing. The drug enhancement ratios were 1.1 to 1.3 for peak carbon ions and 1.2 for peak neon ions.

When misonidazole and $x$-irradiation were administered in four daily fractions, the drug enhancement ratio decreased significantly. This effect would be expected since reoxygenation of hypoxic R1 tumor cells occur within the fractionation interval, i.e., $24 \mathrm{~h}$, and this process reduces the net gain obtained by misonidazole sensitization of hypoxic cells. With carbon and neon beams, dose fractionation had no significant effect on misonidazole enhancement of radiation damage. This finding is not unexpected in view of the reduced dependence on oxygen of cell killing by charged-particle radiation with a high LET. The reduced oxygen effect would clearly diminish the importance of reoxygenation on tumor radiation response in a fractionated dose schedule, and this could account for the similar effects of the hypoxic cell sensitizer misonidazole when used in combination with single and fractionated doses of high-LET radiation.

Preliminary studies have also been undertaken to examine the effect of the 0-desmethyl metabolite of misonidazole, Ro 05-9963 (Flockhart et al., 1979), on the growth delay induced in R1/LBL tumors by both low- and nigh-LET radiation. Initial studies with $X$ rays have yielded singledose enhancement ratios of 2.1 to 2.4 for this compound when injected i.p. at a dose level of $1,500 \mathrm{mg} / \mathrm{kg}$. These values are slightly greater than the enhancement ratios obtained for misonidazole (1.8 to 2.1 ).

\section{POSTIRRADIATION CELL SURVIVAL AND TUMOR REGROWTH CHARACTERISTICS}

Cellular survival curves for R1 tumors (the R2D2 subline) exposed in vivo to $225-k V \times$ rays and to $4-\mathrm{cm}$ extended-peak carbon and neon ions were measured by an in vitro colony-forming assay (Tenforde et al., 1980). These survival curves are shown in Figure 5 for tumors irradiated in situ in both air-breathing and nitrogen-gasasphyxiated rats. In the $X$-ray survival curve, a hypoxic break occurs at a dose of approximately $8 \mathrm{~Gy}$, and the ratio of survival fractions in the air and hypoxic curves at high radiation doses yields a value of $35 \%$ for the fraction of chronically hypoxic cells. Under air-breathing conditions, the RBE values at the $10 \%$ survival level for peak carbon and neon ions were 1.9 and 3.1 , respectively. Parallel studies of radiationinduced growth delay with the same R1 tumor subl ine yielded RBE50 values of 2.3 and 2.9 for the peak carbon and neon ions, respectively. This finding indicates that comparable RBE values are obtained for the growth delay and cell 


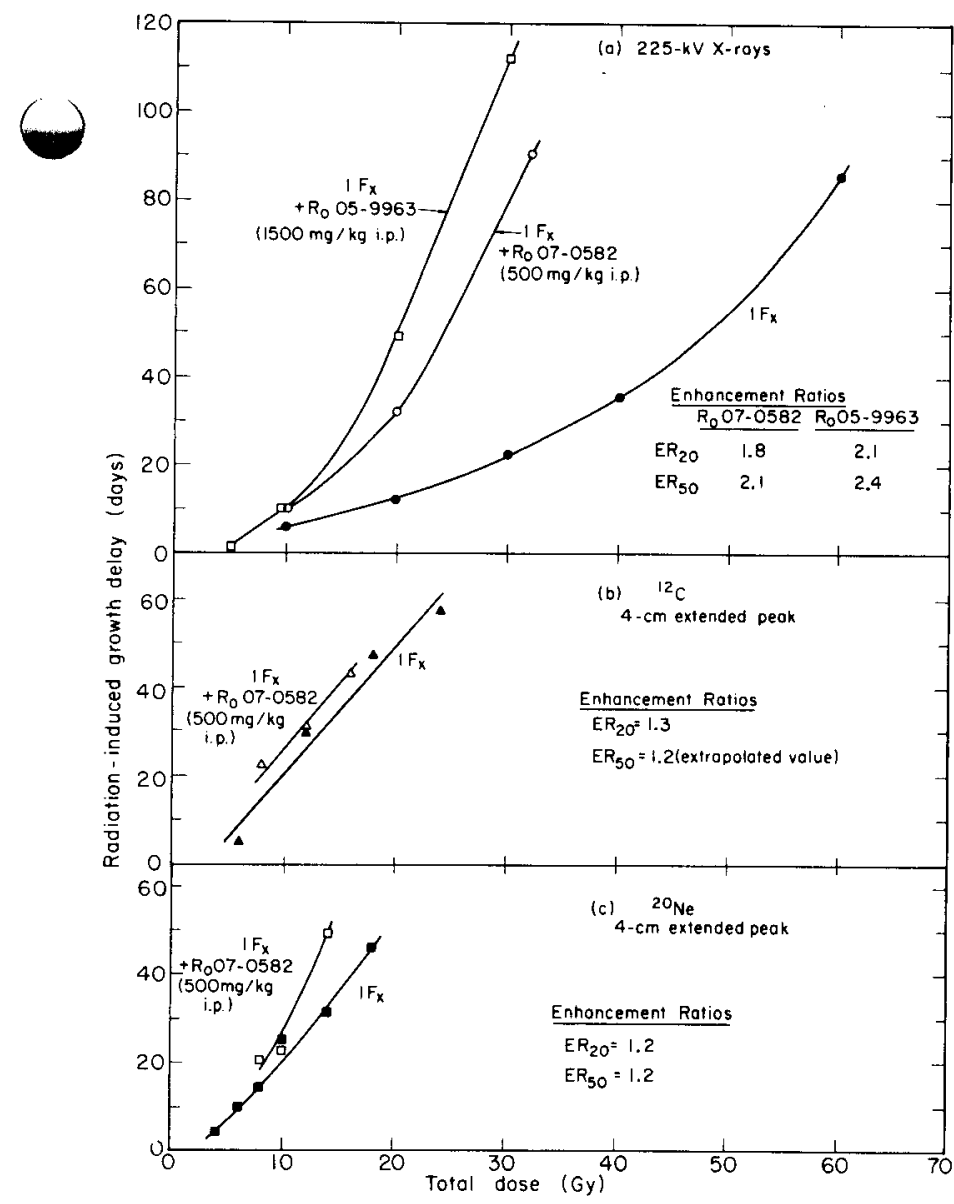

Figure 3. Radiation-induced growth delay curves are shown for $\mathrm{R} 1 / \mathrm{LBL}$ tumors receiving combined treatment with single doses of $X$ rays and the hypoxic cell sensitizers Ro 07-0582 (misonidazole) and Ro 05-9963 (panel a), peak carbon ions and Ro 07-0582 (panel b), and peak neon ions and Ro 07-0582 (panel c). In order to achieve a peak drug concentration in the tumor at the time of irradiation, the sensitizers were administered i.p. 30-60 min prior to radiation. The effect of these compounds on tumor radiation response is indicated by the enhancement ratios calculated at the 20-day (ER20) and 50-day (ER50) growth-delay levels. (XBL 805-3285)

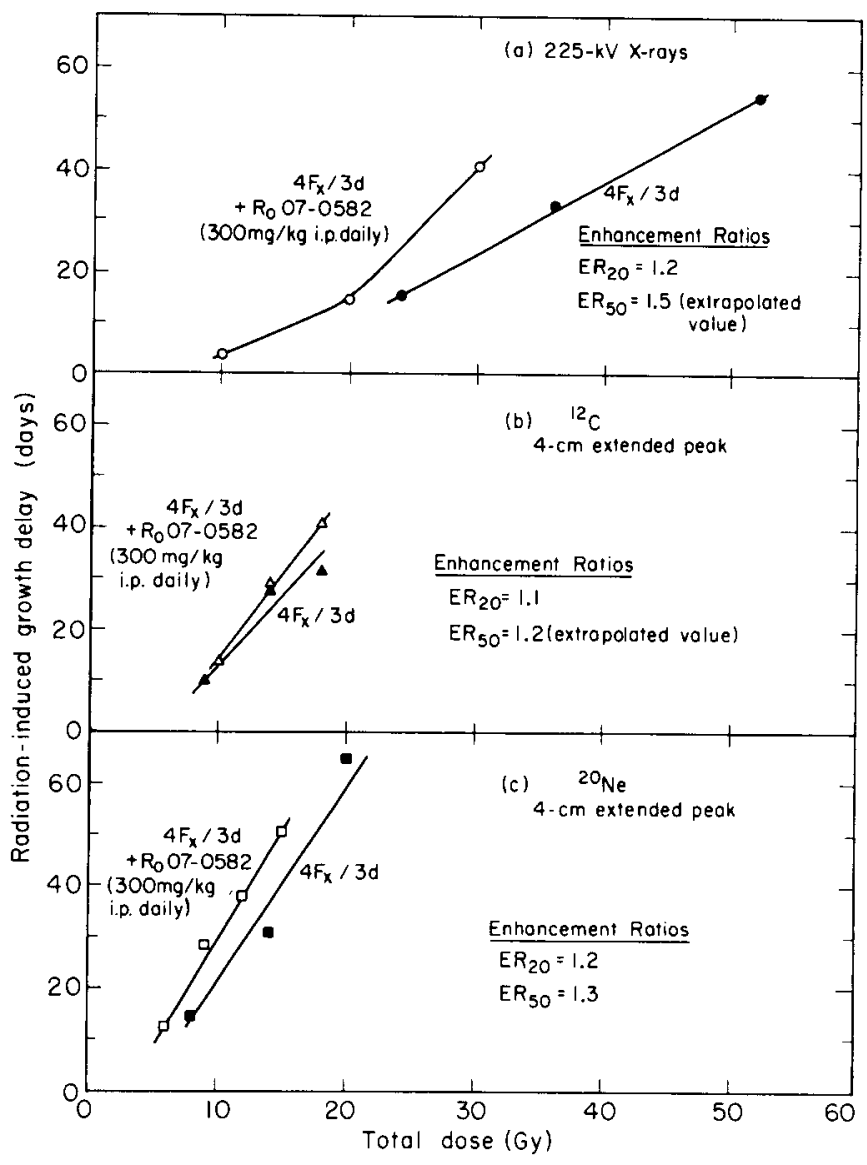

Figure 4. Radiation-induced growth delay curves are shown for R1/LBL tumors receiving fractionated treatments with a combination of misonidazole (Ro 07-0582) and irradiation with $x$ rays (panel a), peak carbon ions (panel b), and peak neon ions (panel $c$ ). The sensitizers were administered as four daily fractions with the drug being given i.p. 30-60 min prior to irradiation. The enhancement ratios demonstrate that the sensitizing effect of Ro 07-5082 is reduced in a fractionated dose regimen with $x$ rays as compared with a single dose schedule (Fig. 3 ). In contrast, the enhancement ratios with peak carbon ions and peak neon ions are similar for single-dose and fractionated-dose schedules. (XBL 805-3286) 
Table 2. RBE Values for Radiation-Induced Growth Delay of R1 Tumors In Vivo Following Combined Treatment with Misonidazole (Ro 07-0582) and Charged-Particle Radiation

\begin{tabular}{|c|c|c|c|c|c|}
\hline $\begin{array}{c}\text { Fractionation } \\
\text { Schedule* }\end{array}$ & $\begin{array}{l}\text { Radiation } \\
\text { Modality }\end{array}$ & $\mathrm{RBE}_{20}{ }^{\ddagger}$ & $\operatorname{RBE}_{50} \neq$ & $E_{20}{ }^{\star \star}$ & $E R_{50}^{* \star}$ \\
\hline $1 \mathrm{Fx}+$ Ro $07-5082$ & $x$ rays & - & -- & 1.8 & 2.1 \\
\hline $1 F x+$ Ro $05-9963$ & $X$ rays & $\ldots$ & -- & 2.1 & 2.4 \\
\hline$(4 \mathrm{Fx} / 3 \mathrm{~d})+$ Ro 07-0582 & $X$ rays & - & -- & 1.2 & 1.5 \\
\hline $1 F x+$ Ro 07-0582 & $12 C$ & 2.1 & $1.4^{\dagger \dagger}$ & 1.3 & $1.2^{++}$ \\
\hline$(4 F x / 3 d)+$ Ro 07-0582 & $12 \mathrm{C}$ & 2.0 & $1.6^{+t}$ & 1.1 & $1.2^{\dagger \dagger}$ \\
\hline $1 F x+R o \quad 07-0582$ & $20 \mathrm{Ne}$ & 1.9 & 1.6 & 1.2 & 1.2 \\
\hline$(4 F x / 3 d)+R 007-0582$ & $20 \mathrm{Ne}$ & 2.9 & 2.3 & 1.2 & 1.3 \\
\hline
\end{tabular}

* For the single fraction schedules, the i.p. dose levels of Ro 07-0582 and Ro $05-9963$ were $500 \mathrm{mg} / \mathrm{kg}$ and $1500 \mathrm{mg} / \mathrm{kg}$, respectively. For schedules of four. fractions in a three-day interval, the daily dose of Ro 07-0582 was $300 \mathrm{mg} / \mathrm{kg}$. In all cases, the i.p. drug injection was given 30-60 min prior to irradiation.

$+\quad$ The peak X-ray energy was $225 \mathrm{kV}$. Both the carbon-ion and neon-ion beams had an initial energy of $400 \mathrm{MeV} / \mathrm{u}$ and the tumors were positioned in the distal 1.5-cm region of a $4-\mathrm{cm}$ extended peak ionization region.

* The RBE 20 and $R B_{50}$ values were calculated from the ratio of the $X$-ray dose to the charged-particle dose required to produce growth delays of 20 days and 50 days, respectively.

$\star * *$ The $E R_{20}$ and ER50 values are drug enhancement ratios at the 20-day and 50-day growth delay levels, respectively. Each enhancement ratio was calculated as the ratio of radiation doses without and with administered sensitizer.

$+t$

Extrapolated value.

survival end points, even though the RBE determinations for the growth delay end point are made at much higher radiation dose levels (Curt is and Tenforde, 1980).

Cellular response to both plateau and 4-cm extended-peak radiation from carbon and neon beams was also measured for 9L gliosarcoma tumors irradiated in situ and assayed for cell survival in vitro (Leith et al., 1975b, 1977; Wheeler et al., 1979). Based on 230-kV X-ray survival curves obtained with tumors from air-breathing and nitrogen-gas-asphyxiated rats, it has been estimated that the fraction of chronically hypoxic cells in the $9 \mathrm{~L}$ tumor is less than $1 \%$ (Leith et al., 1975a). For 9L tumors exposed to plateau carbon and neon ions, the RBE values determined at the $1 \%$ survival level were 1.2 and 1.4 , respectively. In the midposition of a $4-\mathrm{cm}$ extended-peak ionization region, RBE values of 1.6 and 2.6 were obtained for the carbon and neon beams, respectively. The greater response observed for $9 \mathrm{~L}$ tumors exposed to peak neon ions relative to peak carbon ions is in good agreement with the results described above for cell survival of $R 1$ tumors irradiated in situ.

\section{Potentially Lethal Damage Repair}

Cell survival was assayed in vitro following irradiation in situ of human Nall melanoma tumors growing subcutaneously in the flanks of athymic nude mice. These studies were carried out in collaboration with M. Guichard and E. Malaise of the Institut Gustave-Roussy. Tumors were irradiated with neon ions at the midpoint of a $10-\mathrm{cm}$ extended peak, and cell survival was assayed at 0,6 , and $24 \mathrm{~h}$ postirradiation. Parallel experiments were carried out with tumor-bearing mice that had been administered a $1 \mathrm{mg} / \mathrm{kg} i . p$. dose of misonidazole $60 \mathrm{~min}$ prior to irradiation. The 

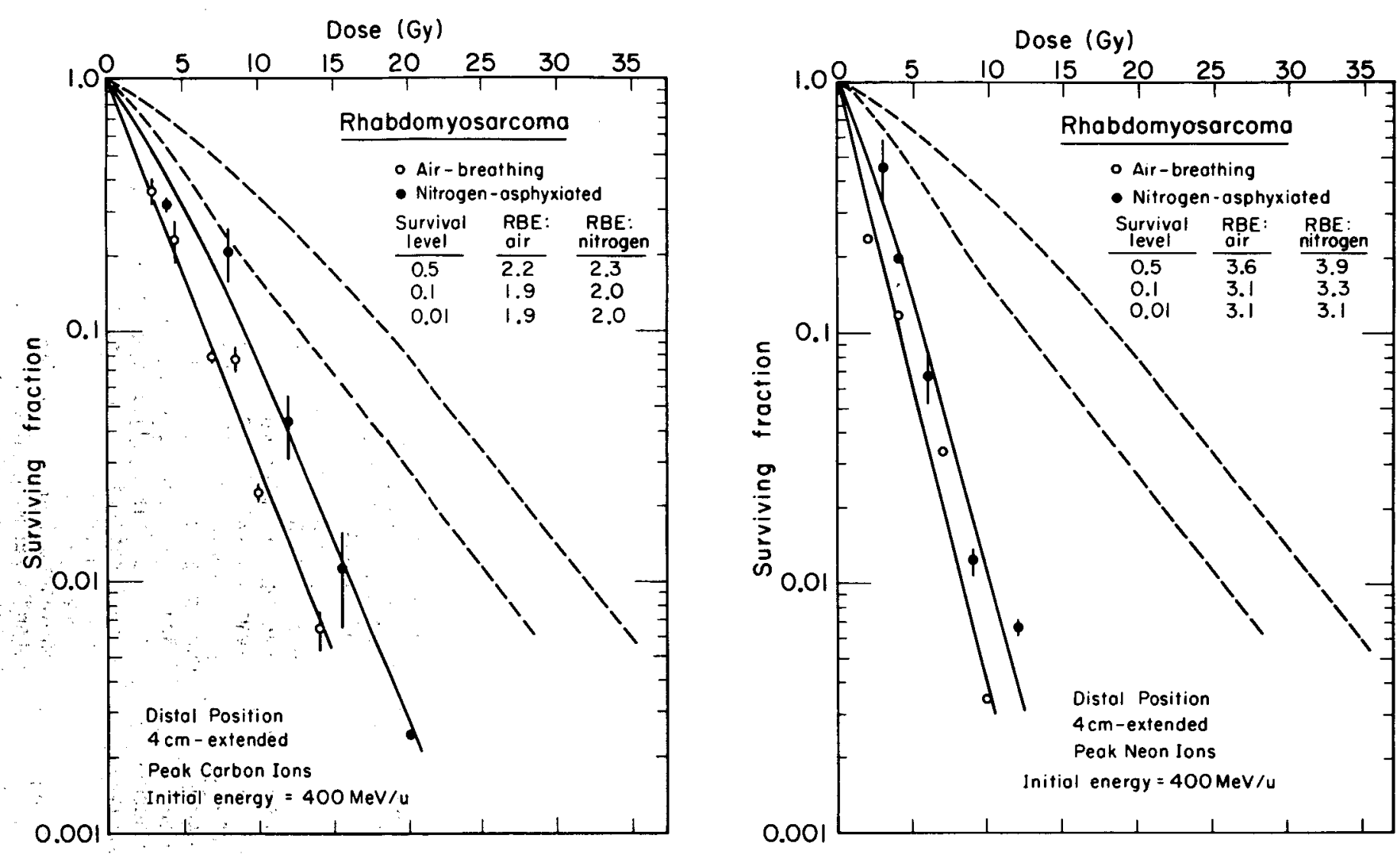

RBE'S FOR CELL KILLING AND GROWTH DELAY

\begin{tabular}{|l|c|c|}
\hline Radiation modality & RBE $_{0.1}$ & RBE $_{50}$ days \\
\hline Peak carbon ions & $1.9 \pm 0.3$ & $2.3 \pm 0.2$ \\
Peak neon ions & $3.1 \pm 0.6$ & $2.9 \pm 0.2$ \\
\hline
\end{tabular}

Figure 5. Cell survival curves under air-breathing and hypoxic conditions are plotted as a function of absorbed dose for R1 tumors (the R2D2 subline) irradiated in situ with carbon-ion and neon-ion beams in the distal position of a 4-cm extended peak. The survival curves for $X$ rays are shown as dashed lines. Tumors were excised immediately following irradiation, and subsequently dissociated enzymatically and plated in vitro for determination of the surviving cell fraction. Control plating efficiencies under air-breathing and nypoxic conditions were $18.2 \cdot 1.7 \%(N=38)$ and $17.0 \pm 1.9 \%(N=27)$. Error bars represent \pm 1 S.E.M. The data presented in the bottom panel demonstrate that comparable RBE values are observed for cell survival and growth delay end points with both charged-particle beams. (XBL 7810-3647) 
principal findings were the following: (1) At the $10 \%$ survival level, the RBE for the peak neon ions relative to ${ }^{60} \mathrm{Co}$ gamma rays was 3.7 , which was higher than the RBE of 3.0 previously obtained for $50 \mathrm{MeV}$ neutrons. (2) Potentially lethal damage (PLD) is repaired after neon ion irradiation and to an extent comparable to that observed following ${ }^{60} \mathrm{Co}$ and neutron irradiation. (3) The enhancement ratio for misonidazole with peak neon ions was 1.4 , which is similar to the values 1.6 and 1.5 obtained for $60 \mathrm{Co}$ and neutron irradiation, respectively. (4) With peak neon ions, as well as with 60 Co and neutron irradiation, preirradiation injection of misonidazole was found to inhibit PLD repair.

\section{Postirradiation Tumor Growth Properties}

Studies have been undertaken to characterize the role of cellular repopulation dynamics, cellular genetic alterations (specifically, heritable nonlethal damage), and tumor-host interactions (specifically, tumor vascularity and immunogenicity) in determining the rate of regrowth of heavily irradiated R1/LBL tumors. Initial results have indicated that heritable nonlethal damage and tumor antigenicity play little, if any, role in the regrowth rate of X-irradiated tumors (Tenforde et al., 1979): Our studies are now focused on the measurement of postirradiation cell proliferation kinetics, and their role in determining the initial rapid rate of regrowth observed following $X$ rays or charged-particle radiation.

\section{CELL KINETICS IN VIVO AND IN VITRO}

\section{In Vivo Studies}

Using the in vivo 3 H-thymidine labeling technique, a comparison was made of cell-kinetic parameters in R1 tumors (the R202 subline) at 8 days following a 20-Gy dose of $x$ rays and a 6-Gy dose of peak neon ions. These studies were carried out in collaboration with $A$. F. Hermens of the Radiobiological Institute in Rijswijk, The Netherlands (Hermans et al., 1979). The cell-cycle times were found to be reduced by approximately $5 \mathrm{~h}$ following $X$ rays and by $4 \mathrm{~h}$ following neon-ion irradiation relative to the value of $17.5 \mathrm{~h}$ obtained for nonirradiated tumors. Rapid cell proliferation was indicated by a significant increase in the labeling index of irradiated tumors. For example, the growth fraction at 8 days following a 6 -Gy dose of peak neon ions was $29 \%$ as compared to the control value of $19 \%$.

\section{In Vitro Studies}

In a collaborative effort with 3 . Gray of the Lawrence Livermore Laboratory, the flow cytofluorometric technique has been used to study the perturbations in cell-kinetic parameters of cultured EMT6 cells exposed to graded single doses of 225-kV $X$ rays, peak carbon ions, or peak neon ions. Preliminary analys is of the data has indicated a significant $G 2$ block at approximately 6-8 $\mathrm{h}$ postirradiation; in addition, all three radiation modalities appear to induce a longlived block at the Gl/S border. The ONA histograms obtained at two-hour intervals for $16 \mathrm{n}$ following irradiation are currently being subjected to analysis with a computer model to predict alterations in cell-cycle phase durations.

Cell-kinetic parameters of EMT6 cells subjected to photon and charged-particle radiation are also being analyzed by the time-lapse cinematographic technique in a second collaborative effort with $E$. Malaise of the Institut Gustave-Roussy. Results indicate that the RBE value for peak neon ions based on the mitotic delay end point is 3.3, and based on the division probability end point, is close to 2 (CollynD'Hooghe et al., personal communication).

\section{ACKNOWLEDGEMENTS}

The authors gratefully acknowledge the excellent technical assistance of K. Crabtree, M. Flynn, S. Hurst, S. Parr, W. Schilling, and S. D. Tenforde. Research support was received from Public Health Service Grants CAl74l1 (Response of rat tumor cells to heavy ions, S. B. Curtis, Principal Investigator) and CA15184 (Heavy-ion radiobiology related to oncology, C. A. Tobias, Principal Investigator).

\section{REFERENCES}

Barendsen, G. W. and J. J. Broerse. 1969. Experimental radiotherapy of a rat rhabdomyosarcoma with $15 \mathrm{MeV}$ neutrons and $300 \mathrm{kV} X$ rays. I. Effects of single exposures. Europ. J. Cancer 5, 373-391.

Barendsen, G. W. and J. J. Broerse. 1970. Experimental radiotherapy of a rat rhabdomyosarcoma with $15 \mathrm{MeV}$ neutrons and $300 \mathrm{kV} X$ rays. II. Effects of fractionated treatments, applied five times a week for several weeks. Europ. J. Cancer 6, 89-109.

Curtis, S. B. and T. S. Tenforde. 1980. Assessment of tumour response in a rat rhabdomyosarcoma. Br. J. Cancer 41, 266-270.

Curtis, S. B., T. S. Tenforde, D. Parks, W. A. Schilling, and J. T. Lyman. 1978. Response of a rat rhabdomyosarcoma to neon- and heliumion irradiation. Radiat. Res. 74, 274-288.

Flockhart, I. R., P. W. Sheldon, I. J. Stratford, and M. E. Watts. 1978. A metabolite of the 2-nitroimidazole misonidazole with radiosensitizing properties. Int. J. Radiat. Biol. $34,91-94$.

Guichard, M., C. Gosse, and E. P. Malaise. 1977. Survival curve of a human melanoma in nude mice. J. Nat1. Cancer Inst. 58, 1665-1669. 
Hermens, A. F. and G. W. Barendsen. 1967. Cellular proliferation patterns in an experimental rhabdomyosarcoma in the rat. Europ. J. Cancer 3, 361-369.

Hermens, A. F. and G. W. Barendsen. 1969. Changes of cell proliferation characteristics in a rat rhabdomyosarcoma before and after $x$-irradiation. Europ. J. Cancer 5, 173-189.

Hermens, A. F. and G. W. Barendsen. 1975. The importance of proliferation kinetics and clonogenicity of tumor cells for volume responses of experimental tumors after irradiation. Proceedings, Fifth International Congress of Radiation Research, Biomedical, Chemical and Physical Prospectives (0. F. Nygaard, H. I. Adler and W. K. Sinclair, eds.), pp. 834-849. Academic Press, New York.

Hermens, A. F., S. B. Curtis, T. S. Tenforde, and G. W. Barendsen. 1979. Analys is of cell proliferation in a rat rhabdomyosarcoma irradiated with neon ions or with $300 \mathrm{kV}$ x-rays. High LET Radiations in Clinical Radiotherapy TG. W. Barendsen, J. Broerse, and K. Breur, eds.) pp. 226-227. Pergamon Press, 0xford.

Leith, J. T., W. A. Schilling, and K. T. Wheeler. 1975a. Cellular radiosensitivity of a rat brain tumor. Cancer 35, 1545-1550.

Leith, J. T., V. Arcellana, J. T. Lyman, and K. T. Wheeler. 1975b. Response of a rat brain tumor to irradiation with accelerated neon ions. Int. J. Radiat. Biol. 28, 91-97.

Leith, J. T., P. Smith, P. Ross-Riveros, and K. T. Wheeler. 1977. Cellular response of a rat brain tumor to a therapeutic neon ion beam. Int. J. Radiat. Biol. 32, 401-407.

Reinhold, H. S. 1965. A cell dispersion technique for use in quantitative transplantation studies with solid tumors. Europ. J. Cancer 1, 67-71.

Reinhold, H. S. 1966. Quantitative evaluation of the radiosensitivity of cells of a transplantable rhabdomyosarcoma in the rat. Europ. J. Cancer 2, 33-42.

Rockwell, S. C., R. F. Kallman, and L. F. Fajardo. 1972. Characteristics of a serially transplanted mouse mammary tumor and its tissue-culture-adapted derivative. J. Nat 1 . Cancer Inst. 49, 735-747.

Tenforde, T. S., S. B. Curtis, K. H. Woodruff, D L. Parks, S. J. Daniels, K. E. Crabtree, W. A. Schilling, and R. J. DeGuzman. 1979. Studies on the regrowth rate, morphological characteristics and transplantation properties of rat rhabdomyosarcoma tumors following large doses of $x$ rays. Int. J. Radiat. Biol. 35, 589-596.

Tenforde, T. S., S. B. Curt is, K. E. Crabtree, S. D. Tenforde, W. A. Schilling, J. Howard, $\therefore$ and J. KT. Lyman. 1980. In vivo cell survival and volume response characteristics of rat rhabdomyosarcoma tumors irradiated in the extended peak region of carbon- and neon-ion beams. Radiat. Res. 83, 42-56.

Wheeler, K. T., D. F. Deen, J. T. Leith, and K. S. Norton. 1979. Cellular response of a rat brain tumor to a therapeutic carbon ion beam. Radiology 133, 757-760. 
176

Blark 


\title{
HEAVY-ION RADIOBIOLOGY OF MULTICELLULAR TUMOR SPHEROIDS
}

\author{
A. Rodriguez and E.L. Alpen
}

Biological investigations with accelerated heavy ions have been carried out at LBL on a regular basis for the past four years. The majority of the cellular investigations have been conducted on cell monolayer and suspension culture systems. The studies to date suggest that heavy charged particle beams may offer some radiotherapeutic advantages over conventional radiotherapy sources. The advantages are thought to lie primarily in an increased RBE, a decrease in OER, and in the advantageous tissue distribution of dose (Raju et al., 1976; Chapman et al., 1978).

Experiments reported here were conducted with $400 \mathrm{MeV} / \mathrm{amu}$ carbon ions, $425 \mathrm{MeV} / \mathrm{amu}$ neon ions, and $570 \mathrm{MeV} / \mathrm{amu}$ argon ions using rat brain gliosarcoma ( $9 \mathrm{~L}$ ) and chinese hamster lung $V 79$ cells grown as multicellular spheroids.

The tumor-like characteristics of multicellular spheroids were initially investigated by Sutherland and co-workers (Sutherland et al., 1971; Inch et a1., 1971; Durand, 1976), who reported that individual spheroids have many characteristics of solid in vivo tumors that are not shared by cells grown in monolayer or suspension culture. In this culture format of nonmonoclonal cells grown in three-dimensional contact, there is variability in cell distribution in the cell cycle, decreasing growth fraction with increasing spheroid size, variation in state of oxygenation in the cells, and some rudimentary forms of intercellular communication. These characteristics have been suggested as the basis for enhanced survival for cells grown and irradiated in the spheroid condition (Durand, 1975; Yuhas and Li, 1978; Durand and Sutherland, 1973, 1975; Sutherland and Durand, 1973; Durand and Biaglow, 1974; Sutherland, 1974; Sutherland et a1., 1970).

Our studies were designed to evaluate highLET radiation survival characteristics of cells grown in this relatively organized tissue-like environment. Our primary objectives were to." determine the RBE values in plateau and spread. Bragg peak regions of the carbon, neon, and argon beams, and evaluate with high and low LET radiation, the role of spheroid architecture in postirradiation survival of cells grown in this format.

RESULTS WITH 9L SPHEROIDS

\section{X-Ray Experiments}

Figure 1 shows the X-ray survival data for ells from large and small spheroids and for cells grown as single cell suspensions. Comparison of survival curves for large and small

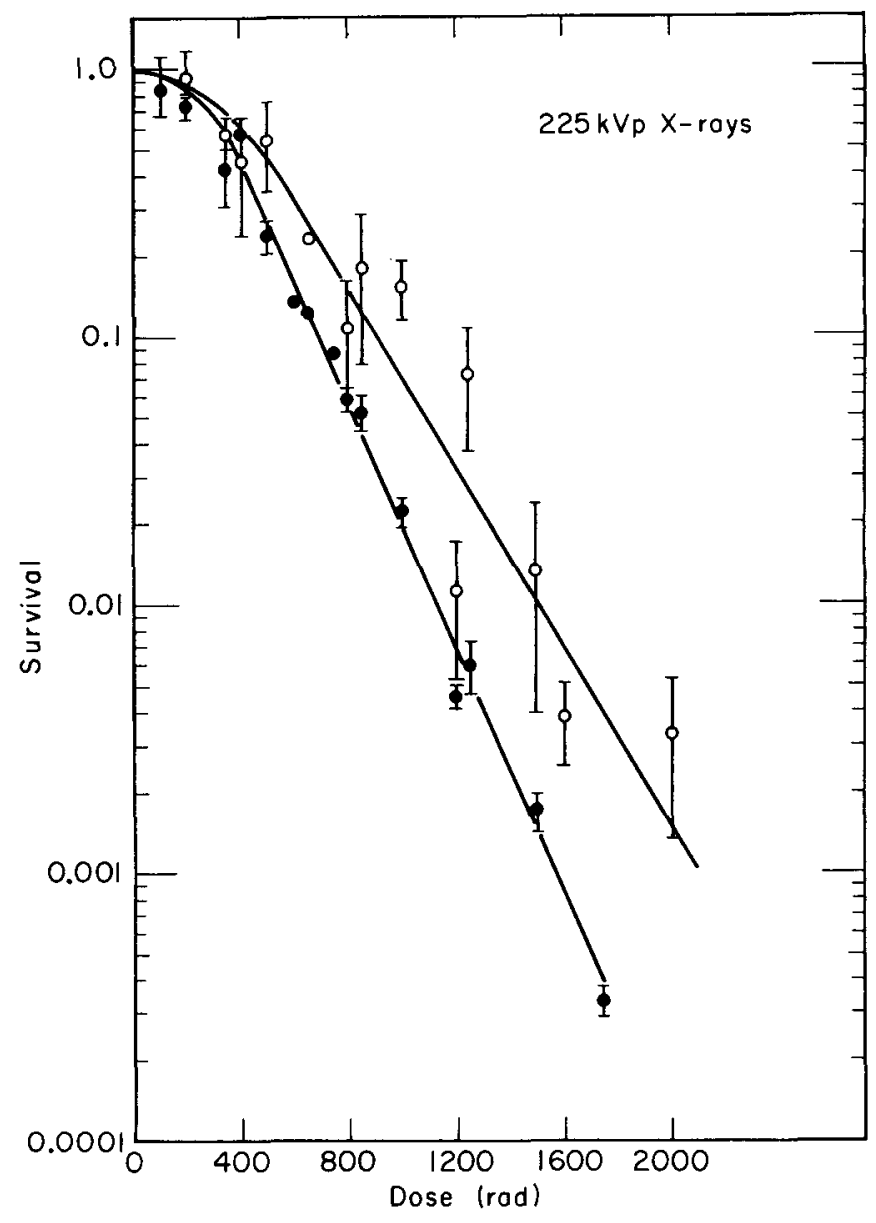

Figure 1. Survival curve for cells irradiated with $X$ rays as spheroids $(0)$ and as cell suspensions (1). The curve for spheroid cell represents the pooled results for small $(100 \mu \mathrm{m})$ and large $(300 \mu \mathrm{m})$ diameter spheroids. Standard deviations are indicated by bars. (XBL 7911-3908)

spheroid cells indicated that there was no significant difference in survival and the data were pooled. The large spheroids also did not appear to have an appreciable fraction of nypoxic cells that might be detected as a deviation of decreased slope at low survival fraction compared to the small spheroid cell survival curve. The survival parameters are derived from the pooled data' from large and 'small spheroids.

Table 1 summarizes the survival parameters for $9 \mathrm{~L}$ cells grown as a single cell suspension and as spheroids for $X$ rays and for carbon, neon, and argon ion radiation. Survival of spheroid cells is characterized primarily by a large $D_{0}$ 
than for cells in suspension, an increase in $D_{Q}$, and little change in $N$. The $X-r a y D_{0}$ for spheroid cells was $260 \mathrm{rad}$ and for suspension cells it was $190 \mathrm{rad}$.

\section{Experiments with Carbon Ions}

Figure 2 shows survival data plotted for single cells and cells from small and large spheroids irradiated with $400 \mathrm{MeV} / \mathrm{amu}$ carbon ions in the plateau region of the Bragg curve (position $P$ in Fig. 3). Cells from both large and small spheroids irradiated in the carbon ion plateau region again show some degree of enhanced radioresistance when compared to single cells irradiated under the same conditions. The $D_{0}$ for spheroids is $260 \mathrm{rad}$ and for cells it is $180 \mathrm{rad}$ (Table 1). This indicates that, at least for this high LET radiation, the protective effect of spheroid architecture is not abolished at an LET of $10 \mathrm{keV} / \mathrm{um}$. The survival parameters for either spheroid cells or suspension cells for

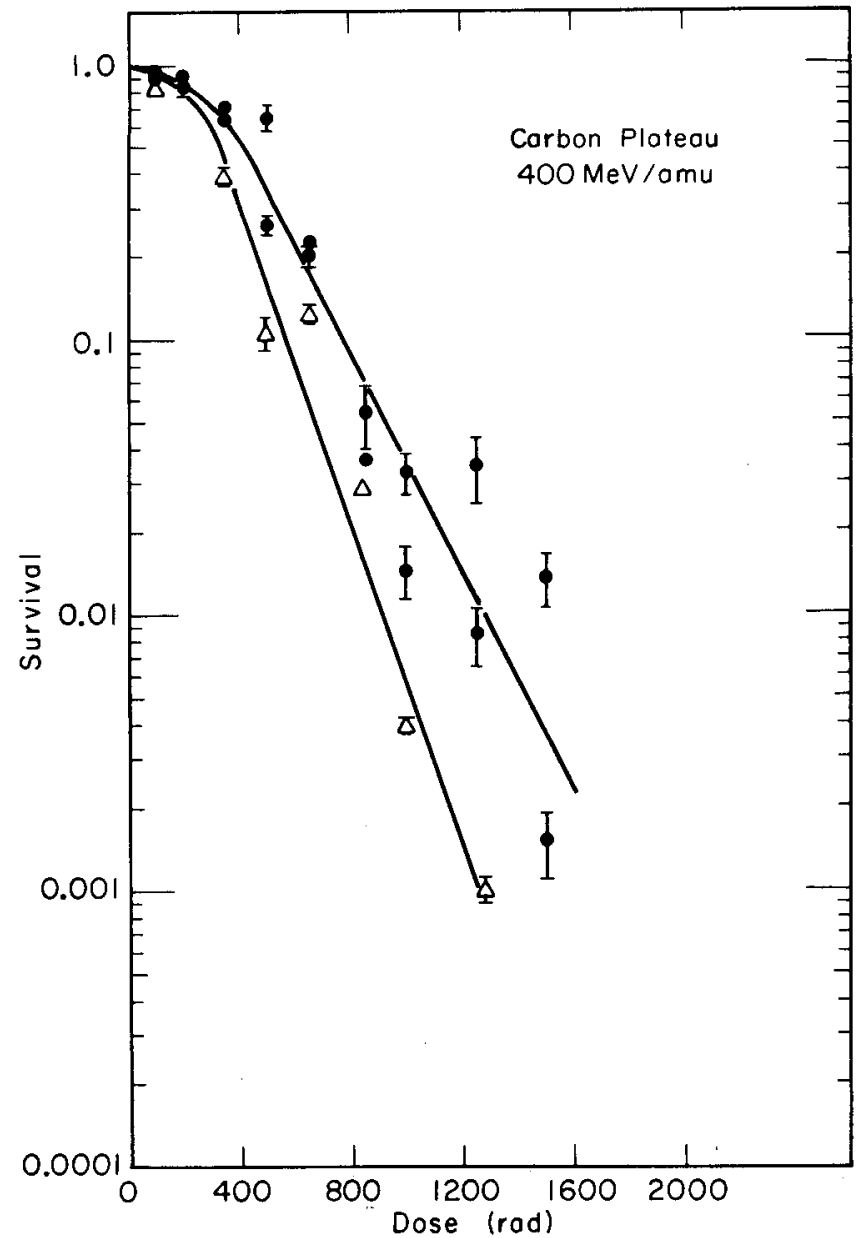

Figure 2. Survival curves for cells irradiated as spheroids ( ) or as cell suspensions ( $\Delta$ ) with $400 \mathrm{MeV} / \mathrm{amu}$ carbon ions in the plateau region of ionization (position P, Fig. 3). The data for spheroid cells represents the combined results for small $(100 \mu \mathrm{m})$ and large $(300 \mu \mathrm{m})$ spheroids. Standard deviations are indicated by bars.

(XBL 7911-3907)
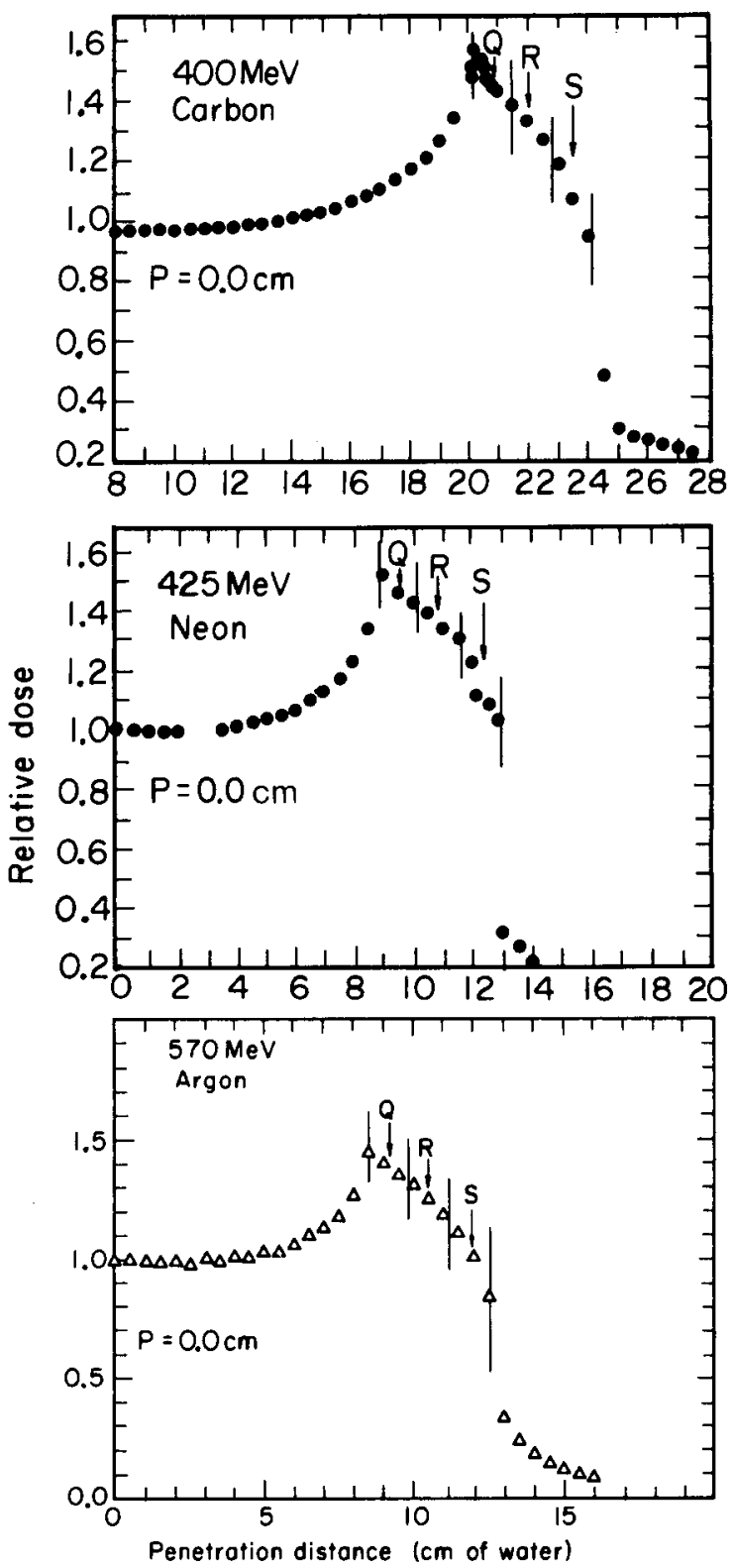

Figure 3. Depth dose distribution curves for the heavy-ion beams. The Bragg peak of the beams was spread to $4 \mathrm{~cm}$ with a brass spiral ridge filter. Samples were placed in the plateau (P) with $0.0 \mathrm{~cm}$ water column and in the proximal (Q), mid (R), and distal (S) portions of the spread Bragg peak. (XBL 808-3648A)

carbon plateau irradiation are not significantly different than for X-irradiation. Since no significant difference exits, the RBE values for carbon plateau irradiation relative to the $225 \mathrm{kVp} X$-ray irradiated spheroid cells and suspension cells are approximately 1.0 at $50 \%$ and $10 \%$ survival, respectively. The values are listed in Table 2 .

To estimate the relative effectiveness for cell killing of the various regions of the spread 
Table 1. Summary of Survival Parameters for $9 L$ Cells Irradiated as Cell Suspensions and Spheroids with X-ray or Heavy Ions

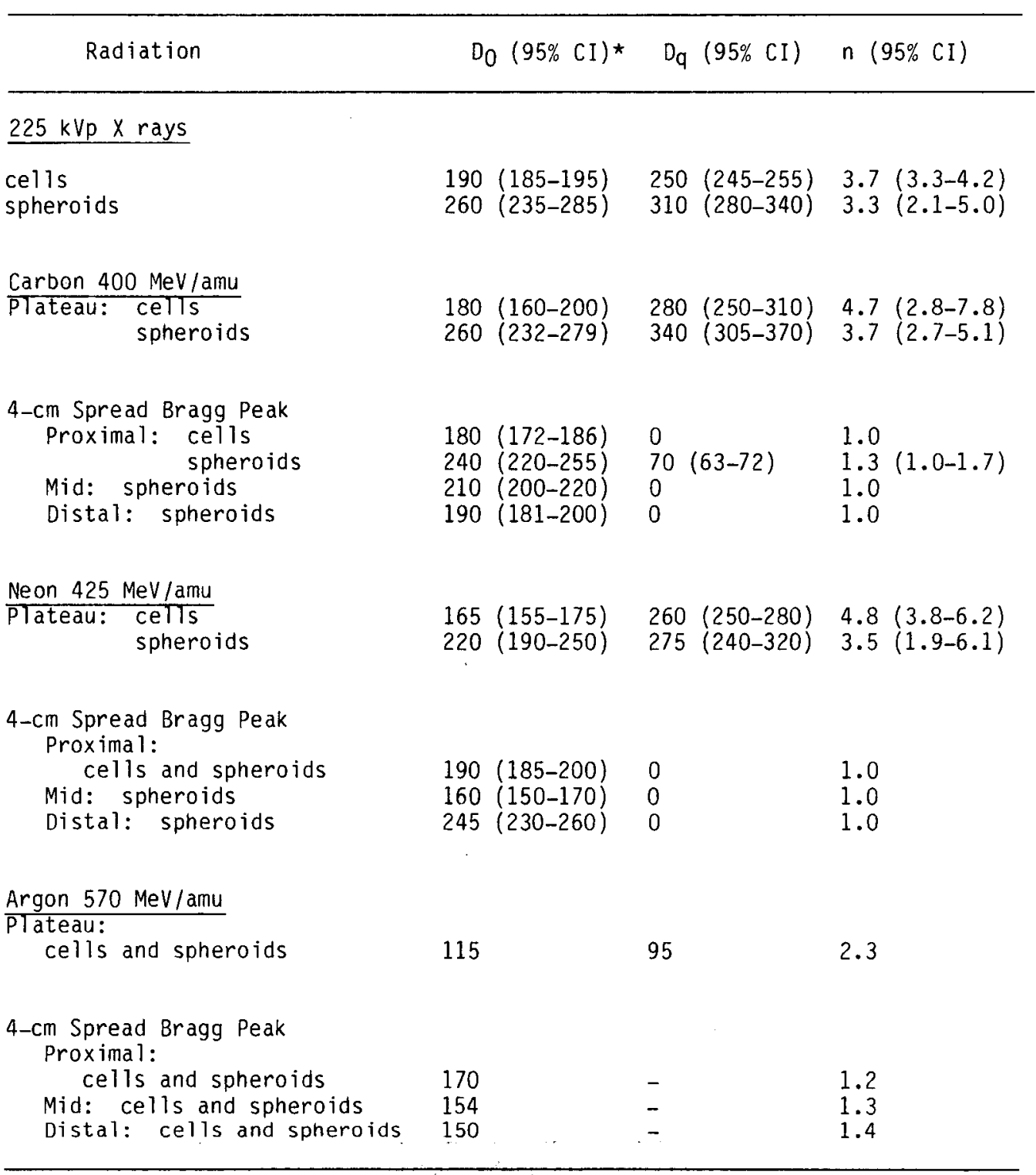

* CI: 95\% confidence interval determined from a least squares regression fit to the data in the exponential region of survival.

Bragg peak of carbon ions, further experients were done comparing the proximal, mid; and distal regions (positions $Q, R$, and $S$, respectively in Fig. 3) of the Bragg curve. These data are shown in Figure 4 for cells from large spheroids and suspension cells. Irradiation of spheroids in, the proximal peak portion of the 4-cm spread. Bragg peak of carbon appears to sharplyireduce the cell survival $D_{0}$ to 70 rad, however cell 1 , survival does appear to be greater for the spheroid cells than for cells from suspensions. The further increases in LET associated with positions closer to the maximum range of the particle lead to survival curves for spheroid cells with smaller $D_{0}$ values as one moves: closer to maximum particle range. The shoulder

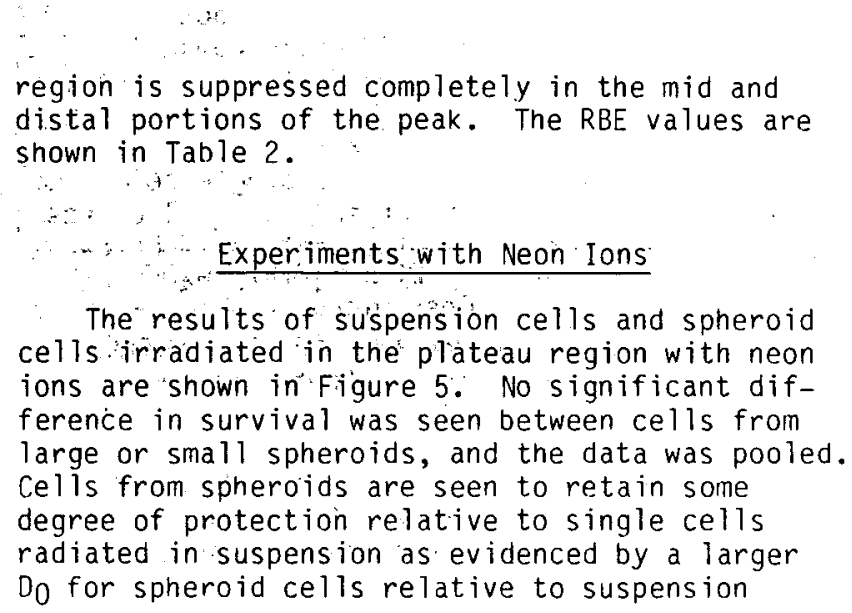
distal portions of the peak. The RBE values are shown in Table 2 .

The results of su'spension cells and spheroid cells irradiated in the plateau region with neon ions are shown in Figure $5 \%$ No significant difference in survival was seen between cells from large or small spheroids, and the data was pooled. Cells from spheroids are seen to retain some degree of protection relative to single cells radiated in suspension as evidenced by a larger $D_{0}$ for spheroid cells relative to suspension 
Table 2. RBE Values for $9 L$ Cells and Spheroids Irradiated with Heavy Ions Relative to $225 \mathrm{kVp} X$ rays

\begin{tabular}{|c|c|c|}
\hline Heavy Ion & $\begin{array}{c}\text { RBE for } \\
50 \% \text { Survival }\end{array}$ & $\begin{array}{l}\text { RBE for } \\
10 \% \text { Survival }\end{array}$ \\
\hline \multicolumn{3}{|l|}{ Carbon $400 \mathrm{MeV} / \mathrm{amu}$} \\
\hline $\begin{aligned} \text { Plateau: } & \text { cells } \\
& \text { spheroids }\end{aligned}$ & $\begin{array}{l}0.94 \\
0.94\end{array}$ & $\begin{array}{l}1.0 \\
0.97\end{array}$ \\
\hline $\begin{array}{cl}\text { 4-cm Spread Bragg Peak } \\
\text { Proximal: } & \text { cel1s } \\
& \text { spheroids } \\
\text { Mid: } & \text { spheroids } \\
\text { Distal: } & \text { spheroids }\end{array}$ & $\begin{array}{l}3.0 \\
2.1 \\
3.4 \\
3.7\end{array}$ & $\begin{array}{l}1.7 \\
1.5 \\
1.9 \\
2.1\end{array}$ \\
\hline \multicolumn{3}{|l|}{ Neon $425 \mathrm{MeV} / \mathrm{amu}$} \\
\hline $\begin{aligned} \text { Plateau: } & \text { cells } \\
& \text { spheroids }\end{aligned}$ & $\begin{array}{l}1.0 \\
1.1\end{array}$ & $\begin{array}{l}1.1 \\
1.2\end{array}$ \\
\hline \multicolumn{3}{|l|}{$\begin{array}{l}\text { 4-cm Spread Bragg Peak } \\
\text { Proximal: }\end{array}$} \\
\hline $\begin{array}{ll} & \text { cells } \\
& \text { spheroids } \\
\text { Mid: } & \text { spheroids } \\
\text { Distal: } & \text { spheroids }\end{array}$ & $\begin{array}{l}2.9 \\
4.0 \\
4.4 \\
2.9\end{array}$ & $\begin{array}{l}1.6 \\
2.1 \\
2.5 \\
1.6\end{array}$ \\
\hline \multicolumn{3}{|l|}{ Argon $570 \mathrm{MeV} / \mathrm{amu}$} \\
\hline $\begin{aligned} \text { Plateau: } & \text { cells } \\
& \text { spheroids }\end{aligned}$ & $\begin{array}{l}2.8 \\
2.7\end{array}$ & $\begin{array}{l}2.6 \\
2.8\end{array}$ \\
\hline \multicolumn{3}{|l|}{$\begin{array}{l}\text { 4-cm Spread Bragg Peak } \\
\text { Proximal: }\end{array}$} \\
\hline $\begin{array}{ll} & \text { cells } \\
\text { Mid: } & \text { spheroids } \\
\text { cells } \\
\text { spheroids } \\
\text { Distal: } & \begin{array}{l}\text { cells } \\
\text { spheroids }\end{array}\end{array}$ & $\begin{array}{l}3.6 \\
3.4 \\
2.8 \\
2.7 \\
2.6 \\
2.5\end{array}$ & $\begin{array}{l}2.0 \\
2.1 \\
2.1 \\
2.2 \\
2.1 \\
2.2\end{array}$ \\
\hline
\end{tabular}

cells. There is a decrease in $D_{0}$ for both cells suspensions and spheroid cells compared with $X$ rays and for the spheroids there may be a reduction in the shoulder $\left(O_{Q}\right)$.

In the proximal peak of neon, single cells and spheroid cells have identical survival curves (Fig. 6A). The implication is that any protection provided by spheroid architecture has been abolished. In both systems the extrapolation number is $1: 0$ and no shoulder exists. The RBE values for the spread Bragg peak of neon are given in Table 2. Figure $6 \mathrm{~B}$ is the data for the comparison of three positions in the spread neon peak. The most effective region of the neon beam is the midpeak region. At this position the RBE values are maximum at 4.4 for a survival fraction of $50 \%$ and 2.5 for a survival fraction of $10 \%$.
We conclude that with the higher LET in the distal region, saturated LET effectiveness is below this value and significant overkill is observed in the distal peak with this cell line.

\section{Experiments with Argon Ions}

Survival in the plateau region of the argon. beam (LET $95 \mathrm{keV} / \mathrm{um}$ ) was much more significantly reduced for both cell suspensions and spheroid cells compared to $X$ rays. Survival appeared to be identical for cell suspensions and spheroid cells (Fig. 7). The RBE values for cell suspensions and spheroid cells. at $10 \%$ survival were 2.6 and 2.8 , respectively, which is significantly higher than the plateau regions of carbon and neon (Table 2). 


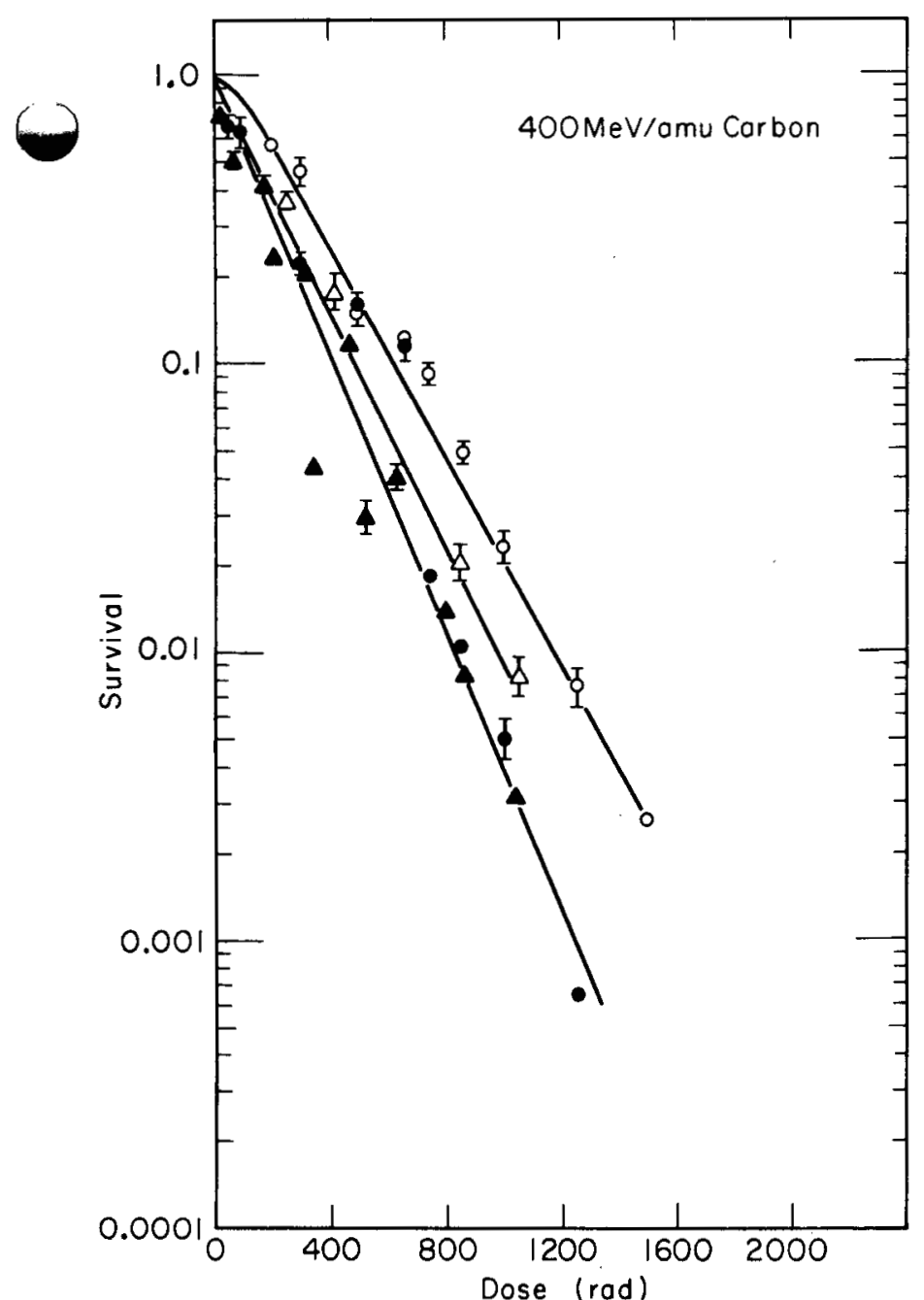

Figure 4. Survival curves for cells irradiated as spheroids in the $4-\mathrm{cm}$ spread Bragg peak of $400 \mathrm{MeV} / \mathrm{amu}$ carbon ions. The positions are proximal (0), position Q; mid ( $\Delta)$, position R; and distal ( $\Delta$ ) position $S$. Letters refer to Figure 3. Survival data points are also shown for cells irradiated in suspension. in the proximal peak (0). (XBL 7911-3906)

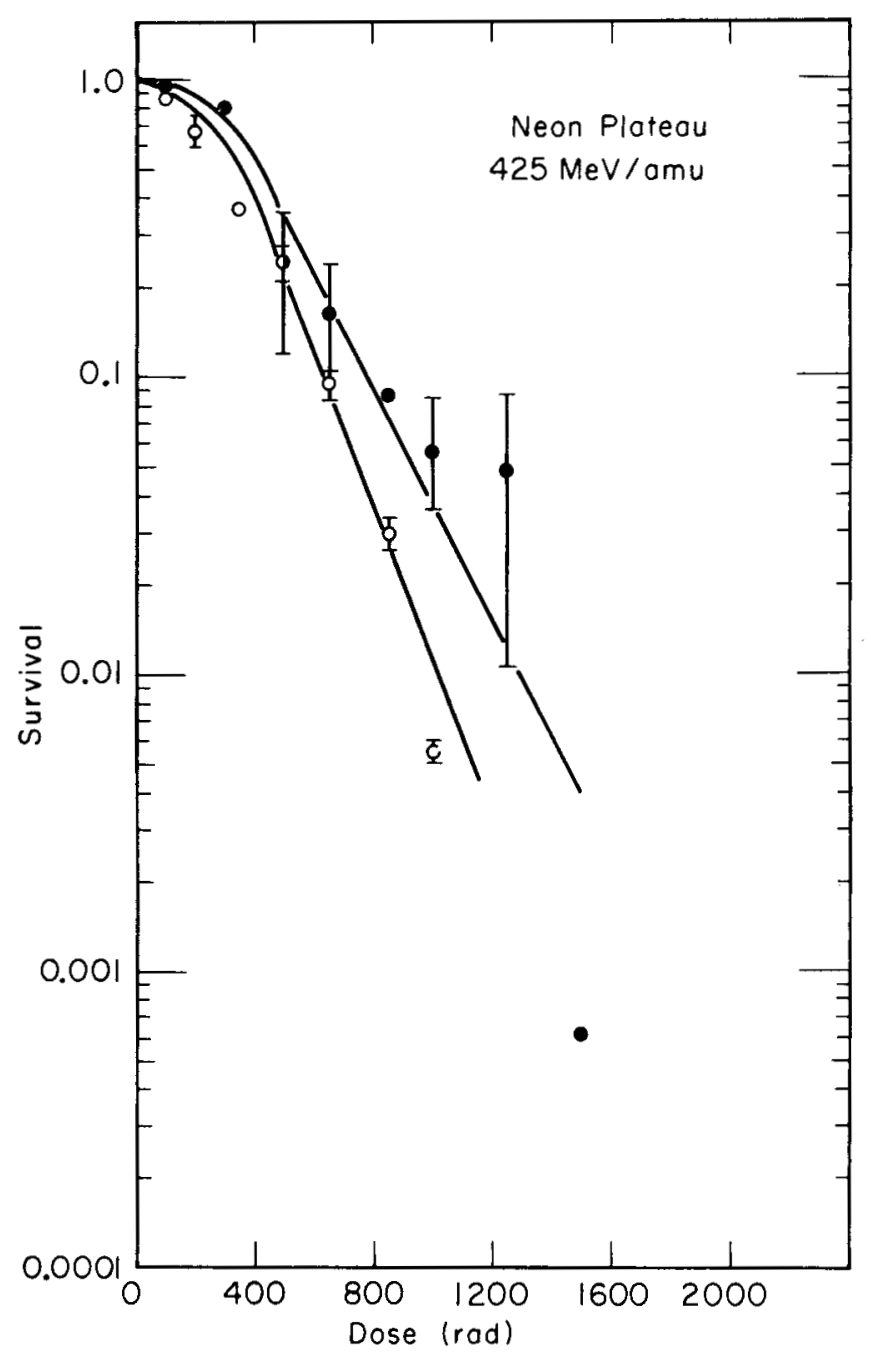

Figure 5. Survival curves for cells irradiated as spheroids (1) or as cell suspensions (0) in the plateau region (position P, Fig. 3) of a $425 \mathrm{MeV} / \mathrm{amu}$ neon-ion beam. (XBL 7911-3905)
Cell survival in the 4-Cm spread Bragg peak of argon was exponential and, as in the plateau, identical results were obtained for both suspension cells and spheroid cells (Fig. 7).. As with: neon, there was reduced effectiveness as the LET. increased above $150 \mathrm{keV} / \mathrm{um}$ from the plateau $(95 \mathrm{keV} / \mu \mathrm{m})$ to the spread peak: $(350-600 \mathrm{keV} / \mathrm{um})$. The RBE at $10 \%$ survival for spheroid cells in the spread peak ranged from 2.1 to 2.2 (Table 2) and was lower than for the plateau.

\section{DISCUSSION}

The X-ray survival we found for $9 \mathrm{~L}$ spheroids is different in some respects from results

\footnotetext{
reported previously with other cell lines. Cells grown as spheroids were more radioresistant than single cells irradiated in suspension. Durand and Sutherland $(1972,1975)$ have reported that Chinese hamster lung cells (V79) grown as spheroids extioit a cell contact mechanism that enhances the ability of the cells to sustain sublethal damage, thereby broadening the shoulder of the survival curve. However, with our cell system the primary effect of low-LET radiation on survival of cells irradiated as spheroids was an increase in the $D_{0}$, whereas Durand and Sutherland have reported that the main effect on V79 cells irradiated as spheroids was seen as a broadening of the shoulder associated with an increase in the extrapolation number.
} 


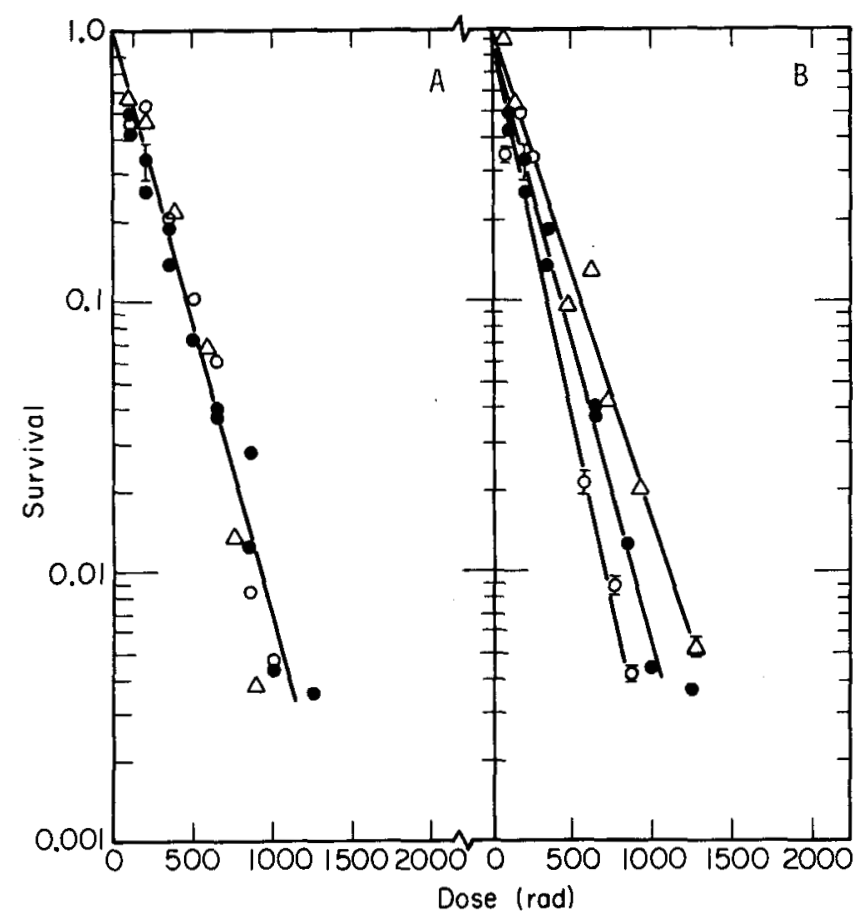

Figure 6. (A) Survival curve for cells irradiated as large (300 $\mu \mathrm{m})$ spheroids (0), small $(100 \mu \mathrm{m})$ spheroids $(0)$, and as cell suspensions $(\Delta)$ in the proximal portion of a $4 \mathrm{~cm}$ spread Bragg peak of a $425 \mathrm{MeV} / \mathrm{amu}$ neon ion beam (position Q). (B) Survival curves for cells irradiated as large $(300 \mu \mathrm{m})$ spheroids in the proximal ( $)$, mid $(0)$, and distal $(\Delta)$ portions (positions $P, Q$, and $R$ ) of a $425 \mathrm{MeV} / \mathrm{amu}$ neonion beam. (XBL 7911-3904)

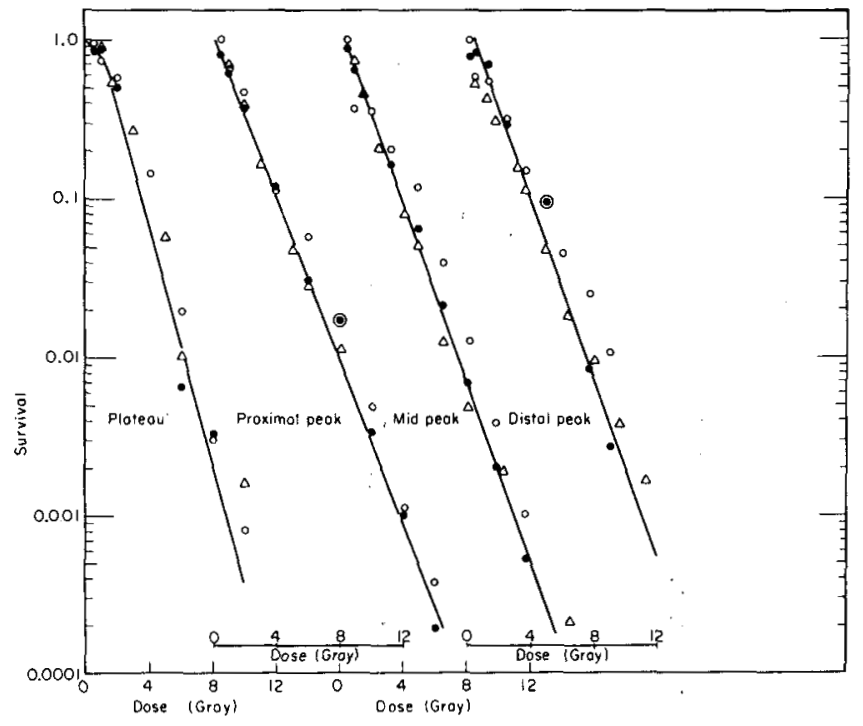

Figure 7. Survival curves for cells irradiated as spheroids $(0)$ and cell suspensions $(\bullet, \Delta)$ in the plateau, proximal, mid, and.distal portion of the 4-cm spread Bragg peak (positions $P, Q$, $R$, and S) of a $570 \mathrm{MeV} / \mathrm{amu}$ argon ion beam. (XBL 797-3640)
As we mentioned earlier, other workers nave observed a number of special characteristics of cells grown in the spheroid culture form. Some have reported that there exist regions of hypoxia and anoxia (Sutherland, 1974; Sutherland and Durand, 1973; Durand and Biaglow, 1974) while others have found no evidence for a higher oxygen tension than expected in the same system (Carlsson et al., 1979; Durand, 1980). The kinetics of cell proliferation in growing spheroids is markedly different from that for monolayer and suspension culture cells (Durand, 1975, 1976; Durand and Sutherland, 1973; Yuhas and Li, 1978). The growth fraction is certainly reduced as spheroids increase in size, and it is also possible that there is significant redistribution of cells in the cell cycle as the spheroids grow. There is, of course, also the demonstrated presence of dead and dying cells in the larger spheroids.

The X-ray cell survival curve for spheroid cells in Figure 1 represents two experiments each for large $(300 \mu \mathrm{m})$ and small $(100 \mu \mathrm{m})$ diameter spheroids. The data were combined because it was not possible to discern any radioresistant fraction in the large spheroids that would be attributable to hypoxia. The large spheroids did exhibit a more variable survival response at the lower survival levels. We have estimated that a hypoxic fraction of less than $3 \%$ would not contribute significantly to the overall cell survival of the spheroids, and, in any case, we cannot find evidence to support partial hypoxia as a probable cause for altered radiosensitivity of spheroid cells.

Histological examination of large spheroids confirmed that they were composed of three distinct regions (Inch et al., 1971; Sutherland et al., 1971). The 9l spheroids, $300 \mu \mathrm{m}$ in diameter, develop an outer zone with many dividing cells, an intermediate zone containing only a few cells in mitosis, and a central zone of necrosis.

It remains to be demonstrated whether the altered distribution of cells in the cell cycle, a reduced proliferative fraction, or the presence of dead and dying cells can possibly contribute to altered spheroid radiosensitivity.

The cellular sensitivity of spheroids grown from this rat gliosarcoma $l$ ine to $X$ rays appears to be similar to that found for the same cells grown as an in vivo rat brain tumor (Leith et al., $1975 a)$. The $X$-ray euoxic $D_{0}$ reported for the in vivo tumor was 295 rad compared to the $D_{0}$ of 260 rad for the spheroids in our experiments. For the tumor cells in a dispersed in vitro suspension culture, the Do was 190 rad. A hypoxic cell fraction was not observed in the in vivo tumor, but the sensitivity of the in vivo to in vitro assay for hypoxic fraction of in vivo tumors would nearly preclude the identificaton of a small hypoxic fraction.

The survival of cells grown as spheroids after carbon and neon irradiation in the plateau region of ionization is significantly enhanced compared to the post-heavy-ion irradiation 
survival of cells maintained in suspension culture. This enhanced survival is primarily seen as an increased $D_{0}$ as with $X$ rays. The degree of protection afforded by the spheroid configuration is about the same as that seen with $X$ rays $\left(D_{0}\right.$ cells $/ D_{0}$ spheroids $\left.=1.3\right)$. Enhanced spheroid survival reported for other high L.ET radiation exposures with Chinese hamster cells (V79) (Durand and 01ive, 1976, 1977; Dertinger et al., 1976) was seen as an increase in extrapolation number with the $D_{0}$ remaining relatively unchanged. Enhancement of spheroid cell survival has been observed with both large and small spheroids for neutron radiation (Durand and 07 ive, 1977) and pion radiation (Dertinger. et al., 1976) as compared to monolayer cultures.

The survival of spheroids in the plateau region of ionization of carbon (LET, $10 \mathrm{keV} / \mu \mathrm{m}$ ) and neon (LET, $30 \mathrm{keV} / \mathrm{\mu m}$ ) is not greatly reduced in comparison to survival with $X$ rays. For carbon the effect is negligible. For neon the $D_{0}$ is reduced for suspension cells and spheroid cells; however, the RBE for the plateau is close to 1.0 for both ions in either growth format. The survival enhancement of spheroids appears to be abolished in the argon plateau where the LET is $95 \mathrm{keV} / \mu \mathrm{m}$.

Irradiation of cells and spheroids in the proximal portions of the 4-cm spread Bragg peak (where specific ionization is approximately $60 \mathrm{keV} / \mu \mathrm{m}$ for carbon, $110 \mathrm{keV} / \mu \mathrm{m}$ for neon, and $370 \mathrm{keV} / \mu \mathrm{m}$ for argon) leads to a marked reduction of the shoulder region of the cell survival curve for either suspension cells or spheroid cells; however, for carbon ions there still appears to be some radioprotection afforded the spheroid configuration. The effectiveness of the spread peaks in relation to the plateau is measured by the RBE gain from the plateau to the peak (Table 3). The RBE gain for spheroid cells at the $10 \%$ survival level in the carbon proximal peak was 1.5 , for neon it was estimated as 1.8 , and for argon 0.71 . For cell suspensions the RBE gain at $10 \%$ survival level was 1.7 for carbon, 1.5 for neon, and 0.71 for argon. The neon beam is certainly more effective for cell killing in spheroids at low survival. However, at the $50 \%$ survival level at which therapeutic doses would be delivered the gain for both neon and carbon is about the same. The highest RBE is seen at the neon midpeak at an LET value of $150 \mathrm{keV} / \mathrm{\mu m}$. In the distal peak, at $200 \mathrm{keV} / \mathrm{\mu m}$, the effectiveness of neon ions is less than in the midpeak, indicating that we have surpassed the LET for maximum effectiveness in this location (Fig. 8). This is further supported by the lower RBE values of the argon beam where the LET is above $95 \mathrm{keV} / \mathrm{\mu m}$ (Fig. 8) in the peak compared to the plateau.

The survival response with carbon, i.e., increased killing effectiveness with increased LET, is as expected. Single cell suspension experiments with Chinese hamster (V79) and human kidney (TI) cells in the $10-\mathrm{cm}$ spread carbon ion Bragg peak have shown that the RBE continues to increase from the proximal to distal peak regions as a function of increasing LET (Chapman et al., 1977, 1978). Survival of cells from spheroids irradiated in the neon spread peak was not as expected. Cell experiments with neon have indicated that the RBE increases and the OER decreases from the proximal to distal peak; this is again a function of increasing LET (Chapman et al., 1978). Our results show a lower RBE in the distal peak than the proximal or mid regions. The argon peak with LET above $300 \mathrm{keV} / \mathrm{\mu m}$ also shows reduced effectiveness. One possible explanation for our findings of reduced cell killing effectiveness for distal neon peak and argon 4-cm spread peak radiation is that for the

Table 3. Plateau-to-Peak RBE Gain for Carbon, Neon, and Argon Beams

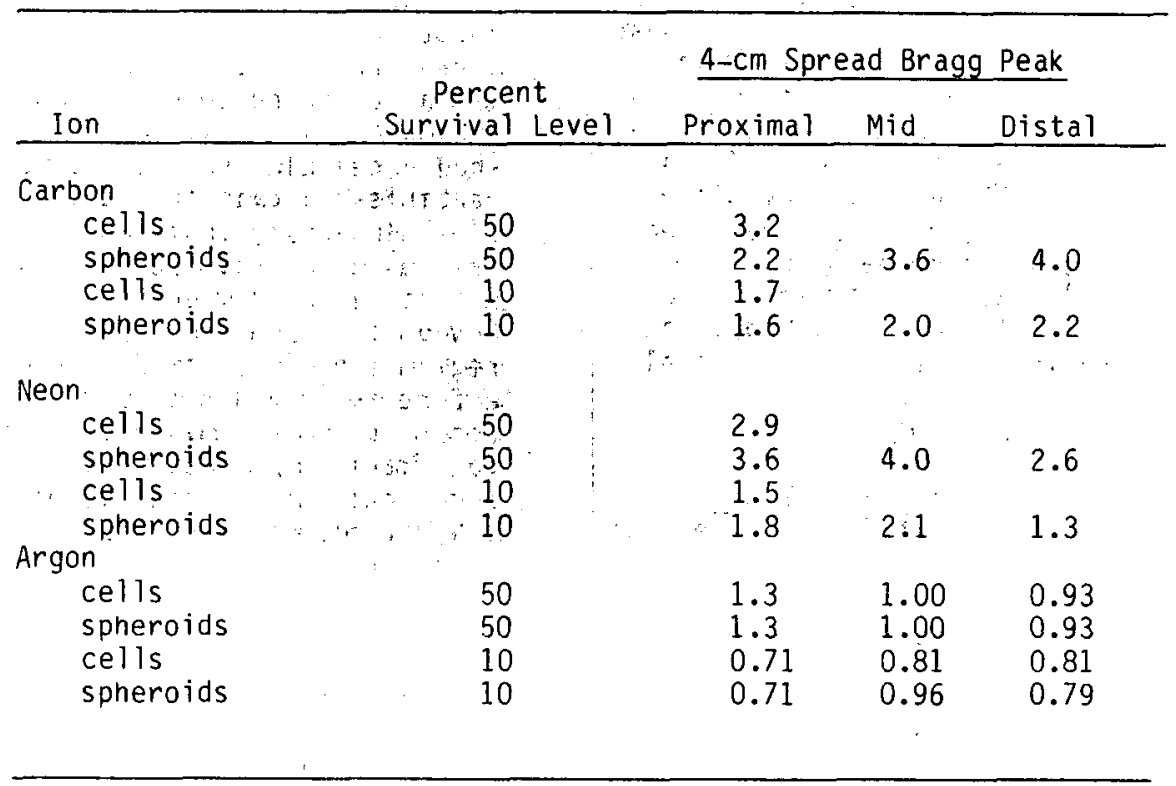




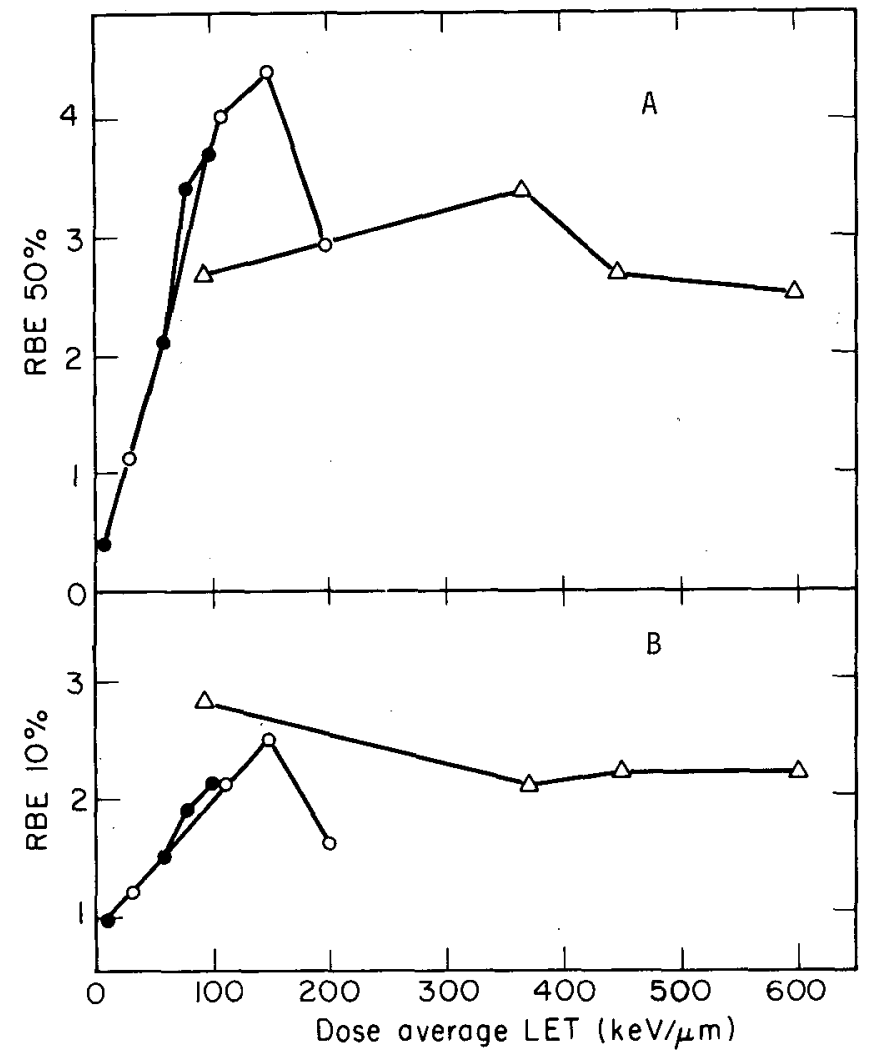

Figure 8. Variation of the RBE for $50 \%$ survival (A) and $10 \%$ survival (B) as a function of dose average LET for a $400 \mathrm{MeV} / \mathrm{amu}$ carbon beam ( ), $425 \mathrm{MeV} / \mathrm{amu}$ neon beam (0), and $570 \mathrm{MeV} / \mathrm{amu}$ argon beam $(\Delta) .($ XBL 808-3649)

spheroid cell configuration the peak effectiveness for the RBE-LET relationship occurs at a lower LET value $(100-150 \mathrm{keV} / \mathrm{\mu m})$ rather than at the higher value seen with single cells

$(200 \mathrm{keV} / \mu \mathrm{m})$. This relationship appears to be true at least at lower doses as seen in Figure $8 \mathrm{~A}$ for $50 \%$ survival RBE. A similar type of response has been observed independently with neon and argon beams in experiments on testes weight loss and gut crypt cell inactivation. It appears that in these normal tissues the RBE decreases with LET above approximately $100 \mathrm{keV} / \mathrm{mm}$. The midpeak neon RBE (2.5) and $D_{0}(120 \mathrm{rad})$ values obtained from the $9 L$ in vivo to in vitro cell survival assay (Leith et al., 1975a) are similar to what we have reported (Tables 1 and 2 ). In addition it should also be pointed out that the survival enhancement and also the shoulder region are abolished at an LET above $80 \mathrm{keV} / \mathrm{um}$.

Our results may indicate that the spheroids approximate the response of organized tissues and tumors more closely than other types of in vitro systems studied so far. An enhancement in survival of spheroid cells compared to single cell survival is seen in the plateau regions of both neon and carbon beams. The survival enhancement has been observed to be due to an increased radioresistance related to a larger
Do value for $9 \mathrm{~L}$ spheroid cells. The proximal and mid portions of the neon peak are more effective than similar regions of the carbon peak in reducing this effect of spheroid morphology on. cell survival.

\section{Studies with V79 Chinese Hamster Cell Spheroids}

Studies on Chinese hamster 179 cells grown as multicell spheroids were conducted by $C$. Lücke-Hühle (Institut für Genetik und für Toxikologie von Spaltstoffen, Kernforschungsgentrum Karlsruhe, Karlsruhe, F.R.G.) and E. A. Blakely, $F$. Ngo, P. Chang and $C$. A. Tobias (LBL). The beams studied and their initial extrapolation energies were carbon ( $400 \mathrm{MeV} / \mathrm{amu}$ ), neon (425 MeV/amu), and argon ( $570 \mathrm{MeV} / \mathrm{amu}$ ). Survival of cells dispersed from spheroids $(390 \pm 20 \mu \mathrm{m}$ diameter) and monolayers was measured at a position in the plateau, and at a position in the middle of the $4-\mathrm{cm}$ extended Bragg peak.

Figure 9 compares survival of V79 spheroid cells with that of $V 7.9$ monolayer cells after exposure to $x$ rays and the three heavy-ion beams, respectively. The difference in radiation dose yielding $10 \%$ survival was used to calculate a survival resistance factor (RF) for spheroid cells compared to monolayer cultures for each type of radiation. Table 4 shows a summary of all RF and RBE data. Unfortunately, no data are available for the argon plateau.

Figure 10 compares survival of $V 79$ cells from spheroids after $X$ rays with survival after exposure to $400 \mathrm{MeV} / \mathrm{amu}$ carbon ions, $425 \mathrm{MeV} / \mathrm{amu}$ neon ions, and $570 \mathrm{MeV} / \mathrm{amu}$ argon ions at plateau and midpeak positions. The great resistance of spheroid cells to $x$ rays is indicated by the pronounced initial shoulder of the $X$-ray survival curve (broken line). Exposure of spheroids at the carbon and neon plateau yielded survival curves with shoulders, however, the survival curves for the Bragg peak showed no shoulder and produced much higher RBE values than for the plateau (Table 4).

The results demonstrate that the increased resistance of $V 79$ spheroids to sparsely ionizing irradiation (possibly due to a cell contact mediated increase in the ability to accumulate sublethal damage as demonstrated by a greater shoulder), decreases with increasing LET of the particles and was absent at very high LET values of 75-450 keV/um. As a consequence the RBE values, which are based on X-ray data for reference, were very high $(4.1$ to 4.2$)$ at the Bragg peaks and considerably lower at the plateau regions, the regions of lower LET. A similar effect was found with $9 L$ spheroid, as previously reported for the midpeaks. The RBE value of 1.5 for the neon plateau is comparable to the value found for pions at an unspread peak and for $7.5 \mathrm{MeV}$ neutrons (Durand and 0live, 1976; Dertinger et al., 1976) while the carbon plateau showed the same killing efficiency as $x$ rays. The RBE values for the V79 monolayers in the range of 1.8 to 2.4 are comparable to data published by others (Chapman et al., 1977, 1978; Blakely et al., 1979) using the same heavy-ion beams at midpeak positions. 

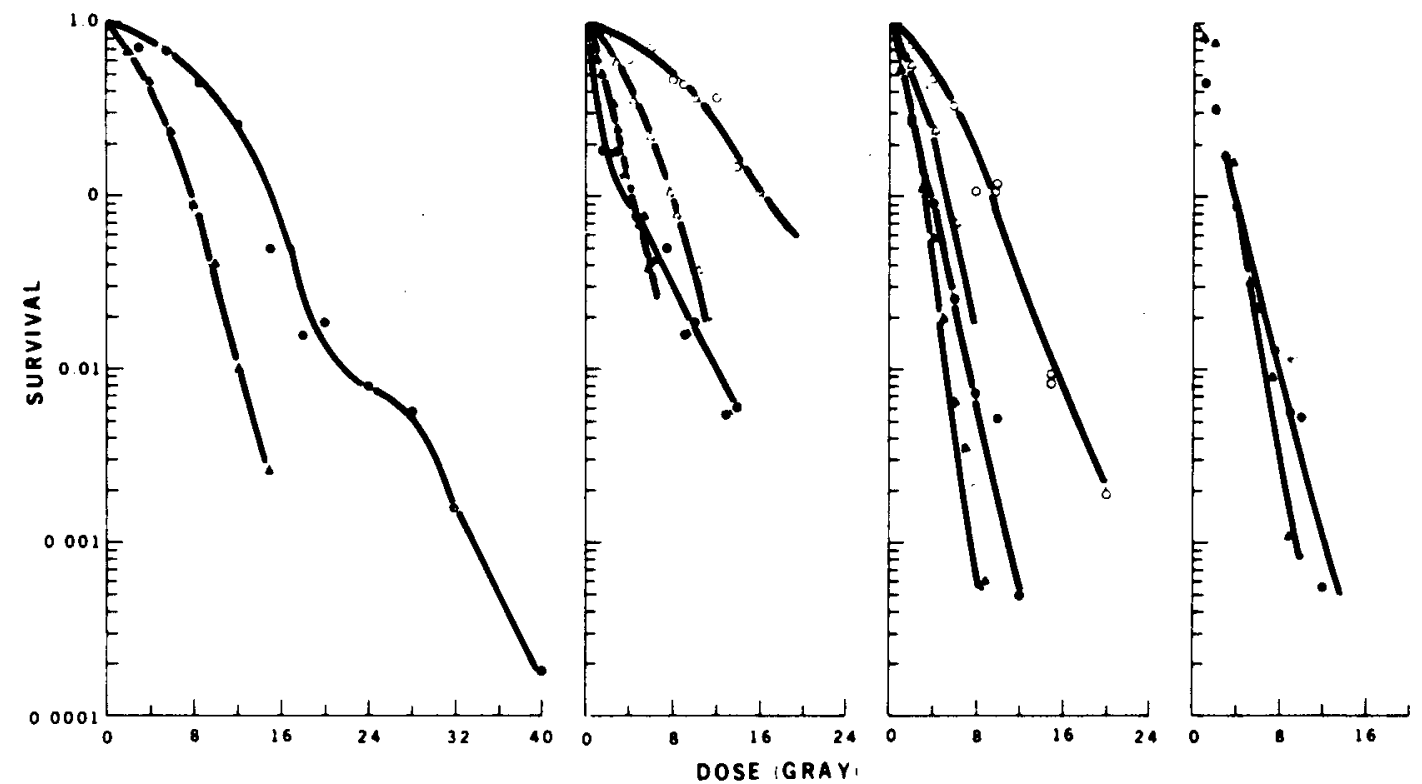

Figure 9. Comparison between survival of spheroid cells (0) and survival of cells from monolayer cultures $(\Delta)$ after exposure to $X$ rays and to the various ions studied at a plateau (open symbols) and midpeak position (closed symbols), respectively. (XBL $808-10893)$

Table 4. Summary of RBE and RF Values

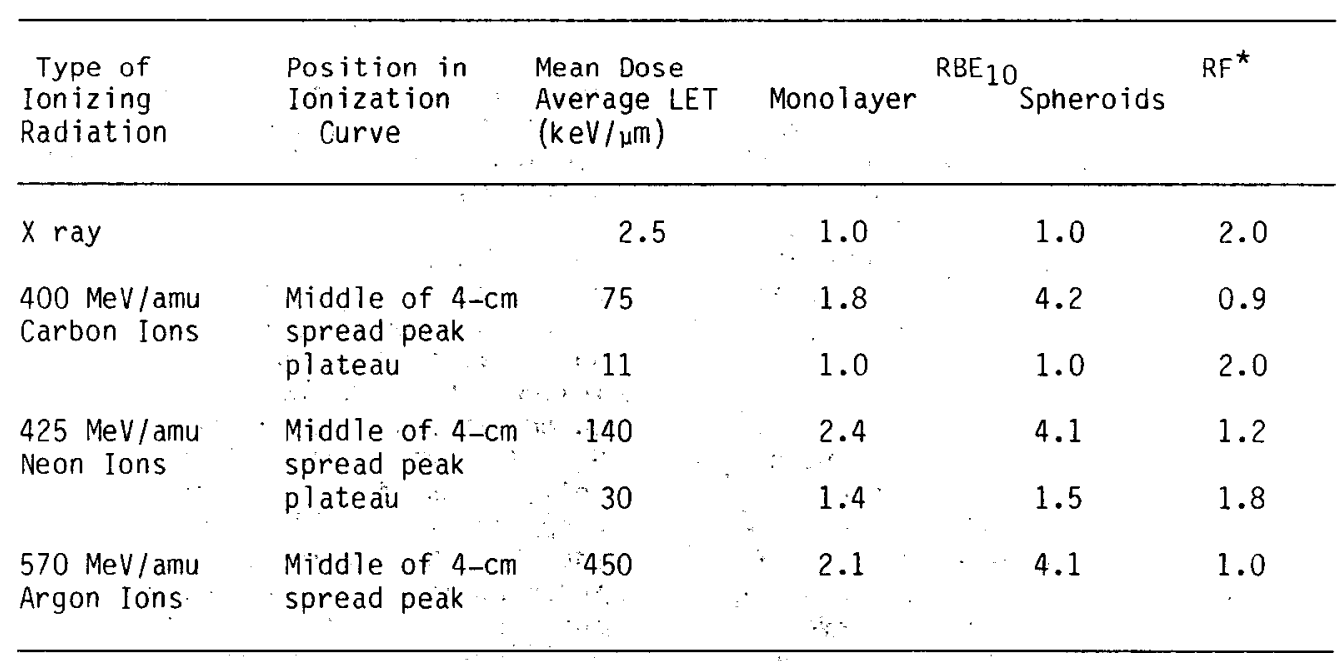

\footnotetext{
* Spheroid to monolayer dose ratio at $10 \%$ survival.
} 
CARBON IONS NEON IONS ARGON IONS

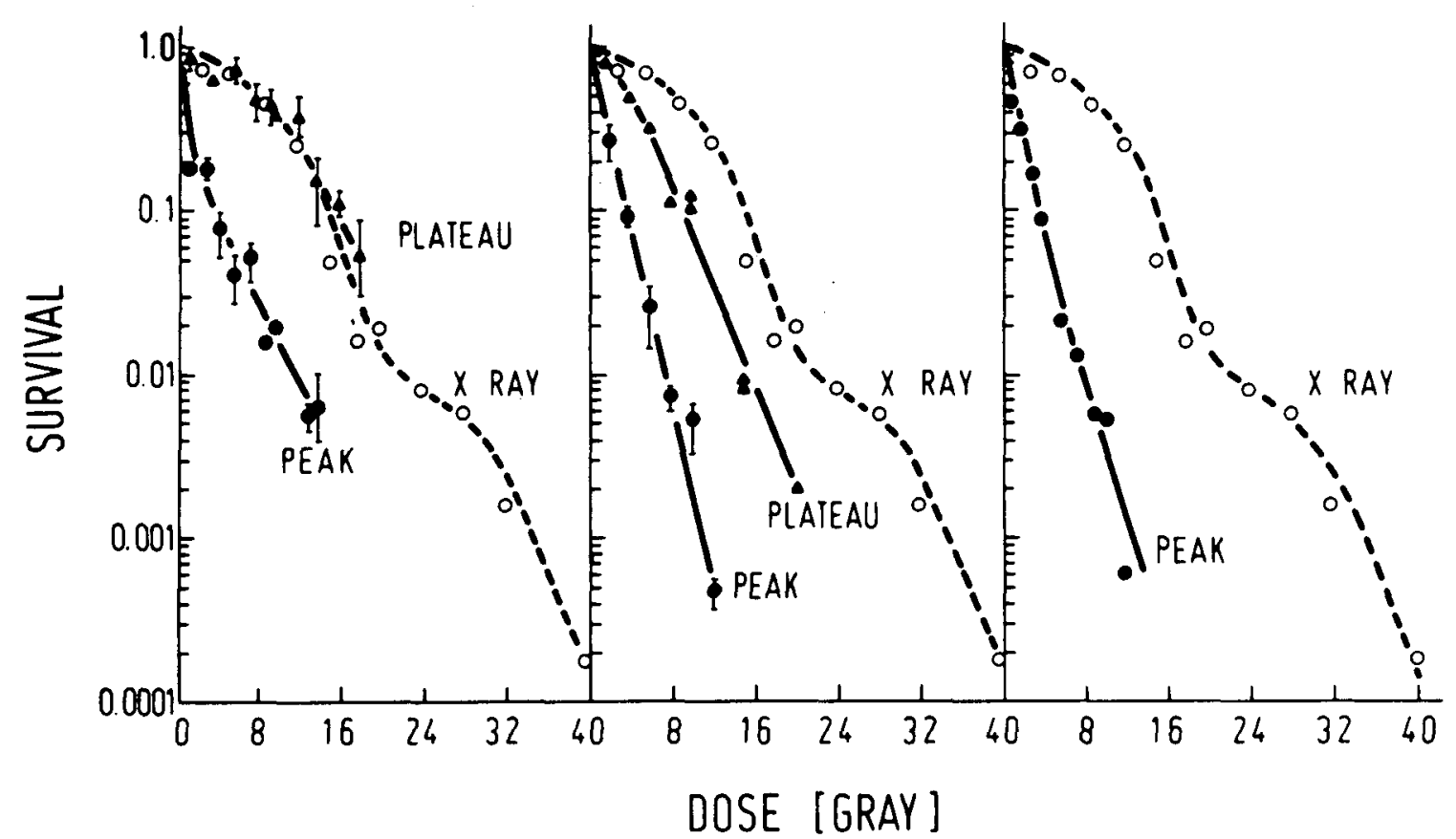

Figure 10. Survival of V79 spheroid cells after exposure to heavy-ion beams at a plateau (1) and midpeak (0) position. Each point represents the mean $\pm S . E$. Argon data are from one experiment only. For comparison, survival after X-ray exposure (0) is included. (XBL 808-10891)

For the radiotherapist, the effectiveness of spread peaks in relation to the plateau is of special interest. Although the beams we used did not have the same range, the peak/plateau RBE ratio in our experiments was most advantageous for the longer ranged carbon beam. Healthy tissue in front of a tumor would be protected by a factor of 4.2 as compared to cell killing of the tumor cells centered within the spread carbon peak. For neon ions the peak/plateau ratio was only 2.7 indicating a higher dose average LET at the neon midpeak compared to carbon. For the argon beam no plateau data were obtained. A maximum RBE gain for the carbon beam agrees with the results of Chapman et al. (1977) using the same beam for V79 suspension cultures and $\mathrm{T}_{1}$ monolayer cultures.

High LET radiation as used in this study is known to eliminate a significant portion of: hypoxic cells (Chapman et al., 1978). Spheroids of a size used in this study have about $4 \%$ to $6 \%$ of such cells as indicated by back extrapolation of the second shoulder in the survival curve (Sutherland and Durand, 1976). The dose-effect curves obtained for spheroids after heavy-ion irradiation certainly demonstrate a marked effect on that population by diminishing the second shoulder of the survival curve. However, doses applied in this set of experiments were not $h i g h$ enough to allow an exact estimation of the amount of hypoxic cells being killed.
Figure 11 shows a series of DNA histograms that demonstrate the cell cycle kinetics of outer cycling and inner noncycling spheroid cells after exposure to 320 rad midpeak neon ions, a dose that may be used in fractionation protocols for radiotherapy. The DNA distributions of outer and inner control cells differ in their amount of $G i$ and $S$ phase cells. After irradiation and subsequent incubation at $37^{\circ} \mathrm{C}$, inner noncycling cells showed hardly any kinetic change while outer cycling cells rapidly began to accumulate in $\mathrm{G}_{2}+M$ phase.

The mathematical analysis of the DNA distributions at 24 hours after exposure to neon revealed a maximum of $G_{2}+M$ accumulation of $35 \%$ at 10 hours postirradiation. The drop in $G_{1}$ followed by a decrease of cells in $S$ phase for outer cells is the result of that block in $G_{2}+M$. There was a slight increase of $S$ phase cells in the inner cell fraction and a very small decrease of $\mathrm{G}_{1}$ cells, however, not significant enough to be interpreted as a recruitment of inner cells due to the block in $G_{2}+M$ of the outer cells. A dose of 800 rad of carbon ions yielded a $\mathrm{G}_{2}+M$ accumulation of $61 \%$, 8 hours postirradiation exposure (data not shown), but still there was no indication for a reactivation of inner noncycling cells until 120 hours after irradiation.

The formation of that block agrees with the findings described for monolayer cells of the 


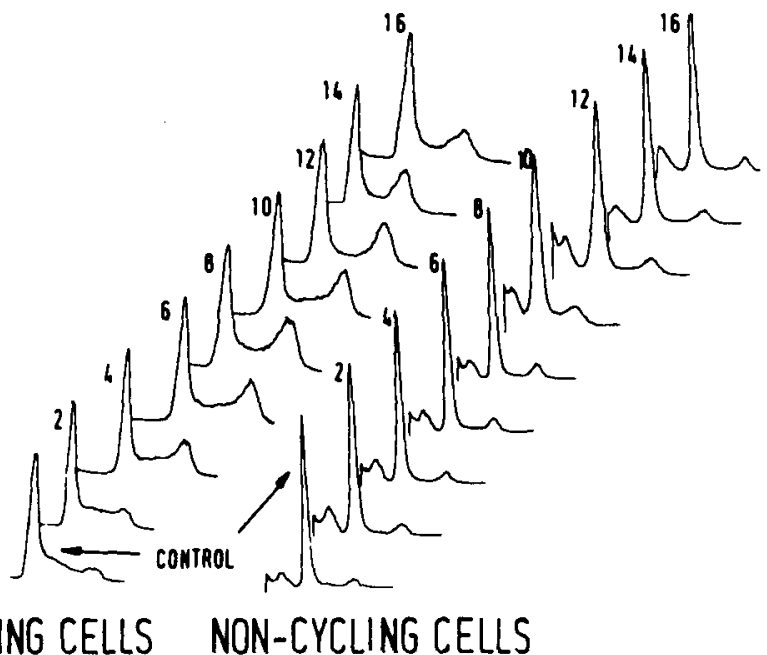

Figure 11. DNA-distributions of outer cycling and inner noncycling spheroid cells before and until $16 \mathrm{~h}$ after exposure to $320 \mathrm{rad}$ midpeak neon ions. (XBL 808-10892)

same cell line. However, the extent of blockage is different at equal doses. Spheroids show a smaller effect with respect to $G_{2}+M$ blockage. An explanation might be that the outer cell fraction of spheroids (obtained by fractionated trypsinization) is somewhat contaminated by noncycling cells leading to quantitatively smaller changes if compared to purely exponentially growing monolayer cells.

However, the block in $G_{2}+M$ caused by high-LET irradiation was considerably higher than that found after Co gamma irradiation (Dertinger and Seiter, 1977), a fact of interest for radiotherapy protocols. Nevertheless, the large number of noncycling cells that did not respond in any way detectable by the method of . flow-microfluorometric evaluation.will be still a problem. The reason for no cycle changes in the inner cells may be that the cells are "crowded." Had we allowed more time for celis to die and lyse, more nutrients may have been available to start the inner cells cycling.:. We know that this occurs in actual tumors.

In conclusion, the fact that the increased resistance of cells growing under extensive three-dimensional contact, which benefits is $\mathrm{u}$ survival after low LET radiation, was not , detected after irradiation with heavy jons at a $\ldots$. spread peak should provide an additional. advantage in the radiotherapy planned with heavy charged particle beams.

\section{GOALS FOR THE COMING YEAR}

For the coming year we will gather more data to complete the single dose survival curves for cells grown in suspension and as spheroids. In addition to cell survival, we will be examining the growth kinetics of intact spheroids and develop models for spheroid growth evaluation. We will also determine a TCD-50 dose for the carbon, neon, argon, and helium beams.

We will initiate studies of cell kinetics in spheroids after irradiation. Perturbations in cell-cycle distribution will be analyzed at selected doses for intact spheroids at periods after irradiation. This analys is will be made using flow-microfluorometric and tritium labeled thymidine techniques.

We will also initiate divided dose experiments to directly evaluate the effects of spheroid architecture on repair of sublethal and potentially lethal injury.

\section{ACKNOWLEDGMENTS}

These studies are supported by a National Research Service Postdoctoral Fellowship (PHS Grant 2-32C009272), A. Rodriguez, recipient; U. S. National Cancer Institute (Grant No. CAA-15184), C. A. Tobias, Principal Investigator; and U. S. National Cancer Institute (Grant No. 1-R01-CA24789-01), E. L. Alpen, Principal Investigator.

\section{REFERENCES}

Blakely, E. A., C. A. Tobias, T. C. Yang, K. C. Smith, and 3. T. Lyman. 1979. Inactivation of human kidney cells by high-energy monoenergetic heavy-ion beams. Radiat. Res. 80 , $122-160$.

Carlsson, C. H., C. G. Stalnacke, H. Acker, M. HajiKarim, S. Nilsson, and B. Larsson. 1979. The influence of oxygen on viability and proliferation in cellular spheroid. Int. J. Radiat. Oncol. Biol. Pnys. 5, 2011-2020.

Chapman, J. D., E. Blakely, K. C. Smith, and R. C. Urtasun. 1977. Radiobiological characterization of the inactivating events produced in mammalian cells by helium and heavy ions: Int. J. Radiat. Oncol. Biol. Phys. 3, 97-102.

Chapman, J. D., E. Blakely, K. C. Smith, R. C. Urtasun, J. T. Lyman, and C. A. Tobias. 1978. Radiation biophysical studies with mammalian cells and a modulated carbon ion beam. Radiat: Res. 74, 101-111.

Dertinger, H. C. and 0. Seiter. 1977. Splitdose survival and cycle progression of proliferating and non-proliferating cells in V79 spheroids. Int. J. Radiat. Bio1. 31, 401-403.

Dertinger, H. C., C. Lücke-Hühle, H. Schlag, and K. T. Weibizahn. 1976. Negative pion irradiation of mammalian cells I. Survival characteristics of monolayers and spheroids of Chinese hamster lung cells. Int. J. Radiat. Biol. 29, 271-277. 
Durand, R. E. 1975. Isolation of cell subpopulations from in vitro tumor models according to sedimentation velocity. Cancer Res. 35, 1295-1300.

Durand, R. E. 1976. Cell cycle kinetics in an in vitro tumor model. Radiat. Res. 81, 85-99.

Durand, R. E. 1980. Variable radiological response of spheroids. Radiat. Res. 81, 85-99.

Durand, R. E. and J. E. Biaglow. 1974. Modification of the radiation response of an in vitro tumor model by control of cellular. respiration. Int. J. Radiat. Biol. 26, 597-601.

Durand, R. E. and P. L. 01 ive. 1976. Irradiation of multicell spheroids with fast neutrons versus x-rays: A qualitative difference in sub-lethal damage repair capacity of kinetics. Int. J. Radiat. Biol. 30, 583-592.

Durand, R. E. and P. L. 01ive. 1977. Fast neutron effects on multicell spheroids. Br. J. Radio 1. 50, 423-429.

Durand, R. E. and R. M. Sutherland. 1972. Effects of intercellular contact on repair of radiation damage. Exp. Ce11. Res. 71, 75-80.

Durand, R. E. and R. M. Sutherland. 1973. Dependence of the radiation response of an in vitro tumor model on cell cycle effects. Cancer Res. 33, 213-219.

Durand, R. E. and R. M. Sutherland. 1975. Intercellular contact: Its influence on the Dq of mammalian cell survival curves. Cell Survival After Low Doses of Radiation: Theoretical and Clinical Implications. Proceedings of the Sixth L. H. Gray Conference, held at Bedford College, London, 16-21 September 1974 (Tikvah Alper ed.) pp. 237-247. John Wiley Sons, New York.
Inch, W. R., J. A. Mccredie, and R. M. Sutherland. 1971. Growth of multicell nodular carcinomas in rodents compared with multicell spheroids in tissue culture. Growth 34 , 271-281.

Leith, J. T., V. Arcellana, J. T. Lyman, and K. T. Wheeler. 1975a. Response of a rat brain tumor to irradiation with accelerated neon ions. Int. J. Radiat. Biol. 28, 91-97.

Leith, J. T., A. A. Schilling, and K. T. Wheeler. 1975b. Cellular radiosensitivity of a rat brain tumor. Cancer 35, 1545-1550.

Raju, M. R., E. Blakely, J. Howard, J. T. Lyman, D. P. Kolofonos, B. Martins, and C. H. Yang. 1976. Human cell survival as a function of depth for a high energy neon ion beam. Radiat. Res. 65, 191-194.

Sutherland, R. M. 1974. Selective chemotherapy of noncycling cells in an in vitro tumor model. Cancer Res. 34, 3501-3503.

Sutherland, R. M. and R. E. Durand. 1973. Hypoxic cells in an in vitro tumor model. Int. J. Radiat. Biol. 23, 235-246.

Sutherland, R. M., W. R. Inch, J. A. McCredie, and J. Kruuv. 1970. A multi-component radiation survival curve using an in vitro tumor model. Int. J. Radiat. Biol. 18, 491-495.

Suther land, R. M., J. A. Mccredie, and W. R. Inch. 1971. Growth of multicell spheroids in tissue culture as a model of nodular carcinomas. J. Nat1. Cancer Inst. 46, 113-120.

Yuhas, J. M. and A. P. Li. 1978. Growth fraction as the major determinants of multicellular tumor spheroid growth rates. Cancer Res. 38, 1528-1532. 


\title{
THE RELATIVE BIOLOGICAL EFFECT OF HIGH Z, HIGH LET CHARGED PARTICLES FOR SPERMATOGONIAL KILLING
}

\author{
E.L. Alpen and P. Powers-Risius
}

Early in the development of radiation biology as a new science the testis was the subject of investigation by research groups. However, Lorenz et a1. (1947) and Eschenbrenner et al. (1948) established the first quantitative basis for the effects of ionizing radiation on the organ. Further developments of definitive models for weight loss of the testes as an analytical tool for cell survival studies of the germinal epithelium of the mouse testis were then set out by Kohn and Kallman $(1954,1955)$. They concluded that weight loss is an exponential function of the radiation dose, and that weight loss is due primarily to cell death in the germinal epithelium, an observation that is well correlated with histological change. The model of Kohn and Kallman has been widely used in examining the relative effectiveness of radiation of varying LET, some of which has been reviewed by Geraci et al. (1977).

A more sophisticated treatment of testis weight loss data was reported by Krebs (1968), but, unfortunately this method is not available in the open literature. The Krebs' method assumes that the residual weight of the testis at minimum postirradiation value is represented by the sum of two exponential relationships: one describing the cell survival of the radiosensitive spermatogonia, and the second describing the cell loss in the radioresistant interstitial cell fraction. Using this model, the first exponential relationship provides the usual parameters, $D_{0}$ and $n$, for the cell survival curves. The Krebs' method is an improvement over the Kohn and Kallman method since it corrects for dose dependent weight loss in the insensitive fraction which Kohn and Kallman assumed to be unaffected by the radiation dose. Interestingly, if one computes the Do from Kohn and Kallman's exponential function it is approximately $90 \mathrm{rad}$, which compares with the Krebs $D_{0}$ of 80 rad for the same radiation source (250 kVp $x$ rays $)$.

Early workers, as well as Kohn and Kállman, have reported that fractionation of the dose over hours to a few days has no effect "on weight loss. Krebs reports an extrapolation number of 1.0 for his testis cell survival function which, in light of more modern interpretations of sublethal injury, would confirm lack of recovery in this cell survival system.

It has been our purpose to examine the : effects of high LET radiations produced with accelerated heavy-ion beams on this cell survival system. If the extrapolation number is indeed shown to be 1.0 for this system, the effects of LET can be examined on the exponential cell survival function, independent of the confounding variable of the presence of a shoulder.

\section{BEAM CHARACTERISTICS AND DOSIMETRY}

For these studies we used helium ions at an initial energy of $228 \mathrm{MeV} / \mathrm{n}$ produced at the 184 inch Synchrocyclotron; the other ions used were produced on the Bevalac, in which the fully stripped ions were first accelerated to $8.5 \mathrm{MeV} /$ nucleon in the SuperHILAC, followed by injection into the Bevatron. The particle energies and associated LET values are shown in Table 1.

Further details of the 184 inch Synchrocyclotron and the Bevalac are given by Curtis et al. (1978) and by Lyman and Howard (1977). Curt is et al. also describe the beam line arrangement and animal exposure facilities in some detail. Further details of dosimetric methods are provided by Blakely et al. (1979). The dosimetry of the ion beams was accomplished with parallel plate ionization chambers in the beam line. The ion chamber closest to the animal was used to determine accumulated dose and to terminate the exposure at the preset value of dose. This system is also described by curtis et al. (1978).

The heavy-ion beams in their unmodulated state have Bragg peaks only a few millimeters in width, an irradiation format that is not particularly useful except for thin layers of tissue, or for cells. To produce more useful beams the Bragg peak is spread by rotating spiral ridge filters of brass. These beams in their modulated form are also used in patient therapy. In the studies reported here, the heavy ions are spread to $4 \mathrm{~cm} ;$ a $10 \mathrm{~cm}$ spread configuration was used for the helium ions. The depth dose distributions are shown in $F$ igure 1 for the ions and energies used. Also shown in Figure 1 are the positions in which radiation exposures were made to plateau, proximal, and distal modified peak regions. The irradiation position in the Bragg peak is controlled by the interposition a var $i$ able water column absorber equivalent to the depth positions shown in Figure 1. These various residual ranges allow the selection of a wide rangé of LET values for a given ion.

Ion irradiations were done individually with a $3 \times 5 \mathrm{~cm}$ collimated field centered on the scrotum and including the lower abdomen of the mouse. The ion dose rates were selected in the range of 25-200 rad per minute by attenuation of the ion beams to lower dose rates, which permitted acceptable exposure times of 1-3 minutes per mouse over the whole dose range used.

Low-LET irradiations were done with a ${ }^{60} \mathrm{Co}$ teletherapy source with the same mouse exposure field, but seven mice were exposed simultaneously. Dosimetry for the 60 Co source was done with a Victoreen condenser R-meter which had been 
Table 1. Physical Characteristics for Heavy-Ion Beams

\begin{tabular}{|c|c|c|c|}
\hline $\begin{array}{c}\text { Ion } \\
\text { (Energy) }\end{array}$ & $\begin{array}{l}\text { Position in } \\
\text { Bragg Peak }\end{array}$ & Designation & $\begin{array}{l}\text { Dose Average } \\
\text { LET (keV/ } / \mu \mathrm{m})\end{array}$ \\
\hline $\begin{array}{l}{ }^{4} \mathrm{He} \text { lium } \\
\text { (228 MeV/amu) }\end{array}$ & $\begin{array}{l}\text { Plateau } \\
\text { Proximal peak } \\
\text { Distal peak }\end{array}$ & $\begin{array}{l}0 \\
D \\
A\end{array}$ & $\begin{array}{l}1.6 \\
4 \\
6\end{array}$ \\
\hline $\begin{array}{l}12 \text { Carbon } \\
(440 \mathrm{MeV} / \mathrm{amu})\end{array}$ & $\begin{array}{l}\text { Plateau } \\
\text { Proximal peak } \\
\text { Distal peak } \\
\text { Distal }+0.5 \mathrm{~cm} \\
\text { Tail }\end{array}$ & $\begin{array}{l}0 \\
D \\
A \\
B \\
C\end{array}$ & $\begin{array}{r}11 \\
55 \\
85 \\
105 \\
30\end{array}$ \\
\hline $\begin{array}{l}20 \text { Neon } \\
(420 \mathrm{MeV} / \mathrm{amu})\end{array}$ & $\begin{array}{l}\text { Plateau } \\
\text { Proximal peak } \\
\text { Distal peak } \\
\text { Distal }+0.5 \mathrm{~cm} \\
\text { Tail. }\end{array}$ & $\begin{array}{l}0 \\
D \\
A \\
B \\
C\end{array}$ & $\begin{array}{r}35 \\
110 \\
150 \\
225 \\
100\end{array}$ \\
\hline $\begin{array}{l}40 \text { Argon } \\
(670 \mathrm{MeV} / \mathrm{amu})\end{array}$ & $\begin{array}{l}\text { Plateau } \\
\text { Proximal peak } \\
\text { Distal peak }\end{array}$ & $\begin{array}{l}0 \\
D \\
A\end{array}$ & $\begin{array}{r}90 \\
380 \\
650\end{array}$ \\
\hline
\end{tabular}

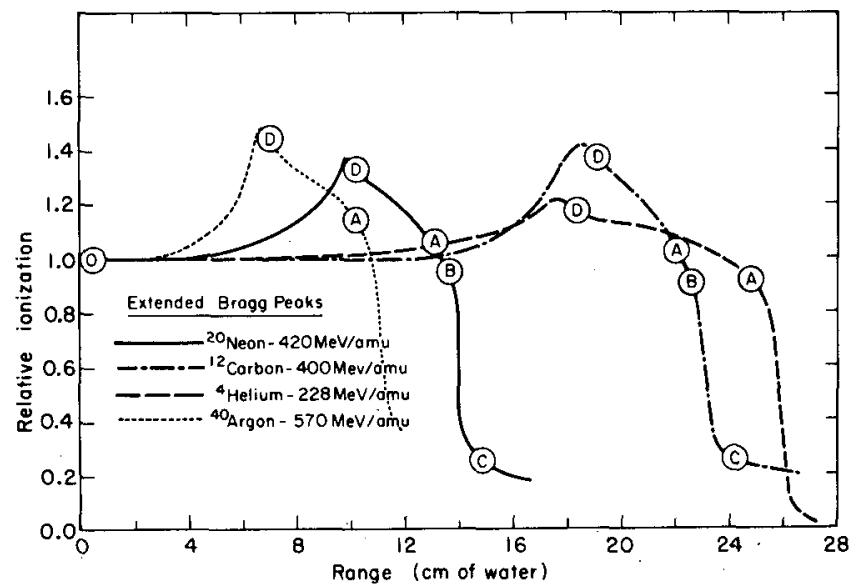

Figure 1. Depth dose distributions for ion beams utilized in this study. The irradiation positions are lettered so that they correspond to the texts and tables: " position " 0 " is in the plateau region of ionization; position " $D$ " is the proximal position of the Bragg peak, position "A" is the distal peak position, position "B" is the far distal region of the peak, and position " $C$ " is the tail region of the Bragg peak. (XBL 809-3669) calibrated against an NBS secondary standard. The dose rate used was in the same range as that used for heavy-ion beams.

\section{METHODS}

The mice used were $\mathrm{B}_{6} \mathrm{D}_{2} \mathrm{f}_{1}$ male mice, 12-15 weeks old at the start of the experiment, which were obtained from Jackson Laboratories, Bar Harbor, Maine.- They were housed seven to a cage with food and water ad libitum, both before and after irradiation. Prior to irradiations, all animals were lightly anesthetized with sodium pentobarbital, as were the control unirradiated mice.

At the time of sacrifice the animals were killed by cervical dislocation, the testes were removed, and dissected free of the epididymides. The two testes were weighed together on a Cahn microbalance to the nearest $0.1 \mathrm{mg}$. Occasionally anatomic defects are noted at sacrifice; for example, single testis, atrophied testis, or grossly abscessed testis. In these infrequent cases both testes are discarded and data were not recorded. 
DATA ANALYSIS

The data were analyzed without grouping. Analys is was done by a computer fitted regression analys is to the data of the form:

$$
W_{D}=W_{S} e^{-k} S^{D}+w_{I} e^{-k} I^{D}
$$

where

$$
\begin{aligned}
& W_{D}=\text { remaining testes weight after dose } D \\
& W_{S}=\text { radiation sensitive weight in the } \\
& \text { testes at } D=0 \\
& W_{I}=\text { radiation insensitive weight in the } \\
& \text { testes at } D=0 \\
& k_{S}=\text { inactivation constant for the radia- } \\
& \text { tion sensitive fraction } \\
& k_{I}=\text { inactivation constant for the radia- } \\
& \text { tion insensitive fraction }
\end{aligned}
$$

Since $W_{S}, W_{I}, k_{S}$ and $k_{I}$ are all unknown, the best fit is achieved by the computer in an iterative fashion. It is assumed that the slope of the response at high dose is due solely to the I subscript terms, and an initial value for $W_{I}$ and $k_{I}$ are computed. With the preliminary parameters for the insensitive fraction, the initial weights for all values of dose are corrected to obtain a first estimate of the regression equation for the sensitive fraction.
With a first estimate of $W_{S}$ and $k_{S}$, the parameters are iteratively adjusted until stability or convergence of estimate of the parameters is obtained. Of course, the parameters of greatest interest are $W_{S}$ and $\mathrm{ks}$, and from these parameters the extrapolation number and the $D_{0}(1 / \mathrm{ks})$ are derived.

Statistical analysis of data so intricately derived from the original source data is, at best, difficult. To achieve homogeneity of variance for the fit of the regression equations, testes weights are weighed by a factor equal to the absolute value of the testes weight. Since the smaller variance is associated with the larger weights, this procedure assigns more importance to the larger weights in determining the best fit regression. Confidence limits for the $D_{0}$ and the extrapolation numbers have been determined by directly calculating the $95 \%$ confidence limit values for the slope of the regression. From these slope limits, the equivalent $95 \%$ confidence limits for the $D_{0}$ are computed from the reciprocal of the slope, and for the extrapolation number from the respective intercepts at $0=0$. Since the confidence limits for these latter values are for derived parameters, we have refrained from statistical comparisons such as student $t$ tests for $D_{0}$ or RBE values.

Figure 2 shows the original data for two typical data sets: ${ }^{60} \mathrm{Co}$ and plateau $20_{\mathrm{Ne}}$.
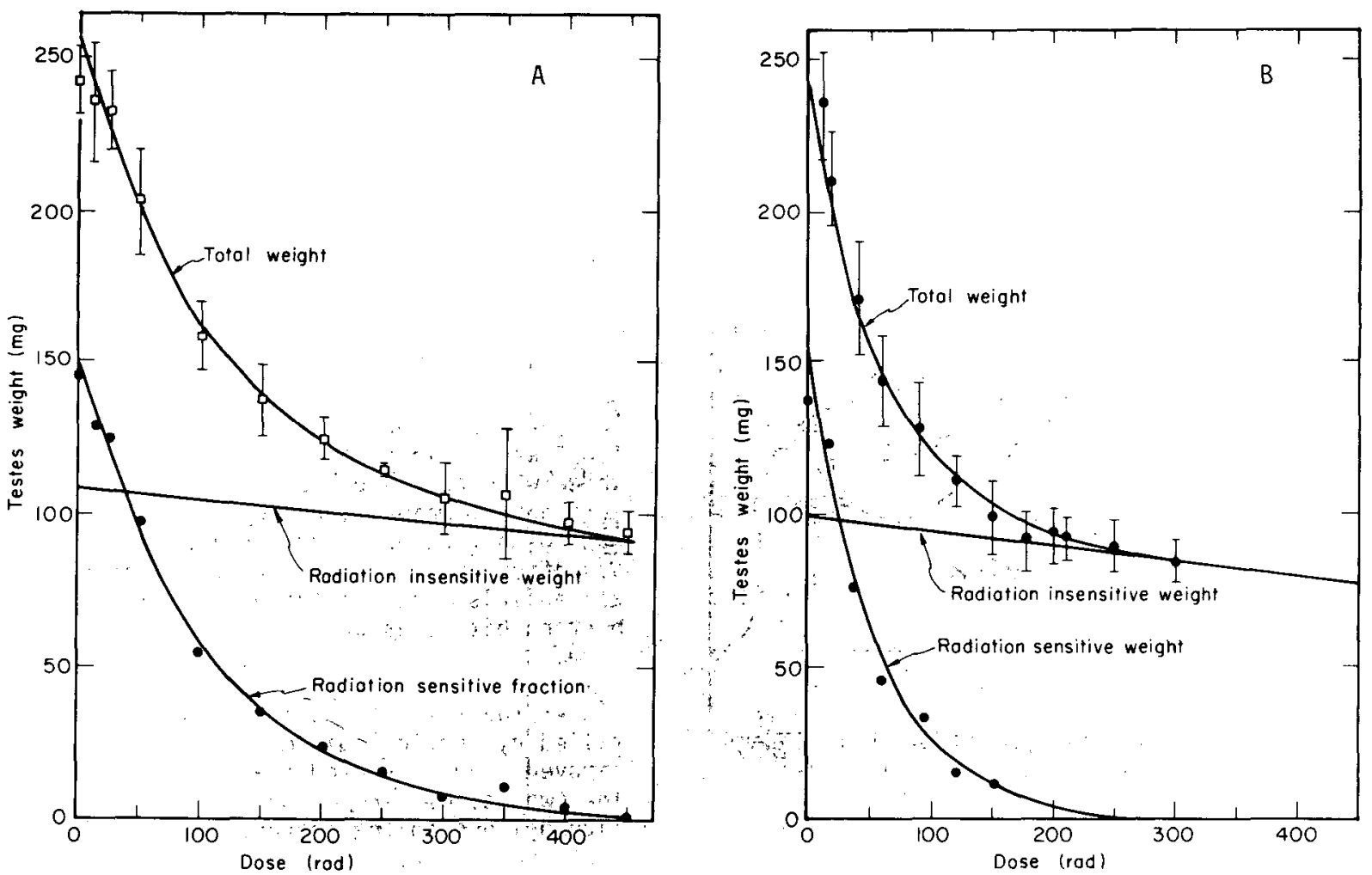

Figure 2. Typical curves for testis weight loss as a function of radiation dose, showing the fitted curves derived from the original data for the sensitive fraction and insensitive fraction. Panel $A$ is data for 60 Co irradiation and Pane $1 B$ is data for irradiation with neon ions in the plateau region of the peak. (XBL 807-3447) 
The $95 \%$ confidence limits for weights associated with each dose point are shown, but it should be noted that the regression analys is is done on nongrouped data.

\section{RESULTS}

Before starting the major studies on effect of LET on testes weight loss, it was necessary to establish appropriate sacrifice times for the high LET exposures. Both Kohn and Kallman (1955) and Krebs (1968) had carefully studied the time dependence of weight loss. Their results show that, for $X$ rays, a nadir is reached between days 24 and 34 and that analytic outcome was not significantly affected by the choice of sacrifice day in this time window. To assure that the same time dependence existed for the heavy-ion irradiations, studies were done on time dependence of weight loss for a wide range of doses and LET values. The data all confirm that the nadir occurs at the same time, regardless of LET. Rather than present these data, which are confirmatory, we have chosen to show graphically in Figure 3, the time dependence of weight loss for ${ }^{60} \mathrm{Co}$ exposure and one $20 \mathrm{Ne}$ exposure condition.
The increased biological effectiveness of the neon beam is easily seen in the increased weight loss at lower dose; however, it is equally clear that the time of the weight-loss nadir is essentially the same for the two radiation types, even though it takes several hundreds of rad more dose to achieve this nadir with the low LET radiation. Given these data we choose to continue to do all animal sacrifices at 28 days after exposure.

The $D_{0}$ values for weight loss of the radiation-sensitive weight fraction of mouse testes for all radiation conditions are shown in Table 2. The $D_{0}$ for 60 Co given in this table is 107 rad, with a 95\% confidence limit of about 6 rad. This can be compared to Krebs' value of $80 \mathrm{rad}$ for $250 \mathrm{kVp} \times$ rays. 0ther values are not easily comparable, since different analytical methods were used for the data. Typically however, Kohn and Kallman (1955) found a value of $90 \mathrm{R}$ and Geraci et al. (1977) report a $0_{50}$ for $250 \mathrm{kVp} \times$ rays, which can be calculated to give a $D_{0}$ of 350 rad.

The Do values for the various heavy ions at wide ranging LET values vary from a low of
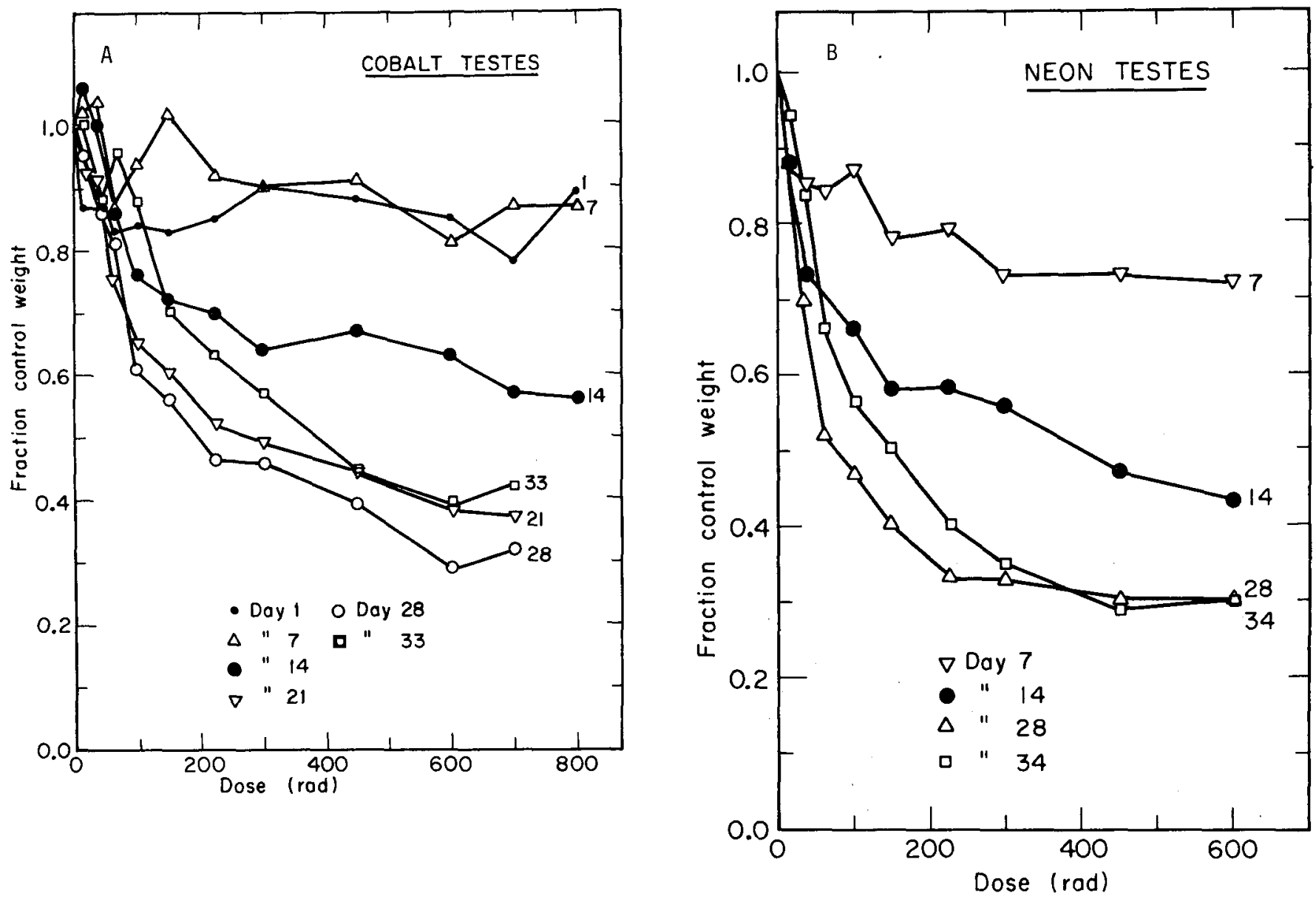

Figure 3. Dose dependency of testes weight loss as a function of the day of sacrifice for (A) ${ }^{60} \mathrm{Co}$ radiation and $(B)$ neon ions in the plateau region of ionization. [(A) XBL 784-3058; (B) XBL 784-3059] 
Table 2. Radiation Survival Curve Parameters for the Radiation Sensitive Weight Loss Fraction of Mouse Testes

\begin{tabular}{|c|c|c|c|}
\hline $\begin{array}{l}\text { Radiation } \\
\text { Modality }\end{array}$ & $D_{0} 95 \% \mathrm{CI}$ & $\begin{array}{l}\text { apolation } \\
\text { umber }\end{array}$ & $\begin{array}{r}\mathrm{RBE}^{\star} \\
95 \% \mathrm{CI}^{\dagger}\end{array}$ \\
\hline${ }^{60} \mathrm{Co}$ & $109(101-113)$ & 1.12 & --- \\
\hline $\begin{array}{l}4_{\text {Helium }} \\
\text { Plateau }(0) \\
\text { Proximal peak (D) } \\
\text { Distal peak (A) }\end{array}$ & $\begin{array}{ll}94.5 & (87.9-102) \\
94.6 & (85.6-106) \\
82.8 & (74.4-93.5)\end{array}$ & $\begin{array}{l}1.06 \\
1.06 \\
1.02\end{array}$ & $\begin{array}{ll}1.13 & (1.05-1.21) \\
1.13 & (1.01-1.25) \\
1.29 & (1.14-1.44)\end{array}$ \\
\hline $\begin{array}{l}{ }^{12} \text { Carbon } \\
\text { Plateau }(0) \\
\text { Proximal peak (D) } \\
\text { Distal peak (A) } \\
\text { Distal +0.5 cm (B) } \\
\text { Tail (C) }\end{array}$ & $\begin{array}{l}75.8(70.1-82.6) \\
71.4(66.6-77.0) \\
64.2(58.3-71.4) \\
55.2(43.8-74.6) \\
68.4(56.4-88.2)\end{array}$ & $\begin{array}{l}1.05 \\
0.96 \\
0.96 \\
0.94 \\
1.18\end{array}$ & $\begin{array}{l}1.41(1.30-1.53) \\
1.49(1.39-1.62) \\
1.69 \\
1.94(1.50-1.83) \\
1.56(1.45-2.45) \\
1.22-1.89)\end{array}$ \\
\hline $\begin{array}{l}20 \text { Neon } \\
\text { Plateau }(0) \\
\text { Proximal peak }(D) \\
\text { Distal peak }(A) \\
\text { Distal }+0.5 \mathrm{~cm}(B) \\
\text { Tail }(C)\end{array}$ & $\begin{array}{l}55.9(49.0-65.2) \\
47.9(42.3-55.2) \\
58.8(54.8-63.4) \\
69.2(58.0-85.9) \\
53.6(44.6-67.2)\end{array}$ & $\begin{array}{r}1.11 \\
1.00 \\
.97 \\
.98 \\
1.10\end{array}$ & $\begin{array}{l}1.91(1.64-2.18) \\
2.20(1.94-2.52) \\
1.82(1.69-1.95) \\
1.55(1.27-1.88) \\
2.00(1.62-2.44)\end{array}$ \\
\hline $\begin{array}{l}{ }^{40} \text { Argon } \\
\text { Plateau }(0) \\
\text { Proximal peak (D) } \\
\text { Distal peak (A) }\end{array}$ & $\begin{array}{ll}35.4 & (29.7-43.8) \\
59.6 & (53.7-67.0) \\
58.3 & (50.2-69.6)\end{array}$ & $\begin{array}{l}0.88 \\
0.87 \\
0.99\end{array}$ & $\begin{array}{l}3.02(2.40-3.67) \\
1.79(1.62-2.02) \\
1.83(1.57-2.17)\end{array}$ \\
\hline \multicolumn{4}{|c|}{$\begin{array}{l}\text { * The RBE is calculated as the ratio of } D_{0}\left({ }^{60} \mathrm{Co}\right) \text { divided by } D_{0} \\
\text { (ion). }\end{array}$} \\
\hline $\begin{array}{l}\text { The confidence } 1 \mathrm{im} \\
\text { computed by taking } \\
\mathrm{D}_{0} \text { and the } 60 \mathrm{Co} \mathrm{D}_{0}\end{array}$ & thas no ri & int & $\begin{array}{l}\text { e, since it } \\
\text { the ion }\end{array}$ \\
\hline
\end{tabular}

30 rad to a high of 95 rad. The ratio of $D_{0}$ values to give derived RBEs are shown in the last column of Table 2, and these range from 1.1 to 3 .

The extrapolation numbers in column 4 of Table 2 show that we have no evidence to reject the hypothesis that the extrapolation number differs significantly from 1.0. The mean extrapolation number for all ions at all positions is $1.01 \bullet 0.06$.

Since an extrapolation number near unity predicts no recovery from sublethal injury, we chose a fractionated exposure design to examine this possibility. A direct comparison was made in three positions of the carbon-ion Bragg peak: plateau, proximal peak, and distal peak. Mice were irradiated either with single doses or with the dose divided in three equal fractions given over five days. The ratio $D_{0}$ (single)/ $/ D_{0}$ (fractionated) for the three positions was found to be $0.85,0.95$, and 1.08 for the plateau, proximal, and distal peaks, respectively. These data offer convincing proof that little or no recovery of sublethal injury occurs in this cell renewal system.

Several other observations can be drawn from the data in Table 2. Initial experiments were done for the exposure conditions as the plateau (0); proximal peak (D); and distal peak, which conform to the regions of plateau ionization, initial rise of the Bragg peak of ionization, and the maximum range, respectively. Each of these positions correspond to increasing LET with movement towards maximum range.

Since the $D_{0}$ appeared to be changing rapidly with LET in the terminal range region of the curve, and since the $D_{0}$ for distal neon was 
nigher than that for the midpeak, we decided to examine in closer detail the dependence of $D_{0}$ on LET in the terminal range regions of the Bragg curve. The additional measurements were made at a position $0.5 \mathrm{~cm}$ (position B) further than the previous distal position, and in the tail of the Bragg curve (position C) for carbon and neon. These data along with the earlier determinations are shown in Figure 4, where position $A$ is the original distal peak exposure, position $B$ is the distal $+0.5 \mathrm{~cm}$ position, and $C$ is the Bragg tail position. To assure comparability with the original data, a repeat determination was done at position A for neon. The original neon distal peak $D_{0}$ was $57.5 \pm 4.9 \mathrm{rad}$. The replicate determination yielded a $D_{0}$ of $56.1 \pm 3.6 \mathrm{rad}$, which is in good agreement with the earlier determination.

For neon (Fig. 4), the more distal position showed a continuing increase in $D_{0}$ over that for the earlier distal position, which confirms that, for neon, the peak effectiveness is aiready surpassed at both positions. The LET values associated with position $A$ and $B$ are $105 \mathrm{keV} / \mu \mathrm{m}$ and $150 \mathrm{keV} / \mathrm{\mu m}$, respectively. In the tail of the neon Bragg peak, there is again increased effectiveness associated with a lower $(100 \mathrm{keV} / \mu \mathrm{m})$ LET value. However, this value is not that significant because a large fraction of the radiation dose in the tail is from nuclear fragments of neon of lower mass than the original ion.

For carbon ions (Fig. 5), the far distal peak (position 8 ) shows a further significant decrease

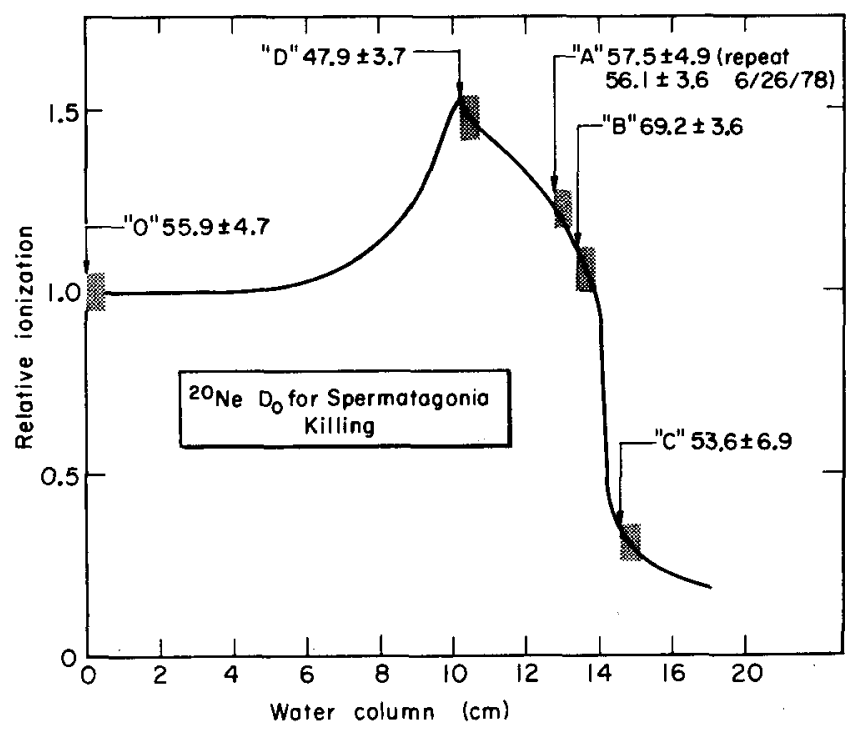

Figure 4. The $D_{0}$ determinants are shown associated with the irradiation position in the neon $4 \mathrm{~cm}$ extended Bragg peak. The positions annotated are as described in the text. The $D_{0}$ values are given with the ir 95\% confidence intervais. At position (A), the $D_{0}$ values are determined in two replicate studies performed several months apart. (XBL 7810-3640)

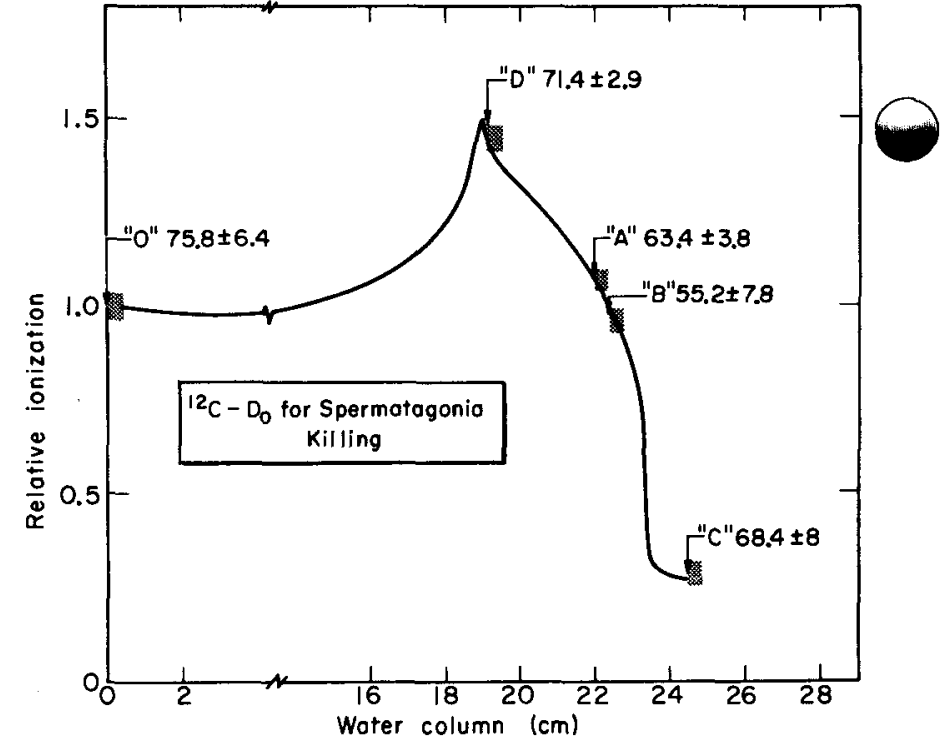

Figure 5. The same as Figure 4, but for carbon ions. The $D_{0}$ values associated with the irradiation position shown are given with their 95\% confidence limit. (XBL 7810-3639)

in $D_{0}$, indicating yet increasing effectiveness at an LET of $100 \mathrm{keV} / \mathrm{um}$ with this ion. The tail again shows decreased effectiveness associated with a lower LET (position C, $70 \mathrm{keV} / \mu \mathrm{m}$ ) and the presence of fragments. Figure 5 also shows the calculated RBE values for the various ions and LET values. The beam positions are indicated by a letter that refers to the beam positions identified in Figures 1,4 , and 5 .

These data are given without confidence limits since, as mentioned earlier, it is not readily possible to determine confidence limits for such highly derived data with sound statistical procedures. The confidence limits for the $D_{0}$ values are obtained by computing the $95 \%$ confidence interval values for the slope of the regression line and taking reciprocals of these limits as limits for $D_{0}$. These limits are further confounded when a ratio is formed with the ${ }^{60} \mathrm{Co} \mathrm{D}_{0}$ which has its own associated error. Confidence limits for ratios are complicated to derive, but when the underlying components of the ratio are derived rather than measured variables the determination of these limits is not a sound statistical procedure.

Generally, the RBE rises continuously and monotonically for all LET values associated with $4 \mathrm{He}$ and $12 \mathrm{C}$ from 6 to $100 \mathrm{keV} / \mathrm{\mu m}$. On the other hand, the data for $20 \mathrm{Ne}$ and $40 \mathrm{Ar}$ do not appear to $f$ it to any possible extension of this curve. The plateau neon RBE is significantly higher than the values for carbon at approximately the same LET. For example, proximal peak and plateau carbon ions yield an RBE of 1.4 and 1.5 associated with LET values of 11 and $55 \mathrm{keV} / \mathrm{\mu m}$, respectively, while neon plateau with an intermediate LET at $30 \mathrm{keV} / \mathrm{um}$ has an: RBE of 1.9 . This discrepancy in RBE at constant LET is even.more 
pronounced when the argon plateau at $100 \mathrm{keV} / \mathrm{\mu m}$ $(R B E=3.0)$ is compared to the neon proximal peak at $110 \mathrm{keV} / \mu \mathrm{m}(\mathrm{RBE}=2.2)$ or the distal peak carbon at $105 \mathrm{keV} / \mathrm{\mu m}(R B E=2.0)$. These data indicate an effect of ion mass or charge that is independent of LET.

Peak effectiveness may or may not have been attained with carbon at $105 \mathrm{keV} / \mathrm{\mu m}$, but for both neon and argon the peak of the RBE-LET curve must fall at or below an LET value of $100 \mathrm{keV} / \mu \mathrm{m}$. Only when further studies are done for these two ions in the range of LET values from 30 to $100 \mathrm{keV} / \mu \mathrm{m}$ can we say with certainty where the peak effectiveness lies for the LET dependence of spermatogonial killing.

Figure 6 shows the RBE values obtained for the tail of the carbon and neon curve. These have not been included in the line connecting the various LET positions because much of the radiation in the tail is contributed by fragments of smaller mass than the original ion, and LET determinations are not reliably computed for this configuration. In both cases, the point falls well off the line: below the line for neon and

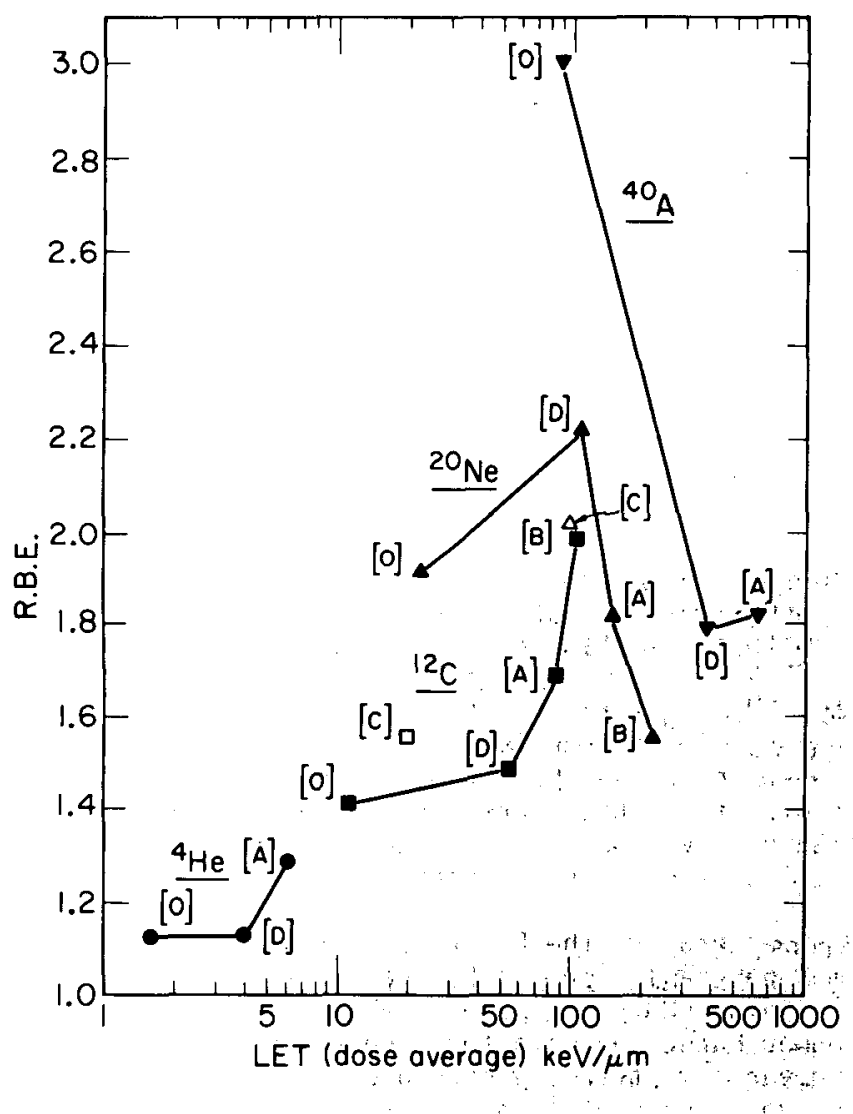

Figure 6. The RBE for various beam positions for: each ion are shown, along with related position notation consistent with Figures 1,4 , and 5 . The [C] values are excluded from the plotted ines because the beam has unusual characteristics in this portion of the Bragg peak. (XBL 807-3444) above the line for carbon. We tentatively attribute this observation to the larger amount of fragmentation in the neon beam, which gives rise to lower mass particles of lesser effectiveness.

\section{DISCUSSION}

The mouse testis has been widely used as a model system for stem cell survival, using a wide range of end points. Residual weight at about 28 days has been used by Lorenz et al. (1947), Eschenbrenner et al. (1948), Kohn and Kallman (1954), and Krebs (1968). More recently, Geraci et al. (1977) used this end point and others, including DNA content of the testes and thymidine incorporation, both at the same postirradiation interval. Withers et a1. (1974) have used a quantitative morphologic end point of regenerating spermatogenic epithelium in individual tubules to develop a cell survival curve. At the large dose levels, excess of $800 \mathrm{rad}$, they are certainly estimating cell survival for a radioresistant population of spermatogenic precursors. These results are consistent with the reported findings of Clow and Gillette (1970). Table 3 lists the $D_{0}$ values from these sources for comparison with our results. In some cases $0_{50}$ values or other parameters were reported in the original paper, but where possible they have been converted to $D_{0}$ values. Others, using similar end points have found similar wide ranging values for testis sensitivity (Bianchi et al., 1969).

Certainly, much of the great variability in the observed end points depends to a great extent upon the wide variability in radiosensitivity of the various developmental stages in the life cycle from stem cell to spermatozoan. This variability as a function of development stage has been well defined in the classic studies of Dakberg (1957; Oakberg and Clark, 1961) and others. The early stages, $A_{0}$ and $A S$, responsible for population maintenance in the stem cell role are quite radioresistant, while late developmental stages, type 8 , are quite radiosensitive. Depending upon whether one is using a regeneration end point such as that of Withers et al. (1974) when the $A_{0} / A_{S}$ population effect would predominate, or a volume or mass end point, in which later, more numerous forms would predominate, a quite different estimate of sensitivity will result.

We bel ieve the 28-day residual weight end point measures mostly the sensitivity of later stages, since these stages make up the bulk of the spermatogonial mass of the testis. The correction for the $h i g h$ radioresistance of Sertoli cells and interstitial cells as determined by subtraction, as we have done, gives a better estimate for $D_{0}$ of the sensitive population, and this correction probably explains the difference between our $D_{0}$ values and those of Geraci et al. (1977).

From the standpoint of the radiobiology of heavy ions, the most significant observation is that LET alone does not account for variation in radiation response of the spermatogonial popula- 
Table 3. Comparison of $D_{0}$ Values

\begin{tabular}{llc}
\hline \multicolumn{1}{c}{ Reference } & Radiation & $D_{0}($ rad $)$ \\
\hline Kohn and Kallman (1954) & $250 \mathrm{kVp} \times$ rays & 90 \\
Krebs (1968) & $250 \mathrm{kVp} \times$ rays & 80 \\
Geraci et al. (1977): weight & $250 \mathrm{kVp} \times$ rays & 350 \\
Geraci et al. (1977): DNA content & $250 \mathrm{kVp} \times$ rays & 540 \\
Geraci et al. (1977): $\mathrm{H}^{3}$ TdR uptake & $250 \mathrm{kVp} \times$ rays & 70 \\
Withers et al. (1974) & $137 \mathrm{Cs}$ & 180 \\
Clow and Gillette (1970) & $300 \mathrm{kVp} \times$ rays & 380 \\
\end{tabular}

tion we are measuring. Clearly, for constant LET but for different particles, the results are quite different; the effectiveness increases with particle mass. These findings are consistent with our observation on the response of the clonogenic stem cells of the small intestine crypts (Alpen et al., 1980). On the other hand, with in vitro systems, Blakely et al. (1979) had found only minor differences for different ions at more or less constant LET. Their end point was survival of human T-1 kidney cells. It remains to be seen whether this finding will be a constantly observed difference when comparing in vitro and in vivo cell survival systems.

Finally, to address the question of RBE with this end point compared to other high LET radiations, we have found RBE values to be relatively low compared to RBE values for fast neutrons using similar systems. Geraci et al. (1977) reports RBE values (for test is weight loss and test is DNA content), ranging from 2.1 to 3.3 . These values for fast neutrons are generally in accord with many other reports. Only with argon ions do we find values as $h i g h$ as those reported for neutrons.

\section{REFERENCES}

Alpen, E. L., P. Powers-Risius, and M. McDonald. 1980. Survival of intestinal crypt cells after exposure to high $Z$, high LET charged particles. Radiat. Res., in press.

Bianchi, M., M. Quintiliani, J. Baarli, and A. H. Sullivan. 1969. Survival of mouse type $B$ spermatogonia for the study of biological effectiveness of very high energy neutrons. Int. J. Radiat. Biol. 15, 185-189.

Blakely, E. A., C. A. Tobias, T.C.H. Yang, K. C. Smith, and J. T. Lyman. 1979. Inactivation of human kidney cells by high energy, monoenergetic heavy ion beams. Radiat. Res. 80 , 122-160.
Clow, D. J., and E. L. Gillette. 1970. Survival of Type A spermatogenic following X-irradiation. Radiat. Res. $42,397-404$.

Curtis, S. B., T. S. Tenforde, I. Parks, W. A. Schilling and J. T. Lyman. 1978. Response of rat rhabdomyosarcoma to neon and helium ion irradiation. Radiat. Res. 74, 274-288.

Eschenbrenner, A. B., E. Miller, and E. Lorenz. 1948. Quantitative histologic analysis of the effect of chronic whole body irradiation with gamma rays on the spermatogenic elements and interstitial tissue of the testes of mice. J. Natl. Cancer Inst. 9, 133-147.

Geraci, J. P., K. L. Jackson, G. M. Christensen, P. D. Thrower, and B. J. Weyer. 1977. Mouse testes as a biological test system for intercomparison of fast neutron therapy beams. Radiat. Res. 71, 377-386.

Kohn, H. I. and R. F. Kallman. 1954. Testes weight loss as a quantitative measure of $X-r$ ay injury in the mouse, hamster, and rat. $\mathrm{Br}$. J. Radiol. 12, 586-591.

Kohn, H. I. and R. F. Kallman. 1955. The effect of fractionated $X-r a y$ dosage upon the mouse testis. I. Maximum weight loss following 80 to $240 \mathrm{r}$ given in 2 to 5 fractions during 1 to 4 days. J. Nat. Cancer Inst. 15, 891-899.

Krebs, J. S. 1968. Analys is of the radiation induced loss of testes and weight in terms of stem cell survival. U.S.N.R.D.L. Technical Report, 68-104. U.S. Naval Radiological Defense Laboratory, San Francisco.

Lorenz, E., W. E. Heston, A. G. Eschenbrenner, and $M$. K. Deringer. 1947. Biological studies in the tolerance range. Radiology 49, 274-285.

Lyman, J. T. and J. Howard. 1977. Dosimetry and instrumentation for helium and heavy ions. Int. J. Radiat. Oncol. 3, 81-85. 
Oakberg, E. F. 1957. Gamma ray sensitivity of the mouse. J. Exp. Zool. 134, 343-356.

Oakberg, E. F. and E. Clark. 1961. Effect of dose and dose rate on radiation damage to mouse spermatogonia and oocytes as measured by cell survival. J. Cell Comp. Physiol. 58 (Suppl. 1), 173-182.
Withers, H. R., N. Hunter, H. T. Barkley, Jr., and B. 0. Reed. 1974. Radiation survival and regeneration characteristics of spermatogenic stem celis of the mouse testis. Radiat. Res. 57; 88-103. 
。

-

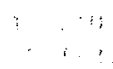

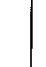




\title{
SURVIVAL OF INTESTINAL CRYPT CELLS AFTER EXPOSURE TO HIGH Z, HIGH ENERGY CHARGED PARTICLES
}

\author{
E. L. Alpen, P. Powers-Risius, and M. McDonald
}

The purpose of the experiments described herein is to evaluate the relative biological effectiveness of high energy charged particles for cell killing in normal tissues. For this evaluation we chose the mouse crypt-cell survival assay of Withers and Elkind (1970).

The intestinal crypt cell is characterized in its response to $x$ and gamma radiation by a broad shoulder on the cell survival curve, and an exponential survival region. It is not possible to measure the breadth of the shoulder as expressed in the usual $D_{0}$ parameter, but Hornsey and Vatistas (1963) have estimated the $D_{0}$ with usual photon irradiation to be $400 \mathrm{rad}$. Their estimate was made by divided dose techniques, but the value is sharply lower than the usual $D_{0}$ estimates of 700 to 900 rad reported by others (Zywietz et al., 1979). The $D_{0}$ for photon irradiation has generally been reported to be on the range of 100 to $150 \mathrm{rad}$.

The gastrointestinal crypt cell survival end point has been widely used for comparison of radiations of various LET values in this rapidly proliferating tissue, principally from exposure to neutrons of various energies (Zywietz et al., 1979; Withers et a1., 1970, 1974; Broerse et al., 1971; Hornsey and Field, 1974; Fu et al., 1979).

In the course of preparing to use accelerated heavy ions from the Berkeley Bevalac for radiotherapy, we have undertaken to study the response of the normal tissues important to the radiotherapist. We report here on the effects of $400-570 \mathrm{MeV}$ per nucleon ${ }^{12} \mathrm{C}, 20 \mathrm{Ne}, 40 \mathrm{Ar}$, and $228 \mathrm{MeV}$ per nucleon ${ }^{4} \mathrm{He}$ ions.

\section{BEAM CHARACTERISTICS AND DOSIMETRY}

The helium-ion beam was produced at the Berkeley 184-inch Synchrocyclotron; the initial energy of these particles was $228 \mathrm{MeV} /$ nucleon. The other ions were produced at the Berkeley Bevalac. The heavier ion beams were first accelerated to $8.5 \mathrm{MeV} /$ nucleon in a linear accelerator, and were then injected and further accelerated to the desired energy in the Bevatron, a machine. built initially as a $2 \mathrm{GeV}$ proton synchrotron. Further details on both the 184-inch synchrocyclotron and the Bevalac are given by Curt is et al. (1978) and Lyman and Howard (1977). Curt is et : a 1 . also describe the beam line arrangements and animal exposure facilities in some detail.

For dosimetry of the ion beams, dual parallel plate ionization chambers were inserted in the beam line. The ion chamber positioned closest to the nimal was used to determine the accumulated dose and to terminate the exposure at the preset value of dose. This system is also described by Curtis et al. (1978).
$X$-irradiation was performed with a Philips orthovoltage generator operated at $225 \mathrm{kVp}$ and 15 $\mathrm{mA}$. The half value layer was $1.1 \mathrm{~mm} \mathrm{Cu}$. Dosimetry was done with a Victoreen condenser $R$ meter calibrated against an NBS secondary standard. The dose rate was approximately $130 \mathrm{rad} / \mathrm{min}$.

For the heavy-ion beams, each mouse was irradiated individually at dose rates varying from 100 to 600 rad per minute. It was assumed that for dose rates of this magnitude there would be no variation in outcome as a function of dose rate. We observed no change in error of estimate when pooled and nonpooled data were analyzed, which suggests that this dose rate range did, indeed, have little effect on our estimate.

The heavy-ion beams were modulated by brass spiral ridge filters to spread the narrow Bragg peak to dimensions useful in radiotherapy. In the studies reported here, a $4-\mathrm{cm}$ spread peak configuration was used for the heavier ions, and a $10-\mathrm{cm}$ spread peak for the helium ions. Irradiations were done in the region of plateau ionization and in the proximal and distal modified peak regions as depicted in Figure 1. The irradiation position in the Bragg peak was controlled by the interposition in the beam line of a variable water column which provided absorber equivalent to the depths shown in Figure I for the various positions. These various residual range values allowed the selection of a wide range of LET values for a given ion.

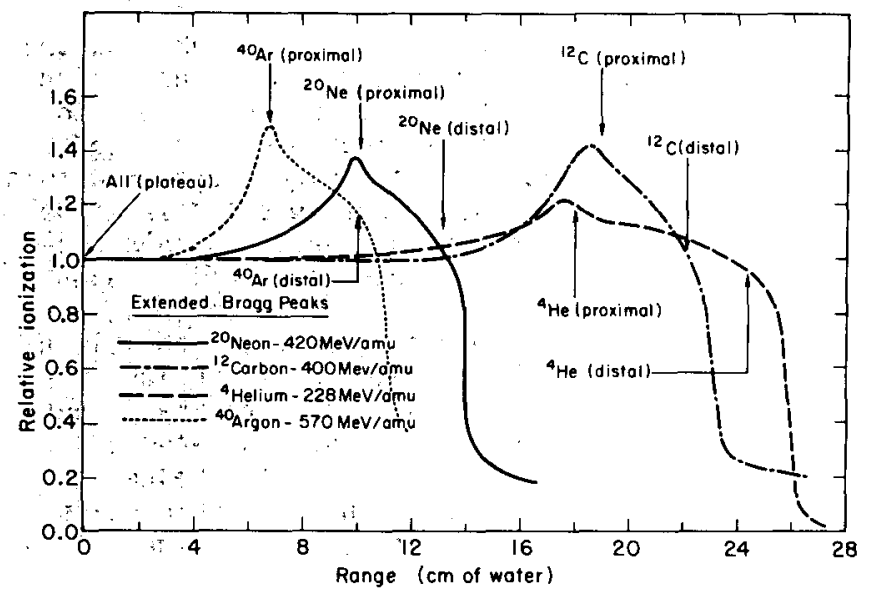

Figure 1. Depth dose distributions for ion beams used. Bragg peaks are spread by use of rotating spiral ridge filters. The irradiation positions are shown in the figure for each ion.

(XBL 784-3062) 
$\mathrm{LAF}_{1}$ female mice (Jackson Labs, Bar Harbor, Maine) $12-17$ weeks old were used for all experiments. They were housed five to a cage, and given food and water ad libitum, both before and after irradiation. Prior to all irradiations, the mice were lightiy anesthetized with i.p.injections of sodium pentobarbital. For both ion beams and $X$ rays, the radiation field was collimated to a $3 \times 5 \mathrm{~cm}$ field which was restricted to the lower two-thirds of the mouse's body excluding the upper thorax and head.

Approximate ly 3 to 3.5 days after irradiation the mice were killed by cervical dislocation; $1 \mathrm{~cm}$ of the jejunum was removed, flushed with physiological saline, and put into Bouin's fixative. The intestinal segment was processed and sections were cut at $5 \mu \mathrm{m}$ and stained with hematoxylin and eosin. Nine intestinal circumferences, each separated by at least $100 \mu \mathrm{m}$ from the previous section to assure no over lap, were counted for each mouse at $200 \mathrm{x}$ magnification. There were usually 5 mice per dose point for each replication, with 3 to 5 replications of each experiment.

The survival of intestinal crypt cells was determined using the microcolony method of Withers and Elkind (1970). The number of regenerating crypts per circumference was counted (greater than 10 living cells in a crypt clone was considered a regenerating crypt). To correct for the probability of more than one cell surviving and regenerating a viable crypt, the number of surviving clonogenic cells per crypt was estimated using the Poisson correction as suggested by Withers and Elkind (1970).

\section{Data Analysis}

Uncorrected crypt cell numbers per circumference were averaged for the nine separate gut sections examined on each mouse. The Poisson correction was made and the data were then computer analyzed for a least-squares fit of the linear regression of the log of surviving clonogenic cells per circumference as a function of dose. The mean corrected surviving clonogenic cell number for each animal was treated as an individual datum, and the variance among the individual estimates on a single animal was ignored. The computer program was so constructed that if a dose group had an average value for clonogenic cells per circumference within $90 \%$ of the control value it was rejected from the analysis. The purpose of introducing such a rejection criterion was to prevent undue influence on the estimation of the $D_{0}$, i.e., the terminal slope, by values that might be on the transitional shoulder region of the curve. The selection of a rejection criterion is, at best, arbitrary; but in preliminary analys is of the data a range of rejection criteria was tested. If the value for a given dose level of surviving clonogenic cells was $90 \%$ or more than the control value, the elimination of this group had no significant effect on the final slope estimate. Using this criterion of stability of slope estimation, we settled upon $90 \%$ of the control value as the level below which a dose group would be included in the analysis.
The calculations provided an estimate of the $D_{0}$ for the terminal exponential portion of the clonogenic cell survival curve and the $95 \%$ confidence interval for the $D_{0}$. Since the $D_{0}$ is only the value of the independent variable (dose) for a $37 \%$ reduction in survival, it was necessary to compute the confidence limit in an indirect manner. We accomplished this by evaluating the confidence limits of the slope of the regression line. The $95 \%$ confidence 1 imit of $D_{0}$ was then obtained by evaluating the regression expression using the $95 \%$ limits for the slope. The extrapolation number was also calculated; however, this parameter did not appear to be particularly informative since small variations in $D_{0}$ were as significant in affecting the extrapolation number as were lateral shifts in the position of the shoulder.

\section{RESULTS}

Data on clonogenic cell survival were analyzed both in terms of the width of the shoulder and the slope of terminal exponential region. The former might be expected to be the most strongly influenced by the higher LET radiations if, in fact, the shoulder represents capacity for recovery from sublethal injury.

The LET values associated with the various exposure positions in the spread Bragg peak ion beams are shown in Table 1 . These values are derived with a computer program developed by Curtis (1977). Typical results and the ranges of values to be expected at any position are discussed in some detail by him in this reference. The values given are the dose average LET at the entrance of the beam into the mouse, the midline of the mouse, and the exit side of the mouse. The present programs that estimate LET distributions are not yet sophisticated enough to provide detailed LET spectra at all locations in the target organ. The shortcomings are, however, not unique to heavy-ion beams, but they are shared with nearly all radiation sources, especially those of higher LET than usually associated with gamma and $X$-ray sources.

Table 1. LET Values for Helium, Carbon, Neon, and Argon Ions*

\begin{tabular}{|c|c|c|c|c|}
\hline \multirow[b]{2}{*}{$\begin{array}{l}\text { Beam } \\
\text { Position }\end{array}$} & \multicolumn{4}{|c|}{ Dose Average LET ( $\mathrm{keV} / \mu \mathrm{m})$} \\
\hline & $\begin{array}{l}\text { Hel ium } \\
(228 \\
\mathrm{MeV} / \mathrm{amu})\end{array}$ & $\begin{array}{c}\text { Carbon } \\
(400 \\
\text { MeV/amu })\end{array}$ & $\begin{array}{c}\text { Neon } \\
(420 \\
\mathrm{MeV} / \mathrm{amu})\end{array}$ & $\begin{array}{l}\text { Argon } \\
(670 \\
\text { MeV/amu) }\end{array}$ \\
\hline Plateau & 1.6 & 11 & 30 & 95 \\
\hline $\begin{array}{l}\text { Proximal } \\
\text { peak }\end{array}$ & 4 & 55 & 100 & 370 \\
\hline Distal peak & 6 & 90 & 180 & 600 \\
\hline
\end{tabular}

*From: Blakely et al. (1979). 
Shown in Figure 2 are typical survival curves plotted along with the calculated regression line. The mean and the 95\% confidence intervals for data collected at each dose are also shown in this figure. Note, however, that the regression line was calculated from ungrouped data, and furthermore, that the varying number of animals per point makes eye fitting difficult at best.

The beam position and energy selected for different ions permit a comparison among ions for biological effectiveness at near constant LET. A good comparison can be made among distal peak carbon at $90-100 \mathrm{keV} / \mu \mathrm{m}$, proximal peak neon at $110-120 \mathrm{keV} / \mathrm{\mu m}$, and plateau region argon at $95 \mathrm{keV} / \mu \mathrm{m}$.

Table 2 lists the relevant parameters derived for clonogenic cell survival with the various radiations. The $D_{0}$ given is one computed for the terminal exponential portion of the survival curve, excluding all data points (groups of mice) in excess of $90 \%$ of the appropriate control. As mentioned earlier, this artifice prevents distortion of the terminal exponential regression by values lying within the transitional region of the shoulder.

As others have noted (Hornsey and Vatistas, 1963) it is not possible to derive valid estimates

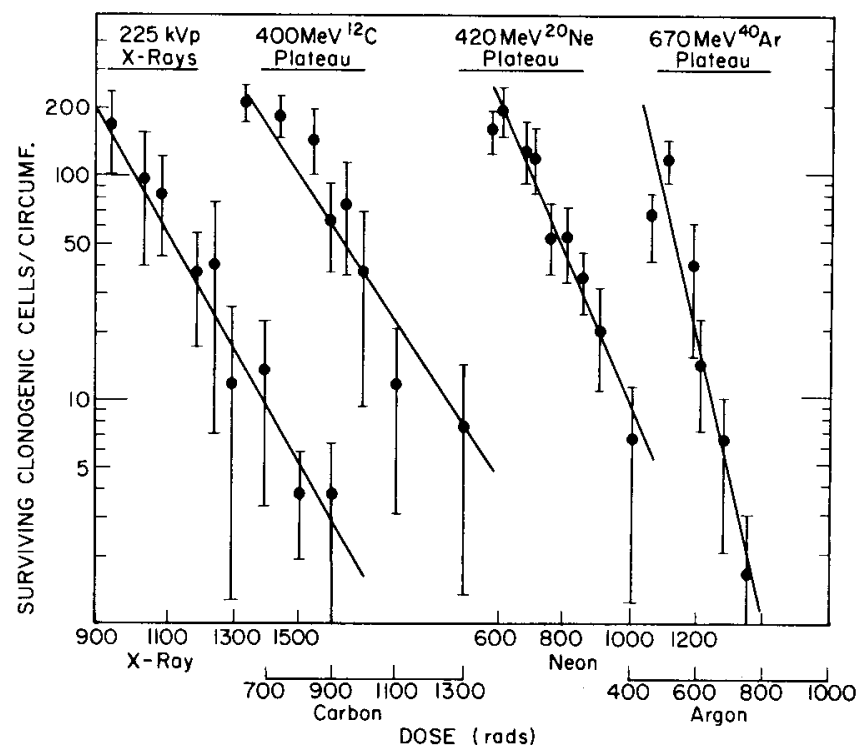

Figure 2. Clonogenic crypt cell survival curves for $225 \mathrm{kVp} X$ rays and for plateau carbon, neon and argon ions. The data points are plotted with their 95\% confidence limits. The calculated regression lines are also shown.

(XBL 802-3122)

Table 2. Crypt Cell Survival Parameters and RBE Values

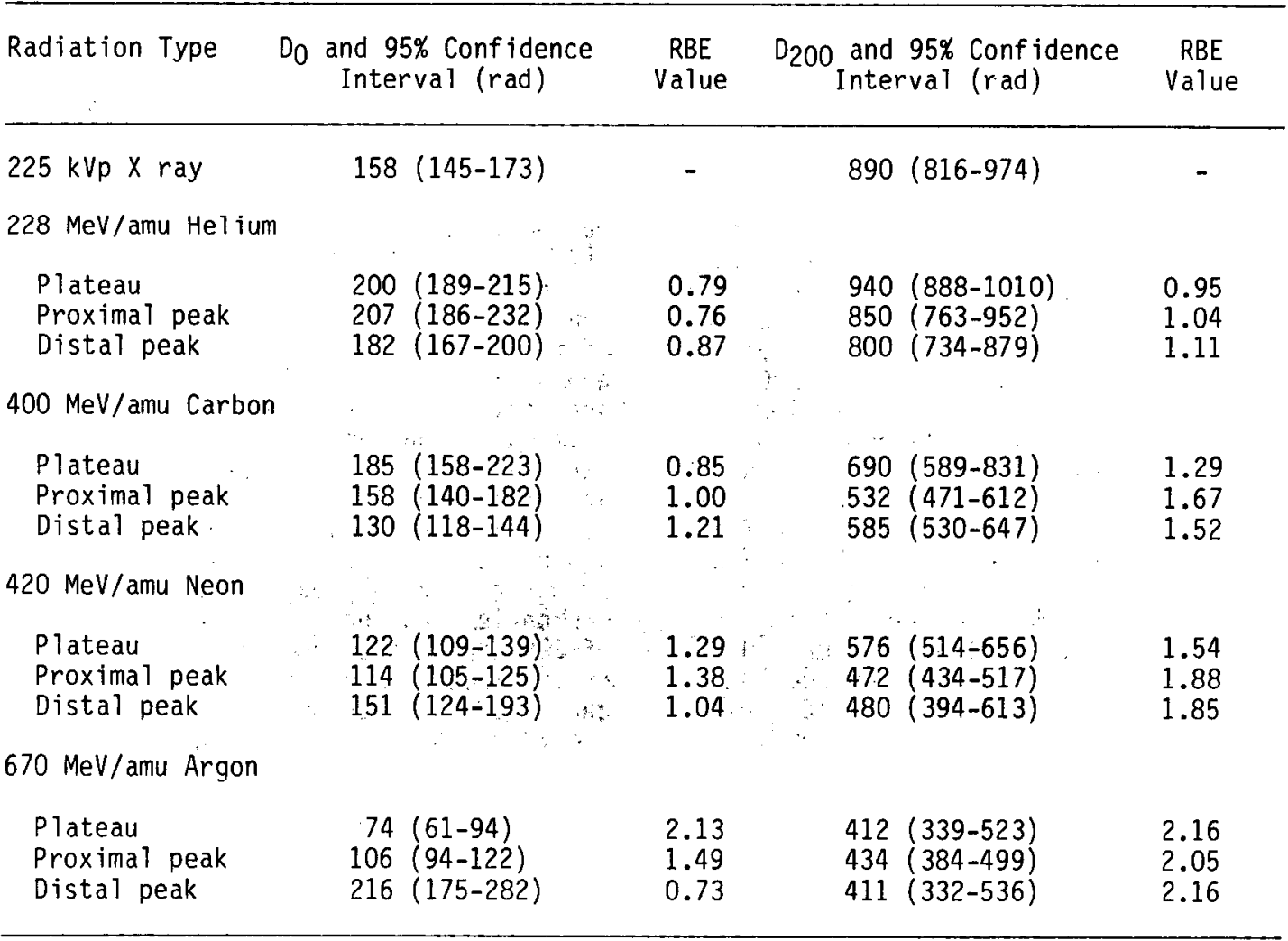


of the $D_{Q}$ except by indirect procedures with the intestinal crypt cell assay. For purposes of shoulder comparison, a useful parameter is the dose associated with a value for clonogenic crypt cell number around or above the control value. This dose is, of course, obtained from the regression expression for the terminal slope. The last column of Table 2 shows the dose for a survival level of 200 clonogenic cells per circumference. This value can be interpreted as an indirect evaluation of $\mathrm{D}_{\mathrm{Q}}$; we will call this value $\mathrm{D}_{200}$.

It is, of course, possible to use our data to construct another estimate of $D_{Q}$ from the value of $n$ derived from the regression equation and the estimate of control clonogenic crypt cell number. Then $D_{0}$ may be estimated from the well-known relationship, in $n=D_{Q} / D_{0}$. As mentioned earlier, these extrapolations through four or five decades of cryptogenic cell survival are quite variable and exquisitely sensitive to small differences in $D_{0}$. The choice of the $D_{200}$ as a substitute for the $D_{Q}$ is made with the full knowledge of the relationship between $D_{0}, n$, and $D_{0}$. However, a survival value at or near the control level of cryptogenic cells will probably be quite insensitive to change in $D_{0}$ since the center of rotation of the exponential portion of the survival curve, as $D_{0}$ changes, must be at or near the control crypt-cell value. In other words, the control level must always be included in the exponential portion, and survival near the control level should be more sensitive to changes in $D_{Q}$ than in $D_{0}$.

Our data permit intercomparison by both ion type and LET as well as an examination of error limits. However, since the $D_{0}$ and $D_{2} 00$ are derived estimates of the independent variable made from the logarithmic regression equations, the usual testing of differences among the estimates is not permitted by sound statistical practice. For example, the " confidence limits are not symmetrical about the $D_{0}$ or $D_{200}$ because the 1 imits are derived from slopes describing a logarithmic dependence of response on dose.

The reference radiation, $225 \mathrm{kVp} X$ rays, is slightly more effective than plateau helium ions, as might be expected. Plateau helium has an LET approximately equivaient to 60 Co gamma rays, and the difference in $D_{0}$ is quite similar to the differences usually expected between $60^{\circ o}$ and $220 \mathrm{kVp}$ $X$ rays in most radiobiological responses (Withers et al., 1970). All positions in the helium-ion beam have $D_{0}$ values somewhat higher than for the $X-r$ ay reference irradiation, and the shoulder, as represented by the $D_{200}$, is not appreciably different from that for $X$ rays. However, at the maximum LET for helium, associated with stopping particles, there is approximately a 10\% decrease in the shoulder value from 890 rad to 800 rad compared to $x$ rays. This small difference may be due to random variation. For the ions heavier than helium there is a continuing decrease in the $D_{0}$ until one examines the $D_{0}$ for distal peak neon. At this beam position and LET one can observe a large increase in $\mathrm{D}_{0}$, with no overlap of the $95 \%$ confidence interval with that for the proximal peak. For argon ions again there is a large increase in the value of $D_{0}$ above those for neon, for all positions in the beam, but plateau and proximal peak argon are much more effective than distal peak argon in terms of $D_{0}$.

The width of the shoulder, as measured by the $D_{200}$, does not follow the same pattern as the slope of the exponential portion of the cell survival curve. There is, from helium through argon, a monotonic decrease in width of the shoulder of the cell survival curve, without any significant peak in the relationship between LET and shoulder width.

For carbon, neon, and argon ions there is a suggestion of a "saturation effect" with increasing LET. The reduced incremental decrease in shoulder with increasing LET is expressed as the plateauing of the RBE curves for each ion. In fact, for argon, a 11 LET values appear to have the same effect on the $\mathrm{D}_{200}$.

\section{DISCUSSION}

The cell survival curve for intestinal clonogenic crypt cells is difficult to interpret in terms of rigorous radiobiological models for cell killing and recovery of sublethal injury. We have already mentioned the difficulties associated with the formal determination of the $D_{Q}$ for which we have chosen to use the $\mathrm{D}_{200}$, the dose for 200 crypt cell survival, as the "stand in" variable. For purposes of discussion we suggest that the data can be best examined by separating the efficiency of direct cell killing, as represented by the $D_{0}$, from possible repair of sublethal injury, which we assume to be related to the width of the shoulder of the cell survival curve as represented here by the $\mathrm{D}_{200}$.

From the data given in Table 2 one may calculate the RBE values relative to $225 \mathrm{kVp} X$ rays for both the $D_{0}$ and the $D_{200}$ as a function of average LET of the particle beam. Figure 3 displays the RBE for the $D_{0}$ values as a function of LET for all of the particle and beam positions studied. Clearly this end point does not follow the expectation that LET alone will predict radiobiological effectiveness for a given radiation quality. The effectiveness of helium ions, which represents a range of LET from 1 to $6 \mathrm{keV} / \mu \mathrm{m}$, is not significantly different from what we might expect for ${ }^{60} \mathrm{Co}$ gamma rays; compared to $225 \mathrm{kVp} X$ rays, helium ions are decidedly less effective. Only with distal peak carbon and heavier ions do we see the significantly increased RBE values that have been associated with the high LET neutron irradiations (Zywietz et al., 1979; Withers et al., 1970, 1974; Broerse et al., 1971; Hornsey and Field, 1974). Even for proximal peak carbon, at $58 \mathrm{keV} / \mu \mathrm{m}$, the carbon-ion beam has not exceeded the effectiveness of $225 \mathrm{kVp} X$ rays insofar as $D_{0}$ is concerned. All of the increased effectiveness of proximal peak carbon can be attributed to a decrease in the shoulder. As far as $D_{0}$ is concerned, these findings corroborate observations of others with neutrons. Zywietz et al. (1979) report from their data on $14 \mathrm{MeV}$ neutrons that the $D_{0}$ is unaffected by the high LET of his radiation source. Clearly, for particles of higher $Z$ there is a significantly increased effectiveness as expressed in smaller Do values or RBE values greater than 1.0 . 


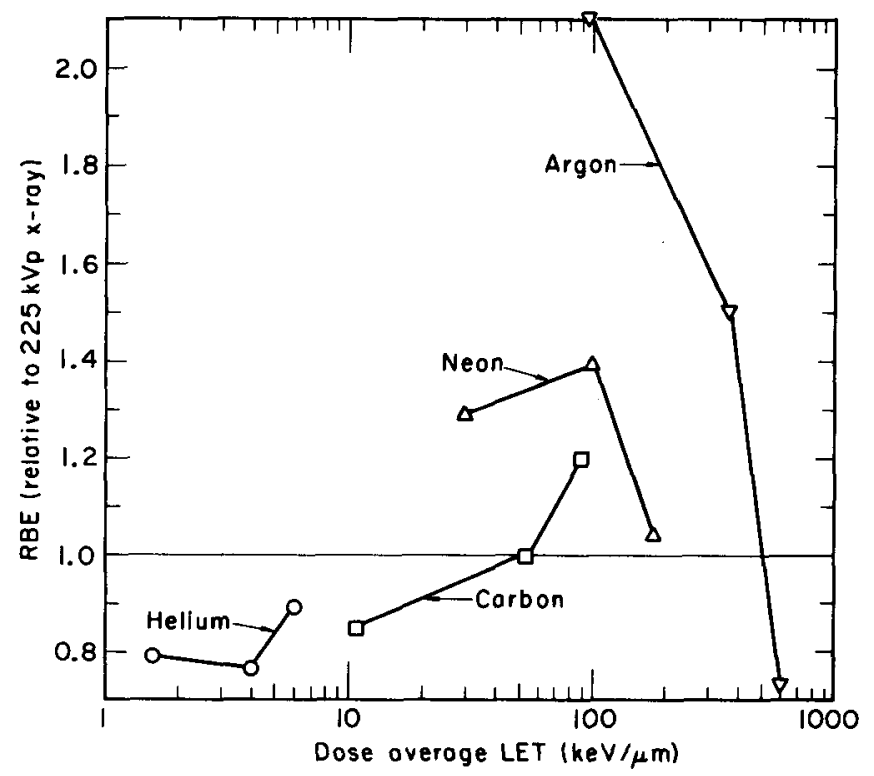

Figure 3. The RBE relative to $225 \mathrm{kVp} X$ rays for the $D_{0}$ for intestinal clonogenic crypt cells. The $D_{0}$ values are determined by best fit regression to the exponential portion of the dose response curve.

(XEL 798-3742)

Another important observation to be made from these data is the large difference in RBE associated with particles of different atomic number or mass at the same average LET. The most direct comparison of LET vs. effectiveness can be made at the $100 \mathrm{keV} / \mu \mathrm{m}$ value of LET. Distal-peak carbon ions with an average LET of $100 \mathrm{keV} / \mu \mathrm{m}$ at an RBE of 1.2 can be compared with proximal peak neon at $100 \mathrm{keV} / \mu \mathrm{m}$ and an RBE of 1.4 , and with plateau argon at $95 \mathrm{keV} / \mathrm{\mu m}$ and an RBE of 2.1. Furthermore, for the comparison chosen, the variations in LET in the exposure region are not as extreme as, for example, distal peak neon or distal peak argon.

The results described here are quite different from those of Todd (1967), who found that a bellshaped single-valued function of LET could describe the RBE for cell killing in vitro. He found maximum effectiveness for his cell line at about 180 $\mathrm{keV} / \mathrm{um}$, regardless of the mass or charge of the particle used for irradiation. Blakely et al. (1979) have generally confirmed Todd's findings with T-1 cells in vitro. They also found a peak effectiveness at around $140 \mathrm{keV} / \mathrm{um}$; although there was evidence that argon ions at about the same : energy we use did not fit the general curve for LET values greater than $100 \mathrm{keV} / \mathrm{\mu m}$. Both Todd and Blakely et al. used unmodified Bragg peak radiation in their studies. We must, therefore, make cross comparisons with some caution until data on cells in vitro irradiated with spread Bragg peaks are available.

We also confirm the decreased biological effectiveness seen by Todd (1967), Blakely et al. 1979), and others for LET values at and above 100 $\mathrm{keV} / \mathrm{um}$, but for our data the LET value associated with maximum effectiveness is certainly at or below $100 \mathrm{keV} / \mu \mathrm{m}$ for neon and argon. The exact location of this peak cannot be ascertained with the data presented here, and further studies must be done at appropriately selected energies to examine the RBE at LET values from $50-100 \mathrm{keV} / \mathrm{\mu m}$ with both neon and carbon. It is clear that for the $D_{0}$, peak effectiveness has been well passed for neon and argon ions beyond $100 \mathrm{keV} / \mu \mathrm{m}$. It remains an enigma why carbon ions have such apparently low effectiveness insofar as $D_{0}$ is concerned. Proximal carbon ions at $55 \mathrm{keV} / \mathrm{\mu m}$ do not approach the effectiveness of neutrons at similar LET values (Zywietz et al., 1979; Fu et al., 1979).

To examine the effect of these radiations on the shoulder region of the crypt cell survival curve, the RBE values for $D_{200}$ are plotted for each ion and LET in Figure 4. Even distal peak helium ions at $6 \mathrm{keV} / \mu \mathrm{m}$ reduce the shoulder as $c$ an be seen by comparison with $225 \mathrm{kVp} X$ rays. As can be seen from Figure 4, all ions used reduce the shoulder of the survival curve appreciably, and, particu1 arly with argon ions, the reduction appears to be reaching a maximum.

The fall in effectiveness for shoulder reduction at higher LET values is similar but not as clear-cut or as dramatic as what is seen for the $D_{0}$ values. Again one can observe that LET is not a complete predictor of RBE as seen in the separation of curves for carbon, neon, and argon. We cannot be sure and are unable to test with this data the reality of the fall-off of the $D_{200}$ RBE above a maximum LET as seen for the DO RBE. Particularly since argon and neon RBE values appear to plateau, we suggest that the small fall with carbon above $50 \mathrm{keV} / \mathrm{\mu m}$ may be due to sample variation. It is not possible to rigorously test these differences by a valid statistical procedure, since

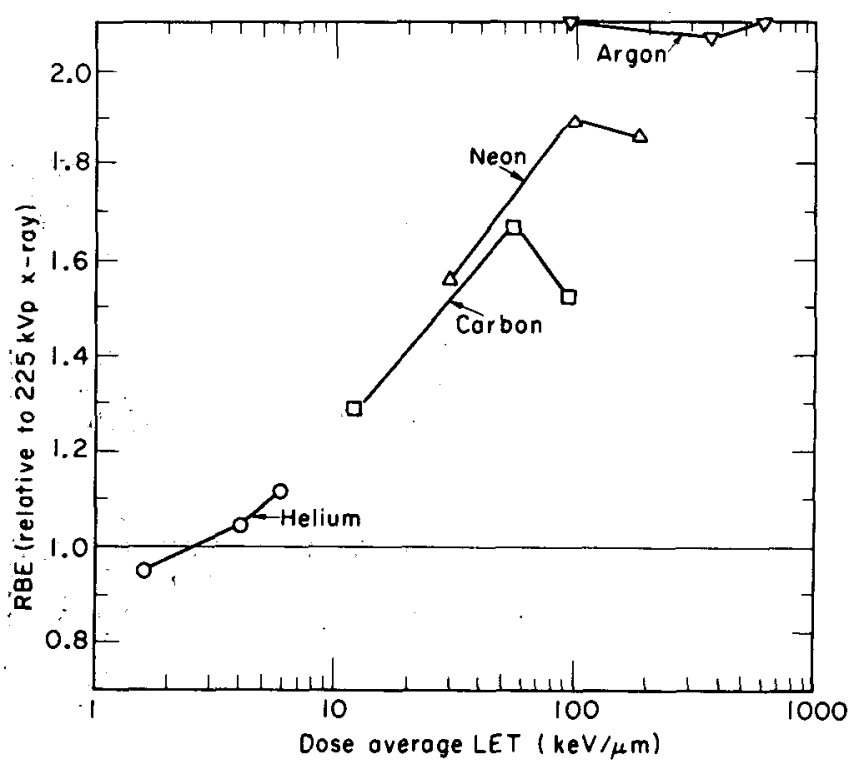

Figure 4 . The $R B E$ relative to $225 \mathrm{kVp} X$ rays for the $\mathrm{D}_{200}$. The $\mathrm{D}_{200}$ is defined in the text as the dose at which 200 clonogenic crypt cells per circumference survive. It is taken as an indirect estimate of $\mathrm{D}_{\mathrm{Q}}$.

(XBL 798-3743) 
the RBE values are ratios derived from data that have already undergone significant mathematical manipulation from the underlying data. However, the confidence limits for the $D_{200}$ values for carbon proximal and distal peak over lap considerably (Table 2).

From these data we must conclude that none of the parameters of the crypt-cell survival curve are uniquely dependent upon LET alone, and that to predict the radiobiological outcome with high energy heavy ions we must look to other properties of the radiation to characterize the biological effectiveness.

Others have already observed that the charge of the ions, as well as possibly the mass or momentum of the particles, may be more important to a description of the biological effects of charged particle beams (Curtis, 1977). We await further theoretical and experimental verification of these concepts to possibly explain our observations.

Finally, we wish to compare our data with a wide range of published results for gastrointestinal crypt-cell assay using neutron beams. Almost all of these workers report their findings in terms of the dose associated with a $10 \%$ survival of clonogenic crypt cells. The appropriate $D_{10}$ values for the heavy-ion data reported here are shown in Table 3.

Table 3. D10 Value for Various Ion Beams

\begin{tabular}{|c|c|c|}
\hline $\begin{array}{l}\text { Radiation } \\
\text { Source }\end{array}$ & $\begin{array}{c}D_{10} \\
(1 \text { S.D. } \\
\text { conf. } \operatorname{limit}_{(\text {rad })}\end{array}$ & $\begin{array}{c}\text { RBE } \\
(1 \text { S.D. } \\
\text { conf. 1imit) }\end{array}$ \\
\hline$X$ ray & 1360 & -- \\
\hline \multicolumn{3}{|l|}{ Helium } \\
\hline $\begin{array}{l}\text { Plateau peak } \\
\text { Proximal peak } \\
\text { Distal peak }\end{array}$ & $\begin{array}{l}1545(1493-1595) \\
1473(1266-1606) \\
1344(1285-1406)\end{array}$ & $\begin{array}{l}0.9(.91-.85) \\
0.9(1.1-.85) \\
1.0(1.1-.96)\end{array}$ \\
\hline
\end{tabular}

Carbon

$\begin{array}{lrl}\text { Plateau peak } & 1250(1149-1368) & 1.1(1.2-1.0) \\ \text { Proximal peak } & 1010(946-1082) & 1.3(1.4-1.2) \\ \text { Distal peak } & 980(931-1029) & 1.4(1.5-1.3)\end{array}$

Neon

$\begin{array}{lll}\text { Plateau peak } & 945(889-1006) & 1.4(1.5-1.3) \\ \text { Proximal peak } & 815(780-852) & 1.7(1.7-1.6) \\ \text { Distal peak } & 934(839-1052) & 1.5(1.6-1.3)\end{array}$

Argon

$\begin{array}{lcl}\text { Plateau peak } & 635(530-804) & 2.1(2.6-1.7) \\ \text { Proximal peak } & 753(707-806) & 1.8(1.9-1.7) \\ \text { Distal peak } & 1063(1003-1106) & 1.3(1.3-1.2)\end{array}$

Zywietz et al. (1979) have summarized most of the pertinent references on neutron effectiveness in the gut crypt assay. For the various studies the reference radiation varied widely, however, in general, for monochromatic $14 \mathrm{MeV} D / T$ neutrons, the RBE values were 1.9 to 2.1. For broad spectrum neutrons the RBE values ranged from 1.3 to 2.2 . Our findings indicate generally similar RBE values for accelerated heavy ions with mean LET values in excess of $50 \mathrm{keV} / \mu \mathrm{m}$. It should be pointed out again, however, that RBE is not a monotonic function of LET, independent of the $Z$ of the particle used.

\section{REFERENCES}

Blakely, E. A., C. A. Tobias, T.C.H. Yang, K. C. Smith, and J. T. Lyman. 1979. Inactivation of human kidney cells by high-energy monoenergetic heavy-ion beams. Radiat. Res. 80, 122-160.

Broerse, J. J., G. W. Barendsen, G. Freriks, and L. M. Van Putten. 1971. RBE values of $15 \mathrm{MeV}$ neutrons for effects on normal tissues. Europ. J. Cancer 7, 171-177.

Curtis, S. B. 1977. Calculated LET distributions of heavy-ion beams. Int. J. Radiat. Oncol. Biol. Phys. 3, 87-91.

Curtis, S. B., T. S. Tenforde, D. Parks, W. A. Schilling, and J. T. Lyman. 1978. Response of rat rhabdomyosarcoma to neon and helium ion irradiation. Radiat. Res. 74, 274-288.

Fu, K. K., T. L. Phillips, D. C. Heilbron, G. Ross, and L.J. Kane. 1979. Relative biological effectiveness of low- and high-LET radiotherapy beams for jejunal crypt cell survival at low doses per fraction. Radiology 132, 205-209.

Hornsey, S. and S. B. Field. 1974. The RBE of cyclotron neutrons for effects on normal tissues. Europ. J. Cancer 10, 231-233.

Hornsey, S. and S. Vatistas. 1963. Some characteristics of the survival curve of crypt cells of the small intestine of the mouse deduced after whole body $X$-irradiation. Br. J. Radiol. 36, $795-800$.

Lyman, J. T. and J. Howard. 1977. Dosimetry and instrumentation for helium and heavy ions. Int. J. Radiat. Oncol. 3, 81-85.

Todd, P. W. 1967. Heavy-ion irradiation of cultured human cells. Radiat. Res. (Supp 1. 7), 196-207.

Withers, H. R. and M. M. Elkind. 1970. Microcolony survival assay for cells of mouse intestinal mucosa exposed to radiation. Int. J. Radiat. Biol. 17, 261-267.

Withers, H. R., J. T. Brennan, and M. M. Elkind. 1970. The response of stem cells of the intes- 
tinal mucosa to irradiation with $14 \mathrm{MeV}$ neutrons. Br. J. Radiol. 43, 796-801.

Withers, H. R., K. Mason, B. 0. Reid, N. Dubravsky, H. T. Bark ley, Jr., B. W. Brown, and J. B. Smathers. 1974. Response of mouse intestine to neutrons and gamma rays in relation to dose fractionation and division cycle. Cancer 34, 39-47.

Zywietz, F., H. Jung, A. Hess, and H. D. Franke. 1979. Response of mouse intestine to $14 \mathrm{MeV}$ neutrons. Int. J. Radiat. Biol. $35,63-72$. 
$\vartheta$

$\vartheta$
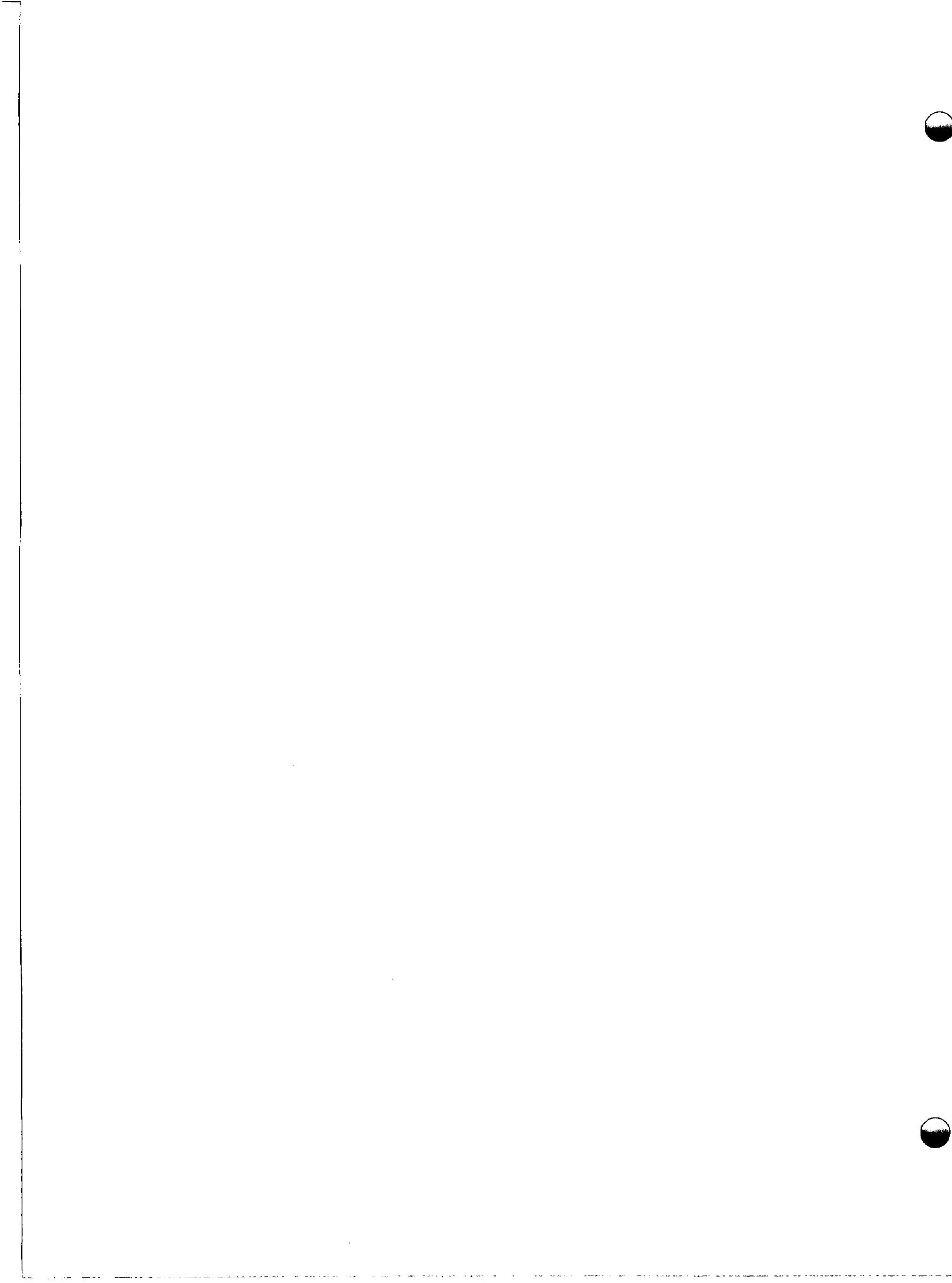


\title{
ESTIMATE OF THE THERAPEUTIC RATIO FOR CHARGED PARTICLE BEAMS
}

\author{
T. S. Phillips* and L. S. Goldstein*
}

The tolerance of normal tissue often limits the total therapeutic dose of low-LET radiation in the treatment of deep-seated; nonresectable tumors. Because of their superior physical and biological depth-dose distributions and high RBE values in hypoxic cells, beams of accelerated heavy ions have obvious clinical potential. It is important therefore to establish the RBE in normal and tumor tissue of these beams in their clinical geometry (where the Bragg peak is modulated to encompass the entire tumor volume) and at doses per fraction anticipated in a treatment plan.

Although evaluating the radioresponse of several normal tissues to $h$ igh-LET radiations delivered in small daily fractions is the most direct way to establish the RBE in a simulated treatment plan, such experiments are often tedious to perform, introduce unwanted variables (e.g., the effect of repeated anaesthesia), and may not conform to the operating schedule at the Bevalac.

Our overall experimental approach has been:

1. establish the dose response for normal tissue, tumors and hypoxic and euoxic cells in vitro to single fraction irradiation and use these data to predict the extent of cellular recovery in high-LET and low-LET regions of the beam;

2. establish the dose response curve for a rapidly proliferating normal tissue to a wide range of doses per fraction with a recovery interval between doses;

3. establish the dose response curve (LD50/28 and LD50/160) for fractionated irradiation of intermediate and slowly dividing normal tissue (esophagus and lung); 4. establish the dose-response of tumors in a multiparametric analys is (cell killing, regrowth, metastatic invasiveness, reoxygenation) to fractionated doses.

MATERIALS AND METHODS
$5 \mu \mathrm{m}$ thick slices were made and stained with Hematoxylin, Phloxine B, and Safran. Surviving crypts were determined using the procedure of Withers and Elkind (1970).

\section{EMT-6/SF Tumors}

The EMT-6/SF tumor originally from Rockwell et a1. (1972) has been maintained by alternate passage in tissue culture and BALB/C mice for several years (see Goldstein et al., 1980 for details). Experiments were performed when tumors implanted on the flanks of BALB/C mice reached a diameter of approximately $0.7 \mathrm{~cm}$. After irradiation, the tumor was removed and separated from host tissue. A single cell suspension was prepared by chopping the tumor and trypsinizing the resultant brei for $10 \mathrm{~min}$. Tumor cells were separated from debris by centrifugation, and a known number of viable cells were plated in complete Dulbecco's medium. Cell survival was determined by viable cell counts after incubating for 9 days at $37^{\circ} \mathrm{C}$.

\section{EMT-6/SF Tumor Cells}

Cells were harvested from flank tumors as described above. An inoculum of $1.0 \times 10^{6}$ cells (euoxic) or $5 \times 10^{6}$ cells (nypoxic) was placed in $1 \mathrm{ml}$ of $\mathrm{Ca}^{++}, \mathrm{Mg}^{++}$-serum-free Dulbecco's medium and incubated for $2 \mathrm{~h}$. The vials to contain hypoxic cells were nermetically sealed before incubation. Following irradiation the cells were diluted and plated as before. Survival was determined by colony counts.

\section{Lung and Esophageal Irradiation}

Damage to the esophagus is measured as the L. $D_{50} / 28$, and damage to the lung is measured as the $\left[D_{50 / 160}\right.$. The mice were anaestnetized and suspended vertically in a lucite holder. A $2.5 \mathrm{~cm} \times 2.5 \mathrm{~cm}$ field (from the xiphoid process to the clavicle) was used.

Male LAF 1 mice, 8-12 weeks old, were given abdominal irradiation in the carbon, neon, or argon beams, or whole-body irradiation in the helium beam or in a $137 \mathrm{Cs}$.irradiator. The mice were killed by cervical dislocation and their. jejunum excised 3.5 days after irradiation. The organ was fixed overnight in Tellysniczky's

fluid. After fixing, eight sections were cut and prepared using routine histological procedures;

\section{Irradiation Procedures}

The following ion beams were used: nelium

Irradiation Procedures
The following ion beams were used: nelium
$(225 \mathrm{MeV} / \mathrm{amu})$, carbon ( $400 \mathrm{MeV} / \mathrm{amu})$, neon $(425$,
557 , or $670 \mathrm{MeV} / \mathrm{amu})$, and argon $(570 \mathrm{MeV} / \mathrm{amu})$.
All beams were modulated. with ridge $\mathrm{fi} 7 \mathrm{ters}$ to
spread the Bragg peak to $4 \mathrm{~cm}$ or $10 \mathrm{~cm}(8 \mathrm{~cm}$ for
helium). Irradiations were done in either the
peak or plateau region. The data were compared
to irradiations with $137 \mathrm{Cs}$ at comparable dose
rates ( 120 rad min-1).


RESULTS

\section{Acute Irradiations of Normal Tissue, Tumor, and Cells in Culture}

Survival of jejunal crypt cells for irradiation in the peak or plateau region of helium, carbon, neon and argon beams with $4 \mathrm{~cm}$ spread Bragg peaks resulted in survival curves shown in Figure 1. The $D_{0}$ for the peak region for helium, carbon, and neon ions is the same as the $D_{0}$ for the plateau region; for argon, the $D_{0}$ of the peak is greater than the $D_{0}$ of the plateau. Survival curve parameters are summarized in Table 1. The RBE in the peak was higher than the RBE in the plateau for helium, carbon, and neon, but the RBE in the peak is lower than the RBE in the plateau for argon. RBE data and the peak-to-plateau RBE ratios are summarized in Tables 2 and 3.

A similar pattern was observed for heavy-ion irradiation of EMT-6/SF tumors (Fig. 2, Table 1). Helium ions appear less effective than photons in this system, but this may reflect differences in the tumor at the time of irradiation ratner than beam effectiveness since the survival characteristics of euoxic and hypoxic cells are essentially the same in both sources (see below). The difference in the peak-to-plateau RBE ratio is not so pronounced for the argon beam in tumor as it was in gut tissue (Fig. 1, Table 3). When assayed at comparable doses, the RBE for carbon, neon and argon beams is higher in tumor than in normal tissue (Table 2).

The dose response curves of the charged particle beams in euoxic (Fig. 3) and hypoxic (Fig. 4) cells show that the RBE values increase with increasing LET. Irradiation in the plateau of the argon beam is again more effective than in the peak region, especially in euoxic cells (Fig. 3). Changes in the $D_{0}$ are more evident for irradiations of nypoxic cells than euoxic cells, and the shoulder is reduced and is decreased with increasing LET (Table 1). Since the snoulder is associated with accumulation of sublethal damage, these data suggest that more nonrepairable lethal damage per unit dose is induced by these charged particle beams.

The OER decreases as the LET of the beam increases (Table 4); the minimum value is about 1.4, which is somewhat nigher than the OER for unmodified beams (Barendsen, 1968). The helium, carbon, and neon beams all maintained a favorable depth-dose distribution, while the peak-toplateau RBE ratio for argon, especially in euoxic cells and gut, indicated an unfavorable depthdose distribution (Table 3 ).

Taken together, these data indicate that the RBE for accelerated heavy ions is higher in tumor

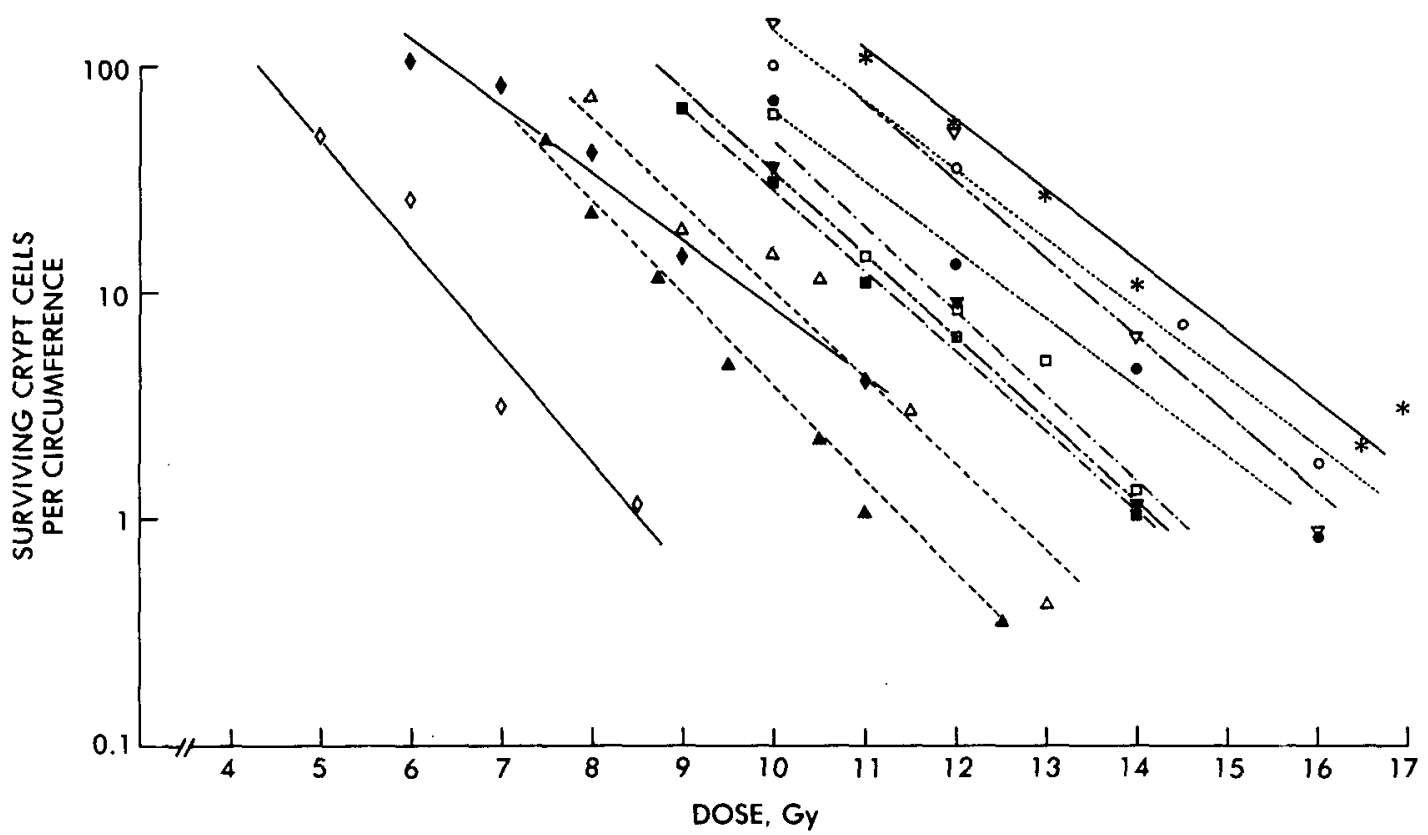

Figure 1. Survival curves (least-squares regression analysis) for jejunal crypt celTs irradiated in the plateau (open symbols) or peak (closed symbols) of nigh LET particle beams: 0--o, plateau, - $\rightarrow$, peak of helium beam with $8-\mathrm{cm}$ spread Bragg peak; $\nabla-.-\nabla$, plateau, $\nabla-\cdots, \nabla$, peak of helium beam with 4-cm spread Bragg peak; 口-.-D, plateau, --.-m, peak of carbon beam with 4-cm spread Bragg peak; $\Delta---\Delta$, plateau, $\Delta--\Delta$, peak of neon beam witn $4-\mathrm{cm}$ spread Bragg peak; $\diamond-\diamond$, plateau, $-\bullet$, peak of argon beam with 4-cm spread Bragg peak; $*-*, 137 \mathrm{Cs}$. (95\% confidence intervals omitted for clarity). (XBL 808-11393) 
Table 1. Calculated Survival Curve Parameters

\begin{tabular}{|c|c|c|c|c|c|c|c|c|c|c|}
\hline \multirow[t]{2}{*}{ Radiation } & & \multicolumn{3}{|c|}{ Crypt Cells } & \multicolumn{2}{|l|}{ EMT-6/SF in vivo } & \multicolumn{2}{|c|}{$\begin{array}{c}\text { Euoxic EMT-6/SF } \\
\text { Cells }\end{array}$} & \multicolumn{2}{|l|}{$\begin{array}{c}\text { Hypoxic EMT-6/SF } \\
\text { Cellls }\end{array}$} \\
\hline & & $D_{0}(95 \%$ Conf. & Int.) & N & $D_{0}(95 \%$ Conf. Int. $)$ & $\mathrm{N}$ & $D_{0}(95 \%$ Conf. Int. $)$ & N & $D_{0}(95 \%$ Conf. Int.) & $\mathrm{N}$ \\
\hline Cesium & & 135 & & 4.37 & 345 & 0.71 & $161(152-170)$ & 5.15 & $442(332-661)$ & 3.66 \\
\hline Helium* & $\begin{array}{l}\text { peak } \\
\text { plateau }\end{array}$ & $\begin{array}{l}139(109-190) \\
137(113-175)\end{array}$ & & $\begin{array}{l}0.87 \\
2.22\end{array}$ & $\begin{array}{l}286(220-409) \\
295(228-417)\end{array}$ & $\begin{array}{l}2.47 \\
3.31\end{array}$ & $\begin{array}{l}116(76-246) \\
174(147-212)\end{array}$ & $\begin{array}{l}7.88 \\
1.93\end{array}$ & $\begin{array}{l}431(354-552) \\
426(394-464)\end{array}$ & $\begin{array}{l}1.43 \\
1.58\end{array}$ \\
\hline Helium ${ }^{\dagger}$ & $\begin{array}{l}\text { peak } \\
\text { plateau }\end{array}$ & $\begin{array}{l}122(93-176) \\
120(73-337)\end{array}$ & $\begin{array}{l}\ddots \\
\ddots \\
\cdot\end{array}$ & $\begin{array}{l}1.16 \\
6.48\end{array}$ & & & & & & \\
\hline Carbon & $\begin{array}{l}\text { peak } \\
\text { plateau }\end{array}$ & $\begin{array}{l}122(109-139) \\
115(84-179)\end{array}$ & & $\begin{array}{l}1.01 \\
3.01\end{array}$ & $\begin{array}{ll}198 & (174-229) \\
175 & (145-221)\end{array}$ & $\begin{array}{l}1.23 \\
1.02\end{array}$ & $\begin{array}{ll}129 & (84-273) \\
148 & (104-253)\end{array}$ & $\begin{array}{l}2.60 \\
2.80\end{array}$ & $\begin{array}{ll}333 & (247-508) \\
352 & (240-664)\end{array}$ & $\begin{array}{l}1.21 \\
1.42\end{array}$ \\
\hline Neon & $\begin{array}{l}\text { peak } \\
\text { plateau }\end{array}$ & $\begin{array}{l}106(91-126) \\
102(79-145)\end{array}$ & $\because \because$ & $\begin{array}{l}0.45 \\
1.94\end{array}$ & $\begin{array}{l}162(135-202) \\
202(169-249)\end{array}$ & $\begin{array}{l}1.82 \\
1.91\end{array}$ & $\begin{array}{ll}123 & (105-145) \\
162 & (137-200)\end{array}$ & $\begin{array}{l}1.33 \\
1.83\end{array}$ & $\begin{array}{ll}259 & (183-447) \\
134 & (119-156)\end{array}$ & $\begin{array}{l}2.16 \\
1.24\end{array}$ \\
\hline Argon & $\begin{array}{l}\text { peak: } \\
\text { plateau }\end{array}$ & $\begin{array}{r}145(114-197) \\
90(66-140)\end{array}$ & & $\begin{array}{l}0.08 \\
0.13\end{array}$ & $\begin{array}{l}158(125-214) \\
144(116-191)\end{array}$ & $\begin{array}{l}0.57 \\
0.56\end{array}$ & $\begin{array}{rr}111 & (89-149) \\
89 & (62-158)\end{array}$ & $\begin{array}{l}1.95 \\
0.58\end{array}$ & $\begin{array}{l}193(134-346) \\
170(111-356)\end{array}$ & $\begin{array}{l}1.20 \\
0.54\end{array}$ \\
\hline
\end{tabular}

* 8-cm spread Bragg peak.

† 4-crm spread Bragg peak. 
Table 2. RBE Values (to $137 \mathrm{CS}$ ) of High-LET Particle Beams Measured at Isoeffect Levels or as the Ratio $D_{0}{ }^{137} \mathrm{CS} / \mathrm{D}_{0}$ Test

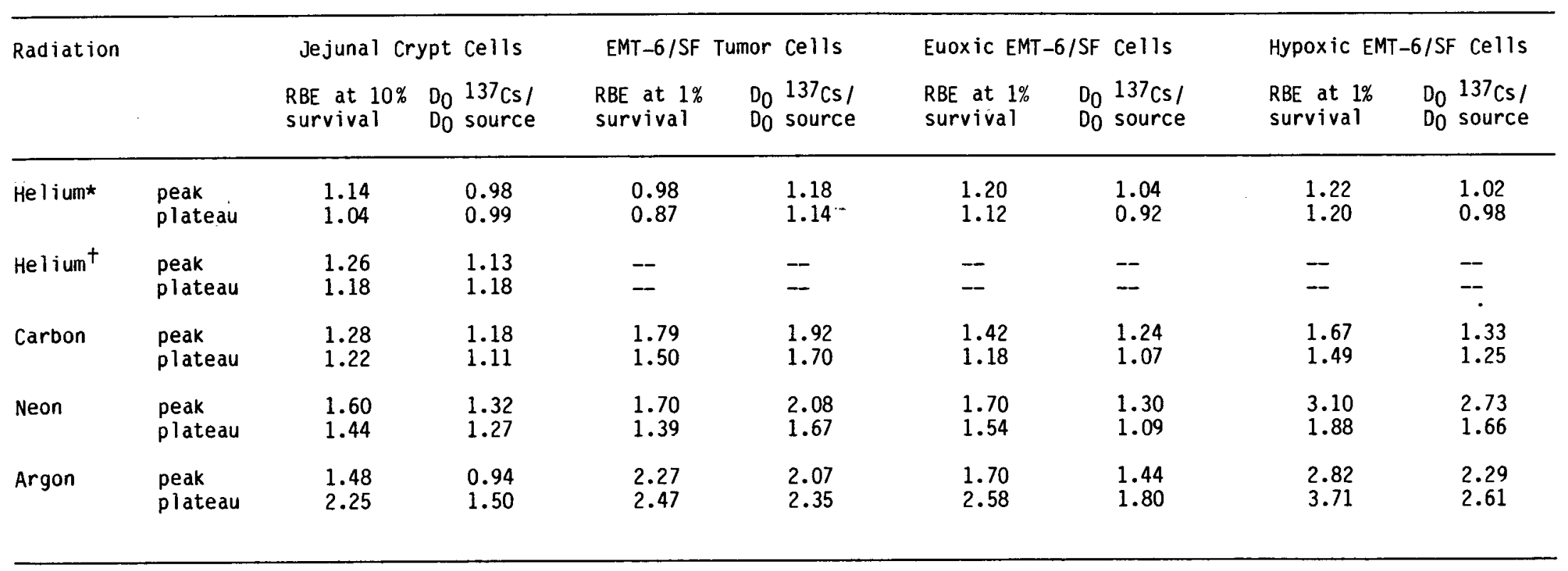

* 8-cm spread Bragg peak.

4-cm spread Bragg peak. 
Table 3. Peak-to-Plateau RBE Ratios for Jejunal crypt Cells or EMT-6/SF Tumor Cells Irradiated in High-LET Particle Beams

Radiation Jejunal Crypt EMT-6/SF Tumor Euoxic EMT-6/SF Tumor Hypoxic EMT-6/SF Tumor Cells Cells In Vivo Cells In Vitro Cells in Vitro

\begin{tabular}{lllll}
\hline Helium & 1.10 & 1.10 & 1.07 & 1.01 \\
& $1.17^{*}$ & & & 1.11 \\
Carbon & 1.05 & 1.18 & 1.20 & 1.65 \\
Neon & 1.11 & 1.24 & 1.10 & 0.76 \\
Argon & 0.66 & 0.93 & 0.66 & \\
\hline
\end{tabular}

* 4-cm spread Bragg peak.

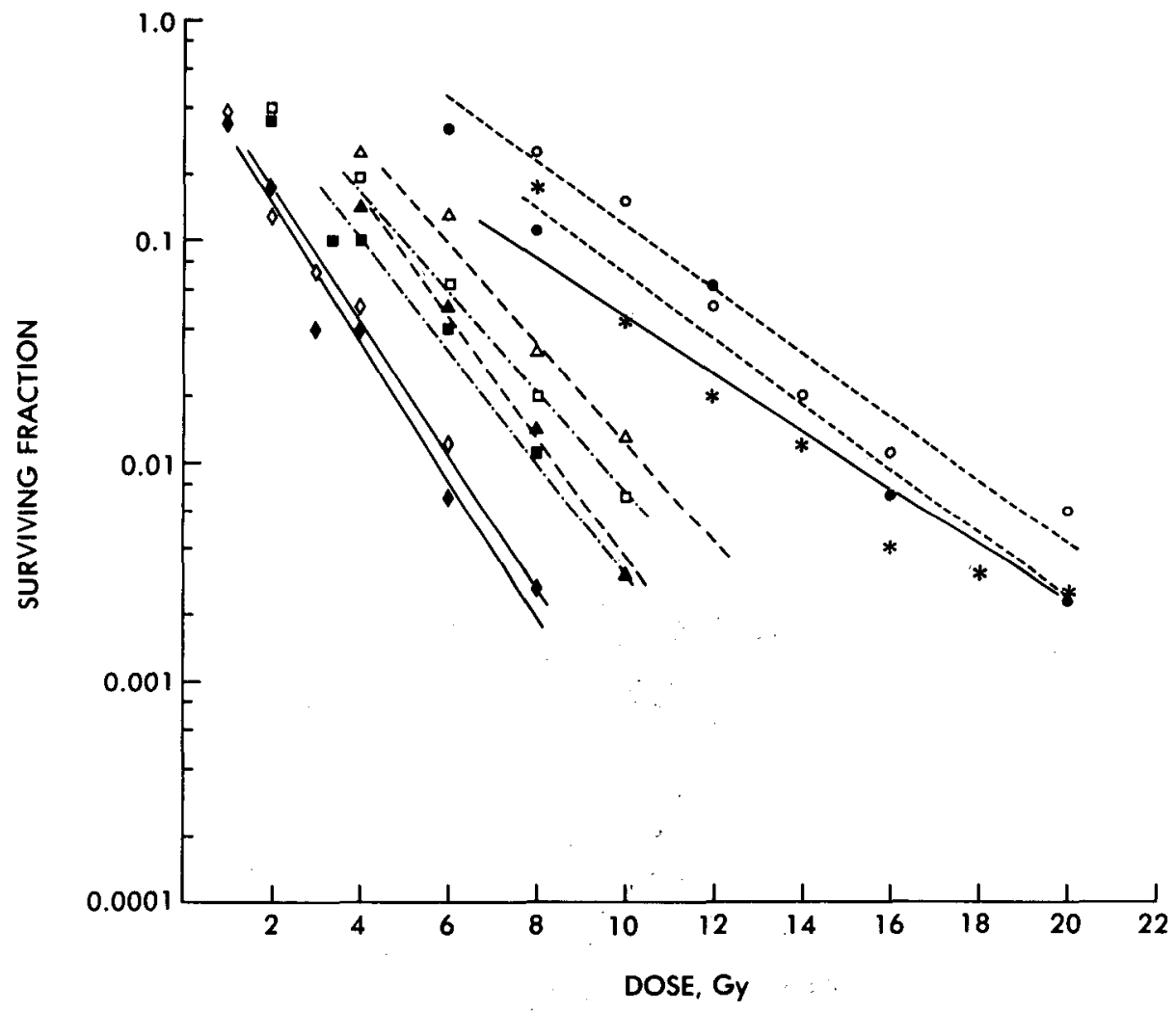

Figure 2. Survival curvès (least squares regression analysis) for EMT-6/SF tumor cells irradiated as flank tumors on BALB/C mice. Symbois (as in Fig. 1) indicate data points used to calculate survival curve. (95\% confidence intervals omitted for clarity.) (XBL 808-11392) 


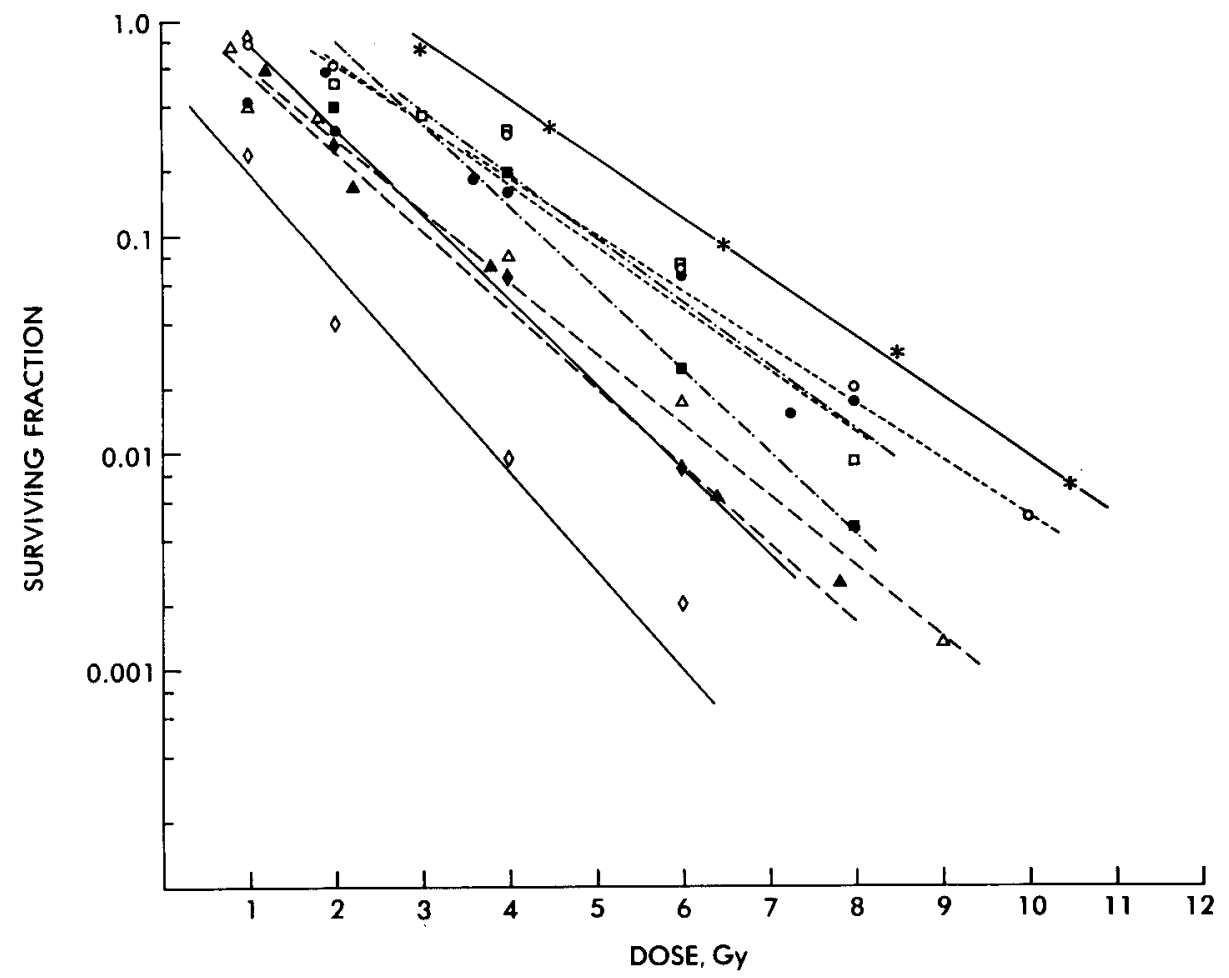

Figure 3. Survival curves (least squares regression analysis) for EMT-6/SF tumor cells irradiated euoxically in high LET beams. Symbols as in Figure 1 ( $95 \%$ confidence intervals omitted for clarity.)

(XBL 808-11394)

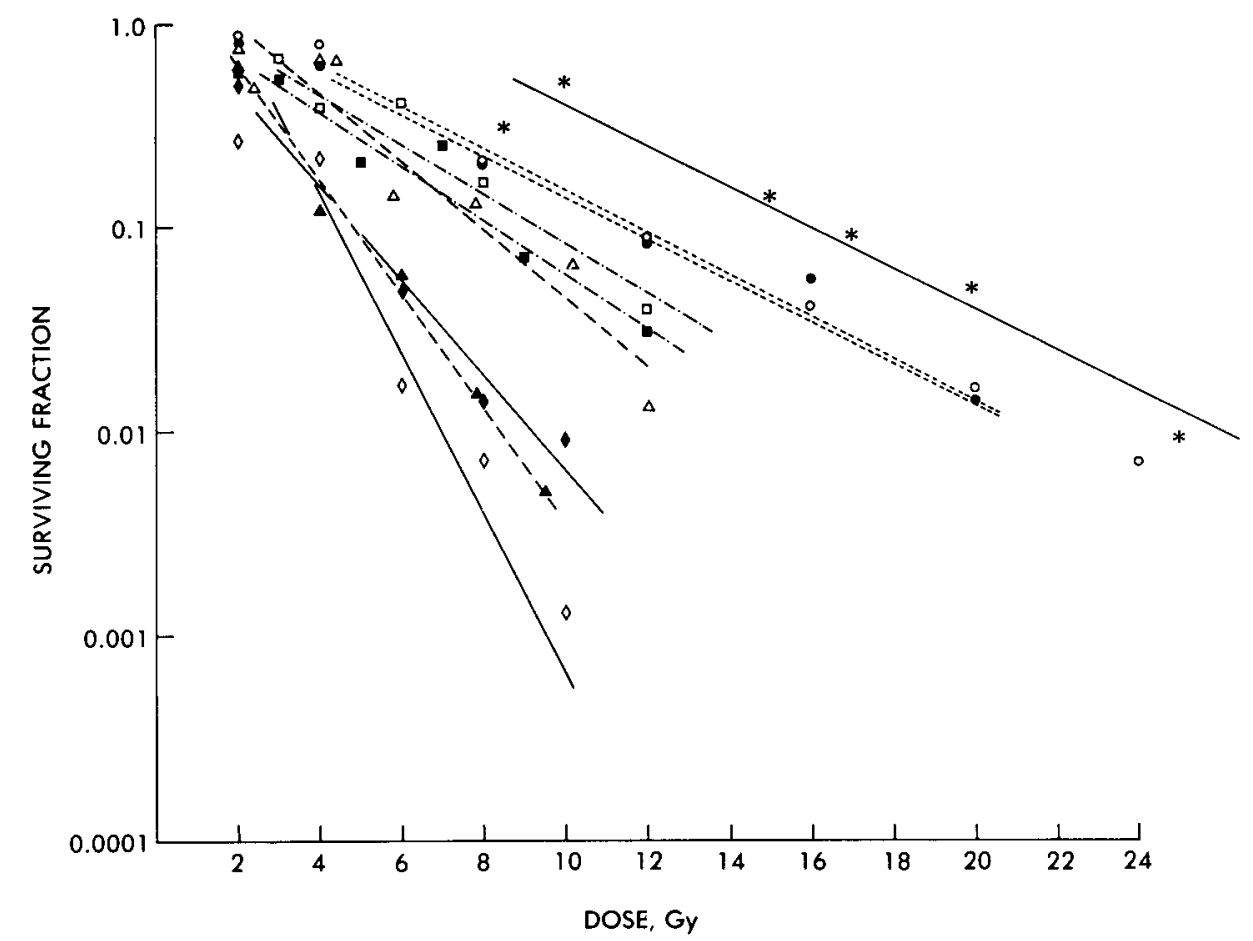

Figure 4. Survival curves (least squares regression analysis) for EMT-6/SF tumor cells irradiated nypoxically in nigh LET beams. Symbols as in Figure 1 (95\% confidence intervals omitted for clarity.) (XBL 808-11396) 
Table 4. OER Values at $1 \%$ Survival for EMT-6/SF Tumor Cells Irradiated In Vitro

\begin{tabular}{lll}
\hline Source & Position & OER \\
\hline 137 CS & & \\
Helium & peak & 2.60 \\
& plateau & 2.43 \\
Carbon & peak & 2.38 \\
& plateau & 2.25 \\
Neon & peak & 2.09 \\
& plateau & 1.42 \\
Argon & peak & 2.15 \\
& plateau & 1.58 \\
\hline
\end{tabular}

cells than in normal tissue. This reflects the enhanced killing of hypoxic cells compared to euoxic cells and the reduced OER. The biological depth-dose distribution for helium, carbon, and neon is favorable and complements their favorable physical depth-dose distribution. The depth-dose distribution of argon, while unfavorable compared to the other ions, is still better than that for low-LET sources. Survival curves with heavy ions have a substantially reduced shoulder compared to survival curves with $137 \mathrm{Cs}$ and this observation suggests that even better depth-dose distribution will result at low doses.

\section{Fractionated Irradiation of Jejunal Crypt Cells}

The beam geometries used in the previous study (with the exception of the argon beam) were investigated to determine the survival curve characteristics of jejunal crypt cells irradiated in a fractionated routine. Additional beam geometries including increased range and a $10-\mathrm{cm}$. spread Bragg peak were included. Fractionation routines were designed to cover a complete range of doses per fraction including those anticipated in clinical trials.

The data are shown in Figures 5 through 9 and the RBE and peak-to-plateau RBE calculations are summarized in Tables 5 and 6 , respectively. Rather than discuss the data for each ion and each geometry, only the most salient features of the experimental results will be described. The data snow consistently that there is less cellular recovery when irradiation is in the peak region than the plateau region of the beams. Since the LET is higher in the peak region, these data indicate that the extent of recovery decreases as the LET increases. Fractionation (. If the heavy-ion dose therefore substantially improves the peak-to-plateau RBE ratio, a finding of obvious clinical significance. For LET such as in the peak of the $4-\mathrm{cm}$ neon beams and all

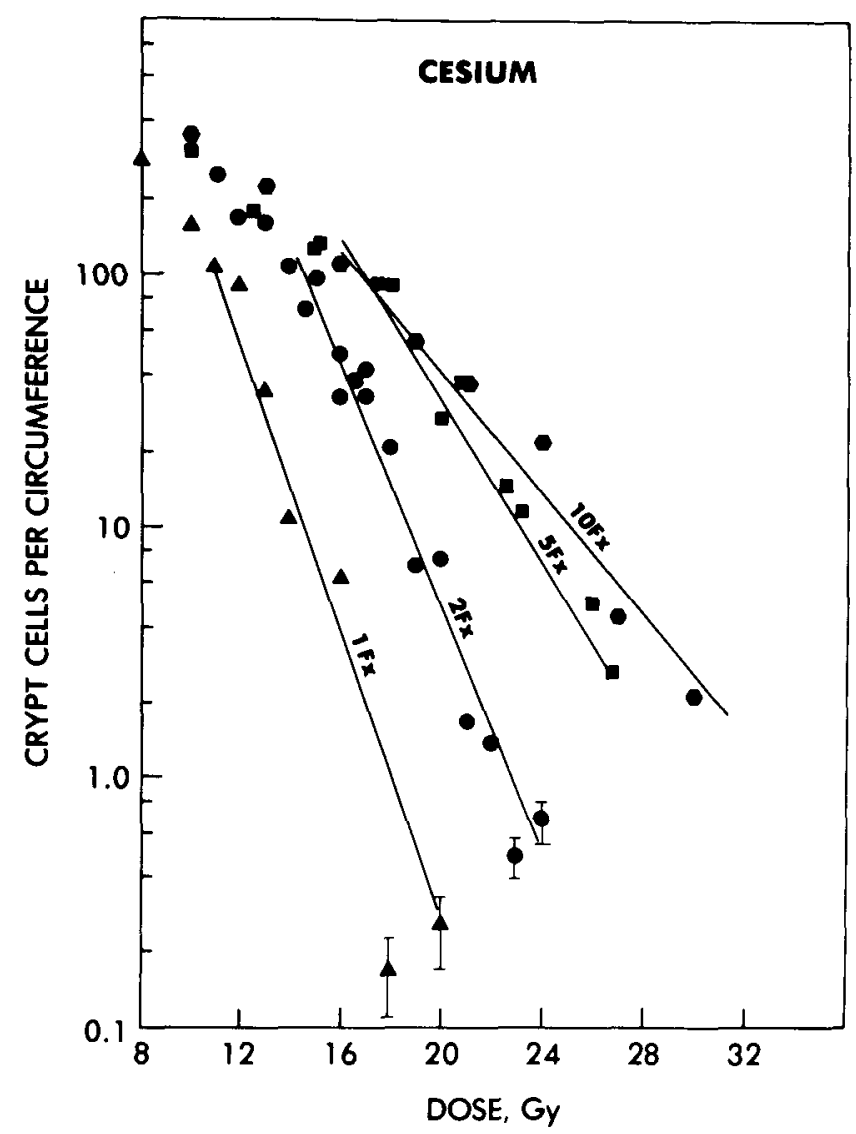

Figure 5. Survival of jejunal crypt cells irradiated with $137 \mathrm{Cs}$ in 1 (A), 2 (O), 5(ם), or 10 (O) fractions. (XBL 808-11375)

positions of the 10-cm argon beam, fractionation enhances cell killing. The mechanism for this may involve a cell cycle dependent radioresponse to the low LET components in these degraded beams. However, because of the pleiotropic changes induced by these beams, which can modify the radioresponse of a cell to subsequent radiation, an explanation of this interesting effect requires further experimentation. Over the dose range examined, the RBE increases with decreasing fraction size (Figs. 10-12).

These data, with the exception of enhanced killing by fractionation, are consistent with predictions based on the shapes of the single fraction dose response curve.

\section{Lung and Esophageal Irradiation}

The RBE for the $4 \mathrm{~cm}$ beams (Table 7 ) are in good agreement with those measured as the $D_{0}$ in the gut except for the neon plateau, which has a substantially lower RBE in the esophagus. The limited data on daily fractionation of the dose indicate that the RBE is entanced, reflecting reduced recovery from sublethal damaged in the high LET beam. As in gut, the extent of recovery appears related to dose per fraction. The RBE 

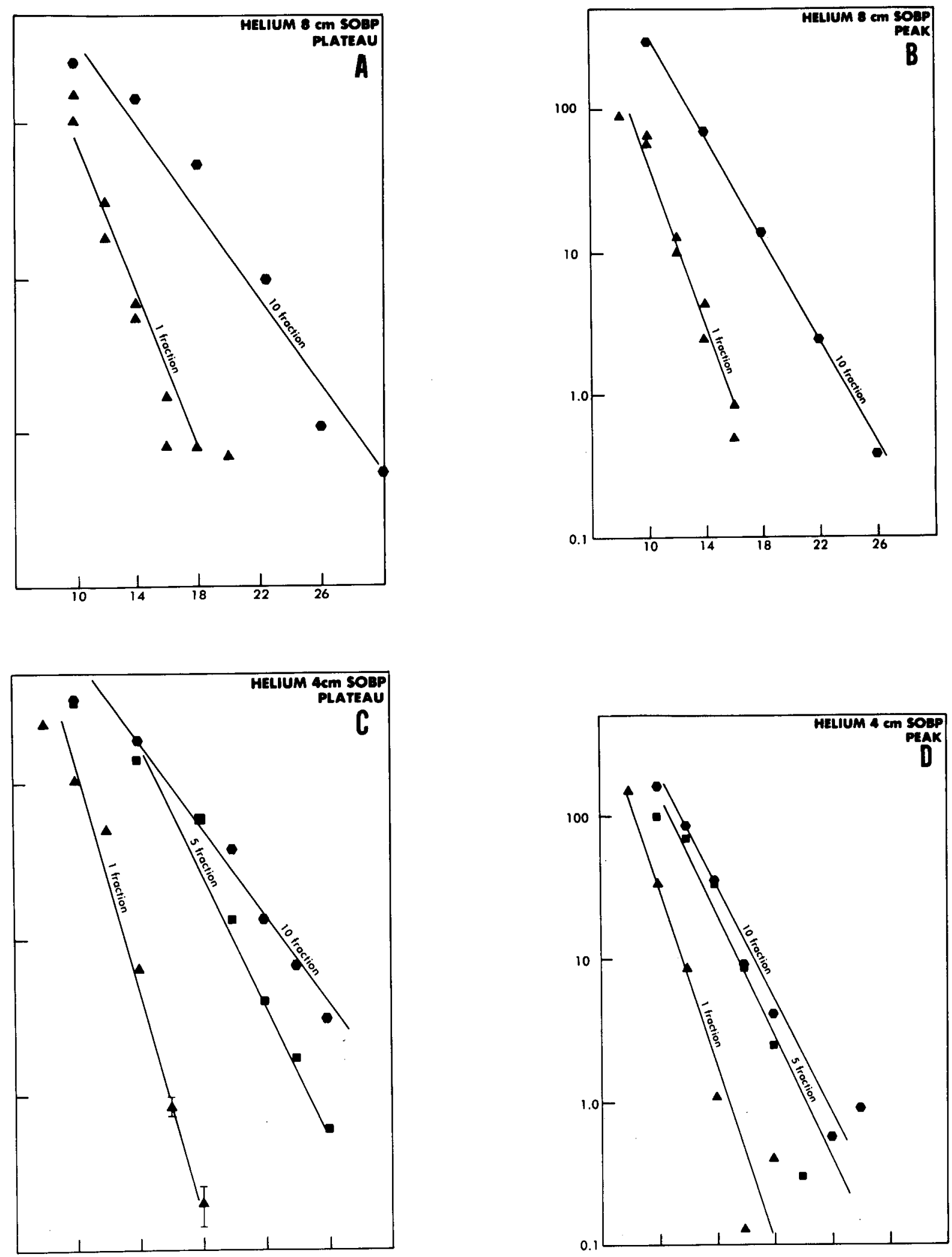

Figure 6. Survival of jejunal crypt cells irradiated in one (triangles), five (squares), or ten (hexagons) fractions in a $225 \mathrm{MeV} / \mathrm{amu}$ helium beam with an $8-\mathrm{cm}$ Bragg peak $(A, B)$ or a $4-\mathrm{cm}$ spread Bragg peak (C,D). [(A) XBL 808-11380; (B) 808-11376; (C) 808-11377; (D) 808-11378]. 

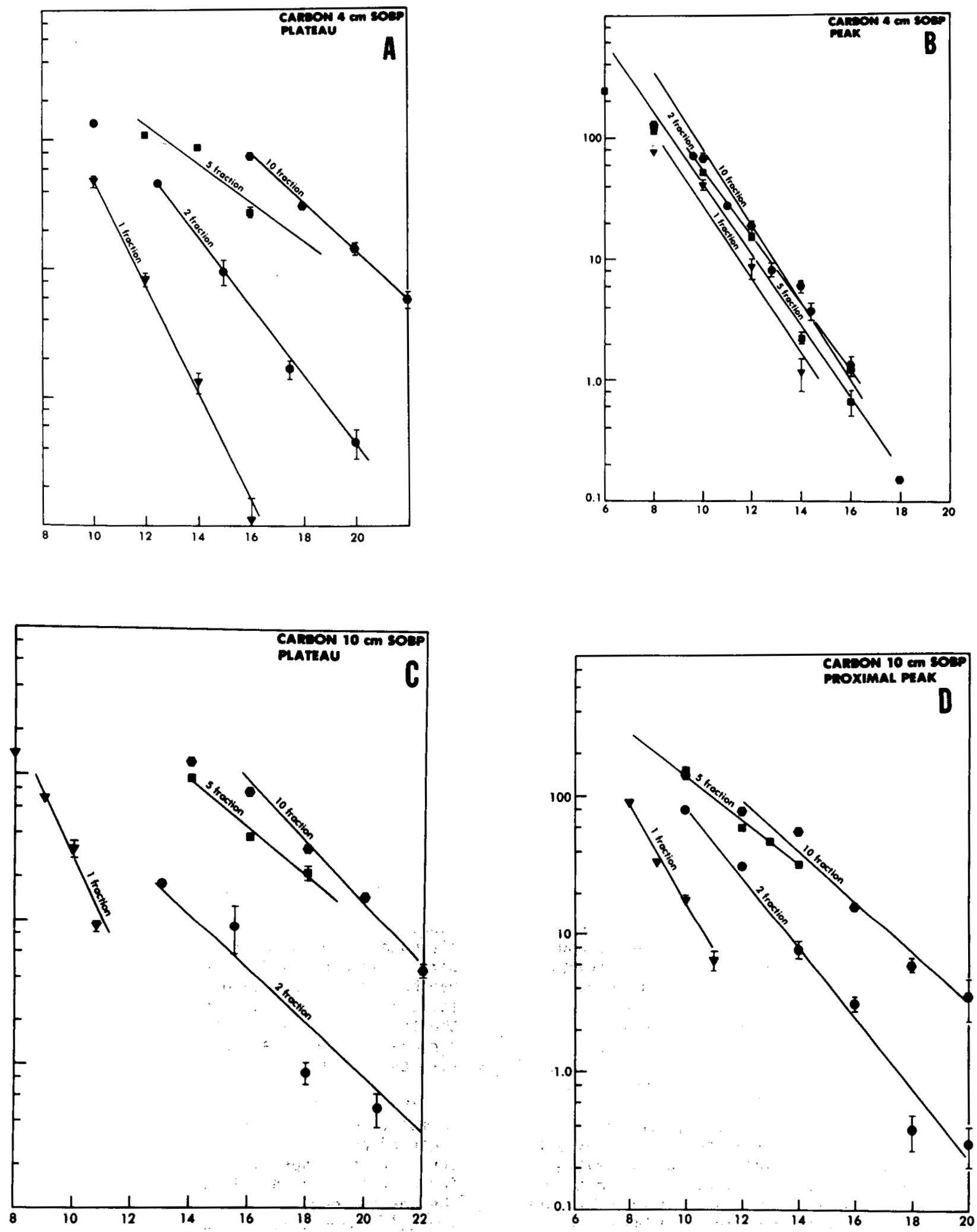

Figure 7. Survival of jejunal crypt cells. irradiated in one (triangles), two (circles), five (squares), or ten (hexagons) fractions in a $400 \mathrm{MeV} / \mathrm{amu}$ carbon beam with a 4-cm spread Bragg peak (A,B) or a 10-cm spread Bragg peak (C-F). [(A) XBL 808-11381; (B) 808-11379; (C) 80811384 ; (D) 808-11383; (E) 808-11382; (F) 808-11385] 


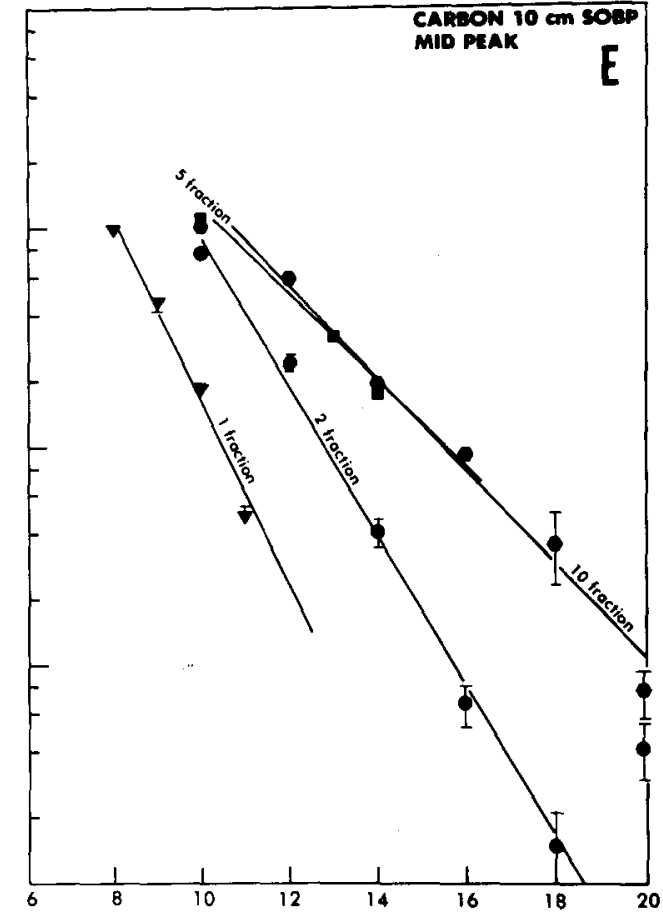

Figure 7. (continued). is lower for pulmonary lethality (Table 8) than gut lethality but the order of effectiveness for the ions remains unchanged. Reduced recovery is again evidenced in the fractionation data, and the unfavorable biological depth-dose distribution of the argon ion is again demonstrated. Data are not available yet for fractionated irradiation with a $10-\mathrm{cm}$ spread Bragg peak.

\section{Irradiation of Other Tumors}

Although the EMT-6/SF tumor system has been used for a long time in radiobiology, it may not be the best system for evaluating a nonimmunogenic, slow growing human tumor. Prior to a large scale investigation with heavy ions, we are screening several different murine tumors for their suitability to such studies. These evaluations are still incomplete.

\section{SUMMARY AND CONCLUSIONS}

Our data consistently demonstrate that the therapeutic ratio (RBE in tumor/RBE in normal

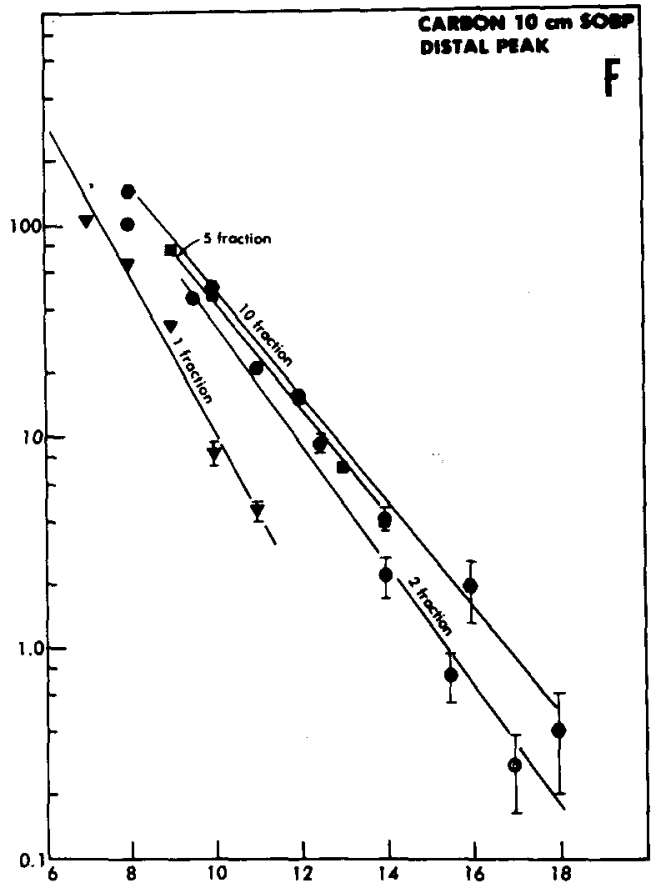

tissue) of the murine systems increases with increasing LET up to the LET of the peak of the modulated neon beam (approximately $100 \mathrm{keV} / \mu \mathrm{m}$ ). Although the argon beam has some features which make it attractive for therapy, its application may be limited because of its unfavorable biological deptn-dose distribution. Increases in the therapeutic ratio reflect the enhanced $R B E$ for charged particle beams in hypoxic cells and tissues relative to euoxic cells and tissues.

The biological depth-dose distribution in normal tissues is markedly enhanced when a recovery period is included in a fractionated irradiation. There is less recovery following irradiation in high-LET beams than low-LET beams. The charged particle beam has both nigh-LET and low-LET regions, and since there is reduced recovery in the peak relative to the plateau, fractionation enhances the peak-to-plateau RBE ratio. The proliferative state of the tissue influences the RBE values as the RBE is nigher in rapidly proliferating tissues.

These data support the continued exploration of charged particle beams for therapeutic applications. 

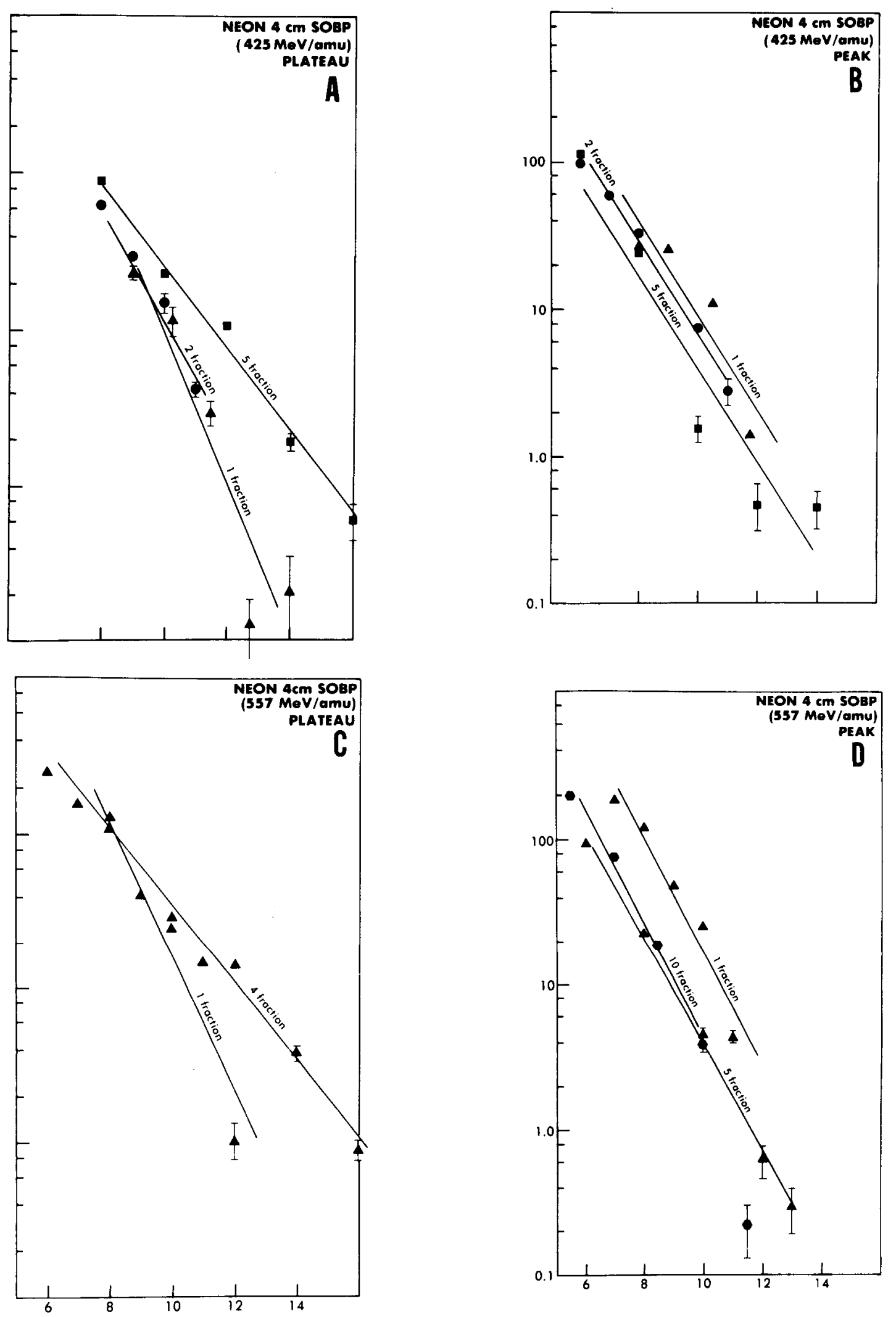

Figure 8. Survival of jejunal crypt cells irradiated in a $400 \mathrm{MeV} / \mathrm{amu}$ neon beam with a 4-cm spread Bragg peak (A,B), or a 557 MeV/amu neon beam with a 4-cm spread Bragg peak $(C, D)$ or a 10-Cm spread Bragg peak (E-H). Symbols for fractionation as in Figure 7 . [(A) 808-11386;

(B) $808-11387$; (C) $808-11388$; (D) $808-11389$; (E) 808-11391; (F) 808-11390; (G) 808-11395; (H) 808-11404 ] 

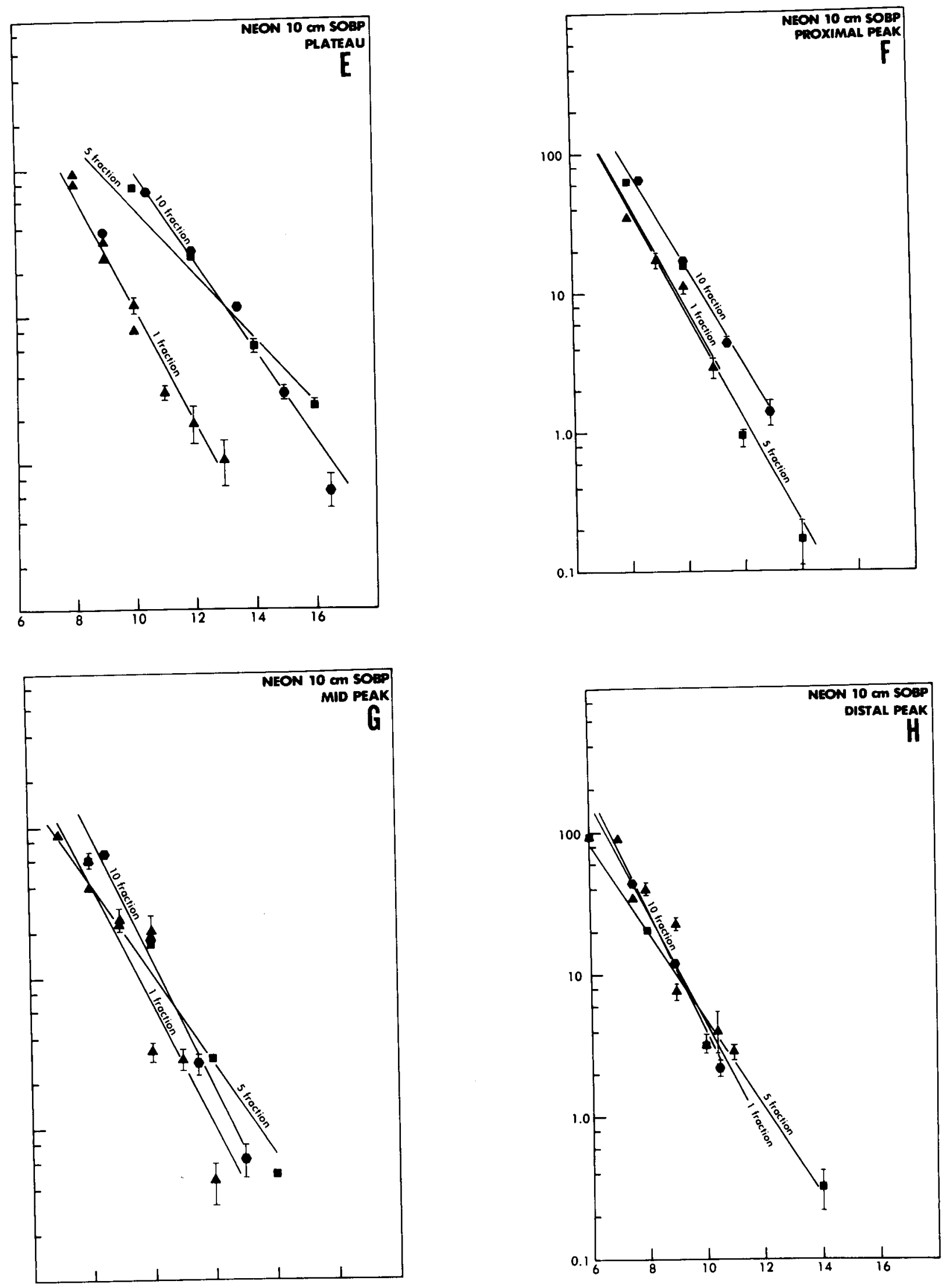

Figure 8. (continued). 

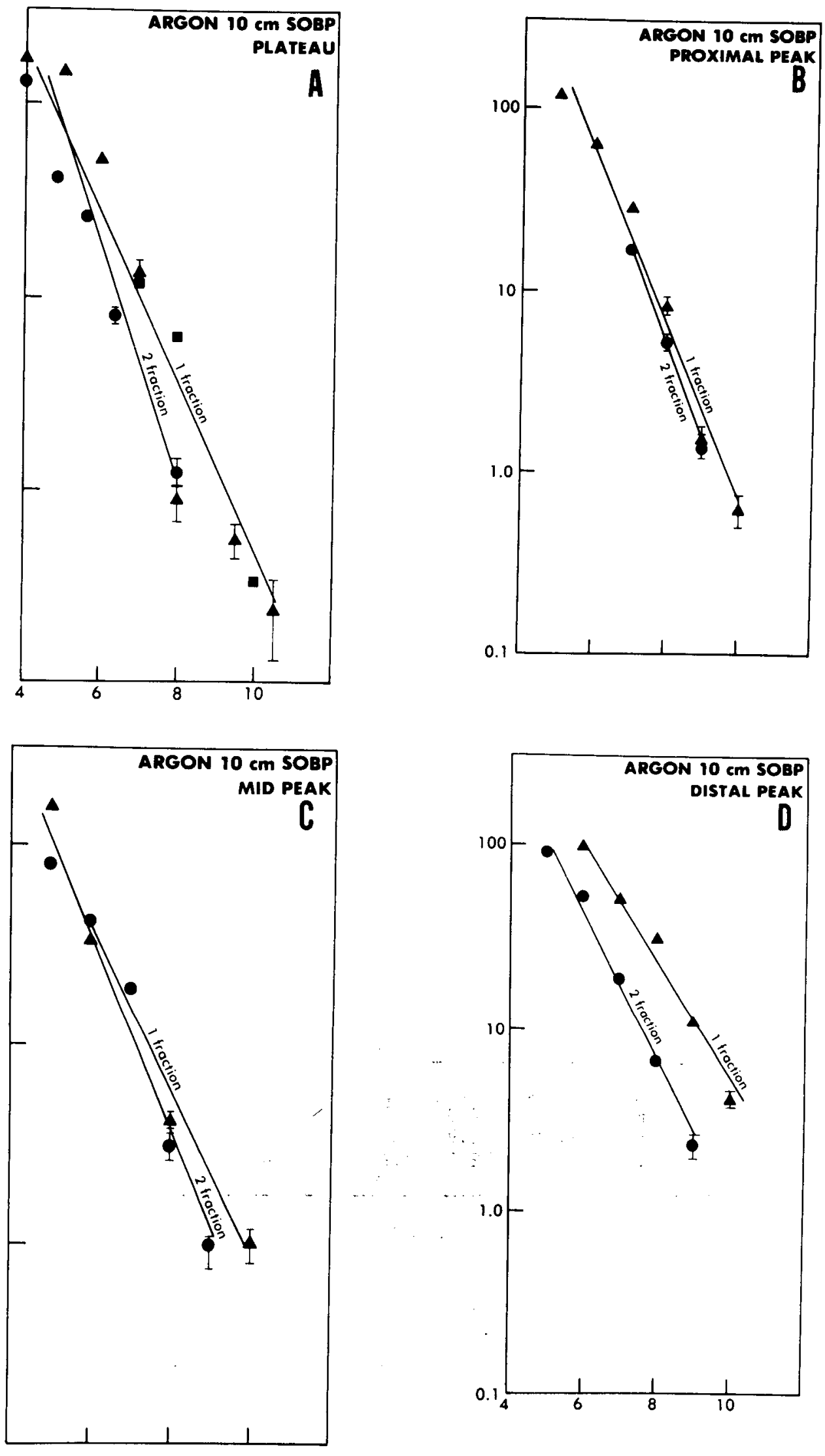

Figure 9. Survival of jejunal crypt cells irradiated in a $570 \mathrm{MeV} / \mathrm{amu}$ argon beam with a $10-\mathrm{cm}$ spread Bragg peak (A-D). Symbols for fractionation as in Figure 7 . [(A) XBL 808-11403;

(B) $808-11402$; (C) $808-11401$; (D) $808-11400]$ 
Table 5. RBE Values for One, Two, Five, or Ten Fraction Doses for Modulated Helium, Carbon, Neon, and Argon Beams

Source $\begin{array}{ccc}\text { Energy } \\ (\mathrm{MeV} / \mathrm{amu})\end{array} \quad \begin{gathered}\text { Ridge } \\ \text { Filter } \\ (\mathrm{cm})\end{gathered} \quad$ Position
Number of Fractions
One Two Five Ten

(10 surviving crypt dose)
One Two Five Ten

$\left(D_{0}{ }^{137} \mathrm{Cs} / D_{0}\right.$ source $)$

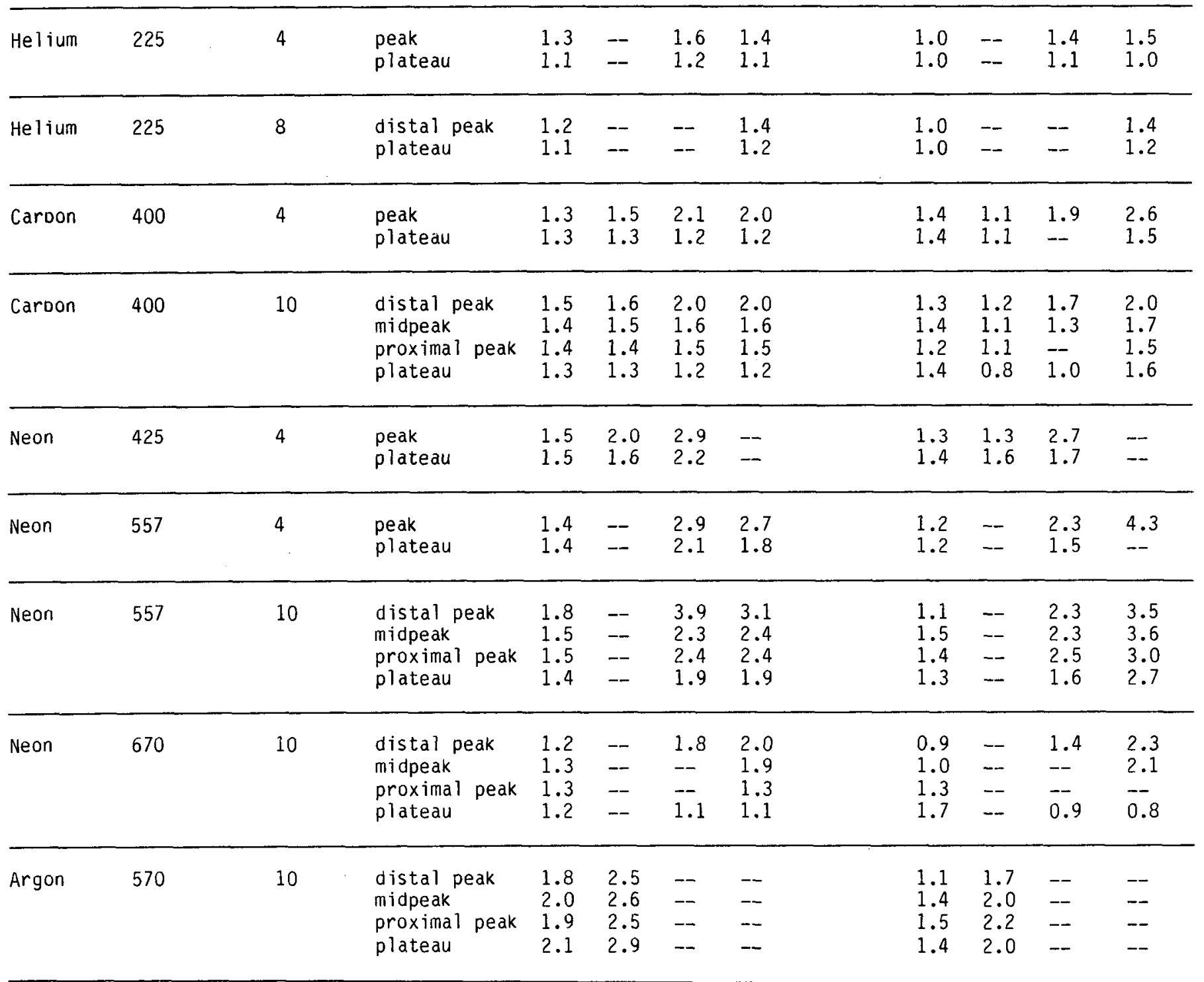


Table 6. Peak-to-Plateau RBE Ratios for One, Two, Five, Ten Fraction Doses for Modulated Helium Carbon, Neon, and Argon Beams

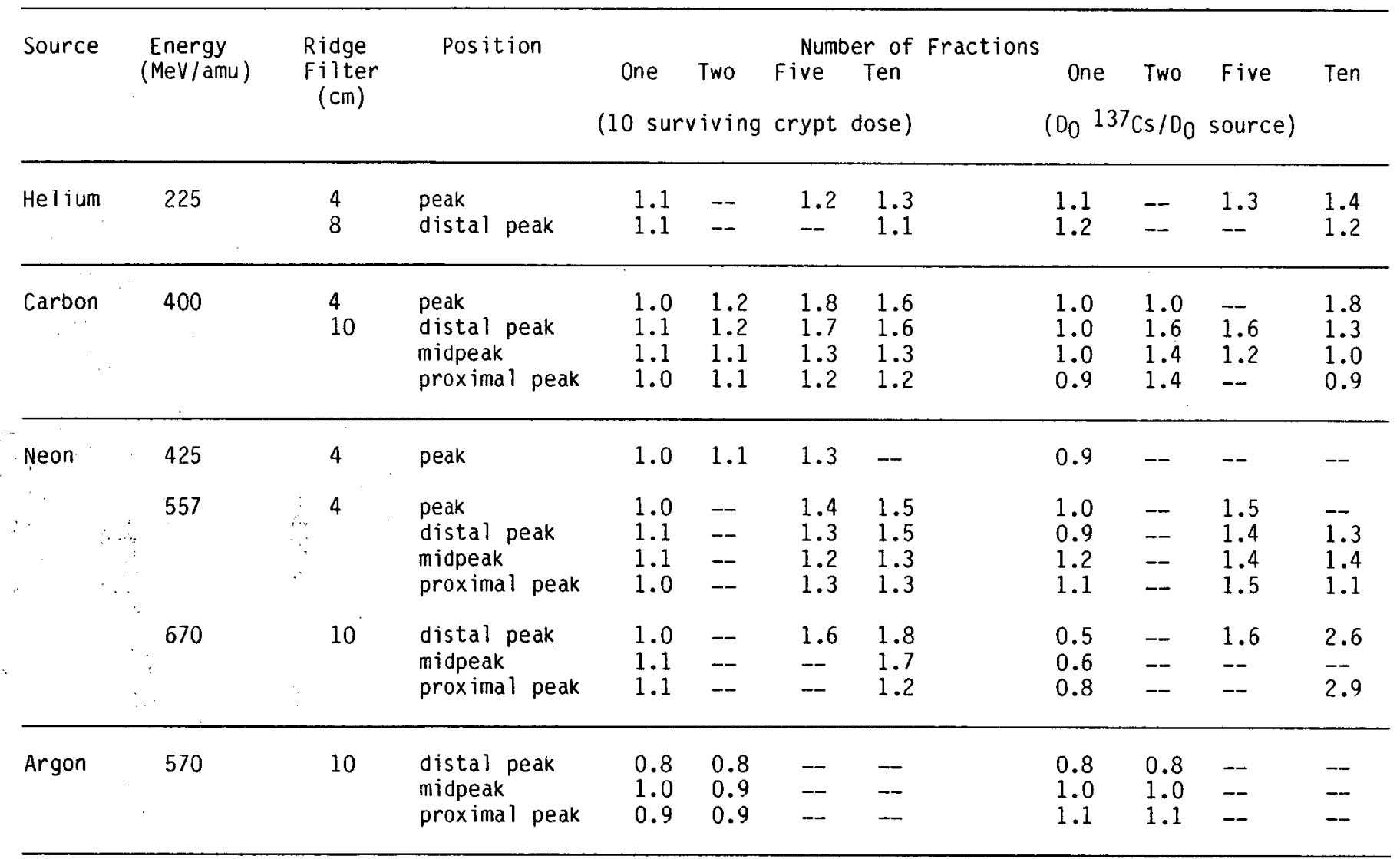




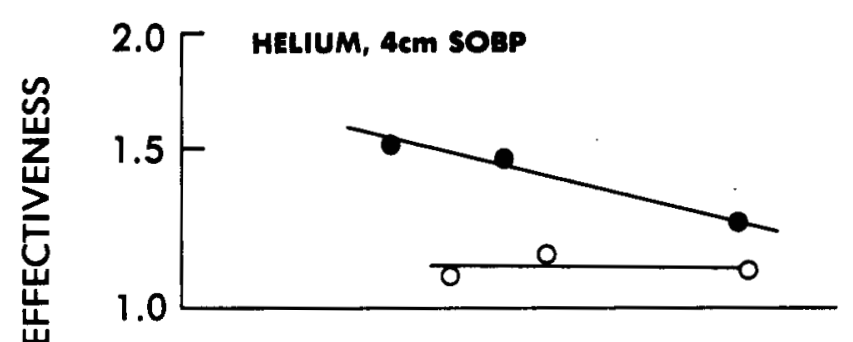

Figure 10. RBE vs. dose per fraction for jejunal crypt celis irradiated in the peak (closed symbols) or plateau (open symbols) of a $4-\mathrm{cm}$ spread Bragg peak (circles) or 8-cm spread Bragg peak (triangles) helium beam. (XBL 808-11399)

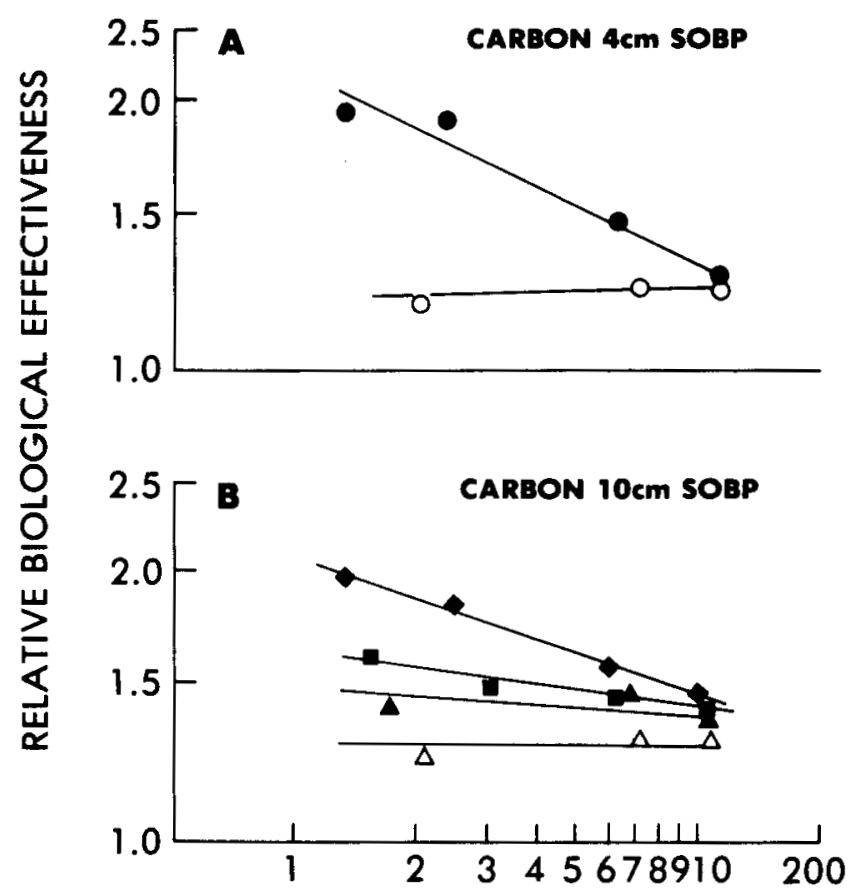

Figure 11. RBE vs. dose per fraction for jejunal crypt cells irradiated in the peak (closed symbols) or plateau (open symbols) of a $4-\mathrm{cm}$ spread Bragg peak $(\bullet, 0)$ or $10-\mathrm{cm}$ spread Bragg peak $(\Delta, \boldsymbol{\Lambda}, \mathbf{\square}, \Delta)$ carbon beam. (XBL 808-11398)

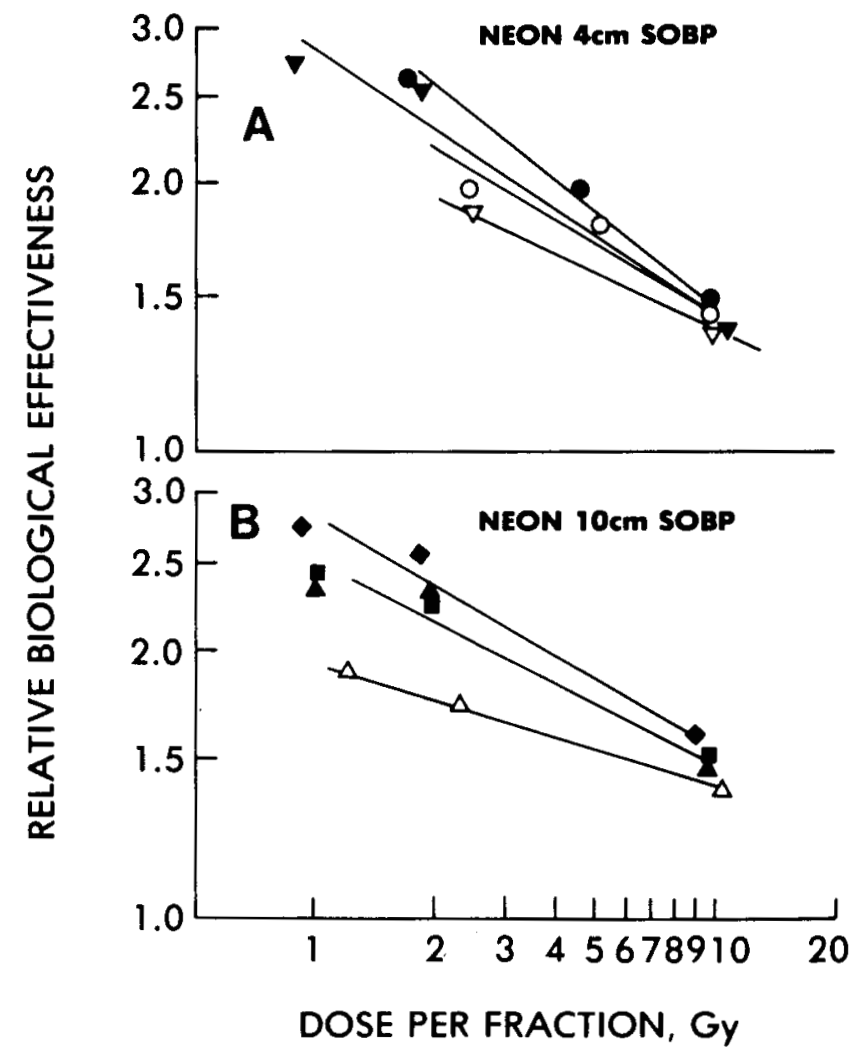

Figure 12. RBE vs. dose per fraction for jejunal crypt ceT1s irradiated in (A) $425 \mathrm{MeV} / \mathrm{amu} 4-\mathrm{cm}$ spread Bragg peak in the plateau $(\nabla)$ and peak ( $\nabla)$; $557 \mathrm{MeV} / \mathrm{amu} 4-\mathrm{cm}$ spread Bragg peak in the plateau (O) and the peak (O). (B) $557 \mathrm{MeV} / \mathrm{amu}$ $10-\mathrm{cm}$ spread Bragg peak in the plateau $(\Delta)$, proximal peak $(\boldsymbol{\Delta})$, midpeak $(\boldsymbol{\square})$, and distal peak $(\Delta)$. 
Table 7. High-LET Data for Esophagus

\begin{tabular}{|c|c|c|c|c|c|c|}
\hline Radiation & Range & $\begin{array}{l}\text { Ridge } \\
\text { Filter } \\
\text { (cm) }\end{array}$ & Position & $\begin{array}{c}\text { Number } \\
\text { of } \\
\text { Fractions }\end{array}$ & $L_{50 / 28}$ & RBE \\
\hline Helium & 26 & 8 & $\begin{array}{l}\text { Plateau } \\
\text { Peak } \\
\text { Plateau } \\
\text { Peak }\end{array}$ & $\begin{array}{r}1 \\
1 \\
10 \\
10\end{array}$ & $\begin{array}{l}3700 \\
3500 \\
-- \\
--\end{array}$ & $\begin{array}{l}1.05 \\
1.10 \\
-- \\
--\end{array}$ \\
\hline Carbon & 26 & 4 & $\begin{array}{l}\text { Plateau } \\
\text { Peak } \\
\text { Plateau } \\
\text { Peak }\end{array}$ & $\begin{array}{r}1 \\
1 \\
10 \\
10\end{array}$ & $\begin{array}{l}3348 \\
3150 * \\
-- \\
3724\end{array}$ & $\begin{array}{l}1.17 \\
1.24 \\
-- \\
2.25\end{array}$ \\
\hline Neon & 15 & 4 & $\begin{array}{l}\text { Plateau } \\
\text { Peak } \\
\text { Plateau } \\
\text { Peak }\end{array}$ & $\begin{array}{r}1 \\
1 \\
10 \\
10\end{array}$ & $\begin{array}{l}3440 \\
2790 \\
-- \\
2550 \star\end{array}$ & $\begin{array}{l}1.13 \\
1.40 \\
-3.30\end{array}$ \\
\hline Argon & 14 & 4 & $\begin{array}{l}\text { Plateau } \\
\text { Peak } \\
\text { Plateau } \\
\text { Peak }\end{array}$ & $\begin{array}{l}1 \\
1 \\
9 \\
9\end{array}$ & $\begin{array}{l}1920 \\
2800 * \\
2100 \\
--\end{array}$ & $\begin{array}{l}2.03 \\
1.40 \\
4.00 \\
--\end{array}$ \\
\hline
\end{tabular}

* Preliminary results, more data needed.

- Not done.

Table 8. High-LET Data for Lung

\begin{tabular}{|c|c|c|c|c|c|c|}
\hline Radiation & Range & $\begin{array}{l}\text { Ridge } \\
\text { Filter } \\
(\mathrm{cm})\end{array}$ & Position & $\begin{array}{c}\text { Number } \\
\text { of } \\
\text { Fractions }\end{array}$ & $L D_{50 / 160}$ & RBE \\
\hline Carbon & 26 & 4 & $\begin{array}{l}\text { Plateau } \\
\text { Peák }\end{array}$ & $\begin{array}{l}1 \\
1\end{array}$ & $\overline{1275}$ & $\overline{1.20}$ \\
\hline Neon & 15 & 4 & $\begin{array}{l}\text { Plateau } \\
\text { Peak } \\
\text { Plateau } \\
\text { Peak }\end{array}$ & $\begin{array}{r}1 \\
1 \\
10 \\
10\end{array}$ & $\begin{array}{l}1260 \\
1220 \\
-- \\
1480\end{array}$ & $\begin{array}{l}1.20 \\
1.25 \\
-- \\
2.90\end{array}$ \\
\hline Argon & 14 & 4 & $\begin{array}{l}\text { Plateau } \\
\text { Peak } \\
\text { Plateau } \\
\text { Peak }\end{array}$ & $\begin{array}{l}1 \\
1 \\
9 \\
9\end{array}$ & $\begin{array}{l}1043 \\
1842 \\
1166^{\star} \\
-\end{array}$ & $\begin{array}{l}1.30 \\
0.80 \\
3.70 \\
--\end{array}$ \\
\hline
\end{tabular}

* Preliminary results, more data needed.

-- Not done. 


\section{ACKNOWLEDGEMENTS}

We thank Mrs. G. Y. Ross, Mr. L. J. Kane, Ms. J. Vennari, Ms. M. Spence, and Ms. H. Kowta for their excellent technical assistance. We also thank the staffs of the Bevalac and 184-inch Synchrocyclotron for the ir invaluable assistance. This work was supported by NIH Grant CA17227 "The therapeutic ratio and experimental radiotherapy," T. L. Phillips, Principal Investigator, and DOE Contract DE AMOS-16SF0 1012 (Laboratory of Radiobiology).

\section{FOOTNOTES AND REFERENCES}

* Department of Radiation Oncology, University of California, San Francisco, CA.

Barendsen, G. W. 1968. Response of cultured cells, tumors, and normal tissues to radiations of different linear energy transfer. Curr. Topics Radiat. Res. Q. 4, 293-355.

Goldstein, L. S., T. L. Phillips, K. K. Fu, G. Y. Ross, and L. J. Kane. 1980. Biological effects of accelerated heavy ions: I. Single doses in normal tissues, tumors and cells in vitro. Submitted to Radiation Research.

Rockwell, S., R. F. Kallman, and L. T. Fajardo. 1972. Characteristics of a serially transplanted mouse mammary tumor and its tissueculture derivative. J. Nat]. Cancer Inst. 49, $739-749$.

Withers, H. R. and M. M. Elkind. 1970. Microcolony survival assay for cells of the mouse intestinal mucosa exposed to radiation. Int. J. Radiat. Biol. 17, 261-267. 


\title{
RESPONSE OF MOUSE SKIN AND BONE MARROW TO HEAVY CHARGED PARTICLES
}

\author{
E. J. Ainsworth
}

The early radiation-induced skin reaction in mouse skin (erythema, moist and dry desquamation) and the survival of nematopoietic stem cells (CFU-S) are two well-known model systems that have been used to explore both fundamental radiobiological questions, and to provide guidelines considered carefully by radiotherapists in the design of their own studies of the tolerance of normal numan tissues to single or fractionated doses of ionizing radiations. The acute skin response in the mouse has been used widely as new radiation modalities, such as nigh energy neutrons or negative $p i$ mesons, are being considered for radiotherapy, and it is essential to determine RBE values in connection with development of treatment plans for patients (Fowler, 1968; Raju and Carpenter, 1978; Leitn et al., $1975,1976)$. Both pig skin and rodent skin, along with other proliferative tissues, have been used in various calibration studies. Mouse skin has the advantage of providing inexpensive answers, with large sample sizes, on a timely basis. The pig skin reaction has the advantage that both early and late reactions can be studied, but in rodent skin the development of a true late-effect end point has proven problematic (Douglas and Fowler, 1976; Broerse et al., 1971; Fowler, 1968, 1969; Geraci, 1976, 1977; Fu et al., 1979; Hopewell et al., 1979).

When radiation therapy alone is used for human cancer treatment, bone marrow tolerance is not a dose-limiting factor. However, when a combination of ionizing radiation and chemotherapeutic drugs are used, acute bone marrow toxicity and damage to hematopoietic stem cells may indeed have direct clinical relevance. In the case of the use of combined modalities on younger patients, late effects on the hematopoietic system and other tissue may be important when either retreatment or cancer induction are considered. Nevertheless, the principal reason for studies with CFU-S are based on the relative ease with which early effects (such as cell kililing) and late effects (such as depression of femur CFU-S content) can be measured accurately.

Previous skin-tolerance studies with heavy charged particles have been conducted by Leith et a 1. (1976) and Raju and Carpenter (1978) using flank skin and foot response as, the end points. Both of these studies involved single doses and : some smal1 number of dose fractions ( 2 or 4 ): Because of the relationships between RBE and dose, and the desirability to determine RBE at therapeutically relevant dose levels, our approach was to use a challenge-dose technique that we have used previously and that Denekamp (1973) has explored extensively using the mouse foot as a model system. This challenge-dose technique assesses injury accumulation and repair or recovery following administration of "comparatively low" doses by challenging animals. This technique can also be used to determine the dose necessary to produce a given response level at different times following low fractionated doses (Ainsworth et al., 1968; Hanks and Ainsworth, 1967; Denek amp, 1973).

No previous studies have been conducted using CFU-S irradiated in vivo as a model system to assess the RBE of heavy charged particles for either cell killing or for late effects. The LET dependence of $\mathrm{D}_{0}$, or the dose necessary to produce a given percent survival, has been reported (Ainsworth et al., 1970; Carsten et al., 1976; Hendry et al., 1979). The goal of the CFU-S studies reported here was to determine the RBE for cell killing, and to use these data to design experiments to explore the effects of HZE particle dose fractionation on femur CFU-S repopulation and on late effects indicated by a reduction in the size of the CFU-S compartment. Some of these studies have been conducted collaboratively with J. C. Schooley, L. S. Kelly, and $D$. A Crouse.

\section{METHODS, PROCEDURES, AND EXPERIMENTAL DESIGN}

\section{Mouse Skin Studies}

To extend the previous work of Leith and associates, we used the same model system, $i . e .$, skin response in mice (BALB/c females, 100-150 days old). Hair on the right flank (both surfaces) was removed with Nair at least three days prior to irradiation. An occasional reaction to the depilatory agent occurred (mild erythema) and all animals showing such a response were rejected from the experiment before irradiation. Mice were anesthetized by i.p. injection of approximately $1.25 \mathrm{mg}$ of sodium pentabarbital, and the desired graded single or fractionated doses of $220 \mathrm{kVp} \times$ rays or charged particles were administered to the right flank; the remainder of the animal was either shielded ( $X$ rays) or out of the radiation field (charged particles). In a few experiments the foot was in the radiation field; which also included the tioia, fibula, and the distal half of the femur. Half of the femur was irradiated because in the radiation configuration used at the Bevalac, exposure of the entire femur was associated with exposure of the pelvic area and bowel, which resulted in intestinal death within a few days after irradiation. Because slightly different jigs were used to expose animals to $220 \mathrm{kVp} X$ rays and to charged particles at the Bevalac, it is likely that a slightly larger area of flank sk in was exposed to $X$ rays than to charged particles; the extent to which this influences the data is currently under study. 
We used the skin scoring protocol described by Leith et al. $(1975,1976)$ in his previous studies with $X$ rays, helium ions, and charged particles. This system, which has grades 0 through 4 to assess damage development and subsequent repopulation, was used to generate response-time curves that were totally consistent with results based on previous publications. The skin reactions were evaluated on each workday for four to eight weeks in two experiments; in all other experiments, the skin reaction was evaluated three times per week for approximately the same period of time: In some experiments mice were individually earmarked, and in other experiments the animals were identified only by cage, but in no case did the scorer know either the dose or the previously recorded reaction for the animal or cage of animals. The results presented here have been analyzed in two ways: the average sk in reaction in relation to dose over a period. of 6-7 to 34-35 days, or the peak reaction where values were averaged before, during, and immediately following the peak observation period. The latter scoring system is referred to as the "three day peak." J. Fowler, Hammersmith Hospital, London, indicated recently that he is considering eliminating average skin scores to focus on future skin reaction studies using end point such as the three day peak (J. Fowler, personal communication). This would eliminate the need for such personnel-intensive evaluation for long periods of time, and would provide adequately steep dose-response curves over a particular dose range that would be suitable for objective assessment. We feel that use of the three day peak is preferable because consideration is given primarily to the killing of skin stem cells rather than a combination of cell killing and repopulation.

It seems appropriate to state here that no further mouse skin studies will be conducted because of deletions from the animal support budget in the NIH grant under which these studies were supported partially. Further evaluations of the data will be done, a manuscript will be submitted for publication in the open literature but there are no plans to extend the studies further at this time.

\section{Hematopoietic Studies}

The CFU-S studies were conducted using the donor technique. 'Animals were given graded radiation doses, sacrificed between 30 and 120 min after irradiation, marrow cell suspensions were prepared, and appropriate aliquots were injected into superlethally irradiated mice (Ainsworth and Larsen, 1969). Using this procedure the CFU-S are irradiated in situ and comparatively few CFU-S are in cell cycle. This is in marked contrast to the situation that occurs when CFU-S harvested from unirradiated animals are given graded radiation doses. Interestingly, both procedures yield similar estimates of $D_{0}$ for photons (Ainsworth and Larsen, 1969). The donor procedure was preferable to the recipient procedure because the Bevalac beam time could be used more economically. Bone marrow was from the femurs using established methods (Schooley, 1966; Ainsworth and Larsen, 1969). The recipient animals were sacrificed ten days after the bone marrow injection, their spleens were removed and fixed in either Bouins or AFA, and nodules with an average diameter greater than $0.5 \mathrm{~mm}$ were counted. Survival curves were constructed based on surviving fraction per $10^{5}$ nucleated cells injected in irradiated animals in comparison with unirradiated controls. Estimates of $\mathrm{D}_{0}$ and extrapolation number, $N$, were obtained using a computer program kindly provided by E. L. Alpen.

Other pilot CFU-S studies are in progress to explore the extent to which single or fractionated radiation doses given to the right $h$ ind limb of the BALB/C mouse produced late alterations in the size and cell population kinetics of the CFU-S compartment. This effort has implications for radiation-induced leukemogenes is and represents an extension of previous studies following total-body irradiation that showed significant reductions in the femur and spleen CFU-S content (Ainsworth et al., 1974). In addition, different levels of reduction were observed when assays were based or, the number of CFU-S extracted from. the femur shaft and when "residual" CFU-S liberated from the bone was assayed (Crouse et al., 1977). Selected animals from the flank sk in studies of $X$-ray tolerance were retained for long-term assessment of CFU-S content. In a 71 cases, the right leg had been irradiated, and the left leg served as the control. Because Werts et al. (1978) have reported late changes in hematopoies is in the contralateral hind limb when the other limb was irradiated, CFU-S assays were also performed on appropriately aged controls. Aliquots of nucleated marrow cells obtained from the femur shaft or residua? bone were injected into syngeneic superlethally irradiated (900 rad gamma radiation) recipients, and spleen nodules were scored at 10 days.

\section{RESULTS AND DISCUSSION}

\section{CFU-S Studies}

The only completed data presented in this report are the CFU-S survival curves following graded doses of photons or heavy charged particles. Two replicate experiments have been conducted with $40 \mathrm{Ar}$ ions, and three or four replications have been completed with the other radiation qualities studied. Because of the limited availability of $40 \mathrm{Ar}$ from the Bevalac, that survival curve contains fewer number of points. For clarity, the computed survival curves for the various radiation qualities are presented without points in Figure 1, and the survival curve parameters and estimates of RBE are presented in Table 1 . The survival curve for ${ }^{60}$ Co gamma radiation conforms generally to expectations based on previously published values for extrapolation number $(\sim 1.3)$ and $D_{0},(\sim 130$ rad), (Til) and MCCulloch, 1961; Grahn et al., 1972; Carsten et al., 1976). The survival curves for heavy charged particles also conform generally to expectations based on previously published responses to densely ionizing radiations: extrapolation numbers of 1 or less were observed in 
Survival Curves for CFU-S Irrodiated in vivo

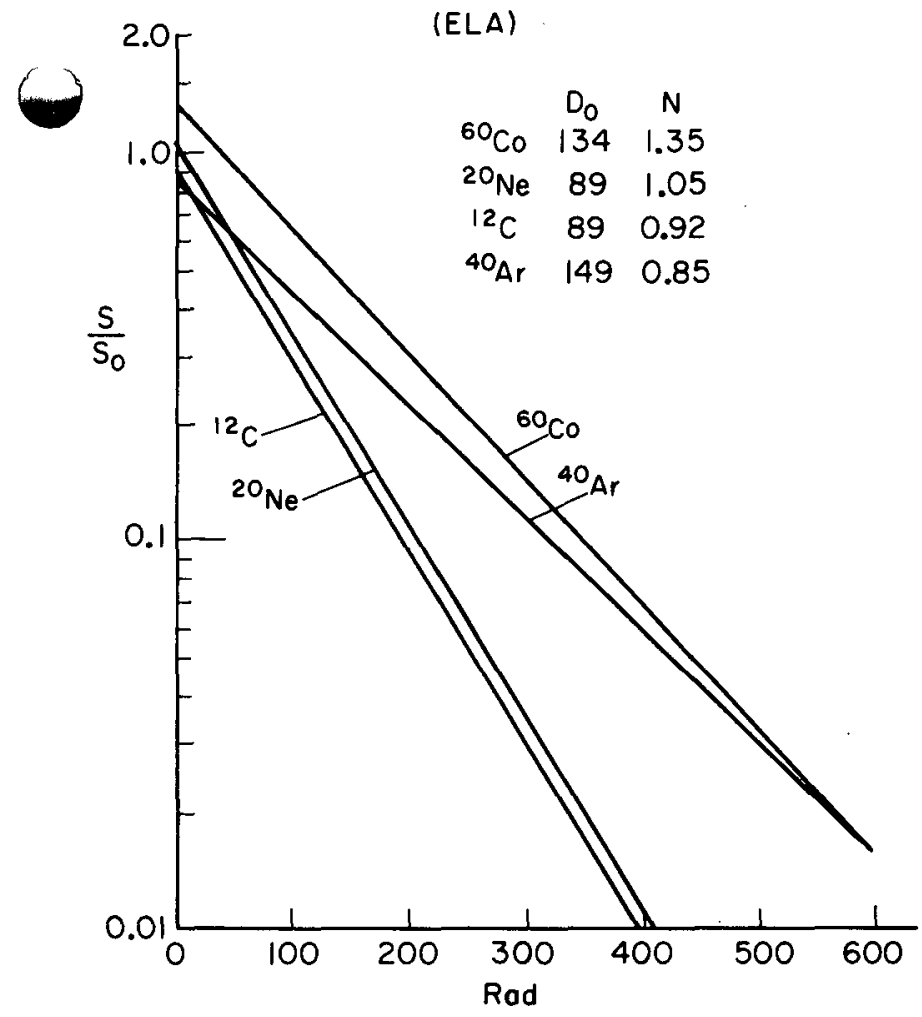

Figure 1. Survival curves for CFU-irradiated in vivo with $60 \mathrm{Co}$ gamma rays, or carbon, neon, or argon heavy charged particles at the midpoint of a 4-cm spread Bragg peak. (XBL 807-3511) all cases and a single exponential probably provides an adequate fit to the data. The significance and veracity of the extrapolation numbers less than 1 for ${ }^{12} \mathrm{C}$ and for $40 \mathrm{Ar}$ cannot be determined at this time, but computed extrapolation numbers of less than 1 were also observed previously by Ainsworth et al. (1976) for fission-spectrum neutrons. A subpopulation of CFU-S particularly sensitive to high LET radiations represents one possible explanation but this is totally speculative. The $D_{0}$ for $40_{A r}$ does not differ significantly from the $D_{0}$ for ${ }^{60} \mathrm{Co}$ gamma radiation, but the $\mathrm{D}_{0}$ for $12 \mathrm{C}$ and $20 \mathrm{Ne}$ are significantly lower than for 60 Co gamma radiation.

Because of the surprisingly low extrapolation number for $40 \mathrm{Ar}(0.85)$ the RBE estimate for $80 \%$ survival approaches 8.0 (Table 1 ). A similar situation prevails for $12 \mathrm{C}$, again because the extrapolation number is 0.92 , and the RBE at $80 \%$ survival approaches the value of 6.0 (Table 1 ). Clearly, uncertainties about survival curve fitting procedures and the absence of experimental points at low survival levels encourages prudence in interpreting these RBE estimates. At $50 \%$ and $10 \%$ survival levels, the RBE estimates decrease with decreasing survival, as expected, and the RBE estimate is the largest for ${ }^{12} \mathrm{C}$ ions. Further statistical evaluation will be necessary to determine if the RBE estimate for carbon differs significantly from the estimate for neon at the $50 \%$ level. Nevertheless, the important point is that the RBE for ${ }^{12} \mathrm{C}$ could be higher (and is at least not lower) than the RBE for $20 \mathrm{Ne}$, where the estimates of dose-

Table 1. Sensitivity of CFU-S Irradiated in situ with photons or Charged Particles

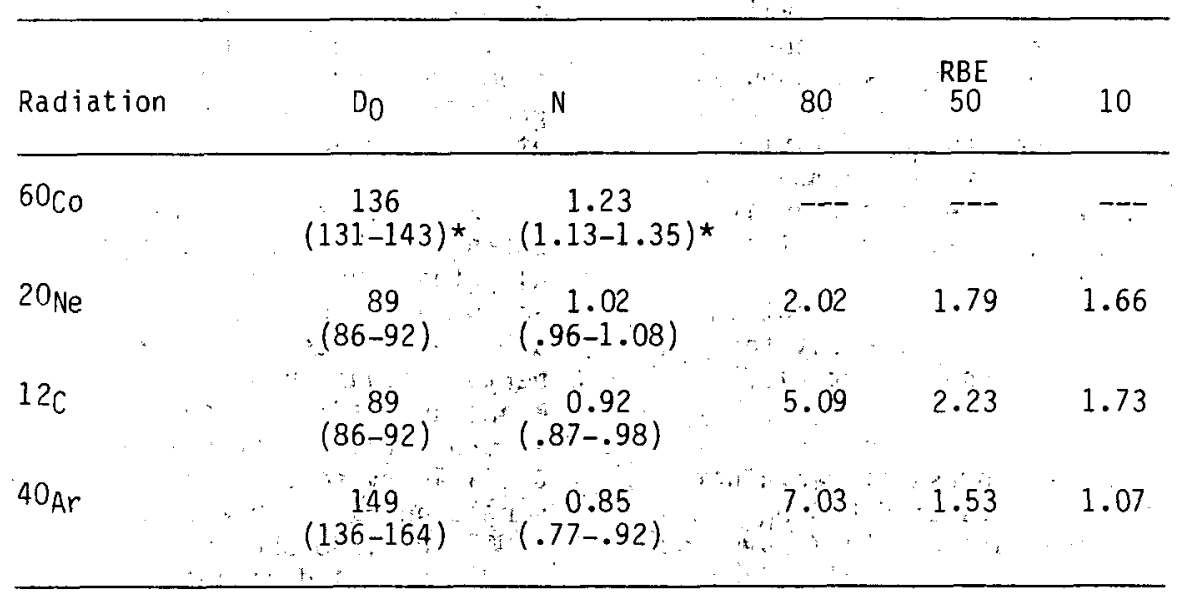

*95\% confidence limit. 
averaged LET are $\sim 40$ and $\sim 90$, $\mathrm{keV} / \mathrm{\mu m}$, respectively (Curtis, 1977). Thus; this model system fails to show a peak RBE at an LET value approaching $100 \mathrm{keV} / \mathrm{\mu m}$, as has been reported by Tobias et al. (1979) for several in vitro model systems. The concept that proliferative systems in vivo may snow a peak RBE at a lower LET value than in vitro systems is also supported by results on testis weight loss and intestinal microcolony survival (see E. L. Alpen et al., Part IV). Saturation or overkill appears to have occurred. In the case of heavy charged particles, charge, velocity, and mass may influence biological response under conditions where LET estimates are similar. The testis and intestinal microcolony data cited above are consistent with the conclusion that factors other than LET are important in radiation-induced cell killing and other end points.

One cautionary note is that a reassessment is in progress on calibration of the Victoreen ion chambers used in the CFU-S studies presented here on $60_{\mathrm{CO}}$ gamma radiation and other studies discussed below on skin using $220 \mathrm{keV} X$ rays. Depending upon the outcome, the estimates of rad dose could be altered, but the overall pattern of LET dependence would not be influenced. Estimates of RBE could be changed, but based on the gamma dose estimates presented here, the RBE estimates for ${ }^{12} \mathrm{C}$ and $20 \mathrm{Ne}$ are in the domain of those reported for fission spectrum neutrons (Grahn et a1., 1972; Carsten et al., 1976).

Future CFU-S experiments directed toward radiation sensitivity in relation to LET $_{\infty}$ will focus on comparison between stopping and nonstopping particles at similar estimated values of LET, and, on a selective bas is measurements of repair sublethal damage. Because the CFU-S has a low capacity for sublethal damage repair, other in vivo or in vitro model systems would be better to use. However, definitive information on repair following irradiation with $12 \mathrm{C}$ ions seems appropriate in order to establish a lower boundary condition for this normal tissue. $\mathrm{Spl}$ it-dose repair data for ${ }^{60}$ Co gamma radiation show, as expected, that the maximum survival ratio observed at $6-8$ hours is -1.4 . 0ther future studies with CFU-S will assess repopulation of the femur following fractionated (daily) doses of carbon ions compared to repopulation following fractionated doses of $60 \mathrm{co}$ gamma radiation when the daily dose per fraction is expected to produce equal cell killing. The objective is to determine if the rate of CFU-S repopulation in the femur is LET dependent in the same way that was observed previously for fission spectrum neutrons (Ainsworth et al., 1974).

Finally, we are in the process of reevaluating earlier unpublished results of CFU-S $D_{0}$ values after in vitro irradiation with $X$ rays and charged particles (L. Kelley, J. Schooley, and Thomas, private communication). Part of this reassessment includes using other survival curve algorithms to estimate RBE values for the in vitro data as well as the data presented here for in vivo irradiation. When completed, a joint publication will be submitted where LET-RBE relationsios for CFU-S killing will be addressed comprehensively based on both sets of data.

Studies of the late effects of ionizing irradiations, or aging, on the hematopoietic stem cell compartment provide insight into the extent to which the normal steady state is either reestablished or altered as a function of age after irradiation. Hematopoietic control systems are important to understanding radiation-induced leukemogenesis. The CFU-S is an adequate model system to study this problem, although CFU-S content per femur and total hematopoietic potential vary independently.

It was once thought that the CFU-S was the hematocytoblast, the most primitive hematopoietic stem cell, or that the CFU-S reflected directly the content of the nemotocytoblast. It is now known that the capacity for nematopoietic regeneration following radiation is not correlated directly with CFU content, especially when the normal steady state has been perturbed by ionizing radiations or otner treatments (Hanks and Ainsworth, 1967; Ainsworth and Larsen, 1969). More than ten years ago we showed that the femur CFU-S content was reduced to less than $5 \%$ of normal 5 days following 450 rad of $250 \mathrm{kVp}$ $X$ rays, but at that time the total hematopoietic capacity for regeneration was estimated to be nearly normal based on LD50/30 (Hanks and Ainsworth, 1967). Our studies have also shown that the fraction of CFU-S and DNA synthes is is increased when total femur CFU-S content is reduced as a consequence of radiation given several hundred days previously (Crouse et al., 1977). Although previous work has been done to assess marrow regeneration after local or partial body irradiation, we are not aware of data that measures explicitly CFU-S content (Knops, 1968; Patt and Maloney, 1976; Werts et a1., 1978). Our pilot project is intended to assess late effects of ionizing radiation on the CFU-S compartment and on the hematopoietic stroma. These studies are being conducted collaboratively with $\mathrm{D}$. A. Crouse at the University of Nebraska Medical Center, Lincoln. Both CFU-S content, the capacity of proliferative cells to form colonies in vitro, and the ability of nematopoietic stroma from aged irradiation animals to support CFU-S proliferation in vitro are being assessed (Crouse et al., 1980).

Representative results on CFU-S content are shown in Table 2. Although we had hoped originally that the unirradiated leg would provide an adequate control for comparison with the irradiated leg, Werts has reported contralateral perturbations when one leg is irradiated; this means unirradiated age control animals should be incorporated into the experimental design. These preliminary results, based on CFU-S assays of single animals, show a femur CFU-S content of 6,000 to $8,500 \mathrm{CFU}$ per femur shaft and 2,000 to 3,500 for residual bone in an animal at approximately 800 days of age. In comparison with this age control, the CFU content was reduced significantly in both the shaft and residual bone in an animal that was assayed 700 days after a single dose of 2,500 rad was applied to the right leg. In fact, the CFU content of the right leg 
Table 2. Late Effects of Irradiation on CFU-S Content in BALB/C Mice

\begin{tabular}{|c|c|c|c|c|}
\hline $\begin{array}{c}\text { Experiment } \\
\text { No. and Description }\end{array}$ & $\begin{array}{l}\text { : Total CFU-S } \\
\text { Shaft }\end{array}$ & $\begin{array}{r}\text { Content } \\
\text { Bone }\end{array}$ & $\begin{array}{l}\text { Spleen } \\
\text { Weight (mg) }\end{array}$ & $\begin{array}{l}\mathrm{HCT} \\
(\%)\end{array}$ \\
\hline \multirow{2}{*}{$\begin{array}{l}\text { CFU-54, Female BALB/C } \\
\text { mice, untreated }\end{array}$} & $6027 \pm 462$ & $1733 \pm 224$ & \multirow[b]{2}{*}{124} & \multirow[b]{2}{*}{38} \\
\hline & $8400 \pm 688$ & $3516 \pm 302$ & & \\
\hline \multirow{2}{*}{$\begin{array}{l}\text { CFU- } 48 \text {, Female BALB/C } \\
\text { mice treated with } \\
2,500 \text { rad single } x \text { ray }\end{array}$} & $\begin{array}{l}\text { Experimental leg } \\
2600 \pm 373\end{array}$ & $\begin{array}{l}\text { Experimental leg } \\
584 \pm 173\end{array}$ & \multirow[b]{2}{*}{75} & \multirow[b]{2}{*}{17} \\
\hline & $\begin{array}{l}\text { Control leg } \\
2000 \pm 494\end{array}$ & $\begin{array}{l}\text { Control leg } \\
916 \bullet 217\end{array}$ & & \\
\hline \multirow{2}{*}{$\begin{array}{l}\text { CFU }-52 \text {, female } B A L B / C \\
\text { mice treated with } \\
2,500 \text { rad single } x \text { ray }\end{array}$} & $\begin{array}{l}\text { Experimental leg } \\
4524 \pm 227\end{array}$ & $\begin{array}{l}\text { Experimental leg } \\
1048 \pm 109\end{array}$ & \multirow[b]{2}{*}{230} & \multirow[b]{2}{*}{50} \\
\hline & $\begin{array}{l}\text { Control leg } \\
5362 \pm 323\end{array}$ & $\begin{array}{l}\text { Control leg } \\
1204 \pm 127\end{array}$ & & \\
\hline
\end{tabular}

was slightly, but not significantly, nigher than the content in the unirradiated leg.

These preliminary results are interpreted to confirm the observation of Werts et a1. (1978) that contralateral responses occur and are reflected in a CFU-S content. The data presented in Table 2 are selected to illustrate a point; they are not representative of other results that were obtained when the CFU-S content was assayed in animals whose spleen weight exceeded $150 \mathrm{mg}$. The situation becomes more complicated when spleen weight is increased due to reticulum cell sarcoma or other neoplastic processes. CFU-S content appears to be highly variable, and no apparent reduction in comparison to the content. in age controls is observed. More data are needed before firm conclusions regarding late effects after partial body irradiation can be drawn.

\section{Mouse Skin Studies}

(a)

The preliminary results of our skin tolerance work are included in this report to illustrate the experimental design and potential information gain when the results are fully analyzed. Eye-fitted curves without individual data points are presented in Figures 2. through 4. Average sk in response (7-35 days or $6-34$ days) is shown when mice receive either single doses or were $\ldots$ challenged with graded single doses at various times after having been given eight $300 \mathrm{rad}$ fractions. The objective was to evaluate repair otential between radiation fractions by chalenging the animals at the time the eighth fractionated dose was given. Challenging animals at various times following completion of the
$X-$ RAY

AVERAGE SCORE vs. DOSE

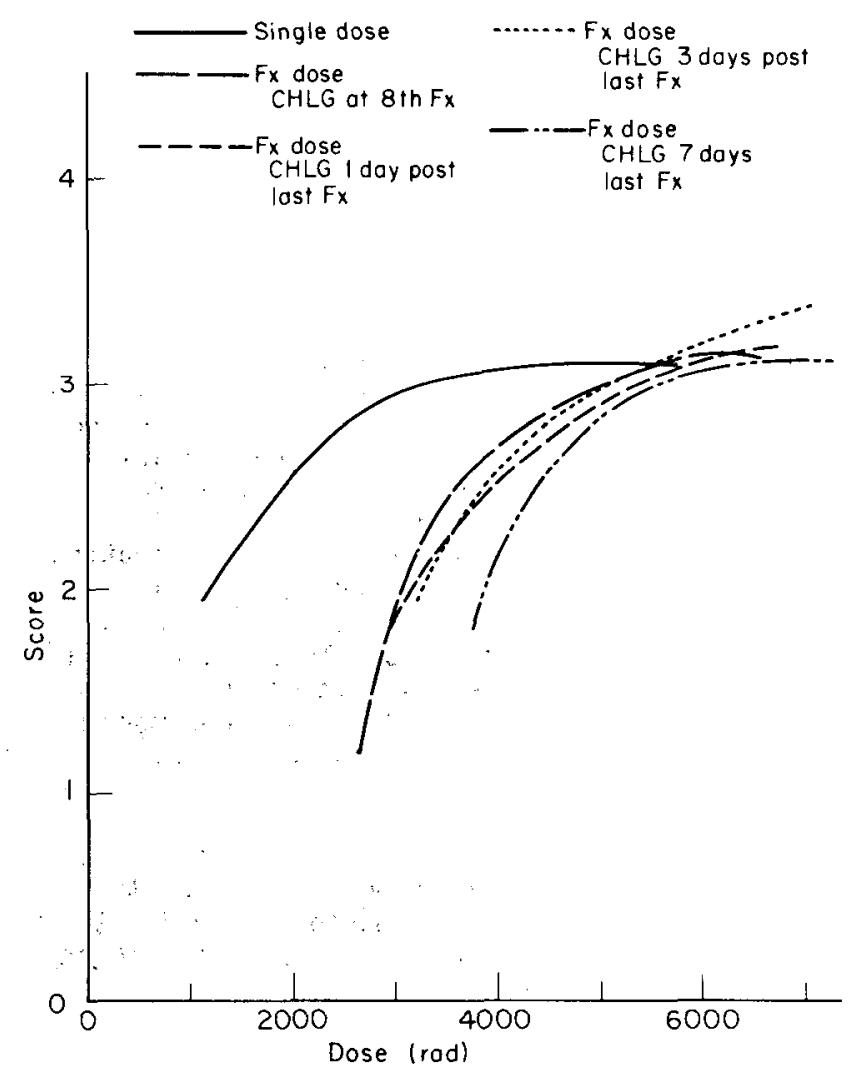

Figure 2. Average skin scores (7-35 days) in BALB/C mice given single or fractionated doses of $X$ rays. (XBL 809-3674) 
CARBON

AVERAGE் SCORE VS. DOSE

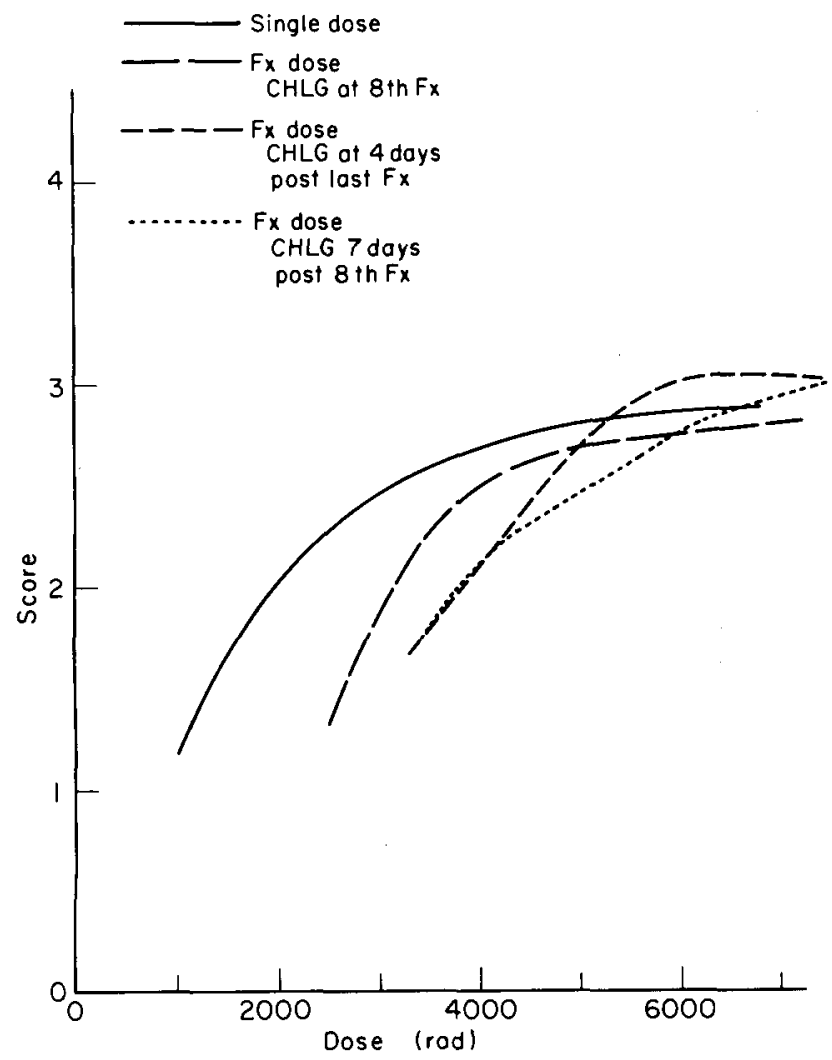

Figure 3. Average skin scores (7-35 days) in BALB/C mice given single or fractionated doses of plateau carbon ions. (XBL 809-3673)

fractionated sequence infers that proliferation of skin stem cells accounts for any increase in skin tolerance. Because statistical evaluations of the results are not complete, and further dosimetric studies are in progress using lithium fluorid chips and Victoreen $R$ meters, we are not prepared to treat quantitatively these results at this time. We have no reason to feel that any of the dose estimates provided in the figures are in serious error, with the exception that at face value the single dose results indicate that the RBE is less than 1 for both plateau neon and carbon ions. This observation is unexpected and contrasts Leitn's results (Leith et al., 1976), therefore, we need more data before firm conclusions can be drawn.

As with the previously published results (Leith et al., 1975, 1976; Denekamp, 1973), the shapes of the dose response curves appear to be linear only at comparatively low average score levels. Thereafter, the response curves tend to saturate with increasing dose, and may actually show a negative slope. These low levels of reaction are a particular scoring challenge, "and. their accuracy and reproducibility is open to question. Leith, using the same mouse strain,
NEON

AVERAGE SCORE vs. DOSE

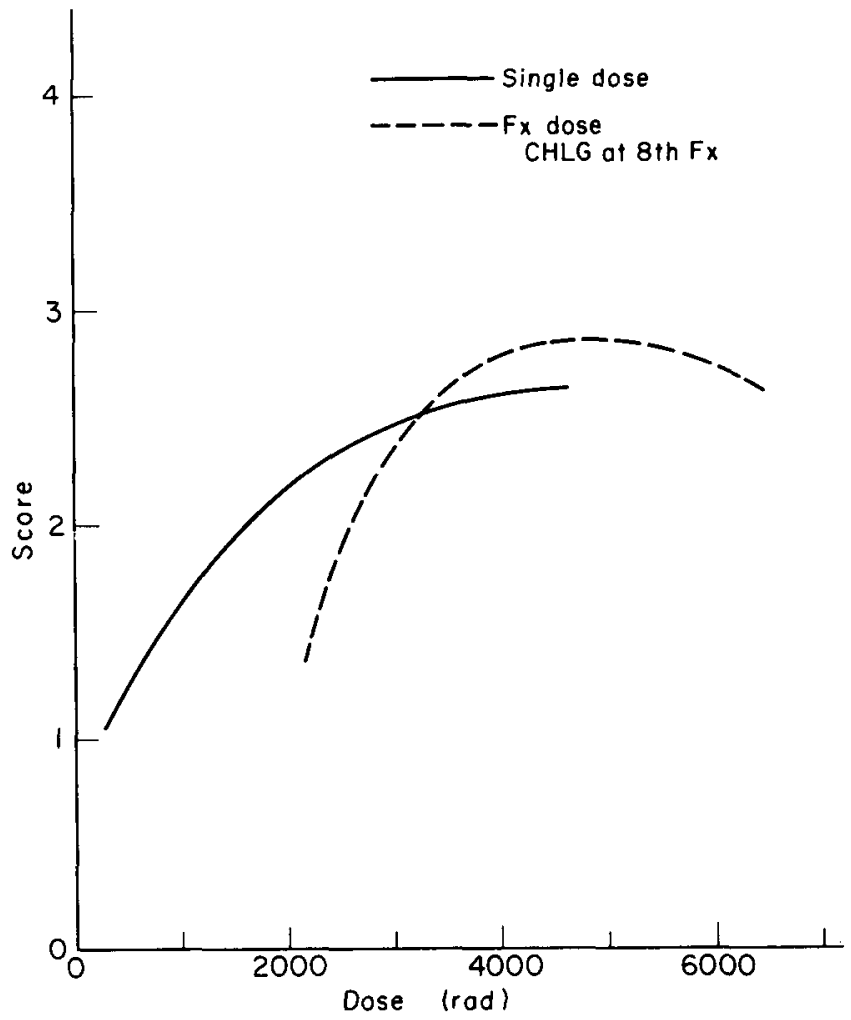

Figure 4. Average skin scores (7-35 days) in BALB/C mice given single or fractionated doses of plateau neon ions. (XBL 809-3672)

same X-ray machine, and same dosimetry methods, reported a 500 rad difference for the doses necessary to produce average reaction levels of 2.0 and 2.5. If the variation amounts to as much as 15-25\% from experiment to experiment, it is imperative to use appropriate sample sizes and to replicate experiments before drawing firm conclusions from skin reaction studies. A fair appraisal is that skin tolerance in the rodent has been used satisfactorily over several decades inspite of the inherent variability, and that both fundamental and clinically relevant conclusions have been drawn from such results (Jacobsen et al., 1958; Douglas and Fowler, 1976; Denekamp, 1973).

Although the shapes of the dose response curves in Figures 2 through 4 are not parallel, and conclusions regarding the degree of repair would be influenced by the reaction levels scored, a first approximation of repair capacity is gained by evaluating the extent to which the dose-response curve for animals challenged at the eighth fraction is displaced to the right in comparison with the single dose-response curve. Qualitatively, it appears that more efficient repair occurs between $X$-ray fractions than between fractions of carbon or neon ions. Irrespective of total dose or fraction size, 
Denekamp (1973) reported a large increase in skin tolerance by days 8 and 15 after an $x$-ray fractionation sequence was complete, due probably to cell proliferation. The present results for both $X$ rays and carbon ions are similar to Denekamp's results: by day 7 , skin tolerance is increased compared to the response in mice challenged at the 8 th fraction. There seems to be no large difference in incremental tolerance for $X-r a y$ and ${ }^{12} \mathrm{C}$ ion irradiation.

We have conducted one fractionated $x$-ray experiment; mice were given 20 graded $X$-ray fractions over 4 weeks, and the average and 3-day-peak reaction were scored. Sk in reactions were evident among these animals before the fractionation sequence was completed, but the temporal distribution of skin reaction was spread over greater time and showed much less of a peak in the mice given fractionated doses vs. those given a single dose. To achieve a reaction level of 2.5 , a total dose of 5,000 rad was required when administered in 20 fractions, but a single dose of 1,400 rad produced the same reaction level. A 3.6-fold increase in tolerance is consistent with a greater repair per fraction than has been detected from either paired dose studies or studies of repair when only a few fractions were given (Denekamp, 1973). Further evaluation is also necessary, but if these data are extrapolated for radiotherapeutic applications involving long-term fractionation sequences, the animal experiments that yield inferences pertaining to repair following only a few fractions could underestimate the total repair or proliferation capacity and skin tolerance.

NOTE: Quantitative estimates of dose-averaged LET $_{\infty}$ values presented here should be considered as approximations only, because different calculational models yield markedly different results. In addition, for spread Bragg peaks the distributions of LET values is broad with the fraction of LET particles rising sharply at the distal peak. Ongoing beam fragmentation studies will yield new data that will contribute to improved estimation of dose-averaged LET . Microdosimetric studies provide a separate measurement of beam quality.

\section{ACKNOWLEDGEMENT}

The assistance of John Prioleau, Andy Shih, Coleen Caudill, and Panna Lee is gratefully acknowledged. The cooperation of Jerry Howard, Tom Criswel], and the Bevalac operations staff is also gratefully acknowledged. These studies were supported in part by Grant CA 15184 from the National Cancer Institute (C: A. Tobias, Principal Investigator).

\section{REFERENCES}

insworth, E. J. 1974. Effects of single or fractionated doses of neutron or gammaradiation on hematopoietic stem cells. Radiat. Res. 59, 314-315. (Abstract).
Ainsworth, E. J. and R. M. Larsen. 1969. Colony-forming units and survival of irradiated mice with AET or endotoxin. Radiat. Res. 40, 149-176.

Ainsworth, E. J., N. P. Page, J. F. Taylor, G. F. Leong, and E. T. Still. 1968. Dose-rate studies in sheep and swine. Symposium on Dose Rate Effects in Mammalian Radiation Biology, pp. 4.1-4.22. USAEC Report CONF 680410. Washington, D. C.

Ainsworth, E. J., R. M. Larsen, K. Kendall, G. F. Leong, J. S. Krebs, and F. A. Mitchel1. 1970. Recovery in the mouse after neutron irradiation. Evaluation of injury and recovery using split-dose lethality and repopulation of colony-forming units. Symposium on Neutrons in Radiobiology, pp. 435-450. USAEC Report CONF 691106. Washington, D. C.

Ainsworth, E. J., R.J.M. Fry, D. Grahn, F. S. Williamson, P. C. Brennan, S. P. Stearner, A. V. Carrano, and J. H. Rust. 1974. Late effects of neutron or gamma irradiation in mice. Biological Effects of Neutron Irradiation, pp. 359-379. International Atomic Energy Agency, Vienna.

Ainsworth, E. J., D. L. Jordan, M. Miller, E. M. Cooke, and J. S. Hulesch. 1976. Dose rate studies with fission spectrum neutrons. Radiat. Res. 67, 30-45.

Broerse, J. J., G. W. Barendsen, G. Freriks, and L. M. Van Patten. 1971. RBE values of $15 \mathrm{MeV}$ neutrons for effects on normal tissues. Europ. 3. Cancer 7, 171-177.

Carsten, A. L., V. P. Bond, and K. Thompson. 1976. The RBE of different energy neutrons as measured by the nematopoietic spleen-colony technique. Int. J. Radiat. Bio 1. 29, 65-70.

Crouse, D. A., E. J. Ainsworth, J. J. Hulesch. 1977. Thymidine suicide studies on nematopoietic stem cells in aging irradiated mice. Anat. Record. 189, 539. (Abstract).

Crouse, D. A., S. L. Mann, and E. J. Ainsworth. 1980. In vitro assessment of acute and late radiation damage in the hematopoietic microenvironment. Radiat. Res."; in press. (Abstract).

Curtis, S. B. 1977. Calculated LET distributions of heavy-ion beams. Int. J. Radiat. Oncol. Biol. Phys. 3, 87-91.

Denekamp, J. 1973. Changes in the rate of repopulation during multifraction irradiation of mouse skin. Br.J. Radiol. 46, 381-387.

Douglas, B. G. and J. F. Fowler. 1976. The effect of multiple small doses of X-rays on skin reactions in the mouse and a basic interpretation. Radiat. Res. 66, 401-426.

Fowler, J. F. 1968. Effects of fractionated irradiation on the skin of pigs and other animals and on tumors in rats. Symposium on 
Dose Rate in Mammalian Radiation Biology, pp. 21.1-21.25. USAEC Report CONF 680410. Washington, D. C.

Fowler, J. F. 1969. Effects of radiation exposure in the therapeutic range: Truncated doses and dose fractionation. Late Effects of Radiation (R.J.M. Fry, D. Grahn, M. C. Griem, and J. H. Rust, eds.), pp. 65-78. Taylor and Francis, London.

Fu, K. K., T. L. Phillips, D. C. Heilbron, G. Ross, and L. J. Kane. 1979. Relative biological effectiveness of low- and high-LET radiotherapy beams for jejunal crypt cell survival at low doses per fraction. Radiology $132,205-209$.

Geraci, J. P., K. L. Jackson, G. M. Christensen, $P$. D. Thrower and B. J. Weyer. 1977. Acute and late damage in the mouse small intestine following multiple fractionations of neutrons or x-rays. Int. J. Radiat. Oncol. Biol. Phys. 2, 693-696.

Grahn, D., E. J. Ainsworth, F. S. Williamson, and R.J.M. Fry. 1972. A program to study fission neutron-induced chronic injury in cells, tissues and animal populations utilizing the JANUS reactor of the Argonne National Laboratory. Radiobiological Applications of Neutron Irradiation, pp. 211-228. International Atomic Energy Agency, Vienna.

Hanks, G. E. and E. J. Ainsworth. 1967. Endotoxin-protection of mice. The relationship to colony-forming units. Radiat. Res. 32 , $367-382$.

Hendry, J. H., J. Baarli, M. Bianchi, and A. H. Sullivan. 1979. The effect of a $600 \mathrm{MeV}$ neutron beam on mouse bone marrow. Int. $\mathrm{J}$. Radiat. Biol. 35, 393-397.

Hopewe 11, J. W., J. L. Foster, C.M.A. Young, and G. Wiernik. 1979. Late radiation damage to pig skin. The effects of overall treatment time and number of fractions. Radiology 130 , $783-788$.

Jacobsen, E. M., A. K. Davis, and E. L. Alpen. 1958. Effect of fractionation of beta irradiation on rat skin. Radiat. Res. 9, 358-368.
Knops, W. H., J. Blom, and W. H. Crosby. 1968. Regeneration of locally irradiated bone marrow. II. Induction of regeneration in permanently aplastic medullary cavities. Blood 31, $400-405$.

Leith, J. T., W. A. Schilling, J. T. Lyman, J. Howard, and D. G. Baker. 1975. Comparison of skin responses of mice after single or fractionated exposure to cyclotron-accelerated helium ions and $230 \mathrm{kV}$ X-irradiation. Radiat. Res. 62, 195-215.

Leith, J. T., K. H. Woodruff, and J. T. Lyman. 1976. Early effects of single doses of $375 \mathrm{MeV} /$ nucleon 20 neon ions on the skin of mice and hamsters. Radiat. Res. 65, 440-450.

Patt, H. M. and M. A. Maloney. 1976. Regulation of stem cell after local bone marrow injury: The tale of an osseous environment. Stem Cells of Renewing Cell Populations, pp. 239-253. Academic Press, New York.

Raju, M. R. and S. G. Carpenter. 1978. Heavy particle comparative study for acute and late reactions. Br. J. Radiol. 51,, 720-727.

Schooley, 3. C. 1966. The effect of erythropoiet in on the growth and development of spleen colony-forming cells. J. Cell. Physiol. 68, 249-262.

Ti11, J. E. and E. A. McCullough. 1961. A direct measurement of radiation sensitivity of normal mouse bone marrow cells. Radiat. Res. $14,213-222$.

Tobias, C. A., E. A. Alpen, E. A. Blakely, J. R. Castro, A. Chatterjee, G.T.Y. Chen, S. B. Curtis, J. Howard, J. T. Lyman, and F.Q.H. Ngo. 1979. Radiobiological bas is for heavy-ion therapy. Treatment of Radioresistant Cancers, pp. 159-183. Elsevier/North-Holland Biomedical Press, Amsterdam.

Werts, E. D., M. J. Johnson, and R. L. DeGowin. 1978. Abscopal supression of bone marrow erythropoiesis. Radiat. Res. 76, 206-218. 


\title{
DOSE RESPONSE FOR RAT SKIN TUMORS INDUCED BY SINGLE AND SPLIT DOSES OF ARGON IONS
}

\author{
F. J. Burns* and R. E. Albert*
}

Considerable attention has been given to establishing the dose response for induction of tumors by low LET radiations, such as $x$ rays and electrons. Skin and otner organs are capable of repairing part of the damage that eventually leads to induction of tumors if the radiation is low LET. It is reasonable to expect on the basis of whole body irradiation with neutrons that oncogenic damage induced by nigh-LET radiation is such that the tissue cannot recover. Certainly numerous studies have shown cells are incapable of repairing the lethal damage produced by nighLET radiation. These lethality studies showed that the LET with the greatest biological effectiveness was about $125 \mathrm{keV} / \mu \mathrm{m}$ above which the energy density becomes so great that energy is wasted and efficiency declines.

With the availability of the argon-ion beam at the Bevalac facility at LBL, it became feasible to study repair or recovery at the LET of maximum biological effectiveness. The plateau region of the Bragg ionization curve has an LET of about $125 \mathrm{keV} / \mathrm{\mu m}$. The existence of extensive data on the dose-response curve for tumor induction in rat skin made this an ideal system for studying the RBE and repair at high-LET values in a specific organ where whole body irradiation can be avoided.

Male CD strain rats (Charles River, Wilmington, MA) were arranged in small boxes in such a way that the skin could be drawn up through the top of the box and the beam passed through double thickness flaps of twenty rats simultaneously. The total skin thickness was about $6 \mathrm{~cm}$ in comparison to the $16 \mathrm{~cm}$ range of the argon ion beam in water. The area irradiated was about $12 \mathrm{~cm}^{2}$.

The rats were irradiated with various doses in a matter of a few minutes. As seen in Figure 1 , tumors began to appear by about 10 weeks in the higher dose groups and continued to appear. more or less steadily until the end of the experiment at 99. weeks when overall survival was about $70 \%$.

Similarly, when the dose was fractionated the tumors began to appear at 10 weeks and continued to appear throughout the experiment (Fig: 2) at both doses. The fractionated exposures, rather than reducing the yield of tumors as for fractionated electron radiation, actually produced a slight increase in tumor yield, especially at $1.5 \mathrm{~h}$. The reason for this slight increase in tumor yield is not clear.

The dose response for all epithelial tumors 49 weeks is shown in Figure 3 . The tumor yield was essentially linear with dose throughout the range up to about 1,000 rad. The fractionated exposures were never less oncogenic than the

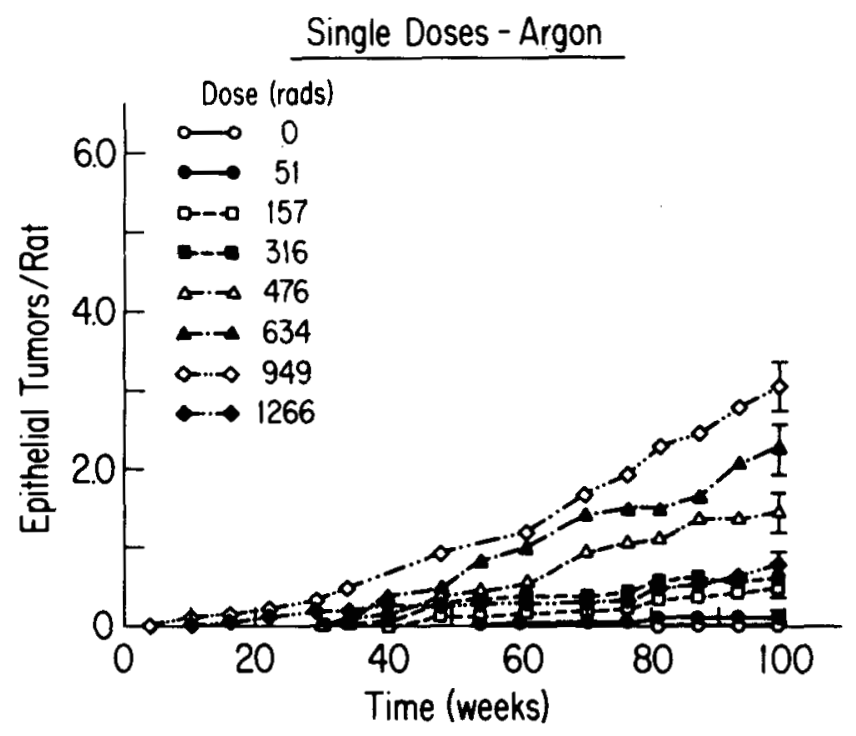

Figure 1. Epithelial tumors in rat skin irradiated with various single doses of argon ions as indicated. There were 20 rats per group except at 51 and 157 rads where there were 60 rats per group. Time zero was the day of irradiation. (XBL 808-11213)

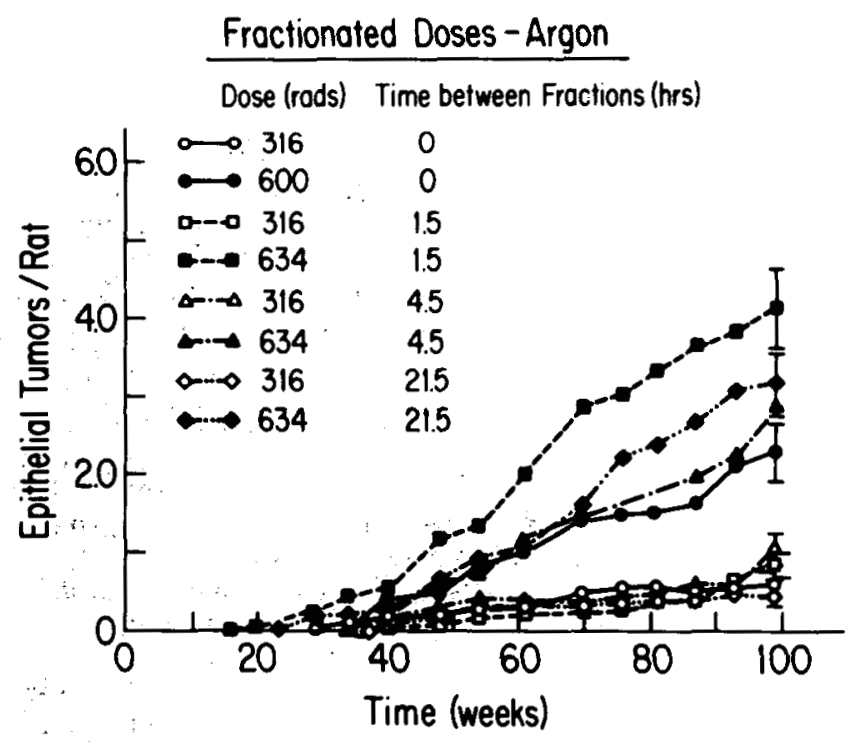

Figure 2. Ep ithelial tumors in rat skin irradiated with one dose or two equal doses split by various periods of time as indicated. Time zero was the day of irradiation and there were 20 rats per dose group. (XBL 808-11212) 


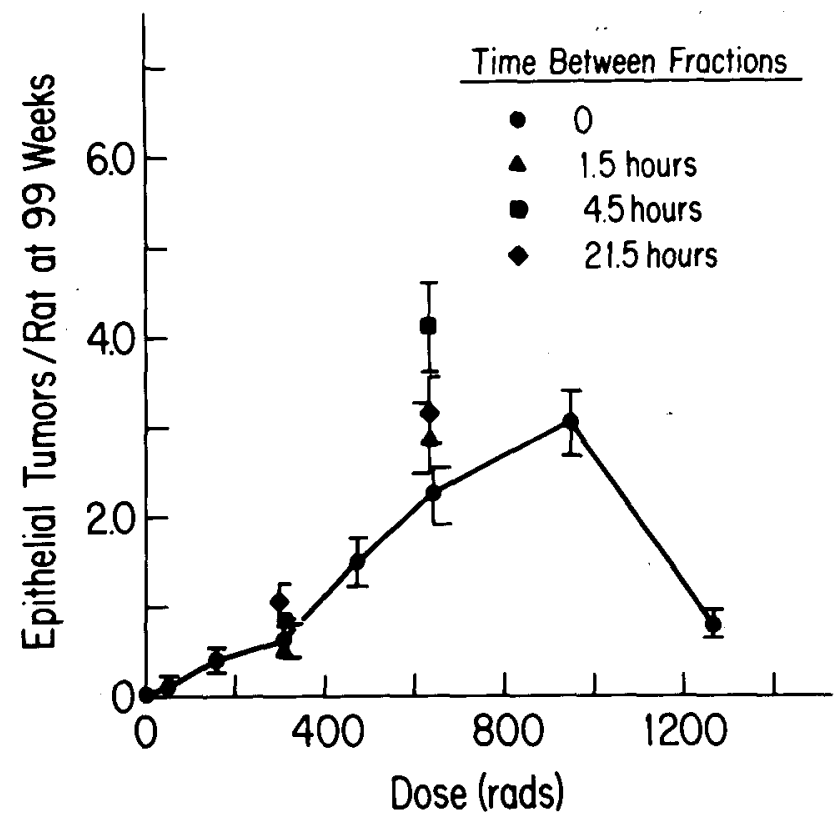

Figure 3. The dose response at 99 weeks for epithelial skin tumors induced by one dose or two equal dose of argon ions as indicated. The error bars are standard deviations derived from the square root of the total number of tumors in each treatment group. (XBL 808-11211)

corresponding single exposures, and in several cases fractionation actually enhanced the tumor yield slightly at the higher dose. No enhancement was observed at the lower dose, which raises a question about its validity at the nigher dose. Not even a suggestion of recovery or repair is apparent in these data.

When the epithelial tumors were classified into various subtypes, the overall pattern for each was about the same as for the epithelial tumors as a whole (Fig. 4). There seems to be a departure from linearity at low doses for the miscellaneous category, but the yield of tumors in this category was comparatively small and statistical variability was correspondingly large. No evidence for recovery or repair was found in any of the subtypes.

Comparatively large numbers of connective tissue tumors were found in the present experiment probably because the straight-through irradiation technique meant that a greater proportion of the dermal cells were irradiated than in earlier experiments with electrons where the penetration was limited. The dose response for fibromas (benign connective tissue tumors) is shown in Figure 5. The curve appears to be linear at lower doses, aithough the maximum tumor yield occurs at a considerably lower dose than the peak for epithelial tumors. The fractionated doses showed no evidence for recovery or repair. There was enhancement of the fibroma yield for fractionation intervals of 1.5 and $4.5 \mathrm{~h}$ but not for $21.5 \mathrm{~h}$. The fibromas were the most numerous type of tumor in the middle dose regions.

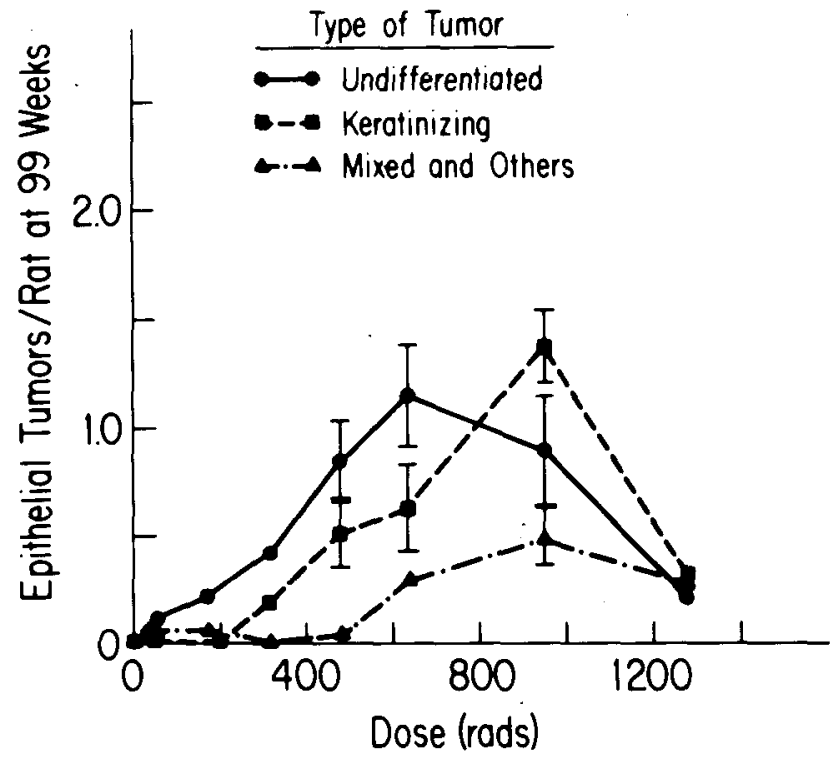

Figure 4. The dose-response at 99 weeks for various types of epithelial skin tumors induced by single doses of argon ions as indicated. Error bars are standard deviations based on the square root of the total number of tumors in each treatment group. (XBL 808-11210)

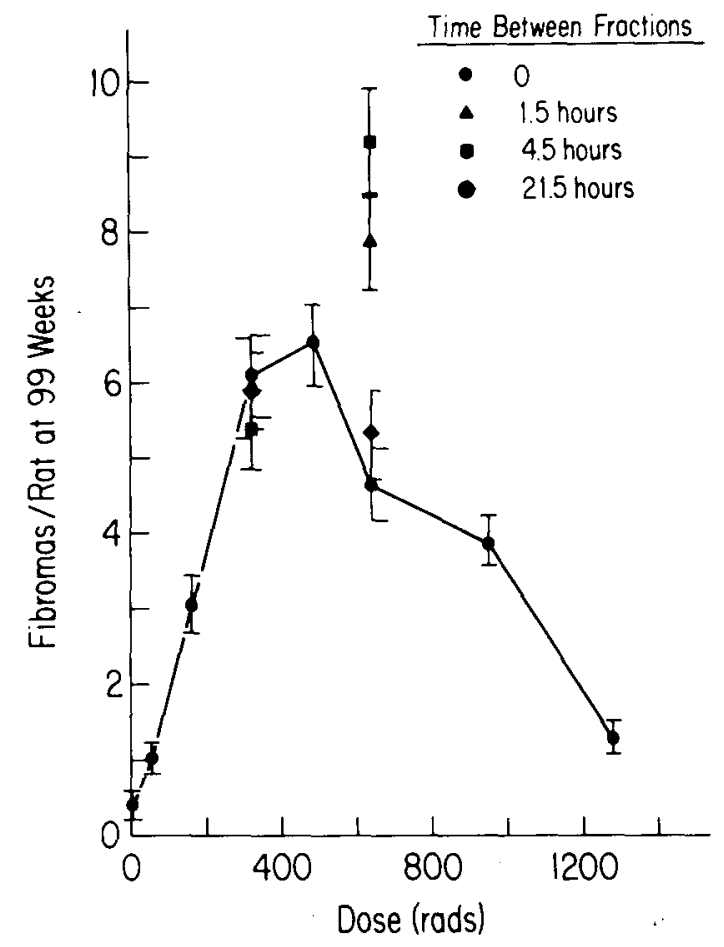

Figure 5. The dose response at 99 weeks for fibromas induced by one or two equal doses of argon ions as indicated. Error bars are standard deviations based on the square root of the total number of tumors in each treatment group.

(XBL $808-11209$ ) 
Sarcomas were seen more frequentiy than in previous experiments with electrons. The sarcoma data are shown in Figure 6 . The error bars are comparatively large but the data are consistent with a linear dependence of tumor yield on dose, and no evidence of repair or split dose recovery can be seen.

Tumor induction in rat skin by argon ions is strikingly similar in dose response and amount of recovery to cell lethality produced in tissue culture by the same radiation beam. It is intriguing that for yet another end point, highLET radiation produces an effect that is approximately linear with dose and from which the tissue is unable to recover. There may have been a slight enhancement in the yield of tumors but this remains to be confirmed.

\section{FOOTNOTE}

* Institute of Environmental Medicine, New York University Medical Center, New York, NY.

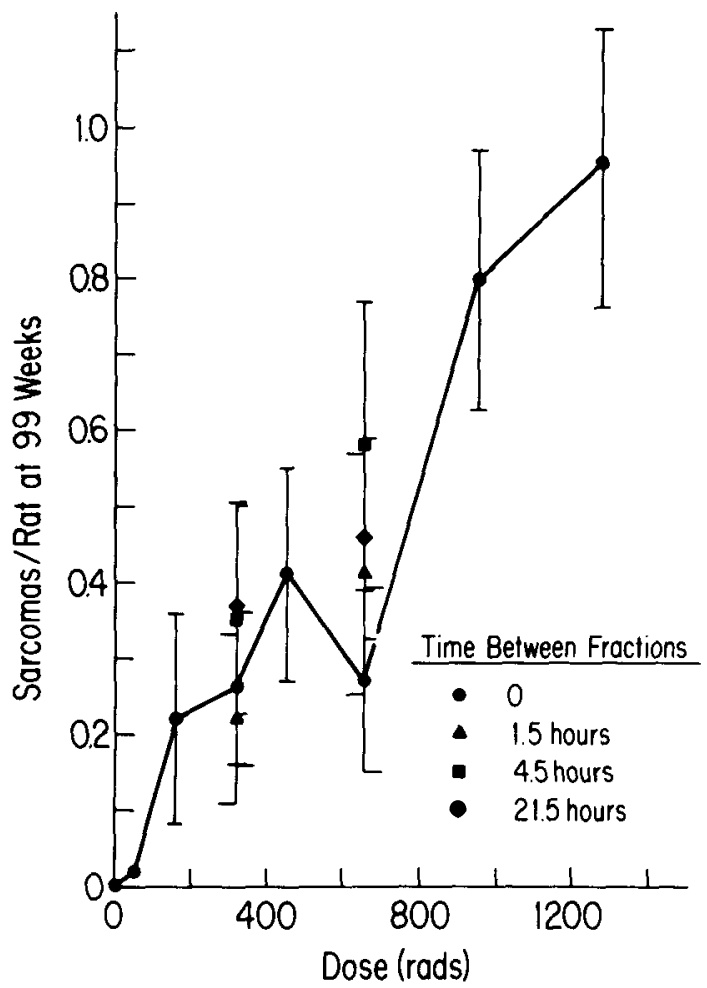

Figure 6. The dose response at 99 weeks for sarcomas induced in rat skin by one or two equal doses of argon ions as indicated. The error bars are standard deviations based on the square root of the total number of tumors. (XBL 808-11208) 


$$
236
$$

Blank 


\title{
RESPONSE OF RAT SPINAL CORD TO SINGLE AND FRACTIONATED DOSES OF ACCELERATED HEAVY IONS
}

\author{
J. L. Leith, ${ }^{*}$ M. McDonald, P. Powers-Risius, S. F. Bliven, ${ }^{*}$ R. E. Walton, \\ K. H. Woodruff, $\dagger$ and J. Howard
}

If the spinal cord is exposed to ionizing radiation, there is the possibility of serious, irreversible injury. To guard against such disastrous possibilities, the radiation tolerance and radiation repair characteristics of the spinal cord must be determined. This has been done using experimental animal model systems such as mice (Geraci et al., 1974; Goffinet et al., 1976) and rats (Asbel1 and Kramer, 1971; Carsten and Zeman, 1966; Van der Koge 1 and Barendsen, 1974; Van der Kogel, 1976, 1977a,b; Leith et al., 1975a,b; White and Hornsey 1978; Masuda and Withers, 1977; Masuda et al., 1977; Hopewe 11 and Wright, 1975). However, with new treatment modalities, such characteristics need to be defined before clinical use. In this regard, we have attempted to study the response of rat spinal cord to irradiation with accelerated heavy ions, in particular carbon and neon, ions. Two different ionization regions in the modified Bragg curve for each ion have been studied for both single and fractionated exposures. We have defined the paralytic response as a function of dose and dose per fraction, and we have determined $\mathrm{RBE}$ and repair values.

\section{MATERIALS AND METHODS}

\section{Animals}

The rats used in these studies were strain CDF females, approximate $7 y 125$ to $150 \mathrm{~g}$ in weight, obtained from either the Charles River Breeding Laboratories, Wilmington, MA, or the Simonson Breeding Laboratories, Gilroy, CA. Animals were given Purina "White Diet" and water ad 1 ibitum. After irradiations, rats were housed three per large cage.

\section{$X$ Irradiations}

Animals were exposed using a Philips therapeutic $X$-ray machine, operated at $230 \mathrm{kVp}$, and $15 \mathrm{~mA}$. Added filtration was $0.25 \mathrm{~mm} \mathrm{Cu}$ and $1.0 \mathrm{~mm} \mathrm{Al}$ and the half-value layer was $0.83 \mathrm{~mm}$ Cu. Rats were anesthetized for irradiations using i.p. sodium pentobarbital at a dose of $50 \mathrm{mg} / \mathrm{kg}$. Rats were positioned in stereotaxic rat holders (used also for the carbon, helium, and neon ion exposures) and were positioned so that the $X$-ray beam passed transversely through the thoracolumbar region of the spinal cord (specifically emcompassing vertebrae T12-L1). The $X-r$ ay beam was collimated using a specially designed Cerrobend collimator so that the aperature was exactly the size used in the heavy ion lexposures (i.e., $16 \mathrm{~mm}$ along the axis of the vertebral column and $11 \mathrm{~mm}$ in height). The Cerrobend applicator was $4.3 \mathrm{~cm}$ long and $0.8 \mathrm{~cm}$ thick and a $3 \mathrm{~cm}$ air gap was maintained between the end of the collimator and the middle of the vertebral column. X-ray dosimetry was done using a Victoreen $250 \mathrm{R}$ probe and exposure doses were taken with the midpoint of the Victoreen thimble chamber $2 \mathrm{~cm}$ from the end of the collimator. Dosimetry was done at the position of the spinal cord with and without the collimator in place. With the collimator in place, the measured exposure dose was typically $94 \%$ of the open field dose. Exposure doses were corrected for temperature and pressure and a Roentgen to rad conversion factor of 0.96 was used to obtain the absorbed dose. Dose rates were about $3.2 \mathrm{~Gy} / \mathrm{min}$. The collimated $x$-ray fields were imaged by taking $x-r a y$ films of the beam spot which were superimposed on an open field film of the rat so that positioning for the multifraction experiments could be as precise as possible. The fractionation experiments were done on four consecutive days at approximately the same time of day.

\section{Helium-Ion Irradiations}

The techniques used for helium-ion irradiation of rat spinal cord have previously been described in detail (Leith et al., 1975a). Briefly, rats were irradiated using the 184-inch Synchrocyclotron at LBL, anesthetized for exposures, and irradiated with helium ions either in the plateau region of ionization, or in the middle of a $6-\mathrm{cm}$ spread Bragg peak region of ionization. Techniques for achieving beam spreading have been described. The irradiation field was exactly the same as for the X-irradiations. In these experiments, both single doses and multiple fractions were given in both positions of the helium-ion ionization regions. For the multifraction experiments, 10 equal fractions given in 22 days were administered.

\section{Carbon- and Neon-Ion Irradiations}

While details for the carbon-ion irradiations have not been previously reported, they are essentially similar to reported techniques for neon ion irradiations (Leith et al., 1975b). The Bevalac was used to accelerate both heavy-ion beams. Ions were produced at the superHILAC with energies of $8.5 \mathrm{MeV} /$ nucleon and injected into the beam transfer line, terminating in a $50 \mathrm{MeV}$ proton injector. The ions were then introduced into the Bevatron and accelerated further to reach final energies of between 0.25 and $2.6 \mathrm{GeV} / \mathrm{n}$. For the spinal cord irradiations, carbon and neon ions were extracted from the Bevatron at $400 \mathrm{MeV} /$ nucleon and transported to the Biomedical irradiation area. The depth dose distributions of the carbon and neon ion beams are shown in Figure $1 A$ and $B$. 


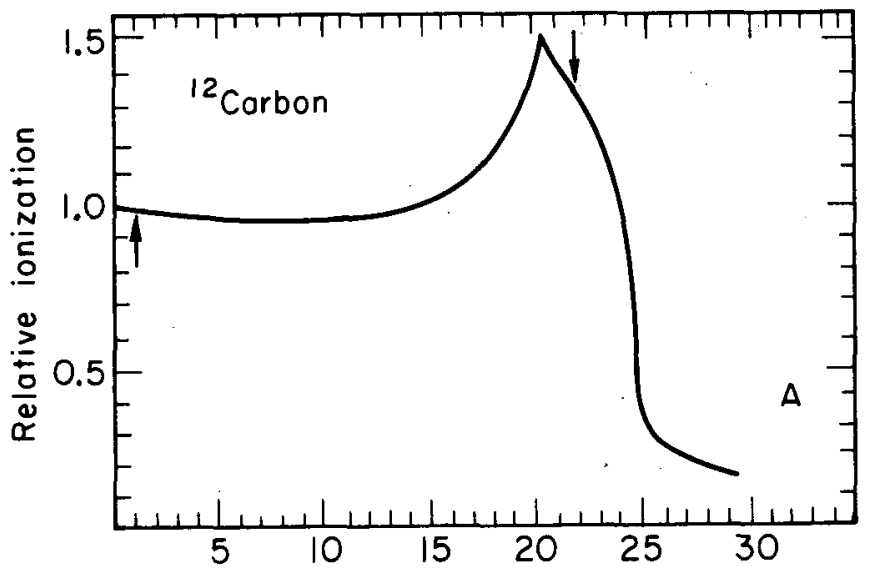

Figure 1. Relative ionization vs. penetration depth curves for the carbon and neon ion beams used. In Figure $1 \mathrm{~A}$, the depth dose pattern for carbon ions is shown, and the arrows indicate the position of the rat spinal cord in either the plateau or the midpeak region of the spread-out Bragg peak. In $F$ igure $1 B$, the depth dose pattern for neon ions is shown, and the arrows indicate the position of the rat spinal cord in either the plateau or the midpeak region of the spread Bragg peak. (XBL 808-3583)

Dosimetry was performed with plane parallel plate transmission ionization chambers. Densitometry tracings of photographic negatives of the beam fields used for the carbon and neon ion irradiations showed that there was less than $7 \%$ variation in density between the center and the periphery of the field.

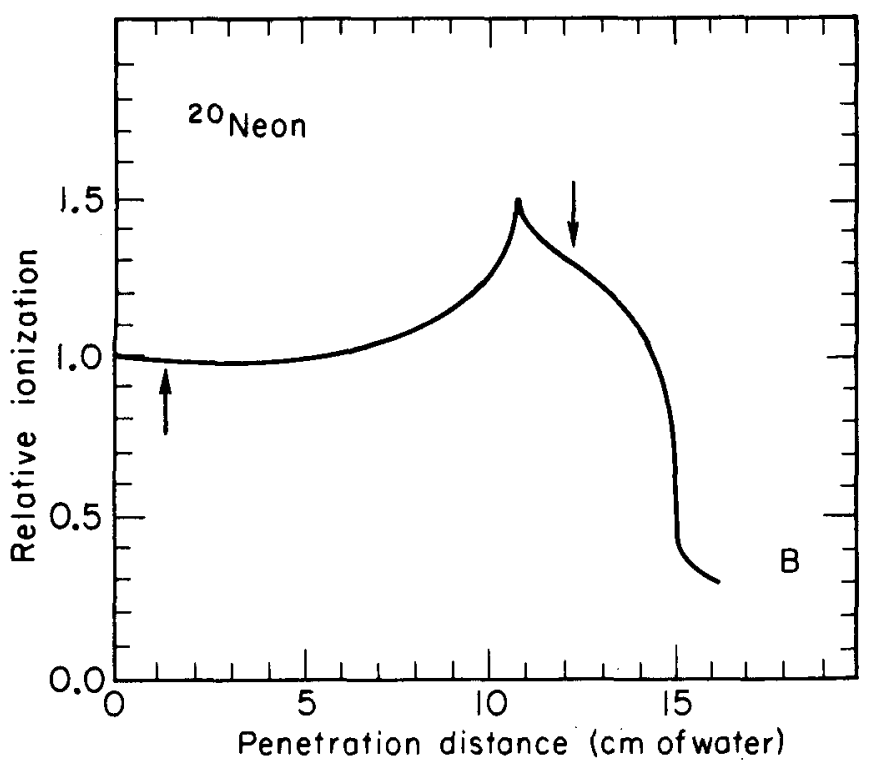

\section{Scoring Techniques}

At weekly intervals after irradiation, rats were assessed for any decrements in hind-limb reflexes, muscle bulk in the legs, leg strength, and paralysis. Impairment of bowel and bladder function was also noted (Leith et a1., 1975; Van der Kogel and Barendsen, 1974). The scoring system by which impairment was evaluated as a function of time is shown in Table 1.

\section{RESULTS}

RBE Values of Heavy-Ion Beams: Single Doses

The percent paralysis curves for single doses of $X$ rays, helium ions, carbon, and neon ions are shown in Figures 2 and 3 . In Table 2, we have summarized all of the data obtained in terms of radiation modalities, number of aninals exposed,

Table 1. Grading System for Assessment of Radiation-Induced Myelopathy

\begin{tabular}{ll}
\hline Grade & Description of Gross Impairment \\
S-0 & No difference from controls \\
S-1 & $\begin{array}{l}\text { Delay in eliciting hindlimb kick response upon pressure to } \\
\text { limbs }\end{array}$ \\
S-2 & $\begin{array}{l}\text { Definite but mild impairment in hindlimb kick response with } \\
\text { obvious curling of toes }\end{array}$ \\
S-3 & $\begin{array}{l}\text { Impairment of kick moderately severe, and rats walk with } \\
\text { slight hunchback, or flatfooted }\end{array}$ \\
S-4 & $\begin{array}{l}\text { Severe impairment, marked hunchback, rats walk on toes } \\
\text { Limb(s) completely useless, total paralysis (either uni- or } \\
\text { bilateral) }\end{array}$
\end{tabular}




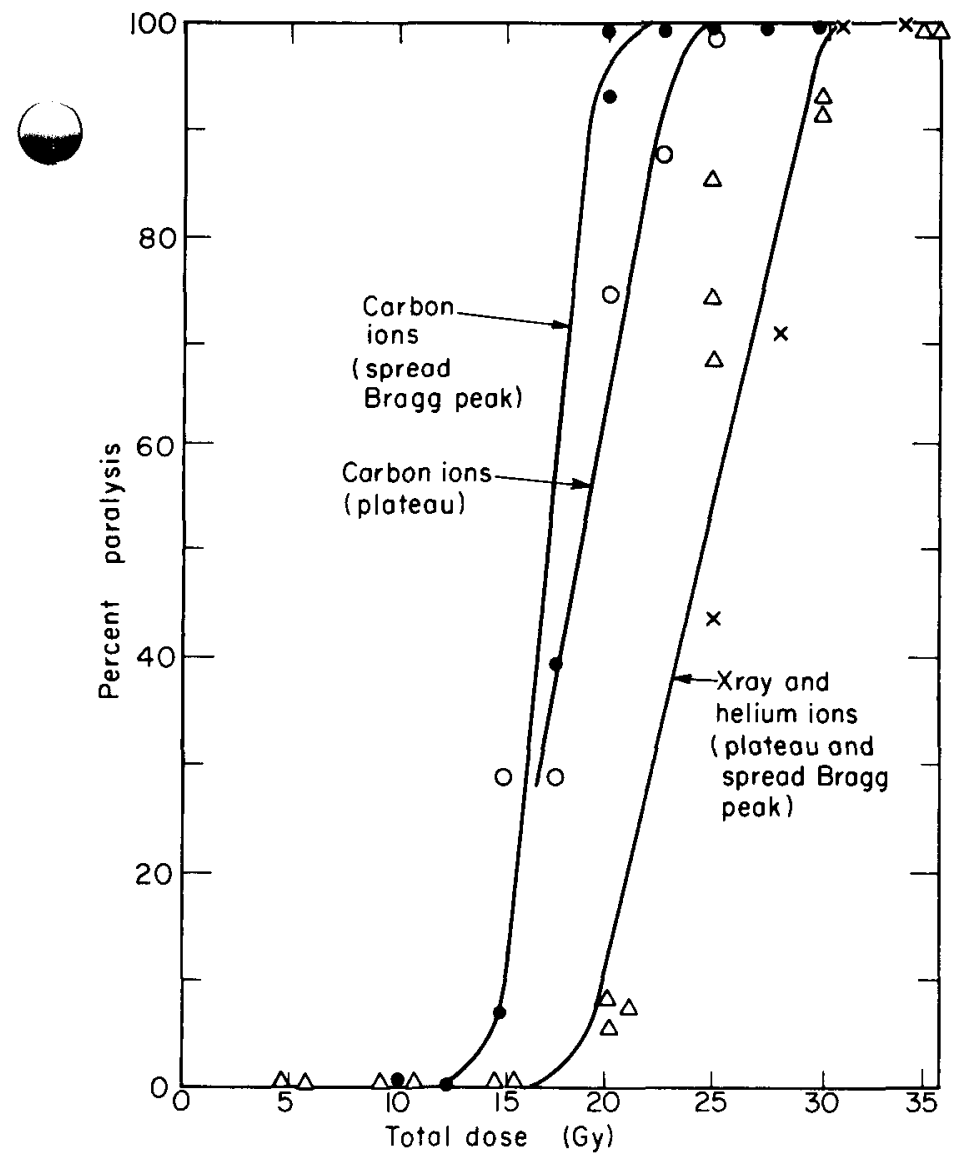

Figure 2. Percent paralysis versus total dose for rats exposed to single doses of low LET radiation ( $X$ rays and helium ions) and to carbon ions in either the plateau or the spread-peak regions of ionization. (XBL 808-3587)

and number of animals paralyzed. The dose needed to produce $50 \%$ paralysis $\left(E_{50}\right)$ in each of the radiation situations is summarized in Table 3 , along with the $95 \%$ confidence limits on the ED50. The data were fitted to a logistic equation in which the probability of paralysis is equal to $1 /\left[1+\left(\operatorname{Ed}_{50} / D\right)^{k}\right]$, where 0 is a variable total dose, and $k$ is the slope of the percent paralysis versus dose curve using a maximum likelihood fitting technique (Bevington, 1969). From these $E D_{50}$ values, the RBE values were determined, and are also summarized in Table $3 \vdots$. In this table, the error limits on the RBE values represent the propagated $95 \%$ confidence limits.

RBE Values of Heavy-I on Beams: Fractionated Doses:

The percent paralys is curves for fractionated doses of $X$ rays, helium, ions, carbon, and neon ions are shown in Figures 4 and 5 . In Table 3 , the total doses needed to produce $50 \%$ paralys is in each of the radiation situations is summarized. Again, the RBE values were determined, and these

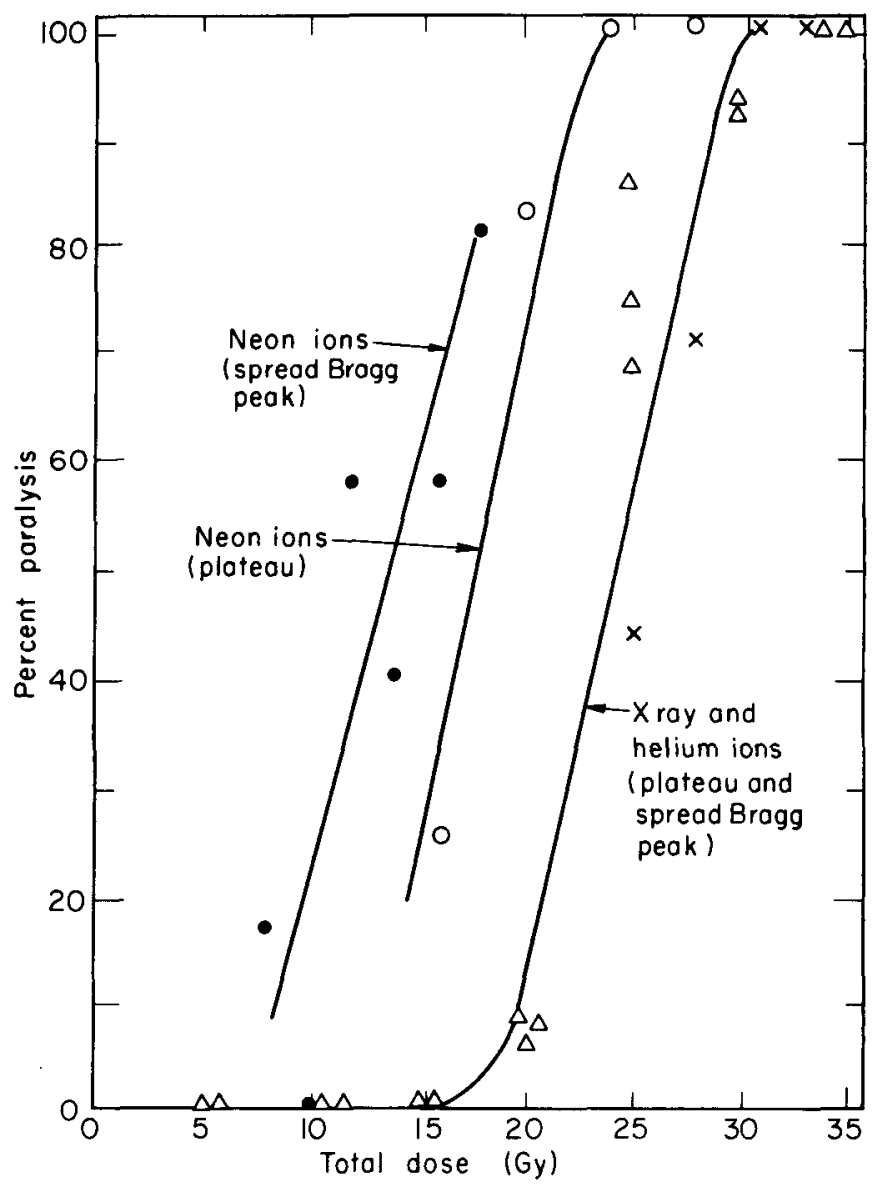

Figure 3. Percent paralys is versus total dose for rats exposed to single doses of low LET radiation ( $X$ rays and helium ions) and to neon ions in either the plateau or the spread-peak regions of ionization. (XBL 808-3586)

values with their $95 \%$ confidence limits are summarized in Table 3 . In Figure 6 , we have plotted the RBE values obtained versus the heavy-ion dose per fraction used.

\section{DISCUSSION}

The pertinent data are listed in Tables 3 and 4 and shown in Figure 6 . From Table 3 and Figure 6, it is apparent that the RBE is increasing with LET after exposure to ions in the carbon spread Bragg peak and neon. plateau and spread Bragg peak ionization regions. As we could only obtain four fractions of the carbon and neon ions used in these studies, the RBE values are not greater than about 2 (neon spread Bragg peak for example). However, we feel that the RBE values at doses/fraction that are likely to be used in patient treatment with heavy ions will be significantly higher.

Using Figure 6 one obtains RBE values at heavy ion doses/fraction of 2 Gy of about 1.5 
Table 2. Response Data for Development of Paralysis

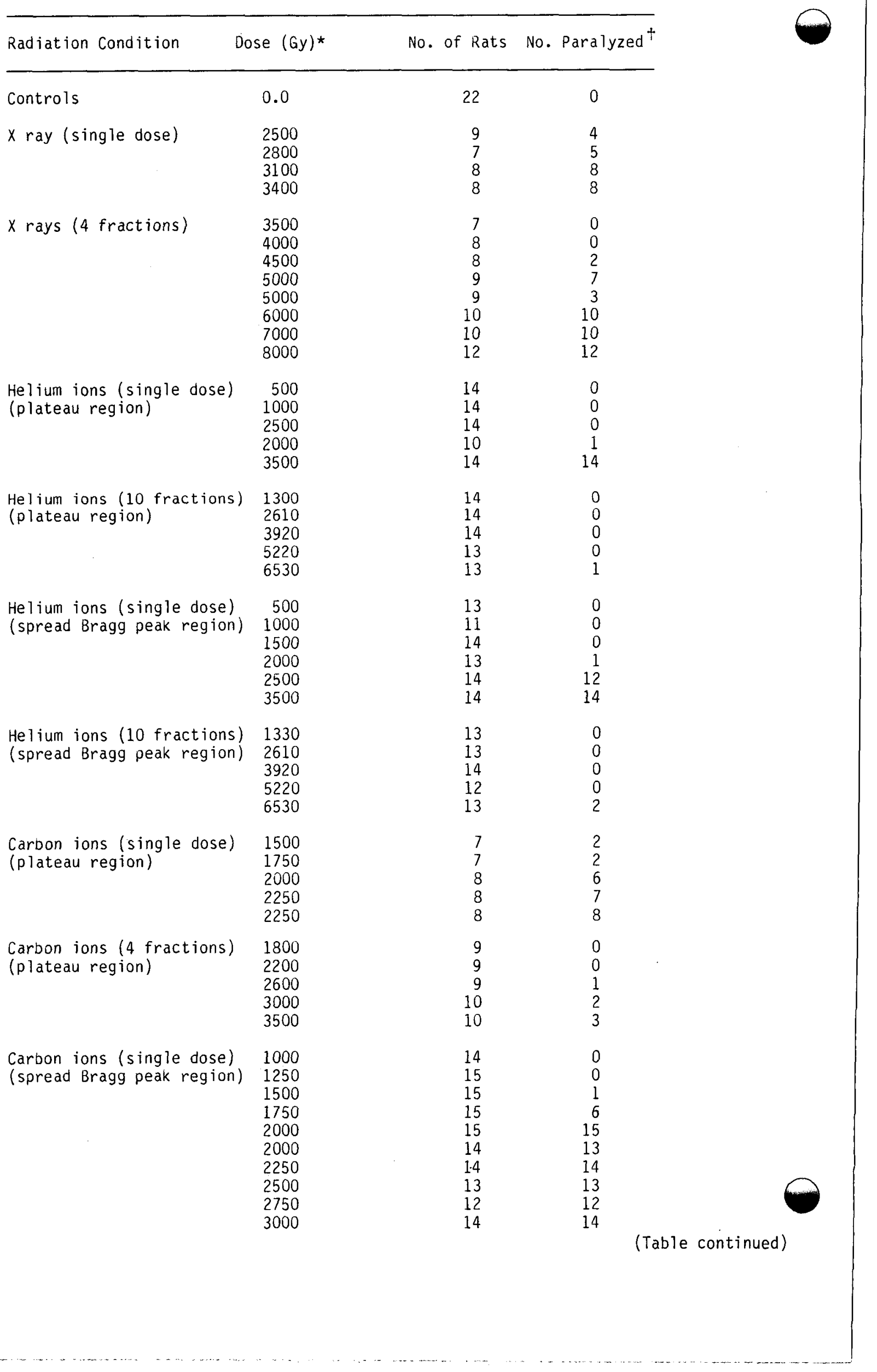


Table 2. (continued)

\begin{tabular}{lrrr}
\hline Radiation Condition & Dose (Gy)* & No. of Rats & No. Paralyzed \\
\hline Carbon ions (4 fractions) & 1600 & 8 & 0 \\
(spread Bragg peak region) & 1900 & 10 & 0 \\
& 2200 & 8 & 1 \\
& 2500 & 6 & 9 \\
& 2800 & 9 & \\
Neon ions (single dose) & 1600 & 4 & 1 \\
(plateau region) & 2000 & 6 & 5 \\
& 2400 & 6 & 6 \\
& 2800 & 6 & 6 \\
Neon ions (4 fractions) & 1800 & 6 & 0 \\
(plateau region) & 2200 & 10 & 1 \\
& 2600 & 7 & 2 \\
& 3000 & 8 & 6 \\
& 3450 & 6 & 6 \\
Neon ions (single dose) & 500 & 16 & 0 \\
(spread Bragg peak region) & 1000 & 12 & 2 \\
& 1200 & 12 & 4 \\
& 1400 & 16 & 4 \\
& 1600 & 12 & 7 \\
& 1800 & 12 & 10 \\
Neon ions (4 fractions) & 1600 & 9 & 0 \\
(spread Bragg peak region) & 1900 & 7 & 1 \\
& 2200 & 9 & 3 \\
& 2500 & 7 & 7 \\
& 2800 & & \\
\hline
\end{tabular}

* The dose given is the total dose.

${ }^{\dagger}$ At one year postirradiation. 
Table 3. Estimated Dose Needed to Produce $50 \%$ Paralys is $\left(E D_{50}\right)$ and RBE Values Obtained from Percent Paralysis Responses

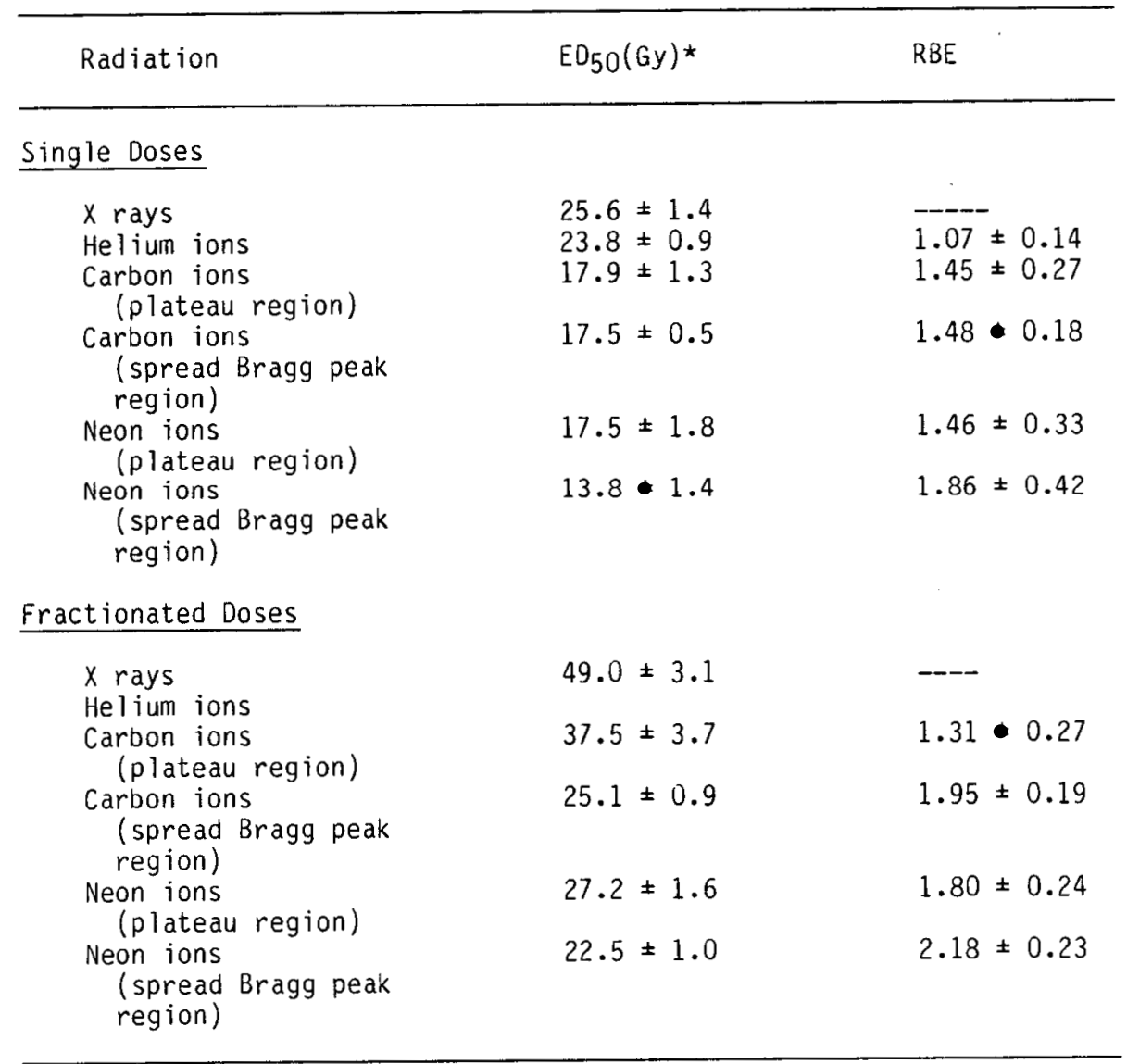

* Values for the $E_{50}$ are the means and the $95 \%$ confidence 1 imits. For RBE values, the errors are the propagated $95 \%$ confidence limits. 


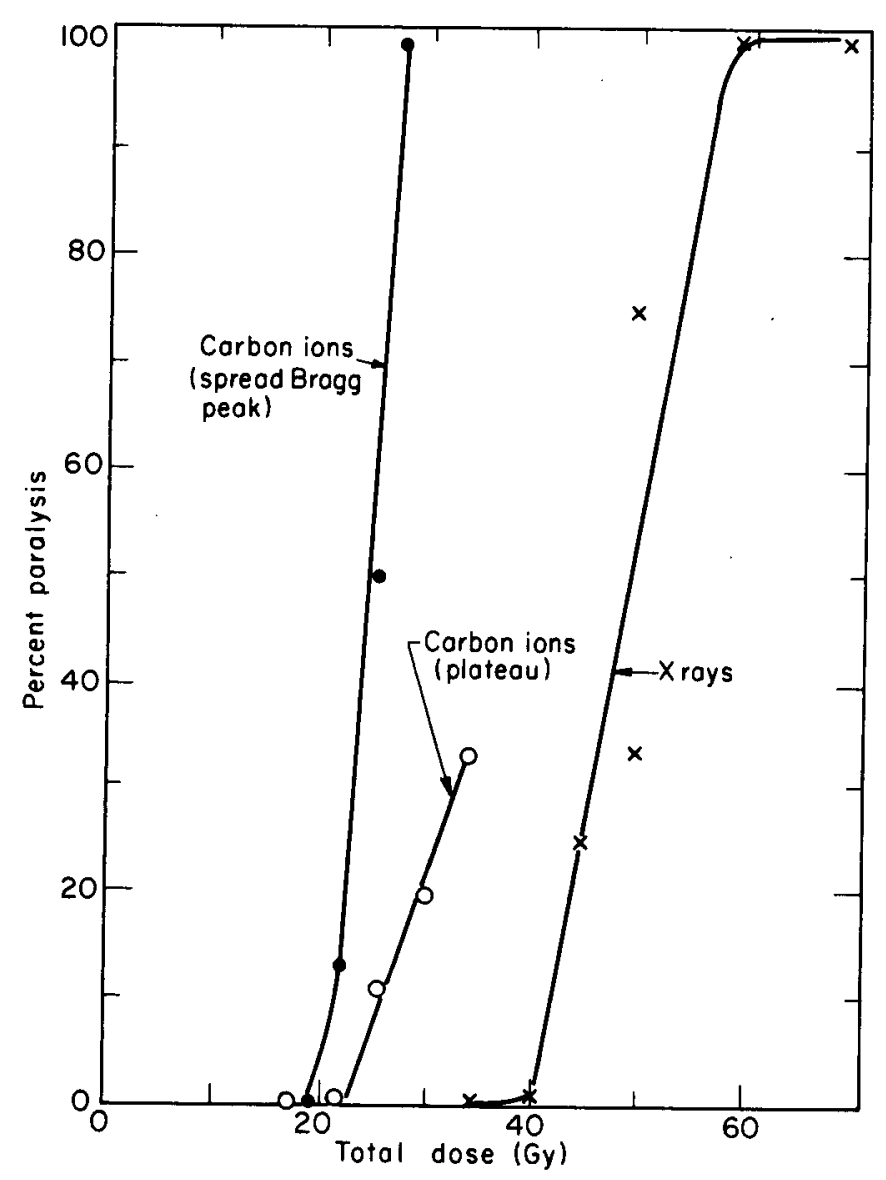

Figure 4. Percent paralysis versus total dose for rats exposed to 4 fractions of $X$ rays or carbon ions in either the plateau or spread-peak regions of ionization. (XBL 808-3585)

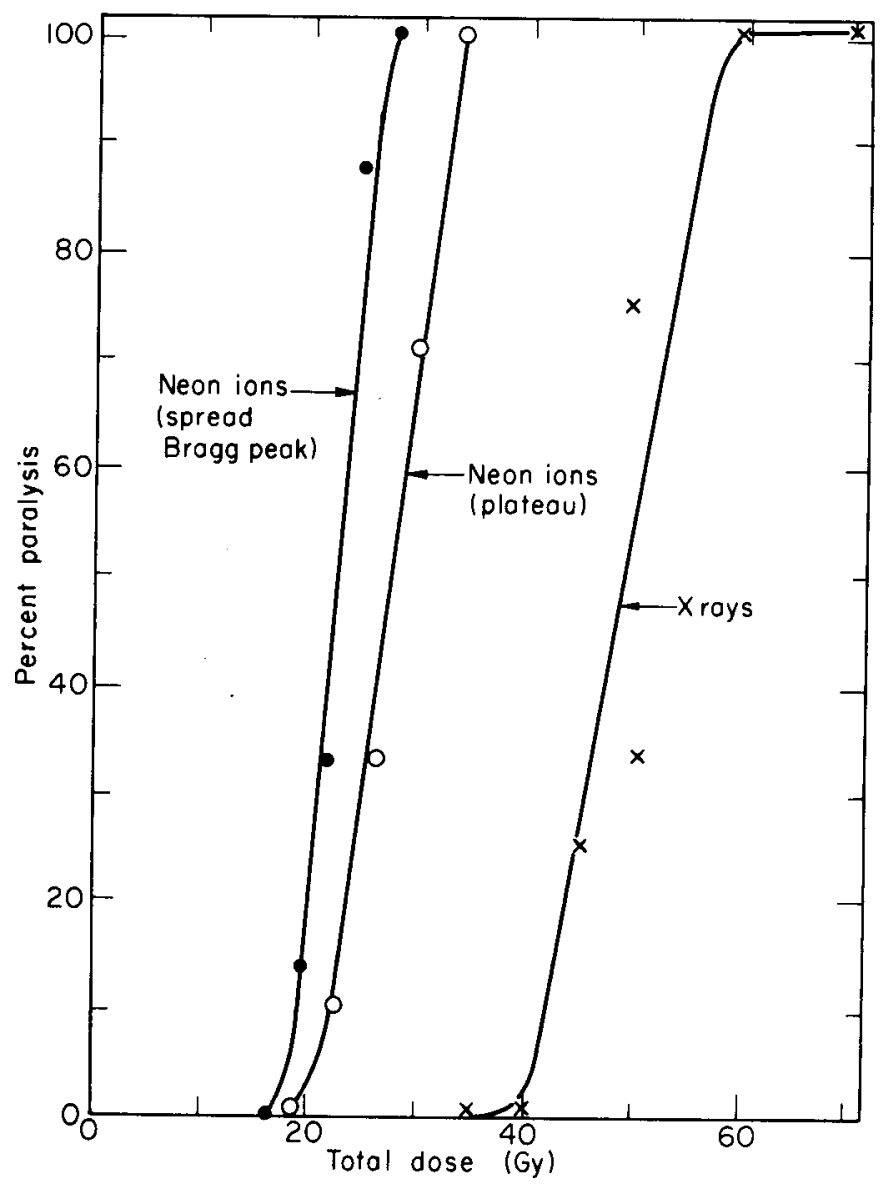

Figure 5. Percent paralysis versus total dose for rats exposed to 4 fractions of $x$ rays or carbon ions in either the plateau or spread-peak aregions of ionization. (XBL 808-3584)

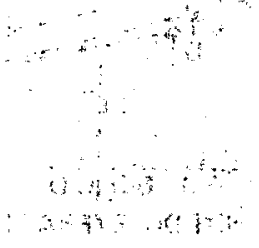

$\because \cdots$ 
Table 4. Fractional Repair $\left(F_{r}\right)$ of Subeffective Radiation Injury in Rat Spinal Cord after Exposure to $220 \mathrm{kVp} X$ rays or Heavy Ions*

\begin{tabular}{lc}
\hline Radiation Modality & $F_{r}\left(\begin{array}{c}\text { (level of comparison }=50 \% \\
\text { paralysis) }\end{array}\right.$ \\
\hline 220 kVp X rays & $0.64(0.54-0.74)^{\dagger}$ \\
$\begin{array}{c}\text { Carbon ions } \\
\text { (plateau ionization region) }\end{array}$ & $0.70(0.43-0.97)$ \\
$\begin{array}{c}\text { Carbon ions } \\
\text { (spread Bragg peak ionization } \\
\text { region) }\end{array}$ & $0.40(0.30-0.50)$ \\
$\begin{array}{c}\text { Neon ions } \\
\text { (plateau ionization region) }\end{array}$ & $0.48(0.28-0.68)$ \\
$\begin{array}{c}\text { Neon ions } \\
\text { (spread Bragg peak ionization } \\
\text { region) }\end{array}$ & $0.52(0.35-0.69)$ \\
\hline
\end{tabular}

* These fractional repair values were calculated from the relationship:

$$
F_{r}=\frac{\left(D_{n}-D_{1}\right) /(n-1)}{D_{n} / n}
$$

where $D_{n}$ and $D_{1}$ represent the doses needed to reach the comparison level of paralysis in either $n$ fractions (in this case 4 ) or 1 fraction, and $n$ is the number of radiation fractions given.

t The values in parentheses indicate the $95 \%$ confidence limits on the range of the mean value of $F_{r}$.

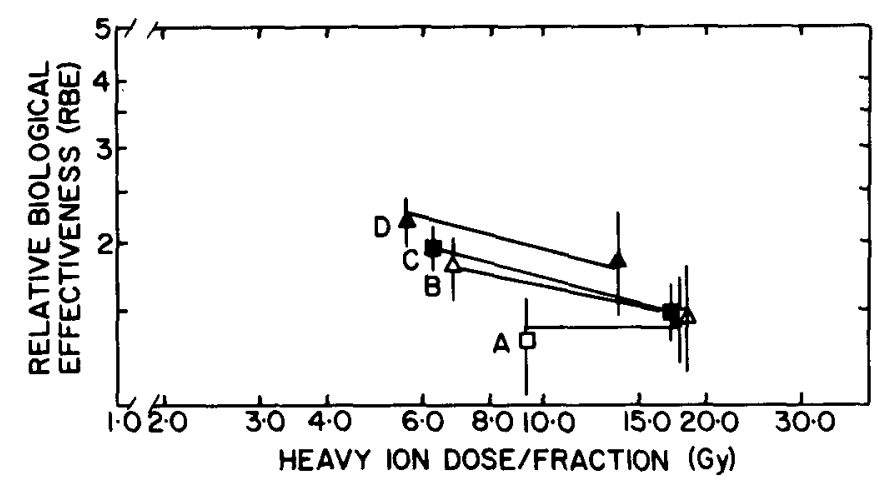

Figure 6. Plot of RBE vS. heavy ion dose/ fractions $(G y)$. Curve A describes the response to irradiation with carbon ions (plateau region of ionization), curve $B$ describes the response to irradiation with neon ions (plateau region of ionization), curve $C$ describes the response to irradiation with carbon ions (spread Bragg peak region of ionization), and curve $D$ describes the response to neon ions (spread Bragg region of ionization). The error bars are the $95 \%$ confidence limits on the RBE values. (XBL 809-11724) 
for carbon ions in the plateau region of ionization, about 2.4 for neon ions in the plateau region of ionization, about 2.7 for carbon ions in the spread Bragg peak region of ionization, and about 3.0 for neon ions in the spread Bragg peak region of ionization. These values are due to the fact that the dose response curve for spinal cord for irradiation with low LET modalities appears to have a large "shoulder" (a very small alpha constant using the linear-quadratic model of cell survival). Therefore, as a corollary, the spinal cord will show large RBE responses to radiation modalities with only a relatively modest increase in LET. We feel that this is an important consideration to be aware of, and to protect against, in any situation in which the spinal cord will be involved in the heavy-ion irradiation field. More accurate determination of the tolerance of spinal cord to inultifraction exposures is dependent on performing experiments in which greater number of fractions are given.

Spinal cord also possesses a large capacity for repair of subeffective damage. In Table 4 we have attempted to characterize this ability by listing the fractional repair $\left(F_{r}\right)$ values obtained for the various radiation treatments. While there are large confidence limits on the data, several facts are apparent. First, high LET radiations do reduce this fractional repair capacity, in a manner roughly correlated with the LET of exposure ion type. Second, although there is a reduction in $F_{r}$, the ability of spinal cord to show repair of subeffective damage is not removed, even at the highest LET beam used (spread Bragg peak neon ions in which the dose average LET is probably about $150 \mathrm{keV} / \mathrm{\mu m}$ ).

While not presented in this paper, we have also obtained data on enzymatic changes in irradiated spinal cord as a function of ion and dose level, on chronic skin responses in rats after irradiation with the different ions and dose levels, on the temporal development of the paralytic response as a function of ion and dose, and on late tumorigenesis. In general, these data tend to corroborate the RBE values obtained for the production of myelopathy in the rat.

In summary, the response of rat spinal cord to exposure with heavy ions in different regions of the ionization curves, is both dose and LET dependent, which allows the derivation of RBE and repair values. At the dose levels and number of fractions studied here, the RBE values are similar to those reported for other normal tissues. However, as we feel that spinal cord has perhaps the widest shoulder of all of the normal tissue systems studied to date, RBE values at low doses/fraction are likely to be significantly higher than other normal tissue systems. This possibility is deserving of further verification and provides a note of caution in situations where the spinal cord may be involved in the heavy-ion irradiation field.

\section{ACKNOWLEDGEMENTS}

This work has been supported by many people in the Biology and Medicine and Accelerator
Divisions of the Lawrence Berkeley Laboratory. Without their help this work could not have been completed, and we express our sincere appreciation. Special thanks are extended to Drs. C. A. Tobias and.E. L. Alpen for the ir continued support during this project. This work was supported by a grant from the NCI (CA15184, C. A. Tobias, Principal Investigator).

\section{FUOTNOTES AND REFERENCES}

* Brown University, Division of Biology and Medicine, and Department of Radiation Oncology, Providence, RI.

+Children's Hospital, San Francisco, CA.

Asbell, S. 0. and S. Kramer 1971. Radiology 98, 678 .

Bevington, P. R. 1969. Data Reduction and Error Analysis for the Physical Sciences, pp. 100-102. McGraw-Hi11, New York.

Carsten, A. and A. Zeman 1966. Int. J. Radiat. Biol. 10, 65 .

Douglas, B. G. and J. F. Fowler. 1976. Radiat. Res. 66,401 .

Geraci, J. P., P. D. Thrower, K. L. Jackson, G. M. Christensen, R. G. Parker, and M. S. Fox. 1974. Radiat. Res. 59, 496.

Goffinet, D. R., G. W. Marsa, and J. M. Brown. 1976. Radiology 119, 709 .

Hopewe 11, J. W. and E. A. Wright 1975. Int. J. Radiat. Biol. 28, 325 .

Leith, J. T., B. S. Lewinsky, K. H. Woodruff, W. A. Schilling, and J. T. Lyman. 1975a. Cancer 35, 1692 .

Leith, J. T., K. H. Woodruff, B. S. Lewinsky, J. T. Lyman, and C. A. Tobias 1975b. Int. J. Radiat. Biol. 28, 393.

Masuda, K. and H. R. Withers. 1977. Radiology $122,233$.

Masuda, K., B. O. Reid, and H. R. Withers. 1977. Radio logy 122, 239.

Van der Kogel, A. J. and G. W. Barendsen. 1974. Br. J. Radiol. 47, 393.

Van der Kogel, A. J. 1976. Radiation tolerance of the spinal cord: The dependence of fractionation and extended overall times, (IAEA Symposium on Radiobiological Research and Radiotherapy, vo 1. I) p. 85-90. IAEA, Vienna.

Van der Kogel, A. J 1977a. Radiology 122, 505.

Van der Koge1, A. J. 1977b. Acta Neuropathol. $39,139$.

White, A. and S Hornsey. 1978. Br. J. Radiol. $\underline{51}, 515$ 
246

Blank 


\title{
GENETIC EFFECTS OF HEAVY-ION BEAMS ON MICE
}

\author{
L. S. Goldstein*
}

Estimates of the genetic risk following irradiation with densely ionizing radiation are based mainly on specific locus mutation rates following neutron irradiation of the 7-locus (0ak Ridge) or 6-locus (Harwel1) mouse. For gammaradiation, the induced rates were $0.78 \times 10^{-7}$ per R-locus for the 6-locus strain and $2.45 \times 10^{-7}$ per R-locus for the 7-locus strain; following neutron irradiation these rates increased approximately five- to sixfold ( $W$. Russe11, 1965). These RBE estimates depend on the total dose, the dose rate, the locus assayed, and the energy of the neutrons.

Other genetic indices that do not require a dedicated national facility, like the specific locus test in mice, include semisterile mutations, dominant visible mutations, and recessive autosomal lethal mutations. When at least one measure of mutation rate, i.e., the doubling dose, is considered, these other systems give risk data that are in general comparable to those found with specific locus testing (Luning and Searle, 1971).

In addition to the genetic effects already mentioned, ionizing radiation induces a defect known as a dominant lethal mutation. Generally assayed following irradiation of the male mouse, the dominant lethal mutation is manifest as a reduction in litter size reflecting embryo death and resorption both before and soon after implantation. Lyon and coworkers have established that the majority of dominant lethal mutations induced in the spermatogonia of mice are expressed as early postimplantation deaths and are induced at a rate of $10.6 \%$ by $1,200 \mathrm{rad}$ of gamma radiation (Lyon et al., 1964).

Dominant lethal mutations have had only limited application in analysis of genetic risk. Since the effect is expressed early, in development, it is difficult to distinguish reduced litter size caused by the early death of embryos from reductions resulting from loss of fertilizing capacity due to aspermia. Since radiation causes killing of progenitor germ cells, especially spermatocytes and late spermatogonia (Oakberg and Diminno, 1960), this latter effect exerts a large influence on analysis of dominant lethal mutations induced in early premeiotic and meiotic stages. Techniques to discriminate between fertilization failures and dominant lethal mutations manifest before implantation (where no decidual reaction occurs) require counting of implants and corpora lutea--and corpora lutea counts are known to be imprecise (Epstein and Röhrborn, 1971). For this reason, dominant lethal mutation analysis has been limited to postmplantation events even though failure to include preimplantation events contributes to the unusually high doubling dose found for this form of mutation.
Recent advances in the culture of mouse embryos have led to the development of a system to assess the dominant lethal mutation rate for preimplantation and early postimplantation events (Goldstein and Spindle, 1976). The developmental potential of embryos sired by irradiated (Goldstein et al., 1978) or chemically treated (Goldstein, 1976) males is measured from the two-cell stage to an early postimplantation stage and compared to the rate in embryos sired by untreated males. The technique also permits a direct assessment of the fertilization rate.

In the experiments to be described, we have used in vitro dominant lethal analysis to determine the mutation rate in male mice irradiated with accelerated particle beams of helium, carbon, or neon ions. We also have assayed the effects of these sources on testis weight, sperm counts, and fertilization rate. The RBE, calculated from low LET data in the literature, is higher for mutagenes is than for cell killing for each source. The mutation rate is higher in postmeiotic than premeiotic cells and the risk to stem cell spermatogonia is much greater than that reported from in vivo photon data.

\section{MATERIALS AND METHOOS}

\section{Irradiation Procedures}

Neon ions ( $557 \mathrm{MeV} / \mathrm{amu}$ ) and carbon ions ( $400 \mathrm{MeV} / \mathrm{amu}$ ) were generated at the Bevalac; helium ions (225 MeV/amu) were accelerated at the 184-inch Synchrocyclotron. The Bevalac beams were degraded by interposing a $4 \mathrm{~cm}$ brass spiral ridge filter, the helium beam by an $8 \mathrm{~cm}$ brass spiral ridge filter. Scattering foils were used to establish dose homogeneity (determined by port films) of at least $85 \%$ from the center to the periphery of the field. "The Bevalac beams were collimated by cerrobend to a $3 \times 5 \mathrm{~cm}$ field and the helium beams was shaped to a $14.5 \mathrm{~cm}$ circle by a lead collimator. A dose rate of approxi-

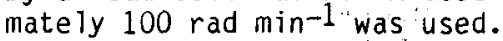

Whole body radiation was given with the helium beam, and lower abdominal irradiation was given with the Bevalac beams. The mice were positioned $0.5 \mathrm{~cm}$ from the distal edge of the Bragg peak in lucite radiation nolders. The residual range of the beam was reduced by a variable thickness water absorber for irradiation in the peak. The dose rate, beam homogeneity and sample position were determined by the wire ionization chambers; dosimetry and Bragg peak determination were performed by the staffs of the Bevalac and the 184-inch Synchrocyclotron. 
Biological Procedures: Dominant Lethal Mutation Assays

Male mice of the random-bred Dub: (ICR) strain (Flow Laboratories, Dublin, VA) 10-12 weeks old were used. They were maintained in our animal facility for at least two weeks prior to their incorporation in the experiment. Forty males were used as controls and forty were used in each of five irradiated groups for each ion. Other males irradiated in the same way were used to determine sperm counts and testis weight loss. As soon as possible after irradiation each mouse was mated to a single female mouse age 8-10 weeks of the same strain. The females were induced to superovulate by injection of $5 \mathrm{I}$.U. of pregnant mare's serum (PMS, Organon, West Orange, NJ), $48 \mathrm{~h}$ before pairing, and $5 \mathrm{I} . U$. human chorionic gonadotropin (HCG, Sigma, St. Lou is, MO), $2 \mathrm{~h}$ before pairing. This was designated Day 0 . On the morning after pairing the females were examined for the presence of a vaginal plug which is taken as evidence of having successfully mated. Successfully mated females from each group were caged together for an additional day.

Females that had mated were $k i l l e d$ by cervical dislocation on Day 1 . The oviducts were excised from each mouse and were placed in individual drops of modified $L-15$ medium (Spindle and Pedersen, 1973). Under 25X magnification, a $30 \mathrm{~g}$ needle was inserted into the ampulla of the oviduct which was then gently irrigated with modified L-15 to flush the two-cell stage embryos and unfertilized ova. Ova and embryos were counted and the number of each recorded. The embryos were transferred in a modified Spemann pipette in groups of 150 to organ culture dishes containing $0.8 \mathrm{ml}$ of modified Biggers' embryo culture medium (Spindle and Pederson, 1973).
The embryos were incubated for 3 days in an atmosphere of $5 \% \mathrm{CO}_{2}$ at $37^{\circ} \mathrm{C}$. During this period the blastomeres of the embryo cleave and the embryo develops from the two-cell stage into the four-cell, eight-cell and morula stage (defined as an embryo in which the individual blastomeres can no longer be distinguished in a dissecting microscope. When a fluid-filled cavity (the blastocoele) forms in the morula the embryo is designated a blastocyst. Blatocysts were counted and transferred in groups of twenty to each well of an eight-chamber slide containing 0.3 to $0.4 \mathrm{ml}$ of "Akiko's" medium (Goldstein and Spindle, 1976). Morulae were counted and transferred separately to one well of the eightchamber slide. Morulae and blastocysts were incubated for an additional 4 days during which time blastocysts expand and eventually break the zona pellucida (hatch) and adhere to the glass substrate (attach). The number of embryos that had escaped from the zona pellucida (trophoblastic outgrowths or TBOG) was recorded.

The entire procedure is performed twice per week for the first five weeks and once per week for the next twenty weeks following irradiation. Using the criteria of 0akberg for timing of stages in the seminiferous epithelium, embryos sired 0-7 days after irradiation were fertilized by irradiated sperm; embryos sired 8-21 days postirradiation were fertilized by sperm that developed from irradiated spermatids; embryos sired 22-52 days postirradiation were fertilized by sperm that developed from irradiated spermatocytes, and embryos sired more than 52 days after irradiation were fertilized by sperm that developed from irradiated spermatogonia (0akberg, 1956).

Dominant lethal mutation rates are determined from the number of morulae and TBOG as:

1 - ( $\left.\frac{\text { fraction of two-cell stage embryos forming morulae, experimental }}{\text { fraction of two-cell stage embryos forming morulae, control }}\right)$

for mutations expressed before blastocyst

formation, and:

$1-\left(\frac{\text { fraction of blastocysts forming } T B O G \text {, experimental }}{\text { fraction of blastocysts forming } T B O G \text {, control }}\right)$

for mutations expressed after blastocyst

formation.

The overall dominant lethal mutation rate is:

$1-\left(\frac{\text { fraction of two-cell embryos forming a TBOG, experimental }}{\text { fraction of two-cell embryos forming a TBOG, control }}\right)$ 
Biological Procedures: Sperm Count and Test is Weight

Random males from the irradiated and control groups were killed by cervical dislocation. Both testes and the caput and cauda epididymes and vasa deferentia were removed and carefully cleaned of fat and other tissue. The testes were separated from the other structures and weighed. The epididymes and vasa deferentia were macerated in saline citrate, and sperm counts were performed in a haemocytometer. The procedure of Searle and Beechey was used (1974). Sperm counts and testis weights were determined weekly for the first 15-18 weeks of the experiment.

RESULTS

$\frac{\text { Sperm Count, Fertilization Rate, }}{\text { Testis Weight Loss }}$

He lium

A loss in the weight of the testis was found beginning three weeks after irradiation. The extent and duration of the weight loss was dosedependent. After reaching a nadir, recovery to control weights took five weeks for the testes of animals given $80 \mathrm{rad}$ and 9 weeks for the testes of animals given $400 \mathrm{rad}$.

Sperm counts were more erratic than testis weights for the first two weeks postirradiation, but a consistent decline in sperm counts was found four weeks after radiation. The extent and duration of oligospermia was dose-dependent. Since the sperm were sampled from the cauda and caput epididymes, a reduction in sperm count would be reflected in the ejaculate in matings that took place one week later. Therefore, the reduction in sperm count is associated with killing progenitor germ cells irradiated at the early spermatocyte and the spermatogonial stages. All sperm counts returned to normal levels by 12 weeks after irradiation.

No reduction in the fertilization rate (i.e.,

two-cell stage embryos

two-cell stage embryos + unfertilized ova

was seen in mice given 80 or 160 rad; erratic rates were seen 4-7 weeks after irradiation in the group given 240 rad; a definite reduction in the fertilization rate was found 4-9 weeks after irradiation with 320 or 400 rad. In the 320 rad group, a low fertilization rate was not associated with aspermia while in the 400 rad group recovery of the fertilization rate preceded the increase in sperm counts.

\section{Carbon}

A decrease in sperm count and a loss in lest is weight was found 4-8 weeks after irradiation with 75 rad of accelerated carbon ions; the fertilization index after this dose remained at the control level. A transient reduction in fertilization rate coincident with reduced testis weight was seen 5 weeks after irradiation with $150 \mathrm{rad}$. After $225 \mathrm{rad}$, a reduction in sperm count at week 4 was followed by a decrease in the fertilization rate 10 days later. A reduced test is weight was found one week before these other phenomena at this dose. The recovery in test is weight preceded recovery of the fertilization rate and sperm count. Reduced sperm counts at 3 weeks postirradiation with 300 or $375 \mathrm{rad}$ was also associated with a reduced fertilization rate 4-7 days later. Loss in test is weight was also seen at these times. Although testis weight and fertilization rate eventually returned to control levels, it is uncertain whether the sperm count ever recovered completely. As with helium irradiations, the data from irradiation in the carbon beam indicate that the effect of radiation on testis weight, sperm count, and fertilization rate are dose dependent.

Neon

The time of the onset of oligospermia following irradiation with neon ions, found 4 days after 250 or $315 \mathrm{rad}$, is dose-dependent. At lower doses the onset is from 4 to 6 weeks. The extent and duration of oligospermia are also dose-dependent. Large variations in sperm count 9-15 weeks postirradiation are apparent. Reductions in the sperm count precede reductions in the fertilization rate by about one week which suggests that oligospermia is the cause. The extent and duration of the reduced fertilization rate are also dose-dependent. Test is weight loss was most apparent 3-4 weeks after irradiation; the time to recover is dose-dependent. Although the fertilization rate eventually returns to control levels, the data suggest that recovery of testis weight and sperm count is either incomplete or very delayed.

The beams are compared for efficiency in cell killing in a plot of the time needed to recover from radiation-induced cell depletion (measured by sperm counts, testis weight or fertilization rate) vs. dose (Fig. 1), an analys is that is analogous to tumor regrowth assays. This approach minimizes the variations found at different' samplings for a specific time after irradiation.

$$
\therefore \text { Dominant Lethal Mutations }
$$

He Tium

Embryos sired within one week by heliumirradiated males resulted from fertilizations by irradiated sperm. A dose-dependent decrease in the frequency of these embryos to develop to morula, blastocyst, and trophoblastic outgrowth stages was found (Fig. 2). Failures were manifest at or before morula formation, and between blastocyst formation and outgrowth. The dominant lethal mutation rate for these data (Fig. 2), fit by a least squares regression analys is for a linear function, was $3.2 \times 10^{-4}$ and $4.0 \times 10^{-4} \mathrm{rad}^{-1}$. The overall dominant lethal mutation rate for helium was $7.5 \times 10^{-4} \mathrm{rad}^{-1}$. 
TESTIS WEIGHT

SPERM COUNT

FERTILIZATION RATE

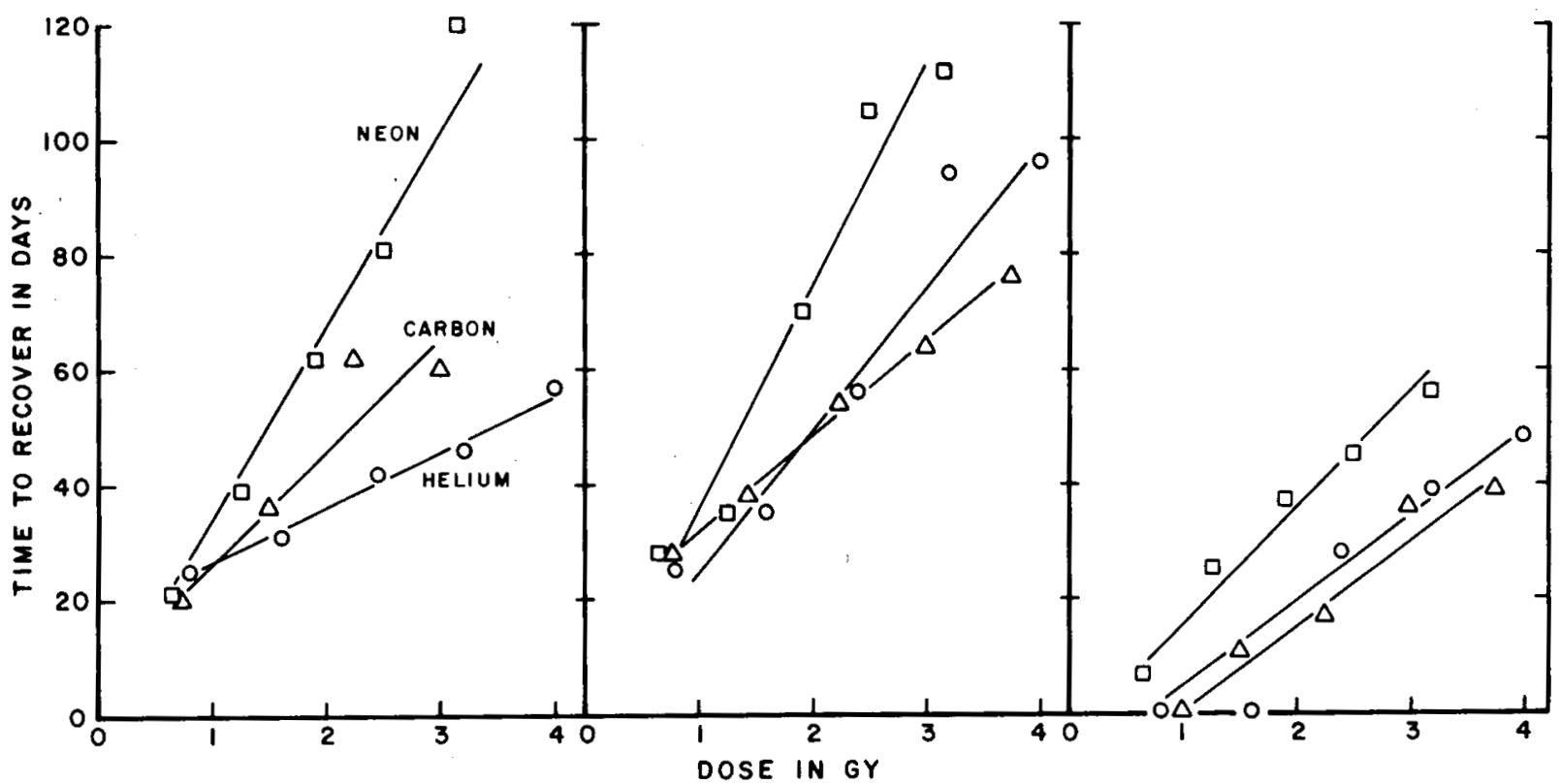

Figure 1. Recovery time to $80 \%$ of controlied testis weight, sperm count, and fertilization rate foliowing irradiation with helium $(0)$, carbon $(\Delta)$, or neon $(\square)$ ions. (XBL 809-11906)

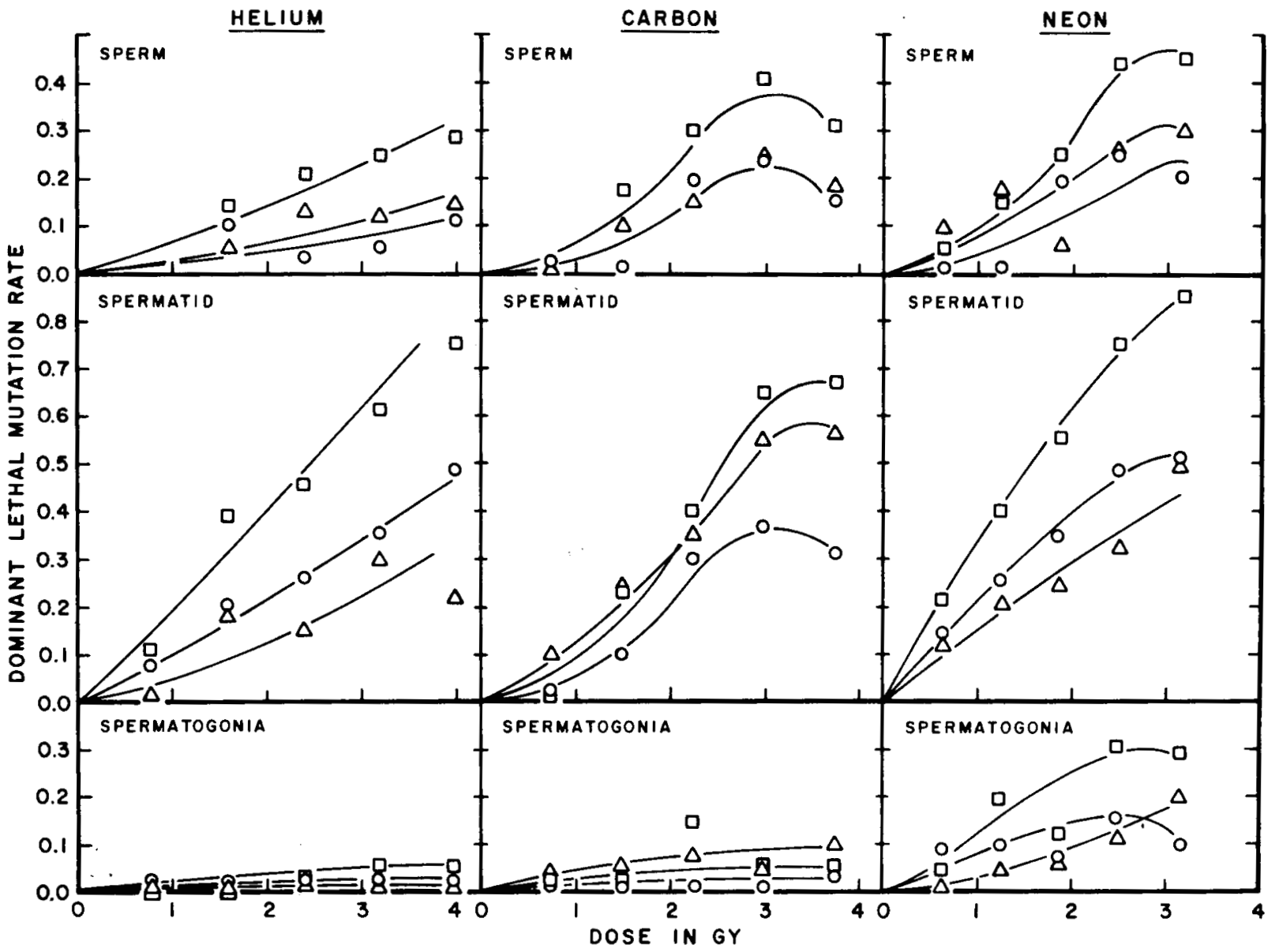

Figure 2. Induction of dominant lethal mutation (DNL) expressed in vitro following irradiation of sperm, spermatids, and spermatogonia by helium, carbon, or neon ions. DLM expressed between two cell and morula $(0)$; OLM expressed between blastocyst and trophoblastic outgrowth ( $\Delta$ ); DLM expressed between two cell and trophoblastic outgrowth (口). (XBL 809-11905). 
Embryos sired 8-20 days after irradiation were fertilized by sperm irradiated in the spermatid stage. Dominant lethal mutations arising from sperm irradiated as spermatids were manifest as failures both before and after blastocyst formation (Fig. 2). The rate of failures before blastocyst formation was $7.2 \times 10^{-4} \mathrm{rad}^{-1}$ and the rate after blastocyst formation was $11.7 \times 10^{-4} \mathrm{rad}^{-1}$; the overall dominant lethal mutation rate was $18.1 \times 10^{-4} \mathrm{rad}-1$ making this stage some 2.4-fold more sensitive than the sperm stage.

Dominant lethal mutations were not detected in cells irradiated as spermatocytes (due to cell killing) or spermatogonia (Fig. 2).

\section{Carbon}

Dominant lethal mutations induced in sperm by accelerated carbon ions were manifest as embryonic failures both before and after blasto-formation (Fig. 1). The dominant lethal rate for events before blastocyst formation is $7.0 \times 10^{-4} \mathrm{rad}^{-1}$ and the rate after blastocyst formation is $5.6 \times 10^{-4} \mathrm{rad}^{-1}$. The overall dominant lethal mutation rate in irradiated sperm is $11.3 \times 10^{-4} \mathrm{rad}^{-1}$.

As with helium, carbon-irradiated spermatids were more sensitive to the induction of dominant lethal mutations than were irradiated sperm

(Fig. 1). The dominant lethal mutation rate was $15.7 \times 10^{-4}$ for losses before blastocyst formation and $11.7 \times 10^{-4} \mathrm{rad}-1$ after blastocyst formation; the overall dominant lethal mutation rate is $18.0 \times 10^{-4} \mathrm{rad}^{-1}$. The irradiated spermatid is therefore about twice as sensitive as irradiated sperm.

Dominant lethal mutations were not detected at doses less than 225 rad in spermatogonia (Fig. 1); the small radiation effect noted for early embryo failures was about balanced by enhanced rates of success for later development in irradiated embryos. Dominant lethal mutation rates of 1.7 to $6.7 \times 10^{-4} \mathrm{rad}^{-1}$ were detected. at higher doses. There is considerable scatter. in these data and they are insufficient for an accurate estimation of the dominant lethal mutation rate in spermatogonia.

\section{Neon}

Dominant lethal mutations arising from neonirradiated sperm were expressed both as failures. before and after blastocyst formation (Fig. 2). . . After 65 or 125 rad, preimpiantation failures predominate; at 250 or 315 rad, the frequency of the two types is about equal. After 190 rad, early postimplantation failures appear to: predominate. This, however, may be artifactual and due to uncertainty in discriminating failures at the very early blastocyst stage from failures at the late morulae stage. The rate of induction of dominant lethal mutations expressed before or after blastocyst formation is $9.2 \times 10^{-4} \mathrm{rad}-1$; the overall rate is $14.7 \times 10^{-4} \mathrm{rad}^{-1}$ (Fig. 1 ).
In spermatids (Fig. 2) the rate of induction of dominant lethal mutation by neon is $16.0 \times 10^{-4} \mathrm{rad}^{-1}$ and $17.3 \times 10^{-4} \mathrm{rad}^{-1}$ for fajlures before and after blastocyst formation. The overa 11 rate of induction of $30.0 \times 10^{-4} \mathrm{rad}^{-1}$, and the neon-irradiated spermatid is again about twice as sensitive as the neon-irradiated sperm.

Dominant lethal mutations are induced by neon ions in spermatogonia (Fig. 2). The overall rate is $11.0 \times 10^{-4} \mathrm{rad}-1$. As with carbon, however, the data vary. It would appear that the testis has not returned to homeostasis, and factors other than those due to genetic effects of the radiation may be influencing the data.

\section{DISCUSSION}

Relatively low doses of radiation by accelerated charged particles $k i l l$ the more radiosensitive germ cell stages and induce mutagenic lesions manifest as dominant lethal mutations in surviving germ cells. The extent and duration for killing and mutation induction depend on the dose and the radiation quality.

Germ cell killing is manifest as a reduction in the testis weight and a reduction in the number of sperm derived from irradiated germ cellis. While both indices are reasonably good qualitative indicators of the lethal effects of the radiation, the inherent variations in sperm count found for the haemocytometer method (Freund and Carol, 1964) or inherent variability in the size of the testis of similarly aged animais limit their application to quantitative questions. These measurements were not the primary focus of our experiments and while the sample size we used (two animals per point) is reported in the literature, a larger sample size would reduce the variation and enhance their sensitivity and accuracy.

Male germ cell stages can be arranged in order of their increasing radiosensitivity to killing by photons as sperm, spermatids, spermatocytes, late spermatogonia (0akberg and Diminno, 1960). Our data (Fig. 1) indicate that the order of their sensitivity to charged particle irradiation is the same as for photon irradiation.

A preliminary estimate of the RBE for the beams when:measured by recovery of test is weight or sperm, counts is given in Table 1 . These data are based on 137. Cs irradiation from Searle and Beechey (1974). The data are in good general agreement with RBE data for cell killing in another normal tissue, the jejunal crypt cell (Goldstein et a $1, \cdots, 1980 a, b)$. It must be emphasized that sperm counts, test is weight, and fertilization. rate are indirect measures of progenitor cell survival.

Aspermia results in a reduced rate of fertilization when the sperm count is less than about $10 \%$ of normal. As with low LET radiation, aspermia ( 3 or more weeks postirradiation) is 
Table 1. RBE Values for Cell Killing Measured as Testis Weight Loss and Sperm Counts

Helium Carbon Neon

$\begin{array}{llll}\text { Test is weight loss } & 1.21 & 1.48 & 2.11 \\ \text { Sperm count } & 1.14 & 1.18 & 1.73\end{array}$

associated with killing of radiosensitive spermatocytes and spermatogonia. Recovery of the fertilization rate is slower for neon-irradiated germ cells than for carbon- or helium-irradiated germ cells. The RBE for this parameter, estimated from corpora lutea counts in vivo by Searle and Beechey (1974), is lower than the RBE for testis weight or sperm counts. These data are included for comparative purposes only since counts of corpora lutea in vivo are inaccurate (Epstein and Röhrborn, 1971), and counts of twocell stage embryos and unfertilized eggs recovered in vitro following mating with $137 \mathrm{Cs}$-irradiated males are not available at this time.

The data suggest that the dose-response curve in sperm for the induction of dominant lethal mutations which are manifest as preimplantation failures has a threshold when the irradiation is by accelerated helium or carbon ions, but is without a threshold when irradiation is by neon ions (Fig. 2). Russell has proposed that failures very early in development (i.e., before blastocyst formation) reflect the induction of chromosome breaks and monosomy in the embryo ( $L$. Russe 11, 1965). For low LET radiation, chromosome breaks are induced by two independent hits and therefore the shape of the mutation induction curves for dominant lethality expressed before blastocyst formation suggests that helium and carbon ions act like photons for this damage, and neon, without a threshold, acts more like fission spectrum neutrons (W. Russel1, 1965). These data support similar conclusions for the quality of these beams from cell survival studies (Goldstein et a $1 ., 1980 \mathrm{a}, \mathrm{b})$.

The presence of a threshold for carbon or helium irradiated sperm suggests that there is repair of the sublethal damage. Since sperm do not exhibit unscheduled DNA syntheses, this repair must take place in the egg. Recognition of damage in the male pronucleus by maternal repair enzyme has been described for alkylating agents (Generoso et al., 1979). Our data suggest that repair occurs following irradiation with accelerated charged particles with LET less than $100 \mathrm{keV} / \mathrm{\mu m}$. These findings are consistent with split-dose and multifraction irradiation studies with charged particle beams in gut $t$ issue where the presence of a repairable component of damage induced by helium and carbon beams is found, but no such component was found for the highest LET region of the neon beam (Goldstein et al., 1980b).

Dominant lethal mutation following high LET irradiation of sperm are also manifest as failure of the blastocyst to escape from the zona pellucida, but the data demonstrate more scatter than those for failures manifest before blastocyst formation. Blastocyst formation and outgrowth are characterized by a de novo protein synthesis (Van Blerkom and Brockway, 1975), which suggests that mutation due to disruption of the gene product (a point mutation) as well as a chromosome-associated lesion may be occurring. These two components of damage, each occurring with its own characteristic induction kinetics, may contribute to uncertainties in the data. Despite uncertainties in describing quantitatively the kinetics of induction for dominant lethal mutations induced in sperm and expressed after blastocyst formation, it is obvious that embryonic failures are manifest late in development following carbon and neon irradiations. It is not clear whether such failures are induced by the helium beam.

The overall dominant lethal mutation rate in vitro:

$1-$

( $\left.\frac{\text { fraction two-cell embryos forming } T B O G \text {, experiment }}{\text { fraction two-ce } 71 \text { embryos forming TBOG, control }}\right)$

can be approximated oy the expression:

$1-\left(\frac{\frac{\text { corpora lutea, implantations, experiment }}{\text { corpora lutea, experiment }}}{\frac{\text { corpora lutea, implantat ions, contro }}{\text { corpora lutea, control }}}\right)$

in vivo when the fertilization rate is unaffected. Since our data indicate that the fertilization rate remains unchanged following moderate doses of radiation to sperm, we have used in vivo data as a basis to estimate the RBE of the charged particle beams for dominant lethal mutation induction in sperm. These data (Table 2) indicate that the RBE for mutation induction is nigher than the RBE for cell killing. Similar conclusions have been made for mutation induction measured as specific locus mutations following neutron irradiation (W. Russe 11, 1965).

Irradiation of spermatids results in dominant lethal mutations which are manifest both before and after implantation without germ cell killing. Quantitatively the spermatid stage is about twice as sensitive to mutation induction as is the sperm, a finding essentially in agreement with mutation induction following photon-irradiation or treatment with mutagenic chemicals (Goldstein et a 1., 1980a,b). The data can be adequately expressed as a linear function without a threshold although the possibility of a threshold for helium (two cell to morula) and carbon (blastocyst outgrowth) cannot be excluded. The neon and carbon beams are more efficient than the helium for the induction of dominant lethal mutations manifest as preimplantation arrest, perhaps reflecting the enhanced high LET components in these beams compared to helium. Neon is more efficient than helium or carbon for inducing dominant lethal mutation manifest later 
Table 2. Dominant Lethal Mutation (DLM) Rate $\left(\times 10^{-4} \mathrm{rad}^{-1}\right)$ Induction by Heavy-Ion Beams

\begin{tabular}{|c|c|c|c|c|c|c|c|c|c|}
\hline $\begin{array}{c}\text { Stage } \\
\text { Irradiated }\end{array}$ & \multicolumn{3}{|c|}{ Preimplantation DLM Rate } & \multicolumn{3}{|c|}{ Postimplantation DLM Rate } & \multicolumn{3}{|c|}{ Overall DLM Rate } \\
\hline $\begin{array}{l}\text { Sperm } \\
\text { Spermatids } \\
\text { Spermatogonia }\end{array}$ & $\begin{array}{l}3.7 \\
7.2 \\
0\end{array}$ & $\begin{array}{r}7.0 \\
15.7 \\
2.6\end{array}$ & $\begin{array}{l}9.1 \\
16 \\
--\end{array}$ & $\begin{array}{l}3.2 \\
11.7 \\
0\end{array}$ & $\begin{array}{l}5.6 \\
11.7 \\
0\end{array}$ & $\begin{array}{l}9.2 \\
17.3 \\
--\end{array}$ & $\begin{array}{r}7.5 \\
18.4 \\
--\end{array}$ & $\begin{array}{r}11.3 \\
18.0 \\
4.2\end{array}$ & $\begin{array}{l}14.7 \\
30.0 \\
11.0\end{array}$ \\
\hline
\end{tabular}

in development. The RBE for the beams for mutagenesis ranges from 3.1 to. 5.1 (Table 2).

Damage to the spermatogonial stem cells represents the greatest potential genetic hazard since it will affect permanently the reproductive integrity of the male. Luning and Searle (1971) have summarized the dominant lethal mutation rate for photons in this stage and found it to range from 5.2 to $15.9 \times 10^{-5} \mathrm{rad}^{-1}$ (data corrected to the formula in Lyon et al., 1964). The RBE for the rate of mutation induction for each charged particle beam calculated from the mean value of Luning's photon data $\left(10.55 \times 10^{-5} \mathrm{rad}^{-1}\right)$ are hel ium, 1.51 ; carbon, 3.63 ; neon, 9.04 . It is not clear whether the dose-response for mutation induction for photons is linear, although this is assumed to be the case in our calculation and those of Luning and Searle (1971). These RBE values are much higher than those for cell killing (calculated at a higher dose) but are in the range described for a variety of mutations following heavy-ion irradiation of cells (Cox et al., 1977).

In summary, our data suggest that sperm, spermatids, and spermatogonia exhibit different sensitivities to mutation induction by high LET beams. They are, in order of their increasing sensitivities: spermatogonia, sperm, and spermatids. The data suggest that there are differences in the shape of the dose-response curves, carbon and helium being curvilinear and neon being linear. Neon has the highest RBE, especially for the spermatogonial stem cell. Cell killing, measured by a variety of end points, is restricted to radiosensitive spermatocytes and spermatogonia at the doses investigated. A dose-dependent extent and duration of oligospermia is found, but the data suggest that other factors may contribute to a decrease in the fertilization rate.

\section{ACKNOWLEDGEMENT}

This work was performed under NIH Grant CA22644. I wish to thank Mrs. J. Florence for her excellent technical assistance.

\section{FOOTNOTES AND REFERENCES}

* Department of Radiation Oncology, University of California, San Francisco, CA.
Cox, R., J. Thacker, D. T. Goodhead, and R. J. Munson. 1977. Mutation and inactivation of mammalian cells by various ionizing radiations. Nature 267, 425-427.

Epstein, S. S. and G. Röhrborn. 1971. Recommended procedures for testing genet ic hazards from chemicals, based on the induction of dominant lethal mutations in mammals. Nature $230,459-460$.

Freud, M. and B. Carol. 1964. Factors affecting haemocytometer counts of sperm concentration in human semen. J. Reprod. Fertil. 8, 149-155.

Generoso, W. M., K. T. Cain, M. Krishina, and S. W. Huff. 1979. Genetic lesions induced by chemicals in spermatozoa and spermatids of mice are repaired in the egg. Proc. Natl. Acad. Sci (USA) 76, 435-437.

Goldstein, L. S. 1976. Methylmethansulfonateinduced dominant lethal mutations in male mice detected in vitro. Mutat. Res. 42, 135-139.

Goldstein, L. S. and A. I. Spindle. 1976. Detection of $X$-ray induced dominant lethal mutations in mice: An in vitro approach. Mutat. Res. 41, 289-296.

Goldstein, L. S., J. Menesis, and R. A. Pedersen. 1978. Dose response relationship for $x-r a y$ induced dominant lethal mutations detected in mouse embryos in vitro. Mutat. Res. 51, 55-59.

Goldstein, L.S., T. L. Phillips, K. K. Fu, G. Y. Ross, and L. J. Kane. 1980a. Biological effects of accelerated heavy ions. I. Single doses in normal tissues, tumors, and cells in vitro. Submitted to Radiat. Res.

Goldstein, L. S., T. L. Phillips and G. Y. Ros5. 1980b. Biological effects of accelerated heavy ions. II. Fractionated doses in normal tissue. Submitted to Radiat. Res.

Luning, K. G. and A. G. Searle. 1971. Estimates of genetic risks from ionizing radiation. Mutat. Res. 12, 291-304.

Lyon, M. F., R.J.S. Philips, and A. G. Searle. 1964. The overall rates of dominant and recessive lethals and visible mutation induced by spermatogonial x-irradiation of mice. Genet. Res. Camb. 5, 448-467. 
Oakberg, E. F. 1956. Duration of spermatogenesis in the mouse and timing of stages of the cycle of the seminiferous epithelium. Am. J. Anat. 99, 507-516.

Oakberg, E. F. and R. L. Diminno. 1960. X-ray sensitivity of primary spermatocytes of the mouse. Int. J. Radiat. Biol. 2, 196-209.

Russe11, L. B. 1965. Death and chromosome damage from irradiation of preimplantation stages. Preimplantation Stages of Pregnancy (G.E.W. Wolsteiholme and M. O'Connor, eds.), pp. 217-241. CIBA Foundation Symposium, J. and A. Churchill, Ltd.
Russe11, W. L. 1965. Studies in mammalian replication genetics. Nucleonics $23,53-56$.

Searle, A. G. and C. V. Beechey. 1974. Sperm count, egg fertilization and dominant lethality after $x$-irradiation of mice. Mutat. Res. 22, $63-72$.

Spindle, A. I. and R. A. Pedersen. 1973. Hatching, attachment and outgrowth of mouse blastocysts in vitro: Fixed nitrogen requirements. J. Exp. Z001. 186, 305-318.

Van Blerkom, J. and G. O. Brockway. 1975. Qualitative patterns of protein synthesis in the preimplantation mouse embryo. I. Normal pregnancy. Dev. Biol. 44, 148-157. 


\title{
CARCINOGENESIS WITH HEAVY-ION RADIATION IN THE HARDERIAN GLAND OF MICE
}

\author{
E. L. Alpen, R. J. M. Fry, ${ }^{*}$ P. Powers-Risius, and E. J. Ainsworth
}

During the initial stages of choice of an appropriate system for evaluation of radiation carcinogenetic effectiveness of heavy ions, we came to consider the mouse harderian gland system developed by Fry and associates (1975) at the Argonne National Laboratory. Fry and his colleagues were able to take advantage of the special characteristics of the mouse narderian gland tumor to evaluate the relative effectiveness of fission neutron and gamma radiation for tumor induction promotion. The principal characteristics of the tumor, which make it so valuable for our purposes are the following. The natural incidence of the tumor in the mice strain chosen is low. A total life time incidence of about $2.5 \%$ is reported by the above authors, and the majority of the tumors in normal mice are benign adenomas. The prevalence in the first 12 to 14 months of the mouse's life is only a percent or two at most. Fry and his colleagues have shown that pituitary hormones (probably prolactin) produced from pituitary jsografts act synergistically with radiation, which increases the incidence and shortens the latent period, but produces little or no effect on incidence prevalance among unirradiated controls. The tumors are epithelial in origin, and occur as adenomas or adenocarcinomas; some are metastatic. Finally, we have a large body of data from Fry's experiments with Janus and Fermi Lab neutron beams with which we can make comparisons.

The studies are designed to test the effect of LET over a range $<1-1,000 \mathrm{keV} / \mu \mathrm{m}$ with ${ }^{60}$ Co photons, or carbon, neon, argon, and iron ions. At the same time, we can compare the carcinogenic effectiveness of ions of different mass at comparable LET. In the design of this. study we assumed that maximum effectiveness would be seen at, or around, $100 \mathrm{keV} / \mathrm{um}$, a value associated with maximum response in a number of: organ and cellular systems (Hirono et al., 197.0; Blakely et al., 1979; Alpen et al., 1980). The. limited data available on LET dependence of. carcinogenic response over a wide LET range, for example the work of Hirono et al. on Aridopsis., (1970), suggests that such a peak effectiveness would be observed at or around $100: \mathrm{keV} / \mathrm{\mu m}$.

\section{METHODS AND MATERIALS}

Male and female $B_{6} C_{1} / A N L$ inybrids (C57B16J $\times$ BALB/CJ) were obtained from the Argonne National Laboratory. Virg in females $6-8$ weeks old at time of implant were used as recipients for the pituitary isografts, and 7 timately as experimental subjects. The donor tuitaries were taken from male mice at or over 00 days of age. The donors were killed by cervical dislocation and the pituitaries were collected. Two pituitaries were taken up into an 18 gauge trocar and transplanted under the capsule of the surgically exposed spleen of the female recipient. Surgery on the recipient was done under ether anesthesia. The activity of the isograft was assessed surgically by examination of both the isograft and the recipient ovaries at the time of scheduled sacrifice.

At 2-3 weeks after pituitary implant, when the recipients were 100-120 days old, the animals were irradiated as described below. After irradiation the animals were housed seven to a cage and allowed acidified water ( $\mathrm{pH} 2.5)$ and food ad libitum.

The heavy-ion irradiations and dosimetry were accomplished as described in detail elsewhere in this report (see Alpen et al., in Part IV). The same $3 \times 5 \mathrm{~cm}$ collimated field was used for all the charged particles except iron. The field included only the nead and upper body. The partial body irradiation technique was used to reduce intercurrent death from mammary tumors and other diseases that would reduce the population at risk. The radiation field relative to the mouse is shown in Figure 1. For carbon, neon, and helium ions the anesthetized animals were irradiated at the distal end of the $10-\mathrm{cm}$ spread Bragg peak. For argon ions, the distal portion of a $4-\mathrm{cm}$ spread Bragg peak was used. The dose rates were adjusted by beam attenuation so that the dose, whatever its magnitude, was given in approximately 1 minute.

Irradiation in the iron beam was a special circumstance. Acceleration of ions of this mass approaches the technical.1 imitations of the Bevalac, and as a result the dose rates are low and field sizes small. The dose rate in the plateau region of ionization was $1-3 \mathrm{rad} / \mathrm{min}$ and the field size was approximately 1.0 to $1.5 \mathrm{~cm}$ in diameter. The beam traversed the head of six mice at a time, and the field was centered on the eye and harderian gland area.

Ions and energies used are as follows: carbon at $400 \mathrm{MeV} / \mathrm{amu}$; neon at $425 \mathrm{MeV} / \mathrm{amu}$, argon at $570 \mathrm{MeV} / \mathrm{amu}$, iron at $600 \mathrm{MeV} / \mathrm{amu}$, all from the Bevalac; and helium at $225 \mathrm{MeV} / \mathrm{amu}$ from the 184-inch Synchrocyclotron. Cobalt irradiations were done with a 60 co te letherapy source at a dose rate of about $40 \mathrm{rad} / \mathrm{min}$. Animals received the irradiation in the same upper body field by exposing them seven at a time while the abdomen and legs were shielded. Dosimetry was performed with a Victoreen condenser R-meter calibrated against an NBS secondary standard. 


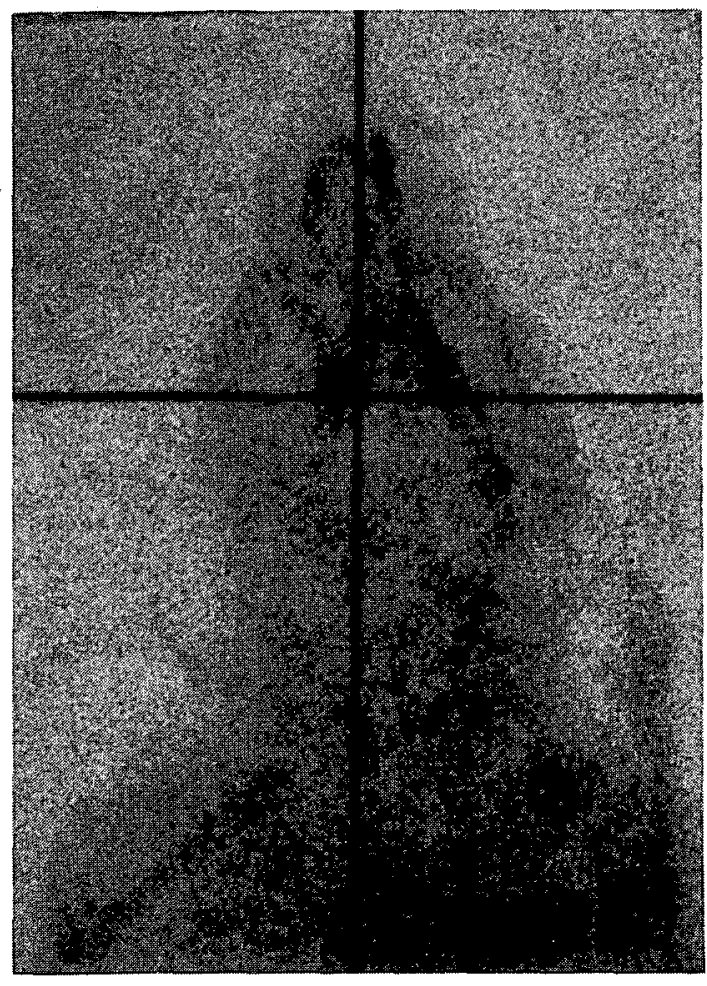

Figure 1. Port film showing the irradiation field relative to the exposed mouse for all heavy ions except iron. (XBB 808-9058)

The mice all were sacrified at a constant time of $16 \pm 0.5$ months after irradiation. Gross observatons at sacrifice included the examination and scoring of the implant and of mammary tissue for signs of hormonal activity. Harderian glands were collected and scored for the presence or absence of gross tumor. Other gross tumor observations were recorded, regardless of location in or out of the radiation field. The following tissues were removed and histologically processed: spleen, pituitary, harderian glands, any tumors, and ovaries if mammary gland activity was reduced or absent. Animals that died before sacrifice were also autopsied if the condition of animal permitted.

\section{Analys is of the Data}

Since this work is still in progress, with some animal sacrifices yet to be completed and much histological scoring yet to be done, it is premature to describe in detail the analys is of the data. We have, however, adopted formal procedures that can be outlined. In the tables that follow, one can observe notations for animals exposed and animals "at risk."

The number of animals at risk will be used in analys is because it has been corrected for animals lost by misadventure unrelated to the experimental design, i.e., escapes, drownings, accidental, or fighting deaths. The values given for prevalence are based on the at-risk number as the denominator for the prevalence fraction. Finally, the number at risk must be further corrected by removing animals with unsuccessful isografts. This final correction must await complete microscopic examination and data correlation. The final prevalence value will be based on the denominator, i.e., mice with active isografts accounted for at sacrifice, and the numerator will be those animals with microscopically confirmed harderian tumors.

\section{RESULTS}

Table 1 lists the total number of animals committed to the study to date. Of these groups several are still to reach appropriate time for sacrifice. The study groups still in progress are listed in Table 2.

An important difference between this study and the earlier fission neutron studies of Fry et al. (1975) is that prevalence at a fixed point in life span, rather than life-time accumulated incidence is observed. This end point is achieved by assigning a fixed date for sacrifice and counting tumors present at that time. This design has great economy in time required for observation and animal handling, and statistically, analys is is simpler. Simple Chi-square analysis for a single time point achieves the analytic goal. No time dependent analysis is required and competing risk corrections are unnecessary. The important fallability of the prevalence design is that if there is a radiation quality dependence of latency for tumor development, it will not be detected by our design. Such a latency change would be seen as altered prevalence, and would be reported as altered tumorigenic potential of the ion under study.

No such prevalence studies have yet been reported, but similar experiments are in progress with high energy neutrons generated at the National Accelerator Laboratory. The prevalence data for these animals have been kindly made available by R.J.M. Fry, and are shown in Table 3. From these data, and the fission neutron data from Janus which are useful for comparison with heavy-ion radiations, it is clear that prevalence in controls is very low, $1-2 \%$ as would be expected from earlier studies (Fry, 1975).

Analysis of the heavy ion-data is far from complete, and we are not prepared to draw conclusions at this time. The data are presented here with the clear understanding that the study is incomplete. In Table 4 are summarized the prevalence results based on gross observations only, and the values would be expected to change when histopathology is done. Also, the activity state of the isograft has not been confirmed by microscopic examination in all cases. The numbers of animals per dose will not be consistent with the count given in Table 1 because of animals lost to follow-up for reasons detailed earlier. Partial, and as yet 
Table 1. Number of Mice Committed to Study Groups

\begin{tabular}{|c|c|c|c|c|c|c|c|c|c|}
\hline & 5 & $\therefore 10$ & 15 & 20 & $\begin{array}{c}\text { Dose } \\
40\end{array}$ & $\begin{array}{c}(\mathrm{rad}) \\
80\end{array}$ & 160 & 320 & 700 \\
\hline${ }^{60} \mathrm{Co}$ & -- & -- & -- & -- & -- & -- & 90 & 90 & 90 \\
\hline Helium & -- & -- & -- & --- & 200 & 123 & 80 & 10 & -- \\
\hline Carbon & 143 & 100 & -- & --- & 194 & 112 & 109 & 63 & -- \\
\hline Neon & -- & 100 & 97 & 104 & 121 & 77 & 48 & 65 & -- \\
\hline Argon & --- & --- & -- & --- & 102 & 74 & 74 & --- & --- \\
\hline Iron & 6 & 24 & -- & 24 & 25 & & & & \\
\hline
\end{tabular}

Unirradiated implant controls: 100

Table 2. Studies in Progress

\begin{tabular}{|c|c|c|c|c|c|}
\hline \multirow{2}{*}{$\begin{array}{c}\text { Experimental } \\
\text { Group }\end{array}$} & \multicolumn{2}{|c|}{ Irradiation } & \multirow{2}{*}{$\begin{array}{c}\text { Number of } \\
\text { Mice }\end{array}$} & \multirow{2}{*}{$\begin{array}{l}\text { Dose } \\
\text { (rad) }\end{array}$} & \multirow{2}{*}{$\begin{array}{l}\text { Sacrifice } \\
\text { Date }\end{array}$} \\
\hline & Date & Ion & & & \\
\hline \multirow[t]{3}{*}{14} & $06 / 07 / 79$ & ${ }^{60} \mathrm{Co}$ & 90 & 160 & $10 / 05 / 80$ \\
\hline & & & 90 & 320 & \\
\hline & & & 90 & 700 & \\
\hline 15 & $02 / 27 / 80$ & ${ }^{12} \mathrm{C}$ & 143 & 5 & $06 / 27 / 81$ \\
\hline 17 & $04 / 26 / 80$ & $20 \mathrm{Ne}$ & 97 & 15 & $08 / 25 / 81$ \\
\hline Control & -- & None & 60 & 0 & $10 / 05 / 80$ \\
\hline
\end{tabular}

Table 3. Prevalence of Histologically Confirmed Harderian Gland Tumors in Mice Irradiated with Neutrons at Fermi Laboratory*

\begin{tabular}{|c|c|c|c|c|}
\hline $\begin{array}{l}\text { Dose } \\
\text { (rad) }\end{array}$ & $\begin{array}{c}\text { Number of } \\
\text { Mice }\end{array}$ & $\begin{array}{c}\text { With Active } \\
\text { Isografts }\end{array}$ & $\begin{array}{l}\text { Active I sografts } \\
\text { and Tumors }\end{array}$ & $\begin{array}{l}\text { Percent } \\
\text { Prevalence }\end{array}$ \\
\hline $\begin{array}{c}0 \\
\text { (control) }\end{array}$ & 98 & 65 & $\therefore 1$ & 1.5 \\
\hline 20 & 125 & 101 & 19 & 18.8 \\
\hline 40 & 108 & 86 & 27 & 31.4 \\
\hline 80 & 76 & $66 \cdot \because$ & 24 & 36.6 \\
\hline 160 & 81 & $\because 69$ & 34 & 49.3 \\
\hline \multicolumn{5}{|c|}{ One Group Isografted After Irradiation } \\
\hline 80 & 85 & 70 & 21 & 30.0 \\
\hline
\end{tabular}

* Courtesy of R.J.M. Fry, study in progress. 
Table 4. Preliminary Estimates of Prevalance, Based only on Gross observations

\begin{tabular}{|c|c|c|c|c|}
\hline Ion & $\begin{array}{l}\text { Dose } \\
\text { (Rad) }\end{array}$ & $\begin{array}{l}\text { Animals } \\
\text { at Risk }\end{array}$ & $\begin{array}{l}\text { Grossly Observed } \\
\text { Harderian Tumors }\end{array}$ & Percent \\
\hline Helium & $\begin{array}{r}40 \\
80 \\
160 \\
320\end{array}$ & $\begin{array}{r}190 \\
119 \\
58 \\
7\end{array}$ & $\begin{array}{r}10 \\
10 \\
23 \\
1\end{array}$ & $\begin{array}{r}5.0 \\
8.4 \\
39.7 \\
14.3\end{array}$ \\
\hline Carbon & $\begin{array}{r}10 \\
40 \\
80 \\
160 \\
320\end{array}$ & $\begin{array}{r}100 \\
189 \\
104 \\
106 \\
62\end{array}$ & $\begin{array}{r}2 \\
47 \\
36 \\
39 \\
20\end{array}$ & $\begin{array}{r}2.0 \\
24.9 \\
34.6 \\
36.8 \\
32.3\end{array}$ \\
\hline Neon & $\begin{array}{r}10 \\
20 \\
40 \\
80 \\
160 \\
320\end{array}$ & $\begin{array}{r}90 \\
104 \\
119 \\
76 \\
46 \\
59\end{array}$ & $\begin{array}{r}3 \\
10 \\
35 \\
30 \\
21 \\
16\end{array}$ & $\begin{array}{r}3.3 \\
9.6 \\
29.4 \\
39.5 \\
45.7 \\
27.1\end{array}$ \\
\hline Argon & $\begin{array}{r}40 \\
80 \\
160\end{array}$ & $\begin{array}{r}102 \\
84 \\
71\end{array}$ & $\begin{array}{l}16 \\
16 \\
20\end{array}$ & $\begin{array}{l}15.7 \\
19.0 \\
28.2\end{array}$ \\
\hline Iron & $\begin{array}{r}5 \\
10 \\
20 \\
40\end{array}$ & $\begin{array}{r}6 \\
24 \\
24 \\
23\end{array}$ & $\begin{array}{l}0 \\
2 \\
6 \\
3\end{array}$ & $\begin{array}{c}0 \\
8.3 \\
25.0 \\
13.0\end{array}$ \\
\hline $\begin{array}{l}\text { Implant } \\
\text { Controls }\end{array}$ & & 36 & 0 & 0 \\
\hline
\end{tabular}

incomplete, microscopic analysis data are given in Table 5 .

Data were also collected on the prevalence of mammary gland tumors and tumors in the lung. The lungs were in the radiation field while only part of the mammary glands were exposed. No signifcant difference as a function of radiation exposure was observed for either tumor types, and no further observations will be made.

\section{DISCUSSION}

Only preliminary and tentative conclusions may be drawn at this time. A complete record audit is in progress to ensure that animal counts and records are complete. Readers will observe differences in tumor numbers when comparing gross and microscopic results. These differences arise both from failure to confirm gross observation as tumors, and finding new tumors visible only at the microscopic level.
Some careful generalizations are possible at this point. The first, and most obvious, is that heavy-ion radiations with LET values of a few $\mathrm{keV} / \mathrm{\mu m}$ and more are capable of producing high yields of harderian gland tumors. The prevalence values for this tumor with heavy-ion radiations cannot, at this time, be distinguished from the results obtained from Fermilab neutrons.

All of these radiations, including neutrons, are capable of producing tumor yields far in excess of the yield resulting from gamma-ray exposure. Our results for gamma rays are not yet in, but from the published data of Fry and associates, we would expect no significant increase above control for gamma-ray doses below 100-200 rad. RBE values have little real significance under these conditions.

Of special significance is the fact that helium ions, at relatively low LET, appear to be 
Table 5. Preliminary Estimate of Harderian Gland Tumor Prevalence Based on Microscopic Confirmation

\begin{tabular}{|c|c|c|c|c|}
\hline Ion & $\begin{array}{l}\text { Dose } \\
\text { (rad) }\end{array}$ & $\begin{array}{l}\text { Animals with } \\
\text { Active Isografts }\end{array}$ & $\begin{array}{c}\text { Active Isografts } \\
\text { and Tumors }\end{array}$ & $\begin{array}{c}\text { Percent } \\
\text { Prevalence }\end{array}$ \\
\hline Helium & $\begin{array}{r}40 \\
80 \\
160 \\
320\end{array}$ & $\begin{array}{c}142 \\
99 \\
45 \\
(5) \star\end{array}$ & $\begin{array}{l}10 \\
13 \\
14 \\
(4)\end{array}$ & $\begin{array}{c}7.0 \\
13.1 \\
31.1 \\
(80.0)\end{array}$ \\
\hline Carbon & $\begin{array}{r}40 \\
80 \\
160 \\
320\end{array}$ & $\begin{array}{c}115 \\
94 \\
(53) \\
(60)\end{array}$ & $\begin{array}{c}20 \\
28 \\
(16) \\
(20)\end{array}$ & $\begin{array}{l}17.4 \\
29.8 \\
(30) \\
(32)\end{array}$ \\
\hline Neon & $\begin{array}{r}20 \\
40 \\
80 \\
160 \\
320\end{array}$ & $\begin{array}{c}98 \\
119 \\
76 \\
46 \\
(59)\end{array}$ & $\begin{array}{c}26 \\
35 \\
30 \\
21 \\
(16)\end{array}$ & $\begin{array}{c}26.5 \\
29.4 \\
39.5 \\
45.7 \\
(27.1)\end{array}$ \\
\hline Argon & $\begin{array}{r}40 \\
80 \\
160\end{array}$ & $\begin{array}{l}89 \\
75 \\
61\end{array}$ & $\begin{array}{l}26 \\
25 \\
31\end{array}$ & $\begin{array}{l}29.2 \\
33 \\
51\end{array}$ \\
\hline Iron & $\begin{array}{r}5 \\
10 \\
20 \\
40\end{array}$ & $\begin{array}{r}4 \\
23 \\
20 \\
20\end{array}$ & $\begin{array}{l}0 \\
2 \\
8 \\
7\end{array}$ & $\begin{array}{r}0 \\
8.7 \\
40.0 \\
35.0\end{array}$ \\
\hline
\end{tabular}

* Parenthetical values require rechecking and an audit of animal numbers.

nearly as effective as the other ions; while for most acute biological end points, the helium ions would be expected to be trivially different from $225 \mathrm{kVp} X$ rays, with RBE values close to 1.0 (Fry, 1975). We must also develop further data to better define the nature of the dose-response curve for all ions at lower doses. This is particularly true for neon, which at this time appears to have the greatest carcinogenic potential and is characterized by an LET of $\sim 100 \mathrm{keV} / \mathrm{\mu m}$.

We must conclude that heavy ions have a high carcinogenic potential in the $\mathrm{B}_{6} \mathrm{CF}_{1}$ mouse harderian gland promoted with pituitary implants. No further conclusions are justified at this time. The lack of dose response for either mammary gland or lung tumors cannot be interpreted other than to speculate that we must go to higher doses to discern any increased effectiveness of heavy ions over gamma rays for these two tumorigenic end points.

\section{FOOTNOTES AND REFERENCES}

* Biology Division, Oak Ridge National Laboratory, Oak Ridge, TN.

Alpen, E. L., P. Powers-Risius, and M. McDonald. 1980. Survival of intestinal crypt cells after exposure to nigh $Z$, nigh energy charged particles. Radiat. Res., in press.

Blakely, E. L., C. A. Tobias, T.C.H. Yang, K. C. Smith, and J. T. Lyman. 1979. Inactivation of human kidney cells by nigh-energy monoenergetic heavy-ion beams. Radiat. Res. $80, .122-160$.

Fry, R.J.M. 1975. Radiation oncogenesis in vivo. Particle Radiation Therapy, pp. 304-329. Pergamon Press, New York.

Fry, R.J.M., A. G. Garcia, K. H. Allen, A. Sallese, T. N. Tahmisian, L. S. Lombach, and 
E. J. Ainsworth. 1975. The effect of pituitary isografts on radiation carcinogenes is in the mammary and harderian glands of mice. Biological Effects of Low Level Radiation Pertinent to Protection of Man and his Environment, pp. 213-227.

International Atomic Energy Agency, Vienna.
Hirono, Y., H. H. Smith, J. T. Lyman, K. H. Thompson, and J. W. Baum. 1970. Relative biological effect of heavy ions in producing mutations, tumors and growth inhibition in the crucifer plant Aridopsis. Radiat. Res. 44, 204-223. 


\title{
CATARACTOGENIC EFFECTS OF HEAVY CHARGED PARTICLES IN MICE
}

\author{
E. J. Ainsworth, J. G. Jose, ${ }^{*}$ V. V. Yang, and M. E. Barker
}

Manned space flights may increase in the future in connection with use of advanced technologies, such as the satellite power systems (SPS), to generate electricity. However, many of the risks associated with extended habitation in a space environment remain undetermined, including the risk of radiation carcinogenesis, mutagenesis, and damage to the eye.

of particular concern at this time are the hazards that might occur from high energy heavy charged particles (HZE) to which space workers will be exposed in a complex radiation environment that consists principally of low LET radiation such as protons and brehmsstrahlung. Although considerable information exists from animal experiments and from human epidemiological studies on the nazards of nigh LET alpna particles and neutrons, less is known about the biological consequences of HZE than about other types of radiation encountered in space (Mays et al., 1979; Rowland et a1., 1978; Ainsworth et al., 1976, 1977; U11rich et a1., 1977; Storer et al., 1979; Isnimaru et al., 1979).

The effects of HZE particles on the crystalline lens of the eye are important because this tissue has proven susceptible to $X$ and gamma rays and particularly susceptible to other forms of high-LET radiation such as neutrons (Bateman and Bond, 1967; Bond, 1960). The high susceptibility of the crystalline lens to densely ionizing radiation suggests that this tissue is also susceptible to the effects of HZE particles.

This report summarizes the results currently available from a prospectively designed study to explore the LET dependence of the cataractogenic process, and presents data collected from mice dedicated to other studies that would not be compromised by slit lamp evaluations for assessment of cataracts. Morphologic studies that were recently initiated may increase our understanding. of the mechanisms of cataract production.

Before financial support.was obtained for. this project, several pilot studies were con-: ducted on mice that survived LD50/30 experiments and were scheduled for sacrifice in studies of harderian gland carcinogenes is by HZE particle irradiation (see Alpen et al. in Part. IV of this report). Some of these results were the subject of a doctoral thesis and are summarized elsewhere (Boomer et al., 1979; Giocolone et al:., 1979). Dose-latency relationships were seen and cataracts were observed at doses as low as 5 rad of $56 \mathrm{Fe}$ ions. Although important experience was ained from these pilot studies, the results did not provide definitive information on LET-RBE relationsnips and thus will not be presented nere.
Previous research, mostly with rodents, established various physical and biological factors that influence the potential for production of radiation-induced lens opacification. These factors are: physical dose, radiation quality, instantaneous dose rate (i.e., exposure time), dose protraction (i.e., fractionation), age at irradiation, and species. Comprenensive studies with neutrons of various energies have shown that RBE values vary inversely with dose. They are in the range of 4 to 7 for high LET neutrons over the dose range of 10 to $40 \mathrm{rad}$, and they are quite nigh, possibly of the order of 100, at lower dose levels of significance for occupational exposures (Bateman and Snead, 1969; Merriam and Focht, 1957; Bond, 1960). The nypothesis is that HZE particles will show an LET dependence and RBE dependence consistent with expectations from previous experiments with photons and neutrons. Because HZE particles have the potential for producing thermophysical lesions or "tunnel lesions," the possibility of unique lens lesions cannot be precluded. However, the role these lesions might play in the cataractogenic process that is attributable to abnormal fiber production by surviving epithelial cells is unknown (Tobjas et al., 1980; Malachowski et al., 1980; Nelson et al., 1980).

\section{METHODS AND PROCEDURES}

The mice used were $\mathrm{LAF}_{1}$ males, or $\mathrm{CB}_{6} \mathrm{~F}_{1}$ females obtained from the R. B. Jackson Memorial Laboratory at 8-10 weeks of age. They were housed ten per cage on No. 6 sawdust bedding, and they were provided food (Purina Lab chow 5008) and acid drinking water (pH 3) ad libitum. Due to Bevalac operations schedules, the age of irradiation varied from 80-120 days.

The mice were irradiated on an optical bench in Bevalac Cave II approximately $3 \mathrm{~m}$ from the thin mylar window where the incident beam enters the exposure room and passes through air to the exposure 10cation: Interposed in the beam line are lead scattering foils, usualiy 12/64 to 1/64 in thick, a collimator, upstream and downstream ionization chambers, a multiwire proportional chamber, and a spiral ridge filter and a water column. when spread Bragg peaks are used. The proportional chamber provides information on localization and size of the incident beam. The collimator reduces the beam size to $3 \times 5 \mathrm{~cm}$. The head and upper chest of vertically positioned mice were exposed to charged particles by "translating" seven mice through the beam spot while mice were affixed to a $24.2 \mathrm{~cm}$ wide lucite holder. The animals were anesthetized with pentabarbital sodium (Diabutal); $1.24 \mathrm{mg} /$ mouse was administered 
approximately $5 \mathrm{~min}$ before irradiation. Dose rates were several hundred $\mathrm{rad} / \mathrm{min}$. Shortly after irradiation animals were transported 0.5 miles to the LBL animal facility, and were subsequently transported an additional $0.5 \mathrm{miles}$ to the animal facility located in the School of Optometry on the Berkeley Campus.

Presented here are observations made on three groups of animals. The experiment central to this project is designated Experiment IV and involves approximately $400 \mathrm{CB}_{6} \mathrm{~F}_{1}$ mice given single radiation doses in March-April 1979. The animals have been observed sequentially following upper body exposure to $220 \cdot \mathrm{kVp} \times$ rays or $12 \mathrm{C}$, $20 \mathrm{Ne}$, or $40 \mathrm{Ar}$ in the plateau portion of the Bragg curve. A second replication is in progress that will increase sample sizes from 16 to 18 per dose to 30 per dose; at the two or three lowest doses, 20 to 30 additional animals are being irradiated for morphologic studies to be done on a serial sacrifice basis. Because data obtained at 9 and 13 months indicated the RBE value is probably $<10$ at 5 rad for all HZE particles studied, the $X$-ray replicate experiment emphasizes doses less than $300 \mathrm{rad}$.

The second group consists of mice irradiated with stopping $425 \mathrm{MeV}$ argon particles in 1978 in support of a doctoral thes is project for $V$. Giocolone. Since that pilot project was completed, two additional evaluations have been done by others to address the question of doselatency relationships (or the time by which average cataract scores plateau at low doses in our mouse strain). Some of these animals have also been sacrificed for morphologic studies.

The third group for which data are reported nere are $60 \mathrm{Co}$ gamma or $225 \mathrm{MeV}{ }^{12} \mathrm{C}$ irradiated mice from the Skyhook Project, which is a small life span study that is described in Part $V$. This pilot study addresses the question of this extent to which gamma or $12 \mathrm{C}$ dose fractionation (24 fractions administered weekly over 24 weeks) influences the cataractogenic process compared to animals that received the same total single dose.

In pilot studies, eye examinations were performed by first sedating the animals with

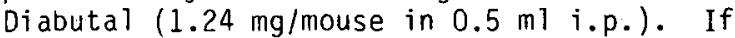
anesthetized animals were replaced on bedding material, foreign objects got in their eyes and interfered with observations made with a slit lamp biomicroscope. Therefore, through careful handling it became possible to perform the evaluations without anestinesia. Dilation was achieved with one drop of $1 \%$ Tropicamide.

The cataract scoring. system and the biomicroscopic appearance of the type of opacification that were observed are shown in Figure 1. The posterior lens opacification was observed primarily as three types which we have called diffuse, amorphous, and stellate. In the diffuse type, the subcapsular region may best be described as similar in size and shape, and lesions were sprinkled across the posterior lens in a sait-and-pepper fashion. Lenses in the amorphous category had lesions that were
Opacification groding: Degrees \& Types Grade

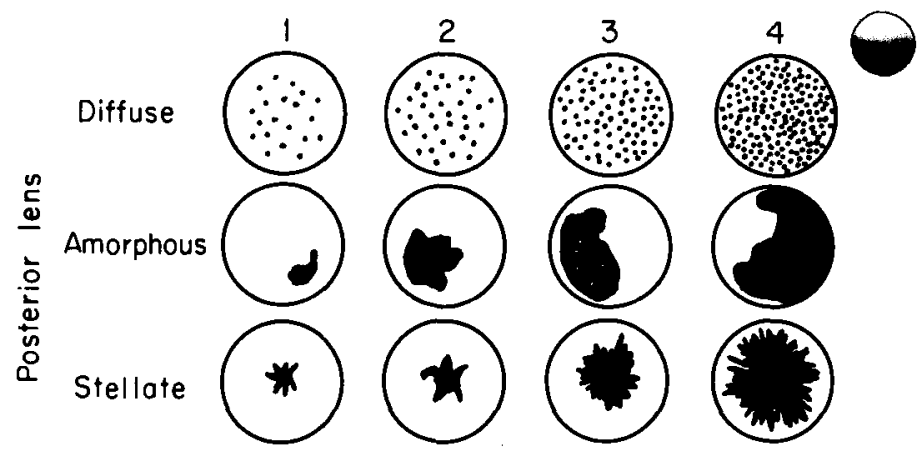

Figure 1. Schematic representation of the types of lens opacities observed and the nature of the grading system that has been developed to describe the degree of the opacification. (XBL 801-3070)

discrete, irregular in shape, and located anywhere in the posterior lens area. Size, shape, and number varied, but the lesions were nearly always opaque. The stellate type was often indistinguishable from the amorphous type. Generally, however, stellate lenses were more central in location and had radiating opaque, thread-like processes resembling the arms of a star distributed radially around the center of the lesion proper.

The scoring system evolved over a period of months, and although subjective, is considered reliable. The scoring was done by three individuals who did not know the animal treatment (dose or quality). Initially, two scorers observed each animal and agreed on the score assigned. Subsequently, because of the large number of examinations, scoring was done by a single individual. To date, scores have only been averaged at each dose and radiation quality and that is now they are presented here. When observations are made to assess dose and qualitylatent period relationships, the data will be handled in the fashion described by Bateman and Snead (1969) such that the frequency of grades 1 and 2 posterior lens cataracts is presented in relation to time after irradiation.

To prepare the lenses for electron microscopy, the mice were killed by cervical dislocation and the whole eye was placed in $2 \%$ glutaraldehyde in $0.075 \mathrm{M}$ phosphate buffer at $37^{\circ} \mathrm{C}$. After 15 minutes a cut was made in the posterior globe. The eyes were neld at $37^{\circ} \mathrm{C}$ in fixative for 15 more minutes, and then the lens was removed from the eye and placed in buffer at $37^{\circ} \mathrm{C}$. The lenses were allowed to equilibrate to room temperature and were then refrigerated overnight. The next morning they were washed in buffer, placed in cold $2 \%$ osmium, and refrigerated one hour. At that time, the lens could easily be cut into pieces with a razor. After dehydration in a series of alcohols and propylene oxide, the lenses were embedded in Araldite and sectioned 
on a Sorvall MT-2B ultramicrotome. They were then stained with uranyl acetate and Reynolds lead citrate, and examined on a Zeiss 10 electron microscope.

\section{RESULTS AND DISCUSSION}

Previous mouse studies that explored LET-RBE dependence of posterior lens cataracts utilized neutrons, and the maximum hazard was observed at an energy of $0.43 \mathrm{MeV}$ where the dose-average $L E T_{\infty}$ was in the range of $\sim 100 \mathrm{keV} / \mathrm{\mu m}$ (Merriam et al., 1965).

The present results from Experiment IV are consistent with a high cataractogenic effect at $\sim 100 \mathrm{keV} / \mu \mathrm{m}$, because plateau $40 \mathrm{Ar}$ ions, with an LET in this range produce consistently higher average cataract scores at 9,11 , and 13 months than do $12 \mathrm{C}$ or $20 \mathrm{Ne}$ ions (Figs. 2 and 3 ).

In Experiment IV mice were screened for lens abnormalities either before or within one month after irradiation; those with more than $1 \%$ spontaneous abnormalities were excluded from the experiment. Approximately $50 \%$ of the mice in each dose group ( $N=7-8$ ) were evaluated monthly, and when $1-5 \%$ opacifications were detected in irradiated animals the entire group was scored. The first complete assessment was done at 6 months, and clear dose-response relationships were not apparent. Except for groups that

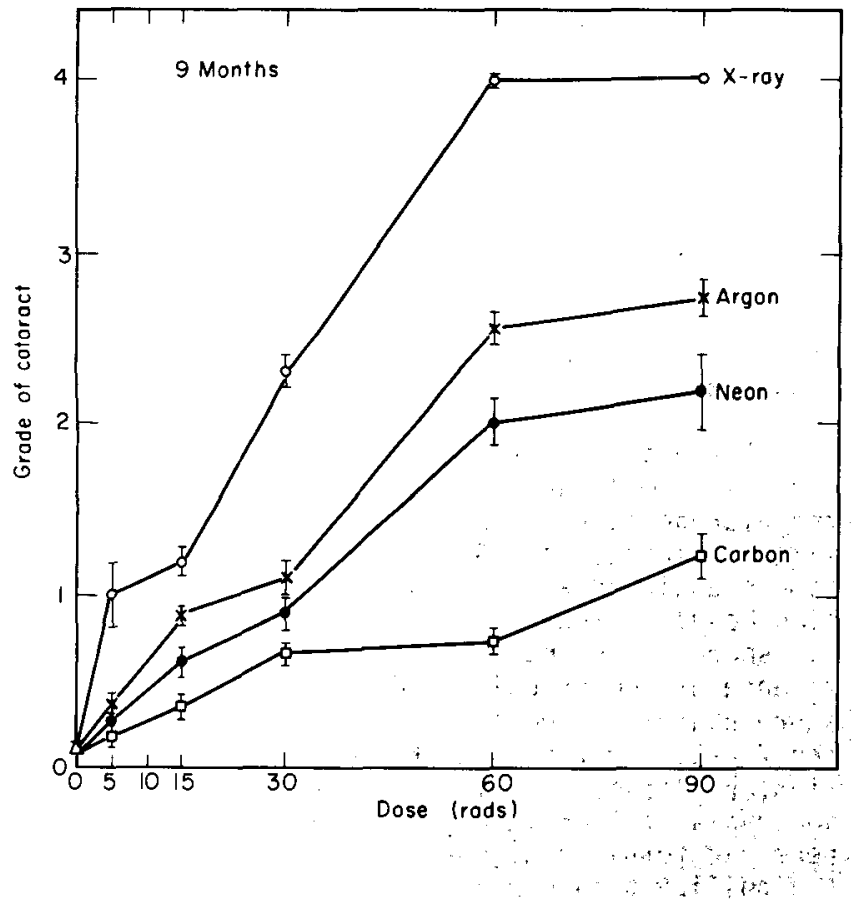

Figure 2. Severity of cataract induced in mice (ordinate) 9 months postirradiation with plateau argon, neon, and carbon ions at the dose indicated on the abscissa. The open circles indicate the degree of opacification in animals irradiated with $x$ rays at ten times the dose indicated on the abscissa. The bars indicate the standard error of the mean. (XBL 801-3072)
CATARACT SCORES OF POSTERIOR LENSES IN EXPERIMENT IIV

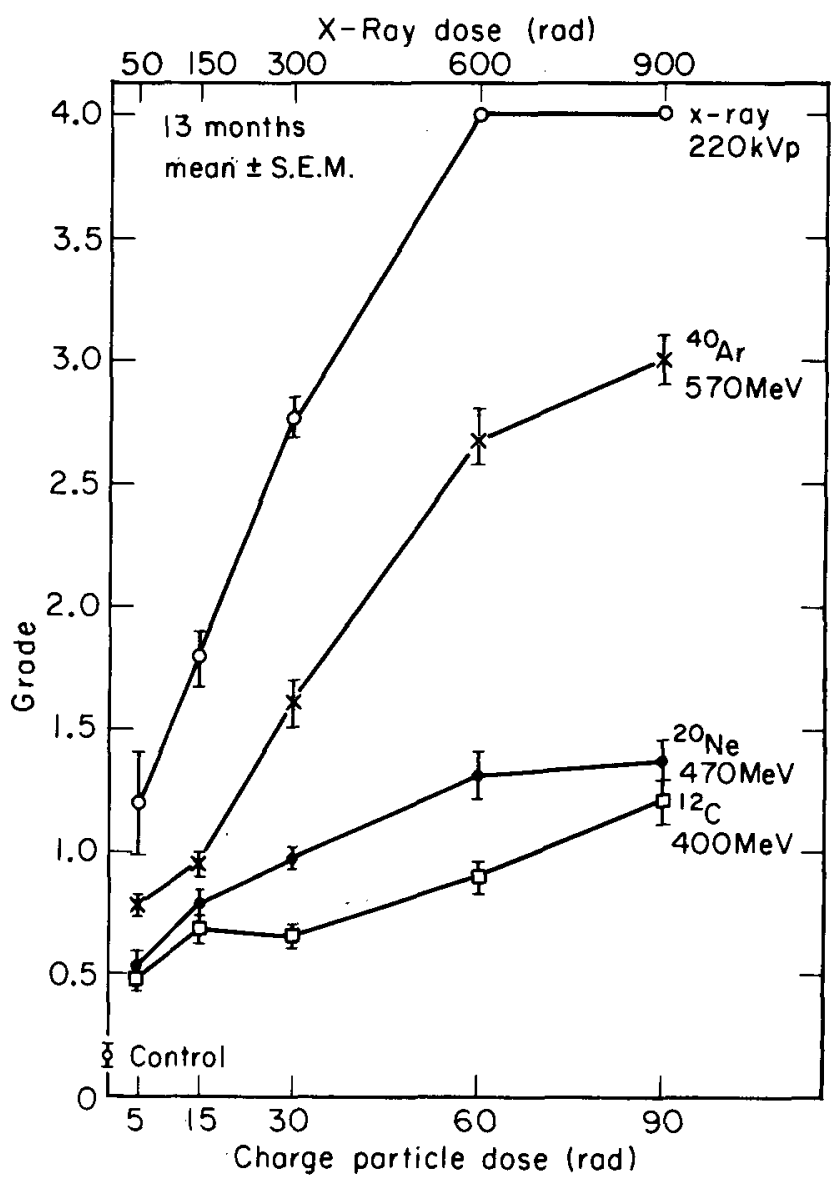

Figure 3. Severity of cataract induced in mice (ordinate) 13 months postirradiation with plateau argon, neon, or carbon ions at the dose indicated on the abscissa. The open circles indicate the degree of opacification in animals irradiated with $x$ rays at ten times the dose indicated on the abscissa. The bars indicated the standard error of the mean. (XBL 809-3671)

received 600 or $900 x$-ray rad, or 90 rad of $40 \mathrm{Ar}$ ions, all average scores were below 0.8 ; the average. score among controls was nil. The evaluation at 6 months postirradiation showed that: a low frequency of abnormalities can be detected after 5 or 15 rad of HZE particles or 50 rad of $X$ rays.

By 9 months the expected LET dependence and dose-response relationships were apparent, but the question of statistical significance of differences between HZE groups was open to question (F.ig.. 2). Based on the dose necessary to produce a grade 1 cataract by 9 months (5-15\% opacification), the RBE for $40 \mathrm{Ar}$ would be 2 to $4(\sim 50-100 \mathrm{rad} / \sim 25 \mathrm{rad})$, and the RBE for $20 \mathrm{Ne}$ and ${ }^{12} \mathrm{C}$ would be lower. Based on data collected at 13 months postirradiation (Fig. 3), the RBE estimate is not vastly different, although average scores tended to increase slightly among 
most groups between 9 and 13 months. Based on grade 2 cataracts (which could be vision impairing if $15-25 \%$ opacification occurred in man), RBE estimates for $40 \mathrm{Ar}$ at 9 and 13 months postirradiation are on the order of 4-5. These values are in the same range reported for high LET neutrons in mice and for HZE particles in rabbits where dose-RBE relationship were not considered (Merriam and Focht, 1965; Lett et al., 1980). Caution in interpreting these data is encouraged by pilot studies with plateau ionization where $56 \mathrm{Fe}$ ions failed to yield definitive results. Observation of animals exposed to 56 Fe radiation were initiated early in the project when the scoring system was not refined and the experience level with mouse cataracts was low (even though our scorers had examined hundreds of human lenses).

To study cataract latency in relation to dose, slit lamp observations were made on $L_{A F}$ female mice that had been exposed to stopping $74 \mathrm{~cm}$ spread Bragg peak) $40 \mathrm{Ar}$ ions and examined initially at 9 months postirradiation and were reexamined at 18 and 23 months. At 9 months following 10 rad, only 1 of 28 lenses examined had any evidence of opacification, but at 18 and 23 months all lens were affected and the average score was $\sim 2.7$ (Table 1 ). Because a different scoring system was used in this pilot study, it

Table 1. Cataracts in the Posterior Lens of LAF 1 Female Mice at 18 Months After Exposure to $40 \mathrm{Ar}$ Ions $(4 \mathrm{~cm}$ Spread Bragg Peak)

Dose (rad)

No. Eyes

$\bar{x}$ SE $\bar{x}$

\begin{tabular}{rll}
\hline 0 & 31 & $0.5 \pm 0.09 \star$ \\
10 & 21 & $2.7 * 0.14$ \\
25 & 20 & $2.9 \pm 0.18$ \\
50 & 22 & $2.6 \pm 0.14$ \\
100 & 13 & $3.6 \pm 0.14$
\end{tabular}

Same Animals Examined 23 Months Postirradiation

Dose (rad)

No. Eyes

$\bar{x} \quad$ SE $\bar{x}$

\begin{tabular}{rrl}
\hline 0 & 26 & $2.2 \pm 0.17$ \\
10 & 18 & $2.6 \pm 0.17$ \\
25 & 18 & $3.1 \pm 0.17$ \\
50 & 24 & $3.0 \pm 0.11$ \\
100 & 6 & 4.0
\end{tabular}

* Significantly different from all irradiated groups $(P=<0.07)$. is impossible to state more quantitatively the progression of cataractogenes is between 9 and 18 months. At low doses the cataractogenic process appears to progress between 9 and 18 months, and might even progress between 18 and 23 months based on average scores among mice given 25-100 rad. However, this interpretation must wait until the scoring reliability and reproducibility question is resolved.

Two separate evaluations have been completed on mice in the life span study designated Skyhook Experiment II, and the results are in part contradictory. The principle objective was to determine the extent to which fractionation of a ${ }^{12} \mathrm{C}$ dose influenced the cornea or lens response. Because the sparing effect of photon dose fractionation is well established, only two groups of gamma-irradiated mice that received the same total dose were evaluated. The results summarized in Table 2 show a large sparing effect when the gamma dose of 417 rad was administered in 24 weekly fractions of 27 rad over approximately six months. In contrast, $12 \mathrm{C}$ ion dose fractionation enhanced or accelerated the cataractogenic process. This was potentially an important observation because fission neutron dose fractionation produces enhancement where life shortening, total incidence or appearance time of some tumors, and vascular injury are the experimental end points (Ainsworth et al., 1974, 1977; Yang et al., 1978). Because cataracts result from abnormal fiber production by surviving cells, one possible inference would be that the number of damaged cells is increased by fractionated doses of high LET radiations, in this case stopping ${ }^{12} \mathrm{C}$ particles where the LET is $\sim 40-50 \mathrm{keV} / \mu \mathrm{m}$. Recruitment of cells into more sensitive stages of the cell cycle by low doses of high LET radiations where little or no shoulder exists on survival curves, could be the enhancement mechanism. However, firm conclusions regarding enhancement of cataractogenes is could not be based on a single set of observations. Consequently, these animals were reevaluated approximately two months later, and the ennancement phenomenon was not confirmed unequivocally (Table 3).

At the time of the second observation of the control animals and animals given a single dose, the average scores were essentially the same or slightly increased. Surprisingly, the average scores assigned animals that received fractionated doses of $12 \mathrm{C}$ ions were lower by 0.3 to 1.5 score units than those recorded previously. At none of the fractionated doses was the average score nigher at the second observation than at the first. At the time of the second examination, replicate observations were also made after intervals of 2 or 10 days on animals that

received total fractionated doses of 40 to $80 \mathrm{rad}$. A significant number of eyes (21) was examined only at the $40 \mathrm{rad}$ dose, and the range in average score was $\sim 0.4$ to 0.8 scoring units. Additional effort is being devoted to this matter of scoring differences between individuals, and the consistency of each individual. Could it be that cataracts that arise in mice that receive fractionated ${ }^{12} \mathrm{C}$ doses are somehow more difficult to score than the other groups? We interpret 
Table 2. Corneal and Lens Response at 334-350 Days Following Single or Fractionated Doses of $225 \mathrm{MeV}$ Carbon Ions (4-cm Spread Bragg Peak) or 60Co Gamma Radiation; Skynook Mice

\begin{tabular}{|c|c|c|c|c|c|c|c|}
\hline \multirow[b]{2}{*}{ Radiation } & \multirow[b]{2}{*}{ Dose (rad) } & \multicolumn{3}{|c|}{$\begin{array}{l}\text { Single Dose } \\
\text { Average Dose S.E. }\end{array}$} & \multicolumn{3}{|c|}{$\begin{array}{l}\text { Fractionated Doses } \\
\text { Average Dose } \pm \text { S.E. }\end{array}$} \\
\hline & & No. Eyes & Cornea & $\begin{array}{l}\text { Postirradiated } \\
\text { Lens }\end{array}$ & No. Eyes & Cornea & $\begin{array}{l}\text { Postirradiated } \\
\text { Lens }\end{array}$ \\
\hline $12 \mathrm{C}$ & $\begin{array}{l}40 \\
80 \\
120\end{array}$ & $\begin{array}{l}40 \\
32 \\
30\end{array}$ & $\begin{array}{l}0 \\
0 \\
0\end{array}$ & $\begin{array}{l}1.2 \pm 0.07 \\
2.5 \pm 0.09 \\
2.7 \pm 0.08\end{array}$ & $\begin{array}{l}32 \\
32 \\
32\end{array}$ & $\begin{array}{l}0.08 \pm 0.06 \\
0.04 \pm 0.09 \\
0.06 \pm 0.07\end{array}$ & $\begin{array}{lll}5 & 2.3 \pm 0.13^{\star} \\
9 & 3.5 \pm 0.10^{\star} \\
7.8 & \pm 0.13^{\star}\end{array}$ \\
\hline $60 c 0$ & 417 & 28 & $0.1 \pm 0.08$ & $3.2 \pm 0.08$ & 42 & $0.07 \pm 0.07$ & $0.70 \pm 0.06^{\dagger}$ \\
\hline Controls & $\begin{array}{l}0 \\
0 \\
0\end{array}$ & $\begin{array}{l}26 \\
16 \\
18\end{array}$ & $\begin{array}{l}0.4 \pm 0.12 \\
0.6 \pm 0.13 \\
0.6 \pm 0.15\end{array}$ & $\begin{array}{l}0.6 \pm 0.10 \\
0.4 \pm 0.06 \\
0.5 \pm 0.08\end{array}$ & & & \\
\hline
\end{tabular}

* Average cataract score at 334-350 days after the first of 24 week iy fractions was increased significantly in comparison with the same total single dose $(P=<0.01)$.

+ Reduced significantly in comparison with the single dose $(P=<0.01)$.

Table 3. Lens Responses at 420 to 470 Days Following Single or Fractionated Doses of $225 \mathrm{MeV}$ Carbon Ions (4- cm Spread Bragg Peak) or ${ }^{60}$ Co Gamma Radiation;* Skynook Mice

\begin{tabular}{|c|c|c|c|c|c|c|}
\hline Radiation & Dose (rad) & Eyes & $\bar{x}$ & SE $\bar{x}$ & Days & at Risk \\
\hline $\begin{array}{r}12 \mathrm{C} \text { (single dose) } \\
\therefore\end{array}$ & $\begin{array}{r}40 \\
80 \\
120\end{array}$ & $\begin{array}{l}40 \\
32 \\
14 \\
16\end{array}$ & $\begin{array}{r}1.45 \\
2.38 \\
3.14 \\
3.06\end{array}$ & $\begin{array}{l}0.09 \\
0.07 \\
0.10 \\
0.06\end{array}$ & & $\begin{array}{l}418 \\
418 \\
418 \\
419\end{array}$ \\
\hline 12C $(24$ fractions $)$ & $\begin{array}{r}40 \\
80 \\
120 \\
10\end{array}$ & $\begin{array}{r}24 \\
8 \\
21 \\
22 \\
8 \\
32 \\
22\end{array}$ & $\begin{array}{r}1.23 \\
1.75 \\
1: 81 \\
2.09 \\
2.75 \\
3.34 \\
0.36\end{array}$ & $\begin{array}{l}0.15 \\
0.16 \\
0.09 \\
0.13 \\
0.16 \\
0.09 \\
0.06\end{array}$ & 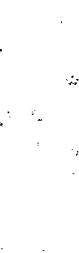 & $\begin{array}{l}460 \\
462 \\
472 \\
462 \\
472 \\
472 \\
460-472\end{array}$ \\
\hline $60 \mathrm{co}$ (single) & 417 & 22 & 3.27 & 0.10 & $\because$ & $\because$ \\
\hline $60 \mathrm{Co}$ (fractionated) & $\because 417$ & 40 & 1.28 & 0.07 & & \\
\hline
\end{tabular}

* Animals from the same dose group were reexamined on different days to estimate observer reliability. 
these data (cautiously) to indicate that in contrast, gamma dose fractionation (where the sparing effect is large), fractionation of the ${ }_{12} \mathrm{C}$ dose produces little or no sparing, and the cataractogenic effect could be increased. By using the highest values recorded for the $12 \mathrm{C}$ fractionated mice; the average scores were slightly higher at the three dose levels, in comparison with the single dose. This important question remains open.

As is true with all other forms of radiation, the major lens changes following irradiation with HZE particles were first observed in the posterior lens. The various forms of the opacifications are diagrammed in Figure 1. At about 18 months postirradiation, several of the animals began to develop an anterior opacification that was fairly discrete and located in the central pupillary area. This opacification will be examined at the light and electron microscopic level in the near future.

The mouse has been criticized as an experimental model for cataract studies because of its high rate of spontaneous opacification. We have not found this problem perhaps because of a difference in the strain selected. We see little opacification in our unirradiated controls at 18 months postirradiation; with longer times, the control lenses show aging changes (Table 1 ). In the controls, the loss of transparency has begun to take up a significant diffuse area of the posterior cortex, but this opacity is certainly not as dense as those that we have seen following irradiation. These changes in the controls seem almost like a change in texture and are much more subtle. The changes in the. irradiated animals are quite discrete and dense.

At this time, we have undertaken limited electron microscopic evaluations of lens opacities induced by HZE particles. These have included lenses only from animals irradiated with argon ions in 1978. Significant changes were observed in these animals (Fig. 4) in comparison to controls (Fig. 5). As is typical of other forms of radiation, there is permanent disruption of the lens bow and the cortex is otherwise abnormal, as evidence by swollen cells and by what appear to be degenerated nuclei and lysosome-like structures. Some of these changes may be observed in the electron micrograph in Figure 4. Deeper layers of the lens were normal in morphology, which is consistent with the suggestion that HZE cataracts, like other radiation cataracts, occur as a consequence of early effects on the epithelial cell layer. We also undertook a preliminary examination of the cornea of these animals.

\section{STATUS AND FUTURE PLANS}

We are continuing with evaluations of the development of opacities in the group II and IV animals in order to determine whether the cataracts progress or plateau at some time. We have also added a replicate experiment for the carbon, neon, and argon irradiated animals. We were prepared (by prior slit lamp examinations

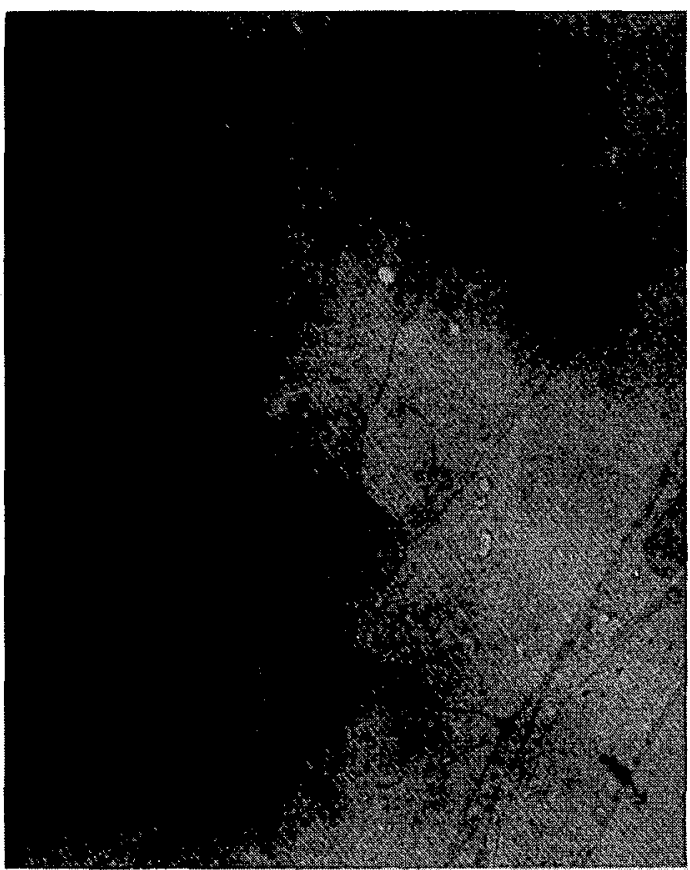

Figure 4. Electron micrograph of region of lens just anterior to bow in an animal that had been irradiated two years earlier with 25 rad of 570 MeV 40 Ar $(4,400 X)$. (XBB 809-10475)

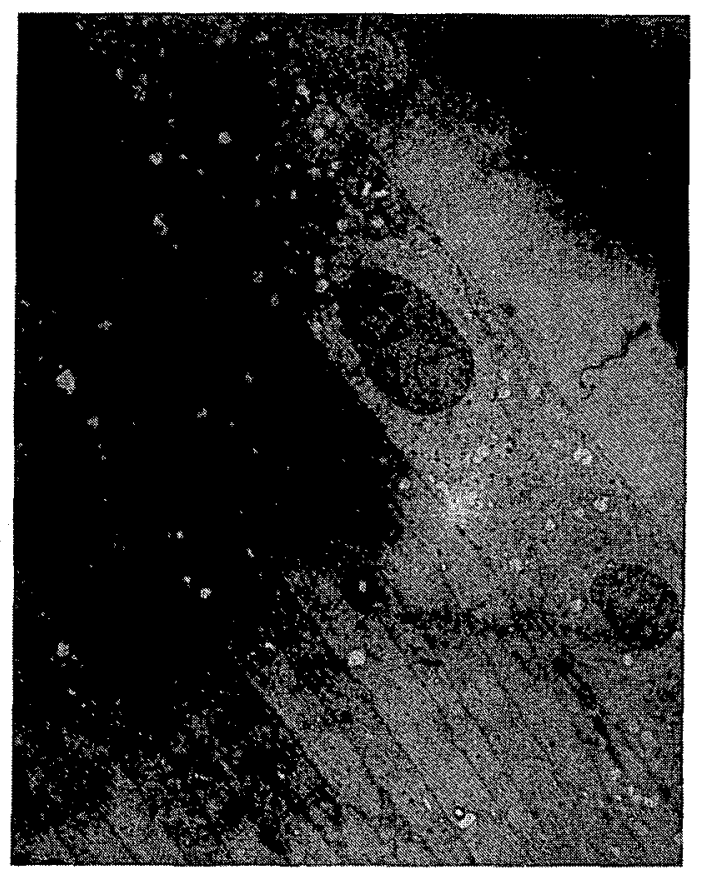

Figure 5. Electronmicrograph of the lens from a control (unirradiated mouse). Note the regularity of the cortical fibers. $(4,400 x)$. (XBB 809-10476) 
of the animals) to irradiate yet another group with iron particles, but an adequate beam was not available.

We also plan to undertake an electron micrograph study of the changes that are induced shortly after X-irradiated and HZE-treated animals. We hope that these studies will determine whether the nature of the radiation damage is similar to the different types of radiation. We also expect that the techniques will eventually provide an early means by which to predict whether a given radiation treatment will result in cataract formation. This suggestion is supported by J. Worgul's studies (personal communication) that demonstrate a correlation between meridional row disorganization and future lens opacification.

NOTE: Quantitative estimates of dose-averaged $\mathrm{LET}_{\infty}$ values presented here should be considered as approximations only, because different calculational models yield markedly different results. In addition, for spread Bragg peaks the distributions of LET $_{\infty}$ values is broad with the fraction of LET particles rising sharply at the distal peak. Ongoing beam fragmentation studies will yield new data that will contribute to improved estimation of dose-averaged $L E T_{\infty}$. Microdosimetric studies provide a separate measurement of beam quality.

\section{ACKNOWLEDGEMENT}

The assistance of Dan Judd, Bennet MCAllister, Steve Detone, John Prioleau, Lynn Mahlmann, Andy Shin, Colleen Caudill, and Panna Lee is gratefully acknowledged. The assistance and cooperation of Jerry Howard, Tom Criswe 11, and the Bevalac operations staff is also gratefully acknowledged. This research was supported by the Department of Energy Contract 31-109-38-5197.

\section{FOOTNOTES AND REFERENCES}

* Schoor of Optometry, University of California, Berkeley, CA.

Ainsworth, E. J., R. J. M. Fry, D. Grahn, F. S. Williamson, P. C. Brennan, S. P. Stearner, A. V. Carrano, and J. H. Rust. 1974. Late effects of neutron or gamma irradiation in mice. Biological Effects of Neutron Irradiation, $p$. 359-379. International Atomic Energy Agency, Vienna.

Ainswortn, E. J., R. J. M. Fry, P. C. Brennan, S. P. Stearner, J. H. Rust, and F. S. Williamson. 1976. Life shortening, neoplasia, and systemic injuries in mice after single or fractionated doses of neutron or gamma radiation. Biological and Environmental Effects of Low-Level Radiation, vol. 1, pp. 77-92. International Atomic Energy, Vienna.
Ainsworth, E. J., R. J. M. Fry, F. S. Williamson, P. C. Brennan, S. P. Stearner, V. V. Yang, D. A. Crouse, J. H. Rust, and T. B. Borak. 1977. Dose-effect relationships for life shortening tumorigenes is and systemic injuries in mice irradiated with fission neutron or 60 Co gamina radiation. Proceedings, Fourth International Congress, International Radiation Protection Association, vol. 4, pp. 1143-1151. Montreuit Press, France.

Bateman, J. L. and V. P. Bond. 1967. Lens opacification in mice exposed to fast neutrons. Radiat. Res. (Supp 1.) 7, 239-249.

Bateman, J. L. and M. L. Snead. 1969. Current research in neutron $R B E$ in mouse lens opacity. Symposium on Neutrons in Radiobiology, Conf. 691106, pp. 192-206. USAEC, 0ak Ridge, TN.

Bond, V. P. 1960. Lens opacification in the mouse: Implications for RBE and QF. Biophysical Aspect of Radiation Quality, pp. 149-160. International Atomic Energy, Vienna.

Boomer, G., B. Singer, and K. Woodburn. 1979. The Effect of Argon Ion Radiation on the Crystalline Lens of Mice. O.D. Thesis, University of California, Berkeley, 1979.

Giacolone, V. I., M. Minnig, and D. Palm. 1979. The Effect of Low Doses of Accelerate Argon Nuclei on the Crystalline Lens of Mice. 0.0 . Thes is, University of California, Berkeley.

Ishimaru, T., M. Otake, and M. Ishimaru. 1979. Dose-response relationship of neutrons and gamma rays to leukemia incidence among atomic bomb survivors in Hiroshima and Nagasaki by type of leukemia, 1950-1971. Radiat. Res. 77, $377-394$.

Lett, J. T., A. B. Cox, P. C. Keng, A. C. Lee, C. M. Su, and D. S. Bergtold. 1980. Late degeneration in rabbit tissues after irradiation by neavy ions. Life Sci. Space Res. 18, in press.

Malachowski, M. J., D. W. Philpott, and R. Corbett. 1980. Retinal tissue response to accelerated iron nuclei. Radiat. Res. 83, 472 (ADstract).

Mays, C. W., G. N. Taylor, S. S. J. Webster, and T.F. Dougherty. 1979. Speculated risk to bone and liver from $239 \mathrm{pu}$. Health Phys. 19, $601-610$.

Merriam, G. R. and E. F. Focht. 1957. A clinical study of radiation cataracts and the relationship to dose: Am. J. Roetgenol. Radium Ther. Nucl. Med. 77, 759-785.

Merriam, G. R., B. J. Biavati, J. L. Bateman, H. H. Rossi, V. P. Bond, L. Goodman, and E. F. Focht. 1965. The dependence of RBE on the energy of fast neutrons. IV. Induction of lens opacities in mice. Radiat. Res. 25 , $123-138$. 
Nelson, A. C., T. L. Hayes, and C. A. Tobias. 1980. Scanning electron microscope study of membrane lesions caused by individual heavy ions. Radiat. Res. 83, 472. (ADstract).

Rowland, R. E., A. F. Stenney, and H. F. Lucas, Jr. 1978. Dose-response relationships for fenale radium dial workers. Radiat. Res. 76 , 368-383.

Storer, J. B., L. J. Serrano, E. B. Darden, Jr., M. C. Jernigan, and R. L. Ullrich. 1979. Life shortening RFM BALB/C mice as a function of radiation quality dose, and dose rate. Radiat. Res. 78, 122-161.
Tobias, C. A., E. A. Blakely, F.Q.H. Ngo, and T.C.H. Yang. 1980. The repair-misrepair model of cell survival. Radiation Biology in Cancer Research (R. E. Meyn and H. R. Withers, eds.) pp. 195-230. Raven Press, New York.

Ulirich, R. L., M. C. Jernigan, and J. B. Storer. 1977. Neutron carcinogenes is: Dose and dose rate effects in BABL/C mice. Radiat. Res. 72, $487-498$.

Yang, V. V., S. P. Stearner, and E. J. Ainsworth. 1978. Late ultrastructural changes in the mouse coronary arteries and aorta after fission neutron or 60 co gamma irradiation. Radiat. Res. 74, 436-456. 
PART V. SHORT- AND

OF LOW FLUENCE HZE PARTICLES 


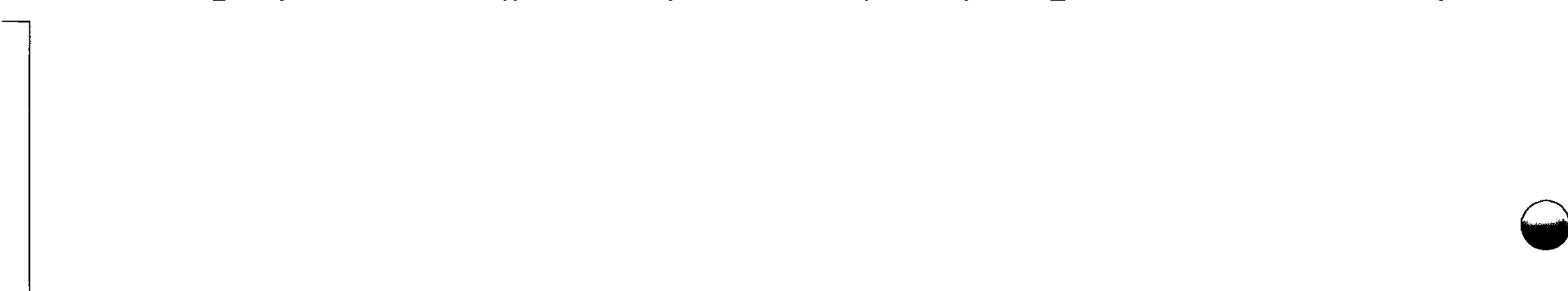
$\theta$ 


\title{
ABUNDANCES, ENERGY AND LET SPECTRA OF HZE PARTICLES IN SPACE
}

\author{
S. B. Curtis and E. V. Benton*
}

In 1948, Freier et al. (1948) reported the discovery of tracks of high energy heavy ions with charge $Z$ greater than two in emulsion stacks carried by balloon to high altitude. It was immediately recognized that these particles were a part of the radiation permeating space near earth and having a source outside our solar system. Since then data have steadily been accumulating from balloons, rockets and satellites on the abundances and energy spectra of this heavy-ion component of the galactic cosmic rays. Heavy ions have also been detected in small numbers in solar particle events and trapped in the earth's magnetic field. All these energetic heavy charged particles with charge greater than two have become known generically as HZE particles (high $Z$ and energy) (Grahn, 1973).

Very early it was recognized that this heavy-ion radiation might pose a hazard to high altitude and space travel (Tobias, 1952). It was, in fact, predicted that visible flashes of light might be caused by the passage of heavy galactic cosmic rays through the eye (Tobias, 1952), a prediction later borne out by the astronauts of several Apollo missions (Chapman et a)., 1972).

The present chapter will review our knowledge of the composition and energy spectra of HZE particles in space and present experimental results of LET spectra measured in several manned satellites.

\section{COMPOSITION OF HZE PARTICLE RADIATION IN FREE SPACE}

Practically all the major elements in the periodic table are represented in the HZE particles measured in space. There is a noticeable maximum in abundance for the iron-cobalt-nickel group $(26<Z \leq 28)$ and then a sharp decline at higher $Z$. The relative composition of the various elements in the HZE flux in free space up to and including the iron group is given in Figure 1, with a 11 abundances normalized to that of carbon (Wefel, 1979). The dashed line shows the universal abundances in the solar system as determined by Cameron (1973). Above $Z=30$, abundances drop several orders of magnitude and for $Z$ greater than 45 , values are five orders of magnitude below that for iron. Another interesting feature of Figure 1 is the increased abundance of even $Z$ nuclei over the ir immediate neighbors with odd $Z$.

\author{
Comparison of the abundances of the elements \\ in the galactic cosmic rays with the solar \\ system abundances
}

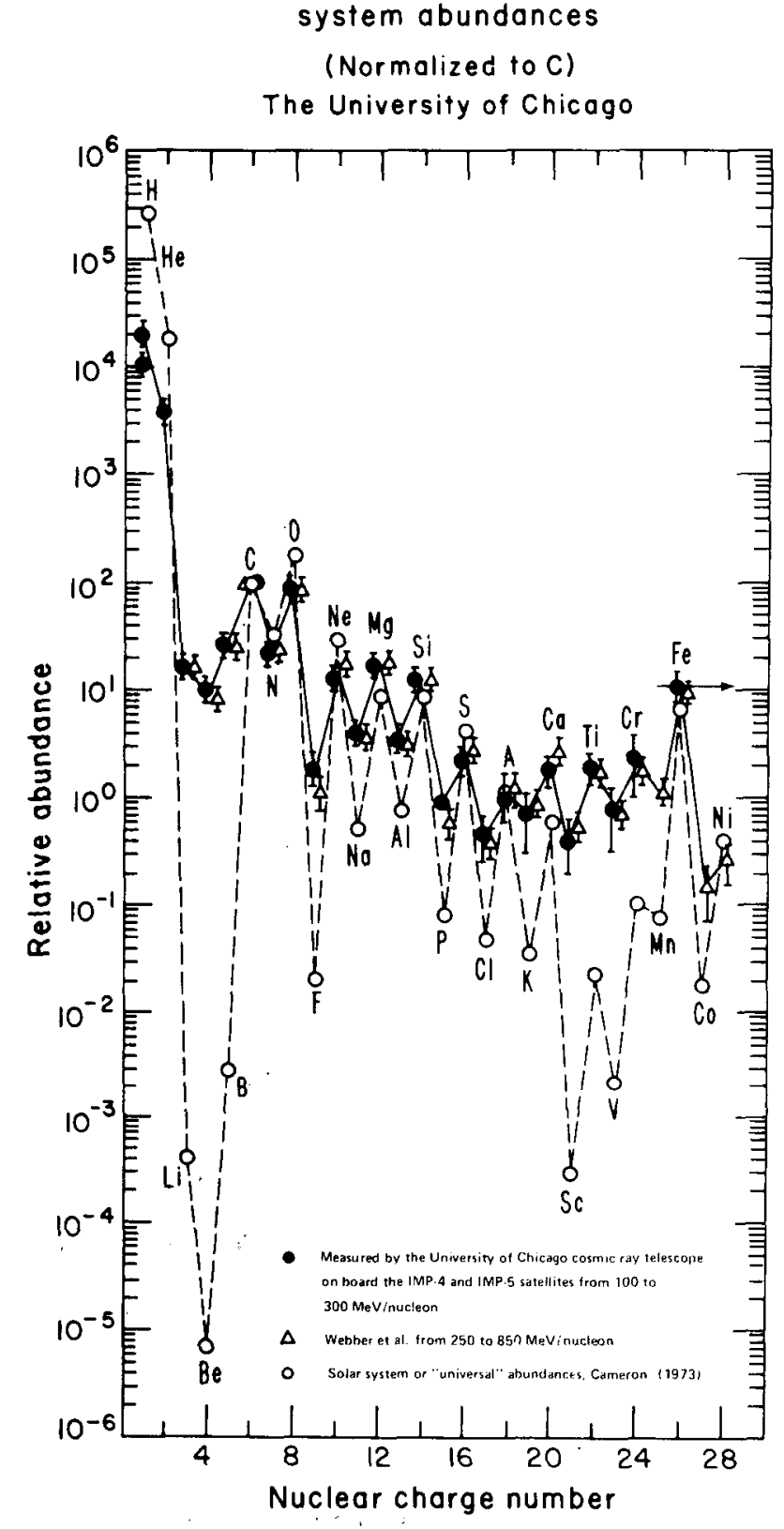

Figure 1. The measured abundances of the elements, relative to carbon, in the galactic cosmic radiation compared to the solar system abundance compilation of Cameron (1973) (dashed line). (From Wefel, 1979). (XBL 803-508) 
ENERGY AND LET SPECTRA IN FREE SPACE

Characteristic energy spectra of any of the HZE particles behind zero shielding at solar minimum (when intensities are highest) comprises a very low energy component (probably of solar origin) in the energy range 0.5 to $2 \mathrm{MeV} /$ nucleon dropping off very rapidly with increasing energy, followed by a local minimum in the spectrum between 2 to $30 \mathrm{MeV} /$ nucleon caused by solar modulation effects, and finaliy a decrease at very high energies falling approximately as E-2.6 where $E$ is the particle $k$ inetic energy. Representative spectra are shown in Figure 2 (from Biswas and Durgaprasad, 1980). An "anomalous component" of the cosmic rays has been observed in the energy range 2-30 MeV/nucleon (Biswas and Durgaprasad, 1980). Energies less than $30 \mathrm{MeV} /$ nucleon are of no concern in the present context, however, because even the lightest ions below this energy travel less than a centimeter in water and will be absorbed in the spacecraft shielding.

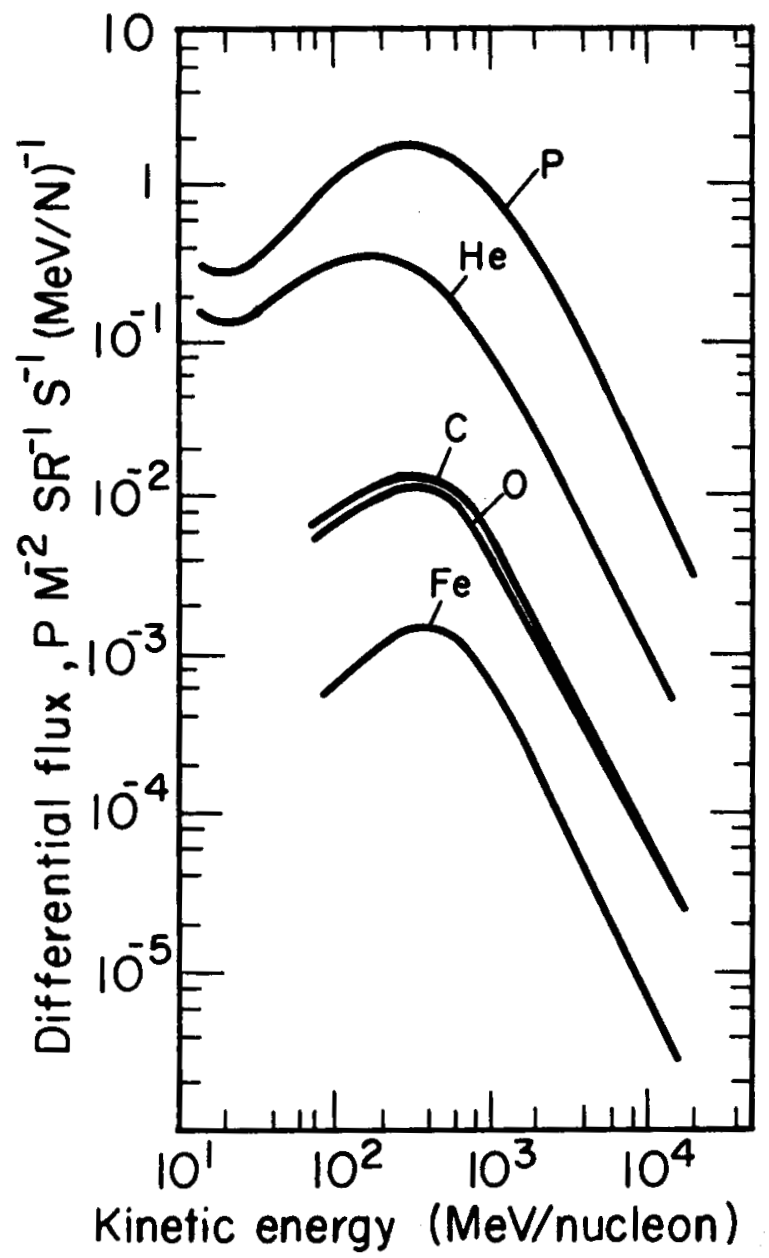

Figure 2. Differential energy spectra of galactic cosmic ray proton; helium, carbon, oxygen, and iron group nuclei during solar minimum. (From Biswas and Durgaprasad, 1980). (XBL 807-3524)
The LET distribution in free space behind just enough shielding to absorb the very low energy component ( $<30 \mathrm{MeV} /$ nucleon) shows a large contribution from the iron group in the high LET range. A calculation of the integral number LET spectrum at solar minimum is given in Figure 3 (Curtis, 1972). We note that the $Z=26$ group, here defined as nuclei with $Z$ between 16 and 28 , contributes a large fraction of the particles with LET greater than $100 \mathrm{keV} / \mu \mathrm{m}$. This fraction will change at depth behind shielding and within the body because these jons have larger cross sections for nuclear fragmentation than the lighter ions; thus, their number will decrease more rapidly as the amount of shielding or material traversed increases.

\section{LET DISTRIBUTIONS IN SPACECRAFT}

To date, only a few measurements of integral LET spectra have been made on actual flight missions. Table 1 lists the mission data for three U.S. space programs, Apol70, Skylab and ASTP, and two Soviet missions, Cosmos 782 and Cosmos 936. Figure 4 shows integral number LET spectra measured in plastic nuclear track detectors [cellulose nitrate (CN) and Lexan polycarbonate] on the three U.S. missions (Benton et al., 1977a, b). The abscissa is LET 350 ,

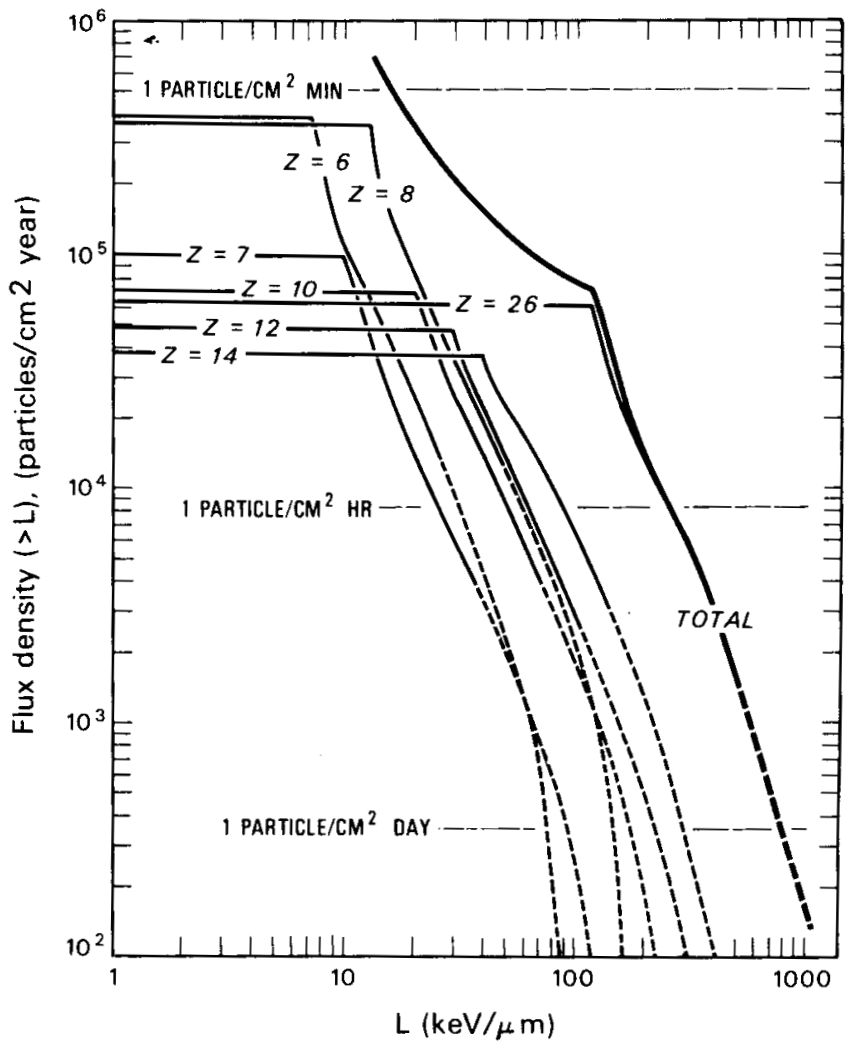

Figure 3. Integral number LET spectrum for the major components of HZE particles at solar minimum under zero shielding. (From Curt is, 1973). (XBL 7811-12342) 
Table 1. Mission Data

\begin{tabular}{lcrrrr}
\hline & Apollo & Skylab & ASTP & Cosmos 782 & Cosmos 936 \\
\hline Altitude $(\mathrm{km})$ & lunar & 435 & 220 & $424 / 220$ & $419 / 224$ \\
Orbit inclination (deg) & $-\ldots$ & 50 & 51.6 & 62.8 & 62.8 \\
\hline
\end{tabular}

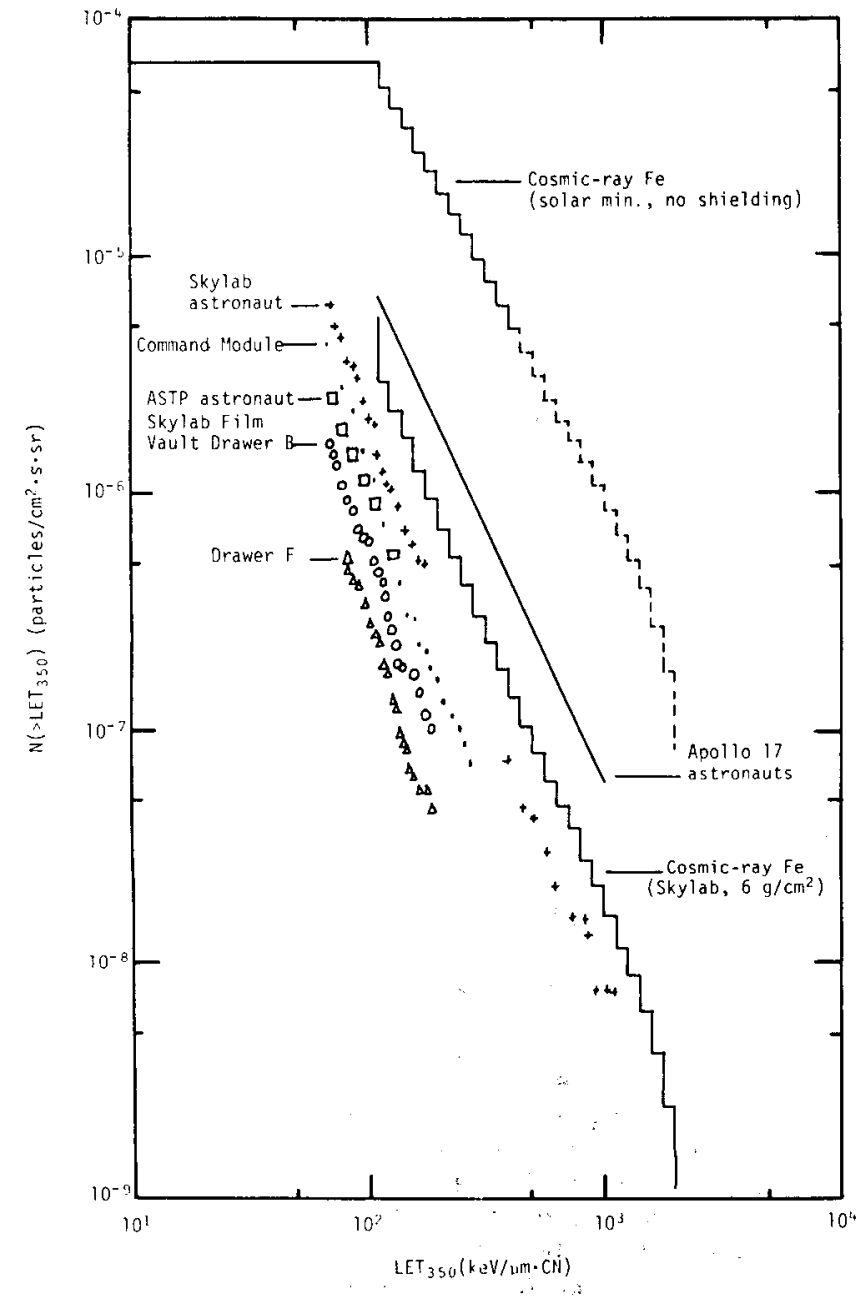

Figure 4. Integral LET spectrum of HZE particles inside spacecraft measured on lunar (Apollo) and near-Earth (Skylab and ASTP) missions, and calculated for cosmic-ray iron (Fe) nuclei in iree space at solar minimum. LET in water (or tissue) is approximately 1.2 times LET350 in CN. (XBL 766-1995) because the threshold for track detection corresponds more closely to LET350 than to $L^{2} T_{\infty}$. The LET $T_{\infty}$ in water (or tissue) is approximately 1.2 times the LET350 in cellulose nitrate. The detectors were either stowed aboard spacecraft or worn as dosimeters in the crew flight suits. In general, the dosimeter locations within the spacecraft were not fixed and estimates of effective shielding are difficult to achieve. The uppermost curve in Figure 4 is calculated for cosmic-ray iron (Fe) nuclei at solar minimum and behind no shielding, i.e., free space. Spectra for Apollo astronauts (lunar missions 8-17) all lie near the line in Figure 4 which falls just below the iron curve. Individual Skylab and ASTP spectra are indicated and fall below the Apollo spectra. A higher particle flux is expected for lunar, i.e., Apollo, missions compared with the near-earth orbiting mission, i.e., Skylab and ASTP. Earth shielding and the geomagnetic field cause a substantial decrease in particle flux compared with that on lunar missions. Integral LET spectra measured in Skylab indicate that the high-LET particle flux decreases with an increase in shielding thickness. The slope of the LET curves for Skylab astronauts $\left(\sim 5-10 \mathrm{~g} / \mathrm{cm}^{2}\right)$, command module $\left(\sim 1-20 \mathrm{~g} / \mathrm{cm}^{2}\right)$, and film vault drawers $B\left(\sim 16-30 \mathrm{~g} / \mathrm{cm}^{2}\right)$ and $F$ $\left(\sim 30-50 \mathrm{~g} / \mathrm{cm}^{2}\right)$ steepens somewhat with increased shielding thickness, being steepest for $f i l m$ vault drawer $F$.

From Figure 4 , the average fluence rate of HZE particles with LET greater than $100 \mathrm{keV} / \mathrm{\mu m}$ measured at the skin of the astronauts for the entire Apollo 17 lunar landing mission was roughly $0.5 \mathrm{~cm}^{-2} \mathrm{~h}^{-1}$. As mentioned above, the variable shielding presented to the detectors over the entire mission prevents an accurate estimation to be made of the average equivalent spherical shielding. At different times during the mission, the earth, moon, and the Apollo spacecraft itself presented varying amounts of shielding to the more or less randomly moving astronauts. With this in mind, a calculated value of 2.6 ions $\mathrm{cm}^{-2} \mathrm{~h}-1$ with LET $_{\infty}>100 \mathrm{keV} / \mu \mathrm{m}$ behind $10 \mathrm{~g} / \mathrm{cm}^{2}$ spherical aluminum shielding at solar minimum (Curtis, 1973) does not seem to be 
at variance with the experimental data. In this calculation, roughly $65 \%$ of the particles were in the charge range 20 to 26 .

In Figure 5 , curves 1 and 2 show a comparison of LET spectra measured inside and outside the Cosmos 936 spacecraft by means of a silicon solid-state detector telescope (Benton et al., 1978; Markelov et a 1., private communication, 1978). Curve 3 is that calculated by $w$. Heinrich of Siegen University, Germany, while curve 4 is the measurement on Cosmos 782 by Benton et al. (1978) using CN track detectors.

The main features of the data gathered to date indicate that the slopes of the integral LET spectra are similar under widely different orbital conditions and shielding. There is reasonable agreement between electronically measured spectra of curves 1 and 2 in Figure 5 and that measured by plastic track detectors, curve 4.

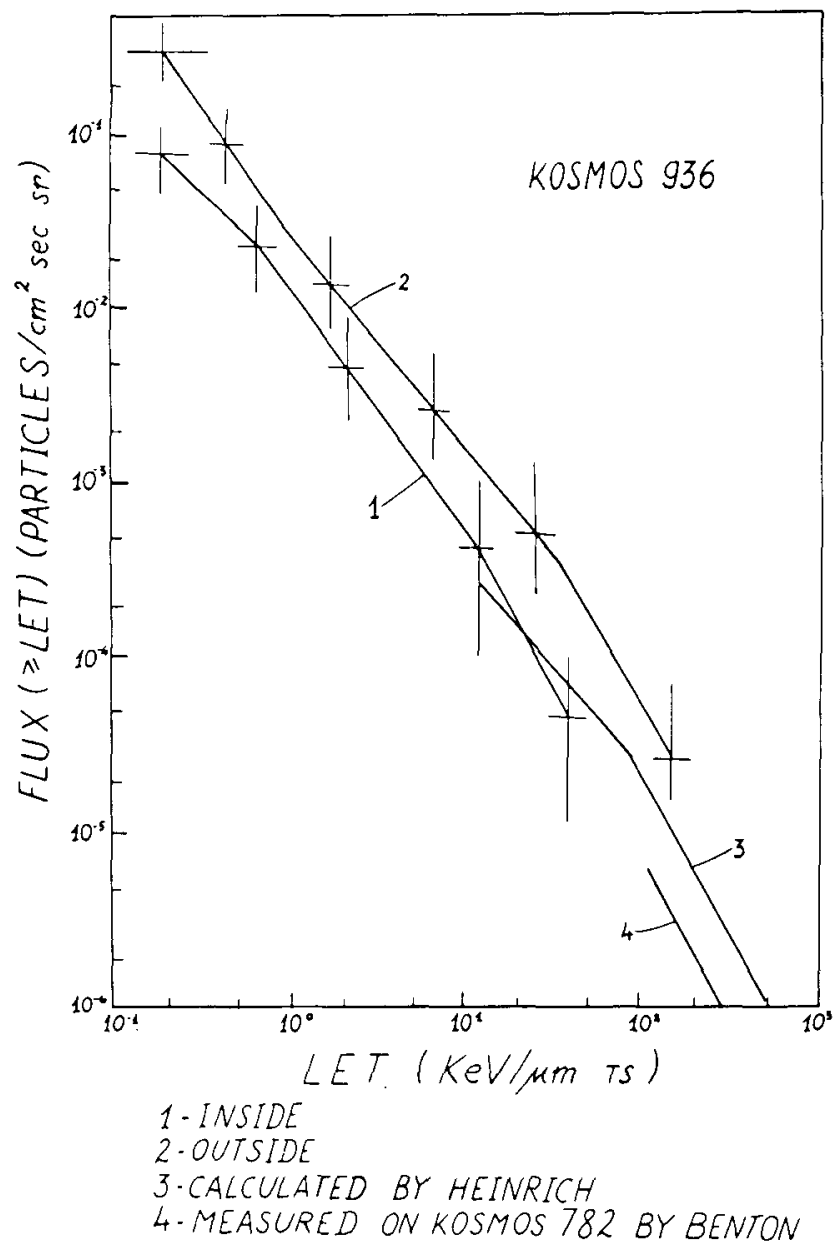

Figure 5. Comparison of LET spectra, measured inside and outside spacecraft, for cosmos 936 and 782. (XBL 807-10715)
HZE PARTICLES IN SOLAR PARTICLE EVENTS AND THE OUTER TRAPPED RADIATION BELT

Heavy ions have been detected in many of the solar particle events which sometimes accompany solar flares. Early work indicated that fluences of the carbon-nitrogen-oxygen (CNO) component, the most numerous of the heavy ions observed, were approximately one sixtieth of the helium component and that this ratio did not vary much from event to event (Biswas et al., 1966). Data from more recent events, in particular from the giant solar particle event of August 1972, indicate that there is an enhancement of heavy ion fluxes at lower energy, i.e., less than $50 \mathrm{MeV} /$ nucleon, but for energies of interest here, above $50 \mathrm{MeV} /$ nucleon, the ratio of helium to CNO nuclei mentioned above was, until recently, considered to be a reasonable average (Bertsch et a1., 1974). There is some recent evidence, nowever, from the rather large 24 September 1977 solar event that preferential acceleration of heavy ions can occur to higher energies, i.e., in the energy region up to at least $300 \mathrm{MeV} / \mathrm{nucleon}$ (Dietrich and Simpson, 1978). In fact in this event, the ratio of iron to oxygen nuclei was close to unity in the energy range 30 to $300 \mathrm{MeV} /$ nucleon; this is roughly twenty times the abundance of iron relative to oxygen nuclei in the solar chromosphere or corona (Cameron, 1973). The shape of the energy spectra of all nuclei were well approximated by a power law falling as E-2.3 ( $E$ is the energy/nucleon) up to energies where the subtraction of the galactic cosmic-ray background became significant.

Thus, it appears that large iron-rich solar particle events can occur, producing significant enhancements of heavy ions over solar abundance values in the energy region above $100 \mathrm{MeV} /$ nucleon. It is not possible, therefore, to rule out the possibility that for giant solar particle events of the type that occurred in August 1972, large enhancements of iron and other heavy ion fluences might produce a measurable contribution to the radiation from solar particles behind moderate spacecraft shielding.

Low energy CNO ions (in the range 13-33 MeV/ nucleon) have also been detected in the outer trapped radiation belt at $L=4$ (Mogro-Compero, 1972). The intensity is roughly one hundred times that of the CNO interplanetary galactic intensities in the same energy region. This region, however, is below the energies of interest to us here. Heavy particles at high energy are thought to be untrappable owing to adiabatic breakdown, i.e., the inhomogeneity of the geomagnetic field at the mirror points keeps particles with large cyclotron radii from becoming trapped. Although the existance of CNO fluxes at 13-33 MeV/nucleon implies higher energy particles could be trapped than thought possible from the proton data, it is not expected that many can become trapped above $40 \mathrm{MeV} /$ nucleon. Data are not available, however, to substantiate this claim. 
SIGNIFICANCE OF HZE RADIATION TO MANNED SPACE MISSIONS

The risk of exposure to HZE particles in long term space missions has yet to be assessed. The light flashes seen by astronauts on Apollo 14 had a mean time between them of around 40 seconds (Chapman et al., 1972). These flashes are not considered hazardous. However, it is not known what damage might accumulate from the lower frequencies of higher LET HZE particles from, for instance, the roughly $0.6 \mathrm{~cm}^{-2} \mathrm{n}^{-1}$ rate of particles measured in Apollo with LET greater than $100 \mathrm{keV} / \mathrm{um}$.

An effort is presently underway by DOE and NASA to determine the feasibility of building a fleet of satellites (the Satellite Power System, SPS) to be deployed in geosynchronous orbit to gather solar energy and beam it to earth via microwaves. This project would entail as many as 50,000 worker-years in orbit with up to $98 \%$ of that time spent in geosynchronous orbit. Because of the very low geomagnetic cutoff at the position of a geosynchronous orbit, essentially all the galactic heavy particles will be impinging on the work and living habitats of the space workers. A workshop was held at LBL in September 1978, which reviewed our current knowledge of the radiation environment to be experienced by space workers on SPS (Schimmerling and Curtis, 1979). The various components of the radiation environment were discussed and it was pointed out that one area needing particular attention was the accurate determination of HZE fluence rates in various critical body organs as a function of shielding thickness in geosynchronous orbit and the effects of these fluence rates on the functional integrity of the organs and the subsequent health of the exposed worker population. Clearly, these important questions must be addressed before an adequate risk assessment of such long term space missions can be made.

\section{FOOTNOTES AND REFERENCES}

* Department of Physics, University of San Francisco, San Francisco, CA.

Benton, E. V., D. D. Peterson and R. P. Henke. 1977a. Summary of measurements of high-LET particle radiation in U.S. manned space

missions. Life Sci. Space Res. 15, 119-127.

Benton, E. V., D. D. Peterson, J. V. Bailey and T. Parne11. 1977b. High-LET particle exposure of Skylab astronauts. Health Phys. 32, 15-19.

Benton, E. V., D. D. Peterson, A. M. Marenny and V. I. Popov. 1978. HZE particle radiation studies aboard Kosmos 782. Health Phys. 35, 643-648.

Bertsch, D. L., S. Biswas, and D. V. Reames. 1974. Solar cosmic ray composition above $10 \mathrm{MeV} /$ nucleon and its energy dependence in the 4 August 1972 event. Solar Phys. 39, $479-491$.
Biswas, S. and N. Durgaprasad. 1980. Skylab measurements of low energy cosmic rays. Space Sci. Rev. 25, 285-327.

Biswas, S., C. E. Fichtel, and D. E. Guss. 1966. Solar cosmic rays multiply charged nuclei and the July 18, 1961, solar event. J. Geophys. Res. 71, 4071-4077.

Cameron, A.G.W. 1973. Abundances of the elements in the solar system. Space Sci. Rev. 15, 121-146.

Chapman, P. K., L. S. Pinsky, R. E. Benson, and T. F. Budginer. 1972. Observations of cosmic-ray induced phosphenes on Apollo 14. Proceedings, National Symposium on Natural and Manmade Radiation in Space, (Warman, E. A., ed.) pp. 1002-1006. NASA TMX-2440, Washington, D.C.

Curtis, S. B. 1972. The heavy particle hazardwhat physical data are needed? Proceedings National symposium on Natural and Manmade Radiation in Space (Warman, E. A., ed.) pp. 1007-1015. NASA TMX-2440, Washington, D.C.

Curtis, S. B. 1973. Frequency of heavy ions in space and their biologically important characteristics. Life Sci. Space Res. 11, 209-214.

Dietrich, W. F. and J. A. Simpson. 1978. Preferential enhancements of the solar flareaccelerated nuclei carbon to zinc from 20-300 MeV nucleon-1. Astrophys. Lett. 225, L41-L45.

Freier, P., E. J. Lofgren, E. P. Ney, F. Oppenheimer, M. L. Bradt, and B. Peters. 1948. Evidence for heavy nuclei in the primary cosmic radiation. Phys. Rev. 74, 213-217.

Grahn, D., ed. 1973. HZE-Particle Effects in Manned Spaceflight. National Academy of Sciences, Washington, D. C.

Markelov, V. V., Y. A. Akatov, S. B. Kozlova and V. H. Bruksin. 1978. Dosimetry measurements using LET telescope. Special report to NASA on joint U.S.-Soviet measurements aboard Kosmos 936, private communication.

Mogro-Campero, A. 1972. Geomagnetically trapped carbon, nitrogen and oxygen nuclei. J. Geophys. Res. 77, 2799-2818.

Schimmerling, W. and S. B. Curtis, eds. 1979. Proceedings of the Workshop on the Radiation Environment of the Satellite Power Systems (SPS), DOE-NASA Conf-7809164, U. S. Department of Energy, Office of Energy Research, Satellite Power System Project Office, Washington, D. C.

Tobias, C. A. 1952. Radiation hazards in high altitude aviation. J. Aviat. Med. 23, 345-372.

Wefe1, J. P. 1979. Instrumentation for radiation measurements in space. Proceedings, Workshop on the Radiation Environment of the Satellite Power System (SPS) (Schimmerling, W. and Curtis, S. B., eds.) pp. 117-183. DOE/NASA Conf-7809164, U. S. Department of Energy office, Washington, D. C. 


$$
276
$$

Blark 


\title{
THERMOPHYSICAL LESIONS CAUSED BY HZE PARTICLES
}

\author{
C.A. Tobias, M. Malachowski, A. Nelson, ${ }^{*}$ and D.E. Philpott ${ }^{\dagger}$
}

Soon after the discovery that stripped atomic nuclei from galactic space could reach the top layers of the earth's atmosphere (Freier et al., 1948), the question was raised whether or not these ions would pose a health hazard for the crews of planes flying at and above the stratosphere or for manned space flight. Tobias predicted that single particles would cause a light sensation in the retina, and speculated on the possibility that single HZE particles might kill or modify a column of living cells in tissues, thus creating columnar lesions (Tobias, 1952). If HZE particles do in fact produce columnar lesions, important functional consequences might result. The brain and the nerve centers responsible for homeostasis especially need to be studied, since the regenerating capacities of brain tissues are low (Grahn, 1973).

Progress was initially slow because the only way to expose living organisms to HZE ions was to fly them at high altitudes for a few hours with helium or hydrogen-fitted balloons. Neverthe less, Chase demonstrated a few years later, that single HZE particles were able to inhibit pigment formation in hair follicles so that black mice whose hair was plucked prior to flying at high altitude exhibited streaks of newly grown white hair during the postflight period after doses of HZE particles of less than 1 rad (Chase and Post, 1956). Slater and Tobias (1963) demonstrated that seeds of Zea mays exposed to cosmic primaries at high altitudes or in space sustained damage from individual HZE ions. Plants grown from these seeds developed necrotic streaks or in other cases streaks of viable cells devoid of chlorophyl1. Since 1971, when heavy particles were first successfully accelerated in the Princeton and Berkeley synchrotrons, it has finally been feasible to plan controlled experiments to investigate the biological effects is produced by these particles.

\section{TRACKS PRODUCED BY HZE PARTICLES IN SOLIDS}

During the last several years, beginning with the work of Fleischer and his associates, it has been shown that HZE particles can produce irreversible lesions in crystalline and amorphous solids (Fleischer and Price 1963, Fleischer et al., 1975). Lesions are more readily produced: in solids with low thermal and electrical conductivity, and those solids that have low fluor-: escence efficiency. Lesions form only if the energy density in the track core exceeds a threshold level and the initial dimensions of the cylindrically shaped lesions are of the same fder of magnitude as the dimensions of the track core. In several plastics, the threshold LET is of the order of $100 \mathrm{keV} / \mathrm{\mu m}$; in mica it is about
$300 \mathrm{keV} / \mathrm{\mu m}$. More recently, special plastics with higher sensitivity have been developed. For example, NP-39, a polycarbonate, can respond to LET values below $100 \mathrm{keV} / \mu \mathrm{m}$. The initial lesions change the internal structure of plastic materials to make it more accessible to hydrolysis. By developing the plastics in concentrated $\mathrm{NaOH}$, enlarged holes or conically shaped depressions are formed wherever there were lesions.

The information supplied by solid nuclear track detectors became so reliable that throughout the manned space-flight program, track detectors (together with special photographic emulsions) became the main passive dosimetry methods employed (Benton et al., 1977; Fleischer et al., 1973).

\section{THERMOPHYSICAL BIOLOGICAL RADIATION INJURY}

When the LET is smaller than $100 \mathrm{keV} / \mu \mathrm{m}$, many of the biological effects are usually ascribed to the actions and cooperative effects between free radicals (Tobias, 1971). The chemistry of dilute solutions of free radicals is now a welldeveloped field of research. However, in this form of radiation injury the portion of transferred energy that results in permanent chemical change is rarely more than $15 \%$. The rest of the transferred energy, $85 \%$ or greater, is dissipated as heat. Beginning with Dessaur in 1918, who wrote of "point heat" effects, the possibilities for thermal effects due to ionizing radiation have often been discussed. Later some of these effects were termed "thermal spike" effects. However, for low-LET radiations in aqueous solutions and in biological systems, the effects that can be ascribed to heat are negligible; the rate of free radical diffusion is usually calculated from ambient temperatures.

When the LET and the locally transferred energy density are high, as in the track core of HZE ions, the possibility exists that macromolecular or structural damage due to vibrational and rotational excited states will contribute to the injury caused by free radicals. The physicochemical structure of the core has been discussed by Chatterjee et al. (1976), and Magee and Chatterjee (1981; Chatterjee and Magee, 1981).

The fine structures of living cells and tissues contain relatively dehydrated macromolecular protein, nucleic structures, and lipid based membranes, all surrounded by an aqueous milieu. Tobias et a1. (1979) suggested that these subcellular structures might be susceptible to a somewhat similar type of damage as what occurs due to HZE particles in solids. We termed these lesions "thermophysical" radiation injury. 
The sequence of events in the damage mechanism is not yet completely understood. However, we can describe the events that may occur in a living cell in six more or less distinct phases.

1. Initial energy deposition. The initial energy deposition from HZE ions is very fast. Much of it is completed in 10-16 seconds, and it results in ionization and coherent excitation of electronic states. At this stage, macromolecular effects cannot be discerned. The energy density of this initial energy deposition can be relatively high. If the energy was instantly and uniformly partitioned between available degrees of freedom, the temperature in the core of heavyion tracks might reach several hundred (or even thousand) degrees $k$.

2. Estaolishment of mechanical stress. During the first $10^{-14}$ seconds, an adiabat ic process occurs chiefly caused by an energy transfer between electronic and vibrational states. In this span of time, there is not much molecular thermal motion. Liquids may be regarded as solids, and the net effect at the end of this period is that considerable mechanical stress is built up in the core region of tracks.

3. Shockwave propagation. Depending on the mechanical properties of the medium and any discontinuities of stress at the edge of the core, some of the energy may escape from the core region as a shockfront, moving with the velocity of sound which in water is about $1.5 \times 10^{5} \mathrm{~cm} \mathrm{sec}-1$. In 10-11 seconds the disturbance can travel $10 \mathrm{~nm}$, a distance on the same order of magnitude as the track core radius. If the medium is near critical temperature and pressure, then cavitation might be initiated. We know that these phenomena actually occur in liquid hydrogen at about $20^{\circ} \mathrm{K}$ in the bubble chamber (Seitz, 1958). Calculations show that it is unlikely that cavitation from heavy particles occurs in liquid water or in the milieu of living cells; in crystals the escape of energy into lattice vibrations is a much more likely process than in polar liquids where the anisotropy of the medium dampens the effect. However, vibrational excitation of macromolecules can lead to molecular dissociation beginning at $10^{-13}$ seconds, and can break some of the hydrogen bonds that are responsible for tertiary helical macromolecular microstructure.

4. Rotational energy transfer. Rotational energy transfer causes radial thermal diffusion, which is considerably slower than the velocity of sound, but faster than the chemical diffusion rate of free radicals and molecules. The thermal diffusion constant of water is about $3 \times 10^{-3} \mathrm{~cm}^{2} \mathrm{sec}^{-1}$. We may regard this phase as one of strong radially expanding thermal agitation that lasts for about $10^{-8} \mathrm{sec}$. In $10^{-9} \mathrm{sec}$ a cylinder of about $30 \mathrm{~nm}$ radius is involved. The thermal agitation will make the disorganization of cellular macromolecular structures that began in phase 3 greater; and water molecules begin to diffuse into microspaces between amino acid chains. The effect might be similar to what is seen in the initial phases of thermal denaturation of biomolecules. In thermal denaturation we know that many enzymes lose their activity at temperatures only a few degrees above $37^{\circ} \mathrm{C}$ if sufficient time is allowed at that temperature. Molecules such as collagen lose their helical configuration at about $60^{\circ} \mathrm{C}$, and DNA meits at $87^{\circ} \mathrm{C}$. Heat treatment can al so cause the coagulation of proteins.

5. Diffusion controlled free radical chemistry. The diffusion of water radicals is slower than thermal diffusion; at room temperature $\mathrm{OH}$ has a diffusion constant of $2 \cdot 10^{-5} \mathrm{~cm}^{2}$ $\mathrm{sec}^{-1}$. In the agitated milieu of the track core, the free radicals can likely exert even greater effects than in the relatively "cold" milieu of low-LET radiations. In the latter case, free radicals usually interact mainly with the external groups of organized protein structures. In heavy-ion tracks, it is likely that free radicals interact with thermally disarranged amino acid chains. One of: the expected effects is establishing cross-links between disarranged structures; the net result is not dissimilar to coagulation in proteins produced by prolonged heat exposure, except that it might occur in a local region at a much accelerated pace. In a cellular domain rich with helical proteins, one may expect order-disorder transitions and gelation.

The interior of biological membranes is usually regarded as liquid, with unsaturated fatty acid chains moving about freely. The heat capacity of this type of membrane is smaller than that of water; electrical and thermal conductivity is also lower. In the interior of these membranes one expects a higher and more local temperature rise that will last for a longer time than in water. Membrane phase transitions are liable to occur, and the free radicals in the track may cross-link the fatty acid chains and cause local rigidity. Such membranes probably develop increased permeability to water and other small molecules. Membrane antioxidants may be locally inactivated by the high free radical density, and autooxidation may proceed as a chain reaction following passage of an HZE particle. Membrane proteins might also undergo cross linking and tertiary structural changes.

Similar changes may occur in the cell nuclei and chromatin structures. Experimental evidence indicates that HZE particles can produce such severe lesions in DNA that lethality is a probable consequence.

6. Stabilized lesions in HZE tracks. The processes described above may result in the appearance of gelled cross-linked tubular structures extending throughout the structural elements of cells. The diameter of these initial lesions is likely to be about two or three times the diameter of the track core, or about 40 to $60 \mathrm{~nm}$. These lesions probably develop in regions of the cell that contain stacked membranes and dense protein fibers or structures, such as the outer segments of visual cells or the endoplasmic reticulum. In regions of the cell that are regarded as dilute, the excess water may quench some of these effects. Tissues rich in proteins 
that possess dense calcified structures might also be susceptible to thermophysical lesions, tissues such as cartilage, tendon, bone, cornea, and the lenses of the eye.

\section{INITIAL EXPERIMENTAL EVIDENCE FOR THERMOPHYSICAL LESIONS IN TISSUES}

Within the last two years, experimental evidence has accumulated on the presence of thermopnysical lesions in mammalian tissues exposed to HZE ions. The most obvious reason for the relatively slow development of this field is that heavier accelerated particles such as argon and iron have only become available at the Bevalac recently and experimental time is at a premium.

Criteria for finding thermophysical lesions are as follows:

1. A single HZE particle should be capable of producing a lesion.

2. There should be an LET "threshold" for thermophysical lesions. Individual particles of low LET, such as protons and electrons might not be able to produce thermophysical lesions at all.

3. Evidence is needed that thermophysical lesions are present at early times following heavy-ion exposure; the process of initial formation is over in 10-4 seconds or less. 4. The lesions may appear in several adjacent cells along the track of a single HZE ion.

5. The lesions may produce immediate functional effects, often related to structural relaxation properties and change in permeability. The effects might be sensory (e.g., visual) or motor (e.g., contraction of a fiber).

6. Biological processes are likely to modify the initial lesions, which may either repair with time or develop into larger defects than existed initially.

Although the above criteria are relatively simple, they were not recognized fully. until recently; definite evidence for thermophysical lesions is accumulating in several systems at. the time of this writing.

\section{The Retina ...}

Philpott et al. $(1972,1973,1978)$ flew rats on two Russian spacecrafts, Cosmos 782 and 936 , in an orbit of $63^{\circ}$ inclination. Plastic nuclear track detectors on flight 782 recorded 80 particles $/ \mathrm{cm}^{2}$ with atomic numbers, between 6 . (carbon) and 28 (nickel). The flight lasted about 20 days, and the rat eyes were fixed" immediately after the flight for visual and .... electron microscopy. In these experiments the most important finding was an occasional necrotic nuclei in the outer nuclear layer of the retina. le cells containing these necrotic areas became wollen concommitantiy with clearing of the cytoplasm (see Fig. 1). In some instances the inner segments directly above these necrotic

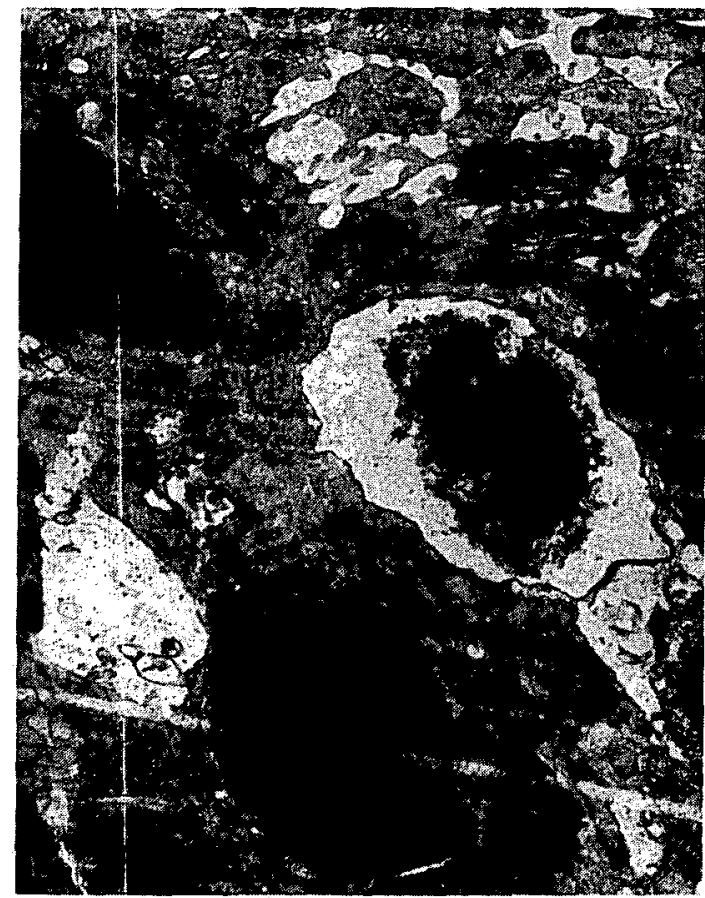

Figure 1. Outer nuclear layer of a rat retina. This rat was flown on COSMOS 782 for 19.5 days in an orbit of $62.8^{\circ}$ inclination. The preparation was made shortly after landing. One necrotic nucleus $c$ an be seen at the center of the picture (from Philpott et al., 1978). (XBB 809-10166)

cells were also swollen and contained myel in fibers and swollen mitochondria. Clear channels were observed especially at the junction of the rod pigment epithelial layer (see Fig. 2). The channels were straight, some as long as $30 \mu \mathrm{m}$ and clearly extended across several cells. Similar channels were discovered earlier in retina cells exposed to neon and argon beams (Philpott et al., 1972, 1973), but these channels were smaller and shorter than those encountered on flight 782. Macrophages were also discovered near the channels. A few nuclei from the outer nuclear layer were displaced into the inner segment area, apparently by passing. through the outer. limiting membrane. Occasionally rod membrane disruption was seen over the entire thickness of rods. Where this was observed the usually densely packed laminar structures were disṕlaced by a large number of irregular vesicles with clear spaces between them. These experiments thus gave clear. indication that straight necrotic channels formed in retinas exposed to low doses of space radiation in flight.

Malachowski (1978) used a different technique for his initial observations (Malachowski et al., 1978). A few anesthetized C57 Bl mice were exposed to a parallel beam of iron particles (600 MeV/amu) at the Bevalac. Two different experiments were done at estimated doses of $1 \mathrm{rad}$ and $10 \mathrm{rad}$. Three months later the animals were perfused through the aorta with triple fix 


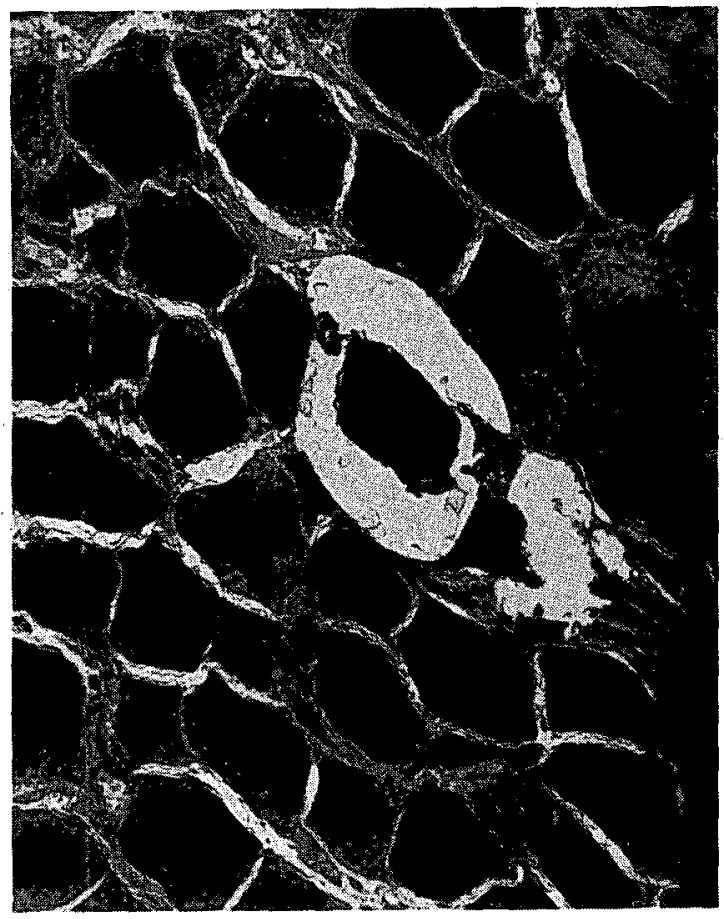

Figure 2. Outer nuclear layer of a mouse retina viewed by transmission electron microscopy. One necrotic nucleus is clearly visible at the center of the picture. The mouse received $1 \mathrm{rad}$ of iron particle radiation, $600 \mathrm{MeV} / \mathrm{amu}$, from the plateau (from Philpott et al., 1978). (XBB 809-10168)

(1978), and after several days on ice the eyes were enucleated, bisected, postfixed in osmium tetroxide, and dried by critical point procedure. The retina was then dry fractured and coated with a conductive coat of $\mathrm{Sn}: \mathrm{Pd}$, and placed into a scanning electron microscope.

The retinas showed relatively large lesions, which we termed "tunnel lesions" because of their physical appearance. The tunnel lesions appear to be almost straight tunnels passing through the outer segment layer of the retina tissue; they can reach lengths up to $50 \mu \mathrm{m}$, with diameters that vary between 1 and $10 \mu \mathrm{m}$. Normal controls and controls that received $x$ rays even at large doses of up to 1000 rad did not produce tunnels. Iron particles appear to cause larger tunnels than argon particles.

Figure 3 shows two fractured sections of a mouse retina. One ( $\mathrm{Fig}$. $3 \mathrm{~A}$ ) received $0.3 \mathrm{rad}$ and the other (Fig. 3B) 10 rad dose of iron particles; they were both sectioned 8 to 9 weeks postirradiation. We estimated that in a parallel beam with kinetic energy of $600 \mathrm{MeV} /$ nucleon the mean distance between individual particles was about $100 \mu \mathrm{m}$ at $0.3 \mathrm{rad}$ and $18 \mu \mathrm{m}$ at $10 \mathrm{rad}$. The lesions seen in Figure 3 are probably due to single iron particles. However, we cannot exclude the possibility that some of the photographed lesions were due to lower energy iron particles with greater energy loss, to a fragment of iron particles that interacted with nuclei of

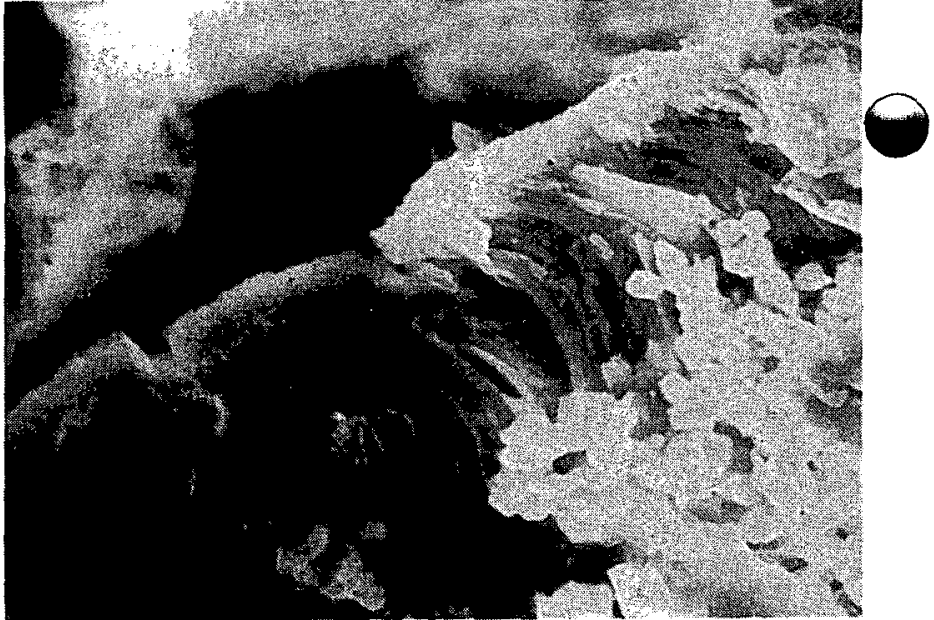

Figure 3A. Mouse retina exposed to less than 1 rad of iron particle radiation. Two to three months postirradiation a characteristic lesion was found by scanning electron microscopy in a retina that had been critical point dried for preservation in vacuo. The tunnel shaped lesions appeared only in the inner and outer segment layers. The radius of the tunnels varied from five to fifteen outer segment diameters in width; a critical diameter of 10 to 30 outer segment widths times the diameter of the outer segments was observed. The photoreceptors close to the tunnel (but beyond the critical diameter area) exhibited little or no radiation damage, smoothing, or degradation of the segments. The general appearance is one of displacement of material and cellular substance around the axis of the tunnel rather than destruction (from Malachowski, 1978). (XBB 760-10234)

the eye, or multiple fragments. Figure 4 is a view of the visual cells of the retina that were mechanically separated from the amacrine cell layer after the retina was fixed. There is an obvious trajectory affecting several visual cells in a straight line path with oblique entry. Malachowski also studied carbon, neon, and argon particle effects. Of these three particle beams, only the argon caused tunnel lesions, and these were much smaller than the lesions caused by iron particles. Thus, these observations suggest the presence of an energy density threshold. No tunnel lesions were observed after large doses of $X$ rays.

In comparing the findings of Philpott and Malachowski, we concluded that they both have observed local effects by demonstrating "channels" in rat retinas flown. in space and "tunnel lesions" in retinas exposed to iron particles. The lesions appear larger when imaged with a scanning electron microscope than they do with a transmission electron microscope. This may be a result of the tissue contraction that occurs during the fixing and embedding for scanning electron microscopy. Future studies will extend and further compare scanning and transmission electron microscope observations. These 


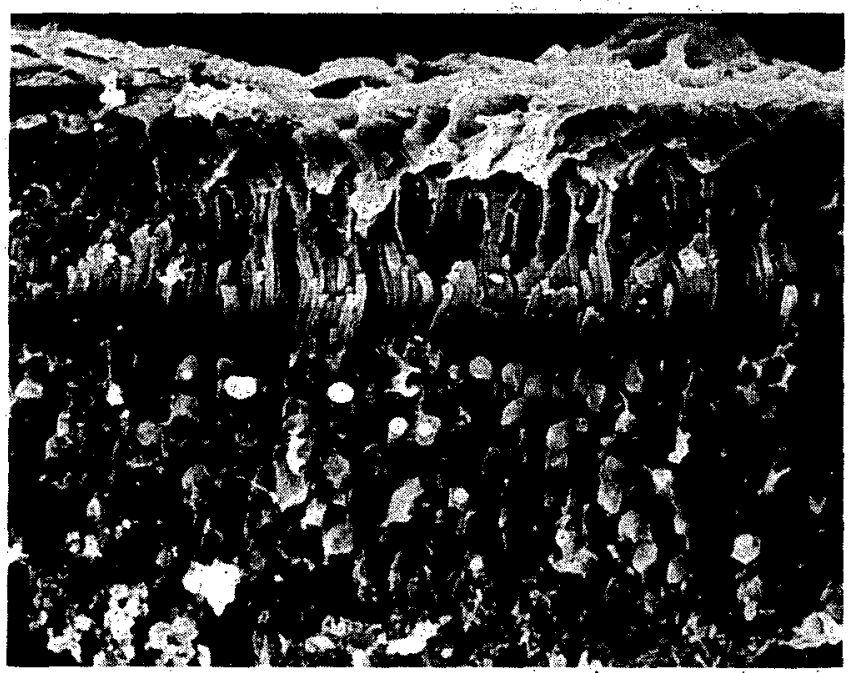

Figure 3B. Mouse retina exposed to $10 \mathrm{rad}$ of iron particle radiation. At higher doses the segments one to five diameters from the axis had degenerated and left a cavity. Membranes were disrupted and degenerated, filaments had retracted, granulated, and degenerated, and the associations and connections of the inner and outer segments were deteriorated and rearranged. At these high doses the areas of influence of each particle appeared to overlap such that the whole field of inner and outer segments under observation appeared to exhibit a degenerate effect while certain locations, which are defined as the axis of the lesion, have disappeared (from Malachowski, 1978). (XBB 790-15436)

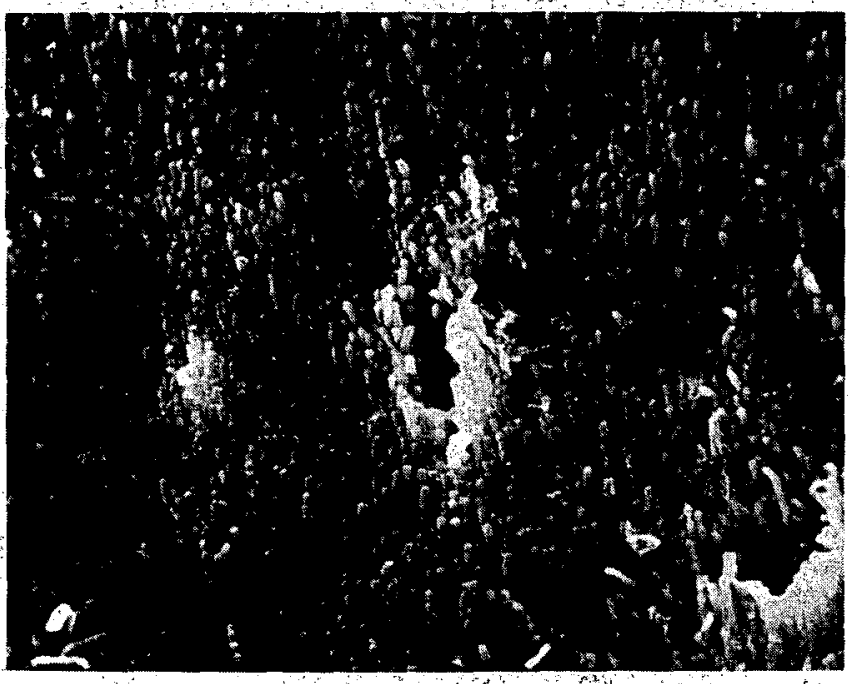

Figure 4. An oblique view of mouse ret ina in the scanning electron microscope three months after exposure to <l rad of iron particles. The light objects are the outer segments of retinal rods.

At the center of the field there is a lesion that may have been produced by an iron particle as it penetrated the retina (from Malachowski, 1978). (XBB 782-1250) observations, striking as they seem, do not fulfill all the criteria listed above; further studies are necessary.

\section{Visual Sensations due to HZE Particles}

We know that single HZE ions produce startling functional effects. The streaks and stars observed by astronauts of the first lunar landing mission, Apollo 11, have been identified as light sensations caused by individual HZE particles crossing the retina. One young investigator has been able to relate light flash events to essentially every neon particle that crossed the regions of the retina, whereas other investigators, all of whom were older, have a 10 to $50 \%$ detection efficiency (Budinger et al., $1971,1972,1977$; Tobias et al.., 1971a,b, 1972). Current studies of visual effects of accelerated heavy ions at the Bevalac are being conducted by McNulty et al. (1975).

\section{HZE Effects in the Brain}

Can HZE ions produce lesions in the brain that extend along heavy particle tracks to several cells. This possibility has been under investigation by $w$. Haymaker and associates in accelerators, high altitude and space flight for a number of years. Perhaps the most direct evidence comes from Miquel et al. (1976) of Ames Laboratory who has observed columnar cystic degeneration in the cephalic ganglionic center (brain) of the adult fruit fly 35 days after it was exposed to krypton particles (Fig. 5). Kraft

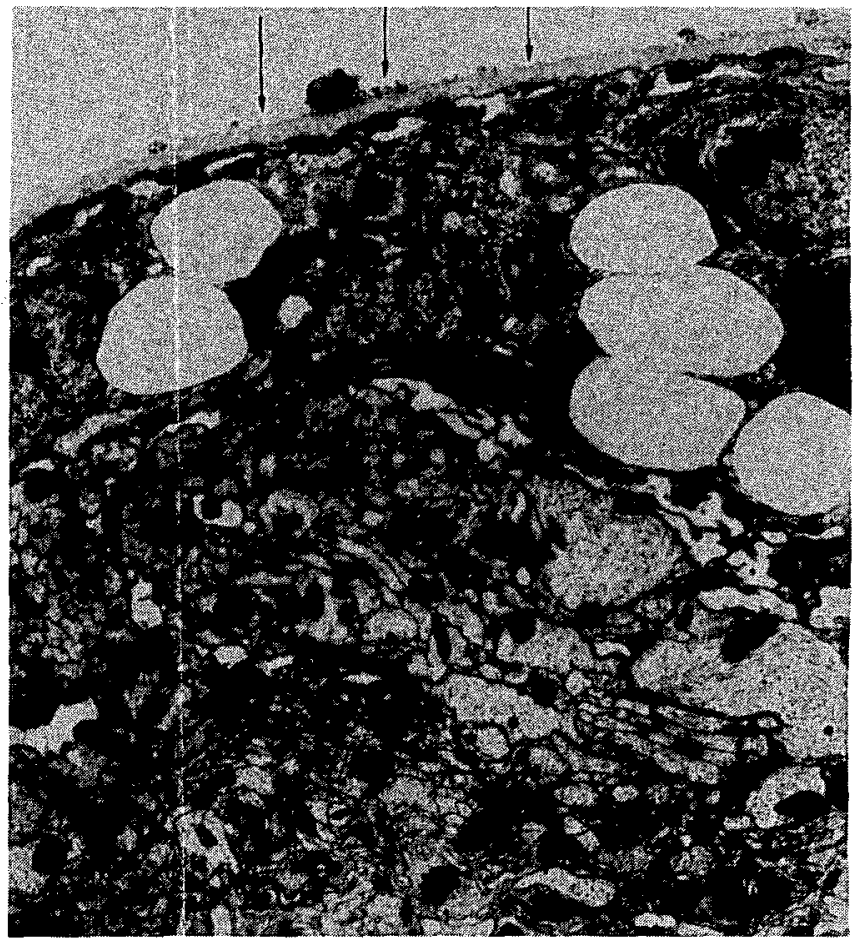

Figure 5. Layers of dead neurons in the path of a low energy, low dose krypton beam (produced in the HILAC) viewed in the transmission electron microscope. (XBB 809-10167) 
and her associates (1979) conducted extensive studies with accelerated neon particles in $\mathrm{C} 57$ B1 mice (1979). At 10 rad no deleterious effects were seen; at $100 \mathrm{rad}$ and higher light microscopy detected increasing numbers of necrotic interstitial cells, mainly neuroglia, cells of the subependynal plate and microneurons of the dentate gyrus. Beginning at about two weeks after exposure, necrotic neurons (coagulative necrosis) appeared in small numbers. These studies are the subject of a separate chapter in Part V (see Kraft et al.).

\section{Cornea}

Perhaps the most convincing evidence for the existence of thermophysical lesions was obtained recently by Alan Nelson (1980), who studied the corneal surface of rats in vivo and in vitro with beams of carbon, neon, and argon particles at the SuperHILAC at kinetic energies of a few MeV/amu. He also made some exposures to $h$ igh energy particles, including $600 \mathrm{MeV} / \mathrm{amu}$ iron. At the same time, particle fluence was monitored with plastic nuclear detector foils. The earliest specimens were fixed for scanning electron microsscopy within a few seconds after exposure; later preparations were made within a $24 \mathrm{~h}$ time interval. Careful studies were made of the electron microscopy of normal corneal surface and of controls that received $x$ rays of 500 rad to 100,000 rad. Figure 6 shows the appearance of a rat cornea fixed immediately after an $X$-ray dose of $500 \mathrm{rad}$. None of the corneas from the $X$-ray animals or unirradiated controls showed any holes or depressions of the kind seen after heavy-ion exposure. Carbon particles produced conical indentations on the corneal surface that sometimes had "holes" at the center that measured $0.08 \mu \mathrm{m}$ for carbon, as shown in Figure 7 . The indentations from the low-energy argon ions accelerated at the SuperHILAC $(3 \mathrm{MeV} / \mathrm{n})$ produced greater damage at the surface but no center holes, as seen in Figure 8 . The initial number of indentations showed good agreement with the flux density of the particles, which was measured independent ly.

These effects demonstrate convincingly that heavy particles make real lesions in the membrane of the cornea, even though lesions may be altered somewhat by the fixing and developing techniques of microscopy. We do not fully understand the mechanism of producing indentations; however, they might be due to the rapid sputtering and sublimation of matter near the ionizing core of particles, which receives a great energy density in a very short time. The diameter of the lesions observed is usually much larger than the initial core diameter, which indicates the importance of secondary transport processes.

\section{Lens}

G. Worgle of Columbia University, working with a team headed by $H$. Rossi, exposed a number of mice to $570 \mathrm{MeV} / \mathrm{amu}$ argon particles (private communication 1979). Scanning electron microscopy showed circular indentations and straight

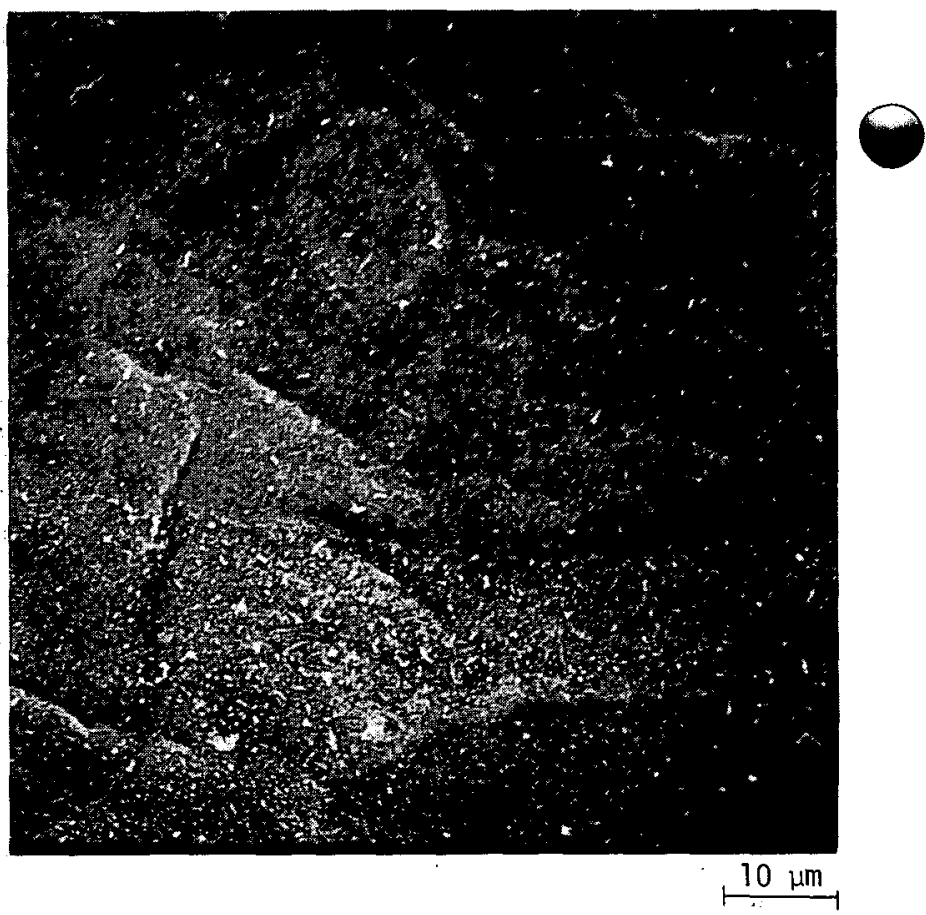

Figure 6. Scanning electron micrograph of a single rat corneal epithelial cell from a sample that received an $x-r a y$ dose of 500 rad. The nuclear bump is in the upper center; the bright specks are contamination crystals. Scale is approximately $3 \mathrm{~cm}=10 \mu \mathrm{m}$ (from Nelson, 1980). (XBB 805-6606)

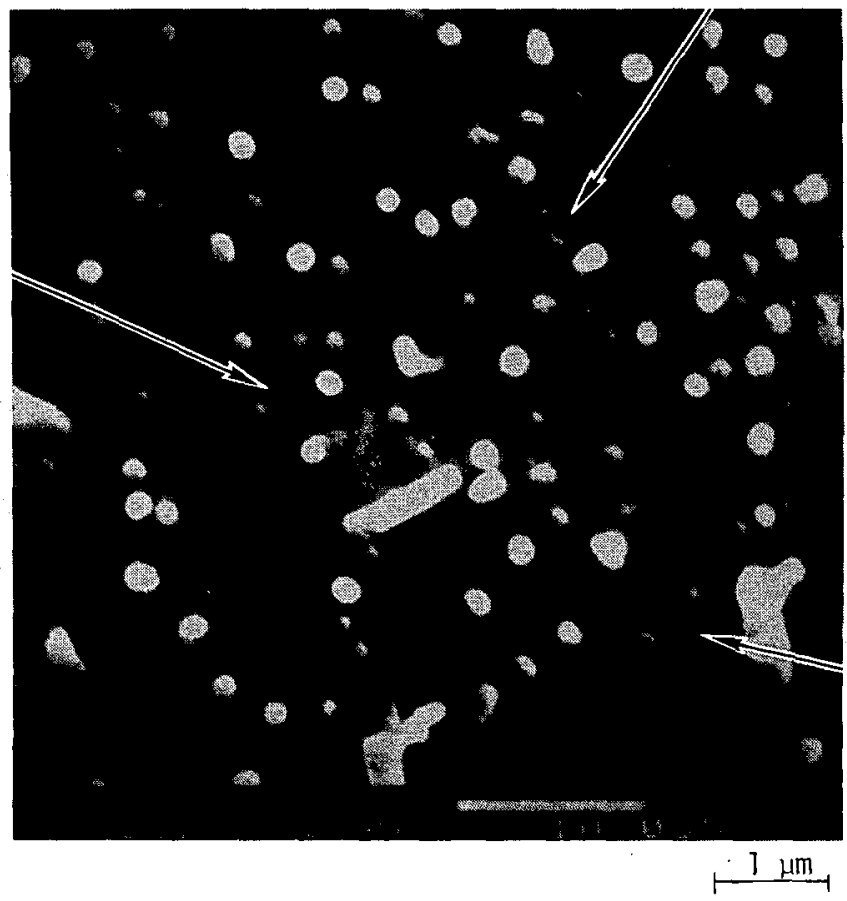

Figure 7. Scanning electron micrograph of a rat corneal epithelial cell plasma membrane that was irradiated with $474 \mathrm{MeV} / \mathrm{amu}$ carbon ions. The membrane lesions measure about $0.08 \mu \mathrm{m}$ in diameter (from Nelson, 1980). (XBB 805-6621) 


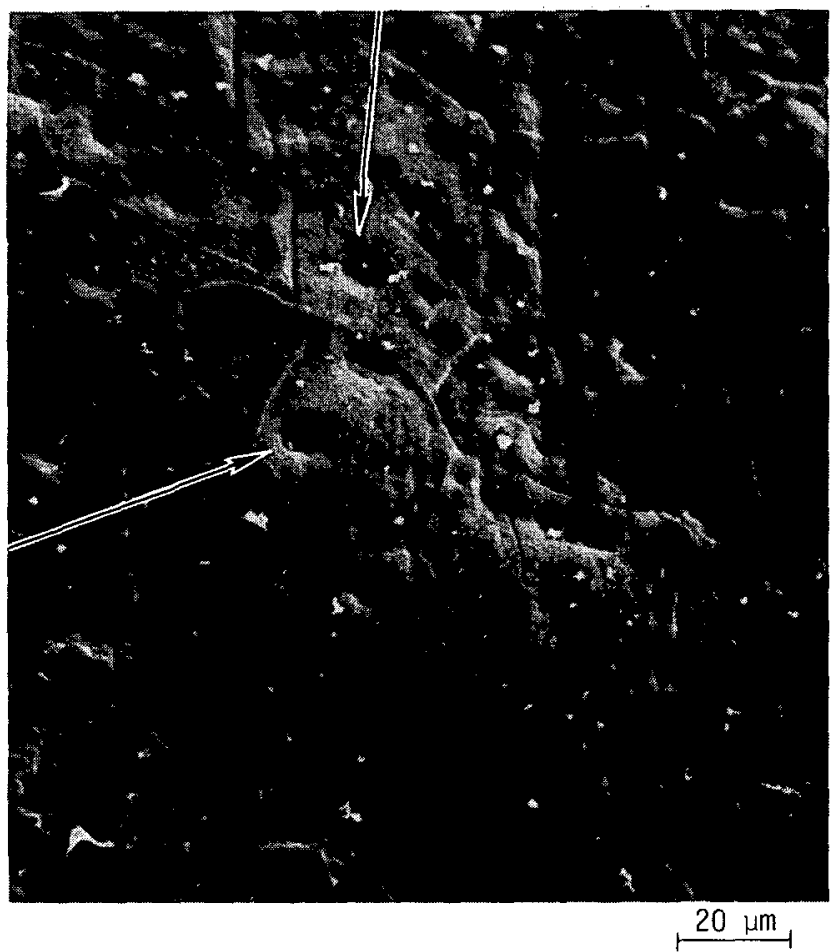

Figure 8. Scanning electron micrograph of a rat cornea irradiated with $3 \mathrm{MeV} / \mathrm{amu}$ argon particles. Lesion diameters measure $5 \mu \mathrm{m}$. Note the relative size of nuclei and whole epithelial cells compared to the heavy-ion lesions (from Nelson, 1980). (XBB 805-14737)

streaks on the lens epithelium, which had been fixed within a few minutes after exposure. This group is also accumulating evidence individual particles passing either through the central or the peripheral portions of the lens causes micro-opacity.

\section{Developmental Effects in Corn (Zea Mays)}

Slater and Tobias (1963) and Tater Yang and Tobias (Tobias et al., 1977), exposed seeds of Zea mays to doses well below 1 rad in balloon flights and in the Biostack space experiments. When plants were grown from irradiated seeds, necrotic and bleached stripes and other:abnormalities appeared in the developing plants'. The evidence is accumulating that these effects are. due to single HZE ions striking one or more cells in the embryonic portion of the seed. These effects cannot be reproduced exactly with Tow-LET radiation. $X$ rays $(1,000$ rad $)$ delivered to the seed produce large numbers of lesions in developing plants; however, the extent and severity of each lesion is orders of magnitude smaller than the lesions produced in space by HZE ions. The largest lesion was found in plants grown from seeds flown in Biostack III (Peterson a.nt a 1., 1976; Bücker et a1., 1977); eleven different lesions were seen in the first five leaves of a single plant grown from one of the seeds (one of 500 exposed). Microscopic nuclear track dosimetry by $E$. Benton identified the particle as a high velocity argon nucleus (or heavier).

SUMMARY OF THE EVIDENCE FOR MICROLESIONS PRODUCED BY HZE PARTICLES

In none of the tissues studied do we find conclusive evidence for microlesions that would satisfy all six criteria stated earlier. However, with transmission and scanning electron microscopy, most of the criteria were fulfilled in the cornea in favor of microlesions produced by single HZE particles. There is evidence of HZE particle-induced lesions in the retina also, however, the size and extent of these lesions are still in doubt because of possible microscopy artefacts.

In seeds of Zea mays, there is strong evidence for developmental effects due to single $\mathrm{HZE}$ tracks; the developmental effects get larger and more elaborate for heavier particles.

\section{Biological Consequences and Potential Hazard from Microlesions}

There are not sufficient data to estimate hazards from HZE particle induced microlesions. However, we should note that in a one year space flight in interplanetary space, the percentage of neural or retinal cells hit varies from about $1 \%$ to $10 \%$. The larger figure is for the largest neurons in the brain (pyramidal or Purkinje cells).

Neural nets have built in redundancy. If some neurons in the net are injured it does not necessarily seriously affect net function. To evaluate the impairment of function, we must evaluate the natural redundancy present in normaliy functioning neural nets, and the effect of injury on electrical noise and discrimination in the net. The only way we can accurately evaluate these effects is by a continued study of the nature and production efficiency of microlesions produced by HZE particles, and by evaluating the relationship of multiple cellular injury to the performance, e.g., visual function in the retina or homeostatic functions in the hypothalamus.

Finally, it will be necessary to evaluate the carcinogenic and cell transforming effects of microlesions. The most likely effect of a microlesion is immediate or delayed cell death and lysis. For this reason it appears likely that the carcinogenic potency of particles of very high LET is less than that of particles of intermediate LET, which cannot form microlesions.

\section{FOOTNOTES AND REFERENCES}

* Now with the Department of Nuclear Engineering, Massachusetts Institute of Technology, Cambridge, MA.

+ Ames Research Center, National Aeronautics and Space Administration, Moffett Field, CA. 
Benton, E. V., D. D. Peterson, and R. P. Henke. 1977. Summary of measurements of high-LET particle radiation in $U$. S. manned space missions. Life Sci. Space Res. 15, 119-127.

Bücker, H., et al. 1977. Biostack III. Experiment MA-107. Apollo-Soyuz Test Project. Summary Science Report, vol. 1, pp. 211-226. Report NASA SP-412. NASA, Washington, D.C

Budinger, T. F., H. Bichsel, and C. A. Tobias. 1971: Visual phenomena noted by human subjects on exposure to neutrons of energies less than $25 \mathrm{MeV}$. Science 17.2, 868-870.

Budinger, T. F., J. T. Lyman, and C. A. Tobias. 1972. Visual perception of accelerated nitrogen nuclei interacting with the human retina. Nature 239, 209-211.

Budinger, T. F., C. A. Tobias, R. H. Huesman, T. F. Upham, T. F. Wieskamp, J. U. Schott, and E. Schopper. 1977. Light-flash observations. Apollo-Soyuz Test Project, Summary Science Report, pp. 193-209. Report NASA SP-412. NASA, Washington, D.C.

Chase, H. B. and J. S. Post. 1956. Damage and repair in mammalian tissues exposed to cosmic ray neavy nuclei. J. Aviat. Med. 27, 533-540.

Chatterjee, A. and J. L. Magee. 1981. Radiation Chemistry of heavy particle tracks. II. Fricke dosimeter system. J. Phys. Chem., in press.

Chatterjee, A., C. A. Tobias, and J. T. Lyman. 1976. Nuclear fragmentation in therapeutic and diagnostic studies with heavy ions. Spallation Nuclear Reactions and the ir Applications (B.P. Shen and M. Merker, eds.) pp. 169-191. Reidel Publishing Co., Dordrecht, Holland.

Fleischer, R. L. and P. B. Price. 1963. Tracks of charged particles in high polymers. Science 140, 1221-1222.

Fleischer, R. L., H. R. Hart, G. M. Comstock, M. Carter, A. Renshaw, and A. Hardy. 1973. Apo 11014 and 16 heavy particle dosimetry experiments. Science $181,436-438$.

Fleischer, R. L., P. B. Price, and R. M. Walker. 1975. Nuclear Tracks in Solids. University of California Press, Berkeley.

Freier, P., E. J. Lofgren, E. P. Ney, and $F$. Oppenheimer. 1948. The heavy component of primary cosmic rays. Phys. Rev. 74, 1818.

Grahn, D., ed. 1973. HZE Particle Effects in Manned Space Flight. National Academy of Sciences, Washington, D. C.

Janssen, P., I. Klatzo, J. Miquel, T. Brustad, A. Behar, W. Haymaker, J. Lyman, and C. A. Tobias. 1962. Effects of alpha particle and proton irradiation on the cerebellum and cerebrum in the rat. Response of the Nervous
System to Ionizing Radiation (T. J. Haley and R. S. Snider, eds.). Academic Press, New York.

Kerr, J. F. R. and J. Searle. 1980. Apoptosis: Its nature and $k$ inetic role. Radiation Biology and Cancer Research (R. E. Meyn and $H$. R. Withers, eds.) pp. 367-384. Raven Press, New York.

Kraft, L. M., M. A. Kelly, J. E. Johnson, Jr., E. V. Benton, R. P. Henke, R. Casson, W. Haymaker, D. E. Philpott, F. S. Vogel, and W. Zenan. 1979. Effects of high LET neon $\left(20_{N C}\right)$ particle radiation on the brain, eyes, and other head structures of the pocket mouse: A.histological study. Int. J. Radiat. Biol. 35, 33-61.

Magee, J. L. and A. Chatterjee. 1981. Radiation chemistry of heavy particle tracks. I. General considerations. J. Phys. Chem., in press.

Malachowski, M. J. 1978. The Effects of Ionizing Radiation on the Light Sensing Elements of the Retina. Ph.D. Thesis, University of California, Berkeley.

Malachowski, M. J., C. A. Tobias, and D. E. Philpott. 1978. Morphological changes in mammalian retina photoreceptors exposed to low doses of accelerated heavy ions. Radiat. Res. 74, 566. (Abstract).

McNulty, P. J., V. P. Pease, and V. P. Bond. 1975. Visual sensations induced by Cerenkov radiation. Science $189,453-454$.

Mique 1, J., M. M. Herman, E. V. Benton, and G. Weich. 1976. Effects of high LET particles $\left(40_{A}\right)$ on the brain of Drosophila melanogaster. Int. J. Radiat. Biol. $29,101-124$.

Mozumder, A. 1969. Charged particle tracks and their structure. Advances in Radiation Chemistry (M. Burton and J. Magee, eds.) vol. 1, pp. 1-102. Wiley Interscience, New York.

Nelson, A. 1980. Theoretical and Observational Analysis of Individual Ionizing Particle Effects in Biological Tissue. Ph.D. Thesis, University of California, Berkeley.

Peterson, D. D., E. V. Benton, and M. Tran. 1976. HZE particle dosimetry, experiment K-103 for Kosmos 782. Technical Report 42. Department of Physics, University of San Francisco, San Francisco, CA.

Philpott, D. E., R. L. Corbett, S. Black, and C. Turnbul1. 1972. Ultrastructural investigation of the light flash phenomenon seen by our astronauts. Proc. Electron Micros. Soc. Amer. 30,52 .

Philpott, D. E., R. L. Corbett, S. Black, and C. Turnbu11. 1973. Ultrastructural Investigation of the Light Flash Phenomenon Seen by Our Astronauts, Through Exposure of Pocket Mouse and Rabbit Retina to X-ray, Nitrogen, and 
Oxygen Irradiation. Lawrence Berkeley Laboratory Report LBL-2016.

Philpott, D. E., R. Corbett, C. Turnbill, G. Harrison, D. Leaffer, S. Black, W. Sapp, G. Klein, and L. F. Savik. 1978. Cosmic ray effects on the eyes of rats flown on cosmos No. 782, Experiment K-007. Aviat. Space Environ. Med.

Seitz, F. 1958. On the theory of the bubble chamber. Phys. Fluids 1, 2-13.

Slater, J. V. and C. A. Tobias. 1963. Effects of cosmic radiation on seed differentiation and development. Radiat. Res. 19, 218.

Tobias, C. A. 1952. Radiation hazards in high altitude aviation. J. Avia. Med. 23, 345-372.

Tobias, C. A. 1971. Physical energy transfer and biological effects. Advances in Medical Physics, (J. S. Laughlin and E.W.Webster, eds.) pp. 28-50.

Tobias, C. A., T. F. Budinger, J. T. Leith, and A. M. Mamoon. 1971a. Visual phenomena induced by cosmic rays and accelerated particles. Proceedings, Advisory Group for Aerospace Research and Development, pp.
1-110. North Atlantic Treaty Organization, Conference Reprint 95.

Tobias, C. A.; T. F. Budinger, and J. T. Lyman. 1971b. Radiation induced light flashes observed by human subjects in fast neutron, $x-r a y$, and positive pion beams. Nature 230, 596-598.

Tobias, C. A., T. F. Budinger, and J. T. Lyman. 1972. Human visual response to nuclear particle exposures. Proceedings, International Symposium and Man-Made Radiation in Space, $p p$. 416-422. NASA TMX 2440, NASA, Washington, D. C.

Tobias, C. A., E. V. Benton, D. D. Peterson, M. Tran, T. Yang, M. Freeling, and L. Craise. 1977. Biological effects of high-LET particles on corn-seed embryos in the Apo 110-Soyuz test project--Biostack III experiment. Life Sci. Space Res. 15, 151-155.

Tobias, C. A., A. Chatterjee, M. J. Malachowski, E. A. Blakely, and T. L. Hayes. 1979. Tracks in condensed systems. Proceedings, Sixth International Congress of Radiation Research, (S. Okada et al. eds.), pp. 146-156. Japanese Association for Radiation Research, Tokyo, Japan. 


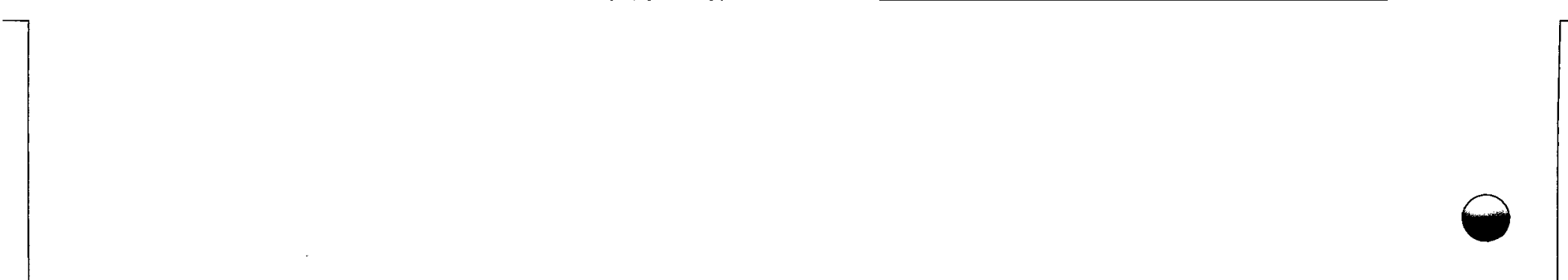

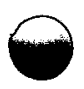
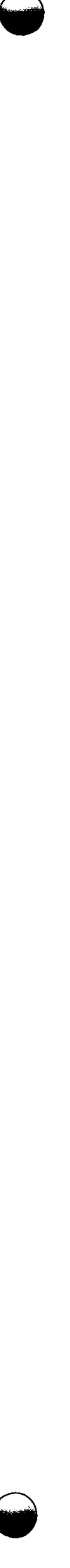


\title{
HZE PARTICLE EFFECTS IN THE MAMMALIAN BRAIN: RELEVANCE TO MANNED SPACE FLIGHT
}

\author{
L.M. Kraft, ${ }^{*}$ F.E. D'Amelio, ${ }^{\dagger}$ and E.V. Benton ${ }^{\dagger}$
}

In order to judge if exposure to $H Z E$ particles during space flight are likely to compromise man's ability to function effectively throughout an extended mission and thereafter, direct, indirect, acute, and chronic effects in the nervous system, especially the brain, need to be determined and quantified. The resulting information should provide the basis for estimating quality factors and RBE values of the various heavy ions for diverse nervous structures, and thus assist in establishing radiation safety standards for manned space flight. Since radiobiological flight experiments in mammals are virtually impossible to conduct and interpret at present, we must rely upon exposure of experimental animals to heavy particles in the Bevalac at $L B L$ to provide the necessary data.

\section{RESULTS IN THE RODENT BRAIN}

We have performed a number of experiments in which the brains of rodents were exposed to high LET neon, argon, or iron particles. Of these, the results of irradiation with neon have been reported (Kraft et al., 1979). Results of argon and iron irradiation are preliminary; nevertheless, some of the data available are presented below.

\section{Results of Irradiation with Neon Particles}

Neon particles ( $400 \mathrm{MeV}$ ), degraded in a water column to about $40 \mathrm{MeV}$, were utilized in a single surface dose of $1,000,100$, or 10 rad in adult C57B1 mice or pocket mice (Perognathus longimembris). Pocket mice were used because they were considered as subjects for future flight experiments since they do not require drinking: water. The beam was directed at the top of the head perpendicular to its long axis; the Bragg peak was situated $2.8 \mathrm{~mm}$ beneath the scalp (about midway through the brain). Animals were killed at intervals between 0.5 and 627 . days.

As seen in the light microscope, within $12 \mathrm{~h}$. after irradiation, increased numbers of necrotic cerebral interstitial cells. (mainly neuroglia, cells of the subependymal plate, and microneurons of the dentate gyrus) were observed after 1,000 and $100 \mathrm{rad}$. Necrotic interstitial cells were $i \ldots$ not seen in the cerebellum or. brain stem.

Beginning about 2 weeks after exposure, necrotic neurons appeared in small numbers. Their peak incidence occurred at 4-5 weeks and they were rarely encountered after 2 months. Ilmost all were seen to be in the state of coagulative necrosis; only rarely was frank lys is observed. At peak incidence the proportion of necrotic neurons of more than
100,000 neurons scanned in the frontal cortex at each dose level was estimated to be $0.04 \%$ at $1,000 \mathrm{rad}, 0.03 \%$ at $100 \mathrm{rad}$, and $<0.0005 \%$ at $10 \mathrm{rad}$. In the cerebellum the incidence was considerably less $(<0.0001 \%)$.

Distribution of necrotic neurons coincided for the most part with the Bragg zone, which is defined as the irradiated region upstream of and including the Bragg peak region. The dose contribution of the primaries ranged from about $1,000,100$, or 10 rad at the calvarium to 2,200, 220 , or 22 rad in the Bragg peak region. Necrotic cells did not, however, constitute a band in this region. Rather, they were scattered more or less at random throughout the Bragg zone (Fig. 1).

Enlarged hyperchromatic neuroglia were first noted in the Bragg zone at 3 weeks after exposure to $1,000 \mathrm{rad}$. They increased in number to about 7 weeks and then declined. At the same dose, axonal damage, as evidenced by uranyl nitratesilver staining, was seen in the deep layers of the cerebrum beginning at 3 months and lasting at least until 10 months after irradiation at $1,000 \mathrm{rad}$.

Glycogen accumulation could not be demonstrated in astrocytes at any time interval. Gliosis was absent from all brains, and the leptomeninges were free from alterations at all times and dose levels. Acute vascular change could not be seen in Lepehne-Pickworth preparations; however, upon perfusion of all mice that received $1,000 \mathrm{rad}$, rare to few petechial hemorrhages were evident in the brain between 14 and 201 days after exposure. These hemorrhages were not seen at the lower dose levels.

Electron microscopic examination of the brain at 36 hours after 1,000 rad showed many distended astroglial end-feet in regions adjacent to compressed vessels, a finding that was not seen in the control mice. Vascular endothelium appeared unaltered, however. In the cerebellum, Purk inje cell perikarya manifested varying numbers of cisternal stacks, most of which evidenced ribosomes an the outermost membrane on ly. The amount of endoplasmic reticulum appeared reduced in comparison to the controls.

At 28 days after irradiation these changes were no Tonger seen:. Only occasional remnants of a necrotic neuron could, by chance, be made out in the molecular layer of the hippocampus. Reactive pericytes with many lipid droplets were encountered; others were clearly necrotic. No ultrastructural changes of any kind could be found at 28 days after exposure to $10 \mathrm{rad}$ surface dose. 
COMPARISON WITH PREVIOUS RADIATION STUDIES IN THE BRAIN

Changes seen in the light microscope similar to those described above have aiso been noted following $X$ and gamma rays, protons, deuterons, and alpha particles (Haymaker, 1969; Zeman, 1968; Zeman and Samorajski, 1971). Doses used, however, were considerably higher than in the neon study. One report (Janssen et a 1., 1962) included data from exposure of the rat brain to alpha particles at Bragg peak using "low" (50 to $1,500 \mathrm{rad})$ as well as high $(3,000$ to $6,000 \mathrm{rad})$ doses. Although postexposure observation intervals, species, and some techniques were different from those of the neon study, distinct points of comparison and divergence do appear. Thus, after 1,500 rad alpha particles, they found a neuronpoor Bragg band at 7 months after exposure. This was not seen in our neon material (1,000 $\mathrm{rad})$. On the other hand, the cerebellum in the ir animals appeared normal, whereas neon-irradiated mice did manifest some necrotic Purk inje cells after 1,000 rad and rare ones after $100 \mathrm{rad}$ irradiation; but these could quite easily have been overlooked in our material had it not been for the fact that tissue sections were scanned cell by cell at high magnification.

Likewise in the cerebrum no changes were noted after 750 rad alpha particles, whereas neuronal necrosis, albeit of low incidence and widespread distribution, occurred after $100 \mathrm{rad}$ of neon particles.

Gliosis and meningeal involvement were absent in both investigations. In evaluating the lack of glycogen in astrocytes after 1,000 rad of neon particles, another study may be examined. Utilizing the glucose oxidase method, Miquel et al. (1966) were able to demonstrate a slight but significant increase in glycogen at $24 \mathrm{~h}$ after irradiation with 1,500 rad of alpha particles, the lowest dose investigated. Again, methodology differed in the two studies. On the other hand, dose alone may have been the effective variable.

Brownson et al. (1963) reported the appearance of necrotic interstitial (predominantly neuroglial) cells within 36 hours after X-irradiation $(50$ to $10,000 \mathrm{rad})$ in the rat brain. Significant numbers of altered oligodendroglia were recorded in rats receiving as little as $150 \mathrm{R}$. They enumerated the necrotic glia and showed that the numbers were directly related to the total $x$-ray dose. Thus, they regarded the effect as primary rather than secondary. Data from the neon study are comparable and tend to support the view that the effect is, morphologically at least, primary.

The acute appearance of necrotic cells in the subependymal plate, Smart (1961) showed autoradiographically that migration of cells in this location is minimal in the adult mouse. Brownson et al. (1963) then speculated that radiation merely accelerates the degeneration of cells in metaphase that can no longer differentiate or migrate and that would ultimately die in any event.
The paucity of reports on "low" dose (less than 1,000 rad) radiation effects in the brain is equalled by the rarity of quantitative evaluations. An exception is the work of Zeman et al. (1961) in which microbeams of deuterons were used to determine the dose at which nerve cells in the visual cortex of mice were totally destroyed in 24 days. When a small volume of tissue was irradiated by means of $0.025 \mathrm{~mm}$ diameter beam, 400,000 rad were required, and there was little variation among animals. However, only about 14,000 rad were needed to attain the end point when the beam was $1 \mathrm{~mm}$ in diameter; here there was considerably greater variation among the mice. Variability decreased in direct relation to the volume of tissue irradiated. Since more vessels were exposed when the larger beam was used, it was thought that the variability was due to the number of vessels irradiated, and neuronal necrosis was regarded as a secondary effect attributable to the radiosensitivity of vessels, rather than to the primary sensitivity of the neurons themselves. At that time neuroglial cell loss was not regarded as contributory to neuronal death, but later Zeman (1968) did discuss this as a possible role of the glia, especially with regards to the demyel ination of axons. Based on our results, we believe that acute interstitial neuroglial cell loss in the adult mouse is perhaps more important than vascular damage at low dose (hundreds of rad and below), since we were unable to demonstrate endothelial changes at the ultrastructural level after 1,000 rad but we could find swollen neuroglial end-feet in association with vessels within hours after irradiation. This observation does not in itself eliminate injury to the endothelium (such as permeability changes) as a factor in the genesis of the alterations in the gliai end-feet.

Acute postexposure oligodendroglial cell loss has been discussed by Brownson et al. (1963). They believed that if injury to surviving oligodendroglia together with the loss of large numbers occurs acutely, there would be an interruption and desynchronization of their renewal cycle with concomitant impaired integrity of the myel in sheath. The question is intriguing and invites intensive investigation.

The presence of hyperchromatic, enlarged, malformed interstitial cells in irradiated brain has occasionally been noted in the literature. Arnold and Bailey (1954) called them "monstrous cells," and described them in the irradiated field of a patient who had received a course of $x-r a y$ therapy (dose was not divulged) 15 months prior to death. Zeman and Samorajski (1971) also recognized such cells in the irradiated human spinal cord where the dose was in excess of $3,500 \mathrm{R}$ of $250 \mathrm{kVp} \times$ rays. In the mice exposed to neon radiation, we regard them as sublethally irradiated neuroglia or the malformed progeny.

Axonal degeneration became evident 3 months after neon particle irradiation $(1,000$ rad), but neuronal necrosis was at peak incidence 1 month thereafter. This observation suggests a continuous slow loss of neurons (perhaps by lysis), 
thus increasing, perhaps significantly even at very low doses, the total number of neurons that are destroyed--numbers that can not be estimated from observation of necrotic neurons alone.

\section{Results of Irradiation with Argon Particles}

Limited data are available in the mouse brain following irradiation with 500 amu argon particles. The irradiation and evaluation were as described above for neon. At peak incidence, following 400 rad surface dose, for example, $0.008 \%$ of the neurons in the cerebral cortex were necrotic (35 days) and at $80 \mathrm{rad}, 0.001 \%$ were seen. In the cerebellum $<0.001 \%$ were found. These results are not markedly different from those in the neon study (Fig. 1).

In the cerebrum of mice irradiated 35 days previously with 2,000 rad argon (surface dose), but not after exposure to 1,000 rad, a definite band devoid of nerve cells could be seen in the Bragg peak region in the hippocampus only. The dose here was about 4,000 rad. The hippocampal gyrus itself was severely damaged. All pyramidal cells in the Bragg band region were destroyed. The neighboring dentate gyrus, on the other hand, rarely manifested necrotic cells, nor was the cerebral cortex lateral to the hippocampal gyrus noticeably involved. This typifies the diverse susceptiblity of various brain regions of structures.

\section{Comparison between Argon and Neon Particle Irradiation}

Figure 1 illustrates the distribution of single necrotic neurons in the frontal, diencephalic, and cerebellar regions of the mouse brain. Note the relative resistance of the cerebellum. The distribution in the diencephalic slice of both mice seems to favor the hippocampus. Because of the small number of necrotic cells involved, however, it is not possible to determine if this distribution is significant.

\section{Results of Irradiation with Iron Particles}

We were able to expose mice to a maximum dose of only 25 rad. Accelerator scheduling further restricted exposure opportunities, therefore, only a few mice could be irradiated. Nevertheless, using the same methods as described, we could find no necrotic neurons at 28 days after irradiation. This end point will not be useful

\section{NEON \\ 1034 RAD \\ $3.7 \times 10^{7}$ PARTICLES $/ \mathrm{cm}^{2}$}
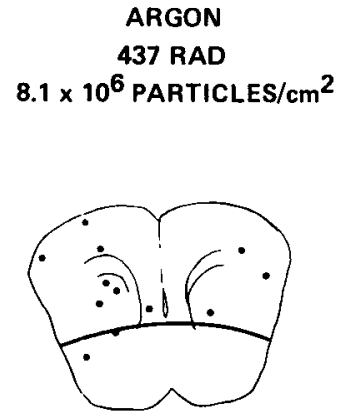

31,000

$(12 \cong 0.008 \%)$

$12 \cong 0.008 \%$

189,000 $(36 \cong 0.024 \%)$

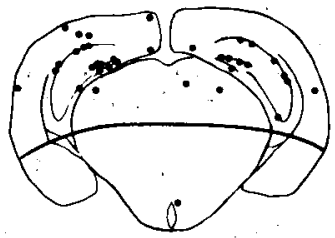

124,000 $(2=<0.001 \%)$

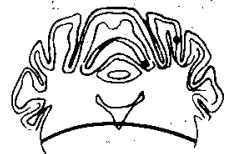

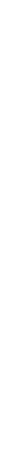
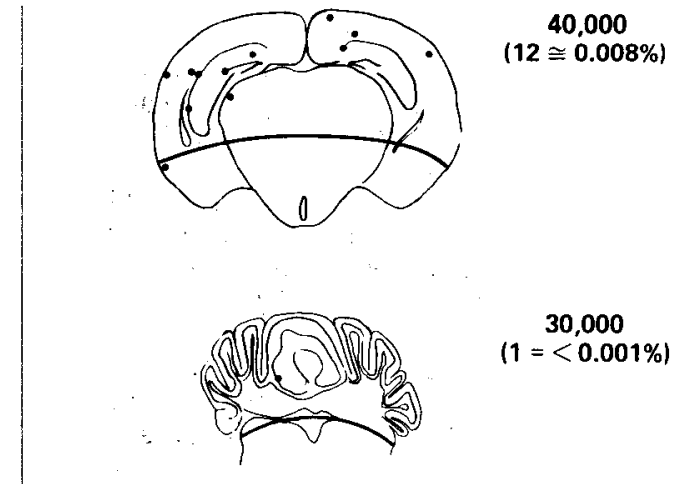

Figure 1. Comparison of results of irradiation of mice with neon or argon particles at the indicated doses (and fluences) 35 days after exposure. The beam was directed from above, parallel with this page. The Bragg peak region is indicated by the curved line in each segment; segments are 48 um thick.

* Total number of particles entering each segment.

$\star \star$ Number of necrotic neurons per segment $\simeq$ percent of total neurons per segment. (XBL 808-11364) 
in comparing iron with other ions until a more intense beam can be made available.

\section{APPLICABILITY OF THE RESULTS TO MAN}

In attempting to extrapolate the findings in the rodent brain to that of man, great caution must be exercised. A principal concern, obviously, is the number of dead neurons that follow irradiation expressed as percent of the total population of nerve cells. Although man's brain is 4,000 times larger in volume than that of the mouse (Comstock et al., 1971) and therefore more likely to be hit by cosmic-ray particles in space, the neuronal concentration in the cerebral cortex, for example, is considerably lower (about 9,000 neurons $/ \mathrm{mm}^{3}$ ) than in the mouse (about 140,000 neurons $/ \mathrm{mm}^{3}$ ) (Tower and Elliott, 1952). The synaptic interconnections in the cortex of man, however, are far more extensive and complex than in the rodent (Holloway, 1968). Thus, in man, single particles would have a greater chance of traversing and ending in gliacytes and neuropil (including vast numbers of synapses containing mitochondria, synaptic clefts, and synaptic vesicles) than they would terminating in the soma of neurons.

Together with endothelial cells, the interstitial glial cells constitute $75 \%$ of the cells of the cerebral cortex in man; the neurons comprise only $25 \%$ of the cellular contingent (Comstock et al., 1971). We therefore feel it is imperative to study the radiobiology of HZE particle effects in the more numerous, more radiosensitive renewing cells of the brain in order to understand the consequences of radiation damage in that compartment to the neurons.

\section{WORK IN PROGRESS AND STUDIES PLANNED}

As we have seen, the results of exposure of mice to 2,000 rad argon particles exemplify the differential radiovulnerability of various neighboring brain structures. The RBE values for neuronal necrosis will, therefore, have to be determined for the most radiosensitive region, perhaps the Sommer sector of the hippocampus. The RBE may turn out to be quite different for more resistant neurons, but for space flight applications, the latter may be academic. clearly, high doses will be required to establish the data in the hippocampus, which we expect to carry out using cell enumeration techniques similar to those of Walker et al. (1980).

For RBE values of interstitial neuroglial cell loss, much lower doses will suffice. The dose levels at which endothelial cells manifest ultrastructural changes have not yet been determined, but we expect to make contributions in this area as well.

Perhaps of importance comparable to possible brain cell loss resulting. from space flight, are the physiologic and abscopal effects of low doses as reflected in ultrastructural and/or biochemical changes. Thus, we have initiated morphologic and endocrine studies after single exposures - the former with respect to synaptic configurations and the latter regarding some of the anterior pituitary hormones. Data, although too fragmentary to warrant description here, are encouraging. We have not yet explored HZE particle effects in the brain after fractionated doses. Nor have the effects of mixed modalities, such as irradiation with the heavy ions followed by $X$ rays, protons, or other types of radiation, been addressed. Considering that radiation exposure is continuous during extended space flight, these fractionated and mixed modality effects will be most important, since they would provide a first approximation to the space radiation environment.

To a considerable extent, exposures of the brain have to date been "range-finding" in character. We look forward to additional utilization of the Bevalac in order to establish more accurately the time/dose/RBE relationships of the effects observed.

\section{SUMMARY}

The brain of rodents has been studied following high LET neon particle irradiation with doses ranging from 10 to 1,000 rad. The changes observed by light microscopy after 1,000 rad irradiation, for example, include acute necrosis of interstitial cells within $36 \mathrm{~h}$ of exposure, necrotic neurons (peak incidence, 4-5 weeks after irradiation; $0.04 \%$ of total cerebral neurons), hyperchromatic neuroglia (peak incidence at 7 weeks), and axonal degeneration beginning at 3 months postexposure. These changes are compared with those reported following exposure to other radiation modalities.

Neuronal necrosis following high LET argon particle irradiation was comparable to that with neon. Low doses (25 rad and below) of iron nuclei resulted in no visible necrotic nerve cells.

Acute neuroglial cell loss or damage is discussed as to its possible role in chronic neuronal necrosis and in loss of axonal integrity. Work in progress and anticipated future studies are indicated.

\section{ACKNOWLEDGEMENT}

This work was supported in part by Grant NSG-2063 and Cooperative Agreement NCC 2-47 from the National Aeronautics and Space Administration to the University of San Francisco.

\section{FOOTNOTES AND REFERENCES}

* Ames Research Center, National Aeronautics and Space Administration, Moffett Field, CA.

$\dagger$ Department of Physics, University of San

Francisco, San Francisco, CA.

Arnold, A. and P. Bailey. 1954. Alterations in the glial cells following irradiation of the brain in primates. Arch. Pathol. 57, 383-391. 
Brownson, R. H., D. B. Suter, and D. A. Diller. 1963. Acute brain damage induced by low dosage X-irradiation. Neurology 13, 181-191.

Comstock, G. N., R. L. Fleischer, W. R. Giard, H. R. Hart, Jr., G. E. Nichols, and P. B. Price. 1971. Science 172, 154-157.

Haymaker, W. 1969. Effects of ionizing radiation on nervous tissue. The Structure and Function of Nervous Tissue (G. Bourne, ed.) vol. 3, pp. 441-518. Academic Press, New York.

Holloway, R. L., Jr. 1968. The evolution of the primate brain: Some aspects of quantitative relations. Brain. Res. 7, 121-172.

Janssen, P., I. Klatzo, J. Miquel, R. Brustad, A. Behar, W. Haymaker, J. Lyman, J. Henry, and C. Tobias. 1962. Pathological changes in the brain from exposure to alpha particles from a 60 inch cyclotron. Responses of Nervous System to Ionizing Radiation (T. J. Haley and R. S. Snider, eds.) pp. 383-409. Academic Press, New York.

Kraft, L. M., M. A. Kelly, J. E. Johnson, Jr., E. V. Benton, R. P. Henke, R. Cassou, W. Haymaker, D. E. Philpott, F. S. Vogel, and W. Zeman. 1979. Effects of high-LET neon particle radiation on the brain, eyes and other head structures of the pocket mouse: $A$ nistological study. Int. J. Radiat. Biol. 35, 33-61.
Miquel, J., P. R. Lundgren, and J. 0. Jenkins. 1966. Effects of roentgen radiation on glycogen metabolism of the rat brain. Acta Radiol. 5, 123-132.

Smart, I. 1961. The subependymal layer of the mouse brain and its cell production as shown by radioautography after thymidine-H3 injection. J. Comp. Neurol. 116, 325-347.

Tower, D. B. and K.A.C. Elliott. 1952. Activity of acetlycholine system in cerebral cortex of various unanesthetized mammals. Am. J. Physiol. 168, 747-759.

Walker, D. W., D. E. Barnes, S. F. Zornetzer, B. E. Hunter, and P. Kubanis. 1980. Neuronal loss in the hippocampus induced by prolonged ethanol consumption in rats. Science 209, $711-713$.

Zeman, W. 1968. The effects of atomic radiation. Pathology of the Nervous System ( $\mathrm{J}$. Minckler, ed.) vol. 1, pp. 864-939.

Zeman, W. and T. Samorajski. 1971. Effects of irradiation on the nervous system. Pathology of Irradiation. (C. C. Berdjis, ed.) pp. 213-277. Williams and Wilkins, Baltimore.

Zeman, W., H. J. Curtis, and C. P. Baker. 1961. Histopathologic effect of high energy particle microbeams on the visual cortex of the mouse brain. Radiat. Res. 15, 496-514 


\title{
LIFE SPAN STUDIES ON MICE EXPOSED TO HEAVY CHARGED PARTICLES OR PHOTONS: PRELIMINARY RESULTS
}

\author{
E. J. Ainsworth
}

Among the plans under consideration to cope with projected national energy needs is the exploitation of solar energy through the Satellite Power System (SPS), which will convert solar energy to microwaves that are collected by rectennas located on the eartn's surface. If such a venture should come to fruition, thousands of workers, over a period of years, will voluntarily live in a space environment for periods up to several months (DOE and NASA, 1978).

During tnis time, they will be subjected to all the rigors of the space environment, including exposures to low and high LET radiations that could be nontrivial in comparison with average doses sustained by occupational workers on earth. Although the rad dose anticipated from nigh-LET neavy charged particles is small (probably less than $5 \%$ ), in comparison with the dose produced by protons and bremsstrahlung, the heavy cnargedparticle dose becomes of greater concern when appropriate quality factors of 10 to 20 (or pernaps nigher values in the future) are assigned to that component of the dose. What are the appropriate quality factors to be assigned to various heavy charged particles characterized by different LET values, where radiation carcinogenes is is the health hazard of principal

interest? The life-span experiments, designated Skynook, that are described in this chapter were designed to yield information on life span shortening and excess mortality rates in relation to LET. These data will be important to determine the appropriate quality factors (Q) by which to assess human health risks in a space radiation environment.

Two critical radiobiological questions concerning HZE particles are: (1) How do HZE particles conform to existing radiobiological theory with regard to the relationships between $\mathrm{LET}_{\infty}$ and RBE for carcinogenes is, life shortening, and other deleterious late effects of radiation? (2) Are unique lesions produced in proliferating or nonproliferating tissues because of the special physical characteristics of HZE particles? The Skyhook project focuses on the first question in the context of radiationinduced life shortening and carcinogenes is. Life shortening was selected as the primary end point in these pilot studies because of a budget compromise. The optimistic expectation is that : future support will provide for complete autopsies and histopathological: assessments that will. permit limited inferences regarding cause of death and the induction-promotion of neoplastic: diseases. These studies could be accomplished with a second generation of experiments with sample sizes that are tailored based on expectaions of radiation-induced tumor frequency. Only in this way can meaningful data be collected to test alternative hypotheses with regard to the shapes of tumor dose-response curves in relation to LET or other physical parameters associated with heavy charged particles.

The experimental design and selection of radiation doses for Skyhook experiments have been influenced largely by the Janus experiments at Argonne National Laboratory. We would like to compare our results with the Janus results, which compare the life-shortening and carcinogenic effects of fission-spectrum neutrons and gamma radiation using a mouse population that numbers in the tens of thousands (Ainsworth et al., 1976). An unequivocal answer is sought with regard to HZE particle hazard in relation to fission neutron hazard, in relation to dose, dose fractionation, and LET. In terms of risk assessment, should the hazard level associated with the most hazardous HZE particle be lower than that determined for fission neutrons, the $Q$ factor assigned for human risk assessment for neutrons at low doses should not underestimate the risk of HZE particles. If the most hazardous HZE particle proves to be surrogate for fission neutrons, with fission neutrons currently considered a more hazardous form of external high LET radiation, the upper boundary condition for hazard and risk assessment would be delineated, and risk assessment problems would be simplified. This assumes that no unique lesions of a noncarcinogenic nature are of particular concern. At the moment that question remains open, and certain emerging data on rodent corneas and retinas, plus theoretical considerations regarding thermophysical lesions, raise important questions about any firm conclusions to the effect that no unique lesions are expected to make HZE particles (Malachowski et al., 1980; Nelson et al., 1980; Tobias et a ., 19790). Based on cumulative mortality data currently available from the Skyhook experiments, stopping $400 \mathrm{MeV}$ carbon particles appear to be no more, and prooably less effective than fission spectrum neutrons for shortening life span.

\section{DESIGN CONSIDERATIONS AND PROCEDURES}

The Skyhook experiments were designed to test three specific hypotheses:

1. The life-snortening response and its associated RBE is LET $T_{\infty}$ dependent. A maximum

occurs at an LET $T_{\infty}$ value of $100 \mathrm{keV} / \mu \mathrm{m}$, and the effect is independent of HZE particle mass, charge, or velocity.

2. HZE particles characterized by LET similar to that of fission spectrum neutrons (40-70 keV/ $/ \mathrm{m})$ snould (a) be as effective as neutrons in the production of life shortening; (b) the relationship between radiation dose and life shortening snould be nonlinear, conform to a model best fitted by a power 
function; $\therefore$ and be characterized by a slope of approximately 0.5 on a log-log-plot; (c) the RBE value for life-shortening response should be inversely proportional to HZE particles dose; (d) fractionated doses of HZE particles that have an LET similar to that of fission neutrons should behave as a neutron surrogate and produce a higher percentage life shortening than the same single dose when fractionated doses are administered over approximately six months.

3. HZE particles and photons produce lifeshortening responses that are totally dose dependent. No damage interaction modality occurs that could serve to influence cell killing, neoplastic transformation, or other radiation responses that might influence the Iife-span effects when single doses of HZE particles and photons are administered with in 0.5 to $2 \mathrm{~h}$. The essential point is that considerations of track - or dose-averaged LET may not be the only way to predict biological response, RBE, or the quality factor for risk assessment.

Biological factors that may influence the relationship between LET and RBE for cell killing (or other end points) are not established fully. Somewhat different relationships may occur after both in vivo and in vitro irradiation (Tobias et al., 1979a). The RBE maximum may not occur at the same value of LET for mammalian cell cultures propagated in vitro and for cellular or tissue responses after in vivo irradiation. In general, for cell killing based on a loss of proliferative capacity, cultured mammalian cells show a peak RBE in vitro at $L_{E}$. values of $100 \mathrm{keV} / \mu \mathrm{m}$ or higher. In vivo cellular or tissue response may peak at a lower $L_{E T}$ value. Interphase death in human 1 ymphocytes irradiated in vitro shows a peak RBE at $\sim 43 \mathrm{keV} / \mathrm{\mu m}$ for the predominant population, and a second peak at $\sim 190 \mathrm{keV} / \mathrm{\mu m}$ for a small subpopulation (Madhvanath et al., 1976).

Emerging results show that intestinal crypt stem cells, testis stem cells, and hematopoietic stem cells (CFU-S) exhibit relationships that are not entirely compatible with the existing theory on the RBE-LET $T_{\infty}$ relationship. For the intestine and testes, estimates of $D_{0}$ differ when animals were irradiated with HZE particles that differed in mass or charge, but were characterized by

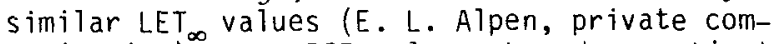
munication). The RBE values, based on estimates of $D_{0}$ for hematopoietic stem cells, are slightly lower than previously published results for fission spectrum neutrons (see Ainsworth et al., Part IV). The RBE value appears to be independent of $L E T_{\infty}$ over the range of 40 to $100 \mathrm{keV} / \mathrm{\mu m}$ because it remains approximately the same at both values of LET. Other data from cell culture experiments have also shown different biological responses caused by changes in ionization track structure, where the $L_{E T}$ remains approximately the same (Cox et al., 1977).

The relationships between $L E T_{\infty}$ and RBE for heavy charged particles are on Ty now being defined accurately for cellular and tissue responses to HZE particle irradiation (Hall et a1., 1977; Guichard et al., 1977; Blakely et al., 1979; Tobias et al., 1979a, Leith et al., 1976; Todd, 1974; Raju et al., 1976; Chapman et a1., 1978). Therefore, the small amount of data on late effects such as life shortening or carcinogenes is is not surprising. Although precise relationships between cell killing, mutagenesis, and carcinogenes is or life shortening in vivo are not understood (Strong, 1977; Mole, 1975), the observations summarized above provide compelling motivation to increase our fundamental knowledge in this area. If mutagenesis is any indication of potential RBE for carcinogenic effects, the information by Cox et al. (1977) described above indicates much higher RBE values for mutation than for cell killing. In addition, the RBE-LET relationship for the two end points specified may show different dependence of. RBE on LET after cells are exposed to low energy HZE particles or photons.

These considerations emphasize the need to characterize life-span responses, in relation to LET $_{\infty}$, for heavy charged particles; they also provide the rationale for specific nypothesis 1 stated above. Within the constraints of the sample sizes available to this experiment, results from the life span study should provide data to test this hypothesis. Departures from strict $L E T_{\infty}$ dependence for 1 ife span response could indicate influences of mass, charge, or particle velocity. Results from the Skyhook experiments should provide information essential to the design of any future experiments that focus more specifically on radiation carcinogenesis, whether they are life-span studies supported by autopsy and histopathological evaluations or experiments that utilize specific tumor model systems.

The protocol for the experiment designated Skyhook-I is shown in Table 1. Radiation quality, doses, estimated values of LET, and sample sizes are shown. Five HZE particle conditions are used to ensure that the same dose-averaged estimate of $L_{E} T_{\infty}$ is provided by both stopping and nonstopping HZE particles at 50 and $100 \mathrm{keV} / \mu \mathrm{m}$, respectively. This should permit specific assessment of any role of ion species, particle mass, or charge. In this way, the shapes of the dose-response curves for life shortening for the five HZE particle conditions can be compared to dose-response curves for 60 Co gamma radiation. Fission spectrum neutrons might not be unique and some HZE particles should be an appropriate surrogate. Should none of the HZE particles evaluated prove to be more nazardous than are fission neutrons, our basic questions will be largely answered, and efforts can be directed to other areas that relate to unique lesions, in particular to critical tissues or to the mechanism by which high LET radiations produce enhancement of tumorigenic response in 1 ife shortening when fractionated doses are given.

Enhancement has significant implications for the underestimation of risk from single-dose studies, and enhancement has been shown convincingly to occur where life shortening, some tumor igenic responses, vascular injury, and chromosomal aberrations are the end points (Ainsworth et al., 
Table 1. Revised Skyhook-I Protocol (2640 CB6 $\mathrm{F}_{1}$ Male Mice)

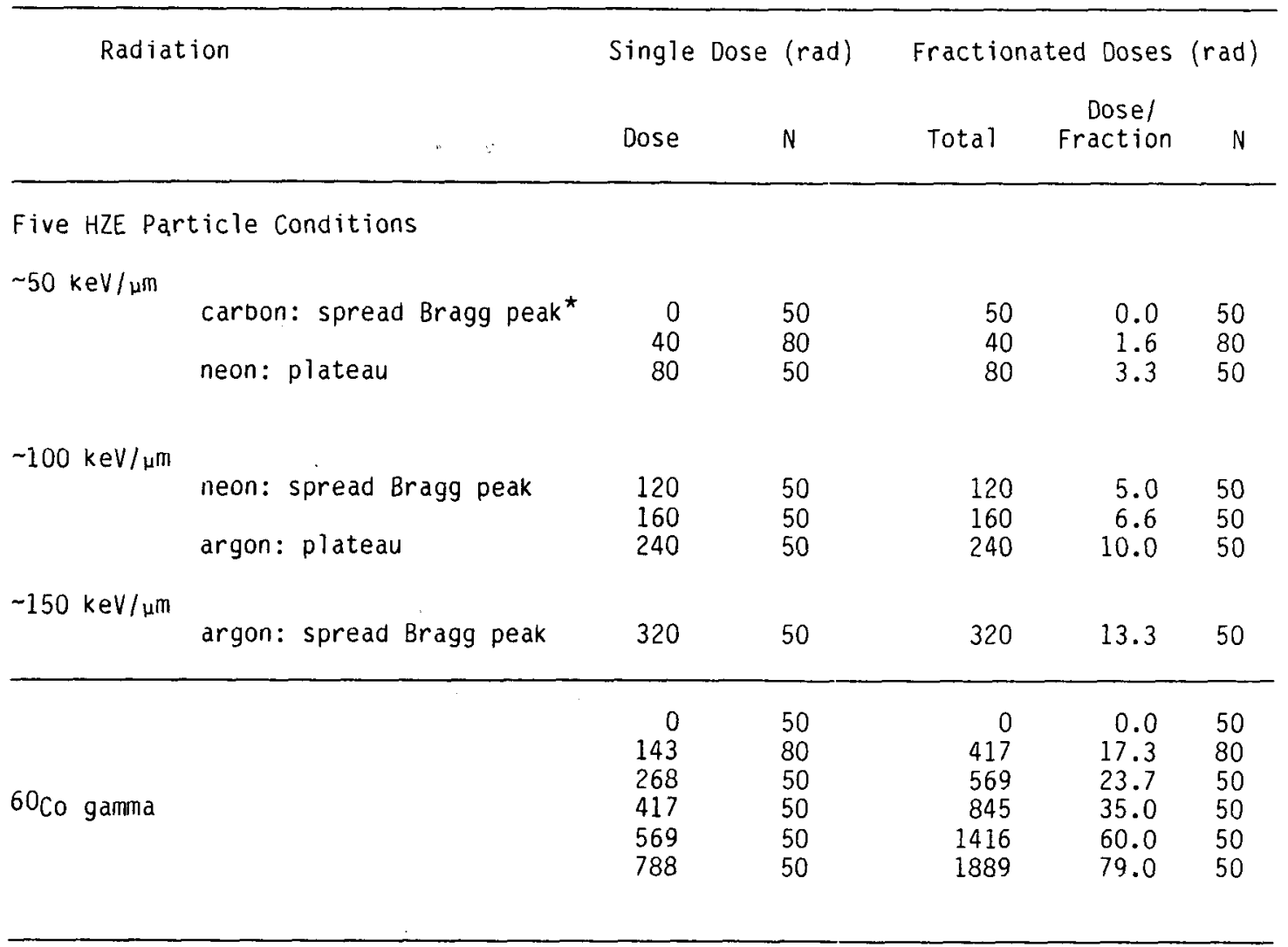

* Total-dose dependence is assessed by exposing groups of 50 mice to single doses of 40 , 80 , or 160 rad of stopping carbon ions, and within 1-2 nours thereafter, administering a single dose of 268 rad gamma radiation.

1974, 1976; Storer et a1., 1979; Fry and Ainsworth, 1977; Yang et al., 1978; Grann et al., 1979). Various national committees concerned with risk estimation have not considered the ennancement phenomenon. When enhancement was.: first demonstrated convincingly (Ainsworth ... et al., 1974), it was assumed to occur only at. high doses where saturation of the tumorigenic. response occurred, and hence had no relevance to the estimation of risk at low doses. However, convincing new data show unequivocally that enhancement occurs at a total fission neutron -. dose of $20 \mathrm{rad}$ (J. F. Thomson, private communica-: tion). The mechanism of enhancement-remains $\therefore$ obscure, al though microdos imetric: explanations have been considered (Ainsworth et al., 1977). Another possibility for the enhancement mechanism is that because of the exponential survival curves that characterize fission spectrum neutrons and some heavy charged particles (at. least in some in vivo and in vitro systems) repeated doses, even low doses, result in cell killing that produces a recruitment of cells into cycle, and this provides for an increased populaion of cells at risk for neoplastic transformaion. When low doses of photons are given, eitner in fractions or continuous exposures at low dose rates, less cell killing and concomitant recruitment should occur, thus the number of cells at risk for neoplastic transformation should not be increased due to actuation of a feedback mechanism triggered by cell killing in organized tissues.

The third specific hypothesis tested in the Skyhook experiments, i.e., the interactions between HZE particles and photons, deserves further explanation. (and indeed, a greater experimental effort than is programmed currently into the experiment). When risk estimates are provided for, exposures to mixtures of high and low LET radiations, e.g., during occupational exposures or in a space environinent, the deleterious effects of high and low. LET radiations are assumed to be totally independent. Rem doses are computed on that basis: If damage interactions were to occur that resulted in a synergistic effect on neoplastic transformation or mutation, computation or rem doses in complex radiation fields would be inaccurate and underestimate radiation risks. The "mixed modality" experiments performed by Ngo et al. (1978) influenced the design of a small fraction of the Skynook experiments. In order to test the nypothes is of total dose dependence, or conversely to consider the question of "additivity," groups of 50 mice 
were given a single dose of carbon ions $(40,80$, or $160 \mathrm{rad}$ ), and then a single dose of $60 \mathrm{Co}$ gamma radiation (268 rad). Simultaneous exposure to the carbon ions and gamma rays would be the preferable means by which to test the hypothesis, but that option is not available to us.

We have done a few preliminary experiments to explore the "additivity" of single doses of high and low LET radiations (Ainsworth et al., 1964). The systematic investigation of potential damage interaction modalities is an emerging area in radiation biophysics (Ngo et al., 1977, 1978). Cellular responses following high-LET radiation were originally thought to represent two conditions: (1) dead or dying cells, and (2) cells that survived without any damage. We now know

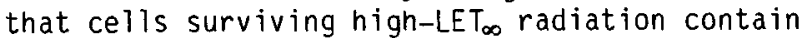
the lesions responsible for mutagenesis, carcinogenesis, and life shortening; RBE values are greater than 1.0. Repair of high-LET damage to DNA does occur following high-LET radiation, albeit to a lesser extent than the repair following low-LET radiation (Roots et al., 1979). Evidence also exists for interaction between damage modalities when cells are exposed to the two radiation qualities over a short time $(30$ to $120 \mathrm{~min})$. Cell cultures irradiated and cultivated in vitro show an ennanced sensitivity to $X$ rays following an initial exposure to fission spectrum neutrons (Ngo et al., 1977). A priming dose of either HZE particles or photons appears to increase the rate of cell killing produced oy the second modality (Ngo et al., 1978). The relationships between cell killing, life shortening, and tumorigenesis are not well understood, but the recent observation that a first dose of nigh-LET radiations (negative pi mesons) influences the sensitivity of cells cultured in vitro to the mutagenic effects of photons ( $J$. Yuhas, private communication) provides a compelling reason to explore the extent to which such phenomena could occur for neoplastic transformation or life shortening and carcinogenesis. This issue is fundamental to radiation risk assessment and computation of rem doses, since damage independence is the bas is of rem dose. Other experiments have shown that the magnitude and duration of the $G_{2}$ block is accentuated following exposure to either negative $p i$ mesons or HZE particles. These studies lend insight to the nature of DNA damage and the sensitivity of surviving cells to a subsequent dose of photons or heavy charged particles (Schlag et al., 1978; Lücke-Hühle et al., 1979). These studies provide the rationale for the third specific hypothesis.

The current Skyhook series of experiments consist of two different experiments conducted under slightly different conditions. The first, Skyhook-P (pilot) used $B_{6} C_{1} /$ ANL mice produced by the animal facility at ANL. These animals were shipped from ANL in connection with a collaborative study on harderian gland carcinogenesis, but they could not be used in those experiments. Most of the 200 animals were in the age range of 120-150 days, but a significant fraction was about 200-250 days old. This age variation is undesirable because the age at irradiation is known to influence radiationinduced life shortening (Ainsworth et al, 1974).
At the time the experiment was initiated these were the only animals projected to be available for six to nine months. We wanted to use the same line of mice as the Janus experiments at ANL so that, although preliminary, a direct comparison between fission neutrons and carbon ions could be made. Pursuant to making direct comparisons between heavy charged particles and fission neutrons, an arrangement had been made with ANL to use the Argonne nybrid mouse in the Skyhook series of experiments, but this could not be accomplished because of administrative and budgetary reasons. At that time, arrangements were made to purchase the reciprocal hybrid, designated $\mathrm{CB}_{6} \mathrm{~F}_{7}$, from the Roscoe $\mathrm{B}$. Jackson Memorial Laboratory, Bar Harbor, Maine. Although females would have been preferable because they are more sensitive to life shortening and they present ovarian tumors and cysts, only males were commercially available. Consequently, the major experimental effort involves the use of $\mathrm{CB}_{6} \mathrm{~F}_{1}$ mice (Table 1). Based on a genetic pedigree, the life shortening and tumor responses in the two hybrids are expected to be the same; indeed, D. Grahn has indicated that radiation induced life shortening under conditions of chronic gamma radiation is similar in the two nybrids (personal communication, 1977).

The Bevalac radiation facilities and dosimetry are described by Lyman et al. in Part VI, Section A. Unanesthetized mice were irradiated four at a time in a $3.5 \times 10.5 \mathrm{~cm}$ lucite animal holder positioned approximately $6 \mathrm{~m}$ from the thin mylar window where the charged particle beam enters Cave II at the Bevalac. Interposed in the beam are lead scattering foils, a collimator, upstream and downstream ionization chambers, a multiwire proportional chamber, and a water column. The variation in dose over the animal holder did not exceed $10 \%$, and the instantaneous dose rates occasionally varied (a few rad/min to approximately $500 \mathrm{rad} / \mathrm{min}$ ). The lowest dose rates were obtained when scheduling considerations at the Bevatron made it necessary to use the Bevatron's local $20 \mathrm{MeV}$ injector rather tnan the SuperHILAC as a source for carbon ion. The $20 \mathrm{MeV}$ injector was used primarily to administer the fractionated doses of carbon ions over six months in the Skyhook-I experiment. A 4-cm spiral ridge filter interposed in the beam line spread the Bragg peak over the anterior-posterior dimensions of the mice. The particle energies used in the Skyhook-I experiment were $225 \mathrm{MeV}$ carbon, $400 \mathrm{MeV}$ neon, or $570 \mathrm{MeV}$ argon. A different carbon energy was used in the Skyhook-P experiment $(400 \mathrm{MeV})$. The lower energy for carbon was used in Skyhook-I because we wanted to minimize beam fragmentation produced in the water column when the range was moderated. The desired residual range was achieved with $225 \mathrm{MeV}$ carbon when the water column setting was minimal $(<0.5 \mathrm{~cm})$. Fragmentation was expected to be greater with carbon, which is the lightest ion used in these experiments, and fragments might be expected to have a lower LET and biological effect than the primary particles. Therefore, use of a lower energy beam provides fewer fragments and focuses the conclusions that could be drawn from the carbon ion studies. The degree of beam fragmentation is a major concern for all 
the beams used in the Skyhook experiments, and ongoing microdosimetric studies should provide information important for interpretation of our results (see Howard et al. in Part II).

For ${ }^{60} \mathrm{Co}$ gamma radiation, two groups of four animals were exposed simultaneously in the same lucite holders used for the HZE particle irradiation. A 2-mm lucite build-up layer was affixed to the proximal surface of the animal holder to achieve electron equilibirum. Single doses over a range of 143-788 rad were given at 80-90 rad/min; the animal holders were positioned $62-72 \mathrm{~cm}$ from the $1,500 \mathrm{Ci}$ source. Fractionated doses ranging from 17-79 rad were given by positioning the holders approximately $200 \mathrm{~cm}$ from the source where the dose rate was approximately 9-10 rad/min; exposure time was varied.

The experimental animals are housed five per cage on sawdust bedding (Sequoia Forest Products); acid water ( $\mathrm{pH} 2.5$ ) and Formulab Chow 5008

(Ralston Purina) were continually available. The animal rooms were maintained at $73-76^{\circ} \mathrm{F}$ with a humidity range of $30-50 \%$. During the first few months after irradiation, death checks were made once or twice weekly, but beginning at six months, the animal census was tallied five days per week. Although there is a clear understanding with the funding agency (NASA) that Skyhook is specifically a life-shortening experiment, we have performed some autopsies and have taken some tissue samples (including tumors) with the hope that future support would provide for some level of histopathological contributions. In February 1980 an informal arrangement was made with $L$. Kraft at the NASA/AMES Research Center (Moffett Field, CA) whereby formaldehyde-preserved carcasses from Skyhook-P and Skyhook-I experiments will be retained for future collection of autopsy data. Although there is currently no support at $L B L$ or NASA/Ames Research Center for this part of the Skyhook studies, we are optimistic that funding will be provided in the future, and we look forward to L. Kraft's collaboration in these studies.

The sample sizes available in the Skyhook-I experiment may be inadequate to provide meaningful data on the shapes of dose-response curves, in relation to LET, for tumors of interest. This matter has been considered by S. A. Tyler, our biomedical consultant, and J. F. Thomson, currently the principal investigator for the Janus Program, has kindly provided us with complete information on total tumor incidence and age specific tumor frequencies from all unirradiated control animals currently available from the Janus program. J. F. Thomson has also con: sented to make available to us comparable tumor data that are available now based on animals that were irradiated in the Janus program between 1971-1974. This information will be critical for projections of the extent to which meaningful tumor data could be gained from the Skyhook-I series of experiments based on the currently orogrammed sample sizes. Consultations have Tready occurred with R.J.M. Fry and R. L. Ullrich, Oak Ridge National Laboratory, concerning the potential information gained from complete histopathological studies on the Skyhook-I animals. Extended collaborative support in this area might be arranged, and should the necessary funding be obtained, we would like to establish a Pathology Advisory Committee that could advise how to proceed most economically and efficiently.

\section{RESULTS AND DISCUSSION}

All irradiations for the Skynook-P experiment were completed in August 1978. As of June 1980, those animals were approximately 700 days at risk, and preliminary information is now available. The current status of the Skyhook-I experiment is summarized in Table 2 and the number of days at risk varies from less than 100 to approximately 570. In order to maximize the experimental variance, and accommodate normal Bevalac operating schedules, the experimental design provided for accumulation of the total sample for any given "condition" by insertion of at least three replicates from different shipment of animals. This is why the number of days at risk is so variable. The number of animals that remain to be irradiated in the Skyhook-I experiment is also shown in Table 2. One exception to the number of replications applies to the group that received 24 fractionated doses of $225 \mathrm{MeV}$ carbon ions over approximately six months. It was necessary to irradiate all 340 animals assigned to that group as a single cohort in order to accommodate the operational schedule at the Bevalac. Heroic scheduling efforts were required on the part of the Bevalac operations staff to comply with our experimental needs for such a long duration of fractionated exposures. To accomplish those fractionated exposures it was necessary to modify appreciably the operations schedule at the Bevalac and to use the local $20 \mathrm{MeV}$ injector week ly during scheduled budgetary or maintenance driven shut-downs of the Bevalac.

The cumulative mortality data from Skyhook-P are compared with results from a Janus experiment, designated (JM-2) initiated at ANL between 1971 and 1973. Figure 1 compares the charged particle and neutron results and Figure 2 compares results obtained after single doses of gamma radiation. Based on the cumulative mortality results available at this time, the mortality curves for Skyhook-P mice that received either 240 or 80 rad are shifted to the right on the $x$-axis, which indicates that the projected mean or medium survival time will probably be longer following exposure to carbon ions. The difference between carbon ions and fission neutrons is particularly pronounced at the total dose of $80 \mathrm{rad}$. If this trend is maintained, it could mean that the shape of the dose-response curve for days lost per rad or percent life shortening. will be different for carbon ions than for fission spectrum neutrons. These preliminary results should be interpreted cautiously. The sample sizes in Skynook-P are small, and the heterogeneity of ages among Skyhook-P animals provides a further complicating variable. Indeed, over the range of 110-278 days, the increased age at irradiation decreases the degree of life shortening particularly for photon irradiations, but probably also has some effect on life shortening produced by nigh LET radiations (Ainsworth et al., 1976). The age hetero- 
Table 2. Skyhook-I Status, July 1980

\begin{tabular}{|c|c|c|c|c|c|c|c|}
\hline Ion & $\begin{array}{l}\text { Energy } \\
(\text { MeV })\end{array}$ & Position & $\begin{array}{l}\text { Radiation } \\
\text { Condition }\end{array}$ & $\begin{array}{l}\text { Total } \\
\text { Programmed } \\
\text { Sample Size }\end{array}$ & $\begin{array}{l}\text { No. Mice } \\
\text { Entered in } \\
\text { Exp. Status }\end{array}$ & $\begin{array}{l}\text { Range of } \\
\text { Days at } \\
\text { at Risk }\end{array}$ & $\begin{array}{l}\text { No. Needed } \\
\text { to Complete } \\
\text { Experiment }\end{array}$ \\
\hline \multirow[t]{2}{*}{$12 \mathrm{C}$} & 225 & Spread Bragg peak & Single & 330 & 330 & $155-555$ & 0 \\
\hline & & & 24 fractions & 3.30 & 340 & 595 & 0 \\
\hline $12 c+60 c o$ & & -- & Mixed Modality & 150 & 85 & $147-393$ & 0 \\
\hline $20 \mathrm{Ne}$ & 557 & Spread Bragg peak & Single & 330 & 108 & $35-239$ & 122 \\
\hline $20 \mathrm{Ne}$ & 557 & Plateau & Single & 330 & 104 & 239 & 226 \\
\hline $40 \mathrm{Ar}$ & 570 & Spread Bragg peak & Single & 330 & 0 & $-\cdots$ & 330 \\
\hline $40 \mathrm{Ar}$ & 570 & Plateau & Single & 330 & 0 & -- & 330 \\
\hline $60 \mathrm{Co}$ & & --- & Single & 280 & 316 & $406-585$ & 0 \\
\hline $60 \mathrm{Co}$ & & -- & 24 Fractions & 280 & 164 & $415-563$ & 16 \\
\hline $\begin{array}{l}\text { Unirradiated } \\
\text { Controls }\end{array}$ & & & & 450 & 224 & $85-595$ & 226 \\
\hline
\end{tabular}

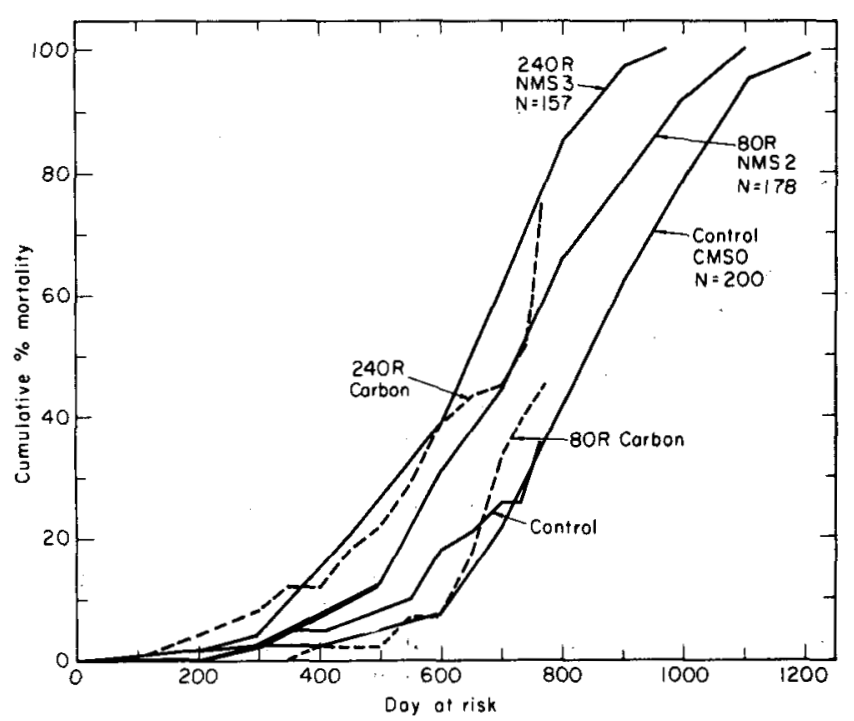

Figure 1. Cumulative percent mortality as a function of days at risk for male $\mathrm{B}_{6} \mathrm{CF}_{1}$ mice irradiated with fission spectrum neutrons (from Janus experiments) and carbon ions (from Skyhook-P experiments). (XBL 808-3639)

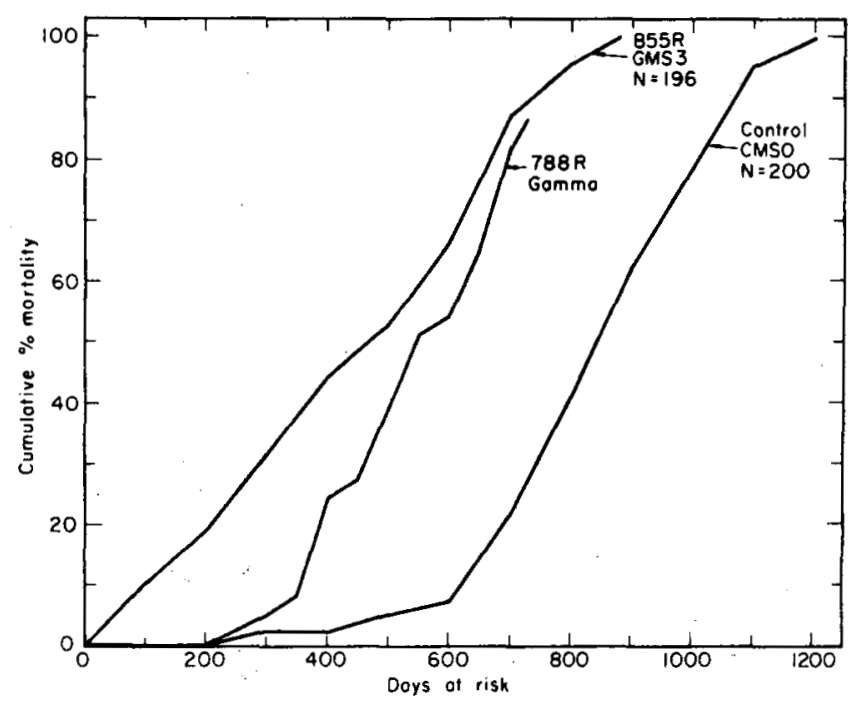

Figure 2. Cumulative percent mortality as a function of days at risk for male $\mathrm{B}_{6} \mathrm{CF}_{1}$ mice irradiated with single doses of $60 \mathrm{Co}$ gamma rays. Data for 855 rad and the unirradiated controls are from the Janus experiments. (XBL 808-3638) 
geneity could also account for the small apparent shift to the left, which indicates a shorter median survival time among the unirradiated controls in the Skyhook-P experiment. Although correct in concept, large variations among mean and median survival times among unirradiated controls have been observed in the Janus experiments, and an approximate 50 day displacement to the left of the single Skyhook control curve is of little significance. The unirradiated control curve assumes considerable importance in critical assessment of the extent to which age neterogeneity at the time of the irradiation (or entrance into the experiment) should influence the Skyhook $-P$ result. Although $225 \mathrm{MeV}$ carbon ions could be more effective than $400 \mathrm{MeV}$ carbon ions, because of diminished fragmentation through virtual elimination of the water column, the data in hand at the moment are consistent with the conclusion that $400 \mathrm{MeV}$ carbon ions are less effective than fission neutrons in producing life shortening. This tentative conclusion is consistent with other results on harderian gland carcinogenesis in the same strain of mouse following exposure to $400 \mathrm{MeV}$ carbon ions (see Alpen et al., Part IV).

Table 3 summarizes the results currently available from Skyhook-I where dose-dependent mortality is compared among the single cohort that received fractionated doses of carbon ions and the first replicate that received single doses. The number of days at risk are greater for the animals that received fractionated doses (565 days) than for animals that received single doses (524 days). Because the dose range is the same for the two radiation conditions, and at this time no dose dependent terms are strongly apparent, comparison of the net percentage mortality between the two groups may be meaningful. At this time, $17 \%$ mortality has occurred in both groups and had enhanced life shortening been produced by fractionation of the carbon ion dose, somewhat higher mortality might be expected. The 41 day excess time at risk among the animals that receive fractionated doses should emphasize any such trend. Again, cautious interpretation of these preliminary results is necessary, but the mortality data available at this time provides no evidence for enhancement by dose fractionation with carbon ions. A11 the mortality comparisons presented here are based on animals presumed to have succumbed from radiation-associated diseases. Animals lost, stolen, strayed or decapitated by cage tops have been deleted; in the case of Skyhook-P this amounts to approximately $3 \%$ of the total sample and in the case of Skyhook-I the fraction is $0.9 \%$.

In sum, at this time, it appears the $Q$ factor used for fission spectrum neutrons could be conservative if it were used for assessment of risk following exposure to $400 \mathrm{MeV}$ carbon ions.

NOTE: Quantitative estimates of dose-averaged $L E T_{\infty}$ values presented here should be considered as approximations only, because different calculational models yield markedly different results. In addition, for spread Bragg peaks the distributions of $L^{2} T_{\infty}$ values is broad with the fraction of LET particles rising sharply at the distal peak. Ongoing beam fragmentation studies will yield new data that will contribute to improved estimation of dose-averaged LET $_{\infty}$. Microdosimetric studies provide a separate measurement of beam quality.

\section{ACKNOWLEDGEMENT}

The assistance of John Prioleau, Lynn Manlmann, Andy Snih, Colleen Caudill, and Panna Lee is gratefully acknowledged. The cooperation of Jerry Howard, Tom Criswell, and the Bevalac operations staff is also gratefully acknowledged. These studies were, in part, supported by NASA.

Table 3. Mortality at:524-565 Days in $\mathrm{CB}_{6} \mathrm{~F}_{1}$ Mice that Received - Single or Fractionated Doses of $225 \mathrm{MeV}$ Carbon Ions

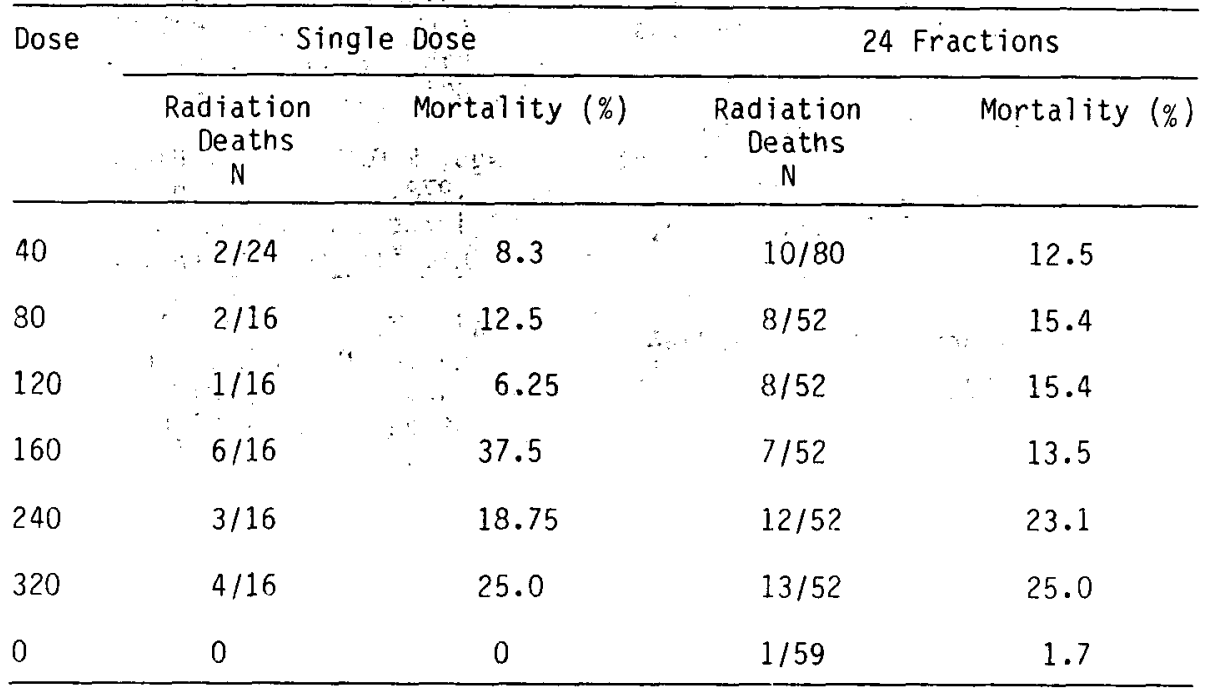


REFERENCES

Ainsworth, E. J., G. F. Leong, K. Kendall, E. L. Alpen, and M. L. Albrignt. Pulsed irradiation studies in mice, rats and dogs. 1964. Biological Effects of Neutron and Proton Irradiations, vol. II, pp. 15-30. International Atomic Energy Agency, Vienna.

Ainsworth, E. J., R.J.M. Fry, D. Grann, F. S. Williarnson, P. C. Brennan, S. P. Stearner, A. V. Carrano, and J. H. Rust. 1974. Late effects of neutron or gamna irradiation on mice. Biological Effects of Neutron Irradiation, pp. 359-379. International Atomic Energy Agency, Vienna.

Ainsworth, E. J., R.J.M. Fry, P. C. Brennan, S. P. Stearner, J. H. Rust, and F. S. Williamson. Life shortening, neoplasia, and systemic injuries in mice after single or fractionated doses of neutron or gamma radiation. 1976. Biological and Environmental Effects of DoseLevel Radiation, vol. 1, pp. 77-92. International Atomic Energy Agency, Vienna.

Ainswortin, E. J., R.J.M. Fry, F. S. Williamson, P. C. Brennan, S. P. Stearner, V. V. Yang, D. A. Crouse, J. H. Rust, and T. B. Bovak. 1977. Dose-effect relationships for life. shortening tumorigenesis and systemic injuried in mice irradiated with fission neutron or 60 Co gamma radiation. Proceedings, Fourth International Congress, International Radiation Protection Association, vol. 4, pp. 1143-1151. Montreuil Press, France.

Blakely, E. A., C. A. Tobias, T.C.H. Yang, K. C. Smith, and J. T. Lyman. 1979. Inactivation of human kidney cells by high energy monoenergetic heavy ion beams. Radiat. Res. 80 , $122-160$.

Chapman, J. D., E. A. Blakely, K. C. Smith, R. C. Urtasun, J. T. Lyman, and C. A. Tobias. 1978. Radiation biophysical studies with mammalian cells and a modulated carbon ion beam. Radiat. Res. 74, 101-111.

Cox, T., J. Thacker, D. T. Goodhead, R. J. Munson. 1977. Mutation and inactivation of mammalian cells by various ionizing radiations. Nature (London) 267, 425-427.

DOE (Department of Energy) and NASA (National Aeronautics and Space Administration). Concept Development and Evaluation Program. SPS Reference System Report No. 0023, Washington, D.C.

Fry, R.J.M. and E. J. Ainsworth. 1977. Radiation injury: Some aspects of the oncogenic effects. Fed. Proc. 36, 1703-1707.

Grahn, D., B. H. Frystak, C. H. Lee, J. J. Russe 11 and A. Lindenbaum. 1979. Dominant lethal mutations and chromosome aberrations induced in male mice by incorporated $239 \mathrm{pu}$ and by external fission neutron and gamma irradiation. Proceedings, International
Symposium on Biological Implications of Radionuclides Released from Nuclear Industries, 26-30 March 1979, vo 7. 1, pp. 163-184. International Atomic Energy Agency, Vienna.

Guichard, M., A. Blachet, and E. P. Malaise. 1977. Measurement of RBE, OER, and recovery of potentially lethal damage of a $645 \mathrm{MeV}$ helium ion beam. using EMT6 cells. Radiat. Res. 71, 413-429.

Hall, E. J., R. P. Bird, H. H. Rossi, R. Coffey, J. Varga and Y. M. Lam. 1977. Biophysical studies with high-energy argon ions.

2. Determinations of the relative biological effectiveness, the oxygen enhancement ratio, and the cell cycle response. Radiat. Res. 70, 469-479.

Leith, J. T., K. H. Woodruff, and J. T. Lyman. 1976. Early effects of single doses of 375 $\mathrm{MeV} /$ nucleon 20 Neon ions on the skin of mice and hamsters. Radiat. Res. 65, 440-450.

Lücke-Hünle, C., E. A. Blakely, P. Y. Cnang, and C. A. Tobias. 1979. Drastic G2, arrest in mammalian cells after irradiation with heavy ion beams. Radiat. Res. 79, 97-112.

Madhvanath, U., M. R. Raju, and L. S. Kelly. 1976. Survival of human lymphocytes after exposure to densely ionizing radiations. ERDA Symposium Series 37, 125-139.

Malachowski, M. J., D. W. Philpott, and R. Corbett. 1980. Retinal tissue response to accelerated iron nuclei. Radiat. Res. 83, 472. (Abstract).

Mole, R. H. 1975. Ionizing radiation as a carcinogen: Practical questions and academic pursuits. Br. J. Radiol. 48, 157-169.

Nelson, A. C., T. L. Hayes, and C. A. Tobias. 1980. Scanning electron microscope study of membrane lesions caused by individual heavy ions. Radiat. Res. 83, 472. (Abstract).

Ngo, F.Q.H., A. Han, and M. M. Elkind. 1977. On the repair of sublethal damage in $V 79$ Chinese hamster cells resulting from irradiation with fast neutrons or fast neutrons combined with X-rays. Int. J. Radiat. Biol. 32, 507-511.

Ngo, F.Q.H., E. A. Blakely, and C. A. Tobias. 1978. Does an exponential survival curve of irradiated mammalian cells imply no repair? Radiat. Res. 74 (Abstract).

Raju, M. R., E. A. B lakely, J. Howard, J. T. Lyman, D. P. Kalofonos, B. Martins, and T.C.H. Yang. 1976. Human cell survival as a function of depth for a high-energy neon ion beam. Radiat. Res. 65, 191-194.

Roots, R., T. C. Yang, L. Craise, E. A. Blakely, and $C$. A. Tobias. 1979. Impaired repair capacity of DNA breaks induced in mammalian cellular DNA by accelerated heavy ions. Radiat. Res. 78, 38-49. 
Schlag, H., K. F. Weibezahn, and C. LückeHühle. 1978. Negative pion irradiation of mammalian cells. II. A comparative analys is of cel1-cycle progression after exposure to $\pi^{-}$mesons and cobalt gamma-rays. Int. J. Radiat. Biol. 33, 1-10.

Storer, J. R., L. J. Serrano, E. B. Darden, J. R. McJernigan, and R. L. Ullrich. 1979. Life shortening in RFM and BALB/C mice as a function of radiation quality, dose, and dose rate. Radiat. Res. 78, 122-161.

Strong, L. C. 1977. Theories of pathogenes ismutation and cancer. Progress in Cancer Research and Therapy. Genetics of Human Cancer (J. MuTvinill et a..) vol. 3, pp. 401-414. Raven Press, New York.

Tobias, C. A., E. L. Alpen, E. Blakely, J. Castro, A. Chatterjee, G. Chen, S. Curtis, J. Howard, J. T. Lyman, and F.Q.H. Ngo. 1979a.
Radiobiological basis for heavy ion therapy. Treatment of Radioresistant Cancers. Elsevier, Natl. Holland Biomedical Press, Amsterdam, the Netherlands.

Tobias, C. A., A. Chatterjee, M. J. Malachowski, E. A. Blakely, and T. L. Hayes. 1979b. Tracks in condensed systems. Proceedings, Sixth International Congress of Radiation Research (S. Okada et al., eds.), pp. 146-156. Japanese Assocociation for Radiation Research, Tokyo, Japan.

Todd, P. 1974. Radiobiology with heavy charged particies directed at radiotherapy. Europ. J. Cancer 10, 207-210.

Yang, V. V., S. P. Stearner, and E. J. Ainsworth. 1968. Late ultrastructural changes in the mouse coronary arteries and aorta after fission neutron or 60 Co $y$ irradiation. Radiat. Res. $74,436-456$. 
PART VI. MEDICAL

APPLICATIONS OF HEAVY IONS

Section A: Radiation Therapy 
(n)

$\bullet$
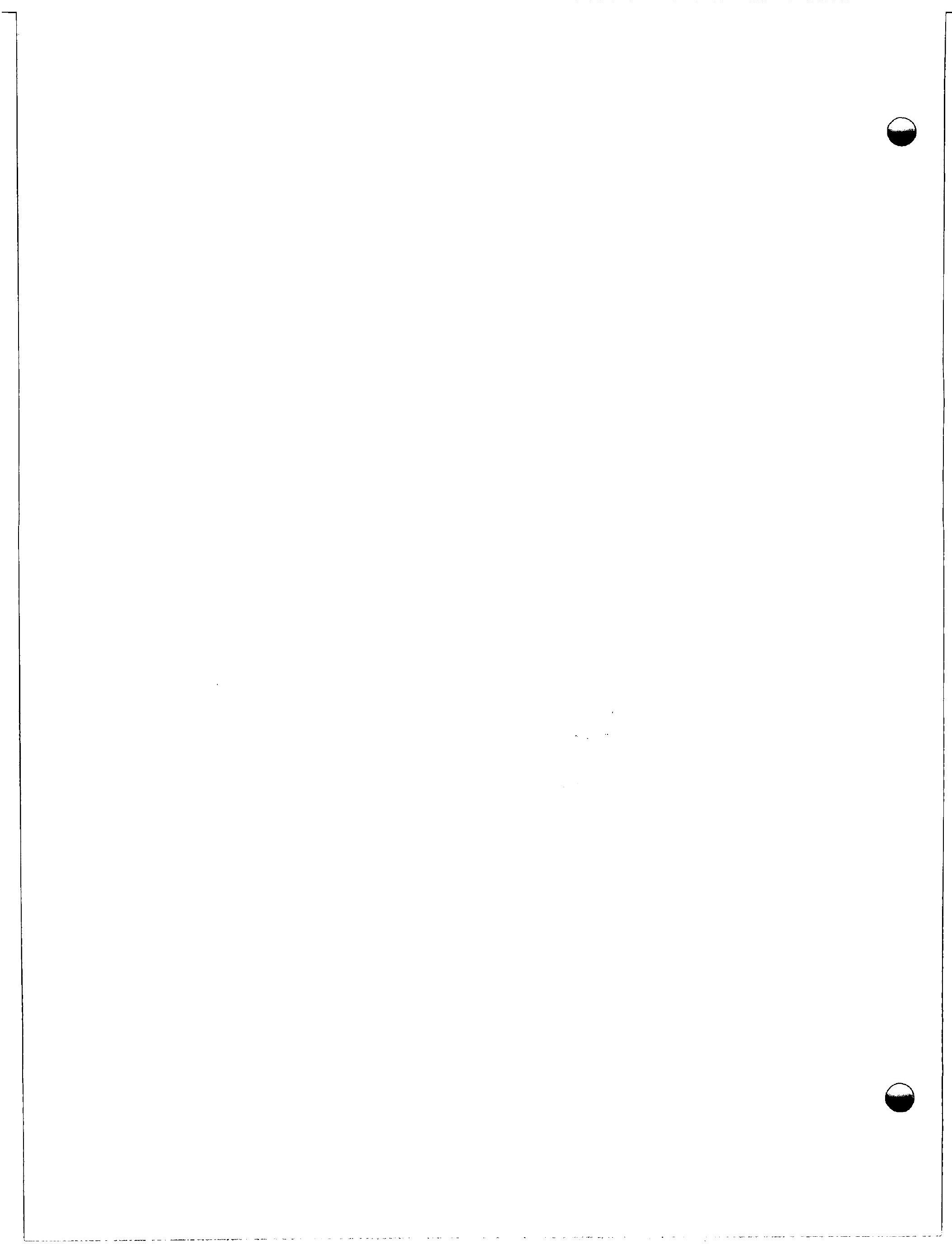


\title{
CLINICAL RESULTS IN HEAVY PARTICLE RADIOTHERAPY
}

\author{
J. R. Castro, ${ }^{\dagger}$ J. M. Quivey, ${ }^{\dagger}$ W. M. Saunders, ${ }^{\dagger}$ K. H. Woodruff, ${ }^{\ddagger}$ G. T. Y. Chen, J. T. Lyman \\ S. Pitluck, C. A. Tobias, R. E. Walton, and T. C. Peters
}

The estimated incidence of new cancer patients in the United States for 1980 approximates 800,000 , with a mortality of nearly 400,000 . About half of these cancer patients will require radiation treatment in their disease management. Radiation therapy used alone or in combination with surgery or chemotherapy is an effective curative treatment modality for a large number of patients with cancers confined to the organ of origin or with direct spread to contiguous anatomical regions, providing the adjacent normal tissues are sufficiently spared to provide continuing function of the affected region.

Normal tissue sparing is enhanced when: (1) the high dose zone more closely approximates the tumor target volume and excludes adjacent structures, and/or (2) the biologic effect on tumor cells is greater than that on surrounding normal cells. Heavy charged particle radiotherapy offers an opportunity to improve both factors over conventional radiation techniques. Although low-LET radiation therapy has been used effectively in many tumors, the factors that diminish the ability of radiation to achieve tumor control (CROS, 1978) include:

1. tumors with cellular characteristics that make conventional irradiation ineffective, e.g., tumors with a high fraction of radioresistant hypoxic cells, tumors with a nigh capacity for repair of sublethal or potentially lethal radiation damage, or tumors with a rapid proliferation such that repopulation occurs between fractions; 2. lesions involving or contiguous with critical dose limiting normal tissues; 3. tumors that nave extensively invaded and damaged the normal structure of origin such that satisfactory healing is precluded and unacceptable sequelae result;

4. tumors so large that the surrounding normal tissues would not tolerate the dose of radiation therapy necessary to kill all malignant cells;

5. poorly reoxygenating tumors and slow growing tumors.

Any modality or combination of modalities that directly affects the tumor factors described above may lead to improved local and regional control. Interest has been growing in recent years in the clinical use of proton, neutron, negative pion, and heavy-ion beams, collectively called particle radiation therapy, to improve local control of various types of tumors. The heavy charged particle radiation therapy clinical trial at $L B L$ is designed to test the clinical ignificance of using helium and heavier particles to irradiate human cancers.

\section{GOALS OF RADIATION THERAPY}

The aim of clinical radiation therapy is to destroy neoplastic cells while the structural integrity of normal tissues is maintained. For most tumors, the dose required to destroy the tumor is close to the tolerance level for normal tissue. Clinical and laboratory evidence have indicated that the larger the tumor, the greater the dose of radiation required for control. Macroscopic tumor may require doses of 7,000 rad over 7 weeks or higher for tumoricidal effect, but this large total dose increases the potential for damage to adjacent normal structures. Surgical removal of gross tumor, leaving only microscopic residual disease, allows delivery of moderate radiation doses in the range of 5,000 rad over 5 weeks for $90 \%$ or higher local control rates (Fletcher, 1977). In general, these dose schedules appear independent of tumor cell type.

Some tumors cannot be exposed to the $\mathrm{nigh}$ radiation doses required for control because the adjacent normal structures and tissues would be unavoidably included in the target volume. (The target volume is defined as the tumor target plus an additional safety zone to include possible microscopic extension.) The nature of the structures included in the high dose zone and the volume of normal tissue irradiated are critical with respect to possible repair of radiation damage. The volume irradiated thus becomes just as important as the particular tissue target.

The overall time of irradiation and the number of fractions delivered are also important factors in most forms of clinical radiation therapy. Sublethal radiation damage can be repaired between fractions, presumably to a greater degree by normal tissues than by tumor cells. Fractionation and protraction are also important considerations in tumor cell reoxygenation, which increases the possibility of local and regional control.

The exact mechanism of radiation damage to cells is not yet known. Radiation may well destroy the bulk of the tumor cells, but the body's own mechanisms may be required to provide permanent control of the disease. The sensitivity of various human tumors in vivo is not reflected accurately in in vitro cultures of mammalian tumor lines. 0ther factors likely to be important in clinical radiation trials include cell population kinetics, tumor vascularity, the particular structure in which the tumor lies, and adjacent normal tissue response.

Current radiation research is directed at increasing the therapeutic gain factor (TGF) which is defined as: 
TGF $=$

damage to the tumor (local control)

damage to normal structures (complications)

If the TGF is $<1.0$, then that radiation has no usefulness in that particular clinical situation. Among the techniques to maximize TGF are the following:

1. debulking surgery with the removal of gross tumor followed by the irradiation of microscopic residual disease;

2. maximized radiation dose in the target volume using combination of different beam portals, wedge filters, electron beams, dynamic treatment planning and other physical techniques;

3. combinations of chemotherapy and radiation therapy;

4. combinations of hyperthermia and radiation therapy;

5. combinations of hypoxic cell sensitizers

and radiation therapy;

6. altered fractionation schedules;

7. high LET particle irradiation.

\section{RATIONALE FOR PARTICLE THERAPY}

Heavy charged particle radiation produces ionization events that are more closely spaced than those of low LET photon radiation, which causes high LET radiation to be more biologically effective.: The energy lost per unit of path length is much increased for high LET radiation, such that: cell sensitivity is less dependent on oxygen concentration; cell killing is more efficient; cells are less capable of repairing radiation damage; sensitivity of the cells is not as dependent on position of the cell in the cell replication cycle; and cells of high ploidy are relatively more sensitive than euploid cells.

For charged high LET particles such as helium, carbon, neon, and argon nuclei, an additional gain results from a more efficient dose distribution, which maximizes the irradiation of a target volume and minimizes the dose to normal structures outside of the target volume. Improved ability to deliver radiation to deeper structures using megavoltage $X$ rays has resulted in higher local and regional control rates than were possible with orthovoltage $x$ rays (U.S. Senate Report, 1970). Thus, further improvement in dose distribution with heavy charged particle radiation therapy might add to current local and regional control rates for some types of tumors.

The physical advantages of heavy charged particle beams result from the fact that they have a charge, and include: a variable but we 11defined range of penetration; sharp lateral edges; sharp distal falloff beyond the extended peak; and favorable peak-to-entrance dose ratios. Protons and helium ions provide the best physical dose distributions, followed by carbon and neon ions. Heavier charged particle beams, such as carbon, neon and argon, do have some secondary fragments that cause a small dose distal to the peak.
The advantage of treating tumors with heavy charged particle radiation is that the beam (or multiple beams from different entry portals) can be arranged such that the extended peak is made to coincide with a specific target volume. Tissues adjacent, superficial, or distal to the tumor are irradiated by the entrance or plateau portion and/or the exit portion of the particle beam, thus affording a lower dose to normal structures. This ability to concentrate the effective high dose zone in the region of a deepseated tumor represents a significant advantage for many clinical situations over dose distributions currently available with conventional photon or electron radiation.

Figure 1 is a schematic illustration of absorbed dose as a function of depth in the body for neutrons and several charged particle beams with peaks spread to $10 \mathrm{~cm}$ (Raju et al., 1978a). The carbon beam has a relatively large exit dose because the beam depicted here had a larger penetration than the others, and thus provided more material upstream for fragmentation.

A comparison of treatment plans with conventional and charged particles is shown in Figure 2. These plans contrast biologically corrected isodose contours of a neon ion beam with low-LET $X$-ray plus electron isodose contours in a deep seated target location for the pancreas. Less dose to normal structures is obtained with the charged particle beam. Other regions in the body show similar advantages with charged particle radiation plans despite the inhomogeneity corrections required for bone, air, and other tissue density changes encountered in the particle path.

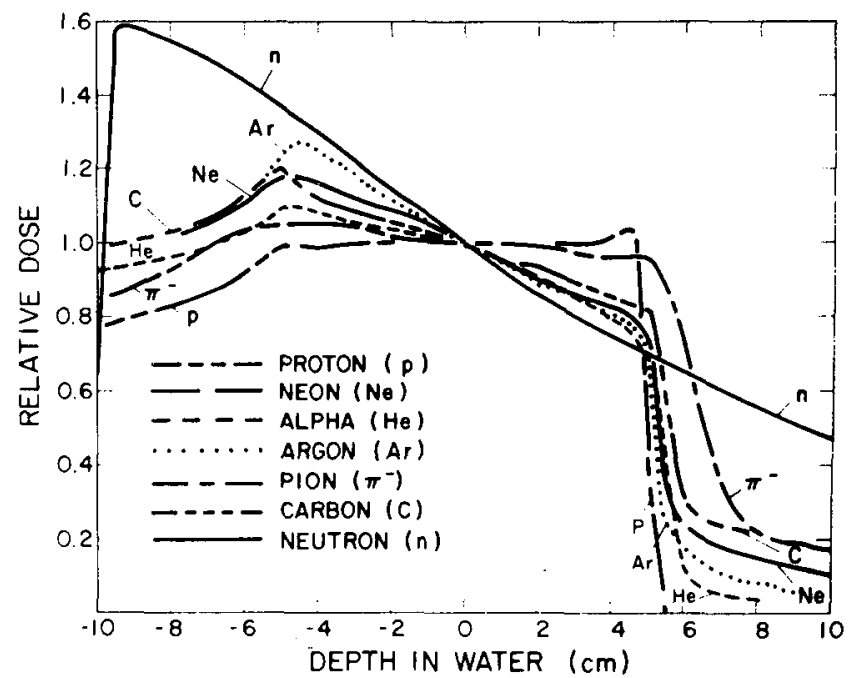

Figure 1. A comparison of experimentally obtained dose vs. depth curves for proton, neutron, pion, helium, carbon, neon and argon beams, normalized to unity at the center of the extended peak region. (XBL 799-11294) 

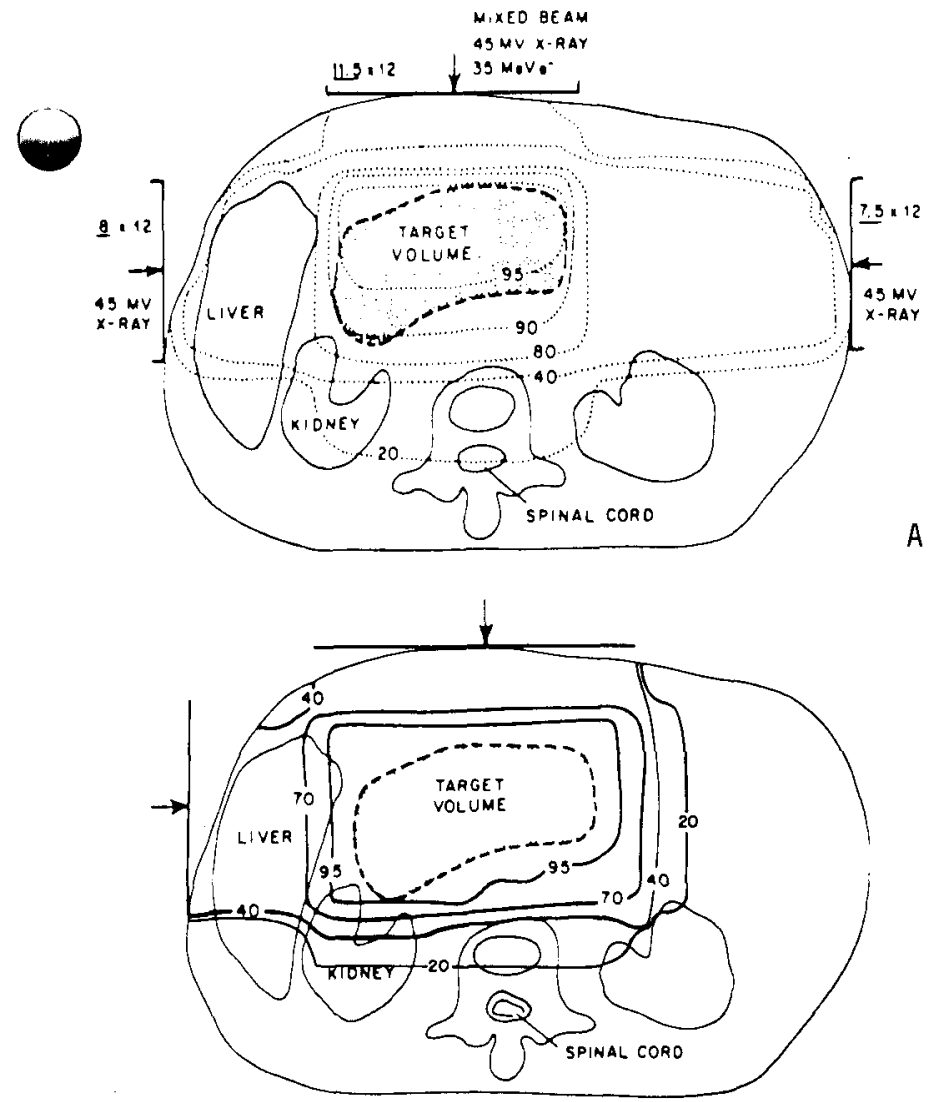

Figure 2. Comparison of plans for treatment of carcinoma of the pancreas. (A) 45 MV X-ray beam (three fields) combined with $35 \mathrm{MV}$ electron beam; (B) 557 MV neon beam (two fields). From CROS, 1978. (XBL 808-11540)

Two significant effects occur: (1) the dose to adjacent critical organs such as spinal cord, kidney, liver, and intestine is reduced; and (2) the integral dose is significantly reduced because smaller volumes accumulate high dose with a consequent reduction of generalized radiation. effects such as nausea and hematologic depression.

\section{BIOPHYSICAL CHARACTERISTICS}

Studies on many different mammalian cell systems in vivo and in vitro suggest that radiation sensitivity to photon low LET radiation is dependent on the oxygen concentration at the time of irradiation. The lower the OER value, the . less the radioprotection to hypoxic cells. Some human tumors may nave a significant component of hypoxic but viable cells that are more resistant to radiation than the cells of surrounding normal tissues. These hypoxic cells are usually found $100 \mu \mathrm{m}$ from the capillary supply. Cells in his region are also often necrotic, which correlates well with the clinical observation that ulcerated or necrotic tumors are difficult to control with radiation. There is some evidence of improved clinical response with irradiation under hyperbaric oxygen for tumors in the head and neck, and for carcinoma of the cervix, at least when used with shortened fractionation schedules and with curie therapy (OER 2.0) (Di sche, 1979).

Heavy charged particle radiation is less dependent than conventional low LET radiation in the presence of oxygen for effective cell killing and consequently may achieve a higher tumor control frequency when the tumor has a large component of hypoxic cells (Gray, 1957).

The lowest reported OER values with heavy charged particle radiation are found in neon and argon irradiation (Hall and Kellerer, 1979; Curtis, 1979; Raju et al., 1978b). These particle beams have OER values ranging from 1.2 to 1.9. Carbon ions have measured OER values of approximately 2.0, while values for nelium nuclei are in the 2.3 to 2.5 range (Curtis, 1979; Raju et a 1., 1978b). LÓw LET radiations have OER values in the range of 2.5 to 3.0 (Raju et a 1 , $1978 b$ )

With heavy charged particles, there is some OER variation across the extended peak region used for therapy, especially for neon ions. Figure 3 shows the variation of OER (and RBE) through the extended peak regions of the carbon, neon, and argon beams for the R-1 rat tumor cell line (Curtis, 1979). The lowest OER values are measured at the distal portion except for argon, where the OER remains relatively constant and low throughout the extended peak. Thus, argon radiation offers the nighest potential for therapy in terms of low OER values. Although argon ions have a less favorable physical dose distribution than other charged particle beams, they may be of interest clinically for relatively superficial, nypoxic tumors because of their low OER.

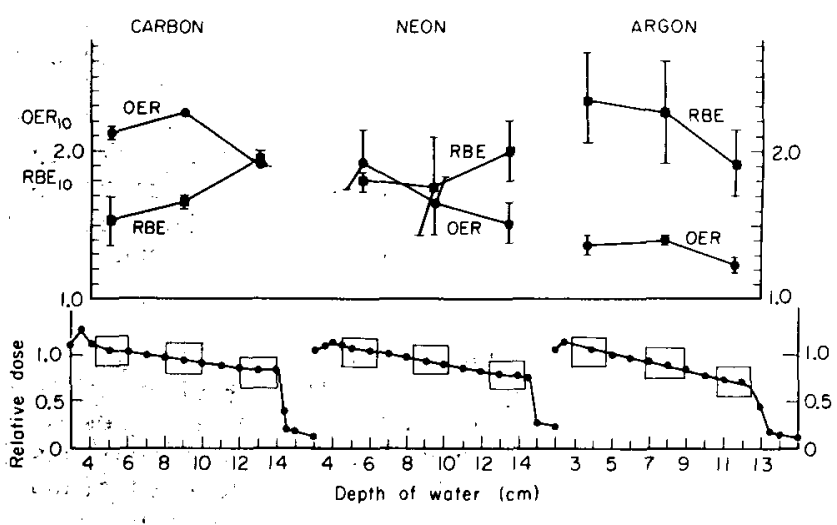

RHABDOMYOSARCOMA CELLS IN SUSPENSION

Figure 3. RBE and OER at $10 \%$ survival for $\mathrm{R}-1$ tumor cells at various depths through the $10 \mathrm{~cm}$ extended-peak regions of carbon, neon and argon beams. The lower panels show the dose vs. depth curves and the positions and extent of the cell suspensions. Courtesy of S. B. Curtis.

(XBL 795-3436) 
The possibility of less variation in sensitivity to particle radiation during different phases of the cell cycle may also be of value. Cell cycle effects have been measured for several nigh LET particles, and they show less variation in sensitivity for heavy particles than for photon radiation. However, in clinical practice, reassortment of tumor cells between irradiation fractions may counteract this possible advantage.

Tumor cells snow less repair of sublethal damage with particle irradiation than with conventional irradiation. This may also be true for normal structures included unavoidably in the target volume, and clinical trials will be necessary to determine if a significant differential exists. The possibility of differential repair in various portions of the particle beam path is also present because of the nigher LET deposition in the extended peak, particularly in the distal region. Little is known at present of the most advantageous fractionation schedules for particle radiation, although a variety of schedules is being studied in clinical practice, including mixing low LET photon beams and high LET particle beams in various combinations. There is evidence of possible potentiation of effect by the addition of particle irradiation to photon irradiation, providing the particle dose is given within a few hours after the photon irradiation (Ngo et al., 1980). There may also be a beneficial effect from a relatively small amount of particle radiation in terms of significantly lowering the OER (Curtis, 1976). In practice, it may be possible to give as small a part as 20 to $30 \%$ of the total dose by particle radiation, thus providing a practical and economic advantage in terms of the clinical radiation plan.

In order to attempt to compare quantitatively the results obtained with different cellular systems, Raju (1980) has calculated oxygen gain factors (OGF) for various beams, defined as the ratio of the OER for $X$ rays to the OER for the beam under study. There is a broad range of values for the charged particle beams (Fig. 4), reflecting the variation of biological responses through the extended peak regions. The OGF values for argon beams extend to the highest values while those for neon and neutron beams are in a similar range. Carbon, pion, and helium OGF values are significantly greater than one, but somewhat lower than for neutrons, neon, and argon ions.

Less desirable characteristics of heavy charged particle radiotherapy that must be addressed are: (1) the need for careful assessment of tissue inhomogeneities and perturbations of the particle beam path; (2) the need to study the LET distribution or other measure of beam quality, since the beam quality can vary significantly through the peak region at least for pion and heavy ion beams; and (3) the need to use biological as well as physical isodose distributions in treatment planning (Fig. 5).

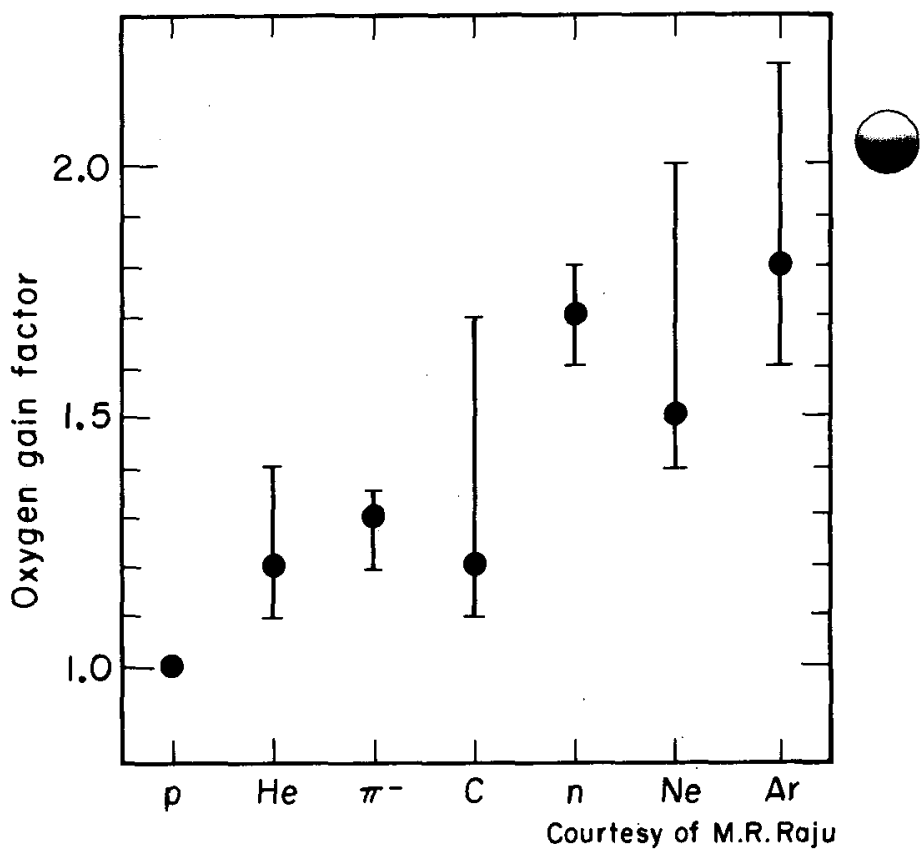

Figure 4. A comparison of oxygen gain factors experimentally determined for the various particles throughout the peak region. The spread of values reflects the fact that beam quality (and therefore the effect of oxygen on cell survival) varies throughout the spread region. (XBL 798-3747)

\section{CLINICAL EXPERIENCE}

Shortly after Rutherford suggested that noncharged particles exist, Chadwick detected neutrons (1932). A remarkable collaboration between E. 0. and J. H. Lawrence and others, following development of the cyclotron, led to pioneering studies on the biological effects of neutrons on normal and neoplastic tissues (Lawrence et al., 1936). By 1938, clinical studies utilizing the 37 -inch (and later the 60-inch) cyclotron were begun at LBL with fast neutrons under the direction of R. S. Stone and J. H. Lawrence (Stone et al., 1940). These studies continued until 1943, during which time about 250 patients were irradiated with neutrons (Stone, 1948; Stone and Larkin, 1942). Although Stone concluded that fast neutrons could $\mathrm{kill}$ cancer cells and cure patients, the late sequelae were so severe that he recommended neutron therapy be discontinued. Parker (1977) and Shel ine and associates (1971) have evaluated the reasons for these late sequelae and conclude that inadequate biological knowledge (including inaccurate RBE estimates) resulted in the administration of excess doses. In addition, many of the patients treated by Stone had been previously treated with $x$ rays, and persisting normal tissue damage was not weighed heavily in planning subsequent neutron dosage. Thus, the results 

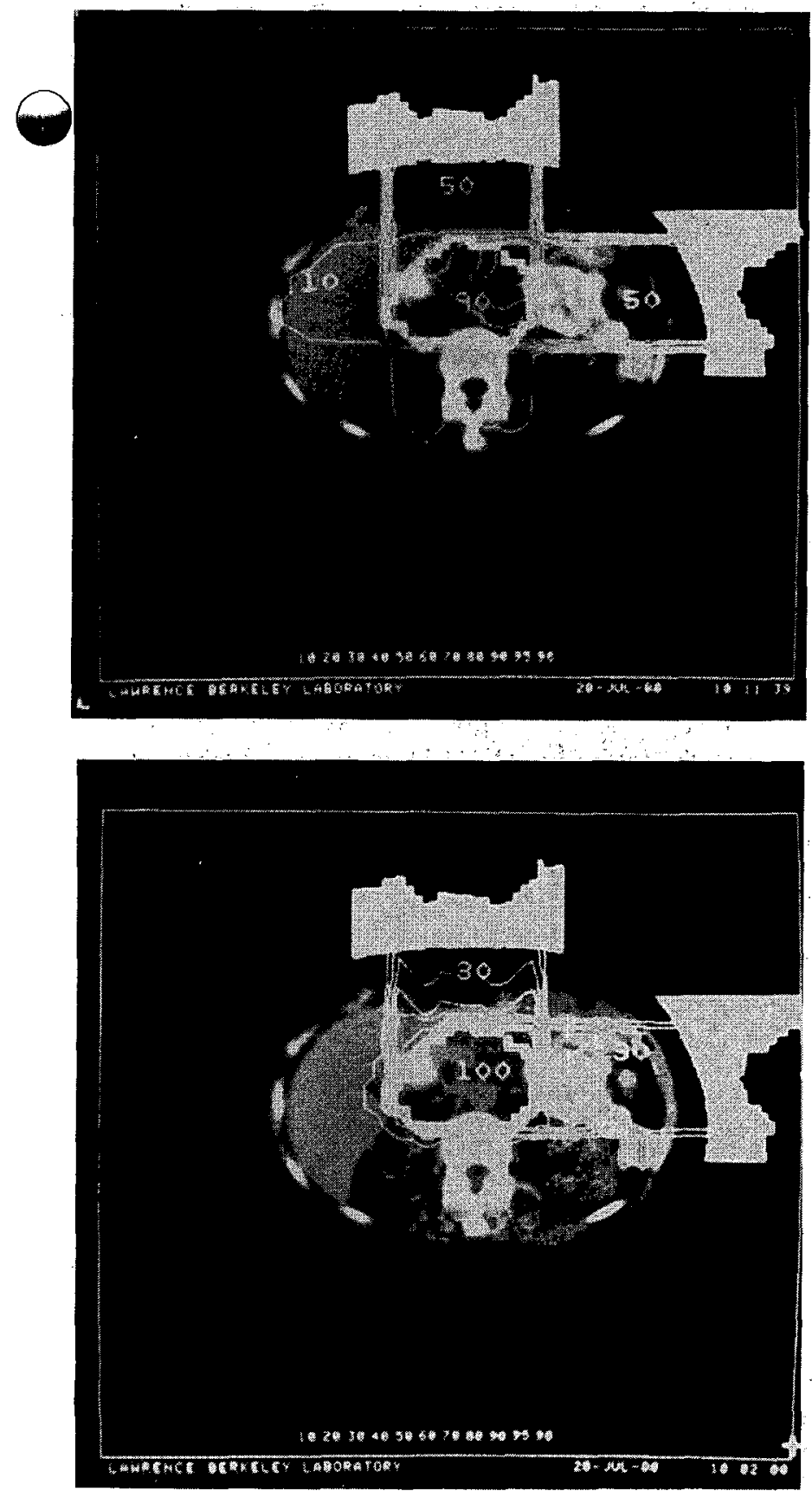

Figure 5. Sample pancreatic treatment plan for helium irradiation, showing physical (upper curve) and biologically corrected (lower curve) isodose lines. Courtesy of G.T.Y. Chen. (XBB 807-8631A)

obtained by Stone are understandable in light of present day knowledge and do not contraindicate further studies of heavy charged particle radiation therapy.

\section{HELIUM-ION RADIATION THERAPY}

In 1954, C. A. Tobias and coworkers began reating pituitary diseases using the Synchrocyclotron at LBL, initially with protons and deuterons and later with the plateau portion of a 934-MeV helium-ion beam. Since 1954, over 1,000 patients have been irradiated with good results in a variety of pituitary related problems, including acromegaly, Cushing's disease and chromophobe adenoma (Boone et al., 1977; Lawrence et a1., 1977).

The use of helium-ion radiation in patients with various malignant tumors was introduced at LBL during the later 1960s through treatment of patients with metastatic carcinoma, including pituitary ablation of patients with widespread breast cancer. Starting in 1975, a more systematic utilization of helium-ion radiation therapy was undertaken in order to study the potential advantage of improved dose localization in clinical radiotherapy (Castro et al., 1977; Castro et al., 1980).

In order to accomplish this, the medical treatment area at the 184-inch Synchrocyclotron was modified to permit large-field (up to $30 \mathrm{~cm}$ in diameter), fractionated 934-MeV helium-ion radiation therapy, utilizing a family of ridge filters to provide a spread Bragg peak ranging in size from 4 to $14 \mathrm{~cm}$. This provides the clinical flexibility to irradiate most tumor targets.

An existing patient positioner, the isocentric stereotaxic apparatus for humans (ISAH) (Lyman et al., 1975) was available, which required only minor modification to allow irradiation of supine, seated, or standing patients. Since the helium-ion beam is a fixed, horizontal beam, about $85 \%$ of the patients have been treated in the upright position in order to irradiate thoracic and abdominal tumors.

Utilizing plaster or Lightcast immobilization, it has been possible to reproduce the patient treatment position daily, with precision to $\pm 5 \mathrm{~mm}$ in the trunk and $\pm 3 \mathrm{~mm}$ in the head and neck region. An individually fabricated lucite head nolder is prepared for patients who will receive irradiation to the head and neck region.

The beam line was also modified by a second scatterer which was introduced inside the medical cave in order to provide a larger diameter $(30 \mathrm{~cm})$, flat field over which homogeneity to $\pm 2 \%$ was available for $90 \%$ of the field area.

A variable water column is available to alter the depth of penetration of the beam, with a maximum range in tissue of $26.5 \mathrm{~cm}$. An adequate dose rate is available to deliver the desired clinical dose of about 150-200 equivalent rad per fraction in approximately one minute. The final collimation of the beam is produced by individually prepared low melting point alloy blocks; that properly shape the beam to the desired target volume.

The Bragg peaks of energetic heavy-ion beams are narrow $(<1 \mathrm{~cm})$, and must be broadened in order to produce a more useful therapeutic depth dose distribution. At LBL this has usually been obtained by the use of specially designed spiral brass ridge filters. The design of these filters depends on the width of the spread Bragg peak 
and the variation of RBE across the modified spread peak. A beam model developed by J. Lyman has allowed us to calculate dose and average LET as a function of penetration distance in order to calculate isosurvival depth dose distributions for approximately $60 \%$ survival level per dose fraction. This is appropriate for typical clinical fractions of 200 rad of photon irradiation. A set of isosurvival ridge filters has been fabricated for helium and heavier particles based on these calculations, which provides a variety of spread Bragg peak widths ranging from. 4 to $14 \mathrm{~cm}$.

Computer assisted tomographic (CAT) scans are utilized for tumor localization, together with other appropriate diagnostic modalities including surgical mapping and clipping of the tumor, routine radiographs, specialized diagnostic studies such as angiography, ultrasound, and helium and heavy particle radiographs. CAT scanning is also used to provide tissue density information so that perturbations of the particle beam path can be evaluated to give accurate clinical treatment planning. A computerized dosimetric system has been developed to provide treatment planning for such patients (Chen et a 1., 1979).

Both physical and biologically corrected isodose distributions are produced (Fig. 5), with RBE corrected isodoses expressed in $60 \mathrm{Co}$ rad equivalent doses (CORE). For convenience of intercomparison with low LET photon irradiation,
CoRE doses and dose distributions are clinically utilized. Careful consideration of RBE data for various tissues and in vitro systems is made in order to modify the CORE model where needed.

The general goal of the helium-ion radiation therapy program has been to test the possible advantage of improved dose localization available with helium ions. Clinically, there has been little evidence of enhanced biological effect in terms of observed tumor regression with helium ions. Observation of skin, intestinal, and mucosal reactions with helium ions has confirmed the clinical RBE of 1.2 (proximal peak) to 1.4 (distal peak). This correlates well with RBE estimates based on in vivo and in vitro pretherapeutic studies.

Dose fraction sizes of 200 CoRE per fraction, 4 fractions per week to total doses of 6,000 to 7,000 equivalent rad have been utilized in order to keep comparable dose prescriptions of heliumion irradiation to low LET photon irradiation. In some instances, helium ions have been utilized after a portion of the total irradiation course has been given with photon irradiation, that is, as a "boost" at the end of therapy. A smaller group of patients has also had helium-ion irradiation initially, followed by use of one of the heavier ions, either carbon or neon, as a boost treatment. Tables 1 through 4 summarize the numbers and types of patients irradiated in the heavy particle trial.

Table 1. Heavy Particle Clinical Trial Patients (July 1975 through July 1980)

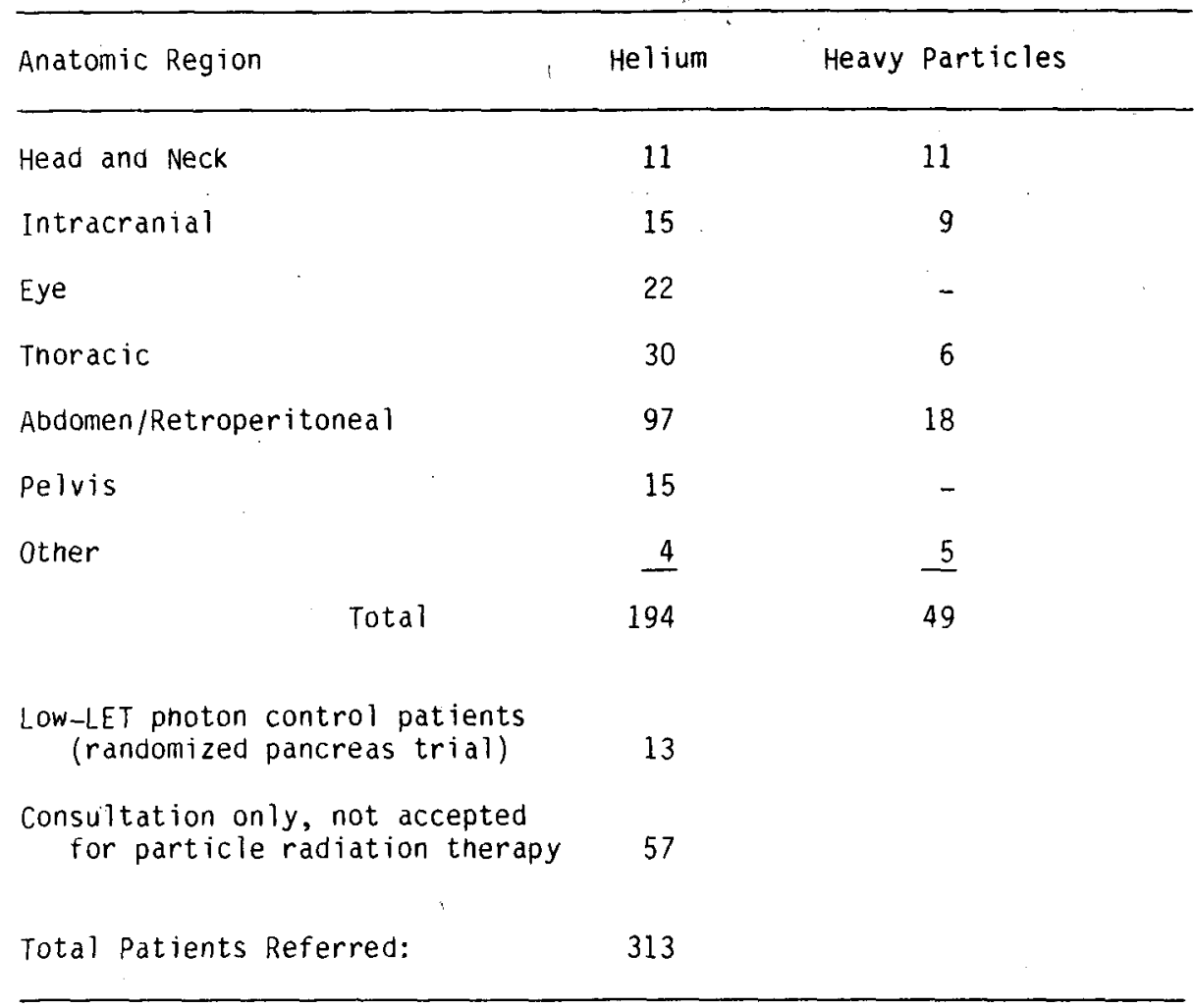


Table 2. Patients Entered Listed by Clinical Protocols Utilized

Phase III NCOG 3P81, RTOG 79-10

Randomized Pancreas Trial (Helium Radiation Therapy)

17 patients (plus 13 controls)

Phase III NCOG 7081, RTOG 79-08

Nonrandomized Ocular Melanoma

(Helium Radiation Therapy)

Phase III NCOG 3E81, RTOG 79-09

Nonrandomized Esophagus

(Helium Radiation Therapy)

Phase II VASOG-LBL

Nonrandomized Pancreas

(Carbon/Neon Radiation Therapy)

2

$\left.\left.\begin{array}{ll}\text { NCOG OR81 } \\ \text { RTOG 79-11 }\end{array}\right\} \begin{array}{ll}\text { Phase I-II } & 65 \\ \text { Miscellaneous Sites } & 42\end{array}\right\} 107$

LBL-BAHIA 1$\}$

LBL-BAHIA 2 (Pilot Pancreatic). $\quad 46$

LBL-BAHIA 3 (Pilot Brain)

LBL-BAHIA 4 (Pilot Esophagus)

LBL-BAHIA 5 (Paraaortic Nodes)

LBL-BAHIA 8 (Pilot Uterine Cervix)

LBL-BAHIA 6 A (Sk in Nodule RBE Study)

46
15
8
3
2
3

Table 3. Distribution of Sites and Ions Used in the Irradiation of Phase I - II Patients (July 1975 through July 1980)

\begin{tabular}{|c|c|c|c|c|c|c|}
\hline $\begin{array}{l}\text { Radiation } \\
\text { Modality }\end{array}$ & Head/Neck & Intracranial & Thorax & $\begin{array}{c}\text { Abdomen or } \\
\text { Retroperitoneal }\end{array}$ & Pelvic & Other \\
\hline Helium alone & 4 & 5 & 8 & 69 & 6 & 4 \\
\hline $\begin{array}{c}\text { Photon (low LET) } \\
\text { plus helium }\end{array}$ & 7 & 10 & 6 & 12 & 9 & - \\
\hline $\begin{array}{l}\text { Photon plus } \\
\text { carbon or neon }\end{array}$ & 1 & 6 & - & 6 & $\therefore$ & - \\
\hline $\begin{array}{l}\text { Helium plus } \\
\text { carbon or neon }\end{array}$ & 3 & 2 & 1 & 11 & - & $\because \cdot$ \\
\hline $\begin{array}{l}\text { Carbon, neon, or } \\
\text { argon alone }\end{array}$ & 7 & 1 & 5 & 1 & - & 5 \\
\hline
\end{tabular}


Table 4. Patient Accrual by Year (Particle Patients Only, Control Patients Excluded)

\begin{tabular}{ll}
1975 & 3 \\
1976 & 11 \\
1977 & 32 \\
1978 & 65 \\
1979 & 78 \\
1980 (estimate) & 85 \\
\hline
\end{tabular}

\section{Pancreatic Carcinoma}

Our largest experience with helium-ion radiation has been for patients with localized, unresectable carcinoma of the pancreas. Sixty patients have been irradiated since 1975, 7 of whom did not complete treatment because of progressive disease. One patient did not have a tissue biopsy. Of the remaining 52 patients, 37 were treated with helium particles only, 9 patients received photon radiation plus helium ions, and 6 patients were treated with helium plus neavier particles, either carbon or neon ions. A minimum dose of 5,000 equivalent rad was delivered, with most patients receiving 6,000 equivalent $\mathrm{rad}$ in 7 weeks. Six of the 52 (12\%) patients receiving definitive therapy are alive and well from 6 to 55 months posttreatment. One patient expired of intercurrent disease without evidence of tumor at 15 months. Three additional patients had control within the irradiated area but died of distant metastases or complications of chemotherapy. Seven patients had evidence of hemorrhagic gastritis, 6 of whom had persistent pancreatic tumor. The mean survival for patients with control in the irradiated area was 21 months; the mean survival for patients with persistent disease was 10 months.

A randomized trial for the treatment of pancreatic carcinoma is now underway which is designed to contrast helium irradiation plus 5-Fluorouracil chemotherapy against low-LET photon irradiation plus 5-Fluorouracil chemotherapy. This clinical cooperative trial is coordinated by the Northern California Oncology Group and the Radiation Therapy Oncology Group who provide the statistical backup, quality control and ancillary services needed for such prospective controlled studies. Thirty patients have been entered to date in the randomized pancreatic carcinoma trial with no significant difference as yet in survival between the study and control arm (Fig. 6). Future trials in carcinoma of the pancreas are planned with heavier particles such as carbon or neon ions, where we hope to see both improved dose distribution and enhanced biological effect.

\section{Esophagea 1 Carcinoma}

A second group of patients of interest who have been irradiated with helium ions are those with carcinoma of the esophagus. Twenty-four patients have been irradiated with nelium ions for this disease. Two patients did not complete treatment because of distant metastases; one patient had a tumor perforation at 3,500 equivalent rad and could not continue irradiation.

of the 21 patients who received definitive therapy, the first 16 patients received 200 CORE per fraction, 4 fractions per week, to total doses ranging from 6,200 to 7,000 CoRE. Eight of these 16 patients have had failure within the

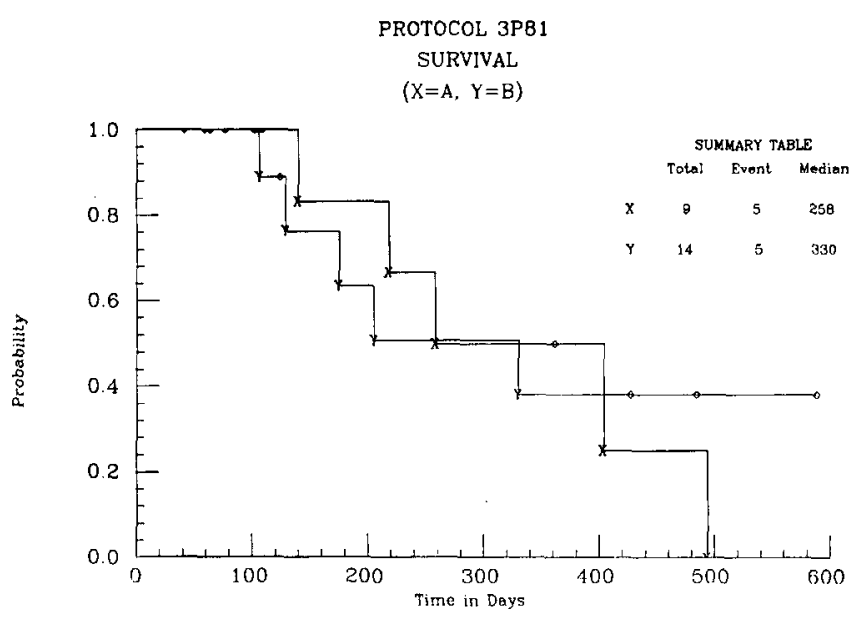

Figure 6. Comparison of survival for study and control arms of a randomized trial of helium irradiation for carcinoma of the pancreas. No difference as yet is evident between photon and helium arms. (XBL 808-11340) 
irradiated area. Subsequent patients have received 6,525 CORE at 225 CORE per fraction, 4 fractions per week.

of the total group of 21 patients receiving definitive therapy, 5 are alive and well without evidence of disease from 4 to 16 months posttreatment. One patient is alive with severe stenosis of the esophagus but without evidence of tumor at 13 months posttreatment. One patient has died of intercurrent disease, 1 of perforation posttreatment with microscopic residual tumor (scored as fatal complication of treatment), and 4 patients have distant metastases but apparent control within the irradiated area. Ten patients of 21 have failure within the radiation field.

This experience suggests little improvement in the treatment of carcinoma of the esophagus with helium ions. Since this is probably related to lack of sufficient enhancement of biological effect with helium-ion radiation therapy despite the good dose localization, we plan to continue the study using a heavier particle, either neon or carbon ions.

\section{Carcinoma of the Stomach}

A smaller group of 10 patients with localized, unresectable carcinoma of the stomach has been irradiated with helium ions. Doses have ranged from a minimum of 4,000 to a maximum of 6,000 CORE given in 4 fractions per week of 200 equivalent rad each. Of the 10 patients, 3 are a] ive and well from 9 to 34 months posttreatment, 1 patient expired of distant metastases but with control in the irradiated area at 10 months posttreatment, and 6 have had persistent disease within the irradiated area. While this group is small, we intend to pursue further studies on localized carcinoma of the stomach, utlizing a neavier ion such as carbon or neon in order to take advantage of enhanced biological effect.

\section{Malignant Glioma of the Brain}

Fourteen patients with malignant glioma of the brain have been irradiated with heavy charged particles. One patient received irradiation solely with helium ions, 10 patients have received whole brain photon irradiation of approximately 5,000 rad followed by reduced volume helium-ion therapy of 1,500 CORE, and 4 patients received photon irradiation plus a carbon ion boost. Of the 14 patients, 3 are al ive without evidence of disease from 10 to 20 months posttherapy. Three of these were treated with photon irradiation plus helium ions; 1 was treated with photon irradiation plus carbon ions. Further studies are planned, aga in with a heavier: ion such as carbon, neon, or argon in order to further explore the potential of heavy charged particle therapy in glioblastoma.
Ocular Melanoma

A group of 22 patients with localized ocular melanoma has been irradiated with helium ions, using a spread Bragg peak of approximately $15 \mathrm{~mm}$ and delivering 7,000 equivalent rad in 5 fractions in 7 to 9 days, later raised to 8,000 equivalent rad in 5 fractions in 7 to 9 days. The tumors are marked surgically with of $2-\mathrm{mm}$ diameter tantalum rings sutured or glued to the sclera, which outline the lesion for beam delivery. The patient is carefully positioned for therapy by using an individually fabricated polystyrene head holder with fixation on a light source. Pre- and posttherapy beam portal films, and continuous television observation during the course of the treatment has assured precise beam delivery.

Ocular melanoma arises in the uveal tract, which includes the iris, the ciliary body of the eye, and the choroid, a vascular envelope between the retina and sclera. Uveal melanomas more commonly arise in the posterior choroid. As these tumors grow, they tend to displace the retina, which leads to visual disturbances and retinal detachments. These symptoms will of ten bring the patient to a physician while the tumor is still relatively small and localized to the eye. While enucleation of the eye is regarded as the most definitive form of management of these lesions, there has also been a concerted effort to develop less radical treatment modalities in the hopes of preserving useful vision. Included among these treatment modalities are photocoagulation, partial eye-wall resection, and radioactive palques utilizing either $60 \mathrm{Co}$ or $125 \mathrm{I}$.

Because of the limited applicability of photocoagulation, and the morbidity associated with partial eye-wall resections and radioactive palques, the possiblity of using the Bragg peak of charged particle beams has been explored, both with protons (Harvard cyclotron) and helium ions (LBL).

We have adapted the Harvard technique to our beam configuration at the 184-inch Synchrocyclotrón and our patient immobilization device (ISAH). The direction of the gaze, the angulation of the beam, the shape of the treatment aperture, and the beam penetration depth and range modulation are established in a planning session using a computerized treatment planning program developed by $M$. Goitein at Harvard. The tumor localization is facilitated by the operative placement of radioopaque tantalum $r$ ings about the base of the tumor. Coordinates of the rings are obtained from diagnostic $x$-ray films. These data and measurements of the tumor height and the AP diameter of the globe as determined from an ultrasound study are used to locate the tumor relative to the structures of the eye. The dose and fractionation have been chosen to facilitate intercomparison of results with the proton treated patients. 
Treatment results to date have been encouraging, with the most significant acute radiation toxicity being a transient lid epitheliitis observed in patients in whom the eyelid could not be completely retracted from the treatment volume. Of 14 patients treated over six months ago, 12 have responded with a reduction in the tumor height of $>0.75 \mathrm{~mm}$ on ultrasound examination. One patient nas stable disease, and one had obvious tumor progression that led to subsequent enucleation. Of the 12 patients who responded, 1 has subsequentiy. been retreated because of presumed residual tumor manifested by progressive fluid collection, despite the fact that the height of his lesion had decreased. Of the total group of 22 patients treated, therefore, 20 appear to have either stable or regressing lesions with followup ranging from 2 to 24 months.

Because the preservation of visual acuity is an important goal of this highly localized technique, we have also been careful to tabulate changes in visual acuity that have occurred since therapy. Two patients have had deterioration of visual acuity that is related to radiation change, including mild cataract formation in 1 patient in whom the tumor arose in the ciliary body adjacent to the lens. The second patient had a large tumor adjacent to the optic disc and has developed central retinal vessel changes 5 months posttherapy with deterioration of vision $(20 / 200)$.

Recent refinements in CT scanning now make it possible to visualize ocular melanomas as they project into the vitreous body. This will be a valuable tool to assist tumor localization, as well as the localization of adjacent critical structures within the eye, such as the optic nerve. Additional radiobiological studies are planned to better evaluate the dose and treatment fractionation schedules, as well as the impact of irradiating different volumes of the eye.

\section{Other Helium Target Sites}

Helium ions have been utilized in a variety of other target sites where improved dose localization has appeared to be of significant potential advantage. These have included selected head and neck tumors around the base of the skull and cervical spine, as well as in the paranasal sinuses, localized soft tissue sarcomata, carcinoma of the biliary tract (localized) and a variety. of locally advanced or recurrent neoplasms in the abdomen and pelvis. The goal of this study has been to ascertain whether improved dose localization can be of significant clinical advantage for selected human tumor sites. While not a prospective controlled trial, this pilot study has indicated a significant value for several such sites, particularly in selected tumors of the head and neck region and soft tissue sarcomata. Further evaluation is planned in order to more accurately determine which such target sites offer the greatest potential.

\section{HEAVY-PARTICLE RADIATION THERAPY}

The Bevalac nas clinical facilities that are similar to those at the 184-inch Synchrocyclotron. A patient positioner has been installed that permits irradiation of supine, seated, or standing patients. Laser localization lights, together with daily beam portal radiographs, assure reproducible and precise immobilization. Lightcast, plaster cast, and similar immobilization. techniques are utilized, as well as wax bolus compensation for tissue inhomogeneities and sloping surface entry. Further attempts at more elaborate compensation await the installation of a variable CT scanner which will permit scanning of patients in the upright position. Approximately 80 of the patients are treated in the upright position because of the fixed horizontal beams provided both at the 184-inch Synchrocyclotron and at the Bevalac.

The goals of the Phase I-Phase II studies with heavy particles such as carbon, neon, or argon ions are: (1) development of effective treatment techniques utilizing carbon, neon, and argon ions based on experience obtained with the helium-ion beam; (2) clinical evaluation of physical and biological dose distributions available with carbon, neon, and argon ions; (3) evaluation of acute and subacute response of normal tissues such as mucosa, skin, and intestine; and (4) initial evaluation of tumor response to heavy particle irradiation.

Forty-nine patients have been irradiated to date with carbon (24 patients), neon (23 patients), or argon (2 patients) ions (Table 2). Most of these patients received carbon or neon ion irradiation because of limited access to the argon ion beam. Our initial clinical efforts have been to confirm the RBE estimates for various tissues provided by pretherapeutic investigations. To this end, we have irradiated several patients with metastatic advanced tumors, paying careful attention to skin, mucosal, and pulmonary reactions.

The first such patient was an individual with Kaposi's sarcoma in whom several fields were irradiated with carbon ions as well as with $10 \mathrm{MeV}$ electrons available at the Zellerbach Saroni Tumor Institute of Mount Zion Hospita] and Medical Center, San Francisco, CA. Sk in reactions were scored by two independent observers and a dose response curve compared. The estimated RBE for 120 physical rad per fraction of carbon ions was approximately 2.7 in this study.

A second patient was irradiated with neon ions for metastatic subcutaneous leiomyosarcoma, with one field irradiated with $400 \mathrm{MeV} / \mathrm{amu}$ neon ions, and compared to a single, adjacent, $15 \mathrm{MeV}$ electron field. Eight fractions for a total of 960 physical rad were delivered with neon, and 8 fractions for a total of 3,200 rad were delivered with electrons. The skin reactions appeared comparable on gross examination. This suggested an RBE of -3.3 for neon in this study. 
Another patient with metastic nodules in both lungs from a primary carcinoma of the uterine cervix was irradiated with $8 \mathrm{MeV} X$ rays to the right lung nodules, and $400 \mathrm{MeV} / \mathrm{amu}$ carbon ions to the left lung nodules. The $X$-ray dose was 4,000 rad in 8 fractions, and the carbon ion dose was 1,480 physical rad in 8 fractions, both treatment courses lasted 11 days. Skin reactions could not be compared because of a coincident allergic dermatitis requiring administration of steroids, but tumor regression was compared using serial chest $X$ rays and serial CT scans. The estimated RBE for carbon ions relative to $8 \mathrm{MeV}$ photons as judged by tumor regression was approximately 2.5 .

An additional patient with multiple metastatic nodules in the lungs was irradiated with $557 \mathrm{MeV} / \mathrm{amu}$ neon ions, to several target sites in the lungs. A total of 1,400 physical rad was delivered with the dose per fraction varying from 85 to 350 physical rad, and the number of fractions varying from 4 to 16 , all given in a period of approximately 30 days. Regression of the nodules as determined by chest $X$ ray and $C T$ scan was essentially similar; thus it was concluded that different treatment schedules and fraction sizes were approximately equal in effectiveness for this particular patient (Table 5).

Only 2 patients have been irradiated with argon ions. The first patient had metastatic sarcoma in the right supraclavicular fossa and neck. This patient was irradiated with 572 physical rad of argon ions given in 4 fractions in 4 days through a single field. There was virtually no skin reaction noted. The patient has been followed for approximately 15 months, with slight regression of the tumor which was noted to be calcified on radiography prior to administration of the therapy. There nas been no evidence of regrowth of the lesion to date. The estimated CORE for this patient is about 1,500 ; the estimated RBE is approximately 3 .

A second patient with metastatic melanoma of the skin of the lower extremities was also treated with argon ions to four different lesions. The doses utilized were 556 and 868 physical rad in 4 fractions over 2. days (two sites at each dose level). Skin reactions were as expected for the predicted. RBE of approximately 2.3 and 2.7 , respectively. Inadequate followup time inhibits any definitive statement about tumor response, although there has been slightly greater regression of the lesions at the higher dose level.

This patient has also had-several lesions irradiated with neon ions, helium ions, and photons. A comparison of skin reactions has suggested that the helium and neon RBE vaiues are approximately correct, although our estimated RBE value for neon, which is based on pretherapeutic data, may be slightly high. These data are summarized in Table 5 .

Additional patients irradiated with neon or carbon ions have included patients with carcinoma of the pancreas too advanced for the randomized nelium clinical trial; primary or metastatic malignancies of the brain; localized, unresectable abdominal or pelvic tumors such as recurrent colonic carcinoma; advanced cancer of the head and neck and a few patients with carcinoma of the lung. We plan to treat at least 100 patients with carbon, neon, or argon ions before completion of the Phase I-Phase II heavy particle trial. Design of future Phase III trials with heavy particles will be based on information accumulated in this clinical study as well as all available pretherapeutic biophysical information.

Clinical irradiation with charged particles at LBL has utilized conventional low LET dosevolume-fractionation techniques as closely as possible in order to reduce the number of variables and afford an easier comparison between low LET and charged particle irradiations. For helium-ion irradiation, this has generally meant doses in the range of 200 CoRE per fraction, 4 to 5 fractions per week, to total doses of 6,000 to 7,000 CORE except for patients with ocular melanomas. Because of difficulties with beam availability at the Bevalac, carbon and neon ions have been given at the rate of 300 to 350 CoRE per fraction to total doses of 4,800 to 5,400 CoRE. Several patients have been excepted from this dose fractionation scheme because they needed rapid irradiation of either pulmonary, or skin and subcutaneous nodules. Gradual escalation of total doses will be carried out in order to assess the highest possible target volume dose that can be delivered with heavy particles while sparing normal structures outside the target volume.

Our current estimate of priority target sites for heavy particles includes glioblastoma, selected advanced head and neck tumors, localized advanced carcinoma of the pancreas, and soft tissue and bone sarcomata. In addition, when the helium-ion pilot study is completed for carcinoma of the esophagus and stomach, we contemplate a Phase I-Phase II study utilizing either carbon or neon ions for these target sites.

In the current Phase I-Phase II studies most patients have received treatment solely with carbon or neon ions. A smaller group of patients nas received heavy particle treatment as a boost following either helium or low LET irradiation. In addition, a few patients have been treated with mixed beams, that is, combining heavy particle irradiation (carbon or neon ions) twice a week with low LET photon irradiation three times per week. The small number of patients in this group precludes any definitive clinical conclusions, but a beginning evaluation of these treatment schedules appears appropriate in order to evaluate patient and physician acceptance, and make some estimate of tumor response. The practical implications of mixed beam or boost schedules in terms of patient accrual and economics are obvious, should these techniques offer clinical value. 
Table 5. Phase I - II Preliminary Heavy-Ion Bragg Peak Patient Data

\begin{tabular}{|c|c|c|c|c|c|c|c|c|}
\hline $\begin{array}{l}\text { Beam and } \\
\text { Initial Energy } \\
(\text { MeV/amu) }\end{array}$ & $\begin{array}{l}\text { Patient } \\
\text { Diagnos is }\end{array}$ & $\begin{array}{l}\text { Radiation } \\
\text { End Point }\end{array}$ & & $\begin{array}{l}\text { Dose/Fraction } \\
\text { (physical rad) }\end{array}$ & $\begin{array}{r}\text { Frac } \\
\mathrm{Ti}\end{array}$ & $\begin{array}{l}\text { ionation } \\
\text { e (days) }\end{array}$ & $\begin{array}{l}\text { RBE } \\
\text { Estimate } \\
\text { (relative to } \\
\text { photons) }\end{array}$ & $\begin{array}{l}\text { Reference } \\
\text { Radiation }\end{array}$ \\
\hline $\begin{array}{l}\text { Carbon } \\
308\end{array}$ & $\begin{array}{l}\text { Kaposi's } \\
\text { sarcoma }\end{array}$ & $\begin{array}{l}\text { Skin } \\
\text { reaction }\end{array}$ & $\begin{array}{l}100 \\
140 \\
170\end{array}$ & $\begin{array}{l}\mathrm{rad} / \mathrm{f} \times \times 10 \mathrm{fx}=1,000 \\
\mathrm{rad} / \mathrm{f} \times \mathrm{x} 10 \mathrm{fx}=1,400 \\
\mathrm{rad} / \mathrm{f} \times \mathrm{x} 10 \mathrm{fx}=1,700\end{array}$ & $\begin{array}{l}\mathrm{rad} \\
\mathrm{rad} \\
\mathrm{rad}\end{array}$ & 11 & (at $\left.120^{2.7} \mathrm{rad} / \mathrm{fx}\right)$ & $\begin{array}{l}10 \mathrm{MeV} \text { electrons } \\
250 \mathrm{rad} / \mathrm{f} x \times 10 \mathrm{fx}=2,500 \mathrm{rad} \\
300 \mathrm{rad} / \mathrm{fx} \times 10 \mathrm{fx}=3,000 \mathrm{rad} \\
350 \mathrm{rad} / \mathrm{f} x \times 10 \mathrm{fx}=3,500 \mathrm{rad}\end{array}$ \\
\hline 400 & $\begin{array}{l}\text { Left lung } \\
\text { metastic } \\
\text { nodules from } \\
\text { carcinoma of } \\
\text { the uterine } \\
\text { cervix }\end{array}$ & $\begin{array}{l}\text { Tumor } \\
\text { regression }\end{array}$ & 185 & $\mathrm{rad} / \mathrm{fx} \times 8 \mathrm{fx}=1,840$ & $\mathrm{rad}$ & 11 & 2.5 & $\begin{array}{l}8 \mathrm{MeV} x \text { rays } \\
500 \mathrm{rad} / \mathrm{fx} \times 8 \mathrm{fx}=4,000 \mathrm{rad}\end{array}$ \\
\hline $\begin{array}{l}\text { Neon } \\
400\end{array}$ & $\begin{array}{l}\text { Metastatic } \\
\text { subcutaneous } \\
\text { leiomyosarcoma }\end{array}$ & $\begin{array}{l}\text { Skin } \\
\text { reaction }\end{array}$ & \multicolumn{3}{|c|}{$120 \mathrm{rad} / \mathrm{fx} \times 8 \mathrm{fx}=960 \mathrm{rad}$} & 10 & 3.3 & $\begin{array}{l}15 \mathrm{MeV} \text { electrons } \\
400 \mathrm{rad} / \mathrm{fx} \times 8 \mathrm{fx}=3,200 \mathrm{rad}\end{array}$ \\
\hline 557 & $\begin{array}{l}\text { Multiple } \\
\text { metastatic } \\
\text { lung nodules }\end{array}$ & $\begin{array}{l}\text { Tumor } \\
\text { regreșsion }\end{array}$ & $\begin{array}{r}350 \\
175 \\
117 \\
85\end{array}$ & $\begin{array}{l}\mathrm{rad} / \mathrm{fx} \times 4 \mathrm{fx}=1,400 \\
\mathrm{rad} / \mathrm{fx} \times 8 \mathrm{fx}=1,400 \\
\mathrm{rad} / \mathrm{f} \times 12 \mathrm{fx}=1,400 \\
\mathrm{rad} / \mathrm{fx} \times 16 \mathrm{fx}=1,400\end{array}$ & $\begin{array}{l}\text { rad } \\
\text { rad } \\
\text { rad } \\
\text { rad }\end{array}$ & $\begin{array}{l}28 \\
30 \\
30 \\
31\end{array}$ & $\begin{array}{l}2.4 \\
2.9 \\
3.2 \\
3.5\end{array}$ & None \\
\hline 670 & $\begin{array}{l}\text { Metastatic } \\
\text { melonoma, sk in }\end{array}$ & Sk in reaction & $\begin{array}{r}85 \\
100\end{array}$ & $\begin{array}{l}\mathrm{rad} / \mathrm{fx} \times 18 \mathrm{fx}=1,530 \\
\mathrm{rad} / \mathrm{fx} \times 16 \mathrm{fx}=1,600\end{array}$ & $\begin{array}{l}\text { rad } \\
\text { rad }\end{array}$ & $\begin{array}{l}34 \\
24\end{array}$ & $\begin{array}{r}3.5 \\
-3.2\end{array}$ & $\begin{array}{l}232 \mathrm{MeV} / \mathrm{amu} \mathrm{Helium} 4,248 \mathrm{rad} / 18 \mathrm{fx} / 30 \text { days } \\
4 \mathrm{MeV} \times \mathrm{ray} 6,350 \mathrm{rad} / 20 \mathrm{fx} / 30 \text { days }\end{array}$ \\
\hline $\begin{array}{r}\text { Argon } \\
570\end{array}$ & $\begin{array}{l}\text { Metastic } \\
\text { sarcoma. } \\
\text { the right } \\
\text { supraclavicular } \\
\text { fossa }\end{array}$ & $\begin{array}{l}\text { Sk in } \\
\text { reaction } \\
\text { and tumor } \\
\text { regression }\end{array}$ & 143 & $\mathrm{rad} / \mathrm{fx} \times 4 \mathrm{fx}=572 \mathrm{rac}$ & & 4 & $\sim 2.7$ & None \\
\hline 570 & $\begin{array}{l}\text { Metastatic } \\
\text { skin melanoma } \\
\text { of lower } \\
\text { extremities }\end{array}$ & $\begin{array}{l}\text { Sk in } \\
\text { reaction }\end{array}$ & $\begin{array}{l}139 \\
217\end{array}$ & $\begin{array}{l}\mathrm{rad} / \mathrm{fx} \times 4 \mathrm{fx}=556 \mathrm{rac} \\
\mathrm{rad} / \mathrm{fx} \times 4 \mathrm{fx}=868 \mathrm{rac}\end{array}$ & & 2 & $\begin{array}{c}2.7 \\
\text { (at } 139 \mathrm{rad} / \mathrm{fx} \text { ) } \\
2.3 \\
\text { (at } 217 \mathrm{rad} / \mathrm{fx} \text { ) }\end{array}$ & None \\
\hline
\end{tabular}


FUTURE STUDIES

Upon completion of Phase I-Phase II studies with carbon, neon and argon ions, design of Phase III trials utilizing one or more of these ions will be undertaken. Ultimately two or three sites will be selected for prospective controlled clinical trials in which low-LET radiation is compared to helium-ion radiation and radiation from a heavier particle such as carbon or neon.

Evaluation of three-dimensional beam scanning will continue in order to improve treatment delivery techniques. 1 Similarly, an autoradioactive beam such as ${ }_{6} \mathrm{C}$ or ${ }_{10}{ }_{\mathrm{N}}$ may assist in localization of the nigh dose zone to the desired target volume (see chatterjee and Alpen, Part VI, Section B). In addition, evaluation of combinations of helium ion irradiation with hypoxic cell sensitizers or nyperthermic techniques is also under consideration for future study.

The design of a hospital based dedicated biomedical accelerator for heavy particle radiotherapy, radiography, and biomedical research will also be undertaken soon, because several years will be needed to properly complete such a design project. This design study should also include a medical evaluation of the particle beam configurations needed for clinical radiotherapy, and consideration of possible location sites, physician and patient populations, and acceptance and referral patterns. This design program should be completed over the next two or three years so that if initial clinical studies show promise for heavy particle radiotherapy, a well-planned construction program can be started with minimum delay.

\section{FOOTNOTES AND REFERENCES}

* Clair Zellerbach Saroni Tumor Institute, Mt. Zion Hospita1, San Francisco, CA.

TDepartment of Radiation Oncology, University of California, San Francisco, CA.

FChidren's Hospital, San Francisco, CA.

Boone, M.L.M., J. H. Lawrence, W. G. Connor, R. Morgado, J. A. Hicks, and R. C. Brown. 1977. Introduction to the use of protons and heavy ions in radiation therapy: Historical perspective. Int. J. Radiat. Oncol. Biol. Phys. 3, 63-64.

Castro, J. R., J. M. Quivey, J. T. Lyman, G.T.Y. Chen, C. A. Tobias, L. L. Kanstein, and R. E. Walton. 1977. Heavy-ion therapy. Biological and Medical Research with Heavy Ions at the Bevalac, 1974-1977, pp. 198-218. Lawrence Berkeley Laboratory Report 5610 .

Castro, J. R., J. M. Quivey, J. T. Lyman, G.T.Y. Chen, T. L. Phillips, C. A. Tobias, and E. L. Alpen. 1980. Current status of particle radiotherapy at Lawrence Berkeley Laboratory. Cancer 46, 633-641.
Chadwick, J. 1932. Possible existence of a neutron particle. Nature (London) 129, 312.

Chen, G.T.Y., R. P. Singh, J. R. Castro, J. T. Lyman, and J. M. Quivey. 1979. Treatment planning for heavy-ion radiotherapy. Int. J. Radiat. Oncol. Biol. Phys. 5, 1809-1819.

CROS (Committee for Radiation Oncology Studies). 1978. Proposal for a program in particle-beam radiation therapy in the United States. Cancer Clinical Trials 1, 153-208.

Curtis, S. B. 1976. The OER of mixed high and low-LET radiation. Radiat. Res. 65, 566-57?.

Curtis, S. B. 1979. The biological properties of high-energy heavy charged particles. Sixth International Congress of Radiation Research. (S. Okada, M. Imamura, T. Terashima, and $H$. Yamaguchi, eds.) p. 780-788. Japanese Association for Radiation Research, Tokyo, Japan.

Dische, S. 1979. Hyperbaric oxygen and hypoxic cell sensitizers in clinical radiotherapy: Present status and prospects. High-LET Radiations in Clinical Radiotherapy. (G.W. Barendsen, J. J. Broerse, and K. Breur, eds.) p. 83-89. Pergamon Press, 0xford.

Fletcher, G. H. 1977. Present status of low-LET radiation in the management of cancers. Int. J. Radiat. Oncol. Biol. Phys. 3, 7-19.

Gray, L. H. 1957. Oxygenation in radiotherapy. I. Radiobiological considerations. Br. J. Radiol. 30, 403-406.

Hall, E. J. and A. Kellerer. 1979. Review of RBE data for cells in culture. High-LET Radiations in Clinical Radiotherapy. (G. W. Barendsen, G.W., J. J. Broerse, and K. Breur, eds.) pp. 171-174. Pergamon Press, 0xford and New York.

Lawrence, J. H., P. C. Aebersold, and E. 0 . Lawrence. 1936. Comparative effects of $X$-rays and neutrons on normal and tumor tissue. Proc. Nat1. Acad. Sci. U.S.A. 22, 543-557.

Lawrence, J. H., C. A. Tobias, C. Chong, J. T. Lyman, J. L. Born, J. Linfoot, and E. Manougian. 1977. Heavy particle irradiation of the pituitary. The Pituitary, A Current Review, pp. 443-451. Academic Press, New York.

Lyman, J. T. and C. Y. Chong. 1975. ISAH: A versatile treatment positioner for external radiation therapy. Cancer 34, 12-16.

Ngo, F.Q.H., E. A. Blakely, C. A. Tobias. 1980. Sequential exposures of mammalian cells to low and nigh-LET radiations. I. Lethal effects following $X$ ray and neon-ion irradiation. Radiat. Res., in press.

Parker, R. G. 1977. Current status of fast neutron teletherapy. Recent Advances in Nuclear Medicine (Lawrence, J. H., and T. F. 
Budinger, eds.) vol. 5, pp. 103-117. Grune and Stratton, New York.

Raju, M. R. 1980. Heavy Particle Radiotherapy. Academic Press, New York.

Raju, M. R., H. I. Amols, J. F. Dicello, J. Howard, J. T. Lyman, A. M. Koehler, R. Graves, and J. B. Smathers. 1978a. A heavy particle comparative study. Part I: Depth-dose distributions. Br. J. Radiol. 51, 699-703.

Raju, M. R., H. I. Amols, E. Bain, S. G. Carpenter, R. A. Cox, and J. B. Robertson. 1978b. A comparative study of heavy charged particles in radiation therapy $\left(n, \pi^{-}, P\right.$, $\mathrm{He}, \mathrm{C}, \mathrm{Ne}, \mathrm{Ar}$ ). III. OER and RBE. Br. J. Radiol. 51, 712-719.

Sheline, G. F., T. L. Phillips, S. B. Field, J. T. Brennan, and A. Raventos, 1971.
Effects of fast neutrons on human skin. Am. 3. Roentgen 3, 31-40.

Stone, R. S. 1948. Neutron therapy and specific ionization (Janeway Memorial Lecture, 1947). Am. J. Roentgeno1. Radiat. Therapy 59, 771-785.

Stone, R. S., and J. C. Larkin Jr. 1942. Treatment of cancer with fast neutrons. Radiology 39, 608-620.

Stone, R. S., J. H. Lawrence, and P. C. Aebersold. 1940. Preliminary report on use of fast neutrons in treatment of malignant disease. Radiology 35, 322-327.

U.S. Senate Report. 1970. Conquest of Cancer. Report of the National Panel of Consultants of the Committee on Labor and Public Welfare of the U.S. Senate, P. 51, Washington, D.C. 


\title{
RADIOLOGICAL PHYSICS OF HEAVY CHARGED-PARTICLE BEAMS USED FOR THERAPY
}

\author{
J. T. Lyman, J. Howard, L. Kanstein, and J. R. Alonso
}

BEAM CHARACTERISTICS

\section{4-Inch Synchrocyclotron Facility}

The 184-inch Synchrocyclotron was fir.st used for biological studies in 1948 (Tobias et al., 1952) and first used to treat patients in 1954 (Tobias et al., 1955). Except for the first thirty patients who received pituitary irradiation with protons, all patients irradiated at this cyclotron have been treated with the helium-ion beam.

The treatment techniques are arbitrarily divided into two categories: small field and large field irradiations. Examples of the small field treatments are pituitary irradiation, which generally utilizes the plateau portion of the helium depth-dose curve, and treatment of ocular melanoma, which uses a modified Bragg peak of the helium beam. Large field treatments (greater than 3-cm beam diameter) for cancer therapy generally require a beam that has a large uniform transverse profile and a modified Bragg peak. The same treatment room is used for both the small and large field irradiations.

\section{Bevalac and Bevatron Facilities}

The beams available for biological investigations at the Bevatron or at the Bevalac range from nelium to iron ions. However, only carbon, neon, and argon beams have been used for therapy. There are two irradiation rooms available: room I is primarily for patient procedures and room II is for the biological and physical investigations (Lyman and Howard, 1977a).

Procedures and instrumentation for patient irradiations at the Bevatron/Bevalac nave been based on the prior experience obtained at the 184-inch Synchrocyclotron, and for that reason both facilities are discussed.

\section{INSTRUMENTATION}

The beams are monitored by large diameter transmission ionization chambers that are flushed with dry nitrogen gas. Segmented collection electrodes permit the beam size and position to be monitored during treatment (Lyman et al., 1975). The ionization chambers are connected to recycling integrators (Snapiro, 1970), which in turn are connected to scalars that interface to a minicomputer. The stability of the ionization cnamber is monitored with a secondary emission monitor that can detect changes in the ionization chamber collection efficiency as well as changes in the gas filling the ionization chamber.
The beam monitoring ionization chambers are calibrated daily with a thimble-type ionization chamber located at the isocenter of the patient positioner. When the dosimetry was compared with measurements made by personnel from other institutions studying heavy charged-particle therapy (neavy, in this context, implies particles heavier than electrons), there was general agreement in the value of the absorbed dose $( \pm 2 \%)$. The accuracy of the dosimetry has also been corroborated by measurements with a calorimeter (Lyman et a1., 1980).

\section{BEAM MODIFICATION FOR THERAPY}

Major modifications may be necessary to transform the small diameter monoenergetic beam that emerges from the accelerator into a beam that can be used to produce the varied dose distributions required for therapy. The primary modifications are to spread the narrow beam over the desired treatment area, and to modulate the depth of penetration of the beam so that all regions of the desired treatment volume receive a biologically equivalent dose.

\section{Beam Broadening and Flattening}

Currently there are two well-proven techniques for forming large uniform fields in the plane perpendicular to the beam direction: magnetically scanned beams and scattered beams.

Magnetically Scanned Beam

A magnetically scanned beanı has been used at Uppsala (Larsson, 1961) where a pair of sweeping magnets scans a pencil beam of protons over a $15 \times 15 \mathrm{~cm}$ region; special attention is given to the pulsed nature of the beam in order to avoid hot spots in the pattern.

A spot scanning system represents another solution to the problem. In this system, it is possible to achieve a radiation field with an irregular shape and intensity distribution, and to correct any fluctuations in the beam intensity (Kanai et al., 1980). This technique has been tested with a $70 \mathrm{MeV}$ proton beam.

Three-dimensional scanning has been proposed as another alternative (Leeman et al., 1977, 1979). This system would combine the spot scanning system with range modulation to enable the irradiation of irregularly shaped volumes. Dose distributions within the volume could be shaped to achieve a uniform response of the tumor cells. Some preliminary design and development have been 
done with this system. A full test at the Bevatron of the three-dimensional scanning system is being proposed. This represents a highly efficient, if complex, solution to the problem.

\section{Scattered Beam}

The second method is a double scattered beam which was initially developed at the Harvard cyclotron (Schneider et a7., 1974; Koehler et al., 1977) and is now also used at both the 184-inch Synchrocyclotron (Crowe et al., 1975) and the Bevalac and Bevatron.

Multiple scattering of charged particles by thin high-Z foils produces a Gaussian-like distribution of small scattering angles. At some distance from the foil this distribution can be projected onto a plane perpendicular to the beam axis to form a gaussian radial flux distribution. At this point, some central portions of the beam may be stopped by a solid beam absorber of an appropriate shape and material. The beam passing around this absorber is then mixed by a second scattering foil. This method has produced a 30-cm diameter helium beam with $\pm 2 \%$ flatness
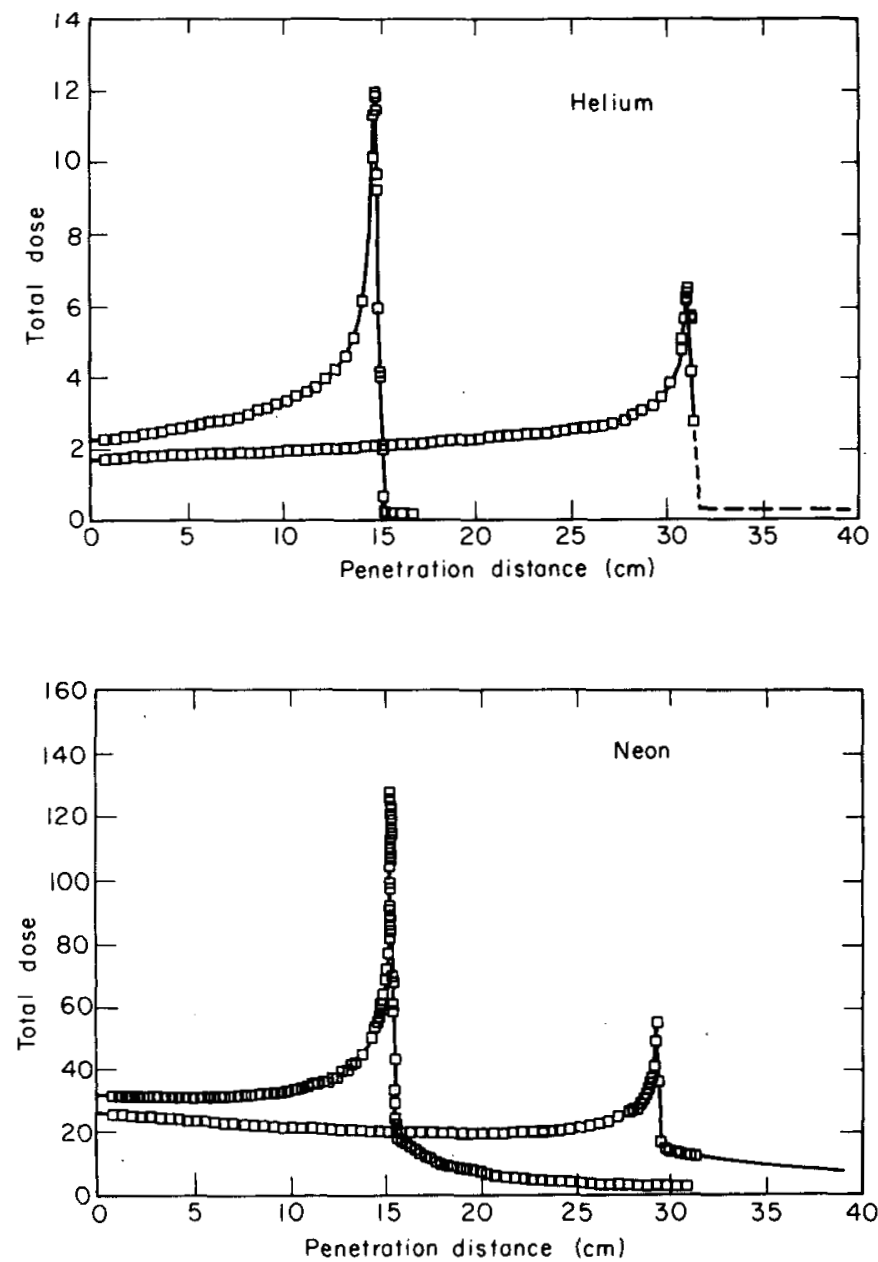

over $90 \%$ of the field (Crowe et a 1., 1975). The beam spreading system behaves approximately like a point source of illumination. The apparent source distance from the isocenter of the patient positioner at the cyclotron is about $6 \mathrm{~m}$, which results in a relatively small divergence of treatment fields with depth in the patient, compared to a $1 \mathrm{~m}$ source-to-skin distance typical for photon sources.

\section{Depth-Dose Modification}

Helium and the heavier ions currently used for radiotherapy at LBL have Bragg peaks characteristic of heavy charged-particle beams (Fig. 1) (Lyman and Howard, 1977b). To optimize the depth-dose distrioution of these Deams for therapy, it was necessary to design a method to superimpose consecutive doses with different Bragg curves in order to obtain the proper dose build-up that will produce the desired dose distribution within a given volume. The method currently used to make this modification is to pass a monoenergetic beam through a ridge filter (Karlsson, 1964) to produce a polychromatic beam. This polychromatic beam can be considered
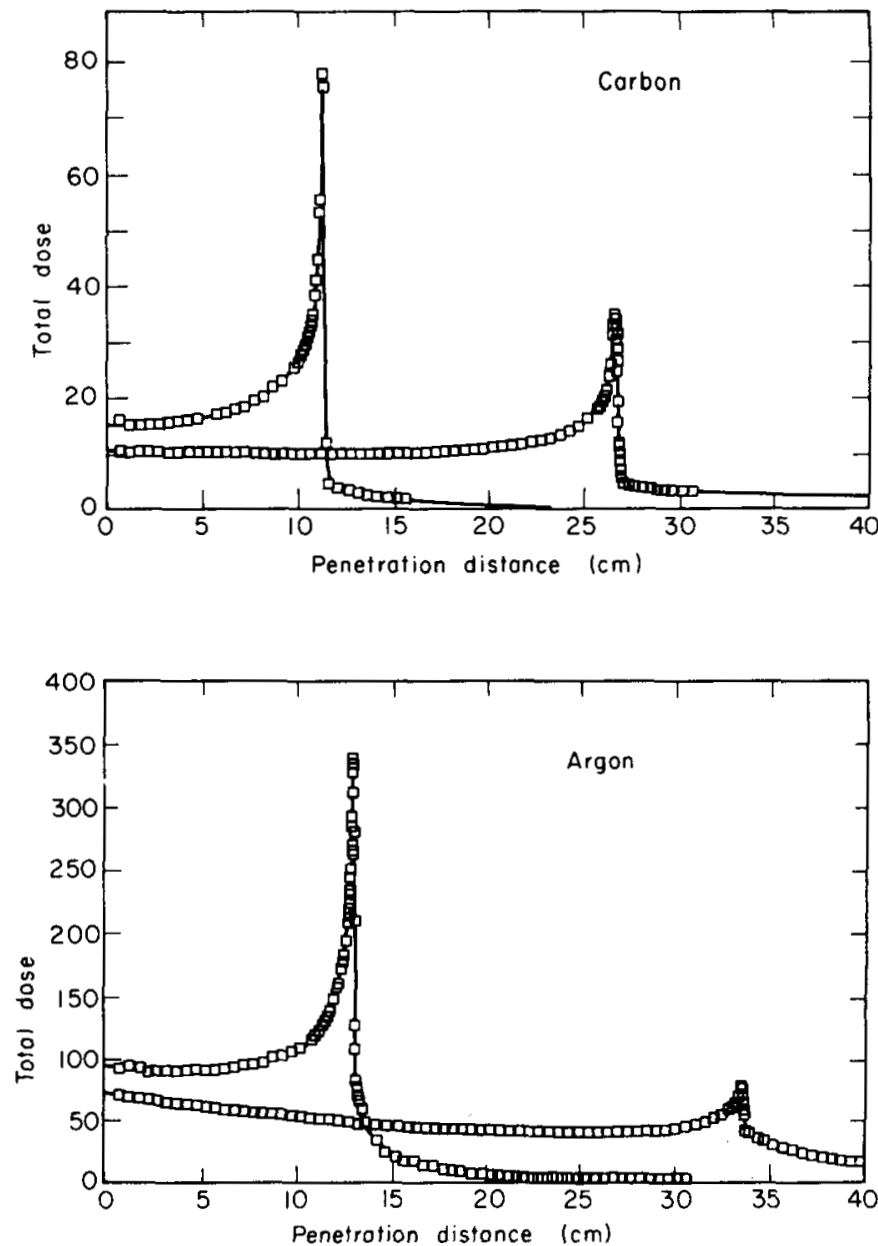

Figure 1. Bragg curves for helium-, carbon-, neon-, and argon-ion beams (Lyman and Howard, 1977b). (XBL 768-9226) 
as being composed of a series of monoenergetic beams with differing ranges. The desired dose distributions are usually not uniform because the RBE values for most beams increase with increasing depth below the entrance surface. The physical dose distribution over the desired treatment volume is shaped to compensate for this increase in RBE.

Ridge filters have been designed for the various heavy charged-particle beams. A different $r$ idge filter is required for each particle beam and for each thickness of the treatment volume in the beam direction. As a practical matter the ridge filters are generally built for treatment volume thicknesses from 4 to $14 \mathrm{~cm}$ in $2-\mathrm{cm}$ increments. The same set of ridge filters has been used for both the carbon and neon beams. This represents a compromise from the theoretically desired dose distributions, however the biologic experiments have not shown conclusively that the dose distribution snould be charged.

At $L B L$ the design of the ridge filter starts with a well-measured Bragg curve. Based on theoretical considerations (Litton et al., 1968), a Bragg curve that is a good representation of the measured curve can be calculated (Lymand and Howard, 1977b). The assumptions used in the beam model are that, with some probability, primary ions are removed from the beam by nuclear interactions which result in fragmentation of the incoming ion. A fixed amount of energy is deposited locally during the fragmentation and a secondary particle (a fragment of the primary) continues in the forward direction with a velocity nearly the same as the primary ion at the time of the collision. All secondary particles proceed to the end of their range without further nuclear interactions, i.e., no tertiary particles are generated.

Two adjustable parameters are needed to adequately match the tneoretical Bragg curves with the experimental data. The first parameter determines the charge conservation in the collision, i.e., what fraction of the original charge is to appear in the secondary fragments. The remaining charge is assumed to have gone to low energy charged fragments that are stopped close to the point of fragmentation. The second parameter determines the relative charge distribution of the secondary ions. The theoretical Bragg curve is composed of the Bragg curve of the primary ions plus the Bragg curves of all the various secondary ions. The dose distribution. can therefore be partitioned to identify the contribution of the primary ions and that of the secondary ions (Fig. 2).

LET values are derived as a function of penetration distance from the beam model that is used to calculate the Bragg curve. The LET values calculated are the dose-weighted averages of the LET of the primary ions and each type of secondary ion. During the design of the iso-

Curvival ridge filter, these LET values are used produce the average LET distribution as a unction of penetration distance for the spread Bragg peak.

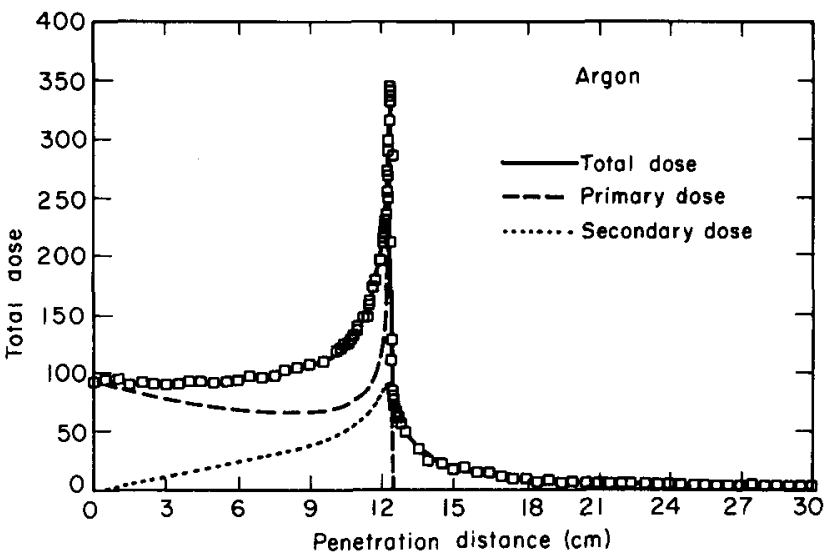

Figure 2. Bragg curve of the $500-\mathrm{MeV} / \mathrm{u}$ argon beam in water. Experimental points are compared with the theoretical curves for the total dose as well as that caused by the primary and secondary particles (Lyman and Howard, 1977b).

(XBL 768-9256)

In order to produce a uniformly effective dose distribution over the desired region, there must be a means of predicting some biologic effect as a function of the average LET and dose at each point in depth along the depth-dose distribution. We use the linear-quadratic model to predict cell survival:

$$
S=S_{0} \cdot \exp \left(-\alpha D-\beta D^{2}\right)
$$

$S$ is the number of surviving cells in a population of $\mathrm{S}_{0}$ cells. The $\alpha$ and $\beta$ terms are both functions of the average LET and the velocity of the particles, and $D$ is the dose. The functions that describe the variations of $a$ and $b$ were chosen to match as closely as possible the experimental RBE values from the known heavy chargedparticle experiments, many of which were summarized in an earlier report (Lawrence Berkeley Laboratory Report 5610, 1977). Experimental conditions of these experiments and the RBE values derived are maintained in a data base. For most experimental conditions several biological systems have been used to test the mutiple experimental conditions (ion, energy, ridge filter, and penetration depth). There is a fairly large range between the lowest and nighest reported RBE values for each condition. The variation is due to the sensitivity of the system, the level of biologic effect at which the RBE is calculated, and the choice of the reference radiation. For the purposes of radiation therapy, the most appropriate RBE values are those derived at a level of biological effect that would be observed following a dose of about 200 rad of high-energy photon irradiation, because this is the size of a typical daily dose. The RBE value at this dose would be higher than the values that are typically reported.

For ridge filter design, it is not important to know the absolute value of the RBE at any point in the beam. However, the relative change 
in $R B E$ between the proximal and the distal peaks must be known so that the ridge filter can be made to produce the proper dose profile.

Once the parameters of the neavy chargedparticle beam and a means of calculating a biologic effect are known, it is a fairly straightforward procedure to calculate the expected biologic effect as a function of depth. The necessary iterations to minimize the deviations from the desired biologic effect can then be performed. The ridge filters are generally designed to produce a region of uniform cell survival if the dose administered is the appropriate dose for a $66 \%$ cell survival in the treatment volume.

The ridge filter design program selects the appropriate weighting factors for a series of monoenergetic beams to produce the specified isosurvival region. In addition, RBE values for the cell survival model are calculated at the $10 \%$ survival level at selected points along the depth-dose curve. These RBE values (Tables 1-3) are compared with RBE values stored in the data base. The RBE values for the cell survival model have been calculated relative to the helium plateau, which is generally thought to be similar in effectiveness to $225-k V \times$ rays.

If the ridge filter is properly designed and built, then the RBE values at one point on the depth-dose curve are sufficient to select the proper dose to achieve the desired biological effect.

\section{PATIENT DOSE SPECIFICATION}

For the helium, carbon, and neon spread Bragg-peak beams, the maximum dose occurs at the proximal peak. For this reason, the proximal

Table 1. RBE Values Relative to the Helium Plateau for a $25 \mathrm{~cm}$ Helium Beam

\begin{tabular}{|c|c|c|c|c|c|c|}
\hline \multirow[b]{2}{*}{ Position } & \multicolumn{6}{|c|}{ Ridge Filter $(\mathrm{cm})$} \\
\hline & 4 & 6 & 8 & 10 & 12 & 14 \\
\hline $\begin{array}{l}\text { Proximal Peak }+0.5 \mathrm{~cm} \\
\text { Midpeak } \\
\text { Distal Peak }-0.5 \mathrm{~cm}\end{array}$ & $\begin{array}{l}1.06 \\
1.08 \\
1.19\end{array}$ & $\begin{array}{l}1.05 \\
1.07 \\
1.19\end{array}$ & $\begin{array}{l}1.04 \\
1.06 \\
1.19\end{array}$ & $\begin{array}{l}1.03 \\
1.06 \\
1.19\end{array}$ & $\begin{array}{l}1.02 \\
1.05 \\
1.20\end{array}$ & $\begin{array}{l}1.02 \\
1.05 \\
1.20\end{array}$ \\
\hline
\end{tabular}

Table 2. RBE Values Relative to the Helium Plateau for a $25 \mathrm{~cm}$ Carbon Beam

\begin{tabular}{|c|c|c|c|c|c|c|}
\hline \multirow[b]{2}{*}{ Position } & \multicolumn{6}{|c|}{ Ridge Filter $(\mathrm{cm})$} \\
\hline & 4 & 6 & 8 & 10 & 12 & 14 \\
\hline $\begin{array}{l}\text { Proximal Peak }+0.5 \mathrm{~cm} \\
\text { Midpeak } \\
\text { Distal Peak }-0.5 \mathrm{~cm}\end{array}$ & $\begin{array}{l}1.92 \\
2.07 \\
2.39\end{array}$ & $\begin{array}{l}1.78 \\
1.94 \\
2.35\end{array}$ & $\begin{array}{l}1.67 \\
1.85 \\
2.32\end{array}$ & $\begin{array}{l}1.60 \\
1.77 \\
2.29\end{array}$ & $\begin{array}{l}1.53 \\
1.71 \\
2.27\end{array}$ & $\begin{array}{l}1.48 \\
1.66 \\
2.25\end{array}$ \\
\hline
\end{tabular}

Table 3. RBE Values Relative to the Helium Plateau for a $25 \mathrm{~cm}$ Neon Beam

\begin{tabular}{|c|c|c|c|c|c|c|}
\hline \multirow[b]{2}{*}{ Position } & \multicolumn{6}{|c|}{ Ridge Filter $(\mathrm{cm})$} \\
\hline & 4 & 6 & 8 & 10 & 12 & 14 \\
\hline $\begin{array}{l}\text { Proximal Peak }+0.5 \mathrm{~cm} \\
\text { Midpeak } \\
\text { Distal Peak }-0.5 \mathrm{~cm}\end{array}$ & $\begin{array}{l}2.54 \\
2.65 \\
2.83\end{array}$ & $\begin{array}{l}2.40 \\
2.54 \\
2.81\end{array}$ & $\begin{array}{l}2.28 \\
2.45 \\
2.79\end{array}$ & $\begin{array}{l}2.18 \\
2.37 \\
2.78\end{array}$ & $\begin{array}{l}2.09 \\
2.30 \\
2.78\end{array}$ & $\begin{array}{l}2.02 \\
2.24 \\
2.77\end{array}$ \\
\hline
\end{tabular}


peak has been selected as the point to which a specified dose is to be delivered. The therapy beam monitors are calibrated so that a given dose $c$ an be delivered to the patient at the proximal peak. A clinical RBE at this proximal peak position is needed to specify the chargedparticle dose for the desired effect.

Until definitive clinical RBE values are obtained, the dose to the proximal peak is specified by the model used to design the ridge filters. Tables $4-6$ give the dose at the

Table 4. Helium Physical Dose

Ridge Filter (cm)

\begin{tabular}{lcccccc} 
CORE & 4 & 6 & 8 & 10 & 12 & 14 \\
\hline 170 & 130 & 133 & 135 & 137 & 139 & 140 \\
200 & 155 & 158 & 160 & 162 & 164 & 165 \\
225 & 175 & 178 & 181 & 183 & 185 & 186 \\
250 & 195 & 199 & 202 & 204 & 206 & 207 \\
300 & 236 & 240 & 243 & 246 & 248 & 249 \\
350 & 278 & 282 & 285 & 288 & 290 & 291 \\
400 & 319 & 323 & 327 & 329 & 332 & 334 \\
500 & 402 & 407 & 411 & 414 & 416 & 418 \\
\hline
\end{tabular}

Taole 5. Carbon Pnysical Dose

$$
\text { Ridge Filter }(\mathrm{cm})
$$

\begin{tabular}{rrrrrrr} 
CORE & 4 & 6 & 8 & 10 & 12 & 14 \\
\hline 170 & 52 & 58 & 63 & 67 & 71 & 75 \\
200 & 65 & 72 & 78 & 83 & 88 & 92 \\
225 & 75 & 83 & 90 & 96 & 101 & 106 \\
250 & 86 & 95 & 103 & 109 & 115 & 121 \\
300 & 111 & 121 & 131 & 139 & 146 & 153 \\
350 & 136 & 149 & 161 & 170 & 178 & 186 \\
400 & 162 & 177 & 190 & 200 & 210 & 219 \\
500 & 221 & 240 & 257 & 269 & 281 & 291 \\
\end{tabular}

Table 6. Neon Pnysical Dose

\begin{tabular}{|c|c|c|c|c|c|c|}
\hline \multirow[b]{2}{*}{ CORE } & \multicolumn{6}{|c|}{ Ridge Filter $(\mathrm{cm})$} \\
\hline & 4 & 6 & 8 & 10 & 12 & 14 \\
\hline 170 & 37 & 40 & 42 & 44 & 47 & 49 \\
\hline 200 & 46 & 49 & 52 & 55 & 58 & 61 \\
\hline 225 & 54 & 57 & 61 & 64 & 68 & 71 \\
\hline 250 & 62 & 66 & 70 & 74 & 78 & 81 \\
\hline 300 & 81 & 85 & 90 & 95 & 100 & 104 \\
\hline 350 & 100 & 106 & 112 & 118 & 123 & 128 \\
\hline 100 & 120 & 127 & 134 & 141 & 147 & 153 \\
\hline 500 & 165 & 175 & 185 & 194 & 202 & 208 \\
\hline
\end{tabular}

proximal peak and the corresponding equivalent dose for a high-energy photon beam for the various ridge filters and ions that are being used. This latter dose is designated as the cobalt-rad-equivalent (CORE) dose.

Isodose plans can be calculated for patient treatment with the heavy charged-particle beams. However, if the RBE values change as a function of the penetration distance, then the isodose plan is not sufficient to illustrate the degree of the biologic effect expected. In general, one would expect to have a lower effective dose in the plateau region and beyond the distal peak, and an increasingly effective dose from the proximal peak to the distal peak. Treatment plans based on the CORE dose are an attempt to provide the minimum information to illustrate the biologic effective dose distribution.

\section{ACKNOWLEDGEMENT}

The 184-inch Synchrocyclotron and Bevatron operations groups have made major contributions in the effort reported here. Special recognition is given to James MacMullen, Fred Yeater, Ken Crebbin, and Tom Criswell. The encouragement and support of $C$. A. Tobias and J. R. Castro has been greatly appreciated. This work is supported by NCI grant CA-19138.

\section{REFERENCES}

Crowe, K., L. Kanstein, J. Lyman, and F. Yeater. 1975. A Large Field Medical Beam at the 184-inch Synchrocyclotron. Lawrence Berkeley Laboratory LBL-4235.

Kanai, T., K. Kawachi, Y. Kumamoto, H. Ogawa, T. Yamada, H. Matsuzawa, and T. Inada. 1980. Spot scanning system for proton radiotherapy. Med. Phys. 7, 365-369.

Karlsson, B. G. 1964. Methoden zur berechnung und erzielung einger fur die tiefentherapie mit hoch-energetischen protonen gunstiger dosisverteilungen. Strahlentherapie 124, $481-92$.

Koehler, A. M., R. J. Schneider, J. M. Sisterson. 1977. Flattening of proton dose distributions for large field radiotherapy. Med. Phys. 4, 297-301.

Larsson, B. 1961. Pre-therapeutic physical experiments with high energy protons. Br. J. Radiol. 34, 143 .

Leeman, C., J. Alonso, H. Grunder, E. Hoyer, G. Kalnis, D. Rondeau, J. Staples, and F. Voelker. 1977. A Three-Dimensional Beam Scanning Device for Biomedical Research. Lawrence Berkeley Laboratory Report LBL-5546.

Leeman, C. H., J. Alonso, H. Grunder, E. Hoyer, J. Staples, and F. Voelker. 1979. A threedimensional beam scanning system for the irradiation of large volumes in particle 
therapy. Proceedings of the First International Seminar on the Uses of Proton Beams in Radiation Therapy, Moscow, December 6-11, 1977.

Litton, G., J. Lyman, and C. Tobias. 1968. Penetration of High-Energy Heavy Ions with the Inclusion of Coulomb, Nuclear, and other Stochastic Processes. Lawrence Berkeley Laboratory Report UCRL-17392.

Lyman, J. T. and J. Howard. 1977a. Biomedical research facilities and dosimetry. Biological and Medical Research with Accelerated Heavy Ions at the Bevalac, 1974-1977, pp. 26-35. Lawrence Berkeley Laboratory Report LBL-5610.

Lyman, J. T. and J. Howard. 1977b. Dosimetry and instrumentation for helium and heavy ions. Int. J. Radiat. Oncol. Biol. Phys. 3 , $81-85$.

Lyman, J. T., J. Howard, and A. A. Windsor. 1975. Heavy charged-particle beam monitoring with segmented ionization chambers. Med. Phys. 2, 163. (Abstract).
Lyman, J. T., H. Blattman, P. Fessenden, K. Y. Lam, J. C. McDonald, A. R. Smith, and L. Verhey. 1980. Report on the Berkeley Dosimetry Intercomparison. Med. Phys.7, 425. (Abstract).

Schneider, R., R. A. Schmidt, and A. M. Koehler. 1974. Physical preparations for Cancer therapy at the Harvard cyclotron. Bull. Amer. Phys. Soc. 19, 31. (Abstract).

Shapiro, E. G. 1970. Linear seven-decade current/voltage-to-frequency converter. IEEE Trans. Nuc 1. Sci. NS-17, 335-344.

Tobias, C. A., H. O. Anger, and J. Lawrence. 1952. Radiological use of the high-energy deuterons and alpha particles. Amer. J. Roent. Radiat. Ther. Nuc1. Med. $\overline{67}, 1-27$.

Tobias, C. A., J. E. Roberts, J. H. Lawrence, B.V.A. Low-Beer, H. O. Anger, J. L. Born, R. McCombs, and C. Huggins. 1955. Radiation Hypophysectomy with High-Energy Proton Beams. Lawrence Berkeley Laboratory Report, UCRL-3035. 


\title{
HEAVY CHARGED-PARTICLE TREATMENT PLANNING
}

\author{
G. T. Y. Chen, S. Pitluck, and J. T. Lyman
}

An essential element of the heavy-ion radiotherapy program is the development of a computerized treatment planning system. In its broadest sense, treatment $\mathrm{planning} \mathrm{includes} \mathrm{development}$ of (1) methods to calculate isodose distributions, (2) dosimetry to verify that such calculations are accurate, and (3) optimized irradiation techniques that confine the $h i g h$ dose region to the prescribed target volume. A computerized tomographic (CT) based system is currently in operation, where sequential scans are first displayed on a dedicated raster graphics display unit. Target contours in appropriate slices are then entered via cursor by the radiotherapist. After the entry angle and ion type are chosen, the treatment planning program uses CT data on a pixel-by-pixel basis to (1) design appropriate compensators to contour the stopping region of the therapy beams, (2) select an appropriate spread Bragg peak for the irradiation, (3) design a collimator aperture for each entry porta1, and (4) generate isoeffect and physical dose distributions overlayed on the CT image. Treatment plans that include the potential effects of compensator misregistration and motion due to respiration may be optionally generated. Computer assisted aids in portal placement are also obtained. In this section, we review progress in the area of clinical physics, and outline future lines of investigation.

\section{ROLE OF CT IN TREATMENT PLANNING}

The value of $C T$ in conventional radiation therapy has been widely recognized (Stewart et a 1., 1978; Hobday et a 1., 1979). CT plays an essential role in charged particle radiotherapy, because it is used in target volume definition, quantitation of inhomogeneities, portal design and placement, and postherapeutic evaluation of treatment effectiveness.

Studies have shown that $C T$ is an important adjunct in tumor volume definition (Munzenrider et a1., 1977; Goitein et a.., 1979), and that without this diagnostic aid, marginalland geometric misses of the target volume occur in over $40 \%$ of cases. In addition, the quantitative information that CT provides is essential in determining the perturbing effects of inhomogeneities on charged particle transport. Photons are exponentially attenuated, and a small error in the determination of the effective depth of the target volume produces only a small dose perturbation. For $4 \mathrm{MeV}$ photons, a $1 \mathrm{~cm}$ error in depth results in a $5 \%$ dose error. In contrast, a $1 \mathrm{~cm}$ error in range overestimation of a charged particle beam will irradiate tissue distal to the primary beam to the $100 \%$ level, thereby compromising the potential benefit from a finite range beam. Underestimation of the range required could lead to underdosing the tumor by about 80 to $90 \%$. Therefore, one of the major objectives has been to develop methods to accurately calculate the appropriate residual range for adequate tumor coverage and to understand the accuracy with which charged particles may be used to produce highly localized dose distributions. The goal is to be able to predict the stopping point of a beam passing through a variety of tissues, to an accuracy of better than $5 \mathrm{~mm}$, and in some cases, possibly to better than 2-3 mm. Typical ranges for charged particle radiotherapy are 20 to $25 \mathrm{~cm}$, which implies that the water equivalent range must be extracted from CT to within $2 \%$.

The quantitative information furnished by $\mathrm{CT}$ is not directly applicable to charged particle treatment planning. CT numbers are a measure of the linear attenuation coefficient of each pixel at diagnostic photon energies, where both photoelectric and compton processes are present. The photoelectric effect is a strong function of both the electron density and effective atomic number, while the Compton process depends primarily on the electron density. Like Compton scatter, the stopping power of charged particles is dominated by the electron density of the medium traversed.

In order to utilize CT data quantitatively, methods to relate CT number to water equivalent pathlength are needed. Figure 1 shows a calibration curve relating $C T$ number to water equivalent range per pixel. The experimental points were determined by CT scanning tissue analogues and then measuring their stopping power in a helium

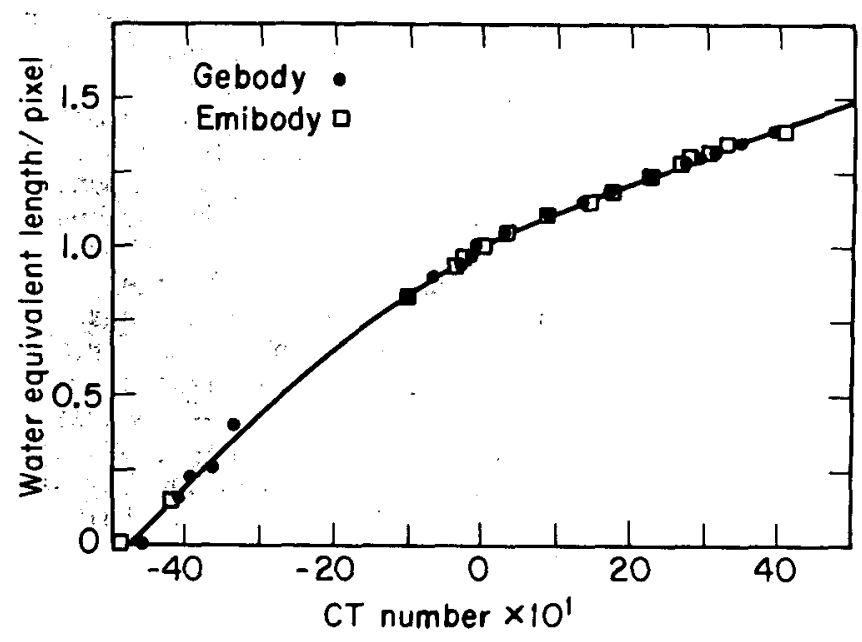

Figure 1. Calibration curve relating $C T$ number and water equivalent range per pixel. (XBL 793-3293) 
ion beam. The calibration curve utilizes CT data at a single scan energy, and implicitly assumes a well-defined relationship between increasing density and increasing effective atomic number of bone. This condition may not always be met (e.g., when metabolic bone diseases are present), but it is intended to be a reasonable approximation that produces sufficient accuracy in most cases. The accuracy of the single energy technique in calculating the water equivalent pathlength in phantoms and in vivo are described in Part VI, Section B of this report. Comparisons between the CT calculated water equivalent pathlength and dosimetry indicate an agreement within $2 \mathrm{~mm}(2 \%)$ - in soft tissue regions of a Rando phantom. In the region of the vertebral body, $5 \%$ differences exist, principally due to partial volume averaging effects.

Dual energy CT scanning is another technique for obtaining the quantitative information needed for treatment planning, and has been used by others for unique determinations of effective atomic number and electron density (Rutherford et al., 1976; Alvarez and Macovski, 1976). Studies evaluating tnis technique are planned. When $C T$ is used quantitatively, CT number drift, beam hardening effects, partial volume sampling, the presence of artifacts, and the transient nature of some inhomogeneities (such as gas in the gastrointestinal tract) must all be considered. Techniques to monitor and correct for scanner drift, and to edit CT data to remove streak artifacts and transient gas pockets, have been developed.

The typical volume element of a high resolution CT scan is $1 \times 1 \times 2 \mathrm{~mm}$ for a $2 \mathrm{~mm}$ slice thickness. This resolution is adequate for most sites of interest. The required spatial resolution for accurate treatment planning depends on the lateral scattering characteristics of the particles used (Goitein and Suit, 1975) and the size of inhomogeneities. In areas such as the abdomen, thorax, or pelvis, neterogeneities traversed are large compared to voxel size. One exception is the detailed structure of the spinal column, for which maximal range shortening has been experimentally determined. Compensation for such a complex inhomogeneity is not performed by a three-dimensional compensator because the design and alignment of such a device would be extraordinarily difficult. Rather, the range from a posterior port is increased by an amount comparable to the maximal shortening of the spin (about $1.5 \mathrm{~cm}$ ) and a small region of overshoot is tolerated. The evaluation of voxel size adequacy for irradiation of tumors in the head requires additional dosimetry. In particular, the irradiation of the base of brain lesions may present treatment planning difficulties because of the presence of relatively thin sheets of bone within the scan plane. Heavy-ion radiography has been an important dosimetric technique in this study (see Part VI, Section B).

The charged particle radiotherapy programs at LBL and Massachusetts General Hospital have an additional constraint imposed by the limitation of fixed horizontal therapy beams. A majority of patients at $L B L$ are treated in the seated or standing position, but current CT scanners can only scan recumbent patients. Therefore, both institutions have ordered a specially modified unit capable of scanning patients who are seated or standing. An artist's conception of the scanner is shown in Figure 2. The gantry is shown in the norizontal mode, which is suitable for an upright patient. In this mode, the gantry is translated upward to obtain a series of scans. Scanning in the treatment position circumvents the tumor and organ shifts, which had previously limited the value of recumbent scans on conventional CT units. The EMI 7070 scanner is capable of 3 second scans with a $2 \mathrm{~mm}$ slice thickness. The scanner was installed in the Research Medicine Building (Bldg. 55) in late summer 1980.

\section{TREATMENT PLANNING: CURRENT STATUS}

Pixel-by-pixel treatment planning programs have been developed at $L B L$ to calculate the dose distribution from multiport charged particle beams (Chen et al., 1979). The process begins by taking a sequential set of CT scans tnrough the region of interest. These scans are then viewed by a team of radiotherapists and physicists on a graphics unit. An interactive graphics program has been developed to facilitate tumor localization and the entry of target volumes into the computer treatment planning system. Options available in this program include (1) standard window and leveling capability to adjust the CT image for optimal tumor delineation; (2) contouring routines that allow the radiotherapist to define the target volume to be treated; (3) the ability to recall and display previous contours, and modify them if needed; (4) the ability to enter reference points on the transaxial view, which later assists in the placement of a computer generated treatment portal; (5) measurement of geometric and water

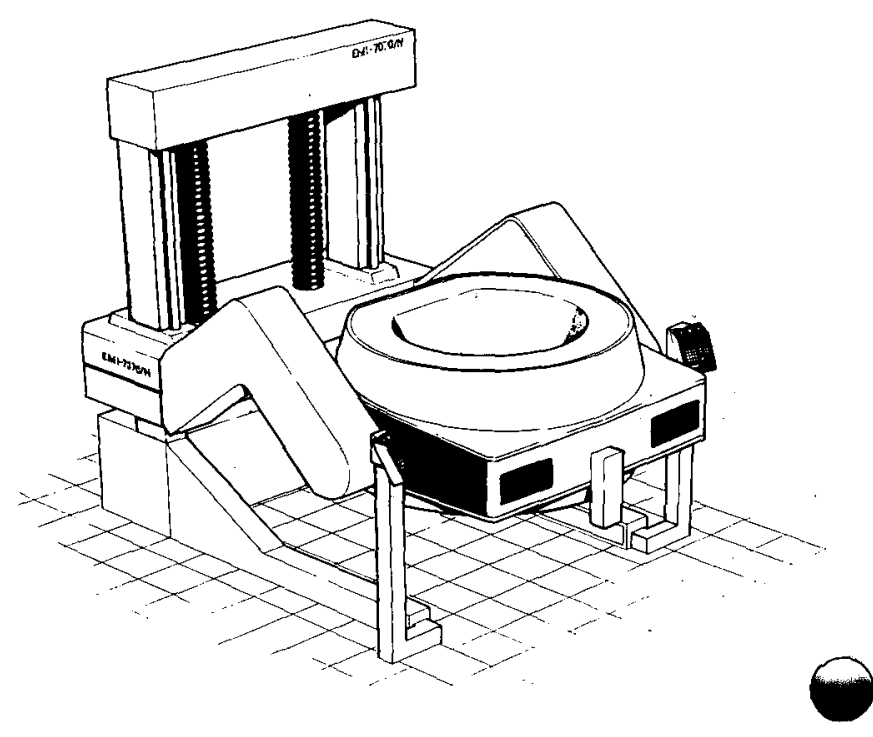

Figure 2. Upright CT scanner. (XBL 7912-13720) 
equivalent pathlengths between arbitrary points; (6) determination of the mean CT number and standard deviation in an irregular region of interest. These features allow the radiation therapy team to define the target volume, make measurements of the lesion size and CT characteristics, and determine the water equivalent depth of structures of interest.

Feedback to verify that target volumes drawn on sequential axial CT scans are reasonable in three-dimensional space is highly desirable. One method to graphically appreciate this point is seen in Figure 3, which shows a series of target contours at different scan levels. The display may be rotated to identify inconsistencies of tumor volume definition from slice to slice. A common inconsistency is the definition of a treatment volume in one slice which is bounded above and below by larger volumes. Contour edit-ing, a feature in the interactive package, may be performed to rectify these inconsistencies.

The CT data are then prepared for treatment planning. Pixel-by-pixel treatment calculations generaliy require some data manipulation prior to calculation. These operations include cleaning the image to remove extraneous apparatus included during the scanning process but not relevant to treatment planning calculations (e.g., head holders, scan table, bolus). In addition, air in the gastrointestinal tract must be edited for proper compensation. An automatic threshold edge detection algorithm (requiring one seed point) has been developed to detect the body air boundary and set all pixel values outside to air density. The algorithm utilizes the raster graphics memory since a $320 \times 320$ matrix cannot be contained in computer core.

After the CT data are prepared, a series of treatment planning programs is run to generate

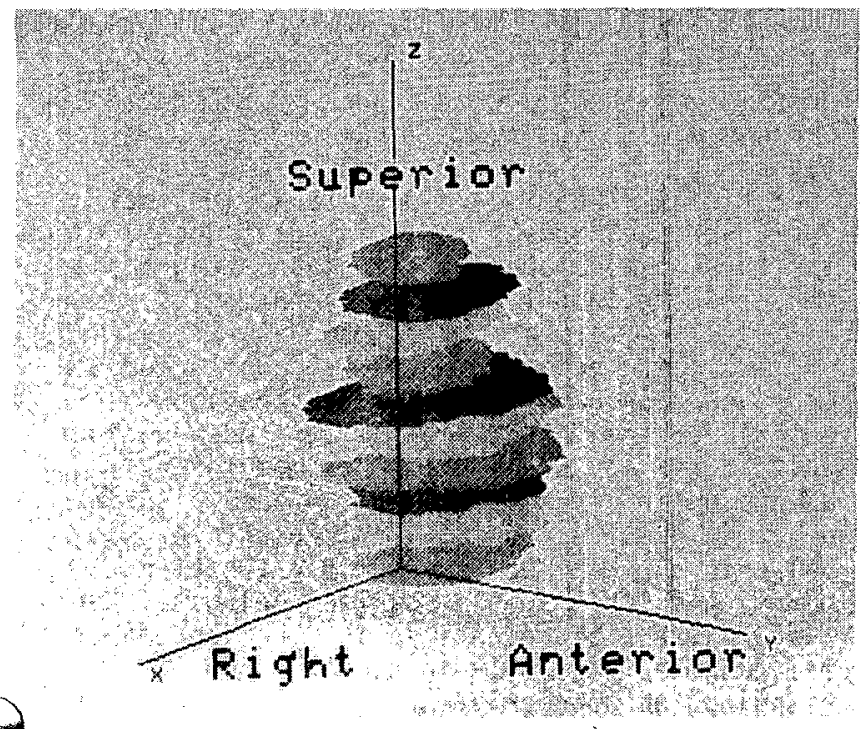

Figure 3. Perspective view of target volumes. (XBB 809-10587)
(1) a port design based on the projected tumor volume, (2) the design of a three-dimensional compensator required to stop the beam at the distal edge of the contour and (3) both physical and isoeffect isodose distributions.

The objective in charged particle dose delivery is to shape a three-dimensional high dose region around the defined target volume. This is done on each slice by calculating the appropriate compensation to stop the primary beam at the distal end of the target contour. The core of the treatment planning algorithm is a subroutine (Huesman et al., 1977) modified to calculate the water equivalent distance from the distal end of the target contour to the body air interface along the specified beam direction. Given this water equivalent range and the maximum range of the heavy-ion beam, the amount of external absorber along that ray is well defined. The calculation is done in a matrix of water equivalent densities per pixel which is derived using a calibration curve such as Figure 1. By examining the target contours in all CT slices, the planning program automatically determines the appropriate spread Bragg peak beam to be consistent with the tumor volume, and calculates the required water column setting for the treatment.

Examples of the program output are shown in Figure 4. Figure $4 \mathrm{~A}$ shows the treatment portal as designed from a series of target contours delineated on fourteen sequential CT scans. The port shape is overlayed on an anterior-posterior projection of multiple CT slices. Figure $4 B$ shows a compensator template, from which a threedimensional compensator may be fabricated. Construction of complex three-dimensional boluses may also be executed by a computer controlled milling machine, as done at the Massachusetts General Hospital cyclotron proton facility.

The isodose distributions are calculated in an $80 \times 80$ matrix by a range shifting algoritnm. Beam models used in the calculation of isodose distributions are discussed in detail by Lyman et al. in the previous paper. Either CORE or physical depth dose distributions may be used in planning, and typical fixed ridge filter beams of carbon are shown in. Figure 5 .

Figure $6 \mathrm{~A}$ is a treatment plan for a two-field irradiation of a pancreatic carcinoma; the broad light line delineates the target volume. Isoeffect 1 ines in $10 \%$ increments show that the high dose region is confined to the target volume Critical organs such as the kidneys and spinal cord are largely spared. In addition, the dose to the gastrointestinal tract from the entering carbon beams is less than $40 \%$. A comparison of the integral dose for the irradiation of this volume with a four-field $25 \mathrm{MeV}$ box irradiation of the same target (Figure $6 \mathrm{C}$ ) shows a reduction in the integral dose of the normal tissue by about $30 \%$. Figure $6 \mathrm{~B}$ illustrates the corresponding physical dose distribution. A $30 \%$ nonuniformity in physical dose is observed within the target volume, but differing RBE values within this region result in an expected uniform biological effect. 

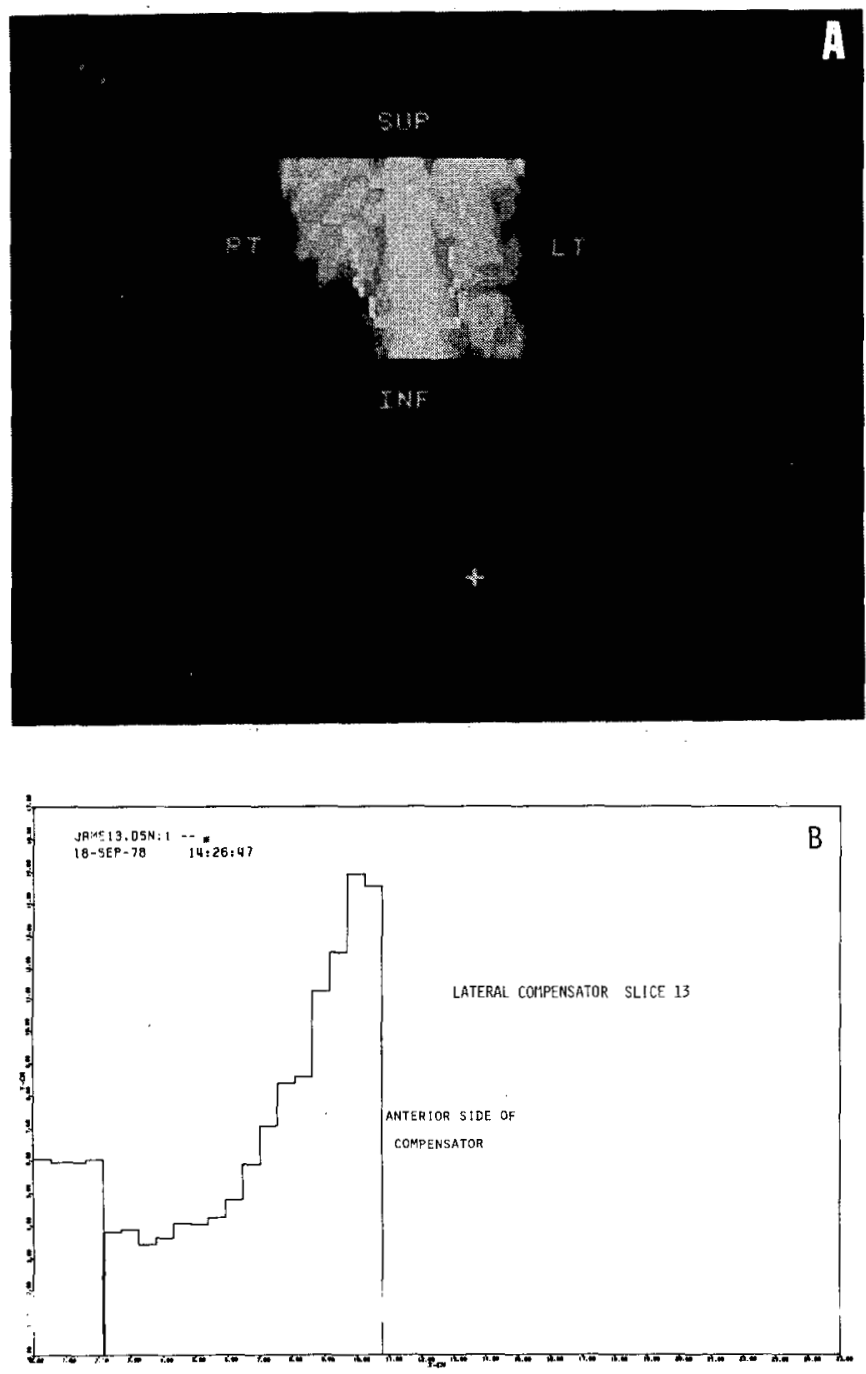

Figure 4. (A) CT derived portal template. (B) Compensator template for one CT slice. [(A) XBB 807-8635; (B) XBL 789-11442]

As with conventional planning, a heavy-ion treatment plan is an idealization of the dose distribution that can actually be achieved. Effects from respiration, multiple scattering of particles, errors in compensation registration, and patient movement during therapy are likely to degrade the sharp dose gradients. Respirationmodified depth-dose distributions and compensation misalignment effects can be included in current dose calculations. Multiple scattering effects on isodose distributions are under study.

\section{Beam Scanning}

Target volumes may be rather irregular in shape in three dimensions. With fixed ridge filter beam delivery, a spread Bragg peak must be chosen to correspond to the maximum target dimension over all slices. This ensures biological dose uniformity over the tumor volume, but results in overdosing normal tissues in other areas.
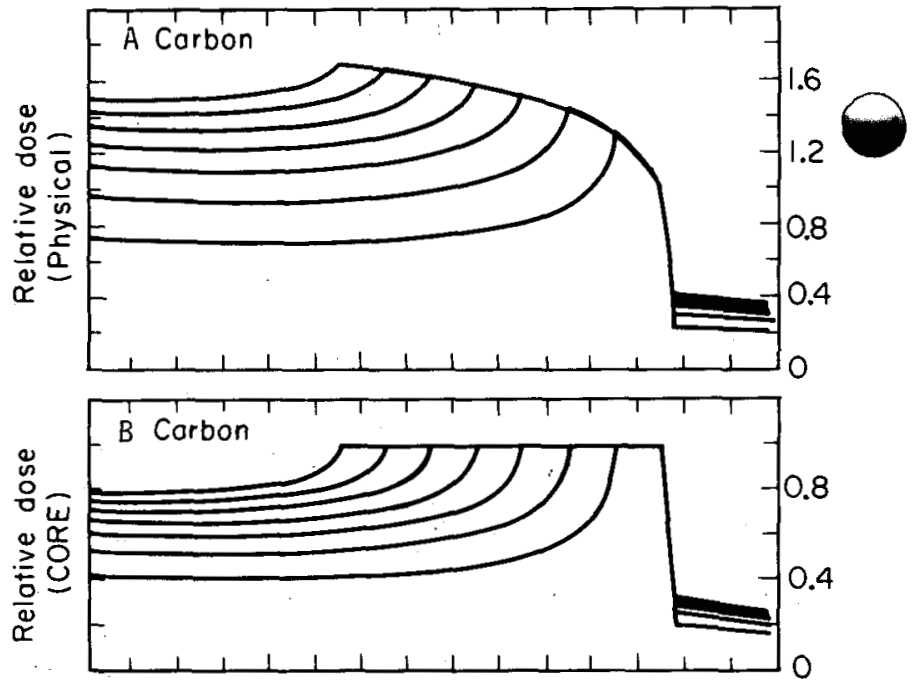

Figure 5. (A) Carbon ion Bragg peaks (pnysical distribution). (B) Spread Bragg peaks of carbon in CORE units. (XBL 787-3372A)

Beam scanning may be used to overcome this disadvantage (Grunder and Leeman, 1977). In beam scanning, a charged particle pencil beam is scanned across the field by magnetic lenses. At each raster point, the depth of penetration, spread Bragg peak dimension, and dose may be delivered to a precalculated value. Hardware for one channel of a scanning system has been assembled for experimental studies at LBL. In this section, we will limit the discussion to the quantitative gain in sparing normal tissues by these techniques over conventional fixed ridge filter delivery methods. Radiobiologic considerations relating to the implicit use of high dose rates to achieve raster beam scanning will not be considered.

The figure used to evaluate the merit of a plan is the ratio of the normal tissue integral dose (NTID) as delivered by beam scanning (BS) versus fixed ridge filter (FRF) methods. A low figure of merit indicates a more favorable condition for beam scanning. Variables that influence the normal tissue integral dose include the three-dimensional target shape, target size, target depth, and field arrangement.

Two computerized treatment planning codes were used in the evaluation. As previously described, the fixed ridge filter code examines al1 target contours and the maximum target dimension in any slice along the beam axis is determined. The ridge filter closest to this value is then chosen and used for treatment planning calculations in all scan planes. The ridge filters are quantized in $2 \mathrm{~cm}$ increments, as in actual therapy. In simulating beam scanning, an appropriate ridge filter for each ray is chosen from a family of ridge filters quantized in $1 \mathrm{~cm}$ units. The spread Bragg peak selected is at least as large as the target size along the ray. 

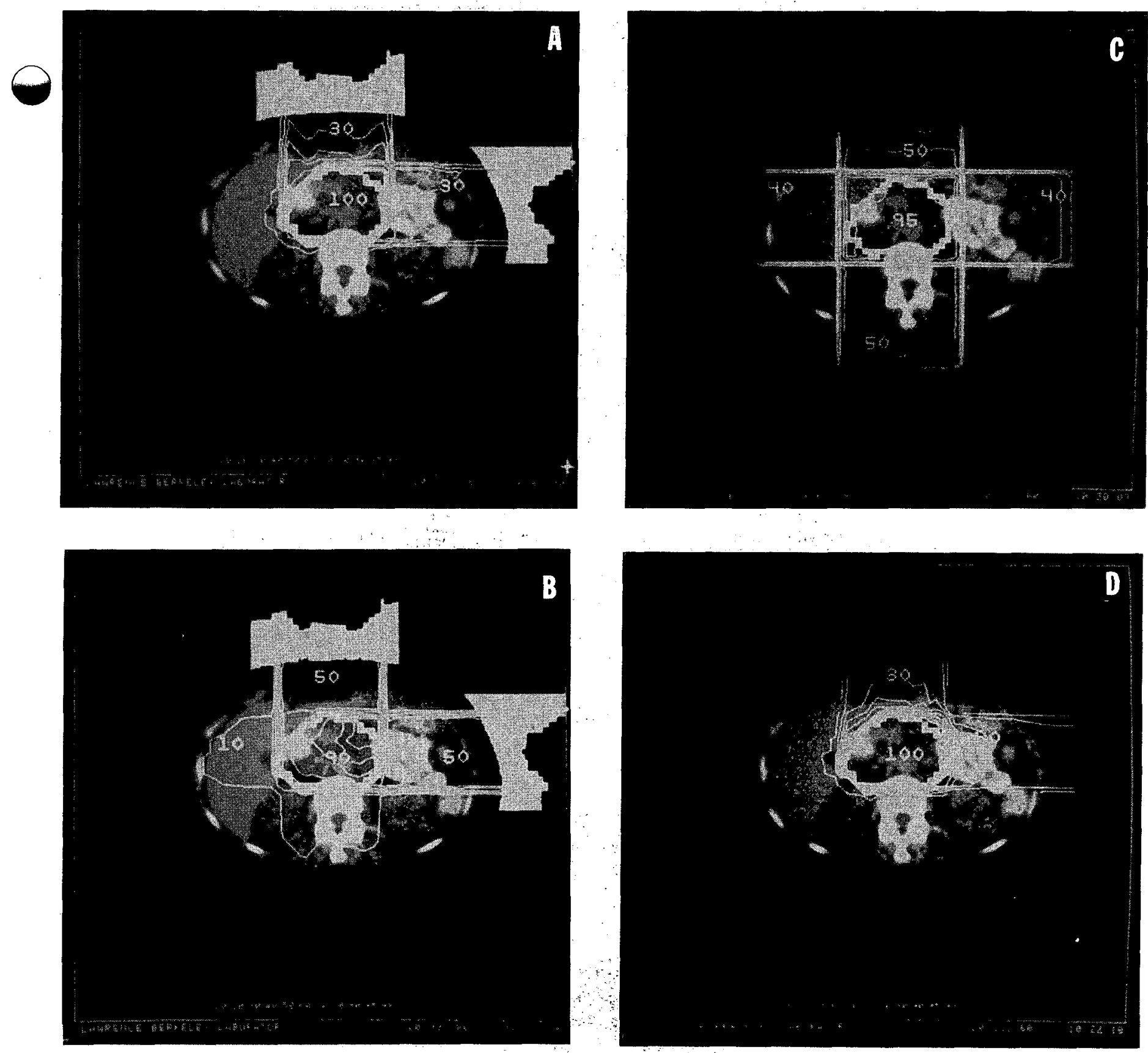

Figure 6. (A) Isoeffect treatment plan for pancreatic lesion (fixed ridge filters);

(B) Treatment plan for pancreatic lesion, phyșical dose; (C) $25 \mathrm{MeV}$ photon four field plan equally weighted; (D) Beam scanned carbon two field plan isoeffect.

[(A) XBB 807-8631; (B) 807-8632; (C) 807-8634; (D) 807-8633]

Treatment plans were calculated using the two codes on pancreatic and brain tumor volumes. The pancreatic tumor consisted of contours outlined on fourteen distinct CT slices. Anterior and one or two latera? fields were used in the planning. The output of the treatment planning program included the total integral dose summed over all slices, the integral dose within the tumor volume, and the normal tissue integral dose.
A representative treatment plan from fixed ridge filter therapy was shown in Figure $6 \mathrm{~A}$. Conformation of the stopping region at the distal edge of the contour with fixed ridge filters and compensators necessarily results in a higher proximal region dose. In elliptical target volumes, as seen in Figure $6 \mathrm{~A}$, the wedge-shaped $40 \%$ dose regions located near the beam edges are due to the use of fixed ridge filters. Use of a 
fixed ridge filter consistent with the maximum target dimension also implies that this ridge filter will not be appropriate for CT levels where the tumor is smaller. Also note that there is a small region outside the target volume treated to $100 \%$. The beam scanned treatment plan (isoeffect isodose) is shown in Figure 6D. Both the intermediate $(50 \%)$ and high isodose (100\%) lines conform much more closely to the target volume. The conformation of the high dose region in other CT planes would be comparable. The fragmentation dose is also reduced because it is dependent upon ridge filter size.

The figure of merit analys is was used on the identical target volume for helium, carbon, and neon ions. The results show that beam scanning with carbon reduces the normal tissue integral dose the greatest, with neon second, and helium third. The normal tissue integral dose is a function of the spread-peak-to-plateau ratio and the size of the fragmentation dose of different ions: From the CORE depth dose currently in use, carbon exhibits the most favorable spread-peakto-plateau ratio with a moderate fragmentation component. The results are summarized in Table 1.
In comparison to photon therapy, both fixed ridge filter and beam scanning substantially reduce the normal tissue integral dose. Again, using the same pancreatic target volume for comparative purposes, the normal tissue integral dose using fixed ridge filters is reduced by $32 \%$ using fixed ridge filters and $50 \%$ by beam scanning. In each case, the target volume received close to $100 \%$ dose uniformly.

Normal tissue integral dose is not a strong function of field arrangement for centrally located lesions, as would be expected from finite range beams. Comparisons of the normal tissue integral dose for two-field right-angle pairs, two-field opposed, and three-field treatment plans all resulted in the same normal tissue integral dose within $2 \%$ (see Table 1 ).

\section{Dosimetry}

Specific aspects of heavy charged particle dosimetry and verification of penetration depth are covered in the heavy-ion radiography dosimetry section and in the radioactive beam

Table 1. Values for Normal Tissue Integral Dose (NTID)

A. NTID: Photons, Fixed Ridge Filters, Beam Scanning

\begin{tabular}{lcc}
\hline Treatment Plan & NTID NTID/NTID (25 MeV) \\
\hline Pnotons (25 MeV) four fields, equal weights & 10,452 & 1.00 \\
$\begin{array}{l}\text { Carbon, fixed ridge filters, two fields } \\
\text { equal weights }\end{array}$ & 7,093 & 0.68 \\
$\begin{array}{l}\text { Carbon, beam scanned two fields, } \\
\text { equal weights }\end{array}$ & 5,278 & 0.50 \\
\hline
\end{tabular}

B. NTID Ratios: Charged Particles

$\begin{array}{ll}\text { Helium } & \frac{\text { NTID }(B S)^{\star}}{\text { NTID }(F R F)^{\dagger}}=0.86 \\ \text { Carbon } & \frac{\text { NTID (BS) }}{\text { NTID (FRF) }}=0.75 \\ \text { Neon } & \frac{\text { NTID (BS) }}{\text { NTID (FRF) }}=0.83\end{array}$

C. NTID as a Function of Field Arrangement for Slice 5 only

(Carbon Ions)

Two fields, right angle pair

Two fields, opposed pair

Three fields (AP, two laterals)
1.0

1.02

1.01

* $B S=$ Deam scanning.

$+F R F=$ fixed ridge filters. 
sections of this report (both found in Part VI; Section B). In addition, photographic film stacks, exit film checks, and in vivo diode measurements are routinely used for patient dosimetry when suitable.

\section{Treatment Planning Computer}

A VAX $11 / 780$ computer will be installed in the Bldg. 55 therapy physics area in late 1980. This computer will allow treatment plans to be performed on $320 \times 320 \mathrm{CT}$ density matrices, thereby increasing calculation accuracy. A hard wired data link between the VAX and the EMI 7070 CT scanner will be installed to facilitate data transfer between the two devices. The VAX will be used for Monte Carlo simulations of beam transport on CT density matrices to evaluate the need to incorporate multiple coulomb scattering algorithms in treatment planning.

\section{Choroidal Melanoma Treatment Planning}

Treatment planning for ocular melanoma is currently done via telephone data link to the Massachusetts General Hospital computer. A code developed by Goitein (Gragoudas et al., 1980) and a graphics terminal allow the visualization of tumor relative to critical structures of the eye. Interactive adjustinent of the position of the eye is an elegant method of optimizing the proper beam entry angle to spare critical structures. At $L B L$, recent interest in the visualization of ocular tumors with high precision CT (available at the University of California, San Francisco) has led to a study to evaluate this means to assist in treatment planning. One particular advantage of CT is its ability to define the relative position of soft tissues surrounding the eye.

\section{ACKNOWLEDGEMENT}

These studies were supported in part by a grant from the National Cancer Institute (Grant No. 5P01CA19138).

\section{REFERENCES}

Alvarez, R. E. and A. Macovski. 1976. Energy selective reconstructions in $X-r a y$ computerized tomography. Phys. Med. Biol. 21, 733-744.
Chen, G.T.Y., R. P. Singh, J. R. Castro, J. T. Lyman, and J. M. Quivey. 1979. Treatment planning for heavy charged particle radiotherapy. Int. J. Radiat. Oncol. Biol. Phys. 5, 1809-1819.

Goitein, M. and H. D. Suit. 1975. The influence of tissue inhomogeneities on the dose distribution of charged particle beams. Proceedings, International Workshop on Particle Radiation Therapy, Key Biscayne, FL. American College of Radiology, Philadelphia, PA.

Goitein, M., J. Wittenberg, M. Mendiond, J. Doucette, C. Fineberg, J. Ferrucci, L. Gunderson, R. Linggood, W. U. Shipley, and H. V. Fineberg. 1979. The value of CT scanning in radiation therapy treatment planning: A prospective study. Int. J. Radiat. Oncol. Biol. Phys. 5, 1787-1798.

Gragoudas, E. S., M. Goitein, L. Verhey, $J$. Munzenrider, $H$. Suit, and $A$. Koehler. 1980. Proton beam irradiation: An alternative to nucleation for intraocular melanomas. Opthamology 87, 571-581.

Grunder, H. and C.W. Leeman. 1977. Present and future sources of protons and heavy ions. Int. 3. Radiat. Oncol. Biol. Phys. 3, 71-80.

Hobday, P., N. J. Hodson, J. Husband, R. P. Parker, and J. S. Macdonald. 1979. Computed tomography applied to radiotherapy treatment planning: Techniques and results. Radiology 133, 477-482.

Huesman, R. H., G. T. Gullberg, W. L. Greenberg, and T.F. Budinger. 1977. Users Manual: Donner Algorithms for Reconstruction Tomography, p. 219. Lawrence Berkeley Laboratory Report 214.

Munzenrider, J. E., M. Pilepich, I. Tchakarova, and B. I. Carter. 1977. Use of body scanner in radiotherapy treatment planning. Cancer $40,170-179$.

Rutherford, R. A., B. R. Pullan, and I. Isherwood. 1976. Measurement of effective atomic number and electron density using an EMI scanner. Neuroradiology 1, 15-21.

Stewart, J.R., J. A. Hicks. M.L.M. Boone, and L. D. Simpson. 1978. Computed tomography in radiation therapy. Int. J. Radiat. Oncol. Biol. Phys. 4, 313-324. 
PART VI. MEDICAL APPLICATIONS OF HEAVY IONS

Section B: Heavy-Ion Radiography and Microscopy 
$\vartheta$

$\bullet$

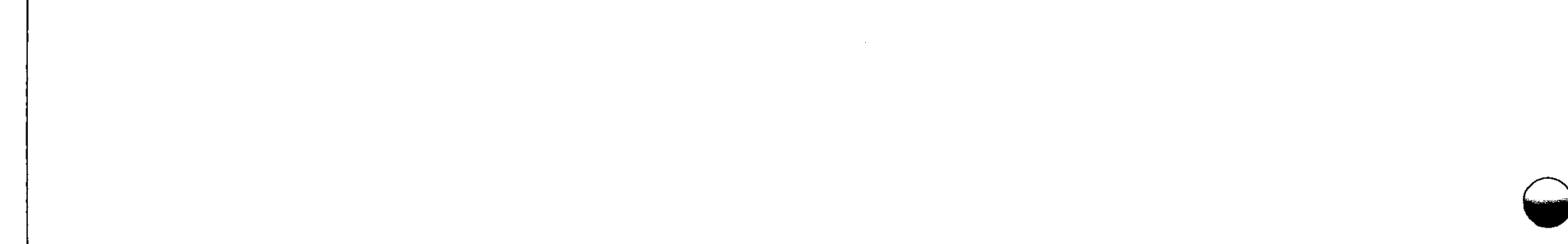




\title{
PROJECTION RADIOGRAPHY AND TOMOGRAPHY
}

\author{
C. A. Tobias, J. I. Fabrikant, * E. V. Benton, ${ }^{\dagger}$ and W. R. Holley
}

Our program on heavy-ion radiography began soon after heavy ions were accelerated to high energies. As we show below, heavy ions are capable of very high electron density discrimination combined with good radial image resolution and low dose. Since heavy-ion beams produce many secondaries, plastic nuclear detectors have an advantage for recording heavy-ion images (Benton, et al., 1973). Projection imaging is now a practical technique that can resolve density differences between normal tissue and tumor tissue in some cases in which $X$ rays could not make a distinction. Initial attempts at heavyion tomography also indicated potential for high resolution in this field (Tobias et al., 1977), and heavy-ion mammography proved capable of high contrast low-dose imaging of abnormal tissue densities in the human breast. The technique is now ready for detailed clinical investigations in cancer diagnosis and therapy.

\section{THE PHYSICAL BASIS FOR HEAVY-ION RADIOGRAPHY}

\section{Range-Energy Relationship}

The passage through water of a beam of monoenergetic carbon ions used for radiography is shown schematically in Figure 1. This figure contains the relative flux density of parent carbon particles, the overall flux density of fragments produced by collisions with nuclei of the absorber, the relative ionization produced by the beam at various depths, the Bragg ionization curve, and the ionization produced by the fragments.

The parent particles are gradually attenuated, due to collisions with nuclei of the absorber, but most of them arrive to the end of their range where there is a precipitous drop in the flux density of particles.

Figure 2 is a schematic drawing of the heavy-ion radiography technique using a stack of plastic nuclear detector sheets. The method is based on precision measurement of the residual: range of initially monoenergetic parent particles, usually carbon or neon, after they pass through the object to be radiographed. This precision measurement is performed for each pixel: of a radiographic image. The measurement can be done in various ways; however, most of ten we measure the distribution of lesions made in the stack of plastic nuclear detector foils by stopping parent particles of the beam. We calculate the mean penetration depth of these particles and the standard deviation of the stopping points in the plastic stack. The plastic nuclear foil

detectors are chosen so that only stopping heavy ions make lesions and that the majority of lower LET particles are not detected.

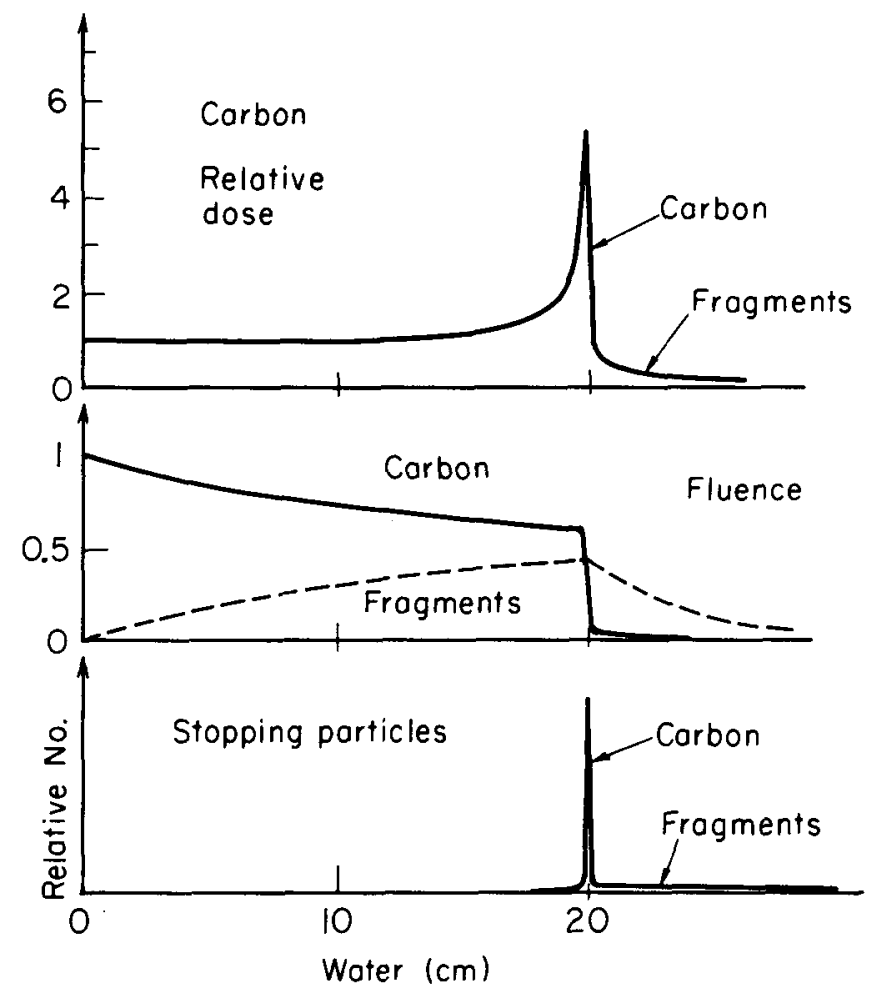

Figure 1. Top: The Bragg ionization curve in time, and ionization from beam fragments. Middle: Flux density of parent carbon and its fragments. The fragment curve is also termed "dome distribution." Bottom: The distribution of stopping carbon particles and fragments. Plastic nuclear emulsions are sensitive to stopping particles only. (XBL 809-3688)

On the basis of these measurements, we calculate the accurate average electronic stopping power through the object at each pixel. These values can be used for resynthesizing stopping power distributions for the purpose of imaging in computerized tomography.

The theoretical basis for energy loss in tissue is given by the well-known Bohr-Bethe equation:

$$
\frac{d E}{d x}=-\frac{4 \pi e^{4} z^{2} n e}{m v^{2}} \ln \frac{2 m v^{2}}{I}=f(E)
$$

(Note: see the glossary at the end of this chapter for a definition of terms.) In this 


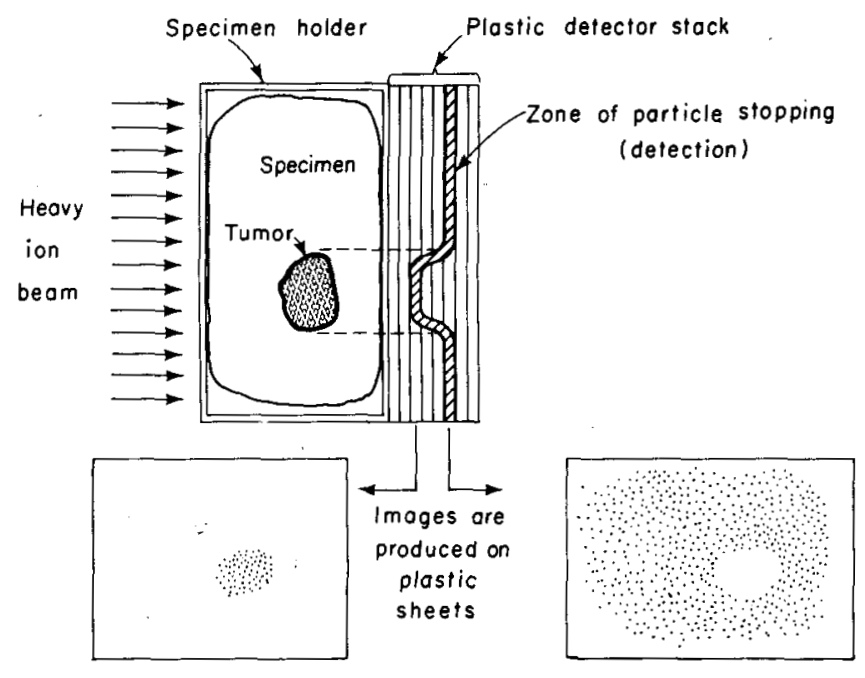

Figure 2. Heavy-ion radiography using plastic nuclear track detectors. The particle beam passes through the tissue and the particles are recorded at their stopping points in the plastic detector stack. The distribution of etched tracks in the detector sheets form the radiographic image. (XBL 774-811)

expression, relativistic terms and low energy correction terms are not shown; these values are given by Mozumder (1969). The integral of this expression gives the range, $R$. We often measure $R$ in units of $\mathrm{cm}$ penetration in the water absorber:

$$
R=\int_{E}^{0} \frac{d E^{\prime}}{f\left(E^{\prime}\right)}
$$

The range-energy relationship for several heavy ions in water were calculated by Stewart (1967). Measurements and calculations of range energy relationships for heavy ions were also made by Northcliffe (1963) and by Barkas and Berger (1964).

As shown in equation 1, our method measures a stopping power quantity that relates essentialiy to the electron density of matter:

$$
n_{e}=\rho N \sum_{i} \frac{Z_{i} v_{i}}{A_{i}}
$$

$\mathrm{n}_{e}$ is the number of electrons per $\mathrm{cm}$ of the absorbing material of density $\rho$, containing molecules composed of atomic species with fractional composition $v_{i}$. Equations 1 and 2 assume linear superimposition of electronic stopping power of atoms. Actually, we know that this is not strictly true, since Thompson (1952) has shown that there is a small effect on equation 2 due to interatomic forces in molecules.

Equation 1 can be also written as:

$$
f(E)=S_{e} e_{e}
$$

where $S_{e}$ is the electronic stopping power. In radiography, it is convenient to compare the stopping power of tissues to that of water. The ratio of stopping powers, or relative electronic stopping power, is defined as:

$$
S_{\text {tw }}=\frac{\left[S_{e}\right]_{\text {water }}}{\left[S_{e}\right]_{\text {tissue }}}
$$

This quantity depends on atomic composition, but is relatively independent of the charge and velocity of particles, except for heavier particles at low velocities. For practical purposes in radiography we usually make the approximation:

$$
\frac{f(E)_{w}}{f(E)_{t}}=\frac{R_{t}}{R_{w}}=\frac{S_{e w} n_{e w}}{S_{e t} n_{e t}}
$$

This leads to the expression for electron density in tissue:

$$
\left[n_{e}\right]_{t}=\frac{\left[n_{e}\right]_{w} R_{w}}{S_{t w} R_{t}}
$$

The relative stopping power has been measured for protons and for deuterons. The value of $S_{t w}$ for soft tissues is close to unity. For purposes of comparison with other modes of radiography we define the heavy-ion number $(\tau)$ as:

$$
\tau=1000\left(\frac{R_{w}}{R_{t}}-1\right)=1000\left(\frac{n_{e t} S_{t w}}{n_{e w}}-1\right)
$$

The heavy-ion number $\tau$ is zero when tissue stopping power is the same as water stopping power. It is positive for materials of high electron density and negative for materials of low electron density. $\tau$ is similar to but not identical with the Hounsfield unit (1980) now generally used in $x$-ray tomography to characterize the absorbtion properties of tissue materials.

In principle a radiographic image with heavy ions is obtained by performing precision residual range measurements at every point of the image plane, which is usually perpendicular to the beam. The basic limitations to the method are straggling and multiple scattering. 
Straggling

Straggling is a dispersion in path length distribution as a result of statistical fluctuations in the energy loss processes. It was shown by Lewis (1952) and by Berger and Seltzer (1964) that the distribution is Gaussian. However; we know that there are small deviations from this distribution.

For a particle of initial energy $E$ and mean range $R$, proceeding in the direction $x$, the range distribution may be written in the form:

$$
s(x)=\frac{1}{\sigma_{x} \sqrt{2 \pi}} \exp \left(-\frac{(x-R)^{2}}{2 \sigma_{x}^{2}}\right)
$$

where $\sigma_{x}$ is the variance in the path length distribution for particles of range R. For particles of initial energy $E$, of is calculated as follows:

$$
\sigma_{x}^{2}=\frac{4 \pi z^{2} A e^{4}}{\left(1+\frac{m}{M}\right)^{2}} n_{e} \int_{E}^{0} \frac{d E^{\prime}}{f(E)^{3}}
$$

There are special corrections to this formula at high and low kinetic energies.

Since the atomic composition of soft tissues is similar to that of water, we may use an approximate practical expression developed for water.

$$
\sigma_{x}(\text { water })=0.0120 \frac{R^{0.951}}{\sqrt{A}}
$$

In the range of validity of this formula $(2 \leq R \leq 40 \mathrm{~cm}), \sigma_{x}$ is almost proportional to range, and it is inversely proportional to the square root of particle mass number, $A . \sigma_{x}$ is one of the main factors to consider in the resolution for electron density in heavy-ion: radiography. For a range of $20 \mathrm{~cm}$ in water, we obtain $\sigma_{x}=0.046 \mathrm{~cm}$ for neon particles, $\sigma_{x}=0.06 \mathrm{~cm}$ for carbon, $\sigma_{x}=0.1 \mathrm{~cm}$ for helium, and $\sigma_{x}=0.2 \mathrm{~cm}$ for protons. The proton value is 4.5 times greater than $\sigma_{x}$ for neon.

\section{Multiple Scattering}

The particles of the beam are deflected in collisions with nuclei of the radiographic object. Many of these collisions result in.small angle deflections, and multiple scattering leads to a divergence of the beam and to a radial preading of the particles away from ideal

traight line trajectories. Multiple scattering then causes lower limits to the sizes of objects that can be resolved by heavy-ion radiography.
Multiple scattering depends fundamentally on the coulomb forces acting between nuclei. Moliere (1948) obtained solutions to the problem of scattering of electrons. He demonstrated that the distribution of scattering angles follows--at small angles-a Gaussian curve, whereas at large angles there are serious deviations from that form. Snyder and Scott (1949) developed the theory for the heavier protons, and Goudsmit and Sanderson (1940) introduced screening by atomic electrons. However, these theories do not fully describe the case of scattering heavy ions on lighter nuclei. Litton (1967) in our laboratory made an attempt to extend calculations to the cases of interest to us. However, uncertainties remain at large scattering angles, where inelastic nuclear collisions and fragmentation of nuclei are taking place, and at very small angles where the screening of atomic nuclei by electrons diminishes the scattering effects. The theoretical screening approximations are not very good for atoms at the low end of the periodic table, such as those similar to tissue composition. In addition, another phenomenon, diffraction scattering, is known to exist. At certain velocities, the magnitude of this phenomenon can be compared to multiple scattering. Finally most of the theoretical treatment is for pure monatomic substances, not for complex molecules. For the Gaussian region, equation 12 defines the rate of change in deflection angle $\phi$ along the path $x$ in the laboratory system:

$$
\phi_{x}^{2}=\frac{d\left\langle\phi^{2}\right\rangle}{d x}
$$

and $\phi_{x}^{2}$ can be used to calculate the change in radial deflection of particles, $\sigma_{y}^{2}$, perpendicular to $x$, that a change in angles $\phi_{x} y_{a t} x$ causes when the particles stop at range $R$ :

$$
\sigma_{y}^{2}=(R-x)^{2} \phi_{x}^{2} \Delta x
$$

At range $R$ the radial distribution of deflections $y$ of the particles is given by:

$$
P(y)=\frac{1}{\left(2 \pi \sigma_{y}^{2}\right)^{1 / 2}} \exp \left(-\frac{y^{2}}{2 \sigma_{y}^{2}}\right)
$$

$\phi^{2}$ and $\sigma^{2}$ are obtained by integrating equations $12^{y}$ and 13 , taking into account the rate of change of particle momentum with beam penetration. This was performed by Litton for the special case of heavy particles (1967).

It is convenient to use for practical purposes an approximate expression for oy. The scatter parameter for liquid water is:

$$
\sigma_{y}=\frac{0.0294 R^{0.896}}{z^{0.207} A^{0.396}}
$$


We regard this formula as an aproximation that is being further tested in the heavy-ion program in view of the uncertainties mentioned above. So far, we find good agreements between equations 11 and 15, and the radiography data obtained. However, exact comparison is difficult because of a variety of factors that contribute to range spread and radial deflection. Table 1 gives $\sigma_{y}$ values for various beams at different range penetrations.

The range straggling and beam deflection limit the theoretical resolution of heavy-ion radiography. From the table it is clear that carbon and neon beams can resolve several times smaller density variations over several times smaller areas than protons can. The ability to resolve small detail in a radiographic image also strongly depends on particle flux density, and therefore the dose. This question will be further discussed below.

\section{Large Angle Scattering}

An advantage of heavier against lighter particles is that in our technique for heavier ions the effects of large angle scattering can be largely eliminated. The reason for this is that when large angle scattering occurs, almost in every case the beam particles fragment to particles of smaller atomic masses. The. sensitivity of the plastic nuclear detectors may be chosen in such a manner that they will entirely miss fragments, or that the fragments make smaller lesions in the detector, which can be discriminated against. In the case of the protons, however, large angle p-p scattering can occur with the nuclei of hydrogen. Since hydrogen is the most abundant atomic species in tissue, it provides many scattering centers. With a large radiography object, the large angle scatters would increase the proton background and decrease radial resolution.

\section{Practical Limitations of Heavy-Ion Radiography}

There are many practicai factors that tend to decrease the resolution of heavy-ion inaging. In addition to straggling, range variations are caused by a finite initial energy spread of the

Table 1. $\sigma_{y}$ Values in Water

\begin{tabular}{|c|c|c|c|c|c|}
\hline \multicolumn{6}{|c|}{ Range $(\mathrm{cm})$} \\
\hline Beam & 2 & 6 & 10 & 20 & 40 \\
\hline $\mathrm{H}$ & 0.055 & 0.15 & 0.23 & 0.43 & 0.80 \\
\hline $\mathrm{He}$ & 0.027 & 0.073 & 0.12 & 0.22 & 0.40 \\
\hline C & 0.014 & 0.038 & 0.06 & 0.11 & 0.21 \\
\hline $\mathrm{Ne}$ & 0.01 & 0.028 & 0.044 & 0.082 & 0.15 \\
\hline
\end{tabular}

beam and by multiple scattering; the beam deflection is increased by angular spread in the beam and by scattering processes upstream from the subject: Noise in the detector system and pixel size in the electronic digitizing and computing processes also limit the resolution. Further work is in progress to reduce the variations caused by these factors.

Modulation Transfer and Resolution of Functions for Heavy-Ion Imaging

Transfer function analysis has been used for the purposes of image analysis for almost twenty years (Rossman, 1969; Metz and Doi, 1979). It is a method of analysis of transfer of properties between the object and image. In simplest terms, modulation transfer coefficients are ratios of Fourier amplitudes that describe the image and the object. We believe that heavy-ion radiographic images are both 1 inear and shift invariant, and therefore satisfy the basic conditions for applicability of transfer function theory.

Assume that a monoenergetic parallel particle beam passes through the phantom shown in Figure 3 which has a series of evenly spaced parallel grooves. The spacing between grooves is $b$ and the width of each groove is b. Between hills and valleys there is stopping power difference of $a$. The shape of this phantom is similar to the shape of actual phantoms used in our experimental study. However, it would be more correct to use sinusoidal phantoms.

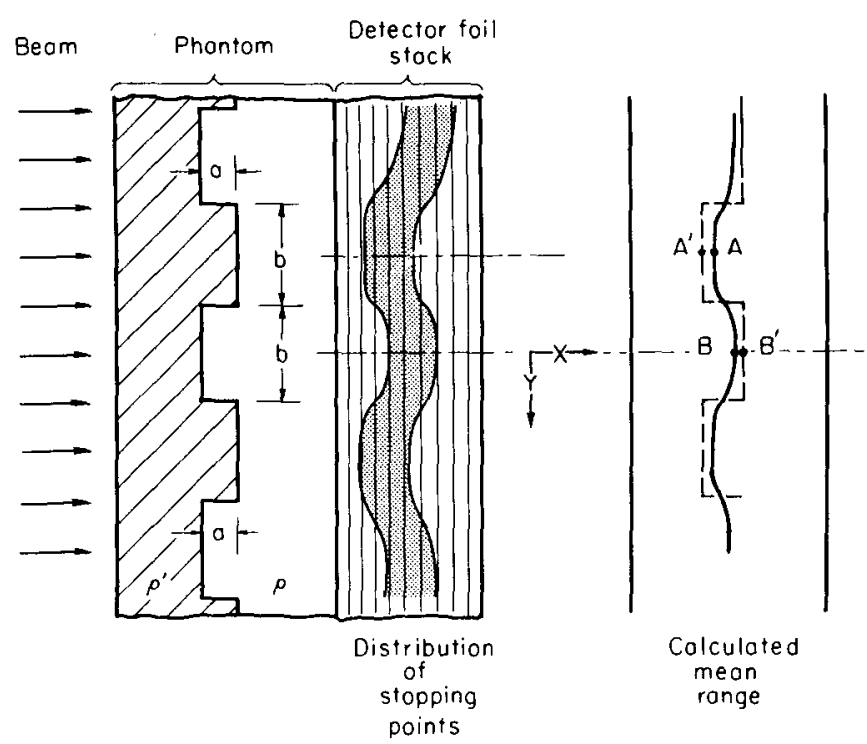

Figure 3. Schematic drawing of a phantom detector foil stack and particle stopping point distribution used to measure and calculate the modulation transfer function and the relationship of dose to resolution. (XBL 809-3687) 
We will represent the distribution $P_{S}(x, y)$ of stopping points of the particles stopping along axis $x$ with range $R$ as:

$$
P_{s}(x, y)=\frac{1}{2 \pi \sigma_{x} \sigma_{y}} \exp \left(-\frac{(x-R)^{2}}{2 \sigma_{x}^{2}}-\frac{y^{2}}{2 \sigma_{y}^{2}}\right) \text {. }
$$

We wish to calculate the probability of finding particles at points: $y=A$ and $y=B$, corresponding to range values of $R$ at the bottom of the valleys and $(R+a)$ the top of the hills.

The fraction of particles that scatter to $A$ from particles of mean range $R$ is:

$$
\begin{aligned}
& m=\frac{1}{\sigma_{y} \sqrt{2 \pi}} \cdot \\
& \qquad \sum_{j=-\infty}^{t \infty} \int_{\frac{b}{2}}^{\frac{b}{2}} \exp \left(-\frac{(A-y-2 b)^{2}}{2 \sigma_{y}^{2}}\right) d y
\end{aligned}
$$

The fraction of particles of mean range $(R+a)$ scattering to $A$ is $1-m$. The particle flux density at depth $x$ is:

$$
\begin{array}{r}
\Delta x n_{A}=\frac{\Delta x}{\sigma_{x} \sqrt{2 \pi}}\left[\operatorname{mexp}\left(-\frac{(x-R)^{2}}{2 \sigma_{x}^{2}}\right)+\right. \\
\left.(1-m) \exp \left(-\frac{(x-R-a)^{2}}{2 \sigma_{x}^{2}}\right)\right]
\end{array}
$$

and $X_{A}$ the mean value of stopping particle distribution is:

$$
x_{A}=\frac{\int_{0}^{\infty} n_{A} x d x}{\int_{0}^{\infty} n_{A} d x}=R+a(1-m) \quad(19) \quad(19)
$$

For $B$, which represents the val ley of the distribution, a similar calculation: leads

$$
X_{B}=R+a m
$$$$
\therefore \text {. }
$$

$$
\text { to: }
$$

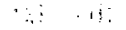

We may now define the modulation transfer factor T as:

$$
T=\frac{\text { observed mean range difference }}{\text { actual stopping power difference }}
$$

$$
=\frac{X_{A}-X_{B}}{a}=1-2 m
$$

The theoretical MTF factors are plotted for particles proton to neon on Figure 4.

Using a variety of phantoms, we measured modulation transfer functions for certain heavyion beams. By courtesy of A. M. Koehler, we were also able to carry out similar measurements at the Harvard proton beam. A representation of some of these measurements is shown in Figure 5 . Since radiographic measurements are done with plastic detector stacks, transfer functions have been measured for each foil, and some of them are shown in the figure. As a function of depth in the detector one first obtains an image that is light where ridges occur in the phantom and dark where valleys are. Deeper in the stack the image reverses. We have shown these MTF distributions with a negative sign to indicate reversal of image contrast, however, the actual MTF coefficients are always positive.

\section{Flux Density and Dose Needed for Resolution}

The knowledge of $T$ allows one to transform observed densities in radiographs to actual densities, and if $T$ is available for all spatial frequencies then an accurate reconstruction of the electron densities of a radiographic object can be made. However, transfer function analys is applies to noise-free images only. In order to calculate resolution in actual physical systems, we must also analyze some of the stochastic aspects of image formation. If we wish to resolve a structure of dimensions $a$ and $b$, such as shown in Figure 3 , it is necessary to apply a beam of sufficiently high particle flux density $F$, measured at a depth where the particle beam leaves the subject and enters the detector stack.

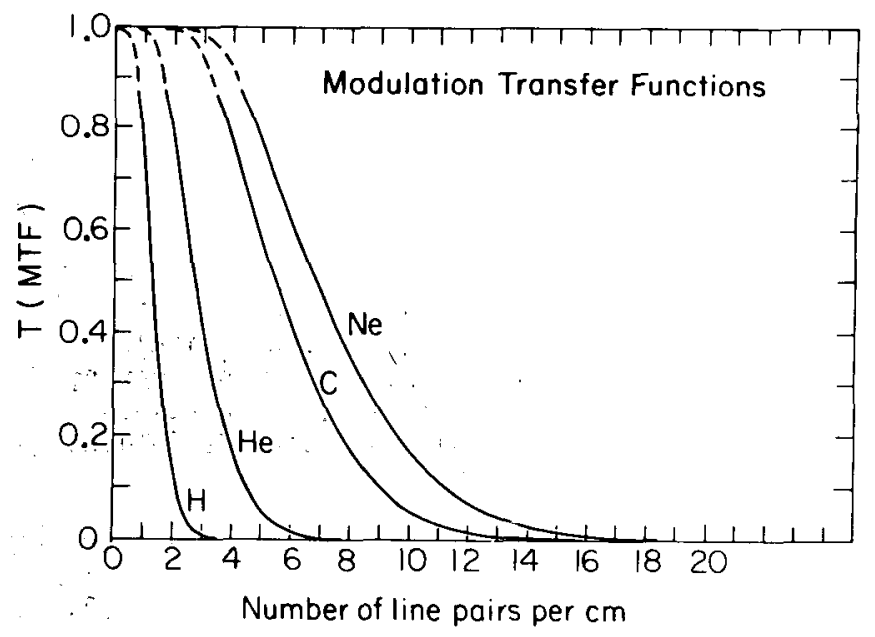

Figure 4. Calculated modulation transfer functions for various particles. The graph is valid for a beam of approximately $20-\mathrm{cm}$ range in water; the inhomogeneity is in the middle of the phantom, about $10 \mathrm{~cm}$ from the detector. We assumed a value corresponding to about $20 \%$ of the statistical error. (XBL 809-1981) 

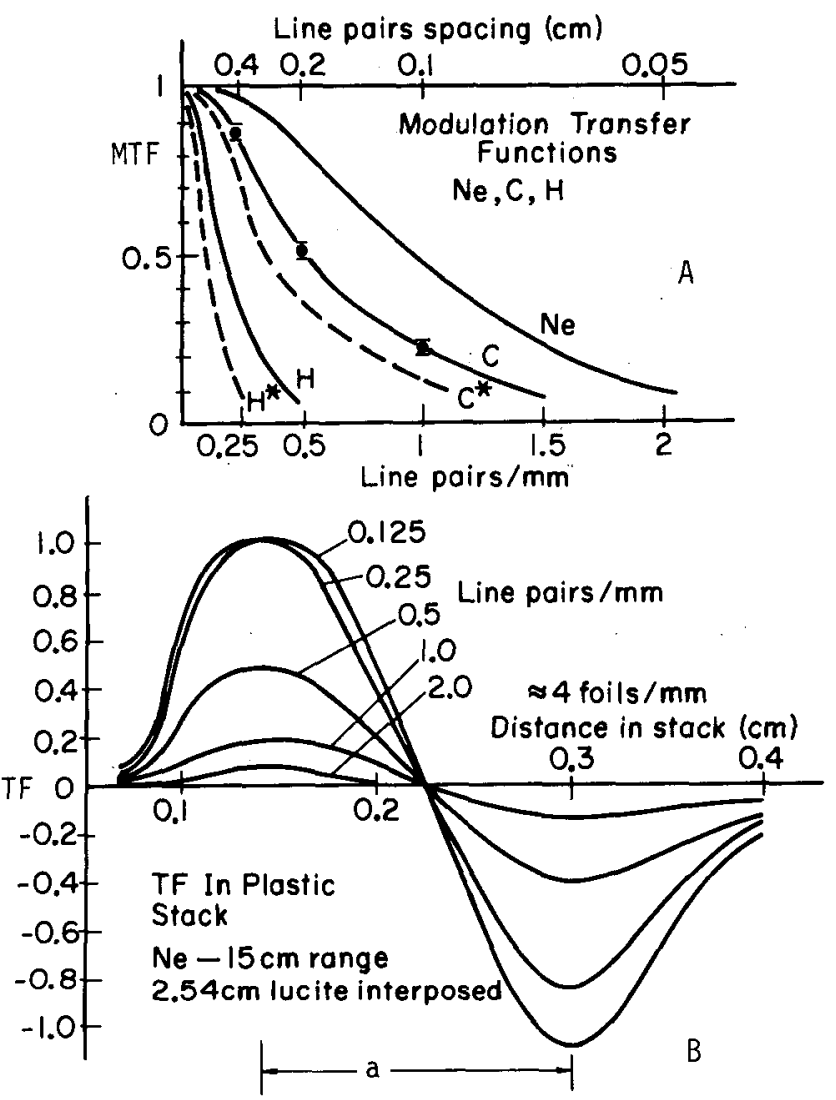

Figure 5. Top: measured and calculated transfer functions for an individual detector sheet. Solid lines are measured values; dashed lines are theoretical curves. Bottom: data from various foils in a stack can be normalized to give relative transfer functions. The inhomogeneity produces first a positive image, then a negative one. We have shown the appropriate functions with positive and negative signs; however, the actual transfer factor is always positive. (XBL 773-3246A)

We may use the well known test employing Student's $t$ for quantitating the statistical significance of the difference in two numbers $n_{1}$ and $n_{2}$ that represent the measured mean values of random distributions with standard deviations of $\sigma_{1}$ and $\sigma_{2}$ :

$$
t=\frac{n_{1}-n_{2}}{\sqrt{\sigma_{1}^{2}+\sigma_{2}^{2}}}
$$

From standard statistical tables one may obtain the value of $t$ necessary for any desired level of significance (Bevington, 1969).
Relating this to heavy-ion radiography, assume that the flux density of particles used is $\mathrm{F} / \mathrm{cm}^{2}$ and that all the particles that cross the area enclosed by a line pair of width $b$ and length of $1 \mathrm{~cm}$ are used for the measurement of $X_{A}$ and $X_{B}$. This particle flux is Fb. Assume further that the standard deviations of $X_{A}$ and $X_{B}$ are $\sigma_{X}$. Referring to Figure 3 , if individual particles $i$ penetrate to depths $X_{A}$ and $x_{B} i$ then from equation 22:

$$
n_{1}-n_{2}=\sum_{i}\left(x_{A} i-x_{B} i\right)=F b T a
$$

and the standard deviation of the measurement:

$$
\sigma_{1}^{2}+\sigma_{2}^{2}=\sum_{i}\left(X_{A} i-X_{B} i-T a\right)^{2}=2 F b \sigma_{X}^{2}
$$

Substituting into equation 22 and rearranging we obtain:

$$
F\left(\text { particles } / c m^{2}\right)=\frac{2 t^{2} \sigma_{x}^{2}}{b T^{2} a^{2}}
$$

If the linear energy transfer in units of MeV $\mathrm{g}^{-1} \mathrm{~cm}^{2}$ is $L$, then we may calculate the dose $D$ in rad for a single radiograph from the flux density $F$ in particles $/ \mathrm{cm}^{2}$ as:

$$
D=\frac{F L}{6.10^{7}}
$$

Figure 6 shows range (or density) resolution against radial resolution for neon and for protons at constant dose leveis. It is clear that better density resolution is achieved at higher dose levels. At the same depth resolution neon has much better radial resolution than protons at the same dose level, and it would take extremely high proton doses to compete with neon.

As many of the radiographs in this report show in agreement with Figure 6 , at about $20 \mathrm{~cm}$ range we obtain a depth resolution down to about $0.1 \%$ and a radial resolution of 0.1 to $0.2 \mathrm{~cm}$ with either carbon or neon beams using particle flux densities between $10^{3}$ and $5.10^{5}$ particles $/ \mathrm{cm}^{2}$. Radial resolution of neon is visibly better than that of carbon, a circumstance that is in part due to statistics and in part due to the fact that neon particles make larger lesions in the plastic materials we use. Using neon beams at high dose, we have actually obtained density resolution of $0.05 \%$ for an object of $1 \mathrm{~cm}$ in size imbeded in a phantom of 12-cm diameter. A dose of $0.020 \mathrm{rad}$ is adequate for neon or carbon-ion mammograms, where radial resolution is on the order of $0.2 \mathrm{~cm}$. 


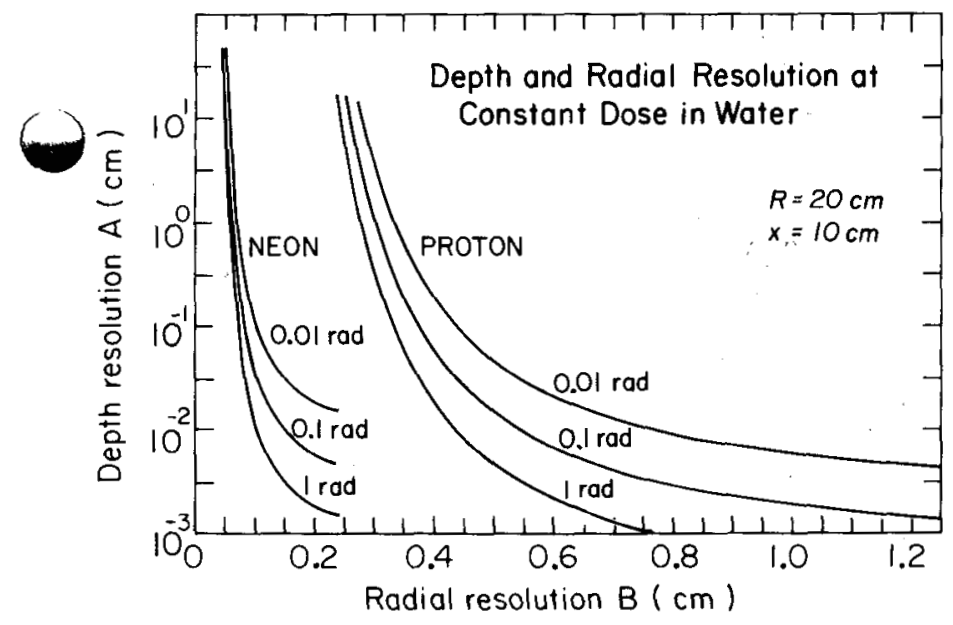

Figure 6. Depth and radial resolutions that can be reached at a constant dose in beams of $20-\mathrm{cm}$ range from objects about $10 \mathrm{~cm}$ from the detector stack. At the same dose, better depth resolution is achieved when the radial dimension of the object is larger. The resolutions that can be reached with neon jons at practical doses are obviously better than those with protons. The calculations were made for $20 \%$ accuracy. (XBL 809-1982)

\section{Comparison with $x$ rays}

$X$-ray radiography is based on the attenuation of $x$-ray quanta in tissues of different atomic composition. $X$ rays are absorbed due to photoelectric and compton absorption processes characterized by $\mu$ and are scattered due to the Compton scattering process. If $X-r a y$ intensity is $I_{x}$ then:

$$
\ln \frac{I_{x}}{I_{0}}=-\int_{0}^{x} \mu d x
$$

Photographic emulsions have a logarithmic behavior, and they are sensitive, to the right side of equation 27. X-ray tomography computes the values of the absorbtion coefficient $\mu$. for each point in a cross-sectional plane. The: process is complicated. Compton scattering produces a background and reduces the ability to resolve radial detail. Also most of the diagnost ic $x$-ray sources are polychromatic the longer wavelength $x$ rays and the softer compton scattered rays are absorbed more rapidly than the shorter wavelength (harder) components of the beam. There are various techniques, e.g., the use of collimating grids, to increase radial resolution at the expense of intensity and other techniques that increase contrast for better viewing at the expense of higher dose. Photoelectric absorption does not involve scattering Ind is therefore inherently capable of nigh adial resolution; however, resolution of small changes in $\mu$ are easier, when $\mu$ is high; for example at low kilovoltages. However, when $\mu$ is nigh, the absorbed dose is also nigh. It is not possible to give a single figure to compare $X$ rays with heavy ions. However, we find that in mammography X-ray diagnostic doses are usually greater than $0.5 \mathrm{rad}$. Carbon-ion mammograms show superior contrast to $X$ rays at twenty-five times lower dose:(20 mrad). The carbon pictures are noisy, but adequate when the radial resolution required is not very high.

In CT scanning, a convenient unit is the Hounsfield unit (1980):

$$
H=\left(\frac{{ }^{\mu} t}{\mu_{w}}-1\right) 1000
$$

where $\mu \mathrm{w}$ is the absorbtion coefficient of water and $\mu \mathrm{t}$ is that of tissue.

We have shown above (equation 8 ) that the relationship of the heavy-ion number $\tau$ to electron density is linear. The $x$-ray absorbtion coefficients are due to the combination of a strongly nonlinear term in energy and atomic number due to the photoelectric effect and a term proportional to the atomic number due to the Compton effect. The $\tau$ number is dependent chiefly on the atomic composition and density of tissue; the $H$ number also changes with the $X-r a y$ spectrum. We expect then that the ratio:

$$
\frac{\mu_{\text {tissue }}}{n_{e_{\text {tissue }}}}=\left(\frac{H+1000}{\tau+1000}\right) \frac{\mu_{w}}{n_{e w}} S_{t w}
$$

might be useful to correct the "hardening artifact" in X-ray CT scans, and, at the same time give us new information on the atomic composition of tissue materials. We have indeed demonstrated experimentally that this is the case.

\section{TOMOGRAPHIC RECONSTRUCTION STUDIES}

We have shown earlier that heavy-ion tomography is feasible and that it has potentially high resolution, but the early reconstructions also had artefacts. We have now investigated the source of some of these artefacts and designed methods to eliminate them. We have consequently obtained heavy-ion reconstructions that are reniarkable for their clarity and high signal-tonoise ratio.

The tomographic reconstructions were made with a "full scale" exposure unit with phantoms and tissue specimens. We hope that, by adding a rotating chair, this same unit will be used with human subjects. The apparatus is shown in Figure 7. The beam is passed through a horizontal slit in a metal shield sufficiently thick to stop all primary particles. The beam then passes through a stationary plastic block with an accurately machined cylindrical hole. The patient's nead-support assembly accurately 


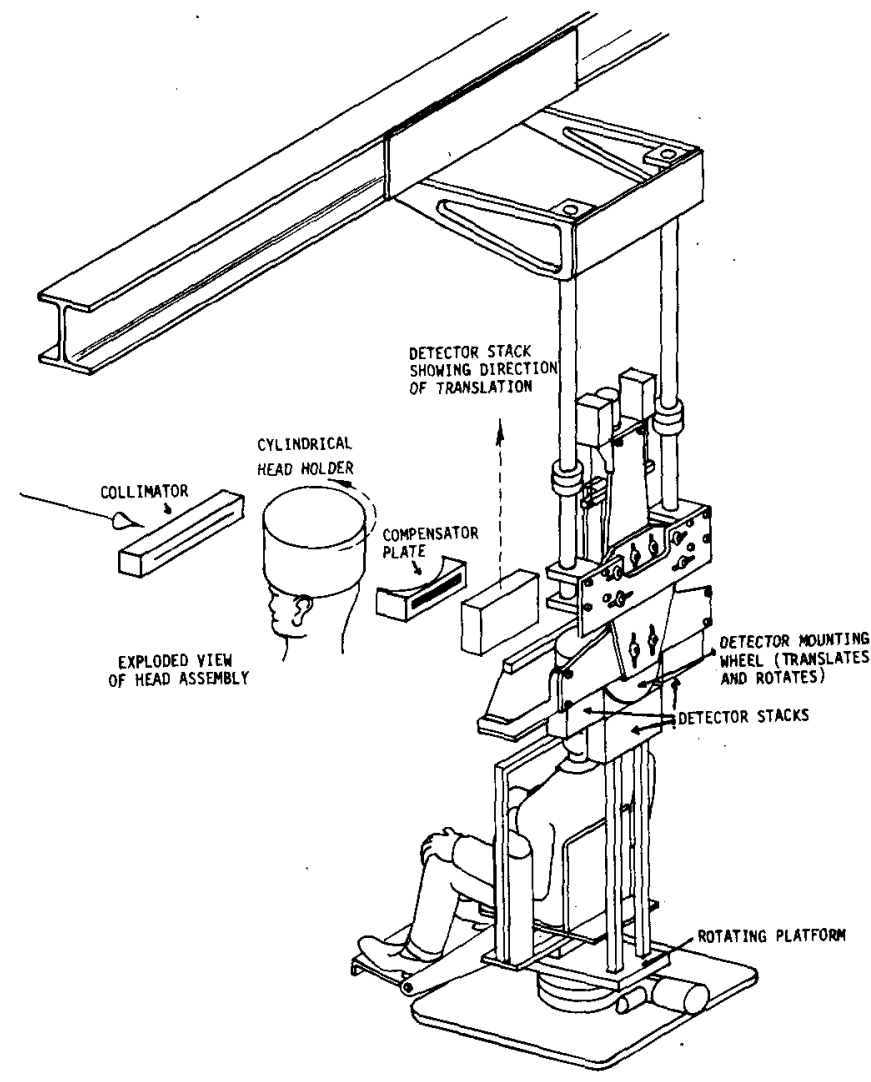

Figure 7. Multiple exposure device for computerized particle tomography. The device is completed and is being used with specimens. The patient rotating chair will be built in the future. (XBL 782-7276)

fits into this hole; above the mask, a cylindrical head assembly is filled with water. At the downstream side of the plastic block a stack of nuclear track detectors is mounted. The particles stop in the plastic stack, and make lesions in a region of approximately the same width as the beam slit, usually $0.2 \mathrm{~cm}$. In the interval between beam pulses, the patient is rotated to a new angular position by about 2 degrees around the vertical axis. At the same time, the plastic stack is moved vertically by about $0.2 \mathrm{~cm}$ so that the next beam pulse records particle transmission for the new angle at an unused portion of the filmstack. The motions are carried out by computer controlled automation linked to the dosimetry system.

After 90 beam pulses, each 2 degrees apart, the plastic stack has 90 strips of data, each corresponding to a different angular position. The patient (or the slit) can then be moved to a new level. Often "control" exposures are also taken on the same plastic stack at a level appropriate to obtain data on water and plastic alone.

Following exposure to the beam, we optically scan and digitize the data in preparation for computer processing. The optical and computer aspects of this process have been described previously.

\section{Foreshortening Distortion}

In order to obtain the best signal to background noise ratio when digitizing it is necessary both to illuminate and view the plastic sheets obliquely. To eliminate the "foreshortening" distortion produced by this tilt angle (usually 45 degrees), a grid of accurately and evenly spaced points (typically $20 \times 20$ ) is placed in exactly the same position as the sheets and digitized. The digitizer produces an image of the grid with the same distortions that are present in the digitized sheets.

A simple pattern recognition procedure is then used to find the position of the center of each grid point in digitized (distorted) space. The corrected image space is related to the true grid space by a simple translation and scale change, and is determined by specifying the location of two corners of the grid in corrected image space.

\section{Correction for Beam Energy Variations}

Tomographic images are obtained with a sequence of beam pulses. Good quality radiography requires about $0.1 \%$ constancy in beam energy. Within a single pulse, energy variations are small; however, at the Bevalac there is about $0.5 \%$ variation in energy from pulse to pulse. In order to correct for this, the projections include a strip on each side of the object where the beam only passes through water. In addition, when possible, a full projection is made with the radiographed object replaced by water so that a true $X$ (object) $-X$ (water) difference can be formed. Possible variations in beam energy from pulse to pulse (i.e., projection to projection) are corrected by adjusting the water correction projection to match the water strips at the edges of each projection before making the subtraction.

\section{Reconstruction Algorithms}

Currently, our computer programs are used to reconstruct images typically of size $128 \times 128$ (sometimes up to $175 \times 175$ ). Usually a filtered back projection algorithm is applied, using a modified Shepp-Logan method from the BudingerHuesmann reconstruction program package (Huesman et a1., 1977). After reconstruction, the results to be displayed are transformed to $256 \times 256$ pixels which are used to display and examine the integrated scans on a RAMTEC color TV display system or a CONRAC black and white system. We can also recall electron densities and standard derivations for further computation.

The actual information obtained by heavy-ion radiography has about one thousand significant shades of grey. Since our eye can only discern about seventy shades, it is not possible to make use of all information simultaneously in a single 
display. Instead the system we use can select electron density windows of any desired width for display of images. In order to interpret heavyion CT scans, we use the expression given in equation 29. Equation 29 is important for two reasons. First, it can be used to correct $x$-ray CT scans for the beam hardening artefact by comparing an X-ray and a heavy-ion CT scan of a water phantom. Second, if tissue imaging by. $X$ rays is compared to tissue imaging by heavy ions, the ratio $\mu \mathrm{t} / \mathrm{met}$ gives information on the atomic composition of tissue.

\section{Test Tube Phantoms}

The simplest phantom consisted of a bowl of water with cylindrical walls. Four polystyrene test tubes were immersed into the water, each containing test solutions. The test tubes had a diameter of $1.0 \mathrm{~cm}$ and wall thickness of $0.07 \mathrm{~cm}$. The density of the test tube wall, compared to water, was 1.04 . In the center of the bowl a nylon rod marked the axis of rotation for the phantom.

Figure 8 shows a reconstruction of the test tube pattern with neon ions. The pattern on Figure 8 may be compared to an X-ray CT reconstruction, Figure 9 , which was made of the same phantom with a General Electric 7800 scanner at the Radiology Department of the University of California, San Francisco. Both tomograms reproduce the pattern, but with notable differences.

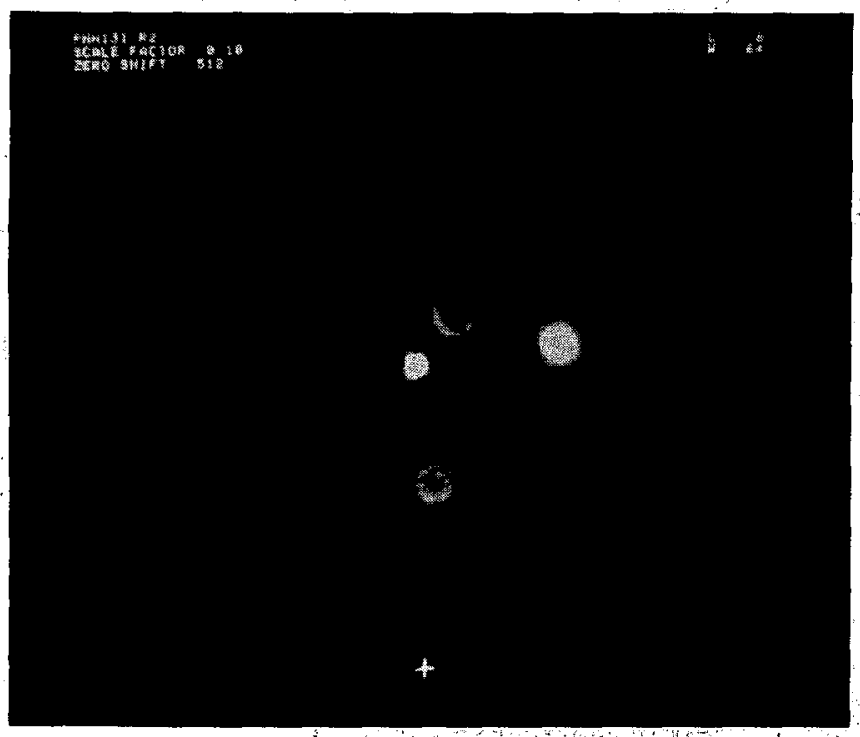

Figure 8. Neon beam CT image of a test tube assembly immersed in water. The diameter of the test tube is $1.0 \mathrm{~cm}$; the walls are polystyrene, $.07 \mathrm{~cm}$ thick. There is also a nylon centering od. The test tube walls are white because their electronic stopping power $\left(n_{e}\right)$ is greater than that of water. (XBB 809-10843)

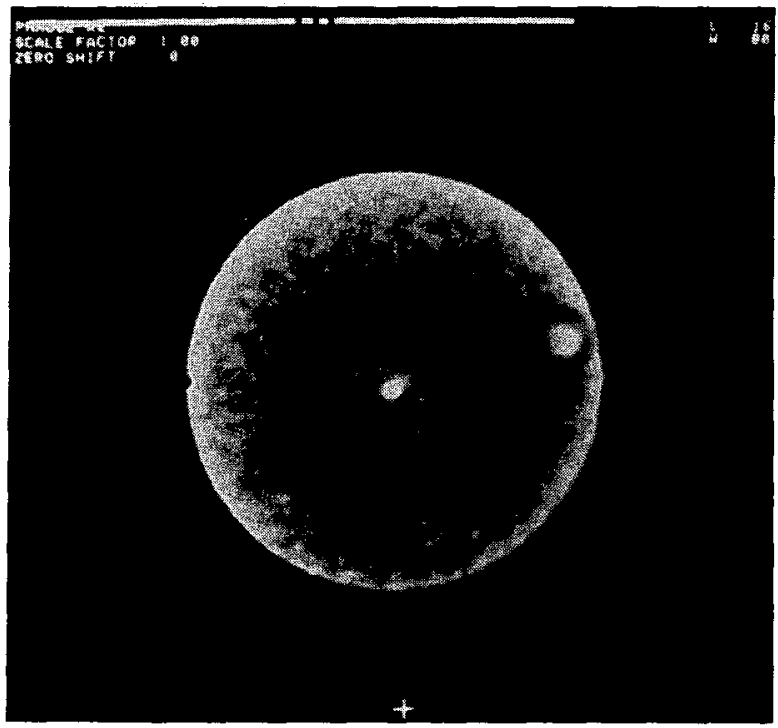

Figure 9. X-ray CT image of the assembly described above. The X-ray background drifts more than the neon background. The test tube walls appear dark because the $x$-ray absorbtion coefficient of polystyrene is smaller than that of water. (XBB 809-10768)

The wall of the test tube appears whiter than the background in the neon CT $\operatorname{scan}(\tau>0)$ and darker than background in the X-ray CT scan $(H<0)$. This is because the electron density of the wall is greater than that of water, whereas the X-ray absorption coefficient of the wall is smaller than that of water. (The wall is composed of polystyrene plastic containing carbon, whereas in water oxygen is a major absorbing constituent.)

Because of the relatively higher absorption coefficient of oxygen than carbon or nitrogen, $x$-ray CT, tomography has some difficulty in differentiating between water and chemical compounds with varying compositions of $H, C, N$, and 0 when the physical density of these compounds is 1.02 to 1.04. Heavy-ion radiography is efficient in that respect; this explains perhaps why in mammography, heavy-jon radiography can image stroma and tumor densities with high contrast, whereas $x$ rays usually cannot. We believe that it is this circumstance that enables heavy-ion radiography to be effective in detecting soft tissue. abnormalities and tumors. These images also show that CT reconstructions of neon images have a high resolution, and can reproduce electron densities more accurately than possible with a good X-ray scanner.

\section{Imaging Tissue Specimens}

The methodology was applied to human tissue specimens in the same full scale apparatus that will be used in vivo on humans. Either neon beams at $557 \mathrm{MeV} /$ amu (15 cm range) or carbon at $450 \mathrm{MeV} / \mathrm{amu}$ (25 $\mathrm{cm}$ range) were utilized. 
A coronal plane of a human brain specimen was studied with $0.2 \mathrm{~cm} \mathrm{~s} 1 \mathrm{it}, 90$ angles and $557 \mathrm{MeV} /$ amu neon beam. Figure 10 shows the initial C.T scan which. is badly distored due to the edge artefact from an air bubble that was lodged in the specimen. The sinogram for this reconstruction is shown in Figure 11. The bubble is shown as a black curved streak; apparently the bubble moved out of the field for the late part. of the exposure.

A program has been written to remove these streaking artefacts by correcting the projected data. An iterative search algorithm first finds the boundaries of the sinusoidal regions by employing gradient determination methods: Once the boundaries are found, the air bubble region is removed and replaced with new values by a linear interpolation. scheme.

After the correction algorithm is applied, the sinogram changes to the form shown on Figure 12. Reconstructing from this level, we obtain the final neon CT SCan in Figure 13 .

The neon CT reconstruction of the brain demonstrates nigh resolution of the soft tissue structures of the brain. Spatial resolution does not limit the precise delineation of the internal structure of the brain. Density resolution appears better than in the X-ray CT scan shown on Figure 14 (taken on a Delta scanner at the Veterans' Hospital, San Francisco) and differences in density are readily determined between the regions of the white and gray matter of the cerebral cortex and the structures of the midbrain (e.g., putamen, internal capsule, caudate nucleus), the corpus collosum, the anterior commissure, the lateral ventricles and the third ventricle, the fornix, and the region of the optic chiasm. The brain specimen was fixed, and

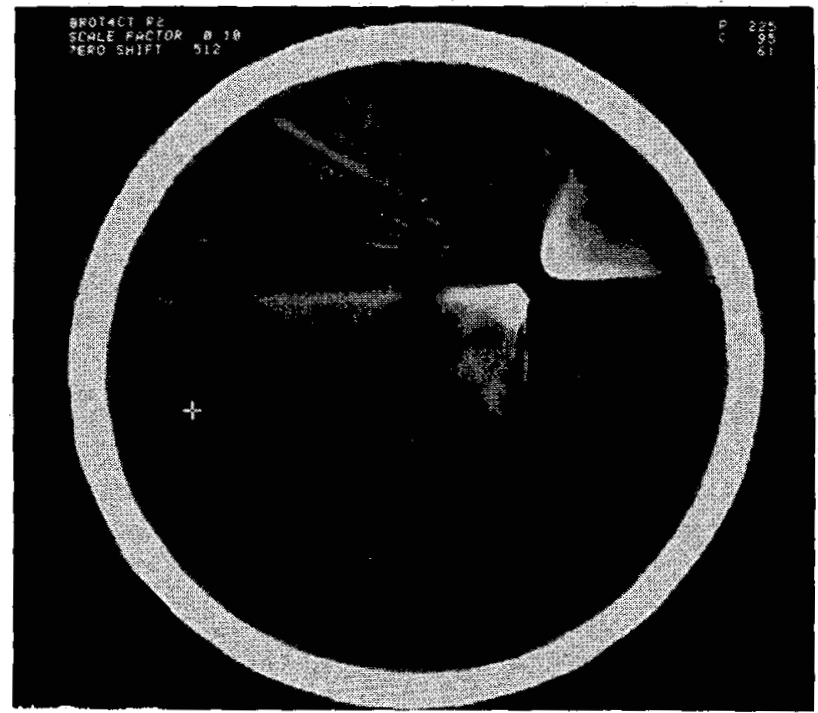

Figure 10. Neon beam CT reconstruction of a human brain specimen. The radiating structures represent artefacts caused by an air bubble. (XBB 809-10867)

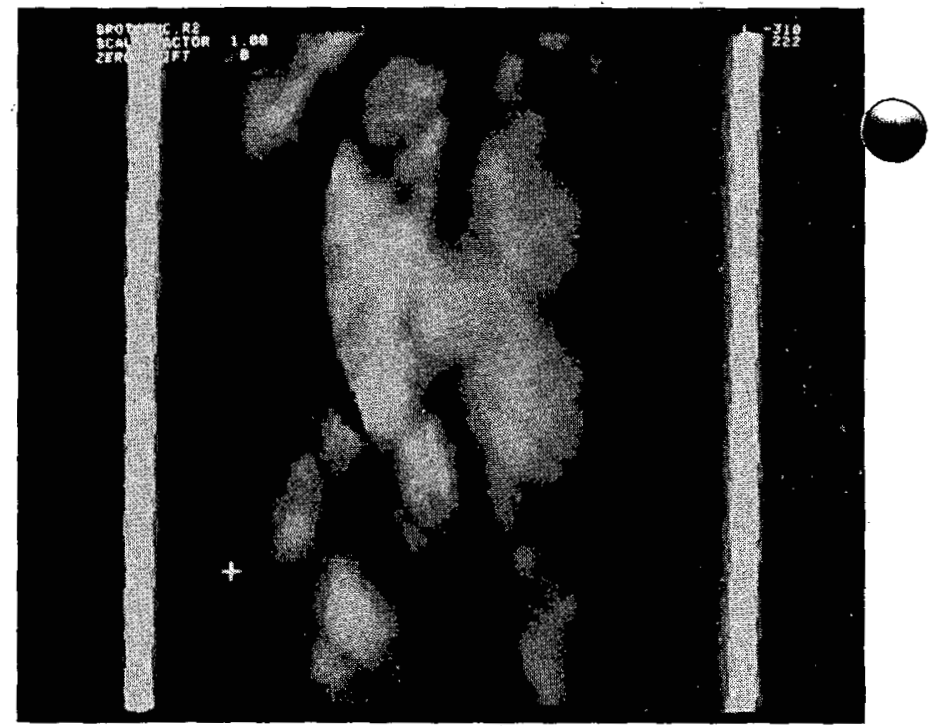

Figure 11. The sinogram for the specimen in Figure 10. The bubble appears as a sharp crescent-shaped dark object. (XBB 809-10868)

we believe that in vivo the actual electron density differences between white and gray matter would have been greater. Study of the inner structure of the brain, including the detection of small tumors, has now come within reach of heavy-particle quantitative densitometry, image enhancement, and CT reconstruction.

We believe that heavy-ion CT scans will have an important role in identifying some of the characteristics of tumor tissues. We have shown that carcinoma of the breast often has stopping

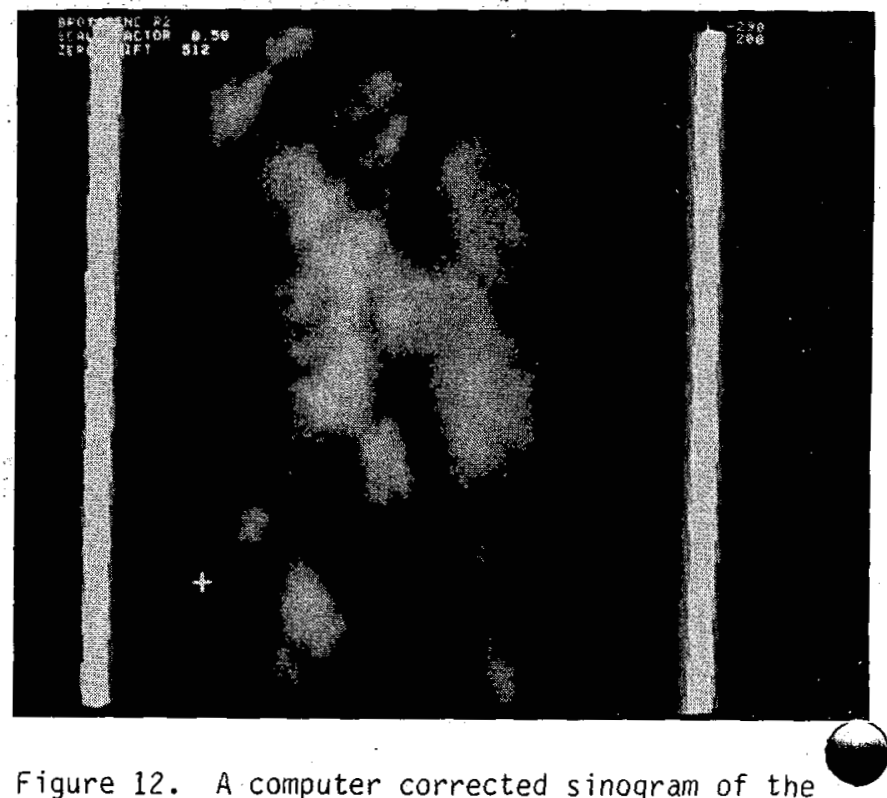
brain shown in Figures 10,11 , and 13 . (XBB 809-10870) 


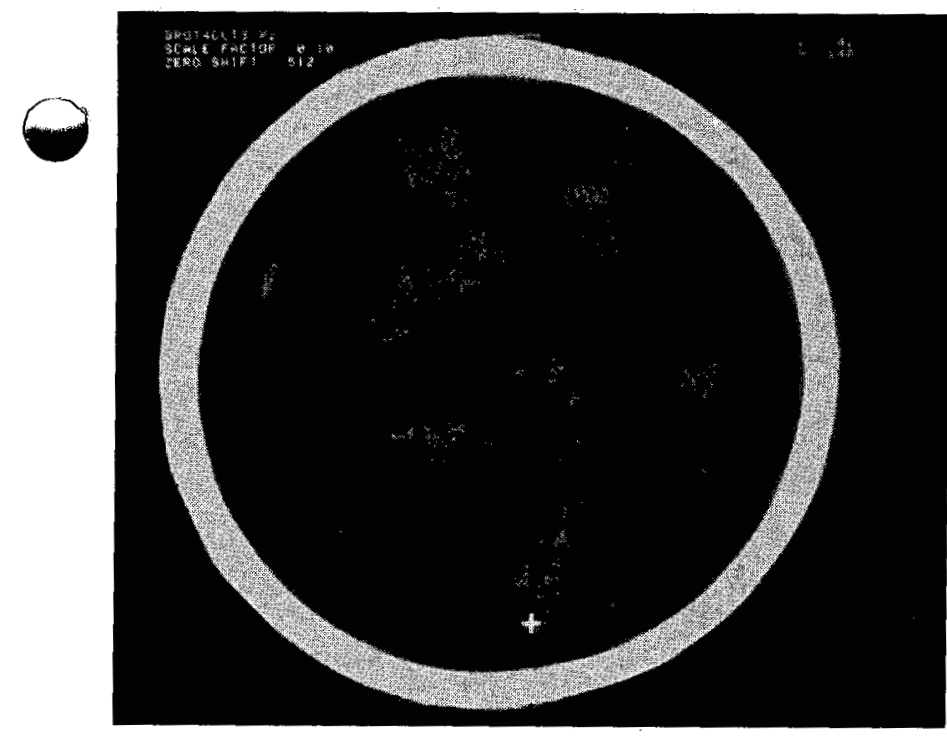

Figure 13. Computer corrected CT reconstruction of a human brain specimen. A neon beam with 90 projections was used, and many details are visible. The demarcation between white and gray matter is very faint; however, we believe this is due to the fact that fixative alters tissue densities. (XBB 809-10849)

power ratios of 1.02 to 1.04 (see the chapter in this section by Fabrikant et al. on mammography). Also, we find that $X$-ray CT scans are particularly insensitive to tissue-like materials in this same stopping power domain. It is likely that $x$-ray and heavy-ion scans together will yield more information than $c$ an be obtained with either one of these techniques.

\section{ACKNOWLEDGEMENT}

The authors acknowledge the many contributions made by their colleagues to the heavy-ion radiography research program. Our coworkers include A. Margulis, H. Genant, E. Sickles, G. Sommer, and $M$. Federle from the Radiology Department, University of California, San Francisco; K. Woodruff, J. Castro, G. Gauger, G. Chen, D. Feinberg, J. Llacer, J. Lyman, J. Howard, M. Pirruccel10, 8. Roman, S. Go, R. Walton, and $G$. Welch of $L B L ; R$. Henke of the Physics Department, University of San Francisco, and $R$. Huesman who, working with $T$. Budinger, developed CT reconstruction programs at the Donner Laboratory. These studies are supported by the National Cancer Institute (Grant No. Y01-CB-40302 and RO1-CA-20721).

\section{GLOSSARY}

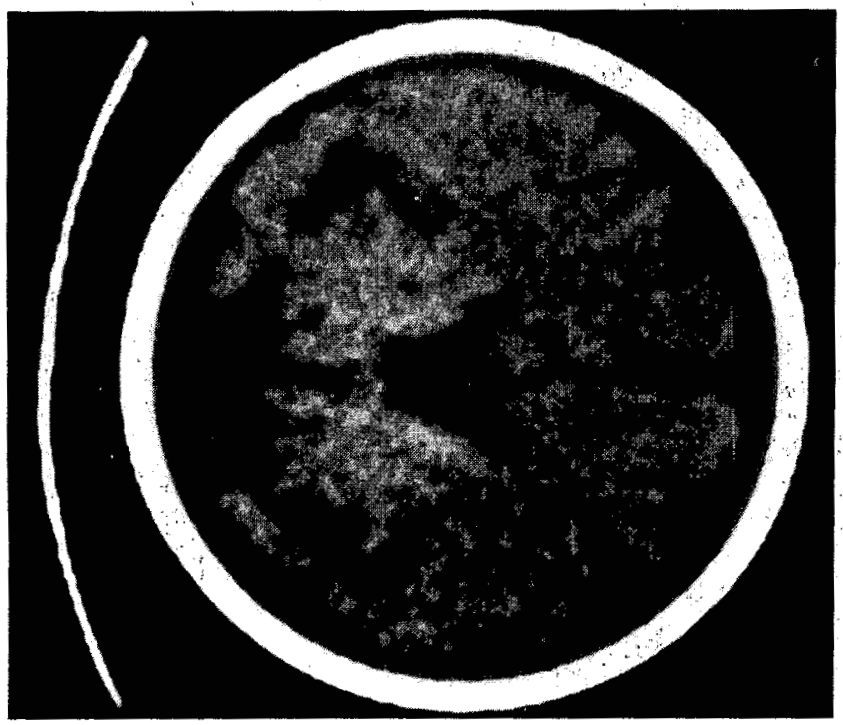

igure 14. X-ray tomographic scan of the brain shown in Figure 13. The diameter of the tank holding the human brain specimen was $12.7 \mathrm{~cm}$. (XBB 809-10951)

$E$
$x$
$e$
$m$
$n_{e}$
$n_{e t,} n_{e w}$
$z$
$v$
$I_{0}$
$R$

kinetic energy of heavy ions coordinate parallel to beam electronic charge electronic man electron density of stopping matter per $\mathrm{cm}^{3}$

$\mathrm{n}_{e}$ in tissue and in water atomic number of heavy ions velocity of heavy ions mean ionization potential range in $3 \mathrm{~cm}$ ( $R_{t}$ and $R_{w}$

range in tissue and water)

\section{$N \quad$ Avogadro's number}

$\mathrm{Z}_{j} \quad$ atomic number of stopping matter A

$A_{j}$

$v_{i}$

$\mathrm{S}_{e}$

$S_{e}$
$S_{t w}$

$\stackrel{a x}{M}$

$\phi_{X}$

$\stackrel{\sigma}{p}(y)$

$P_{S}(X, Y)$

mass number of heavy beam particle mass number of stopping matter mass fraction of component $i$ density of stopping matter, $\mathrm{g} \mathrm{cm}^{-3}$ electronic stopping power relative electronic stopping power heavy-ion number

straggling parameter

mass of heavy beam particle deflection angle at $x$ in the laboratory system

radial deflection parameter

the probability of radial deflection $y$ probability distribution of stopping points

a

b

m

depth of ridges in line phantom width of ridges and vallies in line phantom

fraction of radially scattered particles modulation transfer factor for heavy ions

$t \quad$ Student's $t$

$F$ heavy particle flux density per $\mathrm{cm}^{2}$

$L \quad$ linear energy transfer, $\mathrm{MeV} \mathrm{g}^{-1} \mathrm{~cm}^{2}$ 


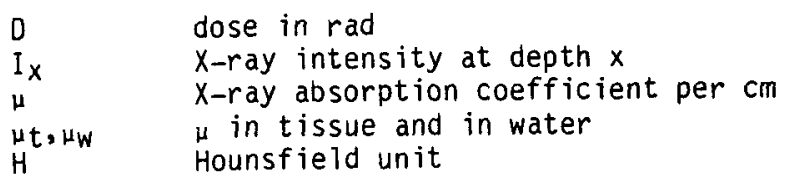

\section{FOOTNOTES AND REFERENCES}

*Department of Radiology, University of California, San Francisco, CA.

Tepartment of Physics, University of San Francisco, San Francisco, CA.

Barkas, W. H. and M. J. Berger. 1964. Studies in penetration of charged particles in matter. NAS-NRC 1133.

Benton, E. V., R. P. Henke, and C. A. Tobias. 1973. Heavy-particle radiography. Science $182,474-476$.

Berger, M. J. and S. M. Seltzer. 1964. Multiple scattering connections for proton range measurements. NAS-NRC 1133.

Bevington, P. R. 1969. Data Reduction and Error Analysis for the Physical Sciences. McGrawHill, New York.

Goudsmit, S. A. and J. L. Sanderson. 1940. Phys. Rev. 57, 24, 36, 58.

Huesman, R. H., C. T. Gullberg, W. L. Greenberg, and T. F. Budinger. 1977. User's Manual: Donner Algorithms for Reconstruction Tomography. Lawrence Berkeley Laboratory Publication 214.

Hounsfield, S. N. 1980. Computed medical imaging. Med. Phys. 2, 283-296.

Lewis, H.W. 1952. Phys. Rev. 85, 20.

Litton, S. M. 1967. Penetration of High Energy Heavy Ions. Lawrence Berkeley Laboratory Report UCRL-17292.
Metz, C. E. and K. Doi. 1979. Transfer function analysis of radiographic imaging systems. Phys. Med. Biol. 24, 2079-1106.

Moliere, Z. 1948. Naturforschung 30, 78 .

Mozumder, A. 1969. Advances in Radiation Chemistry (M. Burton and J. L. Magee, eds.) vol. 1, pp. 1-102. Wiley (Interscience), New York.

Northcliffe, L. C. 1963. Passage of heavy ions through matter. Am. Rev. Nucl. Sci. 13, 67.

Rossi, B. 1956. High Energy Particles. Prentice Hall, Englewood Cliffs, New Jersey.

Rossman, K: 1979. Radiology 93, 257.

Segre, E. 1964. Nuclei and Particles. W. A. Benjamin, Menlo Park, Calif.

Snyder, H. And W. T. Scott. 1949. Phys. Rev. $\underline{76}, 220$.

Steward, P. G.. 1967. Calculation of Stopping Power. Lawrence Berkeley Laboratory Report UCRL-17314.

Thompson, T. J. 1952. Effect of Chemical Structure on Stopping Powers for High Energy Protons. Lawrence Berkeley Laboratory Report UCRL-1910.

Tobias, C. A., H. 0. Anger, and J. H. Lawrence. 1952. Radiological use of high energy deuterons and alpha particles. Am. J. Roentgenol. Radium Ther. Nucl. Med. 67, 1-27.

Tobias, C. A., E. V. Benton, and M. P. Capp. 1977. Heavy-ion radiography. Biological and Medical Research with Accelerated Heavy Ions at the Bevalac, 1974-1977. Lawrence Berkeley Laboratory, Report LBL-5610. 


\title{
HEAVY-ION MAMMOGRAPHY AND BREAST CANCER
}

\author{
J. I. Fabrikant, ${ }^{\star}$ C. A. Tobias, M. P. Capp, ${ }^{\dagger}$ W. R. Holley, \\ K. H. Woodruff,, and E. A. Sickles*
}

The slight difference in radiographic densities of the various tissues of the human female breast that makes X-ray mammography possible, but the range of radiographic densities is quite small. Thus, the diagnosis of benign and malignant breast tumors may depend on recognition of fine shadows and fine gradations of contrast. In order to provide the optimum $X$-ray radiographic contrast in these soft tissues, low kilovoltage, high $x-r a y$ tube current techniques are necessary, imposing high radiation doses to the breast tissues. An additional complicating factor in mammographic diagnosis of breast tumors is the fact that the tissue structure, and hence the radiographic appearances of the female breast, varies with age and physiological condition, i.e., tissue composition changes take place at different ages, during pregnancy and lactation, and in relation to the phase of the menstrual cycle.
Clinical mammography finds its greatest usefulness in the investigation of the lump in the breast, to identify it at the earliest stage, to distinguish benign and malignant tumors, and to identify, if any, a second lesion in the same or opposite breast. It is also of value in the investigation of the nodular breast, particularly when it is difficult to decide where to biopsy. The great majority of malignant breast lesions are diagnosed on the mammographic appearance of a mass, its shape, size, density, and the presence of calcifications. Secondary changes in the breast which can be identified on mammographic studies include skin thickening, increased vascularity, and retraction of the mammary fibrous tissue and diffuse fibrosis. A number of the important mammographic characteristics of breast tumors are listed in Table 1, for potential X-ray differentiation between benign and inalignant breast tumors.

Table 1. Mammographic Diagnosis of Breast Neoplasms

\begin{tabular}{|c|c|c|c|c|}
\hline $\begin{array}{l}\text { Radiographic } \\
\text { Characteristic }\end{array}$ & $\begin{array}{l}\text { Benign } \\
\text { Tumor }\end{array}$ & $\begin{array}{l}\text { Breast } \\
\text { Cancer }\end{array}$ & $\begin{array}{l}\text { X-Ray } \\
\text { Method }\end{array}$ & $\begin{array}{l}\text { Heavy-Ion } \\
\text { Method }\end{array}$ \\
\hline Density & $\begin{array}{l}\text { Slight or } \\
\text { marked increase }\end{array}$ & Marked increase & ++ & ++++ \\
\hline $\begin{array}{l}\text { Character of } \\
\text { Density }\end{array}$ & Homogeneous & $\begin{array}{l}\text { Nonhomogeneous, } \\
\text { center most dense }\end{array}$ & ++ & $+++t$ \\
\hline Shape & $\begin{array}{l}\text { Round, oval, } \\
\text { lobulated }\end{array}$ & $\begin{array}{l}\text { Variable, } \\
\text { spiculated }\end{array}$ & ++ & + \\
\hline Borders & $\begin{array}{l}\text { Well circumscribed, } \\
\text { smooth surrounding } \\
\text { fat layer }\end{array}$ & $\begin{array}{l}\text { Poorly circumscribed } \\
\text { irregular, fuzzy } \\
\text { halo }\end{array}$ & ++ & + \\
\hline $\begin{array}{l}\text { Surrounding } \\
\text { Tissues }\end{array}$ & $\begin{array}{l}\text { Not invaded, } \\
\text { displaced trabeculae, } \\
\text { vascularity normal }\end{array}$ & $\begin{array}{l}\text { Infiltrated, } \\
\text { trabeculae } \\
\text { irregularly retracted } \\
\text { and thickened }\end{array}$ & ++ & +++ \\
\hline Vascularity & Normal & Increased & ++ & +++ \\
\hline Calcification & $\begin{array}{l}\text { Coarse, isolated, } \\
\text { scattered, not } \\
\text { punctate. }\end{array}$ & $\begin{array}{l}\text { Numerous, punctate, } \\
\text { variable density, } \\
\text { central }\end{array}$ & ++ & + \\
\hline $\begin{array}{l}\text { Relative } \\
\text { Size }\end{array}$ & $\begin{array}{l}\text { Same size or larger } \\
\text { than clinical } \\
\text { measurements }\end{array}$ & $\begin{array}{l}\text { Much smaller than } \\
\text { clinical measurements, } \\
\text { often by factor of } \\
2 \text { to } 4\end{array}$ & + & +++ \\
\hline
\end{tabular}

Scale for diagnostic inaging: + poor, ++ fair, +++ good, ++++ excellent. 
Since 1977 , the heavy-ion radiography basic and clinical research program in our laboratory has been systematically investigating the potential of clinical, low-dose, heavy-ion mammography to augment and improve the breast cancer detection rate (Tobias et al., 1978; Sick les, 1979). Heavy-ion mammographic examination of the female breast in our laboratory has now proven to be based on a low-dose, safe, reliable, noninvasive imaging procedure with improved density resolution when compared with $X$ rays (Tobias et al., 1978; Sickles, 1979). The use of heavy-ions for diagnostic imaging of the breast provides valuable quantitative data for image processing, which promises to increase the reliability of the early detection of small cancers in the breast, and to differentiate accurately between benign and malignant tumors. Currently, four areas are under investigation: (1) stopping power values of normal and neoplastic breast tissues:

(2) reduction of radiation dose in the breast;

(3) breast tissue specimen radiography; and

(4) heavy-ion mammography trials in clinical patients with breast disease (Fabrikant et al., 1980).

\section{Stopping Power Values in Breast Tissue Specimens}

Heavy-ion radiographs of postmastectomy breast tissue specimens provide stopping power values along an integrated linear beam path. These data are being used for accurate quantitative densitornetry of the stopping power distributions in the tissue. Table 2 compares characteristic stopping power values (relative to water) in benign and malignant breast lesions derived from digitized heavy-ion mammograms of formalinfixed breast tissues obtained at very low doses. Quantitative differences in stopping power values may provide a quantitative approach for differentiating between benign and malignant tumors. Figure 1 is an oxygen-ion radiograph of a breast specimen containing an infiltrating duct carcinoma; comparison can be made with the conventional $x$ ray. The heavy-ion radiograph demonstrated a far more dense tumor image than the conventional radiograph (Sommer et al., 1978).

Table 2. Stopping Power Values (Relative to Water) of Normal and Neoplastic Breast Tissues

Breast Tissue Stopping Power T Values
values

$\begin{array}{llr}\text { Normal } & 0.957 \pm 0.004 & -43 \pm 4 \\ \text { Carcinoma } & 1.064 \pm 0.003 & 64 \pm 3 \\ \text { Dysplasia } & 1.016 \pm 0.011 & 16 \pm 11\end{array}$
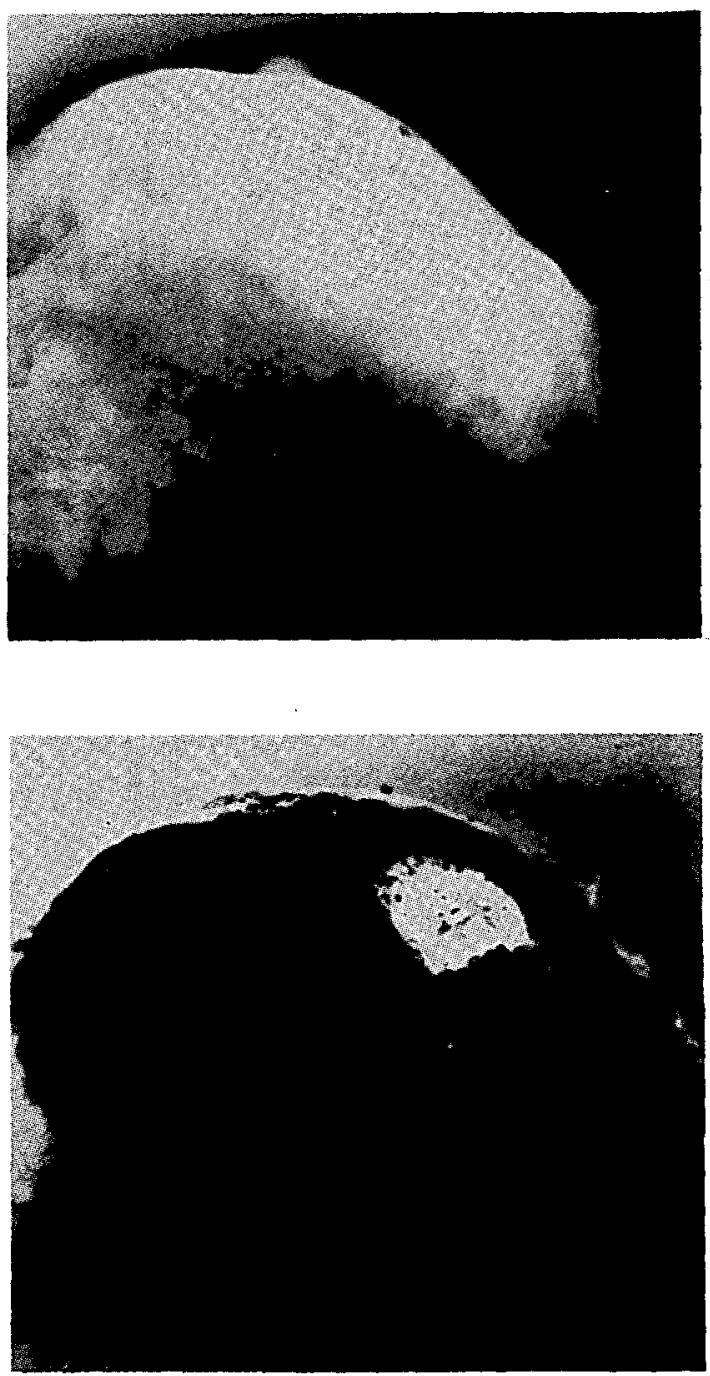

Figure 1. (A) Conventional X-ray radiograph of a mastectomy breast specimen that contained residual infiltrating ductal carcinoma beneath the biopsy site. (B) Oxygen-ion radiograph of the breast specimen; the dense carcinoma is readily identified. (XBB 776-5441)

CLINICAL PATIENT TRIALS WITH HEAVY-ION MAMMOGRAPHY

\section{Objectives}

Since the initiation of the systematic clinical research program, noteworthy advances have been made that support our original observations for the potential development and application of heavy-ion mammography for the early diagnosis of breast cancer in women. In 1978, a clinical science study group in heavy-ion radiography was formed and affiliated with the BAHIA group, which presently consists of some fifty physicians, surgeons, radiologists, pathologists, and physicists from the University of California 
at Berkeley and San Francisco School of Medicine, the Lawrence Berkeley Laboratory, the San Francisco Bay Area community hospitals, and the University of San Francisco.

The specific objectives of the first-stage clinical heavy-ion mammography trial in progress have been: (1) to establish a safe and rêliable method for the examination procedure; (2) to select the heavy-ion with appropriate characteristics for imaging of clinical patients; (3) to determine the degrees of density and spatial resolution necessary for obtaining quality diagnostic images better than or equal to conventional X-ray mammography; (4) to establish the reliability of the nuclear track plastic detectors and the developing methods for producing the diagnostic images; (5) to establish the reliability and reproducibility of imaging processing; (6) to develop methods and programs for computer analysis and image display and for electronic densitometric techniques; and (7) to develop methods for reduction of radiation dose. During the past three years, many of these goals have been achieved.

\section{Selection of Patients}

Patient selection remains carefully controlled by approved clinical protocols, and include the following criteria. (1) The patient is referred for heavy-ion mammography with the clinical diagnosis of breast cancer, a palpable mass, or $X$-ray mammographic breast xerographic evidence of a breast lesion. (2) The patient must have a breast mass determined either by clinical palpation and/or X-ray mammography or breast xerography, and where the diagnosis is uncertain and may be benign or malignant disease. (3) The patient may be in a high-risk group with breast disease suspicious of cancer, where the other breast has had cancer (which has been surgically removed or otherwise treated for cancer). (4) The likelihood exists that the patient will have a breast biopsy or a surgical mastectomy based on the existing clinical and $x-r a y$ mammographic findings. There is full cooperation with the hospital where the breast biopsy or mastectomy is performed in order to obtain pathological verification and correlation of existing breast disease and mammographic examinations. (5) Patients referred to the clinical research program will have had clinical $x$-ray mammograms or breast xerograms, obtained by the referring physician, or by our clinical research protocol. Each patient is interviewed and examined by the research physician of the program. The heavy-ion examination will be arranged so that no delay will occur in the normal course of the patient's management by the referring physician (e.g., biopsy or surgery).

\section{Procedure of Heavy-Ion Mammography Examination}

Patients are examined in the patient medical cave at the Bevalac (Alonso, 1977; Grunder and Leeman, 1977). The patient lies prone on a specially designed mammography examination table. The breast to be examined is immersed in a rectangular lucite water bath (filled with warm water at $37^{\circ} \mathrm{C}$ and a wetting agent). The breast is gently compressed (either in the lateral or in the cephalocaudad direction, depending on the radiographic projection) between parallel plastic plates. The water-bath assembly is positioned perpendicular to the heavy-ion beam line, and parallel to the optical bench rails on which are mounted the ionization chambers and a water-bath variable-beam attenuator. Three-coordinate laser beams provide precise positioning of the breast in the beam line. The patient (except for the breast to be examined) is shielded by brassblock shielding and beam collimation. The nuclear track plastic detector stack of 25-30 sheets is mounted on the downstream side of the water-bath assembly perpendicular to the beam. The patient is left alone in the examination room during the exposure, but she is in continuous view on closed circuit television and there is constant intercom voice communication. A single beam pulse at $0.5 \mathrm{sec}$ is used, e.9., ${ }^{12} \mathrm{c}$,

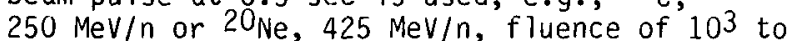
$2 \times 10^{4}$ particles $/ \mathrm{cm}^{2}$. $10^{3}$ neon ions $/ \mathrm{cm}^{2}$ produce about a $20 \mathrm{mrem}$ dose, and $2 \times 10^{4}$ carbon ions $/ \mathrm{cm}^{2}$ about a $150 \mathrm{mrem}$ dose, which is sufficient to expose a nuclear track detector stack of 25 sheets. Both breasts are radiographed. The patient does not move during the interval between the cephalocaudad and lateral projections; instead, the examination couch is moved so that the second position is achieved by rotation of the couch around the vertical axis that intercepts the horizontal heavy-ion beam.

Two heavy-ion mammograms (1atera) and cephalocaudad views) are taken of each breast. A single radiograph is sufficient for a general comparison of breast tissue stopping power to water. For exact measurements of stopping power values, i.e., tissue density in normal and abnormal breast structure, two exposures may be obtained in the same position; two different liquid bath mixtures, water and lipid, are used. This additional radiographic projection of each breast in two different water bath suspensions permits more accurate calculations of tissue electron densities in vivo. The entire heavy-ion mammographic procedure, including patient positioning and radiographic exposures, takes about 30 to 40 minutes. A physician and nurse are always present, informed consent is used, and human use authorization under National Institutes of Health guidelines is followed. Thus far, we have experienced no patient problems, and particularly with regard to the interface between the complex engineering instrumentation and equipment for beam delivery and the patient examination procedure.

\section{Findings of Heavy-Ion Mammography}

The initial stage heavy-ion mammography clinical trial is in progress; thus far, 37 patients have been examined, and approximately 150 heavy-ion mammograms have been obtained. All heavy-ion mammograms are compared with the 
patient's X-ray mammograms or breast xerograms, and pathological examination where available.

The observations on heavy-ion mammography in 35 of 37 patients examined are listed in Tables 3 and 4. Table 3 describes the examinations of patients with benign breast disease diagnosed by $x$-ray mammography or breast xeroradiography and/ or clinical examination, and heavy-ion mammograpny. Table 3 also describes the examinations of patients with clinically benign disease, but where the interpretation of heavy-ion mammograms and the conventional $X$-ray mammograms or breast xerograms were in agreement or may have differed, but carcinoma was not incorrectly diagnosed. Cysts, abscesses, mammary dysplasia, and benign breast tumors were clearly imaged on the heavy-ion mammograms.

Table 4 describes the examinations of patients with breast cancer. In three patients, cancer was not diagnosed by conventional X-ray mammography or breast xerography, but was demonstrated by the carbon-ion mammograms. The imaging efficiencies of conventional $x$-ray mammography on breast xerography and of heavy-ion mammography are qualitatively compared in Table 1. Such important anatomical and pathological characteristics as the density, and character of density, shape, involvement of surrounding tissues, and size are potentially better differentiated by heavy-ion imaging.

The salient observations of the clinical trials include the following:

1. Heavy-ion mammography is a low-dose, safe, reliable, noninvasive imaging procedure. The examination of each patient has provided extensive quantitative image processing, analysis, and image display. 2. The radiation dose to the breast is about $1 / 25$ th to $1 / 250$ th of that on conventiona 1 $X$-ray mammography or breast xerography examinations performed in community-based radiological practice (Table 5). Doses of only 50 mrad for carbon-ion mammograms provide images of good quality. Diagnostic images have been obtained with radiation doses as low as 10 to $20 \mathrm{mrad}$ (Fig. 2). These images are somewhat noisy, however, they can reliably estimate the electron density of tissue regions of about $0.3 \mathrm{~cm}$ in diameter and larger. Carbon-ion mammography is obtained regularly with radiation doses to the breast that are substantially less than those from conventional X-ray mammography procedures. Furthermore, X-ray methods do not provide the wide capabilities of quantitative densitometry, computer analysis and image display for determination of composition of normal and diseased breast tissues in clinical patients.

3. Density resolution in carbon-ion mammographs is equal to or exceeds that of conventional $X$-ray mammography in a 11 patients examined. Carbon-ions demonstrate all abnormal densities in the breasts of patients that can be detected by $x$ rays. However, the carbon-ion mammograms provide greater sensitivity for detection of minute con- trasting tissue densities in the breast compared with $X$-ray mammograms. X rays provide improved lateral (spatial resolution), and are relatively more sensitive to higher atomic number tissue structures, such as microcalcifications in the breast (Fig. 3 ). 4. Carbon-ion mammography detected changes in tissue structure detailed in the breast, and particularly in dense breast tissue, more readily than $X$ rays (Fig. 4). Heavy ions appear to have an advantage in imaging dense breasts, and especially breasts with fibrocystic disease or with a small, dense cancer.

5. Carbon-ion mammography demonstrated small abnormal densities in the breasts of two patients which were not detected by $X$ rays. Both these densities were nonpalpable masses lying deep in the breast tissue, and measured less than $1 \mathrm{~cm}$ in diameter on the heavy-ion mammograms. Thus, in the initial patient trails, in those breasts with potentially detectable cancer, carbonion mammography demonstrated soft-tissue breast cancers less than $1 \mathrm{~cm}$ in diameter which were not demonstrated on conventional $X$-ray mammography.

6. In all patients examined with X-ray mammography and carbon-ion mammography, the heavy-ions confirmed the $x$-ray studies, i.e., either positive or negative for breast cancer (Fabrikant, 1980; Sickles, 1979). Surgical biopsy studies verified these findings in 12 patients ( 8 benign and 4 malignant tumors); 25 patients either did not have biopsies done, or the results of biopsies done are not available at this time. One patient had a nonpalpable mass less than $1 \mathrm{~cm}$ that was demonstrated on the carbon-ion mammograms and not on X-ray examination; biopsy proved the mass to be carcinoma (Fig. 3). In a second patient with a less than $1 \mathrm{~cm}$ nonpalpable mass, less than $1 \mathrm{~cm}$ which was demonstrated only on the carbon-ion mammogram, the results of the biopsy also demonstrated a benign breast tumor.

\section{Radiation Dose and Heavy-Ion Mammography}

At the present time, the improved $x$-ray mammographic and breast xerographic methods can almost always distinguish between benign and malignant tumors in the breast; the experienced radiologist can make this distinction with an accuracy of better than $90 \%$. Any significant improvement in diagnostic accuracy, whether in differentiating among benign diseases, or between benign and malignant tumors, must depend on improved image quality with decreased radiation dose. While there have been significant technical developments in conventional $x$-ray mammography and breast xerography, the majority of mammographic $x$-ray units and procedures still result in skin doses of 2 to $10 \mathrm{rad}$, and a $3 \mathrm{~cm}$ depth dose of about 5 to 7 rad (Egan, 1960; Sutton, 1975), and about similar values, or slightiy greater, for breast xerography. Table 5 lists radiation doses to the breast during the various clinically applicable mammographic procedures in current radiological practice. 
Table 3. Patients with Benign Breast Disease

\begin{tabular}{|c|c|c|c|c|c|}
\hline $\begin{array}{l}\text { Patient } \\
\text { No. }\end{array}$ & Age & Clinical & Heavy-Ion Mammography & X-Ray Mammography & Followup \\
\hline 5 & 34 & $\begin{array}{l}\text { Dense left breast, } \\
2-\mathrm{cm} \text { mass }\end{array}$ & $\begin{array}{l}\text { Symmetrical breasts with- } \\
\text { out mass }\end{array}$ & No masses & $\begin{array}{l}\text { Mass unchanged } \\
\text { in } 5 \text { years }\end{array}$ \\
\hline 6 & 39 & $\begin{array}{l}2-\mathrm{cm} \text { mass, right outer } \\
\text { quadrant, massive fatty } \\
\text { breast }\end{array}$ & $\begin{array}{l}\text { No masses, mass at chest } \\
\text { wall out of beam field }\end{array}$ & No masses & $\begin{array}{l}\text { Biopsy: lymph } \\
\text { node }\end{array}$ \\
\hline 13 & -- & $\begin{array}{l}\text { Mass, left breast, outer } \\
\text { quadrant }\end{array}$ & $\begin{array}{l}\text { Mass in left breast, } \\
\text { outer quadrant }\end{array}$ & $\begin{array}{l}\text { Mass in left breast, } \\
\text { outer quadrant }\end{array}$ & $\begin{array}{l}\text { Biopsy: mammary } \\
\text { dysplasia }\end{array}$ \\
\hline 17 & 48 & Bilateral breast masses & No masses or asymmetry & $\begin{array}{l}2 \text { calcifications in } \\
\text { left breast and } 1 \text { in } \\
\text { right }\end{array}$ & $\begin{array}{l}\text { Biopsy: mammary } \\
\text { dysplasia }\end{array}$ \\
\hline 19 & 39 & Breast mass & No mass & No mass & Biopsy: abcess \\
\hline 20 & 39 & Mass, left breast & No mass & No mass & $\begin{array}{l}\text { Biopsy: fibro- } \\
\text { adenoma }\end{array}$ \\
\hline 21 & 46 & $\begin{array}{l}1-\text { to } 2-\mathrm{cm} \text { mass, } \\
\text { left breast }\end{array}$ & $\begin{array}{l}\text { Mass, increased density, } \\
\text { left breast }\end{array}$ & $\begin{array}{l}\text { Mass, increased } \\
\text { density, left } \\
\text { breast }\end{array}$ & $\begin{array}{l}\text { No change in } 3 \\
\text { years; probably } \\
\text { fibroadenoma }\end{array}$ \\
\hline 22 & 60 & Breast mass & Mass & Mass & $\begin{array}{l}\text { Biopsy: mammary } \\
\text { dysplasia }\end{array}$ \\
\hline 24 & 31 & 4-cm mass, right breast & $\begin{array}{l}\text { Symmetrical dense breasts, } \\
\text { mass }\end{array}$ & $\begin{array}{l}\text { Dense breasts, } \\
\text { mammary dysplasia }\end{array}$ & $\begin{array}{l}\text { Mass unchanged } \\
\text { in } 6 \text { months, } \\
\text { no biopsy }\end{array}$ \\
\hline 25 & 23 & $\begin{array}{l}2-\mathrm{cm} \text { mobile mass, left } \\
\text { breast }\end{array}$ & Asymmetrical dense breasts & $\begin{array}{l}\text { Dense breasts with } \\
\text { mammary dysplasia }\end{array}$ & $\begin{array}{l}\text { Mass unchanged } \\
\text { in } 6 \text { months, } \\
\text { no biopsy }\end{array}$ \\
\hline 27 & 47 & $\begin{array}{l}\text { Right mastectomy for } \\
\text { infiltrating ductal } \\
\text { carcinoma; left breast mass }\end{array}$ & $\begin{array}{l}\text { Mass in left lateral } \\
\text { breast }\end{array}$ & No mass & $\begin{array}{l}\text { Biopsy: focal } \\
\text { fat necrosis }\end{array}$ \\
\hline 28 & 46 & $\begin{array}{l}\text { Breast cyst with recent } \\
\text { appearance of } 2 \text { masses }\end{array}$ & No masses & No masses & $\begin{array}{l}\text { Biopsies: mammary } \\
\text { dysplasia }\end{array}$ \\
\hline
\end{tabular}


Table 3. (continued)

\begin{tabular}{|c|c|c|c|c|c|}
\hline $\begin{array}{l}\text { Patient } \\
\text { No. }\end{array}$ & Age & Clinical & Heavy-Ion Mammography & X-Ray Mammography & Followup \\
\hline 30 & 31 & $\begin{array}{l}\text { Right mastectomy for } \\
\text { infiltrating ductal } \\
\text { carcinoma, left breast } \\
\text { normal }\end{array}$ & No mass, left breast & $\begin{array}{l}\text { No mass, left } \\
\text { breast }\end{array}$ & Norma 1 \\
\hline 31 & 44 & Bilateral breast masses & $\begin{array}{l}\text { Focal area increased } \\
\text { density left breast, } \\
\text { possibly carcinoma }\end{array}$ & $\begin{array}{l}\text { Microcalcifications, } \\
\text { left breast }\end{array}$ & $\begin{array}{l}\text { Biopsies: mammary } \\
\text { dysplasia }\end{array}$ \\
\hline 32 & 32 & Mass, left breast & No mass, dense breasts & $\begin{array}{l}\text { No mass, mammary } \\
\text { dysplasia }\end{array}$ & $\begin{array}{l}\text { Multiple breast } \\
\text { cysts }\end{array}$ \\
\hline 33 & 59 & Bilateral masses & No mass & $\begin{array}{l}\text { No mass, mammary } \\
\text { dysplasia }\end{array}$ & $\begin{array}{l}\text { No change in } \\
5 \text { years }\end{array}$ \\
\hline 34 & -- & Mass cyst & No mass & No mass or cyst & Normal \\
\hline 35 & 24 & $\begin{array}{l}\text { Dense breasts; left breast } \\
\text { mass }\end{array}$ & No mass & $\begin{array}{l}\text { No mass, mammary } \\
\text { dysplasia }\end{array}$ & Normal \\
\hline 36 & 32 & $\begin{array}{l}\text { Breast mass with calcifi- } \\
\text { cation }\end{array}$ & No mass & Mammary dysplasia & \\
\hline 38 & - & Dense breasts & No mass & $\begin{array}{l}\text { Dense breasts, } \\
\text { no mass }\end{array}$ & Normal \\
\hline 39 & -- & Mass, left breast & $\begin{array}{l}\text { Mass, left breast, } \\
\text { mammary dysplasia }\end{array}$ & $\begin{array}{l}\text { Mass, mammary } \\
\text { dysplasia }\end{array}$ & $\begin{array}{l}\text { No change in } \\
2 \text { years }\end{array}$ \\
\hline 41 & -- & Mass & No mass, scar tissue & Small mass & No change \\
\hline 44 & -- & Dense breasts & No mass & No mass & Normal \\
\hline 45 & -- & Dense breasts & No mass & No mass & Normal \\
\hline
\end{tabular}


Taole 4. Patients with Malignant Breast Disease

\begin{tabular}{|c|c|c|c|c|c|}
\hline $\begin{array}{l}\text { Patient } \\
\text { No. }\end{array}$ & Age & Clinical & Heavy-Ion Mammography & X-Ray Mammography & Followup \\
\hline 7 & 60 & $\begin{array}{l}\text { Mastectomy, right breast; } \\
\text { lateral mass, left breast }\end{array}$ & Density left breast & Dense left breast & None \\
\hline 14 & $\begin{array}{l}-- \\
\cdots\end{array}$ & Mass, left breast & No mass & No mass & $\begin{array}{l}\text { Infiltrating } \\
\text { ductal carcinoma }\end{array}$ \\
\hline 15 & 50 & $\begin{array}{c}\text { No mass, left breast } \\
\therefore\end{array}$ & $\begin{array}{l}\text { Mass at site of micro- } \\
\text { calcifications, left } \\
\text { breast }\end{array}$ & $\begin{array}{l}1.5 \times 3 \mathrm{~cm} \text { area of } \\
\text { microcalcification, } \\
\text { left breast }\end{array}$ & $\begin{array}{l}\text { In situ ductal } \\
\text { carcinoma, left } \\
\text { breast }\end{array}$ \\
\hline 18 & 43 & $\begin{array}{l}\text { 2-cm mass, left breast } \\
\end{array}$ & Mass, left breast & Mass, left breast & $\begin{array}{l}\text { Mass larger; } \\
\text { biopsies: atypical } \\
\text { hyperplasia to } \\
\text { lobular in situ } \\
\text { carcinoma }\end{array}$ \\
\hline 37 & 66 & $1-\mathrm{cm}$ mass, left breast & Mass, left breast & Mass, left breast & $\begin{array}{l}1-\mathrm{cm} \text { infiltrating } \\
\text { ductal carcinoma }\end{array}$ \\
\hline 40 & 80 & $1-\mathrm{cm}$ mass, right breast & Mass, right breast & Mass, right breast & $\begin{array}{l}\text { Infiltrating ductal } \\
\text { carcinoma }\end{array}$ \\
\hline 47 & 66 & $4.5-\mathrm{cm}$ mass, right breast & Mass, right breast & Mass, right breast & $\begin{array}{l}\text { Infiltrating ductal } \\
\text { carcinoma }\end{array}$ \\
\hline 48 & 45 & $\begin{array}{l}\text { Grusting lesion, left } \\
\text { breast }\end{array}$ & No masses & No masses & $\begin{array}{l}\text { Infiltrating carci- } \\
\text { noma; Paget's disease } \\
\text { of nipple }\end{array}$ \\
\hline
\end{tabular}


Table 5. Radiation Dose to Breast for Different Mammographic Examinations

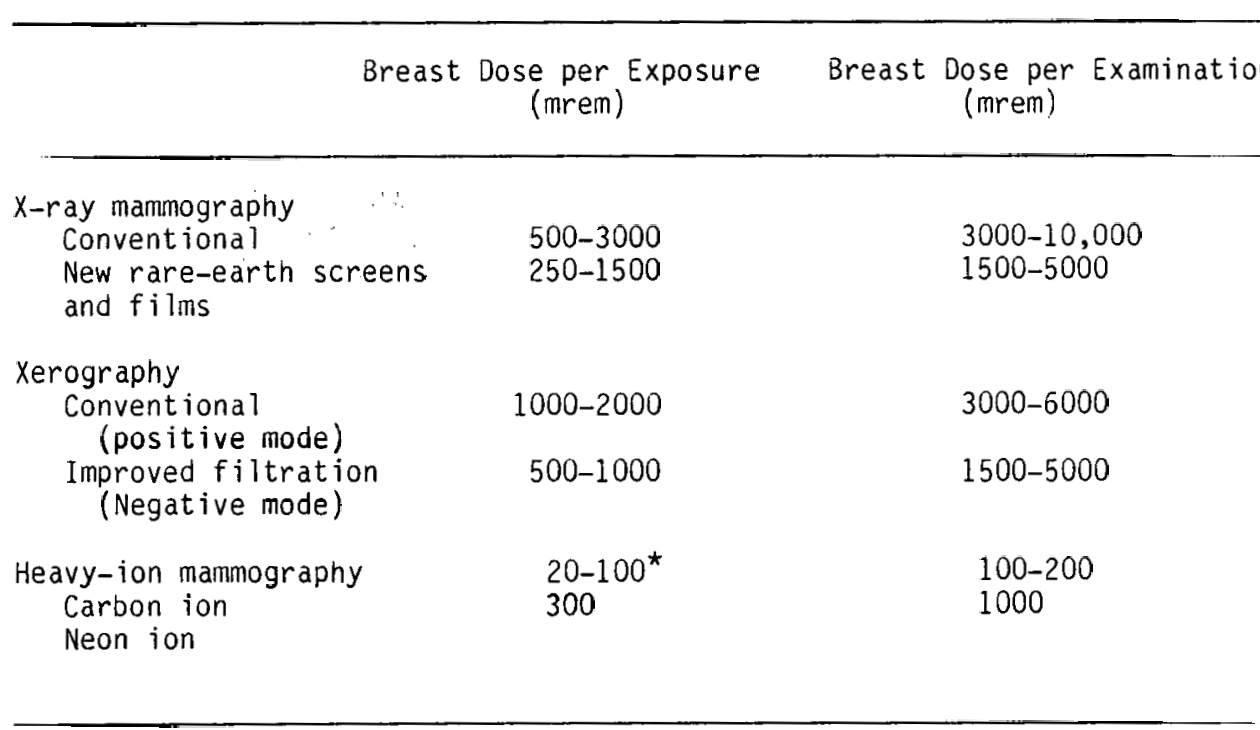

* Diagnostic carbon-ion mammograms have been obtained at a dose of 20 mrad (see Fig. 3).

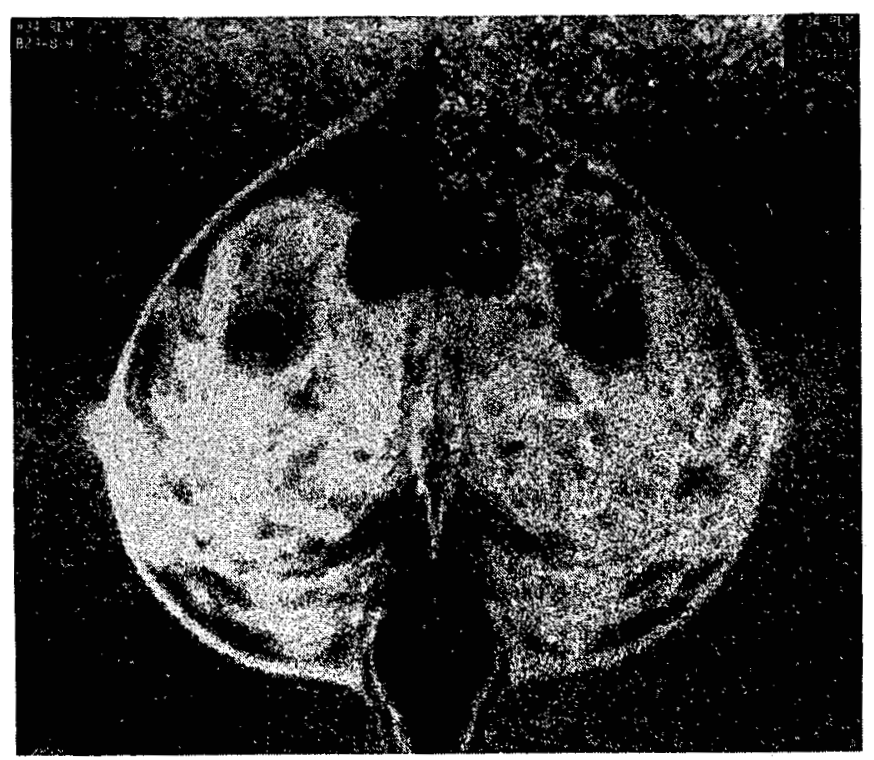

Figure 2. Low-dose carbon-ion mammogram on the same patient: $20 \mathrm{mrad}$ (right) and $60 \mathrm{mrad}$ (left). (XBB 782-2218)

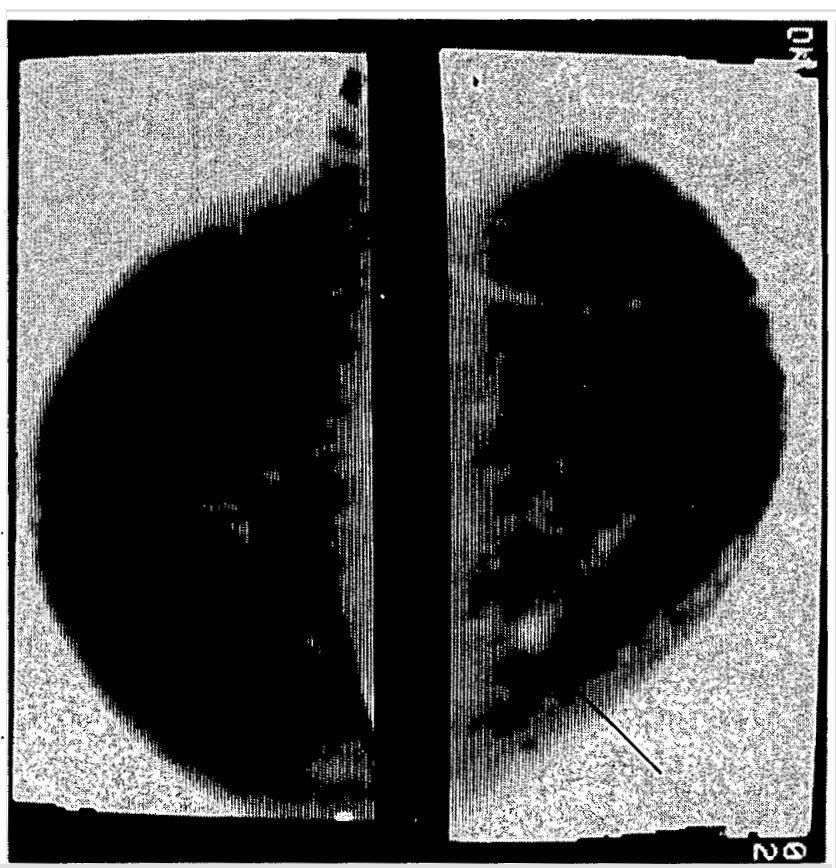

Figure 3. Computer synthesized carbon-ion mammograms of both breasts of the same patient. Left, normal breast; right, breast with $6 \mathrm{~mm}$ carcinoma (arrow). (XBB 780-13077) 
The physical factors that affect the radiation dose in heavy-ion radiography include the particle fluence, the particle energy, loss rate, range straggling, and nuclear disintegrations (Benton et al., 1973). Particle fluence and energy loss rate are the important factors in dose calculations for heavy-ion mammography since low initial energies are used, and the radiation dose in the breast tissue is far removed from the stopping point of the particles. The total number of particles in the heavy-ion beam remains quite low, even if large radiographs are made. For example, a $50 \mathrm{~cm} \times 50 \mathrm{~cm}$ carbon-ion radiograph, with 1000 particles per $\mathrm{cm}^{2}$, uses about $2.5 \times 10^{6}$ particles. This is several orders of magnitude lower than the maximum available from the Bevalac in a single pulse.

In our clinical patient trials, heavy-ion mammography with carbon-ions $(425 \mathrm{MeV} / \mathrm{n}$ ) and using nuclear track detector stacks of $25 \mathrm{film}$ detectors has regularly provided excellent quality diagnostic mammograms with radiation doses of $50 \mathrm{mrad}$ per exposure (Fig. 3); the maximum RBE for plateau phase carbon-ions is estimated to be 1.98 (Blakely et al., 1979). We have demonstrated that carbon-ion mammography can achieve satisfactory radiographic images with doses of $10 \mathrm{mrad}$ per exposure (Fig. 3); the total radiation dose would be $40 \mathrm{mrad}$ for the fourimage examination of both breasts. Thus, diagnostic heavy-ion mammograms can be obtained with radiation dose-reduction factors of some 25 to 250 over conventional and improved $X-r a y$ and breast xerography mammographic procedures.

\section{Stopping Power Density Distribution Histogram Analysis}

The stopping power density distributions determined as a consequence of range distributions are usually employed in some form of visual display. so that the clinician can derive diagnostic information from them. However, it is also possible to attempt statistical analysis of the density distribution patterns, and perhaps eventually use other techniques for mathematical analysis of the internal topography of tissues of the breasts. This is of particular interest, since mammograins of both breasts are usually available and the distribution of normal density patterns between left and right breasts of the same individual exhibit a degree of symmetry, while these patterns are markedly different from one individual to the next. Abnormal densities due to cysts often appear most clearly when deviations between density patterns of the abnormal and the normal breast are under study.

We have made some simple preliminary investigations of breast density patterns. Figure 5 shows the normalized electron density distribution based on lateral radiographs.of the left and of the right breast of the same individual. The shaded area is the left breast which had a small carcinoma that was later jemoved by biopsy. The mean density of both sreasts was about $T=-5 \pm 1$; the values of $\tau=0$ correspond to the same electron density as water.
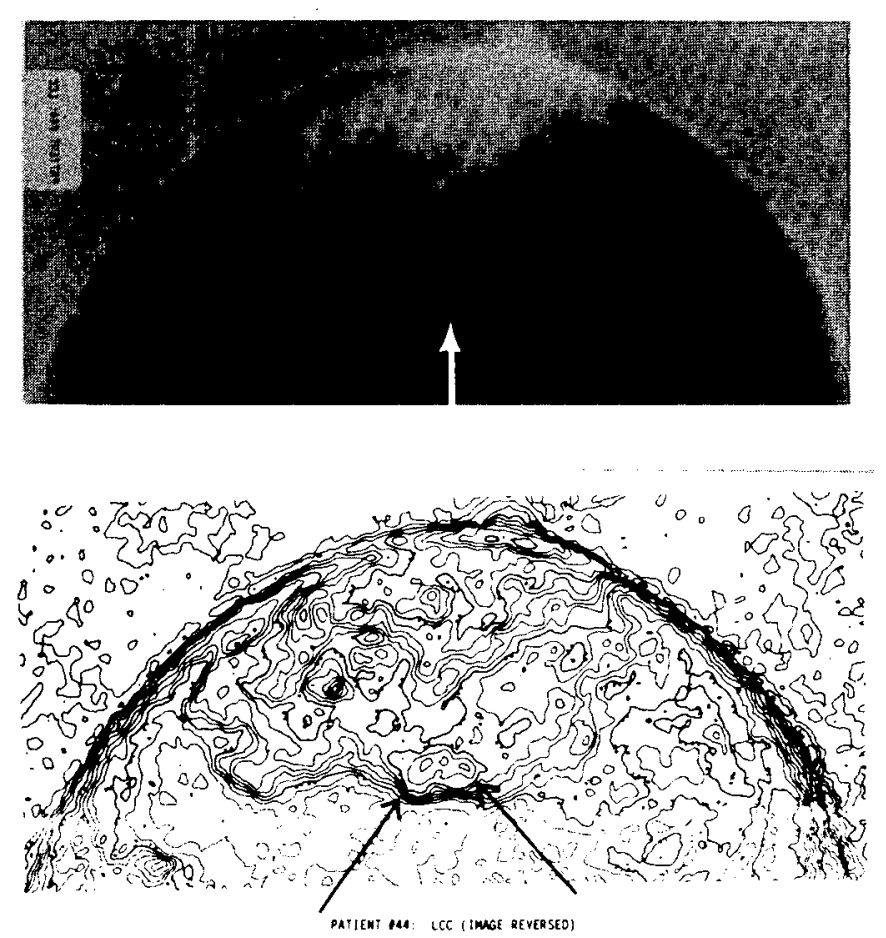

Figure 4. (Upper) Computer synthesized half-tone image of a carbon ion mammogram; the increased density deep in the breast is a carcinoma (arrow). (Lower) Corresponding isodensity contour plot derived from stopping power values of the carbon-ion mammograms. The carcinoma is readily discerned and quantitated. (XBB 782-1823A)

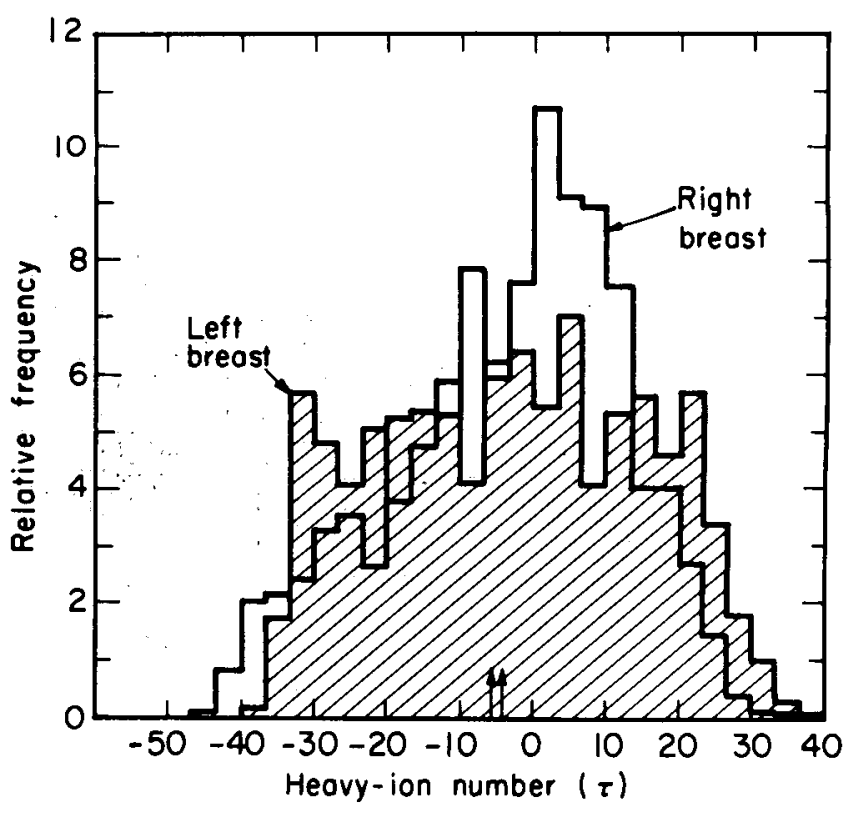

Figure 5. Stopping-power density-distribution histogram of a breast image obtained from a carbon-ion mammogram. (XBL 8010-3718) 
The differences in the electron density distribution are noticeable at the high densities, above water. The left breast had more regions of relatively high density, $T=20$ to 30 , and the right breast had more regions of relatively low density, $T=0$ to 15 . The mean electron density of each breast was about the same. The irregular fluctuations in the graphs are due to density patterns that are reproducible and are not due to statistical variations. With this approach one might be able to detect differences in the densitogram due to diffusively growing tumor, which does not nave a single module large enough for viewing, but which is sufficiently widespread to cause a difference in the spectrum.

We are planning to improve quantitative mammographic analysis techniques. The electron densities used in the frequency plot are not the real densities, but rather they are transformed because of the finite resolution of the method. However, we have increasingly improved information on the modulation transfer function, and we are pianning to reconstruct a more correct image of the breast. The most straightforward method is to perform a Fourier analysis on the data obtained by devising the Fourier coefficients, divide each coefficient with the appropriate MTF factor, and then resynthesize the image. The new image enhances density differences of small structures. We are aware of difficulties with this method at high frequencies, when noise from the statistical distribution of particle densities becomes important. However, high frequency components can be filtered out. The correlated density distributions will give new information on tissue densities in the breast; so $f a r$ our determinations give density values between $\tau=-20$ and $\tau=0$.

\section{Test-Retest Correlation in Fibrocystic Disease}

Heavy-ion mammography has achieved a sufficiently quantitative status so that we plan to correlate mammograms taken on the same patient, several months apart. Since the dose is low (20 mrad per exposure), this does not appear to be hazardous for the patient. Retesting may detect either local changes in tissue density, or statistically significant changes in electron density distribution spectra, including determination of mean density and integrated densities. One group of patients with fibrocystic disease could benefit from improved quantitation so that a measure of the changes that may be occurring in the breast with time could be recorded. Once these techniques have been well established, we plan to include them in a second series of clinical investigations which will begin in 1981 .

\section{FUTURE STUDIES}

Conventional $x$-ray mammography fails to differentiate between dense breast tissue that is normal and benign, and dense cancer tissue that may be masked in the dense breast, unless intervening breast fat is present. Over $50 \%$ of breast cancers do not have microcalcifications, and it is in these patients that dense breast tissue can and frequently does mask small malignant changes. Heavy-ion mammography detects changes in tissue structure in dense breast tissue more readily than $x$ rays. The heavy-ion radiographs can demonstrate considerable detai] in these dense breasts, whereas $x$ rays frequently lack the detail. Quantitative heavy-ion mammography, therefore, appears to have an advantage over $X$ rays in examining dense breast tissues. Determination of stopping power gradients to identify small lesions with only slightly differing density values may lead to differentiation of dense breast tissue and small carcinomas. Some progress has been reported in this regard based on X-ray CT mammography combined with iodinated contrast media infusion; this technique, however requires large radiation doses and potential adverse reactions to iodinated contrast media.

In our heavy-ion mammography clinical trial, in those patients with dense breasts, with or without breast cancer, both breasts are examined. Vigorous criteria are set to establish the smallest size of cancer in the breast that can be determined. It will be important to differentiate between dense breasts and the development of early cancer masked by dense breast tissue. This is being carefully evaluated in the clinical trials and comparisons are being made with conventional $X$-ray mammography in all cases, and with surgical or pathological specimens when available.

Lymphogenous metastatic spread of breast cancer profoundly affects the clinical management and prognosis of the neoplastic disease. Metastases to the draining lymph nodes can occur early, and we are considering the potential of heavy-ion mammography for imaging of axillary lymph node metastases in selected patients. If it is possible to differentiate only slightly differing density values in soft tissues, the potential exists for the identification of dense metastatic breast cancers in the lymph nodes, particularly in the loose areolar tissue of the axilla. Comparisons of image structure and density resolution (stopping power values) may provide quantitative information on the presence of metastatic disease not presently available with conventional X-ray mammography.

\section{CONCLUSIONS}

Heavy-ion radiography is a new diagnostic imaging technique developed in our laboratory that produces superior density resolution at low radiation doses. Heavy-ion mammography has now emerged as a low-dose, safe, reliable, noninvasive diagnostic radiological procedure that can quantitate and image very small differences in soft tissue densities in the breast tissues of patients with clinical breast disease. The improved density resolution of heavy-ion mammography over conventional $X$-ray mammography and breast xerography provides the potential of. detecting small breast cancers of less than $1 \mathrm{~cm}$ diameter; occuit carcinomas as small as $6 \mathrm{~mm}$ have been identified. In dense breasts of younger 
women, the improved density resolution of heavyion mammography should provide improved diagnostic accuracy than does conventional $X$-ray mammography. The radiation dose to the breast from carbon-ion mammography is about $50 \mathrm{mrad}$ or less, and can potentially be only a fraction of this level. The development of computersynthesized diagnostic images, image display and methods of analysis provides additional valuable quantitative informaton, and improved image quality associated with the potential of further reduction in radiation dose to the breast. The results of the present clinical trial in progress of heavy-ion mammography in 37 patients, thus far studied, are extremely encouraging, and warrant continued study for application to the early diagnosis of breast cancer in women.

\section{ACKNOWLEDGEMENTS}

These studies are supported by the National Cancer Institute (Grant No. CA 27021).

\section{FOOTNOTES AND REFERENCES}

*Department of Radiology, University of California, San Francisco, CA.

tDepartment of Radiology, University of Arizona School of Medicine, Tucson, AZ.

FChildren's Hospital, San Francisco, CA.

Alonso, J. 1977. Current status and planned improvements of the Bevalac. Biological and Medical Research with Accelerated Heavy Ions at the Bevalac, 1974-1977, pp. 3-12. Lawrence Berkeley Laboratory Report LBL-5610.
Benton, E. V., R. P. Henke, and C. A. Tobias. 1973. Heavy-particle radiography. Science $182,474-476$.

Blakely, E. A., C. A. Tobias, T.C.H. Yang, K. C. Smith, and J. T. Lyman. 1979. Inactivation of human kidney cells by high-energy monoenergetic heavy-ion beams. Radiat. Res. 80, $122-160$.

Egan, R. L. 1960. Experience with mammography in a tumor institution. Radiology 75, 894-900.

Fabrikant, J. I., C. A. Tobias, M. P. Capp, E. V. Benton, and W. R. Holley. 1980. Heavyion imaging applied to medicine. Soc. PhotoOpt. Instrum. Eng. 233, 255-263.

Grunder, H and C. W. Leemann. 1977. Present and future sources of protons and heavy-ions. Int. J. Radiat. Oncol. Biol. Phys. 3, 71-80.

Sickles, E. A., E. V. Benton, C. A. Tobias, and K. H. Woodruff. 1979. Mammography using Bevalac accelerated heavy particles. Reduced Dose Mammography, pp. 501-505. Masson Publishing Company, New York.

Sommer, F. G., M. P. Capp, C. A. Tobias, E. V. Benton, K. H. Woodruff, R. P. Henke, W. R. Holley and H. K. Genant. 1978. Heavy-ion radiography: Density resolution and specimen radiography. Int. J. Invest. Radiol. 13, 163-170.

Sutton, D. 1975. Textbook of Radiology. Churchill-Livingstone, London.

Tobias, C. A., E. V. Benton and M. P. Capp. 1978. Heavy-ion radiography. Rec. Adv. Nucl. Med. 5, 71-102. 
Blank 


\title{
HEAVY-ION RADIOGRAPHY APPLIED TO CHARGED PARTICLE RADIOTHERAPY
}

\author{
G. T. Y. Chen, J. I. Fabrikant, " W. R. Holley, C. A. Tobias, and J. R. Castro'
}

Successful radiation therapy of clinical cancer depends in large measure on: (1) the ability for precise diagnostic localization and a knowledge of the the size and position of the tumor and of adjacent and surrounding normal tissue structures; (2) optimum utilization of such diagnostic information in radiotherapy treatment planning; and (3) delivery of optimum radiation dose distributions to the neoplasm in the patient, and at the same time, to protect and to spare the normal surrounding tissues or to minimize the radiation dose received by the sensitive normal structures of the body. The use of accelerated heavy-ions (and other charged particles) in highLET radiotherapy of cancer provides unique opportunities for improvement of tumor localization and for accurate delivery of effective heavy-ion dose distributions (Castro et al., $1979,1980)$. Heavy-ion radiography (Tobias et al., 1977, 1978; Holley et al., 1979; Fabrikant et al., 1980) is being developed concurrently at LBL for direct application to problems of tumor localization, tissue inhomogeneities, and treatment planning for charged particle radiotherapy of clinical cancer. The imaging method uses accelerated heavy-ion beams that allow for better localization of deep-seated tumors, for diagnostic imaging adjustment of charged particle therapy beams, for design of irregular-shaped compensators for treatment, and for evaluating tumor response during and following charged particle radiotherapy.

Clinical trials of neutron radiotherapy were instituted by Stone and Lawrence in the 1940s (Stone et al., 1940). Charged particle irradiation of the pituitary for endocrine disease and palliation of advanced cancer patients began in 1955, with protons and deuterons, and subsequently with plateau helium-ion (alpha particle) irradia: tion. In the ensuing twenty-five years, almost 800 pituitary patients have been treated (for review, see Tobias, 1979). Since 1975, the NCI has supported the radiation biophysics and biology research program applied to oncology under C.A. Tobias, (NIH-NCI CA 15184) which has led to preparation for clinical cancer patient trials with helium-ion irradiation at the 184-inch Synchrocyclotron, and now with heavier charged particles (carbon, neon and argon) at the Bevalac. A systematic clinical trial of heavy charged particle radiotherapy began in 1.975 with support from the Department of Energy, and in 1976 with NCI support (NIH-NCI CA 19138 urider J. R. Castro and C. A. Tobias): The clinical trials have now included over 225 patients, and the program has continued strong support. The orincipal core staff include Drs. Castro, Tobias, lpen and Chen. Randomized and nonrandomized clinical protocols with helium-ion radiotherapy now include: carcinoma of the pancreas (random- ized); carcinoma of the esophagus (nonrandomized); advanced carcinoma of the cervix (randomized); and a number of miscellaneous sites of locally advanced cancers (nonrandomized). Approximately 125 patients are treated with helium ions on the 184-inch Synchrocyclotron each year. Nonrandomized clinical trials have now begun at the Bevalac, and it is anticipated that about 75 patients will be treated each year with heavier charged particles, such as carbon, neon, or argon (Castro et al., 1979, 1980).

\section{CLINICAL PHYSICS FOR CHARGED PARTICLE RADIOTHERAPY}

A computerized treatment planning system which used quantitative information from $X$-ray computerized tomographic (CT) scan examinations of the cancer patients has been developed for the heavy-ion radiotherapy trials at LBL (Chen et al., 1979). A range shortening algorithm is used based on water equivalent range to convert $X$-ray CT numbers to electron densities. Dose distributions are then calculated on a pixel-by-pixel basis with appropriate corrections for bone, intestinal gas, and other tissue inhomogeneities in the charged particle beam path. These dose distributions are superimposed on a gray-scale $x$-ray CT scan display and are produced for multiple X-ray CT scan leveis through the tumor target volume. Charged particle isodose distributions are thus produced, Dased on X-ray CT data taken prior to radiation therapy. These plans are developed for isodose distribution in particlerad and the biologically corrected dose distributions in megavoltage photon-rad-equivalents.

Investigations in progress on charged particle treatment planning include an evaluation of physical techniques of compensation of tissue and other inhomogeneities in the particle beam path. Heavy-ion radiography and CT reconstruction imaging provide an important role in this study. For the present, wax bolus compensating devices: are utilized in order to stop the beam at the desired depth in the body. With availability of heavy-ion radiography and CT reconstruction scans in the patjent treatment position, e.g., upright, prone, or supine positions, more precise compensation can be achieved. Such compensation would be based on corrections derived from two-dimensional and three-dimensional heavyion CT imaging with the patient in the treatment position at the time of charged particle radiotherapy, and using the same or similar charged particles. An investigation of motion and respiration effects has also been begun in order to determine the extent of beam range changes in dose distribution occurring during the motion of respiration. 
Verification of charged particle dose distribution in cancer patients has been made by heavy-ion radiography studies using nuclear detector film stacks (e.g., patients with brain tumors and pancreatic carcinoma) as well as in vivo diode measurements (e.g., patients with esophageal carcinoma). Studies comparing irradiation with the static fixed fields with three-dimensional beam scanning techniques are under way in preparation for subsequent development of beam scanning at the Bevalac. Development of dose-distribution graphics for display of three-dimensional isodose distributions obtained by multiple $x$-ray CT reconstructions (based presently on $X$-ray attenuation coefficient data) at $1-\mathrm{cm}$ intervals and less through the tumor target volume are in progress. A continuing programming development for the computerized treatment planning system is being carried out and developed to provide a faster, interactive treatment planning program.

\section{OBJECTIVES OF HEAVY-ION RADIOGRAPHY APPLIED TO CHARGED PARTICLE RADIOTHERAPY OF CANCER}

The objectives of the heavy-ion radiography research program applied to the clinical cancer research program of charged particle radiotherapy have a twofold purpose: (1) to explore the manner in which heavy-ion radiography and CT reconstruction can provide improved tumor localization, treatment planning, and beam delivery for radiotherapy with accelerated heavy charged particles; and (2) to explore the usefulness of heavy-ion radiography in detecting, localizing, and sizing soft tissue cancers in the human body. The techniques and procedures developed for heavy-ion radiography should prove successful in support of charged particle radiotherapy.

Even with the best available diagnostic $X$-ray procedures (e.g., X-ray CT scans) for treatment planning and tumor localization for charged particle radiotherapy, important deficiencies in the necessary quantitative data still exist.

1. Determination of the electron density distribution of the patient's tissues depends on images obtained from X-ray CT tomographic scans; these procedures provide a measure only of $X-r$ ay attenuation coefficients. Uncertainties remain in the stopping-power calculations, since they are determined by assuming tissue structure and tissue composition, and by the presence of computer artifacts. Heavy-ion radiography now aids directly in determining overall stopping power distribution in the beam path, thus allowing corrections in the $X$-ray CT reconstructed image. Ultimately, heavy-ion imaging has the potential to determine precise stopping power distribution within the tissue at each point with the application of heavyion CT scanning, thereby making assumptions and corrections which presently are derived from $X$-ray CT scanning data for the treatment planning no longer necessary.
2. Even with the best available $X-r a y$ methods, including $X-$ ray CT scanning, the tumor volume is poorly defined or often indistinguishable from normal surrounding tissues and organs, and cannot be localized or defined with the precision necessary for charged particle Bragg peak radiotherapy. From current studies in progress we have demonstrated that heavy-ion radiography detects smaller tissue density differences than $X$ rays (Tobias et al., 1978; Fabrikant et al., 1980). Therefore, heavy ions would have a greater chance in detecting normal and abnormal tissue densities within the tumor site and in the normal and abnormal tissues and structures surrounding the neoplasm.

\section{RADIOTHERAPY TREATMENT PLANNING, CORRELATION OF X-RAY.CT NUMBERS, $X$-RAY ATTENUATION COEFFICIENTS, AND HEAVY-ION STOPPING POWER VALUES}

The initial studies of heavy-ion radiography applied to charged particle radiotherapy treatment planning were carried out in brain tumor patients and in pancreatic cancer patients. Figure 1 is a computer-synthesized carbon-ion radiograph of the abdomen of a patient with pancreatic carcinoma. Here, 150 nuclear plastic detector sheets were used. The image clearly demonstrates the region of the head of the pancreas, the liver, the large and small intestine, the ribs, and lumbar vertebral column. A single sheet of the nuclear detector stack is

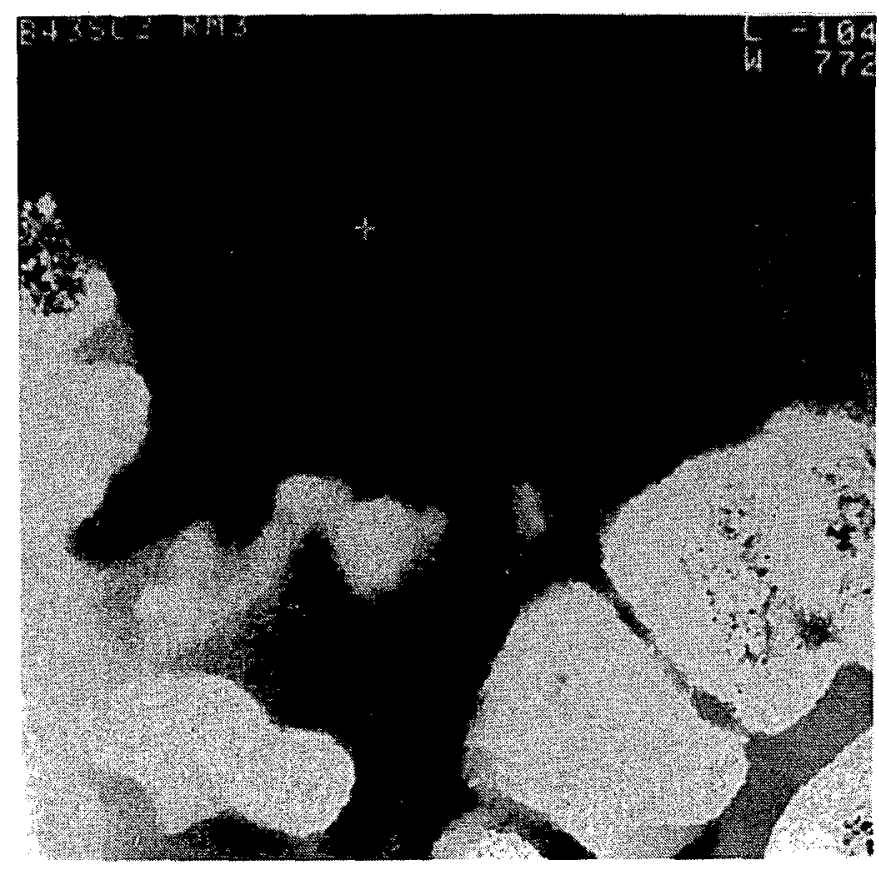

Figure 1. Computer synthesized carbon-ion radiograph of the abdomen of a patient with carcinoma of the head of the pancreas. (XBB 780-13070) 
illustrated in Figure 2, upper; an isodensity contour plot of the distribution of stopping power values of the tissues, by digitizing every third detector sheet is seen in Figure 2,10 wer. The density distribution plot demonstrates the lower stopping power values of the liver parenchyma (L). The localization of the intestinal gas in the colon $(C)$, which is essential for correcting for perturbations arising from tissue inhomogeneities, is readily identified.

We are presently investigating the applications of heavy-ion radiography and CT reconstruction tomography to charged particle radiotherapy treatment planning in two parallel. investigations: in phantoms, organs, and tissue specimens; and patients. We have been studying phantoms and organ and tissue specimens of known density
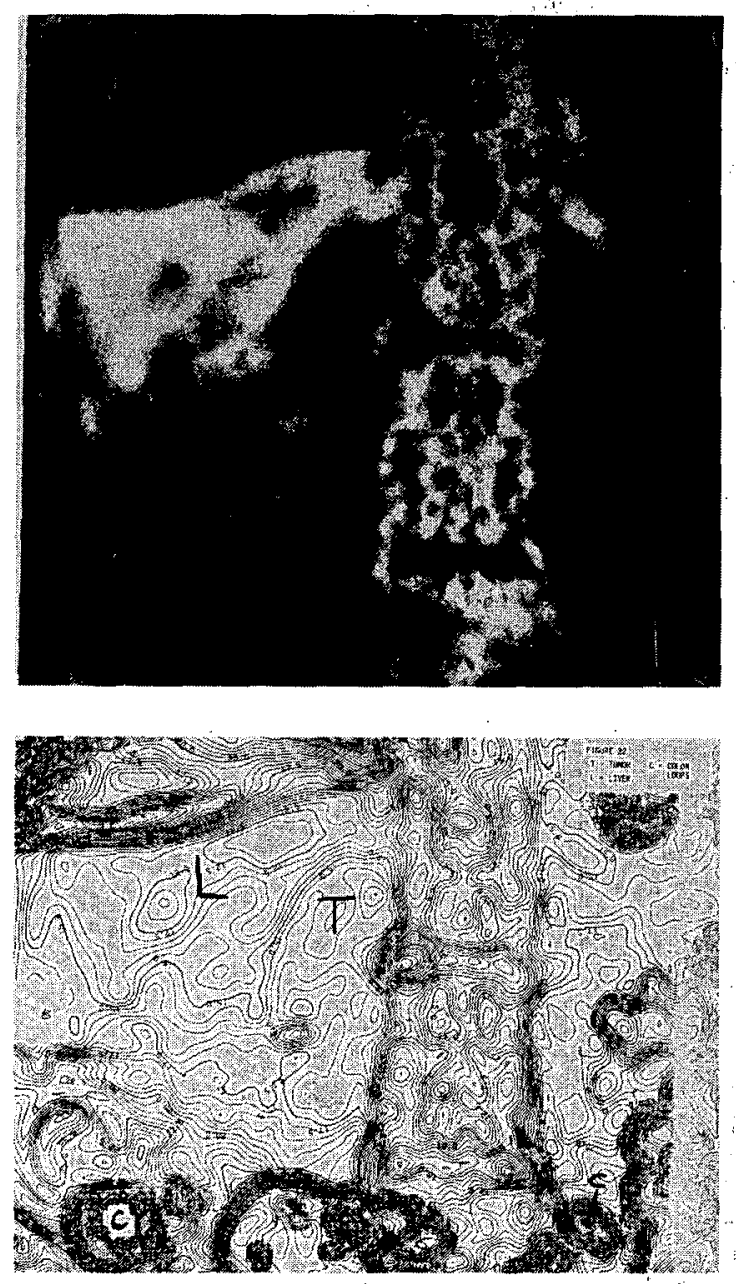

Figure 2. Above: Carbon-ion radiograph of a patient with carcinoma of the head of the pancreas. This is one of the 150 plastic detector-sheets that we exposed. Below: Contour plot of the data obtained from the radiograph. This figure demonstrates the low stopping-power values of the pancreatic neoplasm ( $T$ ); the higher values of the liver $(L)$; and the intestinal gas in the colon (C). (XBB 782 1165A) distribution for comparison of $x$-ray attenuation coefficients obtained from $X$-ray CT scans with stopping power values from heavy-ion radiography and particle CT reconstructions. We are establishing a reliable scale of conversion by comparing integrated $X-r$ ay $C T$ values with planar projection heavy-ion radiographic data, and by comparing, on a pixel-by-pixel basis, X-ray CT values with corresponding particle $C T$ reconstruction values. This is essential both for target delineation, and for the quantitative assessment of tissue and organ structure inhomogeneities in the beam path. We have achieved promising results both in studies in phantoms and in patients during the past year.

We have also been investigating the potential of heavy-ion radiography to verify that the modifications to charged particle treatment plans achieved by detajled calibration of X-ray CT numbers (obtained from clinical X-ray CT scans) to stopping power values are reliable in patients in situ in the clinical situation using the same positioning and the same particle beams for radiotherapy. Here, in addition, heavy-ion imaging will be of considerable value in detecting day-to-day shifts in stopping power values in patients under treatment due to inaccurate beam alignment, movement of air and gas spaces, and changes in size and location of organs and tissues. Accurate detection of these features can be used to adjust the charged particle radiotherapy procedure on a day-to-day basis.

\section{Studies on Phantoms}

Initial studies on phantoms include heavy-ion radiographs of tissue-equivalent radiotherapy phantoms and X-ray CT phantoms. X-ray CT SCan data are used to compare the calculated waterequivalent anteroposterior pathlength with the heavy-ion radiography data, in order to determine the accuracy of the X-ray CT data for purposes of treatment $p$ lanning.

Figure 3 demonstrates the agreement between $X$-ray $C T$ measurements (squares) and measured heavy-ion pathlengths (continuous line) using a tissue equivalent $x$-ray $C T$ phantom containing a human spinal vertebral column. Agreement between the calculated and experimental points is generally within 2-3 $\mathrm{mm}$ over a 19-cm water equivalent pathlength; this represents an accuracy of about 2. However, in the region of the vertebral column (transverse axis, 12-16 cm) lack of agreement approaches $4 \mathrm{~mm}$ or more. This may be due to partial volume averaging effects ( $X$-ray CT reconstruction of a $1-\mathrm{cm}$ slice thickness); to inaccuracies in the X-ray CT to water equivalent length calibration curve; to small errors in registrations between the $X$-ray CT reconstruction and the heavy-ion data; or to a combination of these effects. The sources of these errors are presently being investigated using tissueequivalent phantoms designed for comparison of $X-r a y$ and heavy-ion CT reconstruction images containing solutions of known density. 


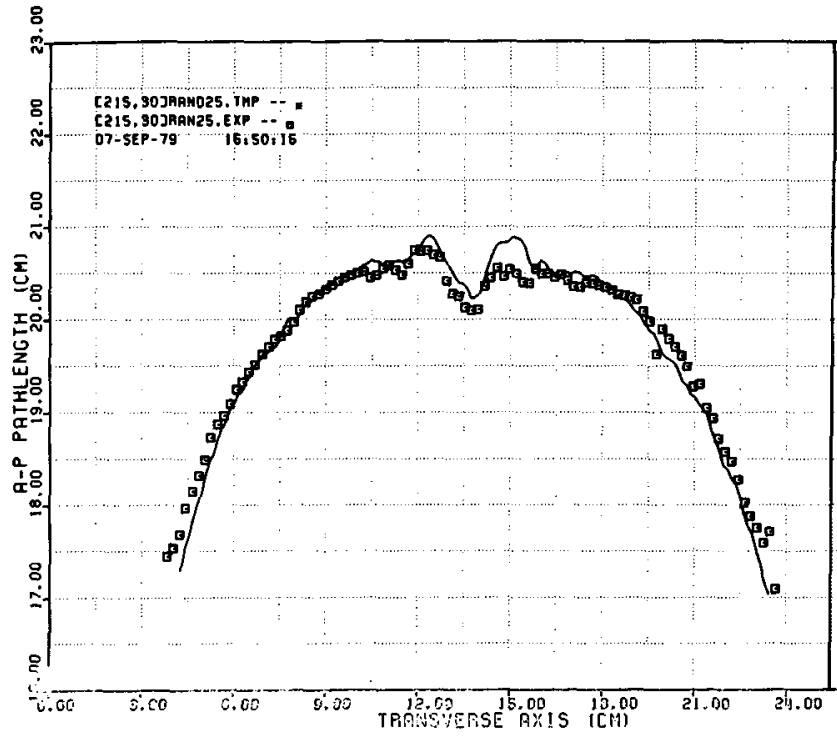

Figure 3. Comparison of $X$ ray CT scan data calculated water-equivalent dose distribution for a two-field plan in the irradiation of a pancreatic carcinoma. The target is delineated by the broad line in the center of the body image. (XBL 809-11823)

\section{Studies on Patients with Carcinoma of the Pancreas}

The largest clinical trial for cancer patients treated with charged particles at LBL is for carcinoma of the pancreas. The treatment procedure, at present, is based on tissue-target two-dimensional contour outline on a diagnostic $X$-ray CT scan of the tumor region. This contour and the $X$-ray CT matrix is used to design a three-dimensional compensator to stop the beam at the distal edge of the contour from each beam direction. Figure 4 illustrates a treatment plan for carbon-ion therapy of pancreatic carcinoma. The plan is designed so that the tumor volume will be treated to a high, homogeneous radiation dose, while the spinal cord, the kidneys, and the gastrointestinal tract will be protected. The accuracy of the treatment plan, and thus the dose distribution on the tissues, is strongly influenced by three important factors: (1) errors due to conversion from $X$-ray CT data to waterequivalent range for heavy-ions (about $3 \mathrm{~mm}$ in water equivalent range); (2) range variations and perturbations from entrance to planned beam stopping point due to tissue inhomogeneities, such as bowel gas (from 5-10 mm) or motion due to respiration (from $5-10 \mathrm{~mm}$ ); (3) compensation misregistration resulting in beam penetration depth errors.

Heavy-ion radiography and CT reconstruction imaging are being applied for the correction of these errors in dose distributions by precise calculation of beam penetration based directly on stopping power points. Eventually, this depth of penetration can be overlayed on a precisely

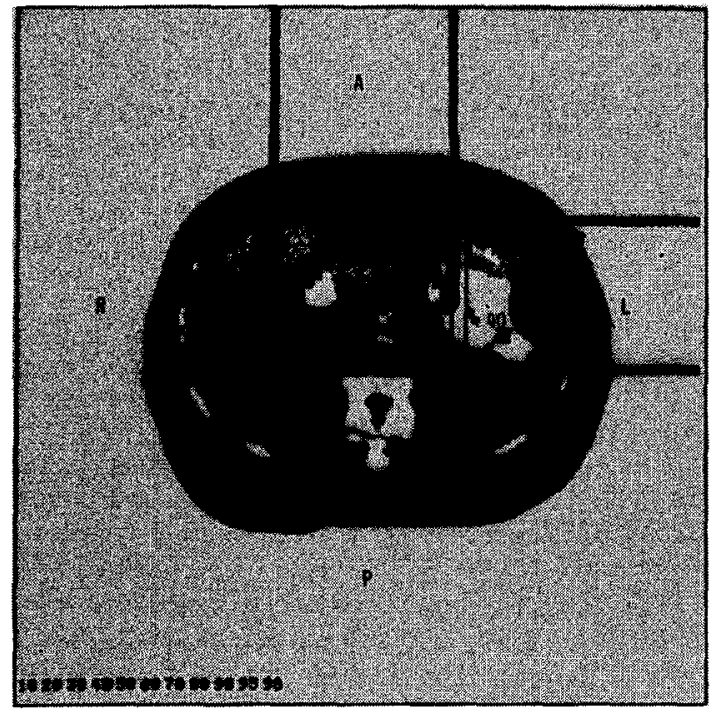

Figure 4. Carbon beam, irregular contour, fixed ridge filters. Isoeffect dose distribution for a two-field plan in the irradiation of a pancreatic carcinoma. The target is delineated by the broad line in the center of the body image. (XBB 794-4289)

registered heavy-ion CT reconstruction of the abdomen, and will include the beam entrance and the stopping points of the beam relative to the organs and tissues of the body, e.g., the tumor, kidneys, spinal cord, and other important structures in the treatment region.

Initial carbon-ion radiographs of three patients with carcinoma of the pancreas undergoing charged particle radiotherapy at $L B L$ have been obtained at the Bevalac. For two patients ( $1 P$ and $2 P$ ), a water bolus technique with entrance and exit flattening surfaces was developed. For the third patient (3P), a wax bolus technique used for treatment was applied, with no exit flattening surface. The heavy-ion data for patient $1 P$ were analyzed and compared with $X$-ray CT data taken two months previously. Difficulties in analysis arose due to:

(1) differences in patient positioning--the $x$-ray CT image procedure was done with the patient in the recumbent position, and the heavy-ion radiograph in the upright position, i.e., the same position for charged particle treatment;

(2) slice thickness of the $X$-ray CT scan was $1.3-\mathrm{cm}$, and part of the body was outside the reconstructed circle. Accordingly, much data correction was necessary for the X-ray CT data for comparison with the heavy-ion radiograph for deriving integrated anteroposterior water equivalent pathlengths.

Figure 5 compares the $1.3 \mathrm{~cm}$ thick $x$-ray $\mathrm{CT}$ scan with a corresponding heavy-ion "slice." There is reasonably good agreement, within $3 \mathrm{~mm}$, between the calculated $X$-ray CT values and the measured heavy-ion integrated pathlengths; one slice showed disagreement of $5 \mathrm{~mm}$. However, the $1.3 \mathrm{~cm} x$-ray CT slice thickness obscured detailed 


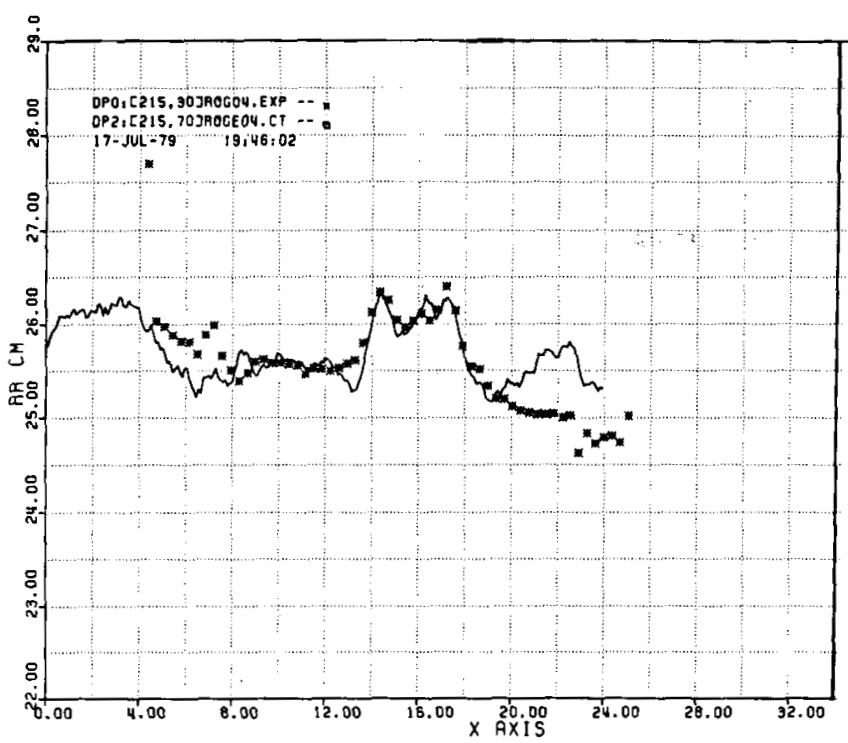

Figure 5. Comparison of the $X$-ray CT scan (1.3-cm thickness) with carbon-ion image "slices." (XBL 809-71824)

range perturbation effects by the vertebral column through partial volume averaging. Heavyion radiography showed much more detail of the beam pul1-back by the complex structures of the vertebral column. Figure 6 contains the heavyion radiography data within the $1.3-\mathrm{cm}$ slice sample by the $X-r a y C T$ reconstruction scan. The water equivalent relative range is plotted against the transverse axis. The curves are arbitrarily displaced by $0.5 \mathrm{~cm}$ for clarity. The greatest perturbation error occurs in the region of the vertebral column (approximately $1.0 \mathrm{~cm}$ to $1.6 \mathrm{~cm})$. Each equivalent slice of heavy-ion data is $1-\mathrm{mm}$ thick; the $1.3-\mathrm{cm}$ X-ray CT slice averages these data with resultant loss of detailed range perturbations. Differences between the uppermost and lowermost curves are as great as $7 \mathrm{~mm}$, which could be important in charged particle treatment planning, particularly in regard to the sharp dose gradient at the distal end of the charged particle beam.

\section{Studies on Patients with Brain Tumors}

Five patients with primary brain tumors and undergoing charged particle radiotherapy thave been radiographed using carbon-ions at the Bevalac. Figure 7 demonstrates the carbon-ion radiograph of the head of a brain tumor patient; the original image is 256 gray leve 1 , but the hard copy illustration consists of 16 gray levels. No wax or water bolus was used for: surface flattening; a metal washer (arrow) is placed over the frontal bone and can be seen in the left lateral projection. The carbon-ion radiograph was made with a 16-mm port with a esidual range of $19 \mathrm{~cm}$. Dense bone complexes (e.g., base of skull), air cavities (e.g., paranasal sinuses and mastoids), soft tissues, cartilage (e.g., pinna of ear) are seen. The

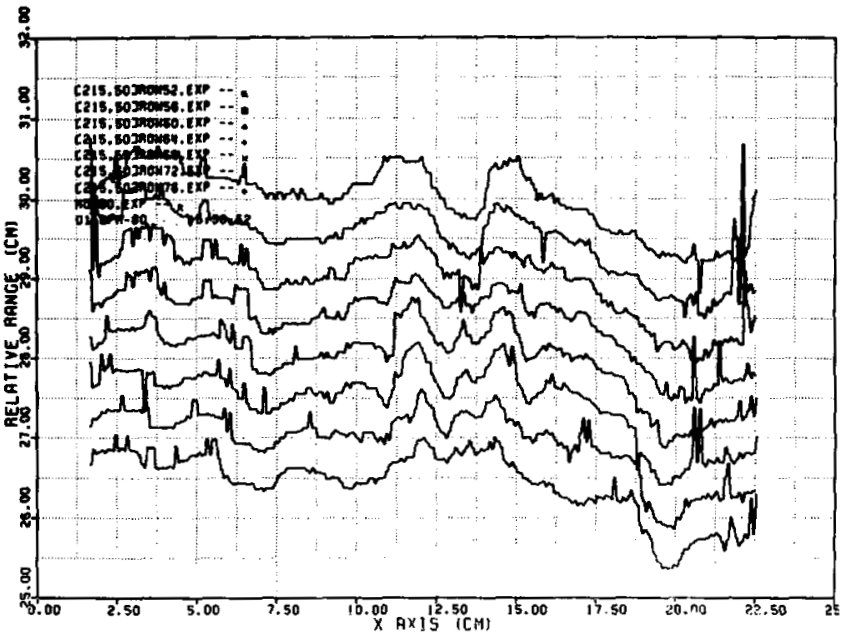

Figure 6. Heavy-ion radiographic within the 1.3-cm scan thickness sample by the $X-r a y C T$ reconstruction. The water equivalent relative range is plotted against the transverse axis. The curves are arbitrarily displaced by $0.5 \mathrm{~cm}$ for clarity. (XBL 809-11825)

video image displays extremely improved detail; each pixel is about $1 \mathrm{~mm}$.

Figure 8 plots the water equivalent pathlength of a 5-mm thick slice through the base of the skull crossing the dense bone; range perturbations of about $4 \mathrm{~cm}$ are seen relative to transit paths. These data are in only fair agreement with X-ray CT calculations; differences average less than $3 \mathrm{~mm}$, but are as great as 6-7 mm. Figure 9 segments and superimposes the data of the 5-mm thick slice into five 1-mm thick

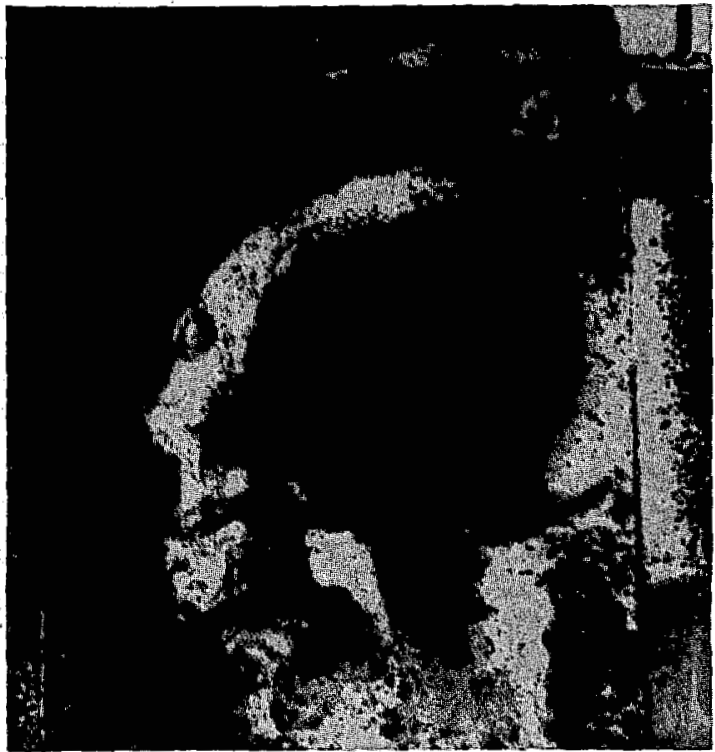

Figure 7. Carbon-ion radiograph of the head of a brain tumor patient undergoing charged particle radiotherapy. (XBB 809-10572) 


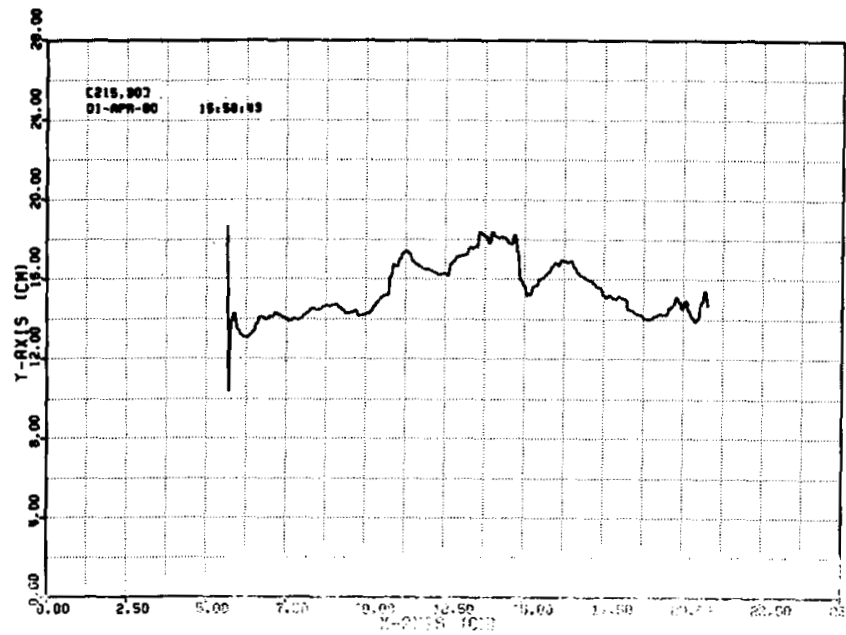

Figure 8. Plot of the water equivalent pathTength of a 5-mm thick slice through the base of the skull crossing dense bone; data derived from carbon-ion radiograph in $\mathrm{F}$ igure 7 . (XBL 809-11821)

slices. Here, differences as much as $2 \mathrm{~cm}$ are seen at the same $x$-axis value and the largest variation is at $x=11 \mathrm{~cm}$. In charged particle radiotherapy of brain tumor patients, it is unlikely that $X$-ray CT scans of less than 2-3 $\mathrm{mm}$ thick will be practical for treatment planning. Here, heavy-ion radiography, and particularly heavy-ion CT tomography reconstruction of the head, will be required where extremely sharp range gradients exist.

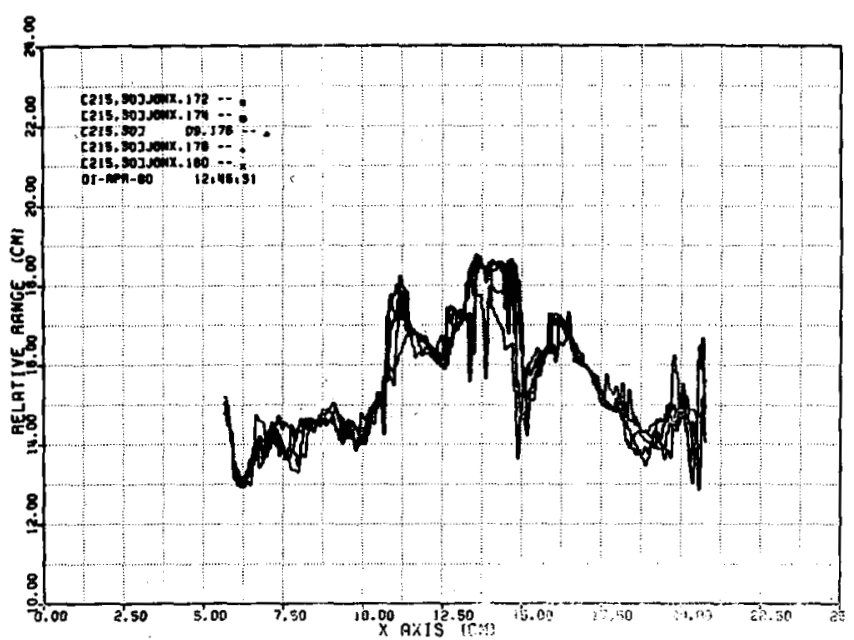

Figure 9. Plot of the 5-mm thick slice illustrated in Figure 8; data are segmented and superimposed. Differences as much as $2 \mathrm{~cm}$ occur at the same $x$-axis value, and the largest variation is at $x=11 \mathrm{~cm}$. (XBL 809-11822)

\section{DISCUSSION}

The technique of heavy-ion radiography is being developed in our laboratory to use carbon and other heavy-ion beams for quantitative radiography of the tissues of the body. The sensitivity of such beams to tissue electron density variations is much greater than that of $X$ rays, and this method is being applied to heavy-ion CT scanning. Potentially it promises to be helpful in delineating the boundaries, structure, and stopping power values of neoplasms under treatment with charged particle beams. The specific objectives of these current investigations are to examine how heavy-ion radiography applied to charged particle radiotherapy aids in treatment planning of patients undergoing accelerated heavy particle radiotherapy; and in detecting, localizing, and sizing the soft tissue tumors in these cancer patients for pretherapy diagnosis and subsequently, for the assessment of response to heavy-ion treatment.

These investigations may help provide the answers to two important questions essential for successful charged particle radiotherapy:

(1) what is the relationship between electron densities measured with $X$-ray $C T$ reconstruction and with heavy ions; and (2) what are typical tissue densities of various human cancers, what is the critical tumor dimension that is detectable, and how do these change under treatment? We have begun experiments to answer these questions. Our initial studies, both in phantoms and with patients are promising, and demonstrate the need for more research on soft tissue tumor localization and delineation with charged particle beam porting with heavy-ion CT reconstruction.

Epp (1977) has urged for continued research in charged particle radiotherapy directed toward tumar localization and delineation with charged particle beam porting using charged particle imaging and CT reconstruction tomography, in order to take full advantage of concurrent pnysics developments in beam delivery, instrumentation, and dose distribution. Goitein (1977) has pointed out that at the present time, information for charged particle treatment planning must require translation of measured $x$-ray CT absorption coefficients to stopping power values. In the LBL charged-particle radiotherapy clinical trials, the data derived from diarrustic $X-$ ray CT reconstructions are used to predict charged particle ranges and tissue densities for measurement of any necessary compensation for tissue inhomogeneities. Furthermore, the spatial resolution of present day X-ray CT scanners in about 1 to $3 \mathrm{~mm}$ may be insufficient for planning for charged particle therapy, where $1 \mathrm{~mm}$ or less may be required. Certain problems arise in relating $X$-ray $C T$ numbers to stopping power values. The $X$-ray $C T$ values saturate in high density regions such as dense bone, and such effects diminish density resolution and introduce ambiguities in relating $X-r a y C T$ numbers to $X$-ray ättenuation coefficients. In addition, beam hardening effects occurring when soft $x$ rays are absorbed in the outer layers of tissue also introduce ambiguities in the correlation of $x$-ray 
CT numbers to the derivation of $X$-ray absorption coefficients and subsequently to charged particles stopping power values. Both the saturation and beam-hardening effects of X-rays are not encountered, or are of little significance, in heavy-ion radiography.

CT reconstruction artifacts affecting treatment planning can arise from sharp interfaces between tissues of widely differing density; these artifacts are inherent in the reconstruction techniques. Both X-ray CT and heavy-ion CT reconstructions contain these artifacts, but they are less for stopping power values than for X-ray attenuation coefficients. Cormack and Koehler (1976) demonstrated charged particle artifacts associated with sharp interfaces due to multiple Coloumb scattering effects and appearing similar to edge enhancements. These are potentially important for proton radiography, but appear less important for heavy-ions, such as carbon and neon. And finally, additional artifacts can result from effects caused by variation in stopping power with particle energy and tissue composition; these effects are probably small, but need to be investigated.

\section{Focal Lesions in the Central Nervous System}

There are additional malignant and nonmalignant diseases in patients who can benefit substantially from the heavy-ion radiography research program, for lesion localization and sizing, for treatment planning with charged particle beams, and for evaluation of response for charged particle therapy. These include patients with pituitary disorders, selected advanced cancer patients, particularly those with carcinoma of the breast or carcinoma of the prostate, and patients with neoplastic and nonneoplastic lesions in the brain, such as metastatic brain tumors, anterovenous malformations, carotid artery-cavernous sinus fistulas, aneurisms, and the radiosurgical treatment of certain enurological diseases, including Parkinson's disease and control of pain.

\section{SUMMARY AND CONCLUSIONS}

The goals of treatment planning for charged particle radiotherapy include precise beam localization and dose distribution within the neoplasm and protection of the surrounding sensitive normal tissues and organs, and correction for perturbations due to tissue inhomogeneities affecting stopping power of the charged particle beams. A computerized treatment planning system presently based on quantitative information in CT scans has been developed for the heavy-ion radiotherapy patient trials at LBL. The CT number of each pixel is converted to water equivalent pathlength and used in the calculation of isoeffect dose distributions from multiport heavy charged-particle irradiation. The developments in heavy-ion radiotherapy and CT scanning re now being applied to charged particle radiotherapy treatment $p l a n n i n g$, and presently the data from electronic tissue density values may be used for quantitatively studying the range shortening effects by innomogeneities. Heavy-ion radiography promises further important developments in the radiation physics of charged particle radiotherapy. Ultimately, it will be possible to deliver greater radiation doses with increased accuracy and distribution of dose to lesions in patients and with greater protection of adjacent normal or sensitive tissue or organ structures than has been possible in the past.

\section{ACKNOWLEDGEMENTS}

These studies were supported in part by grants from the National Cancer Institute (CA 19138 and CA 27021).

\section{FOOTNOTES AND REFERENCES}

${ }^{*}$ Clair Zellerbach Saroni Tumor Institute, Mt. Zion Hospital, San Francisco; and Department of Radiation Oncology, University of California, San Francisco, CA.

tDepartment of Radiology, University of California, San Francisco, CA.

Castro, J. R., C. A. Tobias, J. M Quivey, G.T.Y. Chen, J. T. Lyman, T. L. Phillips, E. L. Alpen, and R.P. Singh. 1979. Results of tumor treatments with alpha particles and heavy ions at Lawrence Berkeley Laboratory. High LET Radiations in Clinical Radiotherapy. Europ. J. Cancer Supp 1., pp. 67-74.

Castro, J. R., J. M. Quivey, J. T. Lyman, G.T.Y. Chen, T. L. Phillips, and C. A. Tobias. 1980. Radiotherapy with heavy charged particles at Lawrence Berkeley Laboratory. J. Assoc. Canad. Radiol. 31, 30-34.

Chen, G.T.Y., R. P. Singh, J. R. Castro, J. T. Lyman, and J. M. Quivey. 1979. Treatment planning for heavy charged particle radiotherapy. Int. J. Rad. Onc. Biol. Phys. 5 , 1809-1819.

Chen, G.T.Y. and S. Pitluck. 1980. Utilization of graphics in heavy ion radiotherapy treatment planning. Workshop on Picture Data Description and Management. IEEE Computer Society, Proceedings No. 80CH1530-S, pp. 172-175. Asilomar, California.

Cormack, A. M. and A. M. Koehler. 1976. Quantitative proton tomography: Preliminary experiments. Phys. Med. Bio1. 21, 560-569.

Epp, E. R. 1977. Physics. Int. J. Radiat. Oncol. Biol. Phys. 3, 419-4 $\overline{21 .}$

Fabrikant, J. I., C. A. Tobias, M. P. Capp, E. V. Benton, and W. R. Holley. 1980. Heavyion imaging applied to medicine. Soc. PhotoOpt. Instrum. Eng. 233, 255-263.

Goitein, M. 1977. The measurement of tissue heterodensity to guide charged particle radiotherapy. Int. J. Radiat. Oncol. Biol. Phys. 3, 27-33. 
Holley, W. R., R. P. Henke, G. E. Gauger, B. Jones, E. V. Benton, J. I. Fabrikant, and C. A. Tobias. 1979. Heavy particle computer tomography. Sixth Computer Radiology, IEEE Trans., pp. 64-70.

Stone, R. S., J. H. Lawrence, and P. C. Aebersold. 1940. Preliminary report on use of fast neutrons in treatment of malignant disease. Radiology 35, 322-327.

Tobias, C. A. 1979. Pituitary radiation: Radiation Physics and Biology. Recent Advances in the Diagnosis and Treatment of Pituitary
Tumors (J. A. Linfoot, ed.) pp. 221-243. Raven Press, New York.

Tobias, C. A., E. V. Benton, and M. P. Capp. 1978. Heavy-ion radiography. Recent Advances in Nuclear Medicine, (J. H. Lawrence and T. F. Budinger, eds.), vol. 5, pp. 71-102. Grune and Stratton, New York.

Tobias, C. A., E. V. Benton, M. P. Capp, A. Chatterjee, M. R. Cruty, and R. P. Henke. 1977. Particle radiography and autoactivation. Int. J. Radiat. Onco ?. Bio. Phys. 3, 35-44. 


\title{
ACTIVE HEAVY-ION RADIOGRAPHY AND COMPUTERIZED TOMOGRAPHY
}

\author{
J. Llacer, W. Chu, C. A. Tobias, J. I. Fabrikant, ${ }^{\star}$ and J. R. Alonso
}

\section{THE USE OF SOLIO-STATE DETECTORS}

\author{
(J. Llacer, J. I. Fabrikant, and C. A. Tobias)
}

Active heavy-ion radiography and computerized tomography (CT) have the potential to generate and process data to obtain CT reconstruction images of a clinical patient. The use of solidstate detectors, fast processing electronics, and an on-line computer with a fast array processor will allow immediate availability of the reconstructed CT image to the physician, both for diagnostic radiology and for therapeutic radiology. The principal advantage of using the same charged particles for radiotherapy treatment planning that are used for the subsequent therapeutic irradiation of the cancer patient is to reduce the systematic and random errors in the calculation of density and composition inherent in X-ray CT. An active heavy-ion CT scanner that could generate a tomographic image within a short time after completing the examination would be valuable to the radiotherapist if the procedure were carried out with a patient already positioned for charged particle therapy. Treatment planning parameters could be verified and corrections for organ position changes would then be done effectively just prior to treatment.

The availability of a silicon ion-implanted resistive sheet, position-sensitive, fully depleted detectors, and a stable surface coating for germanium detectors without any apparent surface dead layers have allowed us to test the configuration of a prototype instrument for the development of an active on-line heavy-ion radiographic and CT scanner. This report reviews the results of the initial investigations and those in progress using selected solid-state detector configuration and design, and discusses the relevant parameters that make the solid-state detectors useful for a high-accuracy low-dose computerized tomographic reconstruction applied to heavy-ion imaging (Llacer et al., 1981).

\section{Materials, and Methods}

Recent developments in solid-state radiation detectors have provided accurate information on the identity, position, and energy of individual heavy ions before and after they transverse an absorber (see Llacer et al., Part II). We have developed in our laboratory thin silicon $\mathrm{dE} / \mathrm{dx}$ detector strips positioned tranverse to the beam path that can detect the LET of a heavy charged particle and separate primary beam charged articles from fragmented nuclei. Furthermore, the silicon detector strips indicate beam position with an accuracy better than $0.15 \mathrm{~mm}$, before and after they traverse a subject.
In addition, the recent development of a stable surface treatment for germanium detectors, with no dead layers, allows the use of long, flat solid-state detectors for full absorption of heavy ions without having to construct expensive detector telescopes. With the new geometric design, the energy of heavy ions can be determined within $0.2 \%$ accuracy, even when as few as 100 particles enter the detector.

Based on these developments, an active heavy-ion computerized tomography detector system is being developed at LBL (Fig. 1). A flat beam of heavy ions with sufficient energy to traverse the object to be imaged is directed to a long strip of thin $(200 \mu \mathrm{m})$ silicon detectors. These detectors deliver signals identifying the time of passage of each primary particle and its position. The heavy ions travel through the object to be imaged and enter the second strip of thin silicon detectors. These detectors determine the nature and position of the particle in time coincidence with the signal from the first silicon detector. Immediately after the second silicon detector strip, a slab of germanium full-absorption detectors measures the residual energy of the charged particle, which is also in time coincidence. Electronic processing of the signals permits testing whether each initial primary particle has or has not undergone nuclear collisions, and determining whether it has been scattered by an excessive angle. When these tests are passed, the residual energy and position of the charged particle are accepted for further processing.

The process of image scanning with the Bevalac is carried out by rotating the objects to be imaged (ultimately, the patient) through about 50-60 angle positions, each about $3^{\circ}$, covering a complete sector of $180^{\circ}$, with the rotation motion occurring as increments between beam pulses. The accelerator pulses occur at a rate of one pulse every 4 seconds; during each

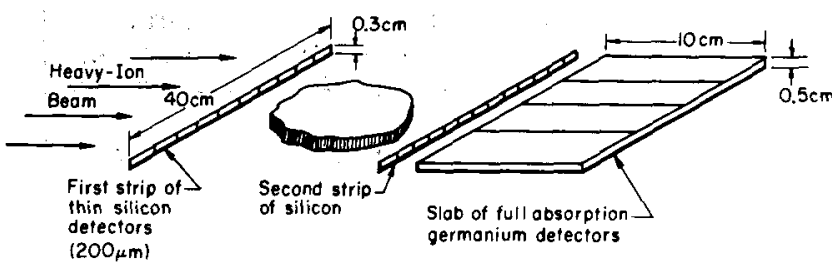

Figure 1. Configuration of solid-state silicon $\mathrm{dE} / \mathrm{dx}$ and germanium detectors for active heavyion CT imaging. (XBL 804-3220) 
pulse, the object or the patient remains stationary and enough data are collected to obtain one complete projection for reconstruction.

Image processing and CT reconstruction from the electronic data proceed in three steps. First, the electronically validated signals of residual energy and position are histogrammed between beam pulses. Second, the histogram of energy vs. position is scanned by an optimal filtering procedure which transforms the large two-dimensional histogram into a simple list of values of absorption (water equivalent) vs. position (Llacer, 1981). This procedure is also carried out between beam pulses. Third, the contribution to the final image of the pulse just completed is calculated before the next beam pulse arrives.

The CT reconstruction can be completed within a few seconds after the end of the $180^{\circ}$ rotation. The results and the final reconstructed $C T$ image can be presented immediately to the diagnostic radiologist, radiotherapist, or clinical physicist. The large dynamic range of the germanium detectors permits computerized tomographic reconstructions without water bags, tissue equivalent materials, or other absorption materials surrounding the patient.

\section{Results}

Initial experiments were designed (1) to determine the response of germanium detectors to energetic heavy ions, including the sensitivity to small changes in water column thickness; and (2) to determine the threshold for radiation damage. A series of preliminary measurements was made with a high-purity $1-\mathrm{cm}$ thick germanium planar detector. $12 \mathrm{C}(400 \mathrm{MeV} / \mathrm{n})$ and $20 \mathrm{Ne}$ ions $(425 \mathrm{MeV} / \mathrm{n})$ were used for these experiments. A water column absorber of variable thickness was placed upstream in front of the detector so that the Bragg peak fell in the detector thickness.

Response of a Planar Germanium Detector to Heavy Ions

The response of the detector to heavy ions was examined both in a pulse-by-pulse mode and in an integral mode. In the pulse-by-pulse mode, pulse height analysis was made of the recorded events. Sensitivity to small changes in water column thickness was measured by the change in the position of the centroid of the Gaussian main peak in the spectrum. In the integral mode, the total current in the detector from the stopping particles was measured. This total current, divided by the number of particles, was used as a measure of the water column thickness.

The pulse-by-pulse method proved superior to the integral method. Here, only those ions that underwent shallow angle ionization collisions, and therefore carried the desired information on density and composition of the absorber along the straight ion path length, deposited their energy in the main Gaussian spectrum peak. The remaining ions underwent nuclear collisions and arrived at the detector in a modified form. These latter ions did not deposit their energy in the Gaussian peak, so the pulse-by-pulse mode discarded these ions in calculating the characteristics of the absorber. In the integral mode, on the other hand, all the particles contributed to the electronic signal, and the statistical fluctuations from all these other processes contributed to a substantial uncertainty to the results. Consequently, the pulse-by-pulse mode of operation was considered the better method for further investigation.

A beam of $12 \mathrm{C}$ ions $(400 \mathrm{MeV} / \mathrm{n})$ was then passed through approximately 21 to $25 \mathrm{~cm}$ of water so that the beam stopped within the $1-\mathrm{cm}$ germanium detector. The beam spectra showed a Gaussian peak with a full-width at half maximum (FWHM) corresponding to approximately $0.25 \mathrm{~cm}$ of water. There were also other lower peaks corresponding to other nuclei.

Five heavy-ion exposures, with 3,600 to 4,500 counts each, were made with $12 \mathrm{C}$ ions ( $400 \mathrm{MeV} / \mathrm{n}$ ) and a water column thickness of $23 \mathrm{~cm}$. Five more irradiations with a similar number of counts were done with a water thickness of $23.1 \mathrm{~cm}$. The centroids of the Gaussian peaks were obtained by least-squares fit to the logarithm of the peak shapes. The means of the two sets of centroids were separated by $4 \mathrm{MeV} / \mathrm{n}$ of collected energy, with a standard deviation of $0.4 \mathrm{MeV} / \mathrm{n}$. This indicates that the expected error in the measurement with approximately 4,000 particles into the detector corresponds to $0.01 \mathrm{~cm}$ of water, or approximately $0.04 \%$.

\section{Threshold for Radiation Damage}

Experiments carried out with $425 \mathrm{MeV} / \mathrm{n} 2{ }^{20} \mathrm{Ne}$ ions were done principally to study the extent to which heavy ions caused radiation damage to the germanium. The main peak widths in the spectra were $0.1 \mathrm{~cm}$ water equivalent $\mathrm{FWHW}$. Sensitivity to small water column thickness change was similar to the one observed for $12 \mathrm{C}$ ions $(400 \mathrm{MeV} / \mathrm{n})$. The characteristics of the energy spectra collected by the germanium detector remained repeatable and stable until a total fluence of $2 \times 10^{8}$ particles $/ \mathrm{cm}^{2}$ was reached. At that point, the germanium detector failed completely. Examination of the shape of the energy spectrum as a function of the water column thickness after the damage occurred showed that holes and electrons failed to traverse a thin layer in the detector where the Bragg peak was located during the heavy-ion irradiation. The detector recovered its initial characteristics after annealing for a few hours at $150^{\circ} \mathrm{C}$.

Experiments with Silicon $\mathrm{dE} / \mathrm{dx}$

Position Sensitive Detectors and a Side Entrance Detector

Based on the results of these sensitivity response and radiation damage experiments, a thin silicon $d E / d x$ position sensitive detector and a 3-cm long, flat germanium detector were 
fabricated. Standard nuclear spectroscopy electronics and a connection to a PDP $11 / 34$ computer were used for a series of experiments

that are described briefly below. A standard nuclear physics data acquisition and analysis program was used.

The aims of the experiments with the silicon detector were: (1) to determine how well the different heavy-ion nuclei could be separated, (2) to assess the levels electronic noise could be achieved inexpensively, and (3) to determine the reliability of the detector design. The fabricated silicon detector has a thickness of $200 \mu \mathrm{m}$, a height of $5 \mathrm{~mm}$, and a width (horizontal, perpendicular to the beam) of $30 \mathrm{~mm}$. It operates fully depleted with an alpha-particle resolution of $70 \mathrm{keV}$ FWHM. The electronic noise resolution is such that positional accuracy can be determined to be approximately $0.6 \%$ FWHM of the detector length for particles depositing approximate $1 \mathrm{y} 5 \mathrm{MeV}$ in the detector, as is the case with ${ }^{12} \mathrm{C}$ ions $(400 \mathrm{MeV} / \mathrm{n})$. Figure 2 illustrates experiments with $20 \mathrm{Ne}$ beams of high energy $(560 \mathrm{MeV} / \mathrm{n}$ ) and a $15-\mathrm{cm}$ water column; the results demonstrate a clean Landau peak for the parent neon nuclei and for the fragments down to helium.

A flat high-purity germanium detector was then fabricated with dimensions of $3.2 \mathrm{~cm}$ in length (in the ion beam direction), $0.5 \mathrm{~cm}$ in height, and $2.2 \mathrm{~cm}$ in transverse width. The aims of the experiments with this new germanium detector were: (1) to understand the effect of detector surface characteristics on heavy-ion spectra when the particles enter the detector from the exposed surface of a flat, long detector, and (2) to verify the initial findings with a 1-cm thick planar germanium detector.
Initial studies demonstrated that the uneven thickness of surface layers in normally treated germanium detectors resulted in broadened peaks in the obtained spectra. The effects of a guard ring were particularly damaging, as they produced double peaking. A new surface treatment has recently been developed at LBL by Hansen et al. (1980). The method leaves the surfaces neutral and stable under a variety of conditions; the new coating (a few angstroms thick) was applied to the detector early in December 1979. Present studies now demonstrate spectra as reliable as those obtained previously with the planar detector (which measures ions as they enter through the thin $\mathrm{p}^{+}$implanted contact).

A series of measurements was carried out with a beam of $20_{\mathrm{Ne}}$ ions $(669 \mathrm{MeV} / \mathrm{n})$; the water-column thickness ranged from 19.6 to $135 \mathrm{~cm}$, and corresponded to the total dynamic range of the germanium detector ( $11.9 \mathrm{~cm}$ water equivalent). Figure 3 illustrates the pulse height distribution for the measurements with a water column thickness of $19.6 \mathrm{~cm}$. The main Gaussian peak, and those generated by other nuclei, can be identified clearly. A plot peak position vs. water column thickness follows the theoretical curve for differential range calculated for germanium particularly well. We have therefore concluded that there are no detrimental effects due to the ions entering the exposed coated surfaces, and that the energy loss of primary heavy ions in germanium detectors follows the theoretical predictions well.

Six more consecutive experiments were done with the $20 \mathrm{Ne}$ ion $(669 \mathrm{MeV} / \mathrm{n})$ beams a $19.8-\mathrm{cm}$ water column, each with exactly 3,000 particles entering the germanium detector. The mean of the

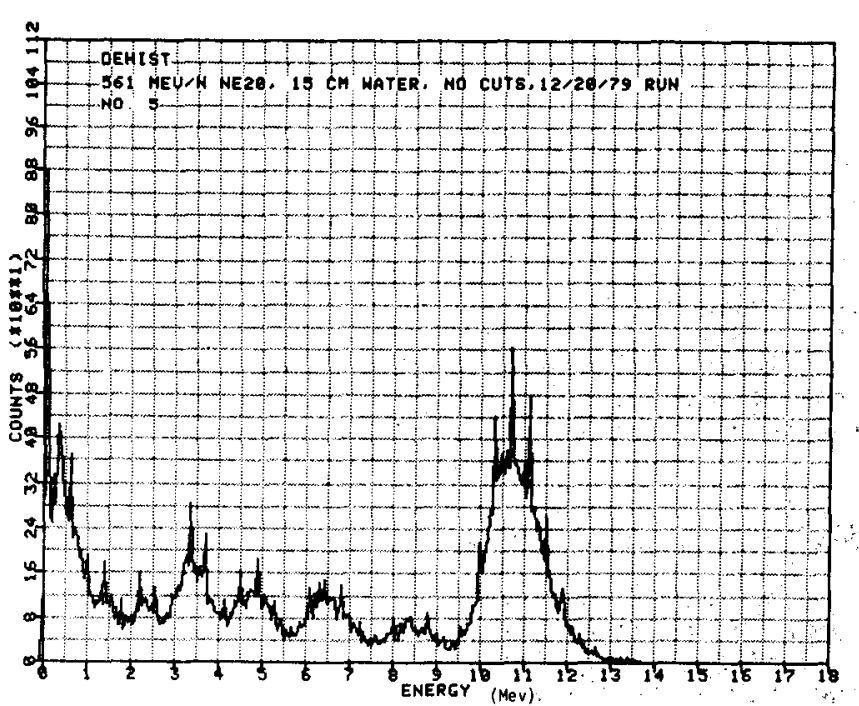

Figure 2. $d E / d x$ spectrum obtained by a $200 \mu \mathrm{m}$ ilicon $\mathrm{dE} / \mathrm{dx}$ detector from a $561 \mathrm{MeV} / \mathrm{n} 20_{\mathrm{Ne}}$ eam after passing through $15 \mathrm{~cm}$ of water. The main Landau-shaped $20 \mathrm{Ne}$ peak and peaks from several nuclei are visible. (XBL 806-10544)

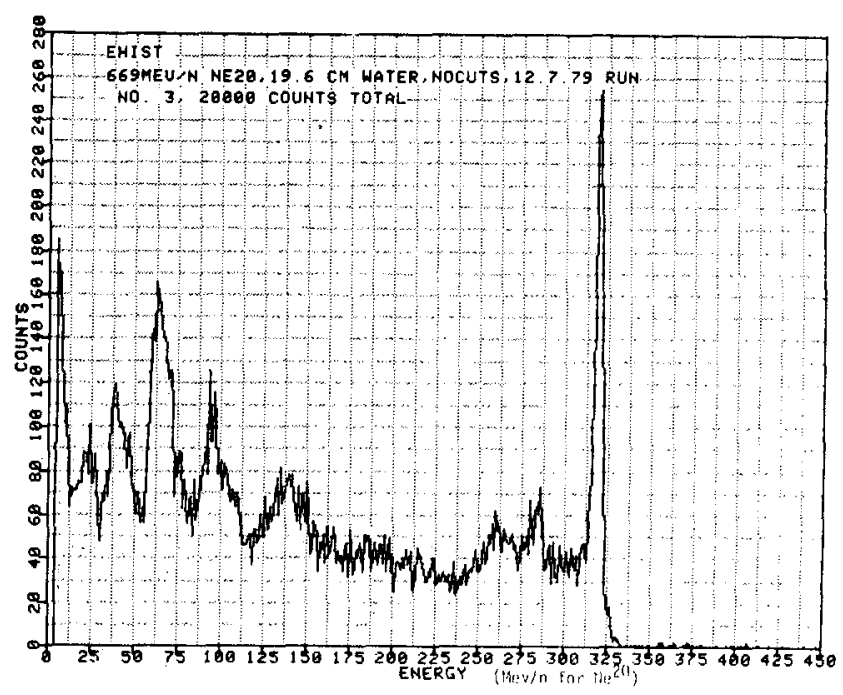

Figure 3. Residual energy spectrum obtained with a $3.2 \mathrm{~cm}$ gerinanium detector from a $669 \mathrm{MeV} / \mathrm{n}$ $20 \mathrm{Ne}$ beam after passing through $19.6 \mathrm{~cm}$ of water. The narrow peak corresponds to the parent neon beam. Peaks corresponding to other nuclei are also visible. (XBL 806-10541) 
Gaussian peak occurred at $315.46 \mathrm{MeV} / \mathrm{n}$, with a standard deviation of $0.1924 \mathrm{MeV} / \mathrm{n}$, which corresponded to $0.011 \mathrm{~cm}$ of water. The expected error was $0.056 \%$, which is in the same range as the results obtained previously with the planar detector.

The capacity of the germanium solid-state detectors to separate and identify a number of different nuclei in an ion beam, as shown in Figure 2 and Figure 3 , is being examined for beam characterization measurements (see Part II).

\section{Beam Scattering Effects}

The addition of a second silicon $d E / d x$ detector to the detector assembly ( $F$ ig. 1 ) has made it possible to analyze quantitatively the geometric scattering of primary particles in traversing a certain thickness of materials, and to determine the percent of surviving primaries from the parent beam to the full absorption detector. Figure 4 illustrates the experimental detector system configuration designed to determine the beam transport characteristics through a $20-\mathrm{cm}$ bar of lucite plastic $(23.80 \mathrm{~cm}$ water equivalent). In the first experiment, the lucite bar was not inserted in the beam in order to determine the characteristics of the beam delivered by the Bevalac. Figure 5 . is a histogram of the offset in traverse position of all the primary charged particles that traversed the two position-sensitive silicon $d E / d x$ detectors. The results demonstrate a near Gaussian distribution with a standard deviation of $0.74 \mathrm{~mm}$. Because the detector noise contrioutes only $0.18 \mathrm{~mm}$ position offset uncertainty, the Gaussian width defines the initial quality of the beam. A particle traversing the first detector at a particular position can be expected to arrive at the second detector, $27 \mathrm{~cm}$ downstream, with a Gaussian distribution offset with $\sigma=0.74 \mathrm{~mm}$. The reasons for this initial beam characteristic may be due to scattering in the several windows in the beam path, in the $d E / d x$ detectors, or characteristics of the beam focusing mechanism of the Bevalac.

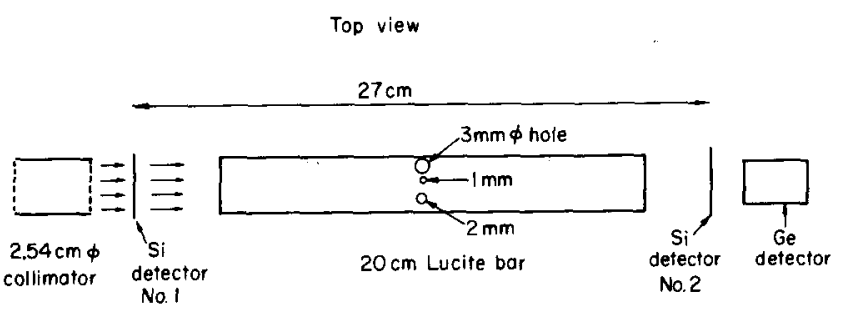

Figure 4. Experimental configuration of solidstate detectors and lucite bar object to study the effects of beam scattering on imaging and for a single-projection imaging experiment using $20 \mathrm{Ne}(560 \mathrm{MeV} / \mathrm{n})$. (XBL 804-3221)

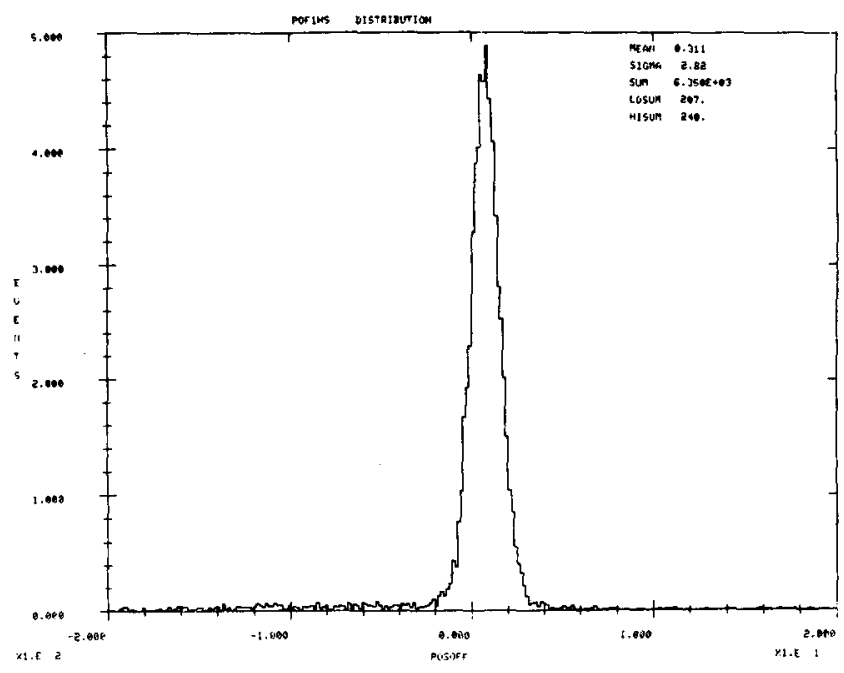

Figure 5. Position off set histogram in a $27-\mathrm{cm}$ air path for single particles traversing the two silicon $\mathrm{dE} / \mathrm{dx}$ detectors of Figure 4 . An ion arriving at the first detector at some position $x$ (transverse to the beam) will traverse the second detector at some position $x-x^{\prime}$, where $x^{\prime}$ is a Gaussian distributed variable with a standard deviation of $0.74 \mathrm{~mm}\left(20_{\mathrm{Ne}}, 560 \mathrm{MeV} / \mathrm{n}\right)$. (XBL 806-10542)

In a subsequent experiment, the 20-cm lucite bar was placed in its holder and positioned in the beam path, and the above measurements were repeated. Only particles that remained $12 \mathrm{C}$ ions upon arrival at the germanium detector provided the data for analysis of distribution characteristics. Figure 6 illustrates the distribution of the resulting position offset Gaussian. Here, the standard deviation is $\sigma=1.37 \mathrm{~mm}$. Because of multiple scattering within the lucite bar, the increase in position offset is $\sigma=1.15 \mathrm{~mm}$, which is probably magnified by the air gap between the bar and second silicon $\mathrm{dE} / \mathrm{dx}$ detector.

The effect of the sample scattering on final image quality should be some loss of contrast (or blurring) at high spatial frequencies when the object to be reconstructed is comparable in size to $24 \mathrm{~cm}$ of water in absorption. Experiments are presently in progress to quantitate the actual effects.

\section{Requirements for Imaging}

In order to determine the approximate minimum number of charged particles required to generate a clinically useful tomogram, data from $20 \mathrm{Ne}$ $(560 \mathrm{MeV} / \mathrm{n})$ experiments were analyzed. The 2-mm sections of the $d E / d x$ detector were defined as data bins, and the positions of the Gaussian peaks measured by the germanium detector as a function of number of particles per bin were obtained. Using a 15-cm water absorber, the standard deviation in six consecutive bins for 


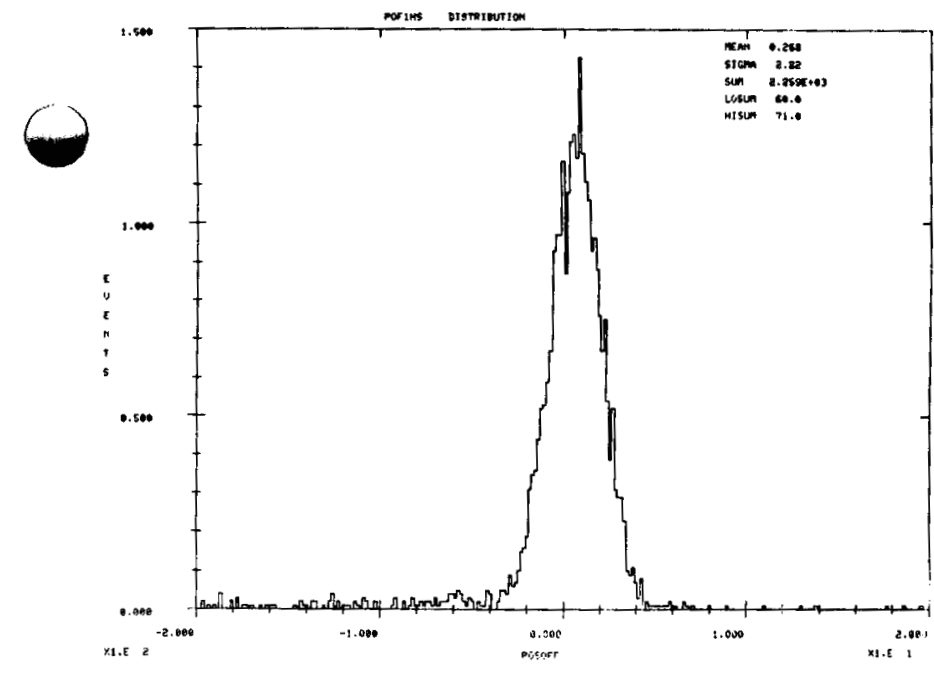

Figure 6. Histogram that is similar to Figure 5 but with ions traversing the $20-\mathrm{cm}$ lucite object. The standard deviation is now $1.37 \mathrm{~mm}$ ( $20 \mathrm{Ne}$, $560 \mathrm{MeV} / \mathrm{n})$. (XBL 806-10543)

200 particles per bin was $0.55 \mathrm{MeV} / \mathrm{n}$, which corresponds to $0.03 \mathrm{~cm}$ water. The expected error is $0.2 \%$. For an extrapolation from a single projection to a complete reconstruction for $3 \times 3 \mathrm{~mm}$ pixels and 50 angles, the error magnification factor is approximately 2 (Huesmann, 1977). Thus, less than a $0.5 \%$ reconstruction error can be obtained with as few as 200 particles in a ion section of useful dimensions (2-4 mm width).

An imaging experiment was then carried out with the same detector and phantom arrangement described in Figure 4. Three holes, $1 \mathrm{~mm}, 2 \mathrm{~mm}$, and $3 \mathrm{~mm}$ diameter, were positioned in a $20-\mathrm{cm}$ lucite bar at approximately midlength transverse to the beam path. The resulting projection was generated in 16 bins of $1-m m$ thickness for approximately 200,400,1000,2000, and 4500 particles per bin. The results are illustrated in Figure 7. With the exception of the $1-\mathrm{mm}$ hole with 200 counts per bin, in which statistical uncertainty would make it difficult to determine whether the hole can be seen in one projection, the results show that multiple scattering effects do not have a strong effect even with the bin size of $1 \mathrm{~mm}$ used. In addition, the expectation of requiring only 200 particles per bin is ascertained for $2 \mathrm{~mm}$ bins.

Examination of the energy spectra of the three solid-state detectors in the present system (Fig. 1) showed that approximately $14 \%$ of the $12 \mathrm{C}$ ion primaries incident in the first silicon $d E / d x$ detector remained primaries in the residual energy silicon $d E / d x$ detector after passing through 24-cm water equivalent absorption and travelling through most of the length of the germanium detector (approximately $11-\mathrm{cm}$ water equivalent). From these experiments, we found hat $3012 \mathrm{C}$ particles in the primary peak are sufficient to determine the residual energy in one of the image bins for an expected error less than $0.2 \%$ in density.

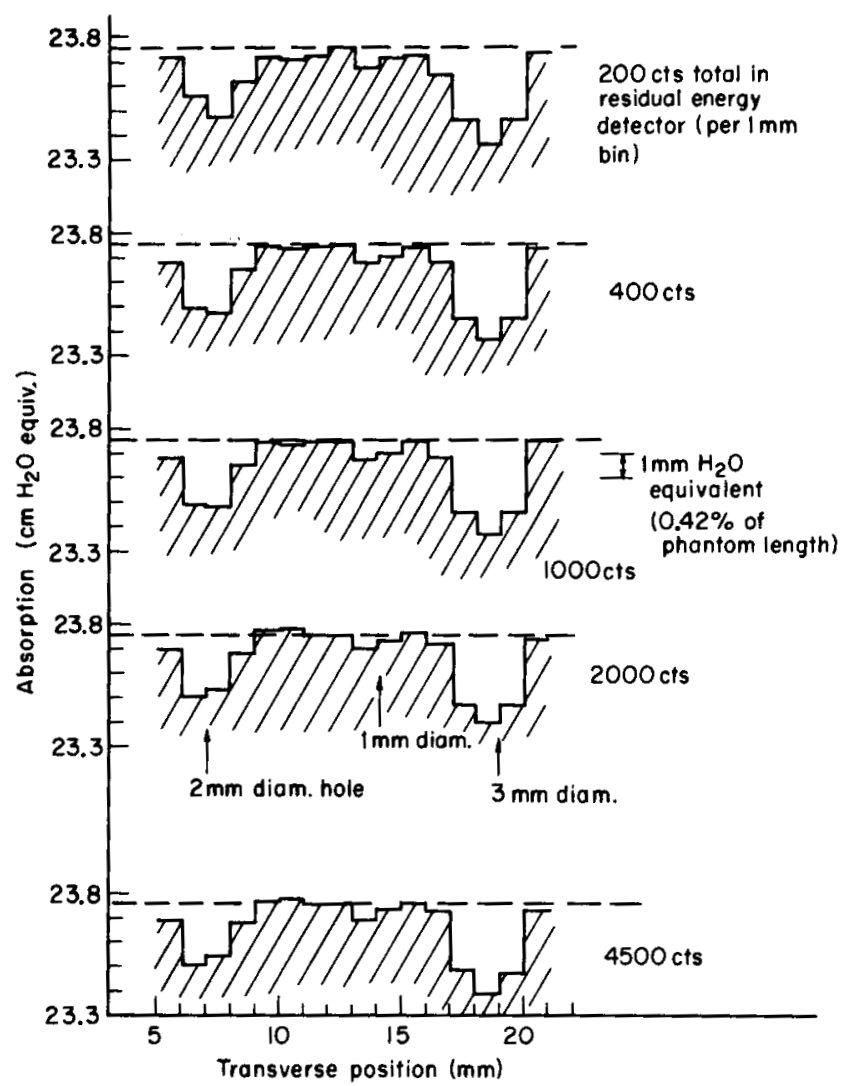

Figure 7. Single projection images of absorption through the experimental configurations illustrated in Figure 4 . The images of the three holes are shown as generated by 200, 400, 1000, 2000 and 4500 particles per $1-\mathrm{mm}$ bin.

(XBL 804-3223)

Radiation Dosimetry for Heavy-Ion Reconstruction

From the above experimental results, it is possible to estimate the radiation dose required for a complete heavy-ion tomographic reconstruction. The average approximate dose, D, for heavy-ion CT imaging is given by:

$$
\text { Dose }(\mathrm{rad})=1.6 \times 10^{-6} \times \operatorname{LET}(\mathrm{eV} / \mathrm{A}) \times\left(\mathrm{Nn} / \mathrm{s}^{2}\right) \text {, }
$$

where LET is the approximate value of that parameter (in $\mathrm{eV} / \mathrm{A}$ ) in the Bragg plateau region, $N$ is the number of particles needed per bin per view, $n$ is the number of views required, and $s$ is the linear size of the pixel in $\mathrm{cm}$. With an approximate LET of $1.5 \mathrm{eV} / A$ for ${ }^{12} \mathrm{C}$ ions, and for $\mathrm{N}=200, n=50$ and $\mathrm{s}=0.3 \times 0.3 \mathrm{~cm}$, the approximate average dose is estimated to be 0.26 rad. A similar reconstruction carried out with protons by Hanson et al. (1978), with $N=625, n=180, S=0.15 \times 0.15 \mathrm{~cm}$, required an average dose of $0.6 \mathrm{rad}$. If the average LET for protons in the energy range of 25 to $250 \mathrm{MeV} / \mathrm{n}$ is taken to be approximately $0.08 \mathrm{eV} / \mathrm{A}$, the calculated dose for the proton reconstruction; based on the above formula $0.64 \mathrm{rad}$, is in good agreement with the dose estimated by Hanson 
et al. (1978). These numbers are not directly related to the modulation transfer functions described in the first chapter of Section $B$, but they represent convenient particle fluences for accurate measurements.

From the radiation damage studies with the planar germanium detector and the above requirements for imaging, it can be estimated that between 3,000 and 5,000 complete reconstructions can be carried out before the germanium detectors are sufficiently degraded to require annealing. By raising the detector temperature to $150^{\circ} \mathrm{C}$ for two or three hours in vacuum, the annealing process can be carried out in the same detector cryostat without disassembling the apparatus.

\section{THE USE OF MULTIWIRE CHAMBERS}

(W. Chu, J. Alonso, and C. A. Tobias)

In Part II of this report we described a novel instrument, the MEDUSA, which was constructed to measure the flux density distribution of a beam of heavy particles (see Alonso et al.). The MEDUSA can measure and display the isoionization contours of a heavy-particle field, and a single beam pulse of $0.2 \mathrm{sec}$ duration or less is sufficient for the measurement. MEDUSA has been purposefully constructed for relatively low resolution and for use chiefly with "plateau" particles. However, some initial tests convinced us that an instrument of the general class of MEDUSA might also be useful for imaging in the Bragg peak region.

The MEDUSA is a multiplane, multiwire, proportional chamber that we are investigating for on-line heavy-ion radiography. Such a device provides two-dimensional projection radiographs in terms of the line integral of the stopping power of the radiographic object. Its use can be extended to produce a three-dimensional representation of the objects in terms of their densities. The exposure time can be extremely short, the data acquisition is simple, and the time between the exposure and the reconstructed image is reduced. Its capability of producing images while the patient is in the exposure position is of clinical value in patient measurement for radiation treatment planning and for verification of the patient setup. The method is easily adjusted to imaging large objects, up to $20 \times 20 \mathrm{~cm}^{2} \times 30 \mathrm{~cm}$ thick or bigger.

In a preliminary study, we used the MEDUSA to produce heavy-ion radiographs of simple phantoms (see Alonso et al., Part II). In the following paragraph, some of the first results from the MEDUSA used for radiography are presented.

By placing a certain thickness of absorber in the heavy-ion beam, we can place the transmitted unmodified Bragg peak slightiy downstream of the multiwire chamber. When a wide, flat beam is sent into the device, it will reconstruct a flat beam profile. If an object is introduced in the beam before it reaches the chamber, the part of the beam impinging on the object will stop within the object and fail to go through the chamber, casting a "shadow" of the object in the chamber. The image of the shadow is readily reconstructed. Each plane of the chamber is made of 64 wires, spaced $4 \mathrm{~mm}$ apart. Figure 8 is a radiograph of two laboratory tools, as reconstructed in the MEDUSA, using a 20 Ne beam of the energy $670 \mathrm{MeV} /$ nucleon. The image is reconstructed in $384 \times 384$ pixels, or 6 pixels per wire spacing of $4 \mathrm{~mm}$. In the reconstructed image of an edge, the transition from minimum to maximum transmission of the heavy-ion beam takes only about $2 \mathrm{~mm}$. A straight edge is reconstructed to an accuracy of about $1.4 \mathrm{~mm}$, which is about one-third the wire spacings.

If a modified Bragg peak (spread by a spira) ridge filter, for example) is used, only the particles in the portion of the peak that have excess energy after passing through the object will enter the chamber. In other words, the transmitted beam intensity is a function of the integrated stopping power of the object along the path of the radiation. The image of the transmitted beam intensities may be translated into an image of the two-dimensional projection radiograph of the object in terms of its residual ranges. This method requires only one exposure per image.

The simple phantom shown in Figure 9 represents the human body (plastic) with lungs (air space cut out in the plastics), and a spinal column (teflon). A heavy-ion beam of $20 \mathrm{Ne}$ with the energy of $670 \mathrm{MeV} / \mathrm{nucleon}$ was used to make the projection radiographs (Figs. $10 A$ and $10 B$ ) of the simple phantom. The beam was laterally broadened with a 28/64 inch $P$ b scatterer, and spread in range using $12 \mathrm{~cm}$ spiral ridge filters and an occluding ring. Figures $10 \mathrm{~A}$ and $10 \mathrm{~B}$ were produced with the water column thickness of 0 and $14 \mathrm{~cm}$, respectively.

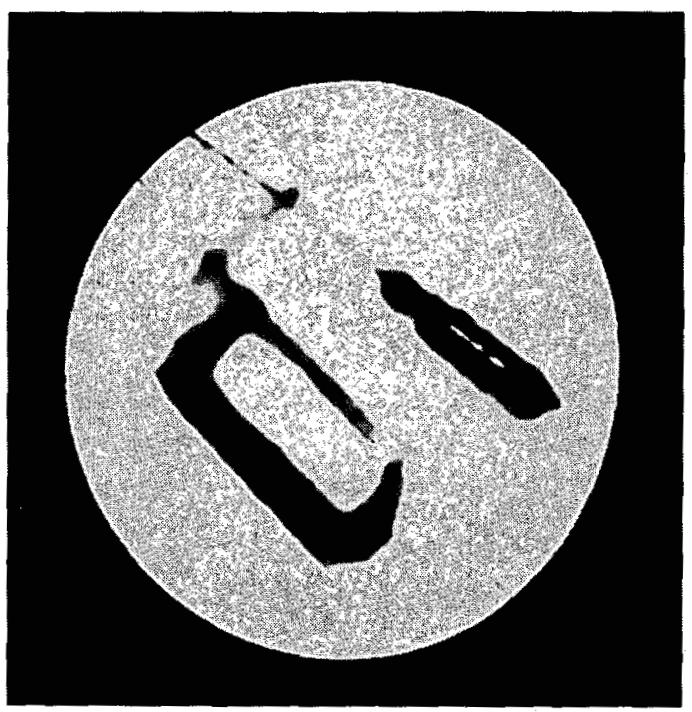

Figure 8. A projection radiograph of laooratory tools produced in the MEDUSA using $20 \mathrm{Ne}$ ions with the energy $670 \mathrm{MeV} /$ nucleon. (CBB 805-5990) 

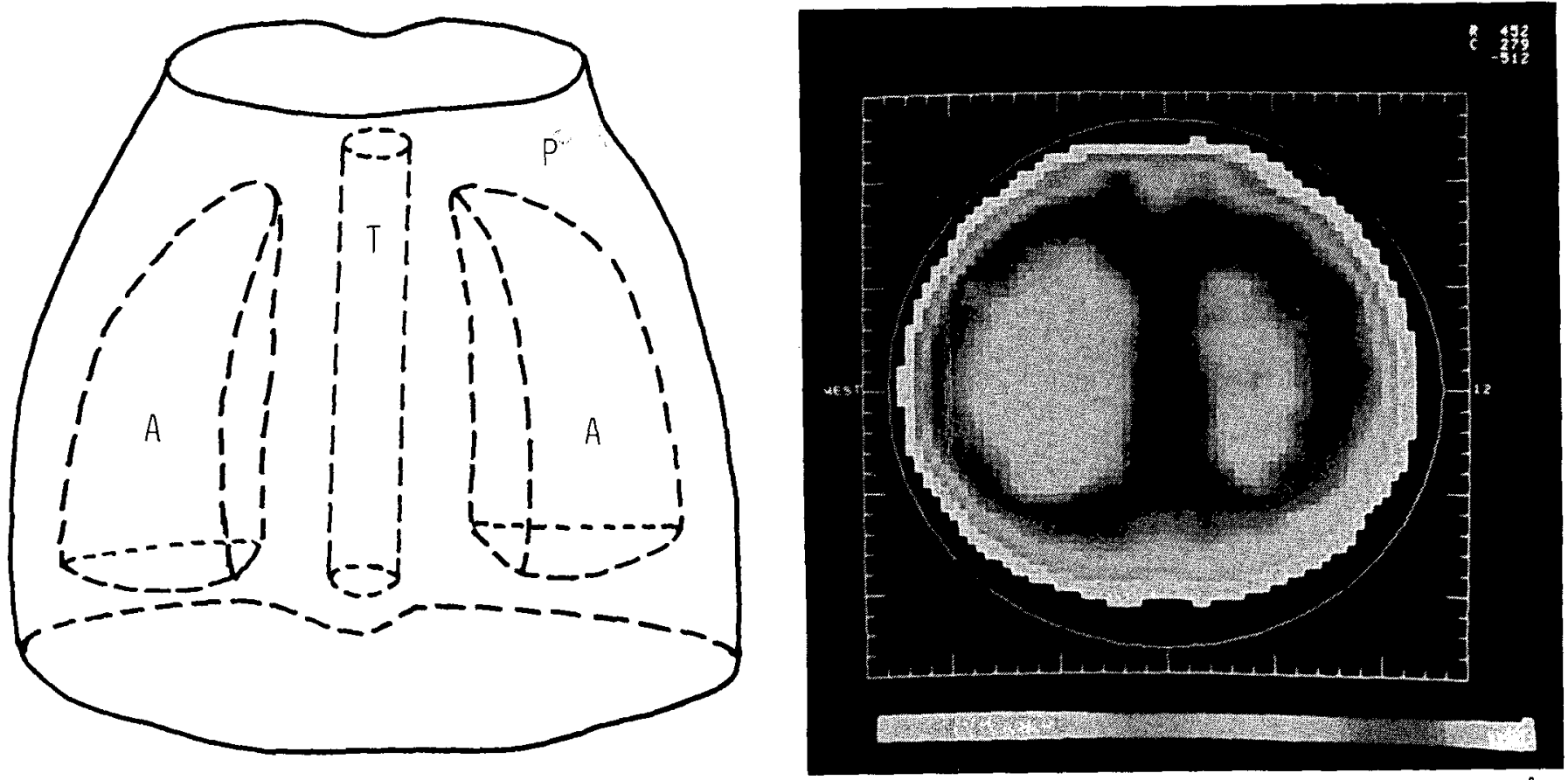

Figure 9. A simple phantom representing the human body $(P=$ plastic $)$, lungs $(A=$ air space $)$, and the spinal column ( $T=$ tefion).

(XBL 808-11466)

To compensate for the beam intensity profiles, variation across the MEDUSA field, the beam intensity profiles without the phantom are also reconstructed at various depths of the modified Bragg peak. At the $0-\mathrm{cm}$ water-column setting, the range of the beam is $23.3 \mathrm{~cm}$ water equivalent, and the structure in the thicker part of the phantom is well analyzed. The entire heavy-ion beams are transmitted through the thin part of the phantom, and the structures in that area are not resolved. On the other hand, at the 14-cm water-column setting, the situation is reversed.

By rotating the object and taking a series of exposures for two-dimensional projection radiographs, we are able to collect data necessary to reconstruct a three-dimensional representation of the object. Note that this method does not produce a three-dimensional image by stacking multiple tomographic siices. Instead, a three-dimensional image is directly synthesized from a series of two-dimensional projections, and therefore, we expect the resolutions of the reconstructed images are uniform in all three dimensions.

The analysis of the data obtained using the MEDUSA and the same phantom described above is

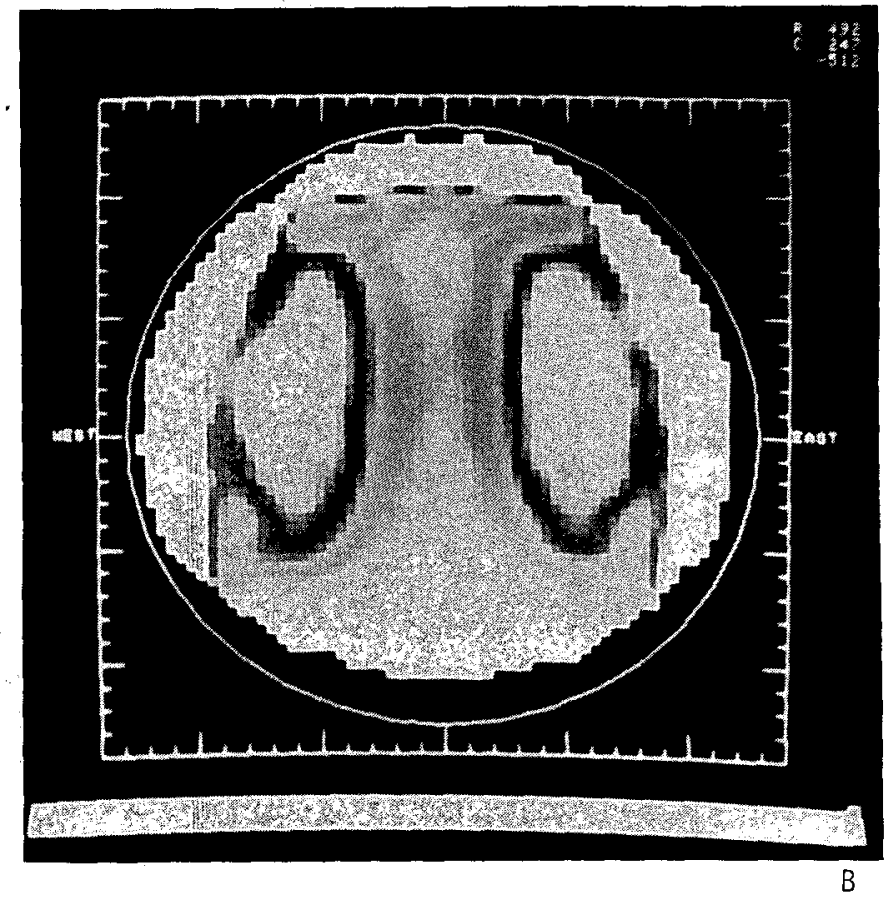

Figure 10. (A) A projection radiograph of the phantom at $0-\mathrm{cm}$ water-column setting. (B) $A$ radiograph of the phantom at $12-\mathrm{Cm}$ water-column setting. [(A) XBB 808-10093; (B) XBB 808-10091] 
in progress. The radiation dose required per exposure is typically less than $1 \times 10^{-5}$ rad. If we use exposures at 16 different angles, and two water-column settings, i.e., a total of 32 exposures, this translates to about $0.3 \mathrm{mrad}$ for the entire three-dimensional reconstruction.

\section{CONCLUSION}

There are a number of possible detector systems and arrangements that can be designed to measure charged particle $\mathrm{dE} / \mathrm{dx}$, position, and residual energy. These include multiwire proportional chambers, array of plastic or alkali halide scintillators, solid-state detectors, or combinations of these detectors. The fast respanse and analog signal processing time of solid-state detectors, their low electronic noise levels, the accuracy with which resistive layers can be ion-implanted for position sensitivity, and their stability under extremely high count rates make them promising for the application to heavy-ion radiographic CT reconstruction imaging. The experiments described and those in progress are encouraging and have the potential for developing heavy-ion CT reconstruction imaging of small objects. Ultimately, we expect to design and construct a prototype detector and electronics modules, and to construct a final imaging system of active heavy-ion CT reconstruction imaging for direct application to diagnostic and therapeutic radiology in the clinical situation.

\section{ACKNOWLEDGEMENT}

These studies are supported in part by the National Cancer Institute (Grant No. CA 27021).

\section{FOOTNOTE AND REFERENCES}

Department of Radiology, University of California, San Francisco, CA

Hansen, W. G., E. E. Haller, and G. S. Hubbard. 1980. Protective surface coatings on semiconductor detectors. IEEE Trans Nat 1. Sci. NS-27 (1), 247-251.

Hanson, U. M. et al. 1978. The application of protons to computed tomography. IEEE Trans. Nat i. Sci. NS-25, 657-660.

Huesman, R. H. 1977. The effects of a finite number of projections angles and finite lateral sampling of projections on the propagation of statistical errors in transverse section reconstruction. Phys. Med. Biol. 22, 511-521.

Llacer, J. 1981. Optimum filter for determination of the position of an arbitrary waveform in the presence of noise. IEEE Trans. Nucl. Sci. NS-28, in press.

Llacer, J., E. E. Halie, W. L. Hansen, and J. T. Walton. 1981. Semiconductor detector for medical tomography with high energy heavy ions. IEEE Trans Nucl. Sci. NS-28, in press. 


\title{
HEAVY-ION MICROSCOPY
}

\author{
G. Kraft, ${ }^{\star}$ T. C. H. Yang, T. Richards, and C. A. Tobias
}

Historically, much of the progress in biological science has been associated with progress in microscopy. Microscopes with high resolution have been developed to obtain a detailed picture from the inside of the cell. In optical microscopy the spatial resolution is limited by the wavelength of the used light which is about 5,000 $\AA$. Therefore, the smallest structures that can be visualized by these methods are about the same size (Table 1).

After the discovery of the defraction properties of material waves, two different modes of electron microscopy, scanning (SEM) and transmission (TEM), were developed. In the SEM, the intensity of the secondary electrons, which is created in the surface of the object, is recorded and almost no information is obtained from the deeper layers. In TEM, the transmitted intensity of the electron beam which has traversed a thin object as target is measured. Due to the multiple scattering of the electrons, only thin layers of target material can be used for high resolution transmission microscopy (Table 1).

It was early recognized that heavy accelerated particles, like protons, alpha particles, or heavy ions have still a much shorter wavelength than optical light or even the electron. They also exhibit much less multiple scattering than electrons and show, therefore, less angular deflection during their path through the target (Magnan, 1949; Belanger and Belanger, 1959; Tobias et al., 1967).
At energies above $10 \mathrm{keV} / \mathrm{u}$ the main interaction of the ions with the target material consists of collisions witn the target electrons. Because the electron have a much lower mass than the heavy ions, the angular deflection and the energy transfer of the projectile is small and the electronic energy loss is a smooth function of the electron density of the target material which is given as the product of atomic number times the number of atoms per unit volume. Therefore, the energy distribution of the particle beam that has traversed the target object reflects the electron density distribution, i.e., the chemical composition and density of the target over the irradiated area.

Up to now it has not been possible to produce enlarged images of the target object by active focussing of the transmitted particle beam. Also, a beam scanning method could not be used up to now because it is not possible to achieve beam spots with sufficiently small diameters $(<1 \mu \mathrm{m})$. Heavy-ion microscopy methods recently published (Yang et al., 1978; Fischer et al., 1979) consists, therefore, of microradiography techniques in which a monoenergetic and parallel beam traverses the object. The energy distribution behind the object is converted in a range distribution in a nuclear track detector. Developing the track detector yields a one-to-one density replica of the object that can be examined in SEM.

This method is similar to soft $X$-ray microscopy (Feder et al., 1977; McGowan et al., 1979)

Table 1. Resolution Obtained with Different Microscopy Techniques ....

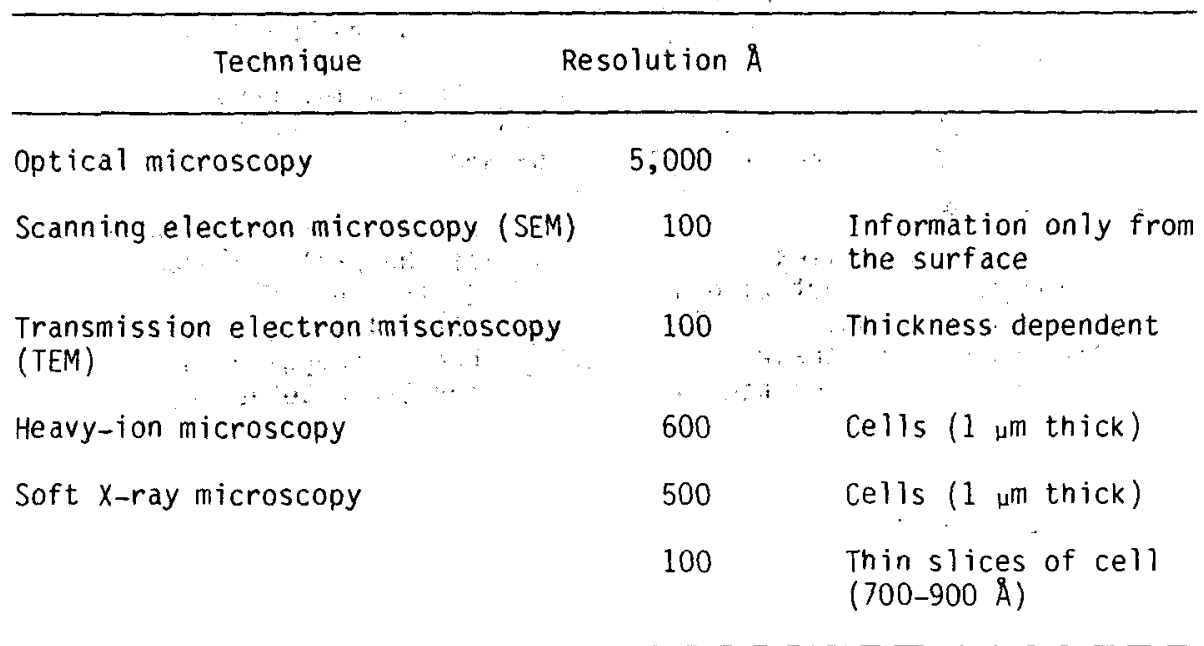


in which a replica is produced by exposing the object to the nearly monochromatic soft $x$ rays eitner from synchrotron radiation or from characterisitc $\mathrm{K}_{\alpha}$ radiation of carbon. The transmitted intensity of the $X$ rays depends on the absorption coefficient of the element and is recorded by a photoresist of nigh nomogeneity.

\section{TECHNIQUE OF HEAVY-ION MICROSCOPY}

A monoenergetic parallel beam of heavy ions traverses an object, e.g., a biological cell, which is in front of a nuclear track detector (Fig. 1). The energy loss $(\Delta E)$ of each particle in the target material is given by $\Delta E=\int d E / d x \cdot d x$. For high energies ( $E>100 \mathrm{keV} / \mathrm{u}$ ) the energy loss $\mathrm{dE} / \mathrm{dx}$ can be approximated by the Bethe-Bloch formula (Bethe, 1930; Bloch, 1933); for lower energies the Lindhard-Scharff-Schiott (Lindhard et al., 1963) theory can be used. In both cases the energy loss is mainly proportional to the product of the atomic number times the number of atom per unit volume, i.e., the electron density. The residual energy $\left(E=E_{0}-\Delta E\right)$ is converted into the range of the particle in the nuclear track detector and the contour envelope of all stopping points reflects the density distribution of the object. When the track detector is etched, the residual range of the particle becomes visible. If the particle tracks are close enough, the tracks overlap and the etching procedure yields a density replica of the object. This replica has the same dimensions as the object, but it also shows the internal structure and it can be magnified by the use of SEM.
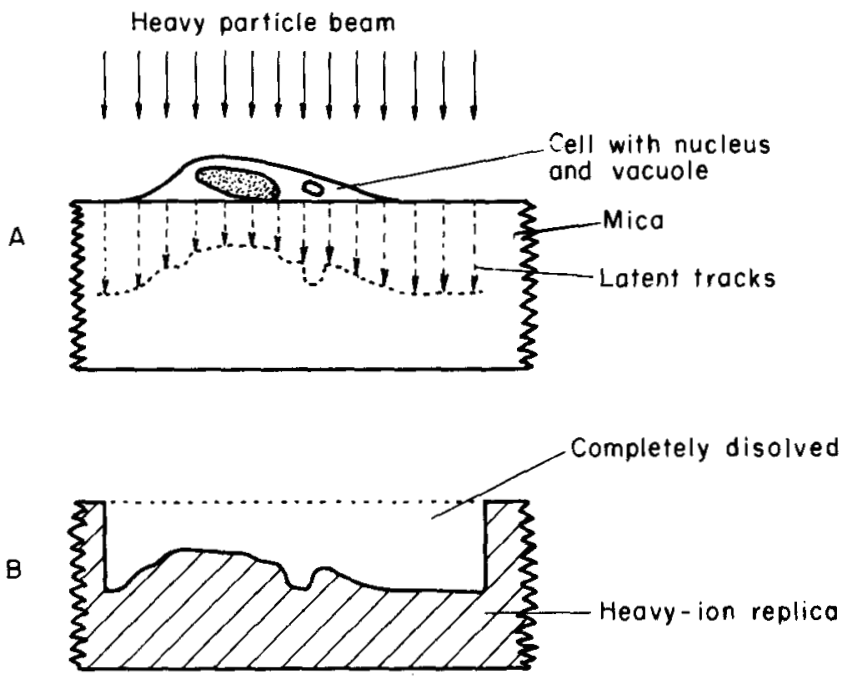

Figure 1. Principles of neavy ion replica formation. (A) Irradiation of the object with a parallel, monoenergetic heavy-ion beam.

Particles perietrating the object lose their energy according to the actual density of the object; residual energy of the particles is converted in residual ranges in the mica and the contour envelope of the latent track is a picture of the density of the imaged object. (B) Etching in a suitable solvent dissolves the particle affected region completely and produces a heavyion replica. (XBL 808-3607)

\section{CELL PREPARATION AND IRRADIATION}

The mammalian cells used in these experiments (CHO and V79) have been grown for 1 or 2 days on sterile mica plates under normal conditions. The cells usually have a better growth rate on mica than on the plastic surface of the Petri dishes. Chromosomes are prepared by arresting the cells in metaphase and subsequent hypotonic treatment. The mica plates with the cells are rinsed with water, dehydrated with ethanol, air dryed for several hours, and irradiated in a vacuum chamber of the 88-inch Synchrocyclotron or the $2 \mathrm{MeV}$ van de Graaf accelerator. The particle dose was controlled by integrating the beam current.

After irradiation, the cells were removed in dichromate sulfuric acid and the mica etched in HF for 30-60 sec. The replicas were covered with a 100 to $200 \AA$ Au-Pd layer and then examined in a field emission SEM (Coates and Welter, Electron Microscopy Laboratory). Although nigh particle doses $\left(10^{14} \mathrm{~cm}^{-2}\right)$ were used, there was no visible change in the morphology of the cells. Figure 2 shows an SEM image of cells of the same sample: irradiated and nonirradiated. The irradiated cells appear slightly brighter in the SEM, which indicates a nigher secondary electron emission yield than in the nonirradiated case. In the optical microscope, the cell appears slightly yellow after irradiation, but in both cases there was no dramatic change found in the morphology of the cells.

Figure 3 is an SEM picture of a heavy ion replica (1 MeV argon) of a mammalian cell, at a magnification of 7,000x. The main part of the cell with the cell nucleus (darker region) and nucleolus is visible in the lower right part of the picture. A long thick fiber stretches to the left, and ends in thin structured region. At the left side at the top of the picture, part of the mica was shadowed from the particle irradiation and the original, unetched surface of the mica is visible. The small white grains at the border line of the cell are replicas of salt crystal remanants of the cell medium which was not totally rinsed off. The resolution of figure 3 is about $600 \AA$.

\section{RESOLUTION OF HEAVY-ION MICROSCOPY}

The maximum resolution obtained with heavy ion microscopy depends on the following parameters:

1. the energy resolution and the divergence of the particle beam;

2. the energy loss straggling and the lateral straggling in the target and the range straggling and lateral straggling in the track detector;

3. the resolution of the etching procedure as given by the track diameter and the steepness of the etched cones;

4. the neat resistivity of the track detector against the $n$ igh intensity electron beam of SEM;

5. the resolution of the SEM. 

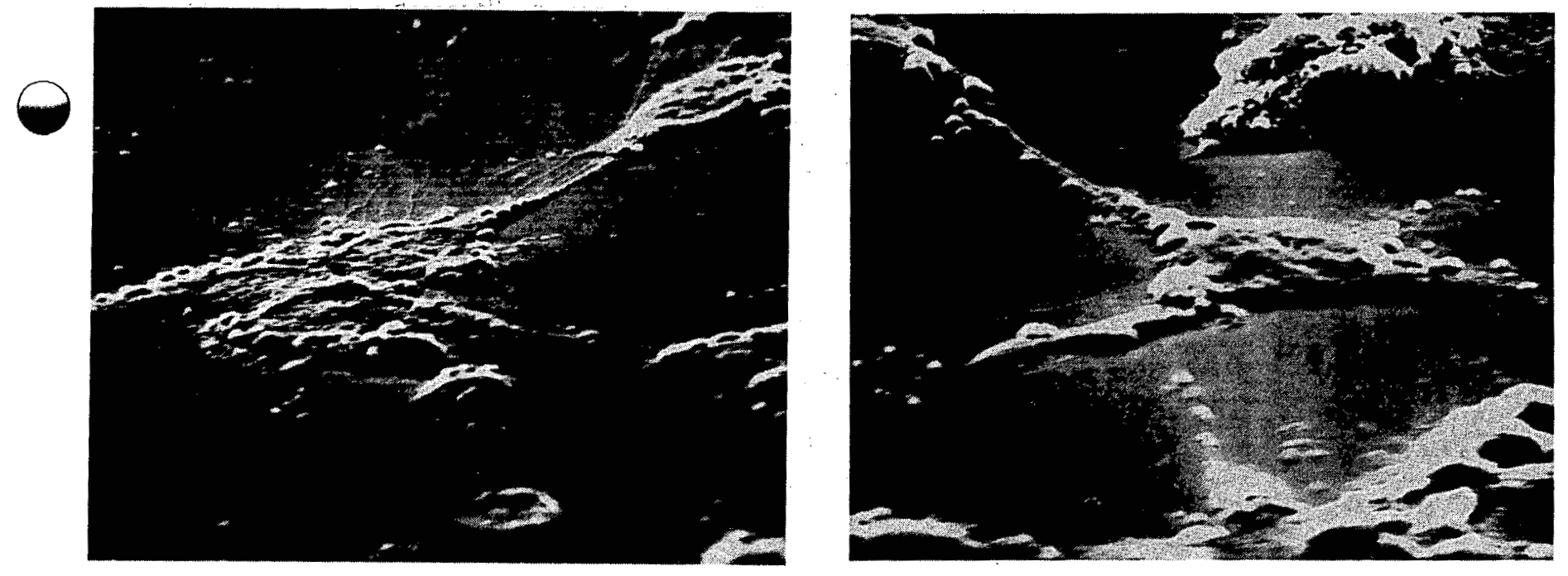

Figure 2. SEM image of $V 79$ cells growing on a mica plate. Left: Not irradiated. Right: Cells irradiated with $10^{13}$ argon ions per $\mathrm{cm}^{2}$ with an energy of $1 \mathrm{MeV}$. (XBB 808-9828)

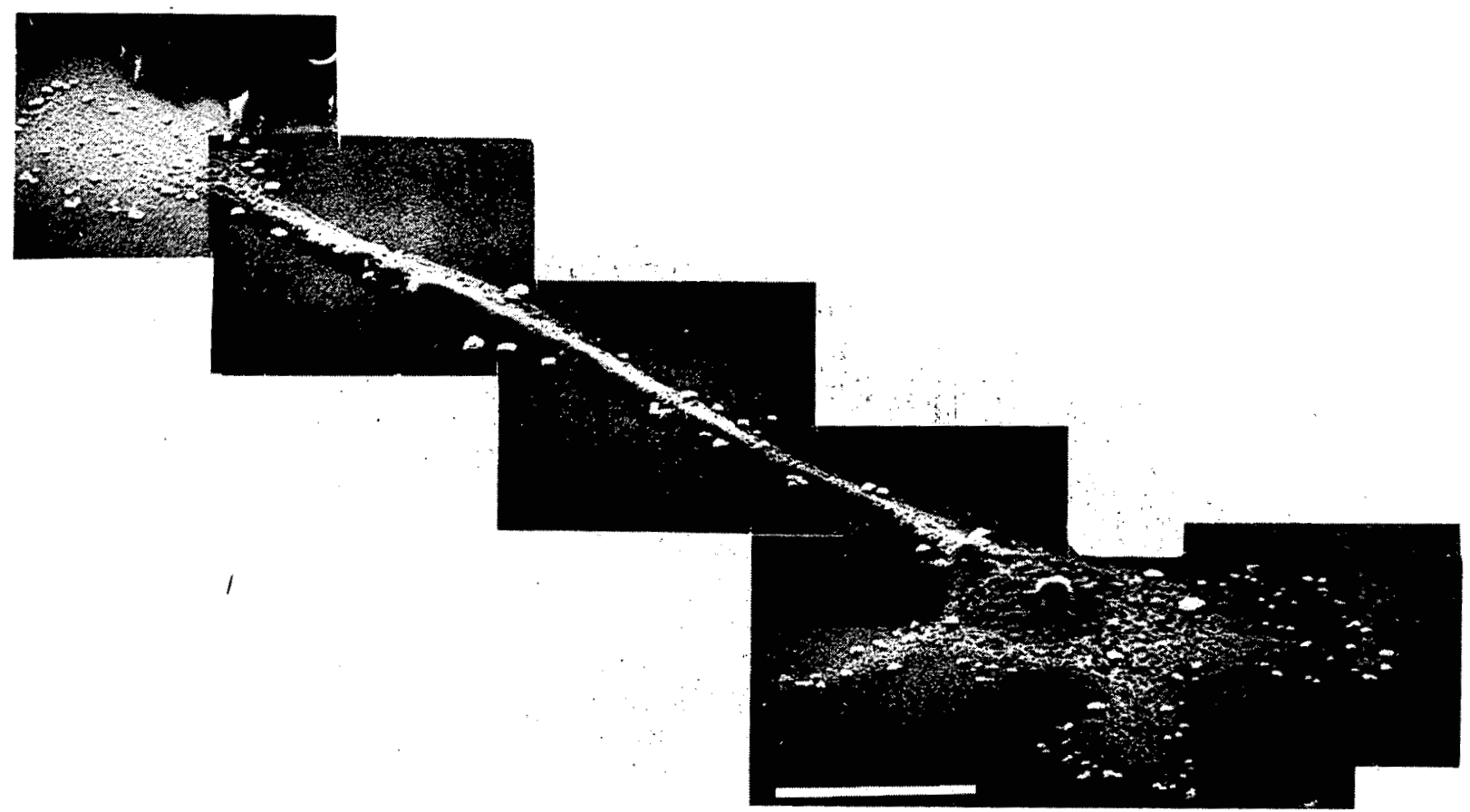

Figure 3. SEM image of neavy-ion replica of a mammalian cell obtained with 1 MeV argon beam. At the right side the nucleus of the cell (dark region) the dense nucleolus is visible. In the upper left, a part of the mica was shadowed from irradiation and the original surface of the mica and the steepness of the etched walls are visible. The marker represents $10 \mu \mathrm{mi}$. (XBB 808-9829) 
Sufficient parailelism of the beam can be easily achieved by enlarging the distance between the last focussing element in the beam pipe and the target. The influence of energy straggling of the primary beam to the range straggling in the detector as well as the intrinsic range straggling and the lateral straggling are the main limiting factors of the resolution (Fig. 4). For the calculation of these curves, the ranges of argon ions in carbon and aluminum have been taken from the Winterbon (1968) tables which are based on the Lindhard-Scharff-Schiott theory. Other tabulated values (J. F. Ziegler, 1980 and private communication) yield about $20 \%$ shorter ranges. But the argon ranges, which are measured in this experiment in mica, are in good agreement with the argon range in carbon of the Winterbon tables... In addition, the energy loss of argon ions in water, which is nearly tissue equivalent, is very close to the energy loss of argon in carbon (Schöpfer et al., 1980). Therefore, when ranges and lateral and longitudinal straggiing were calculated, no distinction was made between the particle track in the biological object and in the mica. The carbon values are used instead of the target material actually used (tissue and mica, both of which have a complex chemical composition).

The intrinsic range straggling (which is not caused by the energy spread of the primary beam) is calculated according to Bohr's theory (1948) which yields a straggling parameter $\Delta R / R=3.3 \%$. Usually this theory overestimates the measured range straggling values, but for the lighter target atoms used here the discrepancy is small and straggling values between 2 and $3 \%$ should be realistic. The lateral straggling in the energy range between 30 and $8 \mathrm{MeV}$ is taken from J. F. Ziegler's tables (1980) and extrapolated to the lower energies using the Sigmund-Meyer theory. The two compounds of the longitudinal straggling caused by the energy straggling of the beam and the intrinsic range straggiing sum up quadratically and the values of the total straggling assuming $0.2 \%$ beam straggling are given in Table 2.

From Table 2 and Figure 4 it is evident that the lowest possible particle energy that is sufficient to penetrate the target object should be used to achieve best resolution. In Figure 5, the replicas of the same type of cells obtained with $14 \mathrm{MeV}$ and $2 \mathrm{MeV}$ argon ion are compared. The decrease of resolution with increasing particle energy and increasing range is clearly visible.

The track etching procedures nave aiways a tnreshold energy below which the particle track cannot be developed. Therefore, it was suggested that tracks should exhioit a range deficit (Fleischer et al., 1975). The formulas used here always refer to the total range and the range deficit is neglected. But in the case of the high particle doses, with which most of the track overlap with each otner, the threshold energy of the etching procedure is no longer determined by the individual track as given in the literature.
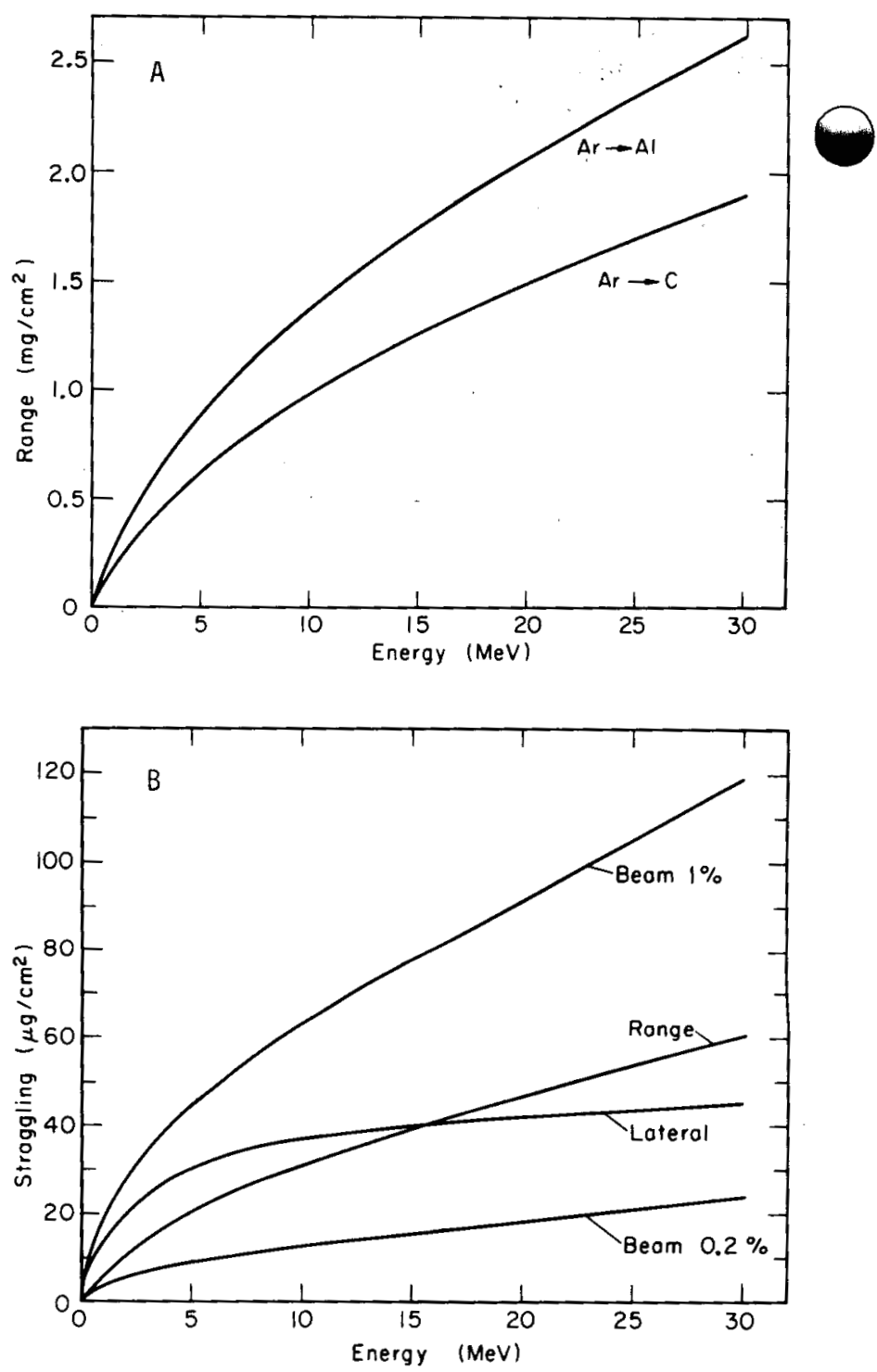

Figure 4. (A) Range of argon ions in carbon and aluminum as a function of particle energy (Winteroon, 1968). (B) Straggling of argon ions as function of particle energy. The lateral straggling in carbon is taken from J. F. Ziegler (1980) and Sigmund et al. (1974), range straggling from Bonr (1948), and the contribution of $1 \%$ and $0.2 \%$ energy spread in the primary beam is calculated according to Figure $4 A$.

[(A) XBL 808-3605; (B) XBL 808-3604]

In addition, these $n$ igh doses are needed to acnieve a good lateral resolution. If a lateral resolution on the order of $100 \AA$ is required then it is necessary that every square of 100 \& length is nit by at least one particle. This sums up to a total particle dose of 1012 particles $/ \mathrm{cm}^{2}$. Considering that the particles are randomly distributed, nigher doses between $10^{13}$ and $10^{14}$ are used in this experiment. 
Table 2. Straggling Parameters for Argon Ions in Carbon

\begin{tabular}{|c|c|c|c|}
\hline \multirow{2}{*}{$\begin{array}{l}\text { Energy } \\
\text { (MeV) }\end{array}$} & \multirow{2}{*}{$\begin{array}{c}\text { Range } \\
\left(\mu 9 \mathrm{~cm}^{-2}\right)\end{array}$} & \multicolumn{2}{|c|}{ Straggling } \\
\hline & & $\begin{array}{l}\text { Longitudinal } \\
(\mu \mathrm{g} \mathrm{cm}-2)\end{array}$ & $\begin{array}{l}\text { Lateral } \\
\left(\mu \mathrm{g} \mathrm{cm}^{-2}\right)\end{array}$ \\
\hline 1 & 0.20 & 7.2 & 16 \\
\hline 2 & 0.34 & 12.5 & 20 \\
\hline 5 & 0.63 & 22.0 & 31 \\
\hline 10 & 0.97 & 33.5 & 38 \\
\hline 20 & 1.50 & 50.3 & 42 \\
\hline 30 & 1.90 & 64.6 & 45 \\
\hline
\end{tabular}

$1,000 \AA=0.1 \mu \mathrm{m}=35 \mu \mathrm{g} / \mathrm{cm}^{2}$ carbon $=28 \mu \mathrm{g} / \mathrm{cm}^{2}$ mica $=10 \mu \mathrm{g} / \mathrm{cm}^{2}$ water

* Assuming $0.2 \%$ energy spread of the primary beam.

\section{THE INFLUENCE OF THE ETCHING PROCEDURE TO THE RESOLUTION}

In most plastic materials and glasses the tracks are etched as cones, which can be easily explained by assuming two different dissolving velocities in the amorphous substrate (Fleischer et al., 1975). One dissolving velocity, $v_{N}$, is the normal dissolving velocity of the material, which is not affected by the radiation. The other dissolving velocity, $v_{t}$, is the dissolving velocity along the particle path (Fig. 6). As long as the ratio of these two velocities remains constant during the etching process, a cone will result as track formation having a cone angle $\theta$ which is given by $\sin \theta=v_{N} / v_{t}$. No structure can be successfully imaged with the replica technique, which has steeper walls than the etched cones (Fig. 6); less steep surfaces will also be affected by the etching procedure. To yield a correct density replica with steep walls, a high etch velocity, vt, is required: Plastic detectors and glass have comparäble etching velocities $\left(v_{t}\right.$ and $\left.v_{N}\right)$ yielding flat cones. In contrast to the plastic detectors, mica snows an extremely high etch velocity in the track $\left(V_{t}=0.3 \mu \mathrm{m} / \mathrm{sec}\right)$ and an extremeliy low velocity $v_{N}=10^{-5} \mu \mathrm{m} / \mathrm{sec}$. This yields particle tracks that have nearly parallel walls (Sophr, 1980). The rhomboidal cross section of the particle track in mica is caused by the. crystalline structure of the mica (Fleischer et a 1., 1975). The steepness of the etching procedure is also visible in Figure 3 , where a part of unirradiated mica is visible in the upper left of the picture. Besides the favored track geometry, mica also has a high heat resistivity and good vacuum properties. Replicas made of
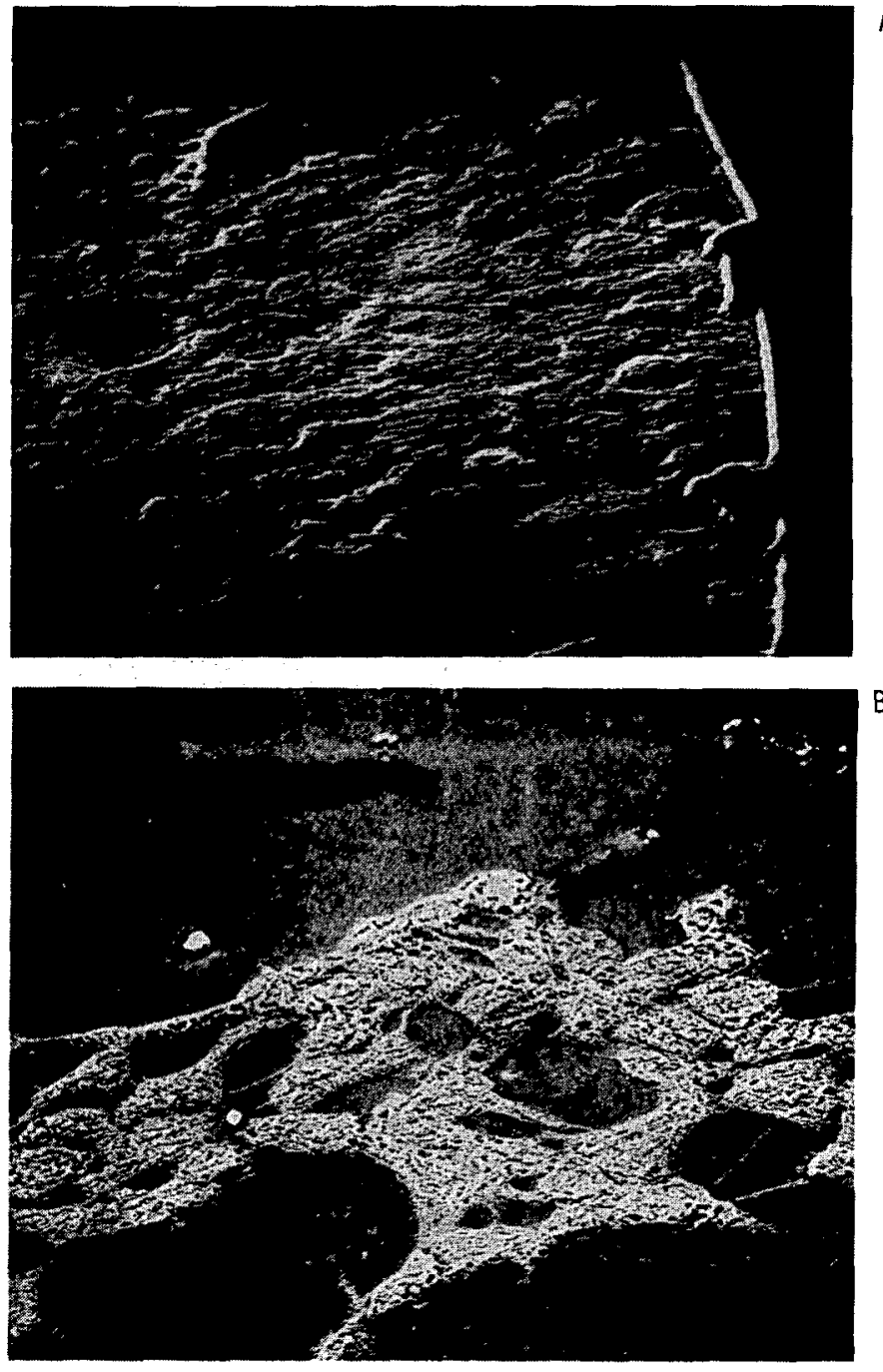

Figure 5. Influence of the primary beam energy to the resolution of heavy-ion microscopy.

(A) Replicas of V79 cells taken with $14 \mathrm{MeV}$ argon ions, SEM magnification 700x. (B) Replicas of V79 cells taken with 2 MeV argon ions, SEM magnification 1,000X. (XBB 808-9827)

mica are not affected by the intense electron beam of the SEM. Therefore, the heat damage and the resolution of the SEM pose no limitations for the resolution of heavy-ion microscopy.

\section{TOTAL RESOLUTION}

Tne total resolution given in Table 2 may be regarded a an upper limit. In this experiment low energies are used to obtain minimal absolute straggling, but in the low energy region only the effect of many overlapping tracks can be developed and the straggling of individual particles in both directions (longitudinal and lateral) may be averaged. The experimental resolution of a $2.4 \mathrm{MeV}$ argon replica is demonstrated by the image of a small fiber of cell (Fig. 7). This fiber nas a diameter of about $900 \AA$ and is shown 


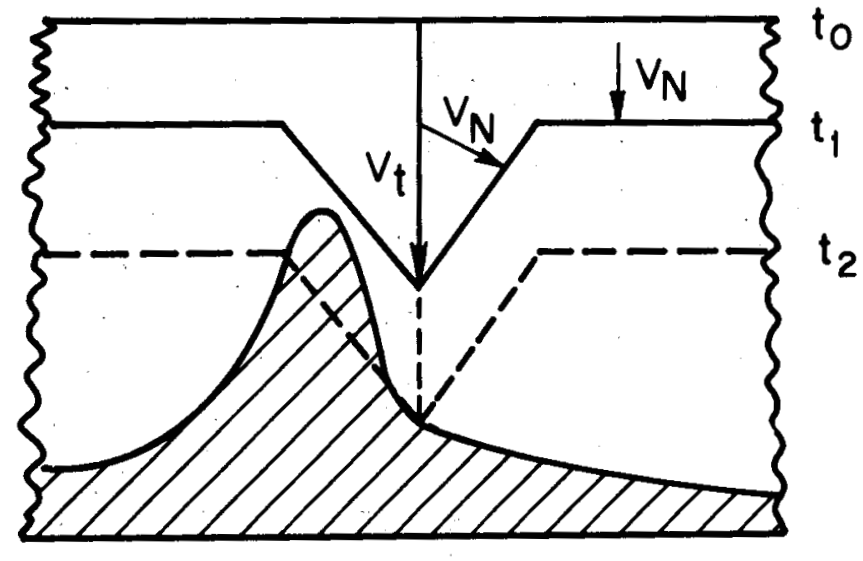

Figure 6. Track geometry for different etching times $\left(t_{0}=0<t_{1}<t_{2}\right)$ assuming two constant etching velocities $v_{N}$ and $v_{t}$. Structures having steeper walls than the etch cones cannot be replicated. (XBL 808-3606) in different magnifications of the SEM. The background ripple caused by the straggling is smaller than the fiber and the grain size of the background is about $500 \mathrm{~A}$. These values may be compared with the calculated values of longitudinal and lateral straggling of 14 and $23 \mu \mathrm{g} / \mathrm{cm}^{2}$, respectively, of Table 2 which are the same magnitudes. Higher resolutions are only possible for tninner targets.

\section{COMPARISON WITH SOFT X-RAY MICROSCOPY}

The structure obtained with the heavy-ion microscopy technique in Figure 3 is similar to the image that has been obtained with the soft $X$-ray replication technique (McGowan et al., 1979). The resolution of the soft X-ray images of whole celis is about the same magnitude (about 500 A). Higher resolution (about $100 \AA$ ) was reported from soft $X$-ray pictures of retina cells (Feder et al., 1977) that were stained and cut in $700 \AA$ thick slices. A further increase of the resolution of heavy-ion microscopy is expected using samples of the same thickness.
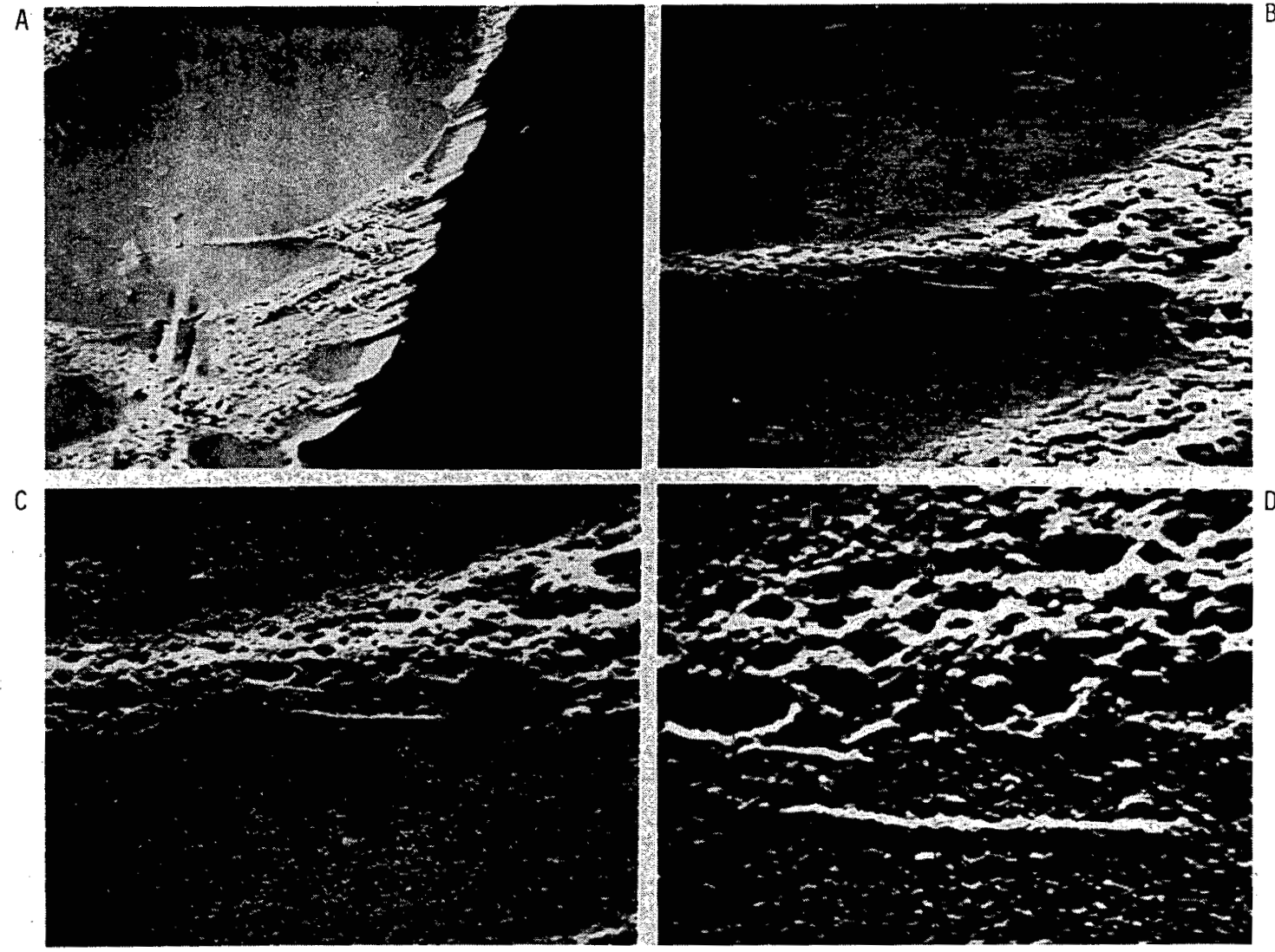

Figure 7. Replicas of a small fiber of $V 79$ cell with different magnification at the SEM: (A) 1,000X; (B) 5,000; (C) 10,000X; (D) 25,000X. (XBB 808-9826) 
The information obtained with soft $x$-ray microscopy is different from heavy-ion microscopy: X-ray absorption is based on the different absorption coefficient of the different atomic numbers; heavy-ion microscopy is sensitive to both chemical composition and density variation. Therefore, internal structures of similar chemical composition but having different densities as, for example, spindle fibers or condensed chromosomes, are easier to detect with heavy-ion microscopy.

Figure 8 is a replica of a dense cell layer taken by an optical microscope with all cells arrested in metaphase. The cells have a close contact to each other; the outline of the individual cells is not visible but the cell nuclei and the chromosomes in some nuclei are visible. With more careful preparation technique such as critical point drying and the incorporation of contrast material, like IUdR which is only bound to the DNA, it should be possible to obtain information of the composition of the chromosomes that are not in a condensed phase.

\section{ACKNOWLEDGEMENT}

The authors would like to acknowledge the excellent cooperation of Mike Stratman at the 2 MeV Van de Graaf accelerator; Martin Murphy and Bob Stockstad at the 88-inch Cyclotron; Don Pardoe at the Electron Microscopy Laboratories; and E. A. Blakely, P. Chang, W. Kraft-Weyrather, and L. Lommel of the Radiation Biophysics group. This investigation was supported by GSI

Darmstadt/Germany, and by the National Cancer Institute (Grant CA15184).

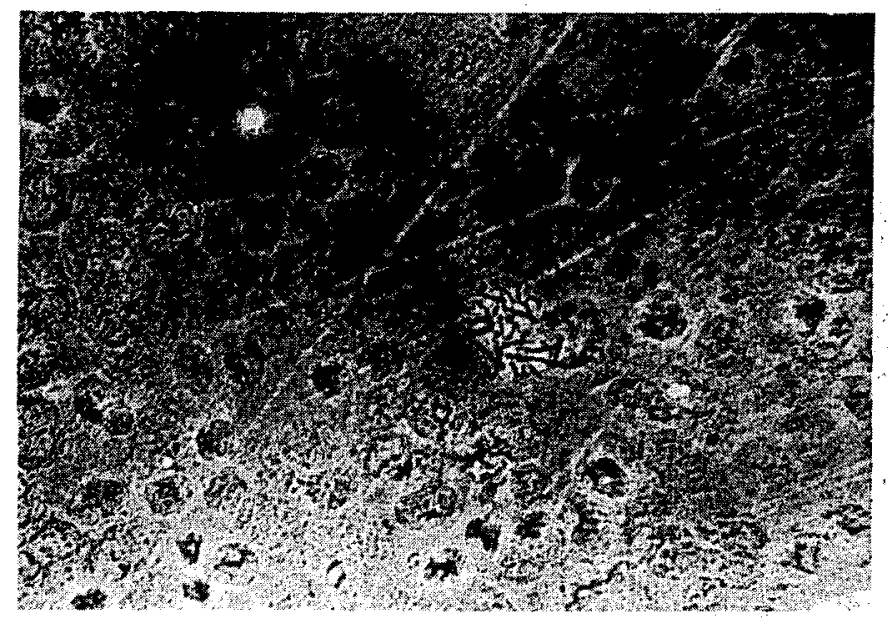

Figure 8. Heavy-ion replica of a dense layer of cells arrested in metaphase taken with an optical microscope. The nuclei in the cells and some dense structures inside the cell nuclei are clearly visible. One set of spread chromosomes can be seen in the middle of the picture. (XBB 805-6639)

\section{FOOTNOTES AND REFERENCES}

*Presently on leave from GSI, Darmstadt, Germany.

Belanger, L. F. and C. Belanger. 1959. J. Biophys. Biochem. Cyto $1.6,197$.

Bethe, H. A. 1930. Zur Theorie des Durchgangs schneller Korpuskularstrahlen durch Materie. Annal. d. Phys. 5, 325-400.

Bloch, F. 1933. Bremsvermögen von Atomen mit mehreren Elektronen Annal. d. Phys. 16, $285-230$.

Bohr, N. 1948. The penetration of atomic particles through matter. Nat. Fys. Medd. Dan. Vid. Selsk. $18,8$.

Feder, R., E. Spiller, J. Topalian, A. N. Broers, W. Gudat, B. J. Panessa, Z. A. Zadumaisky, and J. Sedat, 1977. High resolution soft $X$-ray microscopy. Science 197, 259-260.

Fischer, B. E., B. Genswïrger, and R. Spohr. 1979. Heavy ion microlithography. Report GSI 79-11, 144. GSI, Darmstadt, W. Germany.

Fleischer, R. L., P. B. Price, and R. M. Walker. 1975. Nuclear Tracks in Solids: Principles and Apptications. University of California Press, Berkeley, CA.

Hayes, T. L., C. A. Tobias, and T.C.Y. Yang. 1978. Heavy ion microscopy of hydrated cells with SEM imaging of the contact radiograph. Scanning Electron Micros. 1, 233-239.

Lindhard, J., M. Scharff, and H. E. Scniott. 1963. Range concepts and heavy ion ranges. Mat. Fys. Med. Dan. Vid. Selsk. 33, 14.

Magnan, C. 1949. The proton microscope. Nucleonik 4 , 52-66.

McGowan, J: W., B. Borwein, J. A. Medivos, T. Beveridge, J. D. Brown, E. Spiller, R. Feder, J. Topalian, and W. Gudat. 1979. Towards the development of nigh resolution $X-r$ ay spectroscopy/microscopy of cells. J. Cell Biol. 80, $732-735$.

Schöpfer, F., J. Kiefer, E. Schneider, and G. Kraft. 1980. Inactivation of dried yeast cells by heavy ion of nigh LET. Radiat. Res., in press.

Sigmund, P. and K. B. Winterbon. 1974. Small angle multiple scattering of ions in the screened Coulomb region. Nucl. Instrum. Methods. 119,541 .

Sphor, R. 1980. Nuclear track research activities at GSI. NuCl. Instrum. Methods $173,229-234$. 
Tobias, C. A., T. L. Hayes, H. D. Maccabee, and R. M. Glaeser. 1967. Heavy Ion Radiography and Microscopy in Biomedical Studies with Heavy Ion Beams, Lawrence Berkeley Laboratory Report UCRL 17357, pp. 108-120.

Winterbon, K. B. 1968. Energy Data for MeV Ions in Amorphous Material, Report AECL 3194, Chalk River, Ontario.
Yang, T. C., G. Welch, C. A. Tobias, H. Maccabee, T. L. Hayes, L. Craise, E. V. Benton, and F. Abrams. 1978. The feasibility of heavy charged particle microscopy. Ann. N. Y. Acad. Sci. 306, 322-339.

Ziegler, J. F. and G. I. Iafrate. 1980. Radiat. Effects 46, 199; and private communication. 


\title{
HIGH ENERGY BEAMS OF RADIOACTIVE NUCLEI AND THEIR BIOMEDICAL APPLICATIONS
}

\author{
A. Chatterjee, E. L. Alpen, J. Llacer, J. R. Alonso, and C. A. Tobias
}

Energetic charged particles heavier than electrons are now being used in radiation therapy. The various charged-particle radiation modalities include: pions at Los Alamos, helium through argon at LBL, and protons at Harvard. All of these modalities have one thing in common: they exhibit a favorable depth-dose characteristic when localized tumor volume is concerned. They can deliver a high dose to the treatment volume while minimizing the dose to normal tissues outside the treatment volume (Tobias et al., 1952). This preferential deposition of dose in the tumor region is attributable to the Bragg ionization phenomenon of heavy charged particles. The region of maximum ionization intensity is called the Bragg peak. Figure 1 shows a typical Bragg

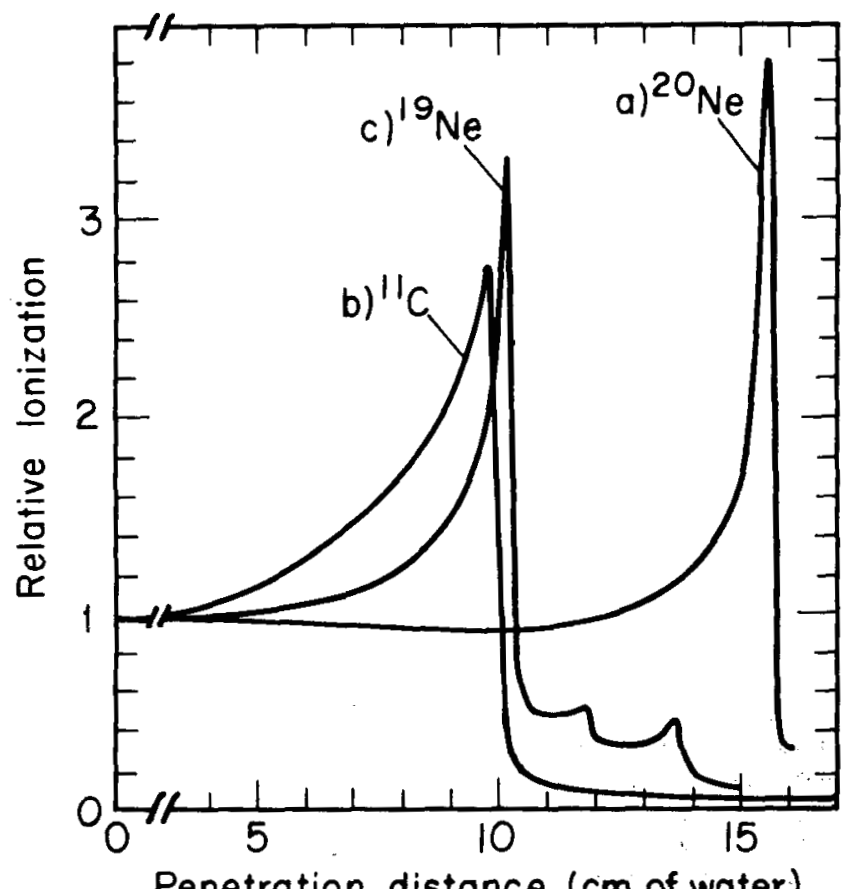

Figure 1. Experimentally measured Bragg ionization curves for high-energy, heavy particle beams (Llacer et al., 1979). (A) $425 \mathrm{MeV} / \mathrm{n}$. $20 \mathrm{Ne}$ beam, the range in water is $15.8 \mathrm{~cm}$, and the peak in ionization occurs at $15.6 \mathrm{~cm}$; these are primary beam particles accelerated in the Bevalac (Gniorso et a1., 1973). (B) Measurements made with a secondary beam of ${ }^{11} \mathrm{C}$ nuclei produced from a primary beam of $12 \mathrm{C}$ particles; the Bragg peak occurs at $9.7 \mathrm{~cm}$. (C) Data for a secondary beam of 19 Ne particles; the Bragg peak occurs at $10.25 \mathrm{~cm}$. There is a small amount of contamination present as indicated by two small peaks of $17 \mathrm{~F}$ and 150 . (XBL 798-3750A) ionization curve for $425 \mathrm{MeV} / \mathrm{n}$ neon beam (i.e., $8.5 \mathrm{GeV}$ ) as measured with a variable thickness water phantom and ionization chamber (Lyman and Howard, 1977).

Since the Bragg peak of monoenergetic neavy particles is quite sharp, and since the degree of ionization in the peax region is several times nigher than the plateau region, caution must be exercised in locating the Bragg peak witnin the tumor volume. Very small errors may give rise to significantly increased dose outside the target volume, but more importantly, it may result in seriously underdosing the tumor volume. In the past, not being able to treat with the Bragg peak nas been a severe limitation in the heavyparticle therapy program (Lawrence et al., 1971). Although the location of a tumor can be well defined througn conventional diagnostic techniques, the placement of the Bragg peak on the tumor depends on knowing the density and composition of intervening substance, bone, tissue, sinus, air, blood, etc., through which the beam must pass. We know from experience with neavy particle therapy that the precise localization of the Bragg peak is an important component of the evaluation of this treatment modality. There are many examples where Bragg peak treatment is not only desirable but essential in order to derive maximum benefit from energetic charged particles. Exact calculation of beam stopping point is impossible because of unknown amounts of intervening bone, tissue, blood, fat, air, etc., in patients.

A program for the radiotherapeutic management of pituitary tumors exists at LBL. These types of tumors have been treated with the plateau region of energetic helium beams with encouraging results. The success of helium-particle pituitary irradiation for the ablation of endocrine disease (Linfoot et al., 1971) results in the delivery of a precise helium beam to the region of sella turcica and the entire pituitary fossa. Care is taken to spare the critical surrounding nervous tissue--particularly the optic chiasm--the cranial nerves, and the temporal lobes. Successful control of the disease occurs in greater than 70 to 80 ' of the cases. Fajlures, when they occur, are primarily in patients with Cushing's disease and Nelson syndrome. These patients frequently have normal to small sellae, and considerable refinement in control of dose and dose distribution is needed for improved control rates. This can be accomplished with Bragg peak rather than plateau ion radiotherapy. There are many examples where Bragg peak treatment is not only desirable but essential in order to derive maximum benefit from energetic charged particles. But so far, such accurate procedures have not been accomplished for lack of precise information 
on effective stopping power of normal tissue between entry port and tumor target. Exact calculation of beam stopping point is impossible because of the unknown amounts of intervening tissue in patients.

The average LET of a heavy charged particle is given by the Bethe stopping power formula (Bethe, 1930):

$$
\begin{array}{r}
-d E / d x=\frac{4 \pi z^{2} e^{4}}{m c^{2} B^{2}} N Z\left[\ln \frac{2 m c^{2} B^{2}}{I\left(1-B^{2}\right)}-B^{2}+\right. \\
\text { correction terms }]
\end{array}
$$

where $z$ is the charge on the incident particle, $\mathrm{NZ}$ is the number of electrons/volume in the medium, $B$ is the velocity of the incident particle in units of the velocity of light, $c$, I is the mean excitation potential of the medium,

$e$ is the electronic charge,

$\mathrm{mc}^{2}$ is the rest energy of an electron.

Correspondingly, the range $\mathrm{R}$ can be calculated as:

$$
R=A \int_{0}^{E} \frac{d E^{\prime}}{\left|d E^{\prime} / d x\right|},
$$

where $E$ is the energy per nucleon of the incident heavy particle, and $A$ is its mass number. For a homogeneous medium, applications of equations 1 and 2 are straightforward, and positioning the Bragg peak at a specified depth is simple. But if the medium is nonhomogeneous (as in a patient), and if we do not know the explicit path length for specific tissues, then equations 1 and 2 are not useful. The electron density and composition of each volume element that a beam penetrates are essential for calculating the depth of penetration of a charged particle for a given energy. An approach to solving this problem is by estimating electron density from $x$-ray computerized tomography information (Chen, 1979). Error in this procedure can be as high as $8 \mathrm{~mm}$ over a pathlength of $20 \mathrm{~cm}$ due to several effects: $X-r a y$ beam hardening, reconstruction artifacts, calibration errors and possible inaccuracies in separating Compton from photoelectric effects (G. T. Y. Chen, private communication). Of course, for smaller pathlengths, the error will be smaller.

Another technique based on tissue activation has been developed by Bennett et al. (1978). Most of their measurements have been restricted to therapeutic proton beams $(200 \mathrm{MeV})$. They detect the decay of 150 (positron emitter with nalf life of $2 \mathrm{~min}$ ) produced from proton nuclear interactions in vivo. In their technique, one has to deal with higher diagnostic dose (about
$20 \mathrm{rad}$ ); and in rotational therapy, such high dose per rotation may not be desirable. Also, in the last $5 \mathrm{~mm}$ of the proton pathlength, no activation is produced because of the energythreshold requirement. A possible alternative approach to this problem is to make direct measurements of the beam-stopping region. This can be done with high-energy radioactive beams from the Bevalac (Ghiorso et al., 1973).

\section{RADIOACTIVE BEAMS}

The availability of high-energy beams of radioactive species is the most recent advancement in the field of accelerator physics. Besides the interest in such beams for nuclear physics, there will also be important applications in therapeutic and diagnostic radiology and in nuclear medicine.

One of the primary interactions experienced by relativistic heavy ions is the peripheral nuclear collision (Greiner et al., 1978; Heckman et al., 1971). The main characteristic of this type of reaction is that only a small amount of excitation energy is imparted to the projectile-. enough to cause a break up of the nucleus, but insufficient to produce significant changes in the projectile's velocity or trajectory (Chatterjee et al., 1976). Thus, radioactive nuclei are produced as secondary particles from peripheral nuclear fragmentation reactions. These nuclei have trajectories and energies differing little from that of the parent particle. Various radioactive beams produced as a result of these reactions, now available on a regular basis from the Bevalac, are: $11 \mathrm{C}, 13 \mathrm{~N}$, 150 , and $19 \mathrm{Ne}$ with sufficient intensity (about $10^{7}$ particles/pulse).

The secondary beam intensity and quality are dependent in large measure on the choice of target material and thickness as well as on the specific properties of the production reaction. For production of radioactive beams, beryllium is the most efficient target.

Since the charge-to-mass ratio of the desired radioactive product nuclei is quite different from most of the other fragments (which are also produced in peripheral nuclear collisions) and from the parent nucleus, isolation of a given radioactive deam nas been achieved quite effectively by magnetic deflection (Alonso et al., 1979). Figure 1 shows a Bragg ionization curve measured for the ${ }^{1} 1_{C}$ beam, a positron emitter, with a nalf life of $20 \mathrm{~min}$. In this type of measurement, made witn a variable thickness water absorber (Tobias et al., 1971), each ion species present in the beam exnibits an identifiable signature: a sharp peak at the end of its range. All the fragments that are produced in a peripheral nuclear callision nave the same energy per nucleon, but because of their different cnarge and/or mass, they extibit different deptns of penetration, as can be deduced from equations 1 and 2 .

The measured Bragg peak of the ${ }^{11} \mathrm{C}$ beam produced from $240 \mathrm{MeV} / \mathrm{n} 12 \mathrm{C}$ occurred at a depth 
of $9.7 \mathrm{~cm}$ of water; the unreacted $12 \mathrm{C}$ beam emerging from the target had been measured earlier to have the Bragg peak at a depth of $10.6 \mathrm{~cm}$. The absence of any peak at $10.6 \mathrm{~cm}$ depth conf irmed the near $100 \%$ purity of the separated $11_{C}$ beam, and decay measurements confirmed the $11 \mathrm{C}$ half life of $20 \mathrm{~min}$. The beam flux monitored at the experimental area was typically $2 \times 10^{7}$ of $11 \mathrm{C}$ particles/pulse compared to the flux of $1.5 \times 10^{10}$ particles of ${ }^{12} \mathrm{C}$ beam--a yield of about one part in 750 .

Results comparing the physical characteristics of $20 \mathrm{Ne}$ (the parent beam) and $19 \mathrm{Ne}$ (the radioactive beam) are both shown in Figure 1 . Data have been plotted in Figure 1 to show the Bragg ionization phenomenon for a pure $20 \mathrm{Ne}$ beam from the Bevalac. The peak of ionization occurs at $15.6 \mathrm{~cm}$ of water, which corresponds to about $425 \mathrm{MeV} / \mathrm{n}$. The Bragg peak is quite sharp, and the separation between the maximum range of the particles and that of the Bragg peak is only about $2 \mathrm{~mm}$.

In order to produce $19 \mathrm{Ne}$, we put $3 \mathrm{~cm}$ of beryllium in the parent beam path so that $20 \mathrm{Ne}$ can undergo nuclear fragmentation. A fraction of the parent beam loses one neutron and is converted into $19 \mathrm{Ne}$ nuclei. Figure 1 shows the data for $19 \mathrm{Ne}$ when this particular beam was tuned into the experimental area instead of $20 \mathrm{Ne}$. The Bragg peak of $19 \mathrm{Ne}$ occurs at $10.25 \mathrm{~cm}$, about $0.95(=19 / 20)$ times the residual range of $20 \mathrm{Ne}$ beam. The residual range of $20_{\mathrm{Ne}}$ after it went through $3 \mathrm{~cm}$ of beryllium was $10.8 \mathrm{~cm}$. Beyond the range of $19 \mathrm{Ne}$, there are a few more small peaks, indicating a small contamination with such products as $17 \mathrm{~F}$ (half life $\sim 60 \mathrm{sec}$ ) and 150 (half life $\sim 2 \mathrm{~min}$ ). All these species have approximately the same charge-to-mass ratio, and hence could not be separated. Nevertheless, the amount of contamination is very small, and their half lives and ranges are so different that the electron density measurements done witn $19 \mathrm{Ne}$ beam are not affected.

\section{SUITABLE RADIOACTIVE BEAMS AND A DETECTOR DEVICE (PEBA)}

Positron emission (the decay mode of ${ }^{11} \mathrm{C}$ and $19 \mathrm{Ne}$ ) allows one to easily pinpoint the location of the nuclear disintegration, i.e., the point where the beam particle stopped. The positron stops within a short distance. (typically less than $2 \mathrm{~mm}$ ) and anninilates, producing two $511-k e V$ photons given off in opposite direction. Radioactive beams that decay by positron emission have special advantages over other radioactive beams for increased efficiency in detection." The two detectable gamma-rays (511- keV) are generated with an angle of $180^{\circ}$ between them, and also in time coincidence. In such a situation a collimator is not necessary, and the effective solid angle is increased enormously.

In spite of these fine advantages of radioctive beams, much of the success of the techique depends upon the availability of a proper detection device. Such a device has been constructed in our laboratory (Llacer et al., 1979).
We call it a "positron emitter beam analyzer" (PEBA). Briefly, there are two banks of detectors, and each detector is made up of large crystals of NaI (Tl). In order to bring the detectors as close as possible to the targets (which can range in diameter from approximately 20 to $50 \mathrm{~cm}$ ), the two banks can be separated by a variable distance. Economics have dictated the use of standard detector sizes and a limitation to $48 \mathrm{NaI}(\mathrm{Tl}$ )-photomultiplier detectors at this time. Figure 2 is a schematic drawing of the arrangements of the detectors which are $1.9 \mathrm{~cm}$ in diameter and $7.62 \mathrm{~cm}$ in length.

Efficiency measurements demonstrate that the system can detect approximately $0.34 \%$ of gamma ray pairs in coincidence for a distance of $37 \mathrm{~cm}$ between planes, or $7,752 \mathrm{cts} / \mathrm{min} / \mu \mathrm{Ci}$ without absorber and $100 \mathrm{keV}$ energy threshold. Efficiency depends approximately on the reciprocal of distance between detector planes. Although PEBA in its first configuration has a low efficiency of detection, it can provide enough accuracy for initial development procedures. A radiation dose of approximately 8 rad at the Bragg peak region, using $19_{\mathrm{Ne}}$, corresponds to about 66,000 gammaray pairs emitted in a measurement time of $5 \mathrm{sec}$. This results in 80 coincidence counts using a phantom similar to a human head. The expected standard deviation for finding the centroid of the end-of-range peak is $0.7 \mathrm{~mm}$ under the above conditions. Doubling the number of detectors, which is feasible, would decrease the dose by a factor of 4 for the same accuracy, or would allow a deviation of $0.5 \mathrm{~mm}$ with 4 rad only at the stopping region.

\section{BRAGG PEAK LOCALIZATION}

Several exploratory measurements have been conducted with radioactive beams to test the feasibility of using these beams to measure effective stopping power of neterogeneous media for heavy charged particles. Such measurements will provide direct information on the average electron density and average stopping number

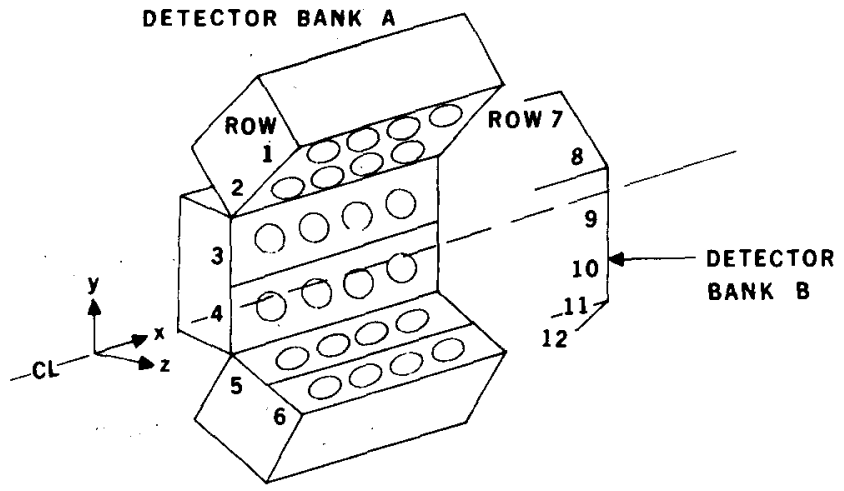

Figure 2. Conceptual design of the detector device (PEBA) showing two banks of 24 detectors, defining a volume along the path of a heavy-ion beam. (XBL 772-7742) 
(see equation 1) of a target with an unknown heterogeneous beam path. Once this information is obtained with a suitable radioactive beam, it can be used in equations 1 and 2 to calculate the energy of any heavy particle of therapeutic choice so that the Bragg peak of the therapeutic beam can be placed on the tumor volume. Figure 3 is a representation of the general geometry used in experiments. A beam of high-energy heavy ions was collimated to a diameter of $1.58 \mathrm{~cm}$ (PEBA has a good positional accuracy as long as the beam diameter is less than $2 \mathrm{~cm}$ ), and made to enter target materials (mixed or homogeneous) positioned between the detector banks and centered along the beam axis.

We have made measurements with $11 \mathrm{C}$ and $19 \mathrm{Ne}$ beams, but the short half life of $19 \mathrm{Ne}(\sim 19 \mathrm{sec})$ allows prompt repeated measurements, making that nucleus very interesting for our purposes. We will report only the results obtained with it.

Prior to the data collection with the $19_{\mathrm{Ne}}$ beam, a $22 \mathrm{Na}$ point source embedded in plastic was used to establish the $x=$ zero reference point on the detector. The activity of the point source was seen by PEBA as a nearly Gaussian distribution with a full width at half maximum of about $1 \mathrm{~cm}$. The centroid of the distribution was established as $x=$ zero. Centroid position was found to be reproducible with $\pm 0.5 \mathrm{~mm}$ accuracy, or better if at least 150 counts were accepted to form an image.

The initial energy of the $20 \mathrm{Ne}$ parent beam was $425 \mathrm{MeV} / \mathrm{n}$, and that of the product $19 \mathrm{Ne}$ beam was $302 \mathrm{MeV} / \mathrm{n}$. In the experiment reported here, ten pulses from the Bevalac (with a period of approximately $4 \mathrm{sec}$ ) were injected into a solid lucite cylinder $25 \mathrm{~cm}$ long and $7.62 \mathrm{~cm}$ in

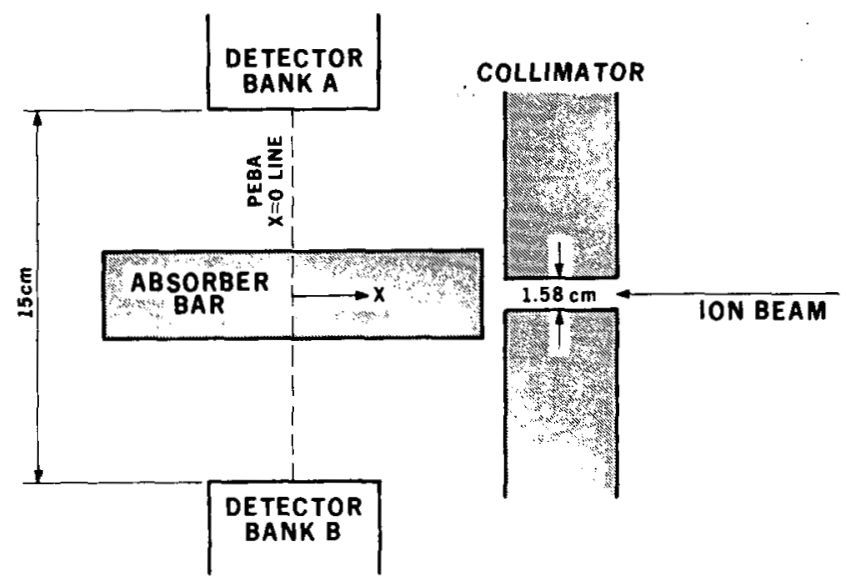

Figure 3. General geometrical setup for experiments with PEBA in the irradiation chamber. The $x=$ zero line is placed in the center of the detector banks. The distance between the banks (shown in the diagram as $15 \mathrm{~cm}$ ) can be varied so that maximum efficiency can be obtained with irradiation subjects of various dimensions. (X81 788-9963) diameter. The purpose was to compare the measured beam-path length with the calculated value for a material of well-known composition. The total number of particles injected was $5.94 \times 10^{5}$, yielding approximately $8.95 \times 10^{4}$ disintegrations in the first $10 \mathrm{sec}$ measurement interval starting $4 \mathrm{sec}$ after the end of the irradiation. The lucite cylinder was placed axially in the beam path, as in Figure 3. The separation between the two banks of crystals was $20 \mathrm{~cm}$, and all the crystals were focused on the beam axis. The zero of the PEBA device was at $10.1 \mathrm{~cm}$ from the (direction of the beam) leading end of the lucite bar.

Figure 4 shows the video output of the PEBA data analys is program. A peak centroid at $x=1.57 \mathrm{~cm}$ (PEBA coordinates) is shown. The average activity detected uncorrected for absorption was $59 \mathrm{nCi}$, with a total of 173 counts collected. The efficiency was found to be $0.26 \%$ for the configuration used, consistent with data obtained with fixed radiation sources. Expected error in the measurement was $a=0.05 \mathrm{~cm}$. The fluctuation depth of the 19 Ne beam in repeated measurements was $(10.1-1.57)=8.53 \mathrm{~cm}$. $0.05 \mathrm{~cm}$.

Since the theoretical mass penetration depth of $19 \mathrm{Ne}$ in lucite is within $1 \%$ that of water, and the density of the plastic has been measured to be 1.186 , the theoretical penetration can be calculated as (penetration in water/1.186). Froin the original data of Figure 1 , we estimate the maximum penetration in water to be $10.3-0.1 \mathrm{~cm}$; the corresponding penetration in lucite would then be $8.68 \pm 0.1 \mathrm{~cm}$. This result agrees well with the PEBA measurements within the expected errors.

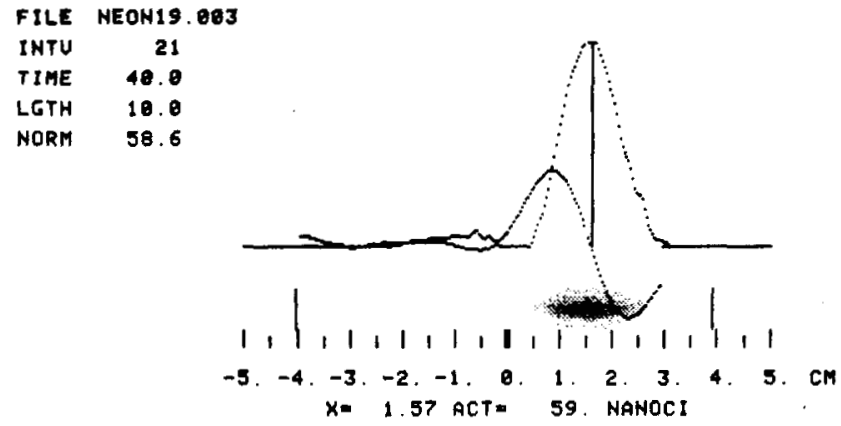

Figure 4. One-dimensional image of the end-ofrange peak of the ${ }^{19} \mathrm{Ne}$ beam of Figure 1 in an absorber of lucite. The centroid of the peak is measured by a convolution of the image with an antisymmetric function selected for insensitivity to statistical fluctuations. The centroid is obtained at the zero crossing of the results of the convolution, also shown in the figure (sinusoidal curve). The activity detected was $59 \mathrm{nCi}$ (uncorrected for absorption); it was the result of $10 \mathrm{sec}$ of counting ( $4 \mathrm{sec}$ after the end of a ten pulse irradiation lasting $40 \mathrm{sec})$. (XBL 7912-13606) 
Similariy, accurate results were obtained when ranges of $19 \mathrm{Ne}$ were measured in pure paraffin (fat-like) and pure $\mathrm{CaSO}_{4}$ (bone-like) materials, establishing the technique with appropriate precision. In another experiment, various thin slices of different materials were interposed between the beam and solid lucite cylinder. Progressively, from the beam entry port, the materials were a $3-\mathrm{mm}$ thick $\mathrm{CaSO}_{4}$ plate, a $1-m m$ lucite plate, a 9-mm paraffin plate, and the long lucite column. As in Figure 4, we examined the video display of the beamstopping region in the reference material lucite rod. The residual range in the lucite rod was $7.54 \mathrm{~cm}$, and the actual calculation from the stopping-power formula for the complex heterogeneous column shows the range should be $7.60 \mathrm{~cm}$.

In the future these measurements will be made with suitable phantoms, with animals, and ultimately with patients. The goal is to demonstrate that the radioactive-beam technique and the PEBA device can provide very useful information for treatment planning, using heavy particle beams in the Bragg peak region.

\section{POTENTIAL APPLICATIONS OF RADIOACTIVE BEAMS}

The medical interest in heavy-particle therapy arises because of the possibility of delivering a maximum dose (Bragg peak) at the tumor volume while minimizing the dose in the surrounding normal tissue region. However, a slight error in localizing the Bragg peak at the appropriate location may lead to undesirable effects. A lack of knowledge in the tissue nonhomogeneity is the major cause of this error. Unless this nonhomogeneity is fully compensated for, the full potential of Bragg peak therapy by heavy particles will never be realized. The radioactive-beam technique, along with a positron imaging device like PEBA can overcome this practical difficulty, even in the regions of the body such as the thorax where the degree of variation in tissue nomogeneity is large. This particular technique may prove to be the most powerful tool available for mapping the beamstopping region with great accuracy.

The dose required for the localization of Bragg peak should eventually be as small as $2 \mathrm{rad}$. The accuracy can be as good as $1 \mathrm{~mm}$ in a measurement time short enough that the elimination of activity through blood flow may not pose any problems in determining the Bragg peak location. Further measurements are needed to evaluate those effects, and such studies are in progress.

It is nighly desirable, and it may even be necessary to acquire all the required diagnostic information leading to Bragg peak localization within a very short time. A data collection time of abou't 10 minutes for ten beam positions seems feasible with a 19 Ne beam, although a maximum ime of $1 \mathrm{~h}$ would be clinically acceptable. This onsideration is especially important when an irregularly shaped compensator has to be used (H. Suit, private communication). In such a situation the radioactive beam is made to scan along the compensator, and the stopping points are determined at each beam position. Interference in activity measurements between neighboring points should be kept to a minimum. Radioactive particles with short half lives are the best candidates for these diagnostic procedures. $19_{\mathrm{Ne}}$ and $15_{0}$ are ideally suited radioactive beams considering that one has to wait about three physical half lives between each measurement. Other radioactive particles of longer half lives may also be suitable if one takes into account the elimination of activity due to tissue perfusion by blood flow.

Irrespective of the choice of the therapeutic beam, ${ }^{19} \mathrm{Ne}$ or 150 can always be used to obtain information which will ensure the placement of the Bragg peak of the thereapy beam at the desired location in a short time. For example, if $12 \mathrm{C}$ particles have been selected to treat a tumor volume, $19 \mathrm{Ne}$ then can be used to determine the average electron density along the beam path. This information will help determine the required energy for $12 \mathrm{C}$ particles to stop at the same place where $19 \mathrm{Ne}$ stopped. We want to verify the correctness of this procedure experimentally by using phantoms and animals. Just for the purpose of demonstration, we may choose $19 \mathrm{Ne}$ and $11 \mathrm{C}$ instead of $19 \mathrm{Ne}$ and $12 \mathrm{C}$ (as mentioned in the above example), because ${ }^{1} I_{C}$ is also a positron emitter, and thus allows us a direct verification of our procedure. The radioactive-beam technique has the potential of being highly useful, and will not be limited by the choice of a particular therapy beam. Also, the Bevalac will have the capability of switching from one beam to another within a very short time.

Bragg peak localization will not be the only use of radioactive beams. Its application in nuclear medicine may give rise to new diagnostic techniques that are not possible at present. Because they are instant tracers, radioactive beams can be implanted quickly into the body without invasive methods--even in a single beam pulse of $1 \mathrm{msec}$ duration; in the next instant, it becomes possible to trace their transport by the help of a suitable detection device. $19 \mathrm{Ne}$ appears to be a good candidate for these applications. Radioactive-beam techniques may prove to have special advantages over the techniques used in conventional nuclear medicine, since these beam particles can be deposited in precise locations witnout the need for injection or surgical approach. Possible applications in detecting microcirculation rates in various parts of the brain will be another milestone in nuclear medicine techniques.

\section{ACKNOWL EDGEMENT}

We wish to thank Mrs. Grace Walpole for editing and typing this manuscript. 
REFERENCES

Alonso, J. R., A. Chatterjee, and C. A. Tobias. 1979. High purity radioactive beams at the Bevalac. IEEE Trans. Nucl. Sci. NS-26(1), 3003-3005.

Bennett, G. W., J. 0. Archambeau, B. E. Archambeau, J. E. Meltz, and C. L. Wingate. 1978. Visualization and transport of positron emission from proton activation in vivo. Science 200, 1151-1153.

Bethe, H. A. 1930. Zur Theorie des Durchgangs schneller Korpuskularstrahlen durcn Materie. Ann. Physik 5, 325-400.

Chatterjee, A., C. A. Tobias, and J. T. Lyman. 1976. Nuclear fragmentation in therapeutic and diagnostic studies with heavy ions. Spallation Nuclear Reactions and The ir Applications (B. P. Shen and M. Kerker, eds.) pp. 169-191. D. Reidel Publishing Company, Dordrecht-Holland.

Chen, G. T. Y., R. P. Singh, J. R. Castro, J. T. Lyman, and J. M. Quivey. 1979. Treatment planning for charged particle radiotherapy. Int. J. Radiat. Onco 1. Biol. Phys. 5, 1809-1819.

Ghiorso, A., H. Grunder, W. Hartsough, G. Lambertson, E. F. Lofgren, K. Lou, R. M. Mair, R. Mobley, R. Morgado, W. Salsig, and F. Selph. 1973. The Bevalac-an economical facility for very high energetic heavy particle research. IEEE Trans. Nucl. Sci. NS-20(3), 155-158.

Greiner, D. E., P. J. Lindstrom, H. H. Heckman, B. Cork, and F. S. Bieser. 1975. Momentum distributions of isotopes produced by fragmentation of relativistic $\mathrm{C}-12$ and $0-16$ projectiles. Phys. Rev. Lett. 35, 152-154.
Heckman, H. H., D. E. Greiner, P. J. Lindstrom, and F. S. Bieser. 1971. Fragmentation of nitrogen-14 nuclei at $2.1 \mathrm{GeV}$ per nucleon. Science 174, 1130-1132.

Lawrence, J. H., C. A. Tobias, and J. A. Linfoot. 1971. Heavy particles in the treatment of acromegaly and Cushing's disease and their potential value in other neoplastic diseases. Nuclear Medicine, 2nd ed. (W. H. Bladh, ed.) pp. 806-820. MCGraw Hill, New York.

Linfoot, J. A., C. Y. Chong, J. F. Garcia, A. S. Cleveland, G. M. Connell, E. Manougian, M. D. Okerlund, J. L. Born, and 3. H. Lawrence. 1971. Heavy particle therapy for acromegaly, Cushing's disease, Nelson's syndrome and nonfunctioning pituitary adenomas. Progress in Atomic Medicine: Recent Advances in Nuclear Medicine, (J. H. Lawrence, ed.) vol. 3, pp. 219-238. Grune and Stratton, New York.

Llacer, J., A. Chatterjee, B. Jackson, J. Lin, and $V$. Zunzunegi. 1979. An imaging instrument for positron emitting heavy ion beam injection. IEEE Trans. Nuc 1. Sci. NA-26(1), 634-647.

Lyman, J. T. and J. Howard. 1977. Dosimetry and instrumentation for helium and heavy ions. Int. J. Radiat. Oncol. Biol. Phys. 3, 81-86.

Tobias, C. A., H. O. Anger, and J. H. Lawrence. 1952. Radiobiological use of high energy deuterons and alpha particles. Am. J. Roentgeno1. Radium Ther. Nucl. Med. 67, 1-27.

Todias, C. A., J. T. Lyman, A. Cnatterjee, J. Howard, H. D. Maccabee, M. R. Raju, A. R. Smith, J. M. Sperinde, and G. P. Welcn. 1971. Radiological pnysics characteristics of the extracted heavy-ion beams of the Bevatron. Science 174, 1131-1134. 
PART VI. MEDICAL APPLICATIONS OF HEAVY IONS Section C: Focal Lesions 


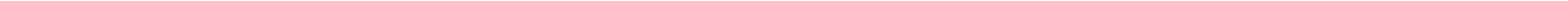




\title{
RECENT ADVANCES IN PITUITARY RESEARCH
}

\author{
J. A. Linfoot
}

The investigational program on human pituitary therapy carried on at LBL has relied on passing plateau phase ions through the pituitary gland rather than using the Bragg ionization peak. There are two general reasons for this (Tobias, 1979). First, there are uncertainties in placing the Bragg peak in the center of the pituitary since the exact stopping power of the tissues along the beam pathway is not known. Erroneous placement of the Bragg peak could result in high doses to the cranial nerves or temporal lobe. Second, the Bragg ionization curve of the $900 \mathrm{MeV}$ helium ions from the 184-inch Synchrocyclotron is unnecessarjly broad due to straggling and multiple scattering. Certain heaky ions available at the Bevalac, such as ${ }_{6} \mathrm{C}$ and $10 \mathrm{Ne}$, promise great improvements in pituitary radiation techniques. The exact localization of the Bragg peak is possible with the aid of the radioactive beams ${ }_{11} \mathrm{C}$ and 19 Ne particles (see Tobias, 1973 and the chapter by Chatterjee et al. in Part VIB). In addition, the multiple scattering and straggling are significantly smaller, so that the dose can be better localized with the heavier particle beams than with helium ions. Finally, the high biological effectiveness of stopping carbon and neon ions would enhance the effects of these particles on the pituitary gland.

Using heavy ions and the Bragg peak, single or multiple laminar lesions or larger lesions using the spread peak should produce focal or total destruction of normal or abnormal pituitary tissue because of the high RBE. The spatial localization of hormone secreting cells within the pituitary of primates (Herbert and Hayashida, 1974) and man (Hardy, 1979) offers a physiological parameter to determine the effects of localized radiation on the pituitary in vivo.

While $910 \mathrm{MeV}$ helium ions have been highly. successful in the treatment of hormone secreting. pituitary tumors; e.g., those associated with acromegaly, Cushing's disease, and amenorrheagalactorrhea syndromes (Linfoot, 1980), there are a number of patients who would benefit from the hormonal effects of rapidablation of either a small selected portion or total destruction of the pituitary gland by a nonsurgical technique, performed on an ambulatory basis.

The aims of the present study are to. investigate the neuroendocrine, neuroanatomicai, and other effects of irradiation on defined $\cdots: 3$ regions of the pituitary with $12 \mathrm{C}$ from the:Bevalac and to correlate the onset of specific hormonal changes to anatomical localization of the focal lesions as well as dose and mode of heavy ion delivery. It is further planned to tevelop methodology for total pituitary destrucion without producing localized or widespread radionecrosis of surrounding neural and neuroendocrine structures such as the cranial nerves, temporal lobes, and hypothalamus.

\section{METHODS}

Three experiments using $250 \mathrm{MeV} / \mathrm{amu}{ }^{12} \mathrm{C}$ have been initiated on Cynomolgus monkeys. In the initial experiment (LBP) a tightly collimated $6 \mathrm{~mm}$ circulator beam from two opposing ports was used to produce bilateral Bragg peak lesions estimated to measure $2 \mathrm{~mm}$. High LET laminar lesions with a plateau dose of 2,000 rad (skin dose) with an estimated peak-to-plateau ratio of $\sim 6$ to 1 (range 5-7 to 1 ) were placed at the margins of the pituitary. Plateau portions of the beam overlapped within the central portion of the gland. The composite peak dose, determined by addition of the entry dose $(\sim 2,000 \mathrm{rad})$ and the estimated peak dose $(12,000$ rad), was $\sim 14,000$ rad, while the central pituitary dose composed of trailing Bragg peaks plus the overlap was estimated to be 10,000 rad. An isodose curve for this experiment is presented in Figure 1.

In the second experiment, bilateral central Bragg peaks (CBP) were superimposed in the

\section{CORONAL VIEW (Mag $=2.0)$ TEST $(6 \mathrm{~mm}$ overlop)}

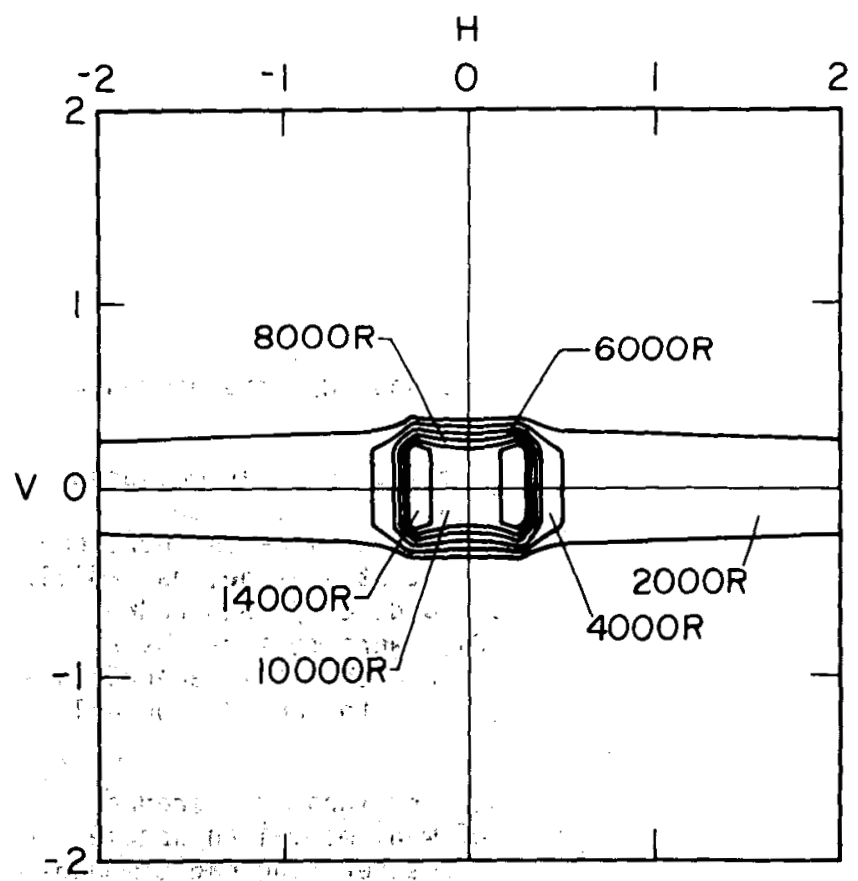

Figure 1. Coronal view of isodose curve employed in experiment I. Bragg peak $250 \mathrm{MeV} / \mathrm{amu}$ 12C were localized to the lateral margin of the pituitary opposite the site of entry. An entry dose of 2,000 rad, $6 \mathrm{~mm}$ circular aperture and bilateral oppositional ports were used. The composite dose was calculated using peak-toplateau ratio of $\sim 5$ to 1 and the addition of the superimposed peak and plateau doses. (XBL 806-3375) 
central portion of the pituitary gland. A similar circular $6 \mathrm{~mm}$ beam and bilateral oppositional ports were used. The plateau entry. dose, however, was reduced to 1,500 rad (sk in dose). The peak-to-plateau ratio was again estimated to be $\sim 6$ to 1 with an estimated Bragg peak dose of $9,000 \mathrm{rad}$, and, assuming both peaks were completely superimposed, resulted in a combined central dose of $-18,000$ rad. The third experiment employed overlapping $5 \mathrm{~mm}$ spread Bragg peaks with the distal peaks localized to the opposite lateral margin of the pituitary and overlap of the spread peaks within the remainder of the gland. $12 \mathrm{C}$ plateau dose of $1,500 \mathrm{rad}$, immobilization, and other techniques were similar to those used in the second experiment.

Correction for variation in tissue density required for accurate localization of the Bragg peak was obtained by means of axial computerized tomographic (CT) scans (Ambrose, 1973), $910 \mathrm{MeV}$ helium ion beams scans, and $250 \mathrm{MeV}{ }^{12} \mathrm{C}$ ion radiography techniques (Tobias et al., 1977). Pituitary localization in the monkeys was achieved using AP and lateral skull $X$ rays and low energy beam spots. The monkeys were anesthetized with ketamine during the radiation procedure and head movement was restricted using a soft cast technique in experiment LBP. A specially designed headholder with a cast bite block inserted in the oral cavity was used in LBP and SBP.

Prior to radiation, baseline measurements were obtained under ketamine, pentobarbital, or ketamine-methoxyflurane anesthesia. Ketamine was determined to be the most satisfactory for short term studies. After 0 and $-15 \mathrm{~min}$ baseline samples, provocative hormonal testing was carried out using a cocktail consisting of thyroid releasing hormone (TRH), gonadotrop in releasing hormone (GnRH), regular U40 insulin, and, in experiment 1 animals, arginine hydrochloride. Peptide hormones for growth hormone $(\mathrm{GH})$, prolactin (PRL), thyroid stimulating hormone (TSH), luteinizing hormone (LH), and, in selected cases, follicle stimulating hormone (FSH) were measured using standard double antibody NIH radioimmunoassay (RIA) kits.

The target hormone, thyroxine, was measured as total thyroxine by RIA as well as free thyroxine by a solid phase technique (Witherspoon et al., 1980). Cortisol, testosterone, estradiol, and the plasma 17-keosteroid, dehydroepiandrosterone sulfate (DHEA-SO $\mathrm{S}_{4}$ ), were measured by RIA using tritiated steroids and charcoal separation (J. Linfoot, H. Hwang, T. Saito, and G. Connel1, unpublished methods).

Because of chemical structural differences of pituitary peptides of many mammalian species (Humme 1 et al., 1974), crossreacting radioimmunoassays are not available for many commonly used animal species. Fortunately, there is sufficient crossreactivity between most primate and human peptide normones so that with modification, currently available human assays can be used in the Cynomolgus monkey.

In order to determine nutritional, seasonal, or stress variations, one male and one female monkey were not irradiated and served as controls. Endocrine studies were performed at the same time interval and in the same fashion as the irradiated animals. Monkeys in the CBP and SBP experiments were irradiated recently, and on ly data obtained at 38 days after irradiation were used to compare the results in the three experiments.

Complete autopsy examinations are planned for a 17 groups and have been performed on a 11 but one monkey from the LBP experiments that is being maintained for long-term radiation effects. Histological samples were prepared from brain, pituitary, target glands, and other major organ systems in order to determine, endocrine or hormonal effects and the presence or absence of radionecrosis. Pituitary tissues were divided and fixed in buffered formalin and mercuric chloride for light microscopy and immunohistochemical staining. Samples for ultrastructural studies were fixed initially in gluteraldehyde and postfixed in osmium tetroxide for both scanning and transmission electron microscopy.

\section{RESULTS}

Serial changes in pituitary peptide hormone concentrations in monkeys treated with LBP are illustrated in Figure 2 and 3 . Because of variations in stress response to anesthesia, each animal's postirradiation data are compared to its own preirradiation value as well as to the mean and standard error of six to eight tests conducted on the control monkeys. The stress response studies comparing ketamine, pentobarbital, and ketamine-methoxyflurane anesthesia show that ketamine alone, while stimulating the release of ACTH (Redgate et al., 1972) is probably the least stressful on the monkeys and, for serial provocative studies such as employed in these experiments, is most satisfactory.

Progressive change in prolactin and TSH was observed, however, complete loss of these peptide hormones was not achieved with the focal lesions in the lateral lobes of the pituitary. Growth hormone (not shown) was also suppressed early in the experiment and remained suppressed throughout the study. There was no significant change in basal LH secretion in any of the male monkeys. provocative responses of $L H$ and FSH to $G n R H$ were not observed in either the control or treated monkeys prior to or after radiation under ketamine, pentobarbital, or ketamine-methoxyflurane (Mori and Hafez, 1973). In spite of efforts to increase sensitivity of the human hormone assays, the problem of poor crossreactivity with simian gonadotropins (Hotchkiss et al., 1971) may not have been completely eliminated in our system and/or the stress release of prolactin may have inhibited LH and FSH. Little change in ACTH (not measured directiy) was implied by the fact that the basal levels of cortisol and DHEA-SO4 did not decrease, and the cortisol response to insulin hypoglycemia was not appreciably blunted in any of the irradiated monkeys. In all three experiments, basal secretion of other target hormones, e.g., gonadal steroids, testosterone, and estradiol showed no consistent or significant 
PROLACTIN RESPONSE TO TRH
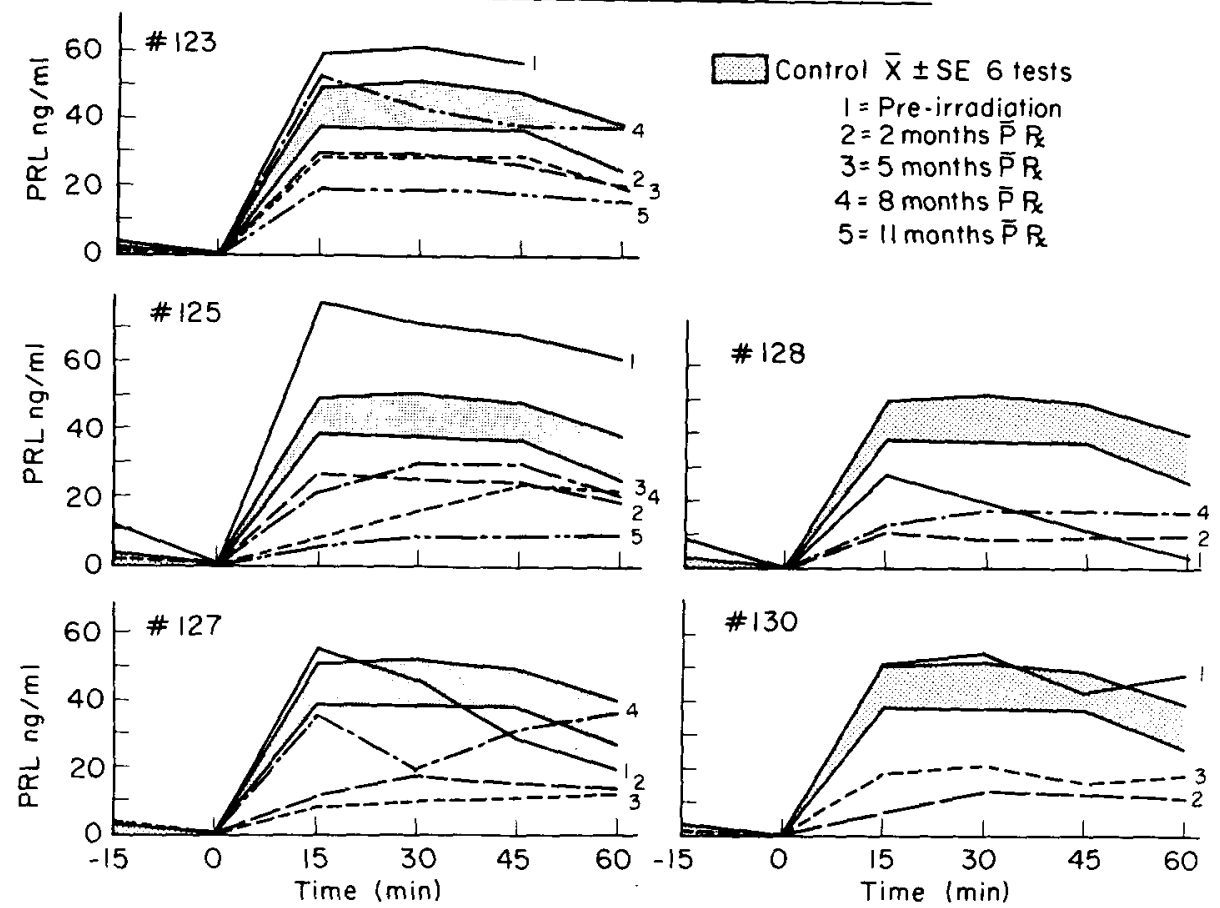

Figure 2. Serial changes in pituitary prolactin hormone secretion before, 2, 5, 8, and 11 months after $250 \mathrm{MeV} / \mathrm{amu}{ }^{12} \mathrm{C}$ irradiation of Cynomolgus monkeys utilized in experiment $I$. The lateral Bragg peak (LBP) (see Fig. 1) was used in these experiments. The hormonal responses to pharmacological stimulation were.measured after the rapid intravenous injection of TRH, GnRH, insulin, and arginine hydrochloride. Normal responses in a control monkey studied similarly under ketamine anesthesia is presented in the shaded area. (XBL 806-3377)

change. Total thyroxine did not change significantly; however, free thyroxine did change coincident with the fall in TSH secretion (see Tables $1 A$ and $1 B)$.

Tables $2 A$ and $2 B$ compare the data from three experiments (LBP, CBP, and SBP) obtained at 38 days after irradiation. Growth hormone response to hypoglycemia appears to fall in all groups except for one monkey in the SBP group, which suggests that the minimal dose, 1,500 rad, , delivered to the lateral margin of the pituitary gland in experiment II (CBP) had sufficient biological effect to substantially decrease GH secretion. Previous studies indicate somatotropes to be the most radiosensitive pituitary cells (Simpson et al., 1959; Van Dyke et al., 1959; Tobias, 1979). It is not clear from these in vivo studies whether the LBP or SBP animals differed in this effect. The CBP; and SBP differed from the LBP monkeys and had little or no blunting of TSH or PRL secretion nor any $f a l l$ in the target hormones, including cortiso 1 and free thyroxine. If complete superimposition of the peaks occurred, a greater effect on cortisol in the CBP animals might have been anticipated since the central necrotic lesions should have involved the corticotropes which tend to localize near the intermediate lobe at the center of the gland. Further post-mortem studies to confirm the accuracy of peak placement will be performed at the end of the experiments, and combined with histological changes in cell composition of the pituitary, will clarify the significance of these in vivo effects.

Lower plateau doses were selected for the CBP and SBP experiments because pigmentary skin lesions and slight epilation of the skin were observed in several monkeys in the LBP group. At the time of autopsy of the LBP monkeys, however, no gross radionecrotic lesions were observed in the cerebral cortex in any of the animals, except for one animal who was improperly aligned during irradiation. In spite of ketamine anesthesia and head immobilization, the animal moved and sustained brain stem as well as cortical irradiation. This animal had atrophy of the third and fifth cranial nerves and had small hemorrhagic radionecrotic lesions at the site of Bragg peak in the hypothalamus but no cortical lesions. The presence of microscopic radionecrotic tracts from plateau irradiation $(\sim 2,000$ rads) of the brain stem suggests a greater radiosensitivity of cranial nerves and hypothalamus than cerebral cortex in this anima?. No gross radionecrosis has been observed in the other autopsied animals. Light and electron microscopic studies are currently in progress.

Previous animal studies with high energy beans focused on the center of the pituitary gland and demonstrated that single central plateau doses of $190 \mathrm{MeV}$ deuterons or $340 \mathrm{MeV}$ 

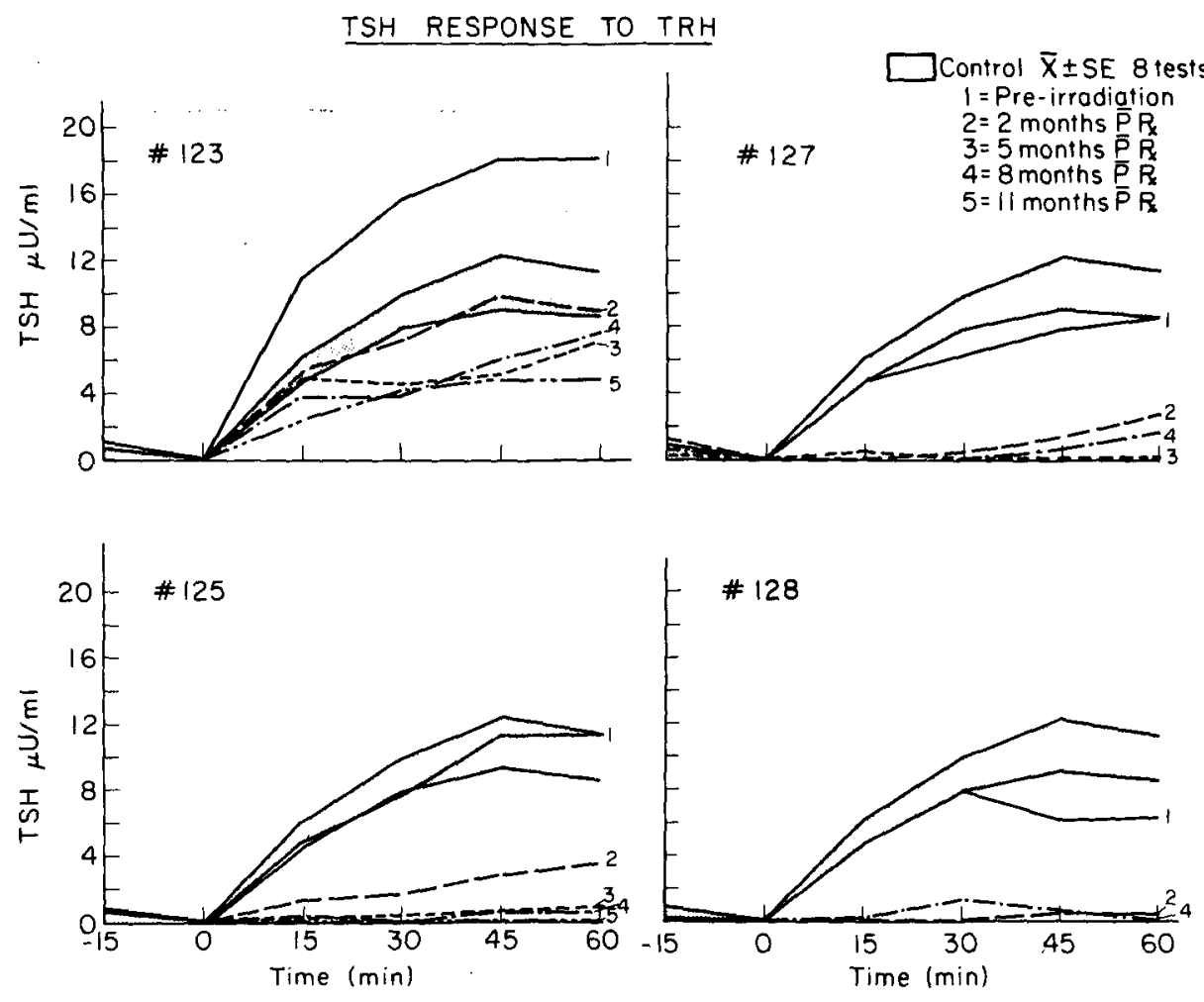

Figure 3. Serial changes in pituitary TSH hormone secretion before, $2,5,8$, and 11 months after $250 \mathrm{MeV} / \mathrm{amu}{ }^{12} \mathrm{C}$ irradiation of Cynomolgus monkeys utilized in experiment $\mathrm{I}$. The lateral Bragg peak (LBP) (see Fig. 1) was employed in these experiments. The hormonal responses to pharmacological stimulation were measured after the rapid intravenous injection of TRH, GnRH, insulin, and arginine hydrochloride. Normal responses in a control monkey studied similarly under ketamine anesthesia is presented in the shaded area. (XEL 806-3376)

Table 1A. Experiment 1: Lateral Bragg Peak (LBP). Mean Maximum Response of Pituitary Peptides to Provocative Pharmacological Testing Mean Increment Change From Zero Time

\begin{tabular}{lccccc}
\hline & No. Tests & $\begin{array}{c}\mathrm{TSH} \\
\mu \mathrm{U} / \mathrm{ml}\end{array}$ & $\begin{array}{c}\mathrm{PRL} \\
\mathrm{ng} / \mathrm{dl}\end{array}$ & $\begin{array}{c}\mathrm{GH} \\
\mathrm{ng} / \mathrm{ml}\end{array}$ & $\begin{array}{c}\mathrm{LH} \\
\mathrm{mIU} / \mathrm{ml}\end{array}$ \\
\hline $\begin{array}{l}\text { Preirradiation } \\
\text { Postirradiation }\end{array}$ & 10 & 11.3 & 69.3 & 4.64 & 1.01 \\
2 months & 6 & 5.5 & 23.6 & 7.68 & 1.35 \\
5 months & 4 & 2.5 & 21.4 & 0.84 & 1.16 \\
8 months & 5 & 2.0 & 29.2 & 0.11 & 1.09 \\
11 months & 3 & 2.3 & 14.1 & 0.08 & 0.02 \\
\hline
\end{tabular}

Changes in mean maximum peptide hormone responses in Cynomolgus monkeys before and after $250 \mathrm{MeV} / \mathrm{amu}{ }^{12} \mathrm{C}$ irradiation of the pituitary. Pharmacological stimulation of the pituitary peptides was. produced using TRH, GnRH, insulin, and arginine hydrochloride administered by rapid, consecutive intravenous injection. 
Table 1B. Experiment 1: Lateral Bragg Peak (LBP) Basal Levels Mean Target Hormone Levels

\begin{tabular}{|c|c|c|c|c|c|}
\hline & $\begin{array}{l}\text { No. } \\
\text { Tests }\end{array}$ & $\begin{array}{c}\text { Testosterone } \\
\mathrm{ng} / \mathrm{dl}\end{array}$ & $\begin{array}{c}\text { Cortisol } \\
\mu \mathrm{g} / \mathrm{dl}\end{array}$ & $\begin{array}{r}\text { DHEA-SO4 } \\
\mu \mathrm{g} / \mathrm{dl}\end{array}$ & $\begin{array}{l}\text { Free } T_{4} \\
\text { ng/di }\end{array}$ \\
\hline Preirradiation & 10 & 718 & 21.6 & 18.12 & 1.16 \\
\hline \multicolumn{6}{|l|}{ Postirradiation } \\
\hline 2 months & 6 & 469 & 17.1 & 22.88 & 0.92 \\
\hline 5 months & 4 & 324 & 10.9 & --- & 0.94 \\
\hline 8 months & 5 & 385 & 16.8 & 11.56 & 1.24 \\
\hline 11 months & 3 & 1006 & 13.4 & 19.57 & 0.70 \\
\hline
\end{tabular}

Changes in basal target hormone levels before and after $250 \mathrm{MeV} / \mathrm{amu}$ ${ }^{12} \mathrm{C}$ irradiation of the pituitary.

Table 2A. Percent Change of Mean Levels Preirradiation and 38 Days Postirradiation

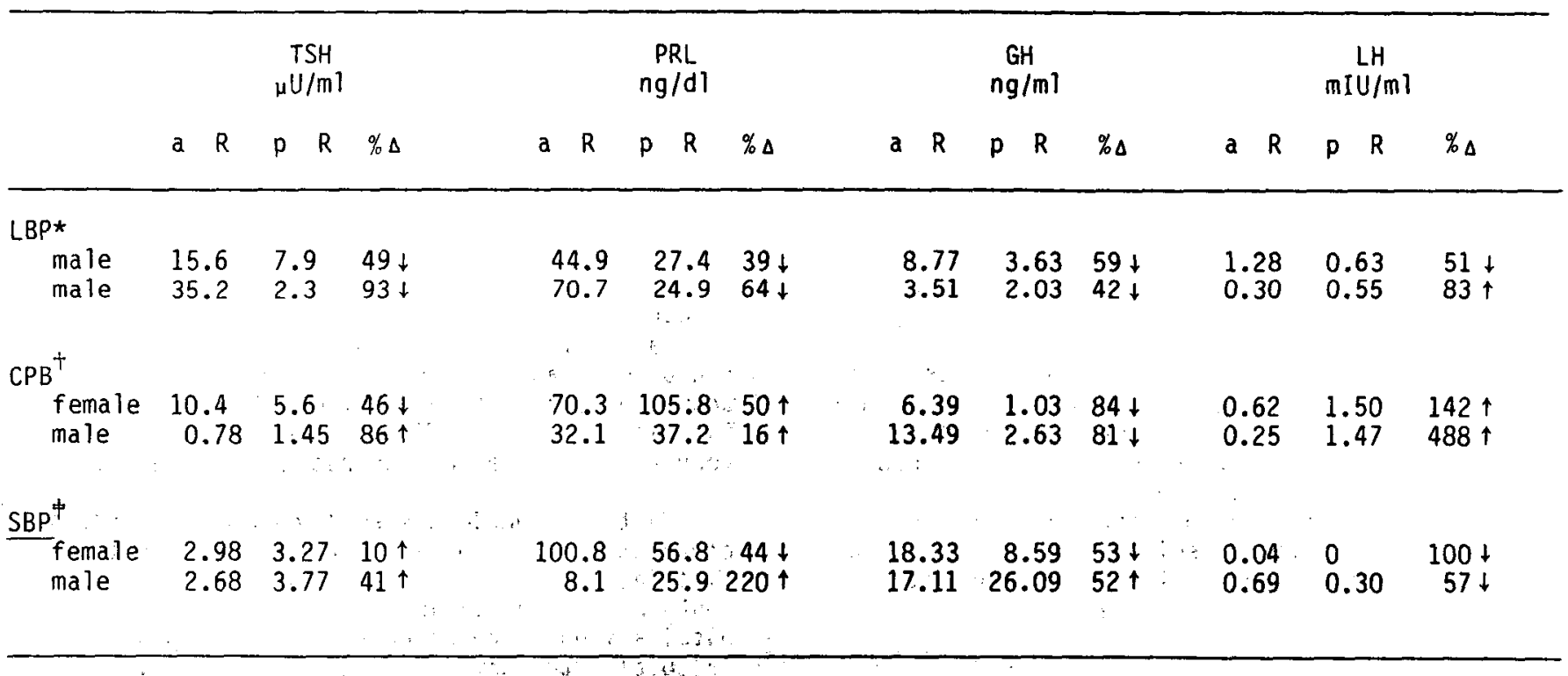

Comparative effects of $250 \mathrm{MeV} / \mathrm{amu} 12 \mathrm{C}$ irradiation of average peptide hormone secretion during pharmacological stimulation with TRH, GñH, and insulin nypoglycemia. The greatest effect at 38 days was observed in the animals treated in Experiment '1. The isodose curve (Fig. 1 ) employed in the LBP animals had a greater effect on GH, PRL, and TSH, which predominate in the lateral portion of the anterior pituitary than on ACTH, which predominates in the central portion of the pituitary. Values are expressed as absolute change from 0 time. Mean level $=(15+30+45+60 \mathrm{~min}$ values $) / 4$.

LBP: Lateral Bragg Peak, experiment 1.

CPB: Central Bragg Peak, experiment 2.

†SBP: Spread Bragg Peak, experiment 3. 
Table 2B. Percent Change of Basal. Levels Preirradiation and 38 Days Postirradiation.

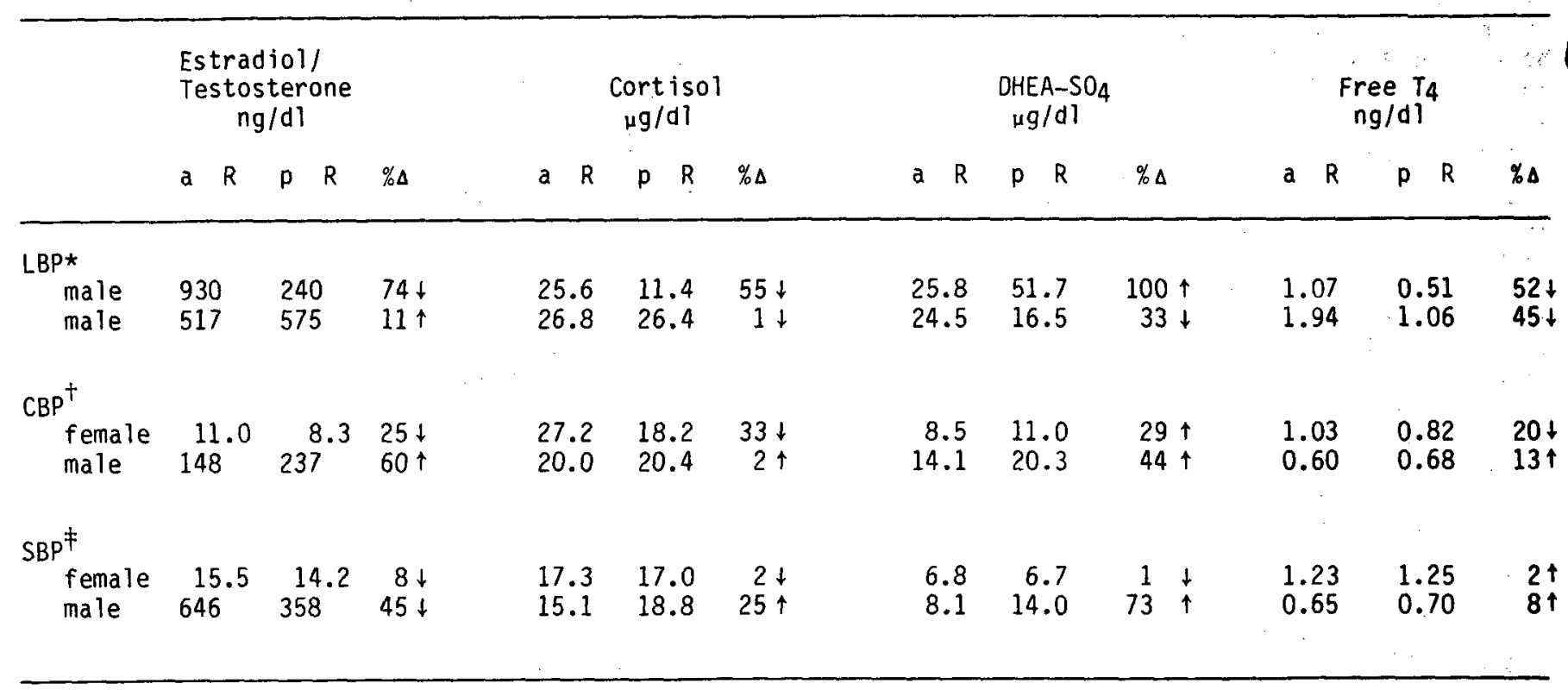

Comparative effects of $250 \mathrm{MeV} / \mathrm{amu}{ }^{12} \mathrm{C}$ irradiation on target hormone secretion. No consistent fall was observed in any experiment except for the fall in free thyroxine, which parallels the decline in TSH in experiment I.

*LBP: Lateral Bragg Peak, experiment 1.

†CBP: Central Bragg Peak, experiment 2.

†SBP: Spread Bragg Peak, experiment 3.

protons in excess of 5,000 rad produced progressive atrophy of the gland. Regardless of the rad per single dose, above 5,000 rad the amount of tissue destruction was eventually the same, although higher doses manifested earlier physiological effects (Simpson et al., 1959; Van Dyke et al., 1959). Because of the rapid fall in dose, it was determined that in the rat, dog, and monkey central doses of 30,000 rad were necessary to approximate the effects of surgical hypophysectomy. Subsequent studies using $340 \mathrm{MeV}$ protons in man demonstrated that fractionated central doses greater than 20,000 rad produced extensive histological changes in the pituitary as well as variable neurological complications. Lower doses, however, resulted in a delay in the destruction of the target tissue (Linfoot et al., 1968; Linfoot, 1979; Tobias, 1979).

\section{CONCLUSION}

From these initial three experiments using $250 \mathrm{MeV} / \mathrm{amu}$ carbon ions and with use of sensitive radioimmunoassay hormone measurements, effects on the in vivo secretion of the pituitary peptide hormones by high LET carbon ions has been demonstrated. A greater and more rapid hormonal ablative effect was observed for LBP with combined peak doses of approximately 14,000 rad in the lateral margins of the pituitary than when either higher central doses (CBP) or lower spread peak doses (SBP) were employed. The in vivo differences observed at 38 days suggest that both dose and volume of irradiated tissues determine the magnitude of the effect on hormone secretion. The greater hormonal effect of Bragg peak lesions in the lateral lobes of the pituitary conforms to other anatomical data and confirms that spatial distribution of cells in the pituitary can be used as an in vivo means of confirming accurate localization of high LET irradiation in the pituitary. Provocative hormone testing is a very sensitive measure of radiation effects.

Further experiments employing in vivo studies correlated with anatomic changes in the pituitary will facilitate the planning of future experiments employing multiple ports and sufficient pituitary doses required for either rapid focal or total pituitary destruction. Finally, these initial experiments confirm the potential role for the use of carbon ion Bragg peak irradiation in the treatment of human disease.

\section{ACKNOWLEDGEMENT}

I would like to acknowledge the invaluable assistance of Jeannette Nakagawa in organizing and coordinating all aspects of this project, the assistance of Ann Yabusaki in the technical work 
and preparation of the manuscript, as well as the excellent collaborative support of John Lyman in the radiation biophysics and Tokuko Saito in the

hormone assays of these monkeys. The cooperation and assistance of Robert Springsteen, Fred Crenshaw, and Bob Walton are especially appreciated. I should also like to acknowledge the assistance of Jack Fabrikant in the radiographic areas, specifically the CT scans and subsequent interpretation of $X$ rays, Kay Woodruff for recent initiation of the nistopathological studies, William Holley and George Chen for the collaboration with the heavy ion radiography, and the technical assistance of Myrtle Foster and Peter Linfoot. Finally, the TRH and GnRH were a gift of the Salk Institute.

\section{REFERENCES}

Ambrose, J. 1973. Computerized transverse axial scanning (tomography) 2. Clinical application. Br.J.Radiol. 46, 1023-1047.

D'Angio, G. J., J. H. Lawrence, A. Gottschalk, and J. Lyman. 1964. Relative efficiency of high-LET radiation (Bragg-peak lithium ions) on normal rabbit skin, using integral dose as a basis for comparison. Nature (London) 204 , 1267-1268.

Hardy, J. 1979. Transsphenoidal microsurgical treatment of pituitary tumors. Recent Advances in the Diagnosis and Treatment of Pituitary Tumors (J. A. Linfoot, ed.) pp. 375-388. Raven Press, New York.

Herbert, D. C. and T. Hayashida. 1974. Histologic identification and immunochemical studies of prolactin and growth hormone in the primate pituitary gland. Gen. Comp. Endocrinol. 24, 381-397.

Hotchkiss, D., L. E. Atk inson, and E. Knobil. 1971. Time course of serum estrogen and luteinizing hormone ( $\mathrm{LH}$ ) concentration during the menstrual cycle of the Rhesus monkey. Endocrinology 89, 177-183.

Hummel, B.C.W., G. M. Brown, B. R. Webster, and J. C. Paice. 1974. Crossreactivity of monkey pituitary extracts in human radioimmunoassays. Gen. Comp. Endocrinol. 23, 143-153.

Linfoot, J. A. 1979. Heavy ion therapy: Alpha particle therapy of pituitary tumors. Recent Advances in the Diagnosis and Treatment of
Pituitary Tumors (J. A. Linfoot, ed.) pp. 245-268. Raven Press, New York.

Linfoot, J. A. 1980. Alpha particle pituitary irradiation in the primary and post-surgical management of pituitary microadenomas. Proceedings of the International Symposium on Pituitary Microadenomas, pp. 515-529. Academic Press, Inc., London.

Linfoot, J. A., J. L. Born, J. F. Garcia, E. Manougian, R. P. Kling, C. Y. Chong, C. A. Tobias, R. A. Carison, and J. H. Lawrence. 1968. Metabolism and ophthalmological observations following heavy particle suppressive therapy in diabetic retinopathy. Symposium on the Treatment of Diabetic Retinopathy (M. J. Goldberg and S. L. Fine, eds.) pp. 277-289. Airlie House, Warrenton, virginia.

Mori, J. and E.S.E. Hafez. 1973. Serum luteinizing hormone concentrations in male monkeys (Macaca fascicularis). Am. J. Vet. Res. 34, $1073-1076$.

Redgate, E. J., E. E. Fahringer, and E. L. Foley. 1972. Ketamine $\mathrm{HCl}$ and ACTH release. Fed. Proc. 31, 811.

Simpson, M. E., G. Van Wagenen, D. C. Van Dyke, A. A. Koneff, and C. A. Tobias. 1959. Deuteron irradiation of the monkey pituitary. Endocrinology 65, 831-857.

Tobias, C. A. 1973. Pretherapeutic investigations with accelerated heavy ions. Radiology $108,145-158$.

Tobias, C. A. 1979. Pituitary radiation: Radiation physics and biology. Recent Advances in the Diagnosis and Treatment of Pituitary Tumors (J. A. Linfoot, ed.) pp. 221-243. Raven Press, New York.

Tobias, C. A., E. V. Benton, M. P. Capp, A. Chatterjee, M. R. Cruty, and R. P. Henke. 1977. Particle radiography and autoactivation. Int. J. Radiat. Oncol. Biol. Phys. 3, 35-44.

Van Dyke, D. C., M. E. Simpson, A. A. Knoeff, and C. A. Tobias. 1959. Longterm effects of deuteron irradiation of the rat pituitary. Endocrinology 64, 240-257.

Witherspoon, L. R., S. E. Shuler, M. M. Garcia, and $L$. A. Zolinger. 1980. An assessment of methods for the estimation of free thyroxine. J. Nuc 1. Med. 21, 529-539. 
398

Blark 


\title{
FOCAL LESIONS IN THE CENTRAL NERVOUS SYSTEM
}

\author{
J. I. Fabrikant, ${ }^{\star}$ T. F. Budinger, C. A. Tobias, and J. L. Born
}

The potential for Bragg peak irradiation with heavy-ion beams as a method for producing discrete focal lesions in the brain is derived from two clinical observations: (1) heavy-ion radiosurgery avoids the morbidity and mortality associated with extensive neurosurgical procedures; and (2) alternative methods, such as cryosurgery, electrothermal surgery, and $X$ rays, provide poor spatial definition and lack of reliability (Budinger, et al., 1977). This capability for inducing focal lesions in the central nervous system (CNS) introduces a valuable neurosurgical probe for investigating mechanisms of radiation injury in nervous tissue at the molecular and cellular level, and for determining limitations in tolerance of radiation by nervous tissues, neurovascular tissues, and supportive glial structures. In addition, the use of the Bragg peak for experimental neuroscience provides a unique tool to investigate the mammalian brain, including nerve pathways, neuroendocrine pathophysiology and the functional anatomy of centers in the brain responsible for homeostasis and regulatory control. Such investigations have potential for treatment of pathological disorders of the CNS in clinical patients.

Budinger and $h$ is colleagues (Pelletier et al., 1978a,b) have been investigating the effects of Bragg peak irradiation with narrow heavy-ion beams in the brain, and particularly the neuroendocrine response of heavy-ion beam Bragg peak irradiation of the anterior hypothalamus in dogs. Tobias and his co-workers are examining mechanisms of heavy-ion beam radiation injury and repair in the central nervous system of the rabbit. Fabrikant and his colleagues have begun to use the Bragg ionization peak of heavyion beams for radiosurgical treatment of selected neurovascular disorders in the central nervous system in patients who are unable to be treated with alternative neurosurgical procedures. This report reviews the animal and human studies cur.rently in progress at $L B L$ with heavy-ion beams to induce focal lesions in the central nervous system, and discusses the potential future prospects of fundamental and applied brain research with heavy-ion beams.

\section{ANIMAL STUDIES}

Budinger and his co-workers (Pelletier et al:, $1978 \mathrm{a}, \mathrm{b})$ are investigating the effects of $2-\mathrm{mm}$. diameter neon-ion beams ( $400 \mathrm{MeV} / \mathrm{amu}$ ) on the anterior hypothalamus in beagle dogs. The plateau dose before final collimation was $8,000 \mathrm{rad}$; the peak-to-plateau ratio was $4: 1$. The dose to a $\left(2 \mathrm{~mm}^{3}\right)$ region at the Bragg ionization peak was about 24,000 rad. The dose of 8,000 rad did not produce cellular changes or other destructive lesions in the hypothalamus

4 months after irradiation, but neurophysiological changes were recorded. There was a marked depression of thyrotropin-releasing hormone (TRH) levels in the serum which began a few days after irradiation, and fell to $20 \%$ of normal range 5 weeks after irradiation; recovery to $80 \%$ of normal occurred by 4 months (Fig. 1). A concurrent fall and recovery of growth hormone occurred; plasma levels fell to $80 \%$ of normal between the 30 th and 50 th day following irradiation. There was a small but significant decrease in plasma growth hormone (GH) levels and a large response to GH-release following L-DOPA infusion in the irradiated animals (Fig. 2). Studies of $99 \mathrm{mT}$ T-TRH transport from the cerebrospinal fluid to the circulating blood demonstrated a bimodal appearance in normal dogs due to both active third ventricle transport and delayed passive transport through the subarachnoid granules. In the irradiated dogs, this bimodal curve was abnormal, and demonstrated a slow washout, when the blood-brain barrier was disrupted in the region of the third ventricle.

These studies demonstrate the value of heavy-ion beams as a neuroscience tool. The implications of these results are that the major production center of TRH in dogs is in the anterior hypothalamus, and not in diffuse regions throughout the brain; that TRH centers can be replenished after destruction of most of the

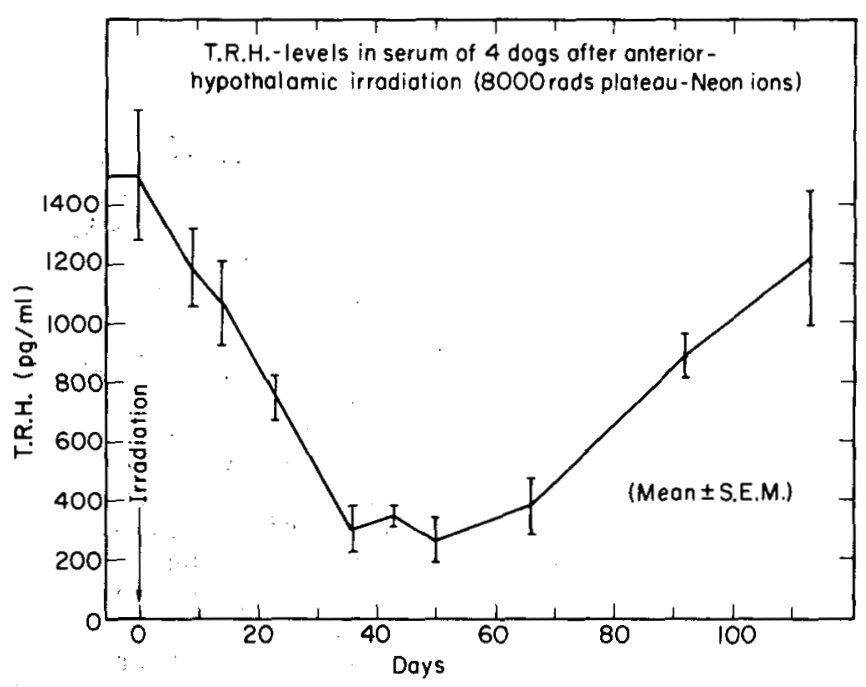

Figure 1. Thyrotropin-releasing hormone (TRH) plasma Tevels in four beagle dogs following Bragg peak irradiation (neon ions, $400 \mathrm{MeV} / \mathrm{amu}, 2 \mathrm{~mm}$ diameter beam of the anterior hypothalamus. The To value is the mean of preirradiation levels obtained up to two weeks prior to irradiation. (XBL 7712-4070) 


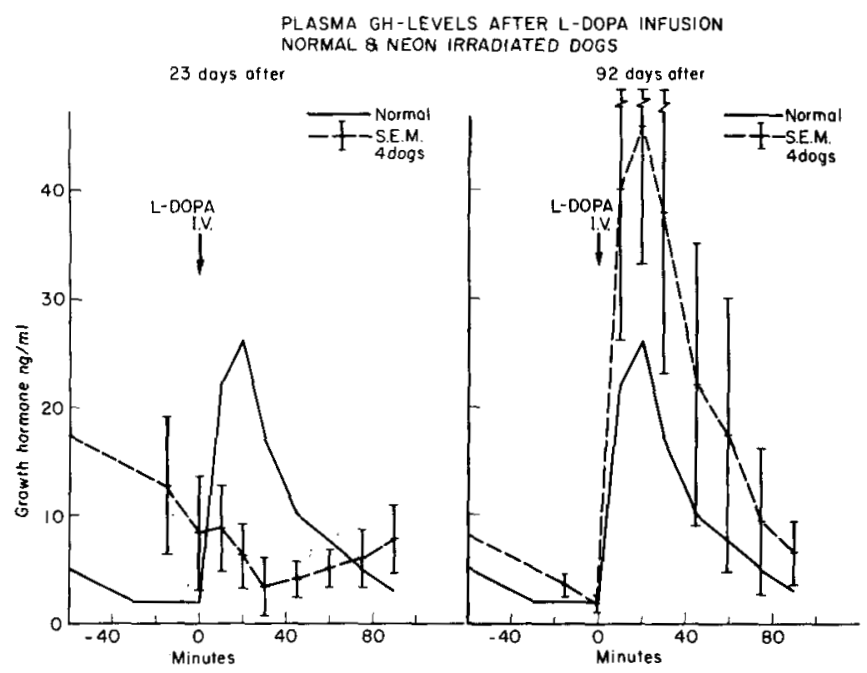

Figure 2. Plasma growth hormone (GH) levels following L-DOPA infusion in both control and Bragg peak irradiated (neon ions, $400 \mathrm{MeV} / \mathrm{amu}$ ) beagle dogs, 23 days and 92 days after irradiation. (XBL 781-2725)

active production centers in the hypothalamus; and that 24,000 rad could be delivered to $0.01 \mathrm{~cm}^{3}$ regions of the anterior hypothalamus without fatal sequelae.

Tobias, Fabrikant, Woodruff, and Lyman are studying the production of discrete focal lesions in the rabbit brain using carbon and neon beams, with an aim to apply Bragg peak irradiation to the treatment of a number of nonneoplastic disorders in the central nervous system. The studies are directed toward investigating the pathophysiology of Bragg peak focal radiation injury in the CNS in relation to correlation of (1) dose, volume and time dependency for induction of damage at various sites; (2) CNS effects of different charged particles with known differences in RBE, particularly carbon, neon, and helium; (3) CNS effects during fractionation of dose, and the extent of repair; (4) sites and nature of cellular and molecular injury with functional neurophysiological alterations. The application of these studies relates to establishing tolerance doses for normal CNS tissues in man for Bragg peak irradiation of CNS disorders, such as vascular abnormalities and tumors. Initial studies in raobits using neon ions (425 MeV/amu, 15-cm range), $1 \mathrm{~cm}^{3}$ volume spread Bragg peak, demonstrated the extent of damage of a $1 \mathrm{~cm}^{3}$ focal lesion in the cerebral cortex (see Fig. 3). Marked leakage of insoluble dye (carbon) is associated with damage to the blood-brain barrier and increased permeability of the cerebrovascular structures. These studies are providing a better understanding of the sequence of events following local brain injury by heavy-ion radiation.

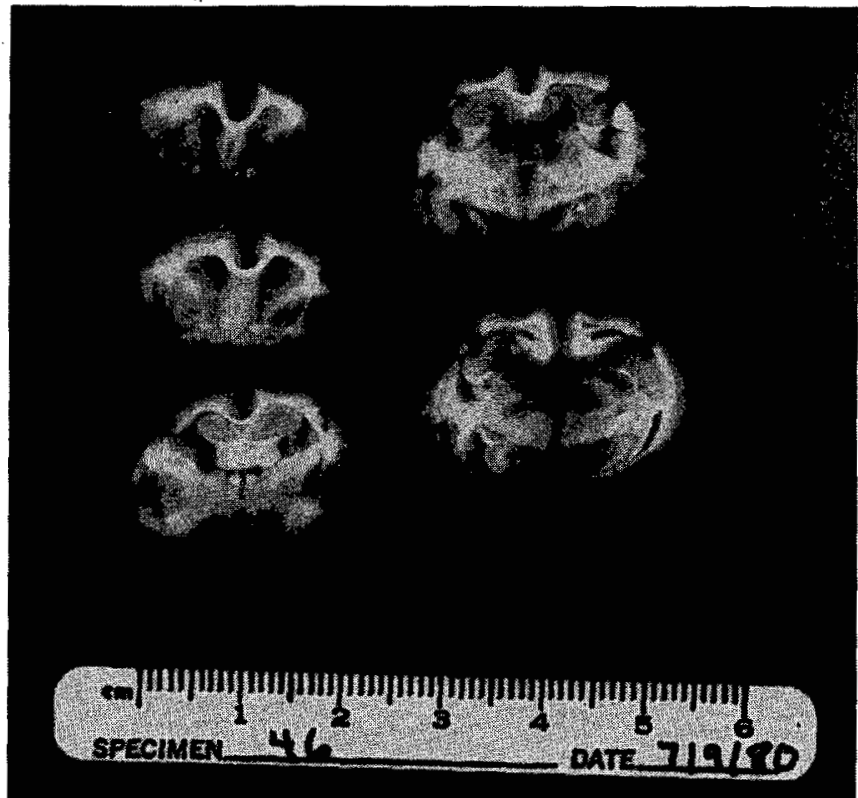

Figure 3. Sections of rabbit brain one week following 8,000 rad neon-ion Bragg peak irradiation, $1 \mathrm{~cm}^{3}$ aperture to the left frontal cortex. A $10 \mathrm{ml}$ carbon dye solution was injected $1 \mathrm{~h}$ prior to killing. A focal area of marked carbon leakage in the irradiated field is noted. (CBB 807-8173)

\section{CLINICAL STUDIES ON PATIENTS}

\section{Intracranial Vascular Disorders}

Surgical excision or surgical ablation are the methods of treatment for intracranial arteriovenous malformations, vascular fistulae, and acoustic neuromas. However, when the CNS lesion is inoperable, or when other factors exist that preclude surgery, alternative methods of treatment are necessary. Neurological radiosurgery for inoperable intracranial arteriovenous malformations, for certain other vascular disorders in the brain, and for large, inoperable acoustic neuromas have become a potential therapeutic procedure in three university centers: Harvard University (Kjellberg, 1979), the Karolinska Institute, Stockholm (Lekse11, 1971; Steiner et al., 1978), and University Hospital, Valencia, Spain (Barcia-Salorio, et al., 1979). The results, using beams of cyclotron-produced protons (Harvard), intense high-dose gammaradiation (Karolinska Institute), or linear accelerator photons (Valencia) appear promising. Approximately 140 patients have been treated, with only minimal morbidity. Conventional photon radiotherapy has been tried in the past, and abandoned.

As part of a collaborative effort between the Department of Neurosurgery, UCSF, and our laboratory, J. I. Fabrikant, Y. Hosobuchi, and their coworkers are applying helium and other heavy-ion 
beams for radiosurgery in intracranical arteriovenous malformations, intracranial vascular. fistulae, and other vascular disorders. The procedure for obliteration of cerebral arteriovenous malformations and interval carotid arterycavernous sinus fistulae using stereotaxically, directed heavy-ion Bragg peak irradiation is now being developed. In the neurological evaluation, it is necessary to select patients for whom direct surgical or other invasive surgical procedures (including cryosurgery and electrothermal cautery) are not possible. Treatment with highenergy heavy-ion beams provides the opportunity for altering the intracerebral hemodynamic condition of these vascular malformations and fistulae and thereby achieving a decrease in the patient's neurological deficiencies, subjective complaints, and frequency of seizures, arresting progressive blindness, and decreasing or arresting episodes of intracranial hemorrhage. The objective, in the case of the intracranial vascular malformations and carotid-cavernous sinus fistulae, is to achieve intravascular obliteration using stereotaxically simulated and directed heavy-ion beams.

Figure 4 illustrates the sequence for a patient-treatment procedure for stereotaxic Bragg peak focal irradiation of the brain. The patient is a 26 year old woman with a large arteriovenous malformation (AVM) of the right thalamus that extends throughout the right lateral ventricle and replaces brain tissue both in the midbrain and the cerebral cortex. The right lateral cerebral angiogram (Fig. $4 \mathrm{~A}$ ) demonstrates the size and location of the AVM in the brain; the patient is wearing the polystyrene head mask used for stereotaxic beam localization, treatment planning, and head immobiliation during the Bragg peak irradiation. Figure $4 B$ is the $X$-ray computerized tomography scan through the AVM lesion; the AVM is enhanced with contrast medium. The AVM is replacing a large part of the dilated right lateral ventricle; the left lateral ventricle is small and compressed by the left thalamus, which is now shifting across the midline. The posterior occipital helium-ion beam port Bragg-peak localization film is illustrated in Figure 4C. . The helium-ion beam is collimated to a $20 \mathrm{~mm} \times 22 \mathrm{~mm} \mathrm{D}$ aperture with a $40 \mathrm{~mm}$ spread Bragg peak, extending posteroanteriorly. The patient received multiport fractionated heliumion (234 MeV/amu) therapy for four days, for a total dose of 2,500 rem. Changes within the AVM are expected to occur within 5-7 months, with occlusion and obliteration of the arteriolar vessels within 9 months to 1 year.

Figure 5 is the pretherapy positron emission tomogram using $82 \mathrm{Rb}$; the dynamic image demonstrates the large blood volume flow transfer from the arterial AVM through to the draining venous system. The objective of Bragg peak therapy is to alter and decrease the high volume intracranial hemodynamic flow pattern to a normal level in order to preclude further intracranial hemorrhage. When clinical changes begin to appear, follow-up positron emission tomograph studies to quantitate changes in intracerebral blood flow dynamics will be carried out.

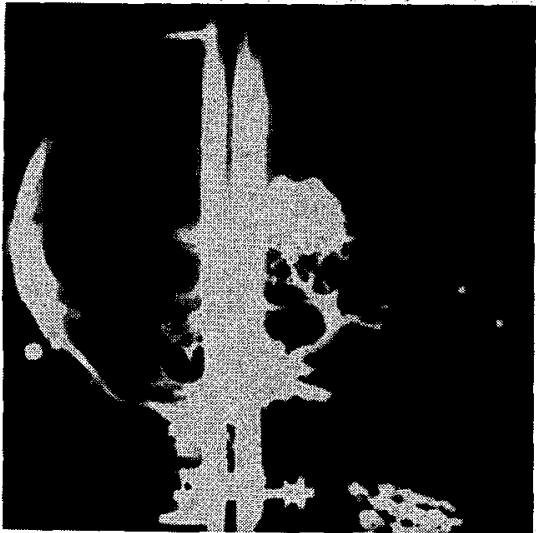

A

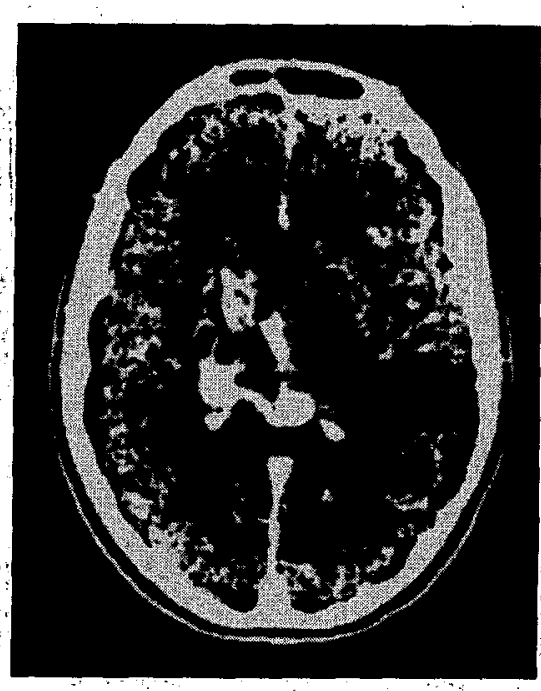

B

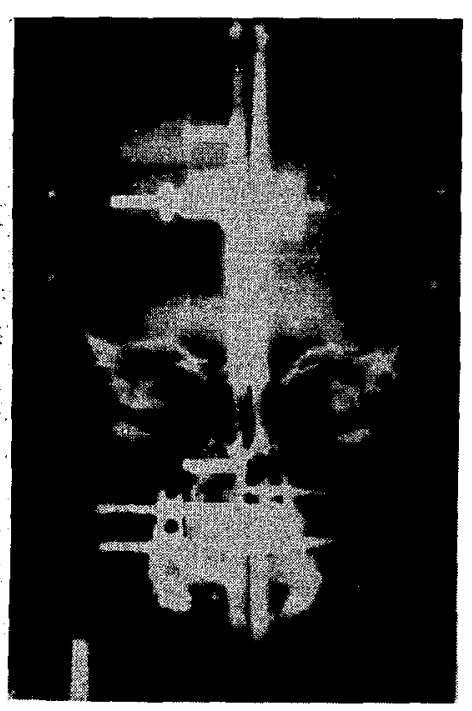

c

Figure 4. Imaging studies of patient who received helium-ion beam Bragg therapy to a 1 arge arteriovenous malformation (AVM) in the brain. (A) Cerebral angiogram of the AVM; the patient's head is immobilized in the polystyrene head mask used for stereotaxic localization, treatment-planning, and heavy-ion radiotherapy. (B) $x$-ray computerized tomographic scan, demonstrating the precise size, location, and extension of the AVM in the thalamus and cerebral cortex necessary for treatment planning for Bragg peak irradiation. (C) Posteroanterior occipital port $\mathrm{film}$ demonstrating the precise volume of the AvM treated with Bragg peak helium-ion irradiation. (XBB 809-10582A) 


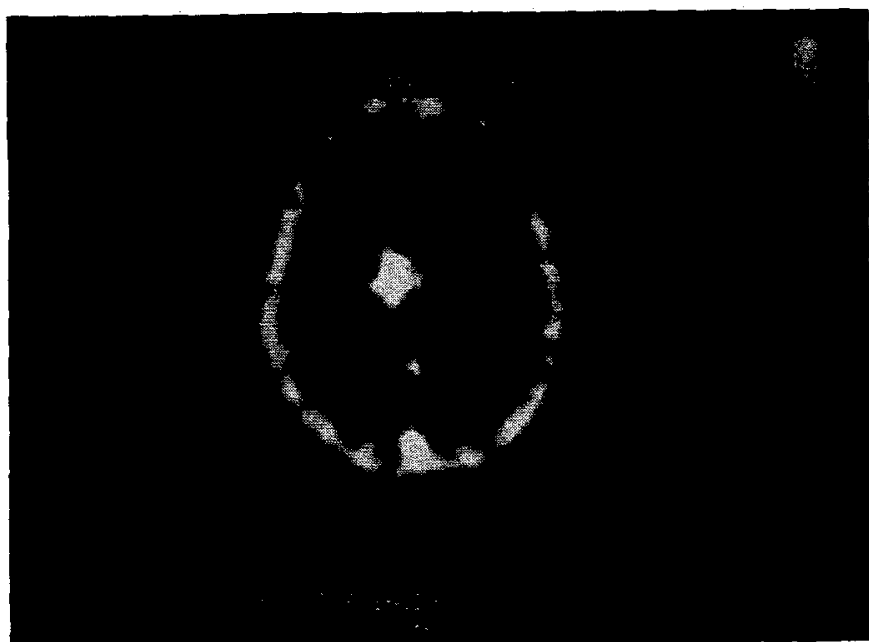

Figure 5. Intracranial arterovenous malformation (AVM) Tocalization using $82 \mathrm{Rb}$ in positron emission tomography with the Donner 280-crystal ring. The large AVM is seen on the patient's right, extending into the region of the midbrain and cerebral cortex. Because $82 \mathrm{RD}$ is a positron emitter, transverse sections that demonstrate the dynamic change of the isotope distribution are obtained using coincidence detection of the annihilation photons. Positron emission tomography provides a quantitative method for investigating blood transit flow and altered intracerebral hemodynamics and blood flow transit patterns following Bragg peak radiosurgery. (From the research medicine laboratory of Budinger, Yen, and coworkers). (XBB 809-10973)

\section{Pituitary Disorders}

The treatment of pituitary disorders, including acromegaly, Cushing's disease, and prolactin-secreting tumors, with helium-ion beams (225 MeV/amu) has been under continuing scientific investigation at $L B L$ for almost twenty-five years, since the initial studies of Lawrence, Tobias, and their colleagues (Lawrence et al., 1937; Tobias et al., 1952, 1959). With high energy particle beams, it is possible to overcome the relative insensitivity of the pituitary gland to externally delivered radiation without irradiating large volumes of surrounding normal brain tissue. In order to achieve adequate radiation dose to the pituitary gland in certain disorders, however, the Bragg peak has only rarely been used at LBL; without knowledge of the details of tissue inhomogeneities and the precise location of vital neural structures relative to the Bragg ionization peak, plateau ions have been used for treatment of pituitary disorders since sufficient energy is available for head penetration.

J. A. Linfoot, J. I. Fabrikant and their colleagues are carrying on the pioneering investigations of J. H. Lawrence and C. A. Tobias and their coworkers on charged particle radiotherapy of pituitary tumors (for review, see Tobias, 1979; Linfoot, 1979). Since 1957, 799 patients have been treated with alpha particle irradiation to the pituitary gland; of these, 467 had pituitary tumors, 157 had metastatic breast cancer, and 169 had diabetic retinopathy. Fractionated doses of high-energy collimated plateau heliumion beams were used, and total doses from 4,500 to about $18,000 \mathrm{rad}$, depending on the pathological condition, were delivered to the pituitary gland; the beams passed through the head without being attenuated. The manner in which a variety of beams in the course of therapy impinge on the pituitary, and three-dimensional dose distribution, is shown in Figure 6 .

Upper limitations to the treatment dose are due to possible injury to cranial nerves and to the temporal lobes nearest the pituitary; the optic nerves, the optic chiasm, and the hypothalamus are protected very efficiently. In acromegaly and Cushing's disease, the aim is not complete ablation of pituitary function, but rather permanent inhibition of the pituitary tumor while preserving some normal pituitary hormone-secreting potential of surviving normal cel1s. Much of the research on the treatment of acromegaly and Cushing's disease may be considered complete, as these procedures are used in routine clinical practice.

FUTURE MEDICAL RESEARCH:

CLINICAL APPLICATIONS OF FOCAL LESIONS IN THE CENTRAL NERVOUS SYSTEM

Accelerated carbon ions have particularly good potential for use in the application of focal lesions in the central nervous system with the Bragg ionization peak. This is because the carbon-ion beam has less scattering and straggling than helium ions and protons by a factor of 1.6 (helium ions) or 3.4 (protons). The Bragg depth ionization curves obtained have greater peak-to-plateau ratios, and can be positioned more accurately, thereby protecting adjacent CNS structures, such as the hypothalamus, thalamus, and brain stem. Furthermore, near the peak of the Bragg curve, the biological effectiveness of the carbon-ion beam is much greater than at the plateau. If the peak is stopped in a CNS lesion, such as in a small vascular disorder in the brain, relatively greater radiobiological effect is achieved with much less dose. In addition, the sensitive neural structures in adjacent regions of the brain are better protected. This is illustrated in the dose-distribution of parallel opposed fields using the Bragg peak or plateau ions. Figure 7 illustrates the lateral dose distribution with transirradiation through the head using plateau heavy ions, and with parallel opposed ports when the Bragg peak is at or near the center of the lesion, e.g., the center of the pituitary gland. In the former, the isodose fall-off within $10 \mathrm{~mm}$ of the center is to $50 \%$ of the maximum dose, whereas in the latter, it is to $5 \%$ of the maximum dose.

It is our belief that heavy-ion beams, such as carbon, will allow improved dose distribution and dose delivery in localized focal irradiation 


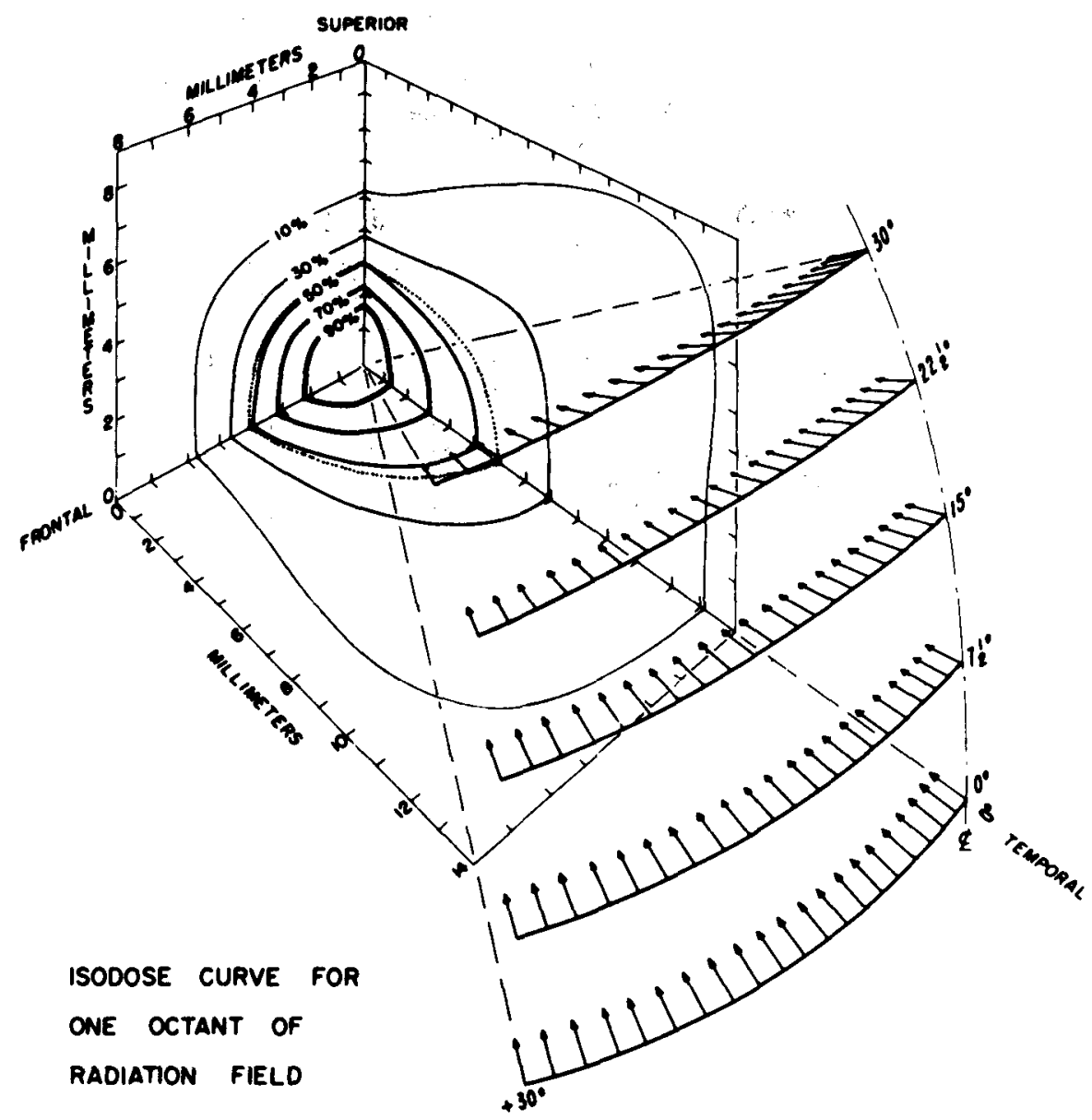

Figure 6. Three-dimensional dose distribution for an octant of the radiation field. (MU-14976)

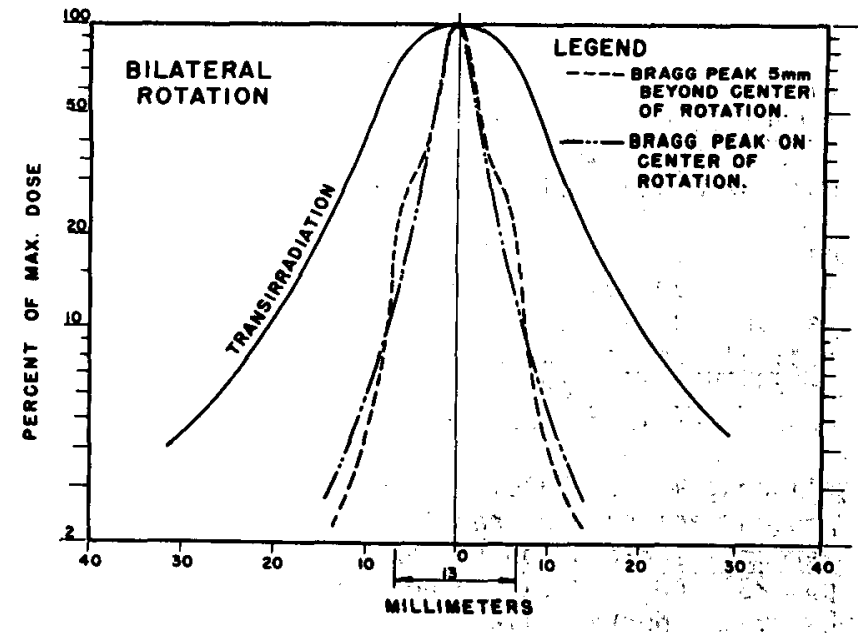

Figure 7. Lateral dose distributions with transirradiation and with parallel opposed ports when the Bragg peak is at or near the center of the pituitary gland. If the Bragg peak can be Fcurately positioned, then the temporal lobes and cranial nerves are much better protected than they are with the current transirradiation (plateau ions) technique. (XBL 785-8710) in the central nervous system. It can be expected that 'small lesions, e.g., vascular fistulae, pituitary microadenomas, and similar radiosurgically amenable disorders, can now be successfully approached. Furthermore, localization of very discrete focal lesions can be achieved in vital brain centers to treat certain diseases, such as Parkinson's disease or for the control. of pain.

\section{APPLICATION OF RADIOACTIVE BEAMS}

$\therefore$ A technique is being developed in our laboratory (A. Chatterjee, E. L. Alpen, J. Llacer, and $C$. A. Tobias) to achieve precise localization of the stopping points of the beam inside the body by the use of a radioactive beam (see Part VI, Section B). This will make it feasible to stop the beam accurately at the appropriate depth in the central nervous system. Here, ${ }^{1} l_{C}$ ion beams, for example, are attractive and provide the potential of accurate positioning not available with helium ions or protons. $19 \mathrm{Ne}$, 150 , and other radioactive beams can also be produced at the Bevalac. Tobias et al. (1971) demonstrated that radioactive ${ }^{11} \mathrm{C}$ nuclei are produced by collisions of $12 \mathrm{C}$ beam particles 
with target nuclei. These ${ }^{11} \mathrm{C}$ nuclei comprise up to $3 \%$ of the total carbon-ion beam, and will decay emitting positrons with a half life of $20.5 \mathrm{~min}$. These positrons are detected with a specially constructed gamma camera. Recent studies have made it possible to form a small beam of pure $1_{1} \mathrm{C}$ particles, which permits determination of the stopping points in an absorber with an accuracy of $1 \mathrm{~mm}$. Figure 8 il lustrates one possible scheme for production of Bragg peak focal lesions in the brain using radioactive heavy particle beams. The positron camera would continually monitor the precise location of the stopping points of the beam in the patient's head. The data are fed back online to a variable absorber which then controls the range penetration of the beam, thereby fully controlling the location and depth dose of the heavy-ion beam.

\section{SUMMARY AND CONCLUSIONS}

The applications of heavy-ion beams for fundamental and applied brain research have unusual potential. Methods are being developed for producing discrete focal lesions in the central nervous system using the Bragg ionization peak to investigate nerve pathways and neuroendocrine responses, and for treating pathological disorders of the brain with Bragg peak heavy-ion radiosurgery.

Studies in animals are demonstrating the value of this neuroscience tool in investigating the response in the mammalian brain following

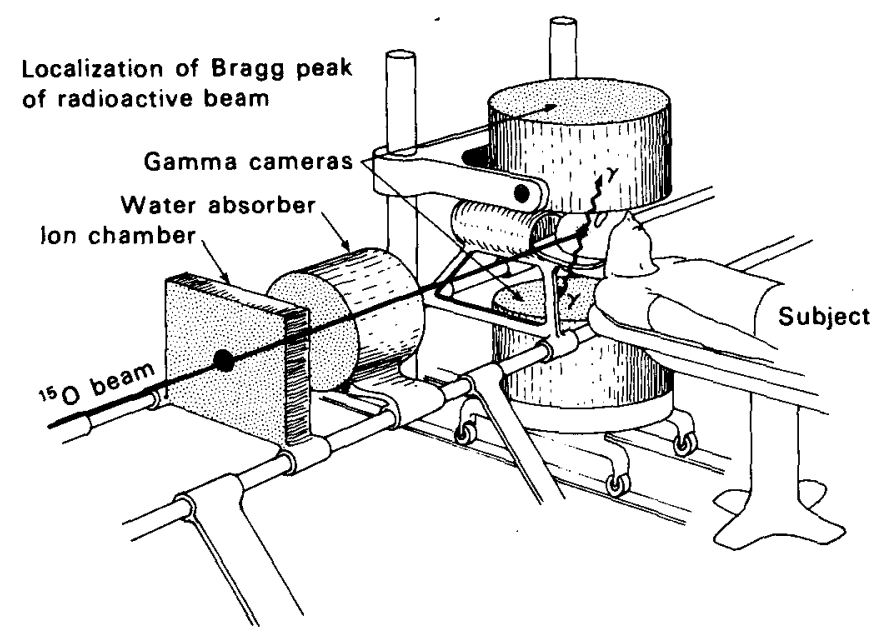

Figure 8. Possible scheme for focal irradiation of the central nervous system with radioactive beams. A positron camera is continually monitoring the stopping point of the radioactive beam at the center of the head. The camera automatically sends signals for range adjustments as the rotational exposures proceed. The scheme illustrates application of an 150 beam, but also applies to other radioactive beams like $11_{C}$ or $19 \mathrm{Ne}$. (XBL 752-4708) induction of discrete focal lesions in the hypothalamus or in the cerebral cortex. These studies are elucidating the neuroendocrinological responses following heavy-ion irradiation of various portions of the midbrain, without the need for complex neurosurgical preparations.

Clinical studies are demonstrating the feasibility of neurological radiosurgery for treating inoperable disorders of the brain, such as arteriovenous malformations and carotid artery-cavenous sinus fistulae in patients who are already demonstrating progressive neurological deficit, including progressive blindness. Further possible applications of heavy-ion focallesion Bragg peak therapy include localized radiation exposure to centers of the brain and spinal cord for treatment of such disorders as parkinson's disease, pituitary microadenomas, and the control of pain. The potential application of radioactive beams will provide accurate localization of the stopping points of the beam, thereby making it feasible to stop the beam accurately at a defined depth within the central nervous system.

\section{ACKNOWLEDGEMENT}

These studies were supported by grants from the National Institute of Health (HL 25840, T. F. Budinger, Principal Investigator), and the National Cancer Institute (CA 27021, C. A. Tobias, Principal Investigator).

\section{FOOTNOTES AND REFERENCES}

*Department of Radiology, University of California, San Francisco, CA.

Barcia-Salorio, J. L., G. Hernandez, J. Broseta; B. Ballester, and G. Masbout. 1979. Radiosurgical treatment of a carotid-cavernous fistula. Case report. Stereotaxic Cerebral Irradiation (G. Szikla, ed.) pp. 251-256, INSERM Symposium No. 12, Elsevier/NorthHolland Biomedical Press, Amsterdam.

Budinger, T. F., J. A. Linfoot, B. R. Moyer, J. T. Lyman, and T. L. Pelletier. 1977. Brain research with heavy-ion beams. Biological and Medical Research with Accelerated Heavy Ions at the Bevalac, 1974-1977. Lawrence Berkeley Laboratory Report LBL-5610, pp. 187-197.

Kjellberg, R. N. 1979. Stereotaxic Bragg peak proton radiosurgery results. Stereotaxic Cerebral Irradiation (G. Szikla, ed.) pp. 233-240. INSERM Symposium No. 12. Elsevier/ North Holland Press, Amsterdam.

Lawrence, J. H., W. O. Nelson, and H. Wilson. 1937. Roentgen irradiation of the hypophysis. Radiology 29, 446-454.

Lekse11, L. 1971. Stereotaxis and Radiosurgery-An Operative System. C. C. Thomas, Springfield, Illinois. 
Linfoot, 3. A. 1979. Heavy-ion therapy: Alpha particle therapy of pituitary tumors. Recent Advances in the Diagnosis and Treatment of Pituitary Tumors (J. A. Linfoot, ed.) pp. 244-267. Raven Press, New York.

Pelletier, J. L., B. R. Moyer, and T. F. Budinger. 1978a. Effects of Hypothalamic Radiation Lesions Induced by a $2 \mathrm{~mm}$ Heavy-Ion Cyclotron Beam. Lawrence Berkeley Laboratory Report LBL-7401.

Pelletier, J. L., B. R. Moyer, and T. F. Budinger. 1978b. Technetium-99m Labelled Thyrotrop in Releasing Hormone Kinetic Studies in Dogs With and Without Anterior Hypothalamic Lesions. Lawrence Berkeley Laboratory Report LBL-7402.

Steiner, L., E.-0. Backlund, T. Greitz, L. Leske11, G. Norén, and T. Rahn. 1978. Radiosurgery in intracranial arteriovenous malformations.

II. A follow-up study. Neurological Surgery with Emphasis on Non-invasive Methods of Diagnos is and Treatment (R. Carrea, ed.). Exerpta Medica International Congress Series No. 433. Amsterdam, The Netherlands.
Tobias, C. A. 1973. Pretherapeutic investigations with accelerated heavy ions. Radiology $108,145-158$.

Tobias, C. A. 1979. Pituitary irradiation: Radiation physics and biology. Recent Advances in the Diagnosis and Treatment of pituitary Tumors (J. A. Linfoot, ed.) pp. 221-253. Raven Press, New York.

Tobias, C. A., H. O. Anger, and J. H. Lawrence. 1952. Radiological use of high energy deuterons and alpha particles. Am. J. Roentgenol. Radium Ther. Nucl. Med. 67, 1-27

Tobias, C. A., D. C. Van Dyke, M. E. Simpson, $H$. O. Anger, R. L. Hugg, and A. A. Koneff. 1959. Irradiation of the pituitary of the rat with high-energy deuterons. Am. J. Roentgenol. Radium Ther. Nuc 1. Med. 72, 1-21.

Tobias, C. A., A. Chatterjee, and A. R. Smith. 1971. Radioactive fragmentation of $\mathrm{N}^{7+}$ ion beam observed in a beryllium target. Phys. Lett. A. 37, 119-120. 
406

Blank 


\title{
RESTRICTION OF THE SPREAD OF EPILEPTOGENIC DISCHARGES IN CATS BY INTRACRANIAL IRRADIATIONS
}

\author{
C. T. Gaffey, V. J. Montoya, J. T. Lyman, and J. Howard
}

Epilepsy is a term that describes a group of disorders caused by sudden, recurrent, highvoltage bursts of electrical activity by some of the brain's hyperexcitable neurons. In certain cases of epilepsy, the presence of a discrete focus is the origin of the brain's paroxysmal, electrical discharges. An example of a focal epilepsy is an occipital scar that produces disruptive effects in one visual field and can spread to involve control centers in both hemispheres of the brain, and thus produce convulsions and loss of consciousness (Huott et al., 1974). Four million Americans have some form of epilepsy. Epilepsy is more widespread than cancer, tuberculosis, muscular dystrophy, cerebral palsy and multiple sclerosis combined. Because $20 \%$ of epileptics have intractable symptoms that can no longer be relieved by drugs or any other known treatment, new approaches to manage epilepsy are needed.

The best approach to the control of epileptic activity involves the least interference with normal brain function. Our aim is to determine in animal studies whether heavy-ion Bragg peak radiation can be used as a noninvasive technique to prevent focal, cortical epilepsy from sending epileptiform electroencephalographic (EEG) discharges to the rest of the brain. The unique advantage of heavy ions is that these particles can be physically controlled to release their energy at a preselected distance inside matter (the Bragg peak radiation effect), even when the target is an intracranial site. Experiments on cats have been used to evaluate if intracranially deposited Bragg peak radiation can render brain tissue incapable of transmitting disruptive EEG seizure signals.

\section{METHODS}

\section{Stereotaxic Implantation Procedure}

The cats are first anesthetized and their heads are immobilized in a David Kapf stereotaxic instrument. Needle electrodes are inserted through burr holes in the skull of the animal to make contact with the cortex. The electrodes are made of nichrome wire (0.0091 in diameter) and they are Formvar insulated except for $1 \mathrm{~mm}$ at the tips. Each cat is stimulated electrically through electrodes in one cortical hemisphere during the implantation procedure. (Gaffey, 1966) while an evoked response in the opposite cortical hemisphere is maximized by finely adjusting the position of the recording electrode. An array of eight electrodes is implanted through the a kull into the cortex of each experimental animal, four homologous leads in each cerebral cortex. A ninth wire lead, inserted into the bony inion, serves as a ground lead. The leads from the right and left hemisphere are soldered to an Amphenol nine pin radiosocket. Leads and sockets are securely held together by Kadon methacrylate dental "plastic. The whole assembly is permanently: anchored to the skull by bone screws and additional dental plastic.

A nine line cable is used to attach the cortical sites in test animals to appropriate stimulating and recording electrophysiological equipment. An eight channel electroencephalographic machine (Grass, Model IIIE) can simultaneously record the spontaneous activity of the eight cortical brain sites of each awake, alert cat. A Tektronix oscilloscope (type 3B4 time base with a T3A3 differential amplifier) in series with a Grass P-5 amplifier is used to monitor fast cortical responses evoked by electrical stimulation in the opposing hemisphere.

\section{Production of an Epileptogenic Site}

To generate an epileptic site in cats a sterile craniotomy is performed and a subpial injection of alumina gel (Caloiochem) is made (Kopeloff, 1960; Kopeloff et a 1., 1955; Fetz and wiler, 1973). The alumina-induced epileptogenic focus in the cortex is located at a known distance from each implanted electrode. A similar surgical procedure is performed on the opposite side without depositing alumina gel. The stereotype EEG structure of the spike and burst pattern serves as evidence of the success of the alumina gel treatment. Eight cats with occipital lobe epilepsy were prepared with a single focal lesion in the left occipital cortex.

\section{Heavy-Ion Irradiation}

Heavy-ion beams are produced in the Bevalac at LBL (Tobias et al. 1971). "Gniorso et al. (1973) have described this facility and its operation. The depth-ionization advantage of heavy-ion beams producing a Bragg peak made this source of radiation most suitable for intracranial radiations. "The set-up for biological experiments and the physical measurements of heavy-ion beams at the Bevalac have been described (Lyman et al:, 1971; Beckman et a 1. , 1974:):

Our purpose is to deposit Bragg peak radiation in a desired brain target. To perform this task a precision alignment apparatus has been constructed (Fig. 1). This device locks the nead of a cat into a supportive, rigid frame. This assembly can position an intracranial target to be irradiated with a precision of $\pm 0.05 \mathrm{~cm}$. Heavy-ion Bragg peak irradiation of cats is per- 


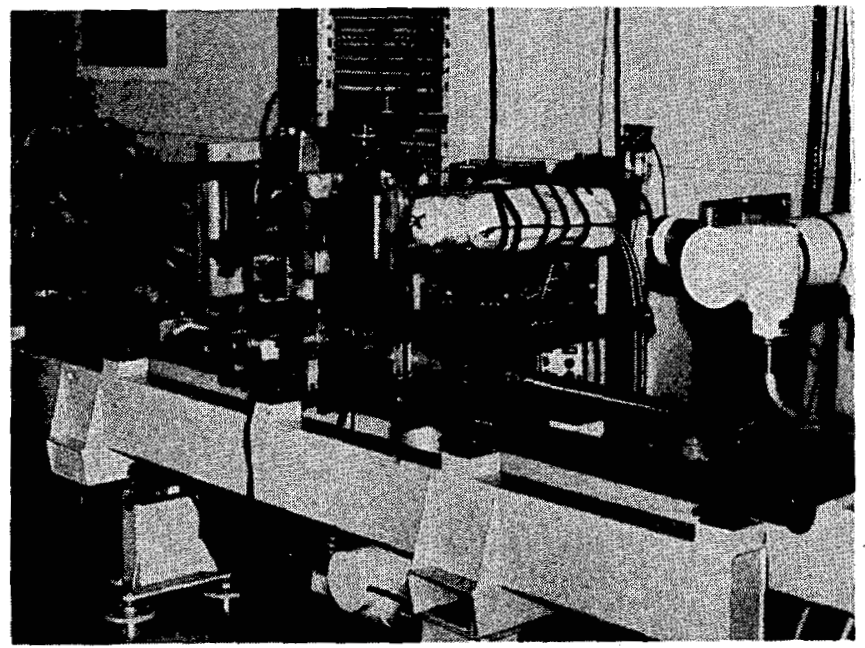

Figure 1. An anesthetized cat in a headholder apparatus at the Bevalac facility. (XBB 766-4939)

formed by remote control operations for the safety of personnel. A closed-circuit TV allows investigators to check the condition of each cat during radiation exposure. An ionization chamber placed immediately in front of an experimental animal measures the plateau dose.

\section{RESULTS}

Eight adult cats had a cortical spot $2-3 \mathrm{~mm}$ in diameter painted with alumina cream in their left occipital cortex. The onset of epileptiform EEG discharge patterns from four locations ( $a, b, c$ and d) in this cortex is presented in Figure 2. High-frequency, cortical spike discharges of about $400 \mu \mathrm{V}$ are present in these EEG records. The amplitude of the discharges in the epileptic cortex is about three to five times greater than the control cortex. In Figure 2 the EEG traces from the control (right) occipital cortex do not show EEG paroxysms, but with time focal spiking from the left cortex spreads to involve the right (contralateral) cortex.

Experiments on six cats used 9-BeV neon ions ( $400 \mathrm{MeV} / \mathrm{nucleon}$ ) generated by the Bevalac. The characteristics of the neon-ion beam are summarized in Table 1. The Bragg peak region of the beam was used in cat brain irradiations. A circular beam $1.77 \mathrm{~cm}$ in diameter was achieved with absorption collumination. The Bragg peak location inside the brain was accomplished by inserting a range-adjusting water column into the neon-ion beam path. Absorbed dose values were obtained from parallel ionization chambers. Precision alignment of the entrance beam to the selected brain target was confirmed by $X$-ray photographs of the cat's head, as given in Figure 3. Six cats had a disc of brain tissue absorb Bragg peak, neon-ion radiation. The disc of irradiated tissue was positioned $3 \mathrm{~mm}$ below the cortical surface and beneath the aluminagenerated focus. The absorbed dose at the Bragg peak was $6,8,10,12,14$, and $16 \mathrm{krad}$ of $400 \mathrm{MeV}$ neon ions/nucleon in the animals studied.

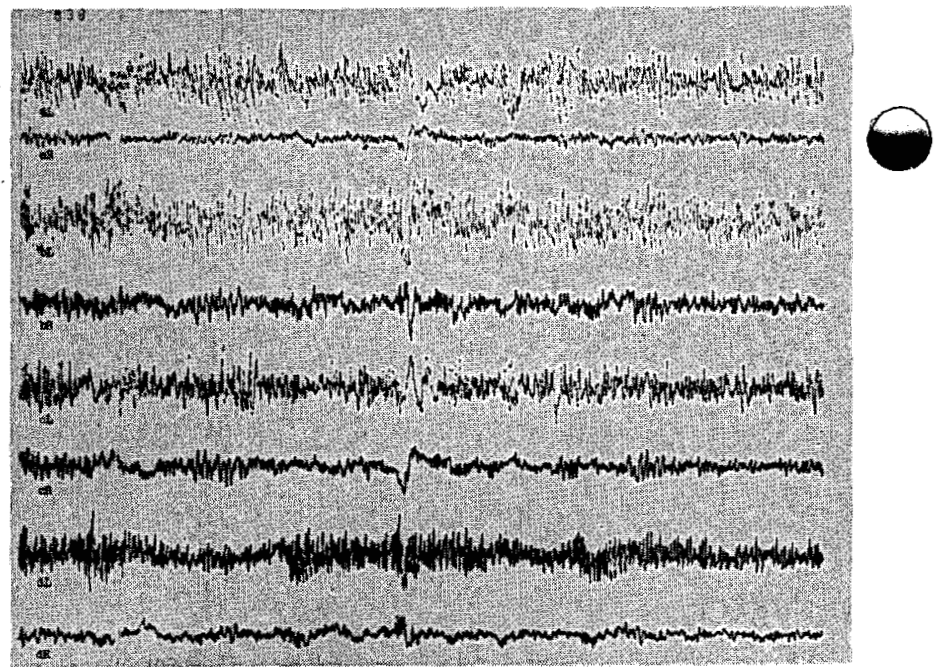

Figure 2. EEG traces from four locations ( $a, b$, $c$, d) in the left (L) and right (R) occipital cortices of a cat. Right cortical traces (aR, $b R, C R, d R$ ) show normal EEG activity while left cortical traces (aL, bL, $C L, d L$ ) show epileptiform EEG discharges. (XBB 775-4285)

In alert, drug-free cats the effect of electrical stimulation through a pair of electrodes in one occipital cortex is seen as an evoked potential difference when measured in the homologous site of the opposite cortex. The term inter-hemispheric response (IHR) is defined as the bioelectric activity recorded in one hemisphere as a consequence of an applied electrical stimulus in the contralateral hemisphere. IHR are used as a physiological index of the conduction pathway between electrodes in the ipsilateral and contralateral occipital cortices.

Table 1. Bevalac Beam Characteristics

Particle

Energy

Charge

Range

LET, plateau

Beam diameter

Particles/pulse

Dose rate

Peak-to-plateau ratio

$$
\text { Neon ion }
$$

$400 \mathrm{MeV} / \mathrm{nucleon}$ $+10$

$14 \mathrm{~cm}$ in water $32 \mathrm{keV} / \mu \mathrm{m}$ of water 0.5 inch $(1.77 \mathrm{~cm})$ $2 \times 10^{8}$ to $1 \times 10^{9}$

$400 \mathrm{rad} / \mathrm{min}$ 4 


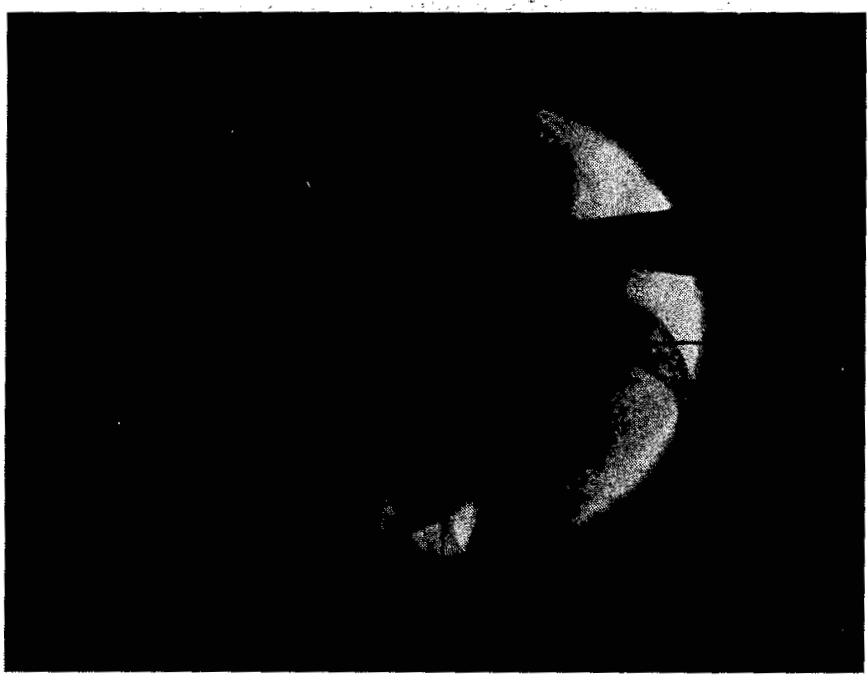

Figure 3. An X-ray picture of the cat's nead in a stereotaxic headholder at the Bevalac facility. cross-hairs locate the entrance of heavy ions into the cat's brain. (XBB 775-4274)

It was found that $8 \mathrm{krad}$ of neon-ion Bragg peak radiation suppressed the IHR 7 days postirradiation. IHR oscillograms in Figure 4 show the IHR control (C) pattern before irradiation. The IHR are measured 4 hours after irradiation and then daily. Figure 5 presents a graph of the time course for the decline of the amplitude of the IHR and its ultimate loss at the seventh day after irradiation.

In these pilot experiments it was observed that the Bragg peak dose was inversely related

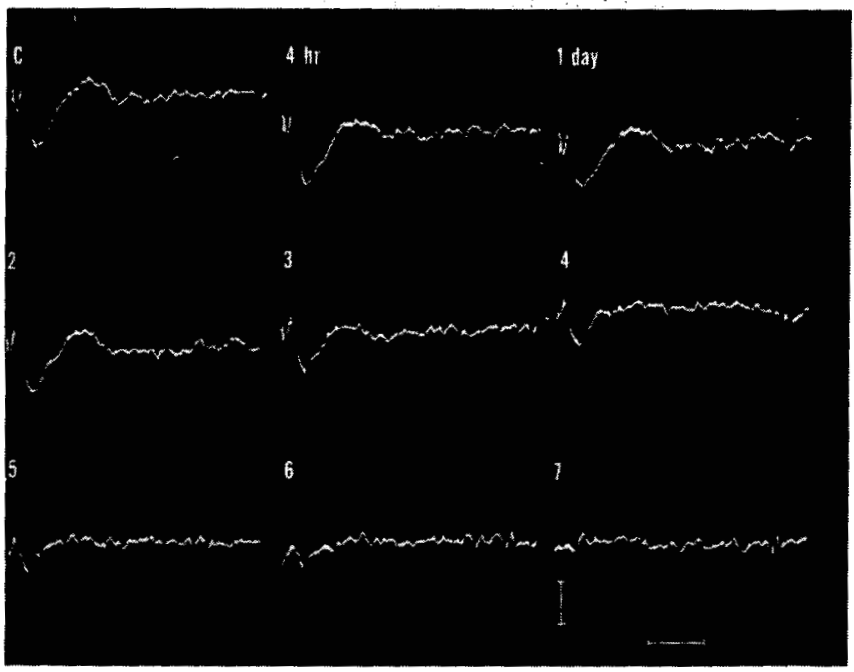

Figure 4. Oscillograms of IHR in the control state $(C=$ prior to irradiation) and at intervals after absorbing $8 \mathrm{krad}$ neon-ion Bragg peak radiation. IHR are from alert, drug-free cats. Calibration bars are $200 \mu \mathrm{V}$ and $50 \mathrm{msec}$ (XBB 774-4272)

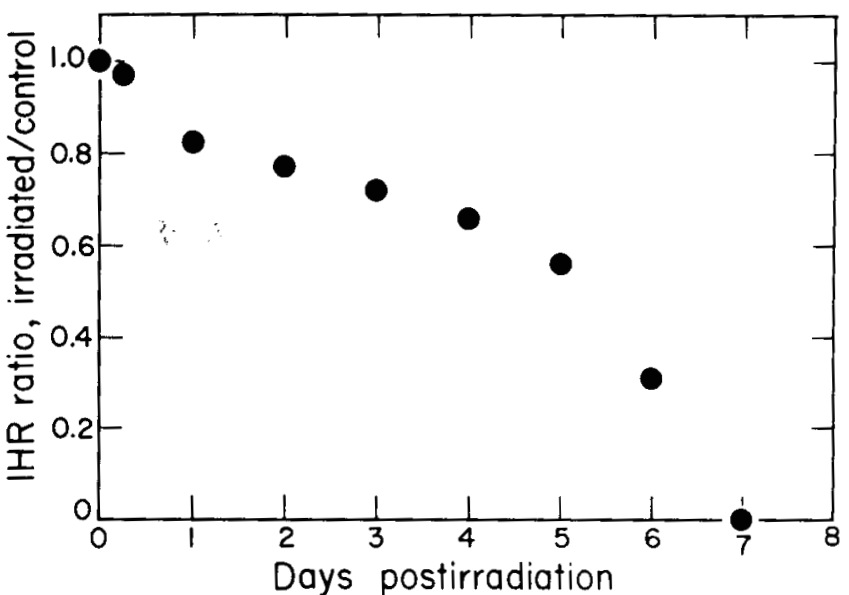

Figure 5. Time course for the attenuation and ultimate loss of the IHR after $8 \mathrm{krad}$ of $\mathrm{Bragg}$ peak neon-ion radiation. (XBL 775-923)

to the postirradiation time for the loss of IHR. Neon dose as a function of postirradiation time for the disappearance of IHR is given in Figure 6 for five cats. We found that $6 \mathrm{krad}$ of neon ions did not cause a loss of IHR even after 30 days postirradiation, although the amplitude of the

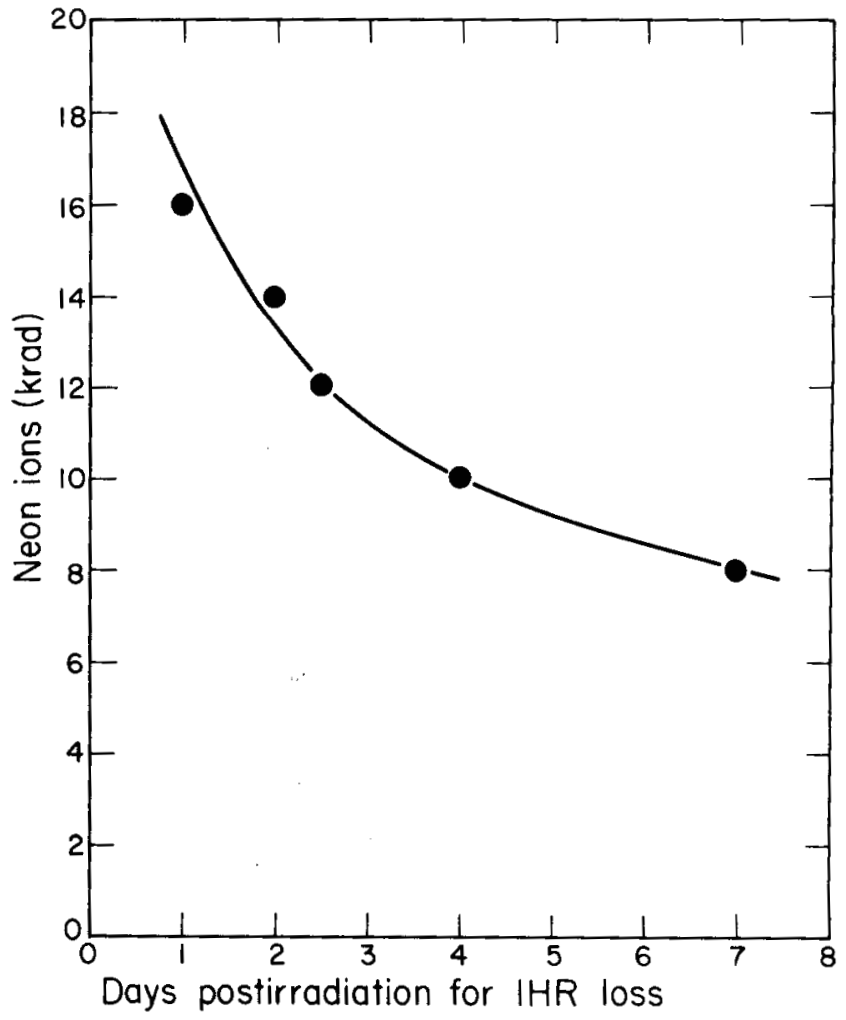

Figure 6. Dose-response relation for the suppression of the IHR. (XBL 775-922) 
IHR was attentuated by $20 \%$ It may be tentatively concluded that the minimum neon-ion dose for the complete suppression of IHR falls between 6 and $8 \mathrm{krad}$.

EEG recordings of constrained, drug-free cats were taken daily after irradiations for two weeks and then EEG sessions were scheduled twice a week for ten weeks. Epileptiform EEG patterns that had spread from the left occipital cortex

(alumina treated) to involve the right occipital cortex prior to the administration of Bragg peak radiation faded from the EEG records after 8 or more krads at postirradiation times comparable to the IHR loss (Fig. 6).

In early studies two cats were exposed to oxygen-ion beams (160) obtained from the Bevalac with an exit energy of $200 \mathrm{MeV} /$ nucleon. The range of these oxygen ions in water was $7.8 \mathrm{~cm}$ and their LET in the plateau region was approximately $23 \mathrm{keV} / \mu \mathrm{m}$ of water. A variable water column was used to place a circular beam (1.77 cm diameter) of Bragg peak oxygen ions $3 \mathrm{~mm}$ below the cortical surface. The intracranial absorbed doses for these two cats were only 425 and 600 rad because of beam failures. No electrophysiological effects, as determined by EEG measurements, were detected by either of these oxygen-ion irradiations.

\section{DISCUSSION}

The cerebral cortex of the cat is a $3-\mathrm{mm}$ deep covering of gray matter containing layers of nerve cells (about 50 to 100 cells thick), glial cells, and blood vessels. Nerve cells are vertically arranged in columns. Each column consists of layers which vary in cell density, in the size of cells they contain, and sometimes in the degree of "columnation." Six cortical layers are generally recognized. Szentagothai (1969) suggested that information processing occurs in successively arranged echelons aiong these vertical columns, with each echelon functioning to extract some meaningful bit of information. Accordingly, EEG activity depends on vertically oriented columnal structure in which excitation occurs predominatly near the top of the column (layer I), which contains mostly fibers, both axonal and dendritic. The cortex sends out and receives associational or commissural fibers from other parts of the cortex. These originate in layer III and $V$ as axons of pyramidal cells. Normally columns of neurons maintain a modulated balance of excitatory and inhibitory influences, but in an epileptogenic area a column of neurons receives excessive, frequent, and almost simultaneous synaptic influences. The common dysfunction at the cellular level in epilepsy is an abnormal, excessive, synchronous discharge of neuronal columns. The feasibility of interrupting transmission of these epileptic signals with Bragg peak, heavy-ion radiation is supported by the findings in this study. Experiments with cats demonstrate that transmission of bioelectrical activity (IHR) in the vertical, columnar direc- tion of the cortex can be innibited by. localiy deposed neon-ion radiation.

The premise that heavy-ion radiation can serve as a tool to block the flow of bioelectric discharges from one part of the brain to another has support in other research. Gaffey and Montoya (1973) demonstrated the efficiency of helium-ion beams in inhibiting interhemispheric transfer after irradiation of the corpus. callosum in the cat. It was found that with low doses corpus callosum transmission could be blocked and no obvious vascular reactions developed. The 1 ack of a vascular response to thin, heavy-ion beams is also supported by others. Malis et al. (1957) noted that laminar lesions in the cat's cerebral cortex could be created by Bragg peak radiation. The striking feature was that a thin band of cerebral cortex devoid of nerve cells was the only sign of radiation. Exquisite lesions were produced, and yet the neurons and the complex vascularity adjacent to these discrete lesions remained intact (Mal is et al., 1960; Malis et a1., 1962; Rose et a 1., 1960; Kruger and Mal is, 1964). As a consequence Van Dyke and Janssen (1963) made a study comparing lesions produced with Bragg peak radiation to those made by a sharp knife, and concluded that Bragg peak radiation produced superior cortical lesions. Like others, they demonstrated an important finding: radiolesions below certain doses were free of circulatory damage for narrow radiation fields. The findings of Janssen et a1. (1962) and Brightman (1959) on the histopathogenes is of brain lesions with Bragg peak radiation agree with this observation. Janssen et al (1962) considered Bragg peak radiation an important tool for the production of lesions in the future because, as the treated tissue disappeared, healing occurred without gross or microscopic evidence of scar formation.

There is intrinsic interest in the application of heavy-ion Bragg peak radiation to the epileptic brain since it is possible that a radiobiological approach may provide information about the nature of epilepsy. The specific aim of this research is to dissociate an epileptic focus from conducting disruptive signals in both the vertical and lateral direction in the cortex while sparing vascular circulation and normal brain tissue. Experiments have yet to be undertaken to see if the lateral spread of epileptic signals can be blocked by narrow-field, heavy-ion radiation. Histopathological examination of brain tissues absorbing Bragg peak heavy-ion radiation is required to determine if normal brain cells are altered by the plateau (entrance dose to the brain) radiation. Also, the task still remains of determining the presence or absence of a vascular involvement as a consequence of the use of heavy ions in intracranial irradiations to correct epilepsy.

Will intracranially deposited Bragg peak radiation be used to restrict the spreading action of epileptic and Parkinsonian signals in man's brain? The relative merits of this approach are still to be determined before such an application can be considered. 


\section{ACKNOWLEDGEMENT}

This work was supported by the National Aeronautics and Space Administration. It is a pleasure to thank Annie E. Gaffey for her assistance with this paper.

\section{REFERENCES}

Beckman, F., C. H. Bonney, D. M. Hunter. 1974. Funduscopic alterations in the Rhesus monkey induced by exposure to heavy ions $(0+8)$ $250 \mathrm{MeV} /$ nucleon. Aerosp. Med. 45, 154-160.

Brightman, M. W. 1959. Early effects of intensive $X$-ray irradiation of the diencephalon in the rat. Exp. Neurol. 1, 97-116.

Fetz, E. E., and A. R. Wiler. 1973. Operantly conditioned firing patterns of epileptic neurons in monkey motor cortex. Exp. Neurol. 40, 586-607.

Gaffey, C. T. 1966. Blockage of pupillodilation with cyclotron accelerated alpha particles. Acta Radiol. Ther. Phys. Biol. 5, 105-117.

Gaffey, C. T., and V. J. Montoya. 1973. Split brain cats prepared by radiosurgery. Int. J. Radiat. Biol. 24, 229-242.

Ghiorso, A., H. Grunder, W. Hartsough, G. Lambertson, $F$. Lofgren, K. Lou, R. Ma in, R. Mobley, R. Morgado, R. Salsig, and F. Selph. 1973. The Bevalac--An Economical Facility for Very Energetic Heavy Particle Research. Lawrence Berkeley Laboratory Report LBL-1386.

Huott, A., D. Madison and E. Niedermeyer. 1974. Occipital epilepsy. Eur. Neurol. 11, $325-339$.

Janssen, P., J. Klatzo, J. Miguel, T. Brustad, A. Behar, J. Lyman, J. Henry, C. A. Tobias, and $W$. Haymaker. 1962. Pathologic changes in the brain from exposure to alpha particles from a 60-inch cyclotron. Response of the Nervous System to Ionizing Radiation (T.J. Haley and R. S. Snider, eds.) pp. 383-409. Academic Press, New York.

Kopeloff, L. M. 1960. Experimental epilepsy in the mouse. Proc. Soc. Exp. Bio1. Med. 104, 500-503.

Kopel off, L. M., C. J. Chusid, and N. Kopeloff. 1955. Epilepsy in Macca mulatta after cortical or intracerebral alumina. Arch. Neurol. Psychiatry 74, 523-526.

Kruger, L., and J. L. Malis. 1964. Distribution of afferent and efferent fibers and cerebral cortex of the rabbit revealed by laminar lesions produced by neavy ionizing particles. Exp. Neurol. 10, 509-524.

Lyman, J. T., J. Howard, G. P. We lch, W. Schimmerling, and C. A. Tobias. 1971. Experimental set-up and physical measurements of a heavy-ion beam for initial irradiation at the Bevatron. Initial Radiobiological Experiments with Accelerated Nitrogen Ions at the Bevatron. Lawrence BerkeTey Laboratory Report LBL-529, pp. 1-19.

Malis, L. I., R. Loevinger, L. Kruger and J. E. Rose. 1957. Production of laminar lesions in the cerebral cortex by heavy ionizing particles. Science $126,302$.

Malis, L. I., C. P. Baker, L. Kruger and J. E. Rose. 1960. Effect of heavy ionizing, monoenergetic particles on the cerebral cortex. I. Production of laminar lesions and dosimetric considerations. J. Comp. Neurol. 115, 212-242.

Malis, L. I., J. E. Rose, L. Kruger, and C. P. Baker. 1962. Production of laminar lesions in the cerebral cortex by deuteron irradiations. Response of the Nervous System to Ionizing Radiation (T. J. Haley and R. S. Snider, eds.) pp. 359-368. Academic Press, New York.

Rose, J. E., L. Malis, L. Kruger, and C. P. Baker. 1960. Effects of heavy ionizing monoenergetic particles on the cerebral cortex. II. Histological appearances of laminar lesions and growth of nerve fibers after laminar destruction. J. Comp. Neurol. 115, 243-295.

Szentagothai, J. 1969. Architecture of the cerebral cortex. Basic Mechanism of the Epilepsies (H. H. Jasper, A. Ward, Jr. and A. Pope, eds.). Little, Brown, Boston.

Tobias, C. A., J. T. Lyman, A. Chatterjee, J. Howard, J. M. Sperinde, and G. P. Welch. 1971. Radiological physical characteristics of the extracted heavy ion beams of the Bevatron. Science 174, 1131-1134.

Van Dyke, D., and P. Janssen. 1963. Removal of cerebral tissue with minimal scar formation in monkeys. J. Neurosurg. 20, 289-298. 


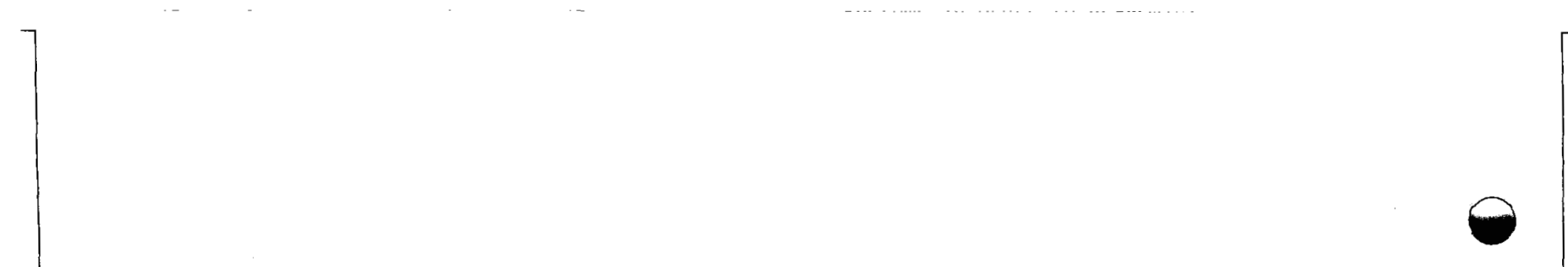

$\vartheta$

$\vartheta$

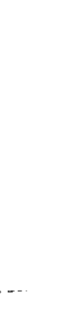




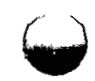




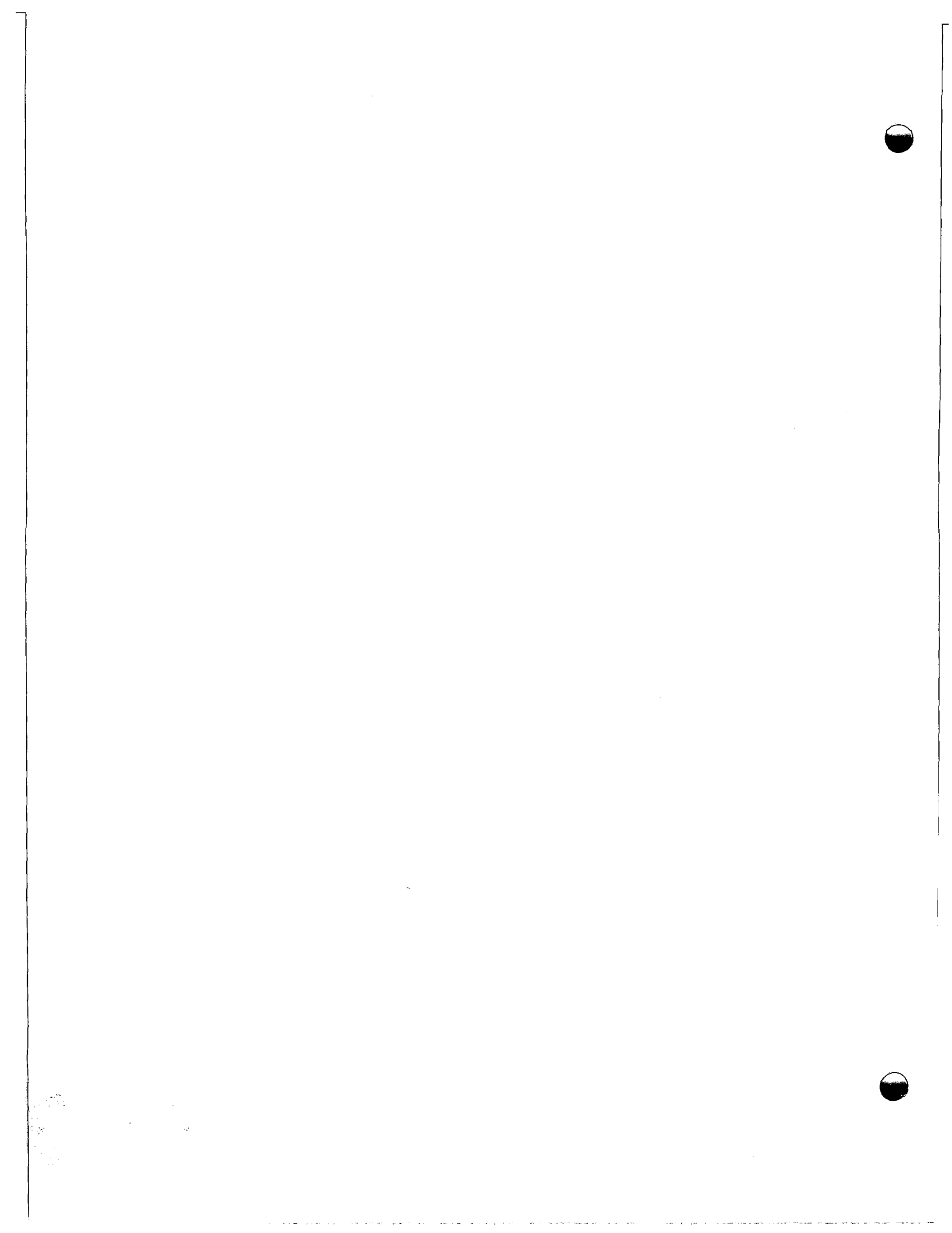




\title{
A FUTURE DEDICATED MEDICAL ION ACCELERATOR
}

\author{
R. A. Gough, J. R. Alonso, T. Elioff, and H. Grunder
}

Current biomedical studies and clinical trials with charged particles rely on accelerators that were designed for physics research. Extending the performance of these machines in terms of beam energies, intensities, and flexibility is often stressed at the expense of high reliability and ease of operation, which are crucial for successful biomedical applications.

In contrast, the design and construction of particle accelerators designed specifically to meet medical requirements would result in costeffective and highly reliable accelerators suitable for routine, hospital-based, clinical service with modest operations and maintenance crews. In fact, as patient trials become fully randomized over more treatment sites, the substantially increased patient loads will make biomedical usage increasingly incompatible with physics programs on existing machines. The construction of dedicated medical ion accelerators such as the facility now being planned in Canada (MARIA, 1980) will be the only feasible solution to permit the large scale evaluation of charged particle radiotherapy. The experience of the $L B L$ 184-Inch Synchrocyclotron, which is dedicated for medical use, indicates that extremely nigh facility availability can be readily achieved when operational standards are optimized for reliability.

Two factors combine to make this a favorable time to pursue the design of a complete heavy-ion therapy facility. First, after considerable experience with 225 MeV/amu alpha particles at the LBL 184-inch Synchrocyclotron, and carbon, nitrogen, oxygen, neon, silicon and argon ions from 400 to $670 \mathrm{MeV} / \mathrm{amu}$ at the Bevalac (LBL, 1977a; Alonso et a1., 1979), radiotherapists are * converging on carbon, neon, and silicon as the particles of choice for the majority of heavy-ion radiotherapy. Second, accelerator designers can build upon the large body of "relevant experience gained at $L B L$, including the extensive survey of appropriate accelerator structures for various particle beams that was completed in 1977 (LBL, $1977 b)$. Since that study there have been substantial technological developments at $L B L$ and elsewtiere in the accelerator-related areas of ion sources, injectors, and computer control systems, as well as the biomedical techniques of beam delivery and shaping, monitoring, and verification both at the LBL 184-inch Synchrocyclotron and the Bevalac.

Therefore, we are in the process of subinitting to the National Cancer Institute a threeyear design proposal (for fiscal years 1981-1983) to perform the requisite research and development and to generate plans and realistic cost estimates for a dedicated heavy-ion medical accelerator suitable for a hospital-based environment.
The specific objectives of this proposal are:

1. optimize the facility design for the desired treatment characteristics, operational reliabililty and cost-effectiveness, and produce preliminary engineering design drawings and cost projections;

2. prototype key components of the accelerator as required;

3. advance beam delivery techniques toward systems that will more fully realize the advantages of heavy ions for radiotherapy.

\section{PRESENT STATUS}

It was the conclusion of the earlier design study that established technology should be used in the conception of a medical accelerator unless novel techniques were judged essential to achieve the design goals or made possible substantial cost savings. This conclusion is valid if a facility is to be created with the highest possible reliability and operational simplicity. Nonetneless, we consider it imperative to evaluate the applicability of new advances in accelerator technology to make the best selection of available techniques in accomplishing the design and operational goals.

If a facility optimized for radiotherapy with beams of silicon and lighter ions were to be built at the conclusion of the earlier design study, the choices for accelerator components would have been:

1. PIG (Penning Ion Gauge) source in $750 \mathrm{kV}$ Cockcroft-Walton power supply;

2. Alvarez linac to inject fully stripped ions into a

3. conventional $700 \mathrm{MeV} / \mathrm{amu}$ strong-focusing synchrotron, of approximately $12 \mathrm{~m}$ radius, as the main accelerator.

Such a configuration could deliver heavy ions through silicon at maximum energies of 700 MeV/amu. As can be seen in Figure 1, this will ensure for silicon approximately $30 \mathrm{~cm}$ range in tissue,- which is sufficient to treat most human tumors. Lighter ions would be available with much longer ranges, which makes them suitable for additional applications such as radiography and associated radiobiological programs, as well as radiotherapy. Since an actual facility will not be constructed for several years, it is advantageous to investigate the potential application of several new technological developments. Among the most appropriate are advanced ion sources designed to produce large beam currents of high charge state ions such as the EBIS source (Arianer et al., 1979) under study at LBL and in other countries, radiofrequency quadrupole (RFQ) (Stokes et a1., 1980; Hamm et al., 1980), 
RANGE-ENERGY CURVES

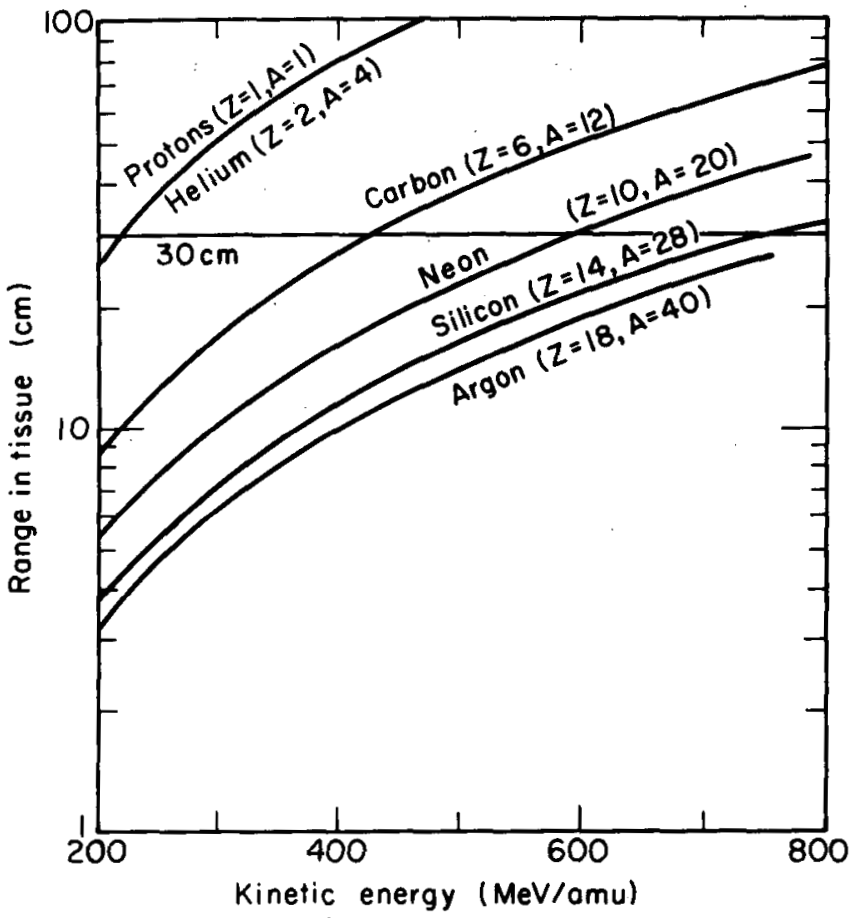

Figure 1. Range-energy curves for charged particle beams showing the kinetic energy as a function of range in tissue. (XBL 809-3667)

and other compact linac structures (Halbach, 1980), all of which might significantly condense and simplify the preinjector and injector schemes, and superconducting magnet technology, which could possibly reduce the size and operating costs of the facility. Evaluation of performance options such as pulse-to-pulse energy variations will be made. Such options might enhance the efficient utilization of the accelerator as long as they are not achieved at the expense of reliability. Considerable flexibility would be possible, depending on the priorities of the user community. Control systems at existing LBL accelerator facilities demonstrate that highly sophisticated beam sharing, beam switching, and multiuser capabilities are presentiy feasible.

One of the most promising recent developments in accelerator technology, demonstrated in a proof-of-principle test (Hamm et al., 1980) at Los Alamos Scientific Laboratory earlier this year, is the radiofrequency quadrupole linac. This structure may provide an elegant method of injection into a medical synchrotron in a hospital-based environment. Among its attractive features are a greatly simplified and smaller preinjector, plus an injector that would consume less electrical power and be much easier to operate than a conventional drift-tube linac.

Some calculations have recentily been performed with the LoS Alamos group (R. H. Stokes and T. P. Wangler, private communication) to determine the specifications and performance of an RFQ linac for ions of charge-to-mass ratio $=$ 0.25 (e.g., $\left.20 \mathrm{Ne}^{+5}\right)$. The results obtained were very encouraging. Further studies of the structures, however, will be necessary before they can confidently be incorporated into a medical accelerator.

The efforts in beam delivery systems will concentrate on advancing beyond the presently used scattering foil-occluding ring technique for producing large and uniform exposure fields. Although this system has been employed for several years at the 184-inch Synchrocyclotron and the Bevalac, and has proved to be effective and reliable, there are certain possibilities for improvement. The lead scattering foils cause a substantial loss of beam energy, up to $20 \%$ for the neon beams, thus higher accelerator energies are required to produce a given depth of penetration in the patient. Also, beam utilization is quite low even though the beam intensities extracted from the Bevalac are high enough to overcome the high beam loss and permit adequate dose rates. Furthermore, field sizes are somewhat smaller than desired, and limitations are placed on treatment planning by the inability to vary either the dose delivered or the extent of Bragg peak spreading across the field.

Thus, to make best use of the inherent properties of heavy ions, and to minimize beam intensity and energy requirements, thereby keeping accelerator costs as low as possible, it is desirable to explore more advanced ideas in beam delivery systems. We propose to direct these efforts toward a scanning system concept: two magnets sweeping the beam across horizontal and vertical axes, with possible added dimensions of range modulation and beam spot size variation.

\section{SPECIFIC AIMS OF THE PROPOSAL}

The purpose of this project is to prepare a preliminary engineering and cost estimate for a dedicated medical ion accelerator. In developing the overall facility design, special attention will be paid to the control system and the beam delivery system. Research and development, and prototyping of key components will be performed where necessary. When these tasks are completed, it will be possible to prepare a comprehensive and reliable projection of both construction and operating costs.

\section{Medical Accelerator Design}

\section{Particle Beam Requirements}

The basic design of the medical ion accelerator proceeds from a single set of quantities describing the desired particle beam: particle choice, charge state, kinetic energy, maximum particle flux, transverse emittance, momentum and energy spread, and duty factor. Some of these quantities are dictated by the medical requirements, while others are determined by costeffective accelerator performance. 
Radiotherapists and radiobiologists are focusing on carbon, neon, and silicon beams as presenting the best combination of physical characteristics and biological effectiveness. Therefore, the appropriate beam parameters to satisfy user requirements can be specified as follows.

Probable heaviest particle ${ }_{14}^{28} \mathrm{Si}$

Maximum kinetic energy $\quad 300-900 \mathrm{Mev} / \mathrm{amu}$

Maximum particle flux 1 to $5 \times 10^{9}$ particles $/ \mathrm{sec}$

Transverse emittance

Momentum and energy spread $4 \times 10^{-3}$

Repetition rate 1 to $2 \mathrm{~Hz}$

Duty factor $25 \%$ to $50 \%$

These parameters provide an intense beam of sufficient range to irradiate even sizeable tumors in treatment times of two minutes or less. The same accelerator configuration can also provide carbon or other lighter ions to readily satisfy all potential radiographic applications. Furthermore, radioactive beams (Alonso et al., 1979) can be generated from the primary ion beams in intensities adequate for many other applications, including treatment verification and nuclear medicine. The final choice of heaviest particle and maximum energy will be a specific objective of the proposed effort.

The relationship between these beam parameters and the accelerator design $c$ an be briefly described. First, particle species and energy are determined by radiobiological studies of the physical and biological dose characteristics and the necessary particle range to reach the tumor sites to be treated. A range of $30 \mathrm{~cm}$ is adequate to reach most deep-seated tumors. Average particle flux is derived from considerations of treatment volume and dose rate. For a conventional beam delivery scheme, an arbitrary standard of $600 \mathrm{rad}-1$ iters/min has been used for small treatment volumes, with a lower dose rate of $200 \mathrm{rad} / \mathrm{min}$ acceptable for a large treatment volume of 13.5 liters of $15 \mathrm{~cm}$ thickness. The required particle flux depends on the depth of the treatment volume, with increased flux required for treatment volumes that are wide and flat rather than deep. Beam and energy losses in the transport and beam handing systems must also be accounted for in determining the intensity and energy of beam to be extracted from the accelerator.

Accelerator beams consist of an ensemble of particles moving in the same general direction but with small internal deviations from the average position and direction. The magnitude of these deviations is related to a quantity: known as the beam emittance. Although medical applications do not require emittance values: smaller than those routinely provided by modern accelerators, the size, cost, and power demand: of beam transport elements is affected by these considerations. Consequently, we expect emittance values of 1 to $2 \times 10^{-5}$ m-radian. Similarly, real beams possess a distribution of nomenta (or energies). For all charged particles except electrons, this corresponds to slightly different stopping points in matter due to the energy-range correlation. A sensible upper limit for this range variation is to require that it not exceed the spread in range due to range straggling that occurs even in an initially monoenerget ic beam. For neón ions, the resulting tolerable energy spread is approximately $4 \times 10^{-3}$. Again, beam handling and transport is greatiy facilitated by keeping the spread even smaller than this value.

Finally, repetition rate and duty factor (the beam on-off periods) specify the macroscopic time scales of the beam. The accelerating (radiofrequency) system sets the microscopic time scale in which the beam appears bunched or modulated. For radiotherapy requirements in the areas of beam monitoring, dosimetry, and beam delivery (especially beam scanning or dynamic isocentric beam delivery), generous duty factors are highly desirable.

\section{Facility Layout}

The detailed design of the overall facility layout must be worked out in consideration of local conditions and site constraints as well as staff preferences. However, it will be important to maximize accelerator utilization by using several parallel treatment rooms, so that the beam may be switched from one room to another during the time needed for patient set-up (approximately $20 \mathrm{~min}$ ). It is entirely possible for a single synchrotron to serve up to eight patient rooms. Assuming five eight-hour treatment days per week, this facility could, as an upper limit, treat nearly 200 patients per day. A major task of this proposal will be to investigate optimal treatment room layouts given different site and economic considerations. With this task and the conceptual accelerator design completed, it will be possible to specify operational modes of the facility and to produce analyses of operating costs.

\section{Engineering for Reliability}

High reliabilty, and the related concept of operational ease and simplicity, are critical for a medical facility from the time the accelerator is first used. The experience at the 184-inch Synchrocyclotron has been an excellent example of high accelerator availability. That machine, which now operates solely for medical studies and patient therapy, consistent ly averages $98+\%$ availability with exceedingly modest operations and maintenance staff. We can also benefit from the experience of industry by using the established methods for qual ity as surance and control. These techniques permit rapid improvements in comporient development by subjecting design procedures and shop practices to critical assessment at:all stages. Since attainment of high reliability means achieving low failure rates and high availability, it is essential to eliminate failure modes requiring long repair times. For a medical accelerator, this means that patient scheduling can proceed in a routine and confident manner. 
Among the important concepts are to construct computer-based reliability models and establish failure rates of known components in order to analyze proposed system designs; to minimize the number of new, untested components; and to evaluate all designs with respect to maintainability and replaceability. Furthermore, the concept of reliabilty should extend over the complete working life of the system. Factors to consider are automatic or built-in diagnostic equipment and systematic test procedures, simplicity in design with fewest different parts, easy accessibility of parts and replacements on site, and as much modular construction as possible.

\section{Prototyping Components}

As mentioned earlier, based on present technology a conventional, strong-focusing synchrotron, injected by a linac (or a cyclotron if neutron and radioisotope production are considered important) is the choice as the most efficient and economical source of ion beams of carbon or heavier. Several components may warrant prototyping to assure reliable cost estimate and project schedules. The priorities for much of this prototyping are yet to be deterinined.

One area where developmental effort is indicated is beam delivery. A beam scanning system appears to be the best technique for biomedical applications of heavy ions. The range of scanning system concepts is wide: from freerunning sweeps of fairly large diameter beams over a rectangular field (using the present ridge filter, water column, and patient collimators for field definition) to a highly sophisticated pixel-by-pixel dose delivery with beams of a few $\mathrm{mm}$ in diameter (Kanai et al., 1980; Leemann et al., 1977). Greatest flexibility is obtained with the latter concept, but its implementation requires substantial technological development of control systems, dosimetry, verification techniques, and the resolution of biological questions about the very high instantaneous dose rates delivered to each pixel.

The proposed plan is to assemble a twodimensional scanning system, largely from existing components, and to install it in a beam line in the Bevalac Biomedical area. It will be used as a research tool to develop and test control systems, to verify dosimetry and beam instrumentation, and to perform radiobiology experiments with the scanned beams. It is anticipated that even in its simplest form, this scanning system will offer substantial, advantages over the occluding ring technique such as larger field sizes, greater uniformity, and higher dose rates. Furthermore, implementation of this system is expected to be straightforward; all the basic components are already in existence and have been shown to easily meet design specifications for the first-order scanning system.

Ultimately, it is anticipated that this beam line may be used for actual patient treatments, and thus may serve as a true prototype for the medical accelerator beam delivery system.

\section{CONCLUSION}

The final phase and ultimate goal of the research and development project will be the development of detailed and reliable cost estimates for the total facility. To accomplish this task, it will be necessary to identify and describe in considerable detail, every significant component of the facility. At the same time, projections of operational costs and required staff will be prepared so that a cost per patient treatment may be generated for comparison purposes.

This preliminary engineering design effort is expected to begin in summer 1981, although considerable preparatory effort has already been expended. It is expected that a proposal to construct such a facility will be submitted in the third year of the design effort. At the completion of the tasks set forth above, the radiotherapy community will be in a confident position to pursue the construction of a fully developed and optimized dedicated medical ion accelerator.

\section{ACKNOWLEDGEMENT}

We wish to express our appreciation to the Los Alamos group for their calculations of RFQ structures relevant to our application.

\section{REFERENCES}

Alonso, J., T. Criswell, and J. Howard. 1979. The Bevalac radiotherapy facility. IEEE Trans. Nucl. Sci. NS-26, 3074.

Alonso, 3., A. Chatterjee, and C. A. Tobias. 1979. High purity radioactive beams at the Bevalac. IEEE Trans. Nucl. Sci NS-26, 3003.

Arianer, J., A. Cabrespine, and C. Goldstein. 1979. CRYEBIS: A multipurpose EBIS for the synchrotron saturne II. IEEE Trans. Nucl. Sci. NS-26, 3713 .

Halbach, K. 1980. Design of permanent multipole magnets with oriented rare earth cobalt material. Nucl. Instr. Methods 169, 1.

Hamm, R.W., K. R. Crandall, C.W. Fuller, L. D. Hansborougn, R. A. Jameson, E. A. Knapp, M. D. Machalek, J. M. Potter, G. W. Rodenz, R. H Stokes, J. E. Stovall, D. A. Swenson, T. P. Wangler, and S. W. Willitiams. 1980. The RF quadrupole linac: A new low-energy accelerator. Proceedings, International Conference on Low Energy Ion Beams II. University of Bath, England.

Kanai, T., K. Kawachi, Y. Kumamoto, H. Ogawa, T. Yamada, and H. Matsuzawa. 1980. Spot 
scanning system for proton radiotherapy. Med. Phys. $7,365$.

LBL (Lawrence Berkeley Laboratory). 1977a. Biological and Medical Research with Accelerated Heavy Ions at the Bevalac, 1974-1977. Lawrence Berkeley Laboratory Report LBL-5610.

LBL (Lawrence Berkeley Laboratory). 1977b. Dedicated Medical Ion Accelerator Design Study, Final Report. Lawrence Berkeley Laboratory Report LBL-7230.

Leemann, C., J. Alonso, H. Grunder, E. Hoyer, G. Kalnins, D. Rondeau, J. Staples, and F. voelker. 1977. A three-dimensional beam scanning system for particle radiation therapy. IEEE Trans. NuCl. Sci NS-24, 1052.
MARIA Workshops. 1980. I. Medical Radionuclide Production (17-18 Sept.); II. Optimal

Parameters of Relativistic Heavy-Ion Beams for Radiotherapy Research; III. Accelerator Systems for Relativistic Heavy Ions in Medical and Scientific Research. University of Alberta, Edmonton, Alberta, Canada.

Stokes, R. H., K. Cranda 11, R. Hamm, F. Humphry, R. Jameson, E. Knapp, J. Potter, G. Rodenz, J. Stovall, D. Swenson, and T. Wrangler. 1980. The radiofrequency quadrupole: General properties and specific applications. Proceedings, XIth International Conference on High-Energy Accelerators. Report LA-UR-80-1855. CERN, Geneva. 
$\bullet$

$\bullet$ 


\section{STAFF LIST}

LAWRENCE BERKELEY LABORATORY STAFF MEMBERS ASSOCIATEO WITH BIOMEOICAL HEAVY-ION RESEARCH

Edward L. Alpen

Director, Donner Laboratory

Associate Director, Biology and Medicine Division

Hermann A. Grunder

Associate Director, Accelerator and Fusion Research Division

Frederick E. Abrams

Kuldip K. Ahluwalia

E. John Ainsworth

Hilda M. Alexander

Jose R. Alonso

Arlette S. Atwater

Jacqueline Barnes

Eleanor Batho

Eleanor A. Blakely

James L. Born

Thomas F. Budinger

Betsy C. Carr

Joseph R. Castro

Polly Y. Chang

Aloke Chatterjee

George T.Y. Chen

William T. Chu

Coleen Caudill Cogorno

Gerald M. Connell

Karen E. Crabtree

Laurie M. Craise

Kenneth C. Crebbin

Tom Criswell

Stanley B. Curtis

Randy J. DeGuzman

Tom Elioff

Jacob I. Fabrikant

Raymond S. Farinato

David Feinberg
Patricia A. Fobair

Robert J. Force

Myrtle L. Foster

Michael Freeling

Cornelius T. Gaffey

Patricia A. Garbutt

Dalia Geller

Catherine R. Gisser

Richard Gough

Linda D. Harrison

Gloria J. Hathaway

Virginia $C$. Havens

Lilian E. Hawkins

Thomas L. Hayes

Lynn R. Hlatky

William R. Holley

Jerry Howard

Steven Hurst

James Judnick

Lea] L. Kanstein

Ben Kapel

William Kennedy

Lennetta G. Lai

Ruth-Mary Larimer

Panna Lee

Christopher $W$. Leeman

John A. Linfoot

Jorge Llacer
Lori Lomme 1

Fred H. Lothrop

Dave Love

Judith A. Lowrie

Joan E. Luna

John T. Lyman

I an S. Madfes

John L. Magee

Lynn J. Mahlmann

Michael J. Malachowski

Peter Mart in

Mara M. McDonald

Brian R. Moyer

Ileen Mulry

Jeannette S. Nakagawa

Alan C. Nelson

Frank Q.H. Ngo

Oksana Oleszko

Shannon S. Parr

Charles C. Pattie

Charles R. Pascale

Theresa $C$. Peters

Theodore L. Phillips

Mary C. Pirruccello

Samuel Pitluck

Patricia Powers-Risius

John C. Prioleau

Jeanne M. Quivey
Michael S. Raybourn

Todd Richards

Adrian Rodriquez

Ruth J. Roots

Pepi Ross-Riveros

Bohdan Roman

Ahren 3. Sadoff

William B. Saunders

walter Schimmerling

Andy Shin

Reba Siero

R. P. Singh

Karen C. Smith

Lore S. Stein

T. S. Subramanian

Susan D. Tenforde

Tom S. Tenforde

Marsh M. Tekawa

Cornelius A. Tobias

Doris S. Tse

Grace V. Walpole

Robert E. Walton

David D. Wei

Graeme P. Welch

Kay H. Woodruff

Tracy C.H. Yang

Michael J. Yezzi

George Young 


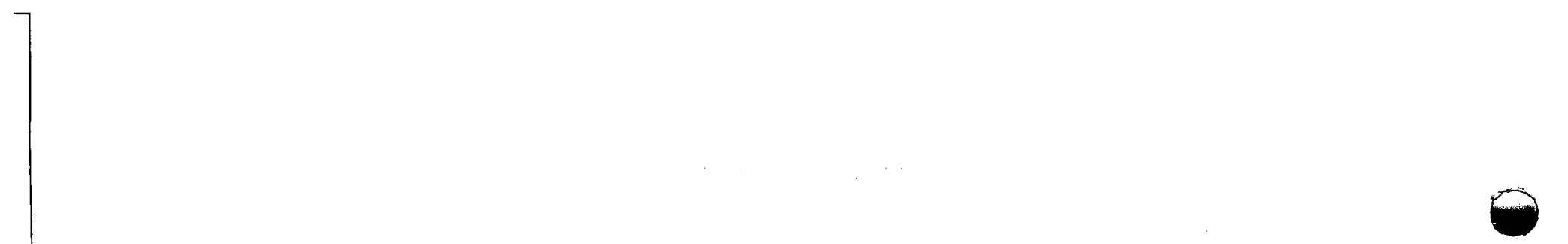

$\theta$

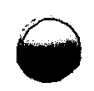




\title{
PROGRAM ADVISORY COMMITTEE
}

\author{
CURRENT BEVATRON/BEVALAC PROGRAM
}

ADVISORY COMMITTEE: BIOMEDICAL PANEL

Herman D. Suit, Chairman

Massachusetts General Hospital

E. John Ainsworth, Executive Secretary

Lawrence Berkeley Laboratory

Edward L. Alpen

Lawrence Berkeley Laboratory

R. J. Michael Fry

Oak Ridge National Laboratory

William A. Glass

Battelle Pacific Northwest Laboratory

Robert F. Kallman

Stanford University Medical Center

Gordon Whitmore

Ontario Cancer Institute 